\title{
DESCRIPTIONS OF WHOI SEDIMENT CORES, VOLUME 5
}

\author{
Prepared by \\ the Staff of the \\ Sea Floor Samples Laboratory \\ Edited by \\ D. A. Johnson and A. H. Driscoll \\ WOODS HOLE OCEANOGRAPHIC INSTITUTION \\ Woods Hole, Massachusetts 02543
}

April 1977

\begin{abstract}
TECHNICAL REPORT
Prepared for the Office of Naval Research under Contract N00014-74-C0262, NR 083-004; and for the National Science Foundation under Grant OCE76-81488.

Reproduction in whole or in part is permitted for any purpose of the United States Government. In citing this manuscript in a bibliography, the reference should be followed by the phrase: UNPUBLISHED MANUSCRIPT.

Approved for public release; distribution unlimited.
\end{abstract}

Approved for Distribution

Elizabeth T. Bunce, Acting Chairman

Department of Geology \& Geophysics 
TABLE OF CONTENTS

$\underline{\text { Page }}$

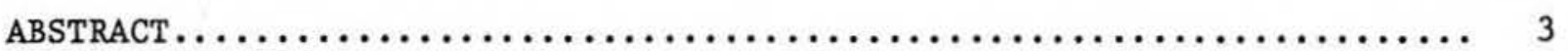

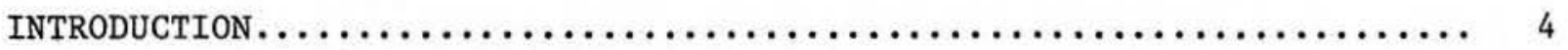

A. Scope and Format of this Report................... 4

B. Summary of Description Procedures and Sediment

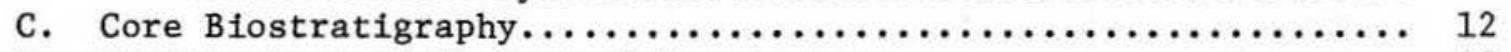

D. Digitization of Geological Sample Data.................. 15

E. Procedures for Obtaining Samples and Additional Core Data..... 18

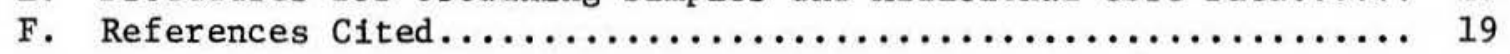

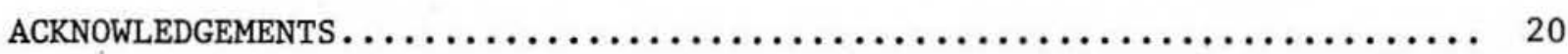

COMPUTER LISTING OF WHOI SEDIMENT CORES, ARRANGED
BY MARSDEN SQUARES $\ldots \ldots \ldots \ldots \ldots \ldots \ldots \ldots \ldots \ldots \ldots \ldots \ldots \ldots \ldots \ldots \ldots \ldots \ldots \ldots \ldots$

DESCRIPTIONS OF WHOI SEDIMENT CORES, VOLUME $5 \ldots \ldots \ldots \ldots \ldots \ldots \ldots \ldots .78$

$\mathrm{R} / \mathrm{V}$ ASTERIAS

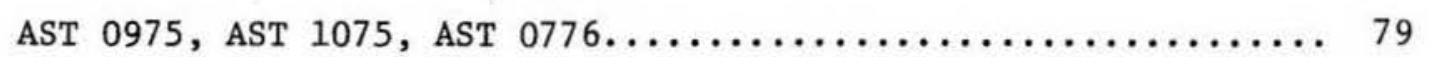

$\mathrm{R} / \mathrm{V}$ ATLANTIS II

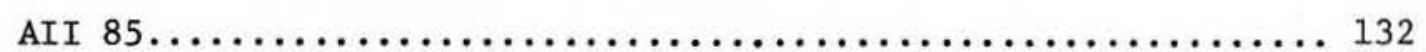

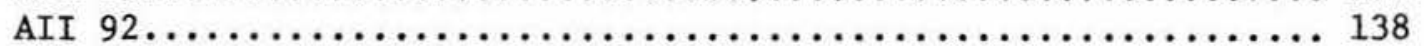

$\mathrm{R} / \mathrm{V}$ CHAIN

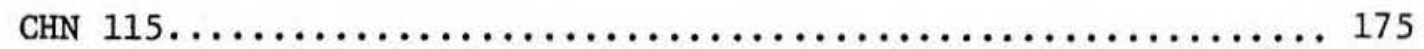

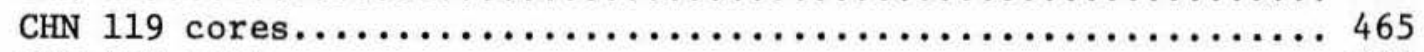

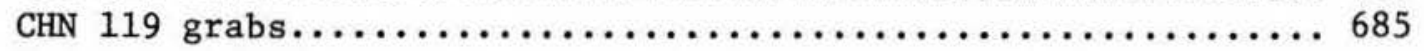

$\mathrm{R} / \mathrm{V}$ KNORR

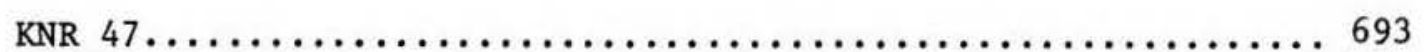

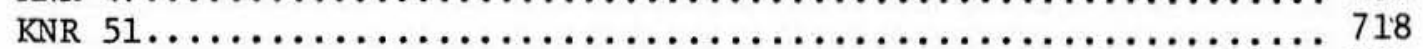

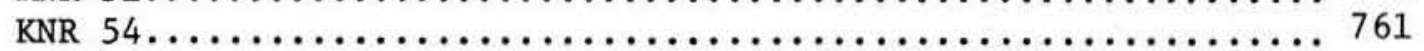

\section{LIST OF TABLES AND FIGURES}

Table 1: $\quad$ Sediment classification system $\ldots \ldots \ldots \ldots \ldots \ldots \ldots \ldots \ldots \ldots$

Table 2: Tertiary cores in WHOI core collection..............13,14

Figure 1a: Sediment classification system................. 9

Figure 1b: Sediment classification system................. 10

Figure 2: Lithologic symbols......................... 11 


\section{ABSTRACT}

This report supplements Volumes 1-4 of the core descriptions published previously in this sequence (Johnson and Driscoll, 1975). It contains visual descriptions and smear slide analyses for all cores received in the geological samples collection of the Woods Hole Oceanographic Institution between November, 1973 and November, 1976. Approximately 368 sample localities from the North Atlantic, Mediterranean, and South Atlantic are represented. Charts of ships' tracks and updated computer listings of all cores in the W.H.O.I. collection are also included. 
INTRODUCTION

\section{A. Scope and Format of this Report}

Approximately two years ago we prepared and distributed a four-volume WHOI Technical Report (Johnson and Driscoll, 1975) containing descriptive and logistical data for all cores comprising the WHOI geological samples collection as of November 1973. The present report, which represents the fifth volume in the series, includes visual core descriptions and smear slide analyses for all WHOI cores obtained between November 1973 and November 1976. Not included are cores obtained during mid-1976 on cruise AII-93 in the Indian Ocean; cores from these and other expeditions in the near future will be included in Volume 6 .

This compilation represents approximately 368 coring stations from the North Atlantic, Mediterranean, and South Atlantic. Core descriptions have been grouped according to ship and cruise number and are arranged chronologically for each cruise. A computer listing of the cores taken on each cruise, together with a chart indicating the ship's track, precedes the core descriptions.

The numbers assigned to the geological samples are followed by a letter (or letters) to indicate the method used in obtaining the samples. The letters which have been used are:

$$
\begin{aligned}
& \text { GC - Gravity core } \\
& \text { PC - Piston core } \\
& \text { PG - Pilot gravity core } \\
& \text { GPC - Giant piston core } \\
& \text { GGC - Giant gravity core } \\
& \text { BC - Box core } \\
& \text { CC - Camera (pogo) core } \\
& \text { FF - Free-fall core } \\
& \text { GR - Van Veen Grab } \\
& \text { HC - Hydro core } \\
& \text { KC - Kasten core } \\
& \text { U/W - Underway bottom sampler }
\end{aligned}
$$

A11 cores in the WHOI collection except the giant cores and Kasten cores were obtained with conventional PVC core liner. These cores have been split and stored at room temperature in sealed polystyrene D-tubes, with moisture-saturated spongy material sealed inside the D-tubes to retard the loss of moisture from the cores. The giant piston cores and giant gravity cores were obtained without the use of core liner inside the core barrels. Upon recovery these cores were extruded into half-round cylindrical shells (150-cm length) and split longitudinally into working and archive halves. Each half was then sealed in plastic sleeving.

We have retained the original station numbers and core numbers insofar as possible. A gap in the core or station numbering sequence for any given expedition indicates either that the core is not now in the WHOI geological collection, or that such a core was never obtained. In some instances, the letter designations for the type of sampling device have been amended or deleted for purposes 
of clarity and consistency within the collection. For example, a core now labeled as a "10a-PC" was originally labeled "10a"; the "PC" has been added to identify it as a piston core, and the " $a$ " has been retained to insure proper correlation with the original coring records.

We have attempted to apply uniform procedures in completing the core descriptions and microscopic analyses of smear slides. A more detailed discussion of the WHOI procedures for shipboard core handling, core archiving, core describing, and core photography is included in the following reports:

(1) Johnson, D. A. and Driscoll, A. H. (1972), "The curating of WHOI's geological collections", Woods Hole Oceanogr. Inst. Tech. Memorandum WHOI-2-72, 20 pp.

(2) Mountain, G. S. (1973), "Procedures for description of WHOI sediment cores", Woods Hole Oceanogr. Inst. Tech. Memorandum WHOI-7-73, 25 pp.

(3) Shephard, F. C. (1976), "Procedures for photographing WHOI sediment cores", unpublished MS.

\section{B. Summary of Description Procedures and Sediment Classification System}

The following is a summary of the descriptive procedures used in preparing this report; a more complete discussion is included in Volume 1 of this series (Johnson and Driscoll, 1975, pp. 7-21).

\section{Visual description}

The entire core is laid out in the correct order of sections, and proper labeling is verified. The core is then subdivided into units, which may be distinguished from each other by lithology, color, texture, or special features. Contacts between units are classified as gradational (G) or sharp (S); sharp bottom contacts are further described as horizontal (H), inclined (I), mottled, irregular, or curved (convex upward or downward). The color of the unit is described by comparison with the Munsell Soil Color Chart. When more than one color is dominant, excluding mottles or burrows, each noteworthy color is recorded. When a multitude of fine laminations is present, only the dominant color is recorded.

Textural notation includes descriptive parameters such as grain size and the amount of dehydration, compaction, and lithification. Grain size parameters used are: lutite $(<4 \mu)$, silt $(4 \mu-62 \mu)$, sand $(62 \mu-2 \mathrm{~mm})$, and gravel ( $>2 \mathrm{~mm})$. Microscopic examination of smear slides is used to determine the lighology and relative abundance of silt- and sand-sized components. Sand and gravel are distinguishable through a magnifying glass and to the unaided eye. Estimation of sizes within the sand range is accomplished by comparison with vials of sieved, standardized sands.

Additional observations fall under the heading of "special features". Graded bedding may be observed in silt-sized or coarser-grained sediment. The range in 
grain size and the depth interval over which grading occurs are noted. Graded beds are often burrowed in the fine upper section and have sharp, eroded bottom contacts. Many graded beds may be turbidites, but this generic term is not used in the descriptions of graded beds. Cross-bedding is rarely observed and is generally restricted to silt- or sand-sized sediment. Beds of alternating colors or textures which truncate each other on a scale as small as one $\mathrm{mm}$ are described as cross bedding. Laminations and microlaminations (up to $1 \mathrm{~mm}$ thickness) are observed and noted.

Poor core recovery, washed sediment, or flow-in are also included as special features. Flow-in may occur in piston cores, and is usually found at the bottom of the core, but may occasionally occur in upper sections as well. It results from insufficient core penetration and subsequent sucking action of the piston upon core pull-out. Verification of flow-in can be obtained by X-radiography when flow-in is suspected but cannot be demonstrated visually. Occasional multiple penetrations of either the pilot core or the piston core have been documented, and are especially well illustrated in some of the CHAIN 119 cores.

\section{Smear slide analysis}

Smear slides have been prepared and analyzed from the top and bottom of each core, at intervals of approximately one meter within the core, and from each major lithologic unit when closer sampling is required. The smear slides are examined through a polarizing binocular microscope, commonly with a magnification of $80 \mathrm{X}-$ 320X. The slide is first scanned at low power for a general indication of its composition. The describer then estimates the percentages of the various components, using standardized smear slides and frequent comparisons between describers in order to give some assurance that percentages are being estimated with some degree of consistency. However, the data tabulated on the smear slide forms should be used only qualitatively as an indication of the relative proportion of various components, and how the relative abundance of each component appears to change within a given core.

The following sediment components represent those most commonly encountered in smear slide analysis, and they are used in identifying the sediment type:

Inorganic Components

(a) Detrital grains

(b) Micronodules

(c) Zeolites

(d) Volcanic shards

(e) Pyrite

(f) Clay
Biogenic Components

$\underline{\text { Calcareous } \quad \text { Siliceous }}$

(g) Foraminifera

(h) Nannofossils

(i) Discoasters

(j) Pteropods

(k) Others
(1) Diatoms

(m) Radiolaria

(n) Sponges

(o) Silico-

flagellates

\section{Designation of sediment type}

A sediment name is assigned to each sample examined, following the sediment classification scheme summarized in Table $I$ and in Figures la-1b. These sediment names are recorded on the smear slide description sheet, and serve as a basis for designating one or more sediment types for each lithologic unit. 
Each principal lithologic unit is described on the visual description sheets, using the following format:

\section{Descriptive Terms}

0-112

CALC OOZE

10 YR 6/4 light yellowish brown common dark brown mottling throughout firm, slightly silty lutite 2 Mn nodules, $2 \mathrm{~cm}$ diam., 95-100 cm $\mathrm{S}$, inclined $10^{\circ}$

\section{Explanation}

Depth interval $(\mathrm{cm})$

Sediment type

Color

Mottling (if present)

Texture

Special features

Basal contact

In the lithologic $10 \mathrm{~g}$ on the left side of the visual description sheets, appropriate symbols are used to summarize the lithology and any special features which are readily observed macroscopically. A key to the symbols used in the lithologic logs is presented in Figure 2. 
TABLE 1: Sediment Classification System

I. MAJOR SEDIMENT NAME: Based on relative proportion of biogenic material $\left(\mathrm{CaCO}_{3}+\mathrm{SiO}_{2}\right)$ and inorganic material.

A. Oozes: Total biogenic material $\geqslant 30 \%$

Calcareous ooze: $\quad \mathrm{CaCO}_{3}>\mathrm{SiO}_{2}$

Calcareous-siliceous ooze: $\mathrm{CaCO}_{3}>\mathrm{SiO}_{2}, \mathrm{SiO}_{2}>58$

Siliceous-calcareous ooze: $\mathrm{SiO}_{2}>\mathrm{CaCO}_{3}, \mathrm{CaCO}_{3}>58$

Siliceous ooze: $\quad \mathrm{SiO}_{2}>\mathrm{CaCO}_{3}$

B. Clays: Total biogenic material $<30 \%$

$$
\begin{aligned}
& \text { Highly } \quad\left\{\begin{array}{l}
\text { calcareous } \\
\text { siliceous }
\end{array}\right\} \text { clay: } 15 \% \leqslant\left\{\begin{array}{l}
\mathrm{CaCO}_{3} \\
\mathrm{SiO}_{2}
\end{array}\right\}<30 \% \\
& \left.\begin{array}{l}
\text { Calcareous } \\
\text { siliceous }
\end{array}\right\} \text { clay } \\
& 58 \leqslant\left\{\begin{array}{l}
\mathrm{CaCO}_{3} \\
\mathrm{SiO}_{2}
\end{array}\right\}<15 \% \\
& \text { Slightly }\left\{\begin{array}{l}
\text { calcareous } \\
\text { siliceous }
\end{array}\right\} \text { clay: } \quad 18 \leqslant\left\{\begin{array}{l}
\mathrm{CaCO}_{3} \\
\mathrm{SiO}_{2}
\end{array}\right\}<58
\end{aligned}
$$

II. SECONDARY SEDIMENT NAMES: Included when silt- or sandsized inorganic components are present in excess of $15 \%$.

(MAJOR SEDIMENT NAME) with $\left\{\begin{array}{l}\text { detrital grains } \\ \text { Mn micronodules } \\ \text { zeolites } \\ \text { volcanic ash } \\ \text { etc. }\end{array}\right\}: 15 \%\left\{\begin{array}{l}\text { detrital grains } \\ \text { Mn micronodules } \\ \text { zeolites } \\ \text { volcanic ash } \\ \text { etc. }\end{array}\right\}: t \geqslant 30 \%$
(MAJOR SEDIMENT NAME) $I\}$ 
FIGURE 1a: Sediment Classification System

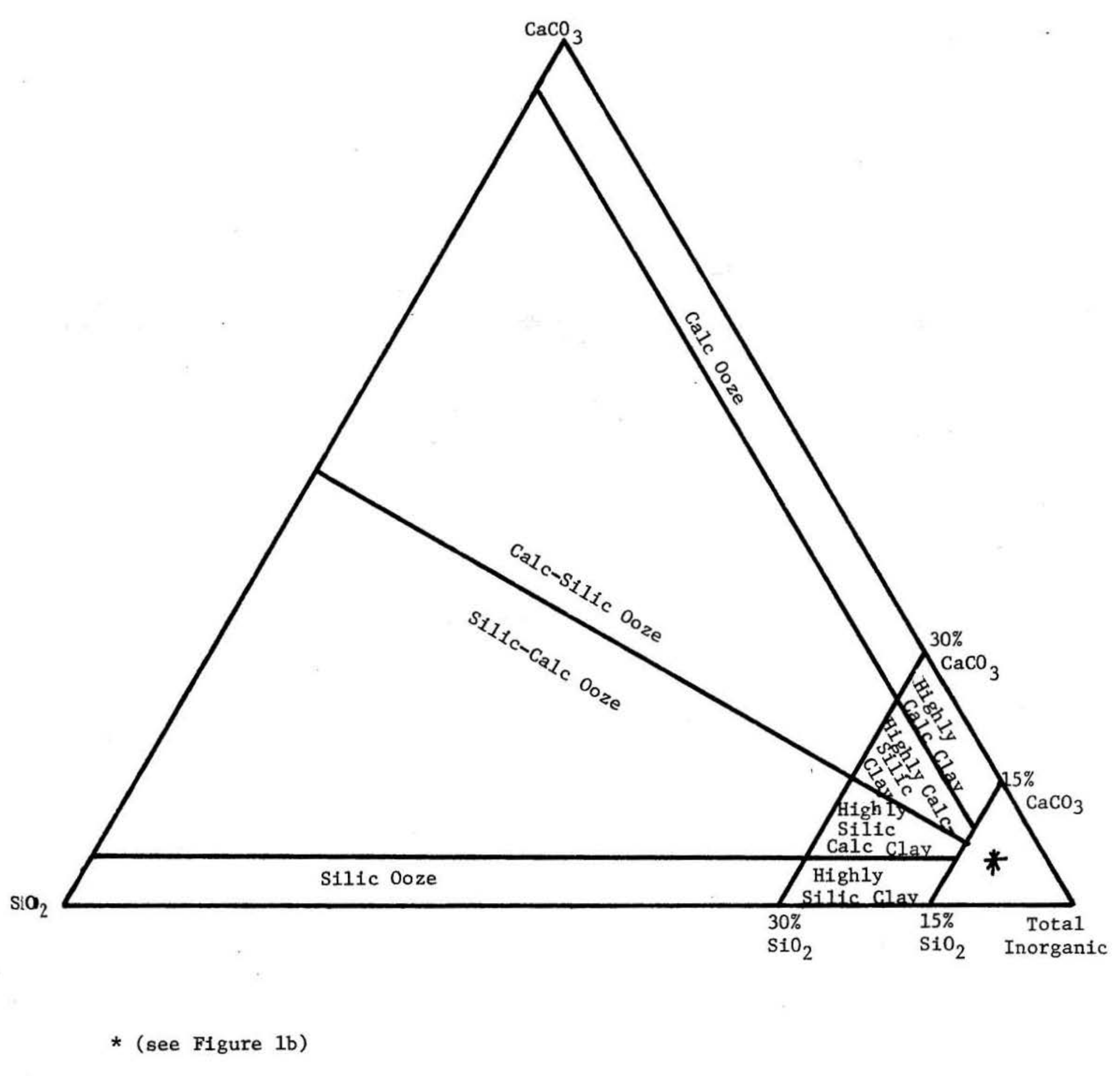


FIGURE 1b: Sediment Classification System

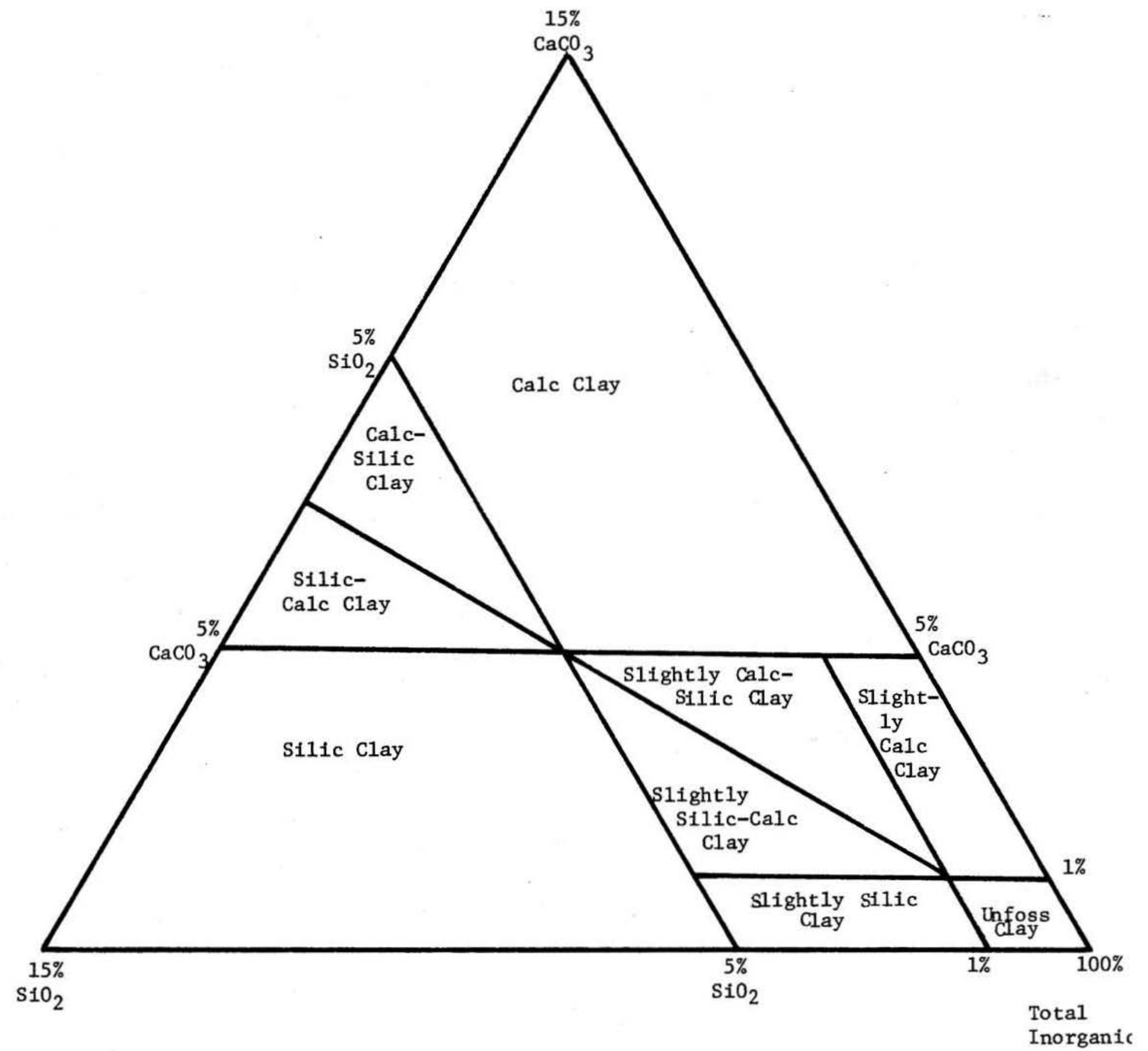


FIGURE 2: Lithologic Symbols

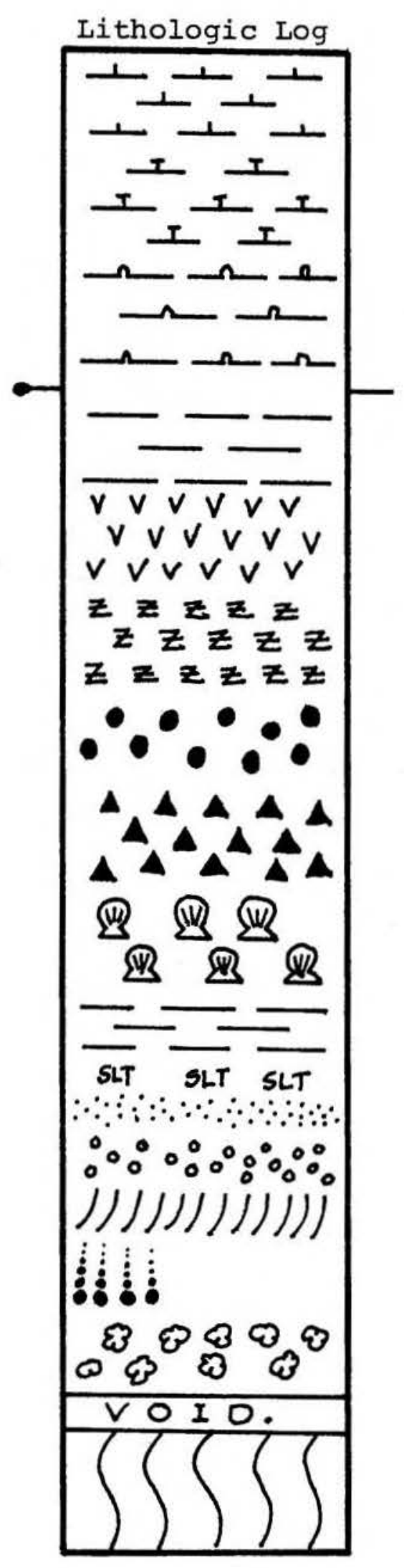

Lithologic Symbols

Calcareous ooze
Foram sand
Siliceous ooze
Unit Contact Mark
Clay

Volcanic ash

Zeolites

Mn nodules

Pyrite

Shell Hash

Clay

Silt

Sand

Granules, pebbles

Cross-bedding

Graded bedding

Mottling (burrowing)

Sediment missing

Flow-in
Pelagic Sediments

Terrigenous Sediments

Special Features 


\section{Core Biostratigraphy}

Calcareous microfossil assemblages have been examined to assign a biostratigraphic age to the top and bottom of each core. An epoch name (e.g. Pleistocene, Pliocene, etc.) was designated for each sample examined; no attempt was made to determine the specific nannofossil or foraminiferal zones. Each visual description sheet contains the age of the top and bottom of the core in the margin next to the lithologic log. Any age which is in question (e.g., Pliocene?) indicates possible reworking, to account for an assemblage of microfossils which have nonoverlapping stratigraphic ranges. In cores where microfossils were absent in the bottom smear slide but were identified in overlying samples, the basal age is assumed to be the same as that of the deepest microfossil-bearing sample which was examined. In this case, the notation used is an asterisk (e.g., Pliocene* ${ }^{*}$. Table 2 lists all Tertiary sediment cores in the WHOI collection, including those described in volumes $1-4$ of this series as well as those included in volume 5 . 
TABLE 2: Tertiary Cores in WHOI Core Collection**

Cruise No.

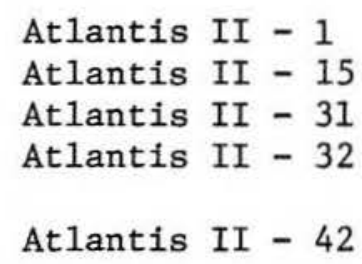

Core No.

$$
\begin{array}{r}
5-P C \\
10-P C \\
15-P C \\
2-G C \\
8-G C \\
11-P C \\
14-P C \\
17-P C \\
18-P C \\
19-P C \\
20-P C
\end{array}
$$

21-PC

40b-PC

41a-PC

5-PC

6-PC

7-PC

$9 B-G C$

$1-G C$

2-PC

4-PC

5-PC

6-PC

7-PC

1-PC

4-PC

13-PC

171-PC

14-PC

22-PC

6-PC

22-PC

32-PC

28-PC

75-PC

78-PC

$80-\mathrm{PC}$

82-PC

$87-\mathrm{PC}$

88-PC

91-PC

93-PC

94-PC

98-PC
Age (base of core)

Pliocene

Pliocene

Pliocene

Paleocene*

Paleocene*

Pliocene

Pliocene

Pliocene

Pliocene*

Pliocene

Pliocene

Pliocene*

Paleocene*

Paleocene*

Upper Pliocene

Middle Miocene

Middle Miocene

Pliocene

Pliocene

Pliocene

Pliocene

Upper Pliocene

Pliocene

Pliocene

Pliocene*

Upper Eocene*

Upper Oligocene

Upper Pliocene

Middle Miocene

Miocene

Lower Miocene

Pliocene*

Pliocene

Pliocene*

Pliocene

Pliocene*

Paleocene

Lower 01igocene

Pliocene

Upper Oligocene

Upper Miocene

Lower Pliocene

Upper Miocene

Lower Pliocene 
TABLE 2: Tertiary Cores in WHOI Core Collection ${ }^{* *}$ (cont'd)

Cruise No.

Chain - 115

Knorr - 31
Core No.

$$
\begin{array}{r}
8-P C \\
23-P C \\
45-P C \\
62-P C \\
64-P C \\
67-P C \\
70-P C \\
73-P C \\
75-P C \\
76-P C \\
79-P C \\
80-P C \\
81-P C \\
82-P C \\
84-P C \\
86-P C \\
26-G G C
\end{array}
$$

Age (base of core)

P1iocene

Upper Miocene

Lower Miocene

Pliocene*

Upper Miocene

Upper Miocene

Eocene

Middle Miocene

Upper Miocene

Lower Pliocene

Middle Miocene

Lower Miocene

Upper Miocene

Oligocene

Upper Miocene

Upper Miocene

Eocene

\footnotetext{
* Bottom sample in core is devoid of microfossils. Basal age is assumed equal to that of lowermost microfossil-bearing horizon.

**Includes all cores obtained prior to November 1976.
} 
D. Digitization of Geological Sample Data

All logistical information about geological samples in the WHOI core collection is stored on magnetic tape and accessible through computer program MUDDIE. In addition to these data, a summary of the descriptive information for each core has been put into digital form to allow rapid retrieval. Stored information about the samples may be retrieved according to combinations of any of the following parameters: ship, cruise, and leg number; latitude and longitude limits; Marsden Square number(s); water depth interval; core lengths; specific or general sampling device; physiographic province; and rock or sediment type.

A complete listing and documentation of the computer program MUDDIE is included in a recent WHOI technical report (Driscoll and Rush, 1975). The following summary explains the coded terms used in the computer listings of samples in this report:

\section{Ship Codes}

$$
\begin{aligned}
& \text { AST - Asterias } \\
& \text { AII - Atlantis II } \\
& \text { CHN - Chain } \\
& \text { KNR - Knorr }
\end{aligned}
$$

Sample Devices

The sampling devices used to collect an individual sample are indicated by the following two-digit codes. In cases where various instruments have been added to the primary sampling device, an entry has been made in the VITA CODE column.

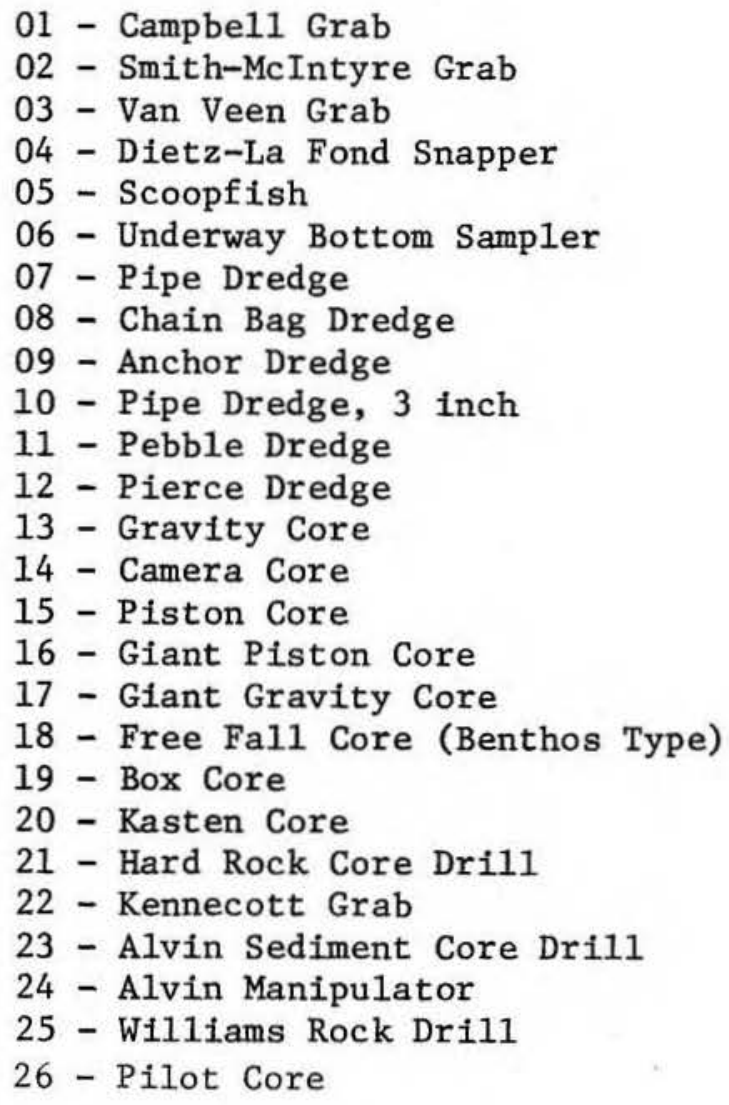


Fix Types

Types of navigational equipment used to determine the sample location are as follows:

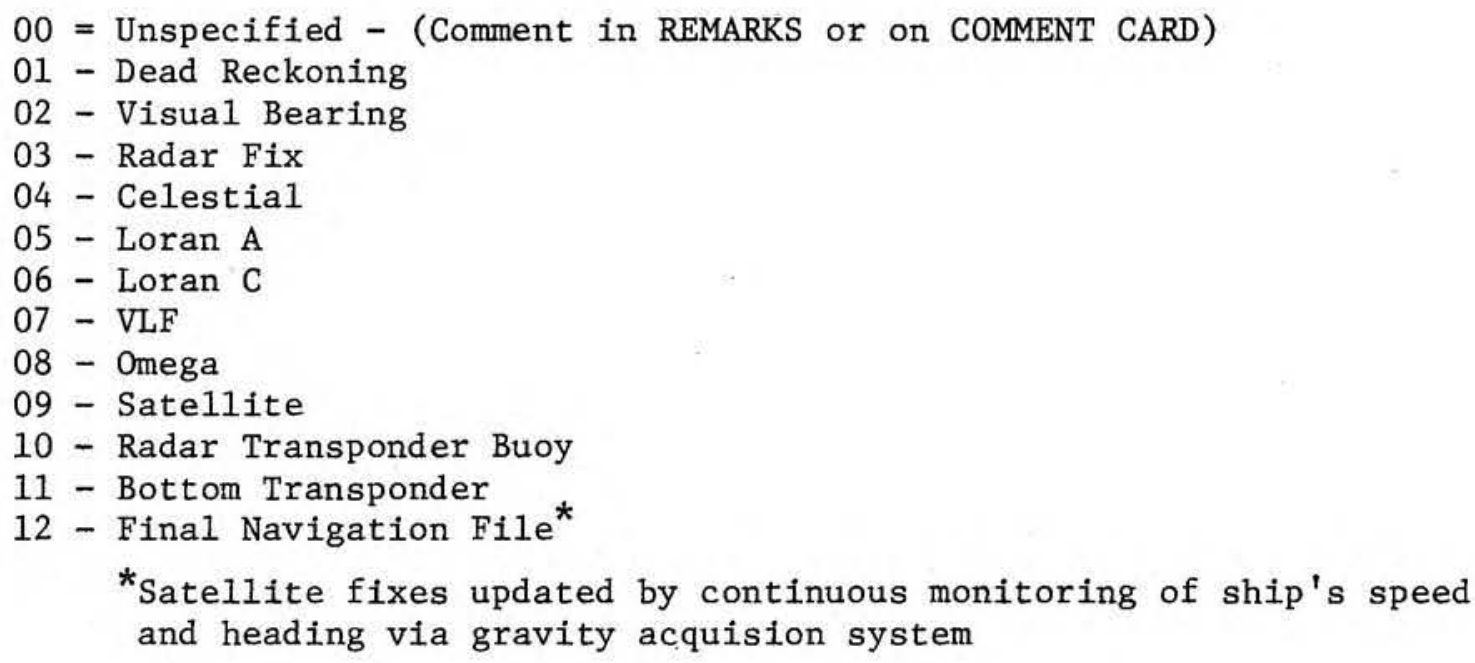

\section{Physiographic Province}

A general physiographic location has been assigned to each of the samples listed, and can be decoded as follows:

01 Insular Shelf

02 Continental Shelf (along continental margin)

03 Insular Slope

04 Continental Slope

05 Insular Rise

06 Continental Rise

07 Marginal Plateau or Borderland, deeper than $100 \mathrm{fms}$ (e.g., Blake Plateau)

08 discontinued

09 Archipelagic Apron

10 Abyssal Plain

11 Abyssal Hills

12 Seamount or Seamount Province

13 Aseismic Oceanic Rise or Ridge (e.g., Rio Grande Rise, Walvis Ridge)

14 Ridge Crest

15 Ridge Flank

16 Axial Valley

17 Trench - Insular

18 Trench - Continental Margin

19 Fracture Zone

20 Marginal Sea (e.g., Sea of Okhotsk, North Sea)

21 Sma11 Ocean Basin (e.g., Red Sea, Caribbean Sea)

22 Inland Fresh Water Lake (e.g., African Lakes)

23 Harbor, Shallow Bay (e.g., Buzzards Bay)

24 Delta or Cone (e.g., Hudson Canyon)

25 Submarine Canyon (e.g., Hudson Canyon)

26 Mid-Ocean Canyon or Channel (e.g., Maury Channe1, N. Atlantic Mid-Ocean Canyon)

99 Unspecified: (Comment in REMARKS or on a COMMENT CARD) 
Rock or Sediment Type

A four-digit code has been utilized to produce a rough description for each sediment sample listed. The first and second digits refer to the primary and secondary sediment types found with the sample. Both digits are from the following list.

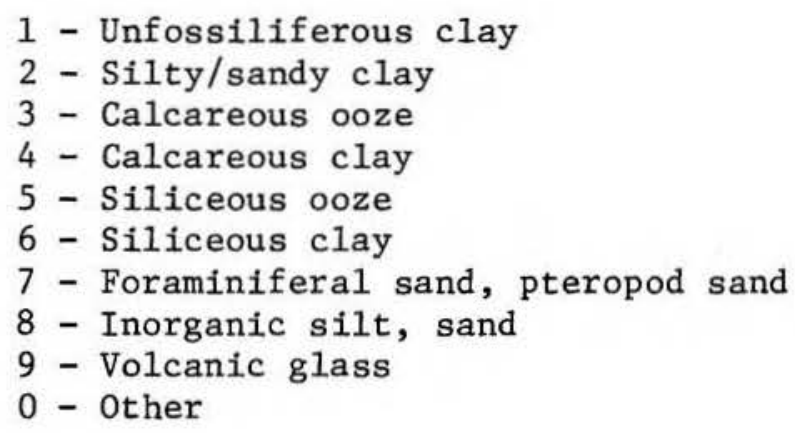

The third digit explains the relationship of the sediment types to one another.

1 - Finely interbedded

2 - Gradational contacts

3 - Sharp contacts

4 - Irregular or disturbed contacts

5 - Entire core of uniform lithology

6 - Contained in the same 1 ithologic unit

7 - Obscured

8 - Visually indistinguishable

0 - other

The fourth digit is used to designate special features occurring within the sample.

1 - Graded bedding or cross-bedding

2 - Extensive mottling or burrowing

3 - Manganese nodules

4 - Granules or pebbles

5 - Shells or shell fragments

6 - Pyrite-rich sediment

7 - Partially lithified sediment

8 - More than one of the above

9 - None

0 - Other

\section{Vita Code}

The VITA CODE is used to amplify the entry made in the DEVICE column and to provide a guide to additional types of data not covered in this listing.

40 - with Camera

41 - with Heat Flow outrigger probes

42 - with Compass

43 - with Nephelometer 
Vita Code (cont'd)

44 - with Velocimeter

45 - with Temperature Pinger

46 - with Compass and Heat Flow

47 - with Camera and Compass

48 - with Camera and Nephelometer

49 - with Camera and Sound Velocimeter

50 - with Camera and Temperature Pinger

51 - with Heat F1ow and Sound Velocimeter

52 - with Heat Flow and Nephelometer

53 - with Heat Flow and Temperature Pinger

54 - with Camera, Heat Flow, and Compass

55 - with Camera, Heat Flow, Compass, and Nephelometer

56 - with Camera, Heat Flow, Compass, Nephelometer, and Sound Velocimeter

57 - with Camera, Heat Flow, Compass, Nephelometer, Sound Velocimeter, and Temperature Pinger

58 - with Camera, Heat Flow, Compass, and Temperature Pinger

\section{E. Procedures for Obtaining Sediment Samples and Additional Core Data}

The WHOI Sea Floor Samples Laboratory is prepared to furnish sediment samples and data to interested scientists, researchers, and students inside or outside WHOI who express a legitimate interest and need. Sediment sampling is normally permitted in reasonable quantities, though sampling of recently acquired cores (taken during the preceding two years) is subject to the approval of the appropriate cruise chief scientist or collector of the samples.

The following procedures will serve as a guide to individuals requesting samples:

\section{PROCEDURES FOR REQUESTING SAMPLES}

(1) Requests for samples may be sent directly to the staff scientist engaged in research on the samples, if this person is known. If not known, sample requests should be sent to the Curator's Office, Data and Earth Sample Center, Woods Hole Oceanographic Institution, Woods Hole, Massachusetts 02543.

(2) A request for samples should include a brief summary of the type of research to be undertaken, the nature of the laboratory facilities available, and the source of financial support available for the work. The names of associated investigators should be given, and the nature of their research, facilities, and funding should be indicated if different from that of the applicant.

(3) If the material requested is within the 2-year period of proprietary access, sample requests will be referred to the appropriate scientists for approval. Otherwise, sample requests will be reviewed by the curator's office.

(4) The curator's office, in consultation with the appropriate WHOI staff scientist, will advise on the availability of material and on any other conditions that may be appropriate to ensure effective utilization of the material. 


\section{RESPONSIBILITIES OF PERSONS RECEIVING SAMPLES}

(1) The original alpha-numeric samples label should be used in published papers, or any departure from this scheme should be clearly equated with the original labeling system in published papers or data summaries. This labeling system will be explained in the information supplied with the samples.

(2) Published papers should acknowledge the source of samples and the appropriate grant or funding agency which supported the cruise recovering the samples. This information will be supplied at the time the samples are sent. These papers should also acknowledge the financial support responsible for maintaining the Woods Hole geological samples (NSF Grant 0CE76-81488, and ONR Contract N00014-74-C-0262).

(3) Copies of all published papers, reports, or data summaries utilizing Woods Hole samples should be sent to the appropriate WHOI staff scientist and the WHOI curator.

(4) The researcher should return all unused samples or portions of samples to the curator at the completion of his work.

(5) Recipients of samples should not co-opt the services of other investigators or undertake research projects which differ substantially from work originally proposed, without obtaining the approval of the curator and the appropriate staff scientist.

\section{F. References Cited}

Driscoll, A. H. and Rush, S. M., 1975, "W.H.O.I. geological samples data file, Volume I", Woods Hole Oceanogr. Inst. Tech. Rept. No. 75-37, 278 pp.

Johnson, D. A. and Driscoll, A. H., 1975, "Descriptions of WHOI sediment cores, Volumes I-IV", Woods Hole Oceanogr. Inst. Tech. Rept. No.75-8, 2937 pp. 
ACKNOWLEDGMENTS

The Woods Hole core lab staff has received continuing support and encouragement from J. R. Heirtzler and J. I. Ewing, who have served as department chairmen of the Geology and Geophysics department. Financial support for the Woods Hole core lab operations have been provided by the Office of Naval Research under Contract N00014-74-C0262, NR083-004; and by the National Science Foundation under Grant No. OCE76-81488.

We have received numerous helpful suggestions from our colleagues concerning procedures for carrying out the descriptive work and presenting the results. We thank Bob Groman for frequent assistance in the operation of the MUDDIE computer program, and Bill Dunkle for his assistance in locating original cruise records and station data.

Full credit for the completion of this report is due to the careful and comprehensive work of the WHOI core lab staff: Jim Broda, Harlow Farmer, Frank Shephard, and Lynn Whiteley. 
COMPUTER LISTING OF WHOI SEDIMENT CORES, ARRANGED BY MARSDEN SQUARES 


\section{2}

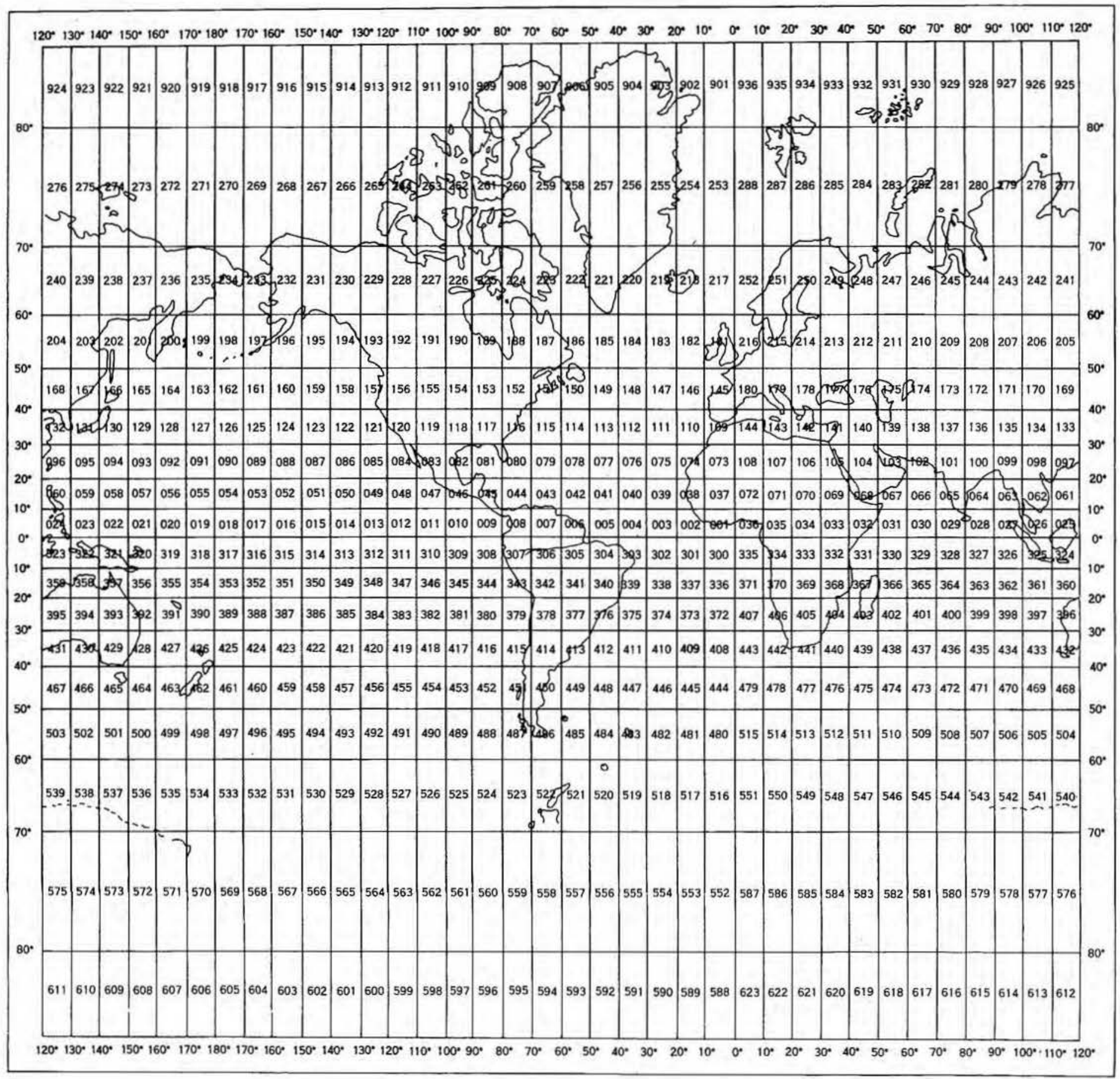


MARSDEN SQUARE \#2

\begin{tabular}{|c|c|c|c|c|c|c|}
\hline CHN & 99 & 3 & 0010 & 0000 & $\begin{array}{c}15 \\
* \text { COMME }\end{array}$ & $\begin{array}{rl}70 & 51 \\
\text { ENTS * * }\end{array}$ \\
\hline $\begin{array}{l}\text { CHN } \\
\text { CHN }\end{array}$ & 115 & $\begin{array}{l}3 \\
1\end{array}$ & $\begin{array}{l}0010 \\
000^{8}\end{array}$ & $\begin{array}{l}0000 \\
0000\end{array}$ & $\begin{array}{l}26 \\
15 \\
* \quad \text { COMME }\end{array}$ & $\begin{array}{r}7051 \\
7312 \\
\text { ENTS* }\end{array}$ \\
\hline $\begin{array}{l}\text { CHN } \\
\text { CHN } \\
\text { CHN } \\
\text { CHN }\end{array}$ & $\begin{array}{l}115 \\
115 \\
115 \\
115\end{array}$ & $\begin{array}{l}1 \\
1 \\
1 \\
1\end{array}$ & $\begin{array}{l}0008 \\
0010 \\
0010 \\
0011\end{array}$ & $\begin{array}{l}0000 \\
0000 \\
0000 \\
0000\end{array}$ & $\begin{array}{l}26 \\
15 \\
26 \\
15\end{array}$ & $\begin{array}{l}7312 \\
7312 \\
7312 \\
7312\end{array}$ \\
\hline CHN & $11_{5}$ & 1 & 0011 & 0000 & & ENT \\
\hline
\end{tabular}

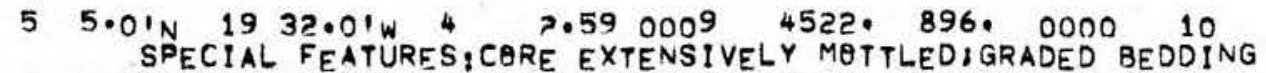
$55.01 \mathrm{~N} 1 \mathrm{G} 32.01 \mathrm{~W} 4 \mathrm{~W}, 5900094522,154,000010$ 91601 IN $1934: 91 W{ }^{2} 1$ 2:99 0008 4572: $881: 000013$ 16.1 IN

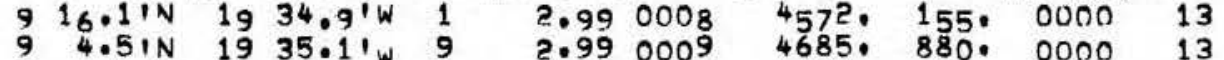
$94.5, N \quad 1935.1$. 9 g 2.990009 4685. 151.000013 $915.71 \mathrm{~N} 1926.41 \mathrm{~W} 92.990010$ 4168. 885. ONOO 13 SECONDARY SED TYPE CALC SILIC CLAY

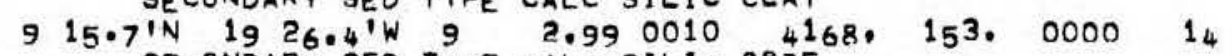
SECONDARY SED TYPE CALC SILIC OQZE

$3738 \quad 54$

466254 343354

$33595^{4}$ 353254 335954 302254

$3032 \quad 54$

MARSDEN SQUARE \#3 4596. $113^{\circ} 5^{\circ} 0000 \quad 11$

$\begin{array}{lrll}\text { CHN } & 99 & 3 & 0011 \\ \text { CHN } & 99 & 3 & 0011 \\ \text { CHN } & 115 & 1 & 0005 \\ \text { CHN } & 115 & 1 & 0005 \\ \text { CHN } & 115 & 1 & 0006 \\ \text { CHN } & 115 & 1 & 0006 \\ \text { CHN } & 115 & 1 & 0007 \\ \text { CHN } & 115 & 1 & 0007\end{array}$

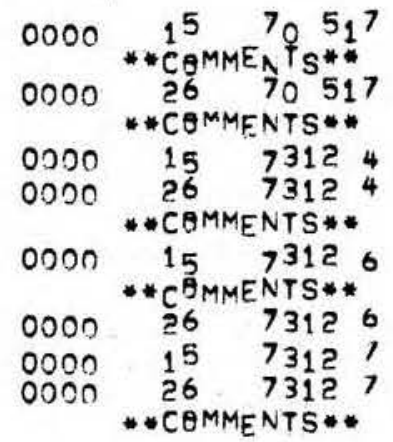

$24.41 \mathrm{~N} 2038.0^{1 \mathrm{~W}}$ 3.500010 FLOW-1N:692-1135 CM 2 $4.41 \mathrm{~N} 2038.01 \mathrm{~W}$ SECONDARY SEDIMENT TYPE, CAL.SILIC OBZE 3.?0 0010 11

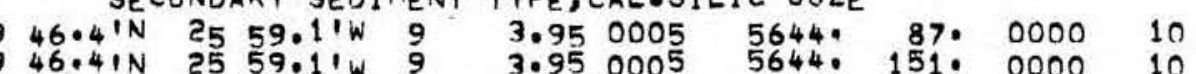
$946.41 \mathrm{~N} 2559.11 \mathrm{~W} 93.950005$ PILET CARE REBOUND SUSPFCTED

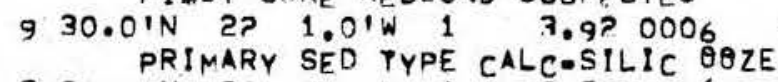

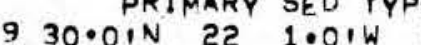
3.920006 4800. 870 . 0000 $4800 \cdot 153 \cdot 0000$

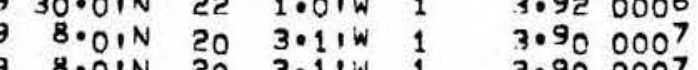
in $20 \quad 3.11$ W 1 3.90 0007

$3806.870^{\circ} 0000 \quad 13$ PILOT CARE REBOUND SUSPFCTED
MARSDEN SQUARE *

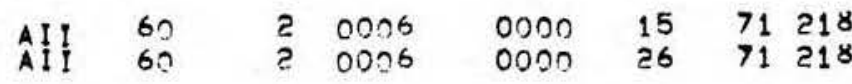

\section{MARSDEN SQUARE *}

O 53.91N 38 23.71W 94.080006 $053.91 N \quad 3823.71 \mathrm{~N} 94.080006$
4488. 290. 0000 4488.49 .0000
332254 308254 $\begin{array}{ll}3359 & 54 \\ 3430 & 54\end{array}$ 062254

646254 $\begin{array}{lll}3582 & 41\end{array}$

$3522 \quad 41$

MARSDEN SQUARE \# 5

$\begin{array}{llllllll}\text { AII } & 31 & 1 & 0006 & 0000 & 15 & 67 & 41 \\ \text { AII } & 31 & 1 & 0009 & 0000 & 15 & 67 & 41 \\ \text { AII } & 31 & 1 & 0009 & 0000 & 26 & 67 & 41 \\ \text { AII } & 31 & 1 & 0010 & 0020 & 15 & 67 & 41\end{array}$

$\begin{array}{lllll}9 & 38.51 N & 43 & 37.01 W & 4 \\ 9 & 35.01 N & 42 & 47.01 W & 1 \\ 9 & 35.01 N & 42 & 47.01 W & 1 \\ 9 & 46.31 N & 41 & 50.01 W & 1\end{array}$

$5 \cdot 93000^{6}$ 5.97 0009 5.92 0009

5.910010
4757.565.

4355. 577, 0000 4350 57.0000 4355. 121. 0000 


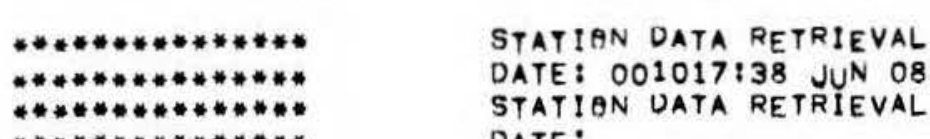

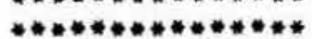

DATE: 001017:38 JUN 08

DATE:

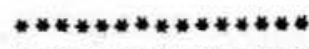

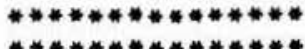

PAGE $?$

** WHOI **

PAGE 1803417591

CERE

LENGTH DREDGE $O R$ OR PHYSIO. RR

MARS- CORE OR END SAMPLE GRAPHIC SED, VITA

FIX DEN DREDGE

SAMPLE DE. DATE SHIP CRUISE LEG STATION NUMBER VICE YRMODA

$\begin{array}{rrrr}\text { AII } & 31 & 1 & 0011 \\ \text { AII } & 31 & 1 & 0011 \\ \text { AII } & 60 & 2 & 0005 \\ \text { III } & 60 & 2 & 0005 \\ \text { CHN } & 115 & 8 & 0159 \\ \text { CHN } & 115 & 8 & 0160\end{array}$

$\begin{array}{cccc} & \text { *COMMENTS * } \\ 0000 & 15 & 67 & 418 \\ 0000 & 26 & 67 & 418 \\ 0000 & 15 & 71 & 216 \\ 0000 & 26 & 71 & 216 \\ 0000 & 13 & 74 & 617 \\ 0000 & 13 & 74 & 617\end{array}$
LATITUDE LONGITUDE TYPE SQUARE NUMBER DEPTH DEPYH VOLUME PRE
PRIMARY AND SECONOARY SEDIMENT TYPE CALC.SILIC OQZE

$\begin{array}{ccccc}9 & 58.01 \mathrm{~N} & 40 & 51.31 \mathrm{~W} & 1 \\ 9 & 58.01 \mathrm{~N} & 40 & 51.31 \mathrm{~W} & 1 \\ 5 & 1001 \mathrm{~N} & 44 & 14.01 \mathrm{~W} & 1 \\ 5 & 1001 \mathrm{~N} & 44 & 14.01 \mathrm{~W} & 1 \\ 1 & 12.21 \mathrm{~N} & 48 & 50.91 \mathrm{~W} & 1 \\ 1 & 37.5, \mathrm{~N} & 49 & 1.01 \mathrm{~W} & 1\end{array}$

$\begin{array}{rl}5 \cdot 90 & 0011 \\ 5.90 & 0011\end{array}$

5.900011

5.540005

5.180093

5.190094

$\begin{array}{rrrr}3469 . & 182 . & 0000 & 19 \\ 34699^{\circ} & 45 . & 0000 & 19 \\ 37060 & 219 . & 0000 & 11 \\ 37060 & 98 . & 0000 & 11 \\ 8 . & 78 . & 0000 & 2 \\ 10 . & 128 . & 0000 & 2\end{array}$

$\begin{array}{rr}3869 & 0 \\ 3322 & 0 \\ 3322 & 41 \\ 3322 & 0 \\ 1819 & 0 \\ 1861 & 0\end{array}$

MARSDEN SQUARE \#8

$\begin{array}{llll}\text { GES } & 96 & 0 & 0004 \\ \text { GOS } & 96 & 0 & 0005 \\ \text { GOS } & 96 & 0 & 0006 \\ \text { OOS } & 96 & 0 & 0009 \\ \text { GOS } & 96 & 0 & 0013\end{array}$

0000

20
20
20

$\begin{array}{ll}67 & 2 \\ 67 & 2 \\ 67 & 2 \\ 67 & 2 \\ 67 & 2\end{array}$

MARSEN SQUAR

0000

0000

0000

0500

$\begin{array}{llll}9 & 45.71 \mathrm{~N} & 7 & 45.71 \mathrm{~W} \\ 9 & 38.11 \mathrm{~N} & 71 & 36.41 \mathrm{~W}\end{array}$

$939.01 N \quad 7129.11 \mathrm{~W}$

$914.71 \mathrm{~N}$

71 23.41 0

$947.9, N 71$ 22051w

6725

$955.4, N \quad 7122.2, W$

$\begin{array}{ll}8.91 & 0001 \\ 8.91 & 0001\end{array}$

8.910001

8.910001

8.910001

8.910001

\section{MARSDEN SQUARE \#9}

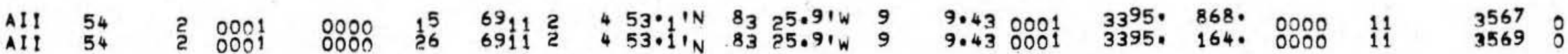

MARSDEN SQUARE \# 10

$\begin{array}{lllllllllllll}\text { CHN } & 21 & 1 & 0003 & 0000 & 15 & 611011 & 39 & 57.81 \mathrm{~N} & 12 & 18.51 \mathrm{E} & 1 & 10.9 ?\end{array}$

MARSDEN SQUARE * 29

$\begin{array}{llll}\text { AII } & 15 & 5 & 0614 \\ \text { AII } & 15 & 5 & 0614 \\ \text { AII } & 15 & 5 & 0614 \\ \text { AII } & 15 & 5 & 0615 \\ \text { AII } & 15 & 5 & 0617 \\ \text { AII } & 15 & 5 & 0618 \\ \text { AII } & 15 & 6 & 0629\end{array}$

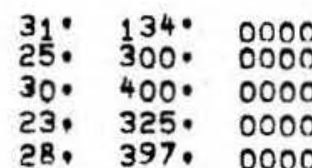

33. 326. 0000
00000

0000

00000

00000

00000

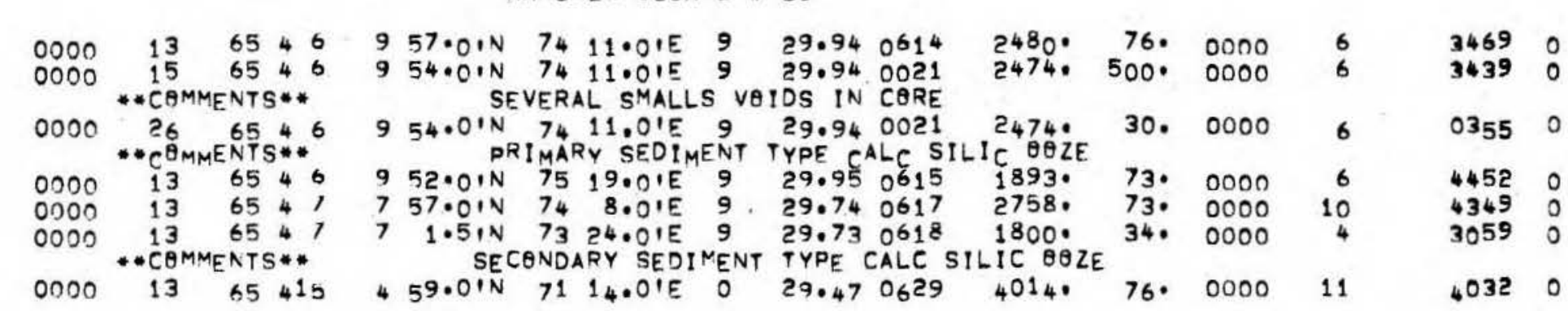




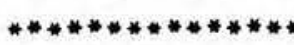

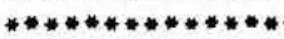

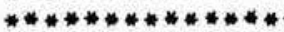

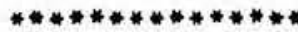

STATIPN UATA RETRIEVAL DATE: 001017:38 JUN OB STATION DATA RETRIEVAL OATE:
$* * * * * * * * * * * * * * *$
$* * * * * * * * * * * * * *$
$* * * * * * * * * * * * * * * *$
PAGE 3

** ${ }^{2} \mathrm{HOl}$ **

PAGE 1803417591

SAMPLE DE- DATE

MARS. CARE $Q R$ LENGTH DREDGE PHYSIO ROCK FIX DEN DREDGE QR OR PHYSIO. QR

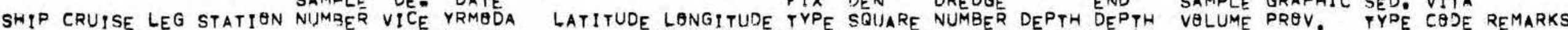

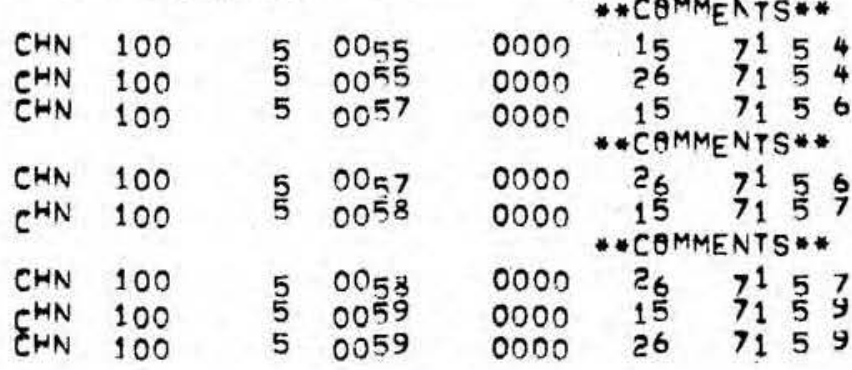
SECENDARY SEDIMENT TYPE CALC SILIC AOZE

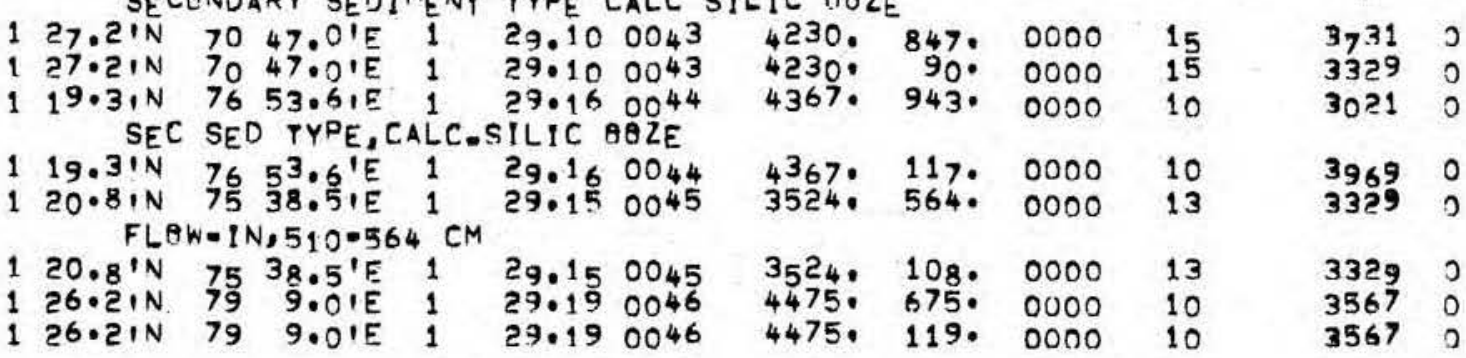

MARSDEN SQUARE \#30

$\begin{array}{lllll}\text { AII } & 15 & 4 & 057 ? \\ \text { CHN } & 100 & 5 & 0053 \\ \text { CHN } & 100 & 5 & 0053 \\ \text { CHN } & 100 & 5 & 0054 \\ \text { CHN } & 100 & 5 & 0054\end{array}$

$0000 \quad 18 \quad 65 \quad 311$

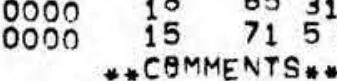
$0000 \quad 26 \quad 715 \frac{2}{15} \quad 715 \frac{5}{3}$ * CAMMENTS**

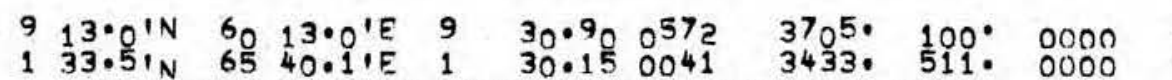

$$
30
$$
SEDIMENT PAND AN HIGH FLATS WO CARLBERG RIDGE

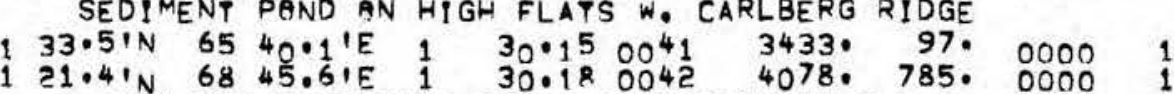
SEC SED TYPE, CALC-SILIC $\theta \theta_{Z}$, FLOW-IN,743-789 CM

$121.41 \mathrm{~N} 6845.615130 .1800424078 .11400000 \quad 15 \quad 33520$

\section{MARSDEN SQUARE \#31}

$\begin{array}{llll}\text { AII } & 15 & 4 & 0558 \\ \text { AII } & 15 & 4 & 0558 \\ \text { AII } & 15 & 4 & 0559 \\ \text { AII } & 15 & 4 & 0560 \\ \text { AII } & 15 & 4 & 0560 \\ \text { AII } & 15 & 4 & 0561 \\ \text { AII } & 15 & 4 & 0563 \\ \text { AII } & 15 & 4 & 0563 \\ \text { AII } & 15 & 4 & 0564 \\ \text { AII } & 15 & 4 & 0565\end{array}$

$859.01 N \quad 5144.01 E 9 \quad 31.810009$ 3985. 870. 0000 PRIMARY AND SECENDARY SEDIMENT TYPE CALC-SILIC BQZE

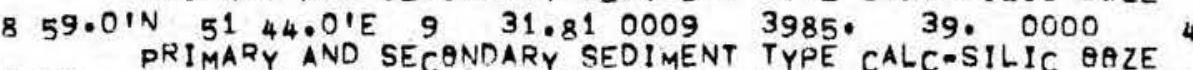
$854.01 \mathrm{~N} 5137.01 \mathrm{E}^{2} \quad 31.810559$ 3797. 104. 0ONO $858.5, N$ 52 2.01E 9 31.82 0010 4350. 665. 0000 6 $858.51 \mathrm{~N} 52$ 2.01E $9 \quad 31.8$ ? 0010 4350. 23. 00006 $858.01 N$ 52 $20.01 E 99$ 31.83 0011 4722. 998. 0000

$0000 \quad 15 \quad 65 \quad 3 \quad 3$ $0000 \quad 26 \quad 6533$ * CAMMENTS** 0000156536 $000026 \quad 6536$ * *COMMENTS** $911.01 \mathrm{~N} 5223.51 \mathrm{E} 93_{1.92} 0012$ 4499. 84900000 PRIMARY SEDIMENT TYPF CALC SILIC OEZE

$911.01 N 5223.51 E 9$ 31.92 0012 4499. 39. 0000

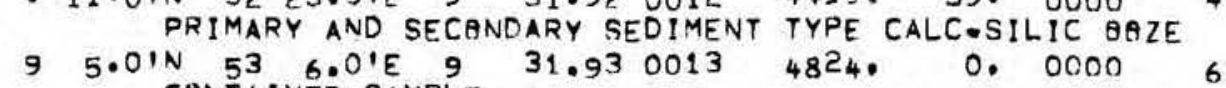
ooon 15 . $65^{3}{ }^{7}$ $0000 \quad 156537$ * COMMENTS** CONTAINER SAMPLE

$92 \cdot 0, N \quad 5340.51 E$ 9 $31.930014 \quad 4852.1050 \cdot 00006$ PRIMARY AND SECANDARY SEDIMEVT TYPE CALC-SILIC GOZE NUMEROUS VAIOS THRQUGHAUT
00190

00290

39590

33420

39590

$\begin{array}{ll}0649 & 0 \\ N & 915-988\end{array}$

03420

$0059 ?$

DOOO O NOSE CONE

$n 0490$ 


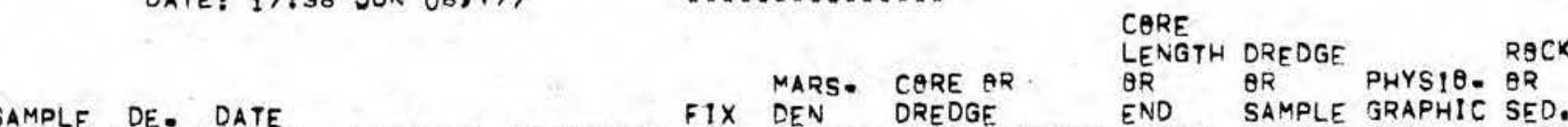

SAMPLE DE. DATE HIC SED, VITA

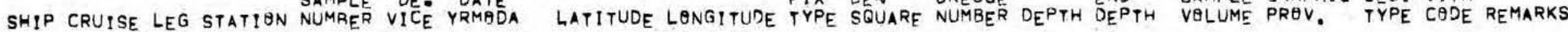

MARSDEN SQUARE *31

\begin{tabular}{|c|c|c|c|c|c|c|c|c|c|c|c|c|c|c|c|c|c|c|c|c|}
\hline AII & 15 & 4 & 0565 & 0000 & ${ }^{26}{ }^{26} \mathrm{MME}$ & $\begin{aligned} & 65 \\
= & N T S\end{aligned}$ & $\begin{array}{ll}31 \\
6 *\end{array}$ & 9 & $\frac{2 \cdot 01 \mathrm{~N}}{\mathrm{SE}}$ & $\begin{array}{c}5340.5 \text { IE } \\
\text { CANDARY SED }\end{array}$ & $\stackrel{9}{I \text { MENT }}$ & $\begin{array}{r}31.93 \\
\text { TYPF }\end{array}$ & $\begin{array}{l}\text { OO14 } \\
\text { CALC }\end{array}$ & $\begin{array}{l}48520 \\
\text { SILIC } \theta \theta\end{array}$ & $\theta_{Z E}{ }^{69 .}$ & 0000 & 6 & 3029 & 0 & \\
\hline AII & 15 & 4 & 0568 & 0000 & 15 & $\begin{aligned} & 65 \\
\text { ENTS } & \text { NTS }\end{aligned}$ & 3 & 8 & $59 \cdot 0, N$ & $\begin{array}{l}5447.0 I E \\
\text { MERAUS VOID }\end{array}$ & $S^{9}$ THR & $\begin{array}{r}31.84 \\
\text { ROUGHOL }\end{array}$ & UT. FL & $\begin{array}{l}4950: \\
\text { IN } 82\end{array}$ & $\begin{array}{c}11790 \\
22=1179\end{array}$ & $\mathrm{CM}_{\mathrm{CM}}$ & 10 & 4661 & 0 & \\
\hline$A 11$ & 15 & 4 & 0568 & 0000 & $\begin{array}{l}26 \\
\text { COMME }\end{array}$ & $\begin{array}{l}65 \\
\text { ENTS }\end{array}$ & $3^{3} y$ & 8 & $59.01 \mathrm{~N}_{\mathrm{PR}}$ & $\begin{array}{l}5447.0 \text { 'E } \\
\text { IMARY AND S }\end{array}$ & $\stackrel{9}{9}$ & $\begin{array}{r}31.84 \\
\text { DARY SE }\end{array}$ & $\begin{array}{l}\text { OO15 } \\
\text { EDIMEN }\end{array}$ & $\begin{array}{l}{ }^{4950^{\circ}} \\
\text { VT TYPE C }\end{array}$ & CALC-SIL & $\begin{array}{l}0000 \\
\text { LIC } \theta \theta_{Z}\end{array}$ & $Z E^{10}$ & 0059 & 0 & \\
\hline AII & 15 & 4 & $0^{569}$ & 0000 & $1^{8}$ & $\begin{aligned} & 65 \\
= & \text { NTS }\end{aligned}$ & $3^{3} 9$ & 8 & $58 \cdot 5, \mathrm{~N}$ & $\begin{array}{l}56 \\
I M A R Y\end{array}$ & $\begin{array}{l}9 \\
\text { IENT }\end{array}$ & $\begin{array}{l}31 \cdot 86 \\
\text { TYPE C }\end{array}$ & $\begin{array}{l}0569 \\
\text { ALC SII }\end{array}$ & $\begin{array}{l}40010 \\
\text { LIC }\end{array}$ & $E^{10^{5 \cdot}}$ & 0000 & 10 & 0369 & 0 & \\
\hline $\begin{array}{l}\text { CHN } \\
\text { CHN }\end{array}$ & $\begin{array}{l}43 \\
43 \\
43\end{array}$ & $\begin{array}{l}1 \\
1 \\
1\end{array}$ & $\begin{array}{l}0027 \\
0029 \\
0069\end{array}$ & $\begin{array}{l}0000 \\
0000 \\
0000\end{array}$ & $\begin{array}{l}15 \\
13 \\
15\end{array}$ & $\begin{array}{l}64 \\
64 \\
64\end{array}$ & $\begin{array}{l}47 \\
47 \\
530\end{array}$ & $\begin{array}{l}5 \\
0 \\
2\end{array}$ & $\begin{array}{l}52.01 N \\
55.01 N \\
49.01 N\end{array}$ & $\begin{array}{ll}53 & 51.01 E \\
51 & 38.01 E \\
59 & 41.01 E\end{array}$ & $\begin{array}{l}1 \\
1 \\
1\end{array}$ & $\begin{array}{l}31 \cdot 53 \\
39 \cdot 01 \\
31 \cdot 29\end{array}$ & $\begin{array}{l}0001 \\
0004 \\
0023\end{array}$ & $\begin{array}{l}49440^{\circ} \\
51140^{\circ} \\
42150^{\circ}\end{array}$ & $\begin{array}{l}582^{\circ} \\
145^{\circ} \\
325^{\circ}\end{array}$ & $\begin{array}{l}0000 \\
0000 \\
0000\end{array}$ & $\begin{array}{l}19 \\
10 \\
15\end{array}$ & $\begin{array}{l}34694 \\
35694 \\
49694\end{array}$ & $\begin{array}{l}41 \\
41 \\
41\end{array}$ & \\
\hline & & & & & - CAMME & ENTS & $5 * \%$ & & $F L \theta$ & $W=I N \quad 7_{0}=325$ & & & & & & & & & & \\
\hline CHN & 100 & 4 & 0036 & 0000 & $\begin{array}{l}15 \\
C_{M N}\end{array}$ & $\begin{aligned} & 71 \\
& \text { ENTS }\end{aligned}$ & 46 & 7 & $\begin{array}{l}48.01 \mathrm{~N} \\
F L \theta\end{array}$ & $\begin{array}{c}56 \quad 12 \cdot 2 \backslash E \\
W=1 N, 355=57\end{array}$ & $10^{1} \mathrm{~cm}$ & 31.76 & 0026 & 4680 . & 142. & 0000 & 15 & 3562 & $0 \mathrm{CHN}$ & RIDGE \\
\hline $\begin{array}{l}\text { CHN } \\
\text { CHN }\end{array}$ & $\begin{array}{l}100 \\
100\end{array}$ & $\begin{array}{l}4 \\
4\end{array}$ & $\begin{array}{l}0036 \\
0037\end{array}$ & $\begin{array}{l}0000 \\
0.000\end{array}$ & 26 & $\begin{array}{l}71 \\
71 \\
\text { ENTs }\end{array}$ & $\begin{array}{ll}4 & 6 \\
4 & 7 \\
5 * *\end{array}$ & $\begin{array}{l}7 \\
7\end{array}$ & $\begin{array}{r}48.0 \text { N } \\
43.9 \text { IN } \\
\text { SEC }\end{array}$ & 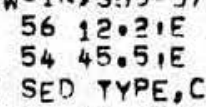 & ${ }^{1}{ }^{1} C=S$ & $\begin{array}{r}31.76 \\
31: 74 \\
\text { SILIC }\end{array}$ & $\begin{array}{l}0026 \\
0027 \\
\theta \theta Z E\end{array}$ & $\begin{array}{l}4680^{\circ} \\
5102 .\end{array}$ & $\begin{array}{r}112 . \\
1149 .\end{array}$ & $\begin{array}{l}0000 \\
0000\end{array}$ & $\begin{array}{l}15 \\
10\end{array}$ & $\begin{array}{l}3569 \\
3021\end{array}$ & ${ }_{0}^{0} \mathrm{CHN}$ & RIDGE \\
\hline HN & 100 & 4 & 0037 & 0000 & 26 & 71 & 47 & 7 & $5^{1} \cdot 9^{\prime N}$ & $5445.5^{\prime} E$ & 1 & 31.74 & 0027 & 5102. & $145^{\circ}$ & 0000 & 10 & 3569 & 0 & \\
\hline $\mathrm{c}^{\mathrm{HN}}$ & 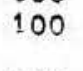 & 4 & 3 & סO & $\begin{array}{l}15 \\
\text { - COMME }\end{array}$ & $\begin{array}{l}71 \\
\text { ENTS }\end{array}$ & 41 & 7 & $\begin{array}{r}4.51 \mathrm{~N} \\
\text { SPE }\end{array}$ & $\begin{array}{l}5557.61 E \\
\text { CIAL FEATUR }\end{array}$ & 1 SE, M & $\begin{array}{l}31.75 \\
\text { MOTTLII }\end{array}$ & $\begin{array}{l}0028 \\
\text { NG AND }\end{array}$ & $\begin{array}{l}42500 \\
\text { MN NEDU }\end{array}$ & $\begin{array}{l}439^{\circ} \\
\text { ULES }\end{array}$ & 0000 & 15 & 3328 & 0 & \\
\hline $\begin{array}{l}\text { CHN } \\
\text { CHN }\end{array}$ & $\begin{array}{l}100 \\
100\end{array}$ & $\begin{array}{l}4 \\
4\end{array}$ & $\begin{array}{l}0038 \\
0040\end{array}$ & $\begin{array}{l}0000 \\
0000\end{array}$ & $\begin{array}{l}26 \\
15 \\
\text { * COMME }\end{array}$ & $\begin{aligned} 71 \\
71 \\
\text { ENTS }\end{aligned}$ & $\begin{array}{l}47 \\
48 \\
6 * *\end{array}$ & $\begin{array}{l}7 \\
6\end{array}$ & $\begin{array}{r}4.51 \mathrm{~N} \\
55.4 \mathrm{~N} \\
\text { PRI }\end{array}$ & $\begin{array}{l}5557.61 E \\
54 \quad 41.71 E \\
M \text { SED TYPE, }\end{array}$ & $\begin{array}{l}1 \\
\text { SILIC }\end{array}$ & $\begin{array}{r}31.75 \\
31.64 \\
\text { C CALC }\end{array}$ & $\begin{array}{l}0028 \\
0029 \\
\theta 02 E\end{array}$ & $\begin{array}{l}4250^{\circ} \\
5106\end{array}$ & $\begin{array}{l}100 . \\
1156 .\end{array}$ & $\begin{array}{l}0000 \\
0000\end{array}$ & $\begin{array}{l}15 \\
10\end{array}$ & $\begin{array}{l}3322 \\
0731\end{array}$ & o & \\
\hline $\mathrm{CHN}$ & 10 ? & 4 & 0040 & 0.000 & 26 & 71 & 48 & 6 & $55 \cdot 4 \mathrm{~N}$ & $5^{4} 41.71 E$ & 1 & $31 \cdot 6^{4}$ & 0029 & $510_{6}$. & 152. & 0000 & 10 & 3359 & 0 & \\
\hline$e^{-H N}$ & 100 & 4 & 042 & ojon & $\begin{array}{l}15 \\
+C O N\end{array}$ & $\begin{array}{l}71 \\
\text { EATs }\end{array}$ & & 4 & $\begin{array}{r}27.41 \mathrm{~N} \\
\text { PRI }\end{array}$ & $\begin{array}{l}51 \\
\text { M SED YYPE, }\end{array}$ & sit & $\begin{array}{r}31.41 \\
C \text { CALC }\end{array}$ & $\begin{array}{l}0030 \\
\theta \theta Z E_{1}\end{array}$ & $\begin{array}{r}5049, \\
\text { SPECIAL }\end{array}$ & $\begin{array}{l}1108 . \\
\text { FEATURE }\end{array}$ & $\begin{array}{l}\text { OOOOO } \\
\text { ES:METT }\end{array}$ & TLING AND & MN & 0 & \\
\hline $\begin{array}{l}\mathrm{CHN} \\
\mathrm{CHN}\end{array}$ & $\begin{array}{l}100 \\
100\end{array}$ & 4 & $\begin{array}{l}00_{4} \text { ? } \\
00^{4} 3\end{array}$ & $\begin{array}{l}0000 \\
0000\end{array}$ & $\begin{array}{l}26 \\
15\end{array}$ & $\begin{array}{l}71 \\
71\end{array}$ & $\begin{array}{l}413 \\
415\end{array}$ & $\frac{4}{3}$ & $\begin{array}{l}27.4 \mathrm{IN} \\
13.7 \mathrm{~N}\end{array}$ & $\begin{array}{l}51.01 \mathrm{E} \\
5223.31 \mathrm{E}\end{array}$ & 1 & $\begin{array}{l}31.41 \\
31.32\end{array}$ & $\begin{array}{l}0030^{\circ} \\
0031\end{array}$ & $\begin{array}{l}50490 \\
5115 .\end{array}$ & $\begin{array}{l}980^{\circ} \\
254 .\end{array}$ & $\begin{array}{l}0000 \\
0000\end{array}$ & $\begin{array}{l}10 \\
10\end{array}$ & $\begin{array}{l}3539 \\
5369\end{array}$ & 0 & \\
\hline & & & & & - CAMME & ENTS & $\theta *$ & & FLO & $W=I N=44 \cdot 254$ & CM & & & & & & & & & \\
\hline CHN & or & 4 & 0043 & 000 & 26 & 71 & 410 & 3 & $13.7 . N$ & $52 \geqslant 3.31 \mathrm{E}$ & 1 & $31 \cdot 32$ & 0031 & 5115 . & 104. & 0000 & 10 & 3569 & J & \\
\hline CHN & 0 & 4 & 0044 & 00 & 15 & 71 & 416 & 3 & $14 \cdot 2, N$ & $5241.01 E$ & 1 & $31 \cdot 32$ & 0032 & 5123. & 959. & 0000 & 10 & 5331 & 0 & \\
\hline CHN & 10 & 4 & 0044 & & ${ }^{26}{ }^{26}$ & ITIS & $41^{6}$ & 3 & $1^{4} \cdot 2$ IN & $\begin{array}{l}5241 \cdot 0: E \\
M . \text { SED. TYP }\end{array}$ & $E^{1}$ & $\begin{array}{r}31 \cdot 32 \\
\text { ILIC.C. }\end{array}$ & $\begin{array}{l}00^{32} \\
\text { ALC } \theta \theta\end{array}$ & $\begin{array}{l}51230 \\
\text { ZE, SEC. }\end{array}$ & $\begin{array}{l}137 . \\
\text { SED. }\end{array}$ & $\begin{array}{l}\text { OOOOO } \\
\text { TYPE, C }\end{array}$ & CALC-SILIS & $\begin{array}{c}0029 \\
C \quad \theta \theta Z E\end{array}$ & 0 & \\
\hline $\mathrm{CHN}$ & 100 & 4 & $00_{45}$ & 0000 & $\begin{array}{l}15 \\
{ }^{15} \\
c^{9}\end{array}$ & ENTS & 416 & 3 & $\begin{array}{c}8.01 \mathrm{~N} \\
\text { VOI }\end{array}$ & $\begin{array}{l}5^{2} 38.3^{\prime \prime E} \\
0.232 .385 \mathrm{C}\end{array}$ & $i_{\text {AND }}$ & $\begin{array}{l}31.32 \\
0 \text { NUMF }\end{array}$ & $\begin{array}{l}0033 \\
R \theta U S ~\end{array}$ & SMALER $\theta$ & $\begin{array}{l}1014^{\circ} \\
\text { ONES AS }\end{array}$ & $\begin{array}{l}\text { OOOO } \\
\text { WELL IF }\end{array}$ & $=L \theta_{W}=I$ & $\begin{array}{c}35^{31} \\
20-1014\end{array}$ & $4^{0} \mathrm{CM}$ & \\
\hline $\begin{array}{l}\text { CHN } \\
\text { CHN }\end{array}$ & 00 & $\begin{array}{l}4 \\
4\end{array}$ & $\begin{array}{l}0045 \\
0046\end{array}$ & $\begin{array}{l}0.00 \\
0000\end{array}$ & $\begin{array}{l}26 \\
15\end{array}$ & $\begin{array}{l}71 \\
71\end{array}$ & $\begin{array}{l}416 \\
417\end{array}$ & $\begin{array}{l}3 \\
2\end{array}$ & $\begin{array}{r}8 \cdot 01 \mathrm{~N} \\
52 \cdot 01 \mathrm{~N}\end{array}$ & $\begin{array}{ll}52 & 38.31 E \\
50 & 13.01 E\end{array}$ & 1 & $\begin{array}{l}31 \cdot 3 ? \\
31 . ? 0\end{array}$ & $\begin{array}{l}0033 \\
0034\end{array}$ & $\begin{array}{l}5126 . \\
50040\end{array}$ & $\begin{array}{l}152 . \\
895 .\end{array}$ & $\begin{array}{l}0000 \\
0000\end{array}$ & $\begin{array}{l}10 \\
10\end{array}$ & $\begin{array}{l}5369 \\
3567\end{array}$ & 0 & \\
\hline ChI & 10 & 4 & 004 & 0000 & 26 & 71 & 417 & 2 & $52 \cdot 01 \mathrm{~N}$ & $50 \quad 13.018$ & 1 & 31.20 & 0034 & 5004 . & 106. & 0000 & 10 & 3469 & 0 & \\
\hline & 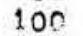 & 4 & 004 & 100 & 15 & 71 & 418 & 0 & $55.8, N$ & $5318.31 E$ & 1 & 31.03 & 0035 & 5101 . & 1064. & 0000 & 10 & 3561 & 0 & \\
\hline CHN & 100 & 4 & 0047 & Do & 26 & 71 & 418 & 0 & $55.8, N$ & $5318.31 E$ & 1 & $31 \cdot 03$ & 0035 & 5101. & 111. & 0000 & 10 & 5739 & 0 & \\
\hline $\mathrm{C}-\mathrm{N}$ & 10 & 5 & 0049 & 100 & 15 & 71 & 427 & 1 & $4.7, \mathrm{~N}$ & $533 n \cdot 0, E$ & 1 & $31 \cdot 13$ & 0037 & $510^{4}$ & 629. & 0000 & 10 & 3561 & 0 & \\
\hline $\operatorname{CHN}$ & 10 & 5 & 0049 & & $\stackrel{26}{* C^{M}}$ & $\begin{array}{l}71 \\
\text { ENTS }\end{array}$ & 427 & 1 & $\begin{array}{r}4.7 \text { IN } \\
\text { PRI }\end{array}$ & $\begin{array}{l}53 \\
\text { MARY SEDIME }\end{array}$ & NT TY & $\begin{array}{r}31.13 \\
Y P_{E} S 1 L\end{array}$ & $\begin{array}{l}0037 \\
L I C-C A L\end{array}$ & $L C^{51040}$ & 107. & 0000 & 10 & 0732 & 0 & \\
\hline $\mathrm{CHN}$ & $10 n$ & 5 & $\mathrm{OO}_{5} \mathrm{O}$ & 0000 & 15 & 71 & 428 & 1 & $56 \cdot 7^{\prime N}$ & $5^{3} 59.11 \mathrm{E}$ & 1 & 31.13 & 0038 & 5126 . & $75^{3}$. & 0000 & 10 & 5369 & 0 & \\
\hline & & 5 & & & 26 & 71 & 428 & 1 & $56.7 . N$ & $5359.11 E$ & 1 & & & & 144. & 0000 & 10 & & 0 & \\
\hline $\mathrm{CHN}$ & 00 & 5 & & 00 & 15 & 71 & $42^{y}$ & 1 & $49.0 . N$ & $5651.71 E$ & 1 & $31 \cdot 16$ & 0039 & 4782 . & 727. & OONO & 10 & 1339 & 0 & \\
\hline
\end{tabular}




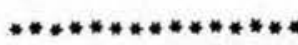

$* * * * * * * * * * * * \bullet *$

$* * * * * * * * * * * * * * *$

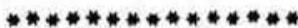

STATIAN UATA RETRIEYAL DATE: 001017:38 JUN OS STATION UATA RETRIEVAL DATE:

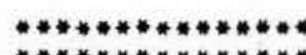

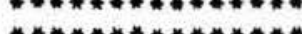

PAGE 5

PAGE 1803417591

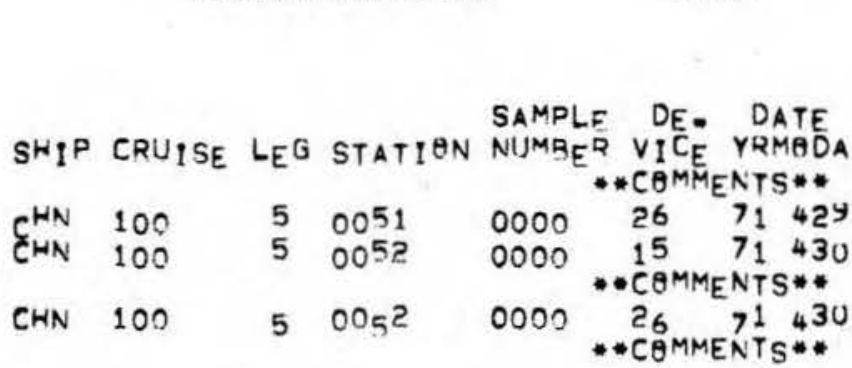

$\begin{array}{ll} & \text { CQRE } \\ & \\ \text { MENGTH DREDGE }\end{array}$

MARS- CORE OR
FIX DEN DREDGE

LENGTH DREDGE RQCK

OR PHYSIO. OR

SAMPLE DE- DATE $\begin{array}{clll}\text { FIX DEN } & \text { DREDGE } & \text { END } & \text { SAMPLE GRAPHIC SED. VITA } \\ \text { LATITUDE LONGITUDE TYPE SQUARE NUMBER DEPTH DEPTH VULUME PROV. TYPE CQDE REMARKS }\end{array}$ SEVERAL VAIDS, FLAW.IN, 1010727 CM

$149.01 \mathrm{~N} 5651.71 \mathrm{E} \quad 1 \quad 31.1600394782 .52 .0000 \quad 10 \quad 13230$

$137.01 \mathrm{~N} 5940.7$ IE 9 31.19 0040 5426. 596. $0000 \quad 15 \quad 50390$

SEC SED TYPE, CALC.SILIC $O Q Z E$

$5426.596 .0000-15-50390$

$137.01 N 5940.71 E$. $51.190040,5426.148 .0000 \quad 15 \quad 50320$

MARSDEN SQUARE *33

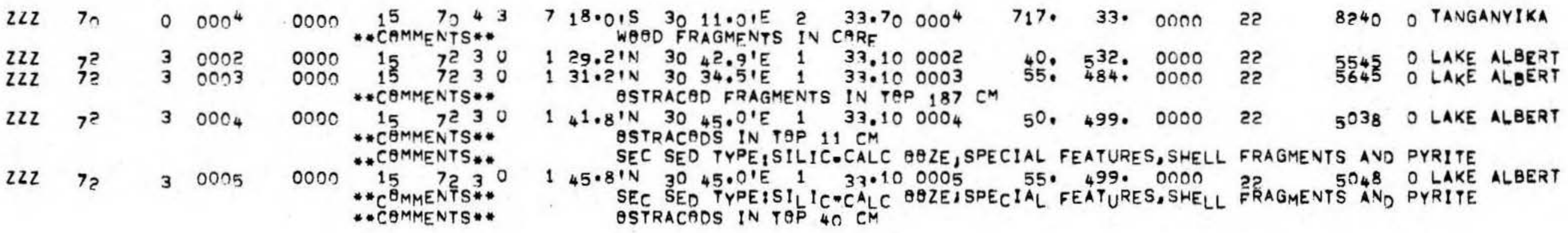

MARSDEN SQUARE \#34

\begin{tabular}{|c|c|c|c|c|c|c|c|c|c|c|c|c|c|c|c|c|c|c|c|}
\hline $\begin{array}{l}222 \\
222\end{array}$ & $\begin{array}{l}70 \\
70\end{array}$ & $\begin{array}{l}0 \\
0\end{array}$ & $\begin{array}{l}0001 \\
0006\end{array}$ & $\begin{array}{l}0000 \\
0000\end{array}$ & $\begin{array}{r}15 \\
15 \\
C \theta\end{array}$ & $\begin{array}{r}70 \\
70 \\
\text { ENTS }\end{array}$ & $\begin{array}{r}43 \\
46 \\
S * *\end{array}$ & $\begin{array}{l}6 \\
6\end{array}$ & $\begin{array}{r}.515 \\
51 \cdot 0 \text { 's } \\
\text { MIO }\end{array}$ & $\begin{array}{r}29 \\
29 \\
\text { REL }\end{array}$ & $\begin{array}{l}30.91 E \\
58.01 E \\
\text { AMINATED }\end{array}$ & $\begin{array}{l}2 \\
2 \\
\text { DIAT }\end{array}$ & $\begin{array}{r}34.69 \\
34.69 \\
\text { TOMITE }\end{array}$ & $\begin{array}{l}0001 \\
\text { OOO6 } \\
\text {, CORE. }\end{array}$ & $I^{2}$ & $\begin{array}{r}0 . \\
245^{\circ} \\
P Q Q R\end{array}$ & $\begin{array}{r}120 \\
1650 \\
\text { CONDI }\end{array}$ & $\begin{array}{l}0000 \\
0000 \\
I O N\end{array}$ & $\begin{array}{l}22 \\
22\end{array}$ \\
\hline $\begin{array}{l}22 \\
22\end{array}$ & $\begin{array}{l}70 \\
70 \\
70\end{array}$ & $\begin{array}{l}0 \\
0 \\
0\end{array}$ & $\begin{array}{l}00 \cap 6 \\
0099 \\
0010\end{array}$ & $\begin{array}{l}0000 \\
0000 \\
\text { 0no? }\end{array}$ & $\begin{array}{r}15 \\
15 \\
15 \\
\text { *CA }\end{array}$ & $\begin{array}{l}70 \\
70 \\
70 \\
\text { ENTS }\end{array}$ & $\begin{array}{rl}4 & 6 \\
4 & 7 \\
4 & 7 \\
5 * *\end{array}$ & $\begin{array}{l}6 \\
6 \\
6\end{array}$ & $\begin{array}{r}51.01 \mathrm{~s} \\
59.01 \mathrm{~s} \\
27.01 \mathrm{~s}\end{array}$ & $\begin{array}{l}29 \\
29 \\
29 \\
201\end{array}$ & $\begin{array}{l}58.01 E \\
51.01 E \\
33.01 E \\
\text { AMINATED }\end{array}$ & $\begin{array}{l}2 \\
2 \\
2 \\
\text { DIAT }\end{array}$ & $\begin{array}{r}34.69 \\
34.69 \\
34.69 \\
\text { THMITE }\end{array}$ & $\begin{array}{l}0006 \\
0009 \\
0010\end{array}$ & & $\begin{array}{l}245 . \\
2850 \\
1140\end{array}$ & $\begin{array}{r}165 . \\
66 \\
122\end{array}$ & $\begin{array}{l}0000 \\
0000 \\
0000\end{array}$ & ?? \\
\hline 222 & 70 & 0 & 0011 & 0000 & 15 & $\begin{array}{l}70 \\
\text { ENTS }\end{array}$ & 5. 6 & 6 & $\begin{array}{r}23.015 \\
C O F\end{array}$ & $E^{29}$ & $\begin{array}{l}35.01 E \\
N \text { PQAR }\end{array}$ & ${ }_{\theta N D I T}^{2}$ & $T^{34} \cdot 6 N^{69}$ & 0011 & & 201. & 123. & 0000 & ट? \\
\hline $\begin{array}{l}Z 2 Z Z \\
Z Z Z\end{array}$ & $\begin{array}{l}70 \\
70\end{array}$ & $\begin{array}{l}0 \\
0\end{array}$ & $\begin{array}{l}0014 \\
0015\end{array}$ & $\begin{array}{l}0000 \\
0000\end{array}$ & & $\begin{array}{r}70 \\
70 \\
\text { EN TS }\end{array}$ & $\begin{array}{r}410 \\
410 \\
5 * *\end{array}$ & $\begin{array}{l}5 \\
5\end{array}$ & $\begin{array}{r}35 \cdot 0 \text { is } \\
33.0 \text { V } \\
\text { VeI }\end{array}$ & $\begin{array}{r}29 \\
29 \\
0,20\end{array}$ & $\begin{array}{l}21.01 E \\
28.01 E \\
00.2290\end{array}$ & $\begin{array}{l}2 \\
2 \\
2 \\
1\end{array}$ & $\begin{array}{r}34.59 \\
34.59 \\
\text { RE IN }\end{array}$ & $\begin{array}{l}0014 \\
0015 \\
P Q \theta R\end{array}$ & CQND & $\begin{array}{r}290 \\
6330 \\
I T I \theta N\end{array}$ & $\begin{array}{r}44^{\circ} \\
229^{\circ}\end{array}$ & $\begin{array}{l}0000 \\
0000\end{array}$ & $\begin{array}{l}22 \\
22\end{array}$ \\
\hline$Z Z$ & $\begin{array}{l}70 \\
70\end{array}$ & $\begin{array}{l}0 \\
0\end{array}$ & $\begin{array}{l}0018 \\
0019\end{array}$ & $\begin{array}{l}0000 \\
0000\end{array}$ & $\begin{array}{l}15 \\
15\end{array}$ & $\begin{array}{l}70 \\
70\end{array}$ & $\begin{array}{l}411 \\
411\end{array}$ & $\begin{array}{l}5 \\
5\end{array}$ & $\begin{array}{l}45.015 \\
56.015\end{array}$ & $\begin{array}{l}29 \\
29\end{array}$ & $\begin{array}{l}25.01 E \\
32.01 E\end{array}$ & $\begin{array}{l}2 \\
2\end{array}$ & $\begin{array}{l}34.59 \\
34.59\end{array}$ & $\begin{array}{l}0018 \\
0019\end{array}$ & & $\begin{array}{r}640^{\circ} \\
361 .\end{array}$ & $\begin{array}{l}141^{\circ} \\
200 .\end{array}$ & $\begin{array}{l}0000 \\
0000\end{array}$ & \\
\hline
\end{tabular}

$\begin{array}{ll}6626 & 0 \text { TANGANYIKA } \\ 1530 & 0 \text { TANGANYIKA } \\ 1530 & 0 \text { TANGANYIKA } \\ 5211 & 0 \text { TANGANYIKA } \\ 52 ? 0 & 0 \text { TANGANYIKA } \\ 1519 & 0 \text { TANGANYIKA } \\ 1229 & 0 \text { TANGANYIKA } \\ 6519 & 0 \text { TANGANYIKA } \\ 6519 & 0 \text { TANGANYIKA } \\ 6819 & 0 \text { TANGANYIKA }\end{array}$


FIX MARS. CQRE $9 R$

CORE R日CK

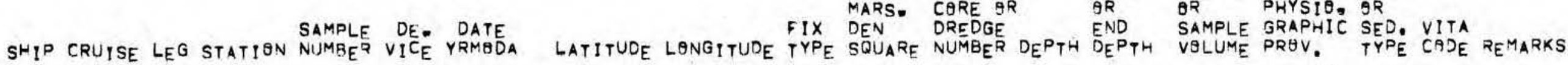

LENGTH DREDGE

OR OR PHYSIO, OR

MARSDEN SQUARE \# 41

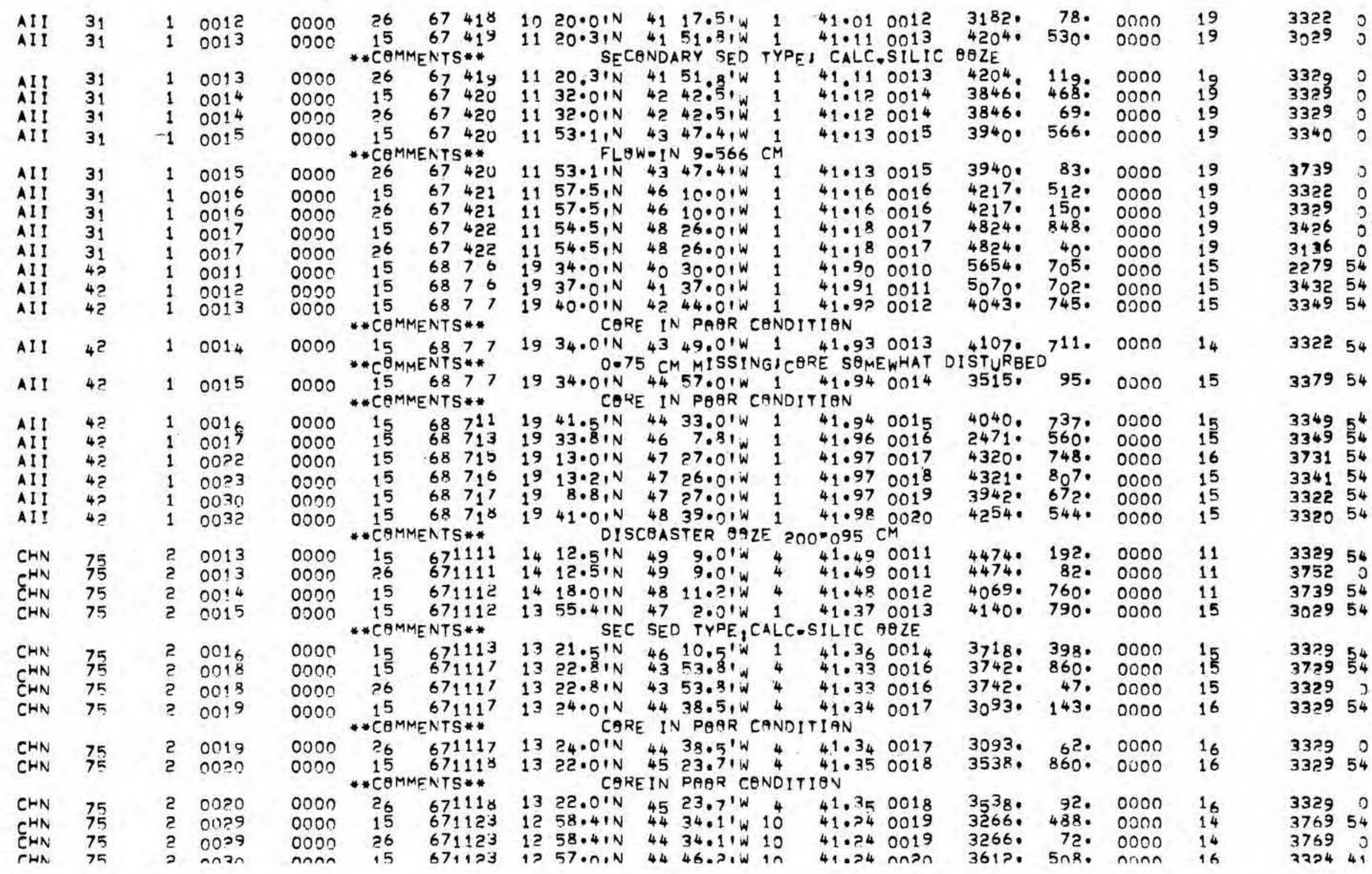




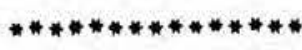

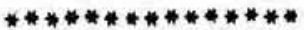

SAMPLE DE. DATE STATION UATA RETRIEVAL DATE: $17: 38$ JUN 08,177

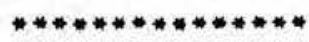

MARS. CORE $Q R$

FIX DEN MREDGE

ATITUDE LONGITUDE TYPE SQU
MARSDEN SQUARE \#41

$\begin{array}{llll}\text { CHN } & 75 & 2 & 0030 \\ \text { CHN } & 75 & 2 & 0031 \\ \text { CHN } & 75 & 2 & 0031 \\ \text { CHN } & 75 & 2 & 0032 \\ \text { CHN } & 75 & 2 & 0032 \\ \text { CHN } & 75 & 2 & 0033 \\ \text { CHN } & 75 & 2 & 0034 \\ \text { CHN } & 75 & 2 & 0034 \\ \text { CHN } & 75 & 2 & 0035 \\ \text { CHN } & 75 & 2 & 0035\end{array}$

$\begin{array}{ll}0000 & 2 \\ 0000 & 1 \\ 0000 & 2 \\ 0000 & 1 \\ 0000 & 2 \\ 0000 & 1 \\ 0000 & 1 \\ 0000 & 2 \\ 0000 & 1 \\ 0000 & 26\end{array}$

$\begin{array}{rrr}671123 & 12 & 57.01 N \\ 671124 & 13 & 0.1 N\end{array}$

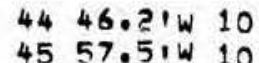

- O IN 4557.5 iw 10

$67112^{4}$

$67112^{4}$

$5 \cdot 0,0$

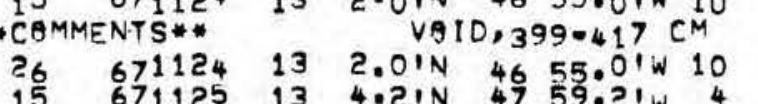

41.240020

$41 \cdot 350021$

$41 \cdot 350021$

41.260022

3229

714.0000

32760.132 .0000

CORE

OR PHYSIO. ROR

END SAMPLE GRAPHIC SED. VITA

***HOI** * COMMENTS*

(15MENTS* CORE IS PREDOMINANTLY MN GRANULES

327601470000014 4067: $45: 0000 \quad 14$ ES

4698. 900. $0000 \quad 15 \quad 343254$

4966. 150. 150000 11 343054

0000 ASH LAYER IN CORE

41.380024

$41 \cdot 290025$

41.290025

4966. 140. $0000 \quad 11$

33290

MARSDEN SQUARE \# 42

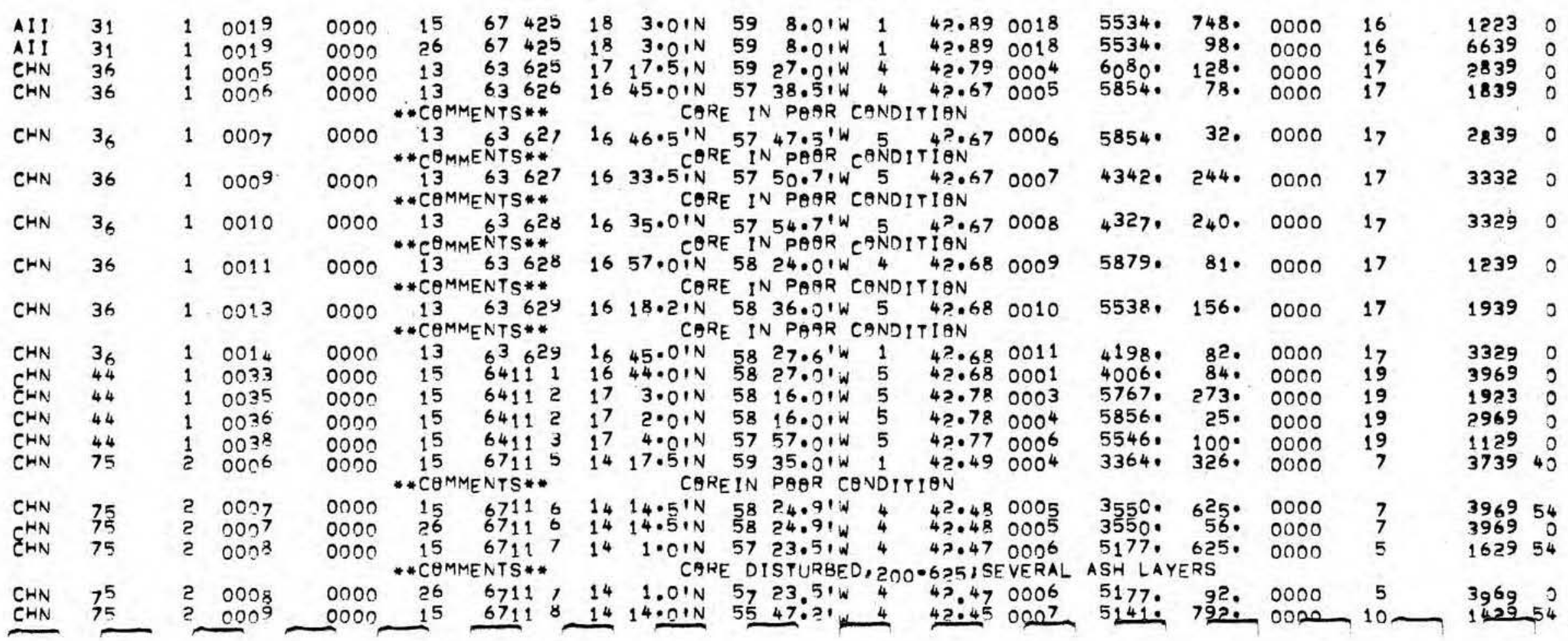




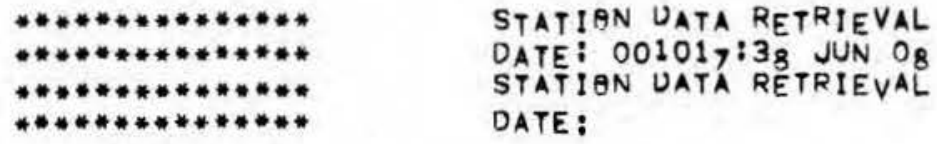

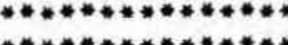

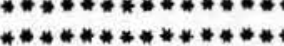

DATE: 001017:38 JUN OB

DATE:

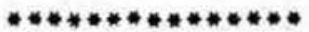

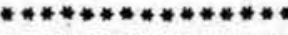

PAGE 9

* WHOI"*

$P A_{G E} 1803417591$

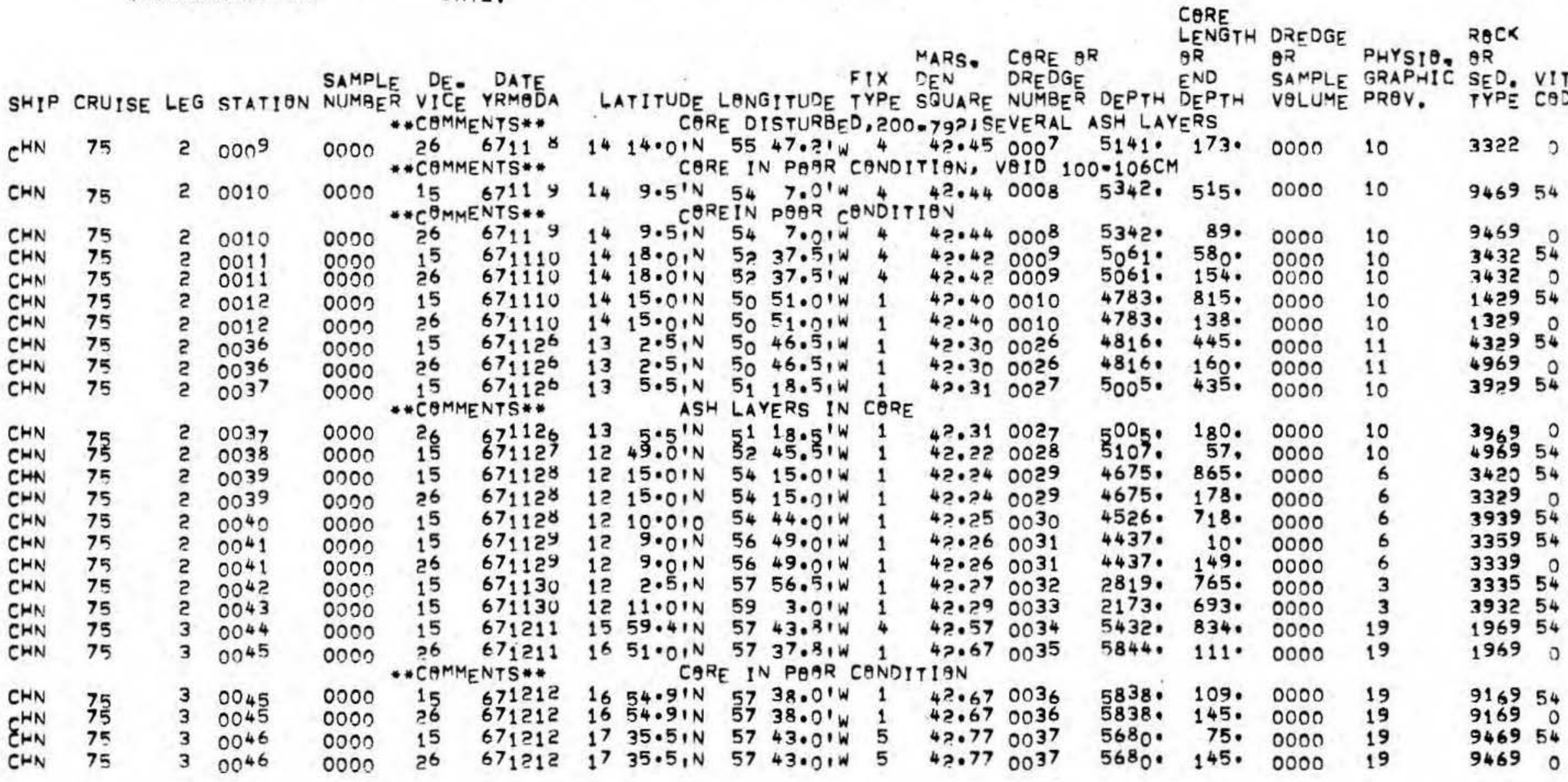

MARSDEN SQUARE \#43

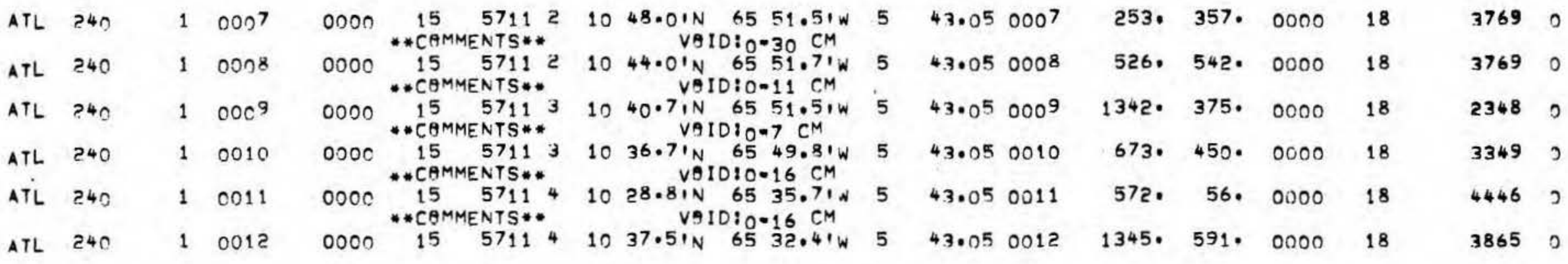




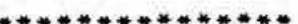

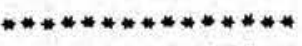

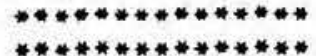

STATIAN UATA RETRIEVAL DATE: $001017: 38$ JUN O8 DATE: STATION UATA RETRIEVAL

PAGE 10

* WWH I**

PAGE 1803417591

CORE

LENGTH DREDGE RECK

MARS. CORE OR ${ }^{-}$LENGTH DREDGE PHYSIO. $O R$

SAMPLE DE- DATE FIX DEN DREDGE ENO SAMPLE GRAPHIC SED. VITA

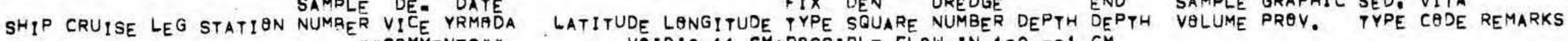
* COMMENTS** VOID:0-11 CM,PESSIBLE FLOW.IN,190-591 CM

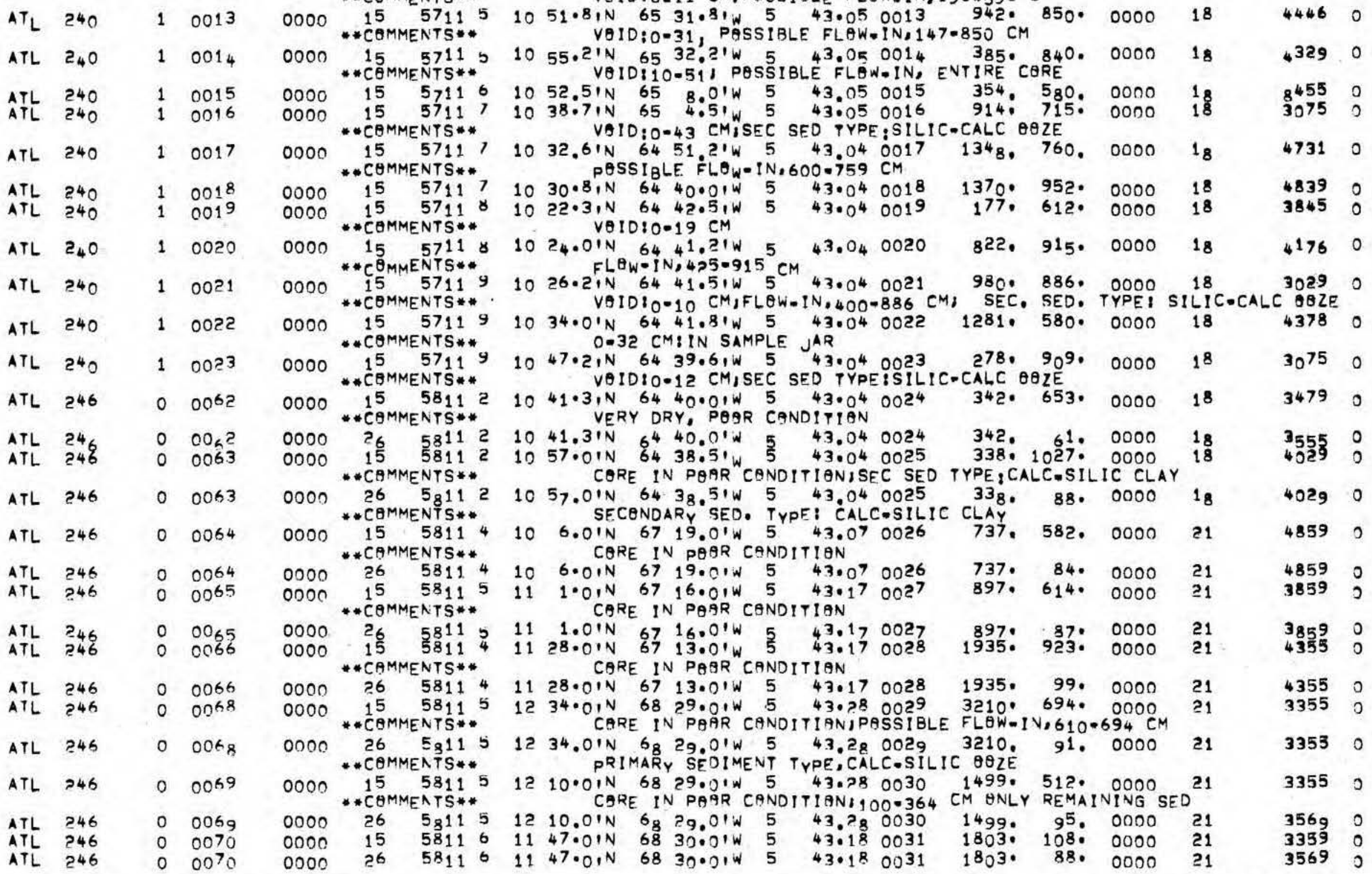




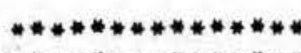

$* * * * * * * * * * * * * * *$
STATION UATA RETRIEVAL DATE: $17: 38$ JUN 08,177

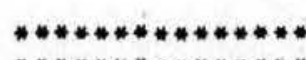

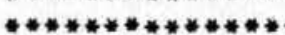

PAGE 11 **NHOI**

MENGTH DREDGE PHYSIO ROCK

SAMPLE DE. DATE FIX DEN DREDGE CORE QR END SAMPLE GRAPHIC SED. VITA

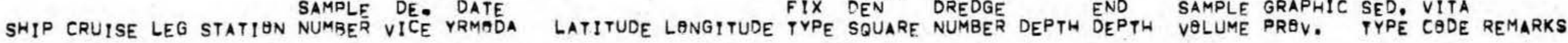

MARSDEN SQUARE *43

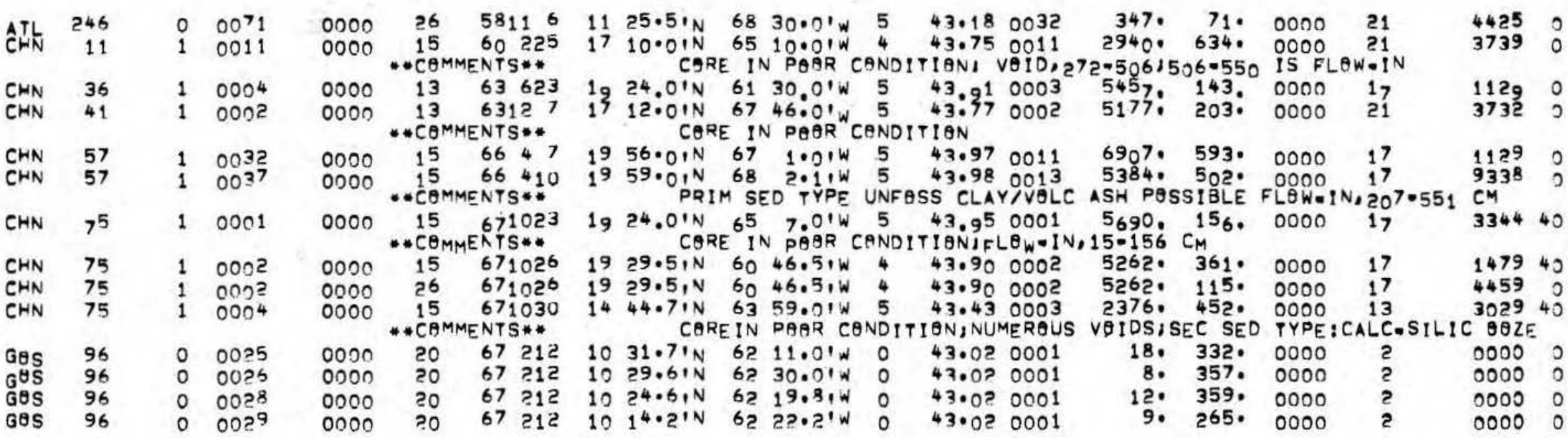

$M_{A}$ RDEN SQUARE * 44

$\begin{array}{llll}\text { ATL } & 246 & 0 & 0214 \\ \text { ATL } & 246 & 0 & 0215 \\ \text { ATL } & 246 & 0 & 0211 \\ \text { ATL } & 246 & 0 & 0217 \\ \text { ATL } & 246 & 0 & 0218 \\ \text { ATL } & 246 & 0 & 0219 \\ \text { CHN } & 41 & 1 & 0003 \\ \text { CHN } & 41 & 1 & 0004 \\ \text { GOS } & 96 & 0 & 0001 \\ \text { GUS } & 96 & 0 & 0002 \\ \text { GOS } & 96 & 0 & 0014\end{array}$

$0000 \quad 26 \quad 581120 \quad 1115.01 N \quad 7135.01 \mathrm{~W} \quad 5 \quad 44.110033$ 0000 0000 0000 0000 Onoc 0000 0000 0000 0000 0000

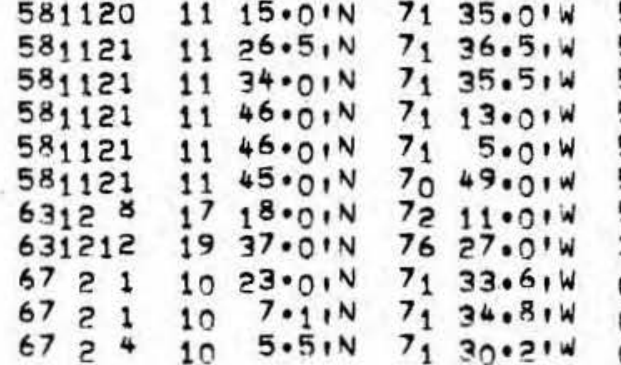
$44 \cdot 110034$ $\begin{array}{ll}44 \cdot 11 & 0035 \\ 44.11 & 0036\end{array}$ 44.110036 $44 \cdot 110037$ $44 \cdot 100038$ $44.7 ? 0002$ 4.960004 44.010001 44.010001 44.010001

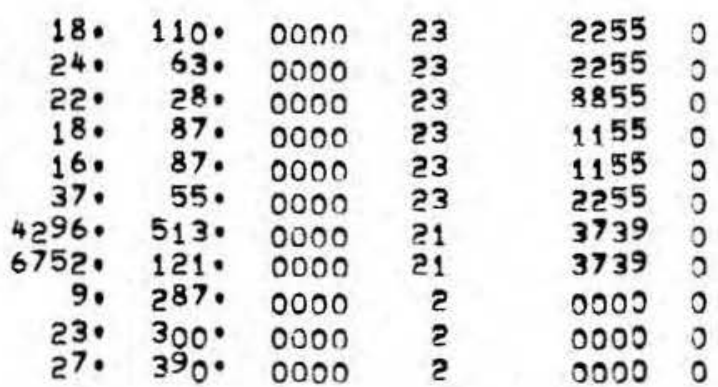

MARSDEN SQUARE \# 45

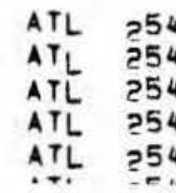

30330 30330 $\begin{array}{ll}3 & 0331 \\ 3 & 0331\end{array}$ 30333
0050

0000

0000

0000

$\begin{array}{ll}3339 & 0 \\ 3339 & 0 \\ 7339 & 0 \\ 3739 & 0 \\ 3849 & 0 \\ 3399 & 0\end{array}$


$* * * * * * * * * * * * * * *$

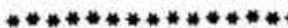

STATION UATA RETRIEVAL DATE: $17: 38$ JUN 08,177

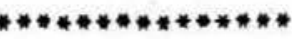

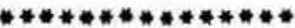

PAGE 12 **WHOI**
MARS. CQRE $Q R$ LENGTH DREDGE PHYSIO.

FIX DEN DREDGE END SAMPLE GRAPHIC SED, VITA

SAMPLE DE- DATE SHIP CRUISE LEG STATION NUMBER VICE YRMAOA LATITUDE LONGITUDE TYPE SQUARE NUMBER DEPTH DEPTH VOLUME PROV. TYPE CODE REMARKS

MARSDEN SQUARE \# 45

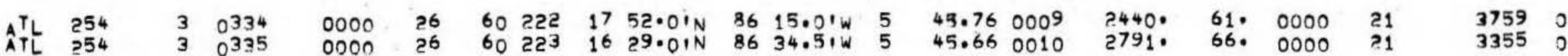

MARSDEN SQUARE \# 65

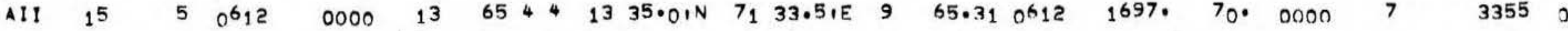

MARSDEN SQUARE \#66

$\begin{array}{llllll}\text { A11 } & 15 & 5 & 596 & 0000 & 1 \\ \text { A1 } & 15 & 5 & 0606 & 0000 & 2 \\ \text { A1 1 } & 15 & 5 & 0607 & 0000 & 1 \\ \text { AII } & 15 & 5 & 0608 & 0000 & 1 \\ & 15 & 5 & 0610 & 0000 & 1\end{array}$

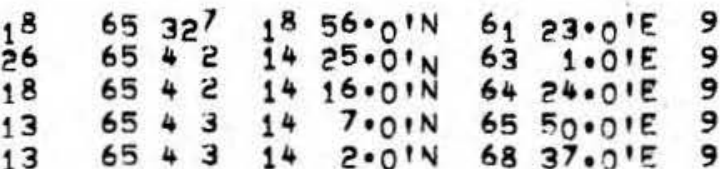

66.810596

66.430020

$66.44060^{7}$

66.450608

66.480610

369.4 $120^{\circ} 0000$

$4023^{\circ} 63^{\circ} 0000$

3965. 87. 0000

$\begin{array}{lll}3957 . & 74, & 0000 \\ 4075, & 28 . & 0000\end{array}$

$24 \quad 3359$

$\begin{array}{lll}3359 & 0 \\ 4 & 3339 & 0\end{array}$

$M_{A}$ RSDEN SQU

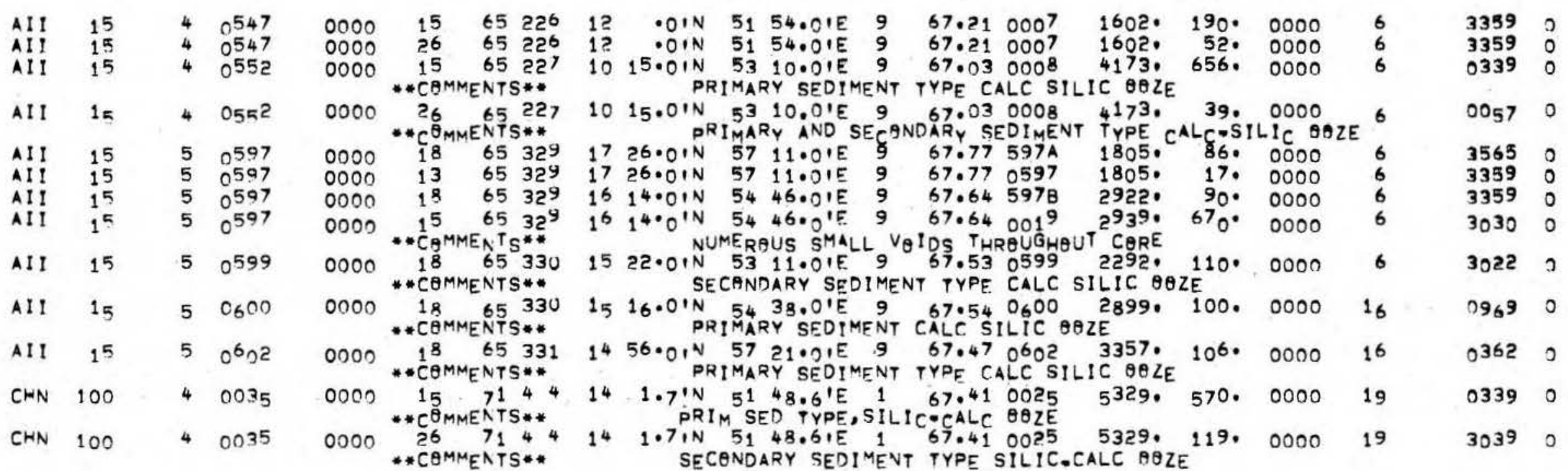



$* * * * * * * * * * * * * * *$
$* * * * * * * * * * * * * *$

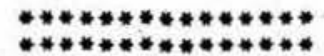

PAGE 13

**WH9I**

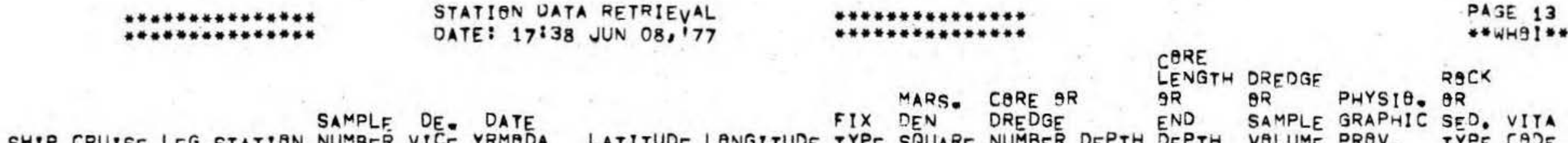

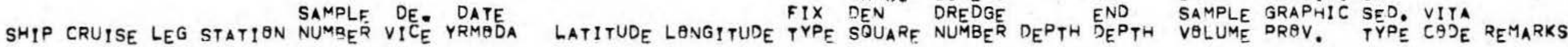

MARSDEN SQUARE \# 68

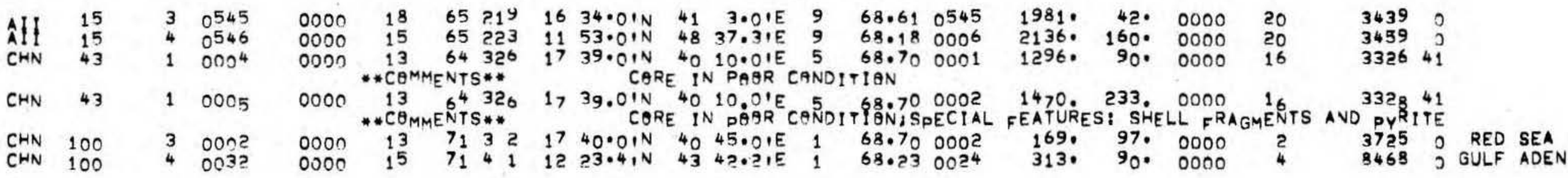

MARSDEN SQUARE \#69

$\begin{array}{lllll}\text { CHN } & 61 & 7 & 0152 \\ \text { CHN } & 61 & 7 & 015 ? \\ \text { CHN } & 61 & 7 & 0153 \\ \text { CHN } & 61 & 7 & 0153 \\ \text { CHN } & 61 & 7 & 0154 \\ \text { CHN } & 61 & 7 & 0154 \\ \text { CHN } & 61 & 7 & 0155 \\ \text { CHN } & 61 & 7 & 0155 \\ \text { CHN } & 100 & 3 & 0001 \\ \text { CHN } & 100 & 3 & 0003 \\ \text { CHN } & 100 & 3 & 0003 \\ \text { CHN } & 100 & 3 & 0005 \\ \text { CHN } & 100 & 3 & 0005 \\ \text { CHN } & 100 & 3 & 0005\end{array}$

\begin{tabular}{|c|c|c|c|c|c|c|c|c|c|c|c|c|c|c|c|c|c|}
\hline $\begin{array}{l}0000 \\
0000 \\
0000\end{array}$ & $\begin{array}{l}15 \\
26 \\
15 \\
\text { CAMME }\end{array}$ & $\begin{array}{l}6611 \\
6611 \\
6611 \\
\text { NTS. }\end{array}$ & $\begin{array}{l}2 \\
2 \\
4\end{array}$ & $\begin{array}{l}19 \\
19 \\
19\end{array}$ & $\begin{array}{l}48.5, N \\
48.5, N \\
43.0, N\end{array}$ & $\begin{array}{l}38 \\
38 \\
38 \\
k=1 N\end{array}$ & $\begin{array}{l}30001 E \\
30001 E \\
41.01 E \\
1,5,3=75\end{array}$ & $\begin{array}{l}1 \\
1 \\
1 \\
\end{array}$ & $\begin{array}{l}69.98 \\
69.98 \\
69.98\end{array}$ & $\begin{array}{l}0000 \\
0000 \\
0000\end{array}$ & $\begin{array}{l}2359 \circ \\
23590 \\
27040\end{array}$ & $\begin{array}{r}711 . \\
45 . \\
755 .\end{array}$ & $\begin{array}{l}0000 \\
0000 \\
0000\end{array}$ & $\begin{array}{l}16 \\
16 \\
21\end{array}$ & $\begin{array}{l}7759 \\
3731 \\
3439\end{array}$ & $\begin{array}{l}41 \\
41 \\
41\end{array}$ & \\
\hline $\begin{array}{l}0000 \\
0000\end{array}$ & $\begin{array}{l}26 \\
15 \\
+C A M M E\end{array}$ & $\begin{array}{r}6611 \\
6611 \\
\text { ENTS. }\end{array}$ & $\begin{array}{r}4 \\
4\end{array}$ & $\begin{array}{l}19 \\
19\end{array}$ & $\begin{array}{r}43.01 \mathrm{~N} \\
34.01 \mathrm{~N} \\
\text { NUN }\end{array}$ & $\begin{array}{r}38 \\
38 \\
E R \theta U\end{array}$ & $\begin{array}{l}41.01 E \\
59.51 E \\
\text { S VAIDS }\end{array}$ & $\begin{array}{l}1 \\
1 \\
I N\end{array}$ & $\begin{array}{l}69.98 \\
69.98 \\
\text { THE CAR }\end{array}$ & $\begin{array}{r}0000 \\
0000 \\
R E\end{array}$ & $\begin{array}{l}27040 \\
1275^{\circ}\end{array}$ & $\begin{array}{l}150^{\circ} \\
890^{\circ}\end{array}$ & $\begin{array}{l}0000 \\
0000\end{array}$ & $\begin{array}{l}? 1 \\
21\end{array}$ & $\begin{array}{l}3732 \\
3439\end{array}$ & 41 & \\
\hline $\begin{array}{l}0000 \\
0000 \\
0000 \\
0000 \\
0000 \\
0000\end{array}$ & $\begin{array}{l}26 \\
15 \\
26 \\
13 \\
15 \\
26 \\
C \theta M M E\end{array}$ & $\begin{array}{l}6611 \\
6611 \\
6611 \\
713 \\
713 \\
71 \\
71 \\
\text { NTS }\end{array}$ & $\begin{array}{l}4 \\
5 \\
5 \\
2 \\
3 \\
3 \\
4\end{array}$ & $\begin{array}{l}19 \\
19 \\
19 \\
17 \\
18 \\
18\end{array}$ & $\begin{array}{l}34.01 \mathrm{~N} \\
23.5, \mathrm{~N} \\
23.5, \mathrm{~N} \\
2.6, \mathrm{~N} \\
9.0, \mathrm{~N} \\
9.01 \mathrm{~N} \\
\text { SPE }\end{array}$ & $\begin{array}{l}38 \\
38 \\
38 \\
39 \\
39 \\
39 \\
\text { CIAL }\end{array}$ & $\begin{array}{l}59.51 E \\
54.01 E \\
54.01 E \\
53.01 E \\
53.01 E \\
53.01 E \\
\text { FEATUR }\end{array}$ & $\begin{array}{l}1 \\
1 \\
1 \\
1 \\
1 \\
1\end{array}$ & $\begin{array}{l}69.98 \\
69.98 \\
69.98 \\
69.79 \\
69.89 \\
69.89 \\
\text { RADED B }\end{array}$ & $\begin{array}{l}0000 \\
0000 \\
0000 \\
0001 \\
0003 \\
0003 \\
\text { BEDDING }\end{array}$ & $\begin{array}{l}1275 \circ \\
2046 . \\
2046 \circ \\
176 . \\
13740^{\circ} \\
13740^{\circ} \\
\text { AND } 5\end{array}$ & $\begin{array}{l}920^{\circ} \\
4240^{\circ} \\
1170 \\
420^{\circ} \\
790^{\circ} \\
1120 \\
M 10 L 1\end{array}$ & $\begin{array}{l}0000 \\
0000 \\
0000 \\
0000 \\
0000 \\
0000 \\
\text { DIFIED. }\end{array}$ & $\begin{array}{l}21 \\
21 \\
21 \\
2 \\
16 \\
16 \\
\text { INDURATED }\end{array}$ & $\begin{array}{l}3329 \\
4817 \\
4867 \\
3359 \\
3868 \\
3232 \\
0 \text { SED }\end{array}$ & $\begin{array}{c}41 \\
41 \\
41 \\
0 \\
0 \\
0 \\
\text { IMENT }\end{array}$ & $\begin{array}{l}\text { RED SEA } \\
\text { RED SEA } \\
\text { RED SEA } \\
\text { TT }\end{array}$ \\
\hline 0000 & $\begin{array}{l}15 \\
\text { COMME }\end{array}$ & $\begin{array}{ll} & 71 \\
\text { ENTS* }\end{array}$ & 3 & 19 & $5.01 \mathrm{~N}$ & $\begin{array}{r}39 \\
C ! A_{L}\end{array}$ & $\begin{array}{l}59.51 E \\
\text { FEATURE }\end{array}$ & Es: & $\begin{array}{l}69.99 \\
H E_{L L} F R\end{array}$ & $\begin{array}{l}\text { O004 } \\
\text { RAGMENTS }\end{array}$ & $\begin{array}{l}328^{\circ} \\
\text { AND }\end{array}$ & 537. & $\begin{array}{l}\text { OOOO } \\
\text { THIFIED }\end{array}$ & SEDIMENT & 3238 & 0 & RED SEA \\
\hline $\begin{array}{l}0000 \\
0000\end{array}$ & $\begin{array}{l}26 \\
13\end{array}$ & $\begin{array}{ll}71 & 3 \\
71 & 3\end{array}$ & $\begin{array}{l}3 \\
5\end{array}$ & $\begin{array}{l}19 \\
19\end{array}$ & $\begin{array}{r}5 \cdot 01 \mathrm{~N} \\
38 \cdot 0 . \mathrm{N}\end{array}$ & $\begin{array}{l}39 \\
38\end{array}$ & $\begin{array}{l}59.51 E \\
36.2, E\end{array}$ & $\begin{array}{l}1 \\
1\end{array}$ & $\begin{array}{l}69.99 \\
69.98\end{array}$ & $\begin{array}{l}000^{4} \\
0005\end{array}$ & $\begin{array}{r}328, \\
2010 .\end{array}$ & $\begin{array}{l}108^{\circ} \\
101 .\end{array}$ & $\begin{array}{l}0000 \\
0000\end{array}$ & $\begin{array}{l}16 \\
16\end{array}$ & $\begin{array}{l}3329 \\
3865\end{array}$ & $\begin{array}{l}0 \\
0\end{array}$ & $\begin{array}{l}\text { RED SEA } \\
\text { RED SEA }\end{array}$ \\
\hline
\end{tabular}

MARSDEN SQUARE \# 75

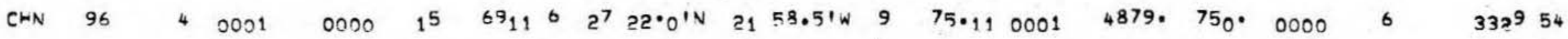

MARSDEN SQUARE \#76

$\begin{array}{llrl}\text { CHN } & 61 & 10 & 0191 \\ \text { CHN } & 61 & 10 & 0172 \\ \text { CHN } & 96 & 4 & 0003 \\ \text { CHN } & 96 & 4 & 0013\end{array}$

$0000 \quad 15 \quad 66126 \quad 2642.01 \mathrm{~N} \quad 3923.01 \mathrm{~W} 1$

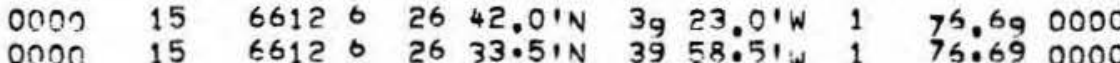

$0000 \quad 15 \quad 66120026 \quad 33.51 N \quad 3958.51$ ' $15 \quad 75.690000$

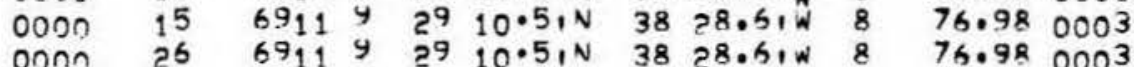

4279. 760. 0000

4356. 160. 0000

4760.558 .0000

$4760.10^{9} .0000$

$\begin{array}{lll}11 & 3322 & 41 \\ 11 & 3329 & 41 \\ 15 & 3731 & 54 \\ 15 & 3329 & 54\end{array}$




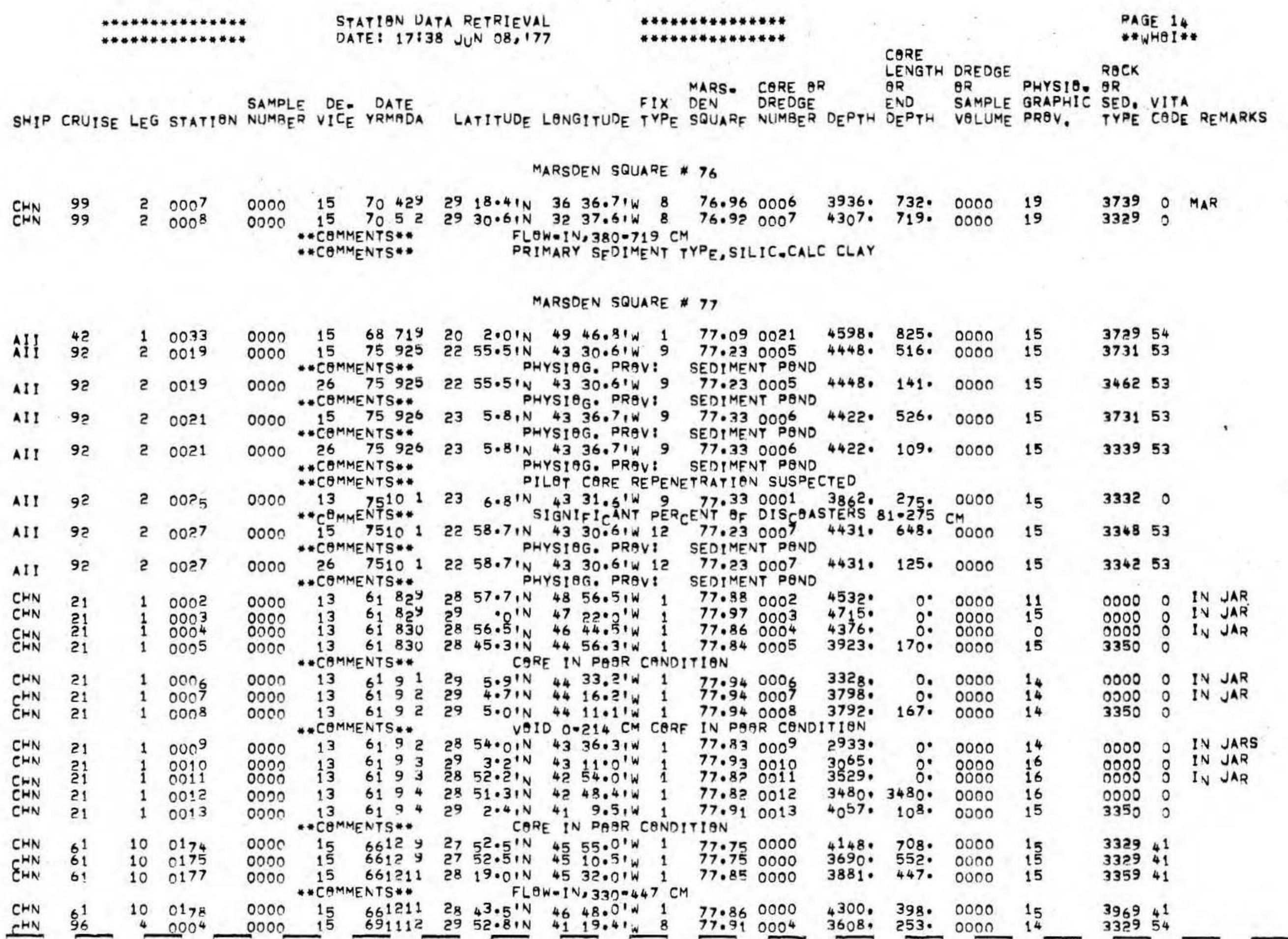


PAGE 15

**WHOl **

MARS. CQRE $\theta R \quad$ LENGTH OREDGE PHYSIO. $9 R$

$\begin{array}{llll} & \text { MARS. CORE OR } & \text { OR } & \text { OR } \\ \text { SAMPLF DE- DATE PIX DEN DREDGE } & \text { END SAMPLE GRAPHIC SED. VITA }\end{array}$

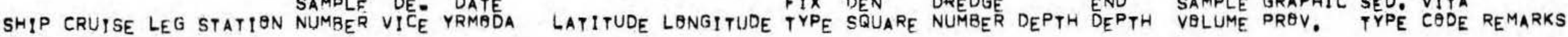

$\begin{array}{llll}\text { CHN } & 96 & 4 & 0004 \\ \text { CHN } & 99 & 2 & 0025 \\ \text { CHN } & 99 & 2 & 0006 \\ & & & \\ & & & \\ \text { III } & 31 & 1 & 0020 \\ \text { III } & 31 & 1 & 0020 \\ \text { AII } & 4 ? & 1 & 0034 \\ \text { AII } & 4 ? & 1 & 0035 \\ \text { AII } & 42 & 1 & 0039 \\ \text { AII } & 42 & 1 & 0039 \\ \text { AII } & 42 & 1 & 0041 \\ \text { III } & 4 ? & 1 & 0042 \\ \text { AII } & 4 ? & 1 & 0042 \\ \text { AII } & 42 & 1 & 0376 \\ \text { CHN } & 21 & 1 & 0001 \\ \text { CHN } & 39 & 2 & 0001 \\ \text { CHN } & 39 & 2 & 0094 \\ \text { CHN } & 39 & 2 & 0007 \\ \text { CHN } & 39 & 2 & 0009 \\ \text { CHN } & 39 & 2 & 0010\end{array}$

\section{0} 0000

0000
MARSDEN SQUARE \#77

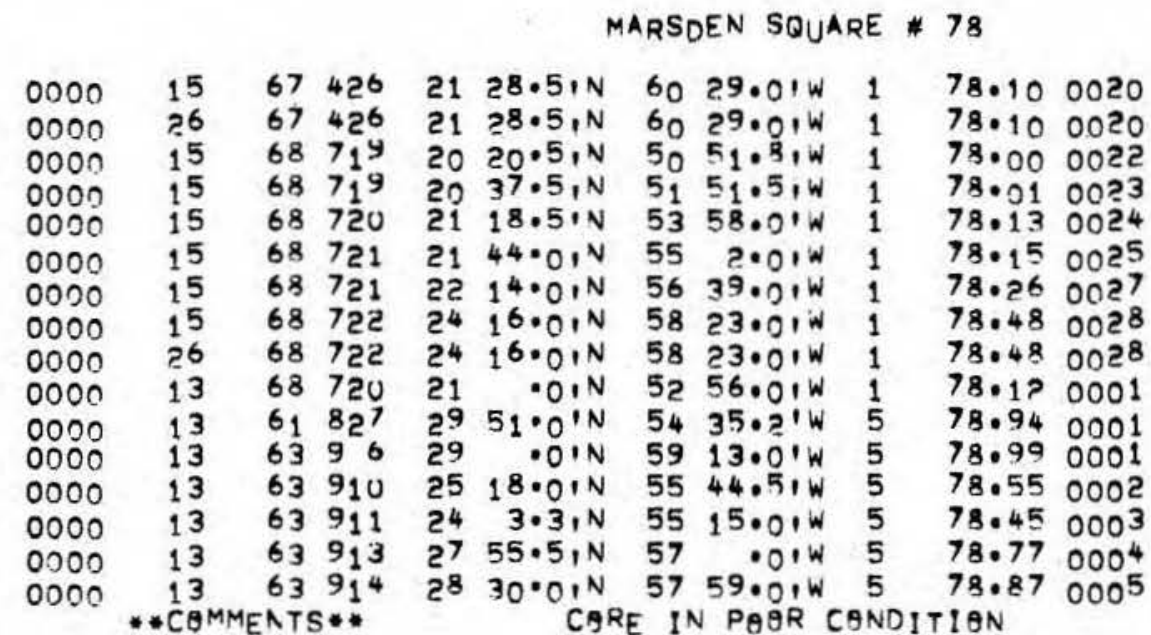

MARSDEN SQUARE \# 79

$\begin{array}{lrrrrrr}3608 . & 40^{\circ} & 0000 & 14 & 3329 & 54 & \\ 3318 . & 830^{\circ} & 0000 & 14 & 3731 & 0 & \\ 3301, & 1940^{\circ} & 0000 & 14 & 3373 & 0 & \text { MAR }\end{array}$

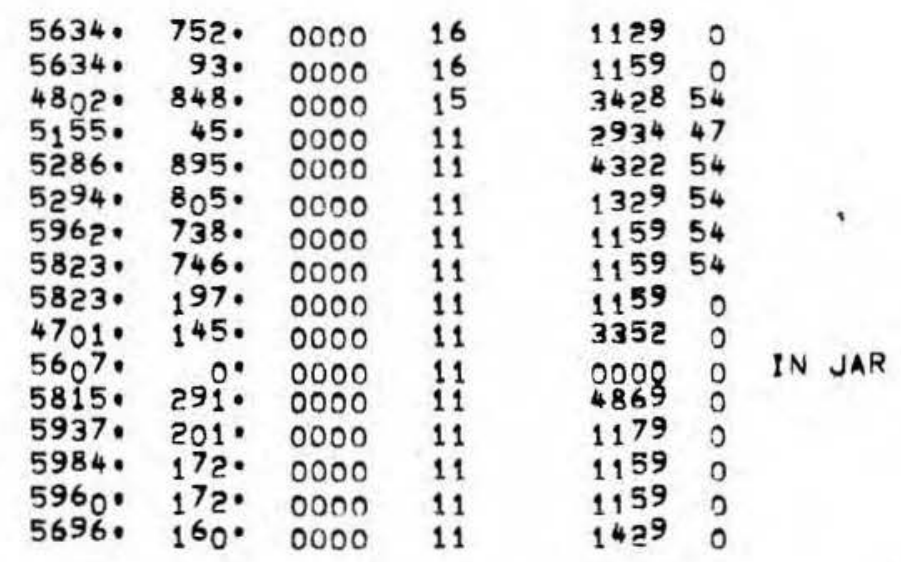

\begin{tabular}{|c|c|c|c|c|c|c|c|c|c|c|c|c|c|c|}
\hline AII & 31 & 1 & 0021 & 0000 & $\begin{array}{r}15 \\
* C \theta r\end{array}$ & $\begin{aligned} & 67 \\
& \text { ENTS }\end{aligned}$ & 428 & 26 & $\begin{array}{r}28.01 \mathrm{~N} \\
\text { CER }\end{array}$ & $\begin{array}{r}61 \\
E I N\end{array}$ & $\begin{array}{l}41.51 W \\
P Q \theta R C \theta\end{array}$ & $\begin{array}{l}1 \\
\text { NDITI }\end{array}$ & $\begin{array}{l}79.61 \\
\text { ION }\end{array}$ & 0021 \\
\hline AII & 31 & 1 & 0022 & 0000 & 15 & 67 & 429 & 27 & $29.5^{1 \mathrm{~N}}$ & $6^{3}$ & $5.5^{1} \mathrm{w}$. & 1 & $79 \cdot 73$ & 0022 \\
\hline AII & 42 & 1 & 0043 & 0000 & 15 & 68 & 723 & 27 & $16.01 \mathrm{~N}$ & 60 & $37.51 \mathrm{w}$ & 1 & 79.70 & 0029 \\
\hline AII & $4 ?$ & 1 & 0043 & 0000 & 26 & 68 & 723 & 27 & $16.01 \mathrm{~N}$ & 60 & $37.51 w$ & 1 & 79.70 & 0029 \\
\hline AII & 4? & 1 & 0044 & 0000 & 15 & 63 & 724 & 29 & $4.0, \mathrm{~N}$ & 6? & $.01 \mathrm{~W}$ & 1 & 79.92 & 0030 \\
\hline AI! & 60 & 8 & $000^{3}$ & 0000 & 13 & 71 & 84 & 23 & $49.0, \mathrm{~N}$ & 69 & $31.8, w$ & 9 & 79.39 & $002 C$ \\
\hline AII & 60 & 8 & $00 ? 5$ & onon & 13 & $7 i$ & 85 & 24 & $8 \cdot 3, N$ & 68 & $20.31 \mathrm{w}$ & 9 & 79.48 & $003 \mathrm{C}$ \\
\hline AII & 60 & 8 & $000^{6}$ & 0000 & 14 & $7 i$ & $8 b$ & 23 & $56.61 \mathrm{~N}$ & 68 & $30.61 \mathrm{w}$ & 9 & $79 \cdot 38$ & 0001 \\
\hline AII & 60 & 8 & 0007 & 0000 & 14 & 71 & 86 & 23 & $46.01 \mathrm{~N}$ & 68 & $43.51 \mathrm{w}$ & 9 & 79.38 & 0002 \\
\hline AII & 60 & 8 & $\cos ^{8}$ & 0000 & 13 & 71 & 86 & 23 & $35.0, \mathrm{~N}$ & 68 & $54.0 .1 \mathrm{~W}$ & 5 & $73 \cdot 38$ & $000^{4}$ \\
\hline AII & 60 & 8 & $\cos ^{\circ}$ & 0000 & 14 & 71 & 86 & 23 & $35 \cdot 6, N$ & 68 & $55.7, W$ & 5 & 79.38 & $000^{3}$ \\
\hline A I I & 60 & 8 & $000^{9}$ & ODCO & 14 & 71 & 81 & 23 & $25 \cdot 6, N$ & 69 & $4.51 \mathrm{~W}$ & 9 & 79.39 & $000^{4}$ \\
\hline A I & 60 & 8 & $000^{9}$ & $000 n$ & 13 & 71 & $\begin{array}{ll}8 & 6 \\
8 & 1\end{array}$ & 23 & $2400, N$ & $\begin{array}{l}69 \\
<0\end{array}$ & $2001 \mathrm{w}$ & $\begin{array}{l}5 \\
5\end{array}$ & $\begin{array}{l}79.39 \\
70 .=0\end{array}$ & $000^{5}$ \\
\hline
\end{tabular}

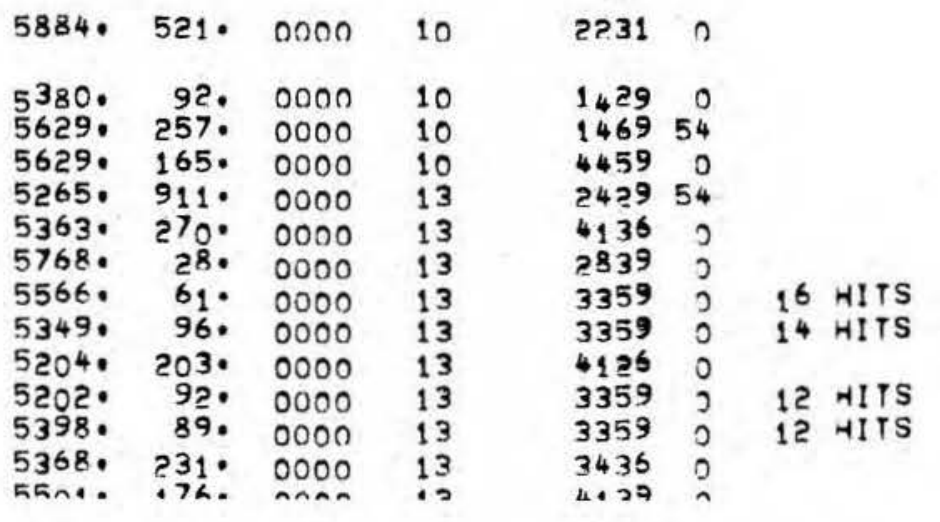


MARS. CORE OR SHIP CRUISE LEG STATION NUMPLE DE VICE YRMODA $\begin{array}{ll}\text { FIX DEN } & \text { MARE OR } \\ \text { TYPE SQUARE NUMBER }\end{array}$
CARE

LENGTH DREDGE RACK

OR OR PHYSIO. OR

$\begin{array}{lll}\text { OR } & \text { OR } & \text { PHYSIOE OR } \\ \text { END } & \text { SAMPLE GRAPHIC SED. VITA }\end{array}$

$\begin{array}{lll}\text { LIX DENTTUDE LONGITUDE TYPE SQUARE NUMBER DEPTH DEPTH VOLUME PROV. TYPE CODE REMARKS } & \text { DREDGE }\end{array}$

MARSDEN SQUARE \#79

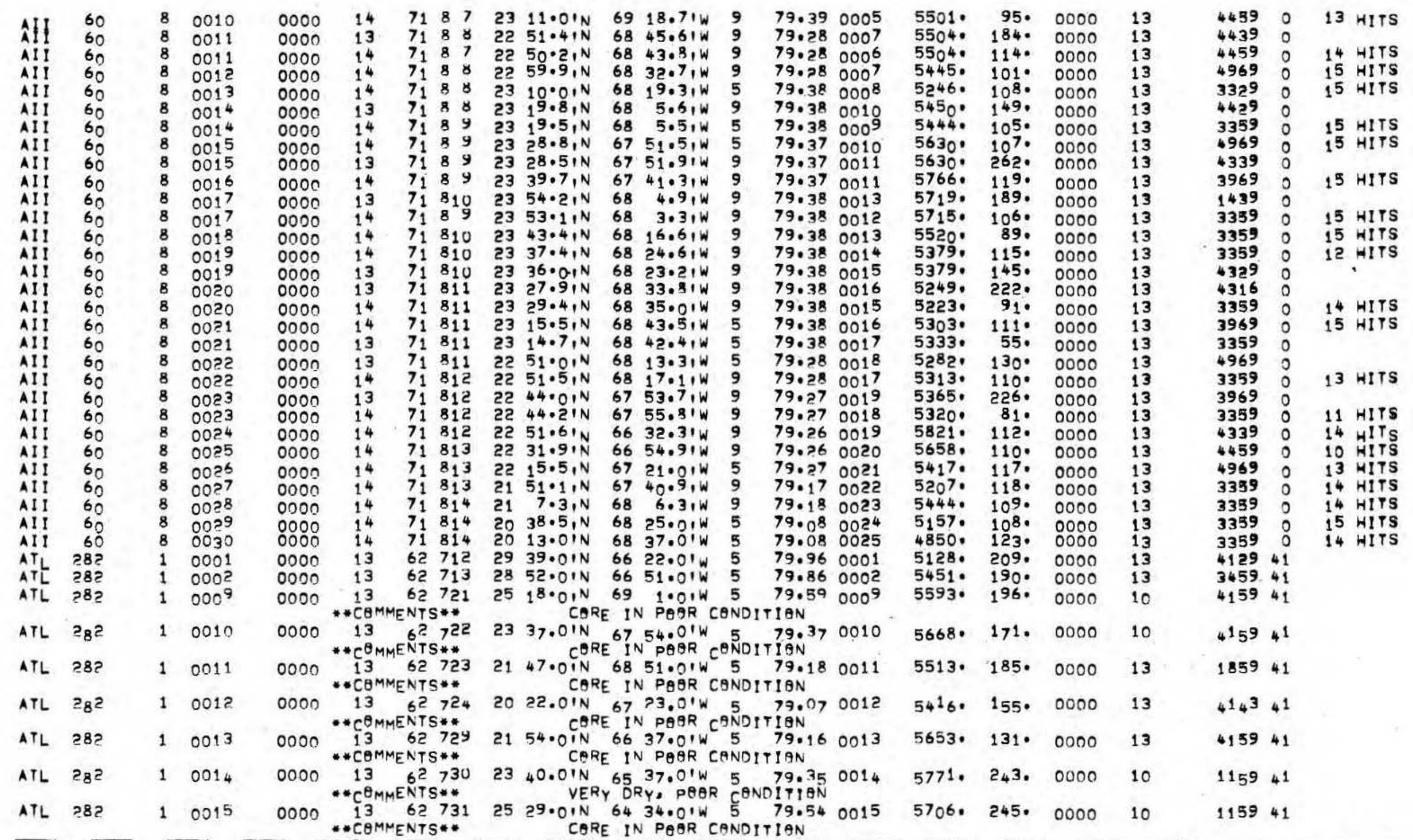




$\begin{array}{llll}\text { ATL } & 282 & 1 & 0016 \\ \text { ATL } & 282 & 1 & 0017 \\ \text { ATL } & 282 & 1 & 0018 \\ \text { ATL } & 282 & 1 & 0020 \\ \text { ATL } & 28 ? & 1 & 0021 \\ \text { ATL } & 282 & 1 & 0022 \\ \text { CHN } & 11 & 1 & 0012 \\ \text { CHN } & 19 & 1 & 0002 \\ \text { CHN } & 19 & 1 & 0003 \\ \text { CHN } & 36 & 1 & 0001 \\ \text { CHN } & 36 & 1 & 0003 \\ \text { CHN } & 39 & 2 & 0011 \\ \text { CHN } & 39 & 2 & 0015 \\ \text { CHN } & 47 & 1 & 0030 \\ \text { CHN } & 57 & 1 & 0014 \\ \text { CHN } & 57 & 1 & 0014 \\ \text { CHN } & 57 & 1 & 0016 \\ \text { CHN } & 57 & 1 & 0016 \\ \text { CHN } & 57 & 1 & 0018 \\ \text { CHN } & 57 & 1 & 0021 \\ \text { CHN } & 57 & 1 & 00 ? 2 \\ \text { CHN } & 57 & 1 & 0022 \\ \text { CHN } & 57 & 1 & 0023 \\ \text { CHN } & 57 & 1 & 0023 \\ \text { CHN } & 57 & 1 & 0024 \\ \text { CHN } & 57 & 1 & 0024 \\ \text { CHN } & 57 & 1 & 0025 \\ \text { CHN } & 57 & 1 & 0023 \\ \text { CHN } & 57 & 1 & 0029 \\ \text { CHN } & 57 & 1 & 0036 \\ \text { CHN } & 57 & 1 & 0038 \\ \text { KNR } & 25 & 1 & 0001 \\ \text { KNR } & 25 & 1 & 0002 \\ \text { KNR } & 25 & 1 & 0002 \\ \text { KNR } & 25 & 1 & 0023 \\ \text { KNR } & 25 & 1 & 0003 \\ & & & \end{array}$

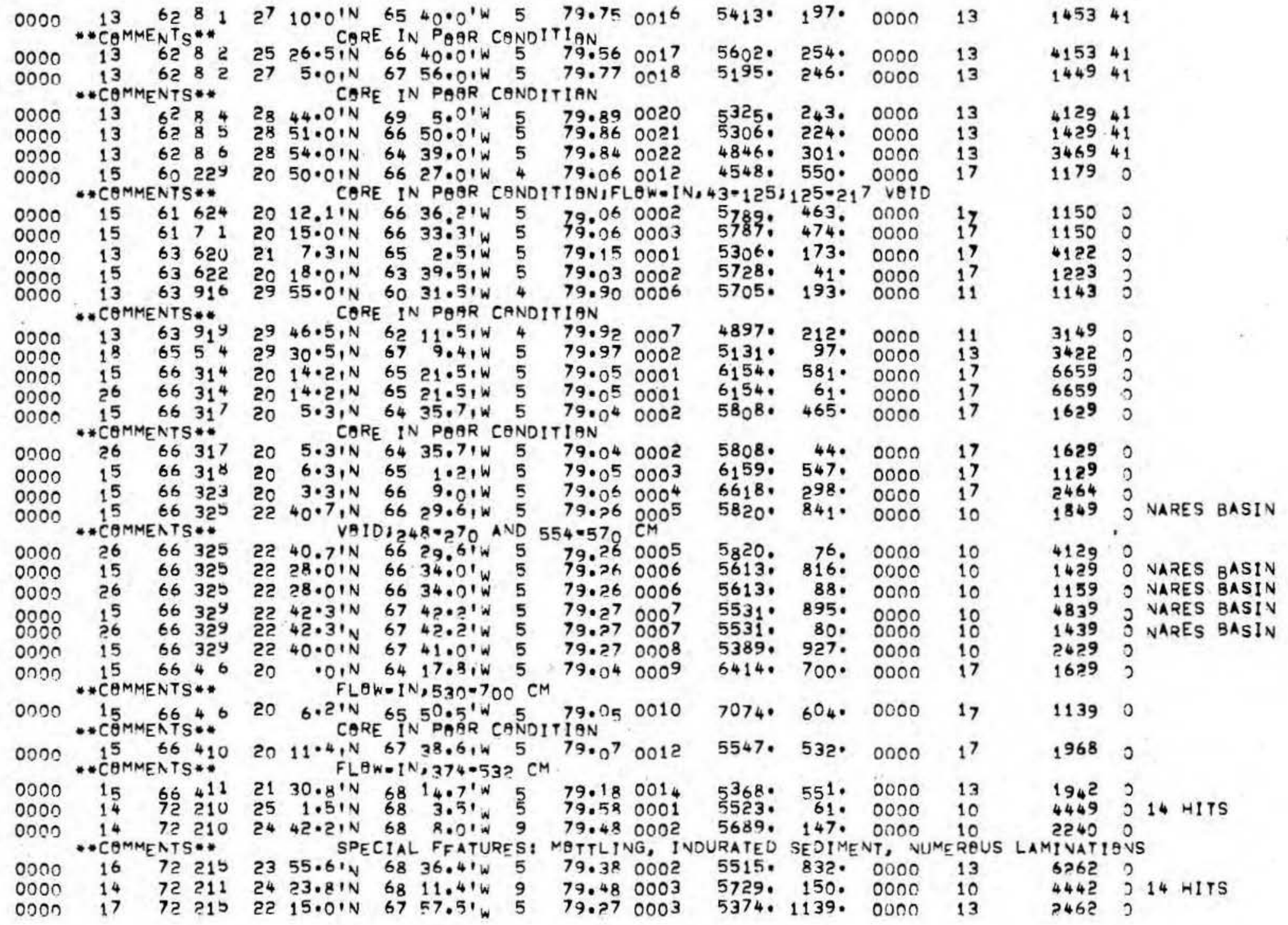




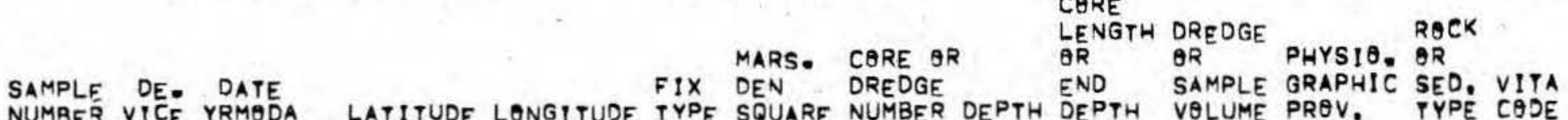

PYPE CODE REMARKS

SHIP CRUISE LEG STATION NUMBER VICE YRMODA

MARSDEN SQUARE * 79

$\begin{array}{llll}\text { KNR } & 25 & 1 & 000^{4} \\ \text { KNR } & 25 & 1 & 000^{4} \\ \text { KNR } & 25 & 1 & 000^{5} \\ \text { KNR } & 25 & 1 & 000^{6} \\ \text { KNR } & 25 & 1 & 0007 \\ \text { KNR } & 25 & 1 & 0008 \\ \text { KNR } & 25 & 1 & 000^{9} \\ \text { KNR } & 25 & 1 & 0012 \\ \text { KNR } & 25 & 1 & 0013\end{array}$

$\begin{array}{ll}0000 & 14 \\ 0000 & 1 \\ 0000 & 1 \\ 0000 & 14 \\ 0000 & 14 \\ 0000 & 14 \\ 0000 & 1 \\ 0000 & 1 \\ 0000 & 1\end{array}$

$\begin{array}{llllllll}14 & 72 & 216 & 23 & 45.21 N & 69 & 40.81 W & 5 \\ 16 & 72 & 216 & 21 & 30.01 N & 67 & 31.01 W & 5 \\ 14 & 72 & 212 & 23 & 48.5, N & 69 & 54.5, W & 5 \\ 14 & 72 & 212 & 23 & 52.61 N & 68 & 35.11 W & 9 \\ 14 & 72 & 213 & 23 & 43.51 & 68 & 41.51 W & 5 \\ 14 & 72 & 213 & 23 & 57.21 N & 68 & 59.01 W & 9 \\ 14 & 72 & 213 & 23 & 24.01 N & 69 & 6.11 W & 9 \\ 13 & 72 & 214 & 23 & 46.91 & 69 & 42.31 W & 5 \\ 13 & 72 & 215 & 22 & 15.41 N & 67 & 56.91 W & 5\end{array}$

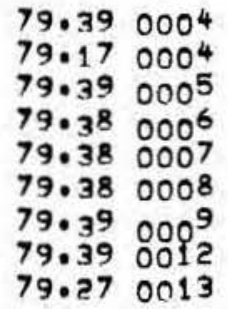

\begin{tabular}{|c|c|c|}
\hline 5392. & 136. & 0000 \\
\hline 63. & 21590 & 0000 \\
\hline $\begin{array}{l}54090^{\circ} \\
54860^{\circ} \\
5306 . \\
5451 .\end{array}$ & $\begin{array}{l}140^{\circ} \\
145^{\circ} \\
145^{\circ} \\
142^{\circ}\end{array}$ & $\begin{array}{l}0000 \\
0000 \\
0000 \\
0000\end{array}$ \\
\hline $\begin{array}{l}54111^{\circ} \\
5419 . \\
5374 .\end{array}$ & $\begin{array}{r}147^{\circ} \\
68^{\circ} \\
39^{\circ}\end{array}$ & $\begin{array}{l}0000 \\
0000 \\
0000\end{array}$ \\
\hline
\end{tabular}

44590

44250

4449 O 30 HITS

44490 30 HITS

44490 2ट HITS

44490 22 HITS

$4449 \quad 0 \quad 2^{7}$ HITS 000

MARSDEN SQUARE * 80

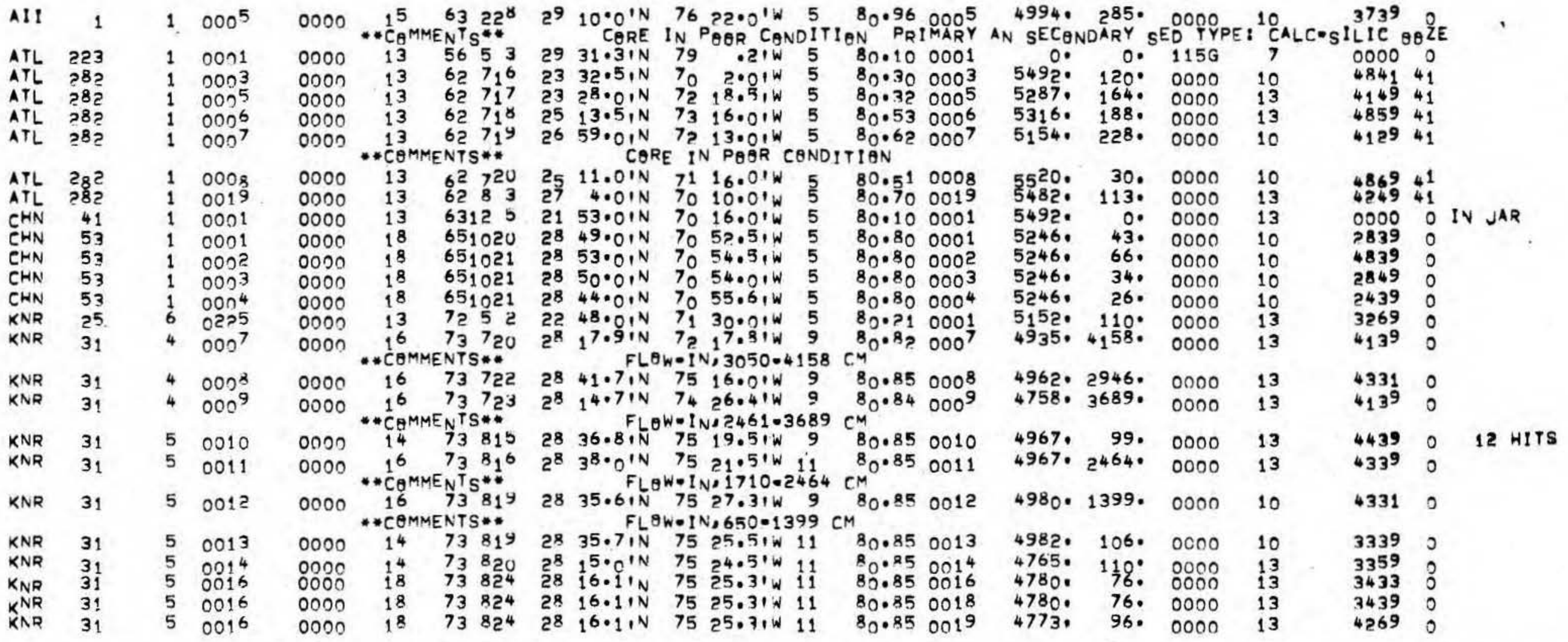




\section{1}

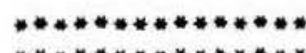

MARSDEN SQUARE *81

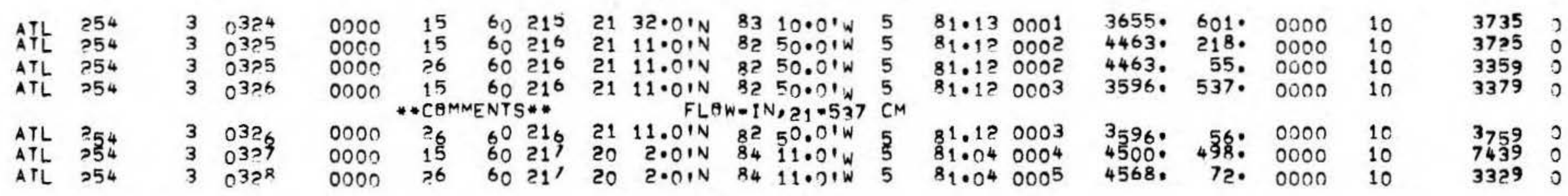

\section{MARSDEN SQUARE \#10?}

$\begin{array}{llll}\text { AII } & 15 & 4 & 0585 \\ \text { AII } & 15 & 5 & 0586 \\ \text { AII } & 15 & 5 & 0586 \\ \text { AII } & 15 & 5 & 0586 \\ \text { AII } & 15 & 5 & 0592 \\ \text { AII } & 15 & 5 & 0594\end{array}$

$\begin{array}{cccc}0000 & 13 & 65 & 321 \\ 0000 & 15 & 65 & 321 \\ & * \text { *CBMMENTS } \\ 0000 & ? 6 & 65 & 321 \\ 0000 & 13 & 65 & 321 \\ 0000 & 18 & 65 & 325 \\ 0000 & 15 & 65 & 325 \\ & 15 & 25 \\ & * C A M M E N T S * *\end{array}$

\section{$9.01 \mathrm{~N} 69$ 50.0'E 902090585}

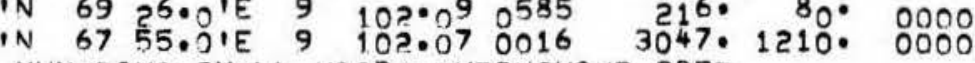
NUMERAUS SMALL VAIDS THRQUGHAUT CORE

$207.01 \mathrm{~N} 6755.0 \mathrm{E}$, $102.070016 \quad 3047.102 .0000$

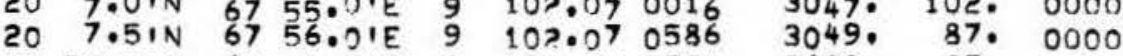
$2050.01 \mathrm{~N} 61$ 1.01E $9 \quad 102.0105922628$. 97. 0000 NUMEROUS SMALL VOIDS THROUGHOUT CORE

3355 3422 , ว

33320

33320

33590

38600

\section{MARSDEN SQUARE \# $10_{3}$}

$\begin{array}{llll}\text { AII } & 15 & 5 & 0529 \\ \text { AII } & 15 & 5 & 0529 \\ \text { AII } & 1= & 5 & 0590 \\ \text { AII } & 15 & 5 & 0591\end{array}$

$\begin{array}{llll}0030 & 15 & 65 & 324 \\ 0000 & 13 & 65 & 324 \\ 0000 & 13 & 65 & 324\end{array}$

$24 \quad 2.01 N$ 59 $53.11 E \quad 9 \quad 103.490017$

3341. 742. 0000 $3341,82.0000$ SEVERAL VAIDS IN CORE

$0000 \quad 13 \quad 65324 \quad 23 \quad 7.01 N \quad 5922.01 E \quad 9 \quad 103.390590 \quad 1805$. 42.0000 $\begin{array}{lll}1805 & 42 . & 0000 \\ 1267: & 70 & 000 n\end{array}$

$\begin{array}{lll}6 & 2230 & 0 \\ 6 & 4239 & 0 \\ & & \\ 4 & 3359 & 0 \\ 4 & 3359 & 0\end{array}$

MARSDEN SQUARE \# 104

$\begin{array}{llll}\text { A11 } \quad 15 & 1^{4} & 0^{7} 9\end{array}$

0000 . 13 CAMMEN $655^{\top} 6_{1}^{\prime}$

2555.5 's

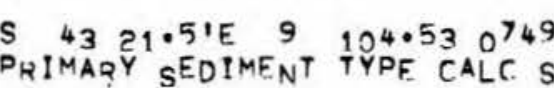

${ }^{3802}$ : 136.0000

6

$0352 \quad 0$

\section{MARSDEN SQUARE \#105}

$\begin{array}{llll}\text { III } & 15 & 3 & 0541 \\ \text { CHN } & 61 & 5 & 0072 \\ \text { CHN } & 6 ! & 5 & 0073\end{array}$

65 218 $2117.01 \mathrm{~N} 38$

65218

661017

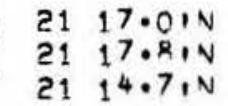

$\begin{array}{ll}21 & 17 \cdot 8, N \\ 21 & 14 \cdot 7, \mathrm{~N}\end{array}$
000015

non 13 $\begin{array}{lllll}38 & 2.01 E & 9 & 105.18 & 0004\end{array}$

38 4.7,E 6 105.18 0000
2089. 2.031.
0.: 0000 $\begin{array}{rr}51 . & 0000 \\ & 0000\end{array}$

$\begin{array}{llll}21 & 0000 & 0 & 3 \text { BAGS } \\ 21 & 4332 & 41 & \\ 21 & 3432 & 41 & \end{array}$


MARS. CORE AR

SAMPLE DE- DATE FIX DEN . DREDGE END OR

SHIP CRUISE LEG STATION NUMBER VICE YRMODA LATITUDE LONGITUDE TYPE SQUARE NUMBER DEPTH DEPTM VOLUME PROV. TYPE CODE REMARKS

MARSDEN SQUARE * 105

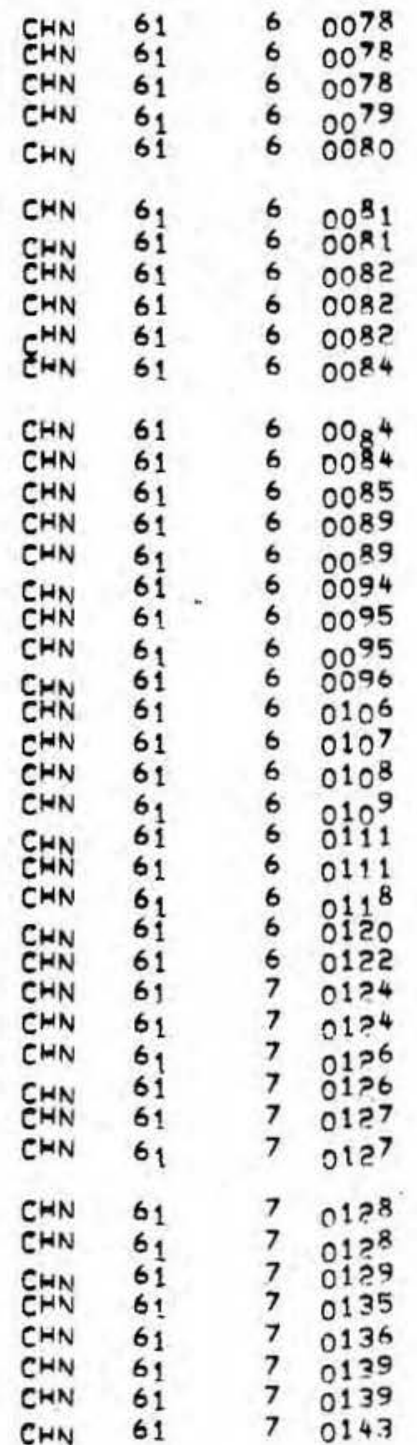

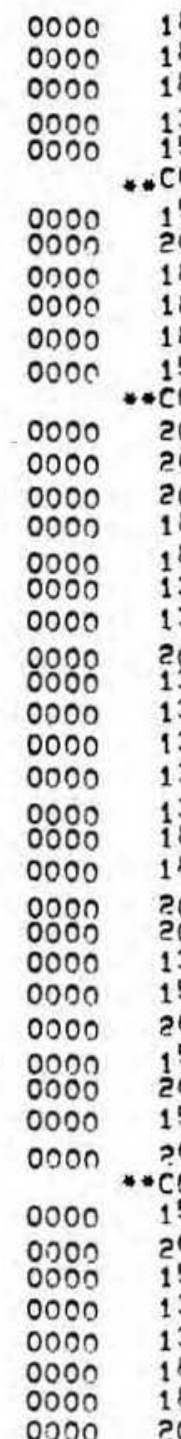
CAMMENTS*

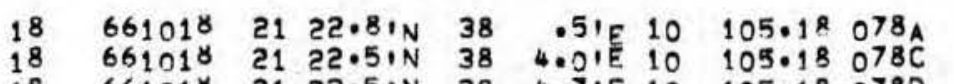
4.0, $105 \cdot 18078 \mathrm{C}$ 661018 CI ?.

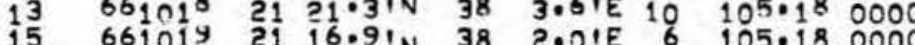
PSSSIBLE FLOW-IN, 55*855 CM

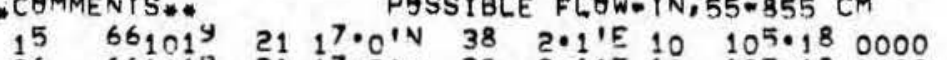

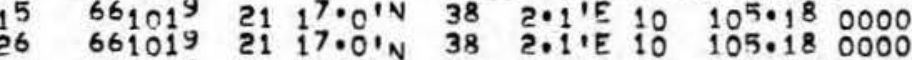
18661019 21 $18.81 \mathrm{~N} \quad 38 \quad 3.81 \mathrm{E} \quad 10 \quad 105.18082 \mathrm{~B}$ $\begin{array}{llllllll}661019 & 21 & 18.81 N & 38 & 3.51 E & 10 & 105.18 & 082 C \\ 661019 & 21 & 18.81 N & 38 & 3.21 E & 10 & 105.18 & 0820\end{array}$ $661050 \quad 2131.0, N \quad 383.81 E 10$ $1947^{\circ} 104.0000$ 1967.121 .0000 VEIDI $0.303 \mathrm{CM}$ 105. 18 OOOO 2167.955 , 0000

1748. 40. 0000 2056. 90.0000 2084.2950000 2154. 219.0000

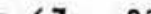
5167. 882,0000 2167. 135. 0000 194401040000 $661020 \quad 2121.01 \mathrm{~N} 3 \mathrm{O}^{\circ} \mathrm{3}^{\prime} \mathrm{F} 10 \quad 105,180000$ 661020 21 ?1.0.N 38 3:8,'E 10 105.18 0000 661020 21 $18.8, N \quad 38 \quad 3.9, E$ I 105.180085 661022 21 $23.31 \mathrm{~N} \quad 38$ 3.41E 10 105.18 0898 (6) 105.180898 661022 21 $22.81 N$ 38 2.31E 10 105.18 0000 $6610222123.31 N$ 38 3.11E $10 \quad 105.180000$ 661053

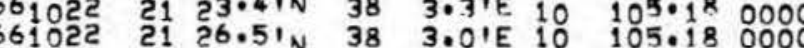
50.180000 $661023 \quad 2121.21 N \quad 38 \quad 3.51 E$ 10 105.180000 $\begin{array}{llllllll}661023 & 21 & 21.7 \text { IN } 38 & 3.31 E & 10 & 105.18 & 0000\end{array}$

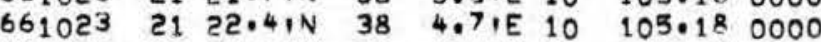
$661023 \quad 212^{4 \cdot 1} 1 \mathrm{~N} \quad 38 \quad 4 \cdot 0^{\prime E} 10 \quad 10^{5 \cdot 18} 0000$ 661024 21 $21.61 N \quad 38$ 5.51E $10 \quad 105.181110$ $661024 \quad 2121.6+N \quad 38 \quad 5.31 E 10 \quad 105.181110$ 661025 21 $4.41 \mathrm{~N} 38 \quad 4.31 E 10 \quad 105.18118$ 661025 21 22.61N 38 4.51E $10 \quad 105.180120$ $\begin{array}{llllllllll}661026 & 21 & 17.61 N & 38 & 1.51 & 10 & 105,18 & 0122\end{array}$ $6610302123.21 \mathrm{~N} 38 \quad 4.31 \mathrm{E} 10$ 105.18 0000 $661030 \quad 2123.2, N$ 38 $4.3, E 10 \quad 105.180000$ $66_{1030} 2123 \cdot 5, N$ 38 $4.31 E 10 \quad 105 \cdot 180000$ $\begin{array}{llllllll}661030 & 21 & 21.91 N & 38 & 4.41 E & 10 & 105.18 & 0000 \\ 661030 & 21 & 21.91 & 38 & 4.41 E & 10 & 105.18 & 0000\end{array}$

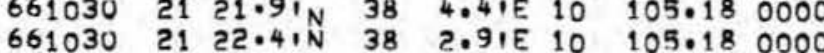
661030 21 $25.4 \mathrm{~N} 38$ 2.91E $10 \quad 105.180000$ ENTS* VOIDI $0.15 \mathrm{cM}$ $6610302125.4, N \quad 38 \quad 3.41 E 10 \quad 105 \cdot 180000$ 661030 2t $25.41 \mathrm{~N} \quad 38 \quad 3.415 \quad 10 \quad 105.180000$ 25.31 38 2.91E $10 \quad 1050180000$ $661112130.01 N \quad 3816.01 E 10 \quad 105.180000$

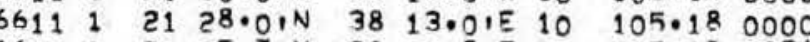
$661112125.7, N \quad 38$ 2.81E $10 \quad 105.18 \quad 139$

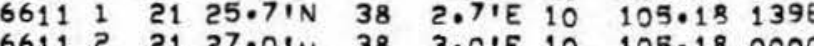

2167: 70: 0000 1939. 400.0000 1917. 90. 0000 $210^{7}: 1211^{\circ} 0000$ 1940.200 .2000 1882 . $305 \cdot 0000$ 2048. 54. 0000 2167. 84. 0000 2153. 78. 0000 1889. 197. 0000 1968. 260. 0000 2017. 122. 0000 $19599^{\circ} 400^{\circ} 0000$ $2020^{\circ} 400000000$ 2057: 125: 0000 2057. 95. 0000 $2068.818^{\circ}, 0000$ 2068, 205. 0000 2106. 834. 0000 $210^{\circ} 148 \cdot 0000$ 2077. 874. 0000 2077.170 .0000 752. 23. 0000 1277. 39. 0000 1972. 122. 0000 $\begin{array}{lll}20077^{\circ} & 122, & 0000 \\ 1882, & 354, & 0000\end{array}$
37292 0059 O HOT BRINE DO39 O HOT BRINE OOI9 HOT BRINE 48694

43410
4079

OO19 O HOT BRINE 44590

O439 O HOT BRINE 001941 HOP BRINE

0000 O HOT BRINE DO59 41 HOT BRINE $0000 \quad 0$

OU32 O HOT BRINE OO59 O HET BRINE 003941 HST BRINE O039 41 HOT BRINE OOOO O AOT BRINE OO59 41 HQT BR!NE DO39 41 HOT BRINE OO39 41 HOT BRINE 003941 HOT BRINE 0339 O HET BR!NE 0000 HOT BRINE O000 O HET BRINE 0331 4I HOT 003941 HaT DO NAT BRINE 003941 HOT BRINE O449 41 HÖT BRINE OO59 41 HOT BRINE

033941 HOT BRINE 033941 HOT BRINE 44741

496941

33524

0447 O HOT BRINE OO17 O HOT BRINE 0000 O HGT BRINE 


\section{MARSDEN SQUARE * 105}

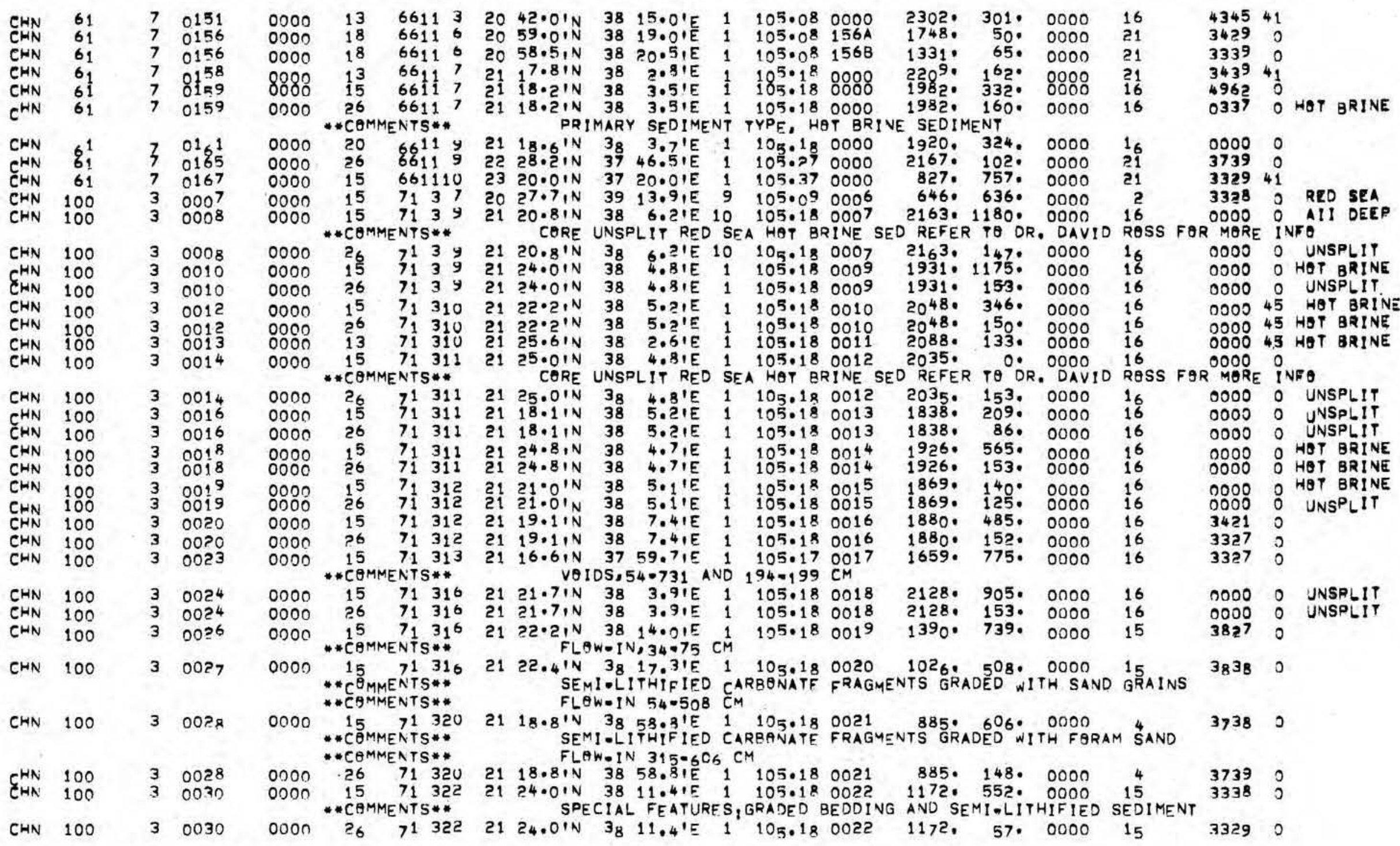




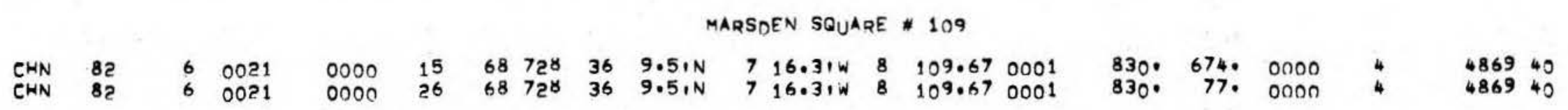

MARSDEN SQUARE * 110

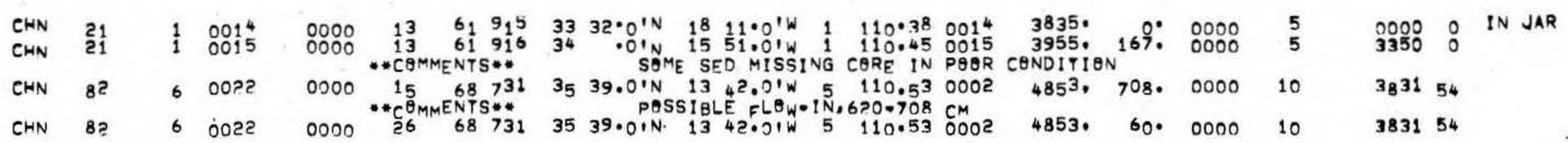

MARSDEN SQUARE \#111

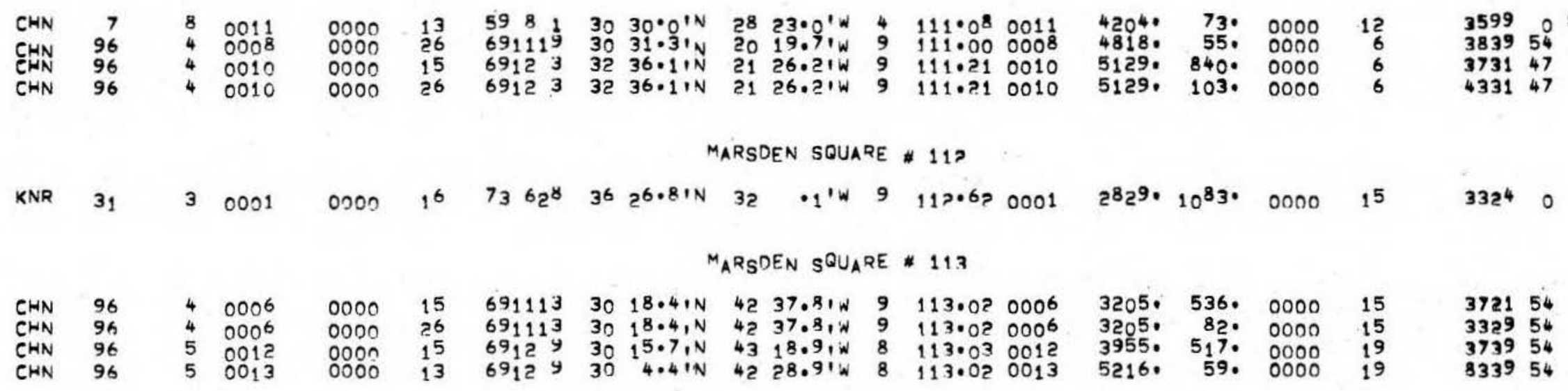


****************)

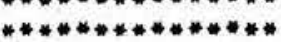
STATIAN UATA RETRIEVAL DATE: $17: 38$ JUN $0_{8}, 177$

SAMPLE DE. DATE SHIP CRUISE LEG STATION NUMBER VICE YRMADA

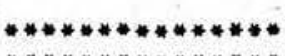

PAGE 23

* WHOI**

\section{RACK}

MARS. CORE AR LENGTH DREDGE PHYSIO. RQR

FIX DEN DREDGE GND SAMPLE GRAPHIC SED. VITA LATITUDE LONGITUDE TYPE SQUARE NUMBER DEPTH DEPTH VOLUME PROV. TYPE CODE REMARKS

MARSDEN SQUARE \# 113

$\begin{array}{llll}\text { CHN } & 96 & 5 & 0014 \\ \text { CHN } & 96 & 5 & 0015 \\ \text { CHN } & 99 & 2 & 0002 \\ \text { CHN } & 99 & 2 & 0003\end{array}$

$\begin{array}{ll}\text { O000 } & 13 \\ \text { ODOn } & 13 \\ \text { OnO0 } & 15 \\ 0000 & 15 \\ \text { ODOO } & 15\end{array}$

691210
691210
70
7023
70 $\begin{array}{llll}30 & 21.9, N & 44 & 7.2, w \\ 30 & 29.9, N & 44 & 50.5, w \\ 30 & 4100, N & 46 & 27.9, w \\ 31 & 17.7, N & 46 & 13.1, w\end{array}$

$8 \quad 113.040014$ $113 \cdot 040015$ $319.7, N \quad 4613.1, W \quad 8 \quad 113.160002$ CWN 99

$\begin{array}{ll}2 & 000^{3} \\ 2 & 000^{4}\end{array}$

0000

$\begin{array}{llll}70 & 42^{4} & 31 & 13 \cdot 5, N\end{array}$

$43 \quad 43 \cdot 1, w$

$\begin{array}{ll}113 \cdot 16 & 0002 \\ 113 \cdot 13 & 0003\end{array}$

$\begin{array}{lll}41190^{\circ} & 293^{\circ} & 0000 \\ 40830^{\circ} & 2866^{\circ} & 0000 \\ 42944^{\circ} & 800^{\circ} & 0000 \\ 4358 . & 5250^{\circ} & 0000\end{array}$

MARSDEN SQUARE \# 114

$\begin{array}{llll}\text { AII } & 85 & 2 & 0004 \\ \text { AII } & 85 & 2 & 0008 \\ \text { KNR } & 31 & 3 & 0003 \\ \text { KNR } & 31 & 3 & 0005 \\ \text { KNR } & 31 & 3 & 0006 \\ \text { KNR } & 47 & 1 & 0001 \\ \text { KNR } & 47 & 1 & 0001 \\ \text { KNR } & 47 & 1 & 0002 \\ \text { KNR } & 47 & 1 & 0002 \\ \text { KNR } & 47 & 1 & 0004 \\ \text { KNR } & 47 & 1 & 0004 \\ \text { KNR } & 47 & 1 & 0005 \\ \text { KNR } & 47 & 1 & 0005 \\ \text { KNR } & 47 & 1 & 0033\end{array}$

0000

57 36.61W 9 114.370004 4530.

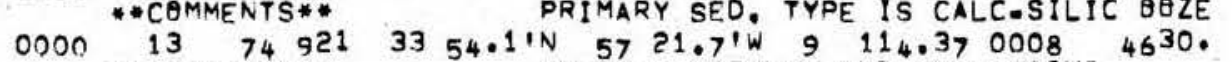

MN.RICH. MOTTLES AND 40370008

0000 * ${ }_{16}^{\theta}$ MMENTS** 737

$3350.8, M_{N}^{M N} 57$ I $2.41 \mathrm{CH} 9 \mathrm{CH} 114.390003$

$F L \theta W=1 N, 2325 \cdot 2.996 \mathrm{CM}$ *CAMMENTS**

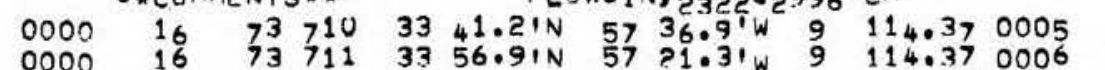

$0000 \quad 15 \quad 75$ (15

$3356.91 N$ N 57 ? $50.31 \mathrm{~W}$, 9 114.37 0006 NUMEROUS HYDROTRO 114.3700014284

$0000 \quad 26 \quad 75210 \quad 3350.61 \mathrm{~N} \quad 5733.11 \mathrm{~W} 91_{11.37} 0001 \quad 4284.122 .0000$

$0000 \quad 15752113340.81 N$ 5 5740.31 W 6 114.37 $0002 \quad 4619.1195 .0000$ NUMEROUS HYDRATROILITE LAMINATIONS

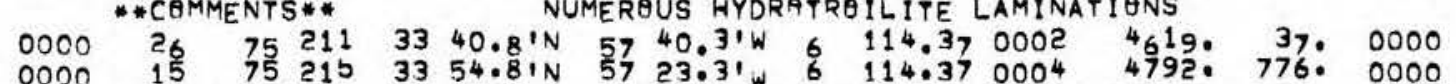

$00001575210 \quad 3354.81 N 5723.31$ W 6114.370004 H 159

$0000 \quad 26 \quad 75215 \quad 3354.81 \mathrm{~N} \quad 5723.31 \mathrm{~W} \quad 6 \quad 114.370004 \quad 4792.62 .0000$

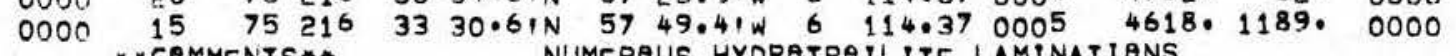

* COMMENTS** NUMERAUS HYDRATRQILITE LAMINATIONS

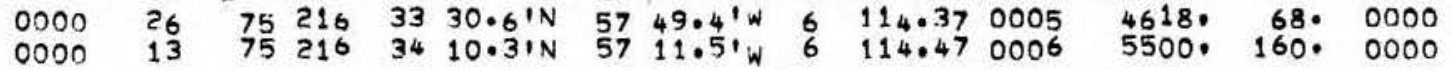

$0000 \quad 13 \quad 75216$

$\begin{array}{llr}19 & 3329 & 54 \\ 19 & 3731 & 54 \\ 19 & 3329 & 0 \\ 19 & 3329 & 0 \\ 19 & 3322 & 0\end{array}$

MARSDEN SQUARE \# 115

$\begin{array}{llll}\text { AII } & 1 & 1 & 0009 \\ \text { AII } & 42 & 1 & 0045 \\ \text { AII } & 92 & 1 & 0004 \\ \text { AII } & 92 & 1 & 0004 \\ \text { AII } & 92 & 1 & 0007\end{array}$

$\begin{array}{ll}0000 & 1 \\ 0000 & 15 \\ 0000 & 15\end{array}$

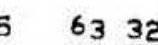
759 * COMMENTS*

$\begin{array}{lllllll}36 & 34.51 N & 67 & 30.51 W & 5 & 115.67 & 0009 \\ 30 & 54.01 N & 63 & 23.01 W & 1 & 115.03 & 0031\end{array}$

4979. 26. 0000

13

33220

0000 ?6 759 PHYST 60 .

4915. 775. 0000

0

* *C WINCH FAILURE: FLOW-IN $46.775 \mathrm{CM}$

AII 9? 10007

0000 . 15759 COMMENTS* * *COMMENTS**

$3016.01 \mathrm{~N} 66 \quad 3.5^{1} \mathrm{~W}, 9115060001$

4915, 49. $0000 \quad 13$

PHYSIOG: PRAV: SEDTMENT POND

PHYSIAG. PREV: SEDIMENT PQND 


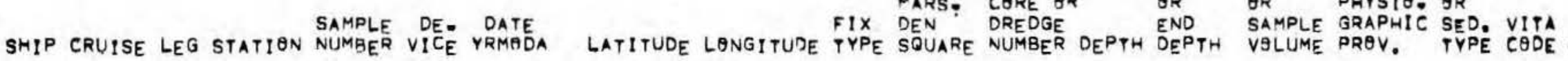

MARS- CORE QR LENGTH DREDGE PHYSIO. $Q R$

CORE

MARS- CORE QR LENGTH DREDGE PHYSIO. OR ORK

MARSDEN SQUARE \# 115

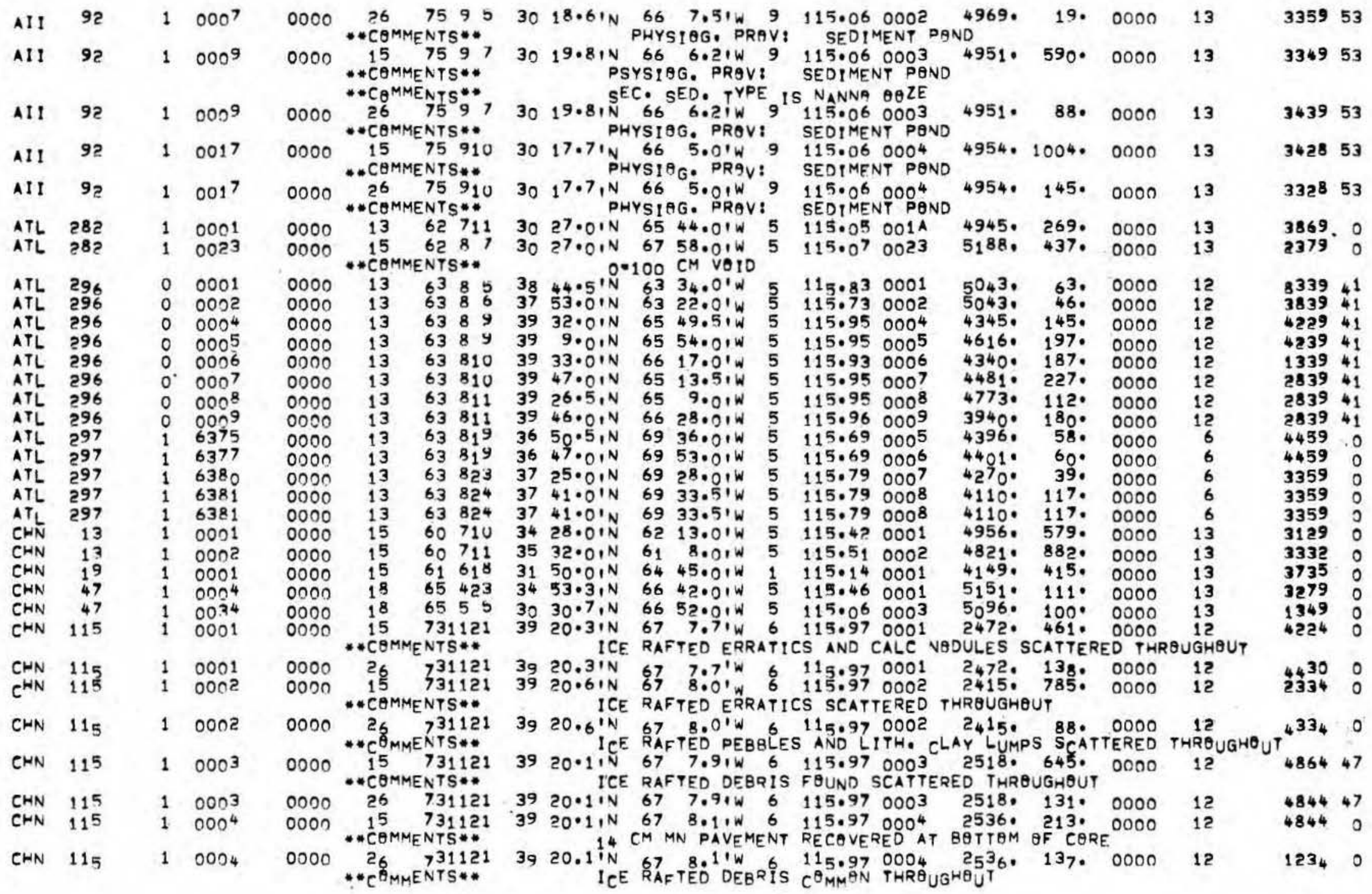




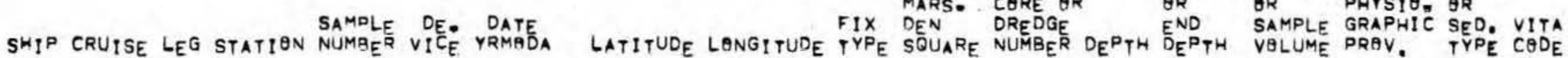

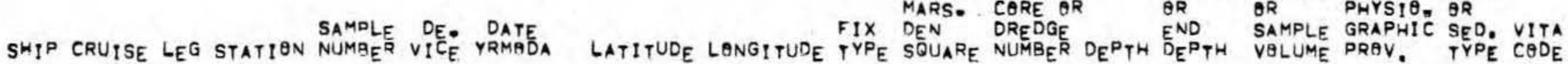
OR OR PHYSIO, OR

MARSDEN SQUARE *115

$\begin{array}{llll}\text { GES } & 102 & 0 & 0001 \\ \text { GOS } & 10 ? & 0 & 0002 \\ \text { GOS } & 137 & 0 & 2755 \\ \text { GES } & 137 & 0 & 2756 \\ \text { KNR } & 31 & 5 & 0017 \\ \text { KNR } & 31 & 5 & 0017 \\ & 31 & & \\ \text { KNR } & 31 & 5 & 0018 \\ \text { KNR } & 31 & 5 & 0019 \\ \text { KNR } & 31 & 5 & 0020\end{array}$

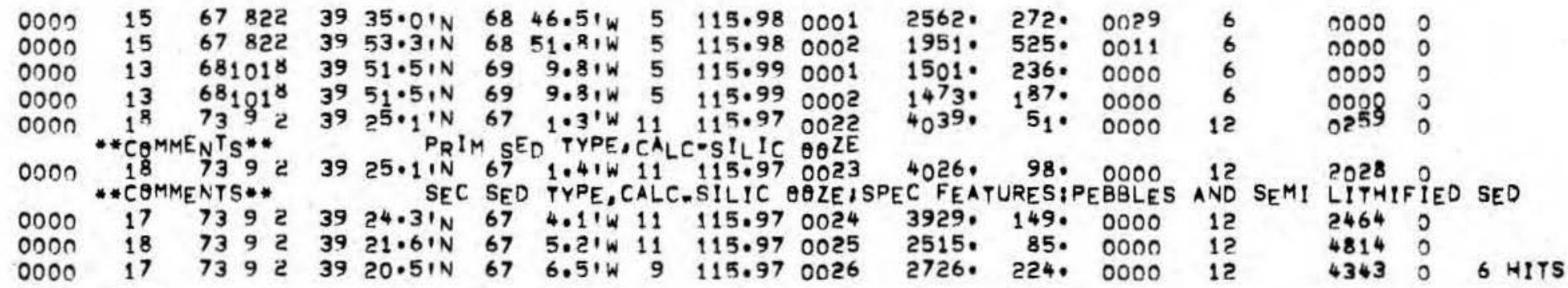

MARSDEN SQUARE \# 116

$\begin{array}{llll}\text { AII } & 1 & 1 & 0001 \\ \text { AII } & 1 & 1 & 0002 \\ \text { AII } & 1 & 1 & 0003 \\ \text { AII } & 1 & 1 & 0008 \\ \text { III } & 7 ? & 1 & 0002 \\ \text { III } & 7 ? & 1 & 0003 \\ \text { AII } & 7 ? & 1 & 0003 \\ \text { AII } & 7 ? & 1 & 000^{4} \\ \text { AII } & 7 ? & 1 & 0004 \\ \text { AII } & 72 & 1 & 0005 \\ \text { AII } & 7 ? & 1 & 0007 \\ \text { AII } & 7 ? & 1 & 0007 \\ \text { AII } & 7 ? & 1 & 0008 \\ \text { AII } & 7 ? & 1 & 0005 \\ \text { AII } & 7 ? & 1 & 0009 \\ \text { AII } & 7 ? & 1 & 0009 \\ \text { AII } & 7 ? & 1 & 0013 \\ \text { AII } & 7 ? & 1 & 0013 \\ \text { AII } & 7 ? & 1 & 0014 \\ \text { AII } & 7 ? & 1 & 0014 \\ \text { AII } & 7 ? & 1 & 0015 \\ \text { AII } & 7 ? & 1 & 0015 \\ \text { AII } & 7 ? & 1 & 0016\end{array}$

\begin{abstract}
$0000 \quad 1^{15} 6322^{5}$
$000015 \quad 6322$

$0000 \quad 1566^{3} 22_{6}$

0000 * *COMMENTS**

$000015 \quad 721021$

$0000 \quad 15 \quad 721021$

$0000 \quad 15 \quad 721021$

$\begin{array}{lll}0000 & 26 & 721021 \\ 0000 & 15 & 721021\end{array}$

$\begin{array}{lll}0000 & 15 & 721021 \\ 0000 & 26 & 721021\end{array}$

$0000 \quad 15 \quad 721021$

$0000 \quad 26 \quad 721021$

0020

15 72102

$0000 \quad 2_{6} \quad 721022$

$\begin{array}{lll}0000 & 15 & 721022 \\ 0000 & 26 & 721022\end{array}$

$0030 \quad 15 \quad 721023$

(1)

000 n

0000

0000

0000

0000

0000

721023

$72102^{3}$

$72102^{3}$

721023
\end{abstract}

15 72102
$32 \quad 2.0^{1 N} 74,9.0^{1 W} 5 \quad 116.240001$ CORE IN POQR CONDITION $3115.01 \mathrm{~N} 7358.01 \mathrm{~W} 5116.130002$ CQRE IN PAAR CANDITIAN

$3056.01 \mathrm{~N} 7436.01 \mathrm{~W} \quad \mathrm{~N}_{16.04} 0003$ CORE IN PQOR CANDITION

$3710.01 N$ TO $13.01 W$ W 116.700008 3958.5 in $7034.51 \mathrm{~W} 5116.900002$ $3949.9, N \quad 7034.3, W \quad 9 \quad 116.90000^{3}$ $39.9, N \quad 7034.3, W \quad 9 \quad 116.900003$ SPECIAL FEATURES. HYDRETROILITE 39 $43.61 \mathrm{~N}$ 70 32.7'W 9116.900004

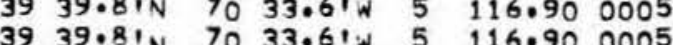

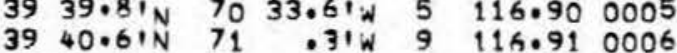
$3940.61 N$ 71 $\quad$.31w 9 1 116.910006 $3949.5, N$ 7 $59.8, W 9116.900007$ $0=40$ DISTURBED

39 $49.5^{1 \mathrm{~N}} 70 \mathrm{59.91 \textrm {W } g} 11 \mathrm{~K} .900007$

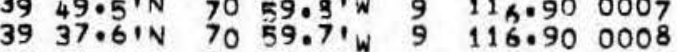
$3937.61 \mathrm{~N} 7059.71 \mathrm{~W} 9116.900008$ $3955.5 \mathrm{~N} 7043.31 \mathrm{~W} 9116.900011$ $3955.5, \mathrm{~N} 7043.3, \mathrm{~W} 9116.9 \mathrm{n} 0011$ $3948.9, N$ 70 43.5, 5116.900012 $3948.9, N \quad 7043.5, w 5114.900012$ $3941.0, N \quad 7045.7, w 5116.900013$ $41.01 N \quad 7045.71 W \quad 5 \quad 116.900013$ FLOW-IN,501-776 CM

\begin{tabular}{|c|c|c|c|c|c|}
\hline $870^{\circ}$ & $222^{\circ}$ & 0000 & 10 & 4250 & 0 \\
\hline 070. & $211^{\circ}$ & 0000 & 10 & ?50 & \\
\hline 3539. & 177. & 0000 & 10 & $443^{\circ}$ & \\
\hline $\begin{array}{l}4291 . \\
2980 \\
8560 \\
8560 \\
19370 \\
\text { METTLES }\end{array}$ & $\begin{array}{r}800^{\circ} \\
3450 \\
8240 \\
990 \\
6370 \\
\text { S, LIT }\end{array}$ & $\begin{array}{l}0000 \\
0000 \\
0000 \\
0000 \\
\text { OOOO } \\
\text { HIFIED }\end{array}$ & $\begin{array}{c}10 \\
4 \\
4 \\
4 \\
4 \\
\text { DEBRIS }\end{array}$ & $\begin{array}{l}3860 \\
2848 \\
2849 \\
2849 \\
2430\end{array}$ & $\begin{array}{r}0 \\
40 \\
0 \\
0 \\
0\end{array}$ \\
\hline $\begin{array}{l}19370 \\
21050 \\
21050 \\
20080 \\
2008 .\end{array}$ & $\begin{array}{r}20 \\
7100 \\
160 \\
612 . \\
900 \\
235^{\circ}\end{array}$ & $\begin{array}{l}0000 \\
0000 \\
0000 \\
0000 \\
0000 \\
0000\end{array}$ & $\begin{array}{l}4 \\
4 \\
4 \\
4 \\
4 \\
4\end{array}$ & $\begin{array}{l}3869 \\
2239 \\
3869 \\
4239 \\
3869 \\
3439\end{array}$ & $\begin{array}{l}40 \\
40 \\
40\end{array}$ \\
\hline $\begin{array}{l}1002 . \\
397 \% \\
397 \% \\
667 .\end{array}$ & $\begin{array}{l}490^{\circ} \\
6930^{\circ} \\
1390^{\circ} \\
482 .\end{array}$ & $\begin{array}{l}000 \\
000 \\
000 \\
000\end{array}$ & $\begin{array}{l}4 \\
4 \\
4\end{array}$ & $\begin{array}{l}3429 \\
2225 \\
2225 \\
2838\end{array}$ & $\begin{array}{l}40 \\
40 \\
40\end{array}$ \\
\hline $\begin{array}{r}667 . \\
12490 \\
1249 . \\
22180 \\
2218, \\
2478 .\end{array}$ & $\begin{array}{l}1030^{\circ} \\
320^{\circ} \\
124^{\circ} \\
467^{\circ} \\
35^{\circ} \\
776^{\circ}\end{array}$ & $\begin{array}{l}0000 \\
0000 \\
0000 \\
0000 \\
0000 \\
0000\end{array}$ & $\begin{array}{l}4 \\
4 \\
4 \\
4 \\
4\end{array}$ & $\begin{array}{l}2830 \\
2439 \\
2259 \\
2255 \\
2329 \\
3869 \\
2339\end{array}$ & $\begin{array}{l}40 \\
40 \\
40 \\
40 \\
40 \\
40 \\
0\end{array}$ \\
\hline
\end{tabular}


SAMPLE DE. DATE

MARS, CQRE $9 R$

CARE

NUMPLE, DE: DATE

FIX DEN DR DEDE

LENGTH DREDGE

END PHYSIO, GR

MARSDEN SQUARE \# 116

$\begin{array}{llll}\text { AII } & 72 & 1 & 0016 \\ \text { II } & 72 & 1 & 0017 \\ \text { AII } & 72 & 1 & 0019 \\ \text { AII } & 72 & 1 & 0019 \\ \text { AII } & 72 & 1 & 0020 \\ \text { III } & 72 & 1 & 0021 \\ \text { AII } & 72 & 1 & 0021 \\ \text { AII } & 72 & 1 & 0022 \\ \text { AII } & 72 & 1 & 0023 \\ \text { AII } & 72 & 1 & 0023 \\ \text { AII } & 72 & 1 & 0024 \\ \text { AII } & 72 & 1 & 0025 \\ \text { AII } & 72 & 1 & 0026 \\ \text { AII } & 72 & 1 & 0026 \\ \text { AII } & 72 & 1 & 0027 \\ \text { AII } & 72 & 1 & 0027 \\ \text { AII } & 72 & 1 & 0029 \\ \text { AII } & 72 & 1 & 0030 \\ \text { AII } & 72 & 1 & 0030 \\ \text { AII } & 72 & 1 & 0030 \\ \text { AII } & 72 & 1 & 0031 \\ \text { ALB } & 18 & 4 & 0007 \\ \text { ALB } & 18 & 4 & 0028 \\ \text { ALB } & 18 & 4 & 0009 \\ \text { ALB } & 18 & 4 & 0010 \\ \text { ATL } & 223 & 1 & 0004 \\ \text { AIL } & 297 & 1 & 6369 \\ \text { ATL } & 297 & 1 & 6371 \\ \text { ATL } & 297 & 1 & 6372 \\ \text { CHN } & 11 & 1 & 0013 \\ & & & \end{array}$

\begin{tabular}{|c|c|c|}
\hline ס סט & $\begin{array}{l}26 \\
15 \\
15 \\
26\end{array}$ & $\begin{array}{l}721026 \\
721026 \\
721028 \\
72105 \\
72105\end{array}$ \\
\hline $\begin{array}{l}000 \\
000 \\
000 \\
000\end{array}$ & 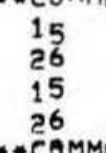 & $\begin{array}{l}029 \\
029 \\
029 \\
02^{9} \\
02^{9}\end{array}$ \\
\hline $\begin{array}{l}000 \\
000 \\
000 \\
000\end{array}$ & 15 & $\begin{array}{r}721029 \\
721029 \\
721029 \\
721030 \\
\text { FNTS }\end{array}$ \\
\hline 000 & - & 721030 \\
\hline $\begin{array}{l}0000 \\
0000 \\
0000 \\
0000 \\
B_{0} \\
C_{0}\end{array}$ & & $\begin{array}{l}721030 \\
721031 \\
721031 \\
721031 \\
7211 \\
7211 \\
7211 \\
7211 \\
7211 \\
681211\end{array}$ \\
\hline $\begin{array}{l}3057 \\
3058 \\
3059 \\
0000 \\
0000 \\
0000 \\
0000 \\
0050\end{array}$ & $\begin{array}{l}13 \\
13 \\
13 \\
13 \\
15\end{array}$ & 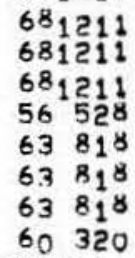 \\
\hline
\end{tabular}

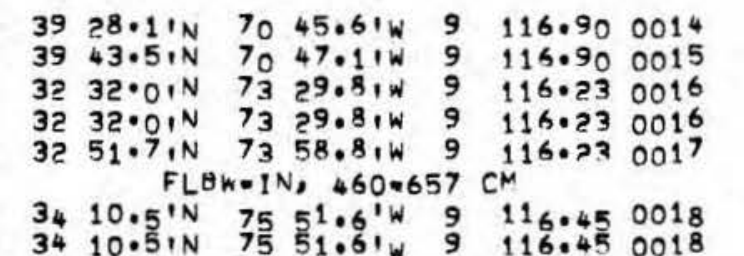

$3413.01 N$ 75 42.01W 9116.450019 $3413 \cdot 0$ IN $7542.01 W$
ENTIRE SAMPLE STERED IN ONE JAR

2478.99 .0000

1331. 565, 0000

$5058,850.0000$

5058.96 .0000

4808 . 657. 0000

481. 812. 0000

481. 10. 0000

1331. 38. 0000

1331. 10. 0000

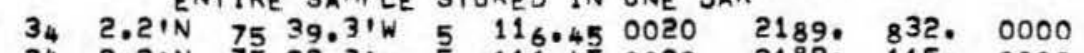

$342.21 N 7539.31$ i 5 116.45 0020 2189, 115. 0000

$343.21 N 7540.01 \mathrm{~W} 5116.450021 \quad 2202.198 .0000$

$34 \quad 1.01 N 7537.21$ IW 5 116.45 OOZ2 294201240.0000

$3349.5^{\prime N} 7518.01 \mathrm{~W} 5116.350023$ 3204. 863. 0000 3349.5 PRIMARY SEOIMENT TYPE CALC SILIC OOZE

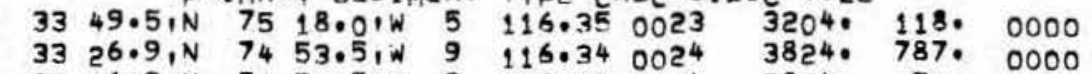

$3326.9, N \quad 7453.5, W \quad 9 \quad 116.340024$

$3413.8, N 7427.81 W$ W 1160340025

3824.787 .0000

$3824^{\circ} 52^{\circ} 0000$

$3414.5, N \quad 7,54.0, W 5116.410268$

$3416.0^{1 N} 77_{1} 53.81 \mathrm{~W} \quad 116.41026 \mathrm{C}$

$3415.01 \mathrm{~N} 7151.51 \mathrm{~W} 5116.410027$

4734. 161\% 0000

4730.53 .0000

$4699^{\circ}$ 63. 6000

4697.062 .0000

556. 202. 0000

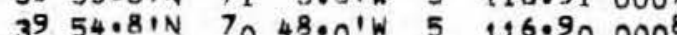

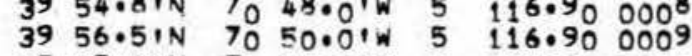

$3959.51 \mathrm{~N} 70 \mathrm{O} 0.01 \mathrm{~W}$

555: 169: 0000

595. 164. 0000

260.170 .0000

$3126.11 \mathrm{~N} 7626.61 \mathrm{~W} 5116.660004$

$3640.01 \mathrm{~N} 7011.01 \mathrm{~W} 5116.600001$

4418: 111: 0000

4261.40 .40000

4243.2100000

5302 . 610. 0000

TYPE CODE REMARKS 


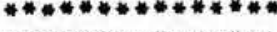

**************)

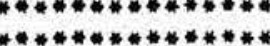

STATION UATA RETRIEVAL STATION UATA RETRIEVAL DATE:

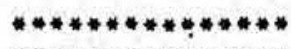

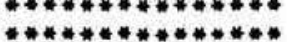

PAGE 27

*WHOI *

PAGE 180341759

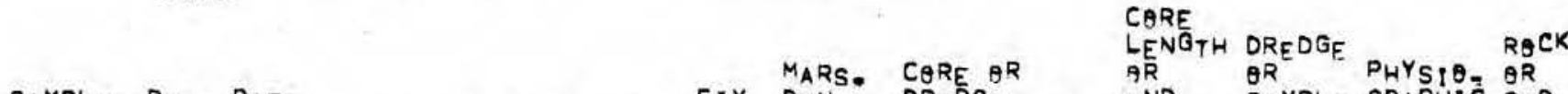

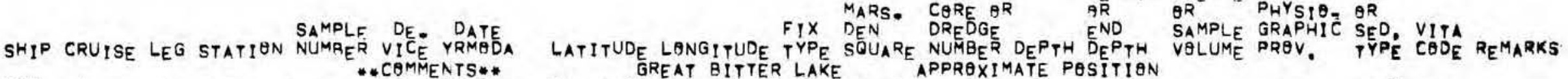

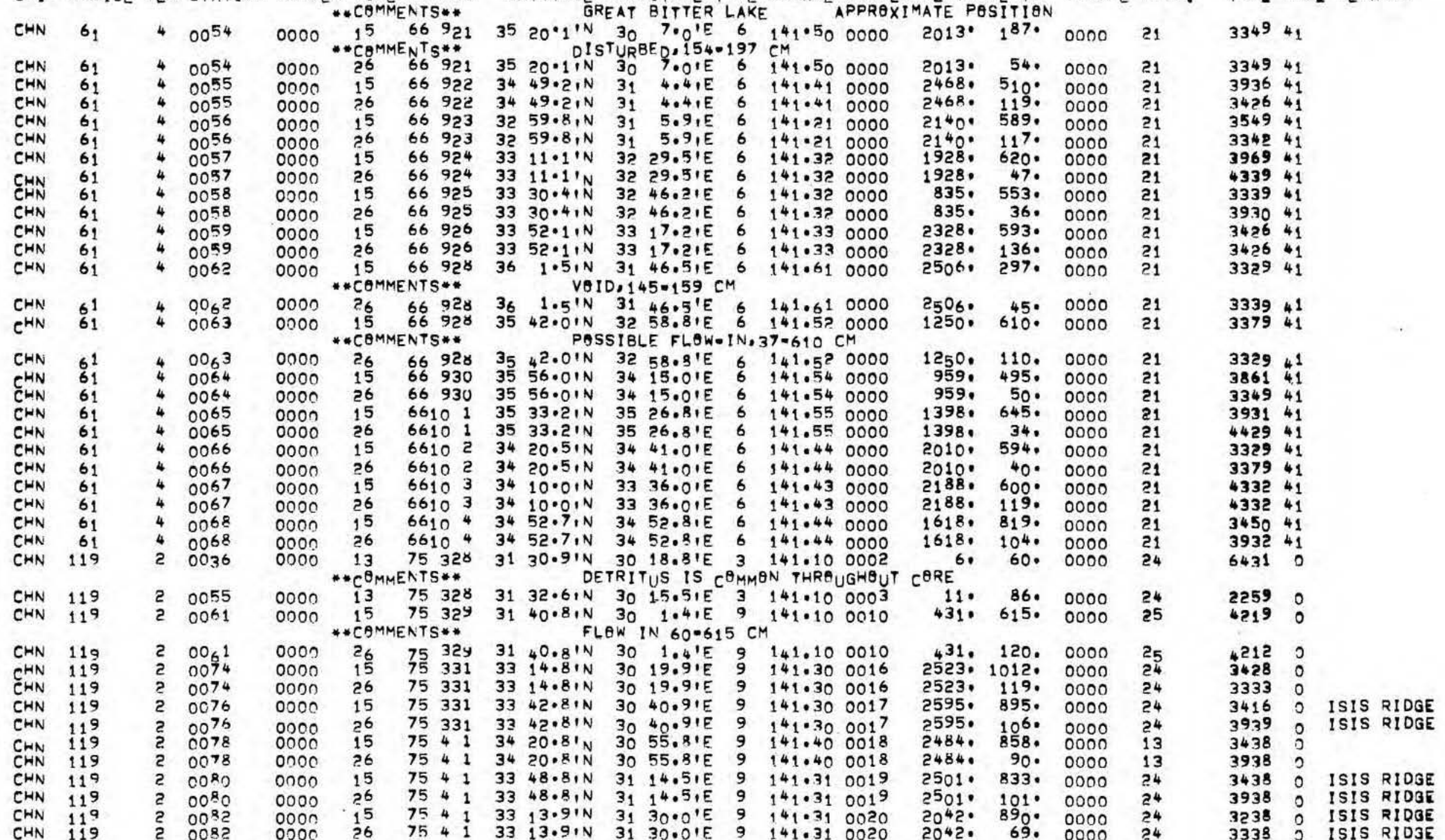




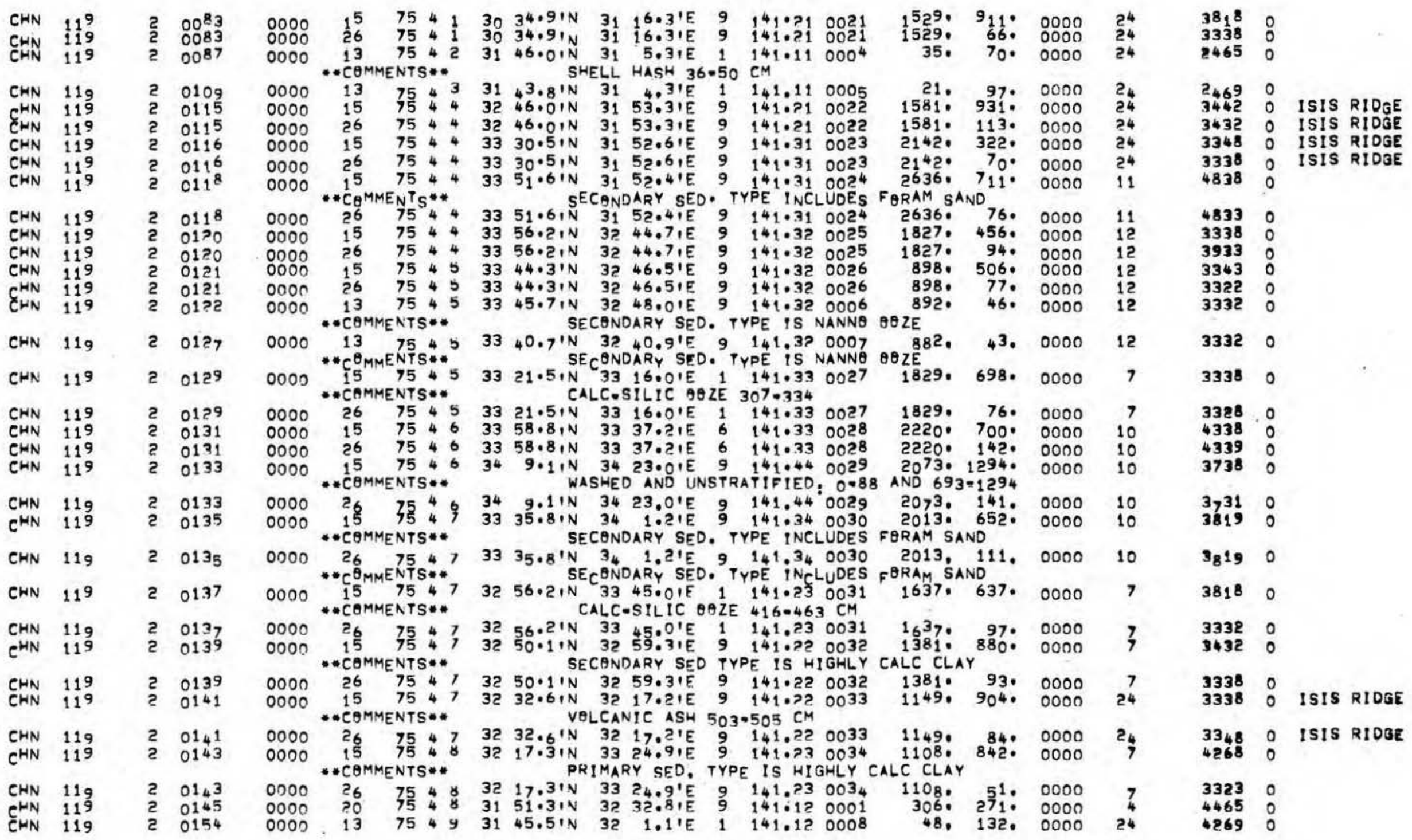


MARSDEN SQUARE * 141

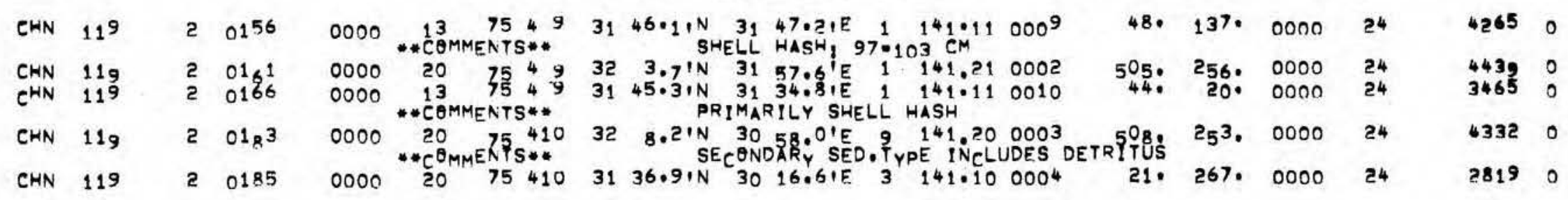

MARSDEN SQUARE * 14?

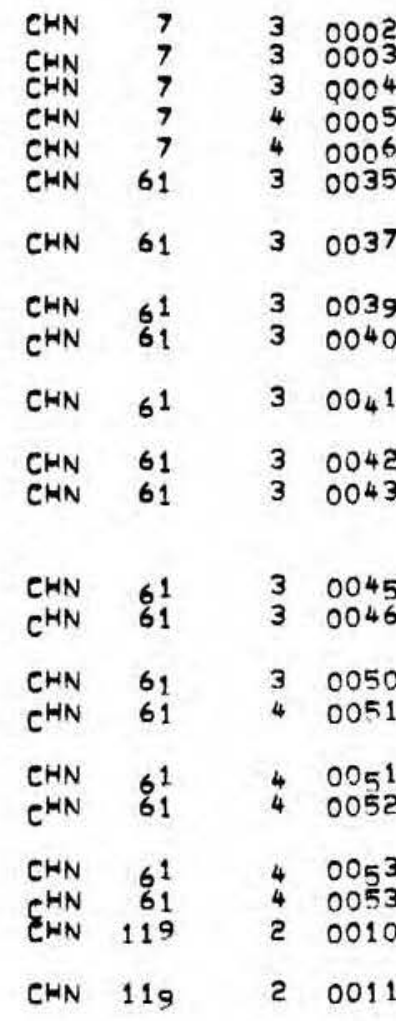

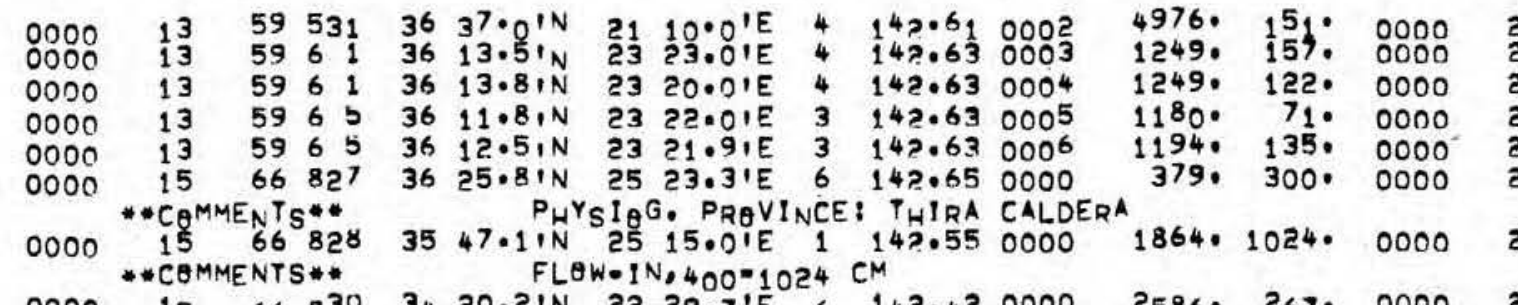

21 3350 O STETSON COR 21 3350 O STETSON COR 21 3350 O STETSAN CAR $\begin{array}{lll}21 & 3350 & 0 \\ 21 & 3330 & \text { S STETSON COR }\end{array}$ 21 986441

496941 AEGEAN SEA

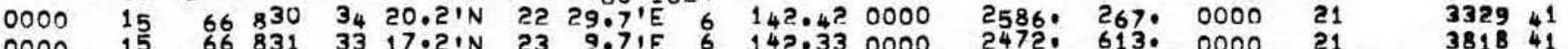

0000 *COMMENTS** SPECIAL FEATURES, GRADED BEDDING, VOLC ASH AND PYRITE, FLOW०IN,446.613 CM

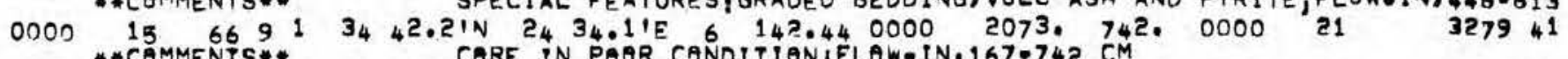

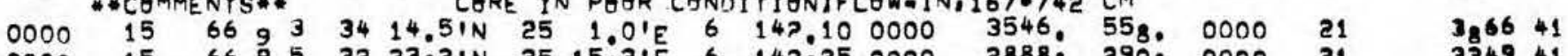

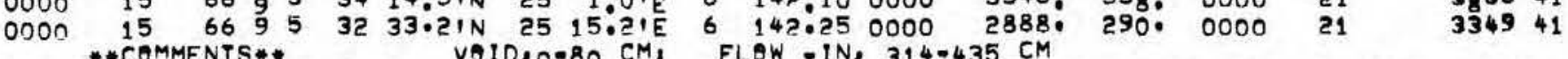

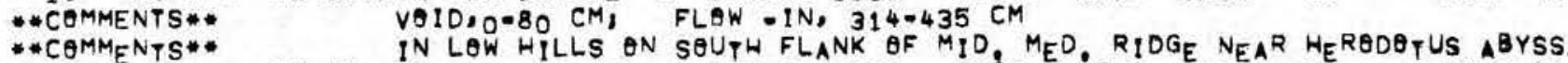

$0000 \quad 15 \quad 66973314.71 N$ 27 51.9'E 6 142.370000 3038. 435. $0000 \quad 21 \quad 449541$

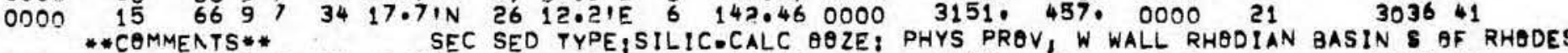

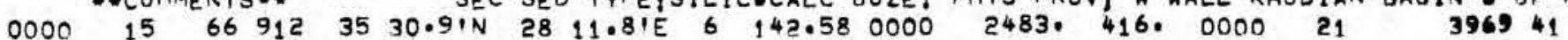

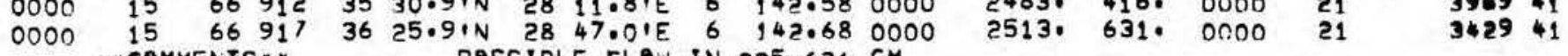

*\#CAMMENTS** PASSIBLE FLOW-IN, $255.631 \mathrm{CM}$

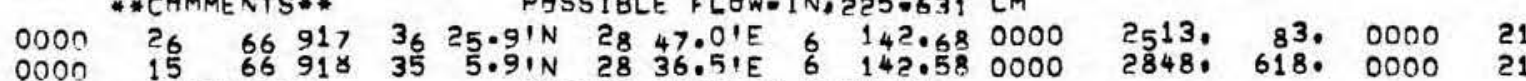

* COMMENTS** ENTIRE CORE IS FLOW-IN

$0000 \quad 15 \quad 66919 \quad 34 \quad 9.01 \mathrm{~N} \quad 2_{8} 51.8^{\prime} \mathrm{E} \quad 6 \quad 142.480000$

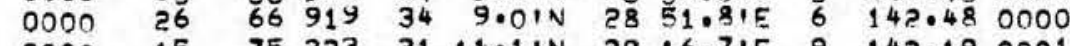

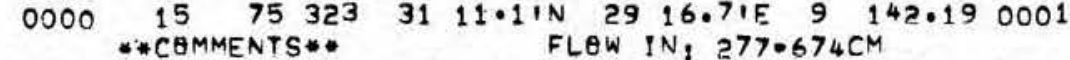

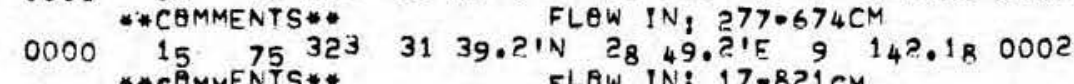
FLOW IN: $17.821 \mathrm{CM}$

$2518,648.000021$

$2518: 101 \%$ : 10000 ?

552. 674. 0000

$\begin{array}{ll}3969 & 41 \\ 3359 & 41\end{array}$

396941

43390 * COMMENTS**

1827. 821. 0000

38690 


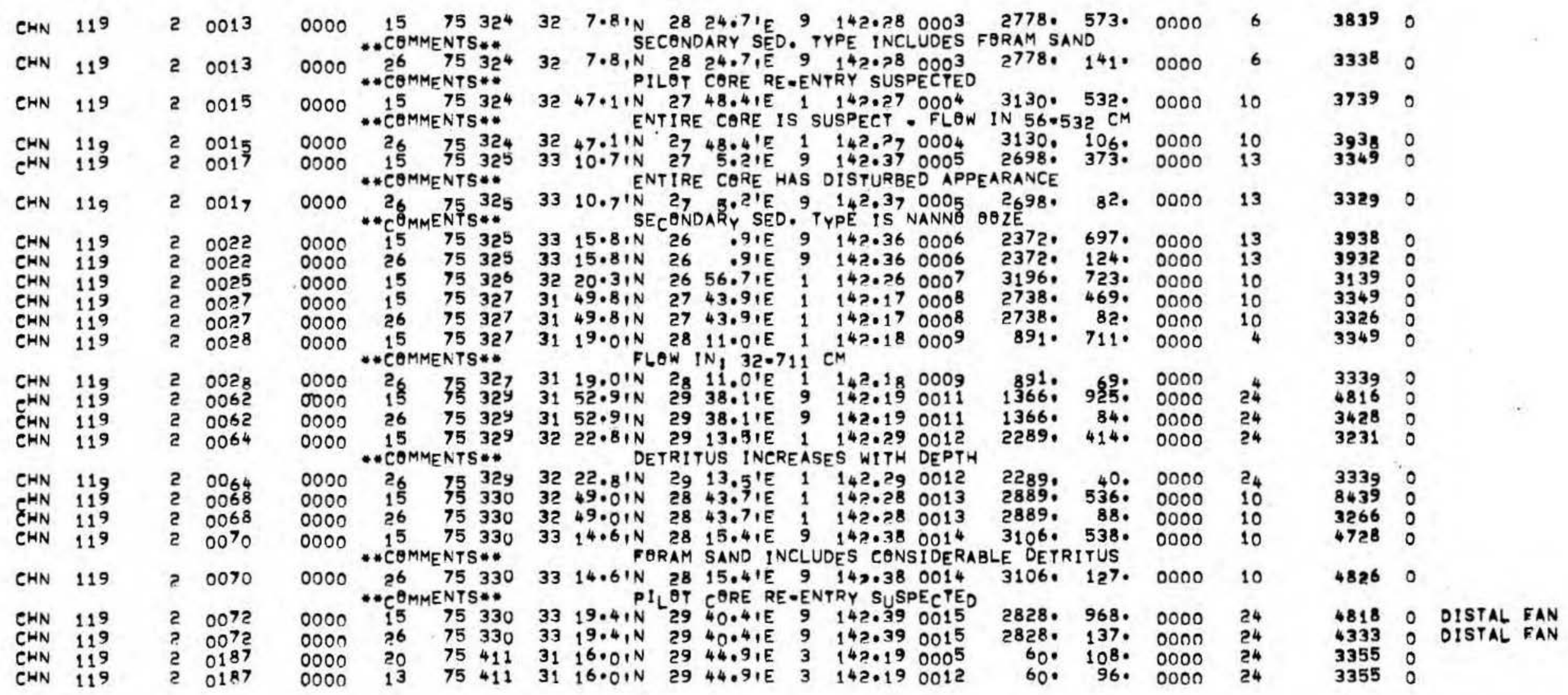

MARSDEN SQUARE \#143

$\begin{array}{rrrr}\text { AII } & 49 & 3 & A 003 \\ \text { AII } & 49 & 3 & A 003 \\ \text { AII } & 49 & 3 & 8003 \\ \text { CHN } & 7 & 3 & 0001 \\ \text { CHN } & 43 & 1 & 0085 \\ \text { CHN } & 61 & 2 & 0019\end{array}$

$\begin{array}{ll}0000 & 1 \\ 0000 & ? \\ 0000 & 1 \\ 0000 & 1 \\ 0000 & 1 \\ 0000 & 1\end{array}$

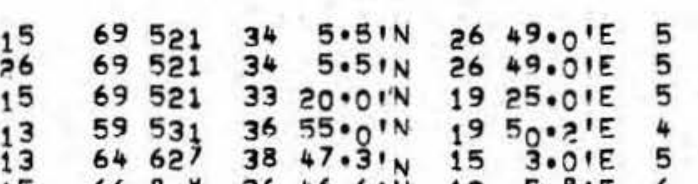

15668 \%
143.46 03.1. 2637. 400. 0000 $\begin{array}{lllll}143.46 & 03.1 & 2637, & 100 . & 0000 \\ 143.3903-2 & 2788 . & 239 . & 439\end{array}$ 1430690001 143.85002 3677 .

0.14 .0000 833: 975. 0000 ON HIGH (1000 FTHMS) IN ATLANTIS II DEEP

$\begin{array}{rll}5 & 3329 & 0 \\ 5 & 3329 & 0 \\ 0 & 3339 & 0 \\ 21 & 3730 & 0 \\ 21 & 4969 & 41 \\ 21 & 3931 & 41\end{array}$




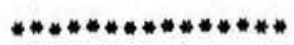

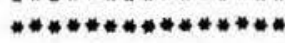

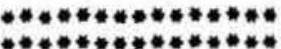

STATIAN DATA RETRIEVAL DATE: O01017:38 JUN O8 STATION UATA RETRIEVAL DATE:

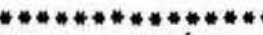

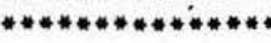

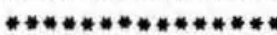

PAGE 31

PAGE 1803417591

\section{CORE
LENGTH DREDGE \\ FIX MARS. CORE OR $Q R$ OR OR PHYSIO. OR} SHIP CRUISE LEG STATION NAMPLE DEE DATE VICE YRMODA LATITUDE LONGITUDE TYPE SQUARE NUMBER DEPTH DEPTH VOLUME PROV EAST END OF SICILY

$\begin{array}{llll}\text { CHN } & 61 & 2 & 0019 \\ \text { CHN } & 61 & 2 & 0025 \\ \text { CHN } & 61 & 2 & 0029 \\ \text { CHN } & 61 & 2 & 0031\end{array}$

$0000 \quad 26 \quad 6688$

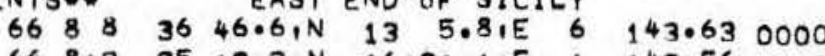
0000 *COMMENTS* 66 $3512 \cdot 3, N$ 16 31.1IE 1 14.3.56 0000 $\begin{array}{rrrr}8333^{\circ} & 104^{\circ} & 0000 & 21 \\ 1460^{\circ} & 732^{\circ} & 0000 & 21\end{array}$

SED. VITA

* \#COMMENTS* FLOW-IN,375.732 CM

3955. 910. 000021

$\begin{array}{ll}3359 & 41 \\ 3346 & 41\end{array}$

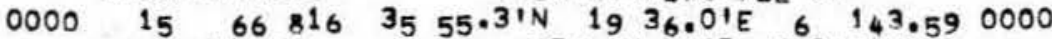

395. 910.

335941 ALL 1 UNIT

$0000 *{ }_{15}^{\theta M} 668_{1}$ \%

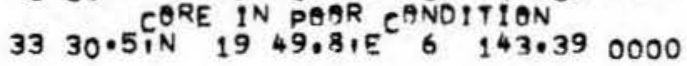

1108 . 338, $0000 \quad 21$

332241

MARSDEN SQUARE \# 144

$\begin{array}{lrrr}\text { CHN } & 7 & 3 & 0008 \\ \text { CHN } & 21 & 1 & 0018\end{array}$

$\begin{array}{lllllllllll}0000 & 14 & 59627 & 39 & 30.01 N & 6 & .01 E & 4 & 144.96 & 0008\end{array}$ CARE IN POAR CONDITION

$2848 \cdot 2848 \cdot 2006 \quad 0 \quad 0000 \quad 0$ - COMMENTS**

MARSDEN SQUARE \#147

$\begin{array}{llll}\text { CHN } & 43 & 1 & 010 \\ \text { CHN } & 43 & 1 & 010 \\ \text { CHN } & 43 & 1 & 010 \\ \text { CHN } & 43 & 1 & 011 \\ \text { CHN } & 82 & 6 & 002 \\ \text { CHN } & 8 ? & 6 & 002 \\ \text { CHN } & 82 & 6 & 002 \\ \text { CHN } & 82 & 6 & 002 \\ \text { CHN } & 82 & 6 & 00 ? \\ \text { CHN } & 82 & 6 & 002 \\ \text { CHN } & 8 ? & 6 & 002 \\ \text { CHN } & 82 & 6 & 002 \\ \text { CHN } & 82 & 7 & 003 \\ \text { CHN } & 8 ? & 7 & 003 \\ \text { CHN } & 8 ? & 7 & 003 \\ \text { CHN } & 82 & 7 & 003 \\ \text { CHN } & 82 & 7 & 003 \\ \text { CHN } & 8 ? & 7 & 003 \\ \text { CHN } & 8 ? & 7 & 003 \\ \text { CHN } & 82 & 7 & 0036\end{array}$

396441 396941 335941 396941

393854

332954

332454

335954

332854

332254

332254

332954

393240

393240

332954

333854

333954

333454

333854

335954 
MARSDEN SQUARE * 147

\begin{tabular}{|c|c|c|c|}
\hline CHN & 82 & 7 & 0041 \\
\hline $\begin{array}{l}\text { CHN } \\
\text { e }^{H N N}\end{array}$ & $\begin{array}{l}82 \\
82\end{array}$ & 7 & $\begin{array}{l}0041 \\
0042\end{array}$ \\
\hline CHN & 82 & 7 & $0_{44}$ \\
\hline $\begin{array}{l}C H N \\
C H N \\
C H N \\
C H N \\
C H N \\
C H N \\
C H N\end{array}$ & $\begin{array}{l}82 \\
82 \\
82 \\
82 \\
82 \\
82\end{array}$ & $\begin{array}{l}7 \\
8 \\
8 \\
8 \\
8 \\
8\end{array}$ & $\begin{array}{l}0044 \\
0045 \\
0049 \\
0049 \\
0050 \\
0050\end{array}$ \\
\hline $\begin{array}{l}\text { CHN } \\
\text { CHN }\end{array}$ & $\begin{array}{l}82 \\
82\end{array}$ & $\begin{array}{l}8 \\
8\end{array}$ & $\begin{array}{l}0051 \\
0051\end{array}$ \\
\hline CHN & 82 & 8 & 0054 \\
\hline
\end{tabular}

0000 - COMMENTS*

$0000 \quad 26 \quad 68826$

0000

0000 15 68 831

\section{0}

0000

0000

0000
0000

.0000

0000

0000

0000 - COMMENTS *. COMMENTS"

156896

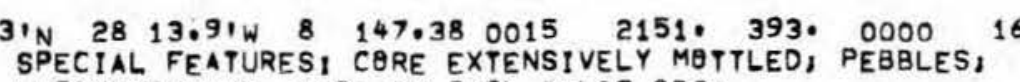

SECONDARY SED. TYPE IS CACL.SILIC OOZE

SPECIAL FEATUREST P PRE EXTENSIVELY MOTTLEDi PEBBLES

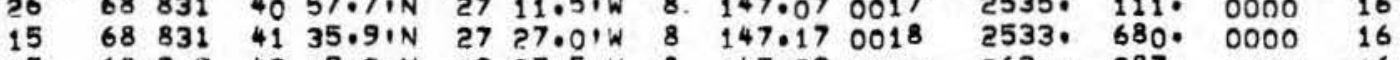

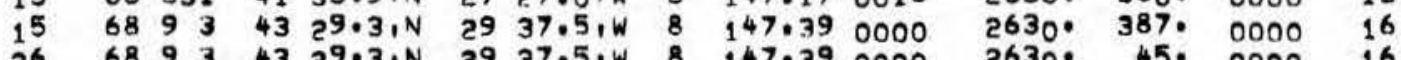

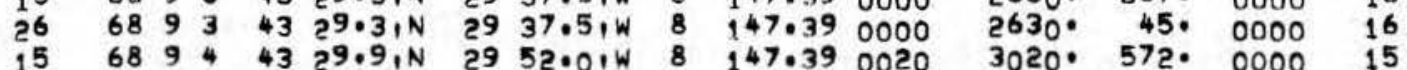

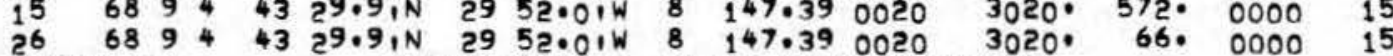
SECONDARY SED. TYPE! CALCOSILIC OOZE

$1568944317.31 \mathrm{~N} 2949.81 \mathrm{~W} 8147.390021$ 2103. 306. 000015 $26 \quad 6894 \quad 4317.31 \mathrm{~N} 2949.81 \mathrm{~W} 8147.390021 \quad 2103.84 .000015$ CORE EXTENSIVELY MOTTLED, PEBBLES

4350.0 IN $2757.5, W$. 8147.37 OO22 2575.
SECONDARY SED. TYPE: CALC.SILIC OQZE

$*$ COMMENTS**

SECONDARY SED. TYPE: CALC.SILIC OQZE 688. 000015 SPECIAL FEATURES: CORE EXTENSIVELY MOTTLEDI PEBBLESI SHELL FRAOMENTSI

$\begin{array}{llll}\text { CHN } & 13 & 1 & 0003 \\ \text { CHN } & 8 ? & 6 & 0024 \\ \text { CHN } & 82 & 6 & 0024 \\ \text { CHN } & 82 & 6 & 0026 \\ \text { CHN } & 82 & 6 & 0026 \\ \text { CHN } & 82 & 7 & 0031 \\ \text { CHN } & 82 & 7 & 0031 \\ \text { CHN } & 82 & 8 & 0056 \\ \text { CHN } & 82 & 8 & 0057 \\ \text { CHN } & 82 & 8 & 0058 \\ \text { CHN } & 82 & 8 & 0059\end{array}$

0000

156079

386854

$\begin{array}{ll}3334 & 54 \\ 3738 & 47\end{array}$

332254

$3838 \quad 47$

302947

373854

303854 
055

\#************* STATION UATA RETRIEVAL

OATE: $17: 3_{8}$ NUN $0_{8,177}$
$* * * * * * * * * * * * * * * *$

AGE 33

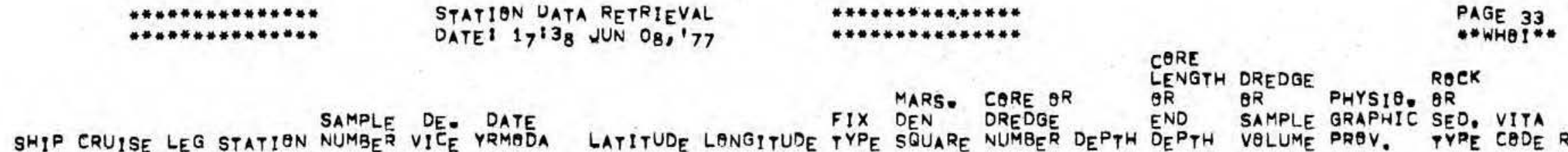

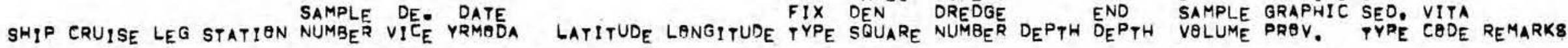

MARSDEN SQUARE * 151

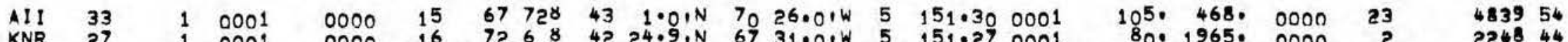

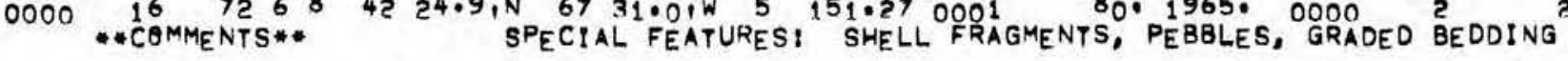

MARSDEN SQUARE *15?

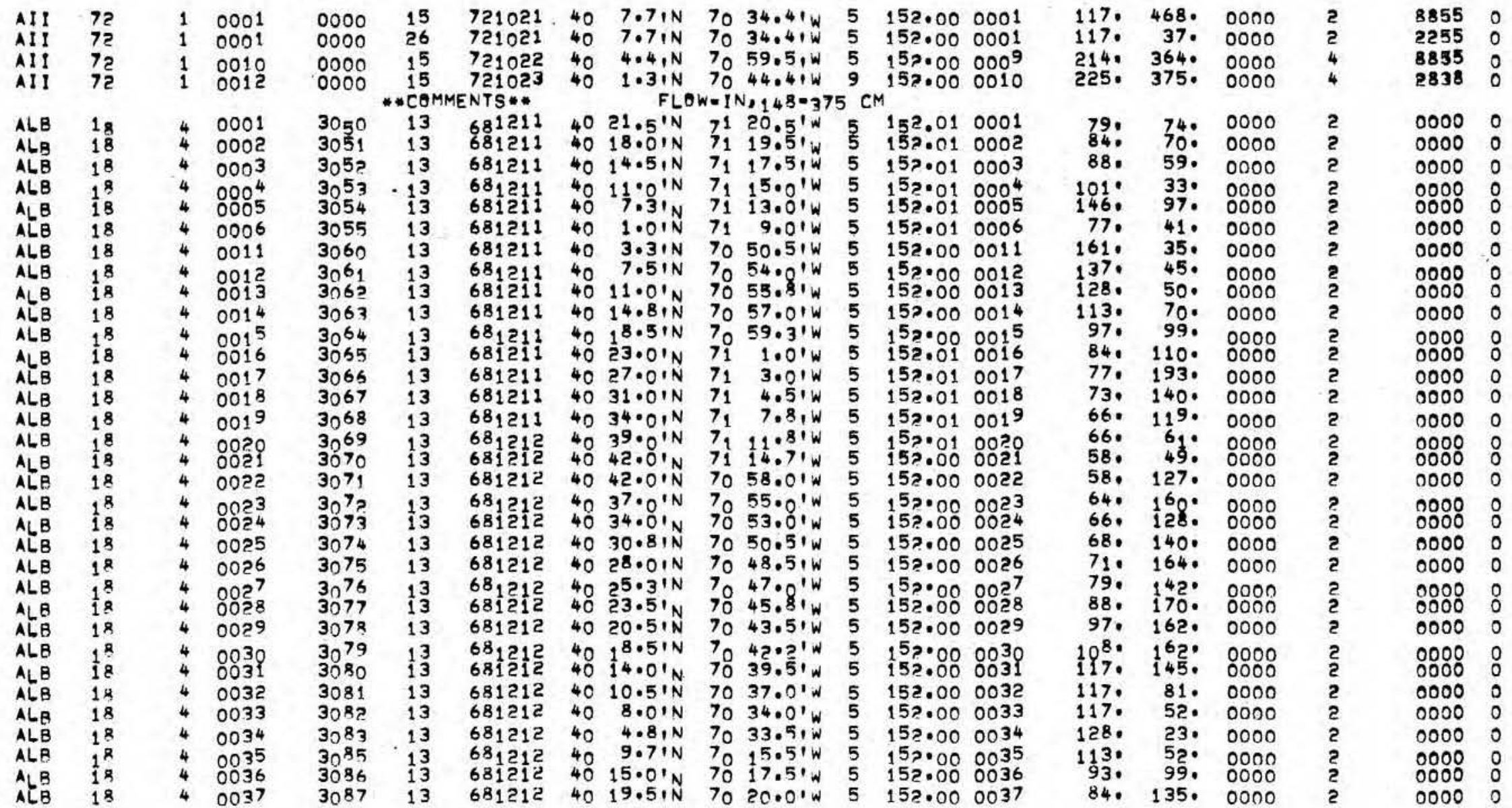


MARS. CORE OR LENGTH DREDGE PHYSIO. ROCK

SAMPLE DE. DATE FIX DEN DREDGE

MARSDEN SQUARE * 15?

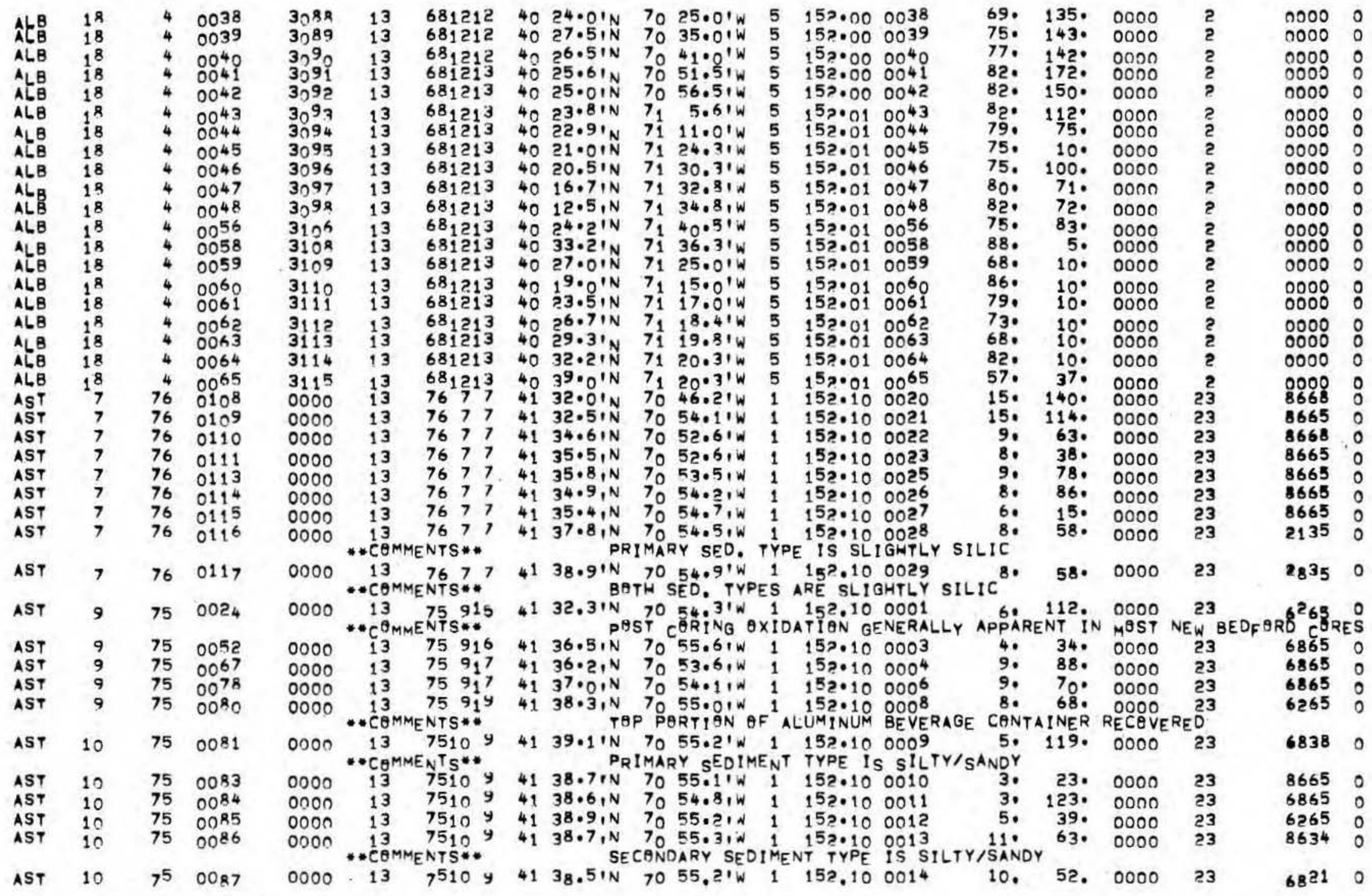




$\begin{array}{ll}* * * * * * * * * * * * * * * & \text { STATION DATA RETRIEVAL } \\ * * * * * * * * * * * * * & \text { DATE! OOIOIT:3B JUN OS } \\ * * * * * * * * * * * * * * & \text { STATION UATA RETRIEVAL } \\ * * * * * * * * * * * * * * & \text { DATE: }\end{array}$

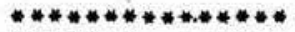

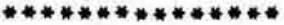

***************
PAGE 35

PAGE 1803417591

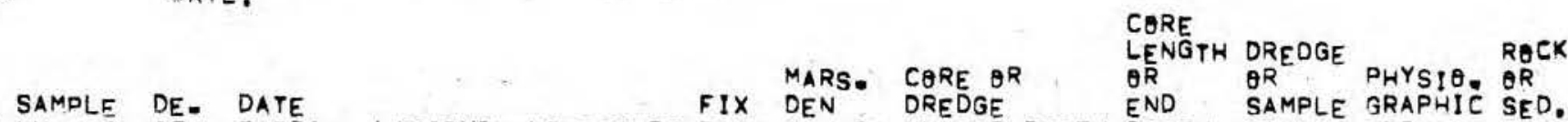

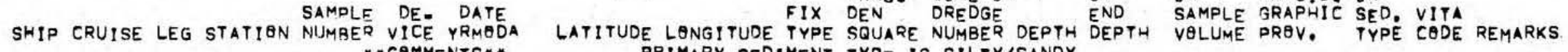
\#CQMMENTS** * PRIMARY SEDIMENT TYPE IS SILTYISANOY

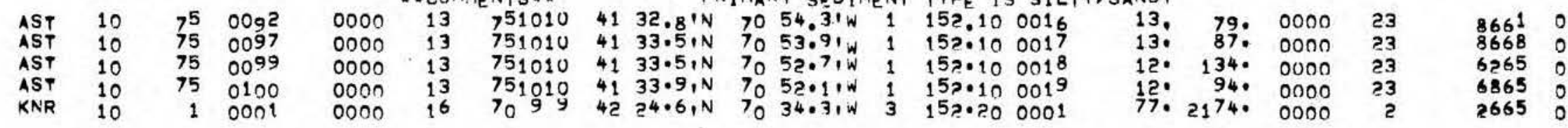

MARSDEN SQUARE * 176

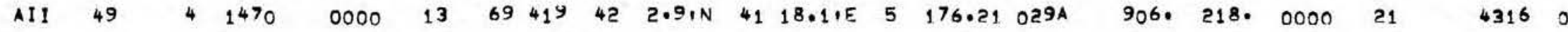

MARSDEN SQUARE \# 177

\begin{tabular}{|c|c|c|c|c|c|c|c|c|c|c|c|c|c|c|c|c|c|c|c|}
\hline AII & $\begin{array}{l}49 \\
49\end{array}$ & $\begin{array}{l}3 \\
3\end{array}$ & $\begin{array}{l}1431 \\
1432\end{array}$ & $\begin{array}{l}0000 \\
0000\end{array}$ & $\begin{array}{l}15 \\
13 \\
\text { COMN }\end{array}$ & $\begin{array}{l}69 \\
69 \\
\text { ENTS }\end{array}$ & $\begin{array}{l}322 \\
323 \\
5 * *\end{array}$ & $\begin{array}{l}42 \\
43\end{array}$ & $\begin{array}{r}14 \cdot 0^{1 N} \\
.61 N \\
C O R\end{array}$ & $\begin{array}{l}33 \\
34 \\
\text { RE IN }\end{array}$ & $\begin{array}{l}4 . Q^{\prime} E \\
4051 E \\
\text { PQAR }\end{array}$ & $\begin{array}{c}5 \\
5 \\
\text { AND }\end{array}$ & $\begin{array}{l}177.23 \\
177.34 \\
\text { ITIAN }\end{array}$ & $\begin{array}{l}002^{A} \\
0003\end{array}$ & $\begin{array}{l}2136, \\
2248 .\end{array}$ & $\begin{array}{l}4910 \\
1240\end{array}$ & $\begin{array}{l}0000 \\
0000\end{array}$ & 21 & $\begin{array}{l}3226 \\
3419\end{array}$ \\
\hline AII & 49 & 3 & 1433 & 0000 & $\begin{array}{l}15 \\
C \theta M\end{array}$ & $\begin{array}{l}69 \\
\text { NTS }\end{array}$ & $\begin{array}{l}32^{4} \\
5 * *\end{array}$ & 44 & $5 \cdot 0 \cdot N_{D R}^{N}$ & $\begin{array}{l}35 \\
\text { IMAR }\end{array}$ & $\begin{array}{l}\text { ODIE } \\
\text { SEDIN }\end{array}$ & $\begin{array}{c}5 \\
\text { IENT }\end{array}$ & $\begin{array}{l}177.45 \\
\text { TYPE CA }\end{array}$ & $00^{4 A}$ & ${ }_{I C}^{2225} \theta \theta Z E$ & 4930 & 0000 & ?1 & 0311 \\
\hline $\begin{array}{l}A I ! \\
\text { AI! } \\
\text { AI! } \\
\text { AI! }\end{array}$ & $\begin{array}{l}49 \\
49 \\
49 \\
49\end{array}$ & $\begin{array}{l}3 \\
3 \\
3 \\
3\end{array}$ & $\begin{array}{l}1434 \\
1435 \\
1436 \\
1436\end{array}$ & $\begin{array}{l}0000 \\
0000 \\
0000 \\
0000\end{array}$ & $\begin{array}{l}15 \\
13 \\
15 \\
26\end{array}$ & $\begin{array}{l}69 \\
69 \\
69 \\
69\end{array}$ & $\begin{array}{l}325 \\
326 \\
326 \\
326\end{array}$ & $\begin{array}{l}44 \\
44 \\
43 \\
43\end{array}$ & $\begin{array}{l}20.01 \mathrm{~N} \\
26.01 \mathrm{~N} \\
24.01 \mathrm{~N} \\
24.01 \mathrm{~N}\end{array}$ & $\begin{array}{l}36 \\
36 \\
36 \\
36\end{array}$ & $\begin{array}{l}\text {.O'E } \\
13.01 E \\
36.01 E \\
36.01 E\end{array}$ & $\begin{array}{l}5 \\
5 \\
5 \\
5\end{array}$ & $\begin{array}{l}177.46 \\
177.46 \\
177.36 \\
177.36\end{array}$ & $\begin{array}{l}005 A \\
0006 \\
007 A \\
007 B\end{array}$ & $\begin{array}{l}1466^{\circ} \\
10330^{\circ} \\
2158^{\circ} \\
2158^{\circ}\end{array}$ & $\begin{array}{l}140^{\circ} \\
160^{\circ} \\
535^{\circ} \\
119^{\circ}\end{array}$ & $\begin{array}{l}0000 \\
0000 \\
0000 \\
0000\end{array}$ & 21 & $\begin{array}{l}2319 \\
2329 \\
2431 \\
4349\end{array}$ \\
\hline AI & $\begin{array}{l}49 \\
49\end{array}$ & $\begin{array}{l}3 \\
3\end{array}$ & $\begin{array}{l}1437 \\
1438\end{array}$ & $\begin{array}{l}0000 \\
0000\end{array}$ & $\begin{array}{l}13 \\
13\end{array}$ & $\begin{array}{l}69 \\
69\end{array}$ & $\begin{array}{l}327 \\
328\end{array}$ & $\begin{array}{l}41 \\
41\end{array}$ & $\begin{array}{l}41^{\circ} 2^{1} \mathrm{~N} \\
58.5^{\prime} \mathrm{N}\end{array}$ & $\begin{array}{l}38 \\
35\end{array}$ & $\begin{array}{l}2^{8 .} .31 E \\
41.01 E\end{array}$ & $\begin{array}{l}5 \\
5\end{array}$ & $\begin{array}{l}177.18 \\
177.15\end{array}$ & $000^{8}$ & $\begin{array}{l}973^{\circ} \\
284^{\circ}\end{array}$ & $\begin{array}{r}620 \\
1450\end{array}$ & $\begin{array}{l}0000 \\
0000\end{array}$ & 21 & $\begin{array}{l}3219 \\
3316\end{array}$ \\
\hline A! & $\begin{array}{l}49 \\
49\end{array}$ & $\begin{array}{l}3 \\
3\end{array}$ & $\begin{array}{l}1439 \\
1440\end{array}$ & $\begin{array}{l}0000 \\
0000\end{array}$ & $\begin{array}{l}15 \\
13\end{array}$ & $\begin{array}{l}69 \\
69\end{array}$ & $\begin{array}{l}32 y \\
32 y\end{array}$ & $\begin{array}{l}42 \\
42\end{array}$ & $\begin{array}{l}21 \cdot 91 N \\
12 \cdot 21 N\end{array}$ & $\begin{array}{l}35 \\
34\end{array}$ & $\begin{array}{l}\text { P9.51E } \\
21.31 E\end{array}$ & $\begin{array}{l}5 \\
5\end{array}$ & $\begin{array}{l}177.25 \\
177.24\end{array}$ & $\begin{array}{l}010 A \\
011 A\end{array}$ & $\begin{array}{r}19430^{\circ} \\
264^{\circ}\end{array}$ & $\begin{array}{l}7000 \\
131 .\end{array}$ & $\begin{array}{l}0000 \\
0000\end{array}$ & 2 & $\begin{array}{l}15 \\
19\end{array}$ \\
\hline & 49 & 3 & 1440 & 0000 & 15 & 69 & 329 & 42 & $12.7, \mathrm{~N}$ & 34 & $21.31 E$ & 5 & 177.24 & 0118 & 207. & 740. & 0000 & & $31<0$ \\
\hline AI & 49 & 3 & $144 n$ & 0000 & ${ }^{2}{ }^{6}$ & 69 & $\begin{array}{c}32^{9} \\
S^{* *}\end{array}$ & 42 & $12 \cdot 71 \mathrm{~N}$ & IMAR & $\begin{array}{l}21 \cdot 31 E \\
Y \text { AND } S\end{array}$ & ${ }^{5}$ & $\begin{array}{l}177 \cdot 24 \\
N D A R Y S E\end{array}$ & $\begin{array}{l}011 \mathrm{C} \\
\text { EDIMEN }\end{array}$ & $\begin{array}{l}20^{7} \cdot \\
T Y{ }^{\circ}\end{array}$ & $\begin{array}{ll}1280 \\
A L C=S 1\end{array}$ & $\mathbb{L}_{\theta \theta Z}$ & 21 & 0059 \\
\hline AlI & 49 & 3 & 1442 & 0000 & & & $\begin{array}{r}331 \\
5 * *\end{array}$ & 44 & $\begin{array}{r}44.31 \mathrm{~N} \\
\text { FLO }\end{array}$ & $\begin{array}{c}31 \\
W=I N\end{array}$ & $\begin{array}{l}57.21 E \\
1.160-27\end{array}$ & ${ }^{5} \mathrm{Cl}$ & $M^{177.41}$ & 0121 & 420. & 278. & 0000 & 21 & 4839 \\
\hline AI! & $\begin{array}{l}49 \\
49\end{array}$ & $\begin{array}{l}3 \\
3\end{array}$ & $\begin{array}{l}1442 \\
1442\end{array}$ & $\begin{array}{l}0000 \\
0000\end{array}$ & $\begin{array}{l}26 \\
13\end{array}$ & $\begin{array}{l}69 \\
69\end{array}$ & $\begin{array}{l}331 \\
331\end{array}$ & $\begin{array}{l}44 \\
44\end{array}$ & $\begin{array}{l}44.31 N \\
35.91 N\end{array}$ & $\begin{array}{l}31 \\
31\end{array}$ & $\begin{array}{l}57.2^{\prime} E \\
54.0^{\prime} E\end{array}$ & 5 & $\begin{array}{l}177.41 \\
177.41\end{array}$ & $\begin{array}{l}0128 \\
012 \mathrm{C}\end{array}$ & $\begin{array}{l}420 . \\
5490\end{array}$ & $\begin{array}{l}132 \\
127\end{array}$ & & 2 & $\begin{array}{l}4326 \\
3419\end{array}$ \\
\hline Al & $\begin{array}{l}49 \\
49\end{array}$ & $\begin{array}{l}3 \\
3\end{array}$ & $\begin{array}{l}1443 \\
1443\end{array}$ & $\begin{array}{l}0000 \\
0000\end{array}$ & $\begin{array}{l}15 \\
26\end{array}$ & $\begin{array}{l}69 \\
69\end{array}$ & $\begin{array}{l}331 \\
331\end{array}$ & $\begin{array}{l}44 \\
44\end{array}$ & $\begin{array}{l}35 \cdot 3, N \\
35 \cdot 3, N\end{array}$ & $\begin{array}{l}31 \\
31\end{array}$ & $\begin{array}{l}55.31 E \\
55.31 E\end{array}$ & $\begin{array}{l}5 \\
5\end{array}$ & $\begin{array}{l}177.41 \\
177.41\end{array}$ & $\begin{array}{l}013 A \\
013 B\end{array}$ & $\begin{array}{l}1057 \% \\
1057 \%\end{array}$ & $\begin{array}{l}766^{\circ} \\
198^{\circ}\end{array}$ & $\begin{array}{l}0000 \\
0000\end{array}$ & 21 & $\begin{array}{l}2416 \\
3429\end{array}$ \\
\hline AI I I & $\begin{array}{l}49 \\
49\end{array}$ & 3 & $\begin{array}{l}1444 \\
1445\end{array}$ & $\begin{array}{l}0000 \\
0000\end{array}$ & $\begin{array}{l}15 \\
15\end{array}$ & $\begin{array}{l}69 \\
69\end{array}$ & $\begin{array}{ll}4 & 1 \\
4 & \end{array}$ & $\begin{array}{l}43 \\
43\end{array}$ & $\begin{array}{l}48.0^{1} \mathrm{~N} \\
16.0^{\prime} \mathrm{N}\end{array}$ & $\begin{array}{l}31 \\
31\end{array}$ & $\begin{array}{l}45.31 E \\
18.01 E\end{array}$ & $\begin{array}{l}5 \\
5\end{array}$ & $\begin{array}{l}177 \cdot 31 \\
177 \cdot 31\end{array}$ & $\begin{array}{l}014 \mathrm{~A} \\
015 \mathrm{~A}\end{array}$ & $\begin{array}{l}1597 . \\
1915 .\end{array}$ & $\begin{array}{l}511 . \\
357 .\end{array}$ & $\begin{array}{l}0000 \\
0000\end{array}$ & 21 & $\begin{array}{l}4216 \\
3716\end{array}$ \\
\hline AI & 49 & 3 & 1445 & 0000 & 26 & 69 & 42 & 43 & $16.01 \mathrm{~N}$ & 31 & $18.01 E$ & 5 & $177 \cdot 31$ & $015 B$ & & 163. & 0000 & 21 & 3419 \\
\hline Al I & $\begin{array}{l}49 \\
49\end{array}$ & $\begin{array}{l}3 \\
3\end{array}$ & $\begin{array}{l}1446 \\
1446\end{array}$ & 0000 & $\begin{array}{l}15 \\
26\end{array}$ & $\begin{array}{l}69 \\
69\end{array}$ & $\begin{array}{ll}4 & 2 \\
4 & 2\end{array}$ & $\begin{array}{l}42 \\
42\end{array}$ & $11.51 \mathrm{~N}$ & 31 & 21.815 & $\begin{array}{l}5 \\
5\end{array}$ & $177 \cdot 21$ & $016 A$ & $\begin{array}{l}21470 \\
2147\end{array}$ & 772. & & ? & 4831 \\
\hline & 49 & 3 & 1446 & & & & & & & MARY & SED TY & & $\begin{array}{l}177.21 \\
\text { CALC-S1 }\end{array}$ & ILICB $\theta 8$ & $Z Z E^{2147}$ & 117. & & & \\
\hline 11 & 49 & 3 & 1447 & 0000 & 15 & 69 & 43 & 41 & $23.01 \mathrm{~N}$ & $3^{1}$ & $3 \cdot 6^{\prime} E$ & 5 & 177.11 & $017 \mathrm{~A}$ & $1256^{\circ}$ & 419. & 0000 & $2^{1}$ & $32^{19}$ \\
\hline
\end{tabular}




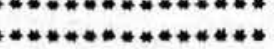

PAGE 36

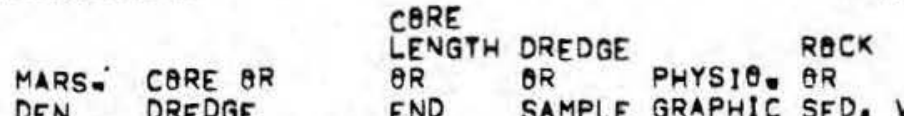

SAMPLE DE. DATE

FIX MARS:- CORE OR

END

* WHOI"*

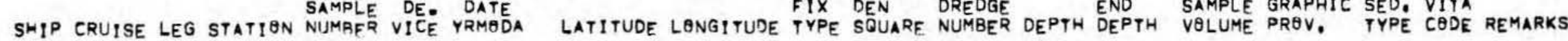

MARSDEN SQUARE \#177

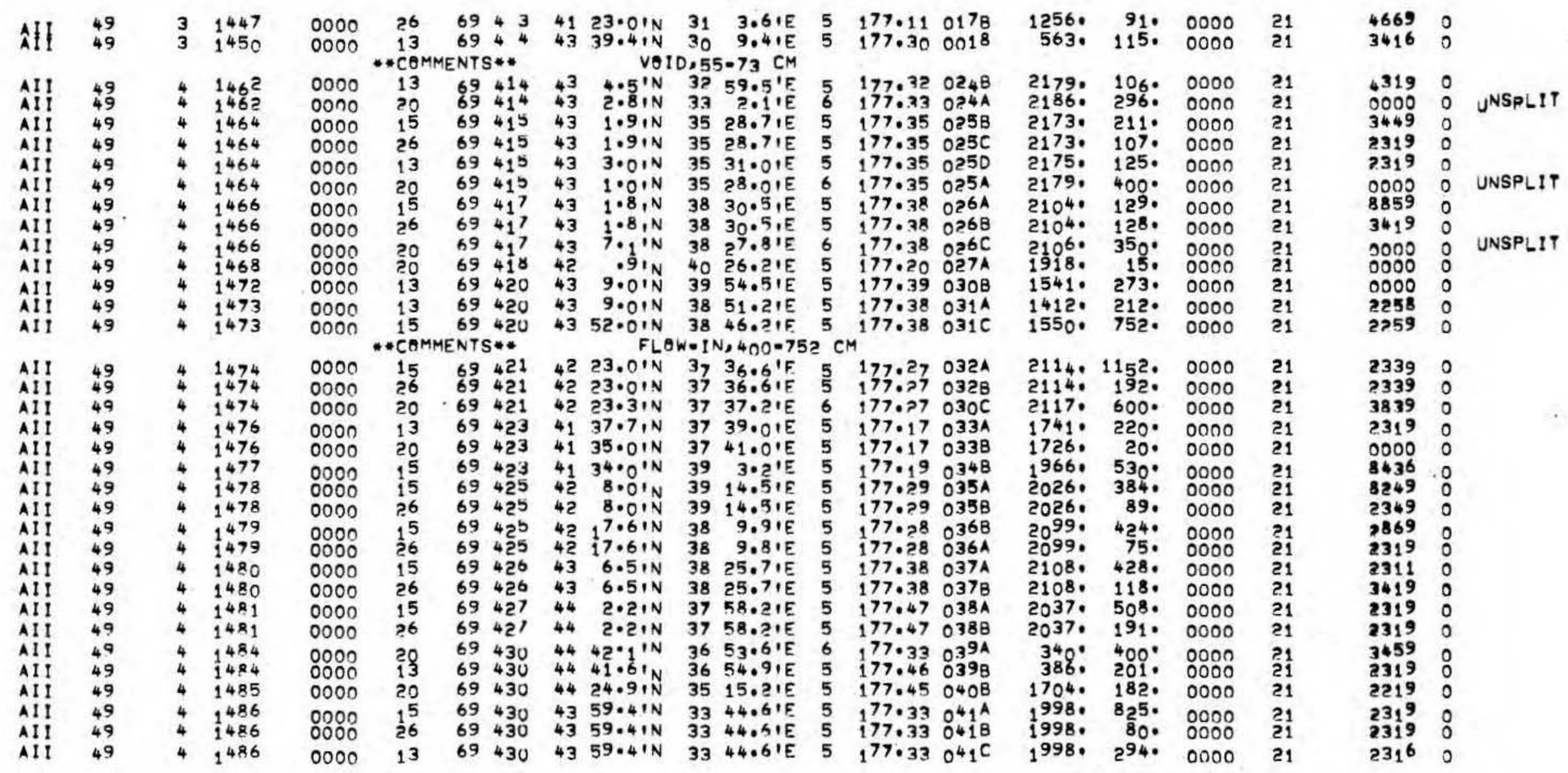

MARSDEN SQUARE * 178

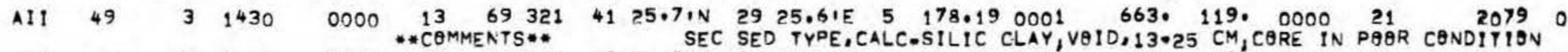

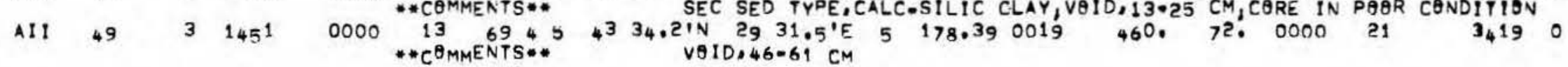




\section{\#************* STATIEN UATA RETRIEVAL

\begin{abstract}
DATE: $17: 38$ JUN O8,177
\end{abstract}

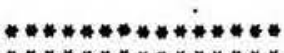

MARS CORE GR

SHIP CRUISE LEG STATION NUMPLE VIEE YRMPE

FIX DEN DREDG

LENGTH DREDGE ROCK

PAGE 37

* WHOI**

$\operatorname{RoCK}$

GND SAMPLE TRYSIOE $O R$

LATITUDE LONGITUDE TYPE SQUARE NUMBER DEPTH DEPTH VULUME PREV. PYPE CODE REMARKS

\section{MARSDEN SQUARE \# 178}

$\begin{array}{llll}\text { All } & 49 & 3 & 1452 \\ \text { AII } & 49 & 3 & 1453 \\ \text { AII } & 49 & 3 & 1461\end{array}$

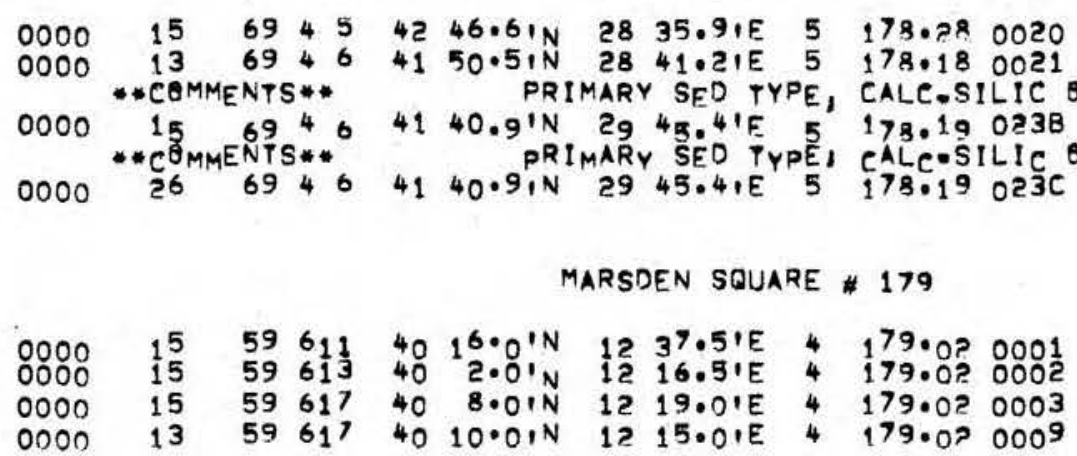

728

848. 0000 $\theta Z E$

1788.890 .0000

$\begin{array}{lll}21 & 4329 & 0 \\ 21 & 0819 & 0\end{array}$

08110

38150

\section{MARSDEN SQUARE \# 180}

$\begin{array}{lrrr}\mathrm{CHN} & 7 & 7 & 0006 \\ \mathrm{CHN} & 21 & 1 & 001\end{array}$

CWN $21 \quad 10020$

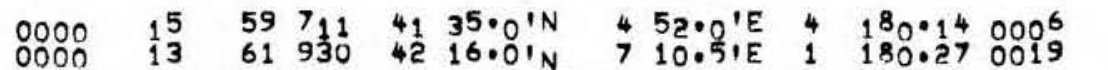
- COMMENTS** CORE IN PQAR CANDITION

$0000 \quad 13611014321.0, N^{3} 8_{15.5, E} 5 \quad 180.380020$

$\begin{array}{lll}35844^{\circ} & 5955^{\circ} & 0000 \\ 3610^{\circ} & 8744^{\circ} & 0000\end{array}$

3612 792, 0000

3453. 190.0000
21
21
21
21

$\begin{array}{ll}3730 & 0 \\ 3960 & 0\end{array}$

32600

3340 O STETSAN COR

MARSDEN SQUARE \#181

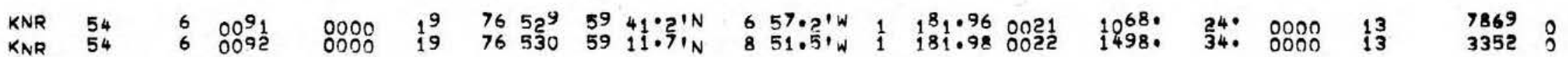

MARSDEN SQUARE \# 182

\begin{tabular}{|c|c|c|c|c|c|c|c|c|c|c|c|c|c|c|c|c|c|c|c|}
\hline $\begin{array}{l}\text { KNR } \\
\text { KNR } \\
K_{N R} \\
\text { KNR }\end{array}$ & $\begin{array}{l}51 \\
51 \\
51 \\
51\end{array}$ & $\begin{array}{l}3 \\
3 \\
3 \\
3\end{array}$ & $\begin{array}{l}0036 \\
0036 \\
0039 \\
0039\end{array}$ & $\begin{array}{l}0000 \\
0000 \\
0000 \\
0000\end{array}$ & $\begin{array}{l}16 \\
26 \\
16 \\
26\end{array}$ & $\begin{array}{l}75 \\
75 \\
75 \\
75\end{array}$ & $\begin{array}{l}824 \\
824 \\
827 \\
827\end{array}$ & $\begin{array}{l}54 \\
54 \\
56 \\
56\end{array}$ & $\begin{array}{l}28.51 \mathrm{~N} \\
28.51 \mathrm{~N} \\
16.51 \mathrm{~N} \\
16.51 \mathrm{~N}\end{array}$ & $\begin{array}{l}15 \\
15 \\
12 \\
12\end{array}$ & $\begin{array}{l}17.91 \mathrm{w} \\
17.91 \mathrm{w} \\
30.21 \mathrm{w} \\
30.21 \mathrm{w}\end{array}$ & $\begin{array}{r}9 \\
9 \\
11 \\
11\end{array}$ & $\begin{array}{l}182 \cdot 45 \\
182 \cdot 45 \\
182.62 \\
182.62\end{array}$ & $\begin{array}{l}0013 \\
0013 \\
0017 \\
0017\end{array}$ & $\begin{array}{l}2665^{\circ} \\
2665^{\circ} \\
2505^{\circ} \\
2505^{\circ}\end{array}$ & $\begin{array}{r}1440^{\circ} \\
139^{\circ} \\
1003 . \\
89^{\circ}\end{array}$ & $\begin{array}{l}0000 \\
0000 \\
0000 \\
0000\end{array}$ & $\begin{array}{l}26 \\
26 \\
26 \\
26\end{array}$ & $\begin{array}{l}4332 \\
3429 \\
4319 \\
3332\end{array}$ \\
\hline $\begin{array}{l}\text { KNR } \\
\text { KNR }\end{array}$ & $\begin{array}{l}5^{1} \\
51\end{array}$ & $\begin{array}{l}3 \\
3\end{array}$ & $\begin{array}{l}\mathrm{OO}_{4} 1 \\
00_{4} 1\end{array}$ & $\begin{array}{l}0000 \\
0000\end{array}$ & $\begin{array}{r}16 \\
26 \\
-C \theta M\end{array}$ & $\begin{array}{r}75 \\
75 \\
\text { ENTS }\end{array}$ & $\begin{array}{r}829 \\
829 \\
S * *\end{array}$ & $\begin{array}{l}56 \\
56\end{array}$ & $\begin{array}{r}17.91 \mathrm{~N} \\
17.91 \mathrm{~N}\end{array}$ & $\begin{array}{l}\text { LI } \\
12 \\
12 \\
\text { LAT }\end{array}$ & $\begin{array}{l}31.31 \mathrm{~W} \\
31.31 \mathrm{~W} \\
\text { CORE RE }\end{array}$ & $\begin{aligned} & 11 \\
11 & \\
= & \bullet N\end{aligned}$ & $\begin{array}{r}18 ? .6 \text { ? } \\
18 ? \cdot 6 ? \\
\text { UTRY SUSF }\end{array}$ & $\begin{array}{l}\text { PECTED } \\
\text { OO19 } \\
\text { OO19 } \\
\text { PECTED }\end{array}$ & $\begin{array}{l}25^{3} 5 \\
2535\end{array}$ & $\begin{array}{r}1492 . \\
82 .\end{array}$ & $\begin{array}{l}0000 \\
0000\end{array}$ & $\begin{array}{l}26 \\
26\end{array}$ & $\begin{array}{r}4319 \\
3389\end{array}$ \\
\hline $\begin{array}{l}\text { KNR } \\
\text { KNR }\end{array}$ & $\begin{array}{l}5^{1} \\
51\end{array}$ & $\begin{array}{l}3 \\
3\end{array}$ & $\begin{array}{l}0041 \\
0041\end{array}$ & $\begin{array}{l}0000 \\
0000\end{array}$ & $\begin{array}{l}18 \\
18\end{array}$ & 35 & $\begin{array}{l}830 \\
830\end{array}$ & $\begin{array}{l}56 \\
56\end{array}$ & $\begin{array}{l}16.81 \mathrm{~N} \\
16.81 \mathrm{~N}\end{array}$ & $\begin{array}{l}12 \\
12\end{array}$ & $\begin{array}{l}32.2^{\prime} w \\
33.2{ }^{\prime} w\end{array}$ & $\begin{array}{l}11 \\
11\end{array}$ & $\begin{array}{l}182.62 \\
182.62\end{array}$ & $\begin{array}{l}0022 \\
0023\end{array}$ & $\begin{array}{l}2930 . \\
2939 .\end{array}$ & 84. & $\begin{array}{l}0000 \\
0000\end{array}$ & $\begin{array}{l}26 \\
26\end{array}$ & $\begin{array}{l}3462 \\
4.359\end{array}$ \\
\hline
\end{tabular}




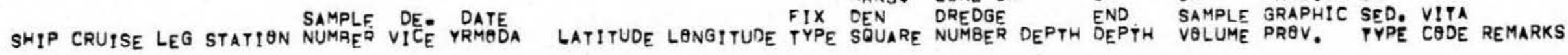

MARS- CQRE OR LENGTH DREDGE PHYSIO. $O R$ ROCK

MARSDEN SQUARE *18?

$\begin{array}{llll}\text { KNR } & \mathbf{5 1} & 3 & 0041 \\ \text { KNR } & \mathbf{5 1} & 3 & 0041 \\ \text { KNR } & \mathbf{5 1} & \mathbf{3} & 0041 \\ \text { KNR } & \mathbf{5 1} & 3 & 00^{4} \\ \text { KNR } & 51 & 3 & 0042 \\ \text { KNR }^{N} & 54 & 6 & 0094\end{array}$

$\begin{array}{ll}0000 & 18 \\ 0000 & 18 \\ 0000 & 18 \\ 0000 & 16 \\ 0000 & 26 \\ 0000 & 19\end{array}$

75830

$5616.61 \mathrm{~N}$

$5616.5, N \quad 1233.81 W 11 \quad 182.620025$ $75830 \quad 56 \quad 16.61 \mathrm{~N}$ 12 $34.01 \mathrm{~W} 11 \quad 182.620026$ $\begin{array}{lllllllll}75 & 830 & 56 & 13.4 \mathrm{~N} & 12 & 38 . \mathrm{O}^{\prime W} & 11 & 182.62 & 0031\end{array}$ $758305613.41 \mathrm{~N} 1238.01 \mathrm{~W} 11$ 18?.6? 0031 $\begin{array}{ll}765315926.01 N \quad 136.61 \mathrm{~W} 1 & 182.93002^{4}\end{array}$

\begin{tabular}{|c|c|}
\hline $\begin{array}{l}2956 . \\
2962 . \\
2619 .\end{array}$ & $\begin{array}{r}85 . \\
91 . \\
51 . \\
1296 .\end{array}$ \\
\hline $\begin{array}{l}619 . \\
290 .\end{array}$ & $\begin{array}{r}123 . \\
39 .\end{array}$ \\
\hline
\end{tabular}

$\begin{array}{lll}26 & 4332 & 0 \\ 26 & 3862 & 0 \\ 26 & 3359 & 0 \\ 26 & 4319 & 0 \\ 26 & 3359 & 0 \\ 13 & 3332 & 0\end{array}$

MARSDEN SQUARE *183

35620

MARSDEN SQUARE \#215

$\begin{array}{llllll}\text { AII } & 32 & 1 & 0004 & 0000 & 13 \\ \text { AII } & 32 & 1 & 0006 & 0000 & 13 \\ \text { AII } & 32 & 1 & 0007 & 0000 & 13 \\ \text { AII } & 32 & 1 & 0008 & 0000 & 13 \\ \text { AII } & 32 & 1 & 0011 & 0000 & 13 \\ \text { AII } & 32 & 1 & 0012 & 0000 & 13 \\ \text { AII } & 32 & 1 & 0013 & 0000 & 13 \\ \text { AII } & 32 & 1 & 0017 & 0000 & 13 \\ \text { AII } & 32 & 1 & 0018 & 0000 & 13 \\ \text { AII } & 32 & 2 & 0020 & 0000 & 13 \\ \text { AII } & 32 & 2 & 0021 & 0000 & 13 \\ \text { AII } & 32 & 2 & 0028 & 0000 & 13 \\ \text { AII } & 32 & 2 & 0029 & 0000 & 13 \\ \text { AII } & 32 & 2 & 0032 & 0000 & 13 \\ \text { AII } & 32 & 2 & 0037 & 0000 & 13 \\ \text { AII } & 32 & 2 & 0038 & 0000 & 13\end{array}$

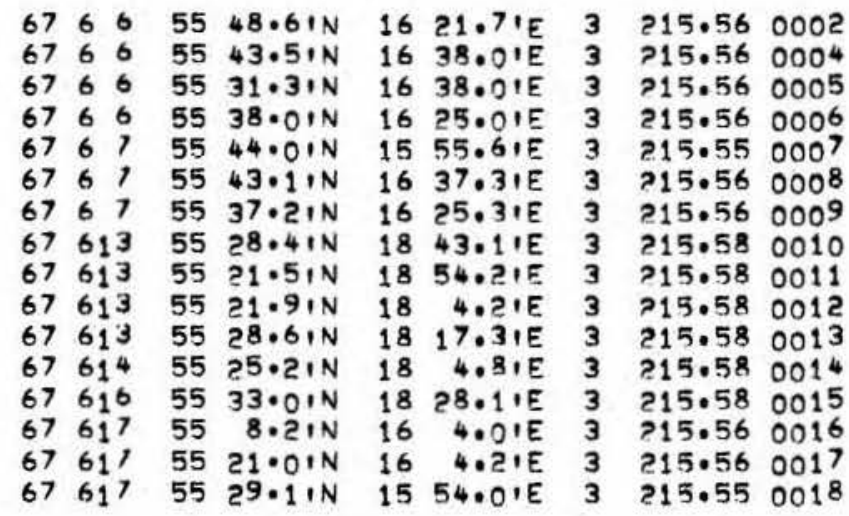

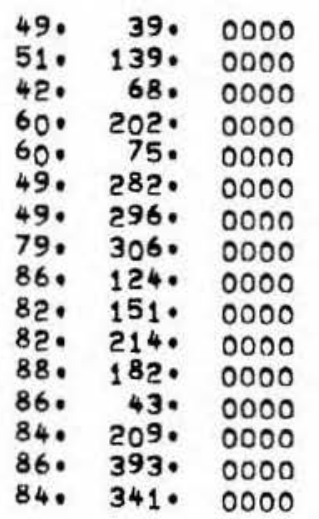

$\begin{array}{lll}20 & 2849 & 0 \\ 20 & 1819 & 0 \\ 20 & 2234 & 0 \\ 20 & 1819 & 0 \\ 20 & 2259 & 0 \\ 20 & 1814 & 0 \\ 20 & 1814 & 0 \\ 20 & 1229 & 0 \\ 20 & 2249 & 0 \\ 20 & 2864 & 0 \\ 20 & 2814 & 0 \\ 20 & 2814 & 0 \\ 20 & 2869 & 0 \\ 20 & 1625 & 0 \\ 20 & 1819 & 0 \\ 20 & 2814 & 0\end{array}$

MARSDEN SQUARE \# 216

$\begin{array}{llll}\text { KNR } & 54 & 6 & 0019 \\ \text { KNR } & 54 & 6 & 0022 \\ \text { KNR } & 54 & 6 & 0030 \\ \text { KNR } & 54 & 6 & 0032 \\ \text { KNR } & 54 & 6 & 0044 \\ \text { KNR } & 54 & 6 & 0059 \\ \text { KNR } & 54 & 6 & 0076\end{array}$

$\begin{array}{ll}0000 & 19 \\ 0000 & 19 \\ 0000 & 19 \\ 0000 & 19 \\ 0000 & 19 \\ 0000 & 19 \\ 0000 & 19\end{array}$

$\begin{array}{llll}76 & 512 & 58 & 26 \cdot O^{\prime N} \\ 76 & 512 & 58 & 26.91 \\ 76 & 513 & 58 & 54.91 N \\ 76 & 514 & 58 & 50.31 N \\ 76 & 515 & 59 & 5.81 N \\ 76 & 519 & 58 & 25.51 N \\ 76 & 52^{6} & 59 & 30.61 N\end{array}$

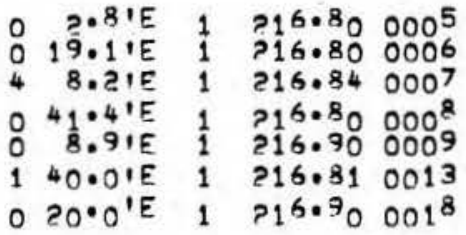

$\begin{array}{lll}1360^{\circ} & 390^{\circ} & 0000 \\ 138 . & 27 \cdot & 0000 \\ 2840^{\circ} & 37 . & 0000 \\ 140^{\circ} & 311^{\circ} & 0000 \\ 1340^{\circ} & 240^{\circ} & 0000 \\ 141 . & 260^{\circ} & 0000 \\ 121^{\circ} & 130^{\circ} & 0000\end{array}$

$\begin{array}{lll}20 & 3266 & 0 \\ 20 & 3269 & 0 \\ 20 & 3269 & 0 \\ 20 & 8369 & 0 \\ 20 & 8469 & 0 \\ 20 & 8369 & 0 \\ 20 & 8859 & 0\end{array}$


OG1

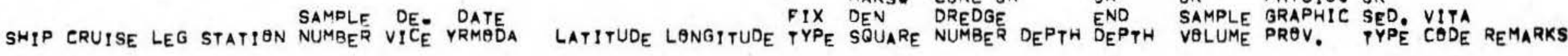

LENGTH DREDGE ROCK

MARS. CQRE $Q R$ OR OR PHYSIO. $Q R$

MARSDEN SQUARE " 218

$\begin{array}{llll}\text { KNR } & 54 & 6 & 0096 \\ \text { KNR } & 54 & 6 & 0098 \\ \text { KNR } & 54 & 6 & 0100 \\ \text { KNR } & 54 & 6 & 0101\end{array}$

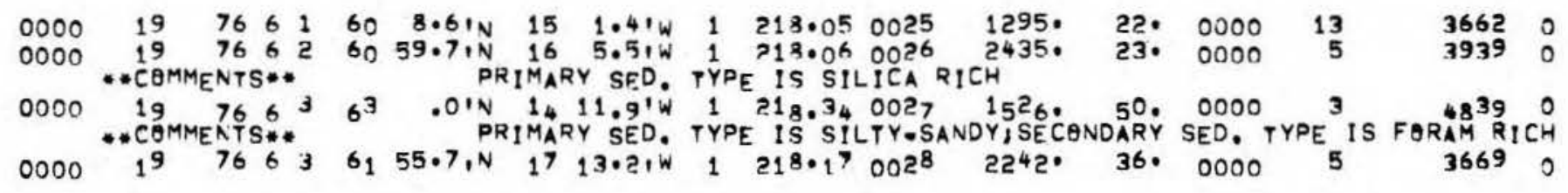

MARSDEN SQUARE * 219

$\begin{array}{llll}\text { KNR } & 51 & 3 & 0021 \\ \text { KNR } & 51 & 3 & 0022 \\ \text { KNR } & 5^{1} & 3 & 0023\end{array}$

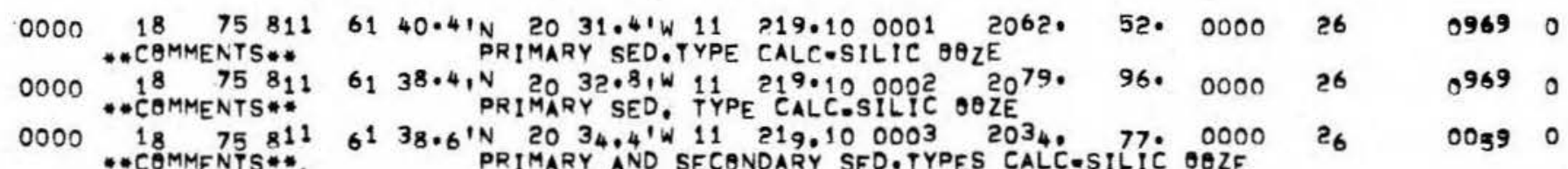

MARSDEN SQUARE \# 25?

$\begin{array}{llll}\text { KNR } & 54 & 6 & 0048 \\ \text { KNR } & 54 & 6 & 00^{7} 0 \\ \text { KNR } & 54 & 6 & 0072\end{array}$

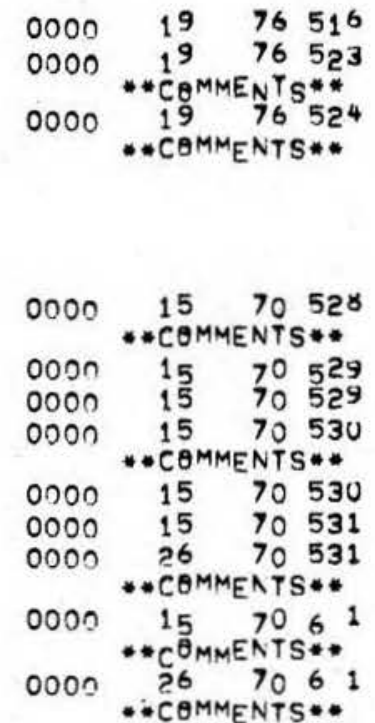

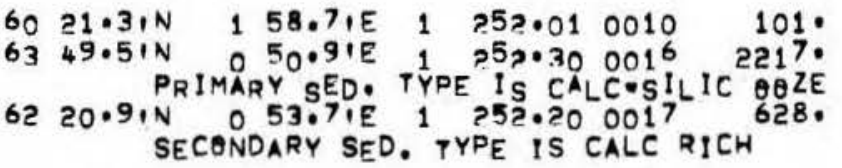

20. 0000

41. 0000

7

88550

39. 0000

MARSDEN SQUARE *300

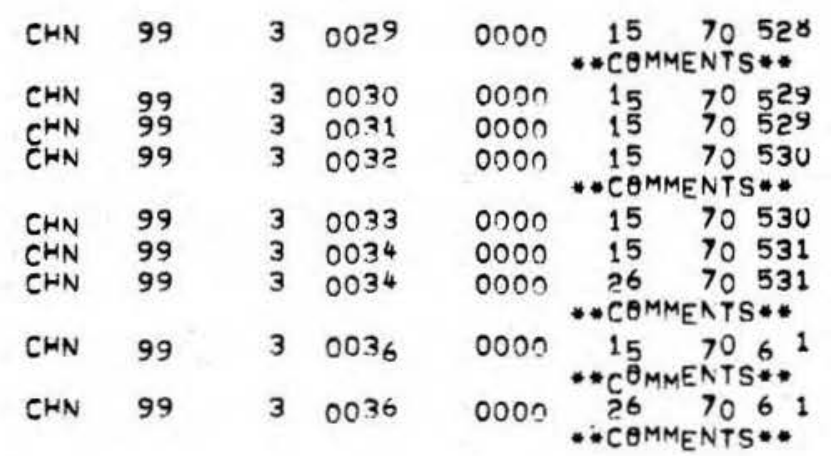

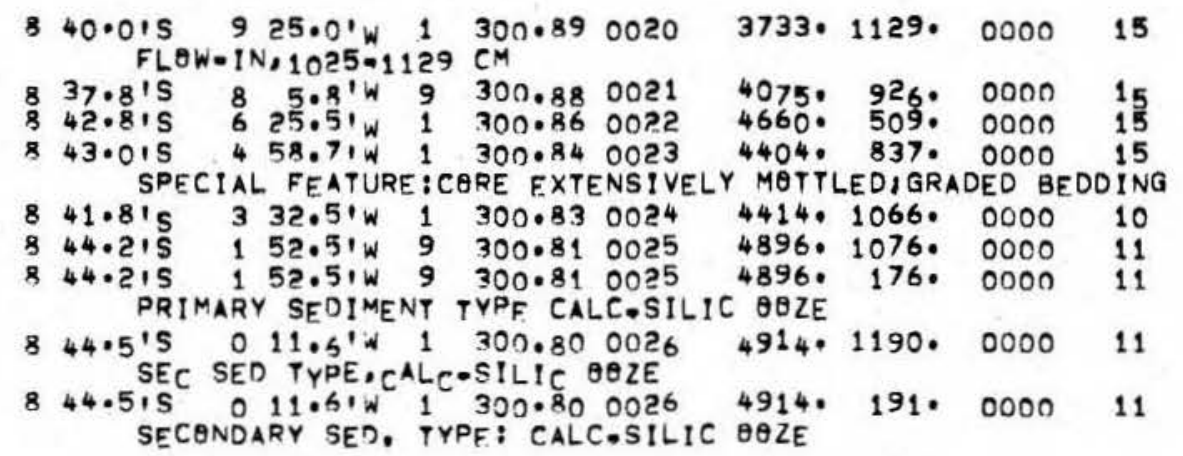

332954 MAR

332954 MAR 133954 MAR 373854 MAR

393254 353254 MAR 054954 MAR 302254 303254 

SHIP CRUISE LEG STATION NUMALE VICE YRMODA $\begin{array}{ll}\text { CORE } \\ \text { MARS: CORE OR } & \text { LENGTH OREDGE PHYSIO. RQR }\end{array}$

FIX DEV DREDGE END SAMPLE GRAPHIC SED, VITA LATITUDE LONGITUDE TYPE SQUARE NUMBER DEPTH DEPTH VOLUME PROV. TYPE CODE REMARKS
MARSDEN SQUARE *301

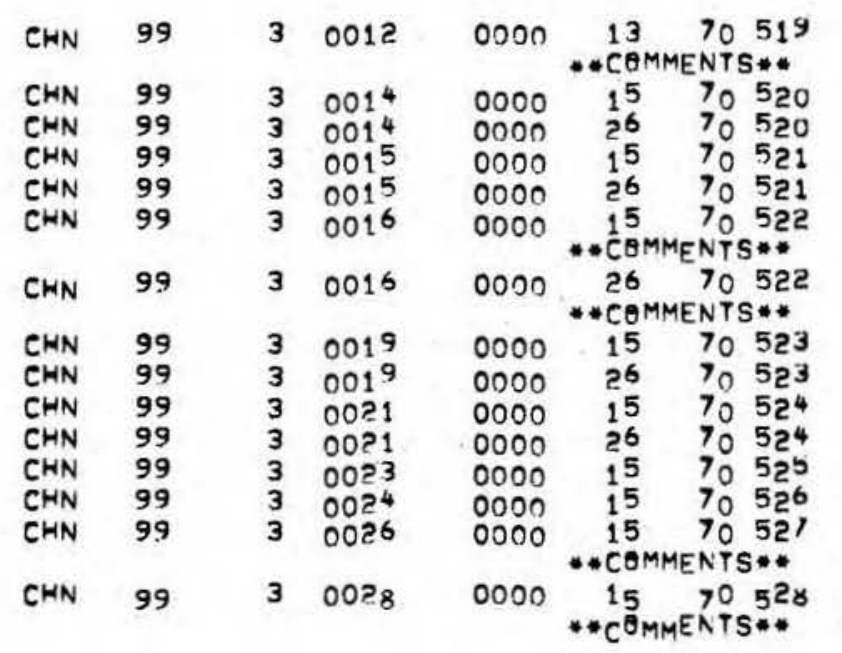

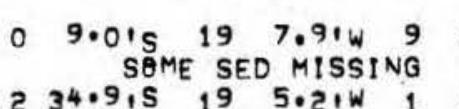

2 $34.9, S \quad 195.2, W \quad 1 \quad 301 \cdot 290011$

$234.9,5$ is 5.2 , 1 1 301.29 0011

$436.015 \quad 193.0, W$ i 301.490012

436.0 , $193.01 \mathrm{~W} 1301.49001$ ? $63^{9} .1$ S 1855.3 , W 9 301.68 0013 O 476

$639.11 \mathrm{~s} 1855.31 \mathrm{~W} 9301.680013$

$815.015 \quad 1740.01 \mathrm{~W} \quad 301.870014$

$815.0151740 .01 \mathrm{~W} 1301.870014$

$810 \cdot 3,5 \quad 1526.9$, 9301.850015

$810.3,5 \quad 1526.9, W \quad 9 \quad 301.850015$

646.7 , S $1247.01 \mathrm{~W}$ 1 301.620016

$\begin{array}{llllll}7 & 2001 \text { is } 14 & 5.01 W & 4 & 301.74 & 0017\end{array}$ POSSIBLE FLOW-IN, $478-856$ CM

$839.7151058 .01 \mathrm{~W} 9301.800019$ $F L \theta W=I N, 700-796 \mathrm{CM}$

MARSDEN SQUARE *308

$\begin{array}{llll}\text { AII } & 54 & 2 & 0053 \\ \text { AII } & 54 & 2 & 0053 \\ \text { AII } & 54 & 2 & 0055 \\ \text { III } & 54 & 2 & 0055\end{array}$

$\begin{array}{lll}0000 & 15 & 69112^{9} \\ 0000 & 26 & 69112^{4} \\ 0000 & 15 & 691130 \\ 0000 & 26 & 691130\end{array}$

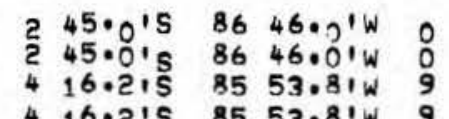

$308 \cdot 26002^{4}$

85 53.81W 9308.450025

308.450025

$\begin{array}{lll}2805, & 818, & 0000 \\ 28050 & 158, & 0000 \\ 3225, & 934, & 0000\end{array}$

3225. 134. 0000

5457. 1097. 0000

5457. 185. 0000

4353. 1095.

353. 188. 0000

$4762 \cdot 1184 \cdot 0000 \quad 15$

4762. 186. $0000 \quad 15$

4029. 1037. 0000

4029, 84. 0000

3652, 911 : 0000

3286, 858. 000

3722. 829, 0000

3116. 856. 0000

3462. $796 \cdot 0000$

33390

1933390

$19 \quad 143254$

$372^{9} 54$

332254

032240 MAR

$334240 \quad M_{A} R$

373154 MAR

332954 MAR

3329 54 MAR

332954 MAR

335954 MAR

$\begin{array}{lll}3729 & 54 & \text { MAR } \\ 3721 & 54 & \text { ASCENSION }\end{array}$

372954

372954 MAR

MARSOEN SQUARE "309

$\begin{array}{llll}\text { AII } & 54 & 2 & 0003 \\ \text { AI } & 54 & 2 & 0003 \\ \text { AII } & 54 & 2 & 0005 \\ \text { AII } & 54 & 2 & 0005 \\ \text { III } & 54 & 2 & 000^{7} \\ \text { III } & 54 & 2 & 000^{7} \\ \text { III } & 54 & 2 & 000^{9} \\ \text { III } & 54 & 2 & 0009 \\ \text { AII } & 54 & 2 & 0011 \\ \text { AII } & 54 & 2 & 0011 \\ \text { AII } & 54 & 2 & 0012\end{array}$

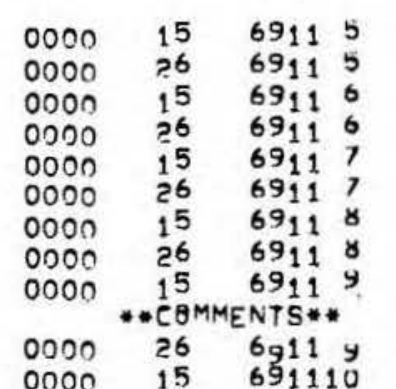

$537.4,5$

$8721.31 \mathrm{~W}$

$537 \cdot 4,5$

$724 \cdot 6,5$

309.570002

9.015 $9113001 \mathrm{~W} 13090910004$

$959.0159113 .01 \mathrm{~W} 1309.910004$

$931.3,59412.6, \mathrm{~W} 9309.940005$

$31 \cdot 3,59412.61$ iw 9309.940005

9 19.01S 9736.2 , W $9309.97000^{6}$ PRIM SED TYPE, CALCOSILIC CLAY

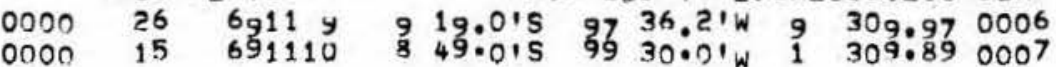

\begin{tabular}{|c|c|c|c|c|}
\hline $\begin{array}{l}37350 \\
37350 \\
4165 \\
4165 \\
41150 \\
41150 \\
3960 \\
3960 \\
4287\end{array}$ & $\begin{array}{r}906 \\
89 . \\
1220 \\
140^{\circ} \\
428 \\
850 \\
771^{\circ} \\
91 . \\
881^{\circ}\end{array}$ & $\begin{array}{l}0000 \\
0000 \\
0000 \\
0000 \\
0000 \\
0000 \\
0000 \\
0000 \\
0000\end{array}$ & $\begin{array}{l}11 \\
11 \\
11 \\
11 \\
11 \\
11 \\
13 \\
13 \\
15\end{array}$ & $\begin{array}{l}3562 \\
3562 \\
6969 \\
3569 \\
3329 \\
3539 \\
3562 \\
3562 \\
0429\end{array}$ \\
\hline $\begin{array}{l}287 \\
3750\end{array}$ & $\begin{array}{l}140 . \\
799\end{array}$ & $\begin{array}{l}0000 \\
0000\end{array}$ & $\begin{array}{l}15 \\
15\end{array}$ & $\begin{array}{l}3562 \\
3032\end{array}$ \\
\hline
\end{tabular}




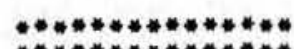

(1)

$* * * * * * * * * * * * *$
STATION DATA RETRIEVAL STATION UATA RETRIEVAL DATE:
***************

(1)
PAGE 41

PAGE 180341759 SHIP CRUISE LEG STATION NUMAER VICE YRMODA

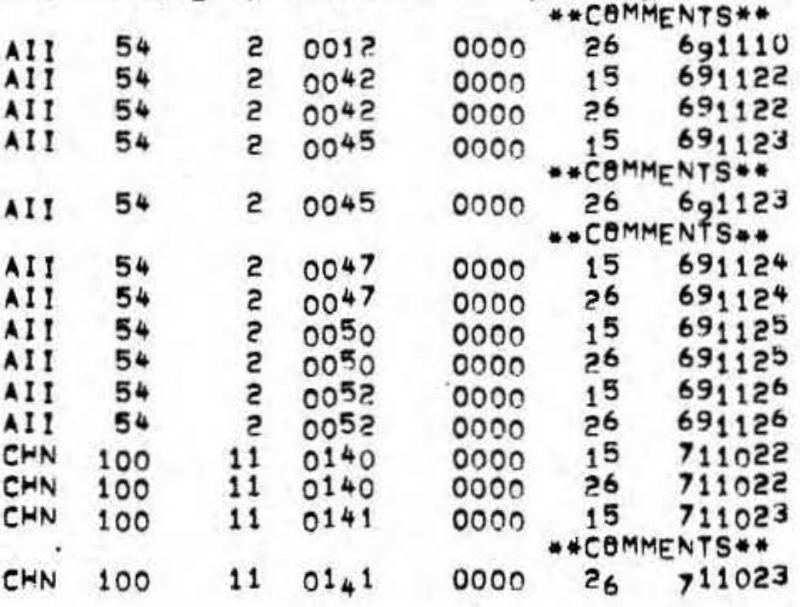

SAMPLE DE DATE

LENGTH DREDGE ROCK

OE

END SAMPLE GRAPHIC STD VITA LATITUDE LONGITUDE TYPE SUUARE NUMBER DEPTH DEPTH VOLUME PROV. TYPE CODE REMARKS SEC SED TYPE, ICALC.SILIC OOZE $849.01 \mathrm{~S} 9930.01 \mathrm{~W}$ 1 309.890007

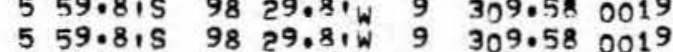
$615.7,59632.5$, 9309.69002 4375: 140. 0000 3659, 961. $000 \quad 15 \quad 53690$ 3659. 127. 0000 15 1503690 6 PRIM SED TYPEICALC.SILIC OBZE

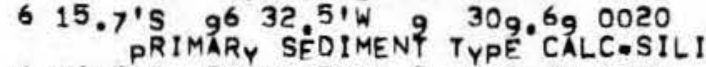
$378^{5}, 145,0000 \quad 15$ $646.9159340 .51 \mathrm{~W} 9309.630021$

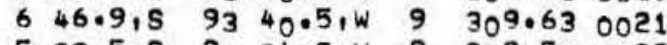
$532.5,599_{1} 24.3, w 99309.510022$ $532.5,599_{1} 24.3, w 9309.510022$ 2 $23.5,5905.2$, w 9 309.20 0023 $223.5,5905.21 \mathrm{~W} 9309.200023$ $105.0159941 .81 w 99309.090094$ $105.0159941 .81 \mathrm{w} 9309.090094$ 834.4159817 .7 iW 9309.880095 PRIM SEO TYPE, CALC.SILIC OQZE $834.41 \mathrm{~S} 9817.71 \mathrm{~W} 9309.880095$

03220

3985. 832. 0000 3985. 155. 0000 4075. 1020. 0000 4075.160 .0000 3230.1046 .0000 3230.67 .0000 4314.739 .0000 4314. 154. 0000 4038.627 .0000 $4038 \cdot 135 \cdot 0000$

15

15
15

35620

\section{MARSDEN SQUARE \#31n}

\begin{tabular}{|c|c|c|c|c|c|c|}
\hline $\begin{array}{l}\text { AII } \\
\text { AII } \\
\text { AII } \\
\text { AII } \\
\text { AIII } \\
\text { AII } \\
\text { AII } \\
\text { AII }\end{array}$ & $\begin{array}{l}54 \\
54 \\
54 \\
54 \\
54 \\
54 \\
54 \\
54\end{array}$ & $\begin{array}{l}2 \\
? \\
2 \\
2 \\
2 \\
2 \\
2 \\
2 \\
2\end{array}$ & $\begin{array}{l}0014 \\
0014 \\
0016 \\
0016 \\
0018 \\
0018 \\
0020 \\
00 ? 0 \\
00 ? 2\end{array}$ & $\begin{array}{l}0000 \\
0000 \\
0000 \\
0000 \\
0000 \\
0000 \\
0000 \\
0000\end{array}$ & $\begin{array}{l}15 \\
26 \\
15 \\
26 \\
15 \\
26 \\
13 \\
15\end{array}$ & $\begin{array}{l}691111 \\
691111 \\
691112 \\
691112 \\
691112 \\
691112 \\
69_{111} \\
69_{111}\end{array}$ \\
\hline AII & 54 & 2 & 0022 & 0000 & $\begin{array}{l}26 \\
\text { COMN }\end{array}$ & $\begin{array}{c}691114 \\
\text { AENTS }\end{array}$ \\
\hline AII & 54 & 2 & 0024 & 0000 & $\begin{array}{ll}15 \\
* \text { COM }\end{array}$ & $\begin{array}{l}691110 \\
\text { AENTS * * }\end{array}$ \\
\hline AII & 54 & 2 & 0024 & 0000 & $\begin{array}{l}26 \\
- \text { COMN }\end{array}$ & $\begin{array}{l}691110 \\
\text { MENTS** }\end{array}$ \\
\hline AII & 54 & 2 & 0025 & 0000 & $\begin{array}{l}15 \\
* \text { COMN }\end{array}$ & $\begin{array}{l}691116 \\
\text { ENTS }\end{array}$ \\
\hline AlI & 54 & 2 & 0025 & 0000 & $\begin{array}{l}26 \\
.\end{array}$ & $\begin{array}{l}691110 \\
\text { IENTS** }\end{array}$ \\
\hline AII & 54 & 2 & 0029 & 0000 & $\begin{aligned} & 15 \\
* & \text { COM }\end{aligned}$ & $\begin{array}{l}691117 \\
\text { IENTS }\end{array}$ \\
\hline Al I & 54 & 2 & $\mathrm{OO}_{2} \mathrm{~g}$ & 0000 & 26 & 691117 \\
\hline
\end{tabular}

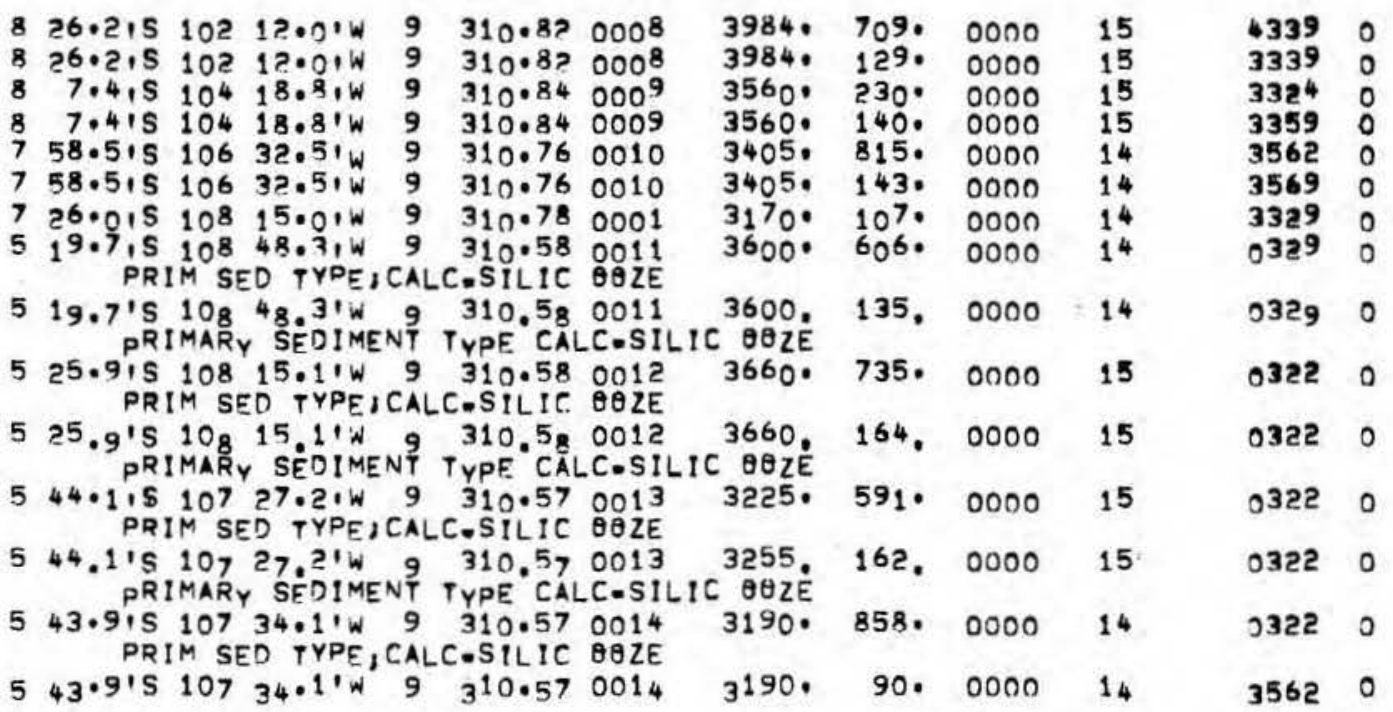


MARSDEN SQUARE *310

$\begin{array}{lrrr}\text { AII } & 54 & 2 & 0031 \\ \text { AII } & 54 & 2 & 0031 \\ \text { AII } & 54 & 2 & 0035 \\ \text { AII } & 54 & 2 & 0035 \\ \text { AII } & 54 & 2 & 0037 \\ \text { AII } & 54 & 2 & 0037 \\ \text { AII } & 54 & 2 & 0039 \\ \text { AII } & 54 & 2 & 0039 \\ \text { CHN } & 100 & 11 & 0142 \\ \text { CHN } & 100 & 11 & 0142 \\ \text { CHN } & 100 & 11 & 0143 \\ \text { CHN } & 100 & 11 & 0143 \\ \text { CHN } & 100 & 11 & 0144 \\ \text { CHN } & 100 & 11 & 0144 \\ \text { CHN } & 100 & 11 & 0145 \\ \text { CHN } & 100 & 11 & 0145 \\ \text { CHN } & 100 & 11 & 0146 \\ \text { CHN } & 100 & 11 & 0146\end{array}$

0000 26 691121
6 24.2'S 10547.31 ' 9 310.65 0015 3497. 741. 0000 PRIMARY SEDIMENT TYPE CALC SILIC OQZE

624.2 , 10547.3 , W 9310.6500153497 .0700000 PRIMARY SEDIMENT TYPE CALC.SILIC OOZE

$0000 \quad 2^{6} \quad 69_{1118}$

0000 .

0000 *CEMMENTS**

0000 "C 15 MMENTS* 691120

0000 " 26 M 691120

$0000 \quad 15 \quad 691121$

0000 "CEMME $\mathrm{TH}_{11023}$

0000 "COMMENTS** 15

$0000 \quad 26 \quad 711024$

$0000 \quad 15 \quad 7_{11024}$

"COMMENTS"*

0000 26 TCOMMENTS**

000015 . 15 MMENTS**

0000 " 26 T11024

$0000 \quad 15 \quad 7_{11025}$

$0000 \quad 2^{6} \quad 7_{11025}$ 6 19.01S 105 39.71'W 9 310.650016 3341. 916. 0000 PRIMARY SEOIMENT TYPE CALC.SILIC OOZE

5 30.61S 10 ? 43.?.W 1 310.5? 0017 3835. 837. 0000 PRIMARY SEDIMENT TYPE CALC SILIC QOZE

5 30.6's 10? 43.21W 1310.5 ? 0017 3835: 170. 0000

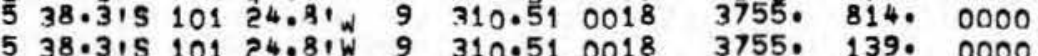

7 51.BIS 100 41.?'W $1310.700096 \quad 4201$ : 833. 0000 PRIM SED TYPE, CALC=SILIC OAZE

$751.81510041 .21 \mathrm{~N} 1310.700096$ 4201. 67. 0000 SECONDARY SEDIMENT TYPE CALC.SILIC OOZE

744.8 's $10124.61 \mathrm{~W} 1$ 310.71 0097 4649. 862. 0000 744.8 'S 10124.61 ' 1 310.71 0097 4649: $110: 0000$ $7.37 .8,510155.4, W$ 1 $310.710098 \quad 3743.678 .0000$ SECONDARY SEDIMENT TYPE CALC SILIC OOZE

737.8 IS 10155.4 IW 1 310.71 0098 3743. 54. 0000 PRIMARY SEDIMENT TYPE CALC.SILIC OOZE

717.8 's $10258.21 \mathrm{~W} 9310,720099$

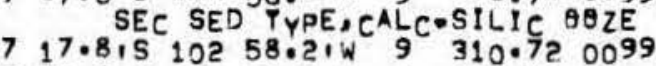

553.6 ,S 10238.8 ,W 1310.520100
$0000 \quad 1571102^{2}$

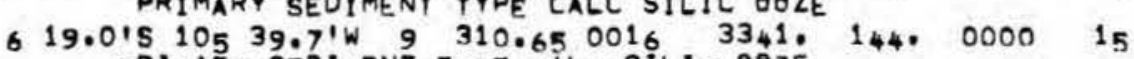

MARSDEN SQUARE \# 316

$\begin{array}{llll}\text { CHN } & 100 & 10 & 0106 \\ \text { CHN } & 100 & 10 & 0107 \\ \text { CHN } & 100 & 10 & 0109 \\ \text { CHN } & 100 & 10 & 0109 \\ \text { CHN } & 100 & 10 & 0110 \\ \text { CHN } & 100 & 10 & 0110 \\ \text { CHN } & 100 & 10 & 0111 \\ C_{H N} & 100 & 10 & 0111 \\ \text { CHN } & 100 & 10 & 0113\end{array}$

$0000 \quad 14 \quad 71910$ $0000 \quad 15 \quad 71911$ ODOO 15 COMMENTS 71 $0000 \quad 2_{6} \quad 71911$ * $C^{8} \theta_{M M E N T S * *}$ 0000 . 15 71912 $\begin{array}{llll}0000 & 26 & 7_{1}^{1} & 912 \\ 0000 & 15 & 71 & 912\end{array}$ $\begin{array}{llll}0000 & 15 & 71 & 912 \\ 0000 & 26 & 7_{1} & 912\end{array}$ $0000 \quad \begin{array}{lll}15 & 7 & 912 \\ 0\end{array}$
$8 \quad 3.7 .516834 .11$ 'W $1 \quad 316.88007$ AXIS SAMOAN PASSAGE SEC. SED

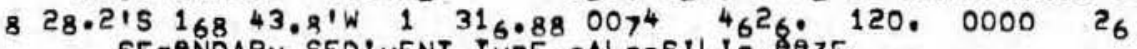
SECONDARY SEDIMENT TYPE CALCESILIC 8 OZE RIDGE EASTERN SIDE OF TME SAMOAN PASSAGE

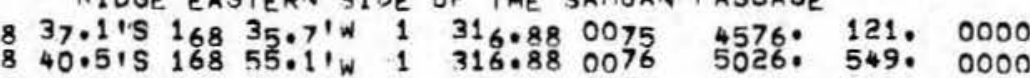

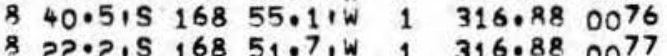

22 . I, 168 M1. I

5026. 549: 0000 5026.112 . 0000 5162 . 354. 0000

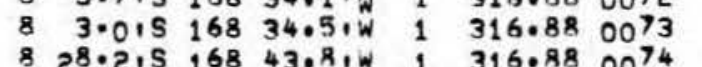

4400 .

4400: 102, 0000

$4440.809 .0000 \quad 10$

4440 . 108. 000010

$\begin{array}{lll}11 & 0322 & 0 \\ 11 & 0322 & 0 \\ 15 & 0322 & 0 \\ 15 & 0329 & 0 \\ 15 & 0322 & 0 \\ 15 & 3332 & 0 \\ 14 & \mathbf{3 5 6 2} & 0 \\ 14 & \mathbf{3 5 6 9} & 0 \\ 10 & \mathbf{4 3 2 9} & 0 \\ 10 & 1039 & 0 \\ 10 & \mathbf{3 5 3 8} & 0 \\ 10 & 6669 & 0 \\ 10 & \mathbf{3 0 2 2} & 0 \\ 10 & 0339 & 0 \\ 10 & 1032 & 0 \\ 10 & \mathbf{5 3 6 2} & 0 \\ 10 & 5362 & 0 \\ 10 & \mathbf{3 3 6 2} & 0\end{array}$

33620

0000 O IN JAR 8853 O SAMOAN PASS $102^{3}$ O SAMOAN PASS 1023 O SAMOAN PASS 3123 O SAMEAN PASS 3129 O SAMOAN MASS 1323 O SAMBAN PASS 1323 O SAMOAN PAS, 1323 O SAMEAN PASS 


\section{MARSDEN SQUARE * 316}

$\begin{array}{llll}\text { CHN } & 100 & 10 & 0113 \\ \text { CHN } & 100 & 10 & 0114 \\ \text { CHN } & 100 & 10 & 0114 \\ \text { CHN } & 100 & 10 & 0116 \\ \text { CHN } & 100 & 10 & 0116 \\ \text { CHN } & 100 & 10 & 0117 \\ \text { CHN } & 100 & 10 & 0117 \\ \text { CHN } & 100 & 10 & 0118 \\ \text { CHN } & 100 & 10 & 0118 \\ \text { CWN } & 100 & 10 & 0123 \\ \text { CHN } & 100 & 10 & 0124 \\ \text { CHN } & 100 & 10 & 0124 \\ \text { CHN } & 100 & 10 & 0126 \\ \text { CHN } & 100 & 10 & 0126 \\ \text { CHN } & 100 & 10 & 0127 \\ \text { CHN } & 100 & 10 & 0128 \\ \text { CHN } & 100 & 10 & 0129 \\ \text { CHN } & 100 & 10 & 0129 \\ \text { CHN } & 100 & 10 & 0131 \\ \text { CHN } & 100 & 10 & 0131 \\ \text { CHN } & 100 & 10 & 0132 \\ \text { CHN } & 100 & 10 & 0132\end{array}$

\begin{tabular}{|c|c|c|}
\hline $\begin{array}{l}000 \\
000\end{array}$ & $\begin{array}{l}26 \\
15\end{array}$ & $\begin{array}{l}71 \\
71\end{array}$ \\
\hline $\begin{array}{l}0000 \\
0000 \\
0000 \\
0000 \\
0000 \\
0000\end{array}$ & $\begin{array}{l}26 \\
15 \\
26 \\
15 \\
26 \\
15\end{array}$ & $\begin{array}{l}7_{1} \\
71 \\
7 \\
71 \\
71 \\
7 \\
71 \\
71\end{array}$ \\
\hline $\begin{array}{l}0000 \\
0000\end{array}$ & & \\
\hline 000 & 15 & 71 \\
\hline $\begin{array}{l}0000 \\
0000\end{array}$ & & \\
\hline $\begin{array}{l}0000 \\
0000 \\
0000\end{array}$ & & \\
\hline 1000 & & \\
\hline $\begin{array}{l}000 \\
0000\end{array}$ & & \\
\hline 000 & & $\begin{array}{l}71 \\
71\end{array}$ \\
\hline
\end{tabular}
825.7 . S 16858.7 iW 9 316.88 0078 $818.7,516833.3$, 9316.880080

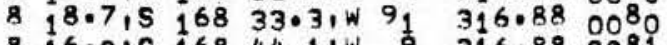
$816.01516844 .11 W 9316.880081$ $816.01516844 .11 \mathrm{~W} 9316.8800^{8} 1$ $715.7, \mathrm{~s} 168$ 27.6, 9316.880083 719.01516821 .6 'W 1 316.78 0082

719.0 is 168 21.6IW 1 316.78 0082 $736.31516757 .0^{1 W} 1316.7700^{87}$ $736.31516757 .01 W 11316.770087$

$\begin{array}{lllllll}7 & 36.715 & 168 & 10.6 & \text { 'w } & 316.78 & 0088 \\ 7 & 27.215 & 168 & 6.71 w & 9 & 316.78 & 0089\end{array}$

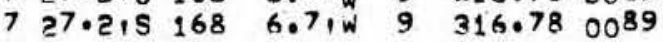

$822.21516851 .71 \mathrm{~W} 1316.880077$ $25.71516858 .71 W 9316.880078$ WESTERN WALL OF SAMEAN PASSAGE $820.315168 \quad 46.01 W \quad 9 \quad 316.88 \quad 0079$ $820^{\circ} 31516846.01 W \quad 9 \quad 3160880079$ SOUTH END OF THE TOKELAU TROUGH SQUTH END $\theta_{F}$ TQHELAU TRQUBH $725.81516832 .01 W \quad 1316.780084$ $725.81516832 \cdot 0^{\prime W} 1316.7800^{84}$ $721.21516831 .21 \mathrm{~W}$ i 316.780085 723.51516841 .31 W 1316.780086 PLATEAU EAST OF THE SOUTHERN END $736.71516810 .61 \mathrm{~W} 9316.780088$ SOUTH END TEKELAU TRQUGH
5162. 85. 0000 4550. 521. 0000 $4550^{\circ} 132^{\circ} 0000$ $5123 \cdot 1118: 0000$ 47.3. $150^{\circ}, 0000$ $4732^{\circ} 17^{\circ} 0000$ 5007. 817. 0000 $500^{7} \cdot 119 \cdot 0000$ $5768^{\circ} 84^{\circ}, 0000$ $5742.160_{6}$. 0000 5742. 84. 0000 5508. 659. 0000 $550^{8} \cdot 74 \cdot 0000$ 5457. 743. 0000 5495. 0.0000 $4430^{\circ} 7120^{\circ} 0000$ F TEKELAU TROUGH 5230. 144. 0000 5289: 135. $000 n$ 5276. 806. 0000 5276. 154. 0000

\begin{tabular}{|c|c|c|c|c|}
\hline $\begin{array}{l}26 \\
26\end{array}$ & $\begin{array}{l}1323 \\
3423\end{array}$ & $\begin{array}{l}0 \\
0\end{array}$ & $\begin{array}{l}\text { SAMEAN } \\
\text { SAMOANN }\end{array}$ & $\begin{array}{l}\text { PASS } \\
\text { PASSS }\end{array}$ \\
\hline $\begin{array}{l}26 \\
26 \\
26 \\
26 \\
26 \\
26 \\
26 \\
26\end{array}$ & $\begin{array}{l}3423 \\
1153 \\
1653 \\
1323 \\
1323 \\
1123 \\
1433 \\
3359\end{array}$ & $\begin{array}{l}0 \\
0 \\
0 \\
0 \\
0 \\
0 \\
0 \\
0\end{array}$ & $\begin{array}{l}\text { SAMEAN } \\
\text { SAMEAN } \\
\text { SAMEAN } \\
\text { SAMQAN } \\
\text { SAMBAN } \\
\text { SAMOAN } \\
\text { SAMOAN } \\
\text { TAKELAUU }\end{array}$ & $\begin{array}{l}\text { PASS } \\
\text { PASS } \\
\text { PASS } \\
\text { PAS } \\
\text { PASS } \\
\text { PASS } \\
\text { PASS } \\
\text { TRE }\end{array}$ \\
\hline 26 & 3631 & 0 & TOKELAU & TRQ \\
\hline $\begin{array}{l}26 \\
26 \\
26 \\
26 \\
26 \\
26\end{array}$ & $\begin{array}{l}1159 \\
1163 \\
1663 \\
1139 \\
0000 \\
3723\end{array}$ & $\begin{array}{l}0 \\
0 \\
0 \\
0 \\
0 \\
0\end{array}$ & $\begin{array}{l}\text { TOKELAU } \\
\text { TOKELANU } \\
\text { TOKELAUU } \\
\text { TOKELAU } \\
\text { IN JAR } \\
\text { TOKELAU }\end{array}$ & $\begin{array}{l}\text { TRO } \\
\text { TRO } \\
\text { TRO } \\
\text { TRO } \\
\text { TRO }\end{array}$ \\
\hline 16 & $\begin{array}{l}3469 \\
6339\end{array}$ & $\begin{array}{l}0 \\
0\end{array}$ & $\begin{array}{l}\text { TOKELAU } \\
\text { TOKELAUU }\end{array}$ & $\begin{array}{l}\text { TRO } \\
\text { TRO }\end{array}$ \\
\hline ?6 & $\begin{array}{l}1529 \\
1663 \\
1163\end{array}$ & $\begin{array}{l}0 \\
0 \\
0\end{array}$ & $\begin{array}{l}\text { TOKELAU } \\
\text { TOKELAAU } \\
\text { TOKELAAU }\end{array}$ & $\begin{array}{l}\text { TRO } \\
\text { FRO } \\
\text { TRO }\end{array}$ \\
\hline
\end{tabular}

MARSDEN SQUARE \# 32R
AII $\quad 15 \quad 15 \quad 0769$

0000 . 13 COMMENTS* $65 \%$

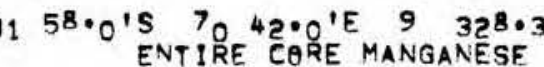

MARSDEN SQUARE *329

CHN $43 \quad 10053$

$\begin{array}{llllllllllll}0 & 000 & 13 & 64517 & 7 & 16.415 & 60 & 32.01 E & 1 & 329.70 & 0018\end{array}$

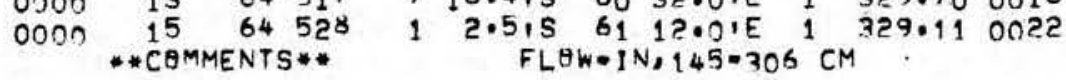

379.. 114. 0000 4565. 306. 0000 15 


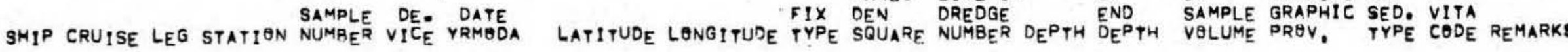

MARS. CORE $\theta R$ LENGTH DREDGE PHYSIO. ${ }_{\theta R}$

CBRE

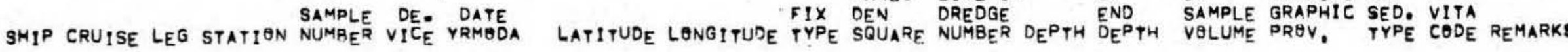

END SAMPLE GRAPHIC SED. VITA

\section{MARSDEN SQUARE \# 330}

$\begin{array}{rrrrr}\text { CHN } & 43 & 1 & 0010 \\ \text { CHN } & 43 & 1 & 0012 \\ \text { CHN } & 43 & 1 & 0015 \\ \text { CHN } & 100 & 4 & 0048 \\ \text { CHN } & 100 & 4 & 0048\end{array}$

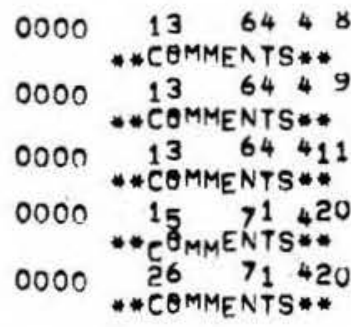

$022.015 \quad 5433.01 E$ 1 330.040005

4864. 264. $0000 \quad 10$

302941

$38.015 \quad 5320.01 E \quad 1 \quad 333^{13} 000^{6}$

4787. 305. $0000 \quad 10$

356941

2 55.0IS 55 43.01E $13300.25000^{8}$

N. OF SEYCHELLES PLATFARM

3697. 301. $0000 \quad 10$

335941

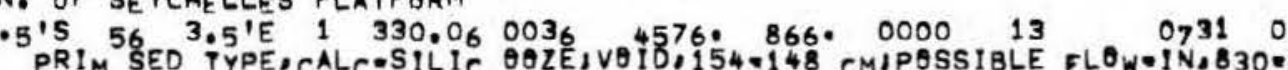

* COMMENTS*

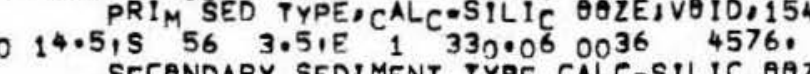

SECONDARY SEDIMENT TYPE CALC.SILIC OOZE

MARSDEN SQUARE *331

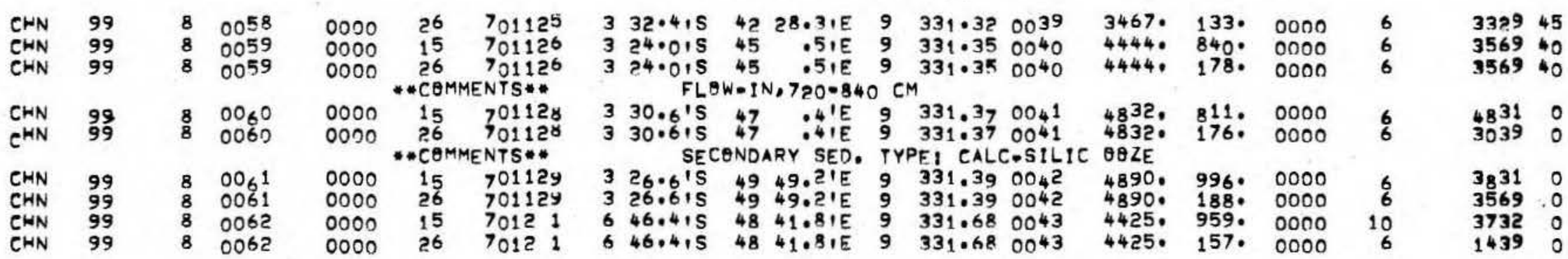

MARSDEN SQUARE *333

\begin{tabular}{|c|c|c|c|c|c|c|c|c|c|c|c|c|c|c|c|c|c|c|c|c|c|c|}
\hline $\begin{array}{l}222 \\
2 Z 2\end{array}$ & $\begin{array}{l}71 \\
71\end{array}$ & 0 & 0001 & $\begin{array}{l}0000 \\
0000\end{array}$ & $\begin{array}{l}15 \\
13\end{array}$ & & $\begin{array}{ll}3 & 0 \\
3 & 0\end{array}$ & $\begin{array}{l}2 \\
1\end{array}$ & $\begin{array}{r}9 \cdot 0^{\prime 5} \\
51.015\end{array}$ & $\begin{array}{l}28 \\
29\end{array}$ & $\begin{array}{r}57 . O^{\prime E} \\
8 . O^{\prime} E\end{array}$ & $\frac{2}{2}$ & $\begin{array}{l}333.28 \\
333.19\end{array}$ & $\begin{array}{l}0001 \\
0002\end{array}$ & $\begin{array}{l}210^{\circ} \\
420^{\circ}\end{array}$ & $\begin{array}{l}680^{\circ} \\
74 .\end{array}$ & $\begin{array}{l}0000 \\
0000\end{array}$ & $\begin{array}{l}22 \\
22\end{array}$ & $\begin{array}{l}5519 \\
9469\end{array}$ & & $\begin{array}{l}\text { LAKE } \\
\text { LAKE }\end{array}$ & $\begin{array}{l}\text { KIVU } \\
\text { KIVU } 1\end{array}$ \\
\hline $2 Z 2$ & 71 & 0 & $000^{5}$ & 0000 & 13 & $\begin{array}{l}71 \\
\text { NTS }\end{array}$ & 30 & 1 & 53.0 is & $\begin{array}{c}29 \\
0.28\end{array}$ & $\begin{array}{r}5.01 E \\
3.54 \mathrm{~cm}\end{array}$ & 2 & $333 \cdot 19$ & $000^{3}$ & 360. & 540 & 0000 & 22 & 5516 & & AKE & KIVU \\
\hline $\begin{array}{l}222 \\
222\end{array}$ & $7_{1}^{1}$ & $\begin{array}{l}0 \\
0\end{array}$ & $\begin{array}{l}0007 \\
0009\end{array}$ & $\begin{array}{l}0000 \\
0000\end{array}$ & $\begin{array}{l}13 \\
15\end{array}$ & $7_{1}^{1}$ & $\begin{array}{ll}3 & 6 \\
3 & 9\end{array}$ & $\begin{array}{l}1 \\
2\end{array}$ & $\begin{array}{l}59.6^{\prime} 5 \\
14.015\end{array}$ & $\begin{array}{l}29 \\
29\end{array}$ & T.3'E & $\begin{array}{l}2 \\
2\end{array}$ & $\begin{array}{l}333.19 \\
333.29\end{array}$ & $\begin{array}{l}0004 \\
0006\end{array}$ & $\begin{array}{l}248 . \\
310\end{array}$ & $\begin{array}{l}90 . \\
4000\end{array}$ & $\begin{array}{l}0000 \\
0000\end{array}$ & $\begin{array}{l}22 \\
22\end{array}$ & $\begin{array}{l}5529 \\
5846\end{array}$ & & $\begin{array}{l}\text { LAKE } \\
\text { LAKE }\end{array}$ & $\begin{array}{l}\text { KIVU } \\
\text { KIVU }\end{array}$ \\
\hline 222 & $7^{1}$ & 0 & 0010 & 0000 & & $\begin{array}{l}\text { NTS } \\
71 \\
\text { RTS }\end{array}$ & S** & 1 & $\begin{array}{l}\text { VeII } \\
\text { BS } \\
\text { VOI }\end{array}$ & 29 & $\begin{array}{l}43 \text { CM } \\
10.21 E \\
5.193\end{array}$ & 2 & 333.19 & 0007 & 473. & $3_{5} 0$. & 0000 & 22 & $55^{16}$ & & AKE & IV \\
\hline 222 & 71 & 0 & 0011 & 0000 & & $\begin{array}{l}71 \\
\text { NTS }\end{array}$ & $\begin{array}{l}310 \\
5 * *\end{array}$ & 1 & $\begin{array}{r}47.0 \text { Is } \\
\text { PAS }\end{array}$ & $\begin{array}{l}29 \\
\text { SI } 1 \text { (日) }\end{array}$ & $\begin{array}{l}10001 E \\
E \text { FLOW. }\end{array}$ & $\stackrel{2}{1 N}, 3^{2}$ & $\begin{array}{r}333.19 \\
20.365\end{array}$ & $\begin{array}{l}0008 \\
\mathrm{CM}\end{array}$ & 450. & 365 . & 0000 & 22 & 5826 & & LAKE & KIVU \\
\hline $\begin{array}{l}Z Z Z \\
Z Z Z\end{array}$ & $\begin{array}{l}71 \\
71\end{array}$ & $\begin{array}{l}0 \\
0\end{array}$ & $\begin{array}{l}0013 \\
0014\end{array}$ & $\begin{array}{l}0000 \\
0000\end{array}$ & $\begin{array}{l}15 \\
15\end{array}$ & $\begin{array}{l}71 \\
71\end{array}$ & $\begin{array}{l}311 \\
311\end{array}$ & $\begin{array}{l}1 \\
1\end{array}$ & $\begin{array}{l}48.915 \\
56.015\end{array}$ & $\begin{array}{l}29 \\
28\end{array}$ & $\begin{array}{l}10.91 E \\
59.01 E\end{array}$ & $\begin{array}{l}2 \\
2\end{array}$ & $\begin{array}{l}333.19 \\
333.18\end{array}$ & $\begin{array}{l}0009 \\
0010\end{array}$ & $\begin{array}{l}4680^{\circ} \\
330^{\circ}\end{array}$ & $\begin{array}{l}275 . \\
500 .\end{array}$ & $\begin{array}{l}0000 \\
0000\end{array}$ & $\begin{array}{l}22 \\
22\end{array}$ & $\begin{array}{l}5246 \\
2544\end{array}$ & & $\begin{array}{l}\text { LAKE } \\
\text { LAKE }\end{array}$ & $\begin{array}{l}\text { KIVU } \\
\text { KIVU }\end{array}$ \\
\hline
\end{tabular}




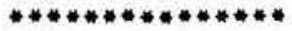

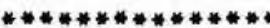

PAGE 45

*WHOI **

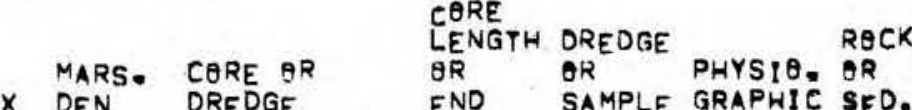

SAMPLE DE. DATE SHIP CRUISE LEG STATION NUMBER VICE YRMODA

\section{MARSDEN SQUARE *333}

$\begin{array}{llll}Z Z Z & 72 & 1 & 000 \\ Z Z Z & 72 & 1 & 000 \\ Z Z Z & 72 & 1 & 000 \\ Z Z Z & 72 & 1 & 001 \\ Z Z Z & 72 & 1 & 001 \\ Z Z Z & 72 & 1 & 001 \\ Z Z Z & 72 & 1 & 001 \\ Z Z Z & 72 & 1 & 001 \\ Z Z Z & 72 & 1 & 001 \\ Z Z Z & 72 & 2 & 000 \\ Z Z Z & 72 & 2 & 000 \\ Z Z Z & 72 & 2 & 000 \\ Z Z Z & 72 & 2 & 000 \\ Z Z Z & 72 & 2 & 000\end{array}$

217.01529 4.7IE 1 333.29 0001 SEVERAL VOIOS IN CARE

$147.6,5$ 29 $12.5, E$ I 333.190002 CARE IN PABR CANDITION

$147.6^{\prime} \mathrm{S} 2912.5^{\prime} \mathrm{E}^{1} 333^{2} 190003$

WORKING AND ARCHIVE HAVLES OF

147.2 IS $2913.71 E_{1} 333.190005$

145.115 29 $15.71 \mathrm{E}$ i $333.19000^{6}$ $147.71 \mathrm{~S} 2915.61 \mathrm{E}$ I 333.190007

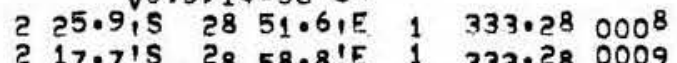

$217.7152858 .8^{\prime}{ }^{\prime} \quad 333.280009$ WORKING AND ARCHIVE HAVLES OF

$137.515293 .61 E_{1} 333.190010$ VO10,0-18 CM

033.518 29 26.4'E 1333.090001 SEC SED TYPEISILICOCALC OQZE

$029.2 . S 2927.81{ }^{2} 1333.090002$

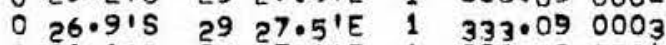
O 21.115 $2927.01 E \quad 1 \quad 333.090004$ VQ10.0-14 CM

O 17.11S 29 28.31E 1333.090005 SEVERAL VOIDS IN CORE
225. 405. $0000 \quad 22$

420. 64. $0000 \quad 22$

420. 950. 000022 400. 145. 0000 22 $1^{5} 0^{\circ} \quad 158.0000 \quad 2 ?$ 70. 452. $0000 \quad 22$ 50. 545. 0000 $1200^{\circ}$
2340
12000 20. 52. 000022 42. 322. 000022 55. 515. 0000 100. 541. 0000 है 110. 545. $0000 \quad 22$
5619 O LAKE KIVU a 5836 O LAKE KIVU 2 OOOO O LAKE KIVU 2 MPLING

5816 O LAKE KIVU ?

58,9 O LAKE KIVU 2

5816 O LAKE KIVU

5816 O LAKE KIVU ? 0000 O LAKE KIVU $?$ 2669 O LAKE KIVU ? 5025 O LAKE EDWARD

5659 O LAKE EDWARD 979 O LAKE EOWARD $\begin{array}{ll}5279 & 0 \text { LAKE EOWARD } \\ 5519 & 0 \text { LAKE EDWARD }\end{array}$ 5519 O LAKE EDWARD

\section{MARSDEN SQUARE \# 334}

\begin{tabular}{|c|c|c|c|c|c|}
\hline $\mathrm{CHN}$ & 99 & 3 & 0041 & $000 n$ & $15 \quad 7065$ \\
\hline $\begin{array}{l}\text { CHN } \\
\text { CHN }\end{array}$ & $\begin{array}{l}99 \\
99\end{array}$ & $\begin{array}{l}3 \\
3\end{array}$ & $\begin{array}{l}0041 \\
0042\end{array}$ & $\begin{array}{l}0000 \\
0000\end{array}$ & $\begin{array}{lll}70 & 6 & 5 \\
70 & 6 & 6\end{array}$ \\
\hline EN & 99 & 3 & 0046 & 0000 & $\begin{array}{l}7066 \\
\text { MMMENTS }\end{array}$ \\
\hline CHN & 99 & 3 & 0046 & 0000 & 7066 \\
\hline
\end{tabular}

$840.415 \quad 1026.71 E 9 \quad 334.800031$ FLOW-IN, 960-1144 CM

$840.41 \mathrm{~S} 1026.71 \mathrm{E} 9 \mathrm{CM} 4.800031$

$840.5, \mathrm{~S} .11$ 49.5IE 1 334.81 0032 FLOW-1N, 340-633 CM

850.51 ' 1149. IIE 9334.810033

$1144^{\circ} 0000$

3855. 124. 0000

1945. 633. 0000

$2209 \cdot 1019 \cdot 0000$

$850.5,51149.2, E 93_{34.31} 00332209.155 .0000$

0000

865354

242954

335954

012954

$5=1019 \mathrm{CM}$

MARSDEN SQUARE \# 3.35

\begin{tabular}{|c|c|c|c|c|c|}
\hline 99 & 3 & $003^{7}$ & 0000 & 15 & \\
\hline IN & 3 & 0037 & & CAMM & FNT \\
\hline $\begin{array}{l}99 . \\
99\end{array}$ & $\begin{array}{l}3 \\
3\end{array}$ & $\begin{array}{l}0038 \\
0038\end{array}$ & $\begin{array}{l}0000 \\
0000\end{array}$ & $\begin{array}{l}15 \\
26\end{array}$ & $\begin{array}{l}70 \\
70\end{array}$ \\
\hline
\end{tabular}

463954

152254 
SAMPLE DE- DATE
SHIP CRUISE LEG STATION NUMBER VICE YRMEDA LATITUDE LONGITUNE TYPE SQUARF NUMBER DEPTH DEPTH VOLUME PROV

MARS. CORE QR

CORE

LENGTH DREDGE RQCK

SAMPLE DE- DATE
SHIP CRUISE LEG STATION NUMBER VICE YRMEDA LATITUDE LONGITUNE TYPE SQUARF NUMBER DEPTH DEPTH VOLUME PROV

SAMPLE DE- DATE
SHIP CRUISE LEG STATION NUMBER VICE YRMEDA LATITUDE LONGITUNE TYPE SQUARF NUMBER DEPTH DEPTH VOLUME PROV

$O R$ OR PHYSIO, $\theta R$

MARSDEN SQUARE * 335

$\begin{array}{llll}\text { CHN } & 99 & 3 & 0039 \\ \text { CHN } & 99 & 3 & 0039 \\ \text { CHN } & 99 & 3 & 0040 \\ \text { CHN } & 99 & 3 & 0040\end{array}$

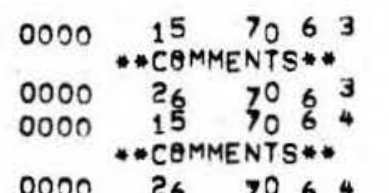

IS $6 \quad 30.01 E \quad 1 \quad 335.860029$ FLOW-IN, 719-1111 CM

CHN $99 \quad 3 \quad 0040$

$0000 \quad 26 \quad 706$

FLOW-IN, $31.01 E$ I 335.870030

$F L \theta W=1 \mathrm{~N}, 957-1163 \mathrm{CM}$

MARSDEN SQUARE *346

$\begin{array}{llll}\text { CHN } & 100 & 11 & 0136 \\ \text { CHN } & 100 & 11 & 0136 \\ \text { CHN } & 100 & 11 & 0137 \\ \text { CHN } & 100 & 11 & 0137 \\ \text { CHN } & 100 & 11 & 0138 \\ \text { CHN } & 100 & 11 & 0138 \\ \text { CHN } & 100 & 11 & 0139 \\ \text { CHN } & 100 & 11 & 0139\end{array}$

$\begin{array}{ll}0000 & 15 \\ 0000 & 26 \\ 0000 & 15 \\ 0000 & 26 \\ 0000 & 15 \\ 0000 & 26 \\ 0000 & 15 \\ 0000 & 26\end{array}$

$\begin{array}{ll}15 & 7 \\ 26 & 7 \\ 15 & 7 \\ 26 & 7 \\ 15 & 7 \\ 26 & 7 \\ 15 & 7 \\ 26 & 7\end{array}$

711020
711020
711021
711021
711021
711021
711022
711022
7
1023.9 's $10530.01 \mathrm{~W}$ 1 346.050090 10 33.91s $10530.01 \mathrm{w}$ 1023.21510348 .216

$1023 \cdot 5,510348.2, W$

$1022 \cdot 01510238.01 \mathrm{~W}$

$1005 \cdot 0,5$ 105 38.0,

$1022 \cdot 1,5$ S $101 \quad 2 \cdot 2, W$

9 346.01 0093

FORAM SAND WITH BASALT AND LITHIFIED SEDIMENT FRAGMENTS

MARSDEN SQUARE *354

$\begin{array}{llll}\text { CHN } & 100 & 9 & 0077 \\ \text { CHN } & 100 & 9 & 0078 \\ \text { CHN } & 100 & 9 & 0078 \\ \text { CHN } & 100 & 9 & 0096 \\ \text { CHN } & 100 & 9 & 0096 \\ \text { CHN } 100 & 9 & 0997 \\ \text { CHN } & 100 & 9 & 0097 \\ \text { CHN } 100 & 9 & 0101 \\ \text { CHN } 100 & 9 & 0101 \\ \text { CHN } 100 & 9 & 0102 \\ \text { CHN } 100 & 9 & 0102\end{array}$

,

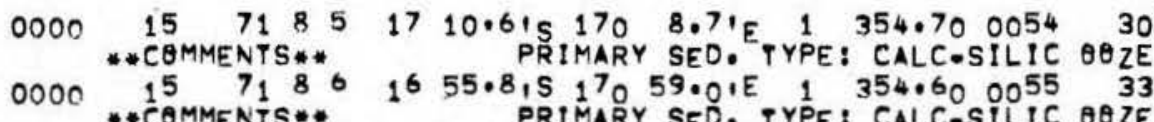

0000 .

*COMMENTS** 1655.8 IS 170 59.0'E 1 PRIMARY SED. 354.600055 YPE: CALC.SILIC OQZ

$0000 \quad 15 \quad 71821 \quad 1320.01517035 .41 E \quad 9 \quad 354.3000663385$.

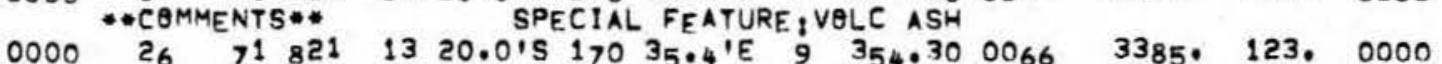

$0000 \quad 15 \quad 71822 \quad 1448.81517044 .71 E$ 9 $354.400067 \quad 35150 \quad 360: 0000$ PRIMARY SED. TYPE: CALC-SILIC OOZE

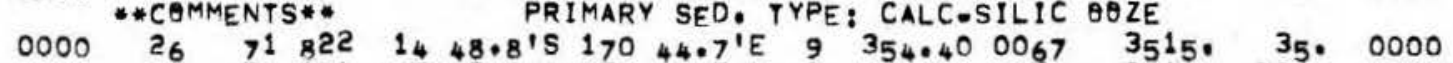

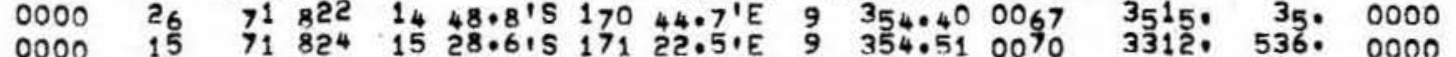

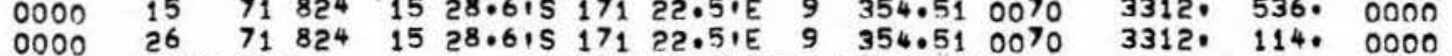
SPECIAL FEATURES VOLC ASH

0000 15 $71824 \quad 1541.61517 ? 8.31 E$ ? 354.520071

3300. 442, 0000 SPECIAL FEATUREIVOLL ISH

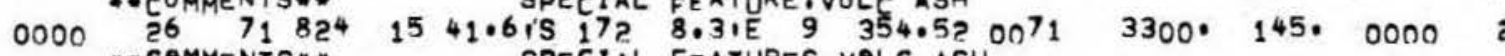
SPECIAL FFATURES VELC ASH
SEO, VITA 


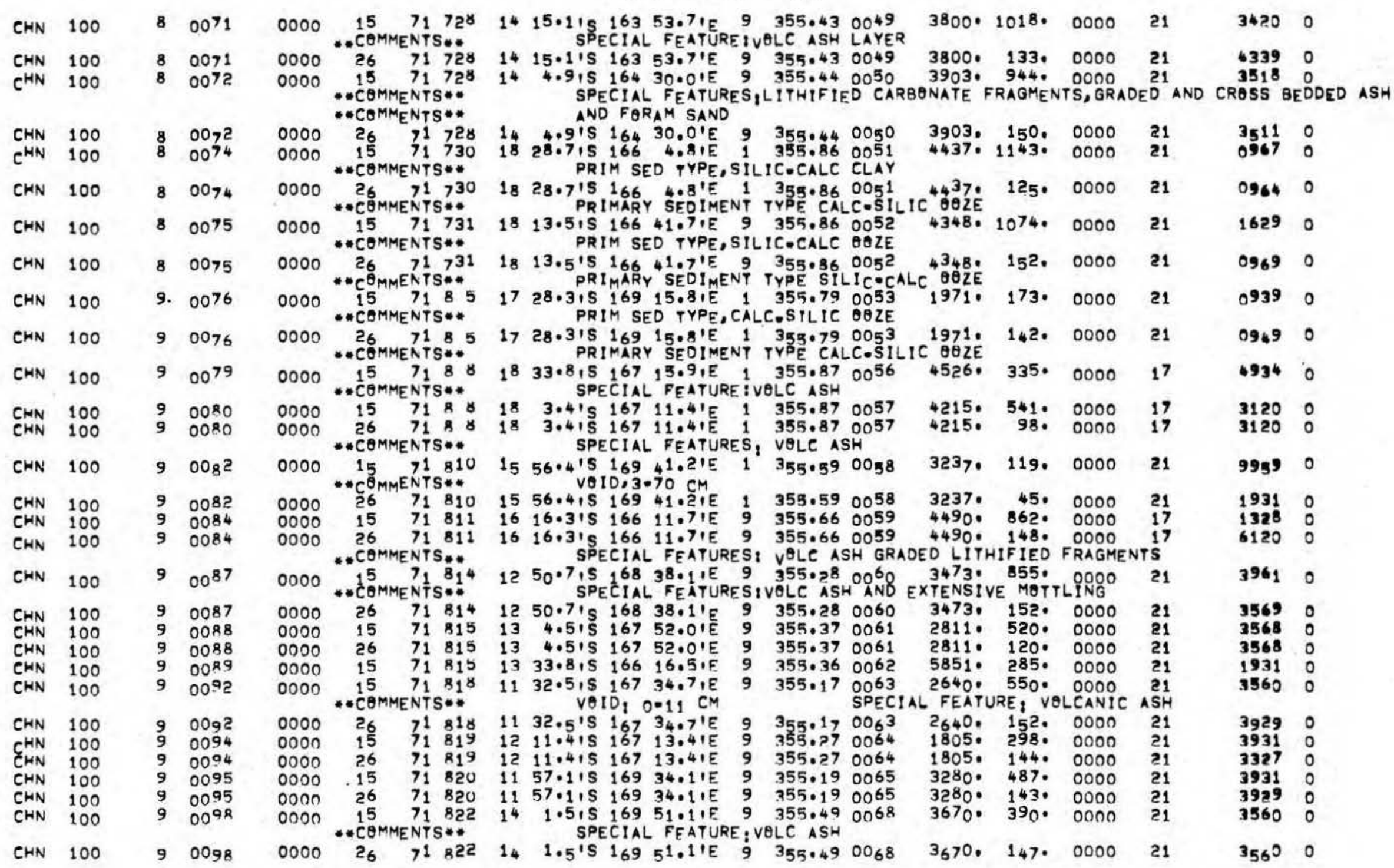




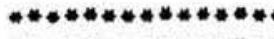

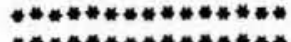

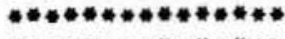

$* * * * * * * * * * * * * * *$
SPATION DATA REPRIEVAL DATE: O01017138 JUN O8 STATION UATA RETRIEVAL DATE:

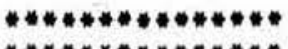

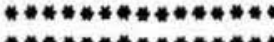

PAGE 48

PAGE 1803417591

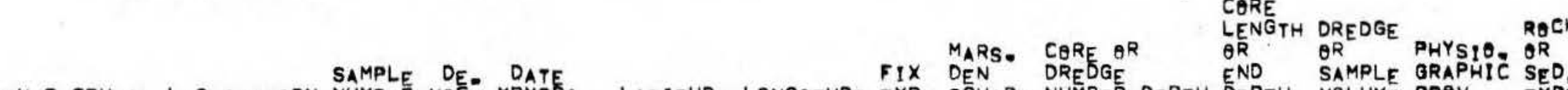

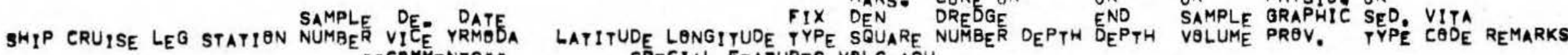

. COMMENTS*" PECIAL FEATURES VALC ASH

CHN $100 \quad 90099$

$41 S^{169} 10.8^{\prime} E$ g 355.4900693248 . 330. 0000

CHN 100

90099

0000 *COMMENTS *

PRIMARY SEO TYPE, CALC-SILIC OOZE

PRIMARY SEDIMENT TYPE CALC SILIC OOZE

09310

*COMMENTS**

MARSDEN SQUARE \#356

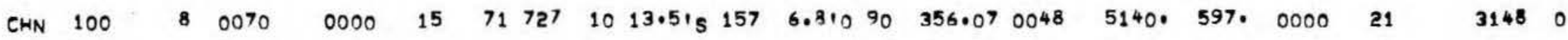

MARSDEN SQUARE *365

CHN $\quad 43 \quad 10027$

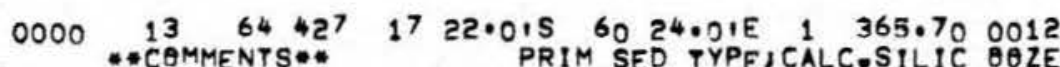

$\begin{array}{lllllllllll}0000 & 13 & 64 & 427 & 14 & 15.015 & 62 & 51.01 E & 1 & 365.42 & 0013\end{array}$

3881, 135. 0000

3820, 107, 000010

0329 41

CWN $43 \quad 10028$

MARSDEN SQUARE \#366

$\begin{array}{llll}\text { AII } & 15 & 13 & 7332 \\ 15 & 13 & 733 \\ 15 & 15 & 13 & 0737\end{array}$

0000

0000

CHN $43 \quad 10023$

$\begin{array}{llll}\text { CHN } & 43 & 1 & 0030 \\ \text { CHN } & 43 & 1 & 0033\end{array}$

0000

0000

$13 \quad 64 \quad 423$

$\begin{array}{lll}64 & 430 \\ 64 & 5 & 2\end{array}$

* 13 COMMENTS**

\section{$65611 \quad 1^{9} 53.0^{\prime S} \quad 56.48 .0^{\prime E} 9366.96 \quad 0^{732}$}

1365611 19 $56.015 \quad 5517.01 E$ 9 $366.950733 \quad 4382 . \quad 139.0000$ PRIMARY SEDIMENT TYPF SILIC CLAY WITH DETRITUS

$\begin{array}{llllllll}17 & 27.0 .5 & 58 & 5.5, E & 1 & 366.78 & 0011\end{array}$

4038 . 127.0000

4095. 262. 0000

$3869.1633^{\circ} 0000$

$\begin{array}{rrr}10 & 0362 & 0 \\ 10 & 889 & 0 \\ 6 & 0459 & 0\end{array}$

$13 \quad 3329$ is

$10 \quad 346941$

MARSDEN SQUARE \#367

$\begin{array}{llll}\text { All } & 15 & 7 & 0673 \\ \text { Al } & 15 & 7 & 0675 \\ \text { CHN } & 99 & 8 & 0063 \\ \text { CHN } & 99 & 8 & 0063 \\ \text { CHN } & 99 & 8 & 0064 \\ \text { CHN } & 99 & 8 & 0064 \\ \text { CHN } & 99 & 8 & 0065\end{array}$

0000
0000
0000
0000
0000
0000
0000

3655

"COMMENTS**

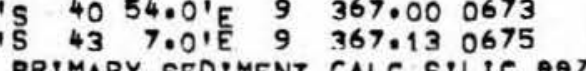

10. 0000

S $4737.71 \mathrm{I} 9367.070044$

7012.21053 .015

$70123 \quad 1318.215 \quad 4658.51 E 99367.360045$

$\begin{array}{llllllll}70123 & 13 & 18.215 & 46 & 58.51 E & 9 & 367.36 & 0045\end{array}$

OnOO

* COMMENTS*

$F L O W=1 N, 635-846 \mathrm{CM}$

$4005 \cdot 858$. 0000

4005.470000

$3243,107 \%$. 0000

3540.896.

000020

0000 ONE JAR

0000 O I VIAL

18390

33590

38190

37310 


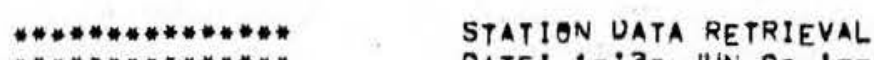

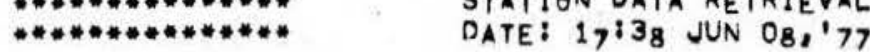

$* * * * * * * * * * * * * * *$
PAGE 49

**WHOI**

\section{MARS. CQRE $O R$ LENGTH DREDGE PHYSIO RQCK}

SAMPLE DE- DATE FIX DEN DREDGE END SAMPLE GRAPHIC SED. VITA

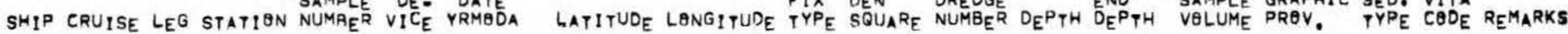

MARSDEN SQUARE *367

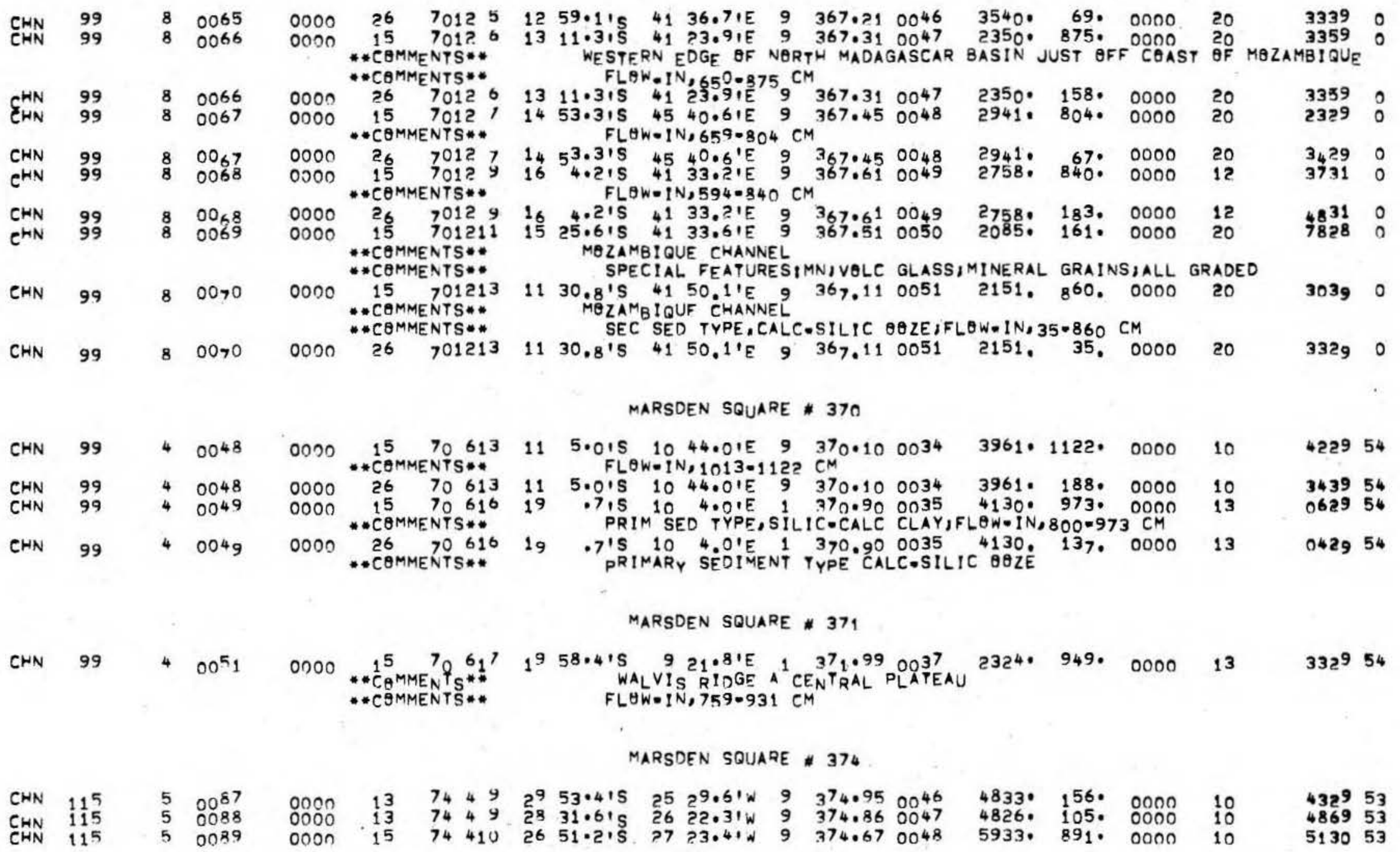


$0000 \quad 5$

$\begin{array}{ll}0000 & 13 \\ 0000 & 15\end{array}$ $\begin{array}{llll}74 & 410 & 26 & 51.21 \\ 74 & 411 & 25 & 17.115\end{array}$

$74 \begin{array}{lll}74 & 411 \\ 74 & 411\end{array}$

\section{5}

53.4
53.04

2412.8 , $396.4,6$

\section{$9 \quad 374.670048$} 9374.580049

MARSDEN SQUARE * 375 $\begin{array}{lllllllllll}0000 & 15 & 71 & 22^{6} & 26 & 39.915 & 34 & .41 & 9 & 375.64 & 0097\end{array}$ $0000 \quad 13 \quad 71$ 228 29 3.4.5 34 42.31W $99375.94009 B$

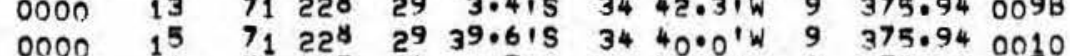

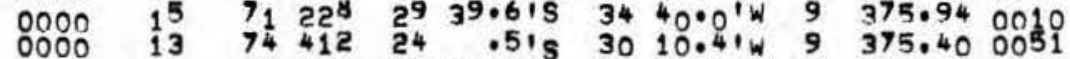
$0000 \quad 13 \quad 74412 \quad 2356.61 \mathrm{~s} 31$ 12.01w 9375.310052 $0000 \quad 13 \quad 74413 \quad 23 \quad 49.015 \quad 3313.5, w \quad 9 \quad 375.33 \quad 0053$

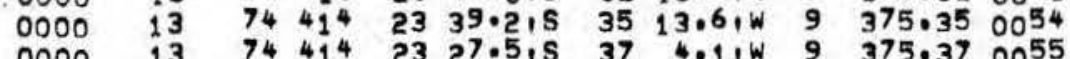

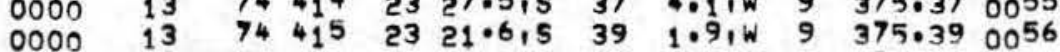
OCOMMENTS * SAMPLE STORED IN JAR

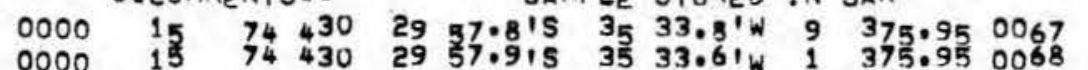

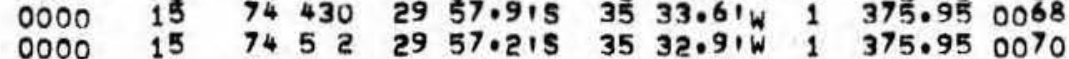

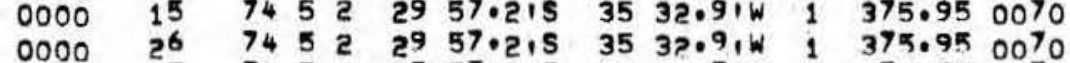
$0000 \quad 15 \quad 7453 \quad 2957.015 \quad 3533.7$.W 1375.950071

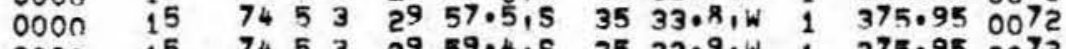

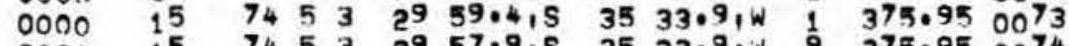

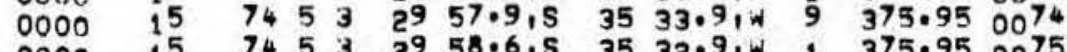

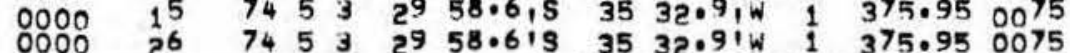

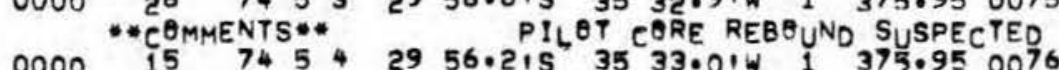

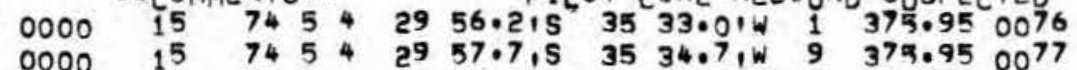

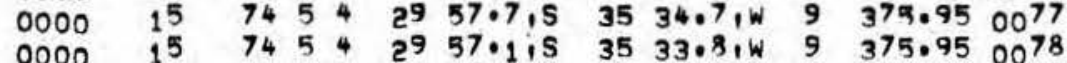
$\begin{array}{lllllllllllllll}0 & 15 & 74 & 5 & 4 & 29 & 57.6,5 & 35 & 33.7, W & 1 & 375.95 & 0079\end{array}$

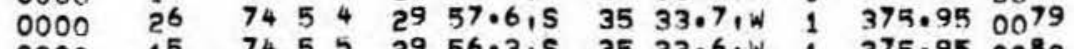
$\begin{array}{lllllllllllll}0000 & 15 & 74 & 5 & 5 & 29 & 56.315 & 35 & 33.6, W & 1 & 375.95 & 0080\end{array}$

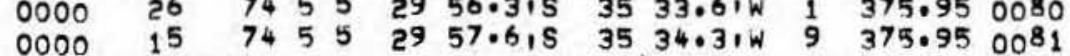
0000 *COMMENTS** 2957.6 IS 35 FLOW $1 \mathrm{~N} 13.31 \mathrm{~W}$. 15

$0000 \quad 15 \quad 74562956.715$ 35 33.5'W 9 375.95 0082 $0000 \quad 15 \quad 7456$ 29 58.015 35 33.71, 1375.950083 $0000 \quad 15 \quad 7456602959.7$ 'S 35 33.5IW 11375.950084

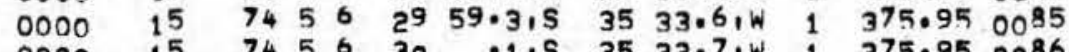

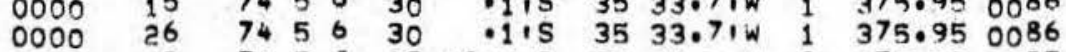

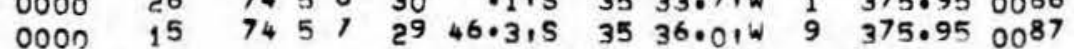

5933. 158, 000010 5460 . 173, 0000 10 314053

\begin{tabular}{|c|c|c|c|c|}
\hline $\begin{array}{l}4626, \\
3273, \\
2990 \\
2940 \\
1840 \\
5313 \\
5179 \\
4757 \\
41530 \\
3944, \\
32060\end{array}$ & $\begin{array}{l}1640^{\circ} \\
223, \\
273, \\
120 \\
118, \\
121 . \\
1170 \\
124, \\
110, \\
50\end{array}$ & $\begin{array}{l}0000 \\
0000 \\
0000 \\
0000 \\
0000 \\
0000 \\
0000 \\
0000 \\
0000 \\
0000\end{array}$ & $\begin{array}{r}10 \\
13 \\
13 \\
13 \\
10 \\
10 \\
10 \\
6 \\
6 \\
6\end{array}$ & $\begin{array}{l}1937 \\
3327 \\
3329 \\
3329 \\
1943 \\
1629 \\
1239 \\
3166 \\
3833 \\
0000\end{array}$ \\
\hline $\begin{array}{l}2195, \\
21920 \\
2340 \\
2340 \\
2235 \\
2235 \\
2209 \\
2150 \\
21920 \\
2260 \\
2260 \\
2260\end{array}$ & $\begin{array}{r}7160 \\
1930 \\
532 . \\
1050 \\
5320 \\
4750 \\
7040 \\
7360 \\
6860 \\
80 .\end{array}$ & $\begin{array}{l}0000 \\
0000 \\
0000 \\
0000 \\
0000 \\
0000 \\
0000 \\
0000 \\
0000 \\
0000\end{array}$ & $\begin{array}{l}13 \\
13 \\
13 \\
13 \\
13 \\
13 \\
13 \\
13 \\
13 \\
13\end{array}$ & $\begin{array}{l}3359 \\
3359 \\
3359 \\
3359 \\
3359 \\
3729 \\
3739 \\
3359 \\
3325 \\
3350\end{array}$ \\
\hline $\begin{array}{l}2315, \\
2330 \\
2230^{\circ} \\
2214, \\
2214, \\
2293, \\
2293, \\
2230^{\circ} \\
2230^{\circ}\end{array}$ & $\begin{array}{r}6710 \\
4230 \\
730 \\
682 \\
240 \\
462 \\
80 \\
876 \\
276\end{array}$ & $\begin{array}{l}0000 \\
0000 \\
0000 \\
0000 \\
0000 \\
0000 \\
0000 \\
0000\end{array}$ & $\begin{array}{l}13 \\
13 \\
13 \\
13 \\
13 \\
13 \\
13 \\
13\end{array}$ & $\begin{array}{l}3329 \\
3729 \\
3359 \\
3339 \\
3359 \\
3731 \\
3739 \\
3730\end{array}$ \\
\hline $\begin{array}{l}2259, \\
2189, \\
2110 \\
2130 \\
2090 \\
2090 \\
2090 \\
3302,\end{array}$ & $\begin{array}{r}5160^{\circ} \\
651^{\circ} \\
667 \\
740^{\circ} \\
740^{\circ} \\
36 . \\
2 .\end{array}$ & $\begin{array}{l}0000 \\
0000 \\
0000 \\
0000 \\
0000 \\
0000 \\
0000\end{array}$ & $\begin{array}{l}13 \\
13 \\
13 \\
13 \\
13 \\
13 \\
13\end{array}$ & 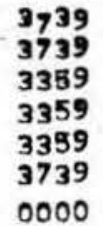 \\
\hline
\end{tabular}




\section{. 073}

********************* STATION UATA RETRIEVAL

*********************** DATE： OOIOIT:38 JUN O8

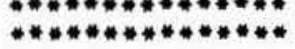
DATE:

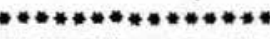

PAGE 51

PAGE 1803417591

SAMPLF DE. DATE SHIP CRUISE LEG STATION NUMAER VICE YRMODA * COMMENTS**

MARS. CQRE AR LENGTH DREDGE PHYSTO ROCK

FIX MARS. CORE AR OR OR PHYSIOQ OR LATE
MODA LATITUE LONGITUDE TYPE SQUARE NUMBER DEPTH DEPTH VOLUME PROV. TYPE CODE REMARKS
S**
NG PENETRATION! MN NODULES AND CRUST SCRAPED UP

\section{MARSDEN SQUARE \# 376}

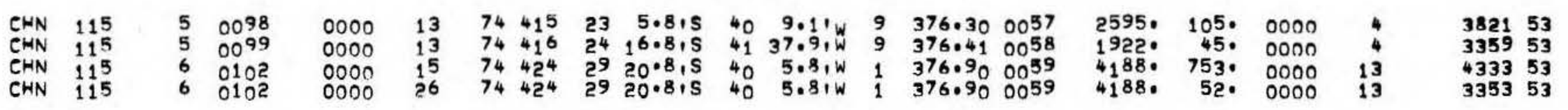

MARSDEN SQUARE * 40?

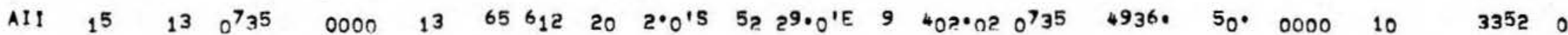

MARDEN SQUARE \#403

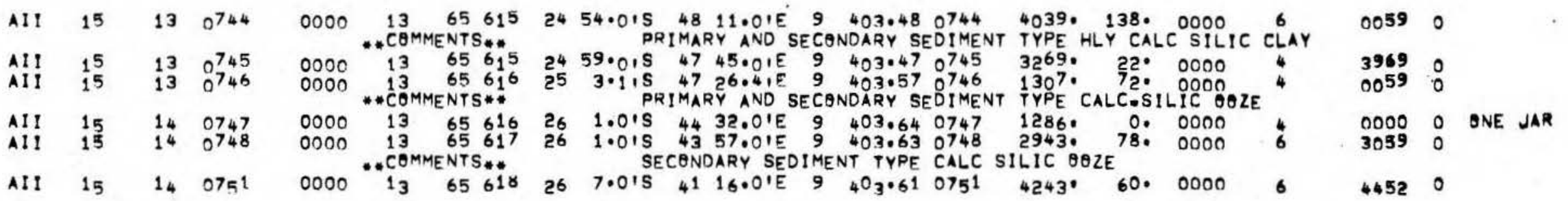

MARSDEN SQUARE *404

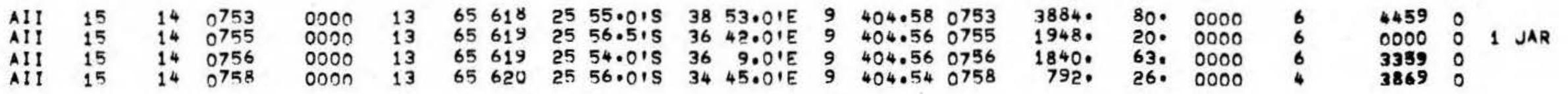

MARSDEN SQUARE \# 406

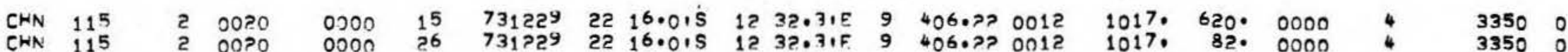




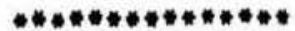

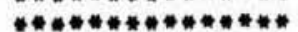

STATION OATA RETRIEVAL DATE: 17138 JUN 08,177

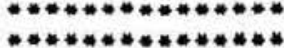

PAGE 58

*WHOI**

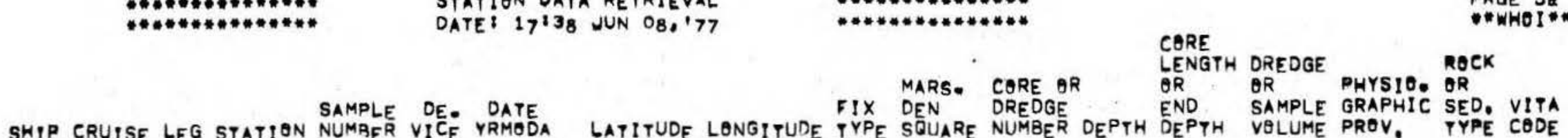

SHIP CRUISE LEG STATION SAMPLE OE. DATE LATITUDE LONGITUDE TYPE SQUARE NUMBER DEPTH DEPYH VELUME PROV.

MARSDEN SQUARE " 406

$\begin{array}{llll}\text { CHN } & 115 & 2 & 0021 \\ \text { CHN } & 115 & 2 & 0021 \\ \text { CHN } & 115 & 2 & 0022 \\ \text { CHN } & 115 & 2 & 0023 \\ \text { CHN } & 115 & 2 & 0023 \\ \text { CHN } & 115 & 2 & 0023 \\ \text { CHN } & 115 & 2 & 0024 \\ \text { CHN } & 115 & 2 & 0024\end{array}$

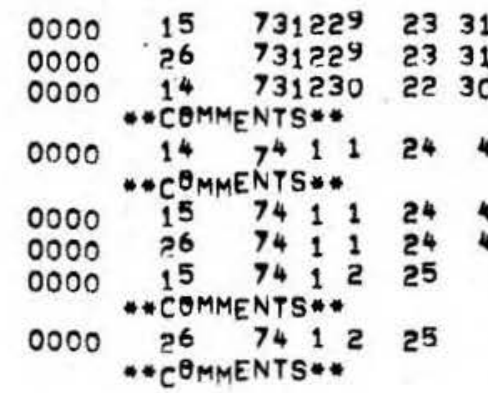

* COMMENTS**

$\begin{array}{lr}\text { CHN } & 99 \\ \text { CHN } & 99 \\ \text { CWN } & 99 \\ \text { CHN } & 99 \\ \text { CWN } & 115 \\ \text { CHN } & 115 \\ \text { CWN } & 115 \\ \text { CHN } & 115\end{array}$

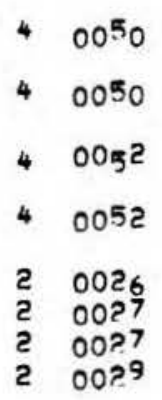

0000
0000
0000
0000
0000
0000
0000
0000

$157067^{7}$

MARSDEN SQUARE * 407

$\begin{array}{llll}\text { CHN } & 115 & 5 & 0069 \\ \text { CHN } & 115 & 5 & 0069 \\ \text { CHN } & 115 & 5 & 0071 \\ \text { CHN } & 115 & 5 & 0071\end{array}$

0000
0000
0000
0000

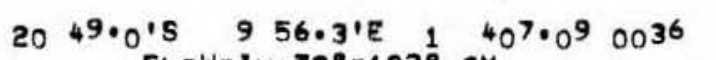

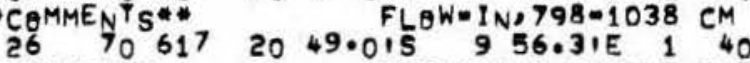
*COMMENTS*

PRIMARY SEDIMENT 4YPE.09 0036

$2640 \cdot 1038 \cdot 0000$

2640 177. 0000

$13 \quad 332^{9} 54$

1570 61Y

is

931. 000013

032954

SOUTH SOMALI BASIN SITE II

$4331,184.0000$. 13

"COMMENTS** PRIMARY SEDIMENT TYPE CALC.SILIC

$\begin{array}{lllll}74 & 1 & 3 & 25 & 25 \\ 74 & 1 & 4 & 25 & 715\end{array}$

$\begin{array}{lllll}74 & 1 & 4 & 25 & 2.415 \\ 74 & 1 & 4 & 25 & 2.415\end{array}$

9 $97.11 \mathrm{E}$

$\begin{array}{ll}407.59 & 0019 \\ 407.57 & 0020\end{array}$

$747.41 E$ I 407.570020

$4685^{\circ} 4760^{\circ}$ OONO 10

4738. 321. 0000

4738, 107. 0000

4792. 877. 0000

10
10
10
10

372154 WALVIS RDG

MARSDEN SQUARE * 408

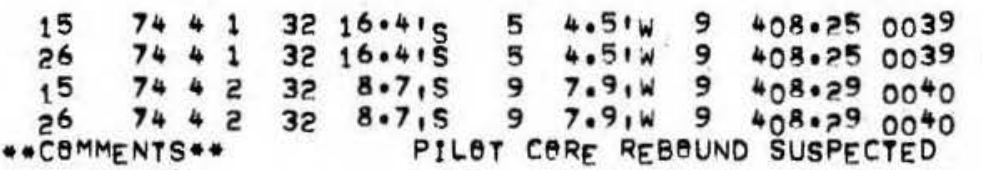

4018. 491. 0000

4018. 121. 0000

4080.485 .0000

$40^{8} 0^{\circ} 146.0000$

10
10
10

003956 WALVIS RDG

CLAY

31390

13300

$\begin{array}{ll}1329 & 0 \\ 3339 & 0\end{array}$

MARSDEN SQUARE * 409

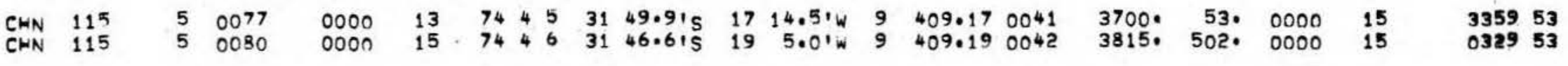

335953

335953

335953

335053 
$* * * * * * * * * * * * * * *$ $* * * * * * * * * * * * * *$

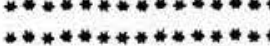

STATION DATA RETRIEVAL DATE: 001017:38 JUA OS STATIAN UATA RETRIEVAL DATE :

SAMPLE DE- DATE SHIP CRUISE LEG STATION NUMRER VICE YRMODA * $\operatorname{COMM}_{E} N T S * *$

\section{$* * * * * * * * * * * * * *$}

PAGE 53

A

CERE LENGTH DKEDGE ROCK

MARS. CQRE AR HR OF PHYSIO, $\theta R$ FIX OFN DREDGE END SAMPLE GRAPHIC SED, VITA LATITUDE LANGITUDE TYPE SUUARF NIJMBER DEPTH DEPTH VOLUME PROV. TYPE CADE PEMARKS PRIMARY SFD TYPE NANNQ AQZE

\section{MARSDEN SQUARE \# 410}

$\begin{array}{llll}\text { CHN } & 115 & 5 & 0084 \\ \text { CHN } & 115 & 5 & 0084 \\ \text { CHN } & 115 & 5 & 0085 \\ \text { CHN } & 115 & 5 & 0085 \\ \text { CHN } & 115 & 5 & 0086 \\ \text { CHN } & 115 & 5 & 0086\end{array}$

0000
0000
0000
0000
0000
0000

2052.41 w $9 \quad 410.100043$

15

$74,73136.5,5$

$7447 \quad 3136.5,5$

$\begin{array}{lllll}74 & 4 & 8 & 31 & 31.115 \\ 74 & 4 & 8 & 31 & 31.115\end{array}$

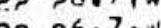

24
24
26.41.

$410 \cdot 10$
410043

$410 \cdot 1=0044$

$\begin{array}{lll}410 \cdot 12 & 0044 \\ 410 \cdot 14 & 0045\end{array}$

$410 \cdot 140045$
7447 31 430515
MARSDEN SQUARE \# 411

$\begin{array}{lllll}\text { AII } & 60 & 2 & C 01 \\ \text { AII } & 60 & 2 & C 01 \\ \text { AII } & 60 & 2 & 001 \\ \text { CHN } & 115 & 6 & 010 \\ \text { CHN } & 115 & 6 & 010 \\ \text { CHN } & 115 & 6 & 010 \\ \text { CHN } & 115 & 6 & 010 \\ \text { CHN } & 115 & 6 & 010 \\ \text { CHN } & 115 & 6 & 010 \\ \text { CHN } & 115 & 6 & 010 \\ \text { CHN } & 115 & 6 & 010 \\ \text { CHN } & 115 & 6 & 010 \\ \text { CHN } & 115 & 6 & 011 \\ \text { CHN } & 115 & 6 & 0137 \\ \text { CHN } & 115 & 6 & 0137 \\ \text { CHN } & 115 & 6 & 012 \\ \text { CHN } & 115 & 6 & 014 \\ \text { CHN } & 115 & 6 & 014 \\ \text { CHN } & 115 & 6 & 014 \\ \text { CHN } & 115 & 6 & 014 \\ \text { CHN } & 115 & 6 & 014 \\ \text { HN } & & & \end{array}$

4141. 699. 0000

4141. 117. 0000

$4411.880^{\circ} 00 n 0$

$411^{\circ}$ 9 000

4305. 591. 0000

4305. 15?. 000n
333953

335953

351253

335253

332253

335953

332941

1129 O VOID $0.80 C M$

$\begin{array}{ll}1129 & 0 \\ 1863 & 53\end{array}$

142253

313353

$31325^{3}$

335253

335953

37395.3

000053

73290

30295

$\begin{array}{ll}3332 & 53 \\ 3322 & 53\end{array}$

33290

33500

33225.7

334053

335258 


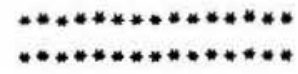

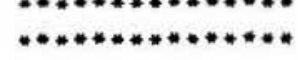

STATIEN UATA RETRIEVAL DATE: $17: 38$ JUN 08,177

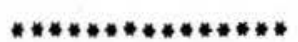

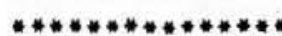

SHIP CRUISE LEG STATION NUMPRER VICE YRMAEA FIX OEN $\begin{array}{ll}\text { ORE } 9 R & 9 R \\ \text { REDGE } & \text { END }\end{array}$ MARSDEN SQUARE \#411

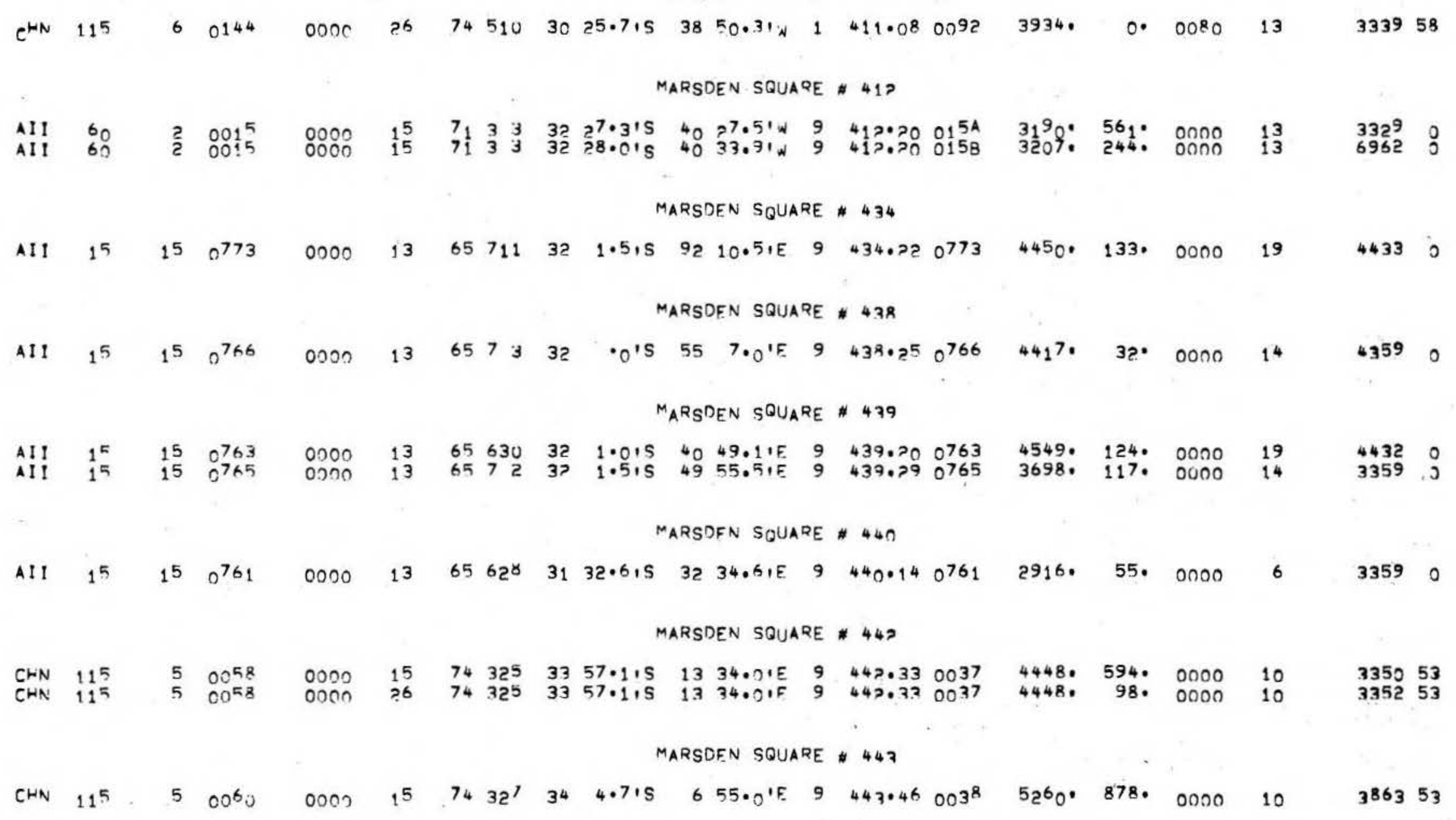
SAMPLE GRAPHIC SED

PAGE 54

**WHOI **

CORE
LENGTH DRFDGE

VOLUME PROV. 
$* * * * * * * * * * * * * * *$

SAMPLE DE. DATE

MARS. CARE $9 R$

CQRE

FIX DEN DREDGE

LENGTH DREDGE PHYSIO. $R$ OR

SHIP CRUISE LEG STATION NIJMRER VICE YRMOD

END SAMPLE GRAPHIC SEO. VITA

\section{MARSDEN SQUURE \# 443}

CHN 115

50060

$2000 \quad 26 \quad 74 \quad 32$
.CEMMENTS**

4.715

PISLAT CARE RFB PUND SUSPECTED
PILATE

$5260 \cdot 160 \cdot 000010$

186053

MARSDEN SQUARE \#478

$\begin{array}{llll}\text { CHN } 115 & 4 & 0032 \\ \text { CHN } & 115 & 4 & 003 ? \\ & & & \\ \text { CHN } 115 & 4 & 0036 \\ \text { CHN } 115 & 4 & 0036 \\ \text { CHN } 115 & 4 & 004 ?\end{array}$

ooon

15
26

1441.715

$\begin{array}{lll}9 & 478.14 & 0024 \\ 9 & 478.14 & 0024\end{array}$

4347. 1176. 00nก

4347. 146. Donn 10

356953

MARSDEN SQUARE \#4R0

$\begin{array}{llll}\text { CHN } & 115 & 4 & 0036 \\ \text { CHN } & 115 & 4 & 004 ?\end{array}$

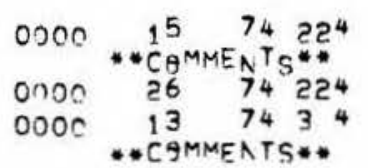

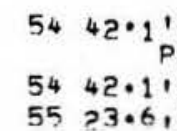

MARSDEN SQUARE \# 515

$\begin{array}{llll}\text { CHN } & 115 & 4 & 0034 \\ \text { CHN } & 115 & 4 & 0035 \\ \text { CHN } & 115 & 4 & 0035 \\ \text { CHN } & 115 & 4 & 0043 \\ \text { CHN } & 115 & 4 & C 047 \\ \text { CHN } 115 & 4 & 0051 \\ \text { CHN } & 115 & 4 & 00^{5} 1\end{array}$

\begin{abstract}
0
o.son

$1574222 \quad 51$

0030 C ${ }^{M}$ MMENTS*

$0020 \quad 26 \quad 74223$
$0000 \quad 137470$
0. CAMMENTS**

$000 n \quad 15 \quad 7437$

. COMMENTS* ODO0 *COMMENTS**
\end{abstract}

- O'S 5 19.81E

CARE DISTUIRBED

PUMICE FRA GS FAUND SCATTERED THREUGHQUT $5436: 0.5$

0 6.O'F 1 1 $15.300026 \quad 2643$.

$515 \cdot 150025 \quad 3788 \cdot 141^{\circ}, 0000$

15

536053

90. 0000

61. 0000 CARE PASSTILLY DISTURBFD DUE TO SPINNING MAGNETOMETER 


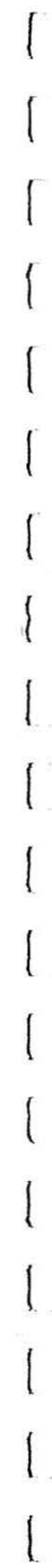

078

DESCRIPTIONS OF WHOI SEDIMENT CORES, VOLUME 5 
I

1

I

1

I

1

1

1

I

1

1

1

1

1

1

I

1

I

1 


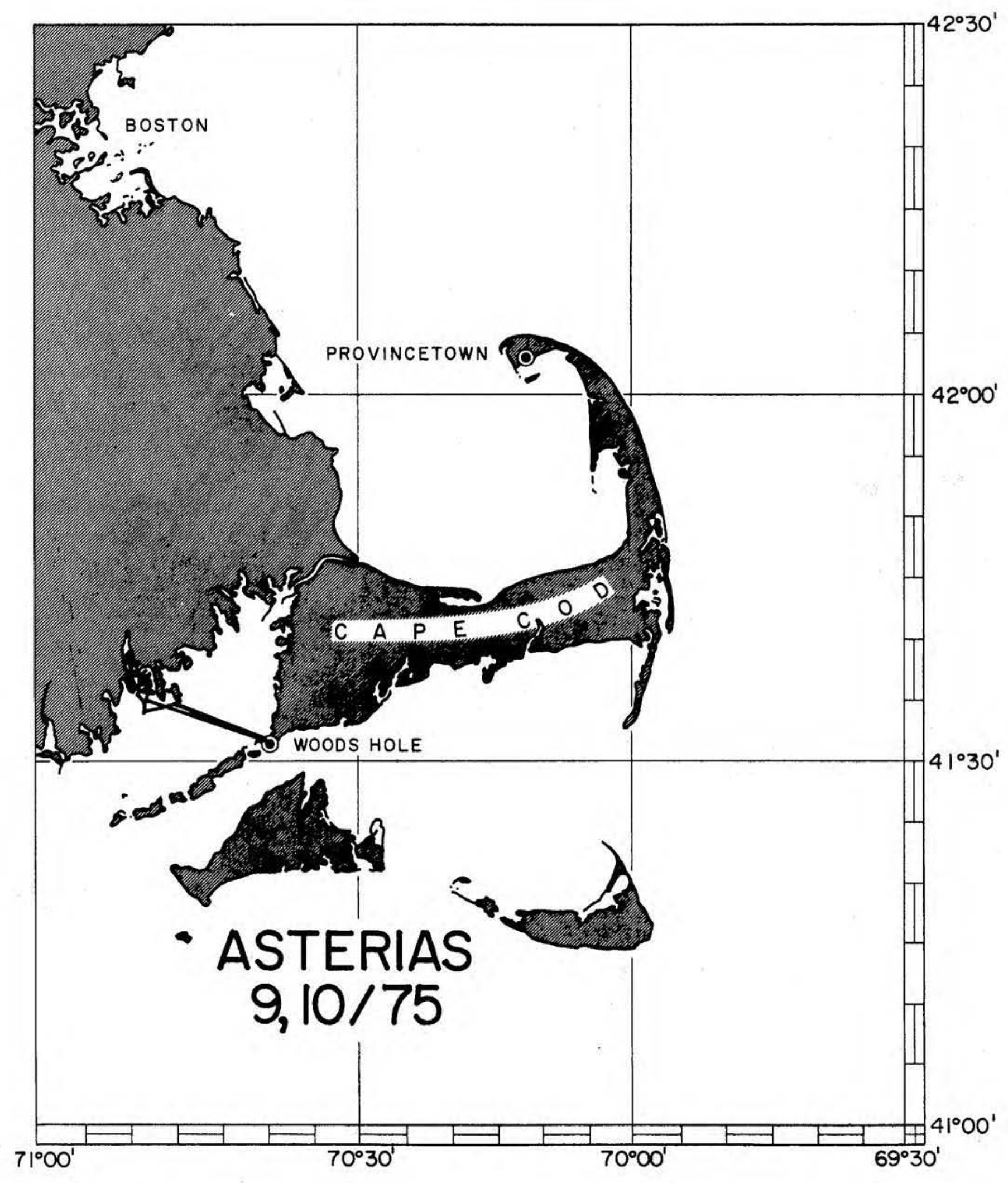


1

1

I

I

I

I

1

1

I

1

1

1

1

1

1

I

1

I

1 


\section{0}

$* * * * * * * * * * * * * * * *$

$* * * * * * * * * * * * * *$

STATION UATA RETRIEVAL

DATE: 17:48 JUN 03,177

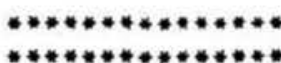

MARS. CARE $9 R$

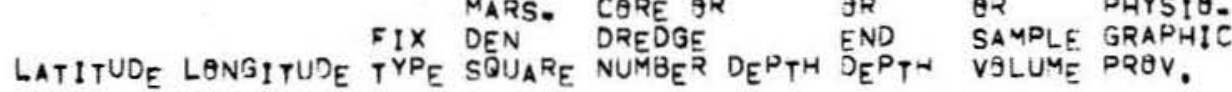

SHIP CRUISE LEG STATION NUMBER VICE YRMODA LATITUDE LONGITUDE TYPE SQUARE NUMBER DEPTH DEPTH VALUME PROV.

LENGTH DREDGE

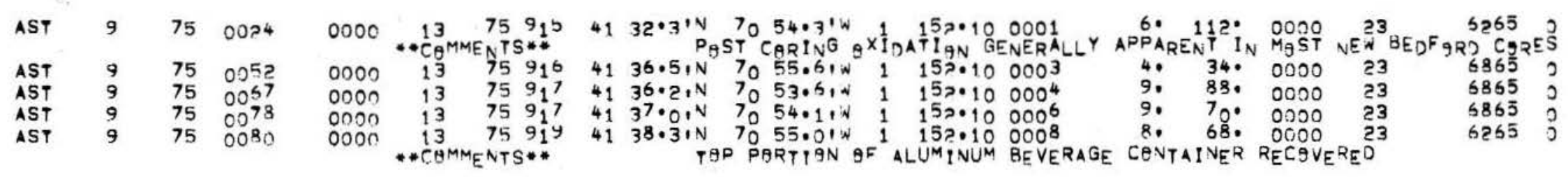

PAGE 1

$* *$ H 


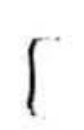

I

$\Gamma$

$\Gamma$

1

I

1

I

I

I

1

I

1

1

I

I

1

I

| 


\section{1}

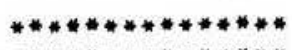

$* * * * * * * * * * * * * * *$
STATION UATA RETRIEVAL DATE: $17: 48$ JUN 03,177

\section{$* * * * * * * * * * * * * * *$ \\ $* * * * * * * * * * * * * * *$}

MARS- CARE $Q R$
FIX DEN DREDGE
SAMPLF DE. DATE SHIP CRUISE LEG STATION NUMAER VICE YRMODA

$\begin{array}{llll}\text { AST } & 10 & 75 & 0091 \\ \text { AST } & 10 & 75 & 0083 \\ \text { AST } & 10 & 75 & 0084 \\ \text { AST } & 10 & 75 & 0085 \\ \text { AST } & 10 & 75 & 0086 \\ \text { AST } & 10 & 75 & 0087 \\ \text { AST } & 10 & 75 & 0092 \\ \text { AST } & 10 & 75 & 0097 \\ \text { AST } & 10 & 75 & 0099 \\ \text { AST } & 10 & 75 & 0100\end{array}$

\begin{tabular}{|c|c|c|}
\hline 0000 & $\begin{array}{l}13 \\
* \text { CAMME }\end{array}$ & $\begin{array}{l}7510 \\
T^{2} S^{*} *\end{array}$ \\
\hline 0000 & 13 & 7510 \\
\hline 0000 & 13 & 7510 \\
\hline 00 & 13 & 7510 \\
\hline 00 & 13 & 7510 \\
\hline 0000 & $\begin{array}{rl} & 13 \\
* & C 9_{M M}\end{array}$ & $\begin{array}{l}7510 \\
\text { ENTS* * }\end{array}$ \\
\hline 0000 & 13 & 751010 \\
\hline 0 & 13 & $75101 i$ \\
\hline 000 & 13 & 751010 \\
\hline & 13 & 751010 \\
\hline
\end{tabular}

$413^{9.1} 1 \mathrm{~N} 7055.3^{1} \mathrm{~W}$

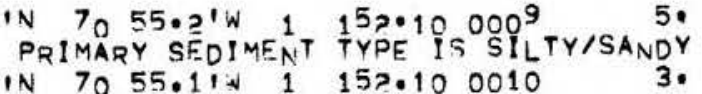
15?.10 0010 $4138.9, N$ TO 55.3, 1 1 153.100011 $4138.7, N$ 7O 55. 3 IN 1 1 1530100012 SECONDARY SEDIMENT TYPE IS SILTY 11. PRIMARY SEDIMENT TYPF IS SILTY/SANDY $4132.8, N$ TO 54.31 . 11152.100016 $4133.5, N \quad 7053.9, W 1152.100017$ $\begin{array}{lllllll}41 & 33.5, N & 70 & 52.7, N & 1 & 152.10 & 0018 \\ 41 & 33.9, N & 70 & 52.11 & 1 & 150.10 & 0019\end{array}$
DA ज̂F 1 ** WHOI**

CQRE

LENGTH DREDGF ROCK IENGTH DRTDGE PHYSIO. AR END SAMPLE GRAPHIC SED. VITA DEPTH VOLUME PROV. TYPE CODE REMARKS 10.

119. 0000

$23 \quad 683^{8} \quad 0$

23. 0000 ?3 123. 000023 39. 000023 63. 000023 52. 0000 23

79. 000023

87. 000023 12. 134. 000023 12. $94^{\circ}$. 000023

$\begin{array}{ll}6838 & 0 \\ 8665 & 0 \\ 6865 & 0 \\ 6265 & 0 \\ 8634 & 0 \\ 6821 & 0 \\ 8661 & 0 \\ 8663 & 0 \\ 6265 & 0 \\ 6865 & 0\end{array}$




\section{2}

$* * * * * * * * * * * * * * *$

$* * * * * * * * * * * * * * *$
STATION UATA RETRIEVAL

DATE: 17:48 JUN 03,177

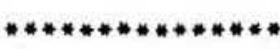

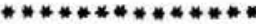

PAGE 1

** WHOI *

SAMPLE DE- DATE

MARS- CARE $9 R$

FIX DEV DREDGE

LENGTH DREDGE PHYSIO. RQCK

SIO. $Q R$

SHIP CRUISE LEG STATION NUMBER VICF YRMADA LATITUDE LONGITUDE. TYPE SQUARE NUMBER DEPTH DEPTH VOLUME PROV.

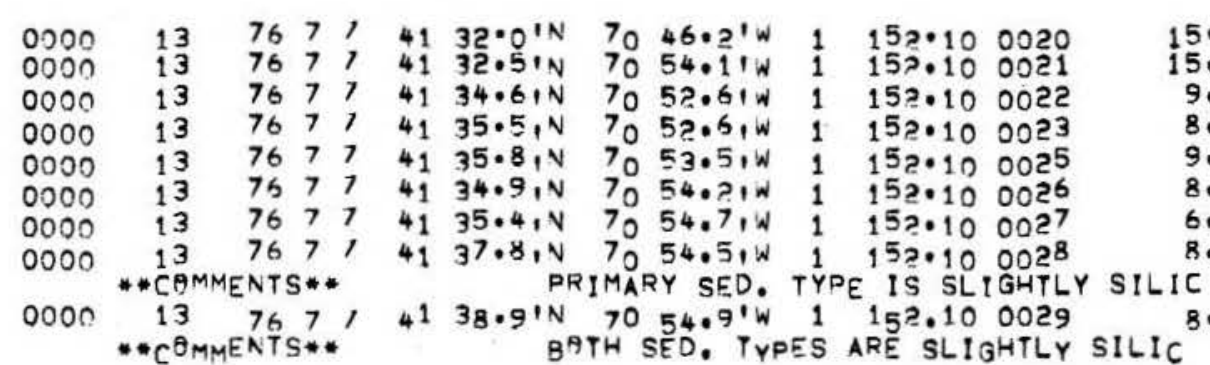

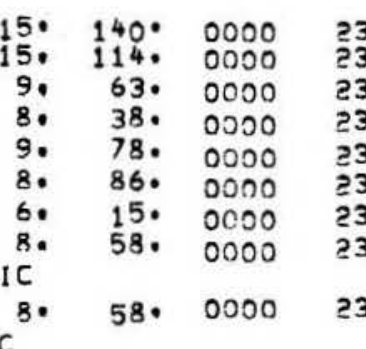

$3668 ?$

3655 ?

$\$ 665$

$\$ 665$

$8665 ?$

366530

3665
3135

$76011^{6}$

$\begin{array}{ll}76 & 0117\end{array}$

* C COMMENTS $7 * *$

BATH SED. TYPES ARE SLIGHTLY SILIC 
Ship BSTERIGS Cruise 9-75 Leg - Sto. 24 Core No. IEC Total Length $1 / 2 \mathrm{~cm}$. Lat. $41 \cdot 32.31 \mathrm{~N}$ Long. $70^{\circ} 5 \%, 30 \mathrm{~W}$ Depth 6.4 meters. Core condition ExcEuEN Date Described 7 man 76 by fholw.

Physiographic location BUZZAROS BAY APPROACHESTO NEW BEOFORO AARBOR.

Lithologic

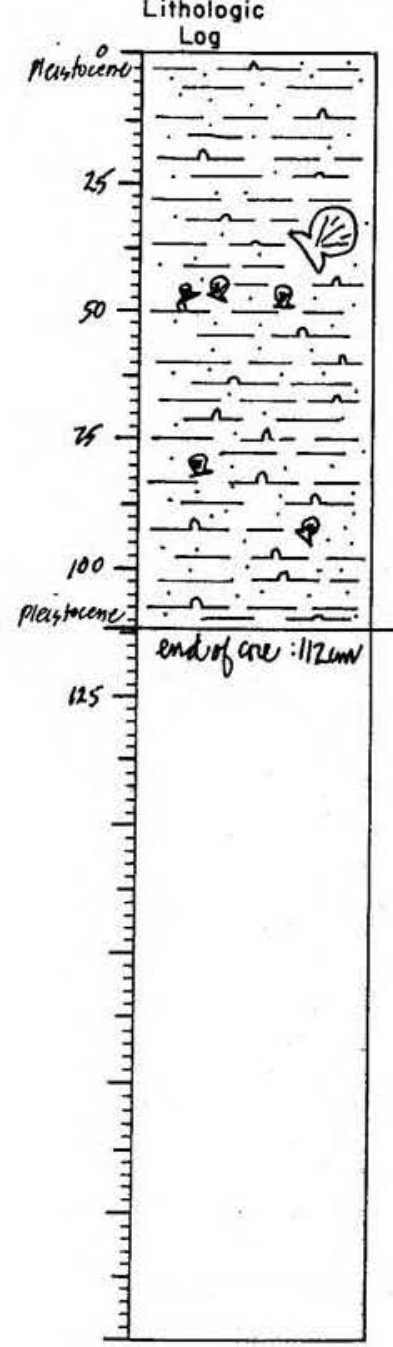

\section{Detailed Description}

$0-112$

SILIC CLAY/DETRITUS GRADES TO HIGLYY SILIC CLAY/ TRITUS

a few gradational color zones found throughout; a faint, gray zone surrounding crushed scallop shell intrudes

firm, quite silty lutite with a number of various pele cypod shell fragments and beds of shell hash found foughout: large scallop she11 $33-38 \mathrm{~cm}$; scattered fragnents $40-44 \mathrm{~cm}$, shell hash $45-47 \mathrm{~cm}$; small bits and fragments scattered $50-85 \mathrm{~cm}$

end of core

SMEAR SLIDE DESCRIPTIONS - W.H.O.I. SEDIMENT CORES

Ship: ASTERTAS

Expedition 9-75

Leg No.

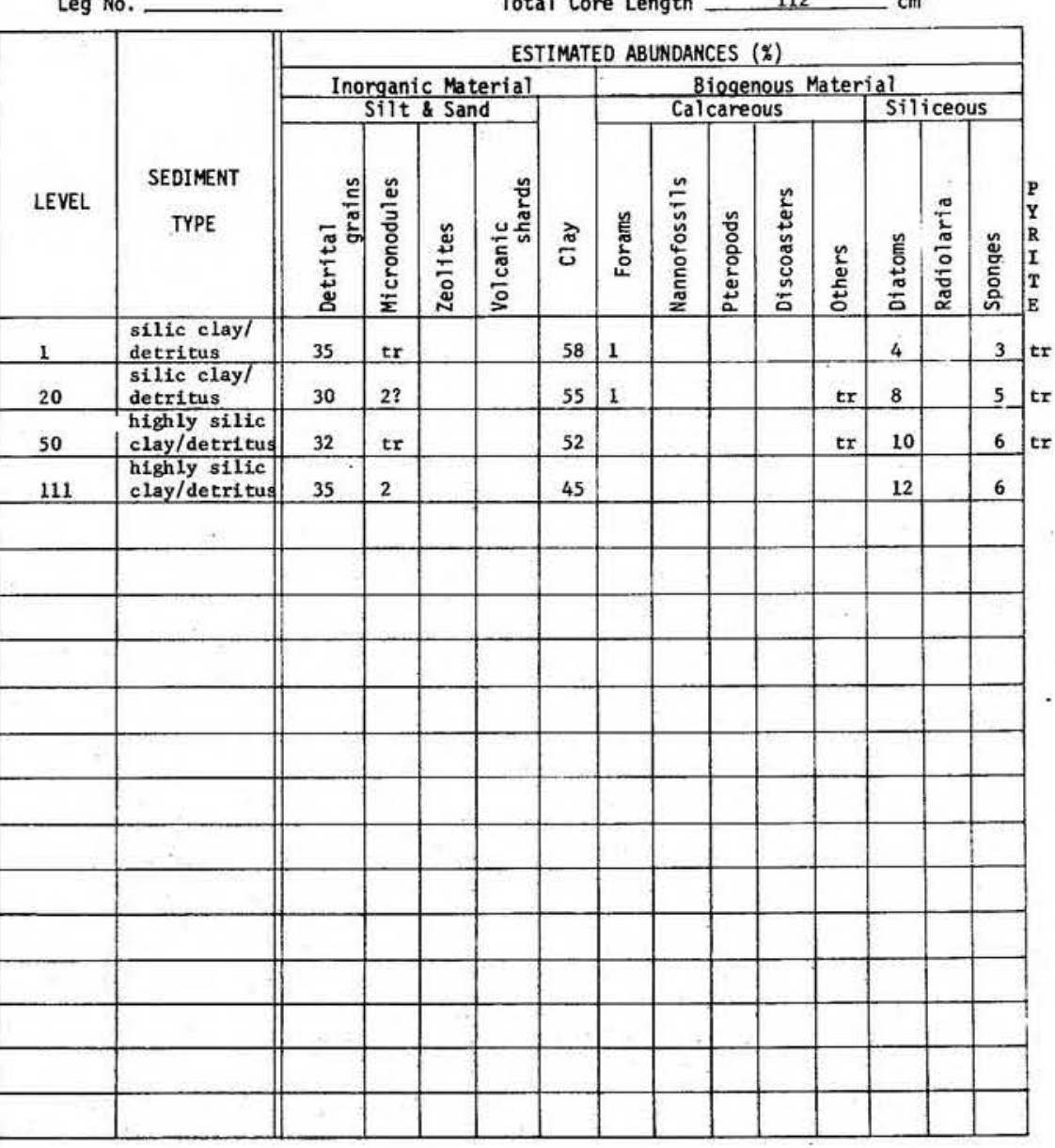

Core No. $1 \mathrm{GC}$

tation No. 
Ship ASTER/AS Cruise 9-75 Leg - Sta. 52 Core No. $36 \mathrm{C}$

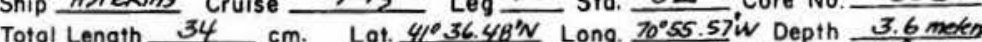

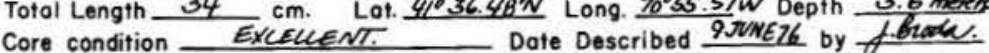

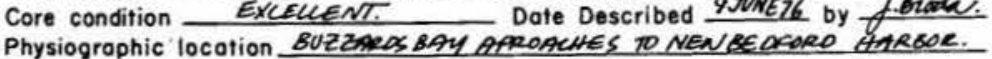
Lithologic

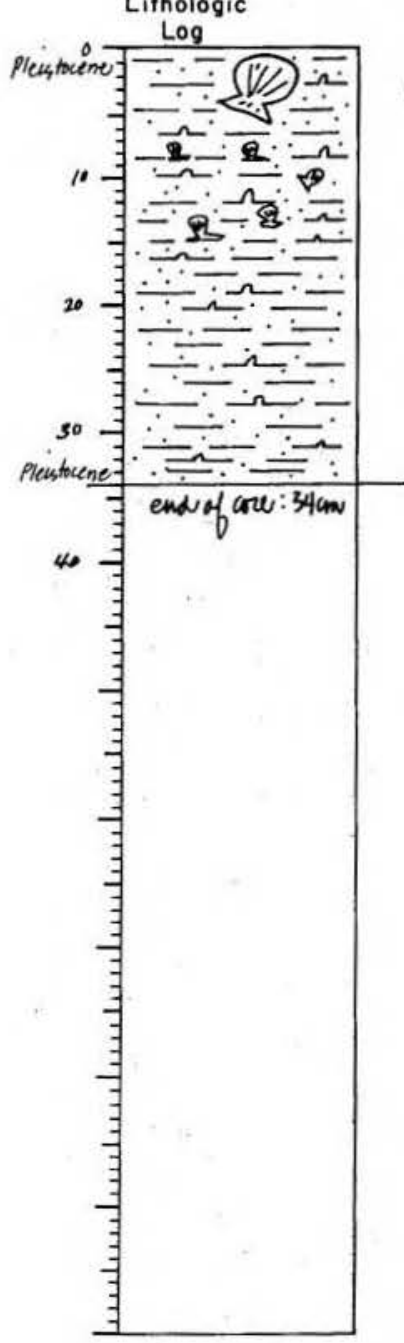

Detailed Description

$0-34$

5 Y $3 / 2$ dark olive gray

a the exception or shells quahog shell 0-5 cr

end of core
SLIGHTLY SILIC CLAY/DETRITUS

a silty lutite matrix with shells

SIEAR SLIDE DESCRIPTIONS - W.H.0.I. SEDIMENT CORES

Ship: _ AST

Expedition 2-75

Leg No.
Core No. $3 \mathrm{CC}$

Station No. 52

Total Core Length _ 34

ESTIMATED ABUNDANCES (\%)

\begin{tabular}{|c|c|c|c|c|c|c|c|c|c|c|c|c|c|c|}
\hline \multirow[b]{3}{*}{ LEVEL } & \multirow[b]{3}{*}{$\begin{array}{c}\text { SEDIMENT } \\
\text { TYPE }\end{array}$} & \multicolumn{13}{|c|}{ ESTIMATED ABUNDANCES ( $(x)$} \\
\hline & & \multicolumn{4}{|c|}{$\begin{array}{c}\text { Inorganic Material } \\
\text { Silt \& Sand }\end{array}$} & & \multicolumn{5}{|c|}{$\begin{array}{l}\text { Biogenous Mater } \\
\text { Calcareous }\end{array}$} & \multicolumn{3}{|c|}{$\begin{array}{l}\text { ial } \\
\text { Siliceous }\end{array}$} \\
\hline & & $\frac{\frac{5}{5}}{\frac{5}{5}}$ & 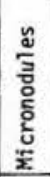 & 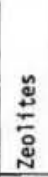 & 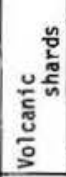 & $\frac{\pi}{U}$ & 点 & 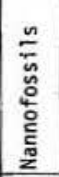 & 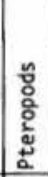 & 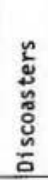 & 蒿 & 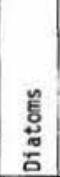 & $\frac{\frac{\pi}{\frac{1}{5}}}{\frac{0}{0}}$ & ڤัँ \\
\hline 1 & $\begin{array}{l}\text { slightly silio } \\
\text { clay/detritus }\end{array}$ & 45 & & & & 50 & & & & & tir & 3 & & 2 \\
\hline 17 & $\begin{array}{l}\text { slightly silic } \\
\text { clay/detritus }\end{array}$ & 60 & & & & 37 & & & & & $\mathrm{tr}$ & 2 & & 1 \\
\hline 33 & $\begin{array}{l}\text { slightly silio } \\
\text { clay/detritus }\end{array}$ & 65 & & & $\theta$ & 30 & & & & & $\mathrm{tr}$ & 2 & & 3 \\
\hline & & & & & & & & & & & & & & \\
\hline & & & & & & & & & & & & & & \\
\hline & & & & & & & & & & & & & & \\
\hline & & & & & & & & & & & & & & \\
\hline & & & & & & & & & & & & & & \\
\hline & & & & & & & & & & & & & & \\
\hline & & & & & & & & & & & & & & \\
\hline 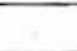 & & & & & & & & & & & & & & \\
\hline & & & & & & & & & & & & & & \\
\hline & & & & & & & & & & & & & & \\
\hline & & & & & & & & & & & & & & \\
\hline & & & & & & & & & & & & & & \\
\hline & & & & & & & & & & & & & & \\
\hline & - & & & & & & & & & & & & & \\
\hline & & & & & & & & & & & & & & \\
\hline
\end{tabular}




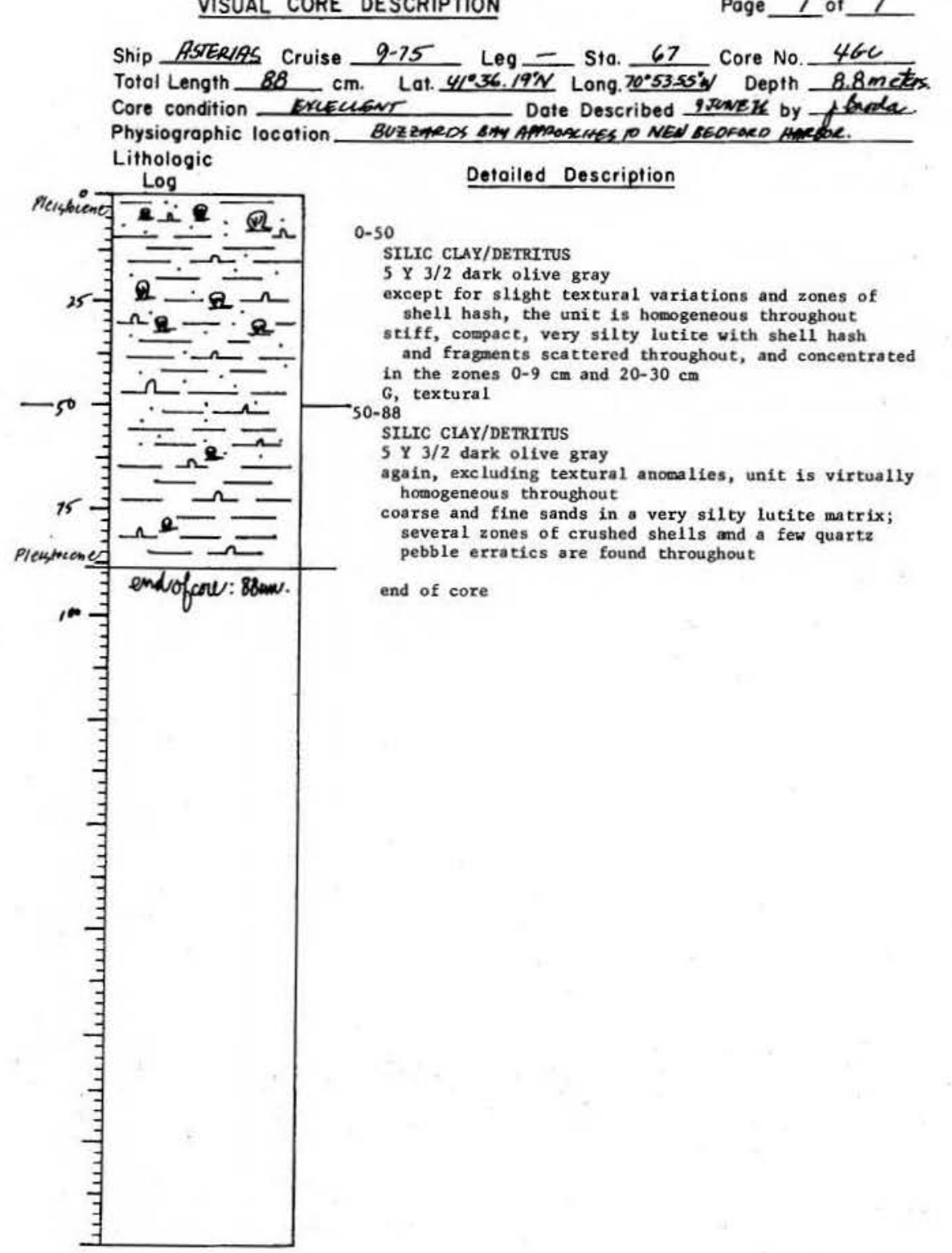

SMEAR SLIDE DESCRIPTIONS - W.H.O.I. SEDIMENT CORES

Ship:_ AST Core No. $4 \mathrm{CC}$

Expedition 9-75

Leg No.

\begin{tabular}{|c|c|c|c|c|c|c|c|c|c|c|c|c|c|c|}
\hline \multirow[b]{3}{*}{ LEVEL. } & \multirow[b]{3}{*}{$\begin{array}{c}\text { SEDIMENT } \\
\text { TYPE }\end{array}$} & \multicolumn{13}{|c|}{ ESTIMATED ABUNDANCES ( $(x)$} \\
\hline & & \multicolumn{4}{|c|}{$\frac{\text { Inorganic Material }}{\text { Silt \& S Sand }}$} & & \multicolumn{5}{|c|}{$\begin{array}{l}\text { Biogenous Mater } \\
\text { Calcareous }\end{array}$} & \multicolumn{3}{|c|}{$\frac{\text { ial }}{\text { Stliceous }}$} \\
\hline & & $\frac{\frac{n}{n}}{5}$ & 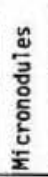 & 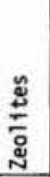 & 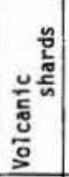 & $\frac{\pi}{\sigma}$ & 宸 & 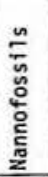 & 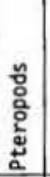 & 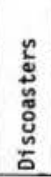 & \begin{tabular}{l}
$\frac{n}{2}$ \\
\multirow{2}{*}{} \\
0
\end{tabular} & 产 & $\frac{\pi}{\frac{\pi}{\pi}}$ & 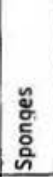 \\
\hline 1 & $\begin{array}{l}\text { silic clay/ } \\
\text { detritus }\end{array}$ & so & tr & & & 40 & & & & & tr & 4 & & 6 \\
\hline 40 & $\begin{array}{l}\text { silic clay/ } \\
\text { detritus }\end{array}$ & 45 & & & & 42 & & & & & tr & 6 & & 7 \\
\hline 55 & $\begin{array}{l}\text { silic clay/ } \\
\text { detritus }\end{array}$ & 60 & & & & 32 & & & & & & 3 & & 5 \\
\hline 87 & $\begin{array}{l}\text { silic clay/ } \\
\text { detritus }\end{array}$ & 65 & tr & & & 27 & & & & & . & 2 & & \\
\hline & & & & & & & & & & & & & & \\
\hline & & & & & & & & & & & & & & \\
\hline & & & & & & & & & & & & & & \\
\hline & & & & & & & & & & & & & & \\
\hline & & & & & & & & & & & & & & \\
\hline & & & & & & & & & & & & & & \\
\hline & & & & & & & & & & & & & & \\
\hline & & & & & & & & & & & & & & \\
\hline & & & & & & & & & & & & & & \\
\hline & & & & & & & & & & & & & & \\
\hline & & & & & & & & & & & & & & \\
\hline & & & & & & & & & & & & & & \\
\hline & & & & & & & & & & & & & & \\
\hline & & & & & & & & & & & & & & \\
\hline
\end{tabular}


Page 1 of 1

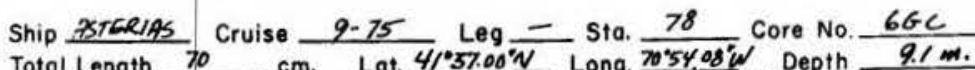

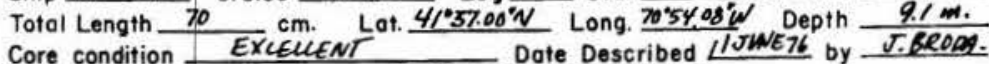
Physiographic location NEN BEOFORO AARBOR.

Lithologic

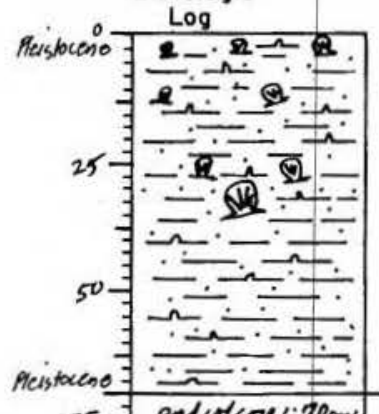

Detoiled Description

0-70

SLICHTLY SILIC CLAY/DETRITUS GRADES TO SILIC CLAY/ DETRITUS

5 Y $3 / 2$ dark olive gray

silght lightening of hue in the textural laminations concentrated 9-13 cm, 26-32 cm large scallop intact crepidula shell; also (detritus and she11 hash) and lenses $32-70 \mathrm{~cm}$ end of core

090

SUEAR SLIDE DESCRIPTIONS - W.H.O.I. SEDINENT CORES

Ship:

Expedition 9-75

Leg No.

\begin{tabular}{|c|c|c|c|c|c|c|c|c|c|c|c|c|c|c|}
\hline \multirow[b]{3}{*}{ LEVEL. } & \multirow[b]{3}{*}{$\begin{array}{c}\text { SEDIMENT } \\
\text { TYPE }\end{array}$} & \multicolumn{13}{|c|}{ ESTIMATED ABUNDANCES ( $($ ) } \\
\hline & & \multicolumn{4}{|c|}{$\frac{\text { Inorganic Material }}{\text { silt \& Sand }}$} & & \multicolumn{5}{|c|}{$\begin{array}{l}\text { Biogenous Mater } \\
\text { Calcareous }\end{array}$} & \multicolumn{3}{|c|}{$\frac{\text { al }}{\text { siliceous }}$} \\
\hline & & 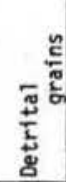 & 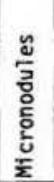 & 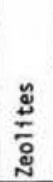 & 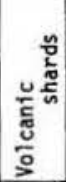 & $\frac{\pi}{0}$ & 产 & 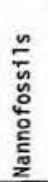 & 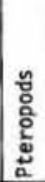 & 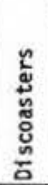 & $\begin{array}{l}\text { č } \\
\text { ș } \\
\text { to }\end{array}$ & $\begin{array}{l}\text { 产 } \\
\frac{\pi}{\sigma}\end{array}$ & $\frac{\pi}{\frac{\pi}{\pi}} \frac{\pi}{\frac{\pi}{0}}$ & $\begin{array}{l}\text { \%̆ } \\
\text { है }\end{array}$ \\
\hline 1 & $\begin{array}{l}\text { slightly silit } \\
\text { clay/detritus }\end{array}$ & 45 & $\mathrm{tr}$ & & & 50 & & & & & tr & 2 & & 3 \\
\hline 36 & $\begin{array}{l}\text { silic clayl } \\
\text { detritus }\end{array}$ & 60 & $t r$ & & & 34 & & & & & $\mathrm{tr}$ & 3 & & 3 \\
\hline 69 & $\begin{array}{l}\text { silic clay/ } \\
\text { detritus }\end{array}$ & 55 & & & & 37 & & & & & & 3 & & 5 \\
\hline & & & & & & & & & & & & & & \\
\hline & & & & & & & & & & & & & & \\
\hline & & & & & & & & & & & & & & \\
\hline & & & & & & & & & & & & & & \\
\hline & & & & & & & & & & & & & & \\
\hline & & & & & & & & & & & & & & \\
\hline & & & & & & & & & & & & & & \\
\hline & & & & & & & & & & & - & & & \\
\hline & & & & & & & & & & & & & & \\
\hline & & & & & & & & & & & & & & \\
\hline & & & & & & & & & & & & & & \\
\hline & & & & & & & & & & & & & & \\
\hline & & & & & & & & & & & & & & \\
\hline & & & & & & & & & & & & & & \\
\hline & & & & & & & & & & & & & & \\
\hline
\end{tabular}

Core No. $\quad 6 \mathrm{GC}$

Station No. 78

Stength

tal Core Length

STIMATED ABUNDANCES ( $x$ firm, compact, very silty lutite with pelecypod shells

157 end of cone: 70 and

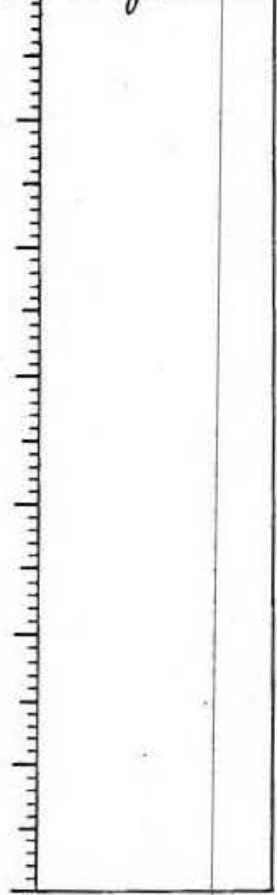


091

VISUAL CORE DESCRIPTION

Page 1 of 1

Ship ASTERIAS. Cruise 9-75 Leg - Sta. 80 Core No. 8GC Total Length $6 B \mathrm{~cm}$. Lat. $41^{\circ} 38.27 \mathrm{~W}$ Long. $70^{\circ} 55.05^{\circ} \mathrm{W}$ Depth $7.9 \mathrm{~mW}$. Core condition EXCELLENT Date Described IIJume 76 by J.Phade.

Physiographic location NEW BCDFORD HARBAC.

Lithologic

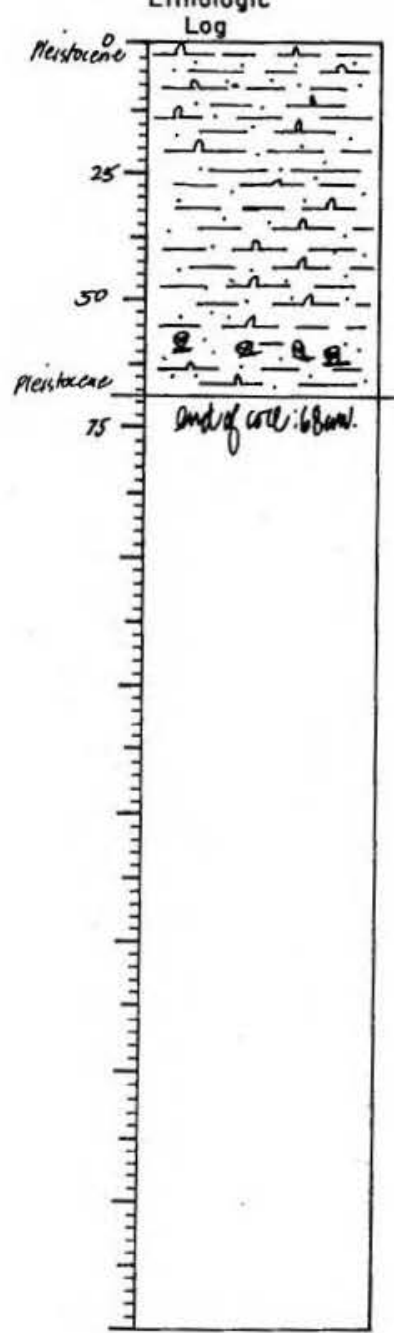

\section{Detailed Description}

$0-68$

HIGHLY SILIC CLAY/DETRITUS GRADES TO SILIC CLAY/ DETRITUS

5 Y $3 / 2$ dark olive gray

numerous and repetative tions throughout the zone $10-58 \mathrm{~cm}$; the majority of the best defined ones are ollve or olive yellow and are $.5-1.5$ cm wide, a few very dark gray laminahash 62-64 cm composed of pelecypods

top portion of aluminum beverage container (partially decomposed) 3-5 cm

end of core
092

SMEAR SLIDE DESCRIPTIONS - W.H.O.1. SEDIMENT CORES
Ship:

Expedition 9-75

Leg No.
Cone No. $\quad B C C$

Station No. 80

Total Core Length $68 \quad \mathrm{~cm}$

\begin{tabular}{|c|c|c|c|c|c|c|c|c|c|c|c|c|c|c|}
\hline \multirow[b]{3}{*}{ LEVEL } & \multirow[b]{3}{*}{$\begin{array}{c}\text { SEDIMENT } \\
\text { TYPE }\end{array}$} & \multicolumn{13}{|c|}{ ESTIMATED ABUNDANCES ( $(x)$} \\
\hline & & \multicolumn{4}{|c|}{$\begin{array}{c}\text { Inorganic Material } \\
\text { Silt \& Sand }\end{array}$} & & \multicolumn{5}{|c|}{$\begin{array}{l}\text { Biegenous Mater } \\
\text { Calcareous }\end{array}$} & \multicolumn{3}{|c|}{$\begin{array}{l}\text { Ial } \\
\text { Siliceous }\end{array}$} \\
\hline & & 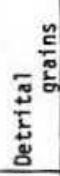 & 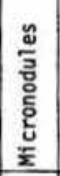 & 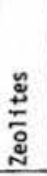 & 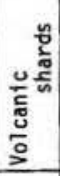 & $\frac{\pi}{0}$ & 产 & 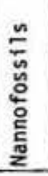 & 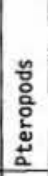 & 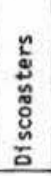 & 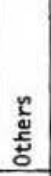 & 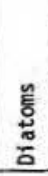 & $\frac{\pi}{\frac{\pi}{2}}$ & 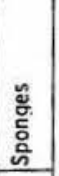 \\
\hline 1 & $\begin{array}{l}\text { highly silic } \\
\text { clay/detritus }\end{array}$ & 45 & $t x$ & & & 40 & & & & & $\mathrm{tr}$ & 8 & & 7 \\
\hline 30 & $\begin{array}{l}\text { hly silic clab } \\
\text { with detritus }\end{array}$ & 25 & 2 & & & 51 & & & & & $\mathrm{tr}$ & 10 & & 12 \\
\hline 67 & \begin{tabular}{|l|}
$\begin{array}{l}\text { silic clay/ } \\
\text { detritus }\end{array}$ \\
\end{tabular} & 35 & 2 & & & 52 & & & & & & 5 & & 6 \\
\hline & & & & & & & & & & & & & & \\
\hline & . & & & & & & & & & & & & & \\
\hline & & & & & & & & & & & & & & \\
\hline & & & & & & & & & & & & & & \\
\hline & & & & & & & & & & & & & & \\
\hline & & & & & & & & & & & & & & \\
\hline & & & & & & & & & & & & & & \\
\hline & & & & & & & & & & & & & & \\
\hline & & & & & & & & & & & & & & \\
\hline & & & & & & & & & & & & & & \\
\hline & & & & & & & & & & & & & & \\
\hline & & & & & & & & & & & & & & \\
\hline & & & & & & & & & & & & & & \\
\hline & & & & & & & & & & & & & & \\
\hline & & & & & & & & & & & & & & \\
\hline
\end{tabular}


. 093

VISUAL CORE DESCRIPTION

Page 1 of 1

Ship AsTER/AS Cruise $10-75$ Leg_Sta. 81 Core No. $9 G C$

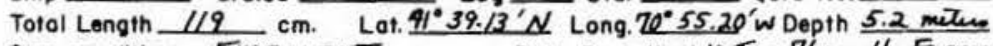
Core condition ExCELLENT Dote Described / 10 Tuen 76 by H. Tarmer

Physiographic location ACusHNET RIVER - NEW BEEFORD HARBar Lithologic

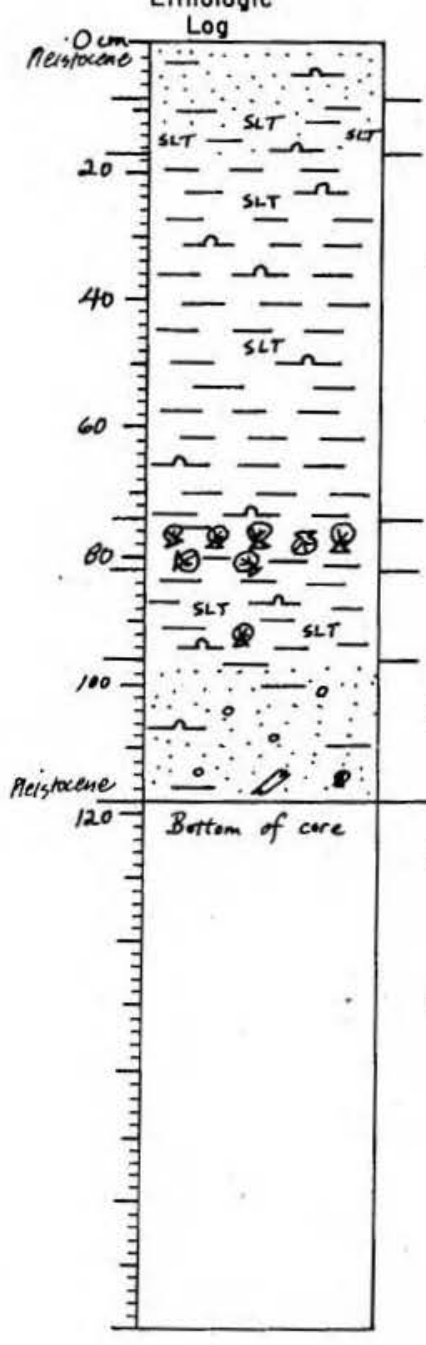

094

SMEAR SLIDE DESCRIPTIONS - W.H.O.1. SEDIMENT CORES

Ship:

Expedition $\quad 10-75$

DETRITUS/SLIGHTLX SILIC CLAY

la sediment is black below exposed surface fine sand

8-17

\section{SLIGATLY SILIC CLAY/DETRITUS}

5 YR $3 / 3$ dark reddish brown

sediment is black below oxidized surface layer very silty/sandy lutite

SIITC CLAY WTTH DETRITUS

10 YR $3 / 1$ very dark gray (black under surface)

slight brownish hue appears from $27-31 \mathrm{~cm}$ in half

of 1 iner and $39-44 \mathrm{~cm}$
very silty/sandy lutite becomes finer and less 44-46 cm; $\mathrm{cm}$ with scattered smaller fragments

$4 \times 4 \mathrm{~cm}$ fragment of concrete block at $23 \mathrm{~cm}$

$74-82$

SHELL HASH IN MATRIX OF SILIC CLAY WITH DETRITUS 5 Y $3 / 2$ dark olive gray

pelecypod shell hash in very silty lutite matrix S2-96

SILIC CIAY WITH DETRITUS

vilform except for a few pecypod fragment.

$\mathrm{s}$
6-119

DETRITUS/SLIGHTLY GILIC CLAY

10 YR $3 / 2$ very dark grayish brown

homogeneous throughout

ard coarse sond with sone small pebbles $(5 \mathrm{~mm})$, a bit of organic detritus $(117 \mathrm{~cm})$ and a few ver

$$
\text { end of core }
$$

Leg No.
Core No. $2 \mathrm{GC}$

Station No. B1

Total Core Length 119

\begin{tabular}{|c|c|c|c|c|c|c|c|c|c|c|c|c|c|c|}
\hline \multirow[b]{4}{*}{ LEVEL } & \multirow[b]{4}{*}{$\begin{array}{c}\text { SEDIMENT } \\
\text { TYPE }\end{array}$} & \multicolumn{13}{|c|}{ ESTIMATED ABUNDANCES ( $(x)$} \\
\hline & & \multirow{2}{*}{\multicolumn{4}{|c|}{$\begin{array}{c}\text { Inorganic Material } \\
\text { Silt \& Sand }\end{array}$}} & \multirow[b]{3}{*}{$\frac{\pi}{6}$} & \multirow{2}{*}{\multicolumn{5}{|c|}{$\begin{array}{l}\text { Biogenous Mater } \\
\text { Calcareous }\end{array}$}} & \multirow{2}{*}{\multicolumn{3}{|c|}{ Siliceous }} \\
\hline & & & & & & & & & & & & & & \\
\hline & & $\frac{\frac{n}{5}}{\frac{5}{5}}$ & 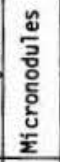 & 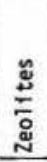 & 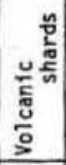 & & 号 & 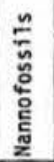 & 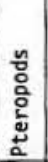 & 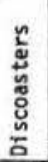 & 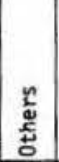 & 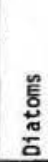 & $\frac{\pi}{\frac{\pi}{0}}$ & 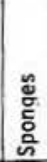 \\
\hline 2 & $\begin{array}{l}\text { detritus/sif } \\
\text { silic clay }\end{array}$ & ${ }_{78}$ & & & & 20 & & & & & tr & 1 & & 1 \\
\hline 20 & $\begin{array}{l}\text { silic clay } \\
\text { with detritus }\end{array}$ & 25 & & & & 66 & & & & & If & 1 & & 1 \\
\hline 65 & \begin{tabular}{l|} 
silic clay \\
with detritus
\end{tabular} & 20 & & & & 75 & & & & & & 3 & & 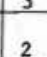 \\
\hline 118 & $\begin{array}{l}\text { detritus/slig } \\
\text { silic clay }\end{array}$ & ${ }_{80}$ & & & & 18 & & & & & & tr & & $\frac{2}{2}$ \\
\hline & & & & & & & & & & & & & & \\
\hline & & & & & & & & & & & & & & \\
\hline & & & & & & & & & & & & & & \\
\hline & & & & & & & & & & & & & & \\
\hline & & & & & & & & & & & & & & \\
\hline & & & & & & & & & & & & & & \\
\hline & & & & & & & & & & & & & & \\
\hline & & & & & & & & & & & & & & \\
\hline & & & & & & & & & & & & & & \\
\hline & & & & & & & & & & & & & & \\
\hline & & & & & & & & & & & & & & \\
\hline & & & & & & & & & & & & & & \\
\hline & & & & & & & & & & & & & & \\
\hline & & & & & & & & & & & & 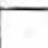 & & \\
\hline
\end{tabular}


Ship AsteRAAS Cruise $10-75$ Leg_Sta. 83 Core No. $10 \mathrm{GC}$ Total Length $23 \mathrm{~cm}$. Lat. $41^{\circ} 38.46^{\prime} \mathrm{N}$ Long. $70^{\circ} 55.08^{\prime}$ 'W Depth 2.7 meters Core condition Excelc Physiographic location ACUSHNET RIVER... NEW BEDFard HARBer

Physiographic location ACUSHNET RIVER ... NEW Belogic Detailed Description

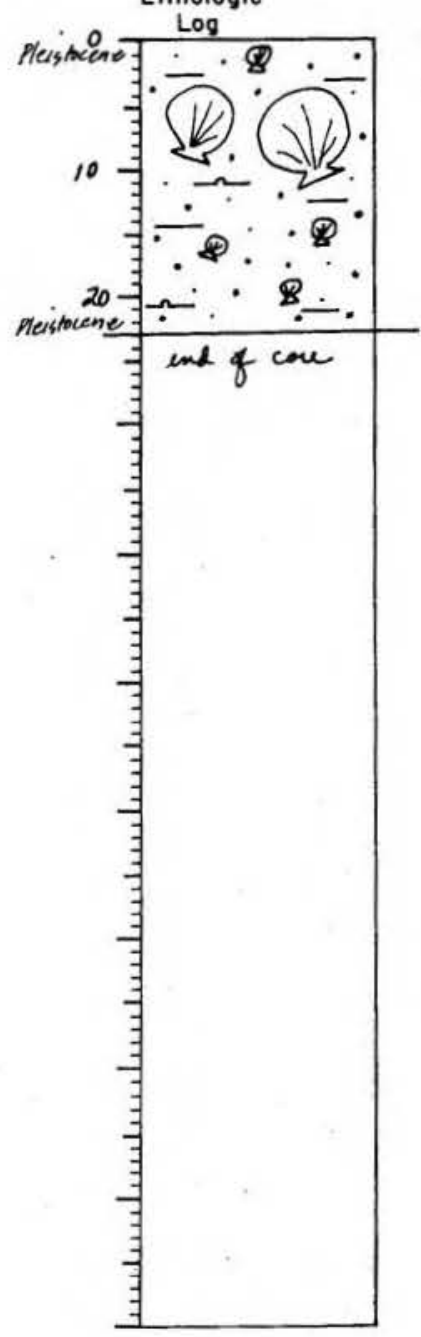

$0-23$

DETRITUS/SLIGHTLY SILIC CLAY

5 Y $3 / 2$ dark olive gray

fill

iner from $4-11 \mathrm{~cm}$

end of core

SMEAR SLIDE DESCRIPTIONS - W.H.O.I. SEDIMENT CORES

Ship:

Expedition

Leg No.

\begin{tabular}{|c|c|c|c|c|c|c|c|c|c|c|c|c|c|c|}
\hline \multirow[b]{3}{*}{ LEVEL } & \multirow[b]{3}{*}{$\begin{array}{c}\text { SEDIMENT } \\
\text { TYPE }\end{array}$} & \multicolumn{13}{|c|}{ ESTIMATED ABUNDANCES (\%) } \\
\hline & & \multicolumn{4}{|c|}{$\begin{array}{c}\text { Inorganic Material } \\
\text { Silt \& Sand }\end{array}$} & & \multicolumn{5}{|c|}{$\begin{array}{l}\text { Biogenous Mater } \\
\text { Calcareous }\end{array}$} & \multicolumn{3}{|c|}{ Siliceous } \\
\hline & & $\frac{5}{\frac{5}{5}}$ & 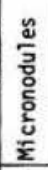 & 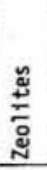 & 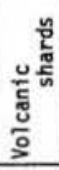 & $\frac{\pi}{6}$ & 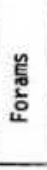 & $\begin{array}{l}\frac{n}{\bar{n}} \\
\frac{\hat{c}}{2} \\
\frac{c}{c} \\
\frac{\sigma}{z} \\
\end{array}$ & 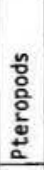 & 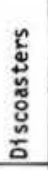 & 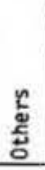 & 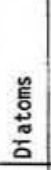 & $\frac{\pi}{\frac{\pi}{\pi}}$ & 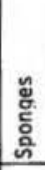 \\
\hline 1 & \begin{tabular}{l|} 
detritus/ \\
slightly silif
\end{tabular} & 66 & & & & 30 & & & & & tr & 2 & & 2 \\
\hline & & & & & & & & & & & & & & \\
\hline 22 & \begin{tabular}{|l|} 
detritus/ \\
sisghtly sili
\end{tabular} & 76 & & & & 20 & & & & & tr & $\mathrm{tr}$ & tr & 4 \\
\hline & & & & & & & & & & & & & & \\
\hline & & & & & & & & & & & & & & \\
\hline & & & & & & & & & & & & & & \\
\hline & & & & & & & & & & & & & & \\
\hline & & & & & & & & & & & & & & \\
\hline & & & & & & & & & & & & & & \\
\hline & & & & & & & & & & & & & & \\
\hline & & & & & & & & & & & & & & \\
\hline & & & & & & & & & & & & & & \\
\hline & & & & & & & & & & & & & & \\
\hline & & & & & & & & & & & & & & \\
\hline & & & & & & & & & & & & & & \\
\hline & & & & & & & & & & & & & & \\
\hline & & & & & & & & & & & & & & \\
\hline & & & & & & & & & & & & & & \\
\hline
\end{tabular}

Core No. $10 \mathrm{GC}$

Station No. 83

(3)

SSTIMATTD ABUNDANCES (6) 
VISUAL CORE DESCRIPTION

Page 1 of 1

Ship AsTERIAS Cruise 10-75 Lto 84 Core No. $11 \mathrm{GC}$ Total Length $123 \mathrm{~cm}$. Lot. $41^{\circ} 38.63^{\prime} \mathrm{N}$ Long. $70^{\circ} 54.79^{\prime} \mathrm{W}$ Depth 2.7 meter Core condition EXCELLENT Date Described 10 Jwne 76 by H. Former Physiographic location ACUSHNET RIVER - NEW BEDFORd HARBOR

Lithologic

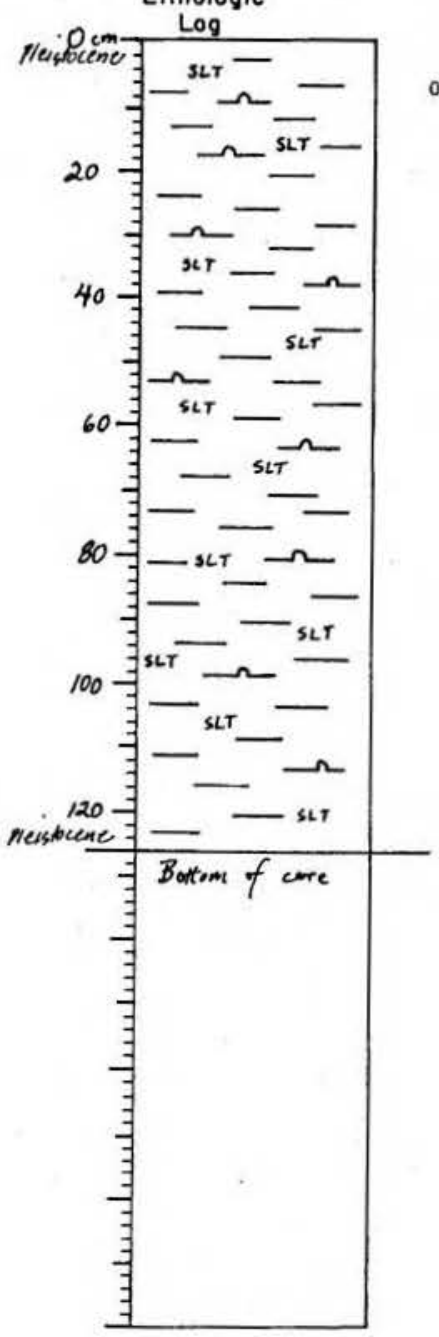

\section{Detailed Description}

$0-123$

SILIC CLAY WITH DETRITUS GRADES TO SILIC CIAY/ DETRITUS

S $X / 2$ dark olive gray - below oxidized surface lap color fairly homogeneous excepting small to medium shell In the interval $15-53 \mathrm{~cm}$

relatively soft, fairly silty lutite, becomes some-
what sandier in basal $15 \mathrm{~cm}$, pebble at $88 \mathrm{~cm}$

end of core
098

SIEAR SLIDE DESCRIPTIONS - W.H.O.I. SEDIMENT CORES

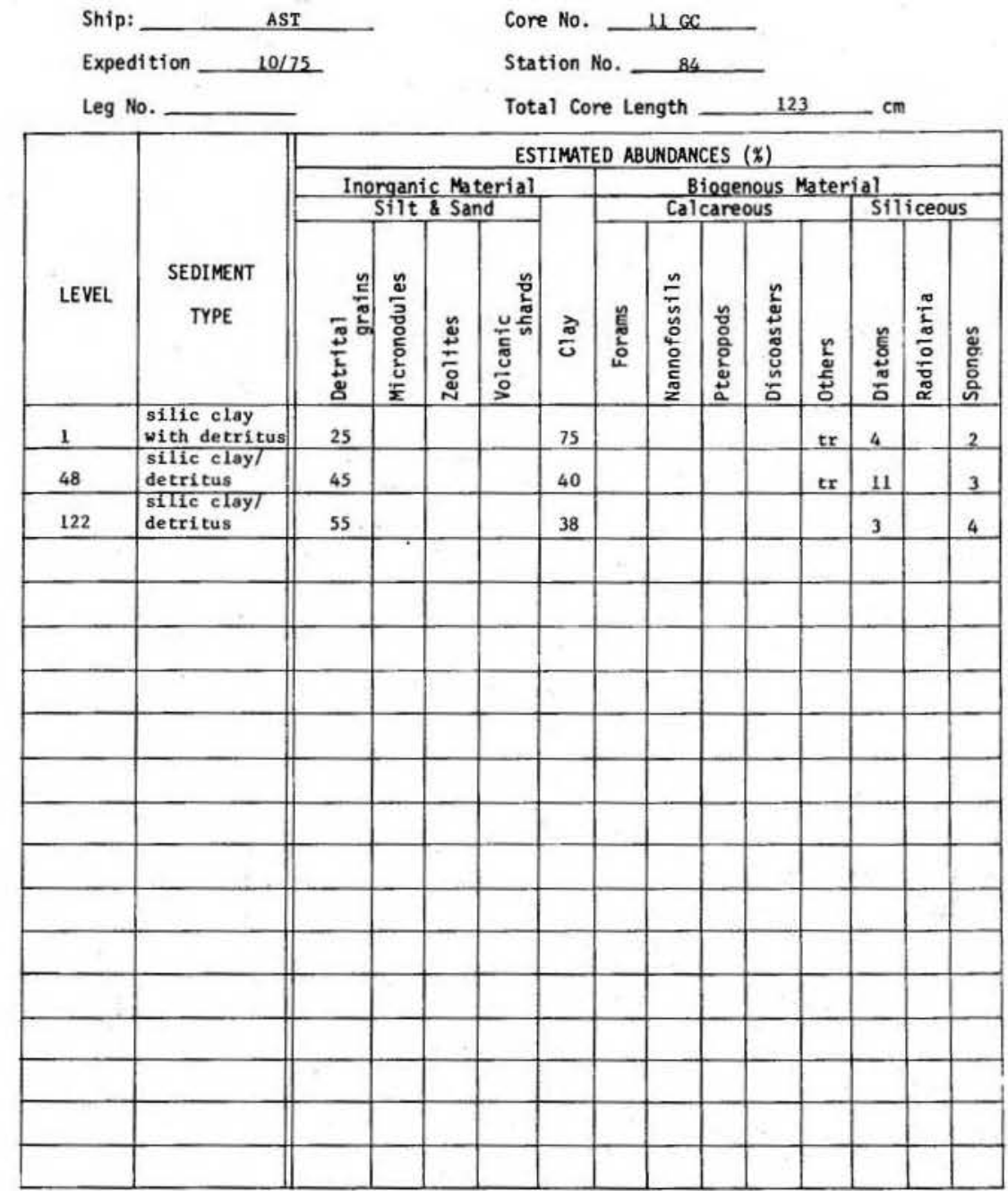


Ship Astersas Cruise 10-75 Leg Sto. 85 Core No. $12 \mathrm{GC}$ Total Length $39 \mathrm{~cm}$. Lat. $41^{\circ} 38.92^{\prime} \mathrm{N}$ Long.70 $55.24^{\prime} \mathrm{W}$ Depth 5.2 meter Core condition ExcEWENT Date Described IIJuex 76 by H. Famer Physiographic location AcusaneT Harsie... NGW BEDFare Marsar

Lithologic

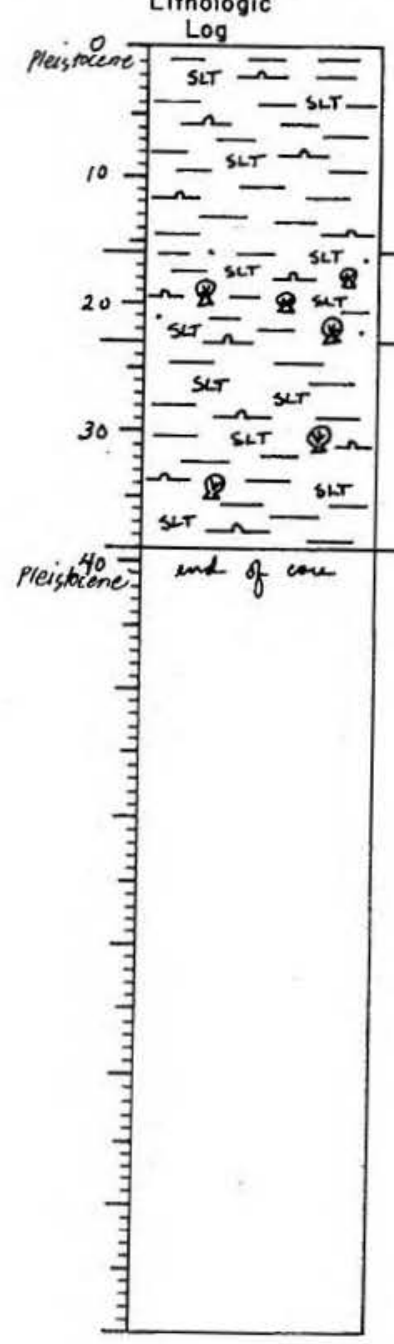

Detailed Description

$0-16$

SILIC CLAY WITH DETRITUS

a distinct rusty red hue is apparent in the top $5 \mathrm{~cm}$ and becomes in the unit

the oxidized surface layer described above, arediment is very dark brown to black subtle $y$ onc-like, falrly silty lutite 16-23. (S, inclined) SILIC CLAY/DETRITUS

again, the exposed surface sediment is someuha 1 ighter colored soft, mulchy, silty lutite with numerous pelecypod S 39 $s^{\text {shell }}$

SILIC CLAY/DETRITU

5 Y $3 / 2$ dark olíve gray; very dark gray below oxidized surface homogeneous excepting scattered pelecypods sorewhat mulchy, silty lutite

end of core

SHEAR SLIDE DESCRIPTIONS - W.H.O.I, SEDIMENT CORES

Ship: AST Expedition $10 / 75$

Leg No.

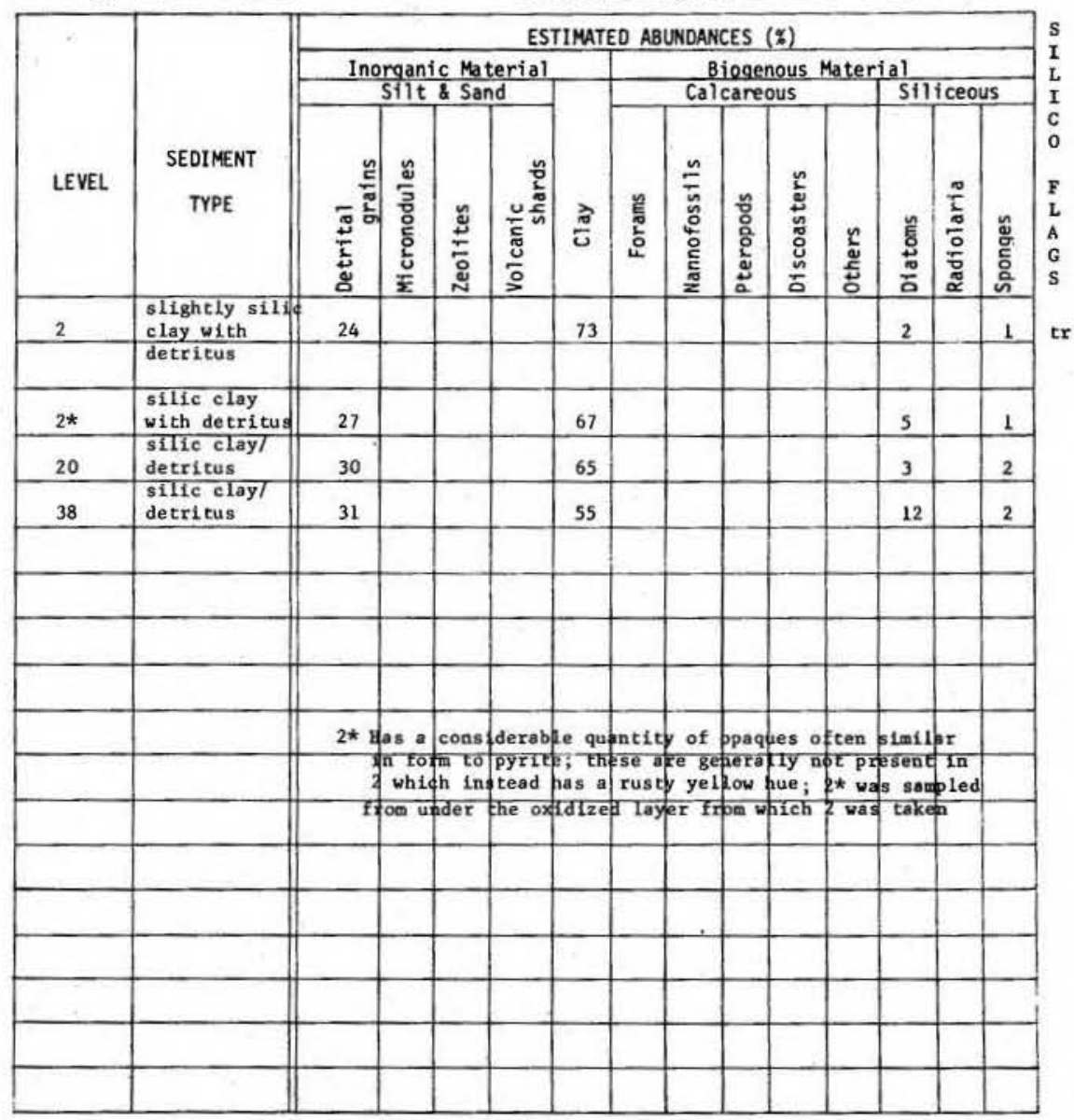

Core No. $12 \mathrm{GC}$

Station No. 85

Total Core Length $\quad 39 \quad \mathrm{~cm}$ 
Ship AstesuAs Cruise $10-75$ Leg_Sto. 86 Core No. $136 \mathrm{C}$ Total Length $63 \mathrm{~cm}$. Lat. $41^{\circ} 38.73^{\prime} \mathrm{N}$ Long. $20^{\circ} 55.28^{\prime}$ wDepth $10.9 \mathrm{metung}$ Core condition Excallent Date Described II Jure 76 by H. Famer Physiographic location ACUSHNET RIVER - NEW BEDFORD HAREOR Lithologic

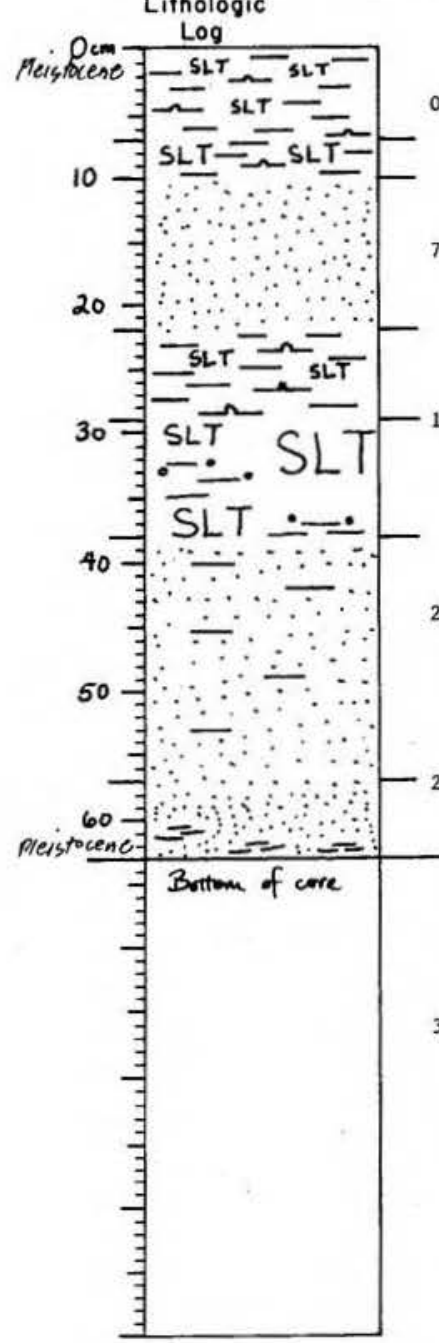

Detailed Description

\section{SILIC CLAY/DETRITUS}

YR $3 / 2$ very dark grayish brown

black moteling with $\mathrm{H}_{2} \mathrm{~S}$ odor below exposed surface $10^{\mathrm{s}}$

SLIGHTLY SILIC CLAY/DETRITUS

SLIGHTLY SILIC CLAY/DETRITUS $4 / 4$ dark yellowish brown, gray to dark gray

below surface layer

marbling of yellow browns is extensive

somewhat spongy, quite silty lutite S

DETRITUS

10 YR $4 / 2$ grayish brown, gray below surface layer

rayish brown and dark yellowish browns: $13-22 \mathrm{~cm}$

$\mathrm{s}$, inclined and somentat distorte

$2-29$

ILIC CLAY WITH DETRITUS

5 Y $3 / 2$ dark olive gray, black below oxidized surface frasments

mulehy, spongy, fairly silty lutite $\mathrm{s}$, very horizonta

DETRITUS/UNFOSSILIFEROUS CLAY

\section{Y $5 / 1$ gray}

YR $5 / 1$ gray brown, mulchy lutite at $32-35 \mathrm{~cm}$ and $37-38 \mathrm{~cm}$;

between these 1s a large, dark gray discoloration quite firm silt excepting the mulchy lutite clasts and a number of very small pebbles that seen to be assos, horizontal

38-57,

.5 Y $6 / 2$ light brownish gray

aclined laminations (about $15^{\circ}$ but slope opposite to laminations in unit \#3 above) of 7.5 YR $5 / 6$ strong

compact, coarse silt except laminations (38-48 cm) which are fine sand, laminations from $48-5$,
fine and texturally indistinguishable

$\mathrm{S}$, very disturbed
Ship ASTERIAS Cruise $10-75$ Leg_ Sta. 86 Core No. $13 \mathrm{GC}$
Lithologic
Detailed Description
Log

57-63

DETRTTUS

$10 \mathrm{YR} S / 2$ grayish brown browner lutite clasts and emphasizes the very dis-

very firm, medium sand with irregular clasts of

end of core 
SMEAR SLIDE DESCRIPTIONS - W.H.O.I. SEDIMENT CORES

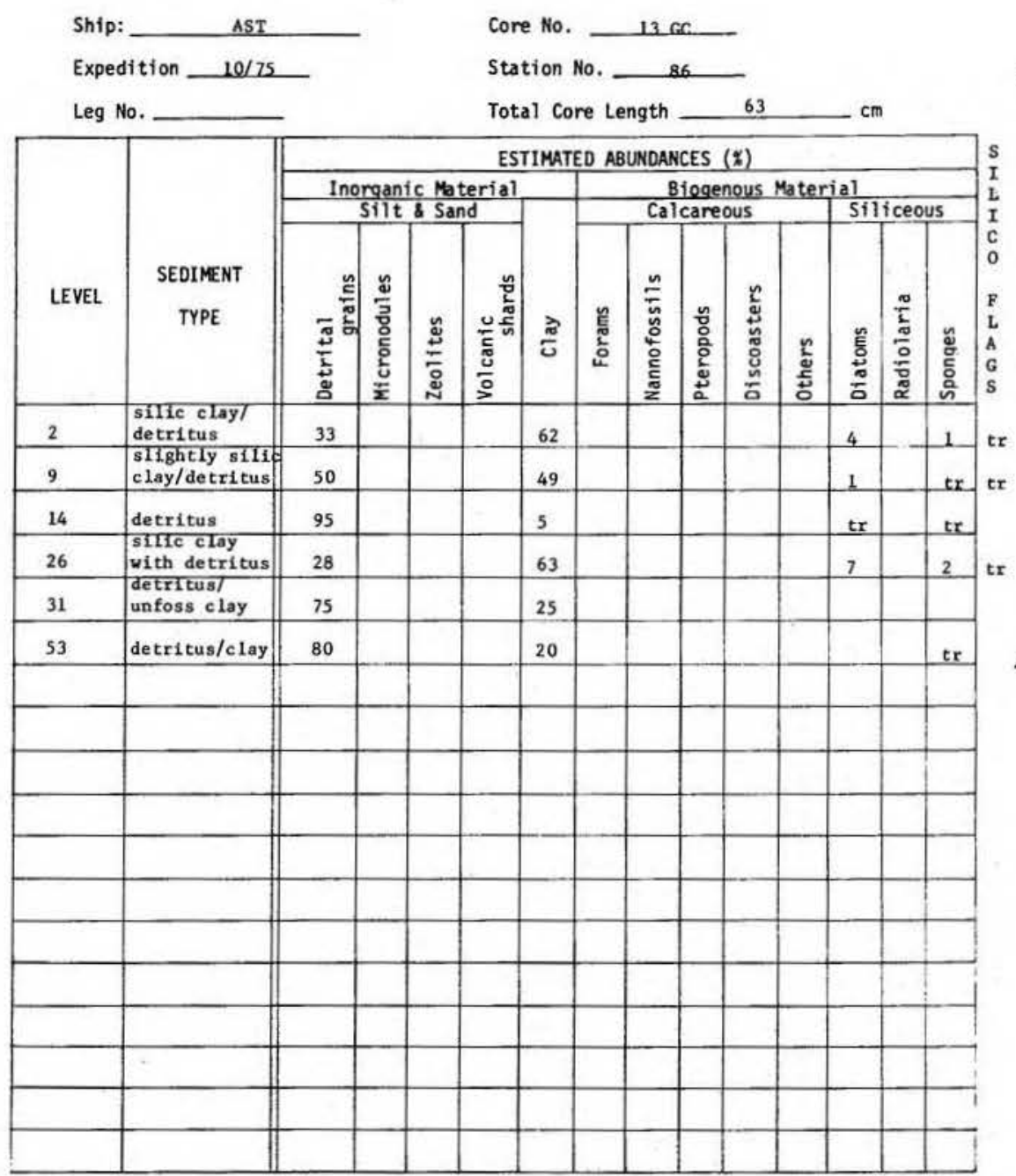

Ship Astexuns Cruise 10-75 Leg_L Sta. 87 Core No. 14 GC Total Length $52 \mathrm{~cm}$. Lot. 开 $38.45^{\prime} \mathrm{N}$ Long. $20^{\circ} 55.22^{\prime}$ W Depth 10 metars Core condition EXCELLENT Date Described"J Juve $7 / 6$ by H. EARenes Physiogrophic location Acusmet River... New Bej Fore Harsor Lithologic

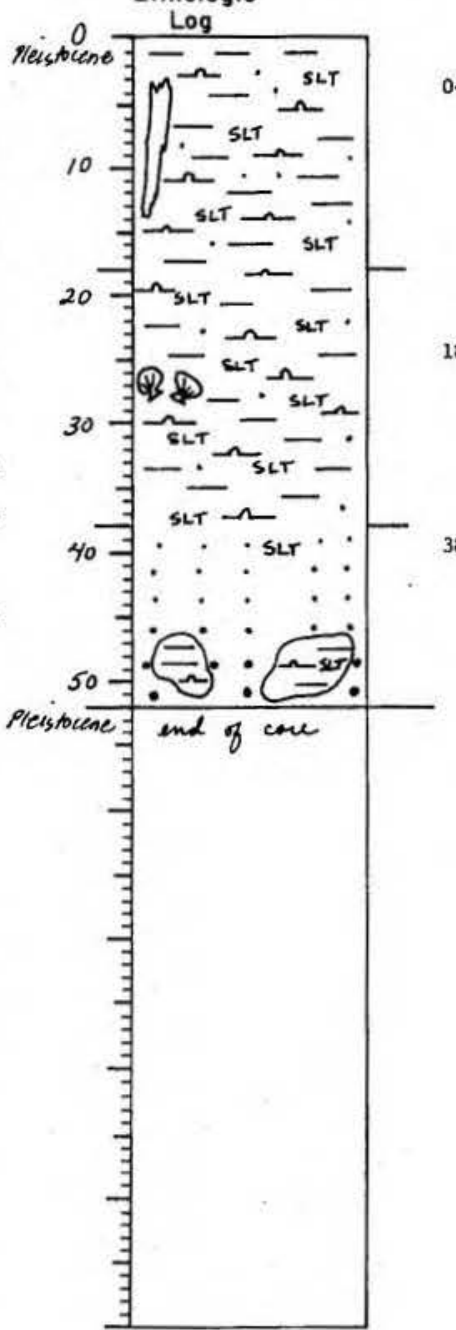

Detailed Description

$0-18$

SIIIC CIAY WITH DETRITUS

10 YR $3 / 2$ very dark grayish brown, black with $\mathrm{H}_{2} \mathrm{~S}$

odor under oxidized surface

subtle rusty brownish oxidation is generally present throughout, but especially notable in a Meuhat spongy, silty lutite

long fragment of wood rests along edge of core from $3-14 \mathrm{~cm}$

8- 38

SILIC CLAY WITH DETRITUS

5 Y $3 / 2$ dark olive gray, black with $\mathrm{H}_{2} \mathrm{~S}$ odor under

shell fragments at $28 \mathrm{~cm}$ 38-52

DRTRITUS/SLIGHTLY SILIC CLAY

10 YR $4 / 1$ dark gray, pales a bit with depth,

sliehtly darker under surface sedfinent

laminations of very dark grayish brown and dark brown are present in top $5 \mathrm{~cm}$; large, dark olive gray clasts appear in basal $6 \mathrm{~cm}$

very fine sand grades to coarse sand; below $46 \mathrm{~cm}$ a couple large $(4 \mathrm{~cm})$, silty lutite clasts are cm: small fragment of

end of core 
SIEAR SLIDE DESCRIPTIONS - W.H.O.I. SEDIVENT CORES

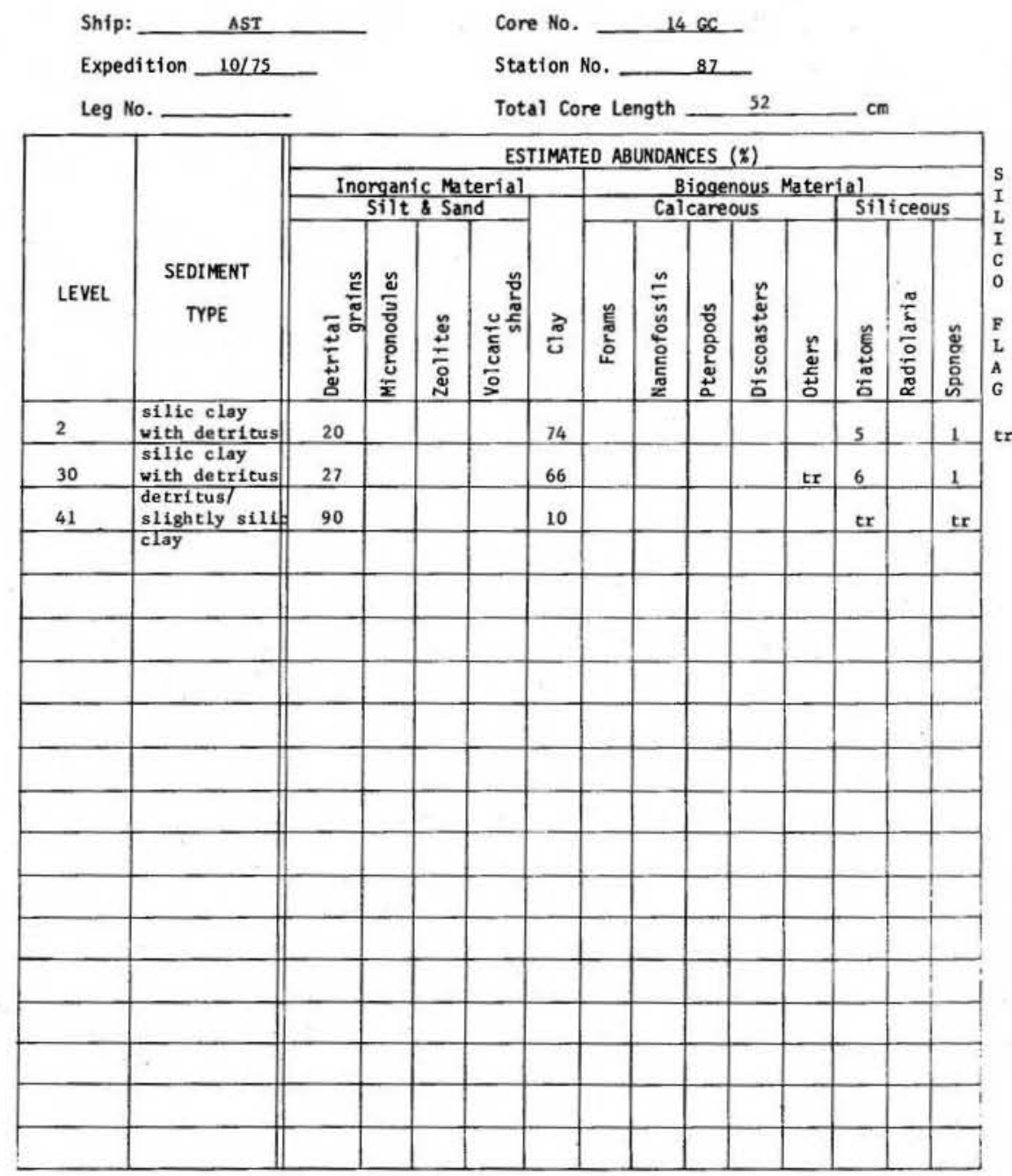

VISUAL CORE DESCRIPTION

Page 1 of 1

Ship AsteR/nts Cruise 10-75 Leg_Sto. 92 Core No. $16-\mathrm{GC}$

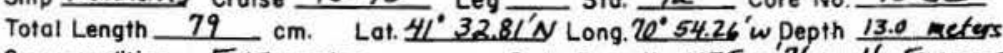
Core condition EXCELCENT Date Described 15 Jume 76 by H. Former Physiographic location BuzZARD's BAY APPROACHES TO NEW BEDFRD HREBR Lithologic

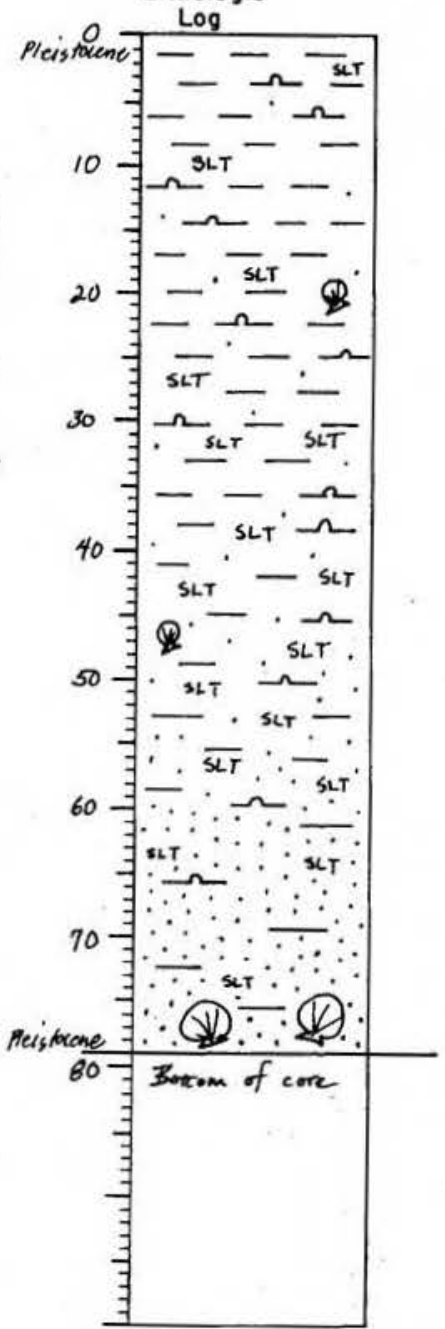

$0-79$ Detailed Description $5 \times 3 / 2$ dark

no mottling, but fine, scattered biotite(?) fragments glitter on the sediment surface quite silty/sandy lutite grades to a fine-
medium sand below $55 \mathrm{~cm}$; a few shell fragments are scattered throughout the core and a couple larger pieces sit at the bottom; $46 \mathrm{~cm}-\mathrm{sma} 11$, rounded pebble end of core 
SIEAR SLIDE DESCRIPTIONS - W.H.O.I. SEDIMENT CORES

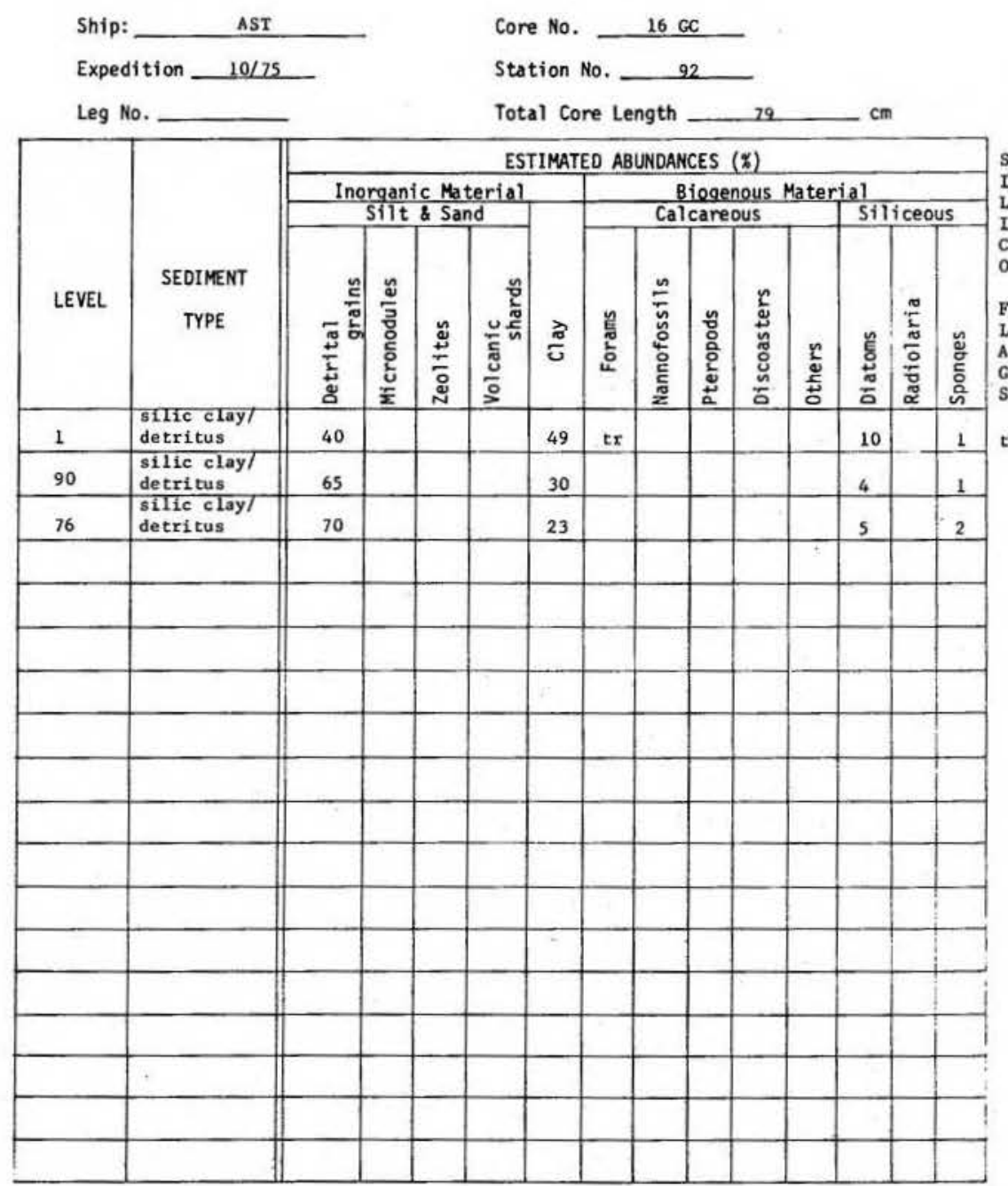

VISUAL CORE DESCRIPTION

Page 1 of 1

Ship AGERAS Cruise 10-75 Leg - Sto 97 Core No. $176 \mathrm{C}$

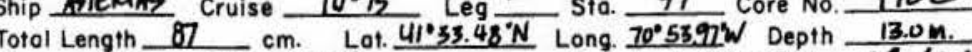
Core condition EXELUENT Lot. $41033.48 \mathrm{~N}$ Long. $70^{\circ} 53.97 \mathrm{~W}$ Depth $\frac{13.0 \mathrm{~h} \text {. }}{\text { f }}$

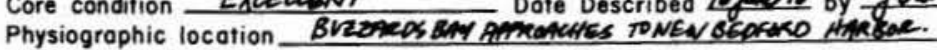

Lithologic

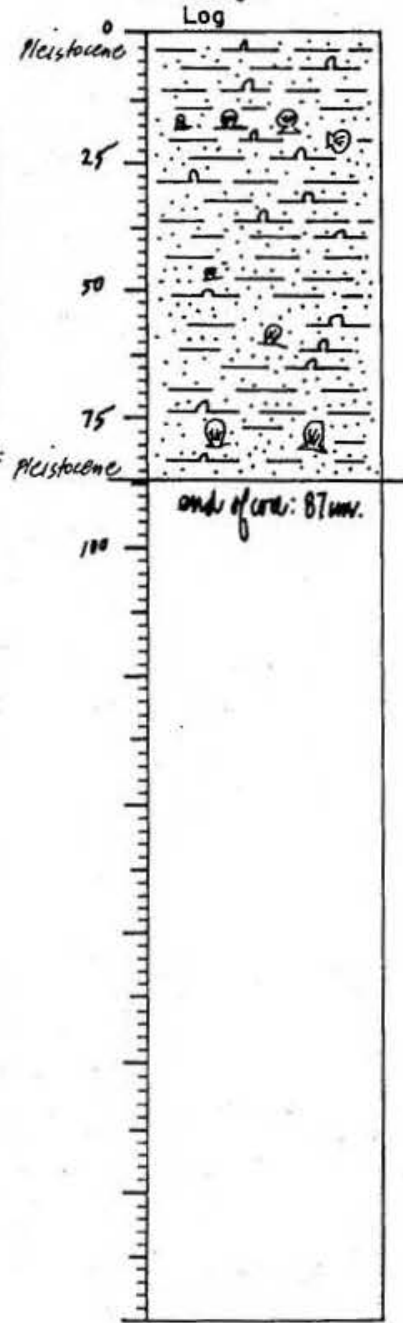

Detoiled Description

$0-87$

DETRITUS/SILIC CLAY

× $4 / 2$ olive gray core is homogeneous throughout

fims, quite silty-sandy lutite with consiscent texture throughout: a few inclusion of pelecypod fragments are found $16-22 \mathrm{~cm}$, creptide crepidula shells are at $38-60$ and $81-83 \mathrm{~cm}$

end of core 
SIEAR SLIDE DESCRIPTIONS - W.H.O.I. SEDIEENT CORES

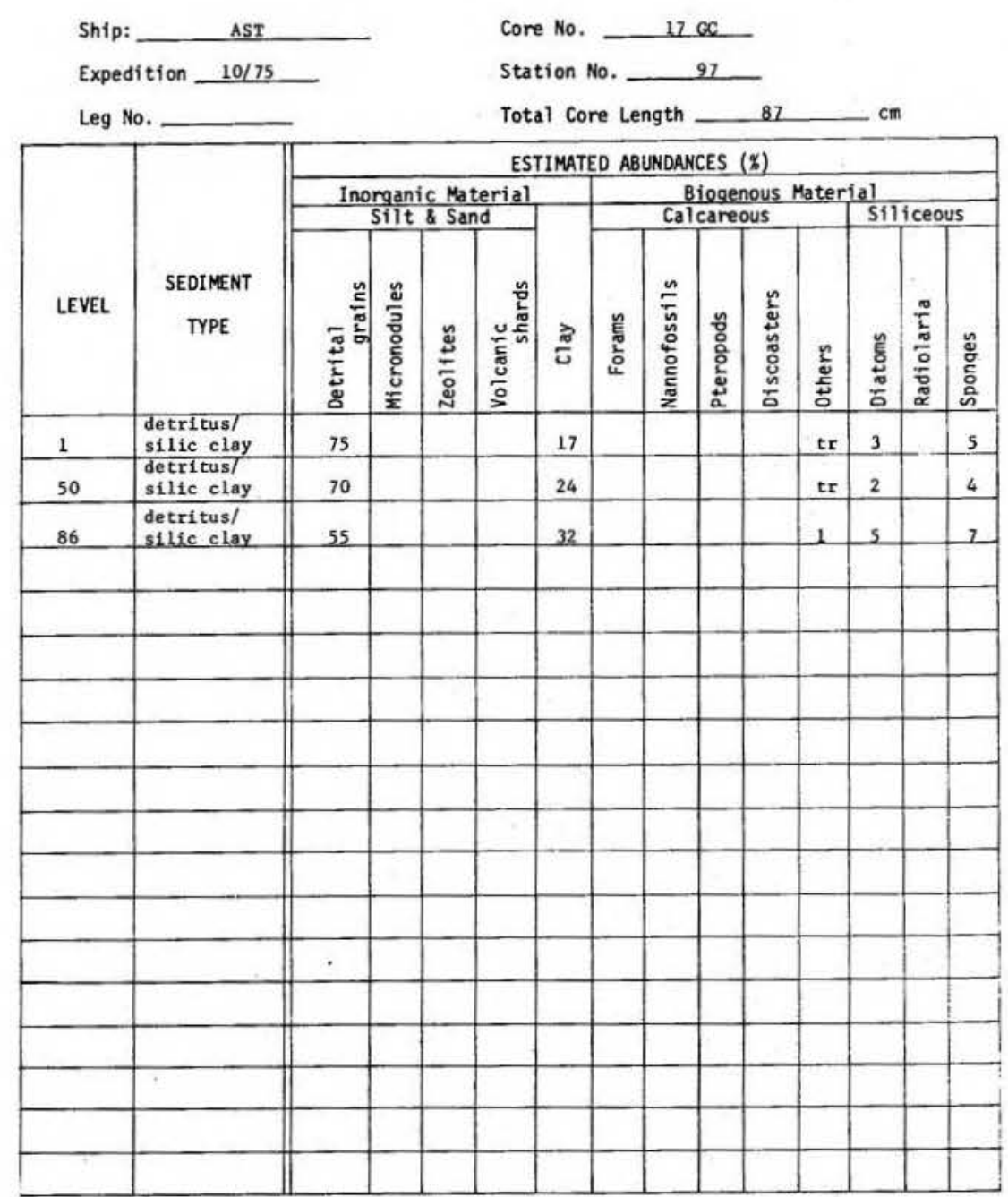

110

VISUAL CORE DESCRIPTION

Page 1 of 1 Ship Cruise 10.75 Leg - Sto 99 Core No. $186 \mathrm{C}$

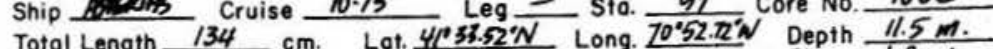

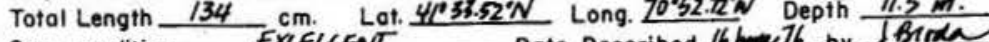
Core condition EXEELENT Date Described 16ye 76 by fBR Physiogrophic location BVZZMEAS BMY MPPRAACHES TO NEW REO HORO HAR BOR. Lithologic

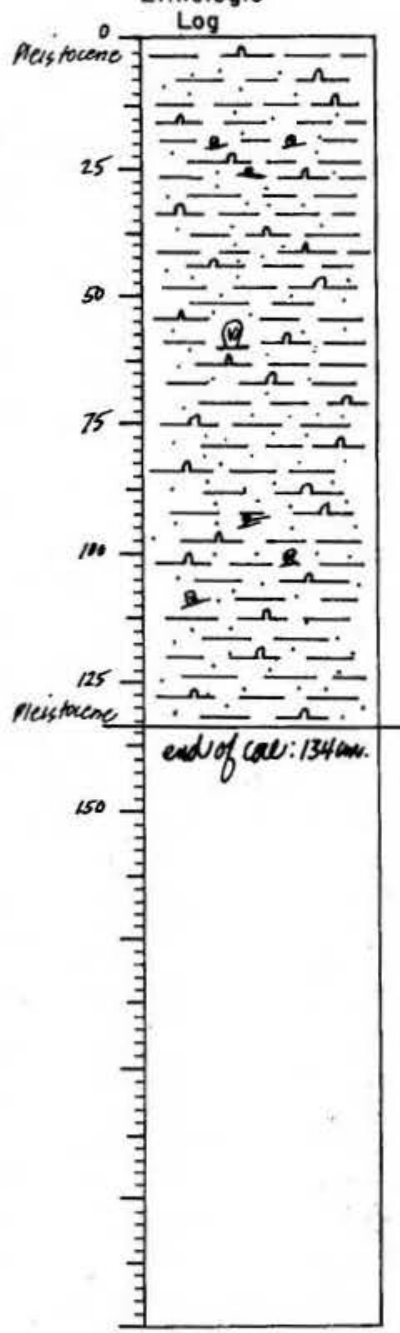

$0-134$

$5 \times 4 / 2$ dark olive gray alteration) $13-23 \mathrm{~cm}$ (faint), $29-50 \mathrm{~cm}$, 75-77 cm, 89-92 cm

very silty lutite with scattered sand grains and shell fragments plus an extremely wellpreserved pteropod at $58 \mathrm{~cm}$

end of core several nebulous, irregular, yellowish 
SMEAR SLIDE DESCRIPTIONS - W.H.0.I. SEDIMENT CORES

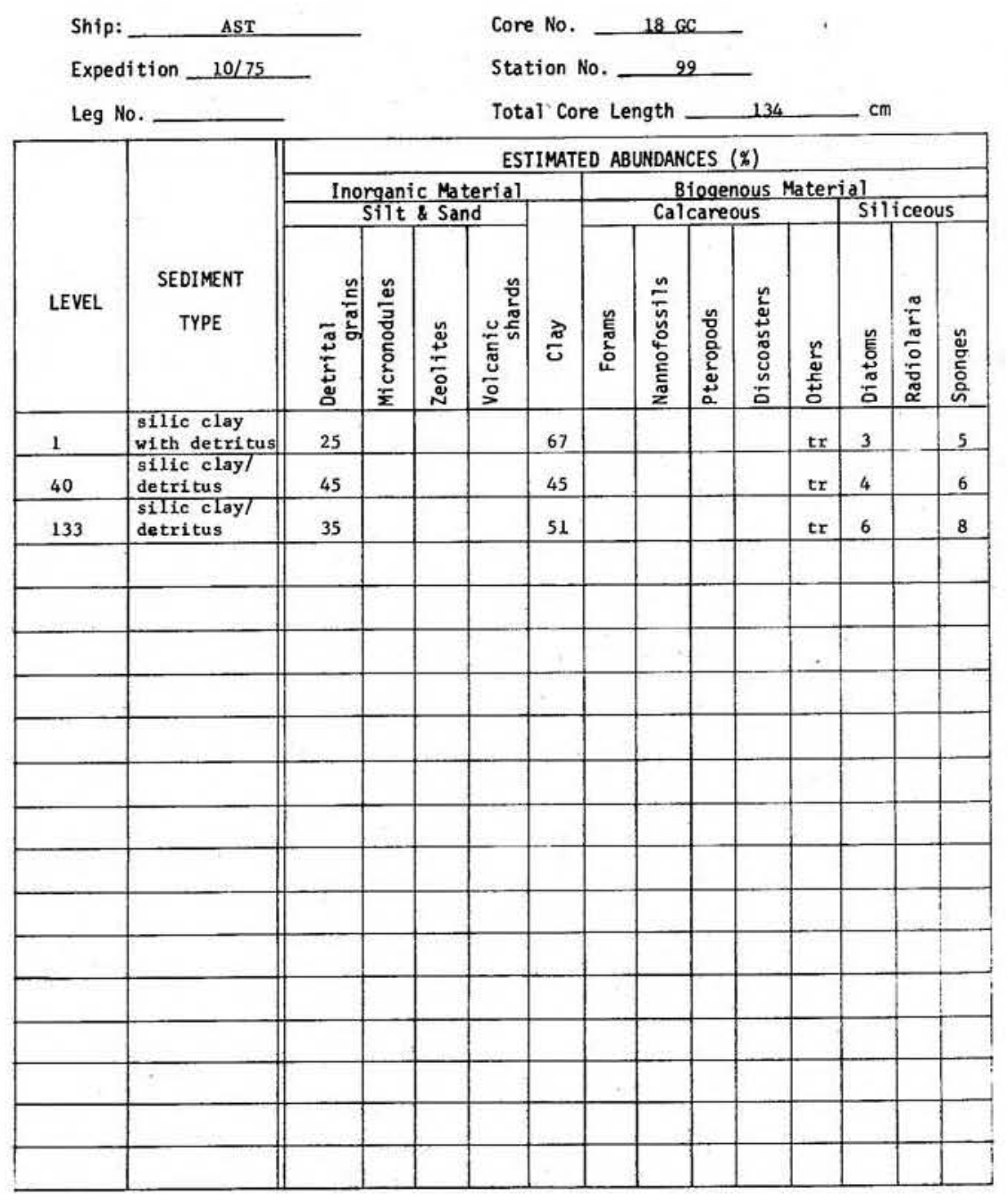

Ship ASTER/AS Cruise 10-75 Leg - Sta. 100 Core No. $19 \mathrm{GC}$ Total Length 94 Lat. $41053.96^{\circ} \mathrm{M}$. $\frac{100}{70^{\circ} 52.09^{2} \mathrm{~V}}$ Depth $11.5 \mathrm{~m}$ Core condition EXCELEN T Dot Described 17 RNEIb by fbunder

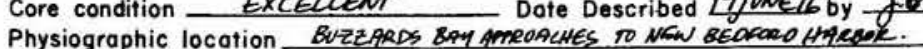
Physiographic location BUZZZRDS EPY AmROACHES TO NEW
Lithologic

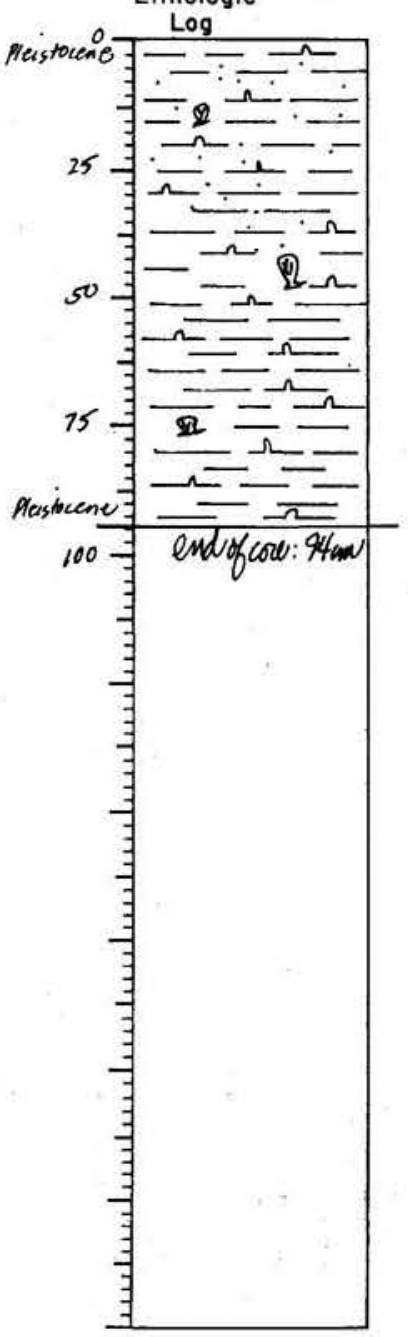

$0-94$ SILIC CIAY/DETRITUS except for occasional shell fragments and faint, rusty (post-coring) zones, core

irm, very silty lutite with varying amounts creasing with depth, but occasionally concentrated in lenses or pockets $20-60 \mathrm{~cm}$

end of core 
SUEAR SLIDE DESCRIPTIONS - W.H.O.I. SEDIEENT CORES

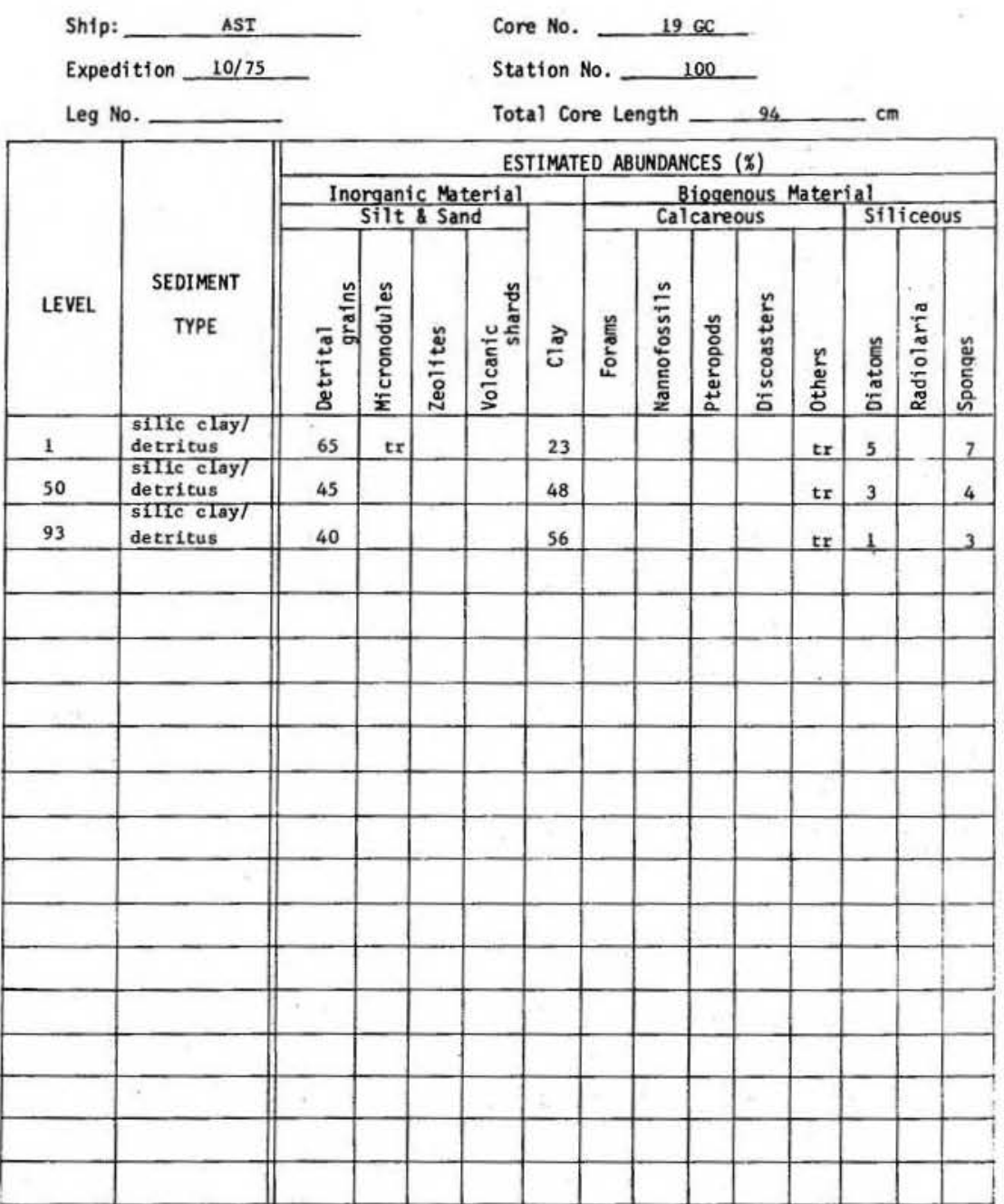

VISUAL CORE DESCRIPTION

Page 1 of 1

Ship Astresas Cruise 7.76 Leg - Sta. 108 Core No. $20 \mathrm{GC}$ Total Length $140 \mathrm{~cm}$. Lat. $41^{\circ} 32 \mathrm{~N}$ Long. $70^{\circ} 46.25^{\prime} \mathrm{W}$ Depth $14.5 \mathrm{Neth}$ Core condition EXCELLENT Date Described 12 Nov 76 by Af. FAermex Physiographic location Buzzerd's Bay epproseles to New Bedford Herbor

Lithologic

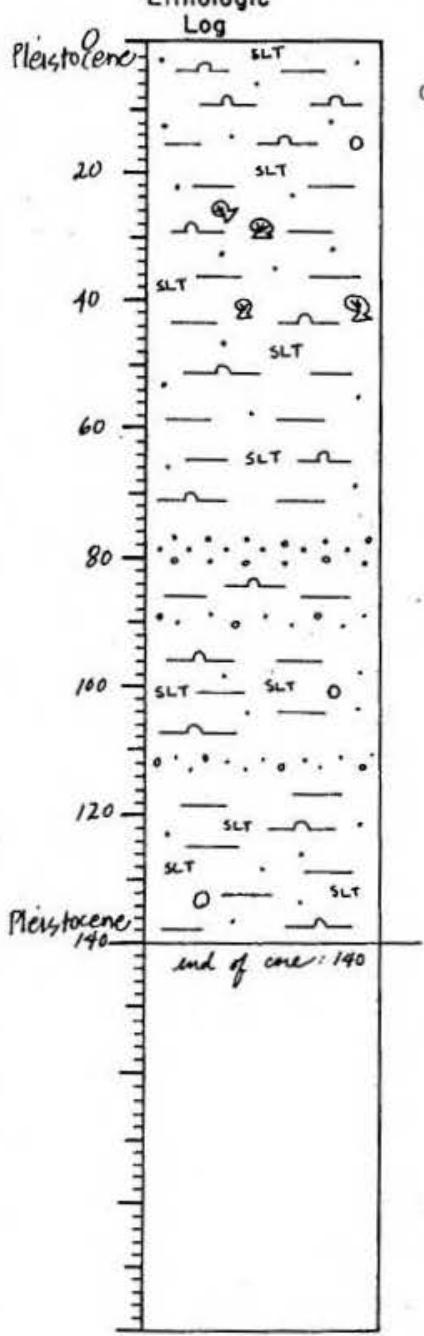

Detailed Description

$0-140$

SILIC CLAY/DETRITUS

5 Y $4 / 1$ dark gray, slightly darker below oxidized

quite homogeneous except for scattered, white bivalves from $28-30$ and $41-45 \mathrm{~cm}$ tions at 79,89 and $112 \mathrm{~cm}$ : occasional small pebbles

end of core 
115

SMEAR SLIDE DESCRIPTIONS - W.H.O.I. SEDIMENT CORES

Ship: $\frac{\text { ASTERIAS }}{\text { Expedition } \quad 7-76}$

Leg No.

\begin{tabular}{|c|c|c|c|c|c|c|c|c|c|c|c|c|c|c|}
\hline \multirow[b]{3}{*}{ LEVEL } & \multirow[b]{3}{*}{$\begin{array}{c}\text { SEDIMENT } \\
\text { TYPE }\end{array}$} & \multicolumn{13}{|c|}{ ESTIMATED ABUNDANCES ( $(q)$} \\
\hline & & \multicolumn{4}{|c|}{\begin{tabular}{|c} 
Inorganic Material \\
Silt \& Sand
\end{tabular}} & \multirow[b]{2}{*}{ 齐 } & \multicolumn{5}{|c|}{$\begin{array}{l}\text { Biegenous Mater } \\
\text { Calcareous }\end{array}$} & \multicolumn{3}{|c|}{ ial } \\
\hline & & 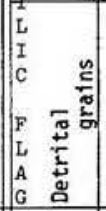 & 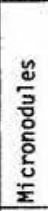 & * & 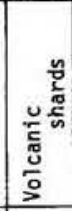 & & 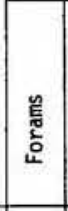 & 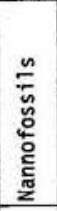 & 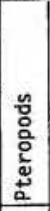 & 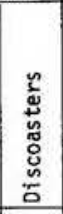 & 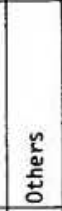 & 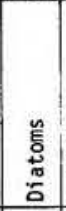 & $\left|\begin{array}{c}\frac{\pi}{2} \\
\frac{\pi}{0} \\
\tilde{0} \\
\tilde{\sigma} \\
\tilde{\alpha}\end{array}\right|$ & 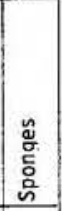 \\
\hline $1 \mathrm{~cm}$ & $\begin{array}{l}\text { silic clay } \\
\text { ith detritus }\end{array}$ & $\operatorname{tr} 30$ & & tr & & 60 & $t r$ & & & & & 5 & tr & 2 \\
\hline 70 & $\begin{array}{l}\text { silic clay/ } \\
\text { detritus }\end{array}$ & $\operatorname{tr} \quad 40$ & & & & 43 & & & & & & 13. & tr & 1 \\
\hline 139 & $\begin{array}{l}\text { silic clay/ } \\
\text { detritus }\end{array}$ & 47 & & & & 40 & & & & & & 2 & & \\
\hline & & & & & & & & & & & & & & \\
\hline & & & & $*_{\mathrm{ir}}$ & ndustr & Lal $f$ & twers. & asb & sto & (2) & & & & \\
\hline & & & & & & & & & & & . & & & \\
\hline & & & & & & & & & & & & & & \\
\hline & & & & & & & & & & & & & & \\
\hline & & & & & & & & & & & & & & \\
\hline & & & & & & & & & & & & & & \\
\hline & & & & & & & & & & & & & & \\
\hline & & & & & & & & & & & & & & \\
\hline & & & & & & & & & & & & & & \\
\hline & & & & & & & & & & & & & & \\
\hline & & & & & & & & & & & & & & \\
\hline & & & & & & & & & & & & & & \\
\hline & & & & & & & & & & & & & & \\
\hline & & & & & & & & & & & & & & \\
\hline
\end{tabular}

\section{6}

VISUAL CORE DESCRIPTION

Page 1 of 1

Ship Asterias Cruise 7.76 Leg - Sto. 109 Core No. 21-GC Ship $32.5 \%$ N Long. $20^{\circ} 54.14^{\prime} \mathrm{w}$ Depth 15.4 meters

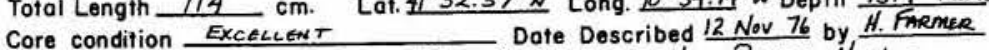
Core condition EXCELLENT Lithologic

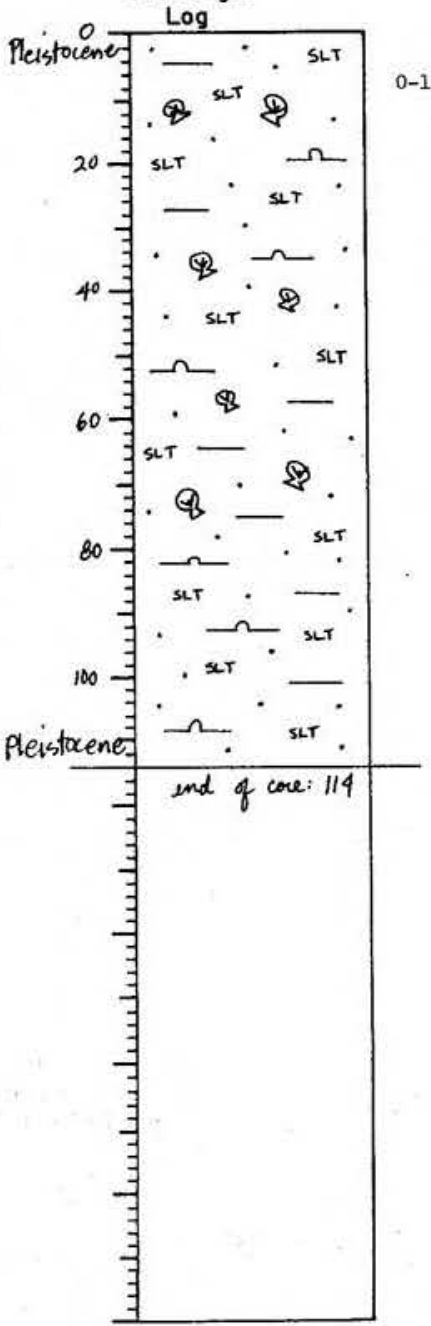

Detailed Description

$0-114$ CLAY/DETRITUS Y $4 / 1$ dark gray, slightly darker below oxidized surface throughout; also, scattered bivalve fragments are
present in the following intervals; $7-17,34-46$, nd $59-80 \mathrm{~cm}$

very silty lutite with an occasional pebble

end of core mottling, but tiny black flecks are abundant 
SMEAR SLIDE DESCRIPTIONS - W.H.O.I. SEDIMENT CORES

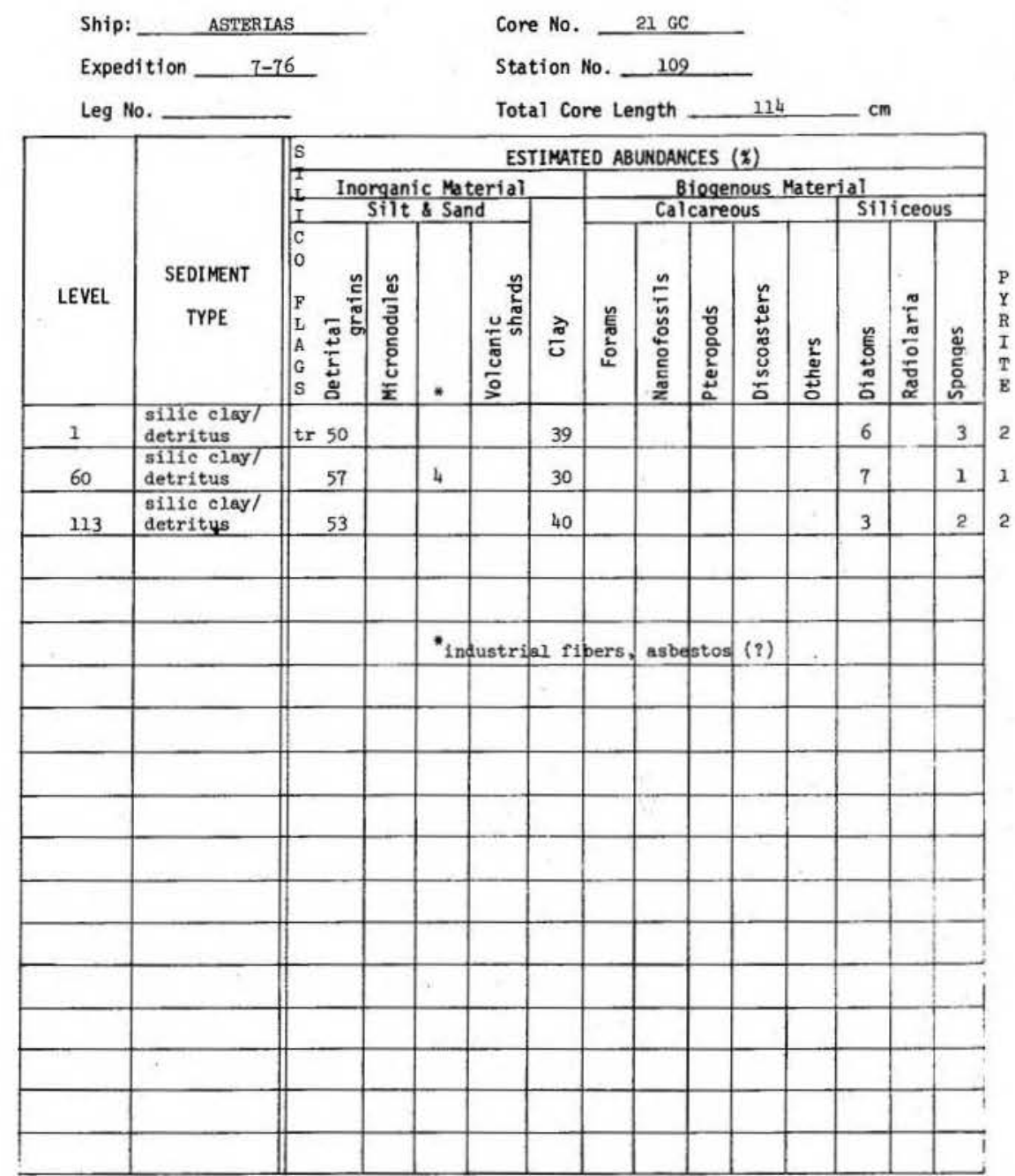

VISUAL CORE DESCRIPTION

Page 1 of 1

Ship Astreias Cruise 9.76 - Sta. 110 Core No. 22-GC

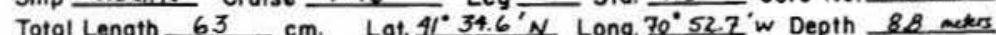
Core condition ExCELLENT 11 Date Described 12 Nov 76 by H. Fremss.

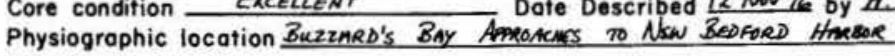

Lithologic

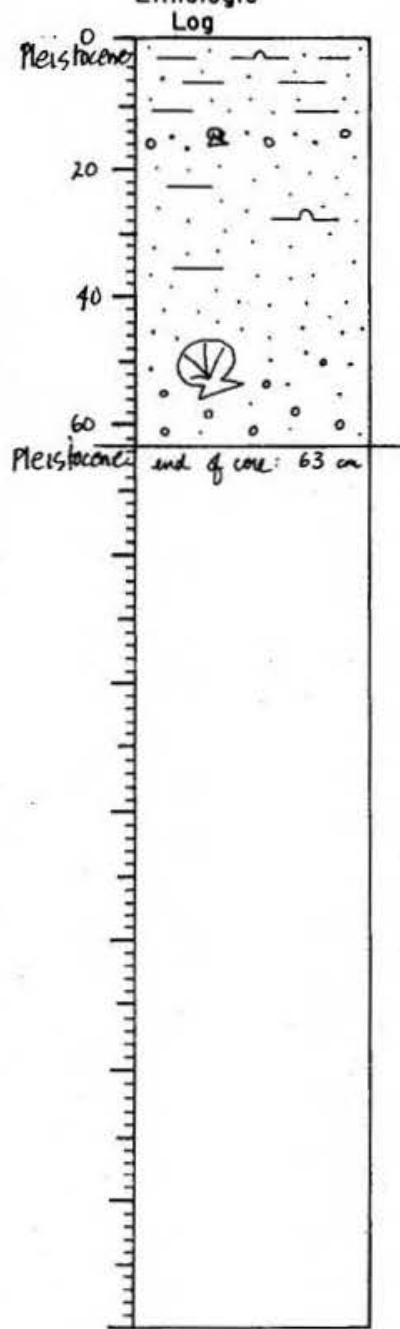

\section{Detailed Description}

$0-63$

5 Y $2.5 / 2$ black oxidizes to $3 / 2$ dark olive gray no mottling

very silty/sandy 1 ut ( 10 cin); coarse sand, pebbles, and shell rragto small bravel in lower $20 \mathrm{~cm}$; several large ors ter shells appear in this same interval

end of core 
SIEAR SLIDE DESCRIPTIONS - W.H.O.I. SEDIMENT CORES

Ship: ___ ASTERIAS

Expedition $7-76$

Leg No.

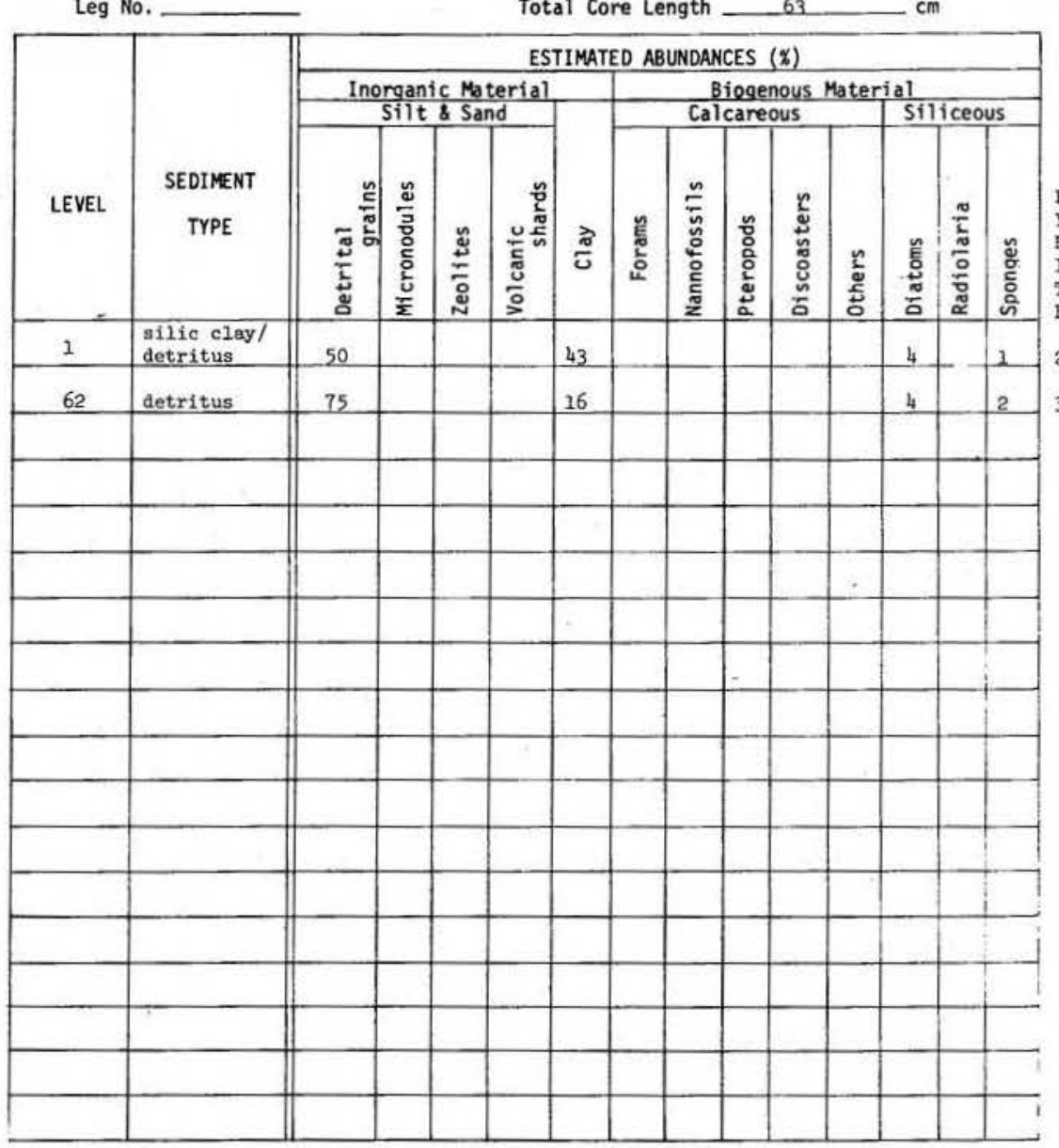

Core No. $22 \mathrm{GC}$

Station No. 210

Total core length \begin{tabular}{l|l|l} 
Siliceous & \\
\end{tabular} $\underset{\mathrm{Y}}{\mathrm{Y}}$ Pleis Pleistorent

Ship AstrRiAs Cruise 7.76 Leg - Sto. 111 Core No. 23.6C Tolol Length 38 rom

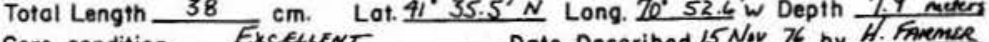

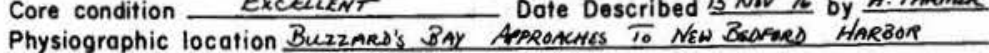
Lithologic Detailed Description

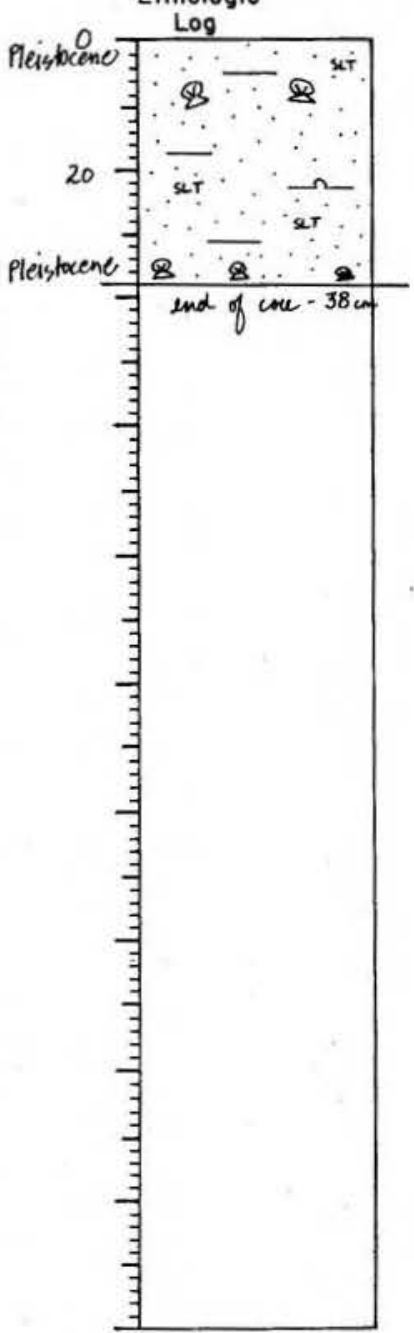

$-38$

5 Y $2.5 / 2$ black, oxidized surface layer is $3 / 2$ dark olive gray

no mottling

bivalve frearse sand vith jelte matrix; a sev bile numerous pieces are found in the basal $2 \mathrm{~cm}$

end of core 
SMEAR SLIDE OESCRIPTIONS - W.H.O.I. SEDIMENT CORES

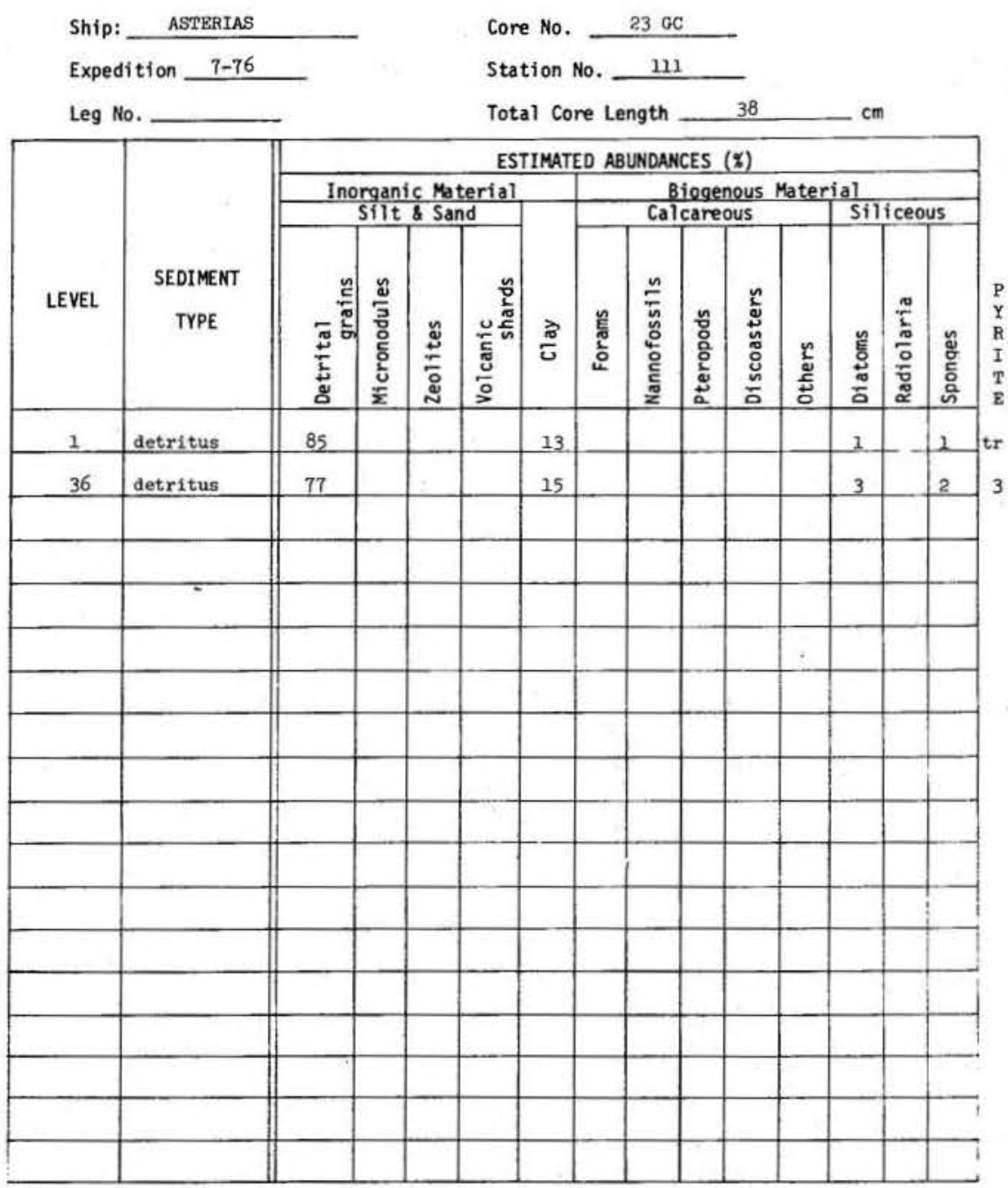

VISUAL CORE DESCRIPTION

Page 1 of 1

Ship A TrenAs Cruise 7.76 - sto 113 Core No. 25 QC Lat. $41^{\circ} 34.9^{\circ} \mathrm{N}$ Long. $70^{\circ} 53.6^{\prime} \mathrm{W}$ Depth 8.8 meters

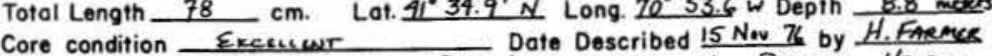

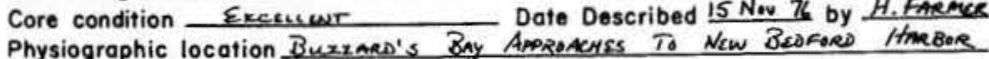

Physiographic location Buzzand's Bay AapzoAcHEs To
Lithologic Detailed Description

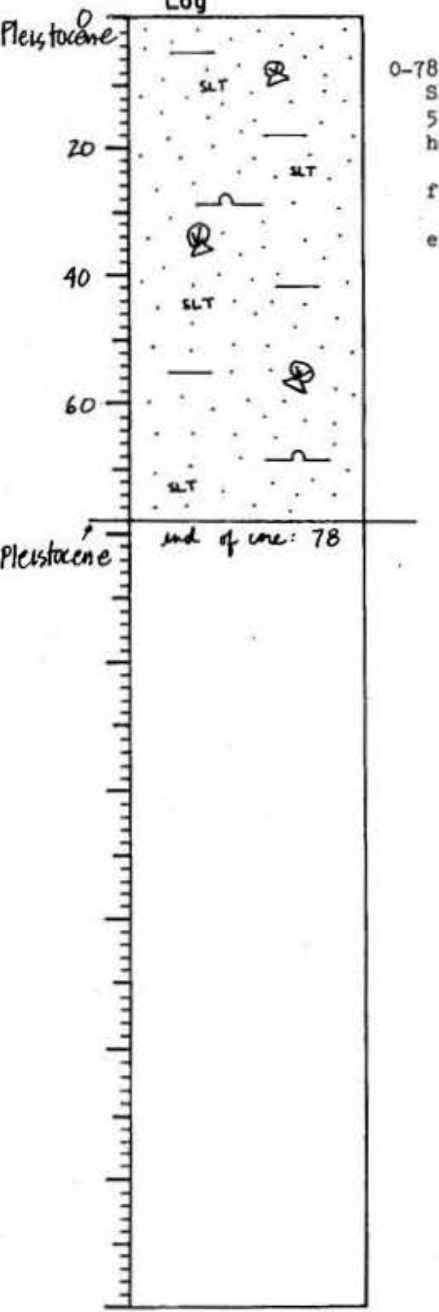

SILIC CLAY/DETRITUS

5 Y 2.5 black oxidizes to $3 / 2$ dark olive gray comogeneous except for scattered bivalve fragnents throughout
fine to medium sand in lutite matrix end of core 
SHEAR SLIDE DESCRIPTIONS - K.H.O.I. SEDINENT CORES

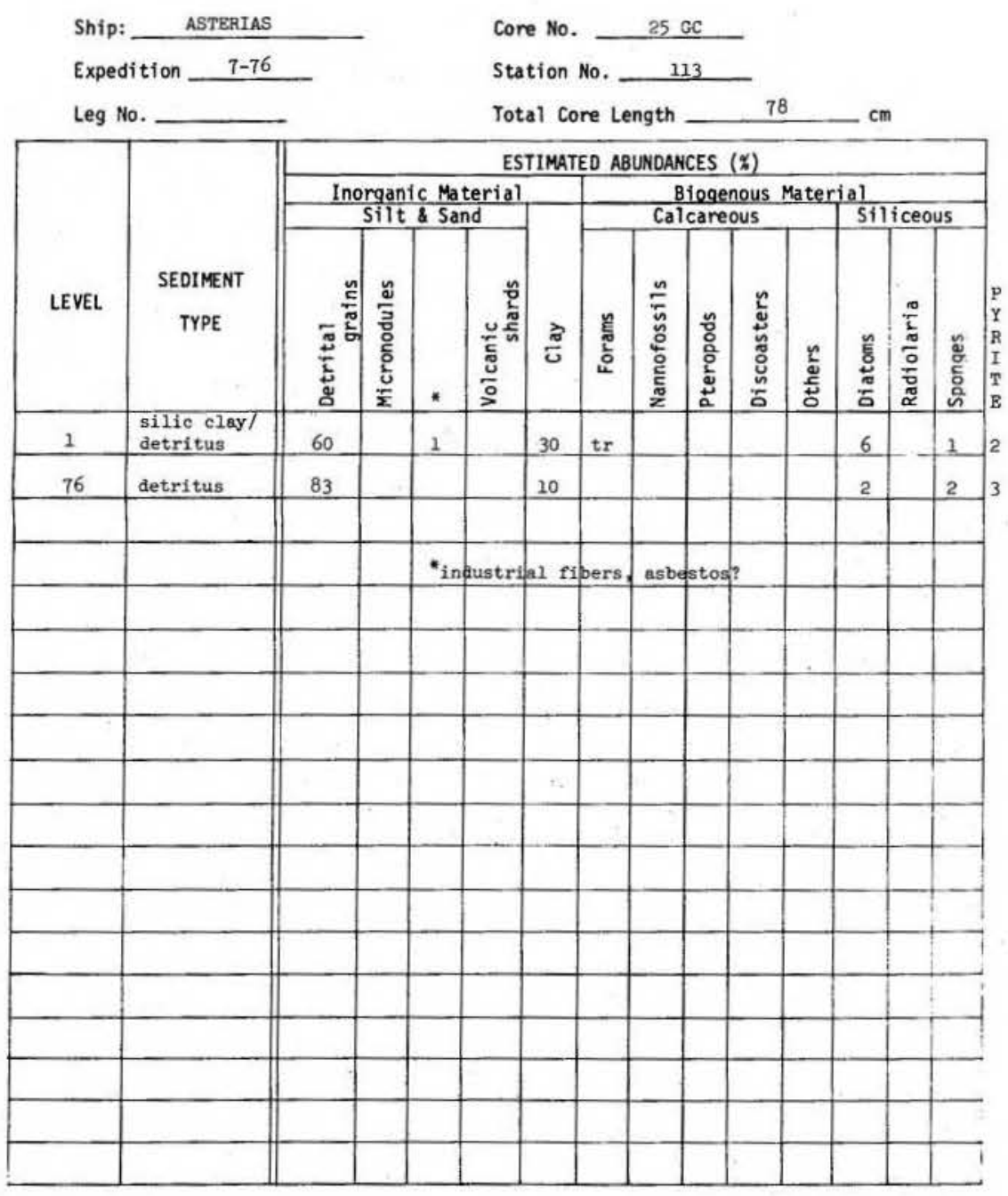

Ship Asterias Cruise 7.76 Leg - Sto. 114 Core No. 26 \& Total Length $86 \mathrm{~cm}$. Lat. $41^{\circ} 34.9^{\circ} \mathrm{N}$ Long. $\overline{70^{\circ} 54.2}$ ' w Depth 7.9 mades Core condition Excellent Dote Described 15 Nov 78 by H. Faremer

Physiographic location BuzzARDS BAY APPRoneres no New Bedface Haebor

Lithologic

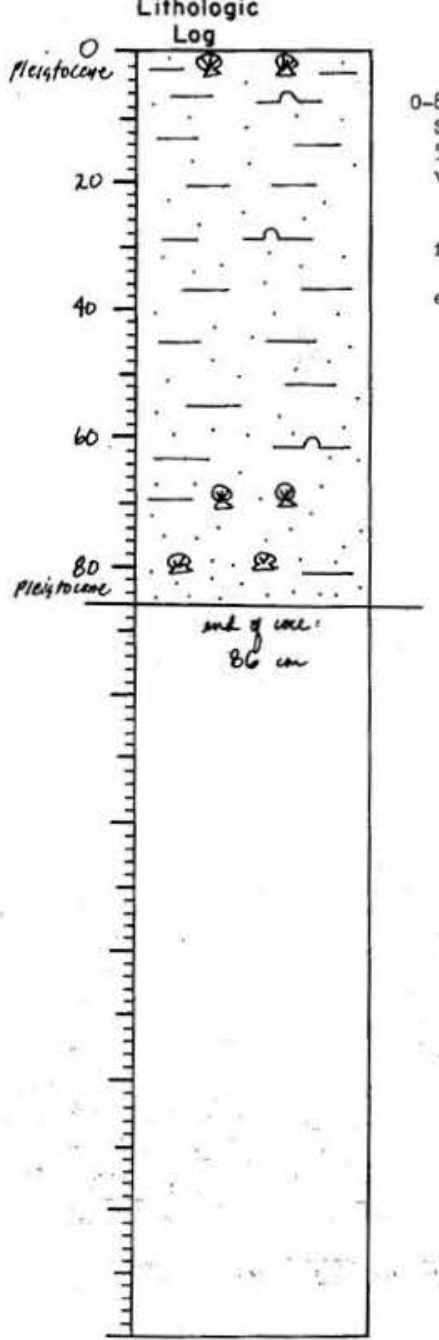

\section{Detailed Description}

$0-86$

SILIC CLAY/DEIRITUS

which are prinarily concentrated in the intervals

$0-3,72-73$ and $79-82 \mathrm{~cm}$
fine to medium detritel sand in lutite matrix

end of core
Y 
SEEAR SLIDE DESCRIPTIONS - W.H.0.I. SEDIMENT CORES

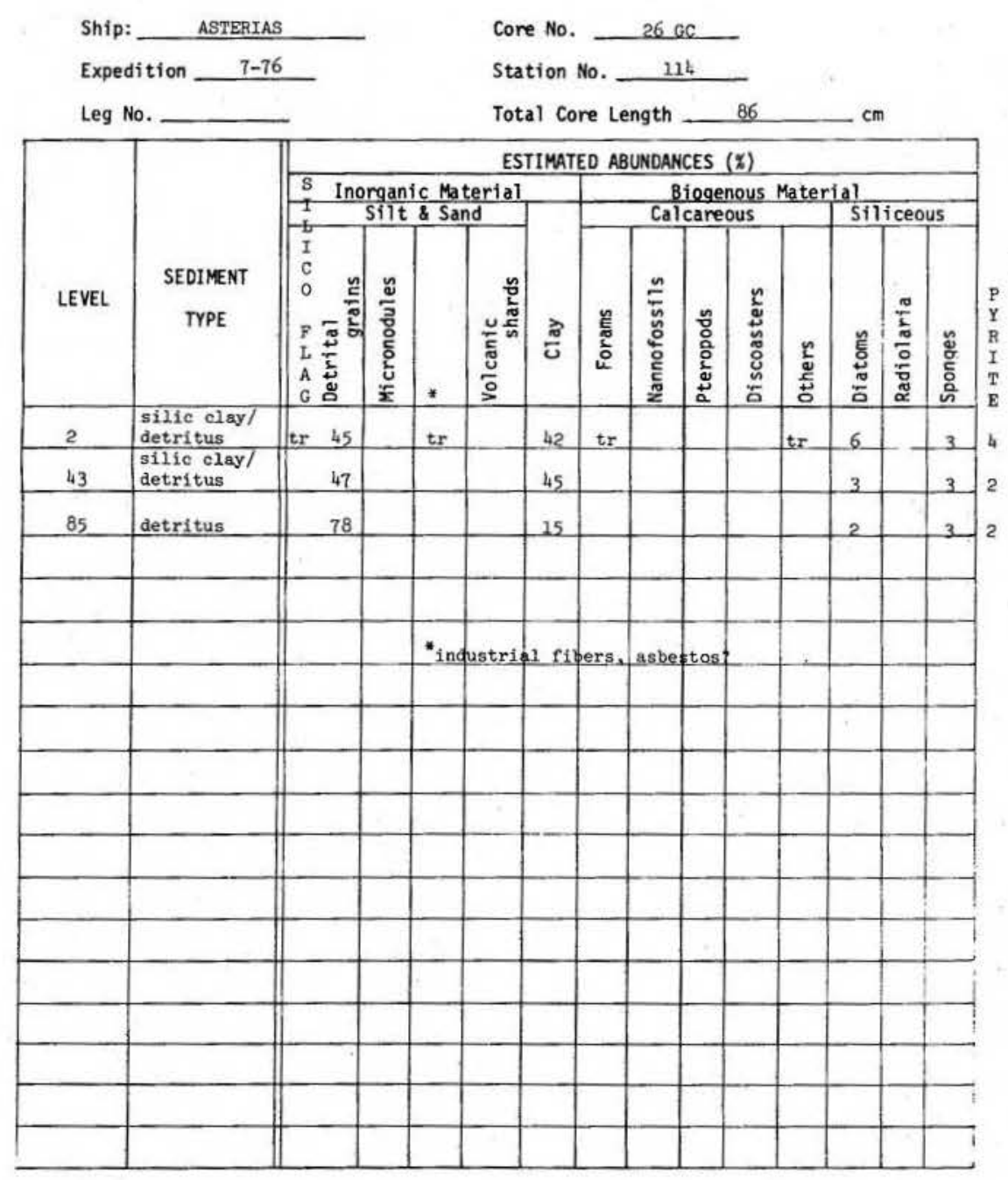

Ship ASTERIAS Cruise 7-76 Leg - Sta. 115 Core No. $27 \mathrm{GC}$ Total Length $15 \mathrm{~cm}$ Lat. $41^{\circ} 35.4^{\prime} \mathrm{N}$ Long $70^{\circ} 54.7^{\circ} \mathrm{W}$ Depth 5.8 mekers Core condition EXCELLENT T. Date Described 15 NWV 76 by T. Brade. Physiographic location BUZZARDS EAY APPREACHES TO NEN BSPPORD HARBORLithologic

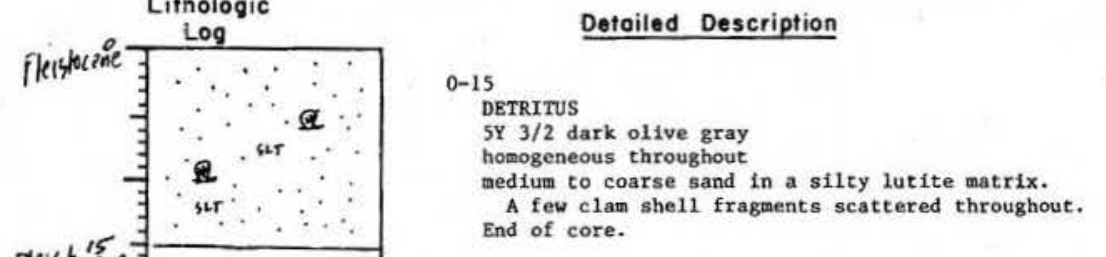


SMEAR SLIDE DESCRIPTIONS - W.H.O.I. SEDIMENT CORES

Ship: Asterias

Expedition 7.76

Leg No. _ _

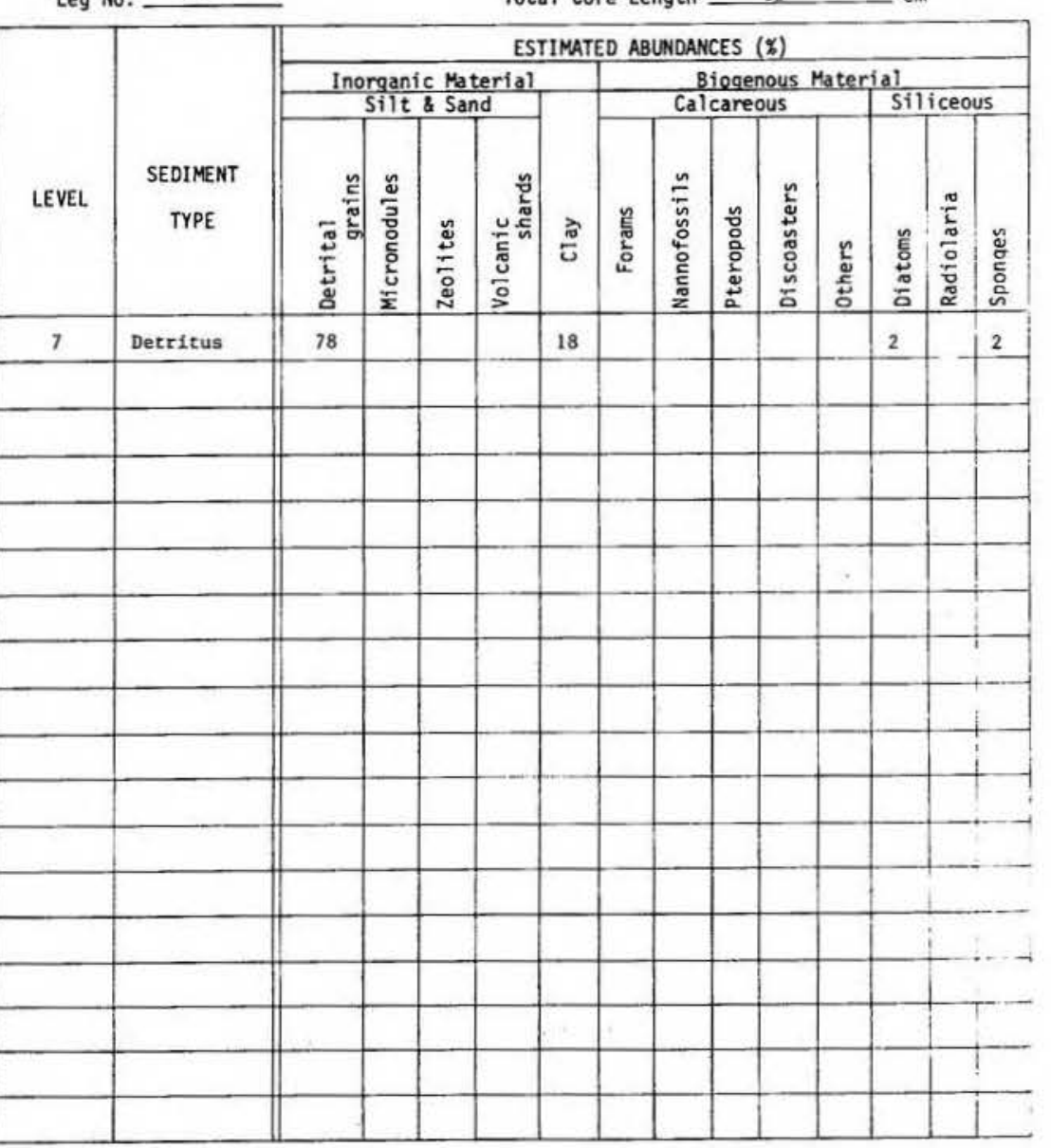

Ship AsteriAs Cruise $7-76$ Leg - Sto. 116 Core No. 28.GC Total Length $58 \mathrm{~cm}$. Lat. $41^{\circ} 379^{\circ} \mathrm{N}$ Long. $70^{\circ} 54.6^{\prime} \mathrm{w}$ Depth 7.6 meter Core condition Excedlent Dote Described I 5 Nov 76 by H. Fnemer Physiographic location NEW BEDFORD HARBOR

Lithologic

Detailed Description

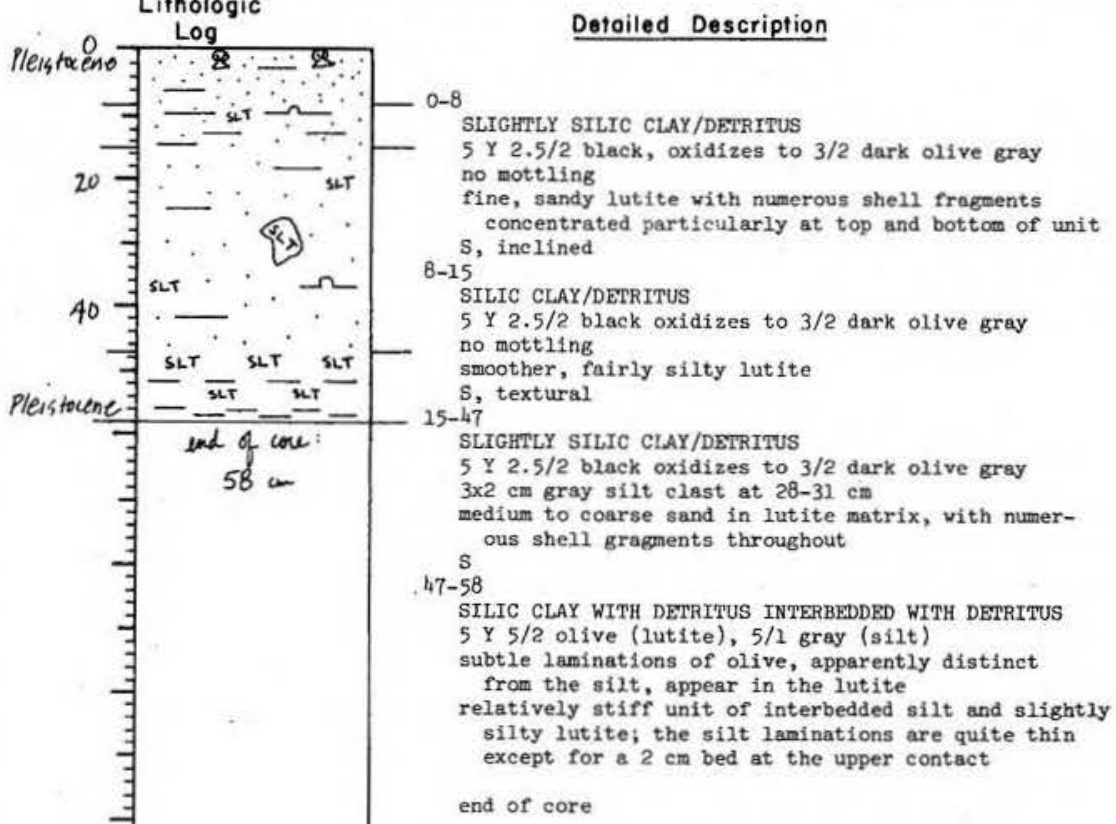

end of core
Station No. 115

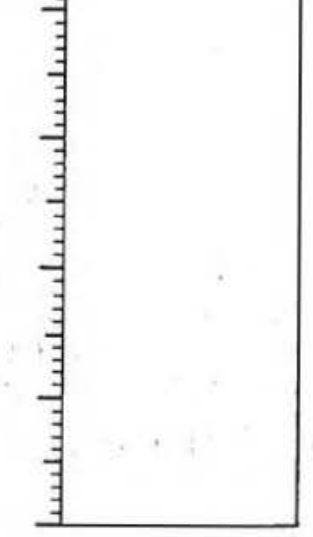




\section{SMEAR SLIDE DESCRIPTIONS - W.H.O.I. SEDIMENT CORES}

Ship: ASTERIAS

Expedition $7-76$

Leg No.

\begin{tabular}{|c|c|c|c|c|c|c|c|c|c|c|c|c|c|c|}
\hline \multirow[b]{3}{*}{ LEVEL. } & \multirow[b]{3}{*}{$\begin{array}{c}\text { SEDIMENT } \\
\text { TYPE }\end{array}$} & \multicolumn{13}{|c|}{ ESTIMATED ABUNDANCES (q) } \\
\hline & & \multicolumn{4}{|c|}{$\frac{\text { Inorganic Material }}{\text { Silt \& Sand }}$} & \multirow[b]{2}{*}{ ढ़ } & \multicolumn{5}{|c|}{$\begin{array}{l}\text { Biogenous Mater } \\
\text { Calcareous }\end{array}$} & \multicolumn{3}{|c|}{$\frac{\text { al }}{\text { Siliceous }}$} \\
\hline & & 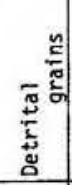 & $\begin{array}{l}\frac{a}{3} \\
\frac{3}{3} \\
\frac{0}{0} \\
\frac{0}{2} \\
\frac{0}{2}\end{array}$ & * & 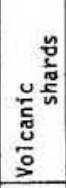 & & 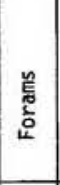 & 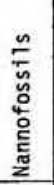 & 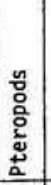 & 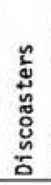 & 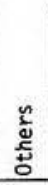 & 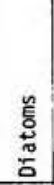 & 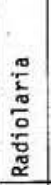 & 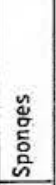 \\
\hline $2 \mathrm{~cm}$ & $\begin{array}{l}\text { Plightly silid } \\
\text { lay/detritus }\end{array}$ & 70 & & & & 24 & & & & & & 2 & & 2 \\
\hline 13 & $\begin{array}{l}\text { Silic clay } \\
\text { detritus }\end{array}$ & 42 & & $\operatorname{tr}$ & & 50 & tr & & & & & 4 & & 2 \\
\hline 48 & detritus & 88 & & & & 12 & & & & & & & & \\
\hline 57 & $\begin{array}{l}\text { unfossiliferou } \\
\text { clay }\end{array}$ & 5 & & & & 95 & & & & & & & & \\
\hline & & & & & & & & & & & & & & \\
\hline & & & & *in & Lustri & al $\mathrm{fi}$ & pers, & asbe & stos? & & . & & & \\
\hline & & & & & & & & & & & & & & \\
\hline & & & & & & & & & & & & & & \\
\hline & & & & & & & & & & & & & & \\
\hline & & & & & & & & & & & & & & . \\
\hline & & & & & & & & & & & & & & \\
\hline & & & & & & & & & & & & & & \\
\hline & & & & & & & & & & & & & & \\
\hline & & & & & & & & & & & & & & \\
\hline & & & & & & & & & & & & & & 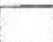 \\
\hline & & & & & & & & & & & & & & \\
\hline & & & & & & & & & & & & & & \\
\hline & & & & & & & & & & & & & & \\
\hline
\end{tabular}

VISUAL CORE DESCRIPTION

Page 1 of 1

Ship AsTERIAS Cruise $7.76 \quad$ Sto. 117 Core No. $29 \mathrm{GC}$

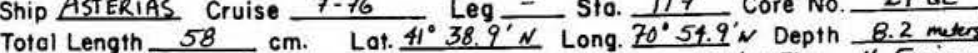

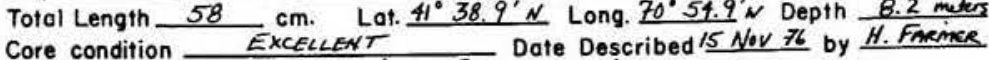
Core condition $\frac{\text { EXCELLENT }}{\text { Physiographic location DEW BEDFORD HARBDR }}$

Lithologic

Detailed Description

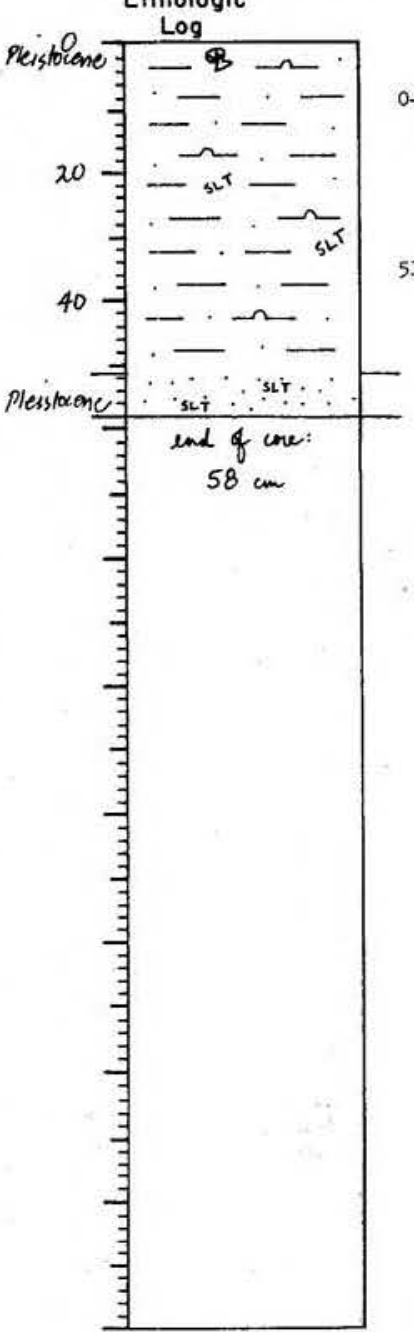

SILIC CIAY WITH DETRITUS smooth, fairly silty lutite, molluscan fragments in top $5 \mathrm{~cm}$ $\mathrm{S}$
$51-58$ DETRITUS

5 Y $3 / 2$ dark olive gra

small $(1 \mathrm{~cm})$, black, organic-rich spot, $57 \mathrm{~cm}$; maller, light gray, sandy lutite also at $58 \mathrm{~cm}$, small wood fragment shell fragments

end of core
5 Y $3 / 2$ dark olive gray 
SMEAR SLIDE DESCRIPTIONS - W.H.O.I. SEDIMENT CORES

Ship: ASTERIAS

Expedition $7-76$

Leg No.
Core No. $22 \mathrm{GC}$

Station No. 117

Total Core Length 58 $\mathrm{cm}$

\begin{tabular}{|c|c|c|c|c|c|c|c|c|c|c|c|c|c|c|}
\hline \multirow[b]{4}{*}{ LEVEL } & \multirow[b]{4}{*}{$\begin{array}{c}\text { SEDIMENT } \\
\text { TYPE }\end{array}$} & \multicolumn{13}{|c|}{ ESTIMATED ABUNDANCES (\%) } \\
\hline & & \multirow{2}{*}{\multicolumn{4}{|c|}{$\begin{array}{c}\text { Inorganic Material } \\
\text { Silt \& Sand }\end{array}$}} & & \multicolumn{8}{|c|}{ Biogenous Material } \\
\hline & & & & & & & & & care & & & Sil & iceo & \\
\hline & & 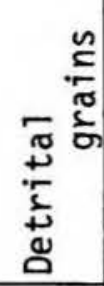 & 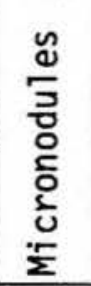 & 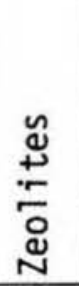 & 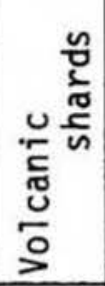 & $\frac{\vec{\sigma}}{\sigma}$ & 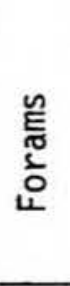 & 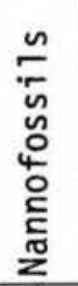 & 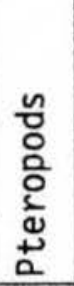 & 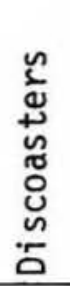 & $\begin{array}{l}n \\
\frac{n}{\omega} \\
\frac{n}{0}\end{array}$ & 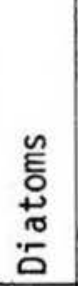 & $\begin{array}{l}\frac{\pi}{2} \\
\frac{\pi}{0} \\
\frac{0}{\pi} \\
\tilde{\pi}\end{array}$ & $\begin{array}{l}\text { o. } \\
\text { o. } \\
\text { ò } \\
\text { in }\end{array}$ \\
\hline 2 & $\begin{array}{l}\text { silic clay } \\
\text { with detritus }\end{array}$ & 25 & & & & 58 & & & & & $\operatorname{tr}$ & 9 & & 3 \\
\hline 57 & detritus & 75 & & & & 16 & & & & & & 3 & & 3 \\
\hline & & & & & & & & & & & & & & \\
\hline & & & & & & & & & & & & & & \\
\hline & & & & & & & & & & & & & & \\
\hline & & & & & & & & & & & & & & \\
\hline & & & & & & & & & & & & & & \\
\hline & & & & & & 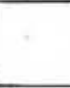 & & & & & & & & \\
\hline & & & & & & & & & & & & & & \\
\hline & & & & & & & & & & & & & & \\
\hline & & & & & & & & & & & & & & \\
\hline & & & & & & & & & & & & & & \\
\hline & & & & . & & & & & & & & & & \\
\hline & & & & & & & & & & & & & & \\
\hline & & & & & & & & & & & & & & \\
\hline & & & & & & & & & & & & & & \\
\hline & & & & & & & & & & & & & & \\
\hline
\end{tabular}


1
I
1
'
1
1
1
1

I

I

I

1

1.

I

1

1

1

I

l 


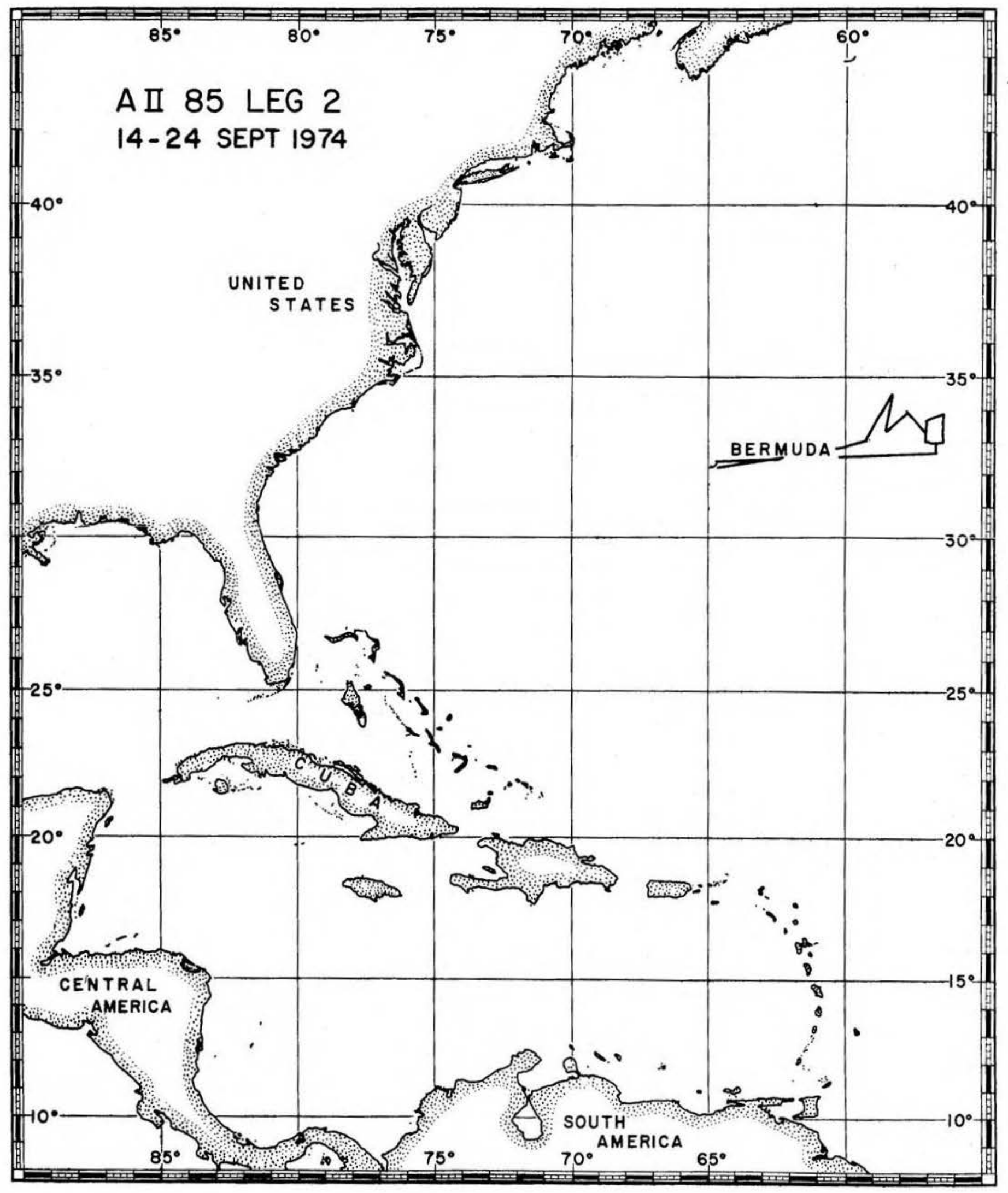




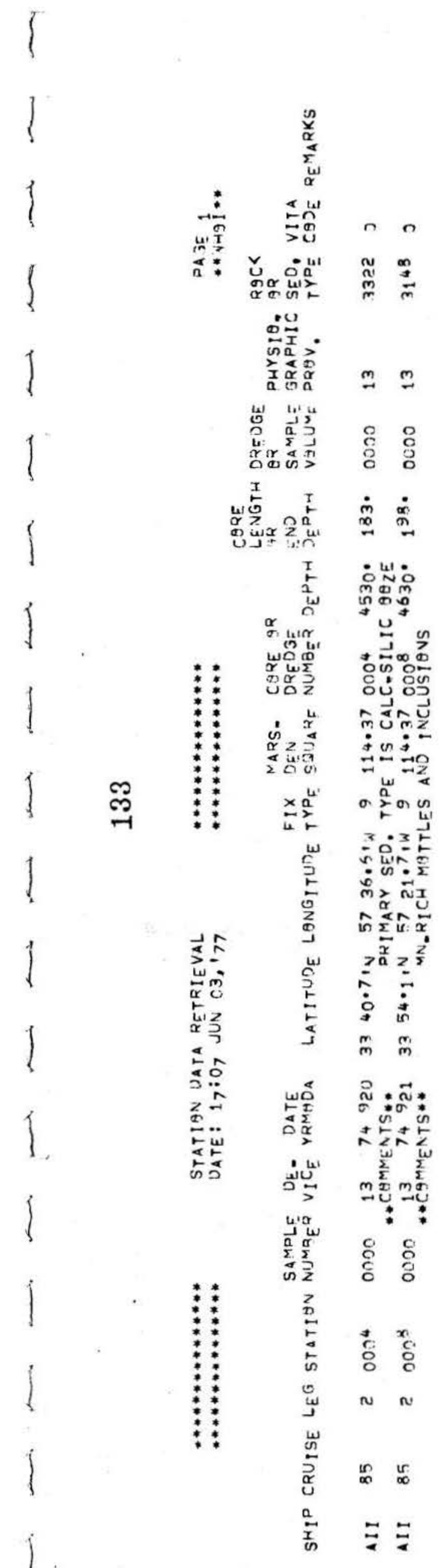


1
1
1
1

1

I

I

1

I

1

I

I

I

I

1

1

1

I

l 
Page 1 of 1

Ship AII Cruise 85 Leg II Sta. 4 Core No. $\frac{4 \mathrm{GC}}{4530 \mathrm{a}}$ Total Length $183 \mathrm{~cm}$. Lat. $33^{\circ} 40.7 \mathrm{~N}$ Long. $57^{\circ} 36.6^{\circ} \mathrm{V}$ Depth $4530 \mathrm{~m} . \mathrm{cos}$ Core condition ExCEUEN Date Described 22 Je 76 by f-brode. Physiographic location

Lithologic

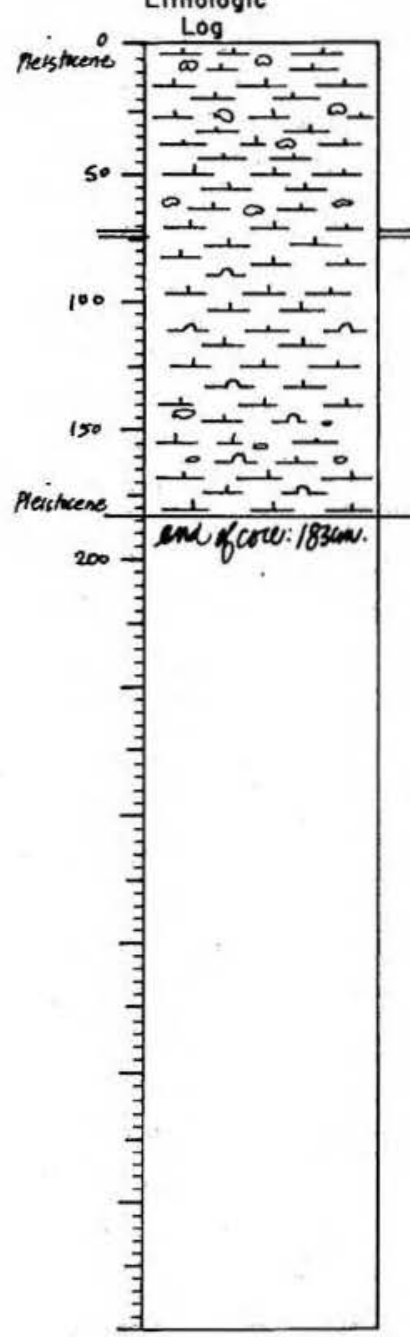

\section{Detailed Description}

0-72

CAIC OOZE

5 YR $5 / 4$ brown grades to 10 YR $6 / 3$ pale brown and back to 7.5 YR $5 / 4$ brown tional zones and many sma 11, gray and ollve green, freckle-1ike mottles forams and occa(clay)

$2-74$

CALC OOZE

$2.5 \times 4 / 4$ olive brown and 7.5 YR $5 / 4$ brown

vertil, hake a compact cly silty lutite ${ }^{6}$

CALC-SILIC OOZE

10 YR $5 / 3$ brown grades to $5 / 2$ grayish brown few freckle-like olive mottles and specks $140-169 \mathrm{~cm}$

very molst, slick lutite with a trace of forams and

end of core
SIEAR SLIDE DESCRIPTIONS - W.H.O.I. SEDIMENT CORES

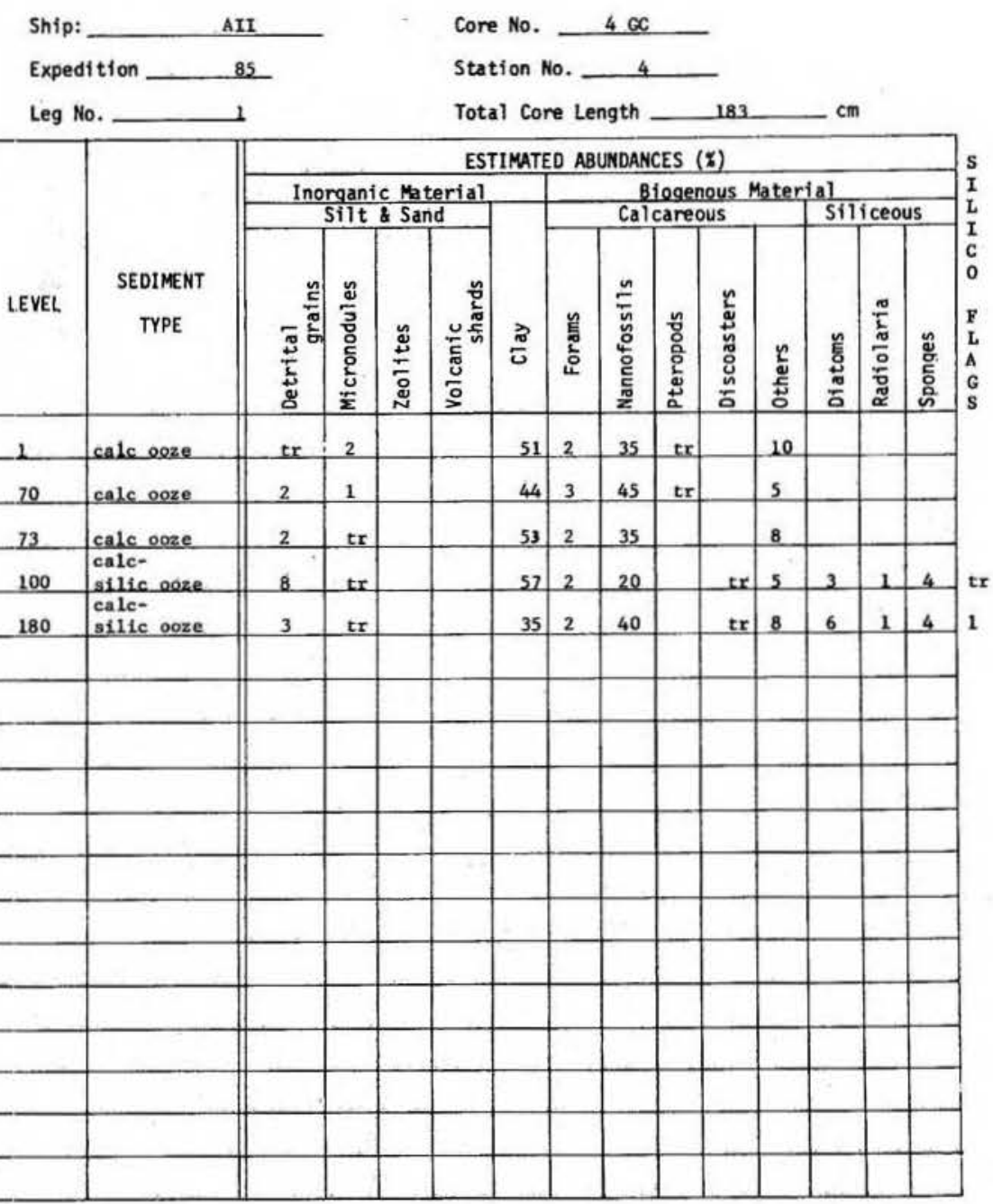


136

VISUAL CORE DESCRIPTION

Ship AII Cruise 85 Leg II Sto. 8 Core No. $86 \mathrm{C}$

Total Length $198 \mathrm{~cm}$. Lat. $33^{\circ} 54.1^{\circ} \mathrm{N}$ Long. $77^{\circ} \mathrm{Z1.7^{ \circ } \mathrm { W }}$ Depth $4630 \mathrm{mcom}$

Core condition EXCELLENT Date Described 22 yene 76 by f.Broder Physiographic location BERMVDA RISE.

Lithologic

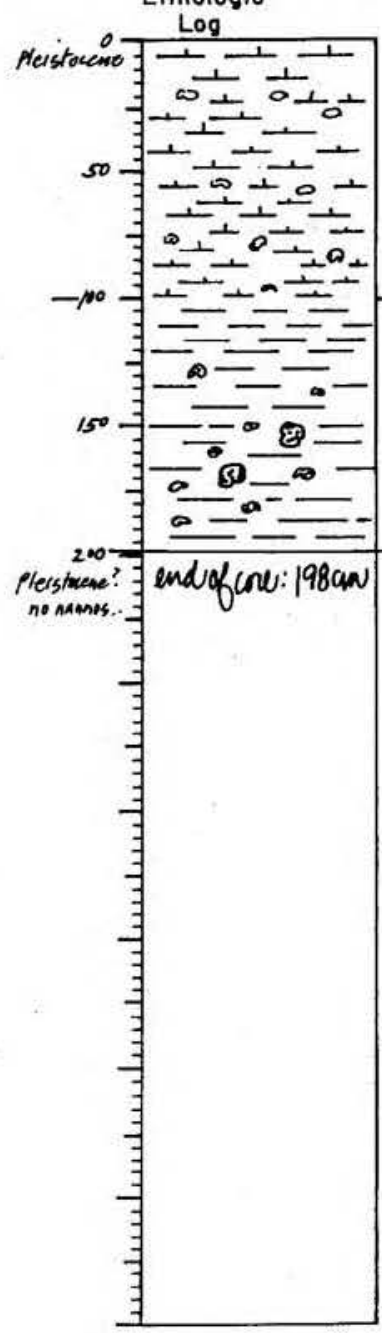

0-100 brown and back to 7.5 YR $5 / 4$ brown in two sequential patterns

moist, slightly silty lutite with foram content ranging from scattered to abundant

Sharp - irregular and mottled

100-198

FFOSSILIFEROUS CLAY WITH MOTTIES AND INCLUSIONS

OF Mn MICRONODULES

5 Y $6 / 3$ pale olive and $2.5 \times 6 / 4$ light yellowish brown common alternation of small zones of the above colors throughout the undt; heavily mottled with dark olive gray and black, slick, Mn-rich lutite; a bit of more compact but slick lutite with a few scattered, more compact but s throughout
lithified lumps the

end of core

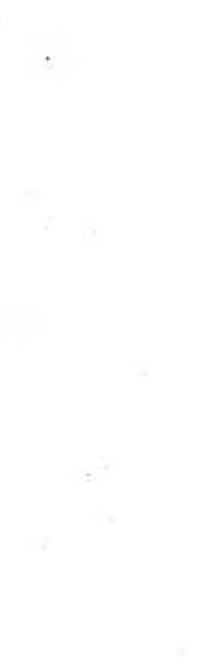

137

SMEAR SLIDE DESCRIPTIONS - W.H.O.I. SEDINENT CORES

\begin{tabular}{|c|c|c|c|c|c|c|c|c|c|c|c|c|c|c|}
\hline \multirow[b]{3}{*}{ LEVEL } & \multirow[b]{3}{*}{$\begin{array}{c}\text { SEDIMENT } \\
\text { TYPE }\end{array}$} & \multicolumn{13}{|c|}{ ESTIMATED ABUNDANCES ( $(x)$} \\
\hline & & \multicolumn{4}{|c|}{$\begin{array}{l}\text { Inorganic Material } \\
\text { Silt \& Sand }\end{array}$} & & \multicolumn{5}{|c|}{$\begin{array}{l}\text { Biegenous Mater } \\
\text { Calcareous }\end{array}$} & \multicolumn{3}{|c|}{ Siliceous } \\
\hline & & 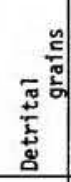 & 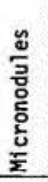 & 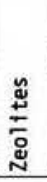 & 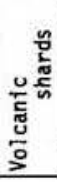 & ङ & 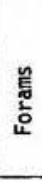 & 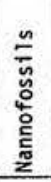 & 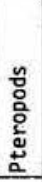 & 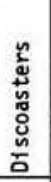 & 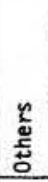 & 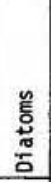 & $\frac{\pi}{\frac{\pi}{\pi}}$ & 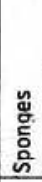 \\
\hline 1 & calc ooze & 3 & tx & & & 32 & 8 & 45 & & & 12 & & & \\
\hline 95 & calc ooze & 4 & 5 & & & 23 & 12 & 50 & & & 6 & & & \\
\hline 105 & unfoss_clay & 6 & 5 & & & 89 & & & & & tr & & & \\
\hline 134 & $\begin{array}{l}\text { unfoss clay/ } \\
\text { Mn micronod1s }\end{array}$ & tr & 55 & & & 45 & & & & & tr & & & \\
\hline 197 & unfoss clay & 8 & 5 & & & 87 & & & & & $t x$ & & & \\
\hline & & & & & & & & & & & & & & \\
\hline & & & & & & & & & & & & & & \\
\hline & . & & & & & & & & & & & & & \\
\hline & & & & & & & & & & & & & & \\
\hline & & & & & & & & & & & & & & \\
\hline & & & & & & & & & & & & & & \\
\hline & & & & & & & & & & & & & & \\
\hline & & & & & & & & & & & & & & \\
\hline & & & & & & & & & & & & & & \\
\hline & & & & & & & & & & & & & & \\
\hline & & & & & & & & & & & & & & \\
\hline & & & & & & & & & & & & & & \\
\hline & & & & & & & & & & & & & & \\
\hline
\end{tabular}

Detailed Description

$5 / 4$ brown grades to 10 YR $6 / 4$ light yellowish

faint intermottling of the colors above especiall

Ship:

Expeditio AII

Leg No. 1

Core No. $\quad 8 \mathrm{ec}$

8

ATED ABLWDANCES ( 


\section{8}

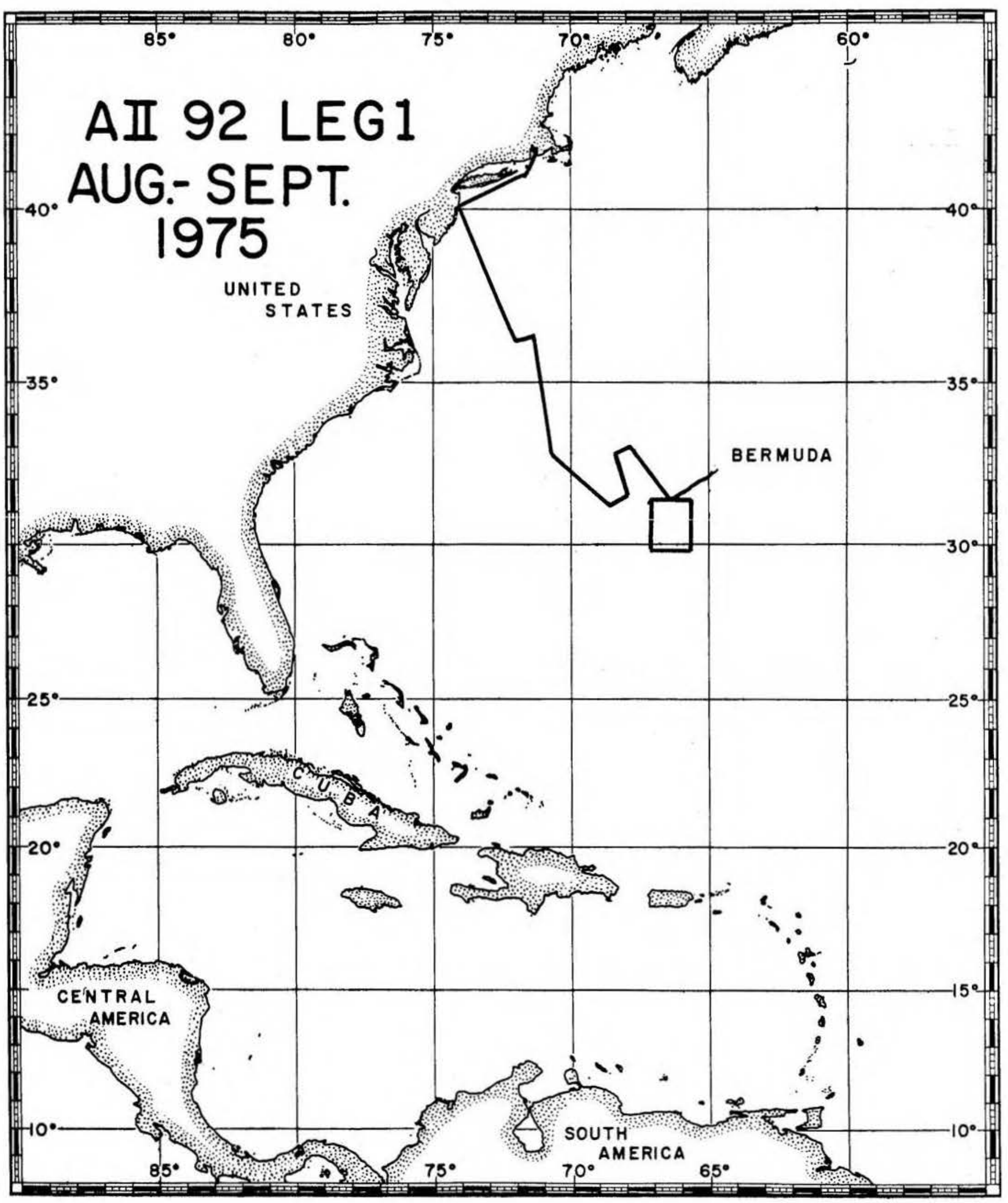




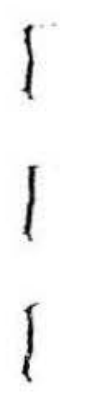

I

I

1

I

1

1

1

1

1

I

1

1

I

I

? 
194

VISUAL CORE DESCRIPTION

Page 3 of 3

Ship CHAIN Cruise 115 Leg 1 Sta. 2 Core No. 2 PC

Lithologic

Log

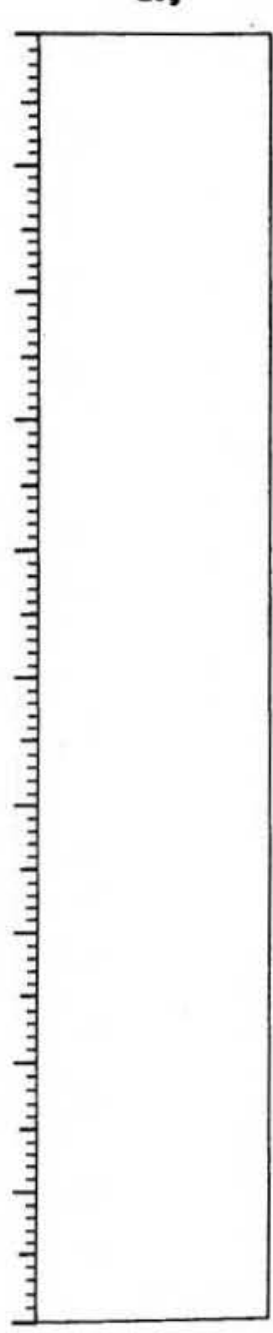

Detailed Description

679-785

SLIGHTLY CAIC CLAY
1OTR $4 / 2$ dark brownish gray

faint shadowy dark gray and redalsh brown irregular mottle moist slightly silty lutite with a few scattered forams end of core
195

SMEAR SLIDE DESCRIPTIONS - W.H.O.I. SEDIMENT CORES

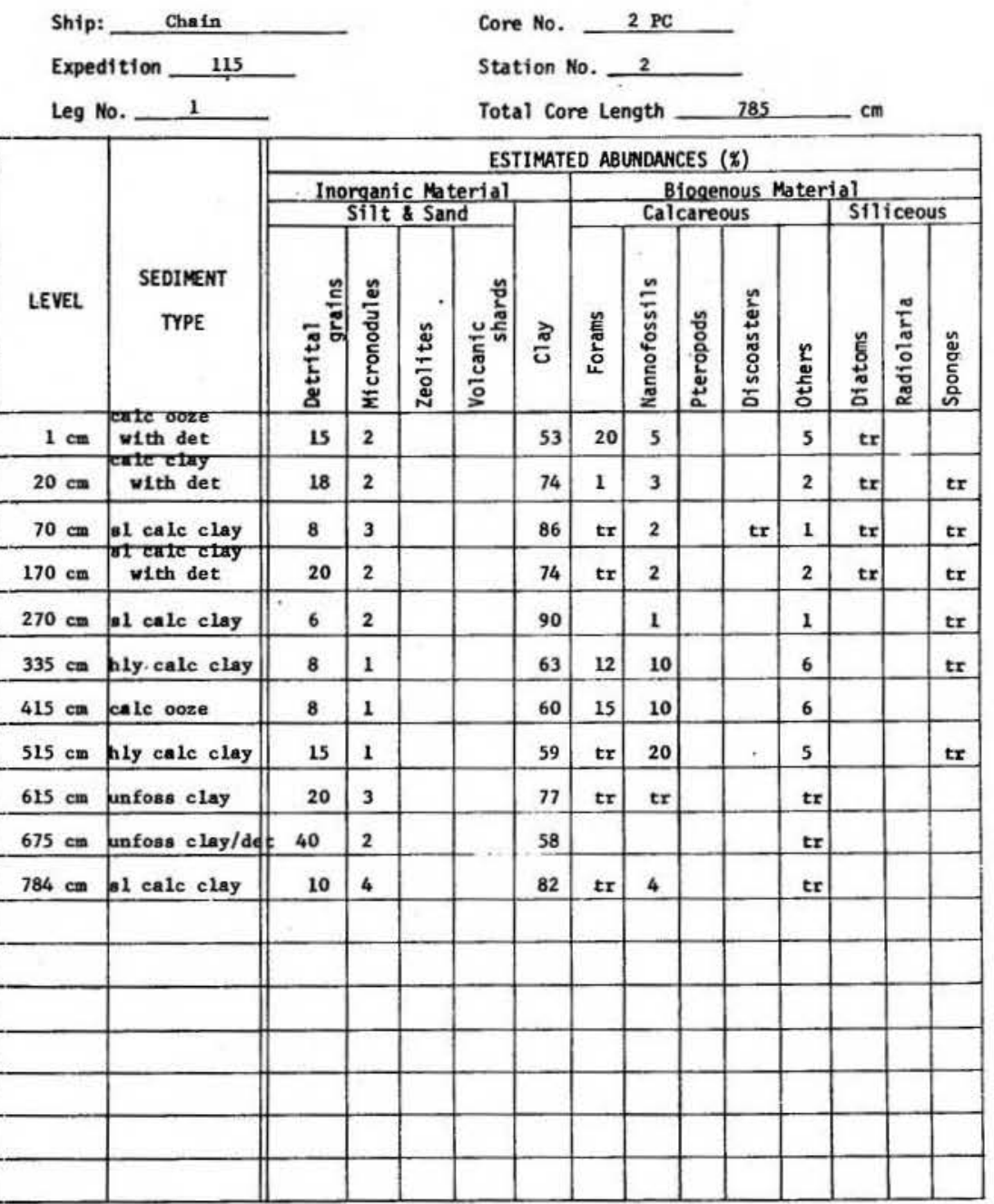


196

VISUAL CORE DESCRIPTION

Page 1 of 1

Ship CHAIN Cruise 115 Leg 1 Sto. 2 Core No. 2 PG

Total Length $88^{\circ} \mathrm{cm}$. Lat. $37^{\circ} 206^{\circ} \mathrm{N}$ Long. $17^{\circ} 080^{\circ} \mathrm{W}$ Depth $2415 \mathrm{~m}$.corr

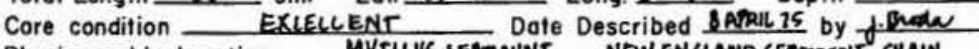
Physiographic locotion MYTILUS SEMMOUNT... NEN ENGLAND SEMHOUNI CHAIN

Lithologic

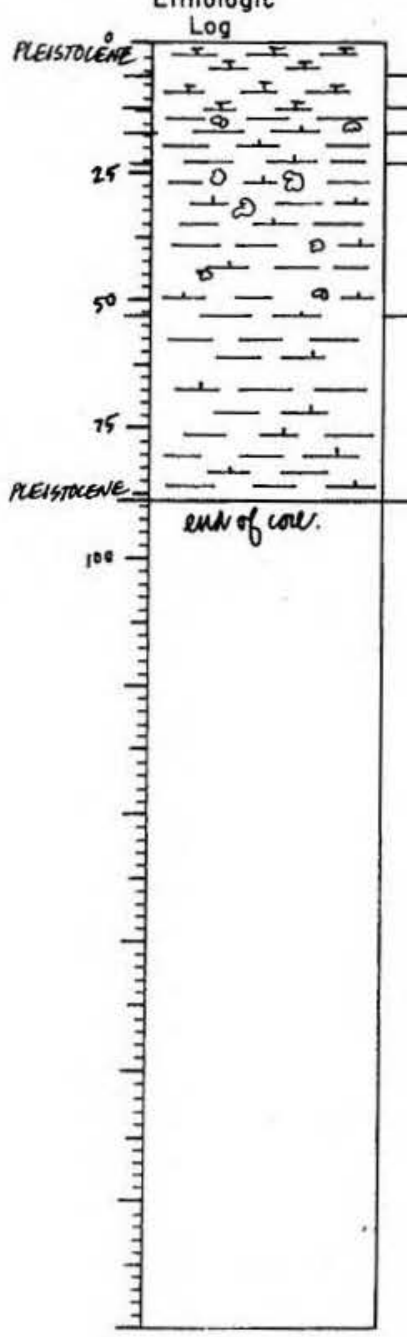

Detailed Description

\section{CR. C OOZE}

$2.5 \mathrm{~mm}$ to $5 \mathrm{~mm}$ diameter black pebbles scattered throughout

6-12

CAIC OOZE

10XR $6 / 4$ light yellowish brown

menter sand

$5 \mathrm{~m}$ diameter, also one large $3 \mathrm{~cm}$ 17 mottled

12-17

SL CALC CIAY

$7.5 Y R 5 / 4$ brown

scattered forms in silty lutite, abund. forms in mottles at upper unit contact

17-23

SL CAIC CIAY

1 TOYR $5 / 3$ brown

tiffer silty lutite with scattered forams, black flecks and $\operatorname{amal1}$ pebile

23-53

\section{AIC CIAY}

$2.5 \times 5 / 2$ grayloh brawn

comon, large, brown mottiling $23-33 \mathrm{~cm}$, very faint brown coumon moteling $30.46 \mathrm{~cm}$

ilecks throughout 53-88

\section{SL CALC CLAY}

SY $5 / 1$ gray

bomogeneour throughout

very silck molst lutite

at $54 \mathrm{~cm}, 11$

end of core

\section{YR $5 / 2$ grayish brom}

197

SMEAR SLIDE DESCRIPTIONS - W.H.O.I. SEDIMENT CORES

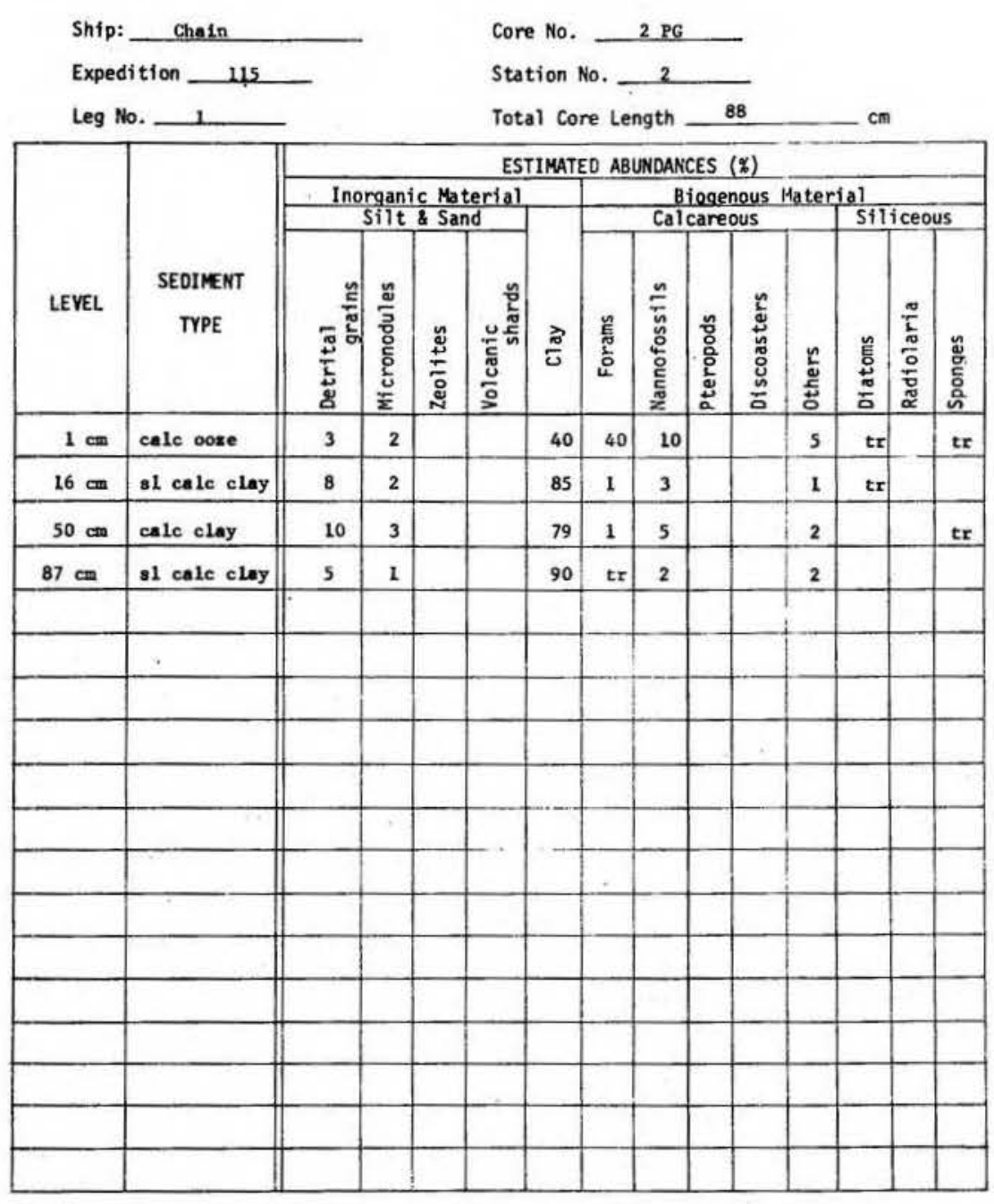


Ship CHAIN Cruise $115 \quad$ Leg 1 Sta. 3 Core No. 3 PC

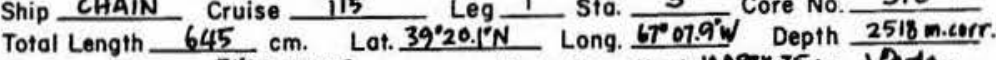
Core condition EXEELENT Date Described 10 APRM 75 by Dede

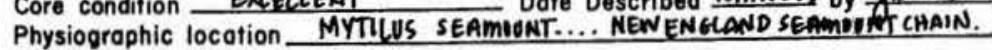

Lithologic

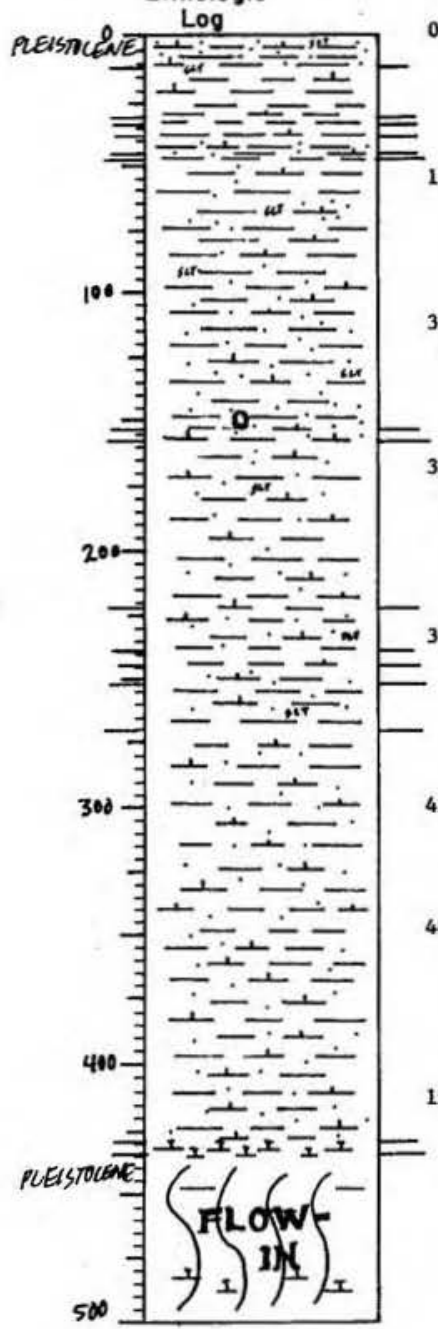

Detailed Description

SLICaTLY CALC CLAY WTI DERTRIUS OrR $5 / 1$ gray

$0=3 \mathrm{~cm}$ 6 textura 1

SLIGRTLY CAIC CLAY

$7.5 Y 5 / 2$ brow $n$

of ailty gray lutite throughou dark gray 11thified nodules at 18 and $24 \mathrm{~cm}$

30-33

UNPOSS CLAY

$5 Y 5 / 2$ ollve gray

tite with intrusions of light brownish gray

33-37

SLIGATLY CALC CTAY

$575 / 3$ olive gray

comon marbling $5 / 6$ olive

tered forams

1ithifled lanination at $37 \mathrm{~cm}$ 5
-45

SLICATLY CALC CLAY WTTH DETRTTUS

.5Y 5/2 graylsh brown

lack flecks and foran

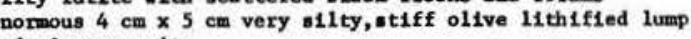

s

URFOSS CLYY

$5 Y 5 / 3$ ollve

silick compact lutite

S

SLIGATLY CALC CLAY WIMH DETRITUS

5Y $4 / 2$ olive gray

molst lutite with varying degrees of silt content $48-80 \mathrm{~cm}$, consistently silty from $80-154 \mathrm{~cm}$ cattered mall pebbles and 11thified lumps, 100-145 cm 4-157

CALC CLAY/DETRITUS

silty, almost pebbly lutite (very stiff)

large $3 \mathrm{~cm} \times 3 \mathrm{~cm} \times 1 \mathrm{~cm}$ glacial erratic within
199

VISUAL CORE DESCRIPTION

Page 2 of 2

Ship CHAIM Cruise 115 Leg 1 Sta. 3 Core No. 3PC

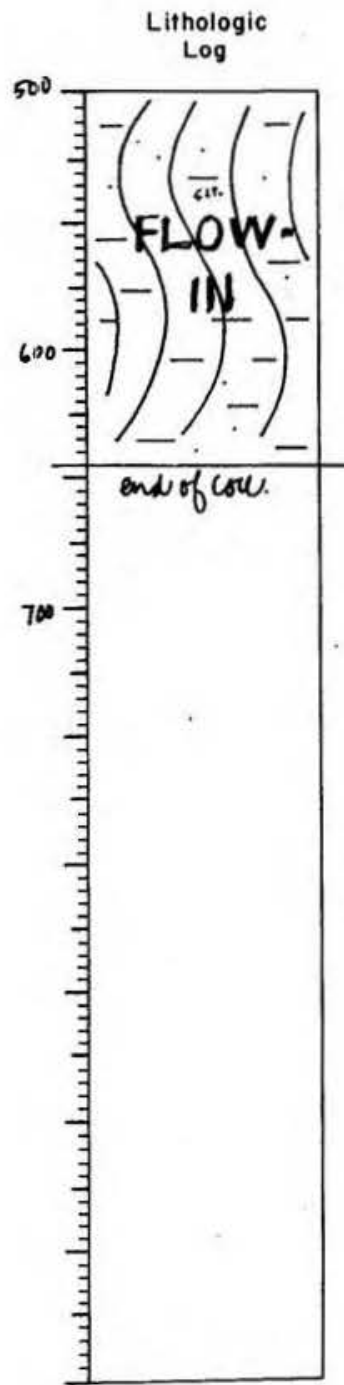

Detailed Description

157-222

CALC CLAY MITH DETRTTUS

SY $4 / 2$ olive gray grades to 7.5 YR $4 / 2$ dark brow

very faint brown laminations and marbling throughout moist silty lutite with scattered forams and black fleck splotches and clusters of black flecks 190-210 cm

222-238

SLIGATLY CAIL CLAX WITH DETRITUS

with scattered forams grading to very abund. G

38-244

SLIGATLY CALC CLAY HITH DETRITUS

10YR $7 / 3$ very pale brown and $5 / 4$ yellowish brown

very pale brown foram sand mixed with yellowish brown $\mathrm{s}$

$-252$

SLIGHTIY CALC CLAY UITH DETRITUS

$5 \mathrm{YR} 4 / 4$ reddish brow

foram sand with above unit at upper contact

$\stackrel{5}{252-270}$

SLICATLY CALC CLAX WITH DETRITU

$2.5 \times 4 / 4$ olive brow

large detrital grains scattered

270-429

varying hues of 10YR 4/2 dark grayish brown and 7.5YR $4 / 4$ dark

slight faint light gray and dark gray mottling found silty lutite vith for

varying slightly throughout 5 textural

429-434

CALC OOZE

10YR $6 / 2$ 11ght browalah gray

silty foram sand mixed a bit with lutite at base 33-645

UNFOSS CLAX WITH DETRITUS

$5 Y \quad 3 / 2$ dark olive gray and $4 / 2$ olive gray

(bete) appen

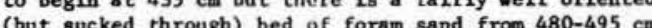


SMEAR SLIDE DESCRIPTIONS - W.H.0.I. SEDIMENT CORES

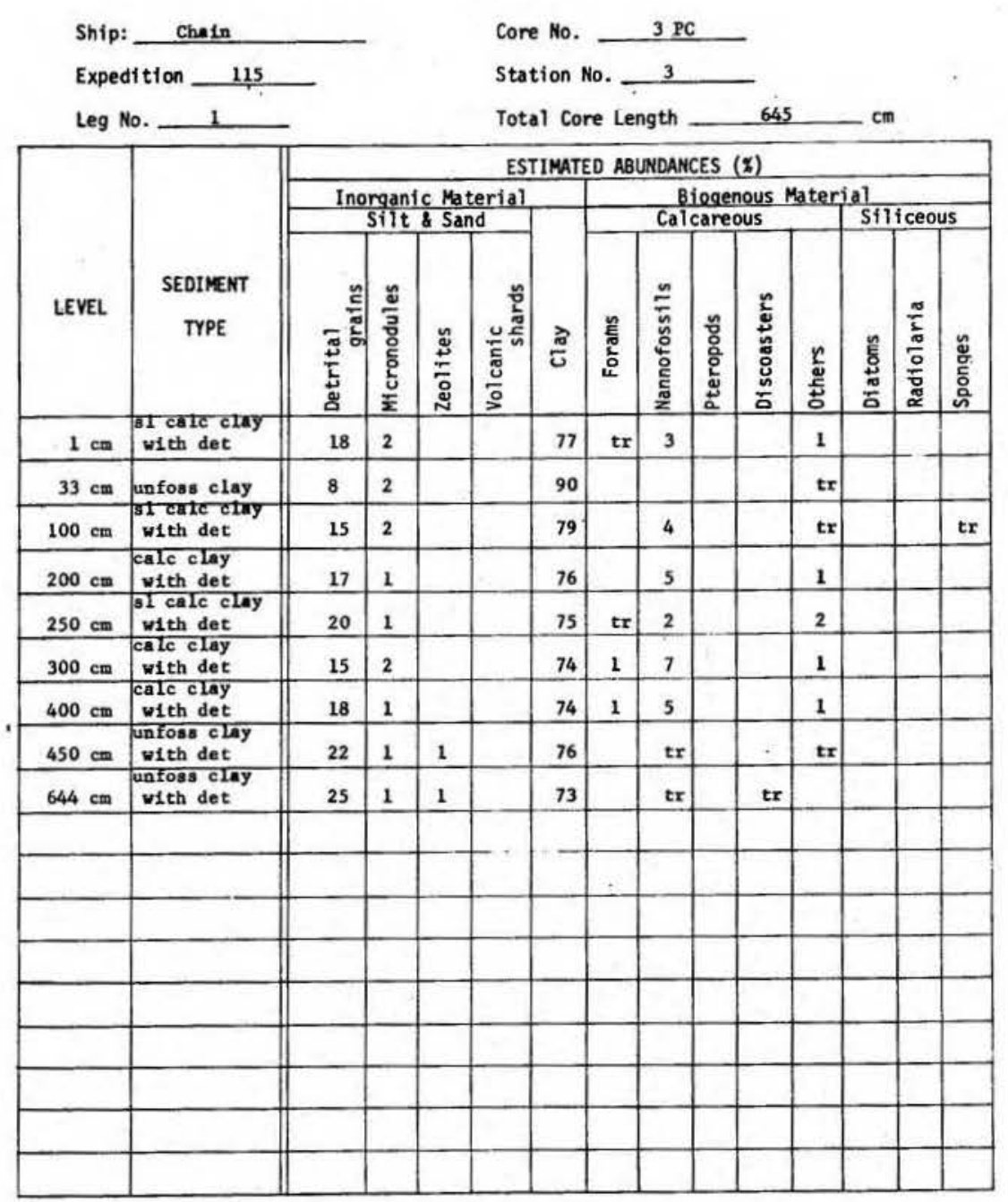

Ship CHAIN Cruise 115 Leg 1 Sta. 3 Core No. 3PG Total Lengith 131 cm. Lat. $30^{\circ} 20.1 \mathrm{~N}$ Long. $67^{\circ} 079^{2} \mathrm{~V}$ Depth $2518 \mathrm{mcer}$ Core condition EXCELLENT Date Described 11Aftil 75 by f. Brodar

Physiographic location MYTILWS SEAMMOUNT... NEW ENGLANO SEAMOUNT CHAIN.

Lithologic

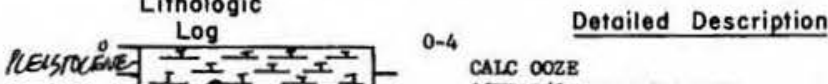

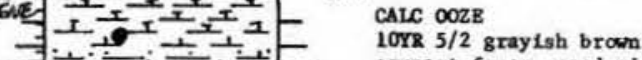

A

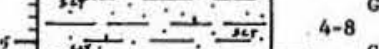

25

$2.5 Y 5 / 2$ grayish brown

foram sand grades to molst slick lutite vith very abund.

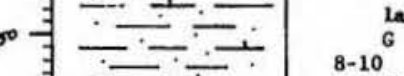

large $3 \mathrm{~cm} \times 1 \mathrm{~cm}$ ice rafted glacial erratic $4-7 \mathrm{~cm}$

SLIGATLY CALC CLAY WITH DETRFTUS

… 10YR $5 / 2$ graylsh brown flecks

10-27,

SLIERTLY CALC CIAY WITH DETRITUS

10YR $5 / 1$ gray

moist slick lutite with a thin $(5 \mathrm{~mm})$ band of extremely abund.

forams $(13 \mathrm{~cm})$ and 1ithified Inminations at $23 \mathrm{~cm} \& 25 \mathrm{~cm}$ S textural

$1007 \div \div$

我 $27-127$

CAIC CIAY WITH DETRITUS

1OYR $5 / 1$ gray

12s

3ilty lutite yith abund, black flecks except for band 100-112 cin of very molst slick lutite with no flecks

scattered small (2.5-5 ice rafted debris throughout,

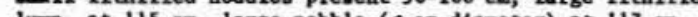

150 年 $\underset{127-131}{s}$

131

2.5 YMA4/ dark gray WITH DETRUTUS

slick lutite with dowinant very stiff lithified mottle at upper valt contact

pebble (ice rafted) $128-131 \mathrm{~cm}$ end of core 
SIEAR SLIDE DESCRIPTIONS - W.H.0.1. SEDIMENT CORES

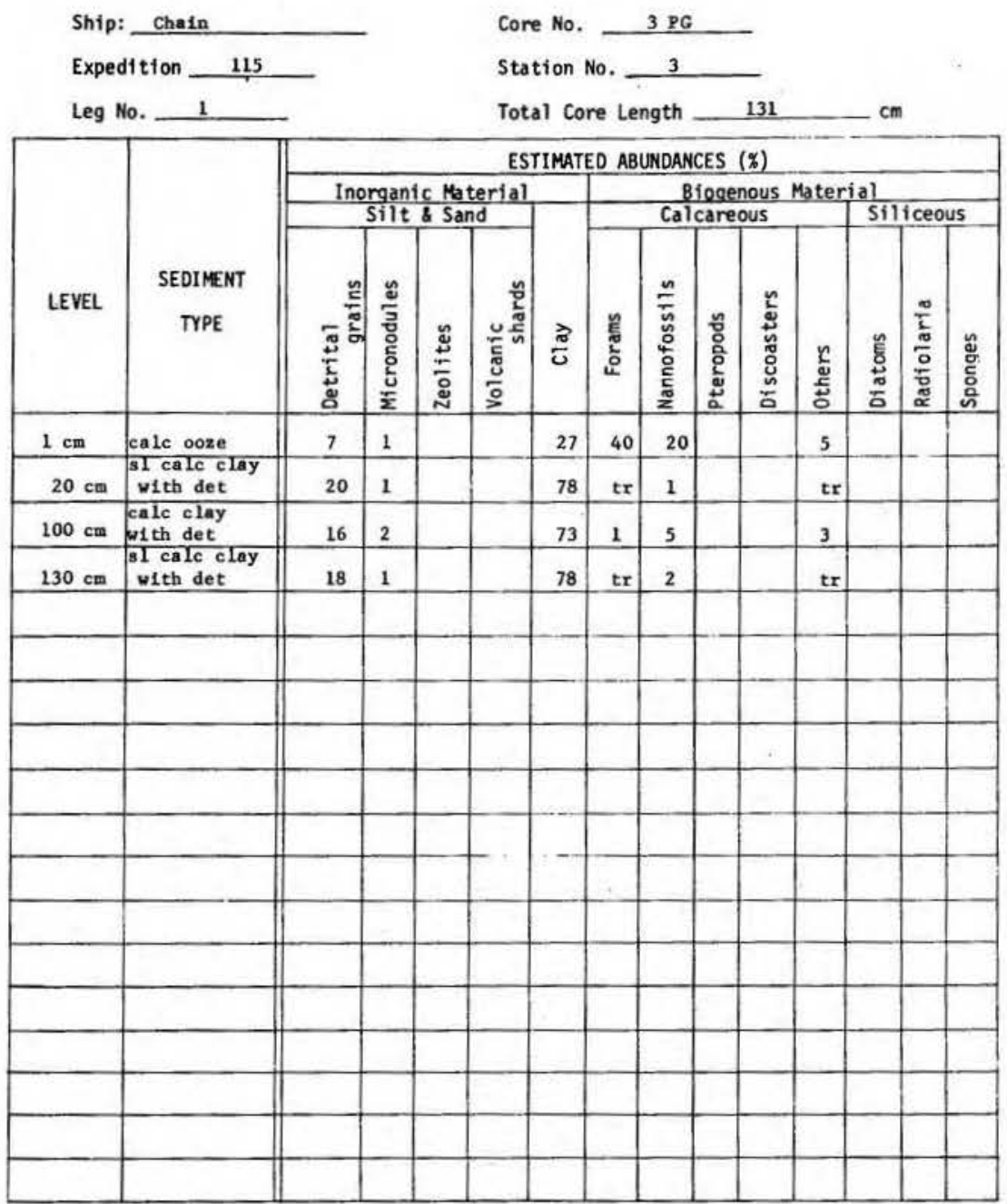

203

VISUAL CORE DESCRIPTION

Page 1 of 2

Ship CHAIN Cruise 115 Leg 1 Sta. 4 Core No. 4 PC Total Length $213 \mathrm{~cm}$. Lot. $399^{\circ} 20.1^{\prime} \mathrm{N}$ Long. $67^{\circ} 08.1 \mathrm{~W}$ Depth $2536 \mathrm{mearr}$. Core condition EXLELLENT Date Described $11 \mathrm{H}$ WIL 75 by froda

Physiographic location MYTILUS SEAMUUNT... NEW ENGLAND SEAMUUNT CHAIN.

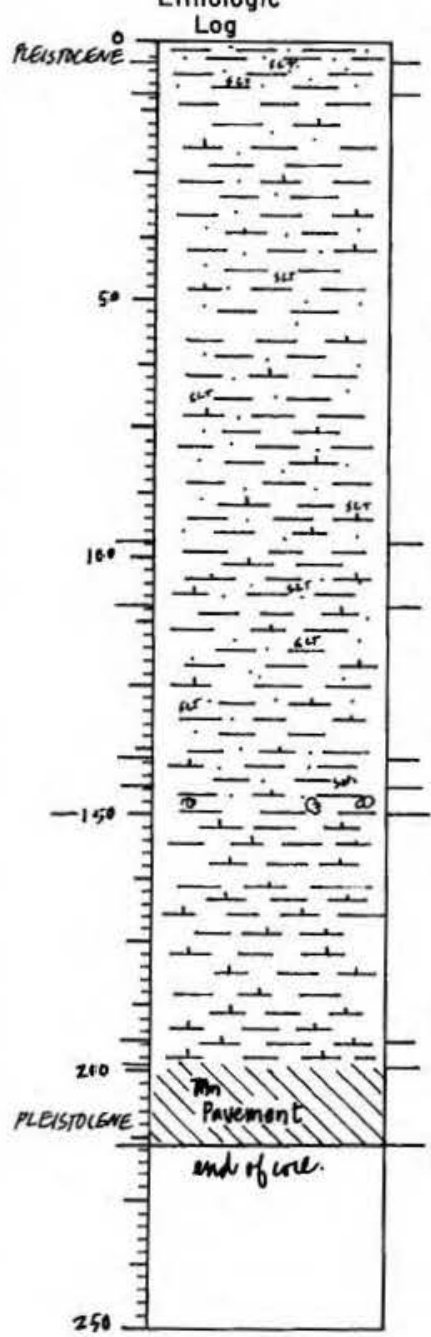

Detailed Description

0-3 UNPOSS CLAY WITH DETRTTUS $5 \times 4 / 3$ olive compact silty lutite with bh ck flecks thinly laminated $s$ throughout

UNPOSS CLAY WITH DETRITUS

silty lutite uith onalitifthifed olive and brow nodules scattered throughout $8-97^{\mathrm{S}, \mathrm{H}}$

SLICHTLY CAIC CUYY HITH DETRITUS

varying hues of $5 Y 4 / 2$ olive gray and $10 Y R \quad 4 / 2$ dark graylsh brown

nodules scattered throughou

at $92 \mathrm{~cm}$ a $5 Y 2 / 1$ black very silty lithified inclusion (m) 1 can diameter

$\mathrm{s}$
$97-109$

SLIGHTLY CALC CLAY
1OYR $3 / 3$ dark brown

mooth lutite with scattered forams and 1-2 min olive gray $6^{139}$

109-139

SLIGRTLY CALC CIAY WITH DETRITUS

the lutite with one slick gray band 134-139 can S

139-144

SLIGHTLY CALC CLAY WITH DETRITUS

.5Y 6/2 11ght brownish gray

oram sand with $\operatorname{smal1}$ percentage of lutite at base and $\mathrm{s}^{\mathrm{nu}}$

SLICHILY CALC CLAY HITH DETRITUS

7.SYR $5 / 4$ brown

inn exmpact lutite with abund. forams s

HLY CALC CLAY WITH DET

7.5YR $5 / 2$ brown

arbling throughout

compact lutite with abund. forams and thin laminations and streaks of black flecks 
204

VISUAL CORE DESCRIPTION

Page 2 of 2 Ship CHAIN Cruise 115 Leg 1 Sta. 4 Core No. 4PC

Lithologic Log

\section{Detailed Description}
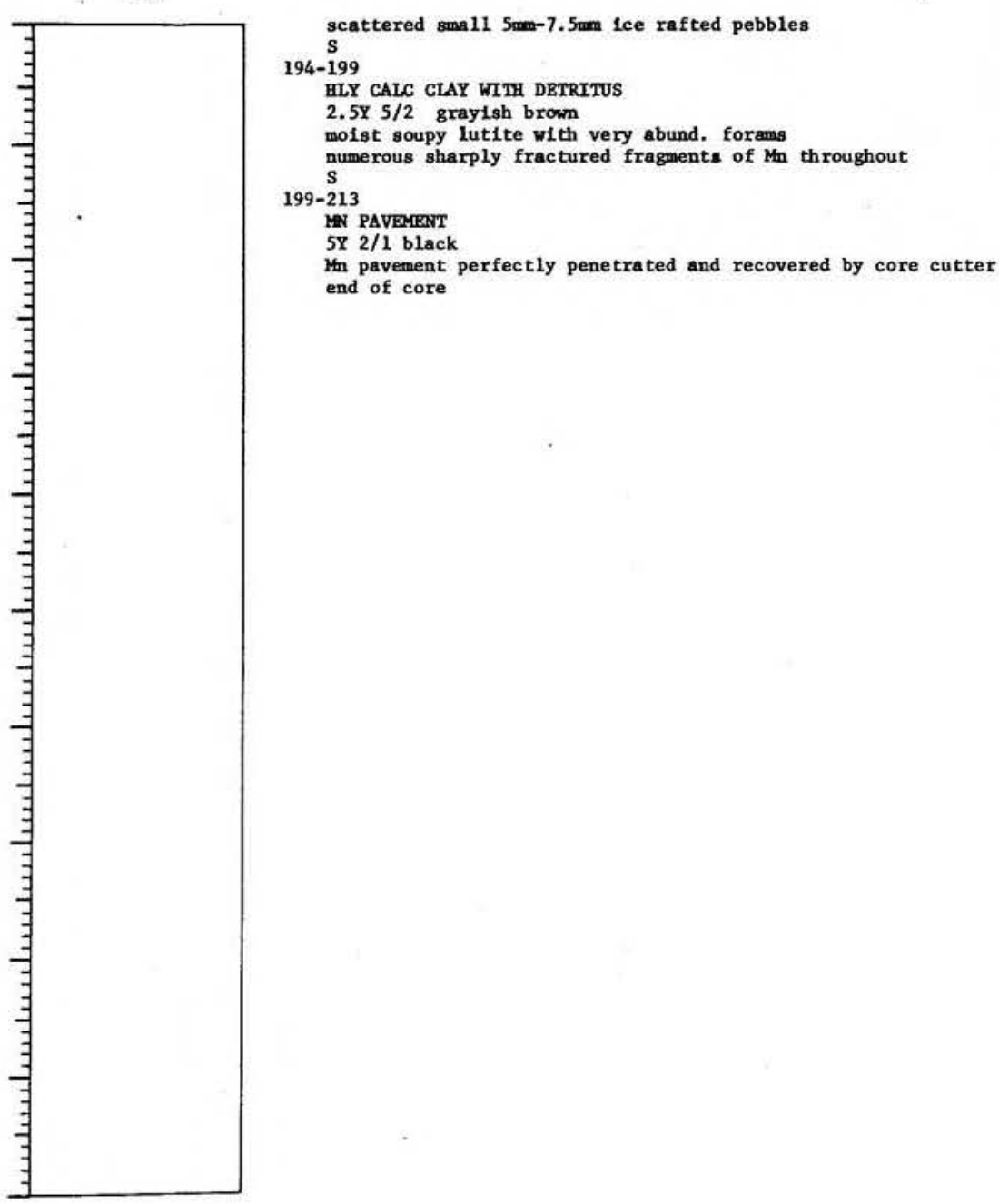

205

SHEAR SLIDE DESCRIPTIONS - W.H.O.I. SEDIMENT CORES

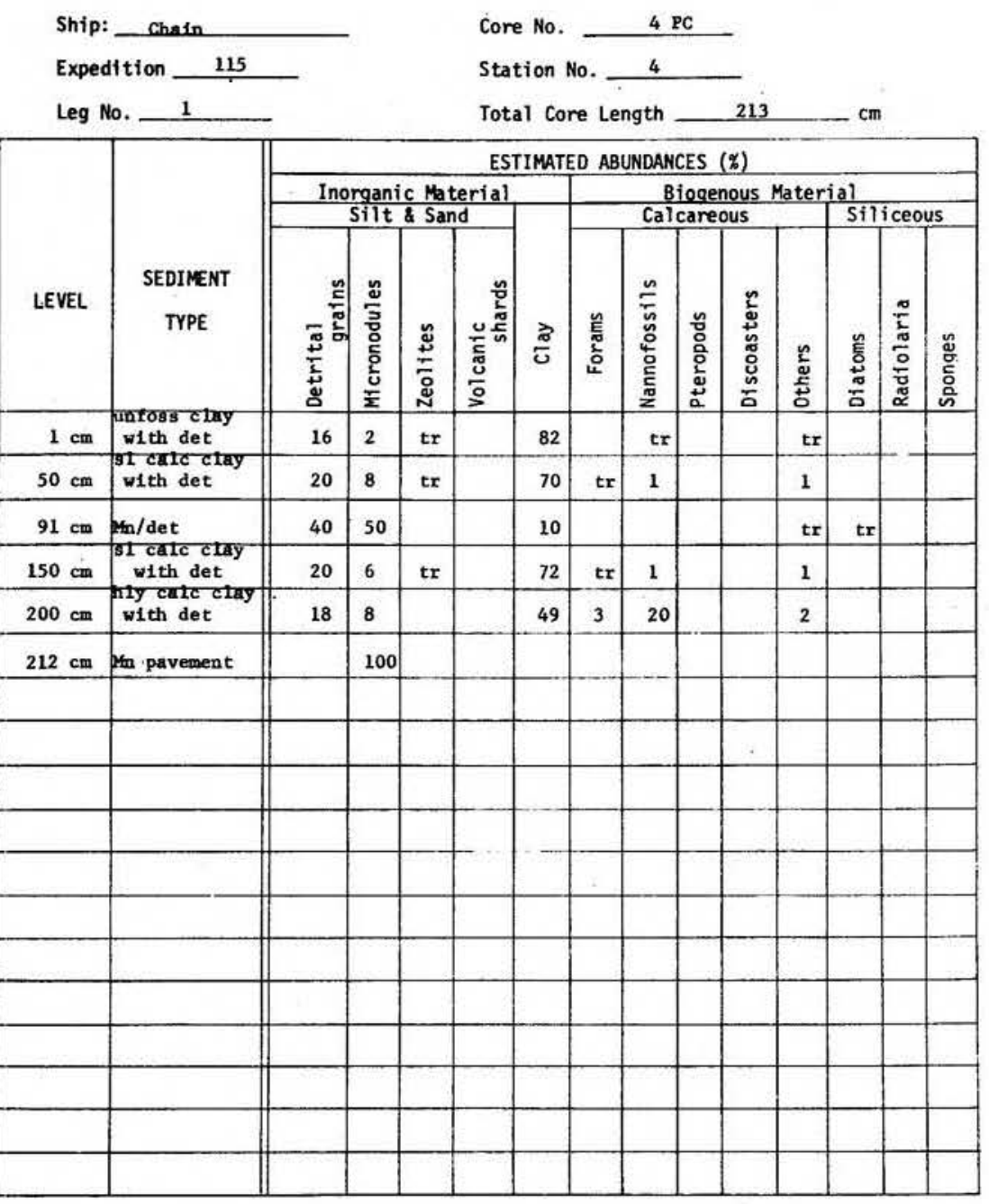


206

VISUAL CORE DESCRIPTION

Page 1 of 1

Ship CHAIN Cruise $115 \quad 4 \quad 15$ Sto 4 Core No 4 PG Total Length $137 \mathrm{~cm}$. Lat. $39^{\circ} 20 . \mathrm{I}^{\mathrm{N}}$ Long $17^{\circ} \mathrm{OS} .1 \mathrm{~W}$ Depth $2536 \mathrm{~m}$.com

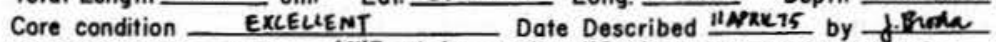
Physiogrophic location MYTILUS SEAMOVNT... NEWRENLLAND SEAMOVUNT CHATN.

Lithologic

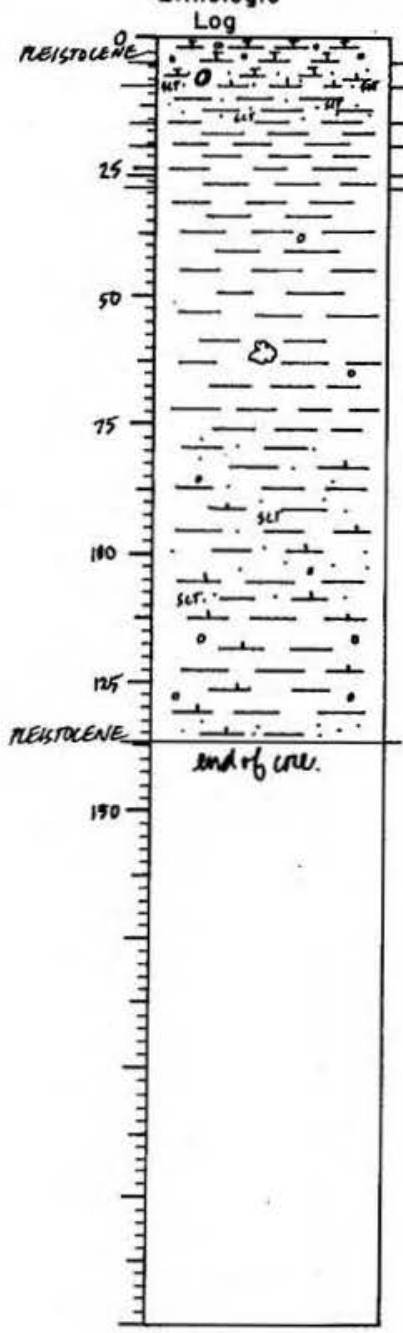

\section{Detailed Description}

\section{CALC OOZE}

10YR $6 / 2$ 11ght brownish gray

mo1st foram sand with 1-2 mm sized pebble gralns courson $G$

\section{CALC OOZE WITI DETRITUS}

foras sand srades to silick lutite with abund. forams, $2.5-5$ m dimeter pebbles scattered throughiout ice rafted pebble, $1.5 \mathrm{~cm} \times 1 \mathrm{~cm}$ between 5 and $7 \mathrm{~cm}$

-16 textural

UNFOSS CLAY WITH DETRITUS

1OYR $5 / 4$ yellowish brown

$5 \mathrm{~mm}$ lamination of partially 1ithified lutite, $7.5 \times 5 / 4$ brow at $10 \mathrm{~cm}$ also fine marbling and laminae of same color mo1st slick lutite $\stackrel{\mathrm{m}}{\mathrm{G}}$

16-21

UNFOSS CLAY

$5 \mathrm{Y} 5 / 2$ olive gray

very fine brown lanination at $17 \mathrm{~cm}$

molst silck lutite

21-26

UNPOSS CLAY

UNPOSS CLAY

silty lutite with abund. black fleck $S$ textural

26-29

SY $5 / 2$ ollve gray

and bleck flecks, alo a somentat graded bed of 1-2 mized grains $28-28.5 \mathrm{~cm}$ 5 textural

29-137

UNPOSS CIAY GRADES TO CALC CLAY

2.SY $5 / 2$ graylsh brown

oist lutite with varying amounts of silt, more compect at 117

cee rafted debris cormon the $77-117 \mathrm{~cm}, 133-135 \mathrm{~cm}$

1ithified lump at $60 \mathrm{~cm}$

end of core
207

SIEAR SLIDE DESCRIPTIONS - W.H.0.I. SEDIMENT CORES
Ship: Chain

Expedition 115

Leg No.
Core No. 4 PG

Station No. 4

Total Core Length -132

\begin{tabular}{|c|c|c|c|c|c|c|c|c|c|c|c|c|c|c|}
\hline \multirow[b]{3}{*}{ LEVEL } & \multirow[b]{3}{*}{$\begin{array}{c}\text { SEDIMENT } \\
\text { TYPE }\end{array}$} & \multicolumn{13}{|c|}{ ESTIMATED ABUNDANCES ( $(x)$} \\
\hline & & \multicolumn{4}{|c|}{$\begin{array}{c}\text { Inorganic Material } \\
\text { Silt \& Sand }\end{array}$} & \multirow[b]{2}{*}{$\frac{\pi}{6}$} & \multicolumn{5}{|c|}{$\begin{array}{l}\text { Biogenous Mater } \\
\text { Calcareous }\end{array}$} & \multicolumn{3}{|c|}{ Siliceous } \\
\hline & & $\frac{5}{5}$ & $\begin{array}{l}\frac{\omega}{3} \\
\frac{5}{0} \\
\vdots \\
\frac{0}{2} \\
\frac{5}{2}\end{array}$ & 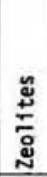 & 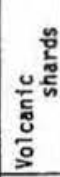 & & 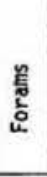 & 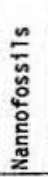 & 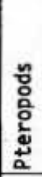 & 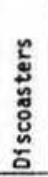 & 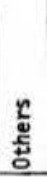 & 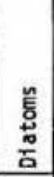 & $\frac{\pi}{\frac{\pi}{L}}$ & 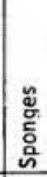 \\
\hline $1 \mathrm{~cm}$ & calc ooze & 2 & 1 & & & 17 & 50 & 25 & & & 5 & & & \\
\hline $10 \mathrm{~cm}$ & $\begin{array}{l}\text { Unfoss clay } \\
\text { with det }\end{array}$ & 16 & 2 & & & 82 & & tr & & & tr & & & \\
\hline $70 \mathrm{~cm}$ & unfoss clay & 14 & 2 & & & 84 & & tr & & & tr & & & \\
\hline $132 \mathrm{~cm}$ & cale clay & 4 & 1 & & & 78 & tr & 15 & & & 2 & & & \\
\hline & & & & & & & & & & & & & & \\
\hline & & & & & & & & & & & & & & \\
\hline & 7 & & & & & & & & & & & & & \\
\hline & & & & & & & & & & . & & & & \\
\hline & & & & & & & & & & & & & & \\
\hline & & & & & & & & & & & & & & \\
\hline & & & & & & & . & & & & & & & \\
\hline & & & & & & & & & & & & & & \\
\hline & & & & & & & & & & & & & & \\
\hline & & & & & & & & & & & & & & \\
\hline & & & & & & & & & & & & & & \\
\hline & & & & & & & & & & & & & & \\
\hline & & & & & & & & & & & & & & \\
\hline & & & & & & & & & & & & & & \\
\hline
\end{tabular}


208

VISUAL CORE DESCRIPTION

Page 1 of 1

Ship CHAIN Cruise 115 Leg 1 Sta. 5 Core No. 5 PC

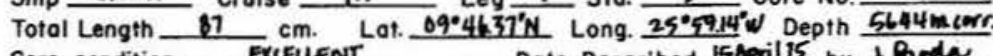
Core condition EXCELENT Date Described 15 April 15 by fhoda Physiogrophic location GAMBIA ABYSSAL PLAIN.

Lithologic

Detailed Description

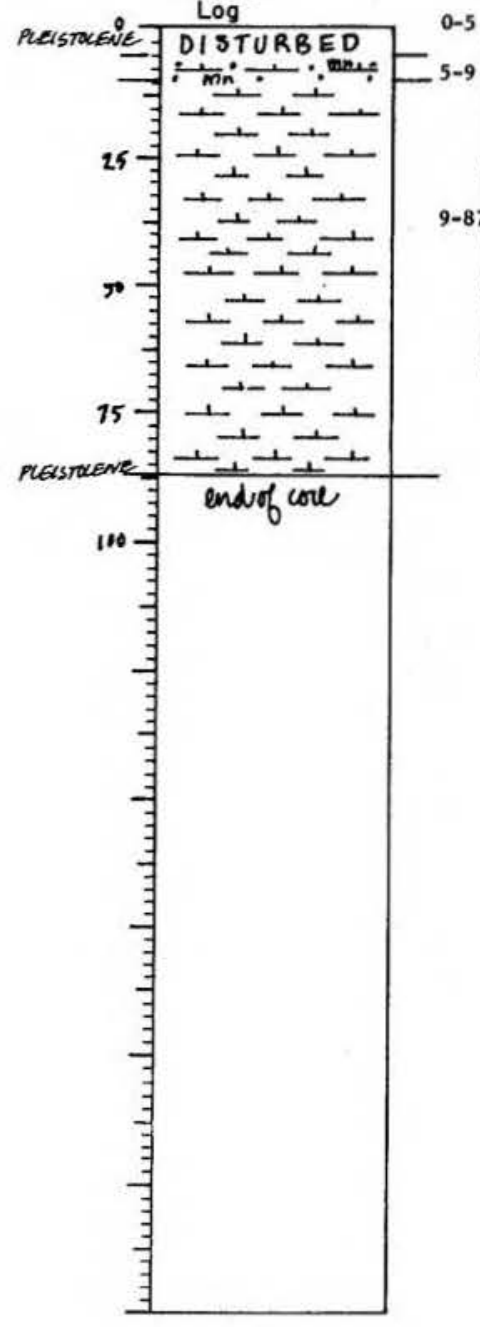

UASHED AND DISTURBET

CALC OOZE

above molst slick lutite

S, 1

\section{CALC OOZE.}

$2.5 \times 5 / 2$ gray1sh brown

very moist slick lutit

end of core

top tost

during recovery as core cutter sheared off upon pullowt.

\section{9}

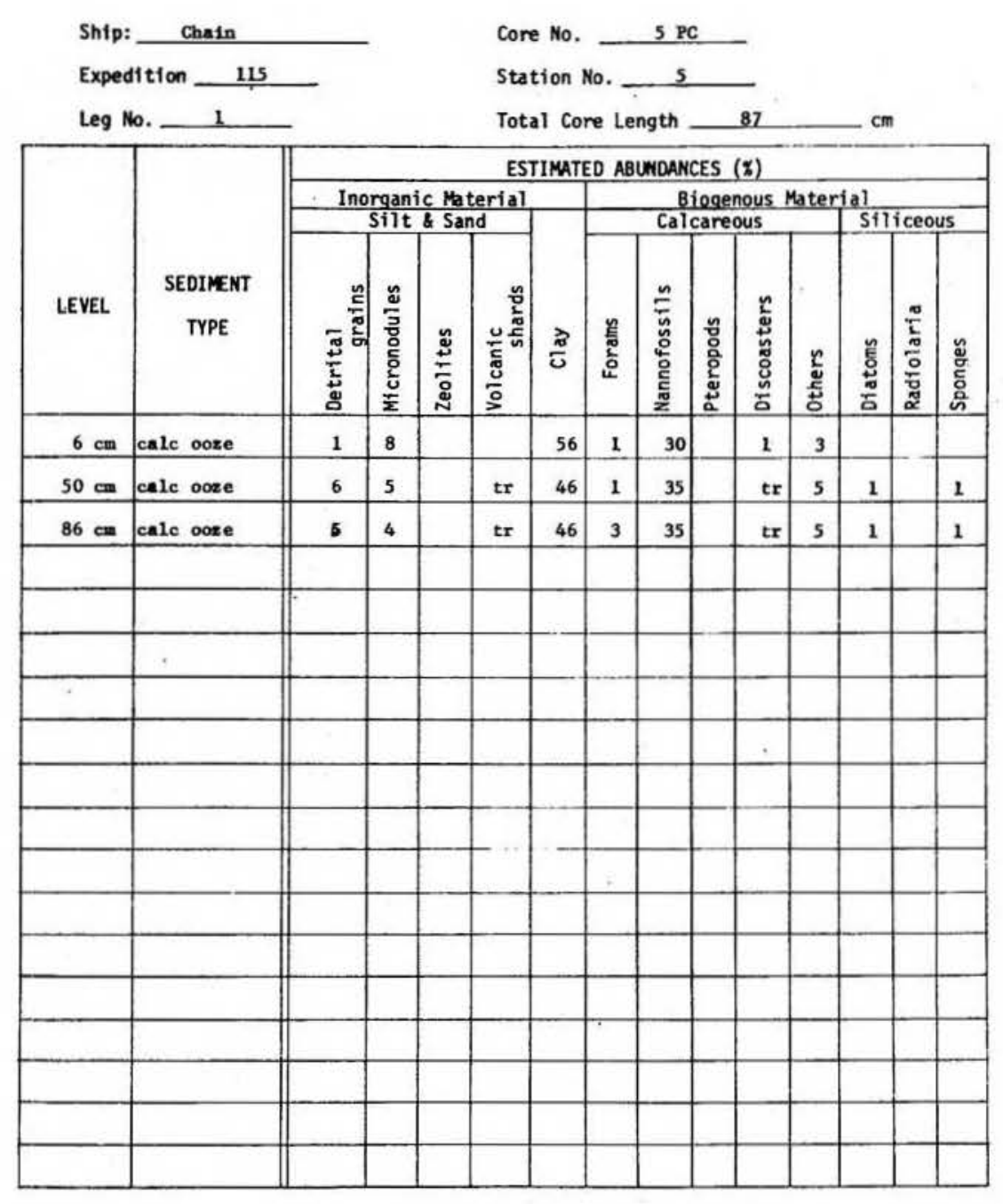

\section{SHEAR SLIDE DESCRIPTIONS - W.H.O.I. SEDIIENT CORES}


210

VISUAL CORE DESCRIPTION

Page 1 of 1

Ship CHAIN Cruise 115 Leg $t$ Sto. 5 Core No. $5 P_{G}$

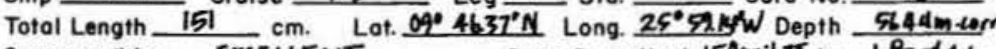

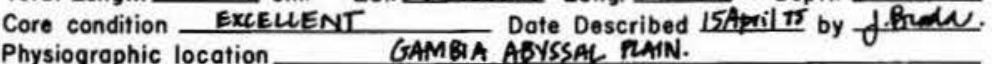

Physiographic location

Lithologic

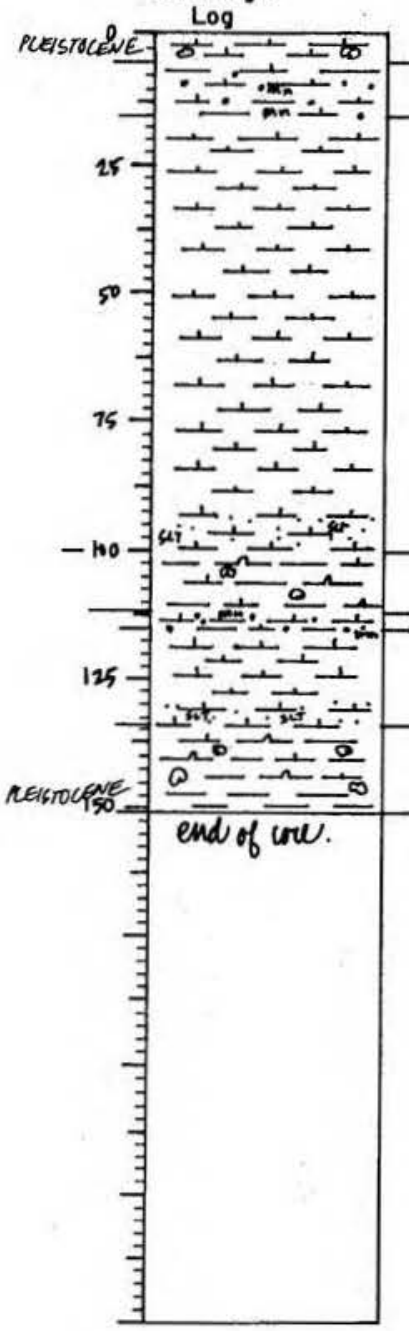

$0-5$

Detailed Description

CALC OOZE

fatn

moist slightly silty lutite with scattered foram very $G$

HGHLY CALC CLAY WITH MU MICRO MODULES

OYR $6 / 3$ pale brown, 20 RR $5 / 3$ brown and $4 / 3$ dark brown

15-100

\section{CALC OOZE}

$2.5 \mathrm{Y} 5 / 2$ graylsh brow

homogeneous $15-90 \mathrm{~cm}$, inclined and irregular, lighter silty

very molst s1ick lutite $15-90 \mathrm{~cm}$, very silty lutite $90-100 \mathrm{~cm}$ s,

HIGALY CALC SILIC CTAY

10YR 6/3 pale brown

firm compact slightly allty lutite with falrly abund. forams

-115

HIELLY CAIC CLAY WITH Ma MICROBODULRS

6/3 pale brom, $5 / 3$ brown, $4 / 3$ derk brova

coeplete fine intermicrolamination of the colon above

\section{CALC OOZE}

$2.5 \times 5 / 2$ grayish brown $133-134 \mathrm{~cm}$ cm mo1st slick lutite $115-133 \mathrm{~cm}$, very silty $133-134 \mathrm{~cm}$

134-151

HIGHLY CALC -SILIC CIAY, GRADES TO UNFOSS CLAY 10XR $6 / 3$ pale brown

firm, compact, slightly silty lutite vith abund, foram end of core

Note; Due to a repetitive occurrence of similar lithologies ch1s core is suspected to be an example of a multiple pen-

$0-112 \mathrm{~cm}, 2$ d pente

has all features of the ftrat but has been greatly cotration due to the resistance offered by the sediment already in the 1iner.

211

SIEAR SLIDE DESCRIPTIONS - W.H.O.1. SEDIMENT CORES

Ship: Chain

Expedition 115

Leg No. 1
Core No. 5 PG

Station No.

Total Core Length 151

ESTIMATED ABUNDANCES ( $(x)$

\begin{tabular}{|c|c|c|c|c|c|c|c|c|c|c|c|c|c|c|}
\hline \multirow[b]{3}{*}{ LEVEL } & \multirow[b]{3}{*}{$\begin{array}{c}\text { SEDIMENT } \\
\text { TYPE }\end{array}$} & \multicolumn{13}{|c|}{ ESTIMATED ABUNDANCES ( $x$ ) } \\
\hline & & \multicolumn{4}{|c|}{$\begin{array}{l}\text { Inorganic Material } \\
\text { Silt \& Sand }\end{array}$} & & \multicolumn{5}{|c|}{$\begin{array}{l}\text { Biogenous Mater } \\
\text { Calcareous }\end{array}$} & \multicolumn{3}{|c|}{ al } \\
\hline & & 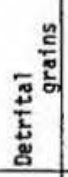 & 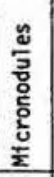 & 岕 & 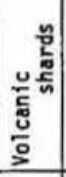 & $\frac{\pi}{5}$ & 䇾 & 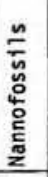 & 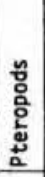 & 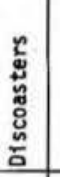 & $\begin{array}{l}\mathfrak{k} \\
\text { E⿱ } \\
\text { cे } \\
\end{array}$ & 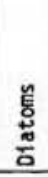 & 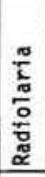 & 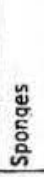 \\
\hline $1 \mathrm{~cm}$ & calc cose & 5 & 10 & & tr & 49 & 1 & 30 & & $\mathrm{tr}$ & 5 & & & \\
\hline $12 \mathrm{~cm}$ & $\begin{array}{l}\text { hiy calc clay } \\
\text { with He micro }\end{array}$ & 2 & 18 & & & 54 & 1 & 22 & & $t x$ & 3 & & & \\
\hline $50 \mathrm{~cm}$ & calc ooze & 5 & 5 & & $\operatorname{tr}$ & 48 & 1 & 35 & & & 5 & 1 & & tr \\
\hline $95 \mathrm{~cm}$ & detritus & 80 & 4 & & & 5 & 8 & & & & 3 & & & \\
\hline $110 \mathrm{~cm}$ & $\begin{array}{l}\text { highly calc- } \\
\text { silic clay } \\
\end{array}$ & 2 & 1 & & & 67 & 10 & 15 & & $\mathrm{tr}$ & 3. & 1 & & 1 \\
\hline $125 \mathrm{~cm}$ & calc ooze & 5 & 4 & & & 46 & 1 & 35 & & $\mathrm{tr}$ & 5 & 1 & & 1 \\
\hline $150 \mathrm{~cm}$ & unfoss clay & 2 & 2 & & & 96 & $\mathrm{tr}$ & $\mathrm{tr}$ & & $\mathrm{tr}$ & $t x$ & tr & & tr \\
\hline & & & & & & & & & & . & & & & \\
\hline & & & & & & & & & & & & & & \\
\hline & & & & & & & & & & & & & & \\
\hline & & & & & & & & & & & & & & \\
\hline & & & & & & & & & & & & & & \\
\hline & & & & & & & & & & & & & & \\
\hline & & & & & & & & & & & & & & \\
\hline & & & & & & & & & & & & & & \\
\hline & & & & & & & & & & & & & & \\
\hline & & & & & & & & & & & & & & \\
\hline & & & & & & & & & & & & & & \\
\hline
\end{tabular}


Ship CHAIN Cruise 115 Leg 1 sto. 6 Core No. 6 PC Total Length $870^{\circ} \mathrm{cm}$. Lat. $09.30 .0^{\circ} \mathrm{N}$ Long. $22^{\circ} \mathrm{OL} .0^{\circ} \mathrm{W}$ Depth A8002.Cerr.

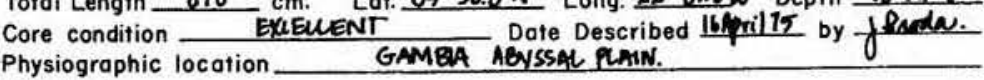
Lithologic

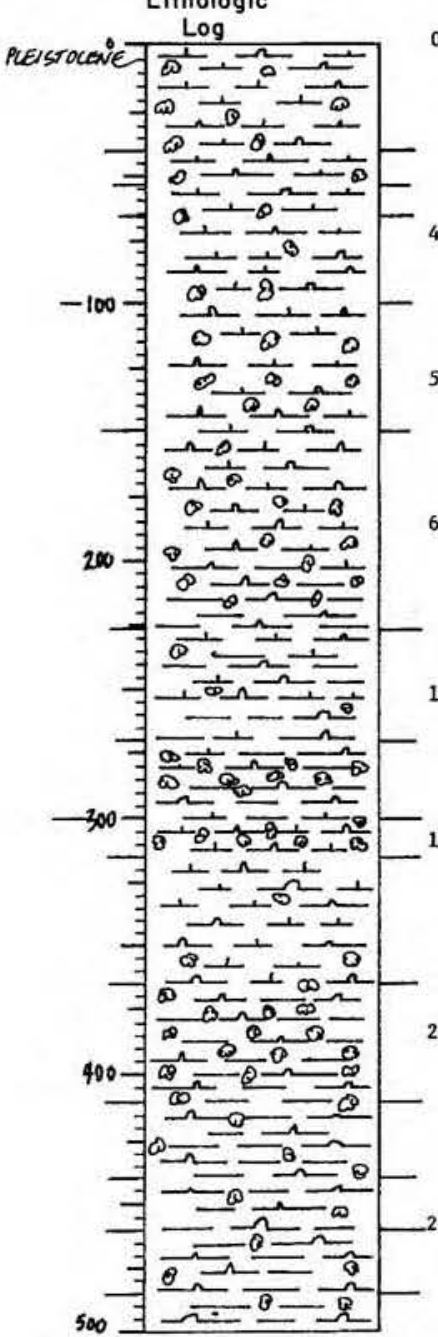

Detailed Description

0-40 Detailed OXR $4 / 4$ extensive marbling and mottling with dark brown, very dark grayish brown and gray very molst slick lutite with scattered forams, $0-6 \mathrm{~cm}$ and pocket of foram and 35-39 ca

40-5 CALC SILIC OOZE

1OYR $4 / 2$ dark grayish brown

extensive brown and graylsh brown mottling throughout moist slick lutite with scattered foram

CALC SILIC OOZE 1OYR $6 / 4$ light yellowish brown

extensive gray and grayish brown mottling and marbling moist slick lutite with a few foram 5 mottled

\section{SILIC CALC OOZB}

\section{$2.5 \mathrm{Y} 3 / 2$ very dark gray 1 sh brown}

extensive light yellowish brown and light brownish gray mottling $82-100 \mathrm{~cm}$ moist slightly silty lutite with scattered forams moist
S mot
$00-150$
SIIIIC

ILIC CALC OOZD

LOXR 6/3 pale brown grades to $5 / 4$ yellowish brown pale brown and 1ight brownish gray mottling $S$ mottled

150-226

SILIC CALC OOZE GRADES TO SILIC CIAY

YYR $6 / 4$ light yellowish brown grades to $6 / 2$ light bmenals ery ex marbling dive dark gray and grayish brown mottling and moist slick lutite with a few forams $\underset{226-270}{\text { S }}$

HIGHLY CALC SILIC CLAY

(o $5 / 4$ ight yellowish brown grades to $5 / 6$ yellowish brown light gray and light gray mottilng

excellent foram rich gray bu unit basal contac 5 inclined $10^{\circ}$

HIGHLY CAIC SIEIC CIAY

1OYR $5 / 8$ yellowlsh brom

intensively mottled with light and dark grays $273-290 \mathrm{~cm}$ moist slightly silty lutite with abund. forams

Ship CHAIN Cruise 115 Leg 1 Sta. 6 Core No. GPC

Lithologic

Log

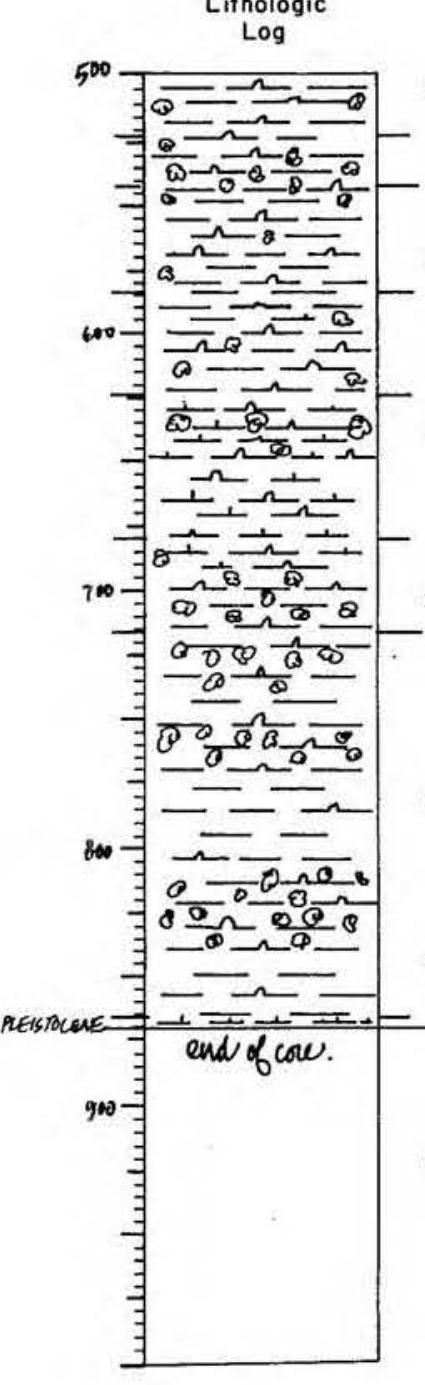

\section{Detailed Description}

$G$ mottled

300-315

HTC OOZE

CyR $6 / 3$ pale brown grades to $5 / 2$ grayish brown

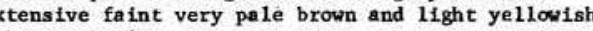
very abund. forams in moist silty lutite 15- 365

CALC SILIC OOZ

10YR $6 / 6$ brownish yellow grades to $5 / 4$ yellowish bown silght faint light gray and very pale brown mottling slick moist lutite with a few forams included in the mottles large $1 \mathrm{~cm} \times 2 \mathrm{~cm}$ lithified lump at $340 \mathrm{~cm}$

$\underset{365-410}{6 \text { mott }}$

SIUIC CIAY

1OYR $5 / 4$ yellowish brown

very extensive large and small dark gray and grayish brown

mottling tiffer more compact lutite with very few forams 6 mottled
mate

SILIC CIAY

10XR $5 / 6$ yellowish brown grades to $4 / 3$ brown

cormon 1ight gray and dark grayish brown mottling compact lutite with a few partially lithified inclusion am lithified concave upward yellowish brown lamination at $431 \mathrm{~cm}$ $440-460$

SILIC CIAY

1OYR $5 / 2$ gray 1sh brown grades to $3 / 1$ very dark gray

slight faint pale brown motting throughout smooth compact lutite grades to slightly silty lutite with scattered foram

$460-485$

SITIC CLAY

10YR $6 / 3$ pale brown grades to $6 / 4$ light yellowish brown slight gray mottling throughout, extensive in the zone $477-482 \mathrm{~cm}$

very compact lutite with a few forams scattered, more abund. at upper contact

485-522

SILIC CLAY

IOYR $5 / 1$ gray and $4 / 1$ dark gray

a few la rge elliptical dark gray moteles

few forams $\mathrm{S}$ mottled
repeatelified, almost dry, olive gray $2 \mathrm{~mm}$ thick laminae 
VISUAL CORE DESCRIPTION

Ship CHAiN Cruise 115
Lithologic

Log

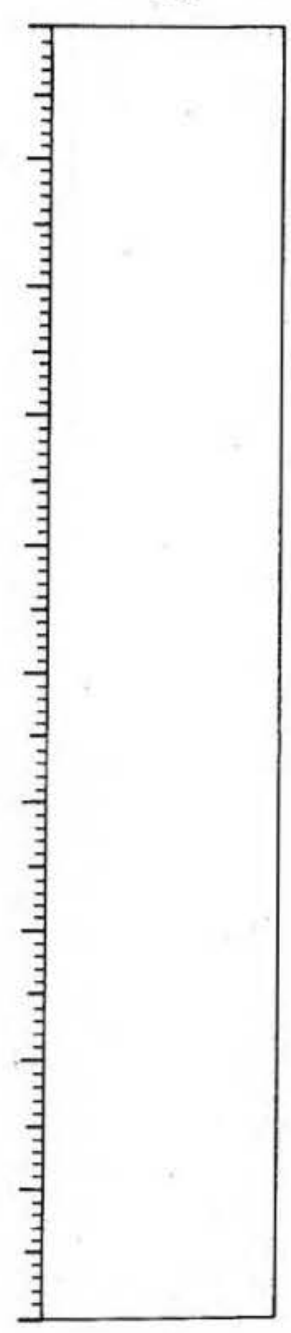

Page 3 of 3 6 Core No. 6 PC
$522-542$

SILIC CLAY

10YR $5 / 2$ grayish brown grades to $6 / 3$ pale brown extensive large, gray and gray - brown mottling throughout
slick but compact lutite with scattered forams in mottles $542-584$

SILIC CIAY GRADBS TO SLIGHTLY SILIC CLAY

1OYR $5 / 4$ yellowtsh brown grades to $6 / 3$ pale brown

very fatnt light gray mottiling found comonly $557-575 \mathrm{~cm}$ molst compact slightly silty lutite with scattered foram $G^{8}$

584-624

SLICHTLX SILIC CLAY GRADES TO SILIC CLAY $7.5 Y R 5 / 6$ strong brown grades to $5 / 4$ yellow 1 sh brown silght gray and grayish brom mottling

smooth compset lutite

very fine, brittle black lamination $0.5 a k$, apana entire liner in $598 \mathrm{~cm}$, sewilithified sediment found $2 \mathrm{~mm}$ above and belo $602-604 \mathrm{~cm}$ spans liner, ocese vhat concave upuard entire zone is very compact, almost dry and slightly dis-

$\underset{624-680}{G}$

CALC SILIC OOZE

(n)

mansive dark gray $630-645 \mathrm{~cm}$

abund. $624-645$ a

6 mottled

-716

CALC SILIC DOZE GRADES TO SLIGaTIY SILIC CLAY

10YR 5/1 gray grades to $5 / 2$ gxayish brown

extensive 11ght gray and dark gray marbling and mottiling

compact slightly silty foram rich lutit 116-866

SLIGHILY SILIC CLAY

IOYR $6 / 4$ light yellowish brown grades to $6 / 3$ pale brown

common gray and dark gray mottling throughout, very exteng

and doninant in the zones, $716-738,750-776,815-840 \mathrm{~cm}$

firm very compact silty lutite with scattered forams mostly $c^{10}$

866-870

BANNO OOZE

10YR $7 / 4$ very pale brown

ve light gray mottling and burrowing

fin sirghty silty lutite uith scattered foram

end of core

\section{5}

SMEAR SLIDE DESCRIPTIONS - W.H.O.I. SEDIMENT CORES

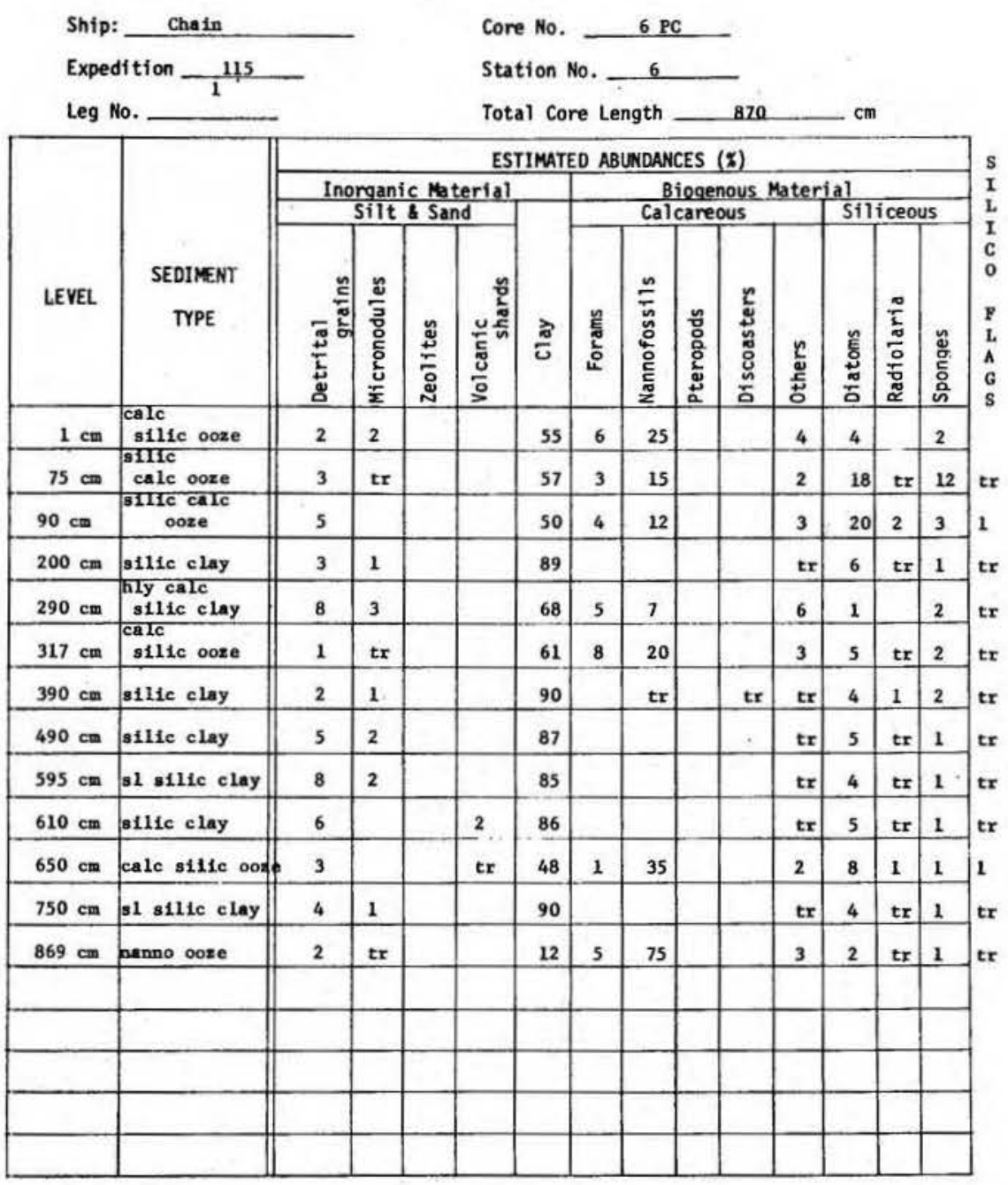


216

VISUAL CORE DESCRIPTION

Page 1 of 1

Ship CHAN Cruise 115 Leg 1 Sta. 6 Core No. 6 PG Total Length $153 \mathrm{~cm}$. Lat. $093^{\circ} 30.0^{\circ} \mathrm{N}$ Long. $\frac{6}{22^{\circ} 01.04 \mathrm{~W}}$ Depth $4800 \mathrm{~m} .0 \mathrm{mr}$. Core condition ExcEuENT. Date Described theril7s by feroda. Physiographic location GAMBIA ABYSSAL RAAIN.

Lithologic

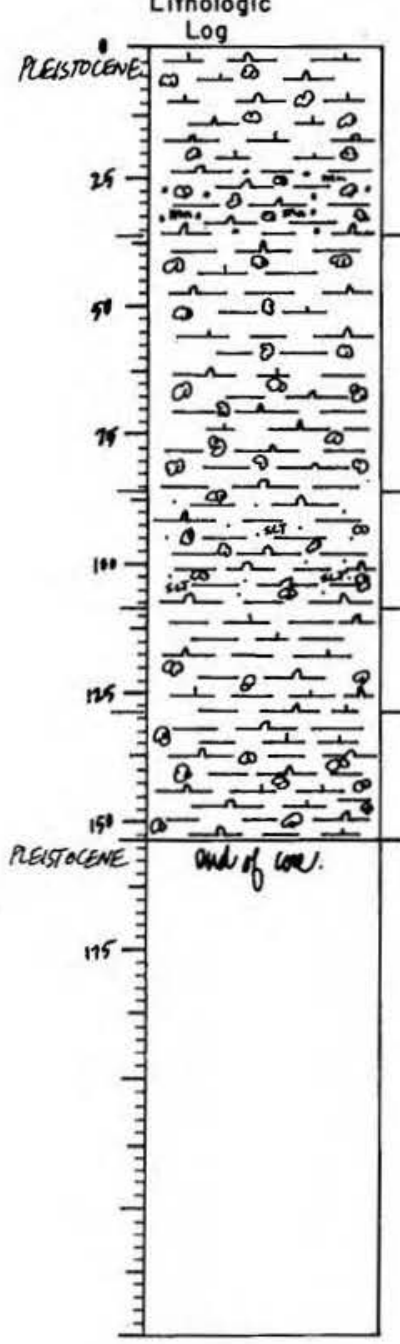

CALC SILIC DOZE GRADES TO SILIC CLAY WITH Mn MICRONODULRS OrR $4 / 4$ dark yellowish brow Jlowish brown mottling and marbling at lite withered forams most abund. at top core amination of slick black flecks $20-22 \mathrm{~cm}, 34-36 \mathrm{~cm}$ 6 mottled

\section{HIGHLY SILIC CALC CIAY}

$2.5 \times 3 / 2$ very dark grayish brown grades to $4 / 2$ dark graylsh brown very ex

ing slick molat lutite with a few scattered forams 6 mottled

(108)

ILIC CLAY WITH DETRITUS

OXR $6 / 3$ pale bmon grades to $5 / 6$ yellowish brown enstve brownish yellow, 11ght yellowlah brown and 1ight gray wetting and marbing throughout mo1st silghtly silty lutite, forams very abund. $86-93 \mathrm{~cm}$,

$108-128$ HIGHIX CAIC SHIXC CLAY 10YR $4 / 2$ dark grayish brow

conson pale brown mottiling $120-128 \mathrm{~cm}$ molst silghtly silty lutite with a few foram 8-. 15 HLY CALC SILIC CLAY

iight yellowish brown grades to $6 / 3$ pale brom very extensive 1ight gray and grayish brown mottling and noist slightly silty lutite with scattered forams end of cor

\section{7}

SIEAR SLIDE DESCRIPTIONS - W.H.O.I. SEDIMENT CORES

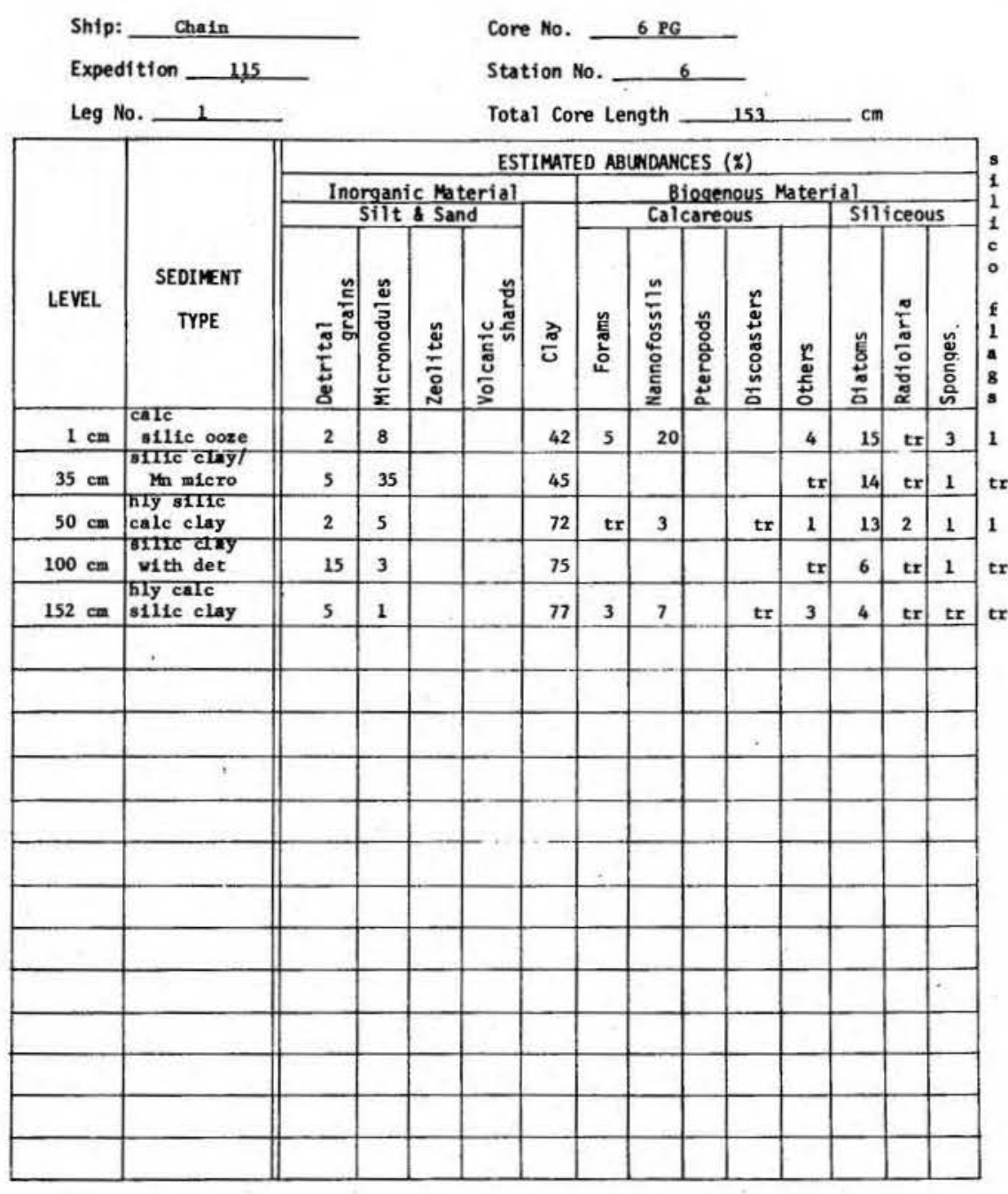


218

VISUAL CORE DESCRIPTION

Page 1 of 3

Ship CHAIN Cruise 115 Leg 1 Sta. 7 Core No. TPC Total Length $870 \mathrm{~cm}$. Lat. $09 \cdot 08.0^{\circ} \mathrm{N}$ Long. $20^{\circ} 03.1 \mathrm{~W}$ Depth $3806 \mathrm{m.corr}$.

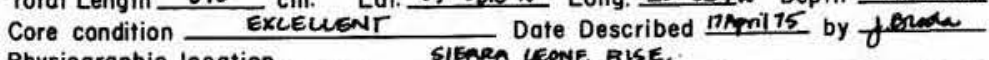
Physiographic location SIERRAn LEONE RISE.

Lithologic

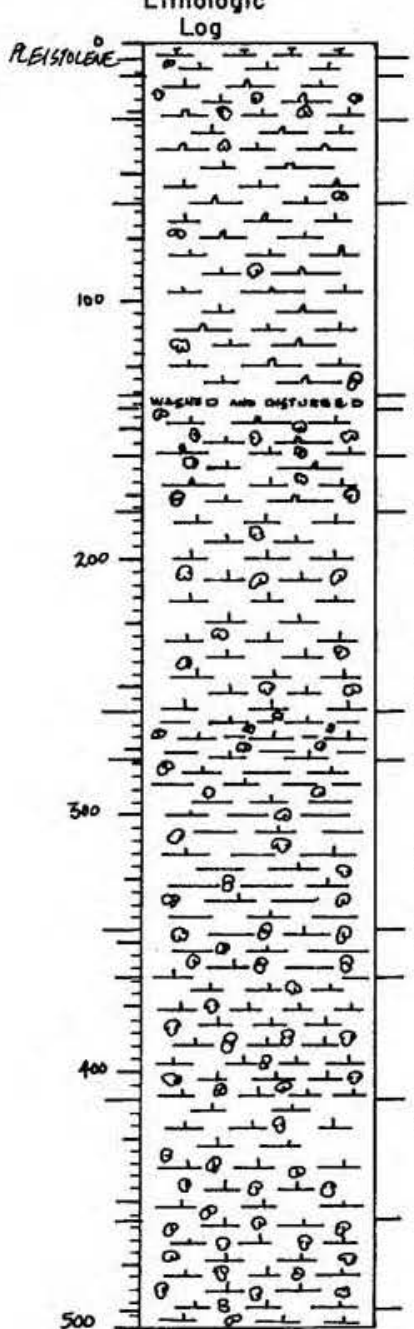

$0-5$

Detailed Description

\section{CALC OOZE}

IOYR $7 / 3$ very pale brown

entire unit someshat washed and reworked

5-17

CALC OOZE

fark $6 / 4$ light yellowish brown

列 moist unconsolidated lutite with scattered forams, 12-27 $G$ mottled

CALC SILIC OOZE

$2.5 \mathrm{Y} 4 / 2$ dark grayish brown grades to $3 / 2$ very dark grayish extensive light gray, pale brown and light yellowish brown

mottling

firmer, slightly silty lutite with scattered foram 27-62

CALC SILIC OOZE

10YR $6 / 4$ light yellowish brown grades to $6 / 3$ pale brown moist, slightly silty lutite with abundant foram very

CALC SILIC OOZE

OYR $6 / 4$ 11ght yellowish brown grades to $6 / 3$ pale brown then and very pale brown mottling s 36-142 Washed and disturbed pocket of silty lutite with very $142-160$

CALC SILIC OOZE

10YR $6 / 4$ 11ght yellowish brown

common to extensive 11ght and dark gray mottling and burrowing more compact, slightly silty lutite with scattered forams (1) $\mathrm{rlch}, 1.5 \mathrm{~cm}$ wi 160-182 $\mathrm{r}$ at $153-155 \mathrm{~cm}$

SILIC OOZE

J/2 grayish brown grades to $4 / 1$ dark gray

chered very dark gray motties, three excellent very pale

compact slightly silty lutite with scattered foram

$S$ mottled

219

VISUAL CORE DESCRIPTION

Page 2 of 3

Ship CHAIN Cruise 115 Leg 1 Sta. 7 Core No. IPC

Lithologic

Log

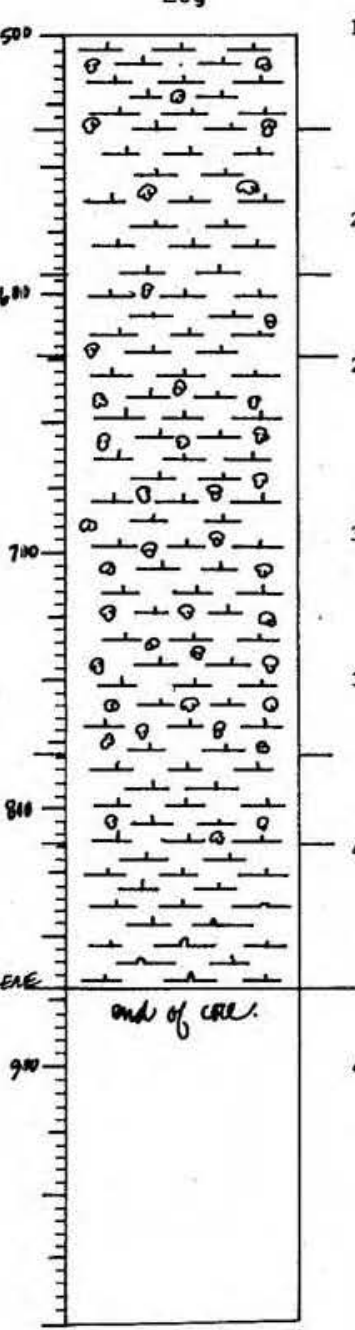

Detailed Description

CALC OOZE

varying hues of 10YR 6/2 light bromish gxay, 6/3 pale brown and $6 / 4$ 11ght yellowish brows

cournon light and dark gray mottling throughout

11ghtly silty lutite

260-279

CALC CLAY

10YR $5 / 6$ yellowish brow

extensive pale brown and very pale brown mottling compact silty lutite with scattered forams

279-34.5

CALC GLAY

10YR $6 / 3$ pale brown

extensive faint gray and light gray maxbling and mottling

throughout
scattered forams in firm slightly silty lutite

$\underset{345-364}{G \text { mottled }}$

CAIC CIAY

1OYR $5 / 4$ yellowish brown

extensive large gray and dark gray mottling throughout

silty lutite with very few forams except scattered in mottles

S64 mot

CALC $007 \mathrm{E}$

CALC OOZE
10YR $6 / 2$ 1ight brownish gray

extensive dark gray, light yellowish brown and gray mottling

and burrowing

inm slightly silty lutite with scattered foram S irregular

$-457$

CALC $6 / 4$ 11ght brownish gray grades to $6 / 3$ pale brown

faint gray shadowy marbling $410-430 \mathrm{~cm}$, tiny gray spherica 1 mottles extensive 430-457 cm

firm, oompact, silghtly silty lutite with scattered forams large, dark gray, $1 \mathrm{~cm}$ wide burrow spans entire liner at $414 \mathrm{~cm}$. $\stackrel{6}{6}$

CALC OOZE

$2.5 \mathrm{Y} 5 / 2$ grayish brown

extensive light gray, gray and pale brown mottling and

compact lutite with very few forams except scattered in $S$ irreguh $\mathrm{r}$ 
220

VISUAL CORE DESCRIPTION

Page 3 of 3

Ship CHAIN Cruise 115 Leg 1 Sta. 7 Core No. IPC

Lithologic Log

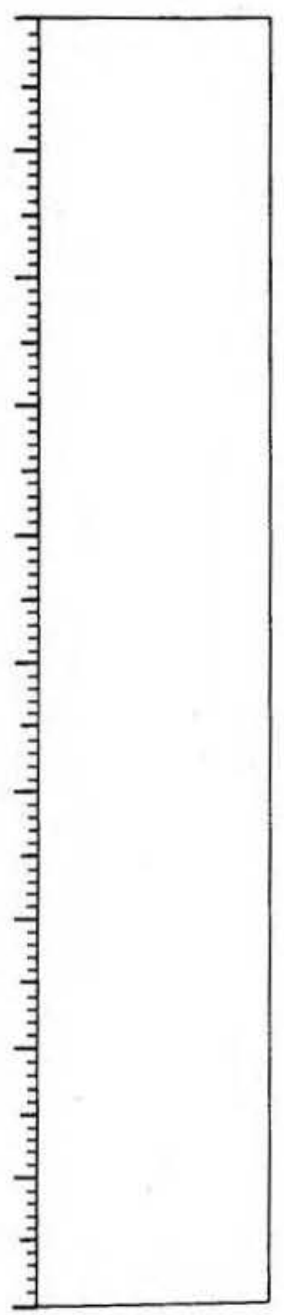

Detailed Description

493-535 CALC 0028

10YR $6 / 3$ pale brown grades to $6 / 4$ 11ght yellowish brown comon, faint, gray and light gray mottling compact slightly ailty lutite with scattered forams atery gray foram rich pocket at $510 \mathrm{~cm}$

CALC OOZZ

OYR $7 / 2$ 11ght gray grades to $6 / 3$ pale brown, then to $5 / 3$ brown a few very pale brown burrowa in the zone 559-572 cm very firm smooth lutite with abund. forams grades to very fer $2-624$

CAIC 002

1OYR $4 / 1$ dk. gray grades to $6 / 3$ pale brown

a few 11ght gray and pale brown mottles scattered throughout, extensive light yeliowish brown maxbling in the zone 603 $612 \mathrm{~cm}$

somevhat less consolidated, silightly silty lutite with s

624-778

of 10YR 6/2 11ght bromish gray and $6 / 3$ pale varying extensive, faint 11ght gray and gray marbling and mottling darker in the zonea $695-724$ gan

scattered forams more tougrds unit basal contact

78-814

OYR $5 / 4$ yellow1sh: brow

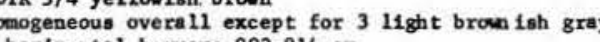
horlantal burrous $803-814 \mathrm{cad}$

very fer foras

$14-870$

CALC OOZR GRADES TO CALC SILIC OOZE

OYR $6 / 2$ light brownish gray grades to $6 / 4$ 11ght yellowish

very firm, compact, s1ightly silty lutite with abund. forams

\section{1}

SHEAR SLIDE DESCRIPTIONS - W.H.O.I. SEDIMENT CORES

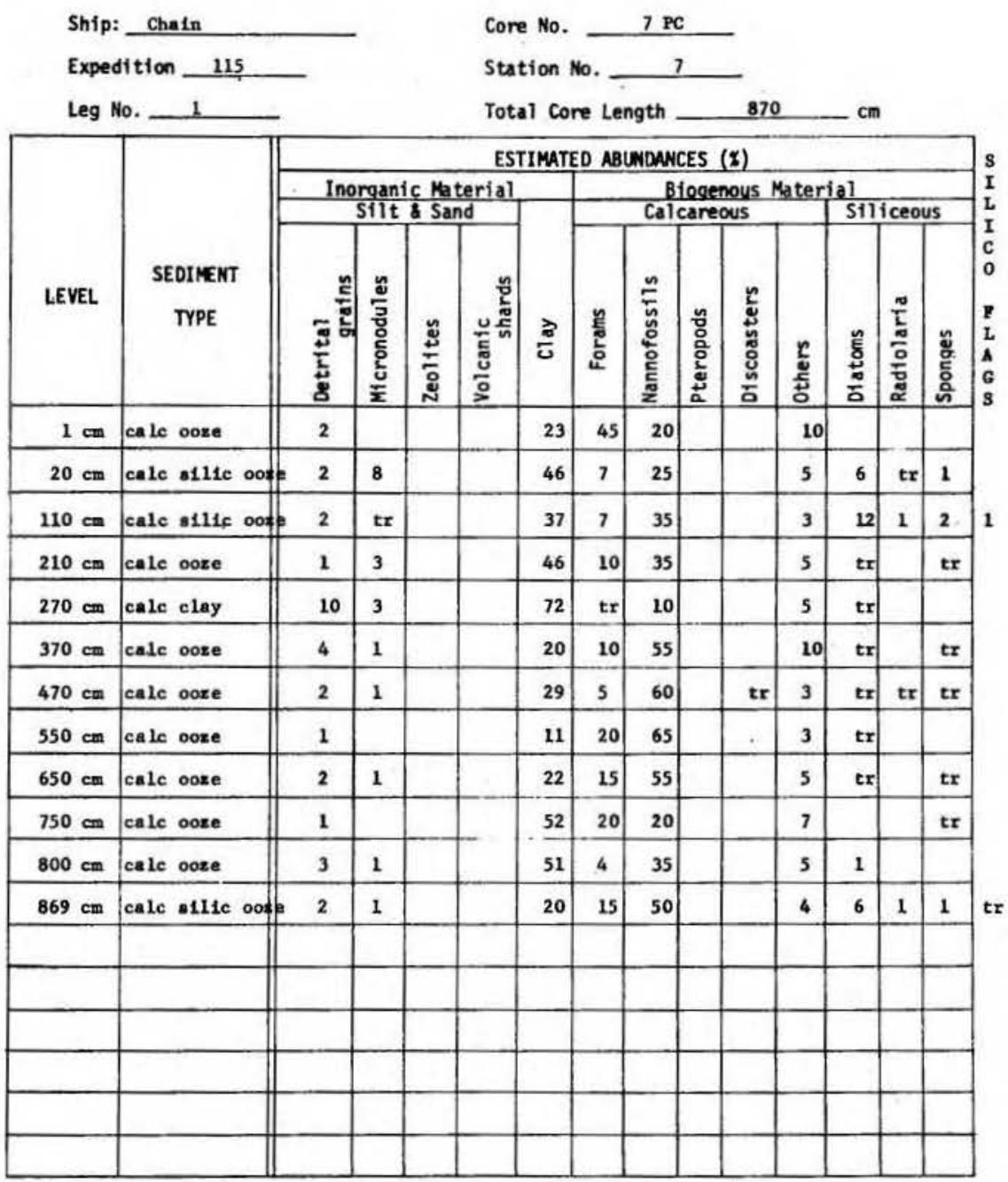


222

VISUAL CORE DESCRIPTION

Page 1 of 1

Ship Cthin Cruise 115 Leg 1 sta. 7 Core No. $7 \%$

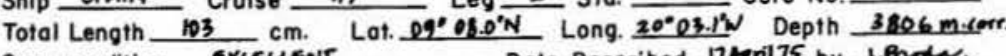
Core condition ExUEUE⿰亻⿱丶⿻工二木

Physiographic location

Lithologic

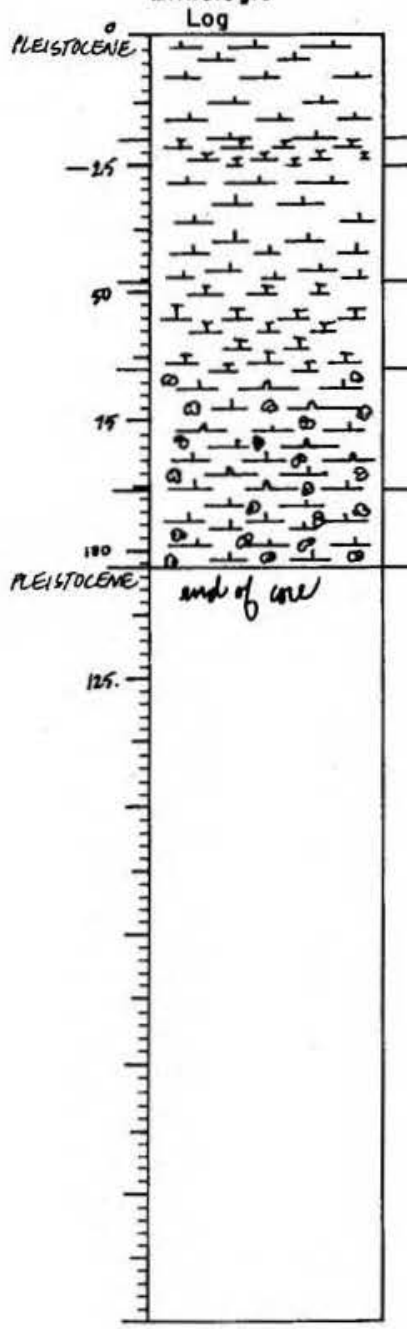

Detailed Description

\section{CALC OOZE}

(o $5 / 3$ pale brown moist silty lutite vith very abund. forams

${ }_{-25}^{6}$ textura 1

CALC OOZE

cham

$S$ inclined $15^{\circ}$

$25-48$

\section{CALC OOZE}

10YR $5 / 2$ grayish brown grades to $6 / 3$ pale brow

molst silty lutite with very abund. forams

48-65

\section{CALC OOZE}

very pale brown

$S$ inclined $15^{\circ}$

65-88

CALC SILIC OOZE

OYR $6 / 41$ t. yellowish brow

extensive faint 1ight gray mottling and marbling futite with scattered forams

CAIC
$2.5 Y$
$4 / 2$ dark grayish brown grades to $3 / 2$ very dark graylsh brown and back to $4 / 2$

gray, and 1ight yellowish brown mottling and marbling
firmer slightly silty lutite with scattered forams end of core

Note; Due to a repetitive occurrence of similar lithologies this core ls suspected to be an example or a mintelple penetration (pilot core rebound) recovery, 1 st $0-25 \mathrm{~cm}$,
223

SHEAR SLIDE DESCRIPTIONS - W.H.O.I. SEDIMENT CORES

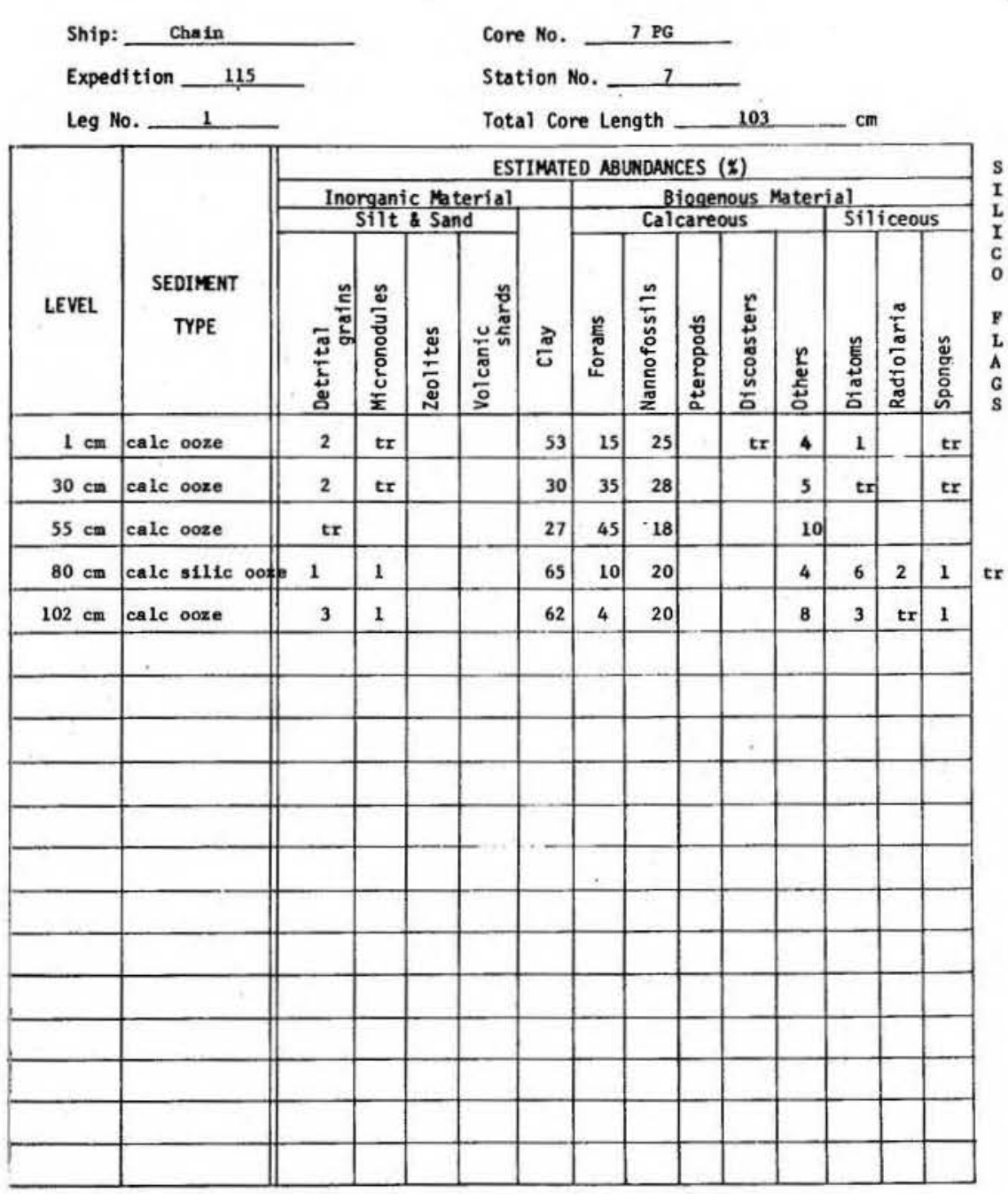


Ship CHAIN Cruise 115 Leg 1 Sto. 8 Core No. $8 \mathrm{PC}$ Total Length $88_{1} \mathrm{~cm}$. Lat. $09^{\circ}{ }^{\circ}$. 1. $\mathrm{N}$ Long. $19^{\circ} 34.9^{\prime} \mathrm{W}$ Depth $4572 \mathrm{~m}$.cem Core condition EXCEUENT. Date Described 21AmiL75 by j.bhodu. Physiographic location Lithologic

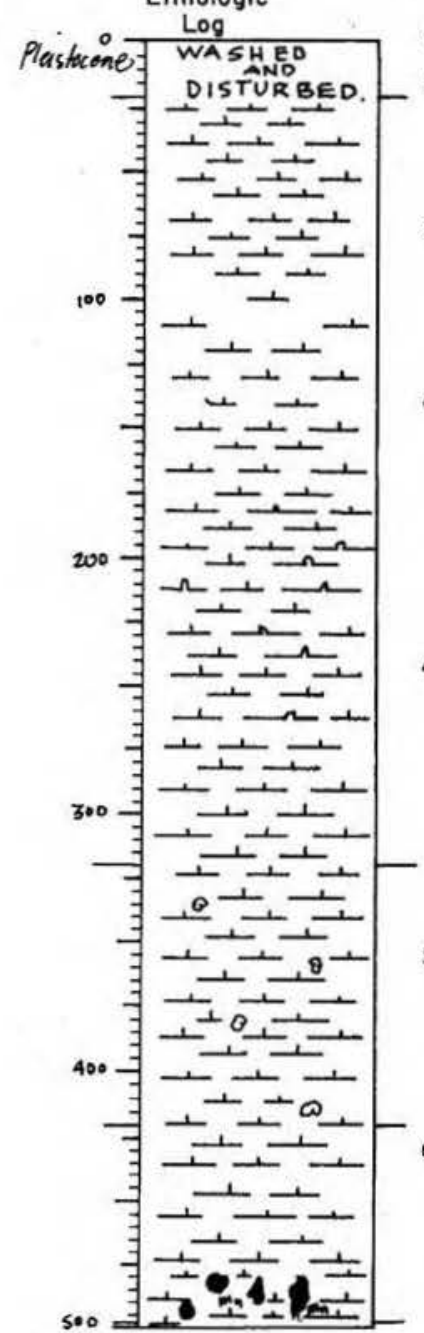

\section{Detailed Description}

DISTURBED AND HASHED

CALC OOZE GRADES TO CALC SILIC OOZE AAD BACK TO CALC OOZE $2.5 \mathrm{Y} 5 / 2$ gray1sh brown

very moist unconsolidated lutite

ver
$320-420$

CALC OOZE

$2.5 \times 5 / 2$ gray 1 sh brown grades to $6 / 2$ 1ight grayish brown

faint light gray and graylish brown marbling

slightly silty moist lutite grades to very silty (calc and for am fragmenta) lutit

420-498

CALC OOZE

rregularly spaced, sorbewhat inclined laminations of the

ragmented forams grade to foram sand

somewhat disturbed 473-498 $\mathrm{cm}$, four large, $4-6 \mathrm{~cm}$ diameter of olive 1ithified calc anterial identical to lumps and $s$
8-591

CALC SILIC OOZE GRADES TO HIGHLY CALC CLAY

LYR $5 / 4$ yellowish brown

and light yellowish brom marbling mottiling throughout

scattered silty lutite vith scattered forams throughout lumps $580-590 \mathrm{~cm}$

two grayish brown zones of extremely abund. forams $526-530 \mathrm{~cm}$ 作 very

CAIC OOZE

1OYR $8 / 3$ very pale brom

foram sand with scattered 1rregular lans-1.5 cm diameter pale yell ow 11thified lums

列, also $s$

1-621

HIGHLY CALC CLAY GRADES TO CALC OOZE

10YR $6 / 3$ pale brown

mo1st silty lutite with extremely abund. forams and scattered,

S irregular
$2.5 \mathrm{Y} 5 / 2$ graylsh brown and $6 / 2$ 11ght grayish brow
VISUAL CORE DESCRIPTION

Page 2 of 2

Ship CHAIN Cruise 115 Leg 1 Sta. D Core No. 8PC

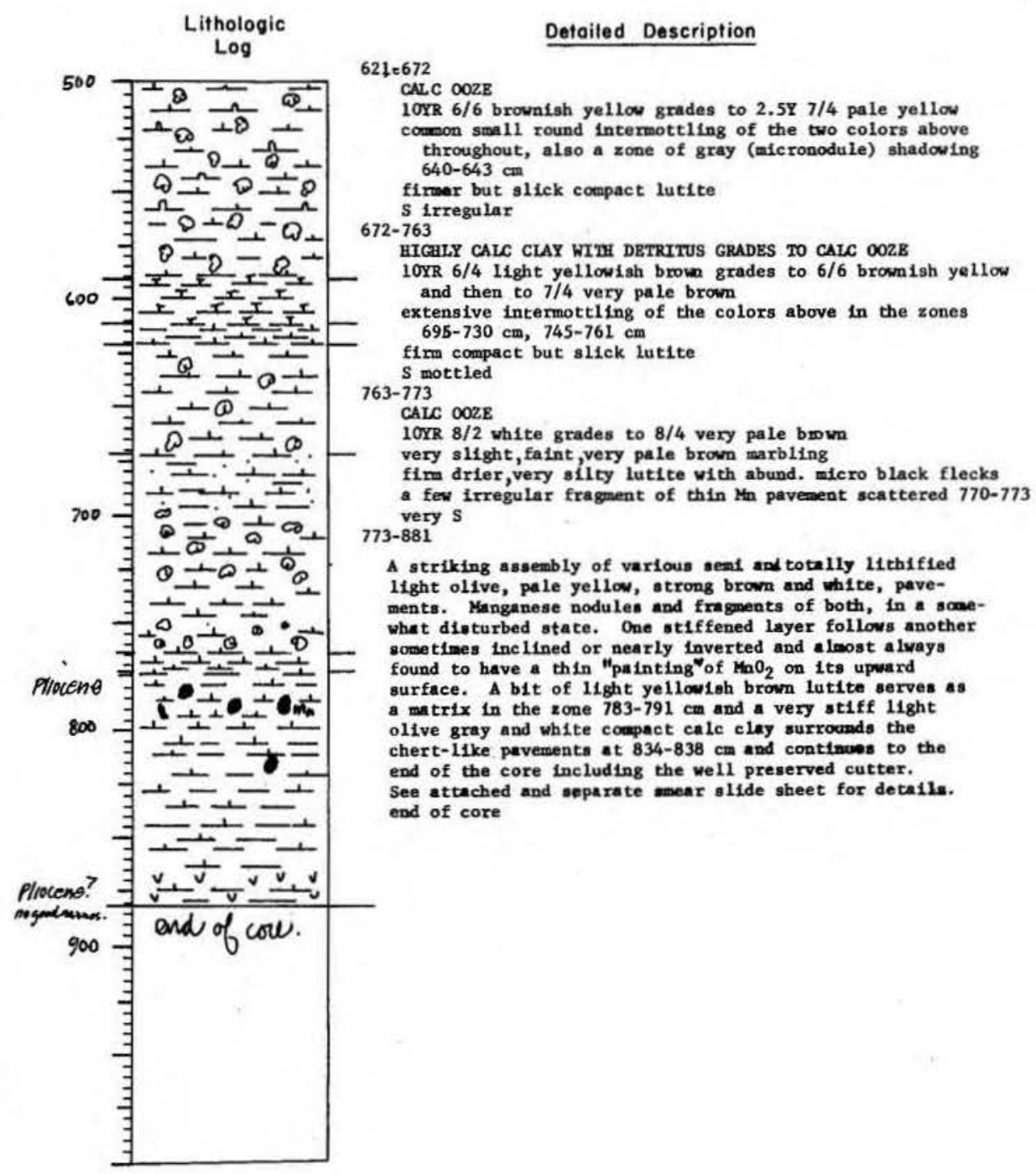


226

SIEAR SLIDE DESCRIPTIONS - W.H.O.I. SEDIMENT CORES

Ship: Couln

Expedition 115

Leg No. 1

Core No. $8 \mathrm{PC}$

Station No. 8

Total Core Length $\quad 881 \quad \mathrm{~cm}$

\begin{tabular}{|c|c|c|c|c|c|c|c|c|c|c|c|c|c|c|}
\hline \multirow[b]{3}{*}{ LEVEL } & \multirow[b]{3}{*}{$\begin{array}{c}\text { SEDIMENT } \\
\text { TYPE }\end{array}$} & \multicolumn{13}{|c|}{ ESTIMATED ABUNDANCES (\%) } \\
\hline & & \multicolumn{4}{|c|}{$\begin{array}{c}\text { Inorganic Material } \\
\text { Silt \& Sand }\end{array}$} & & \multicolumn{5}{|c|}{$\begin{array}{l}\text { Biegenous Mater } \\
\text { Calcareous }\end{array}$} & \multicolumn{3}{|c|}{ Siliceous } \\
\hline & & $\frac{5}{\frac{5}{5}}$ & 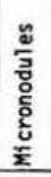 & 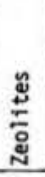 & 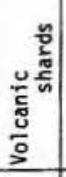 & $\frac{\vec{\pi}}{\tilde{u}}$ & $\begin{array}{l}\text { 崑 } \\
\text { s. }\end{array}$ & 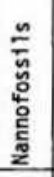 & 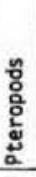 & 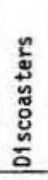 & 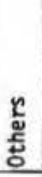 & 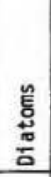 & $\begin{array}{l}\frac{\pi}{\pi} \\
\frac{\pi}{\sigma} \\
\frac{0}{0} \\
\tilde{\alpha} \\
\end{array}$ & 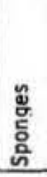 \\
\hline $30 \mathrm{~cm}$ & calc ooze & 3 & 1 & & $\mathrm{tr}$ & 40 & 5 & 40 & & 3 & 5 & 2 & $\underline{t x}$ & 1 \\
\hline $100 \mathrm{~cm}$ & calc ooze & 3 & 2 & & $\mathrm{tr}$ & 45 & 6 & 35 & & 2 & 4 & 2 & & 1 \\
\hline $200 \mathrm{~cm}$ & calc silic of & 2 & 2 & & $\mathrm{tr}$ & 37 & 7 & 40 & & 2 & 5 & 4 & tr & 1 \\
\hline $300 \mathrm{~cm}$ & calc ooze & 2 & 1 & & & 33 & 8 & 45 & & 2 & 6 & 2 & & 1 \\
\hline $350 \mathrm{~cm}$ & cale ooze & 3 & 1 & & & 15 & 50 & 25 & & 2 & & 3 & tr & 1 \\
\hline $400 \mathrm{~cm}$ & calc ooze & 2 & tr & & $\mathbf{t r}$ & 10 & 60 & 20 & & 1 & 6 & 1 & tr & tr \\
\hline $450 \mathrm{~cm}$ & calc ooze & 2 & tr & & & 10 & 65 & 17 & & 1 & 5 & tr & & tx \\
\hline $502 \mathrm{~cm}$ & cale silic oof & 2 & 2 & & & 24 & 8 & 40 & & 1 & 5 & 12 & 1 & 4 \\
\hline $585 \mathrm{~cm}$ & hly calc clas & 7 & 1 & tr & $\mathrm{tr}$ & 67 & 2 & 12 & & 1 & 10 & & & \\
\hline $606 \mathrm{~cm}$ & hly calc clay & 3 & & & & 82 & $\mathrm{tr}$ & 5 & & tr & 10 & & & \\
\hline $625 \mathrm{~cm}$ & calc ooze & 2 & & & & 40 & 10 & 40 & & 2 & 6 & & & \\
\hline $675 \mathrm{~cm}$ & $\begin{array}{l}\text { hly calc clay } \\
\text { with det }\end{array}$ & 15 & 3 & & & 66 & tr & 7 & & 8 & 1 & & & \\
\hline $725 \mathrm{~cm}$ & calc ooze & 1 & tr & & & 59 & 1 & 10 & & 25 & 4 & & & \\
\hline $750 \mathrm{~cm}$ & calc ooze & 1 & tr & & & 43 & 1 & 20 & & 30 & 5 & & & \\
\hline $765 \mathrm{~cm}$ & calc ooze & 1 & tr & & & 53 & 20 & 5 & & 6 & 15 & & & \\
\hline $772 \mathrm{~cm}$ & calc ooze & 2 & 1 & & & 40 & 20 & 20 & & 10 & 8 & & & \\
\hline $880 \mathrm{~cm}$ & calc clay/det & 5 & tr & & 302 & 58 & tr & 2 & & & 5 & & & \\
\hline & & & & & & & & & & & & & & \\
\hline
\end{tabular}

227

SIEAR SLIDE DESCRIPTIONS - W.H.O.I. SEDIIENT CORES

Ship: _Chain

Expedition 115

Leg No. 1
Core No. 8 PC (AVENENT ZouE)

Station No.

Total Core Length $\_881$

\begin{tabular}{|c|c|c|c|c|c|c|c|c|c|c|c|c|c|c|}
\hline \multirow[b]{3}{*}{ LEVEL } & \multirow[b]{3}{*}{$\begin{array}{c}\text { SEDIMENT } \\
\text { TYPE }\end{array}$} & \multicolumn{13}{|c|}{ ESTIMATED ABUNDANCES ( $(\boldsymbol{)})$} \\
\hline & & \multicolumn{4}{|c|}{$\begin{array}{c}\text { Inorganic Material } \\
\text { Silt \& Sand }\end{array}$} & & \multicolumn{5}{|c|}{$\begin{array}{l}\text { Biogenous } \\
\text { Calcareous }\end{array}$} & \multicolumn{3}{|c|}{$\begin{array}{l}\text { ial } \\
\text { Siliceous }\end{array}$} \\
\hline & & $\frac{5}{5}$ & 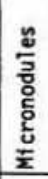 & $\stackrel{ \pm}{\frac{ \pm}{2}}$ & 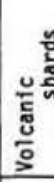 & $\frac{\pi}{\omega}$ & 高 & 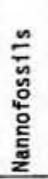 & 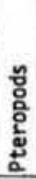 & 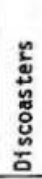 & 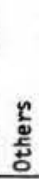 & 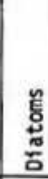 & $\begin{array}{l}\frac{\pi}{i} \\
\frac{\pi}{0} \\
\frac{0}{0} \\
\frac{\pi}{0}\end{array}$ & $\begin{array}{l}\text { ŏ } \\
\text { cे } \\
\text { ò } \\
\text { ñ }\end{array}$ \\
\hline 1. $774 \mathrm{~cm}$ & calc clay & 2 & & & 4 & 82 & 3 & 3 & & & 6 & & & \\
\hline 2. $780 \mathrm{~cm}$ & calc ooze & 1 & & 1 & 2 & 51 & 35 & 5 & & & 5 & tr & & \\
\hline 3. $785 \mathrm{~cm}$ & calc ooze & 8 & 2 & 10 & tr & 48 & 5 & 25 & & & 2 & & & \\
\hline 4. $795 \mathrm{~cm}$ & calc ooxe & 3 & & tr & 2 & 54 & 30 & 6 & & & 5 & & & \\
\hline 5. $800 \mathrm{~cm}$ & calc clay & 2 & & tr & 3 & 81 & 3 & 5 & & & 6 & & & \\
\hline 6. $810 \mathrm{~cm}$ & bly calc clas & 2 & 2 & $\mathrm{tr}$ & 1 & 76 & 5 & 10 & & & 4 & & & \\
\hline 7. $811 \mathrm{~cm}$ & hly cale clay & & & tr & 1 & 79 & 2 & 8 & & & 10 & & & \\
\hline 8. $825 \mathrm{~cm}$ & hly calc clay & 1 & & & 4 & 70 & 5 & 15 & & . & 5 & & & \\
\hline 9. $835 \mathrm{~cm}$ & calc clay & 3 & & & 3 & 80 & 3 & 5 & & & 6 & & & \\
\hline $10.836 \mathrm{~cm}$ & hly cale clay & 4 & tr & & 2 & & 10 & 5 & & & 5 & & & \\
\hline & & & & & & & . & & & & & & & \\
\hline & & & & & & & & & & & & & & \\
\hline & & & & & & & & & & & & & & \\
\hline & & & & & & & & & & & & & & \\
\hline & & & & & & & & & & & & & & \\
\hline & & & & & & & & & & & & & & \\
\hline & & & & & & & & & & & & & & \\
\hline & & & & & & & & & & & & & & \\
\hline
\end{tabular}


Ship CHAIN Cruise 115 Leg 1 Sta. $B$ Core No. BPG Total Length $155 \mathrm{~cm}$. Lat. $09{ }^{\circ} 16.1 \% \mathrm{~N}$ Long. $19 \cdot 34^{\circ} 9^{\circ} \mathrm{W}$ Depth $4572 \mathrm{m.corr}$ Core condition EXCELLENT Date Described ZIARTiLTS by f.Buder.

Physiographic location GIERRA LEONE RISE.

Lithologic

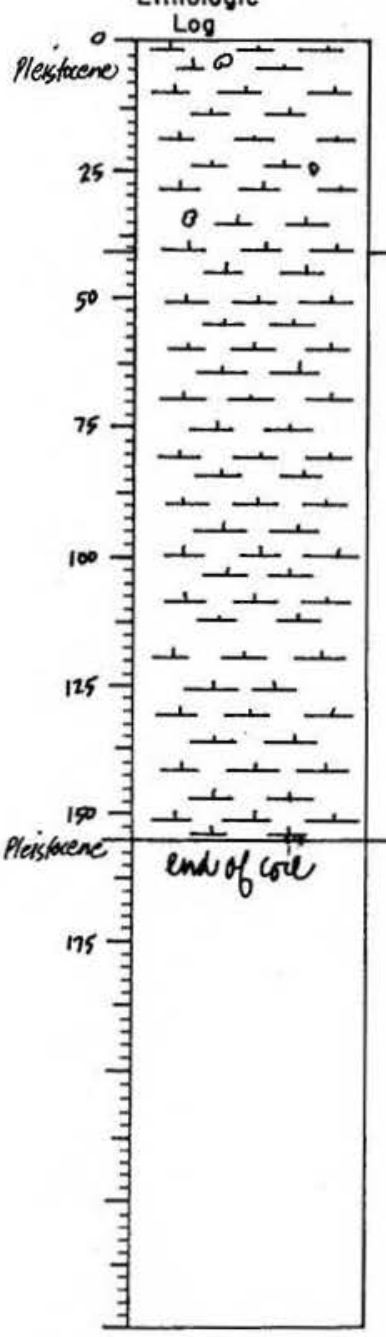

Detailed Description

CALC $002 \mathrm{E}$

a very few scattered grayish bown foram-rich mottles scattered forams at core top and in mottles, otherwise very moist slick unconsolidated lutite $S$
-155

CALC OOZE

CALC OOZE
$2.5 \mathrm{Y}$ 5/2 grayish brown

homogeneous throughout

very molst slick unconsolidated lutite

end of core

SIEAR SLIDE DESCRIPTIONS - W.H.O.I. SEDINENT CORES

Ship: Chain

Expedition 115

Leg No. 1
Core No. 8 PG

Station No.

Total Core Length

ESTIMATED ABUWDANCES (*)

\begin{tabular}{|c|c|c|c|c|c|c|c|c|c|c|c|c|c|c|}
\hline \multirow[b]{3}{*}{ LEVEL } & \multirow[b]{3}{*}{$\begin{array}{c}\text { SEDINENT } \\
\text { TYPE }\end{array}$} & \multicolumn{13}{|c|}{ ESTIMATED ABUNDANCES ( $(x)$} \\
\hline & & \multicolumn{4}{|c|}{$\begin{array}{c}\text { Inorganic Material } \\
\text { Silt \& Sand }\end{array}$} & & \multicolumn{5}{|c|}{$\begin{array}{l}\text { Biogenous Mate } \\
\text { Calcareous }\end{array}$} & \multicolumn{3}{|c|}{$\frac{\text { al }}{\text { Silliceous }}$} \\
\hline & & 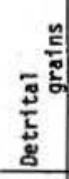 & 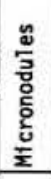 & $\frac{ \pm}{\frac{\pi}{2}}$ & $\frac{\text { to }}{\frac{n}{2}}$ & $\frac{\pi}{0}$ & 总 & 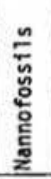 & 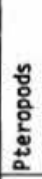 & 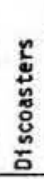 & $\begin{array}{l}r \\
0 \\
\text { ș } \\
0\end{array}$ & 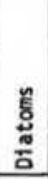 & 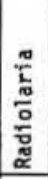 & ڤัँ \\
\hline $1 c$ & calc ooze & 2 & & & tr & 51 & 15 & 25 & & 1 & 3 & 2 & & 1 \\
\hline $42 \mathrm{~cm}$ & calc ooze & 3 & 1 & & tr & 40 & 6 & 40 & & 2 & 4 & 3 & & 1 \\
\hline $154 \mathrm{~cm}$ & calc ooze & 2 & 3 & & tr & 46 & 6 & 34 & & 2 & 4 & 2 & tr & 1 \\
\hline & & & & & & & & & & & & & & \\
\hline & & & & & & & & & & & & & & \\
\hline & & & & & & & & & & & & & & \\
\hline & & & & & & & & & & & & & & \\
\hline & & & & & & & & & & . & & & & \\
\hline & & & & & & & & & & & & & & \\
\hline & & & & & & & & & & & & & & \\
\hline & & & & & & & . & & & & & & & \\
\hline & & & & & & & & & & & & & & \\
\hline & & & & & & & & & & & & & & \\
\hline & & & & & & & & & & & & & & \\
\hline & & & & & & & & & & & & & & \\
\hline & & & & & & & & & & & & & & \\
\hline & & & & & & & & & & & & & & \\
\hline & & & & & & & & & & & & & & \\
\hline
\end{tabular}


Ship CHAIN Cruise 115 Leg 1 sto. 10 Core No. gPC Total Length $880 \mathrm{~cm}$. Lat. $090^{\circ} 04.5^{\prime} \mathrm{N}$ Long. $19^{\circ} 35.1^{2} \mathrm{~V}$ Depth $4685 \mathrm{~m}$.Corr.

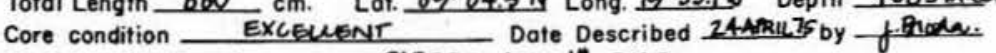
Physiographic location GIERRA LEONE RISE.

Lithologic

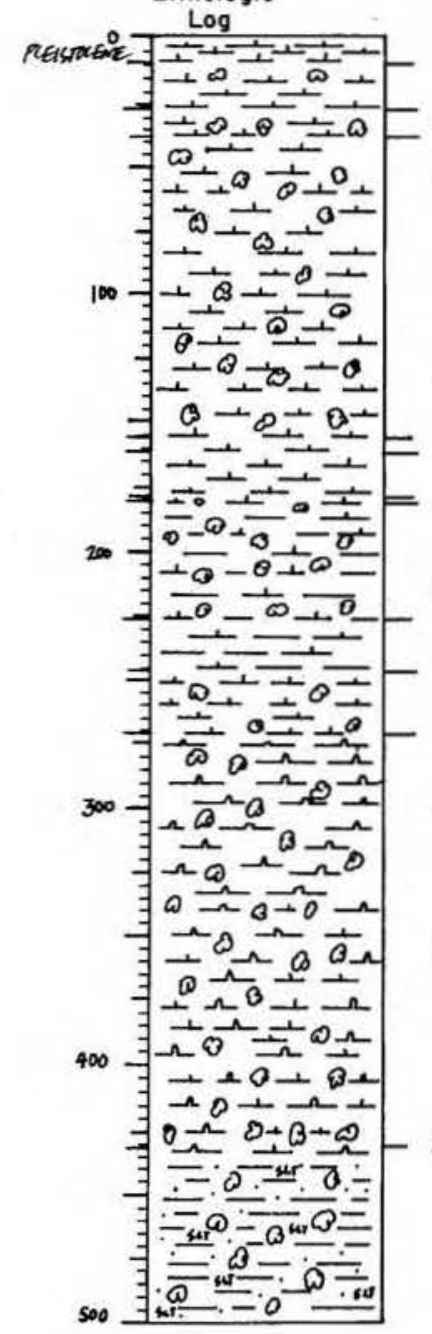

0-9

\section{Detailed Description}

\section{CALC OOZE}

$2.5 Y 5 / 2$ grayish brow

moist, unconsolidated, slightly silty lutite

$9-26$

CALC OOZE

6/3 pale brown

several dark gray burrows span entire liner $10-15 \mathrm{~cm}$ nearly completed, but someuhat disturbed band of fora at upper contact

6-37 irregular

CALC OOZE

10YR $8 / 3$ very pale brown, 10YR $6 / 4$ 11ght yellowish brown and $4 / 1$ dark gray

complete 1rregular internottling of the 3 colors above compact lutite with gcattered foram $31-34 \mathrm{~cm}$ has 5 ma $S$
$37-156$ mottled

CAIC OOZZ

10YR $6 / 3$ pale brown

extensive, irregular $t$ inclined, large light yellowish brown, 列

moist, but coepact, slightly silty lutite with foram content S-161

\section{CALC OOZE}

10YR $7 / 4$ very pale brown and $6 / 4$ 11ght yellowish brown complete intemottling of the two colors above lutite with aburd, foram

161-1

CALC OOZE

$2,5 Y 5 / 2$ grayigh brown

1rregular swirling of dark gray, very pale brown and light yellowish brown

irregular distribution of foram none to scattered

78-180

CALC OOZE

1OYR $7 / 4$ very pale brown and $6 / 3$ pale brown

common mixing of the two colors above

$s$ irregular
VISUAL CORE DESCRIPTION

Page 2. of 3 Ship CHAIN Cruise 115 Leg I Sto. 10 Core No. 9PC

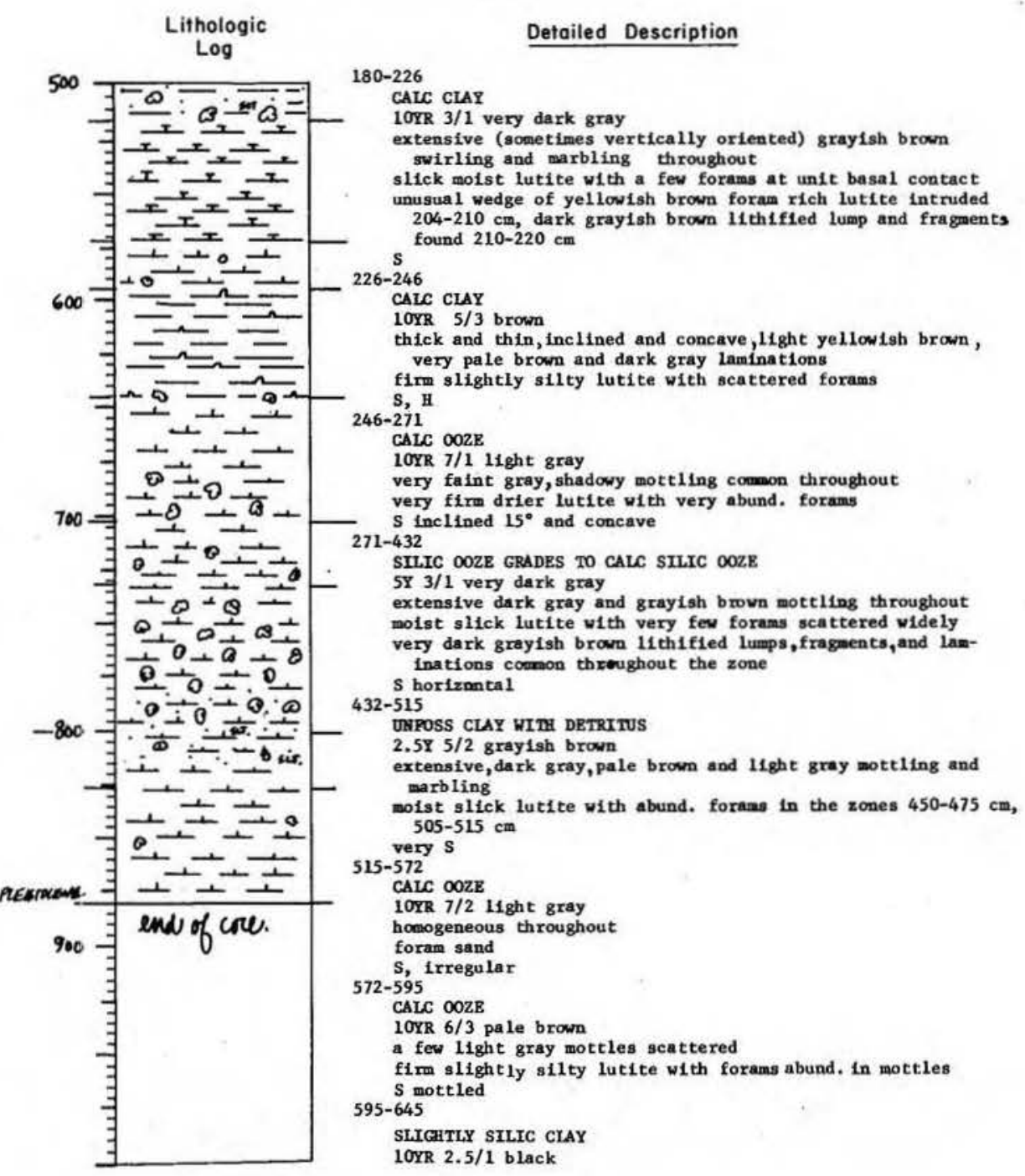


Ship CHAIN Cruise IIS Leg 1 Sta. 10 Core No. gPC

\section{Lithologic}

Log

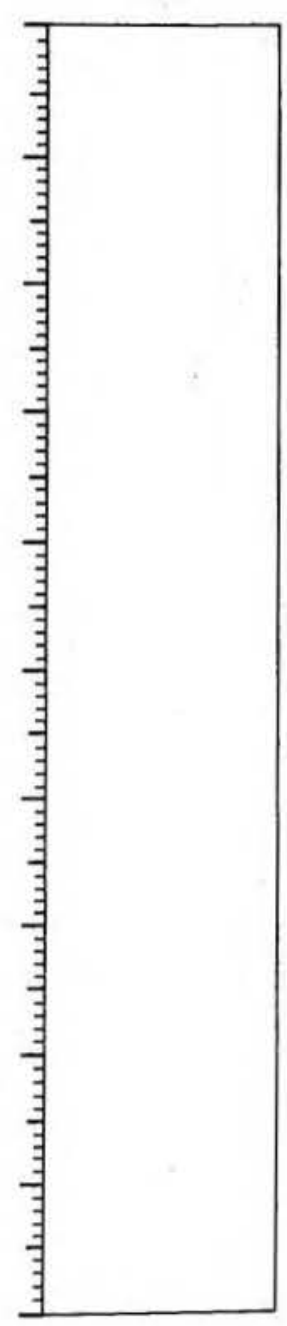

\section{Detailed Description}

few hazy dark gray motel oist slick lutite with a few forams in mottles at basa contact

645-702

$$
\text { CALC } O O Z B
$$

10YR $4 / 1$ dark gray

coanson large 11ght gray and very dark gray mottles, 672-704c firm slick lutite with a few forams, more abund. in mottles $S$ mott 1
732

CALC OOZB

$2.5 \mathrm{Y} 5 / 2$ graylsh brown grades to $6 / 211$ ght brown1sh gray sight 1ight gray mottling $G$

732-800

CALC OOZE GRADES TO CALC OOZR HITH DETRTTUS $3 / 1$ very dark gray

extensive irregular gray and pale brown mottling throughout $S, \mathrm{H}$

800-826

CALC OOZE WITH DETRITUS GRADES TO CALC OOZE

$2.5 Y 5 / 2$ grayish brown grades to $6 / 2$ 11ght branish gray cornon gray mottling $800-810 \mathrm{~cm}$

number of 2 ite $w i$ th abund. forams grades to foram sand foram and $810-826 \mathrm{~cm}$

S6-880

CALC OOZE

feu scattered 1ithified 5/4 olive mottles

fin sightly silty lutite with very few forams and scattered

end of core
SIEAR SLIDE DESCRIPTIONS - W.H.0.I. SEDIIENT CORES

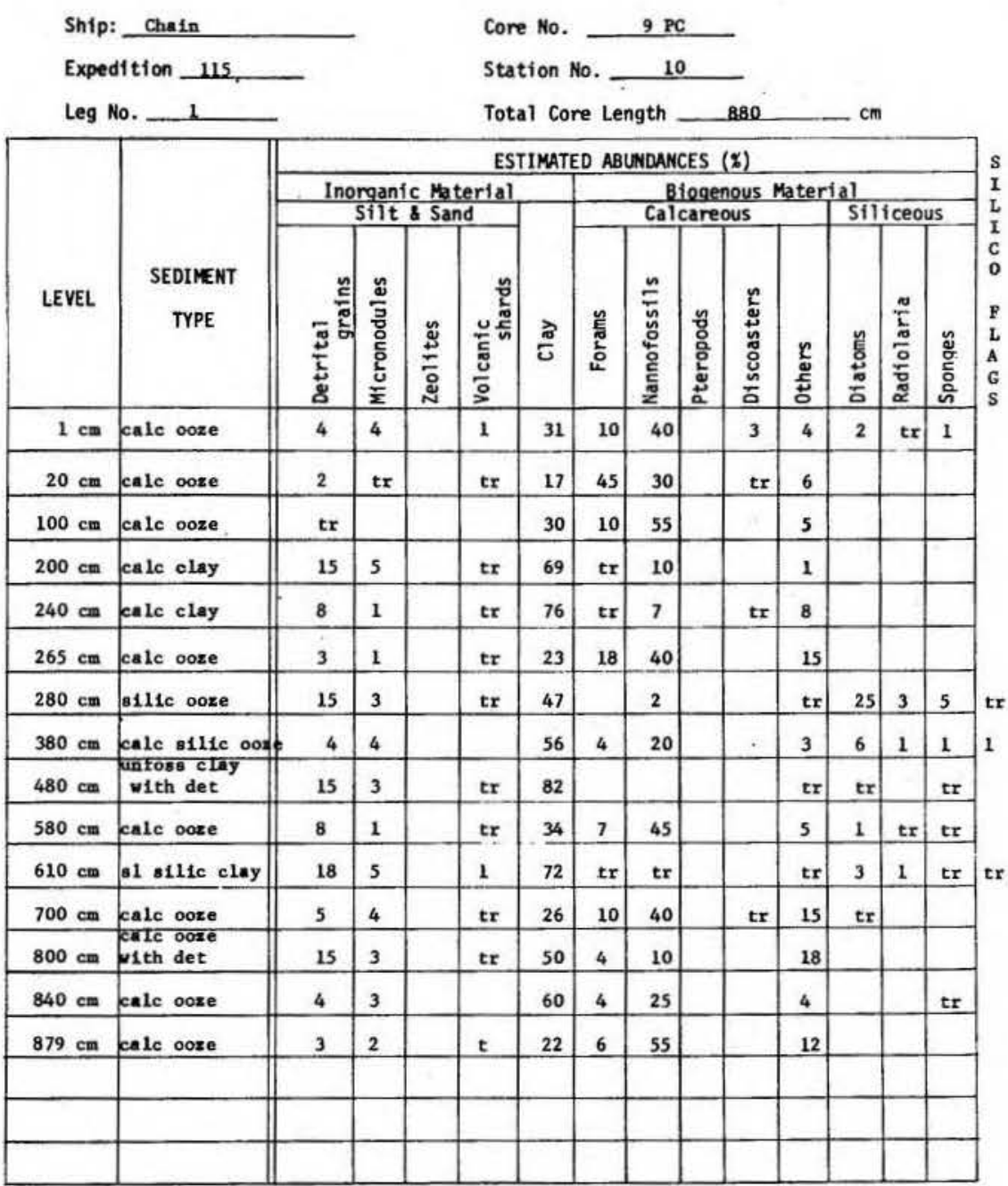


Ship CHAIN Cruise 115 Leg 1 Sta. $\frac{10}{19^{\circ} 35.1^{\circ} \mathrm{W}}$ Core No. $\frac{9 P G}{4685 \mathrm{~m} \text {. }}$

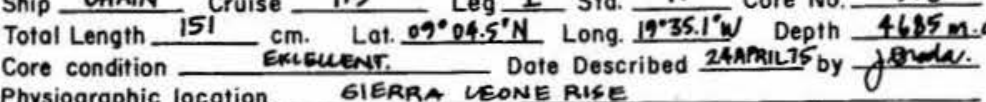

Lithologic

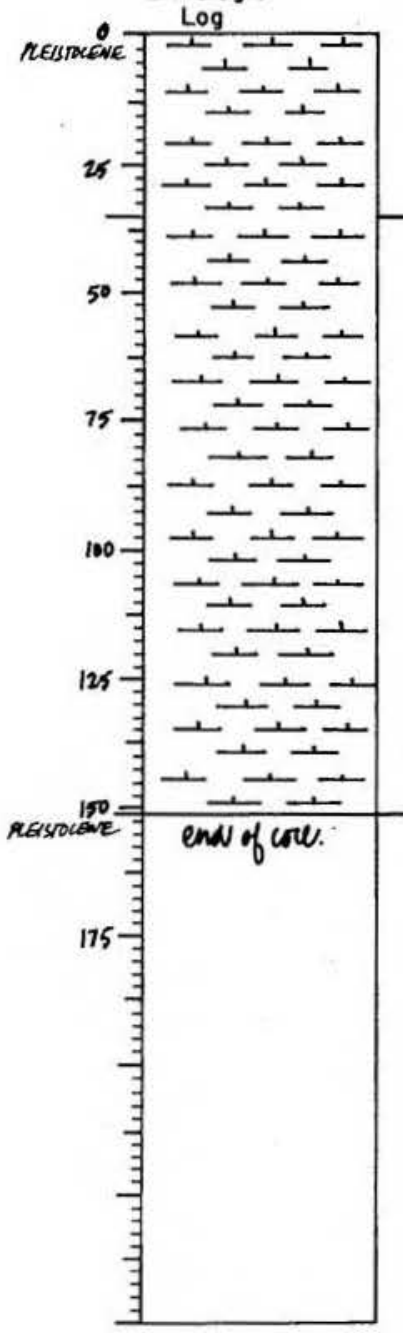

Detailed Description

\section{CALC OOZE}

OYR $6 / 3$ pale brown

howogeneous throughout in the zone $0-10 \mathrm{~cm}$ 6 mottled 35-151

CAIC OOZE

$2.5 \times 5 / 2$ grayish brow

molst firmer, slightly

lutite, siltier at base

nd of core
Ship: Chain

Expedition 115

Leg No.

\begin{tabular}{|c|c|c|c|c|c|c|c|c|c|c|c|c|c|c|}
\hline & \multicolumn{13}{|c|}{ (5) } \\
\hline \multirow[b]{3}{*}{ LEVELL } & \multirow[b]{3}{*}{$\begin{array}{c}\text { SEDIYENT } \\
\text { TYPE }\end{array}$} & \multicolumn{13}{|c|}{ ESTIMATED ABUNDANCES ( $(\chi)$} \\
\hline & & \multicolumn{4}{|c|}{$\begin{array}{c}\text { Inorganic Material } \\
\text { Silt \& Sand }\end{array}$} & & \multicolumn{5}{|c|}{$\begin{array}{l}\text { Biogenous Mater } \\
\text { Calcareous }\end{array}$} & \multicolumn{3}{|c|}{ sil } \\
\hline & & 琣 & 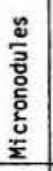 & 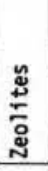 & 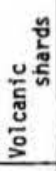 & $\frac{\pi}{6}$ & $\begin{array}{l}\text { 宸 } \\
\text { 5 }\end{array}$ & 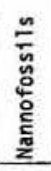 & 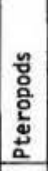 & 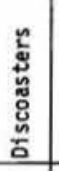 & $\begin{array}{l}c \\
\text { v } \\
\text { ș } \\
\end{array}$ & 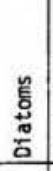 & $\frac{\pi}{i}$ & 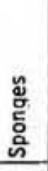 \\
\hline $1 \mathrm{~cm}$ & calc ooze & 1 & 4 & & 1 & 36 & 8 & 40 & & 3 & 4 & 2 & $\mathrm{tr}$ & 1 \\
\hline $50 \mathrm{~cm}$ & calc ooze & 1 & 3 & & tr & 43 & 10 & 35 & & 3 & 3 & 1 & $\operatorname{tr}$ & 1 \\
\hline $150 \mathrm{~cm}$ & calc ooze & 5 & 1 & & $\mathrm{tr}$ & 27 & 45 & 25 & & 2 & 5 & & & $\mathrm{tr}$ \\
\hline & & & & & & & & & & & & & & \\
\hline & & & & & & & & & & & & & & \\
\hline & & & & & & & & & & & & & & \\
\hline & & & & & & & & & & & & & & \\
\hline & & & & & & & & & & . & & & & \\
\hline & & & & & & & & & & & & & & \\
\hline & & & & & & & & & & & & & & \\
\hline & & & & & & & . & & & & & & & \\
\hline & & & & & & & & & & & & & & \\
\hline & & & & & & & & & & & & & & \\
\hline & & & & & & & & & & & & & & \\
\hline & & & & & & & & & & & & & & \\
\hline & & & & & & & & & & & & & & \\
\hline & & & & & & & & & & & & & & \\
\hline & & & & & & & & & & & & & & \\
\hline
\end{tabular}

SHEAR SLIDE DESCRIPTIONS - W.H.O.1. SEDINENT CORES

Core No. 9 PG

Station No. 10

Total Core Length _L_LS

$\mathrm{con}$ 
Ship CHAIN Cruise 115 Leg 1 Sto. 11 Core No. 10 PC Total Length $885 \mathrm{~cm}$. Lat. $09 \cdot 15.7 \mathrm{~N}$ Long. 19026.37 W Depth 4168m-corr Core condition ExCEUENT. Date Described 28 aran 15 by fonden. Physiographic location SIERRA LEONE RISE.

Lithologic

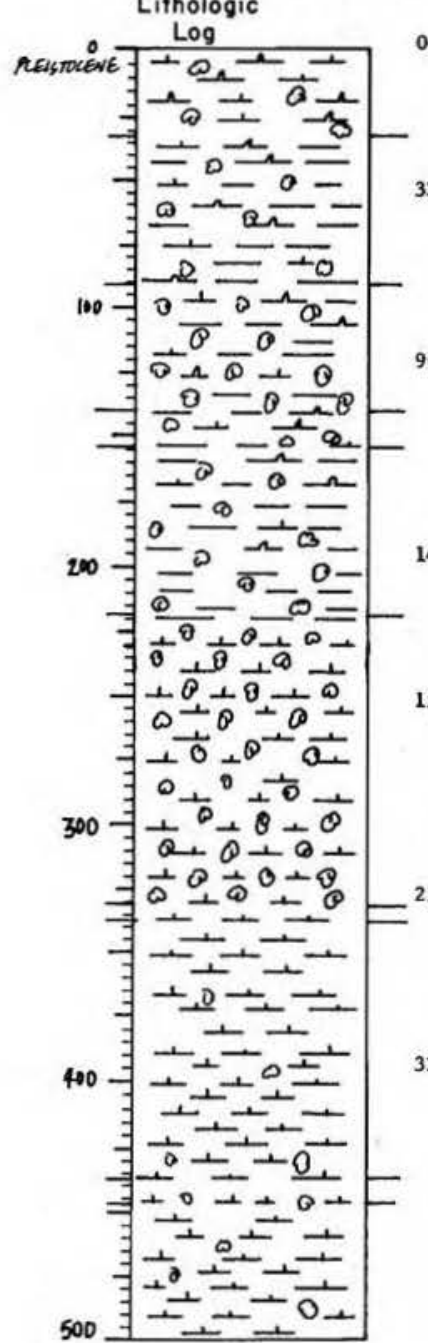

0-32 Detailed Description

CALC SILIC OOZE

moist unconsolidated lutite; very few forams 6 mottled

CALC SILIC CIAY

10YR $6 / 4$ light yellowish brown grades to $6 / 6$ broumish yello and back to $6 / 4$ 11ght yellowish brown . comonly throughout molst slightly silty latite with abund. forams $0-140$

CAIC SILIC CLAY

10YR $5 / 4$ yellowish brown; $6 / 6$ brownish yellow; and $5 / 2$ gray1sh

moist slighity silty lutite vith very fey forams grading to $140-154$

CALC SIIIC CLAY

$2.5 \mathrm{Y} 4 / 2$ dark gray1sh brown

common pale brown and bromian yellow mottling

mo1st lutite with very few forams

S, irregular

4-218

CALC STUTC CIAY GRADES TO UNPOSS CIAY

10YR $6 / 4$ light yellow 1ah brown grades to $7.5 Y R$ S/6 strong comson, brown yellowish brown, and gray mottling and maxbling mo1st slightly silty lutite with forams scattered in the zone 154-184 only 218-331

CALC DOZE

varying hues of 10YR $6 / 3$ pale brown, $6 / 4$ 1ight yellowish brown dark gray

complete and extensive intermottling of the colors above scattered to few forams throughout, moist slightly silty 31-33

GALC OOZE

OYR $7 / 4$ very pale brown

firmer slightly silty lutite with scattered forams

$\mathrm{S}$ mottled

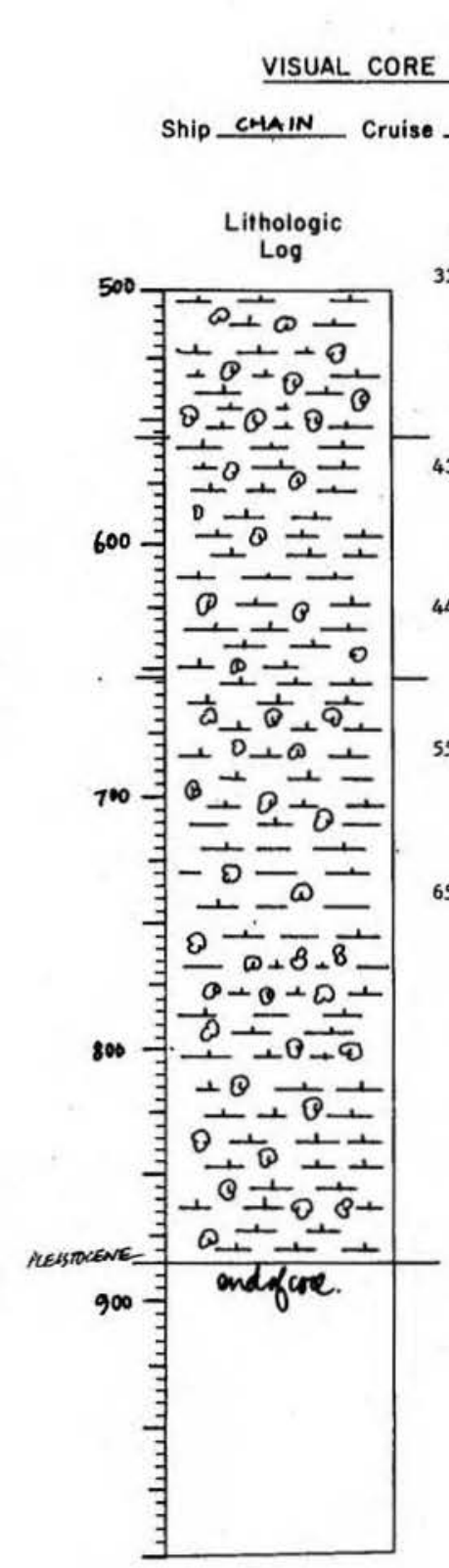

115

1 Sta. 11 Core No. $10 \mathrm{PL}$

Detailed Description

CAIC DOz:

10YR $5 / 3$ brown grades to $5 / 6$ yellowish brown and then to $6 / 3$

pale brown
faint light gray motting throughout plus a bit of inter-

mottling of the colors above

molst silck lutite with a few forams

437-447 mottled

CALC OOZE

$2.5 \mathrm{Y} 3 / 2$ very dark grayish brom

slight dark gray marbling and mottling

very slick lutite

$\mathrm{G} \mathrm{mo}$
$447-557$

CALC OOZE
1OYR $6 / 3$ pale brown grades to $6 / 4$ 11ght yellowlsh brown

couron to extensive graylah brown and gray mottling throughout firm slick lutite with forams ranging from few to abund. $\underset{557-653}{S \text { irregular }}$

CALC OOZE

CALC $002 B$
1OYR $6 / 3$ pale brown repeatedly grades to $5 / 4$ yellowish brow 10YR $6 / 3$ pale brawn repentedly grades to $5 / 4$ yellowlsh brown
coumon light gray and gray mottling in the yellowish brown sones

53-885

CALC OOZE GRADES TO HIGHLY CAIC CIAY ABD EACK TO CALC 002 1OYR $6 / 4$ 1ight yellowinh brown repeatedly grades to $5 / 4$ yellowish brown

(a) extensive, of 11ght gray and fim alishtly allty lutite oith acater

scattered to abund. forams, end of core. in mottled zones. 
238

SIEAR SLIDE DESCRIPTIONS - W.H.O.I. SEDINENT CORES

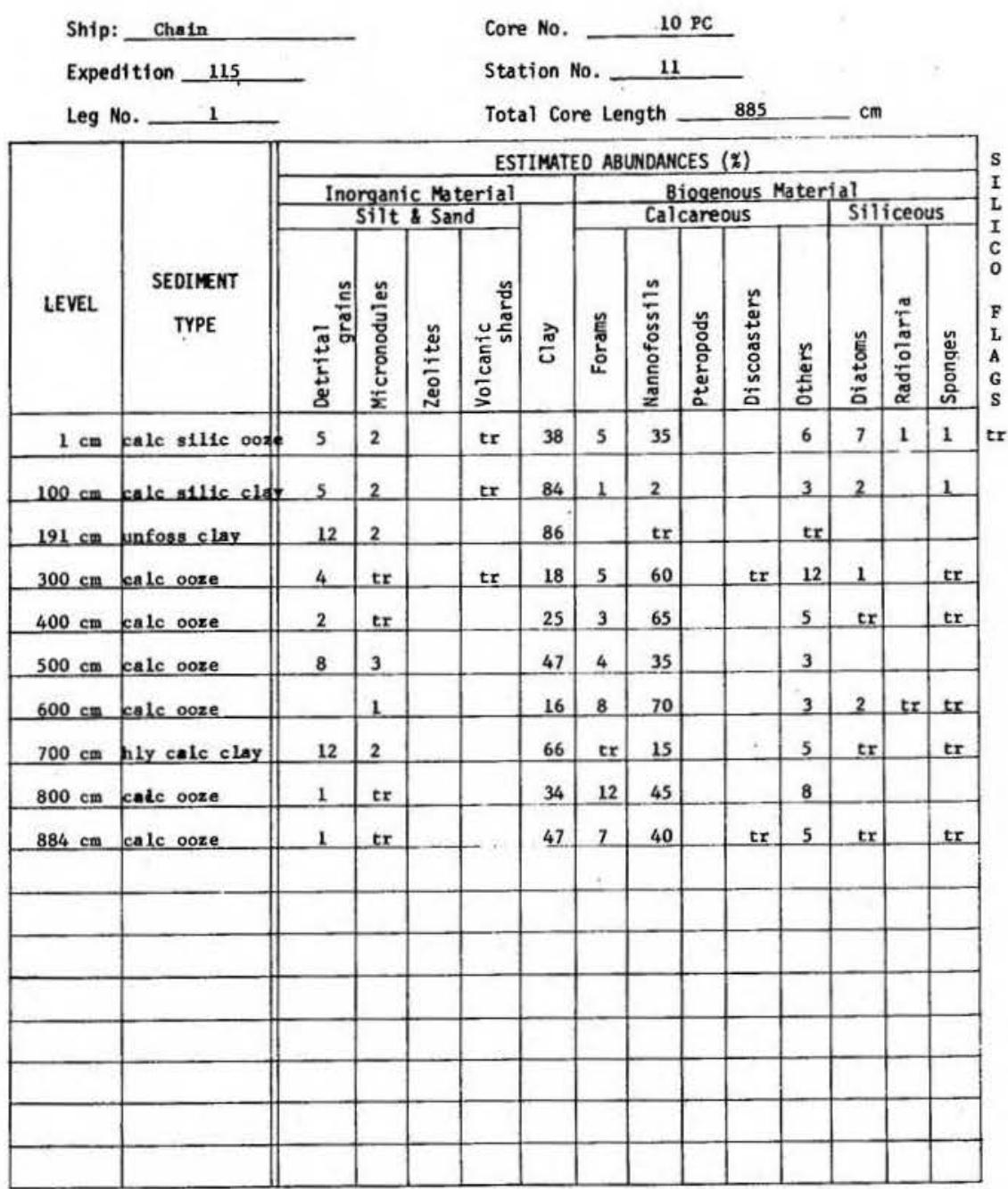

Page 1 of 1

Ship CHAIN Cruise 115 Le 1 Sta 11 Core No. IOPG Total Length $153 \mathrm{~cm}$. Lat. $09^{\circ} 15.7^{\prime} \mathrm{N}$ Long. $19^{\circ} 26.37 \mathrm{~W}$ Depth $4168 \mathrm{~m} \cdot \mathrm{corr}$. Core condition EXCEULENT Date Described 25ankn Ts by f.Boda. Physiographic location SIERRA LEONE RISE.

Lithologic

$0 \quad$ Log

Log $\quad 0-5$

-50 Detailed Description

CALC OOZE

comon light yellowis h brown and grayish brown marbling and mottlin

$++\infty-$

- $\triangle B \pm$

15 开士 는

$+\div \overline{0}$

$\div 0 \div$

$-507 \frac{1}{0}+\frac{1}{0}+\frac{5}{0}$

ID \pm 0 二

$\exists \vec{B}+\vec{Q}+\vec{Q}$

75 工本立立

月:0 0 -

$7 . \frac{10}{0} \theta$

100

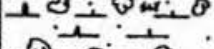

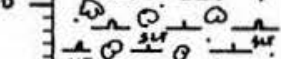

sur 0.0.

$125=\frac{\theta+\theta}{B+\theta-1}$

- $B \stackrel{0}{0}$

$=0 B=-2$

Rersousve

175.

end of coces. 50-95

CALC OOZE GRADES TO CALC SILIC OOZE WITH DETRTTUS tiaht yellowish brow

extensive brownish yellow and gray mottling throughout coupact lutite with very abund. forams

$S$ mottled

CAIC SILIC DOZE WITH DETRITUS GRADES TO SLICHTLY SILIC CLAY 10rk $5 / 3$ broum grades to $S / 2$ graylsh brow

very pale brom zone of very abund, forams 111-117 cm end of core

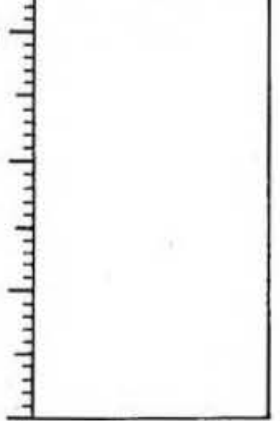


240

SHEAR SLIDE DESCRIPTIONS - W.H.O.I. SEDIVENT CORES

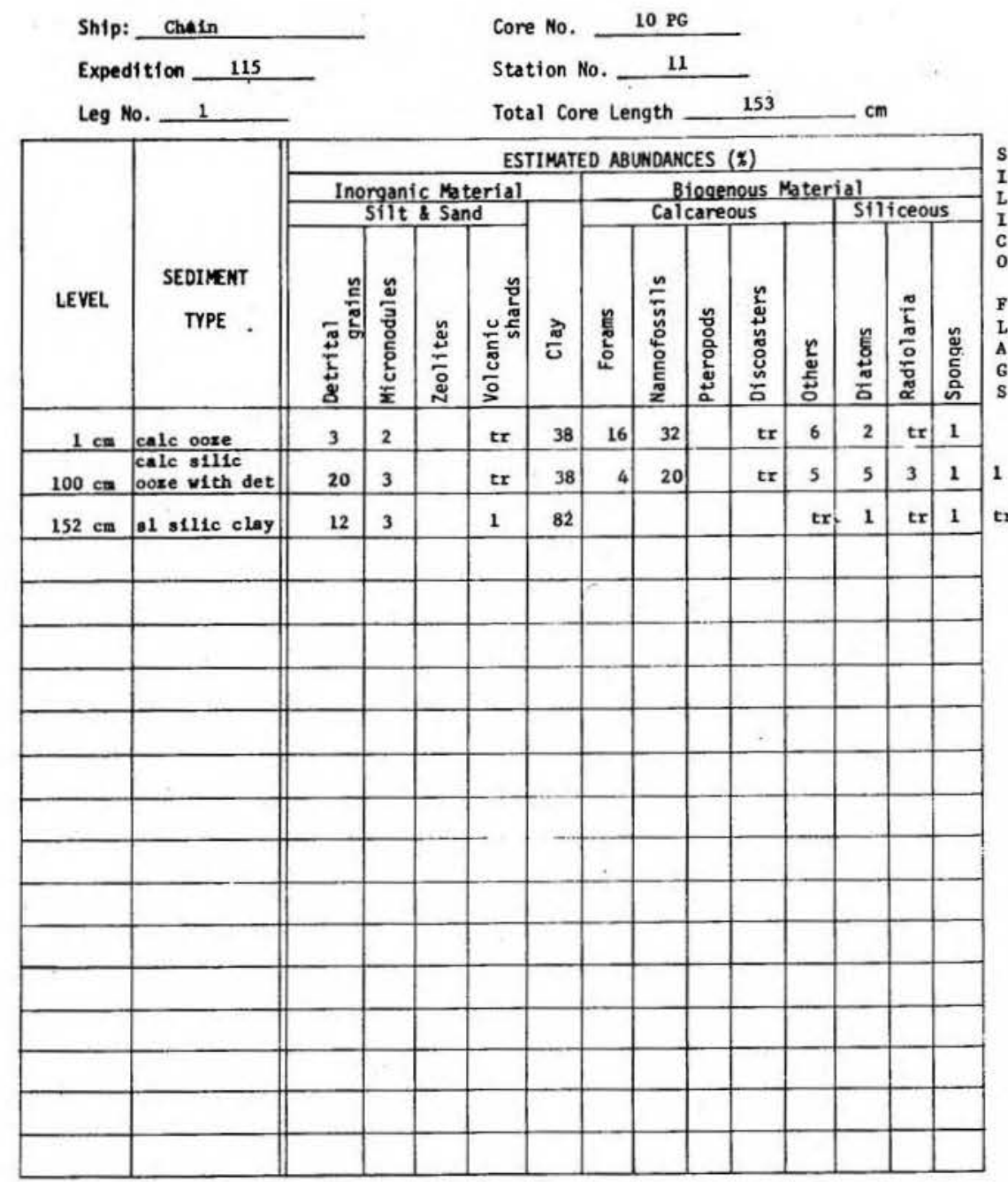

241

VISUAL CORE DESCRIPTION

Page 1 of 1

Ship GHAIN Cruise 115 Leg 2 Sta. 20 Core No. 12 PC

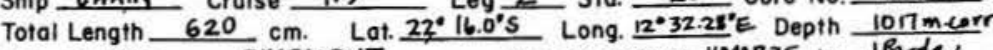
Core condition EXCEUENT Date Described IMAR7S by firnta Physiogrophic location_SOUTHWEST AFRICAN SCOFE: WALVIS BAII

Lithologic

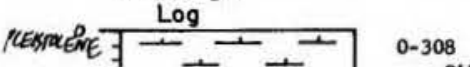

H $\infty+\quad$ CALC OOZE

faint laminationgray

丰 $+\infty+$

$\exists+\overline{0}+$

fons, bands, and mottles of olive gray $5 Y 4 / 2$ and

olive $4 / 4$ found coemonly throughout

moist slick, rubbery lutite with abund. Hiny white flecks

79-81 cm band of extremely abund. white flecks, distinct but

$200=1+\infty-108-456$

田- CALC OOZE

D十 - 1 5Y $4 / 2$ olive gray

extensive olive and dark olive gray mottiling $310-340 \mathrm{~cm}$, carmon

$\exists \square+$

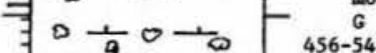
alick, nubbery lutite vith witite flecka cas

壬古宁 ${ }_{\text {CALC OOZE }}^{456-540}$

市 1 -

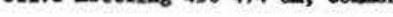

moist rubbery lutite with common uhite flecks

正十 $\frac{1}{9}-540-620$

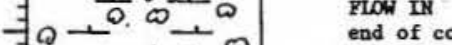

त $+\overrightarrow{0}$ end of core

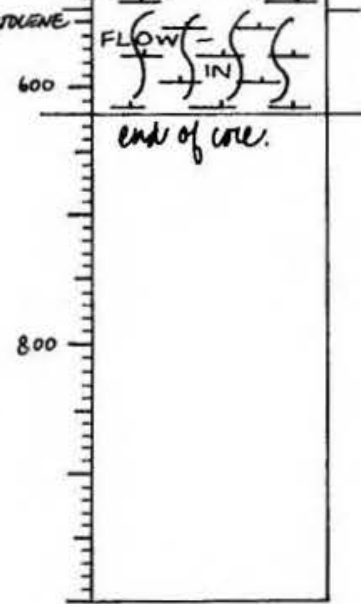


242

SMEAR SLIDE DESCRIPTIONS - W.H.O.I. SEDINENT CORES

Ship: _Chain

Expedition 115

Core No. 12. PC

Leg No. 2

Station No. 20

Total Core Length $\quad 620$

\begin{tabular}{|c|c|c|c|c|c|c|c|c|c|c|c|c|c|c|}
\hline \multirow[b]{3}{*}{ LEVEL } & \multirow[b]{3}{*}{$\begin{array}{c}\text { SEDIMENT } \\
\text { TYPE }\end{array}$} & \multicolumn{13}{|c|}{ ESTIMATED ABUNDANCES ( $(\not)$} \\
\hline & & \multicolumn{4}{|c|}{$\begin{array}{c}\text { Inorganic Material } \\
\text { Silt \& Sand }\end{array}$} & \multirow[b]{2}{*}{$\frac{\pi}{\tilde{U}}$} & \multicolumn{5}{|c|}{$\begin{array}{l}\text { Biogenous Mater } \\
\text { Calcareous }\end{array}$} & \multicolumn{3}{|c|}{ Siliceous } \\
\hline & & 㺼 & 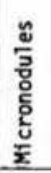 & 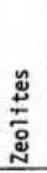 & 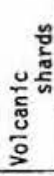 & & 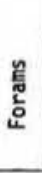 & 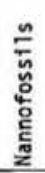 & 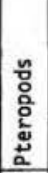 & 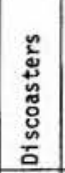 & 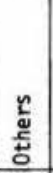 & 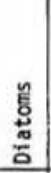 & $\begin{array}{l}\frac{\pi}{L} \\
\frac{0}{0} \\
\frac{0}{0} \\
\tilde{\pi} \\
\end{array}$ & 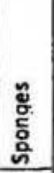 \\
\hline $1 \mathrm{~cm}$ & calc ooze & 1 & & & $\operatorname{tr}$ & 51 & 3 & 40 & 2 & & 3 & & & $t r$ \\
\hline $80 \mathrm{~cm}$ & calc ooze & 1 & & & 1 & 50 & 4 & 30 & 1 & & $12 *$ & & & 1 \\
\hline $150 \mathrm{~cm}$ & calc ooze & 2 & 1 & & tr & so & 3 & 38 & $\mathrm{tr}$ & $\mathrm{tr}$ & 6 & & & tr \\
\hline $250 \mathrm{~cm}$ & calc ooze & 2 & 1 & & $\mathrm{tr}$ & 45 & 10 & 33 & & & $9 *$ & & & tx \\
\hline $350 \mathrm{~cm}$ & calc ooxe & 2 & 2 & & 1 & 40 & 8 & 40 & & & 7 & $t x$ & & $\mathrm{tr}$ \\
\hline $460 \mathrm{~cm}$ & calc ooze & 2 & 2 & & 1 & 40 & 10 & 40 & & & 5 & $t r$ & & $t x$ \\
\hline $560 \mathrm{~cm}$ & calc ooze & 3 & 2 & & 1 & so & 5 & 30 & & & 9 & & & $t x$ \\
\hline $619 \mathrm{~cm}$ & calc ooze & 2 & 2 & & 1 & 45 & 10 & 35 & & - & 5 & $t \pi$ & & $\mathrm{tr}$ \\
\hline & & & & & & & & & & & & & & \\
\hline & & & & & & $\star$ & mee & Lish & one & debrit & $s$ & & & \\
\hline & & & & & & & & & & & & & & \\
\hline & & & & & & & & & & & & & & \\
\hline & & & & & & & & & & & & & & \\
\hline & & & & & & & & & & & & & & \\
\hline & & & & & & & & & & & & & & \\
\hline & & & & & & & & & & & & & & \\
\hline & & & & & & & & & & & & & & \\
\hline & & & & & & & & & & & & & & \\
\hline
\end{tabular}

243

VISUAL CORE DESCRIPTION

Page 1 of 1

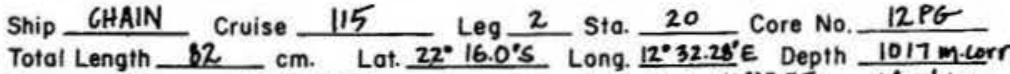

Leg 2 Sta. 20 Core No. $12 \mathrm{PG}$

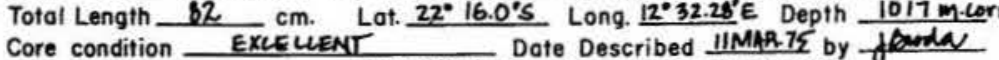
Core condition $\frac{\text { EXLE WENT }}{\text { Physiographic location SOUTHWEST AFRICAN SLOFE : WALVIS BAII }}$

Lithologic

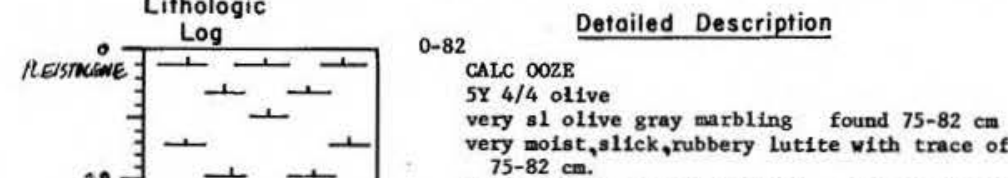
CALC OOZE

of white flecks $75-82 \mathrm{~cm}$. Note: when split aboard ship, odor of hydrogen sulfide was end of core 
244

SIFAR SLIDE DESCRIPTIONS - W.H.O.I. SEDIMENT CORES

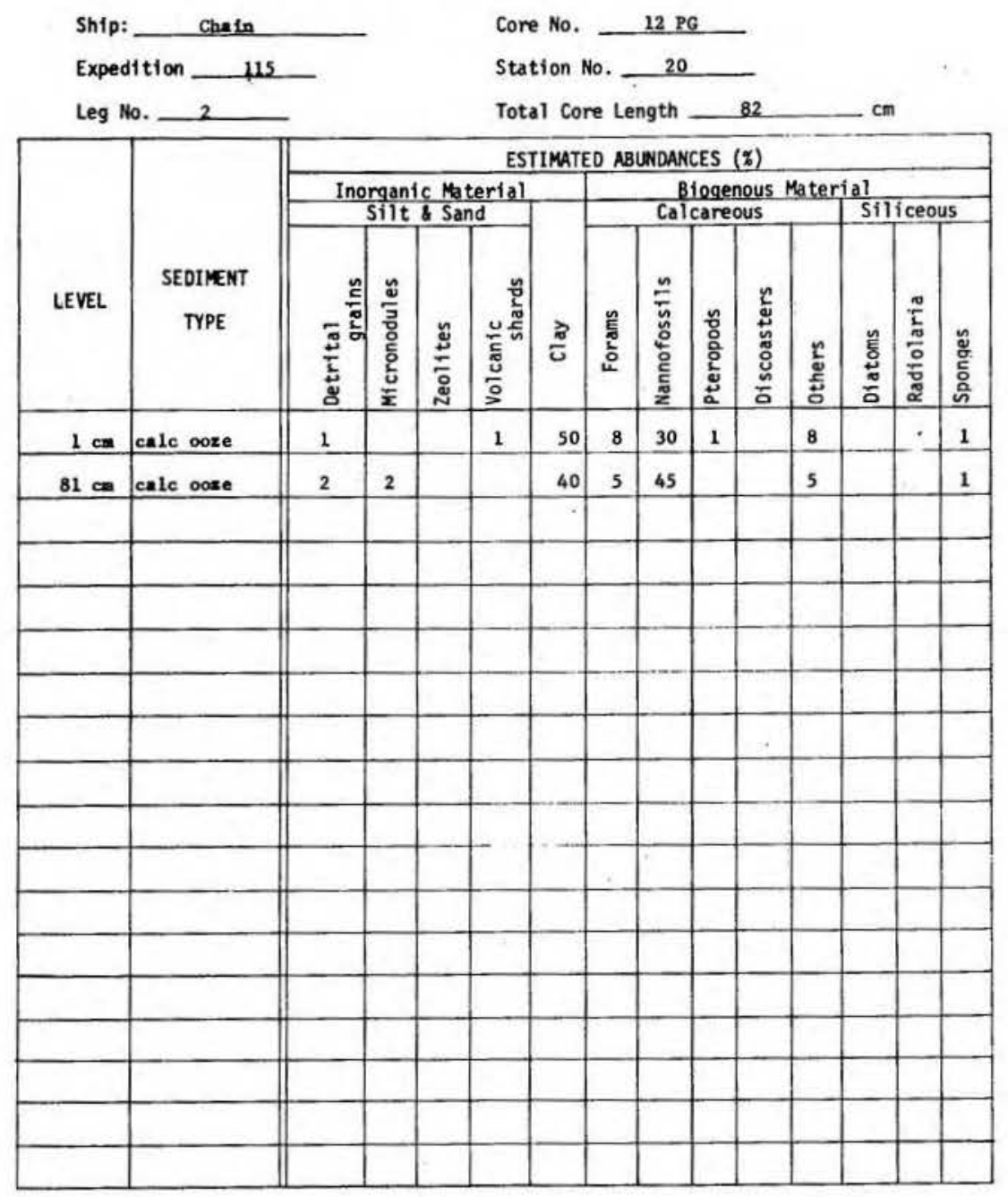

245

VISUAL CORE DESCRIPTION

Page 1 of 1

Ship CHAIN Cruise 115 Leg $\frac{2}{5}$ Sta. $\frac{21}{1}$ Core No. $13 P C$ Total Length $510 \mathrm{~cm}$. Lat. $23^{\circ} 31.35^{\circ} \mathrm{S}$ Long. $\frac{12^{\circ} 14.9^{\circ} \mathrm{E}}{\text { Depth } 2132 \mathrm{~m} \cdot \mathrm{com} \text {. }}$ Core condition EXCEUENT. Date Described RMMa 15 by folpodar

Physiographic location IN AREA OF SLUMPS OFF SOUTANEST AERILA

Lithologic

REISTOCOE

DISTURBED

$\perp$ AND $\perp$

DSORIENTED

寻宫市迹

100

-

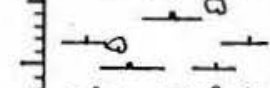

$\overrightarrow{-}+\vec{C}+$

- 1 - $1+1$

$200=101000$

$=\underbrace{\infty}+\frac{1}{0}+\frac{1}{2}$

$\exists+1$,

丰的士

버도.

$300 \exists+,+8+$

当 $-\frac{1}{\bar{\theta}}+1$

我安

$\div \div=$

$\omega \rightarrow \bar{\omega}$

$\exists \rightarrow 0-1$

400 丰

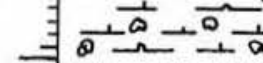

$\exists \rightarrow \longrightarrow$

두두

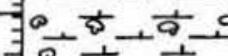

而古,

\section{Detailed Description}

0-69

Sediment disturbed and dis oriented. Sample was extrided one. Overall color $5 \mathrm{Y} 5 / 4$ ollve marbled w1th $4 / 3$ olfve; 69-205 sediment type calc ooze.

CAIC SILIC OOZE GRADES TO CALC OOZE

$4 / 3$ olive

extensive pale olive $6 / 4$ and olive 5/4 mottling $69-85 \mathrm{~cm}$ and 200-205 cm; common ollve burrowing and banding $85-200 \mathrm{~cm}$ moist slick rubbery lutite with scattered white flecks

205-226

KABRO OOZE

$5 Y 6 / 3$ pale ollve

extensive pale yellow and olive moteli ng throughout

excellent elongated burrow spans entire 1iner at $219 \mathrm{con}$

26-35

KANamo DO2E

6/4 pele oltve grades to 5/3 oltwe

occasional vell formed pale yellow and pale olive burrows

$G$
$6-430$

$350-430$

CAIC SILIC $00 Z$

$5 Y 5 / 3$ ollve

olive and pele ollive burrowing and mottling

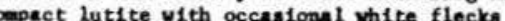

$430-459$

CALC SILIC OOZE

$5 Y 5 / 2$ ollve gray

v. sl ollve mott1ing

6
$459-510$

MARMO OOZE

5Y $5 / 3$ olive

extensive fal it olive and pale olive mottling at upper unit contact

lutite with occasionsl white flecks

end of core

- - J eneofcone 
246

SIEAR SLIDE DESCRIPTIONS - W.H.O.I. SEDIMENT CORES

Ship:

Leg No. 2

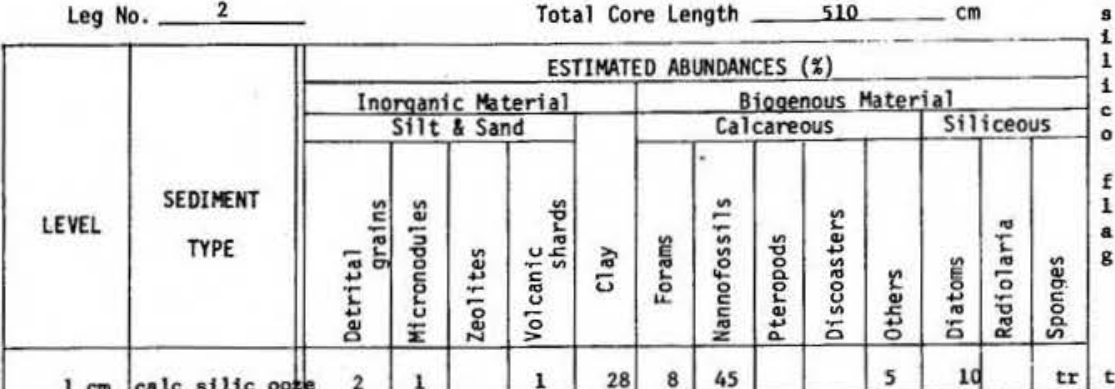

\begin{tabular}{|l|l|l|l|l|l|l|l|l|l|l}
$1 \mathrm{~cm}$ Calc silic oofe 2 & 1 & & 1 & 28 & 8 & 45 & & 5 & 10 & $\mathrm{tr}$ \\
$\mathrm{tr}$
\end{tabular}

\begin{tabular}{l|l|l|l|l|l|l|l|l}
$75 \mathrm{~cm}$ & calc allic oose 1 & 1 & & tr & 30 & 5 & 50 \\
\hline
\end{tabular}

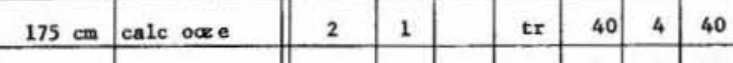

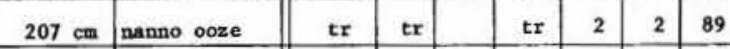

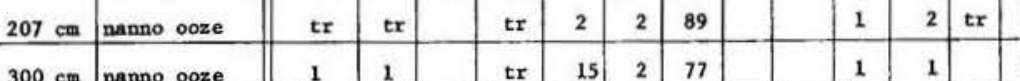

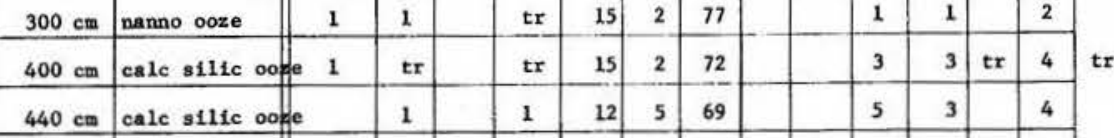

$509 \mathrm{~cm}$ nanno ooze

\begin{tabular}{|l|l||l|l|l|l|l|l|l|l|l|l|l|l|l|}
\hline $509 \mathrm{~cm}$ & nanno ooze & 1 & 1 & & & 17 & 2 & 75 & & & 1 & 1 & 2 \\
\hline & & & & & & & & & & & & & & \\
\hline & & & & & & & & & & & & & & \\
\hline & & & & & & & & & & & & & & \\
\hline & & & & & & & & & & & & & & \\
\hline & & & & & & & & & & & & & & \\
\hline & & & & & & & & & & & & & & \\
\hline
\end{tabular}

247

VISUAL CORE DESCRIPTION

Page 1 of 1

Ship CHAIN 15 L 21 Core No. I3PG

Total Length $76 \mathrm{~cm}$. Lat. $23^{\circ} 31.35^{\circ} \mathrm{S}$ Long. $2^{\circ} 14.9^{\circ} \mathrm{E}$ Depth $2132 \mathrm{~m}$.corr

Core condition EXCELLENT Date Described 12 MAR.75 by bada

Physiographic location IN AREA OF SLUMPS OFF SOUTHWEST AEFKA.

Lithologic

Detailed Description

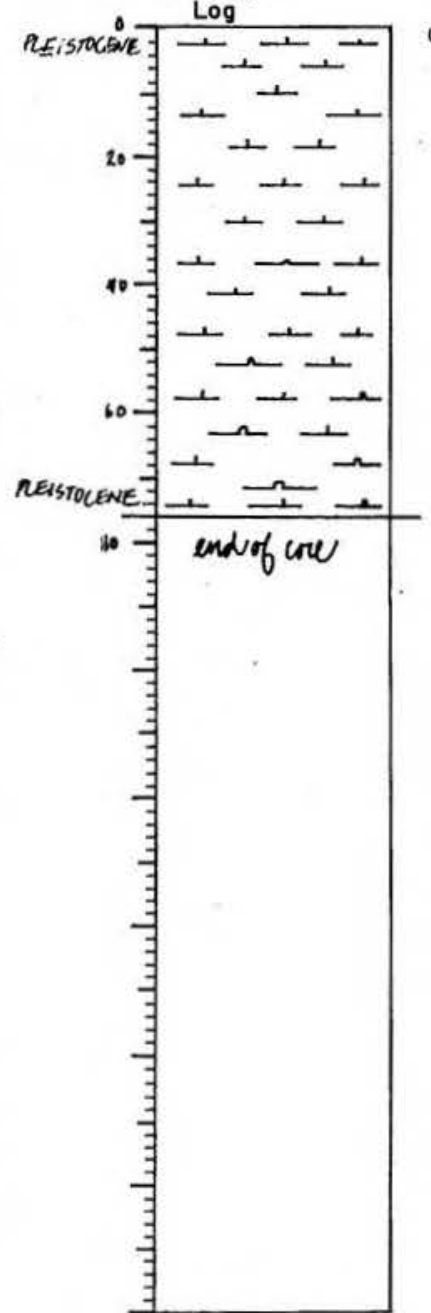
SY $6 / 4$ pale olive

molat al ailty lutite uith scattered forang

end of core
CALC OOZE GRADES 20 CALC SILIC OOZE 
248

SMEAR SLIDE DESCRIPTIONS - W.H.O.I. SEDIMENT CORES

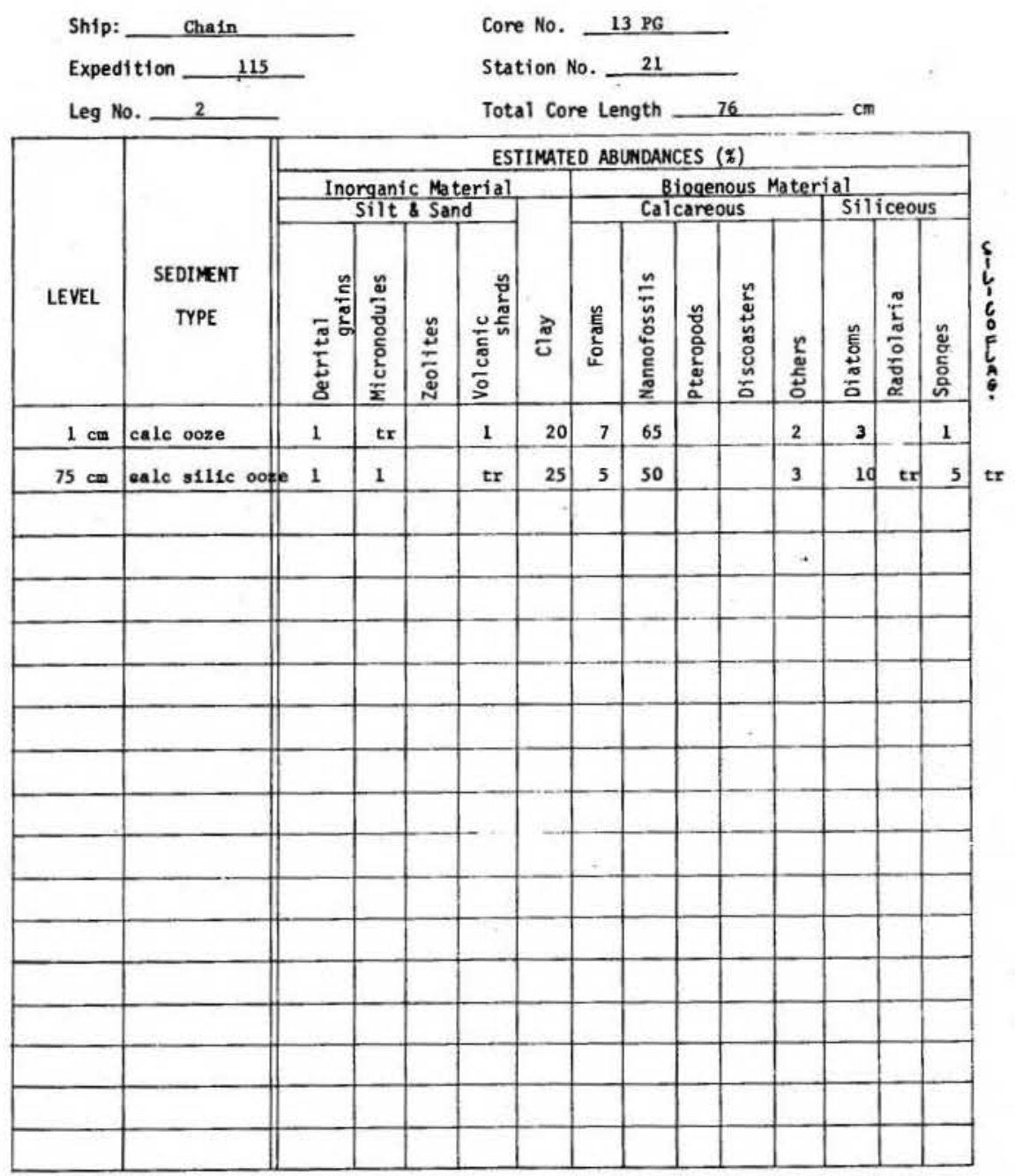

249

VISUAL CORE DESCRIPTION

Page 1 of 1

Ship CHAIN Cruise 115 Leg 2 sto. 22 Core No. $14 \mathrm{CC}$

Total Length $53 \mathrm{~cm}$. Lat. $22^{\circ} 30.0^{\circ} \mathrm{S}$ Long. $Q^{\circ} 084^{\circ} \mathrm{E}$ Depth 2429 m.corr.

Core condition EXLEVENT Date Described 13 Max.75 by Bumbe

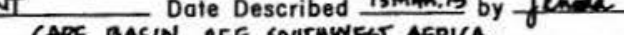

Physiographic location

Lithologic

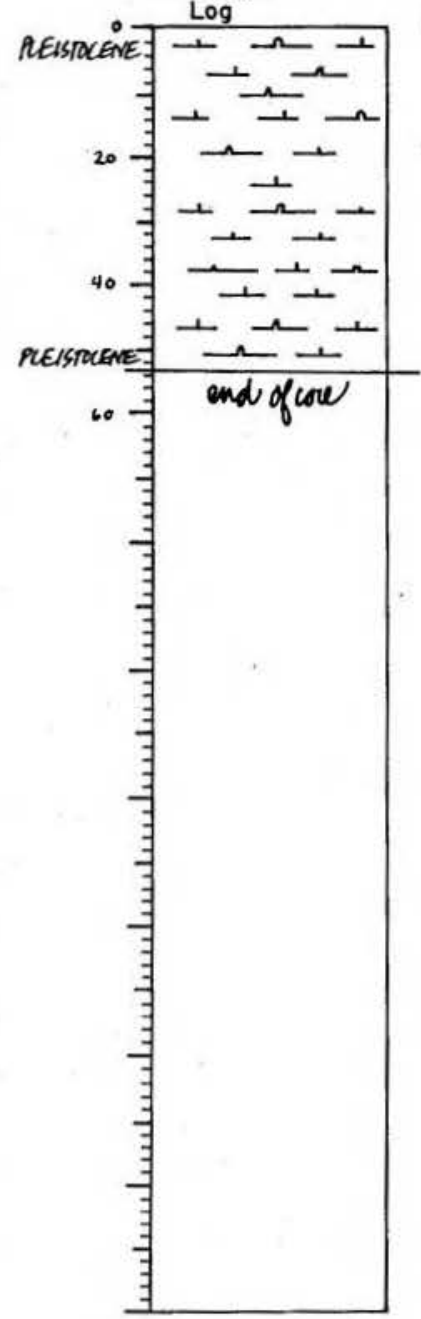

$0-53$

Detailed Description

\section{CAIC SILIC DOZE}

a bit of pale ollve marbling and banding found scattered

v. moist slick sl silty lutite with acattered forams

core is cunulative, represents three successive hits of pogo camera frame. 
250

SHEAR SLIDE DESCRIPTIONS - W.H.O.I. SEDIMENT CORES

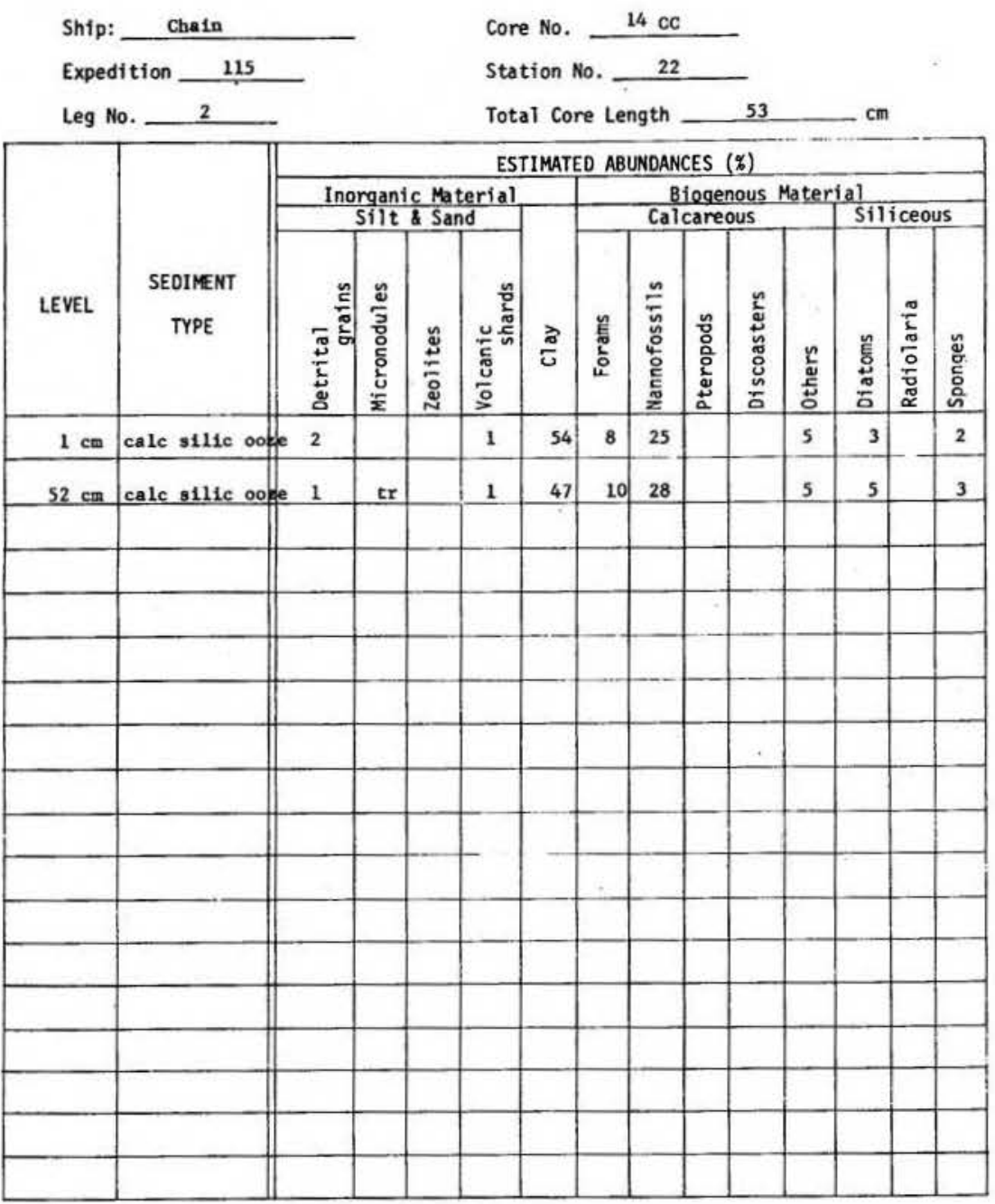

251

VISUAL CORE DESCRIPTION

Page 1 of 1 Ship CHAIN Cruise 115 Leg 2 sta. 23 Core No. $15 \mathrm{CC}$ Total Length $83 \mathrm{~cm}$. Lat. $24^{\circ} 04.17^{\circ} \mathrm{S}$ Long. $12^{\circ} 39.39^{\prime} \mathrm{E}$ Depth $2194 \mathrm{~m} . \mathrm{kar}$ Core condition EXCELENT Date Described 13 MAR75 by Jenda Physiographic location IN AREA OF SUUMPS OFF SOUTHWEST AFDICA. Lithologic

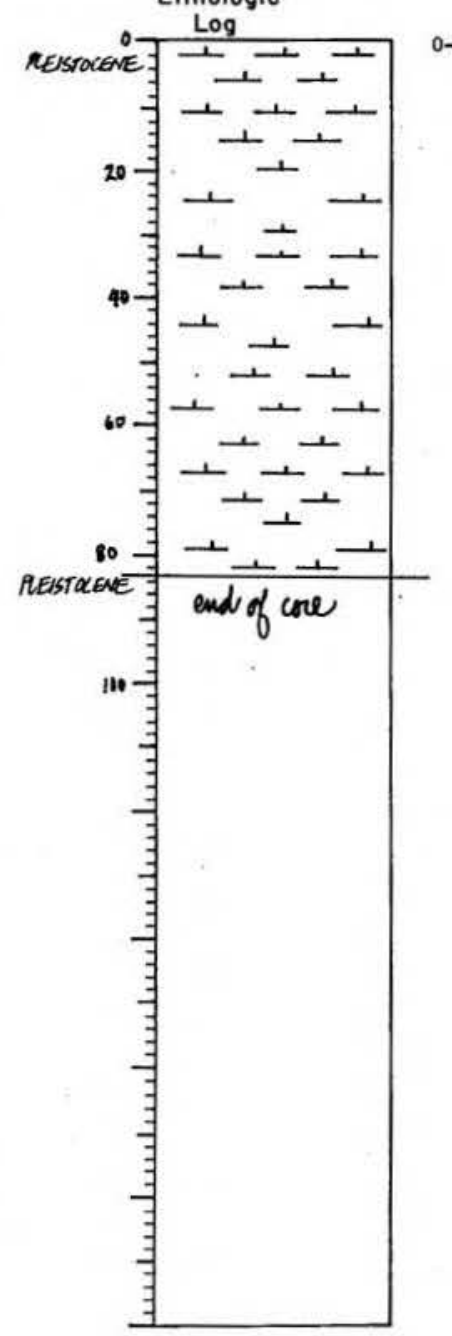

CALC OOZE

SYR $6 / 4$ pale ollve

very poist si silty lutite with scattered forams

NOTE: core is cumulative, represents 10 successive hits

of pogo camera frame.

end of core 
252

SIEAR SLIDE DESCRIPTIONS - W.H.O.I. SEDIEENT CORES

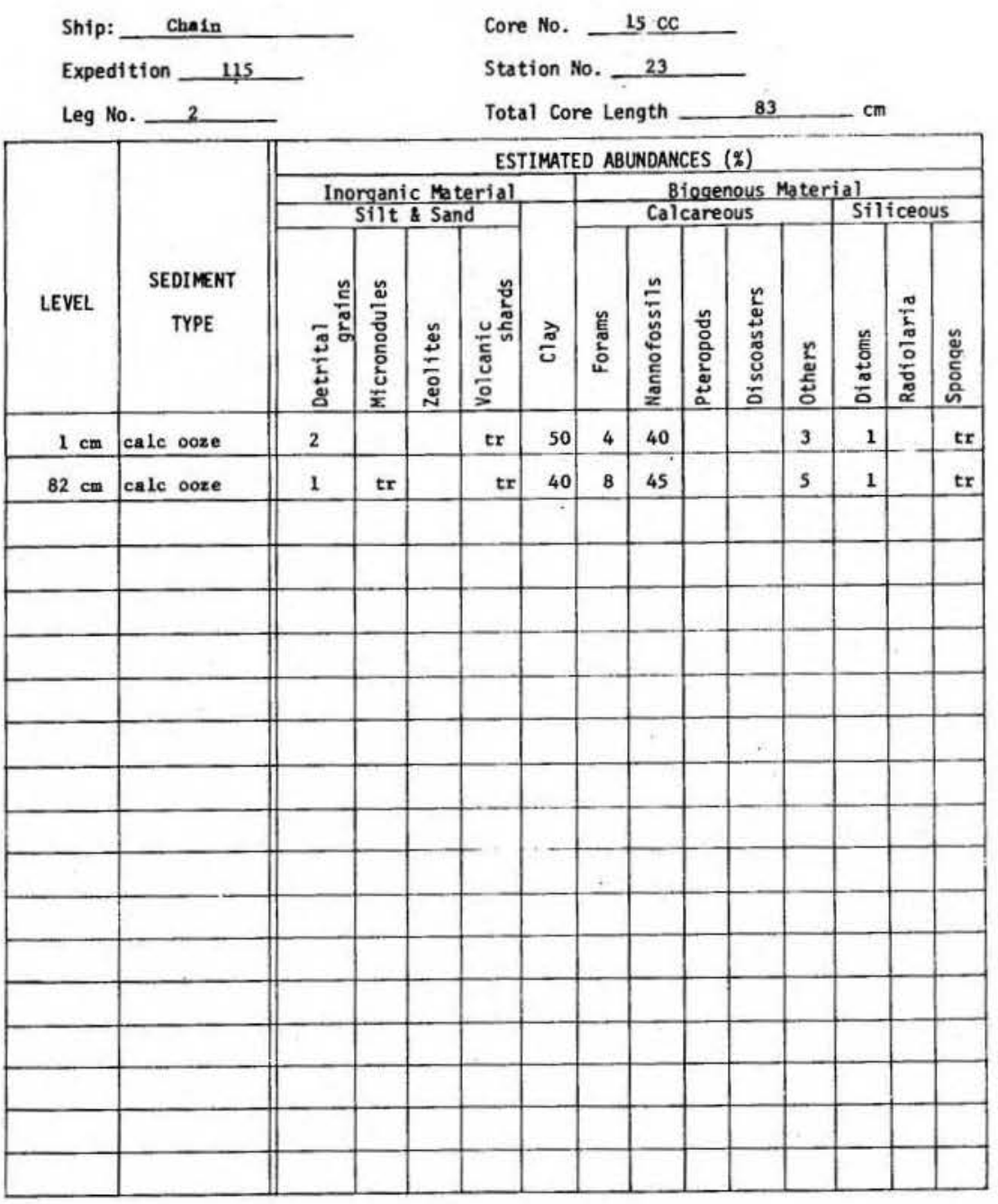

253

VISUAL CORE DESCRIPTION

Page 1 of 1

Ship CHAIN Cruise 115 Sto 23 Core No. I6PC

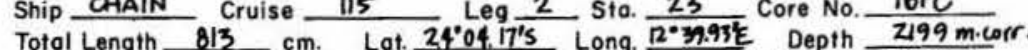
Core condition ExLELENTI Date Described zomane 15 by fouda. Physiographic location_IN AREA OF SWMPS MOMSOUTHWEST AFRKA

Lithologic

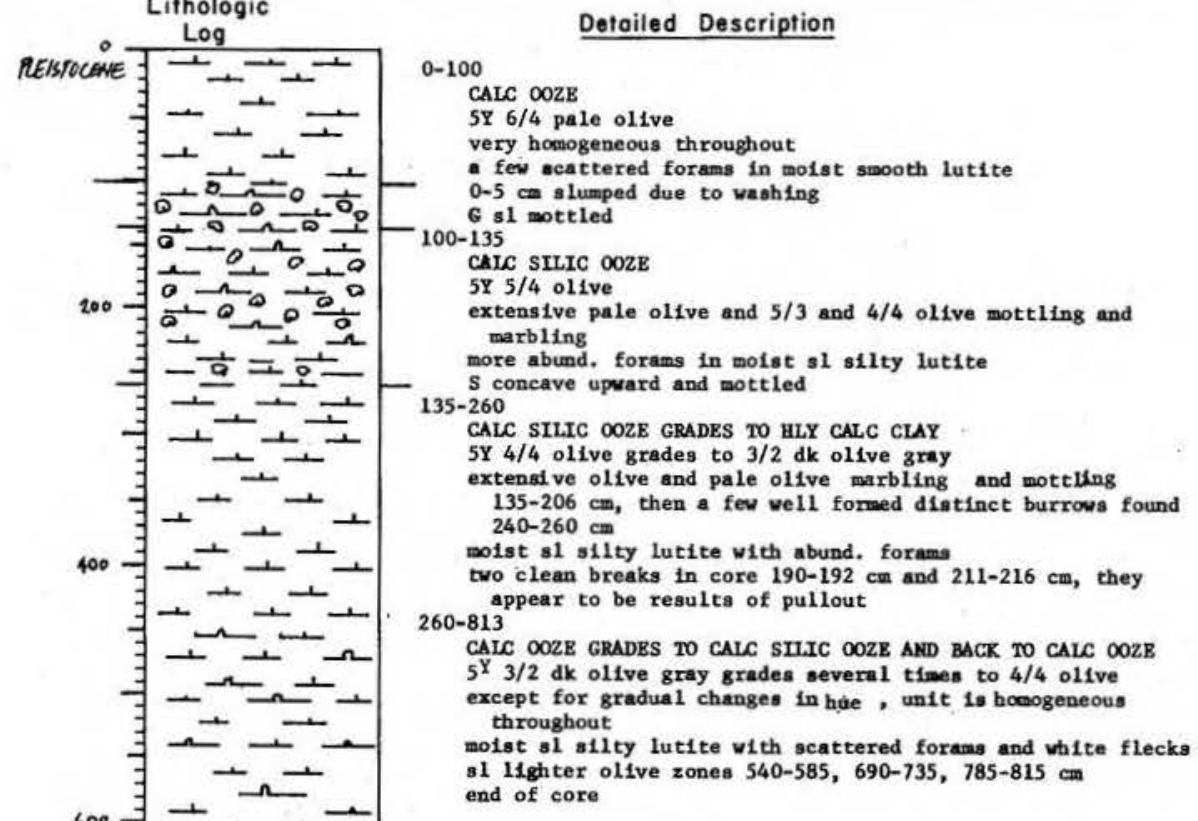

$600 \exists+1+$

斗

$\exists+I^{2}+$

든

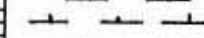

$+\frac{1}{1}+$

RESTRESE

end of core.

900

将 
254

SHEAR SLIDE DESCRIPTIONS - W.H.O.I. SEDIMENT CORES

Ship: Chain

Core No. $16 \mathrm{PC}$

Expedition 115

Station No. 23

Leg No.

Total Core Length

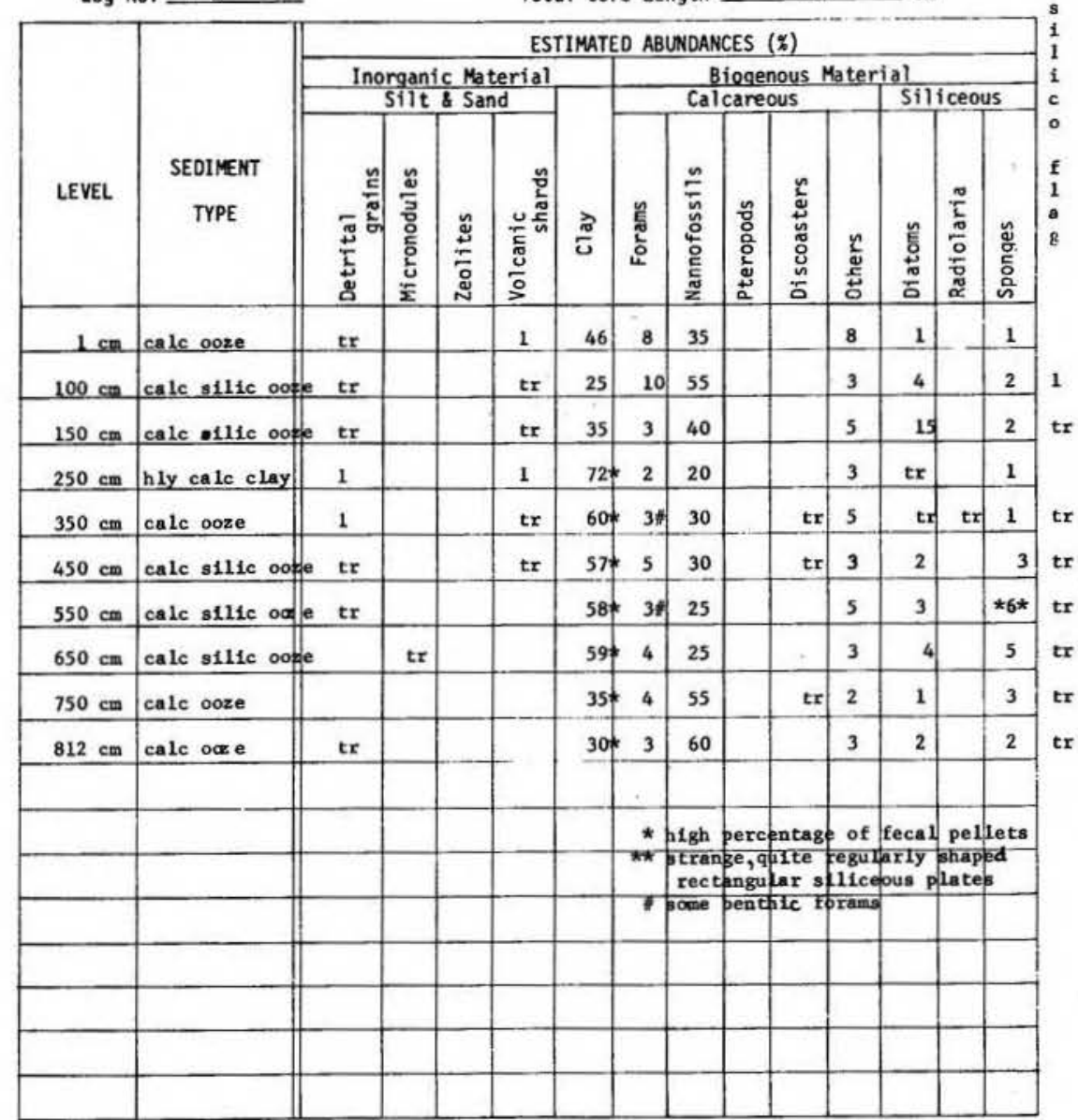

255

VISUAL CORE DESCRIPTION

Page 1 of 1

Ship CHAIN Cruise 115 Leg 2 Sto. 23 Core No. I6PG Total Length $77 \mathrm{~cm}$. Lat. $24^{\circ} 04.7^{\prime} \mathrm{S}$ Long. $12^{\circ} 39.93^{\circ} \mathrm{E}$ Depth $2199 \mathrm{meor}$. Core condition EXEUENT Date Described 20 Mane 75 by forda. Physiogrophic locotion INAREA OF SUUMPS: OFF SOMUWEST AFRleA. Lithologic

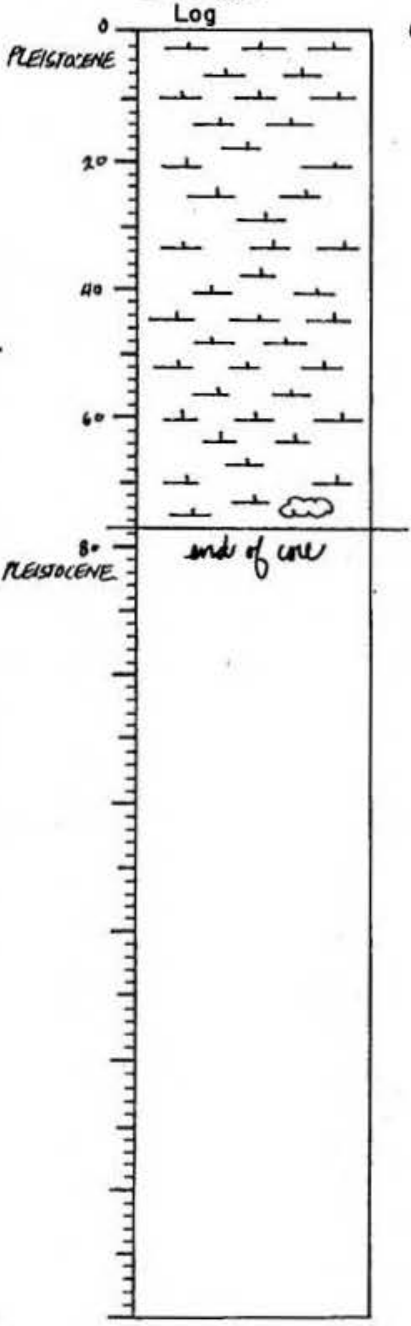

0-77

Detailed Description

CALC OOZE

Pare olive burro extending $2 / 3$ 's the width of the liner at $75 \mathrm{~cm}$ molst swooth lutite with a few scattered forams end of core 
256

SMEAR SLIDE DESCRIPTIONS - W.H.O.I. SEDIMENT CORES

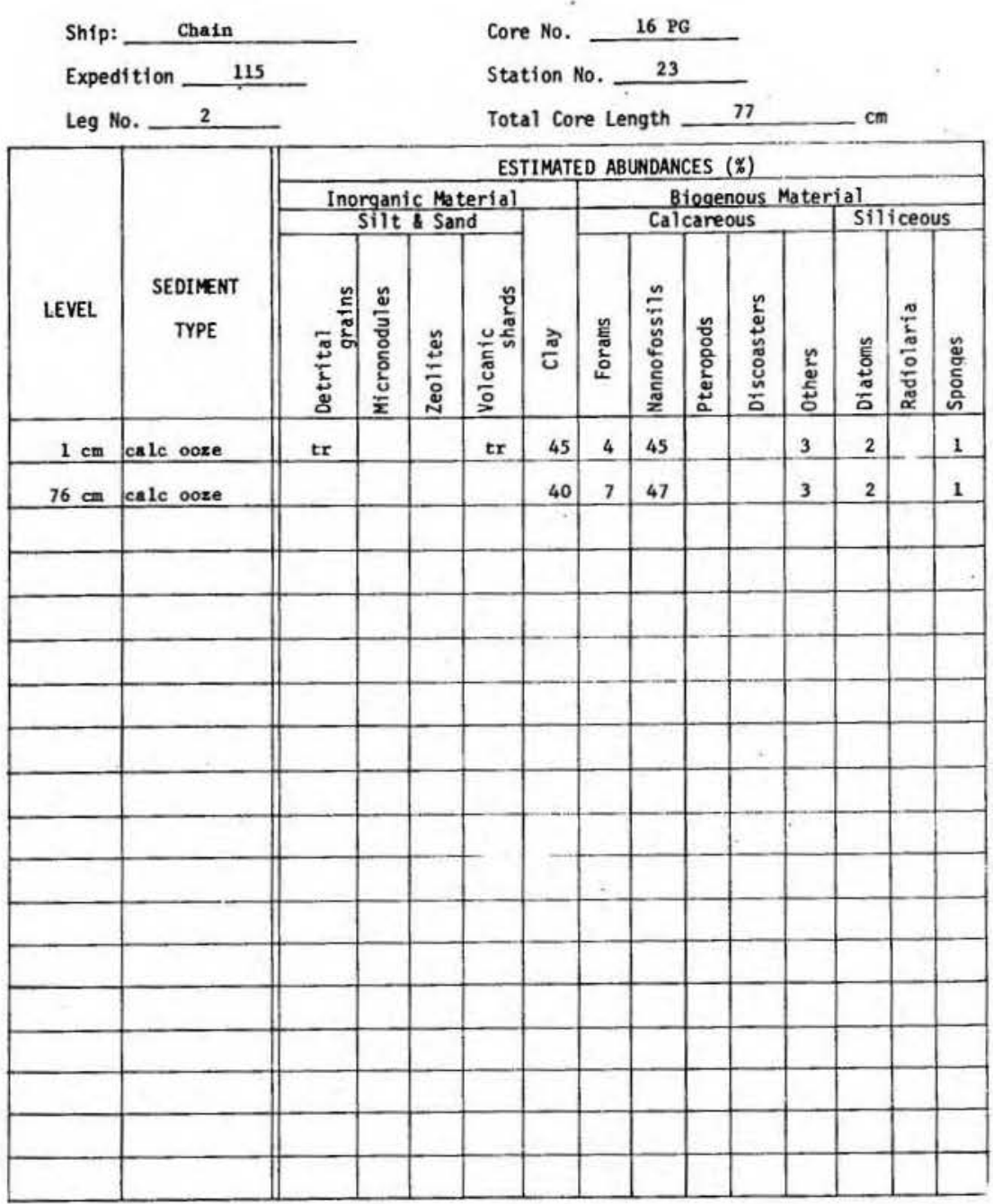

257

VISUAL CORE DESCRIPTION

Page 1 of 3

Ship CHAIN Cruise 115 Leg 2 Sta. 24 Core No. I7PC Totol Length $872 \mathrm{~cm}$. Lat. $2500.3^{\prime} \mathrm{S}$ Long. $10^{\circ} 4 \mathrm{R}^{\prime} \mathrm{E}$ Depth $4254 \mathrm{~m}$.corr Core condition EXLELLENT. Date Described 21 MMR75 by froder. Physiogrophic location_CONIINENTAL RISE OFF SOUTH WEST APRICA. Lithologic

Detailed Description

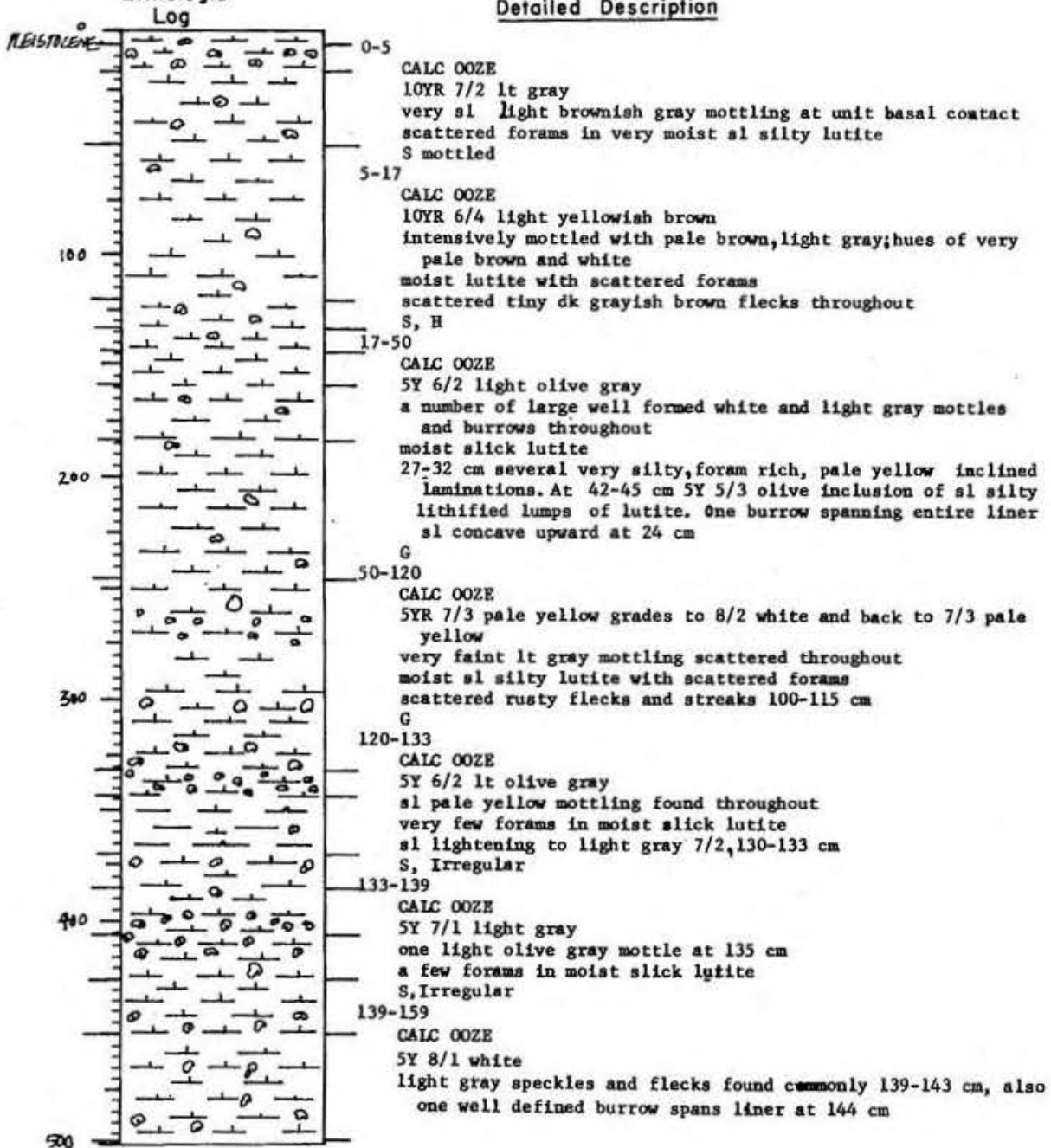




\section{8}

VISUAL CORE DESCRIPTION

Ship CHAIN Cruise 115 Leg 2 Sta. 24 Core No. 17 P

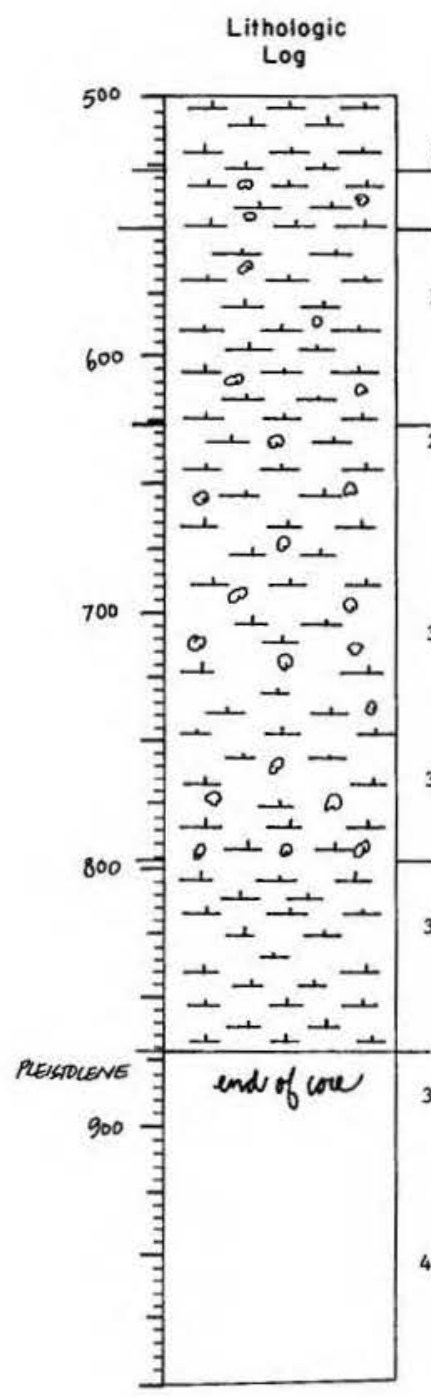

Detailed Description

moist s1 silty lutite with scattered forams 5 mottled

CALC OOZE

$5 \times 7 / 1$ 1ight gray

faint pale yellow aarbling and burrowing found 31 throughout molst lutite with scattered forams

5,
170-245

$$
\text { CALC OOZR }
$$

very faint light g y burrows and mott 1 ing moist lutite with scattered forams

occasional rusty flecks found scattered throughout - 6 mottled

SY $6 / 2$ LER (a) white mottles 260-272 cm, common olfve gray mottling and maxbling elsewhere $G$ mot
$2-343$

CALC OQE E

Y $6 / 4$ pale olive

very extensive 'tiny light olive gray mottling throughout $S$ mottled

343-370

SILIC CALC CLAY

SY $4 / 3$ olive grades to $5 / 3$ olive

In gradationsl zone ist slick lutite, no foram

370-385

385

CALC OOZE

SX $7 / 2$ 1ight gray

abund. motting $370-380 \mathrm{~cm}$ with above unit

a few tiny black flecks and specks scattered

regular

CALC OOZE

SY $8 / 2$ white

common large 11ght olive gray mottles $385-394 \mathrm{~cm}$, extensive

tiny light olive gray mottling $394-406 \mathrm{~cm}$
scattered forams in firm silick lutite

06-425

SY $6 / 2$ light olive gray

very extensive tiny light gray mottles $406-417 \mathrm{~cm}, 1 \mathrm{~g}$. burrous
and 11ght gray maxbling $417-425 \mathrm{~cm}$

VISUAL CORE DESCRIPTION 259

Page 3 of 3

Ship CHAIN Cruise 115 Leg 2 Sta. 24 Core No. 17PC

Lithologic

Log

\section{Detailed Description}

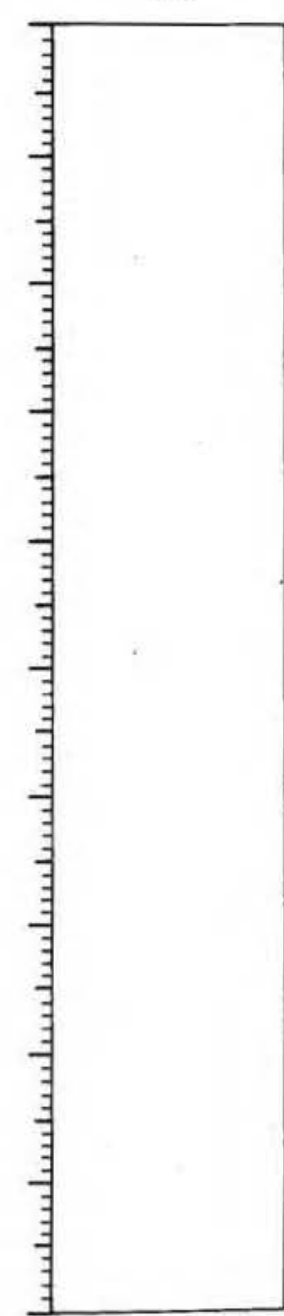

moist smooth lutite, no forams $\underset{425-450}{\text { S ve }}$ $25-450$

NAN\$O OOZE
$5 \mathrm{Y} 8 / 2$ white grades to $7 / 1$ 1ight gray burd. white mottling in light gray zone $440-450 \mathrm{~cm}$

$G$ mottled

CALC OOZE

$5 Y 6 / 2$ light olive gray grades to $5 / 2$ olive gray extensive intermottling and marbling of the two color above plus a few white mottles $480-490 \mathrm{~cm}$ iny black flecks tork

scattered $470-480 \mathrm{~cm}$

498-527

NANMO OOZE

$5 \times 8 / 1$ white

irm smooth lutite

hue varies slightly throughou

527-550

CALC OOZE

$5 Y 7 / 2$ light gray grades to $6 / 2$ light olive gray, back to $7 / 2$ slight light gray motting

imm amooth lutite with a few scattered forams a few black flecks and streaks, $530-540 \mathrm{~cm}$

550-626

NANMO OOZE

SY $8 / 2$ white

faint pale yellow marbling throughout

firm almost plastic-1ike lutite with a few scattered forams and black flecks

626-796

NANAO OOZE GRADES TO CALC OOZE

$5 \times 6 / 2$ 1ight olive gray grades to $7 / 2$ light gray cominon vilte, 1ight gray and pale yellow motting, a few odd $2.5 Y 6 / 2$ 11.ght brownish gray mottles $660-670 \mathrm{~cm}$

firm smooth lutice with a few forams

grey and 11ght gray mottling abund. 725-740 with 11ght olive $766-780 \mathrm{~cm}$ very C
$96-872$

NANNO OOZE

$5 Y 8 / 1$ wh1te

few black flecks and streaks found scattered

end of core 
260

SIEAR SLIDE DESCRIPTIONS - W.H.O.I. SEDIENT CORES

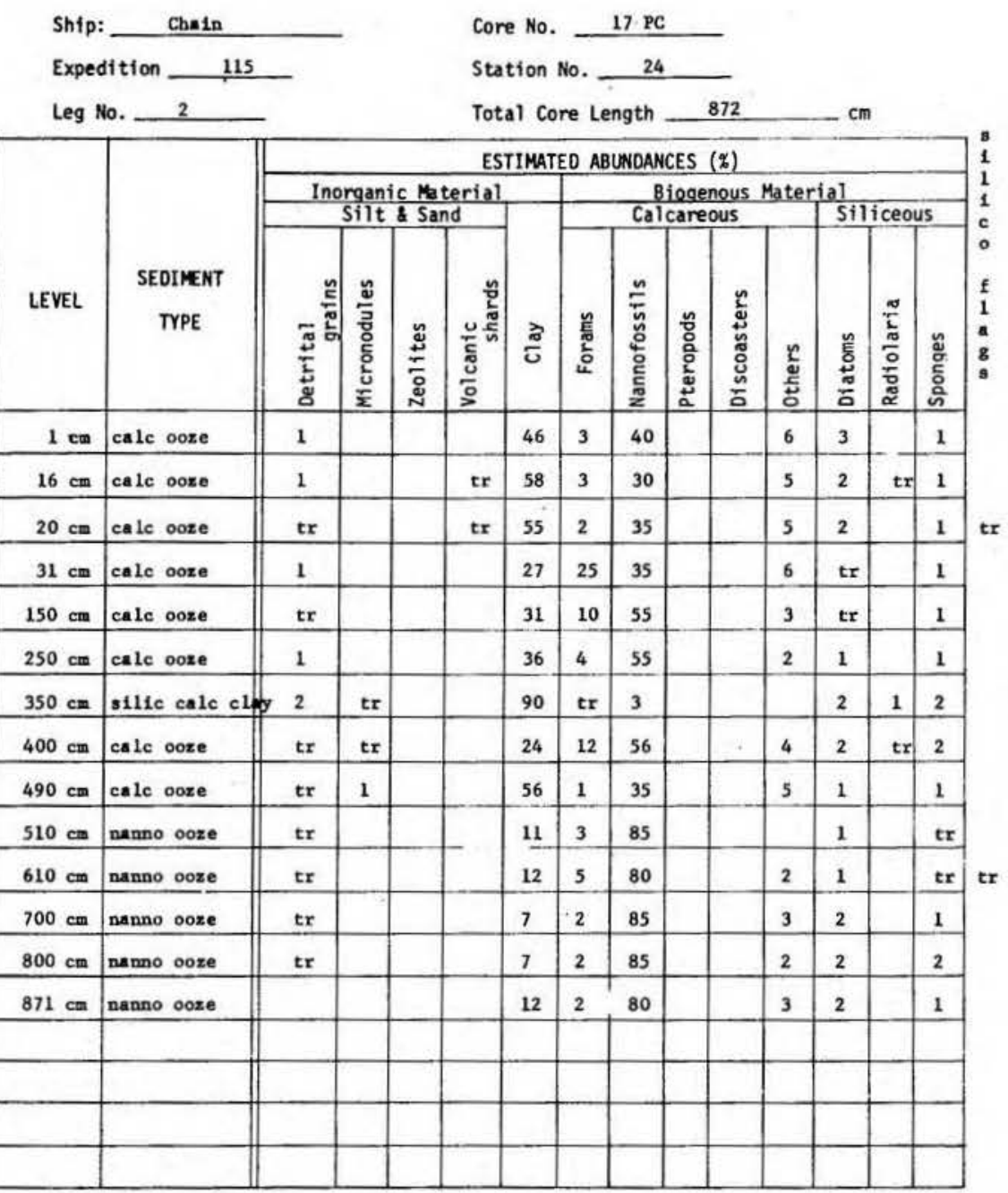

VISUAL CORE DESCRIPTION

Page_ 1 of 1

Ship GHAIN Cruise 115 Leg 2 sto. 24 Core No. $17 \mathrm{PG}$ Total Length $100 \mathrm{~cm}$. Lat. $25^{\circ} 00.3^{\prime} \mathrm{S}^{\mathrm{S}}$ Long. $10^{\circ} 40.3^{\prime} \mathrm{E}$ Depth $4254 \mathrm{mem}$ Core condition Excellent Date Described 24Mur.75 by fonde Physiographic location CONFINENTAL RKE OFF SUUTHWEST ABRICA. Physiographic location CONIINEarat Rus of

Lithologic

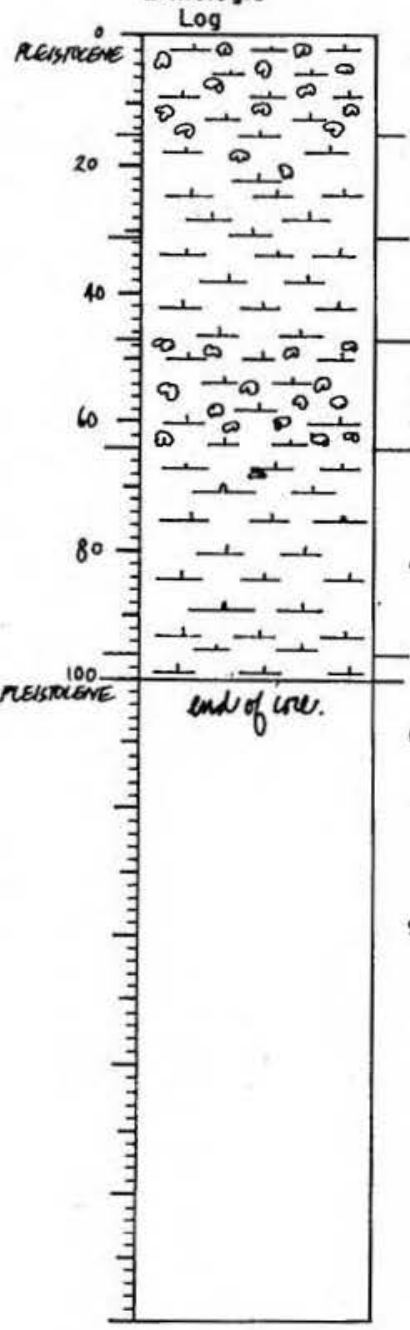

Detailed Description

0-15

CAIC OOZE

1OYR $6 / 4$ it yellowish brown

extensively mottled with pale brown, hues of very pale brown, scattered forams in mo1st lutite

15-31, CALC OOZE

5Y $6 / 2$ 1t olive gray

three vell formed large white mottles $18-24 \mathrm{~cm}$

oist slick lutite

28-30 ca a very silty pale yellow inclined lami nation spans $\mathrm{s}, \mathrm{I}^{\circ}$ $\mathrm{S}, \mathrm{I}^{\circ}$

CALC OO2E

$10 Y R 7 / 2$ 1t gray

hosogeneous throughout

47-64 6 mottled

CAIC OOZE

10YR 6/4 1t yellowish brown

extensive pale brown, very pale brown, white and it gray motting

lutite with scattered forams

64-96

CAIC SILIC OOZE

$5 Y 6 / 2$ it olive gray

one stan 11 white mottle $65 \mathrm{~cm}$

moist silck lutite

pale yellow inclined laminations

$\mathrm{S}_{6-100}^{\text {sp }}$

CAIC OOEE

IOYR $7 / 2$ 1t gray

onogeneous throughout

mith scattered foram

and repetitive series suspected to be

1st penetration $0-31 \mathrm{~cm}$

3rd penetration. 31-96

ad penetration $96-100 \mathrm{~cm}$

end of core 


\section{2}

SMEAR SLIDE DESCRIPTIONS - W.H.0.I. SEDIMENT CORES

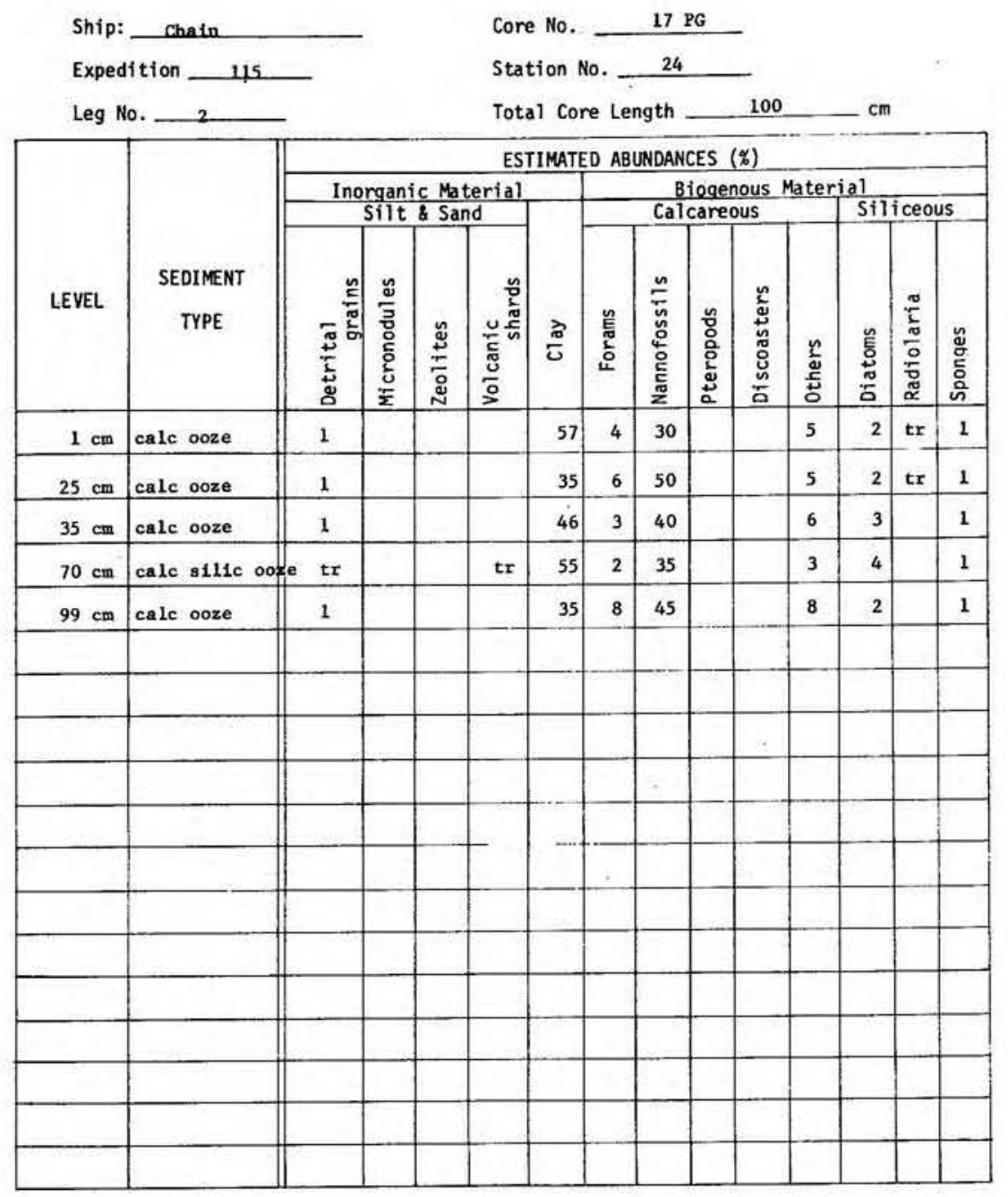

263

VISUAL CORE DESCRIPTION

Page 1 of 2

Ship CHAIN Cruise 115 Leg 2 Sto. 26 Core No. $19 \mathrm{PC}$

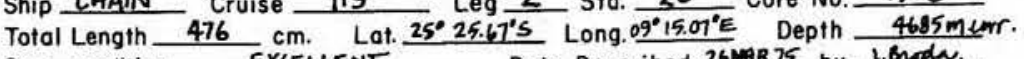
Core condition EXLELLENT Date Described 26MAR75 by forodu. Physiographic location ABYSSAL PLAOY: CAPE BASM

Lithologic

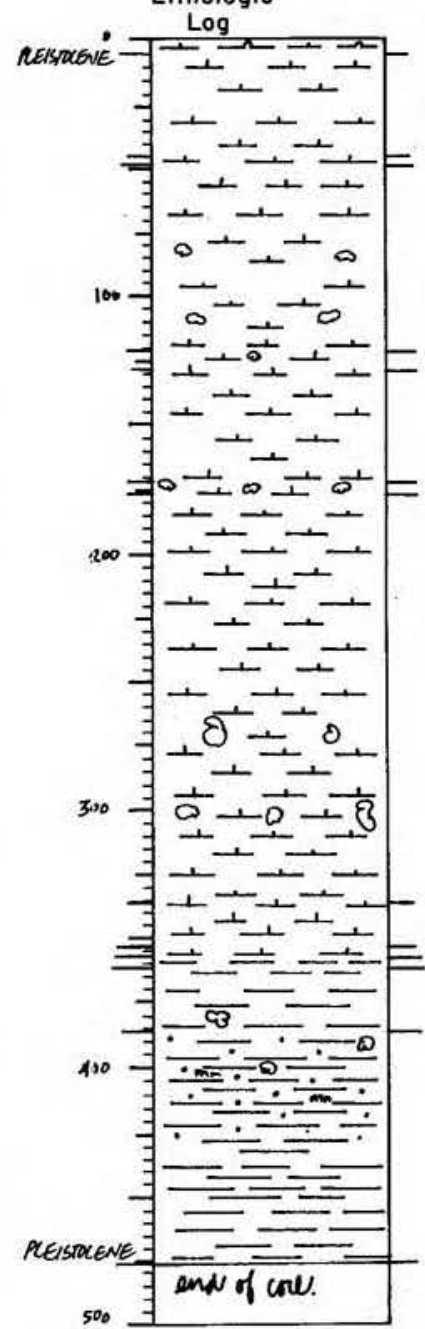

\section{0-5 Detailed Description}

CALC SILIC OOZE

10YR $6 / 3$ pale brow

grayish brown $5 / 2$ marbling throughout scattered forams in very moist sl silty lutite ashed and disturbed

-45 S, I

CALC OOZE

10YR $7 / 2$ light gray

extensive- /n-rich black and dk gray flecks and streaks throughout
fimmer 81 silty lutite with scattered forams 45-49,

CALC OOZE

10YR $7 / 3$ very pale brown

numerous dark gray and gray laminations and common marbling

inm smooth lutite with a few foram S, si concave dowmard

CALC OOZE

$2.5 \times 8 / 2$ white

light gray $10 \mathrm{XR} 7 / 1$ shadowy marbling found virtually throughout, missing only in laminated zone

firm smooth lutite of th occssions 1 forams
an unlikely but seemingly undisturbed, totally enclosed an unlikely but seemingly undisturbed, totally enclosed
$1.5 \mathrm{~cm}$ diameter, 11thified, hly calc clay nodule at 100 $\mathrm{cm}$, also a very small $2 \mathrm{~mm}$ piece of the same found at $60 \mathrm{~cm}$. 111-118 cm a series of 11ght gray and white laminations $1-4 \mathrm{~mm}$ thick $\mathrm{S}$ concave upward

121-128

CAT OOZE
$2.5 Y$ Y $7 / 2$ light gray

(uhirled) yith $2.5 \times 8 / 2$ white

firm smooth lutite with a few foram $\mathrm{S}, \mathrm{I}$

128-172

$2.5 \mathrm{Y} 8 / 2$ white

occasional faint 1OYR $8 / 1$ white or light gray marbling and mottling

irm smooth lutite with scattered forams

S,

LYR $7 / 3$ very pale brown

extensive marbling (whirled) with $2.5 \mathrm{Y} 8 / 2$ white

sl silty luttte with scattered forams

$s$, maxbled 
VISUAL CORE DESCRIPTION

Page 2 of 2

Ship CHAIN Cruise 115 Leg 2 Sto. 26 Core No. 19PC

Lithologic

Log

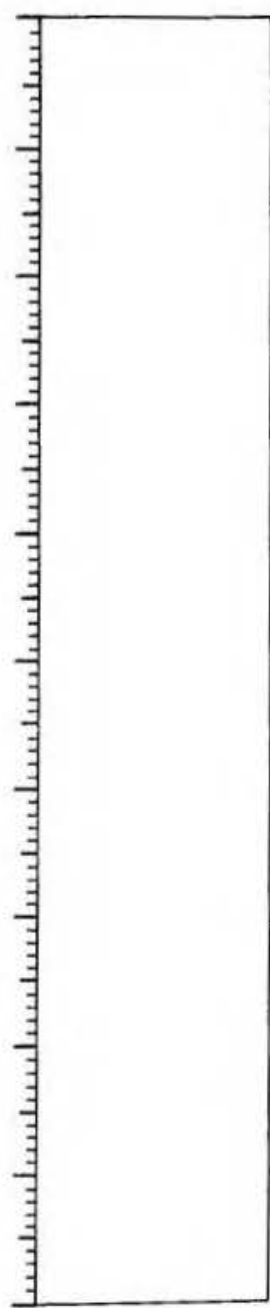

Detailed Description

176-336

\section{CALC OOZE WITH NANAOO OOZE INCLUSIONS}

$.5 \mathrm{Y} 8 / 2$ white
various inclined swirls, laminations and marbling with 10YR 8/1 white, pale ollve and light gray

NOTB: Th1s entire unit appears sosevhat disturbed and disoriented. Highly inclined, non-continous and

sediments exist below this section in the core.
sion 336-353

$$
\text { CALC OOZE }
$$

SY $8 / 1$ and $8 / 2$ white

liternating sometimes incomplete laminations of color

above (all concave upuard).

very finm smooth lutite, no forams

S very concave upwar

CALC 002

$5 \mathrm{Y} 6 / 2$ 11ght olive gray

f1mo smooth lutite, no forang

$\underset{356-361}{S, ~}{ }^{B}$

UNFOSS CLAY

X $6 / 4$ pale olive

slight fa int 10YR $5 / 3$ brown marblin

th lutite, no forams

unit contac:

G,
$361-386$

$1-386$ insoss ciay

\section{UNFOSS CLAY}

OYr $5 / 3$ brown

$\mathrm{firm}$
6
$386-476$

UNFOSS CLAY/Mn GRADES TO UNPOSS CLAY

10YR $4 / 3$ dark brown

very large $5 / 3$ brown mottles found commonly $386-440 \mathrm{~cm}$,

throughou

flecks suggests flow in

end of core
265

SMEAR SLIDE DESCRIPTIONS - W.H.O.I. SEDIMENT CORES

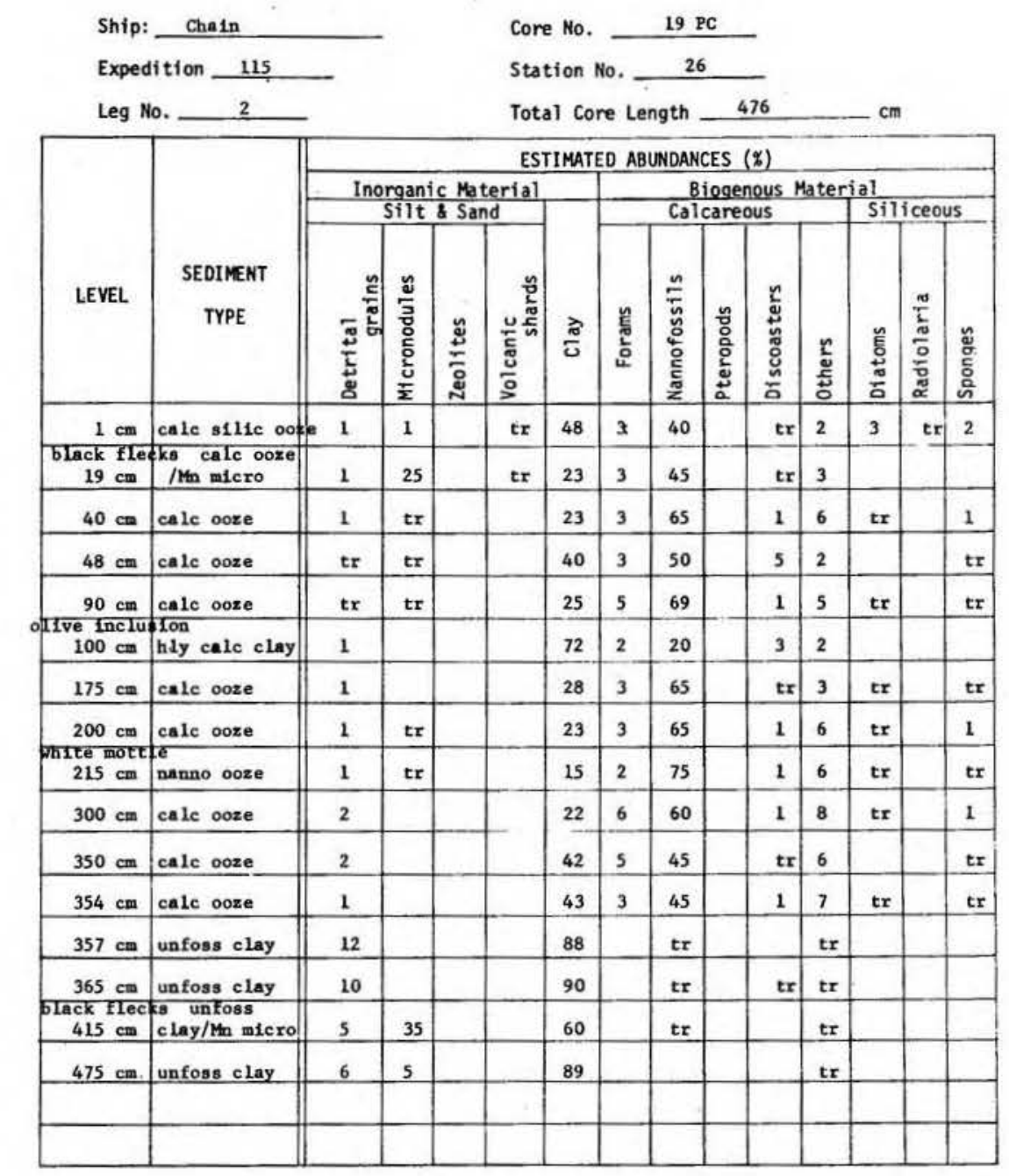


266

VISUAL CORE DESCRIPTION

Page 1 of 1

Ship CHAIN Cruise 115 Leg 2 Sta. 27 Core No. 20PC

Total Length $321 \mathrm{~cm}$. Lat. $25^{\circ} 02.4^{\prime} \mathrm{S}$ Long. $07^{\circ} 47.38^{\prime} \mathrm{E}$ Depth $4738 \mathrm{~m}$

Core condition EXCELLENT Date Described 2bMaB 75 by

Physiogrophic location CAPE BASIN NEAR WPLUS RIDGE.

Lithologic

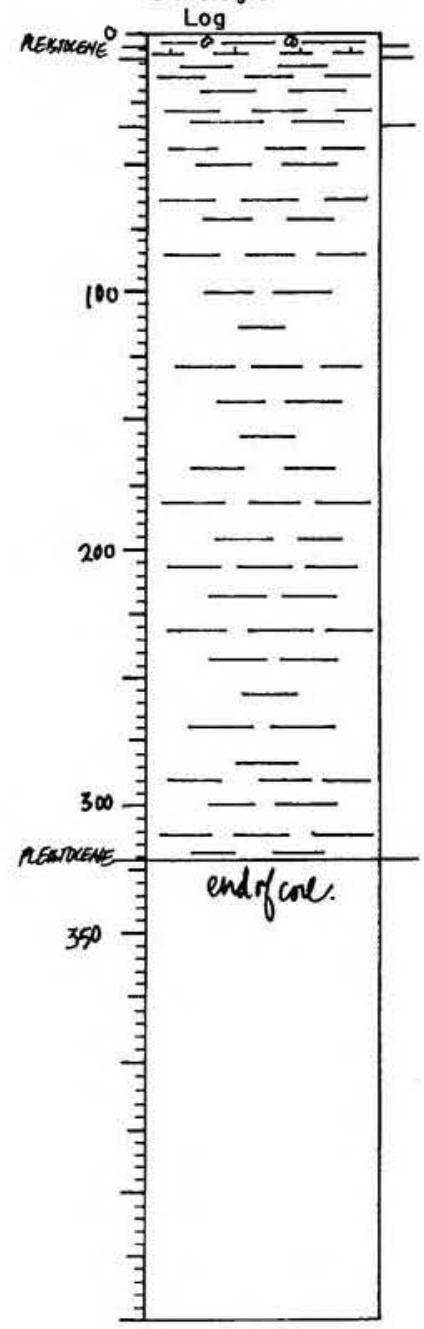

\section{Detailed Description}

$0-4$

UNFOSS CIAY

extensive pale brown marbling and black flecks fim slick lutite

CALC OCZ E

10YR $6 / 3$ pale brown

marbling throughout

silty lutite with abund. foram

8-35 ve upwar

UNFOSS CIAY

10YR $4 / 3$ brown

ches and fleck

5-321

UNFOSS CIAY

10YR $4 / 4$ dark yellowish brown

very homogeneous throughout

firm silck lutite

voTe: core did

corer essed into mud, red to trip upon pullout.

end of core.
267

SMEAR SLIDE DESCRIPTIONS - W.H.O.I. SEDIIENT CORES

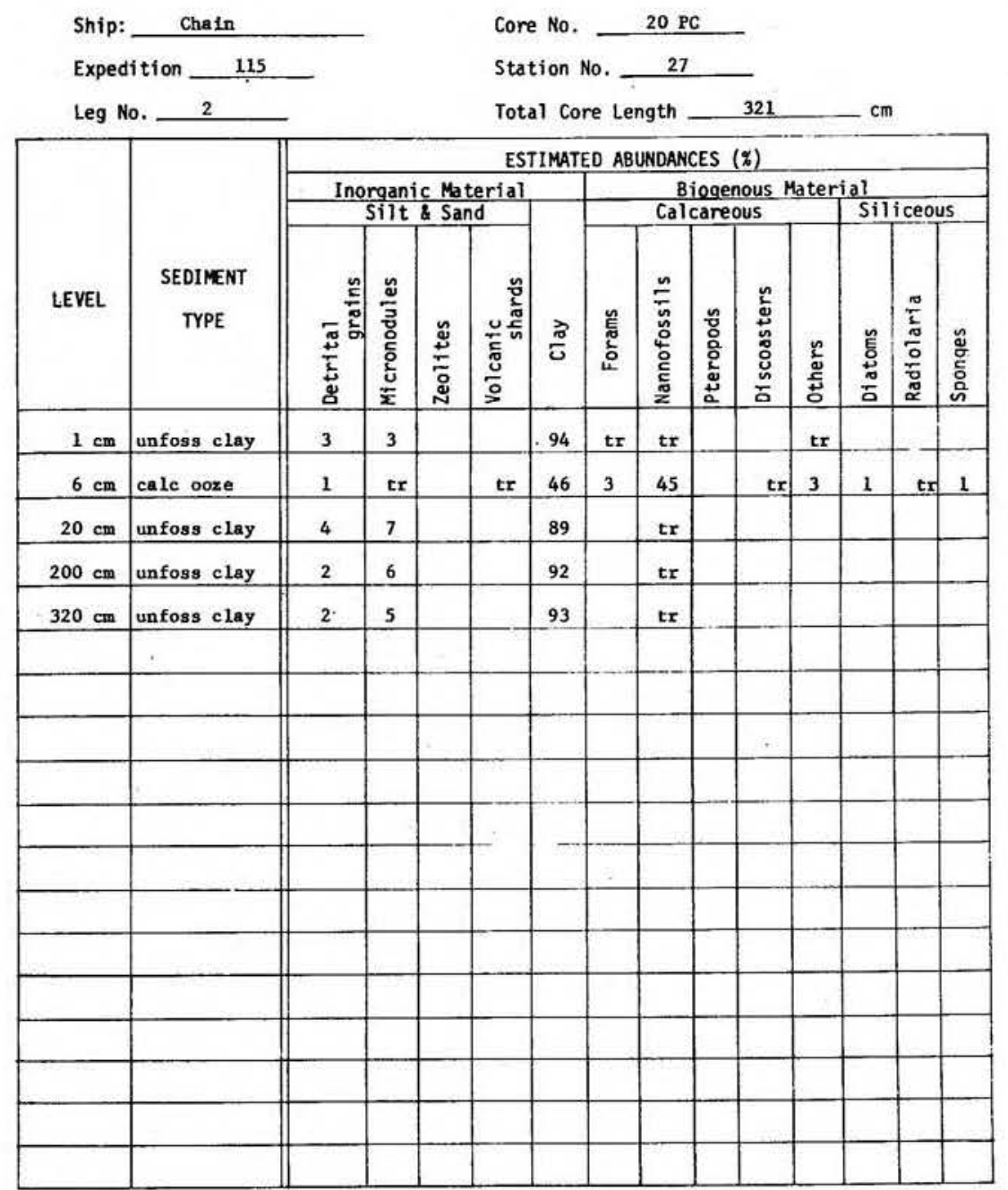


Ship CHAIN Cruise 115 Leg 2 Sto. 27 Core No. 20PG Total Length $107 \mathrm{~cm}$. Lot. $25^{\circ} 024^{\circ} \mathrm{S}$ Long. $07^{\circ} 4730^{\circ} \mathrm{E}$ Depth $4738 \mathrm{~m}-\mathrm{wer}$. Core condition ExuEUENI Date Described 29MAR.75 by Jendw.

Physiographic location CAPE BASIN NEAR WAUNS RDEE. Lithologic

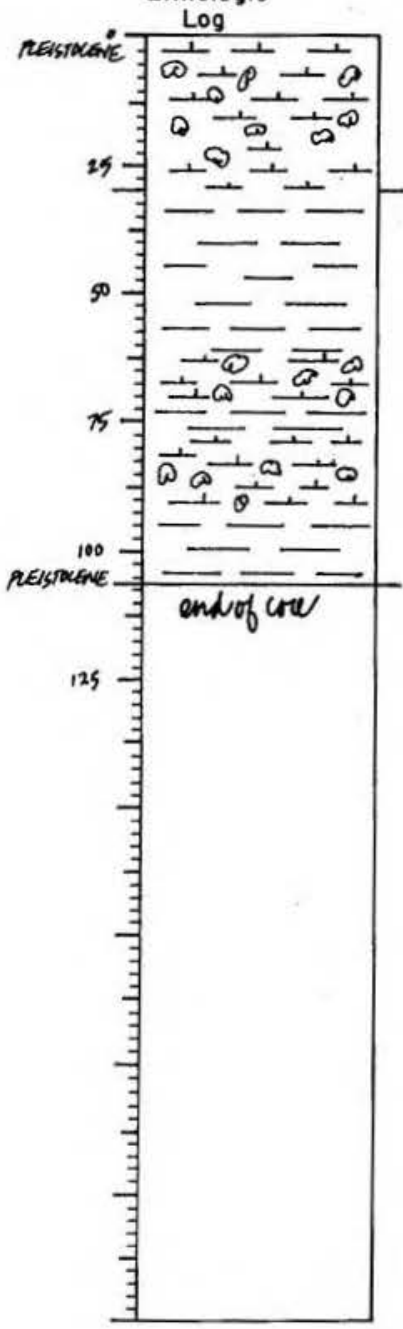

Detailed Description

0-35

CALC OOZB

(OFin $6 / 3$ pale brown grades to $6 / 4$ 11ght yellowish brown extensive very pale brown mottling throughout mo1st s1 silty lutite

35-107

UNFOSS CLAX HTTH MOTILING ZONES OP CALC OOZE 10YR $5 / 3$ brown

yellowish brown mottling $60-70 \mathrm{~cm}$ f1rm sltcker lutite with scattered forams and black flecks end of core

SMEAR SLIDE DESCRIPTIONS - W.H.O.I. SEDIMENT CORES

Ship: Chain

Expedition 115

Leg No.

$$
\text { Leg No. }
$$

\begin{tabular}{|c|c|c|c|c|c|c|c|c|c|c|c|c|c|c|}
\hline & & & & & & & & & & & & & & \\
\hline \multirow[b]{3}{*}{ LEVEL } & \multirow[b]{3}{*}{$\begin{array}{c}\text { SEDIMENT } \\
\text { TYPE }\end{array}$} & \multicolumn{13}{|c|}{ ESTIMATED ABUNDANCES $(x)$} \\
\hline & & \multicolumn{4}{|c|}{$\begin{array}{c}\text { Inorganic Material } \\
\text { Silt \& Sand }\end{array}$} & \multirow[b]{2}{*}{$\frac{\pi}{\sigma}$} & \multicolumn{5}{|c|}{$\begin{array}{l}\text { Biegenous Mater } \\
\text { Calcareous }\end{array}$} & \multicolumn{2}{|c|}{$\frac{\text { al }}{\text { Siliceous }}$} & \\
\hline & & 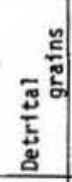 & $\begin{array}{l}\frac{u}{3} \\
\frac{\partial}{0} \\
\frac{5}{0} \\
\frac{0}{\Sigma}\end{array}$ & 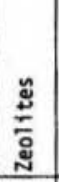 & 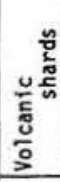 & & 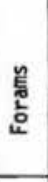 & 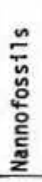 & 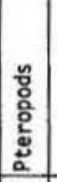 & 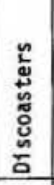 & 产 & 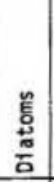 & $\begin{array}{l}\frac{\pi}{2} \\
\frac{\pi}{0} \\
\frac{0}{0} \\
\frac{\tilde{\alpha}}{\alpha} \\
\end{array}$ & 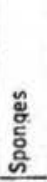 \\
\hline $1 \mathrm{~cm}$ & calc ooze & 1 & 1 & & & 44 & 6 & 40 & & 1 & 5 & 1 & $\operatorname{tr}$ & 4 \\
\hline $50 \mathrm{~cm}$ & unfoss clay & 4 & 7 & & & 89 & & & & & & & & \\
\hline $106 \mathrm{~cm}$ & unfoss elay & 2 & 7 & & & 91 & & & & & & & & \\
\hline & & & & & & & & & & & & & & \\
\hline & & & & & & & & & & & & & & \\
\hline & . & & & & & & & & & & & & & \\
\hline & & & & & & & & & & & & & & \\
\hline & & & & & & & & & & . & & & & \\
\hline & & & & & & & & & & & & & & \\
\hline & & & & & & & & & & & & & & \\
\hline & & & & & & & & & & & & & & \\
\hline & & & & & & & & & & & & & & \\
\hline & & & & & & & & & & & & & & \\
\hline & & & & & & & & & & & & & & \\
\hline & & & & & & & & & & & & & & \\
\hline & & & & & & & & & & & & & & \\
\hline & & & & & & & & & & & & & & \\
\hline & & & & & & & & & & & & & & \\
\hline
\end{tabular}

Core No. 20-PG

Station No. 27

otal Core Length $\quad 107 \quad \mathrm{~cm}$ ESTIMATED ABUNDANCES ( $(x)$ 
Ship CHAIN Cruise 115 Leg 2 Sta. 29 Core No. 23 PC Ship CHAIN Cruise $\frac{115}{877} \mathrm{~cm}$. Lat. $25^{\circ} 03.71^{\circ} \mathrm{S}$ Long. $\frac{27^{\circ} 55^{\circ} .25^{\prime} \mathrm{E} \text { Copth }}{4792 \text { convmetars }}$ Core condition EXUCUENT Date Described 3MAR2 75 by fondes. Physiographic location CAFE OFIN WAWIS AAPEE

Lithologic

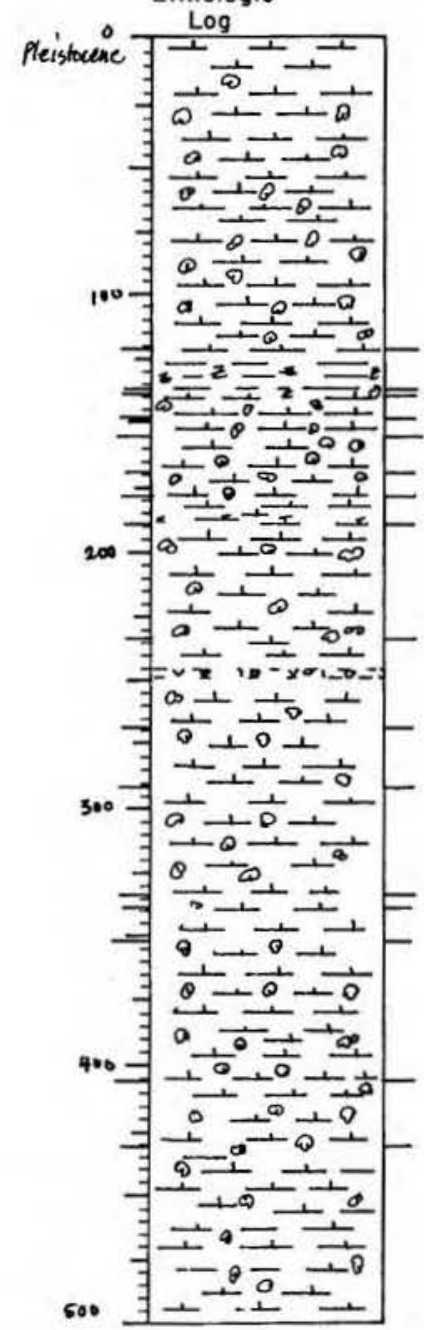

\section{C-121 CALC OOZE GRADES TO NANNO DOZE} 10YR $8 / 1$ white

extensively mottled w1th 1 OXR $8 / 3$ very pale brown and very fad nt light gray very silty plastic lutite with extremely abnd. forams s, I

UNFOSS CLAY/ZEOLITES

1OYR $4 / 4$ dark yellowish brow

extensive intermarbling, 132-137, of 11ght yellowish brown and 7.5Y $5 / 4$ brown, texturally distinct sediments tled zone hes portione with abund fornes and others of slick lutite

$S, I$
$137-139$

NAMNO OOZE

10YR $8 / 1$ white

motes found with unit

lutite with very abond, forams

139-148

\section{CR. C OOZE}

10YR $6 / 4$ 1ight yellowish brown grades-to $5 / 3$ brom extensive theterting of two colors above and black 6 motecks

148-155

NANMO DOZE

1OYR $8 / 1$ white

very extensive light yellowish brown and pale brown moist somem

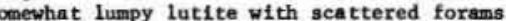
S,

10YR $6 / 4$ 11ght yellowish brown grades to $5 / 3$ brown extensive intermottling of two colors above $G$ mottled

$169-178$ 178 mottled

IOYR $8 / 1$ wh1t

very extensive ilght yellowlsh brown moteling moist sonewhat lumpy lutite with scattered fora 5 mottled

CALC OOZE IOYR $8 / 1$ white

VISUAL CORE DESCRIPTION

Page 2 of 4

Ship CHAIN Cruise 115 Leg 2 Sto. 29 Core No. 23 P C

Lithologic

Log

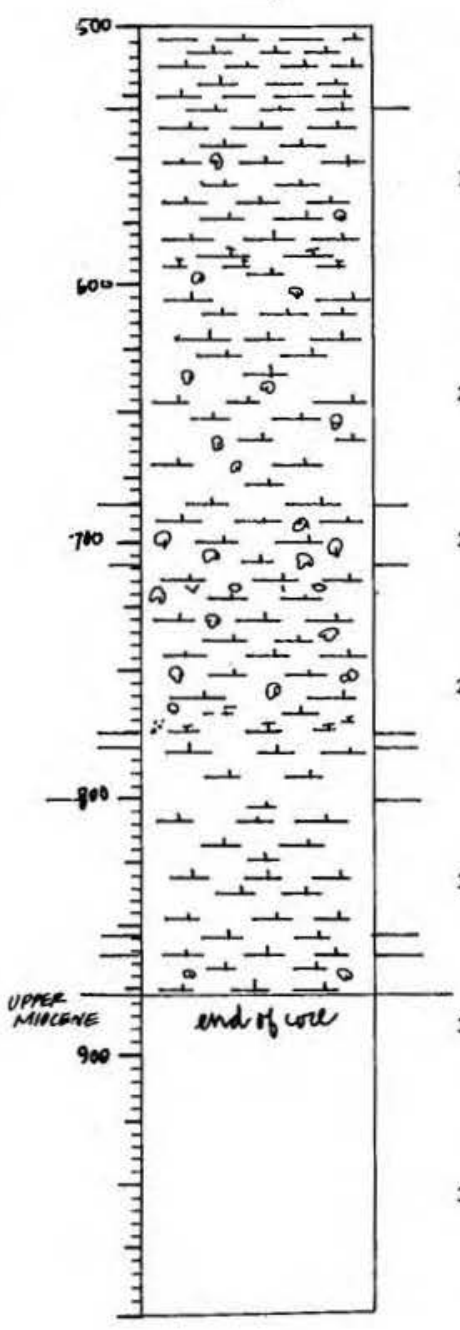

\section{Detailed Description}

shadowy paile brwwn mottles found s1 throughout very firm plastic lutite with increasing foram content has shapp horizontal contact with $1 \mathrm{~cm}$ foram sand bed at unit basal contact
weathered reddish and black detrital grains found coumonly in foram san

CALC $002 E$

10YR $6 / 4$ 1ight yellowish brown and 5/3 brown

extensive intermottling of two colors above plus a zone moist firw lutite with scattered forams, $189-222 \mathrm{~cm}$ a gradand very abund.

33- 268 at bottom
mottled

NANHO OOZB

10 YR $8 / 1$ white

comann light yellowish brown mottling throughou

S,
$268-292$

CAIC 002

10YR $5 / 4$ yellowish brown

coman white moceling grad

moist lumpy lutite with a few foram

G mo

NANHO OOZE

10YR $8 / 1$ whit

cominon light yellowish brown mottling throughout

firm plastic lutite with a few forams

$330-333 \mathrm{~cm}$ foram content rapidly increases and grades to $s$ nearly sand

$S$ mottled
333-337

CALC $002 E$

10YR $6 / 4$ 11ght yellowish brown

s1 very pale brown mottling throughout

moist lutite with scattered forams

$337-352$

NANAO OOZE

NANAO OOZE
IOYR $8 / 1$ wh 1

extensive very pale brown mottling grades to sl scattered firm plastic lutite,scattered forams

graded bedding of forams and fragments $347-352 \mathrm{~cm}$ $S$ in
CALC

\section{CALC OOZB}

CALC 0 OZB
1 OYR $4 / 3$ dark brown

extensive white foram-rich mottling $370-384 \mathrm{~cm}$ moist slick lutite with silty foram-rich mottles 
VISUAL CORE DESCRIPTION Page 3 of 4 Ship CHAIN Cruise 115 Leg 2 Sta. 29 Core No. $23 \mathrm{PC}$

Lithologic

Log

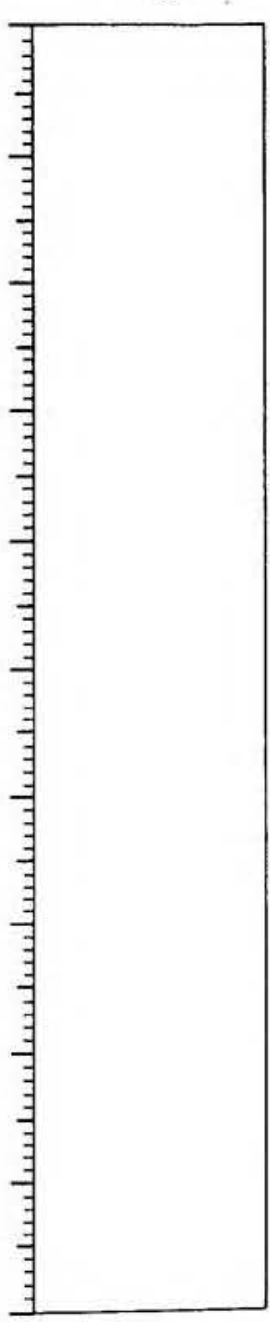

Detailed Description

406-431

CAIC OOZE

very extensive small thite and yellowish brown mottling throughout

fim sl silty lutite; foram content increase with depth from scattered to ahund.

431-530

CALC OOZE (WHITE ZONIS) AND SL CALC CLAY (MARK BROWN ZONES) 1OYR $4 / 3$ dark brown and $8 / 1$ white

extensively mottled chalky ooze, mottling is mostly very
enting

firm compact lutite with varying foram content, most abund. lighter zones $437-450 \mathrm{~cm}, 466-473 \mathrm{~cm}, 485-502 \mathrm{~cm}, 524-530$ $G$ mottled

$530-685$

NAMNO OOZE

LOYR $8 / 1$ white

11 faint very pale brown and light yellowish brown

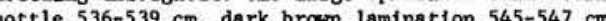
very pale brown lamination $590-593 \mathrm{~cm}, 11 \mathrm{ght}$ yellowish browm band $670-675 \mathrm{~cm}$

firm plastic lutite with a few forams and black fleck throughout, graded foram sand bed $587-591 \mathrm{~cm}$ faint concave upward black fleck laminations scattered

$685-708$

CALC OOZE

1OYR $6 / 4$ 11ght yellowish brom

xtensive large white and very pale brown mottle throughout

compact sl silty lutite with scattered forams $\mathrm{S}$ mo
$708-775$

OYR $5 / 4$ bm wn grades to $6 / 4$ 11ght yellowlsh brown to $7 / 4$ very pale brow

extensive intermottling of colors above $715-735 \mathrm{~cm}$,

31 thereafter
firm compact lut

void $717-721 \mathrm{~cm}$, somevhat a raded bedding of forams and black flecks $770-775 \mathrm{~cm}$ S, B

CALC OOZE

CALC OOZE
LOYR $4 / 4$ dark brown

moist silty lutite with abund. forams and black det.grains

273

VISUAL CORE DESCRIPTION

Poge 4 of 4

Ship CHAIN Cruise 115 Leg 2 Sta. 29 Core No. 23 Pe

Lithologic

Log

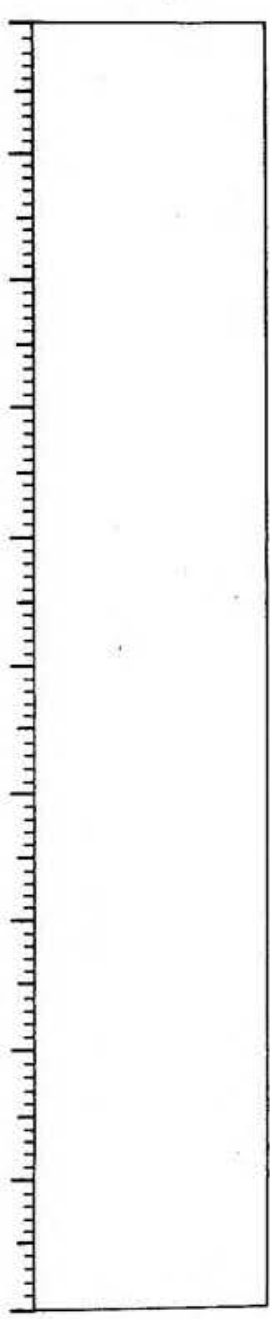

Detailed Description

$780-800$

CALC OOZB

10YR $8 / 1$,

well graded foram-det, sand:coarse to fine

numerous dark red and black det.grains mixed throughout $\underset{5, \text {, textural }}{853}$

\section{MAXNO OOZE}

OYR $8 / 1$ white

ficy stiff plastic lutite with abund. forams and black $s$, textural

853-861

CALC OOZE
$10 \mathrm{XR} 8 / 1$ white

10XR $8 / 1$ white

sowewhat graded foram-det. sand exactly ss above
numerous dark red and black det. gratns mixed throughout very $\mathrm{S}, \mathrm{B}$

861-877

CALC 00 ZZE
10YR $4 / 4$ dark brown

s1 faint light yellowish brown mottling

could be disturbed or flow in during late pullout, upper end of core 
274

SIEAR SLIDE DESCRIPTIONS - W.H.O.T. SEDIMENT CORES

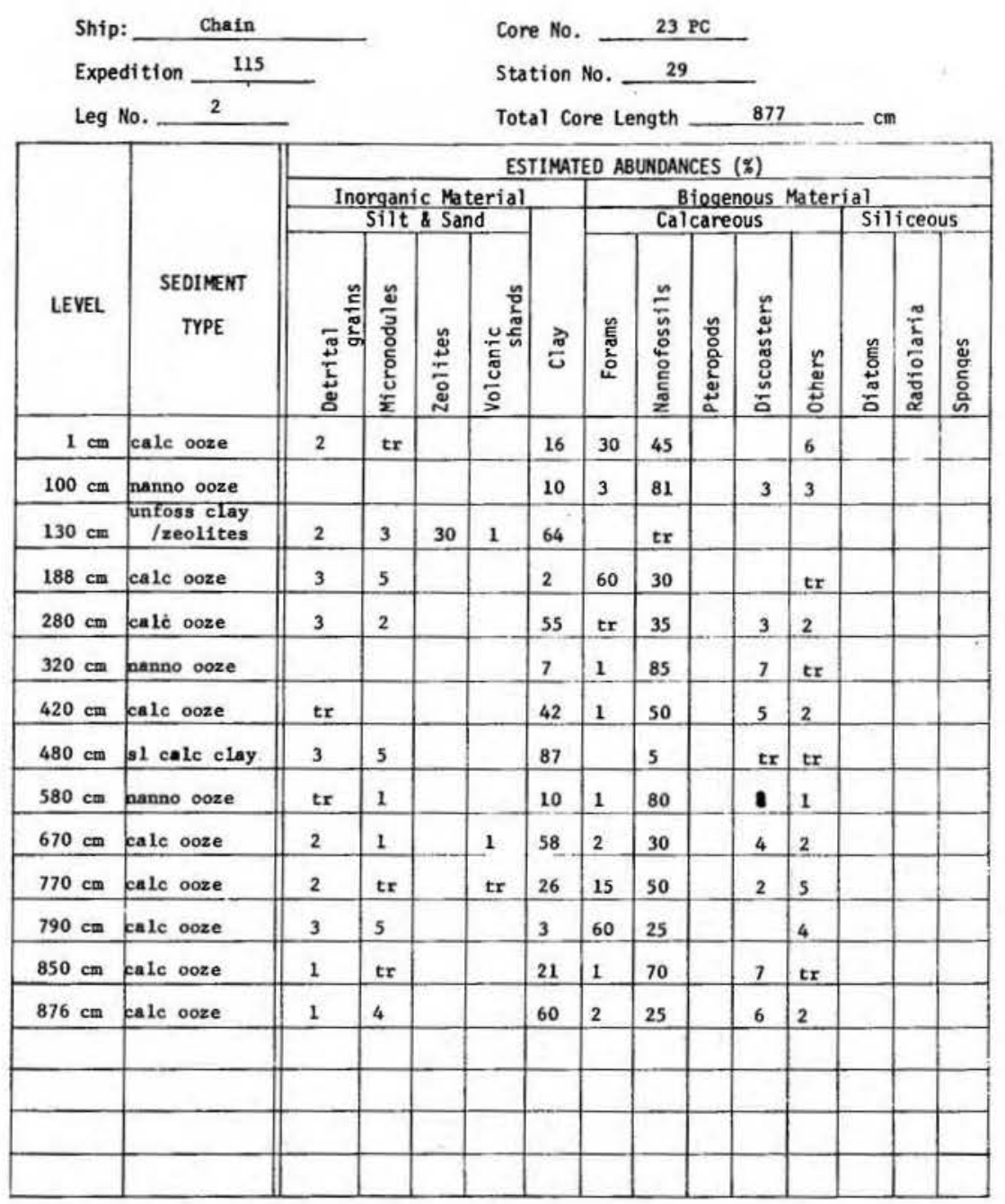

\section{5}

VISUAL CORE DESCRIPTION

Page 1 of 2

Ship CHAWN Cruise 115 Leg 4 sta. 32 Core No. 24 PC Total Length $1176^{2} \mathrm{~cm}$. Lat. $111^{\circ} 019^{\prime} \frac{4}{5}$ Long. $\frac{32}{14^{\circ} 41.3^{\prime} E}$ Core No. $\frac{24 P C}{4347 \text { CeBs m }}$ Core condition Excellent Physiographic location Lithologic

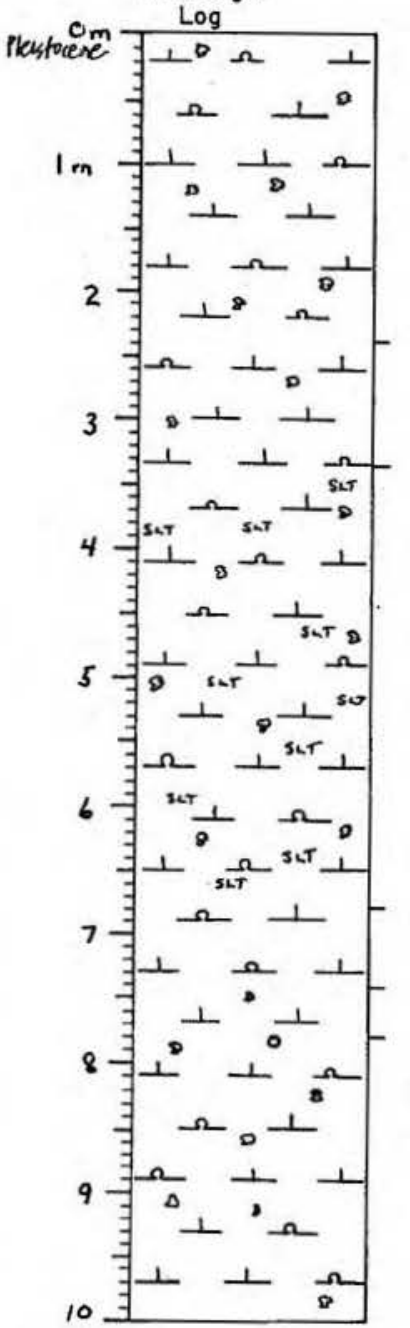

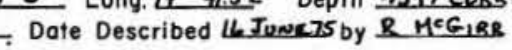
Cape Basin

0-236 Detoiled Description

CAIC SILIC OOZE

(t) 5 Y $5 / 2$ olive gray with many shades in

various sizes of mottles throughout of gray and olive hues scattered to coumon, this burrowing probably has resulted in the mottled appearance of the unit which at one time may have consisted of many smaller, visually distinct somevhat slick occasional fuzzy mear-hory lutite greenish; top $3 \mathrm{~cm}$ are brown and an laminations usually G 36-332 ALC SILIC DOZE

$5 / 2$ olive gray to $4 / 2$ at $318 \mathrm{~cm}$

ghout jor colors omewhat slick fairly silty lutite.

332-678 CALC SILIC DOZE WITH DETRITUS

$5 Y 6 / 1$ gray, $7 / 1$ light gray and siac lar hues of gray and comon mottling throughout of the major colors, definte signs of bioturbstion in places

unit may in reality be composite of many saall units of differing lithologies that have since been partially G

$678-740$

\section{CALC SILIC DOZE}

$5 Y 7 / 1$

faint mottling only throughout of gray G

740-779

CALC SILIC 0028

gottles olive gray

$1 / 2 \mathrm{~cm}$ diameter basalt pebble at $769 \mathrm{~cm}$ 5 irregular

CALC SILIC OOZE

gray, 5/2 olive gray and intermediate shades variable density,

general lightening and darkening of unit color about 6 times
common mottling (burrowing) between these different-color areas, occasional homogeneous aress as vell 1161-1176 end of core 
VISUAL CORE DESCRIPTION 276

Page 2 of 2

Ship CMAIN Cruise 115 Leg 4 Sto. 32 Core No. 24 PC

Lithologic

Log

Detailed Description

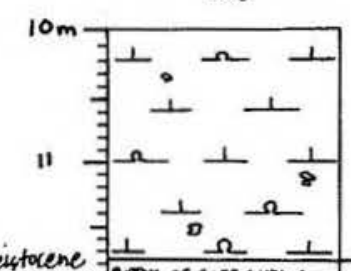

12

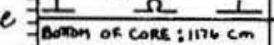

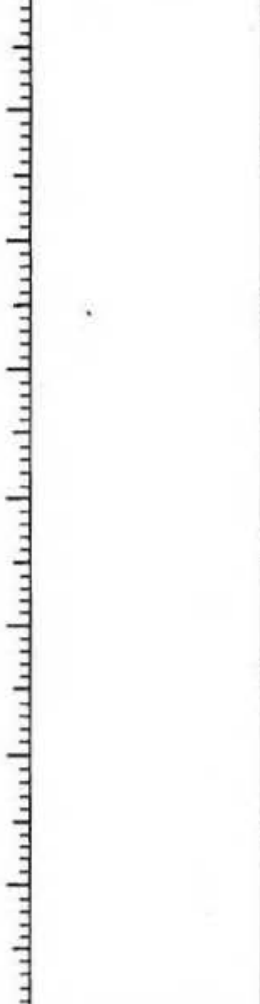

277

SHEAR SLIDE DESCRIPTIONS - W.H.O.I. SEDIMENT CORES

Ship: _Chain

Expedition 115

Leg No. 4

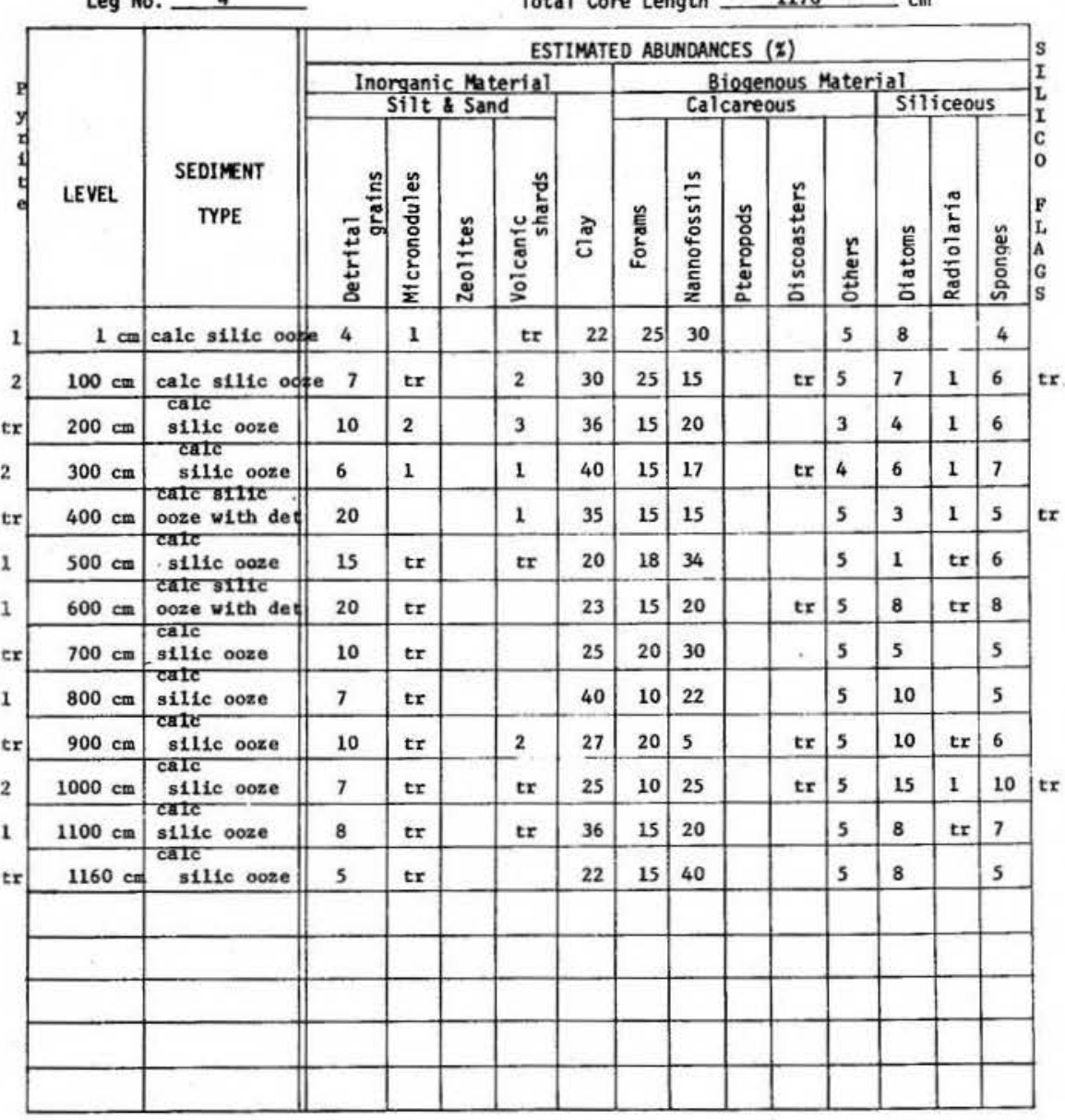

Core No. 24 PC

Station No. 32 . 
Ship CHAIN Cruise 115 Leg 4 Sta. 32 Core No. 24 PG Total Length $146 \mathrm{~cm}$. Lat. $41^{\circ} 01.9^{\prime} \mathrm{s}$ Long. $14^{\circ} 41 .^{\prime} \mathrm{E}$ Depth $4347 \mathrm{CORR} \mathrm{m}$ Core condition EXCEUEAT Dote Described 16 JUNE 75 by R Meciles Physiogrophic location_Cape Basin

Physiographic

Lithologic

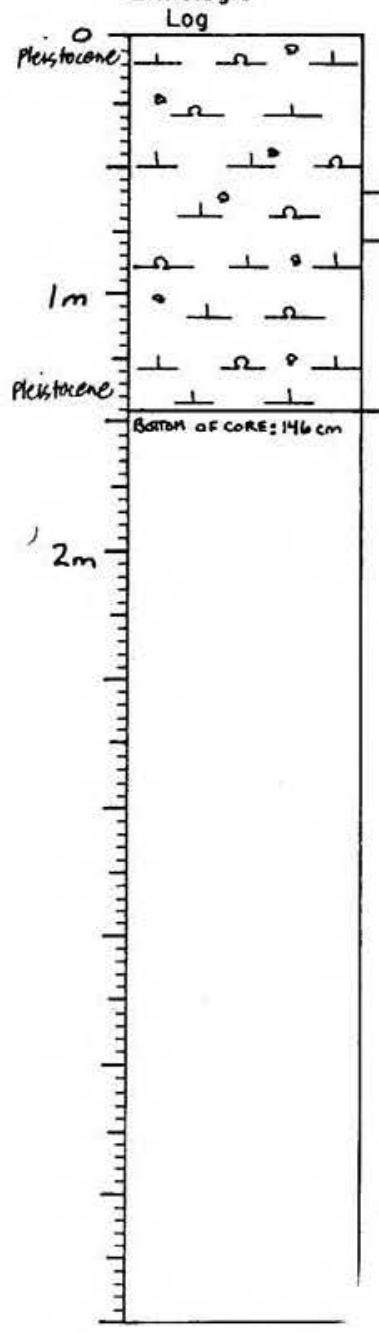

Detailed Description

CALC SILIC OOZE

scattered mottling throughout, $1 \mathrm{~mm}$ to $2 \mathrm{~cm}$ across, gray and olive gray S hortzon silty lutite ontal

CAIC SILIC OOZE

1OYR $7 / 2$ light gray and $5 / 3$ brown

scattered mottling throughout of two major colors

of the two anjor colors and related shades $78-146$

\section{CAX SILIC OOZE}

5Y 5/3 ollve, 7/1 light gray and related shades occasional mottles throughout of major colors

列 end of core
SMEAR SLIDE DESCRIPTIONS - W.H.O.I. SEDIMENT CORES

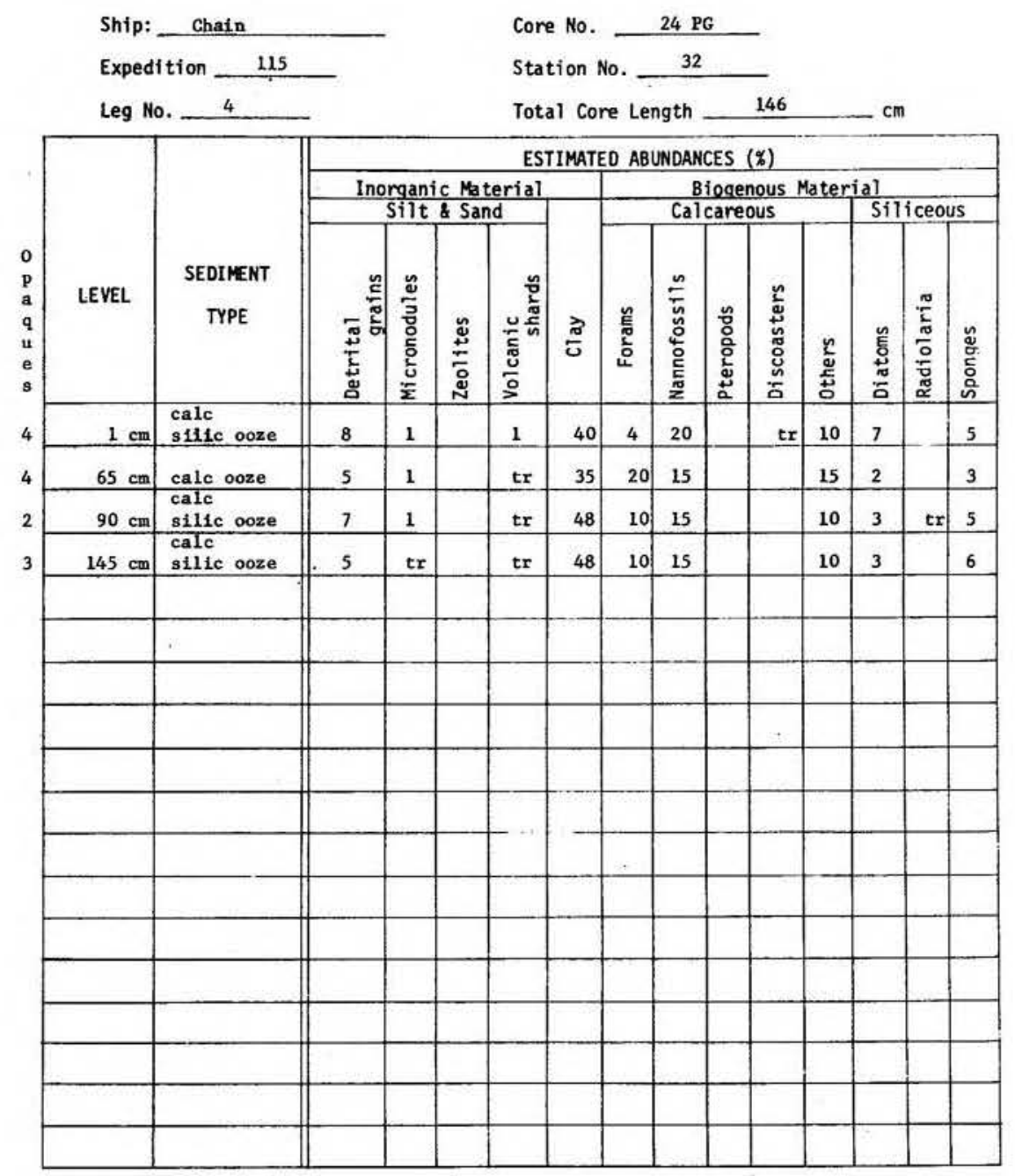


280

VISUAL CORE DESCRIPTION

Page 1 of 1

Ship CHAIN Cruise 115 Leg $\frac{4}{5}$ sta. $\frac{34}{195^{\circ}}$ Core No. $25 \mathrm{PC}$

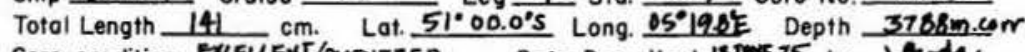
Core condition EXLEUENT/OSSTUKESD. Dote Described ISTWE 75 by frode. Phusiographic location NOGTHEAGT OF TRINE JONCTION.

Lithorogic

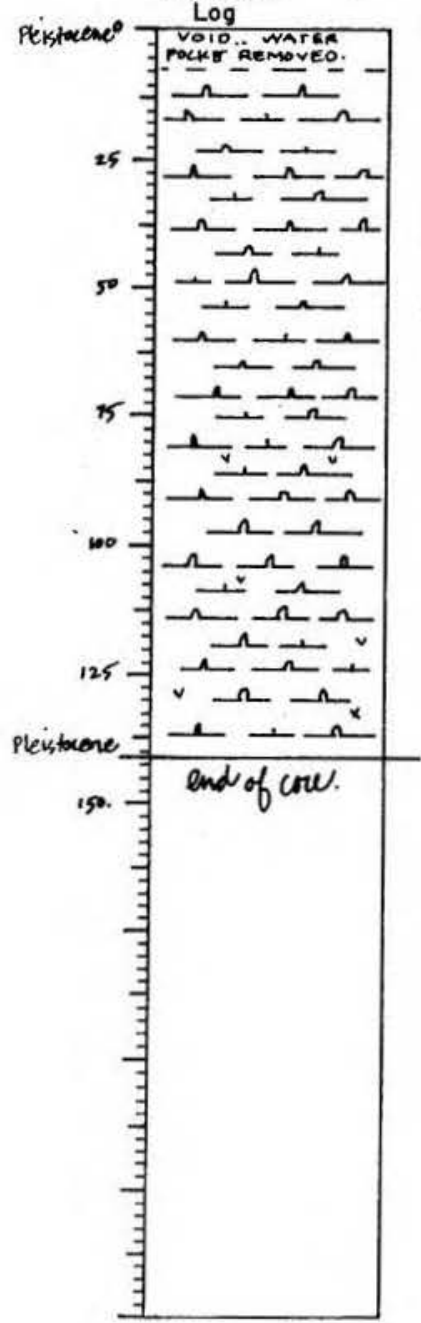

Piston core pretripped but was nonetheless lowered to the As a result of the pretrip, hourever, the core cumple. catcher and botton two sections of liner vere lost

(retaining screws having shesred); thus th1s sediment (25PC) had to be extruded from the stalniess core pipe. It is highty disturbed and unstratified and represents no better

$0-7$

$$
\begin{aligned}
& \text { Water pocket removed after core had settled, (11ner also } \\
& \text { shortened by } 7 \mathrm{~cm} \text { ) }
\end{aligned}
$$

shortened by $7 \mathrm{~cm}$ )

SIIIC CALC OOZE

$2.5 Y 7 / 211$ ght 8 ray gradeg to $6 / 2$ light bromish gray unstratified swirling and marbling of above colors in the result of numerous pumice and ash fragments end of core
281

SMEAR SLIDE DESCRIPTIONS - W.H.O.I. SEDIMENT CORES

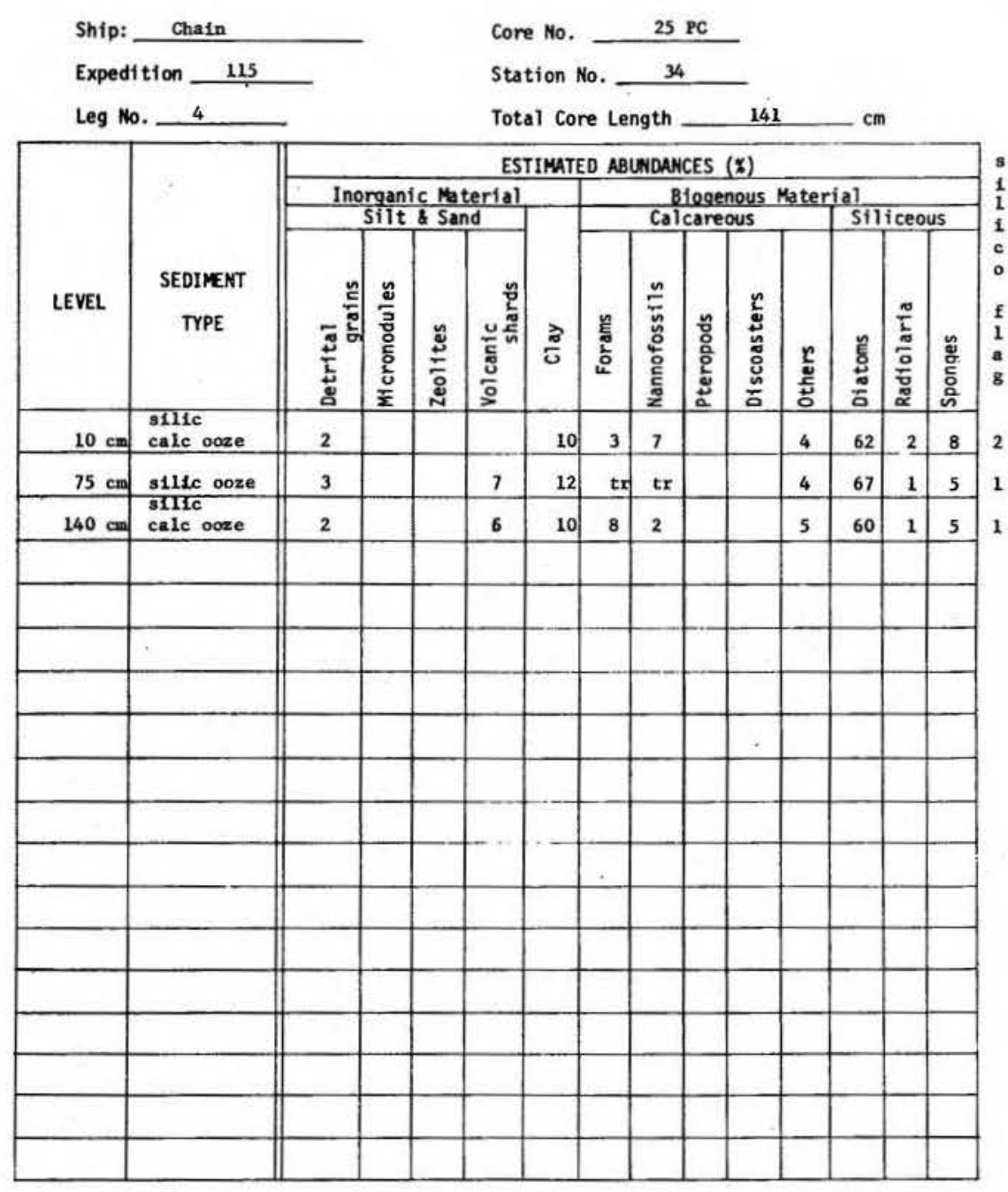


Ship CHAIN Cruise 115 Leg 4 Sta. 35 Core No. 2 GPC Total Length $1190 \mathrm{~cm}$. Lat. $53^{\circ} 360^{\circ} \mathrm{S}$ Long. $00^{\circ} 06.0^{\circ} \mathrm{E}$ Depth $2643 \mathrm{~m}$. Corr. EXCELLENT. Physiographic location EAST FLANK OF MIO-ARANTLL RIDEEAXIS. TRIPLE JUNRTIONL. MAGNETC

Lithologic

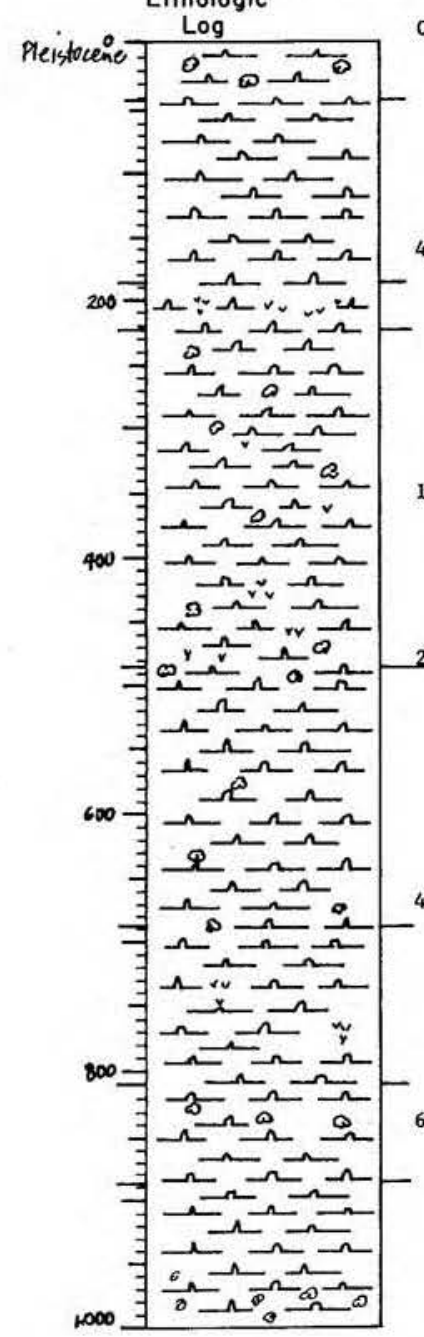

0-41 Detailed Description

DIATOM OOZE
$2.5 \mathrm{Y} 6 / 4$ light yellowish brown grades to $5 / 4$ light olive

common intermotti

a little wash ing along 1 iner $20-45 \mathrm{~cm}$

41-185

DIATOM OOZE

7.5YR $8 / 4$ pink and $5 \mathrm{Y} 6 / 3$ pale olive

intermittent zones of the two colors above throughout:most dominant olive zones $78-85 \mathrm{~cm}, 110-117,175-185$, plus many smaller ones

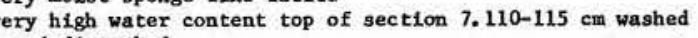
$85-222$

\section{DIATOM OOZE}

$2.5 \mathrm{Y} 7 / 4$ pale yellow

very abund. clusters of black ash flecks and fragments

and liners edge

DIATCM OOZE

occasional 1ight brownish gray and light gray mottling and

arbing plus scattereday

and pumice fragments

several large (1 cm diameter) chunks of pumice found

486-688 $420-485 \mathrm{~cm}$

DTATOM OOZE

10YR $8 / 4$ very pale brown grades to $7 / 4$ very pale brown

a few grayish brom mottles at upper unit contact; elsewhere fint shadowy irregula

of unit 88-810

\section{DIATOM OOZE}

dark gray clusters of ash and pumice

fragments

stiffer more compact but still sponge-1ike lutit

Ship CHAIN Cruise 115 Leg 4 Sta. 35 Core No. 26 PC

Lithologic

Log

1000

1203

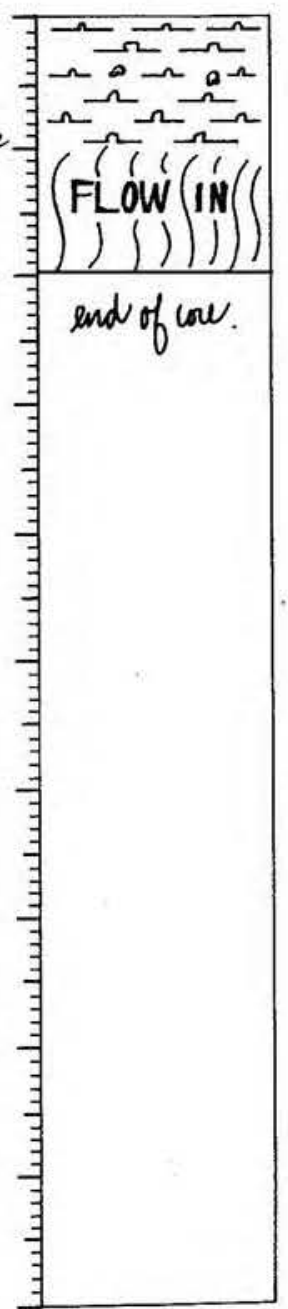

Detailed Description

810-888

DIATOM OOZ:

10YR $7 / 3$ very pale brown grades to $8 / 4$ very pale brown scattered light gray mott1ing $810-835 \mathrm{~cm}$

stiff compact sponge-1ike lutite

pumice fragnents and ash clusters comnon; some vertical orientation of

in horizontal storage before splitting 888-948 DTATOM $00 Z$

$5 \mathrm{Y} 5 / 2$ olive gray grades to $6 / 3$ pale olive

slight intennottling of the two colors above in gradational zone

11ty (ash) athe sponge-11ke lutite

abund. black ash and pumice fragments throughout

948-1010

DIATOM OOZE

10 R 84 very pale brown

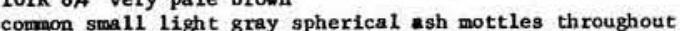
very moist sponge-like lutite

1010-1100

DIATOK OOZE

10YR $5 / 2$ olive gray

mottles found scattered throughout tiff silty lutite with a few $1 \mathrm{~cm}$ diameter irregular chunks $100-1190$ of pumice

FLON IN 


\section{4}

SIEAR SLIDE DESCRIPTIONS - W.H.O.I. SEDIMENT CORES

Ship: Chain

Expedition 115

Leg No.

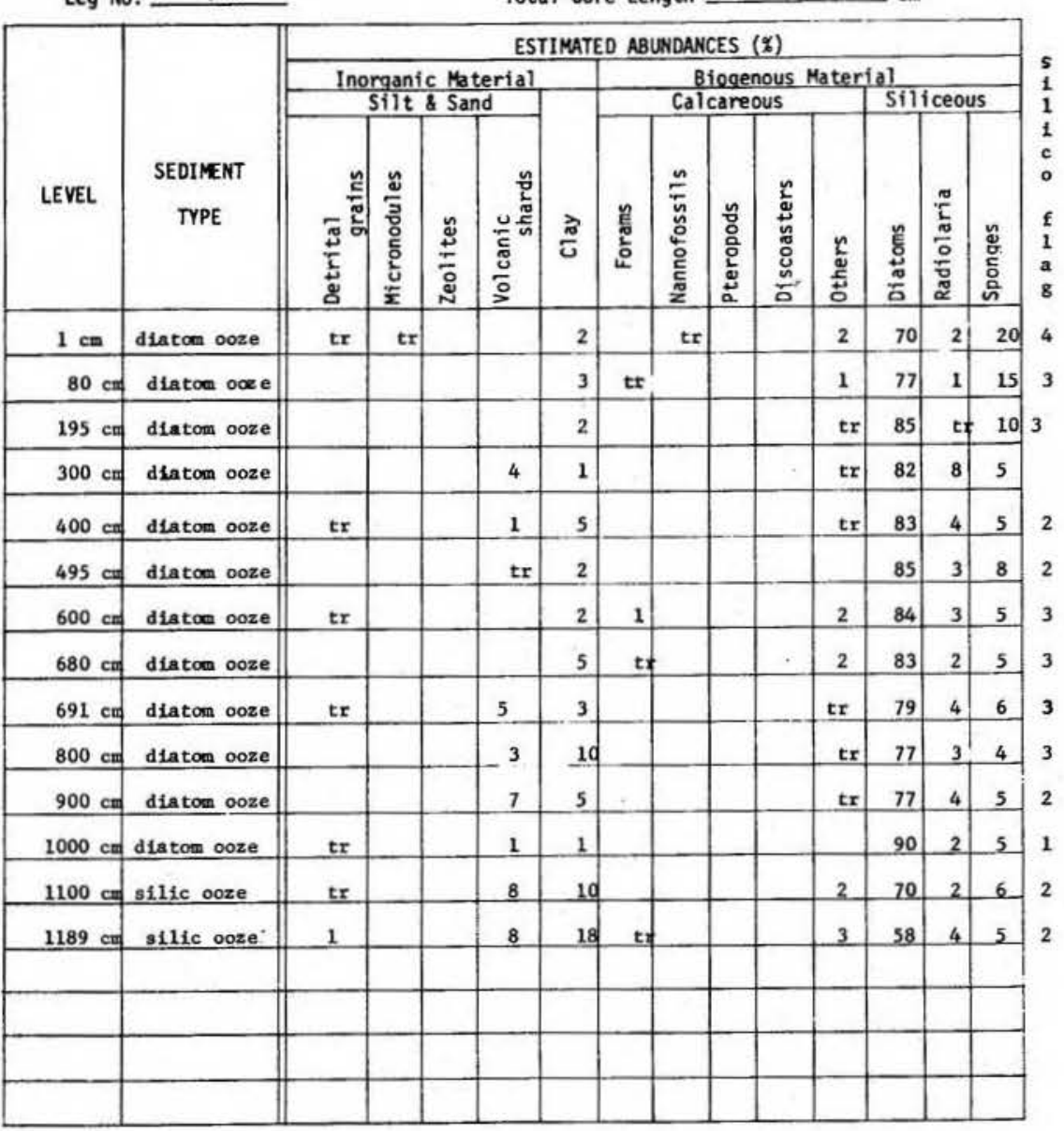

285

VISUAL CORE DESCRIPTION

Page 1 of 1

Ship CHAIN Cruise 115 Leg 4 sto 35 Core No. 26PG Total Length $61 \quad \mathrm{~cm}$. Lat. 550 36.0\% CXCELLENT.

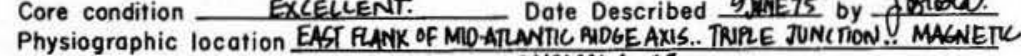

Lithologic

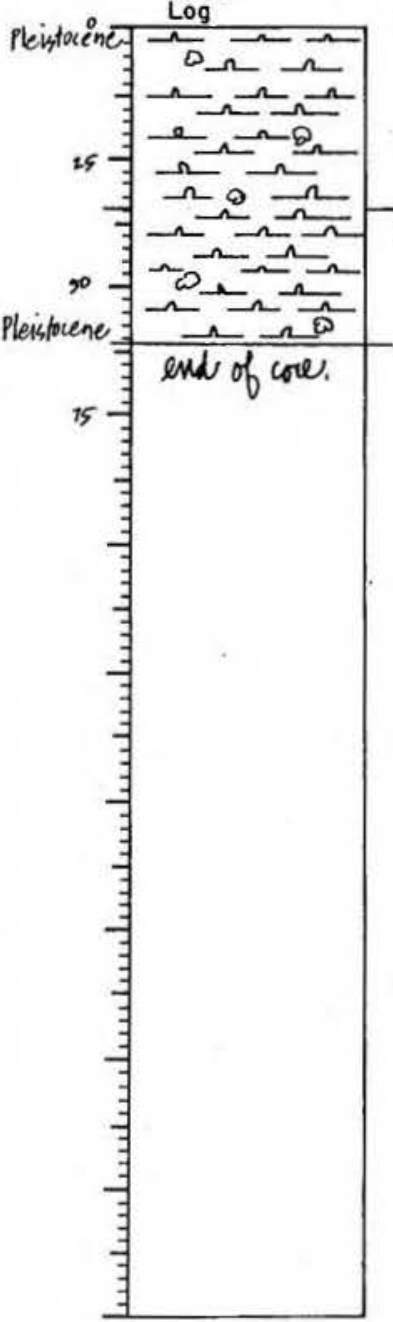

$0-34$ Detailed

10YR $7 / 4$ very pale brom grades to $6 / 4$ 1ight yellowish brown slight faint pale olive and brom mottling and marbling throughout

two seperate pyritized worm tubes; one fragmented, found at

34- 6 inclined $10^{\circ}$

\section{DATOM OOZE}

DYR $7 / 4$ very pale brown grades to $6 / 4$ light yellowinh brown slight faint ollve and grayish brown mottling

end of core 
286

SHEAR SLIDE DESCRIPTIONS - W.H.O.I. SEDIMENT CORES

Ship: Chain

Expedition 115

Leg No. 4

\begin{tabular}{|c|c|c|c|c|c|c|c|c|c|c|c|c|c|c|}
\hline \multirow[b]{3}{*}{ LEVEL } & \multirow[b]{3}{*}{$\begin{array}{c}\text { SEDIMENT } \\
\text { TYPE }\end{array}$} & \multicolumn{13}{|c|}{ ESTIMATED ABUNDANCES (ซ) } \\
\hline & & \multicolumn{4}{|c|}{$\frac{\text { Inorganic Material }}{\text { Silt \& Sand }}$} & \multirow[b]{2}{*}{ 党 } & \multicolumn{5}{|c|}{$\begin{array}{l}\text { Biogenous Mater } \\
\text { Calcareous }\end{array}$} & \multicolumn{3}{|c|}{$\frac{\text { al }}{\text { Siliceous }}$} \\
\hline & & 吾 & 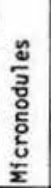 & 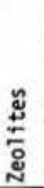 & 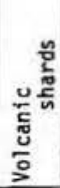 & & 黄 & 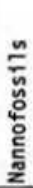 & 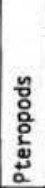 & 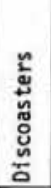 & 点 & 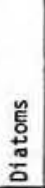 & 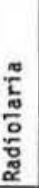 & $\begin{array}{l}\text { के } \\
\text { हूँ } \\
\text { जั }\end{array}$ \\
\hline $1 \mathrm{~cm}$ & diatom ooze & 1 & & & & & 3 & & & & 4 & 83 & 2 & 5 \\
\hline $61 \mathrm{~cm}$ & diatom ooze & tx & & & & & 1 & & & & 2 & 89 & 1 & 5 \\
\hline & & & & & & & & & & & & & & \\
\hline & & & & & & & & & & & & & & \\
\hline & & & & & & & & & & & & & & \\
\hline & & & & & & & & & & & & & & \\
\hline & & & & & & & & & & & & & & \\
\hline & & & & & & & & & & 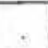 & & & & \\
\hline & & & & & & & & & & & & . & & \\
\hline & & & & & & & & & & & & & & \\
\hline & & & & & & & & & & & & & & \\
\hline & & & & & & & & & & & & & & \\
\hline & & & & & & & & & & & & & & \\
\hline & & & & & & & & & & & & & & \\
\hline & & & & & & & & & & & & & & \\
\hline & & & & & & & & & & & & & & \\
\hline & & & & & & & & & & - & & & & \\
\hline & & & & & & & & & & & & & & \\
\hline
\end{tabular}

Core No. 26 PG

Station No. 35

Station No. 35

Total Core Length $\quad 61+\mathrm{cm}$

ESTIMATED ABUNDANCES (\%)

VISUAL CORE DESCRIPTION

Page 1 of 2

Ship CHAIN Cruise 115 Leg 4 Sta. 36 Core No. $27 \mathrm{PC}$

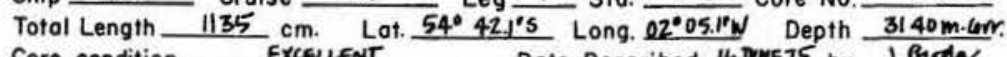

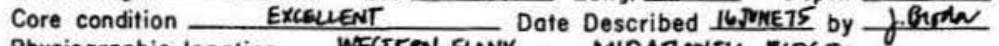

Physiographic location WESTERN FLANK ... MIOARANTIC NIDGE

Lithologic

Pleistocene Log

Log

\section{Detailed Description}

DIATOH OOZE GRADES TO SILIC CALC OOZE AND BACK TO DIATOR TY $6 / 3$ pale ollve and $S / 3$ ollve 002 two colco

moist generally unconsolidated sponge-1ike siliceous mulch with occasionsl pockets of ash and punice fragments
$0-80 \mathrm{~cm}$ very soupy

200 ก

$\exists \overrightarrow{0} \rightarrow \overrightarrow{0}=$ $\exists \rightarrow \infty \rightarrow$ $\exists 0 \Omega 0=$ 는 $\exists \Omega$ $=0 \Omega$ -400 교

$\exists$ $\exists \cap \Omega$ $\exists \sim \Omega$ $\Omega \Omega^{2} \Omega$ $\exists \Omega$
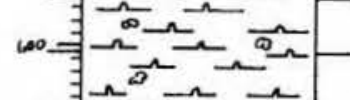

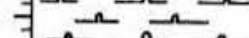

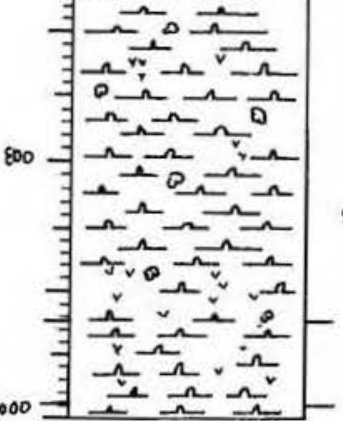

DIATOM OOZE

$5 Y 7 / 3$ pale yellow grades to $2.5 \times 7 / 4$ pale yellow 作 faghinations 561-603 co firmer sponge-like silic mulch

\section{DTA TOM OOZE}

DIA TOM OOZE $6 / 2$ Hitht

slight faint pale yellow marbling and $.5 \mathrm{~cm}-1 \mathrm{~cm}$ laminations (concave up) stiffer more compact lutite with abund. black pumice flecks 6 marbelled

$-603-922$

DIATOM OOZE GRADES TO SILIC OOZE

$2.5 Y 5 / 2$ grayish brown grades slowly to $4 / 2$ dark grayish

occasional olive 1ight gray and dark grayish brown marbling and motriling

relatively stiff compact lutite with varying ash and pumice content; generally slight with a few pockets rich in ash

large sharp irregular $3 \mathrm{~cm} \times 5 \mathrm{~cm}$ pumice fragpent (ice rafted) 922-987

\section{STLIC OOZE WITH LAMINATIONS OP DIATOM OOZE}

repeated and quite distinct laminations $1-1.5 \mathrm{~cm}$ wide of throug bou $965-987 \mathrm{~cm}$.

background matrix between beds is moist unconsolidated lutite with scattered ash and pumice fragents 987-1012 Horizontal

DIATOM OOZE

2 grayish brown

.

moist silty lutite with abund. ashipumice fragments 
VISUAL CORE DESCRIPTION 288

Ship CHAiN Cruise 115 Leg 4 Sto. 36 Core No. 27PC

Lithologic

Log

Detailed Description

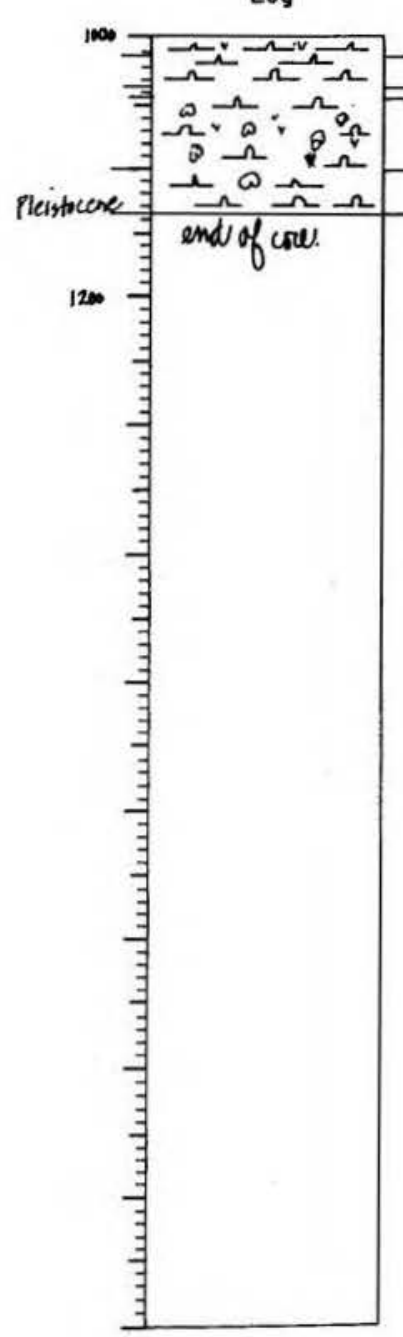

1012-1034 SILIC 00ZE

$2.5 \times 6 / 2$ light brownish gray of the same hue soist slightly silty lutite with ash and pumice only in mottles 1034-1043

\section{SILIC OOZE}

2.5Y $4 / 2$ dark grayish brown and N 2.5/ black to pure with very abund. ash and pumice fragments grades $s$ horizontal

1043-1100

$2.5 Y 6 / 2$ light brownith gray grades to $5 / 2$ grayish brow extensive internarbelining of colors above plus sobe light texturally uncontinuous conbinsetio

of moist lutite, sponge$c$

1100-1135

SILIC OOZE

sllic mulch with scattered ash and end of core
SIIIC OOZE AND ASH

$2.5 \times 7 / 2$ 11ght gray
289

SHEAR SLIDE DESCRIPTIONS - W.H.0.1. SEDIMENT CORES

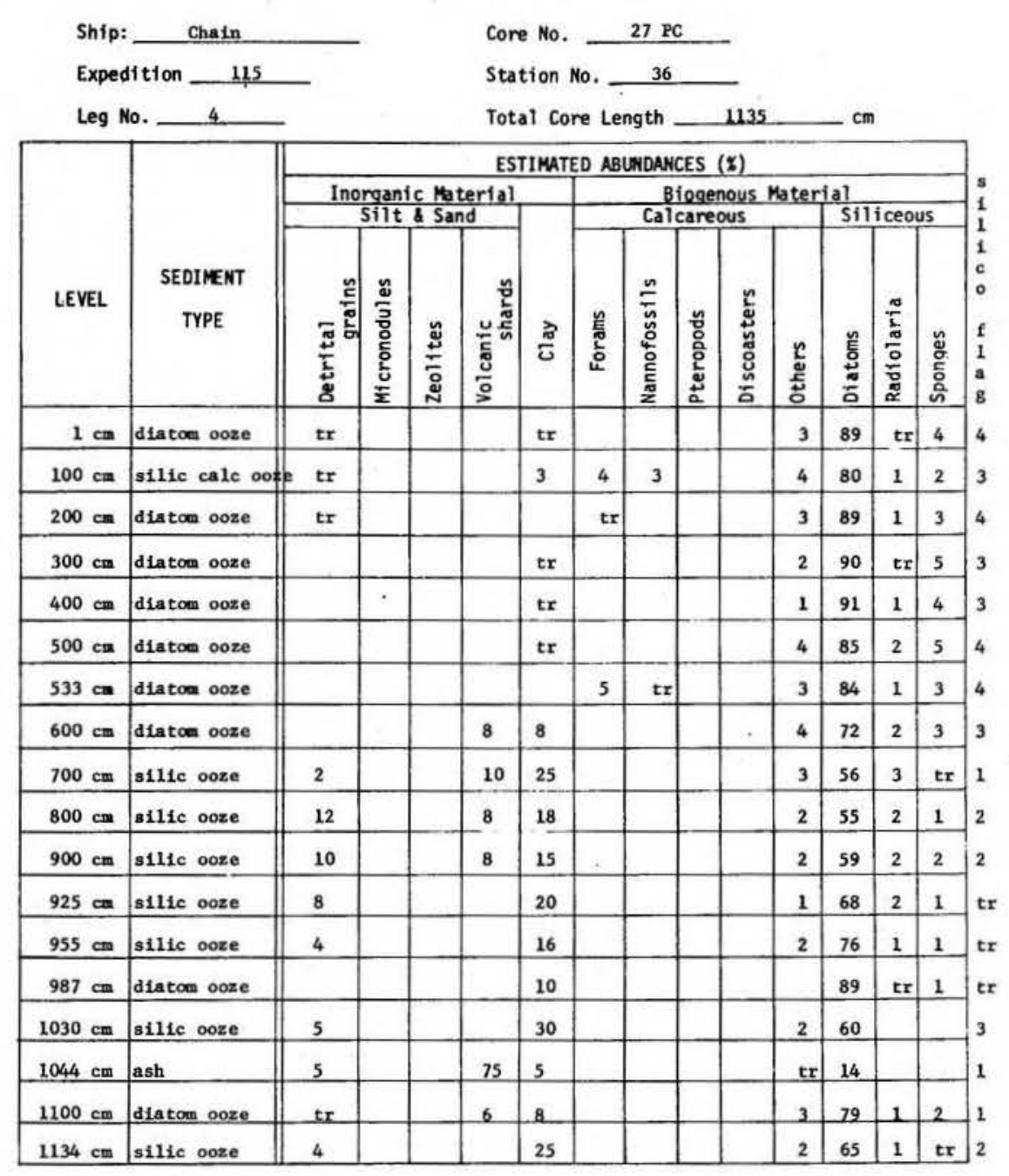


Ship CHAIN Cruise 115 Leg 4 sta. 36 Core No. 27 PG

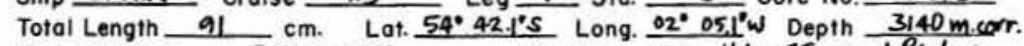
Core condition ExCELENT Date Described 16 puc 15 by forda.

Physiogrophic location WESTERN FLANK... MIDATLANTIC KIDEE

Lithologic

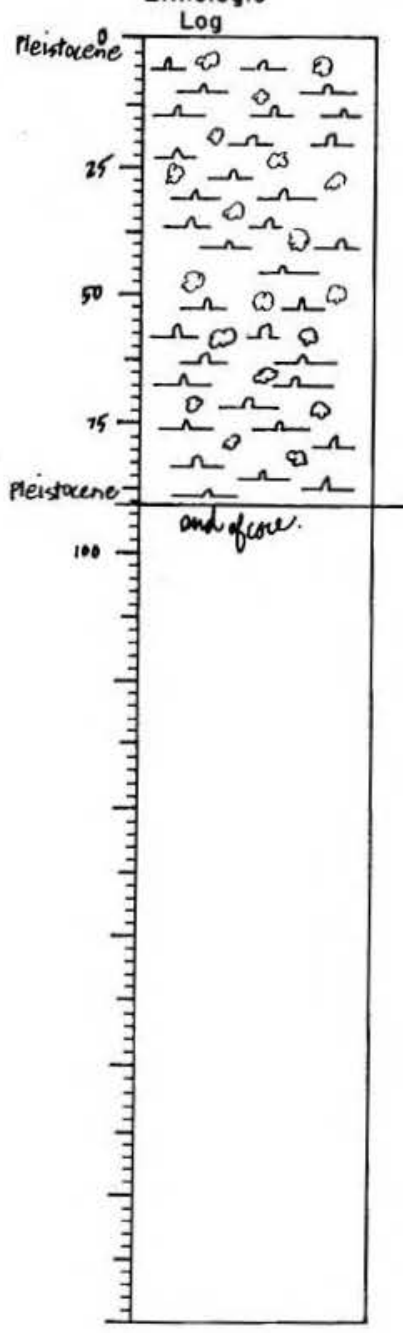

0-91 Detailed Description

\section{DIATCM OOZE}

$5 Y 6 / 3$ pale olive and $5 / 3$ ollve

extensive and complete intermottling and marbling of the

very molst unconsolidated sponge-1ike allic mulch

SMEAR SLIDE DESCRIPTIONS - W.H.O.I. SEDIMENT CORES

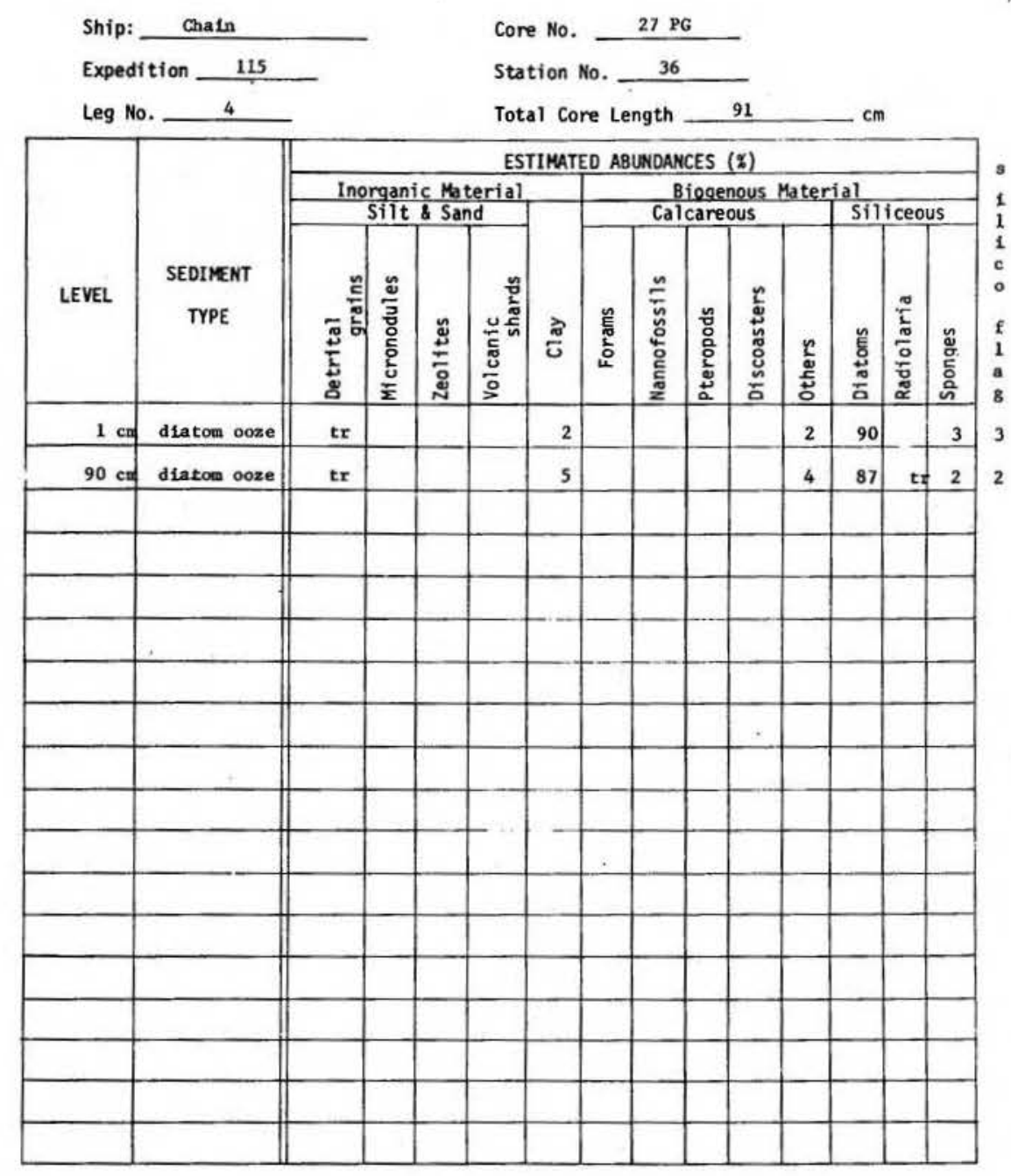


292

VISUAL CORE DESCRIPTION
Page_ 1 of 1

Ship Crain Cruise 115 Leg 4 Sta. 43 Core No. 32 GC Total Length $143 \mathrm{~cm}$. Lat. $54^{\circ} 35.5^{\prime}$ 'S Long. $00^{\circ}$ O4.8 8 Depth 1260 cose m Core condition EXCEHENT Date Described 16 Thne 75 by $R$ MC GIRR

Physiographic location Spiess Ridge, Slightly East of Central Anomdy Triple Junction

Lithologic Detailed Description

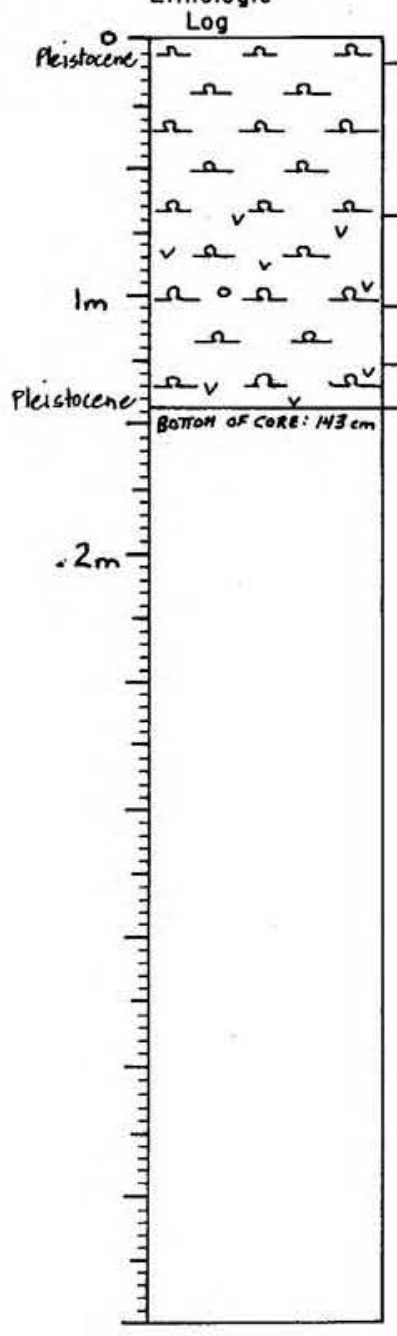
SILIC OOZE

sandy silty, spongy, lutite material sma11 black specks throughout

9-67 DIA

DY $6 / 3$ pale olive and $6 / 4$

molst, spongy lut1te mot
6

SILIC OOZE/VOLCANICS

N2.5/ black

sandy, spongy lutit

probable disturbance of unit due to spin magnetometer work, it apparently has resettled horizontally with heavier $x$ and finer material on 列

$104-126$ ${ }_{126} 1$ rregular

$2.5 \mathrm{Y} 8 / 4$ pale yellow

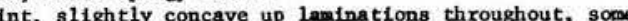
contamination with upper unit 104-109 cm

126-143 143 .

5Y $6 / 3$ OOZB/VOLCANICS $6 / 4$

several large inclusions of black sand, posstbly washed and not several Large 1nclusions of bfuck
stratigraphically meaning ful

f1 $1 \mathrm{~m}$, moist spongy lutite

I sample taken at $139 \mathrm{~cm}$ unit possibly disturbed due to spin magnetometer

end of core
293

SMEAR SLIDE DESCRIPTIONS - W.H.0.1. SEDIMENT CORES

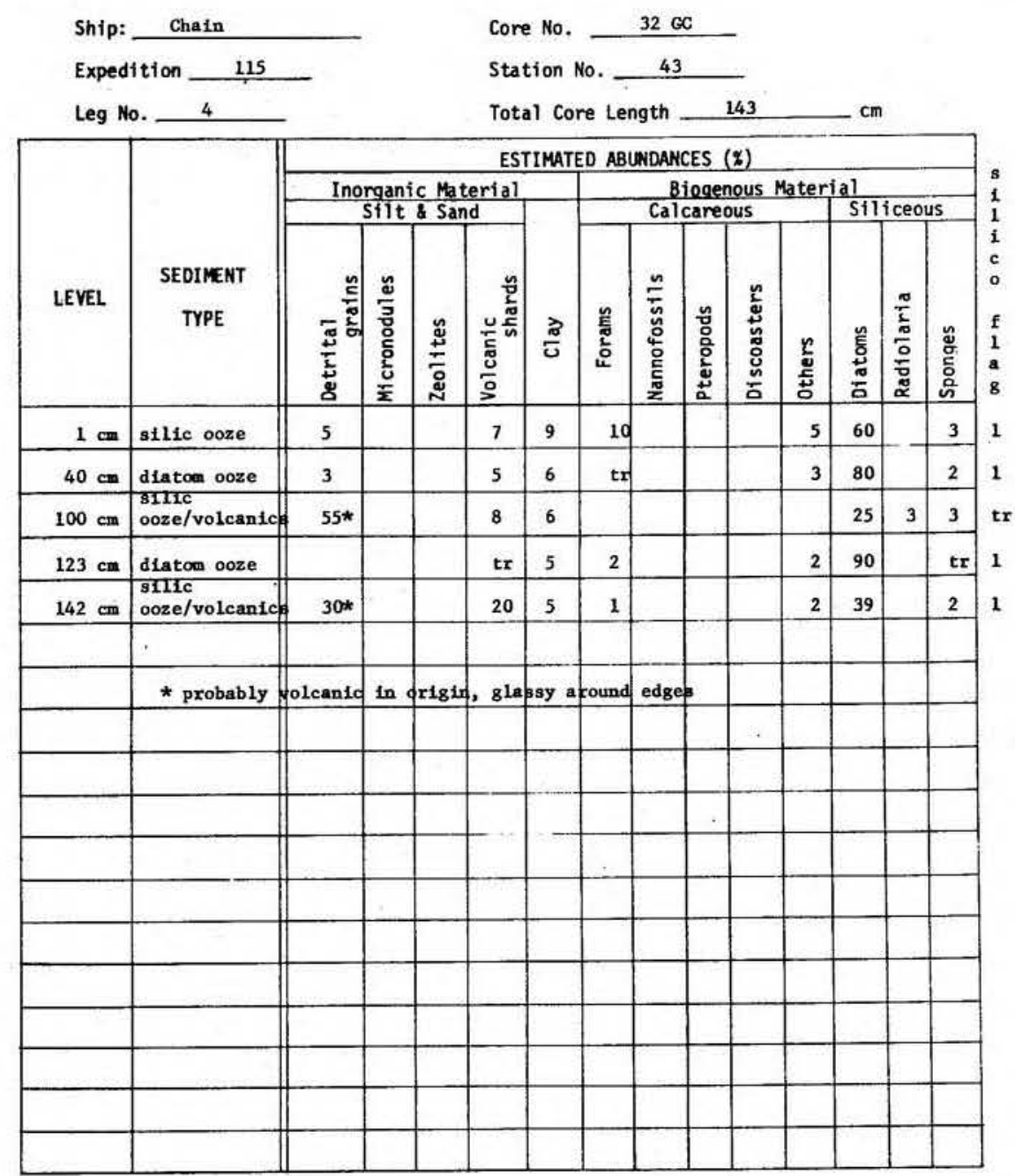




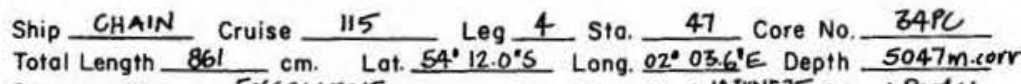

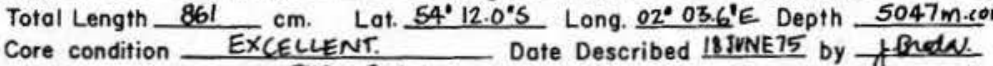
Physiographic location. TRIRE JUNCTIIN.... AFRKA - ANTARCTCA FRACTUREZZONE SOUTH-

Lithologic

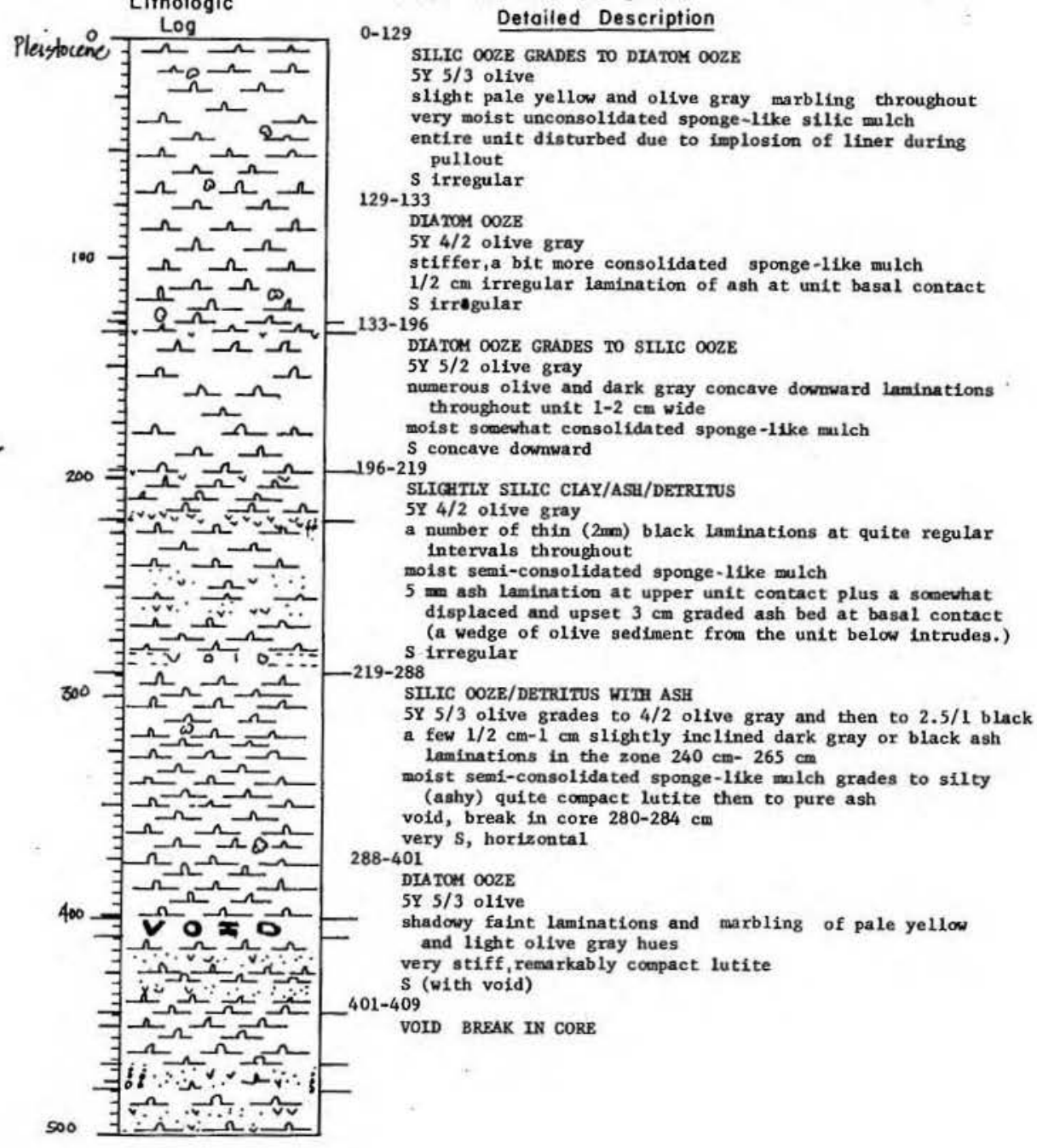

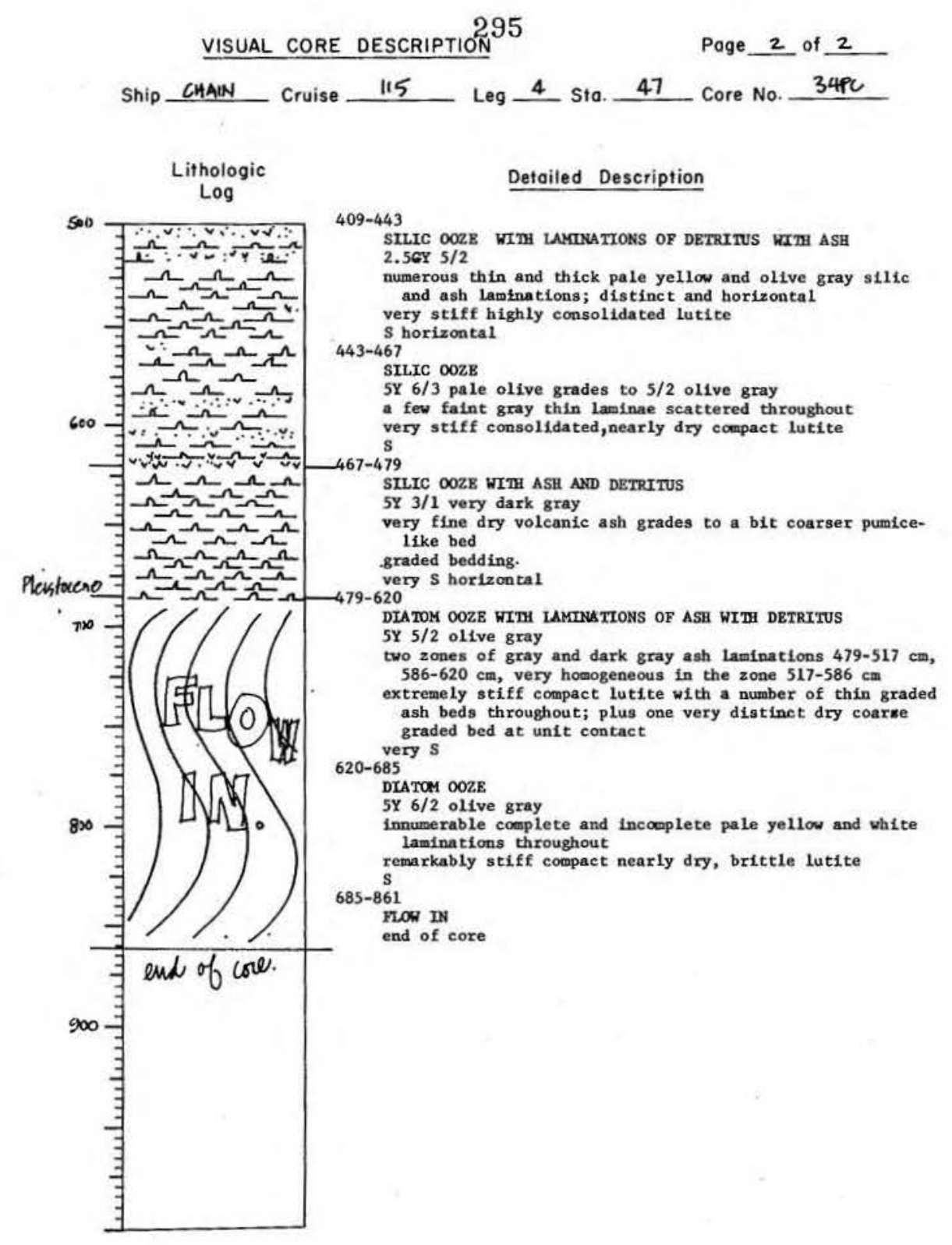


296

SIEAR SLIDE DESCRIPTIONS - W.H.O.I. SEDIMENT CORES

Ship: Chain

Expedition 115

Leg No. 4

\begin{tabular}{|c|c|c|c|c|c|c|c|c|c|c|c|c|c|c|}
\hline \multirow[b]{3}{*}{ LEVEL } & \multirow[b]{3}{*}{$\begin{array}{c}\text { SEDIMENT } \\
\text { TYPE }\end{array}$} & \multicolumn{13}{|c|}{ ESTIMATED ABUNDANCES ( $($ ) } \\
\hline & & \multicolumn{4}{|c|}{$\begin{array}{c}\text { Inorganic Material } \\
\text { Silt \& Sand }\end{array}$} & \multirow[b]{2}{*}{$\frac{\pi}{U}$} & \multicolumn{5}{|c|}{$\begin{array}{l}\text { Biogenous Mater } \\
\text { Calcareous }\end{array}$} & \multicolumn{3}{|c|}{ Silliceous } \\
\hline & & 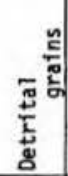 & 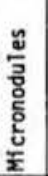 & $\frac{\stackrel{u}{ \pm}}{\frac{ \pm}{2}}$ & 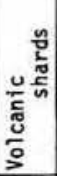 & & $\begin{array}{l}\text { 䇺 } \\
\text { c }\end{array}$ & $\begin{array}{l}\frac{n}{\bar{n}} \\
\stackrel{\underline{c}}{\circ} \\
\frac{c}{c} \\
\frac{\pi}{z}\end{array}$ & 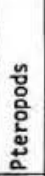 & 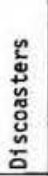 & 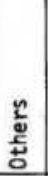 & 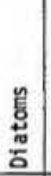 & $\frac{\pi}{\frac{\pi}{k}}$ & 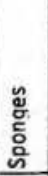 \\
\hline $1 \mathrm{~cm}$ & silic ooze & 8 & & & & 30 & $t$ & & & & 2 & 56. & 1 & 1 \\
\hline $100 \mathrm{~cm}$ & diatom ooze & 2 & & & & 8 & $\operatorname{tr}$ & & & & 1 & 86 & tr & 1 \\
\hline $200 \mathrm{~cm}$ & silic ooze & 8 & & & 3 & 26 & & & & & 4 & 56 & $\mathrm{tr}$ & 1 \\
\hline $215 \mathrm{~cm}$ & $\begin{array}{l}\text { ash/det } \\
\text { ashetsy }\end{array}$ & 35 & & & 40 & 9 & & & & & 3 & 12 & $\mathrm{tr}$ & $\mathrm{tr}$ \\
\hline $287 \mathrm{~cm}$ & $\begin{array}{l}\text { Sific ooze } \\
\text { with ash/det }\end{array}$ & 30 & & & 20 & 8 & 3. & & & & 4 & 34 & $t x$ & tr \\
\hline $390 \mathrm{~cm}$ & diatom ooze & tr & & & $\mathrm{tr}$ & 15 & & & & & & 83 & $\mathrm{tr}$ & $t x$ \\
\hline $422 \mathrm{~cm}$ & det with ash & 65 & & & 20 & 4 & & & & & 10 & 1 & & \\
\hline $425 \mathrm{~cm}$ & silic ooze & 10 & & & 3 & 40 & & & & $\cdot$ & 2 & 43 & $\mathrm{tr}$ & 1 \\
\hline $475 \mathrm{~cm}$ & $\begin{array}{l}\text { S1110 ooze } \\
\text { with ash and }\end{array}$ & 15 & & & 15 & 5 & 2 & & & & 3 & 55 & 1 & 2 \\
\hline $525 \mathrm{~cm}$ & diatom ooze & & & & & & 5 & & & & 2 & 91 & $t \mathbf{x}$ & tr \\
\hline $619 \mathrm{~cm}$ & ash with det & 18 & & & 70 & & 2 & & & & & 10 & & \\
\hline $650 \mathrm{~cm}$ & diaton ooze & & & & & & 5 & & & & 3 & 90 & & \\
\hline $845 \mathrm{~cm}$ & diatom ooze & $\mathrm{tr}$ & & & & & 15 & & & & 4 & 80 & & \\
\hline & & & & & & & & & & & & & & \\
\hline & & & & & & & & & & & & & & \\
\hline & & & & & & & & & & & & & & \\
\hline & & & & & & & & & & & & & & \\
\hline & & & & & & & & & & & & & & \\
\hline
\end{tabular}

297

VISUAL CORE DESCRIPTION

Page 1 of 1

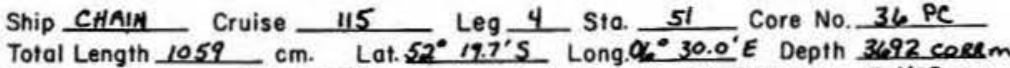

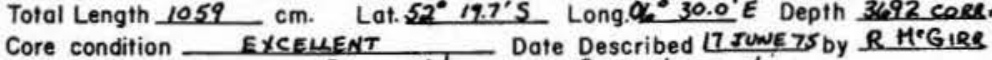
Physiographic location African Plate NE of Triple Jinction

Lithologic

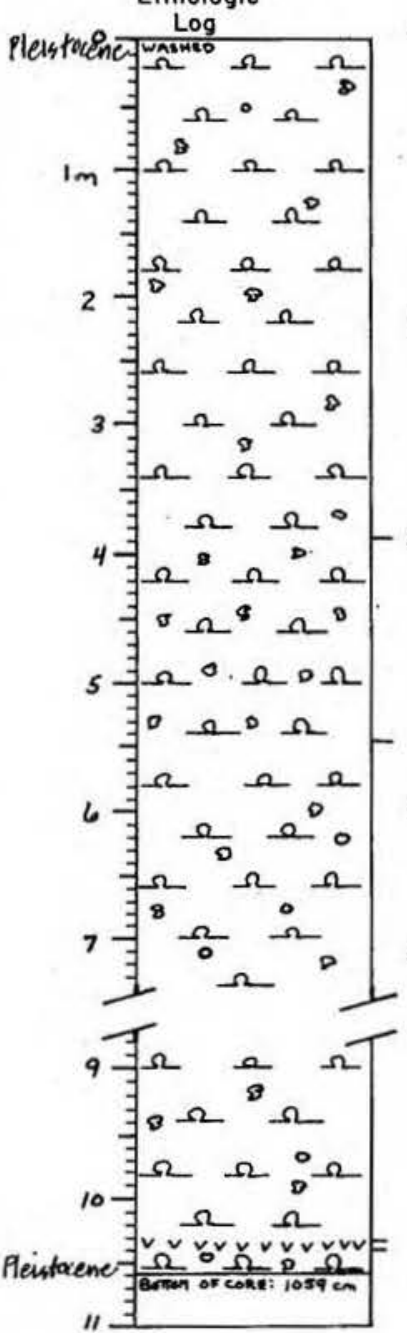

0-384 Detailed Description

Dray $5 Y 4 / 2$ and $6 / 2$ 1ight olive gray

intermotting of the 2 major colors and similar hues throughout

spongy slightly silty silic lutite

brobably pumice,

large size; occasional chunks of volce to their relatively in diameter scattered throughout, pocket of broken $G$

\section{DIATAM OOZE}

DIATCM OOZE
$2.5 \mathrm{Y} 7 / 2$ 11ght gray

extensive fuzzy mottling and marbling throughout, gray and ollve gray

apongy silic milch materis

numerous black specks and rock fragsents throughout, most probably pumlce, to $1 / 2 \mathrm{~cm}$ in diamet

spongy silic milch, in places silty
slight variations in the 0 lor throughout; numerous black

specks and mall rock fragments, (probably pumice

groups of puntce chunks at $664,705,782 \mathrm{~cm}$, $616 \mathrm{~cm}$,

(2 $\mathrm{cm} \times 3 \mathrm{~cm})$ at $968 \mathrm{~cm}$

032-1036 Irregular

VOLCAMIC GIASS

$5 Y 5 / 2$ olive gray

ed small pebbles

$S$ irregular

36-1059

DIATOM OOZE

$5 Y 6 / 3$ pale olive

spongy silic lutite $\times 2 \mathrm{~cm})$ at 1042 ocattered black

specks throughout $1045-1059 \mathrm{~cm}$, core cutter

end of core marbling of major colors and similar bues throughout 
298

SIEAR SLIDE DESCRIPTIONS - W.H.0.1. SEDIMENT CORES

Ship: Chain

Expedition 115

Leg No. 4

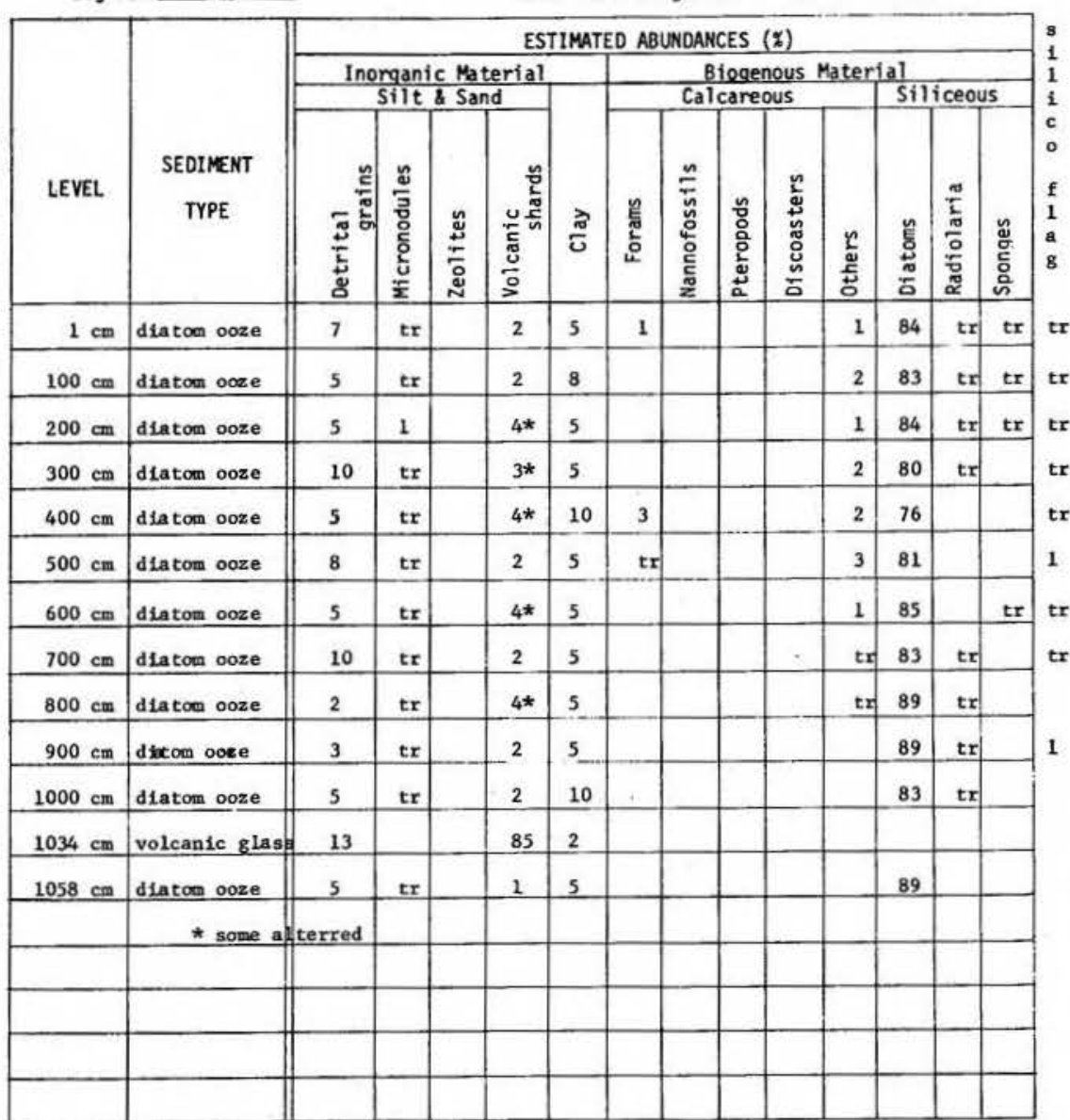

299

VISUAL CORE DESCRIPTION

Page 1 of 1

Ship CHN Cruise 115 Leg 4 Sto. 51 Core No. 36 PG Total Length $105-\mathrm{cm}$. Lat. $52^{\circ} 197^{\prime} \mathrm{S}$ Long $06^{\circ} 30.0^{\prime} \mathrm{E}$ Depth 3692 Cose m COre condition EYCELENT

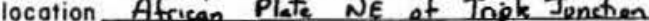
Lithologic

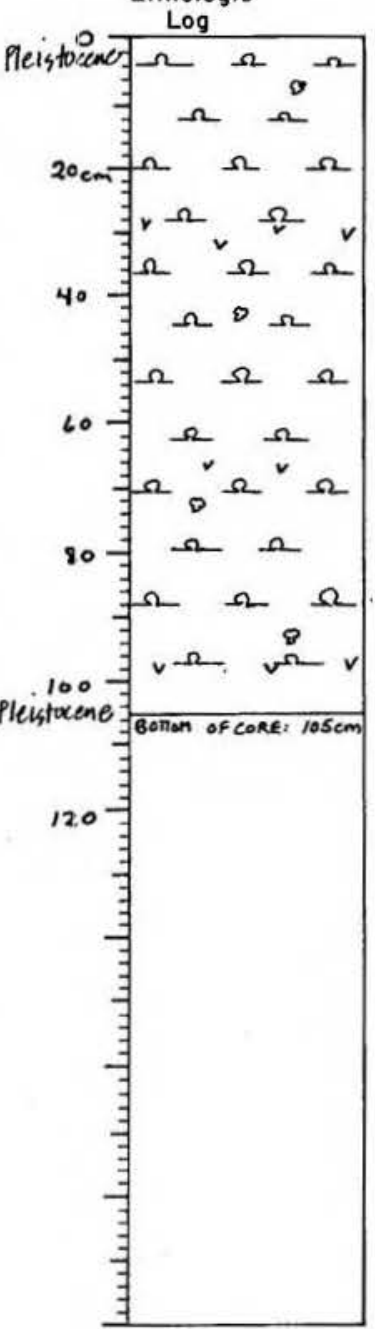

\section{Detailed Description}

\section{DIATOM OOZE AND CHUNKS OF PUMICE} $5 Y 5 / 3$ olive
occasional swirls and mottles, olive gray molh, sock sillc wleh

throughout; three pockets of vuggy "pumice 1ike chunks" $25-31,66,97 \mathrm{~cm}$, several black rock fragments at $97 \mathrm{~cm}$

end of core 
300

SIEAR SLIDE DESCRIPTIONS - W.H.O.I, SEDIMENT CORES

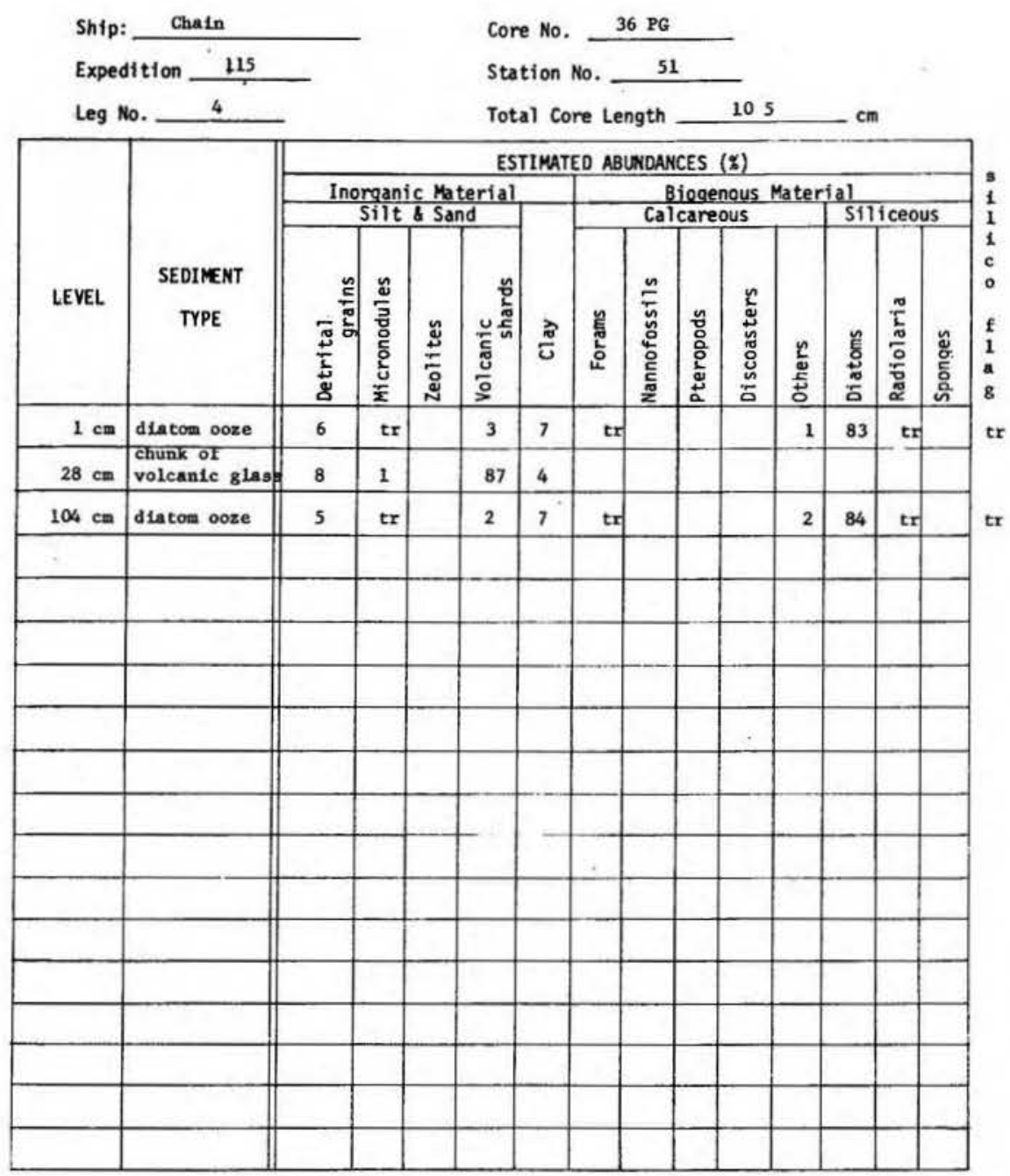

301

VISUAL CORE DESCRIPTION

Page 1 of 2

Ship CHAIN Cruise 115 Leg 5 sto. 58 Core No. 37 PC

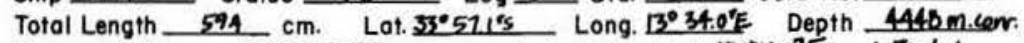

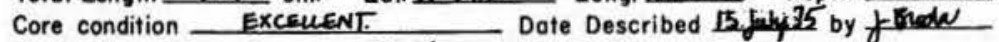
Physiographic location NIRTHEACA CAPE BASIN Lithologic

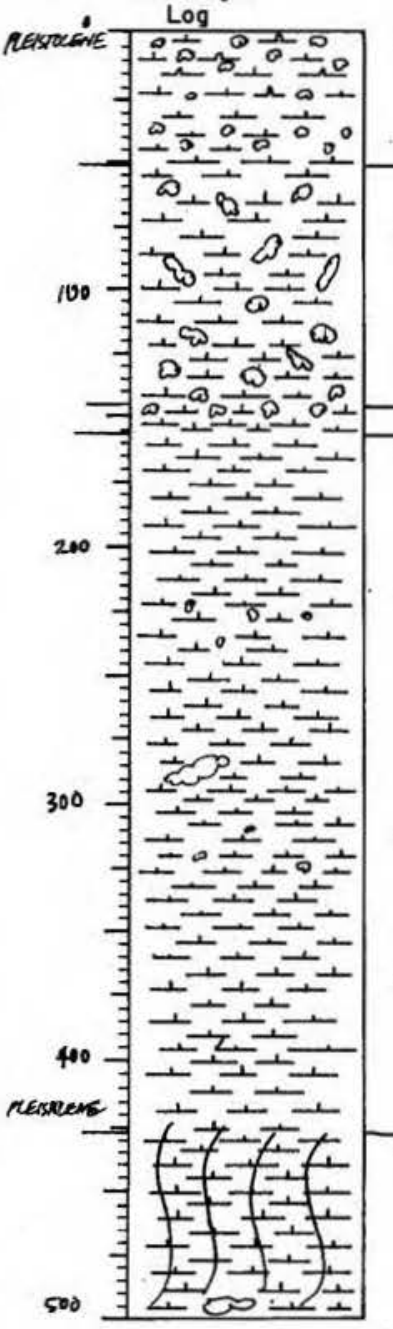

Detailed Description

0-51

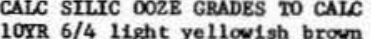

extensive brown and grayish brown mottling and marbling in the zones $0-15,29-48 \mathrm{~cm}$, coumon pale brom maxbling moist silty lutite with faint abund. forams 51-145 CALC OOZE

10YR $6 / 3$ pale brown

extensive very pale brown burrowing throughout, thin well preserved burrows of ten span entire vidth of 1iner at stiffer, but st111 mo1st, silght1y silty lutite vith a few $G$ mottled

145-156

MUNA OOZE

10YR 7/2 11ght gray

extensive intermotting with unit above at upper contact oth compact

156-327

$$
\text { NANASO OOZE }
$$

10YR $8 / 1$ white

numerous large and gmall light gray zones and burrows, curlously inclined in opposite directions throughout most $262-267$. sphertcal motiles acat of the ligte inclined $2^{\circ}$

$327-356$ CAIC OOZE $5 Y 7 / 2$ 11ght gray

very stiff compact brittle lutite S irregular

356-422 CALC DOZE 1OYR $8 / 1$ white two large 1rregular $5 \times 7 / 2$ 11ght gray inclusions (burrows?)

very gtiff conpact brittle lutite

422-594 94

end of core 
VISUAL CORE DESCRIPTION 302

Ship_CHAIN Cruise 115 Leg 5 Sta. 58 Core No. 3 TPC

Lithologic

Log

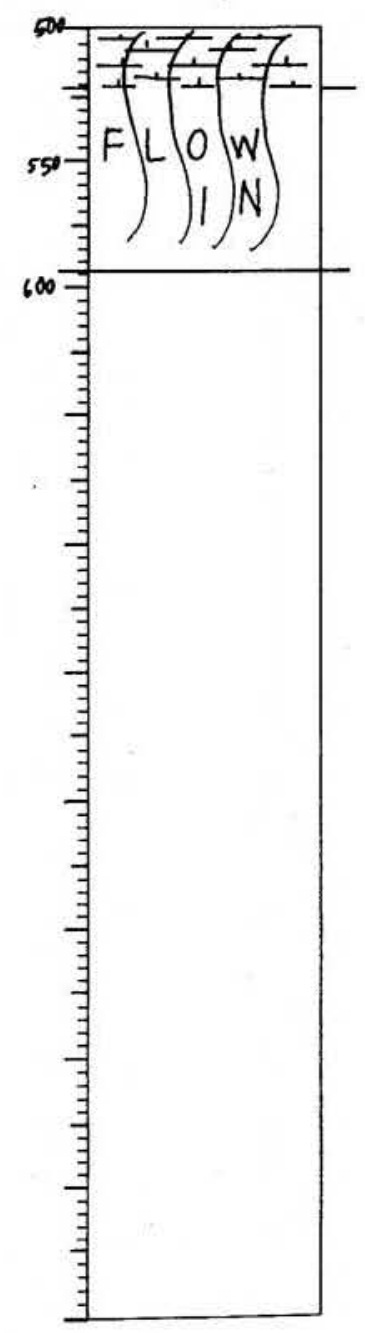

Detailed Description

Ship: _Chain

Expedition 115

Leg No.

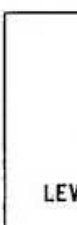

303

SMEAR SLIDE DESCRIPTIONS - W.H.O.I. SEDIMENT CORES
Core No. 37 PC

Station No. 58

Total Core Length $594 \quad \mathrm{~cm}$

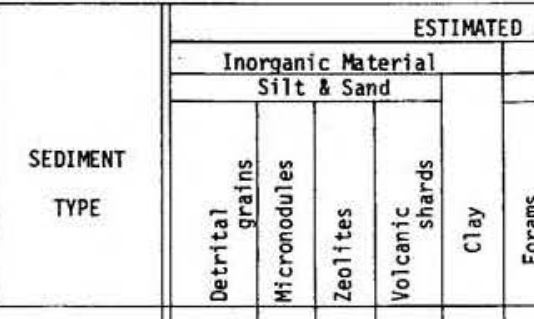

$$
\text { DANCES (\%) }
$$

Biogenous Materia
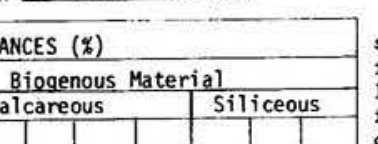

\begin{tabular}{|l|l|l|l|l|l|l|l|l|l|l|l|l|l|l|l}
$55 \mathrm{~cm}$ & calc ooze & 10 & & & $\operatorname{tr}$ & 40 & 1 & 35 & & $\mathrm{tr}$ & 12 & $\mathrm{tr}$ & & 2 \\
$\mathrm{c}$ & $\mathrm{tr}$
\end{tabular}

$200 \mathrm{~cm}$ nanno ooze

15 . 5 (15

\begin{tabular}{l|l}
$240 \mathrm{~cm}$ & nanno ooze \\
\hline $300 \mathrm{~cm}$ & nanno ooze
\end{tabular}

$350 \mathrm{~cm}$ namno ooze

$385 \mathrm{~cm}$ calc ooxe

$420 \mathrm{~cm}$ calc ooze

$575 \mathrm{~cm}$ calc ooze

\begin{tabular}{|l|l||l|}
$575 \mathrm{~cm}$ & calc ooze & 2 \\
\hline & &
\end{tabular}


304

VISUAL CORE DESCRIPTION

Page_1 of _ 1

Ship CHAIN Cruise 115 Leg 5 sta. 58 Core No. $37 \mathrm{PG}$

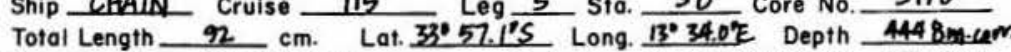

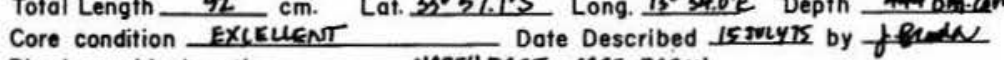
Physiographic location NIRTHEAGT CAPE RASIN.

Lithologic

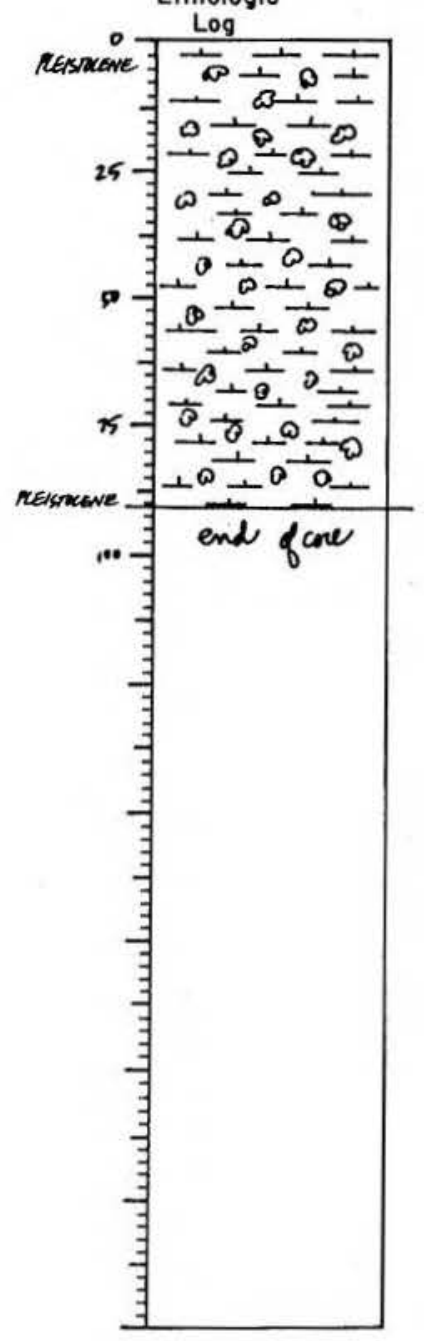

\section{Detailed Description}

CALC OOZE

10YR $6 / 3$ pale brown grades to $5 / 3$ brown and back to $6 / 3$ extensive very pale brown mottling and burrowing throughout

molst silty lutite with a few scattered forams
end of core
305

SMEAR SLIDE DESCRIPTIONS - W.H.O.I. SEDIMENT CORES
Ship: Chain

Expedition 115

Leg No. 5
Core No. $37 \mathrm{PG}$

Station No. 58

Total Core Length $\quad 92$

ESTIMATED ABUNDANCES (q)

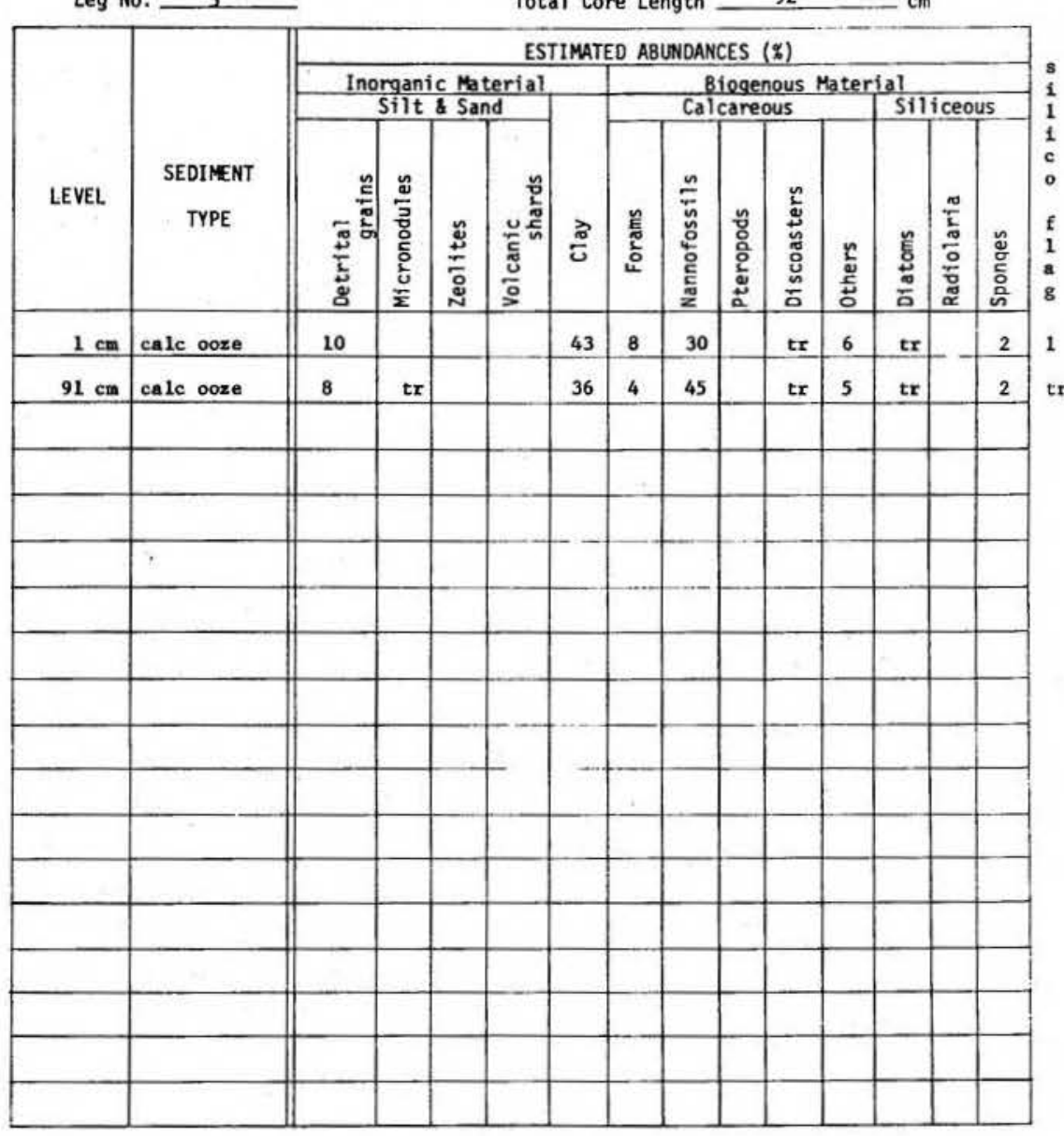


Ship CHAiN Cruise 115 Leg 5 Sta. 60 Core No. 38 PC Total Length $878^{\circ} \mathrm{cm}$. Lat. $34^{\circ}$ oy.74's Long. $26^{\circ} 55.00^{\prime} E$ Depth 5260 coRR Core condition EXCELLENT Date Described 16 JOWY75 by R MEGIRR Physiogrophic location Mid-Cape Basin

Lithologic

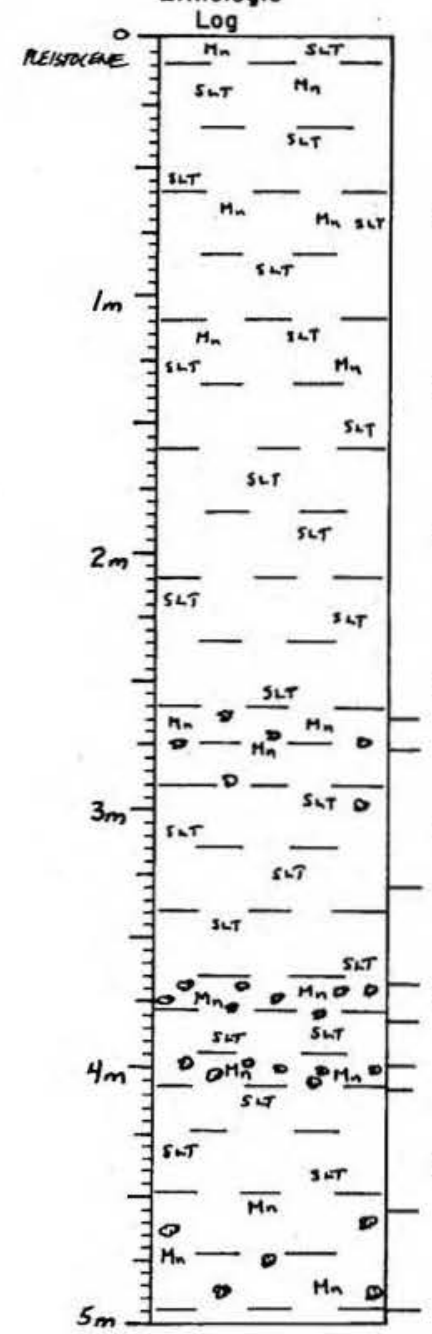

\section{0-265 Detailed Description}

\section{UNPOSS CLAY WITH DETRIIUS}

10YR $6 / 4$ lisht yelloutsh brow

moist, firm lutite

St horlzontal, black fuzzy laminations and patche. $0-23$ ing in sections within unit, probably manganese

S Irregular
65-277

UNFOSS CLAY/MN MICRONODULES

SY $2.5 / 1$ black

mottling and maxbling scattered at top to extensive at bottom, 11ght yellowish brown molst firm lutite

277-330

UNPOSS CLAY WTH DETRITUS

10YR $6 / 411$ ght yellowish brown

several small black mottles and elongated burrows 277-295 cm molst finim lutite

330-368

\section{UTPOSS CLAY WITH DETRITUS}

three very small black patches of silt sized grains moist finm lutite

368-382

URPOSS CIAY/Re MICRONODULES

SY $2.5 / 1$ black

ing thwoughout, light yellowish brom

moteled lutite

$32-398$

UNFOSS CLAY WITH DETRITUS

10rR $6 / 4$ 11ght yellowlsh brown

1 very mall, black mottle of silt sized grains $386 \mathrm{~cm}$

very homogeneous

S silghtly couvex up

$8-408$

UNFOSS CIAY/YN MICRONODULES

SY $2.5 / 1$ black

extensive mottling 398-403, common 403-408, 1ight yellowish

8-454

ONFOSS CLAY WITH DETRITU

10YR 6/4 11ght yellowish brow

molat firn Lutite

very homogeneous
Ship CHAIN Cruise 115 Leg 5 Sta. 60 Core No. 38 PC

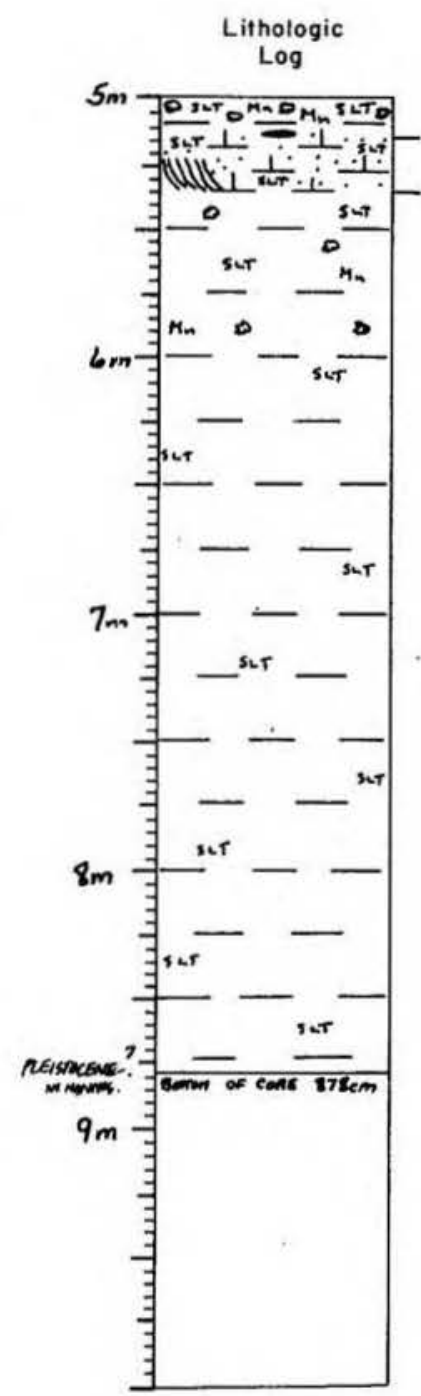

Detailed Description

454-493

UNPOSS CLAY/MN MICROHODULES

5Y 2.5/1 black

common mottling throughout, 1ight yellowish brown

moist finm lutite

ilghter and darker bues throughout

493-514

UNPOSS CLAX WITH DETRITUS AND UNFOSS CLAY/MN MICRONODULES 10YR $6 / 4$ light yellowish brown, 5Y $2.5 / 1$ black severe 1stermottling and marbling of the tyo major color moist firm lutite

14-535 CALC OOZR WITH DETRITUS

10YR $6 / 4$ 11ght yellowish brown and $5 Y 2.5 / 1$ black

$1 \mathrm{~cm}$ chunk of black silt and lutite grains cemented togethe $518 \mathrm{~cm}$ alternating microlaminations of the 2 major colors, laminations vary from very convex up in shape to nearly flat and horizontal; cross bedding

535-878

UWFOSS CLAY WTTH DETRITUS

10TR 6/4 litht yellowis

fuzzy black patch $547-589 \mathrm{~cm}, 1$ black lamination at $608 \mathrm{~cm}$ molst fim lutite

unit homogeneous except for 2 areas of black mottling, core cutter 863-878 ca s textural, convex up 
308

. SMEAR SLIDE DESCRIPTIONS - W.H.O.I. SEDIMENT CORES

Ship: Chain

Expedition 115

Core No. $38 \mathrm{PC}$

Leg No. 5

ESTIMATED ABUNDANCES (\%)

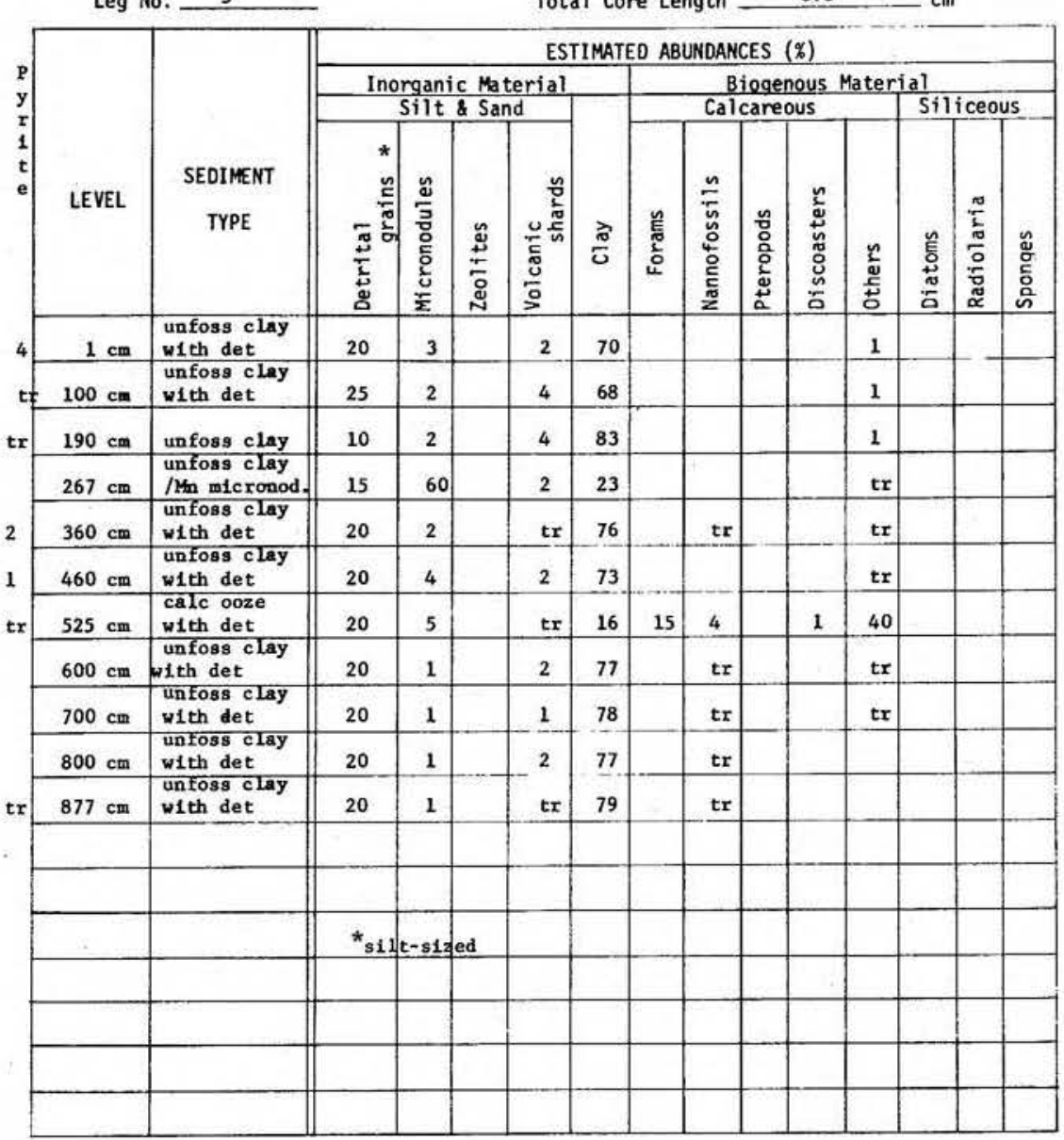

309

VISUAL CORE DESCRIPTION

Page 1 of 1

Ship CHaw Cruise 115 Leg 5 sta 60 Core No. 38 PG Total Length $160^{\circ} \mathrm{cm}$. Lat. $34^{\circ} 04.74^{\prime} \mathrm{S}$ Long. $06^{\circ} 550^{\circ}$ E Depth $5260 \mathrm{CORR} \mathrm{m}$ Core condition EXCELENT Date Described 16 JULY 75 by $R M^{C} G$ IRR Physiographic location Mid-Cape Basin

Lithologic

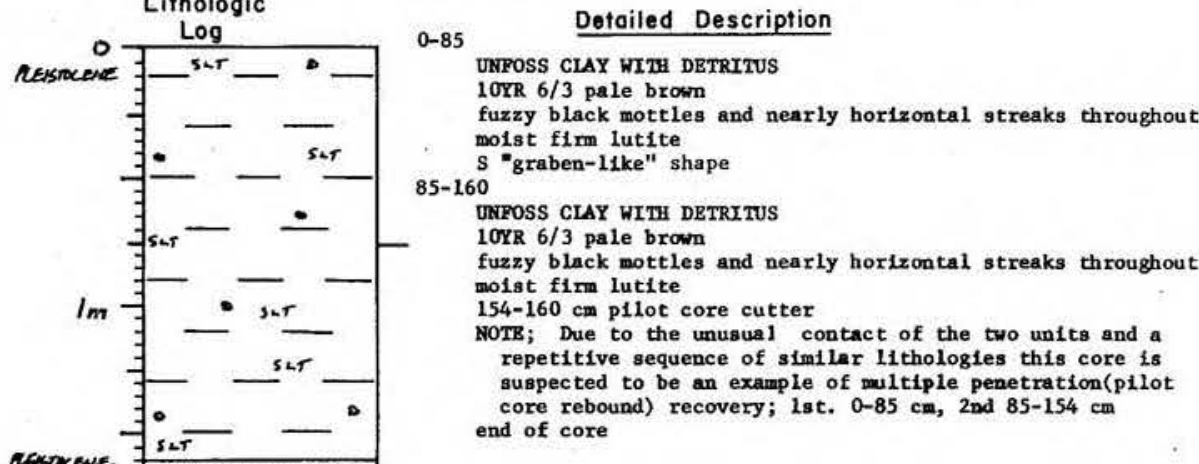

rasrovene

scion of core: $110 \mathrm{~cm}$

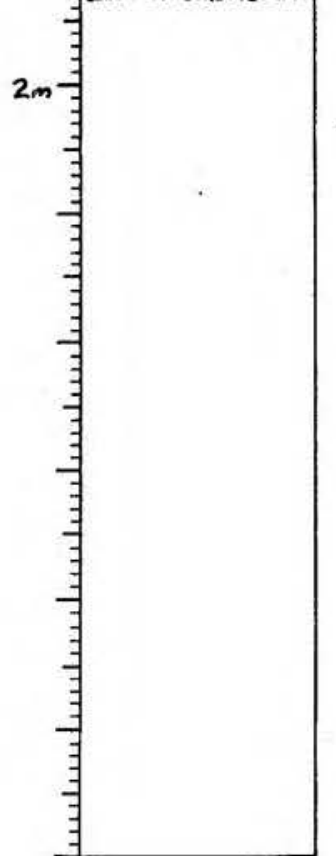


310

SMEAR SLIDE DESCRIPTIONS - W.H.O.I. SEDIMENT CORES

Ship: Chain

Expedition 115

Core No. 38 PG

Leg No. 5

Station No. 60

\begin{tabular}{|c|c|c|c|c|c|c|c|c|c|c|c|c|c|c|}
\hline & & & & & & & & & & & & & & \\
\hline \multirow[b]{3}{*}{ LEVEL } & \multirow[b]{3}{*}{$\begin{array}{c}\text { SEDIMENT } \\
\text { TYPE }\end{array}$} & \multicolumn{13}{|c|}{ ESTIMATED ABUNDANCES ( $(\%)$} \\
\hline & & \multicolumn{4}{|c|}{$\begin{array}{c}\text { Inorganic Material } \\
\text { Silt \& Sand }\end{array}$} & \multirow[b]{2}{*}{$\frac{\pi}{0}$} & \multicolumn{5}{|c|}{$\begin{array}{l}\text { Biogenous } \mathrm{K} \\
\text { Calcareous }\end{array}$} & \multicolumn{3}{|c|}{$\frac{\text { ial }}{\text { Silliceous }}$} \\
\hline & & 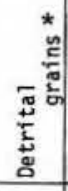 & $\begin{array}{l}\frac{\omega}{3} \\
\frac{5}{0} \\
\frac{0}{0} \\
\frac{0}{2} \\
\end{array}$ & 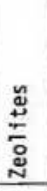 & 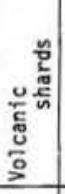 & & $\begin{array}{l}\text { 哀 } \\
\text { bे }\end{array}$ & 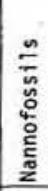 & 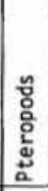 & 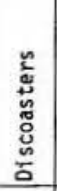 & $\begin{array}{l}r \\
\text { E⿱亠幺 } \\
0 \\
\end{array}$ & $\begin{array}{l}\breve{n} \\
\vdots \\
0 \\
\vdots \\
\end{array}$ & $\begin{array}{l}\frac{\pi}{i} \\
\frac{\pi}{0} \\
\frac{0}{0} \\
\frac{\tilde{\alpha}}{\alpha} \\
\end{array}$ & 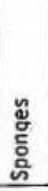 \\
\hline $1 \mathrm{~cm}$ & w1th det & 20 & 1 & & 5 & 72 & & 2 & & & $t x$ & & & \\
\hline $85 \mathrm{~cm}$ & $\begin{array}{l}\text { unfoss clay } \\
\text { with det }\end{array}$ & 25 & 2 & & 3 & 65 & & 1 & & & $t x$ & & & \\
\hline $153 \mathrm{~cm}$ & $\begin{array}{l}\text { unfoss clay } \\
\text { with det }\end{array}$ & 25 & 7 & & 3 & 65 & & $t$ & & & tr & & & \\
\hline & & & & & & & & & & & & & & \\
\hline & * sillt-sized & & & & & & & & & & & & & \\
\hline & & & & & & & & & & & & & & \\
\hline & & & & & & & & & & & & & & \\
\hline & & & & & & & & & & & & & & \\
\hline & & & & & & & & & & & & & & \\
\hline & & & & & & & & & & & & & & \\
\hline & & & & & & & & & & & & & & \\
\hline & & & & & & & & & & & & & & \\
\hline & & & & & & & & & & & & & & \\
\hline & & & & & & & & & & & & & & \\
\hline & & & & & & & & & & & & & & \\
\hline & & & & & & & & & & & & & & \\
\hline & & & & & & & & & & & & & & \\
\hline & & & & & & & & & & & & & & \\
\hline
\end{tabular}

311

VISUAL CORE DESCRIPTION

Page 1 of 1

Ship CHAin Cruise $115 \quad$ Leg 5 sto 69 Core No 39 PC

Total Length 491 cm. Lot. $32^{\circ} 16.43^{\prime} \mathrm{S}$ Long. $5^{\circ}$ 04.55'WDepth 4018 corem Core condition EXCELLENT Date Described 15 JULY 75 by R MC GIRR

Physiographic location. Southern Angole Basin

$0-178 \quad \begin{gathered}\text { Lithologic } \\ \text { Log }\end{gathered} \quad$ Detailed Description

$1^{0}+1 \quad$ CALC OOZE

10YR $7 / 3$ very pale brown

$1+1$

$\exists \perp \perp \perp$

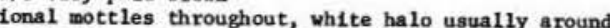

very pale brown center, mottled at bottom contact

moist firm silty lutite

slight variations in hue throughout, somewhat darker 170-178

$\underset{178-361}{S \text { mottled }}$

CALC OOZE

1OYR $8 / 2$ white grades to $7 / 2$ light gray

scattered mottles throughout, white, usually haloed around gray center, mottled at contact slight variations in color throughout
S mottled

Im

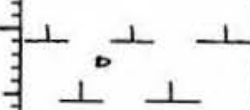

361-491

CAIC DOZE

LOYR $8 / 1$ white grades to $7 / 3$ very pale brown

$\exists \perp 1$

large white mottles scattered throughout, thin black streaks

throughout

moist very finm slightly silty lutite

end of core core cutter

$2 \mathrm{~m} \neq \perp \perp \circ \quad \begin{aligned} & 478-491 \mathrm{~cm}, \\ & \text { end of core }\end{aligned}$

$\exists \perp \perp$

$\exists 111^{\circ}$

$11 \quad 1$

$3 m-1$

$\exists 1^{\circ} \perp \frac{1}{0}$

$\exists \perp 1^{\circ}$

$\exists \perp \perp 1$

录, 1

$4 m$

111
111
$110+1$
$1+\frac{1}{0}+1$ 


\section{2}

SHEAR SLIDE DESCRIPTIONS - W.H.0.I. SEDIMENT CORES

Ship: Chein

Expedition 115

Leg No. 5

\begin{tabular}{|c|c|c|c|c|c|c|c|c|c|c|c|c|c|c|}
\hline \multirow[b]{3}{*}{ LEVEL } & \multirow[b]{3}{*}{$\begin{array}{c}\text { SEDIMENT } \\
\text { TYPE }\end{array}$} & \multicolumn{13}{|c|}{ ESTIMATED ABUNDANCES (\%) } \\
\hline & & \multicolumn{4}{|c|}{$\begin{array}{c}\text { Inorganic Material } \\
\text { Silt Sand }\end{array}$} & & \multicolumn{5}{|c|}{$\begin{array}{l}\text { Biogenous Mater } \\
\text { Calcareous }\end{array}$} & \multicolumn{3}{|c|}{$\frac{\text { al }}{\text { siliceous }}$} \\
\hline & & 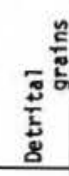 & 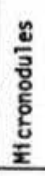 & $\frac{ \pm}{\frac{\tilde{a}}{2}}$ & 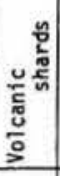 & $\frac{\pi}{6}$ & 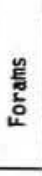 & 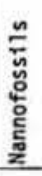 & 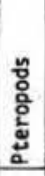 & 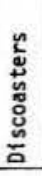 & $\begin{array}{l}c \\
\text { हैँ } \\
\text { ثँ } \\
\end{array}$ & 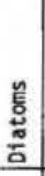 & $\frac{\pi}{\frac{\pi}{2}}$ & $\begin{array}{l}\text { ๕ัँ } \\
\text { ํㅡㄹ }\end{array}$ \\
\hline $2 \mathrm{~cm}$ & calc ooze & 5 & & & tr & 20 & 25 & 40 & & & 10 & $\mathrm{tr}$ & & \\
\hline $100 \mathrm{~cm}$ & calc ooze & 5 & 2 & & tr & 53 & 15 & 15 & $\mathrm{tr}$ & tr & 10 & & & \\
\hline $200 \mathrm{~cm}$ & calc ooze & 3 & tr & & 1 & 16 & 10 & 60 & & tr & 10 & & & \\
\hline $300 \mathrm{~cm}$ & calc ooze & 3 & 1 & & tr & 10 & 5 & 71 & & tr & 10 & & & \\
\hline $400 \mathrm{~cm}$ & calc ooze & 5 & 1 & & 2 & 15 & 20 & 52 & & & 5 & & & \\
\hline $490 \mathrm{~cm}$ & calc ooze & 5 & $\operatorname{tr}$ & & 10 & 15 & 2 & 63 & & tr & 5 & & & \\
\hline & & & & & & & & & & & & & & \\
\hline & & & & & & & & & & . & & & & \\
\hline & & & & & & & & & & & & & & \\
\hline & & & & & & & & & & & & & & \\
\hline & & & & & & & & & & & & & & \\
\hline & & & & & & & & & & & & & & \\
\hline & & & & & & & & & & & & & & \\
\hline & & & & & & & & & & & & & & \\
\hline & & & & & & & & & & & & & & \\
\hline & & & & & & & & & & & & & & \\
\hline & & & & & & & & & & & & & & \\
\hline & & & & & & & & & & & & & & \\
\hline
\end{tabular}

313

VISUAL CORE DESCRIPTION

Page 1 of 1

Ship CHAIN Cruise 115 Leg 5 Sta. 69 Core No. $39 P G$ Total Length $121 \mathrm{~cm}$. Lat. $32^{\circ} 16.43^{\prime}$ 's Long. $5^{\circ}$ Ox.55' Wepth $4018 \mathrm{ceph}$ Core condition EXCELLENT Date Described 16 TULY7S by R M'GiRe

Physiographic location Southean Angale Besin

Lithologic

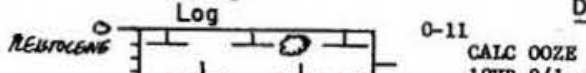

Detailed Description

$\perp \perp-\quad \begin{aligned} & \text { CAIC OOZE } \\ & \text { 10YR } 8 / 1 \text { white }\end{aligned}$

large mottle in center of unit, $3 \times 3 \mathrm{~cm}, 11$ ght gray

$\perp \perp \perp$ s 1 irregular

$\exists, \perp+1$, CALC OOZE

Im $1 \perp \div$ several Iight gray Im

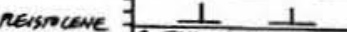

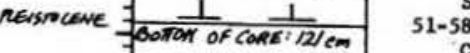
5 concave up

CALC OOZR

10YR $6 / 3$ pale brow

firm silty lutite

58-79

CALC DOZE

10YR $7 / 1$ gray grades to $7 / 3$ very pale brow

several small white and gray patches 58-64 cat $s$ irregular

79-121

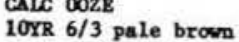

scattered vh1te mottles throughout

firm silty lutite

end of core 
314

SIEAR SLIDE DESCRIPTIONS - W.H.O.I. SEDINENT CORES

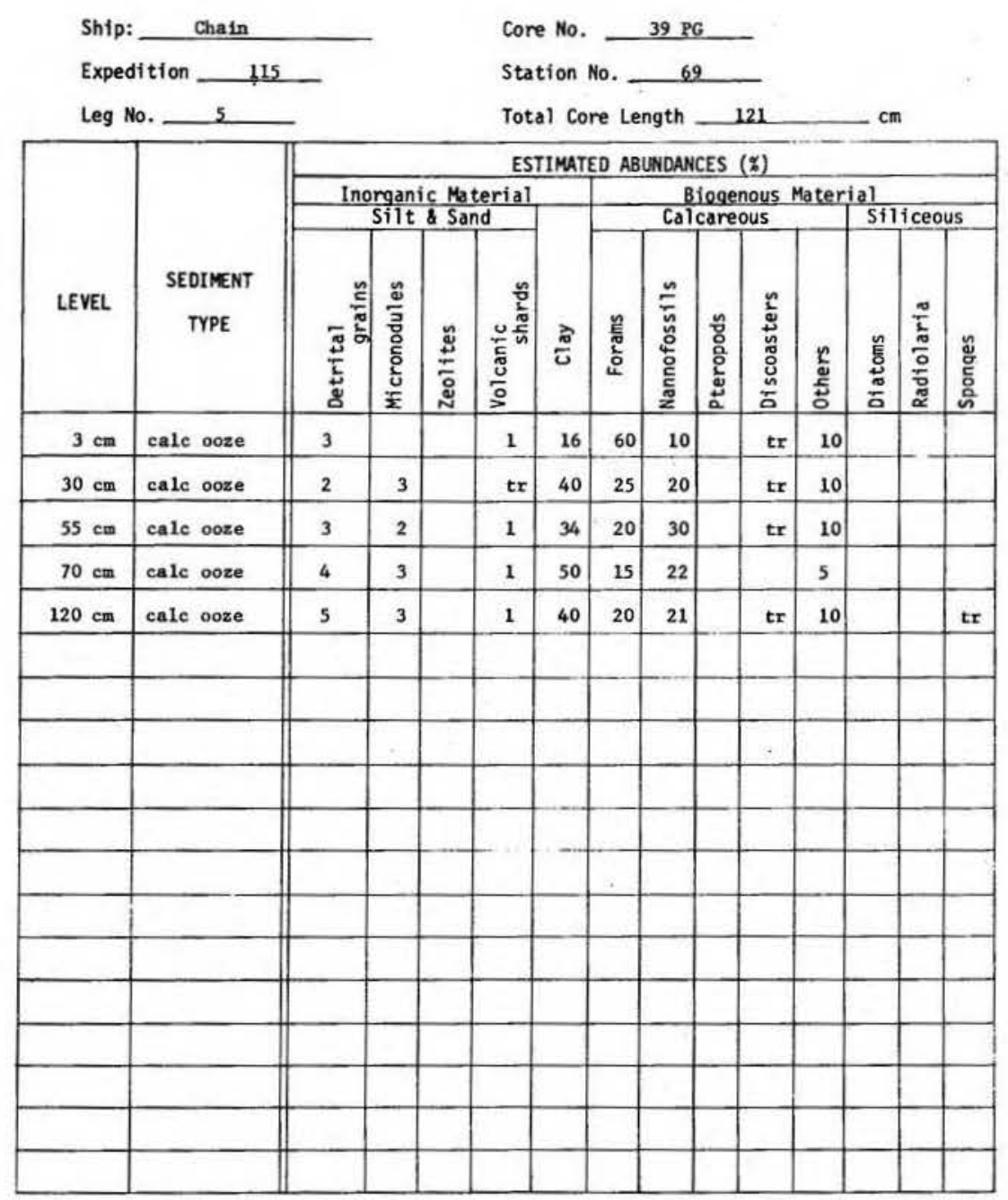

315

VISUAL CORE DESCRIPTION

Page 1 of 1

Ship CHAIN Cruise 115 Leg 5 Sta. 71 Core No. HOPC

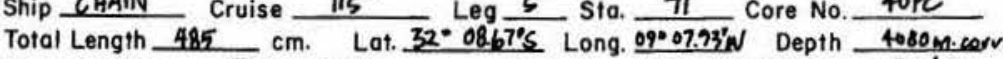
Core condition EXCELLENT. Date Described MNVLY7S by finde Physiographic location SOUIIHERN ANGOLA BASN.

Resrocene: $\begin{gathered}\text { Lithologic } \\ \text { Log }\end{gathered}$

1 OYR $7 / 3$ very pale brown gently grades repeatedly to $7 / 4$ very pale brow

sight intermottling of the two colors above in gradationa zones plus scattered white mottling throughout

firm slightly silty lutite with scattered forams; becoming at base of unit scattered streaked pockets of black flecks $50-180 \mathrm{~cm}$ 235-251

CALC OOZE

10YR $6 / 3$ pale brown

extensive 11ght gray and white mottling throughou $G$

$251-27$

\section{MANNO OOZE}

1OYR $7 / 3$ very pale brown

11ght gray mottling and

astic-like lutite

black flecks abundant in light gray mottles

200

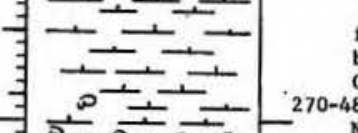
485

NAKFO OOZE

10YR $8 / 1$ white

mottles found scattered throughout

firm, very plastic-11ke lutite with very few forams

$\exists=0 \div 0+0$

30 志士田 end of core 


\section{6}

SMEAR SLIDE DESCRIPTIONS - W.H.O.I. SEDIMENT CORES

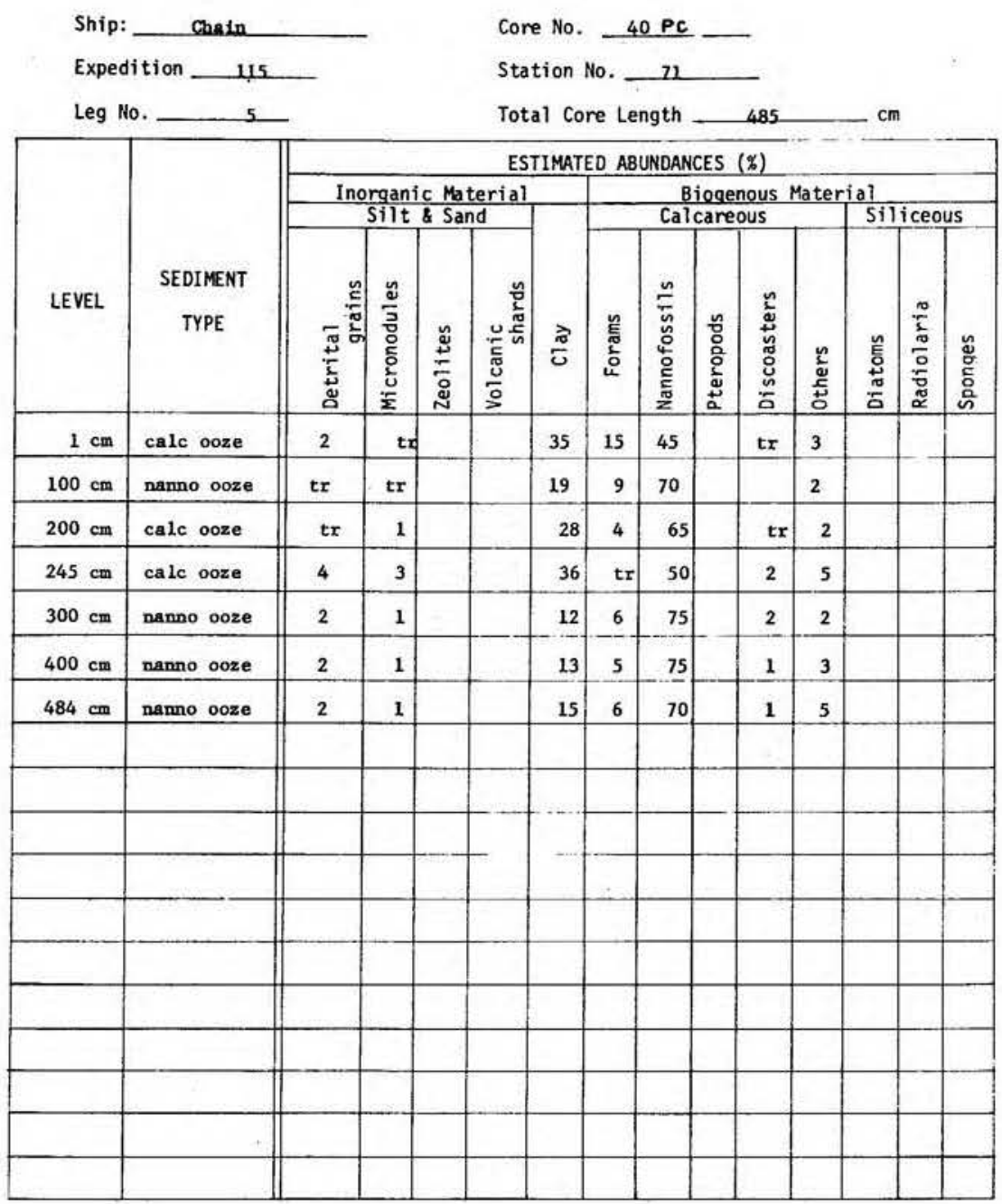

317

VISUAL CORE DESCRIPTION

Page 1 of 1

Ship CHAIN Cruise 115 Leg 5 sta. 71 Core No. 4OPG Total Length $146 \mathrm{~cm}$. Lat. $32^{\circ} 08.67^{\prime \prime S}$ Long. $09^{\circ} 07.93^{\prime} W^{\prime}$ Depth Hopm.cour Core condition ExCELLENT. Date Described 4702475 by f. Brow Physiographic location CWTHERA ENGOA BASIN.

Lithologic
Log NoIB; Detailed Description

REISTOCENE

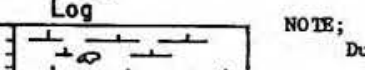

Detailed Description

1s suspected to be an example of a multiple penetration
(p1lot core rebound) recovery; 1st. 0-87, 2nd 87-146 cm

s.

10XR $7 / 3$ very pale brown

firm silty lutite with very abund. forams

50 -

CALC 0OZB

10YR $6 / 4$ light yellowish brown

fim alish sly ght white wotting

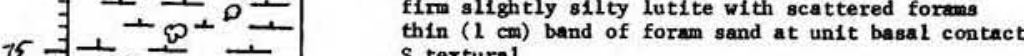

计士 1 S textural

CALC OOZE

10YR $8 / 2$ wh1te grades to $7 / 3$ very pale brown

at $=$ - 1 - very firm plastic 1ike lutite with vers

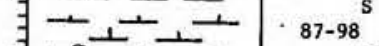

10YR $7 / 3$ very pale brown

125 very slight white mottling foumd throughout

寻立立宁

neisracene

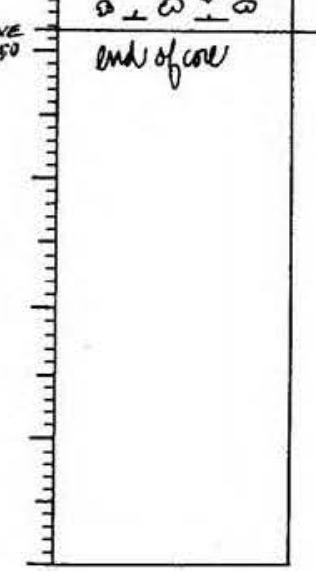
G

CALC OOZR

OYR $6 / 4$ 1ight yellowish brom

occasional faint slight white mottling inm slightly silty lutite with scattered forams thin zone of very abund. forams at unit basal contact

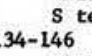

\section{CACC OOZE}

1OYR $8 / 2$ white grades to $7 / 3$ very pale brown

coumon white mottling found throughout

very fim plastic like lutite

end of core 


\section{8}

SMEAR SLIDE DESCRIPTIONS - W.H.0.1. SEDIMENT CORES

Ship: Chain

Expedition 115

Leg No. 5

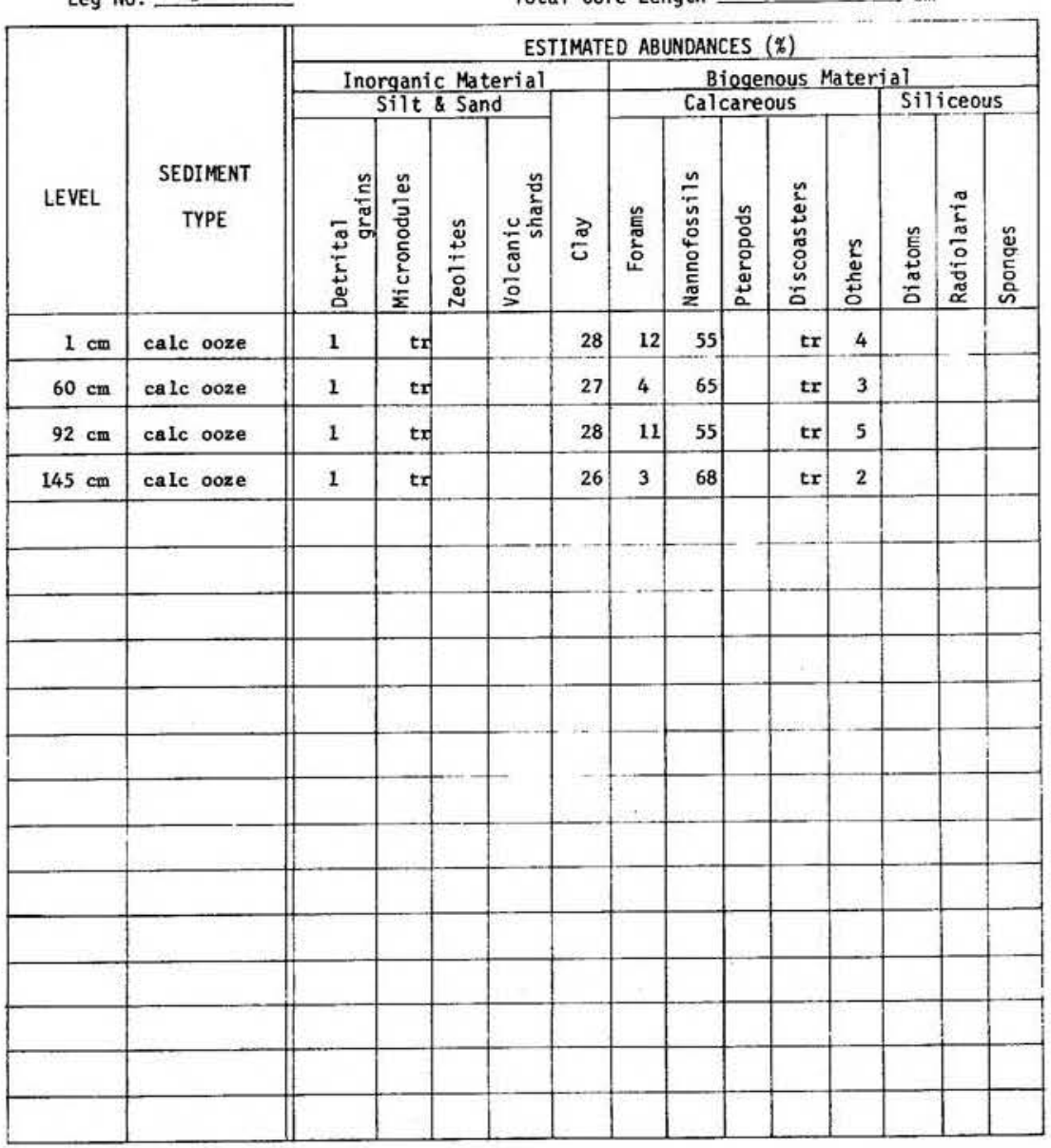

319

VISUAL CORE DESCRIPTION

Page 1 of 1

Ship CHAIN Cruise 115 Leg 5 Sta. 77 Core No. 41 GC Totol Length $53 \mathrm{~cm} \quad$ Lat $31^{\circ} 499^{\prime} \mathrm{S}$ Long. $17^{\circ} 145^{\prime} \mathrm{W}$ Depth $3700 \mathrm{cosem}$ Date Described $\angle 5 J u y t 5$ by $R$ Physiographic location West Flank, Mid-Atlantic Ridge

Lithologic

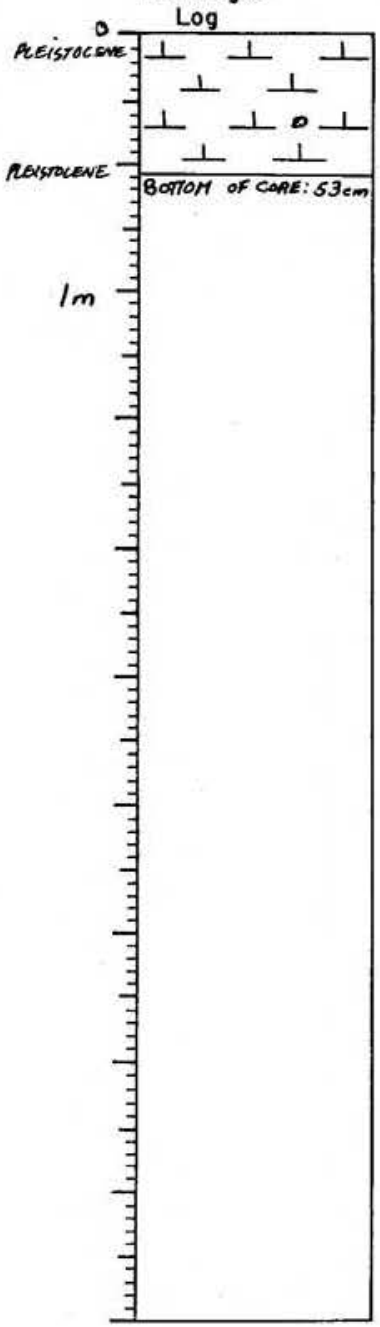

Detailed Description

CALC OOZE

10YR $6 / 3$ pale brown

three medion white mottles, 19, 37, $42 \mathrm{~cm}$

slitht darkening in color, $13-22 \mathrm{~cm}$

end of core 
SIEAR SLIDE DESCRIPTIONS - W.H.O.I. SEDIMENT CORES

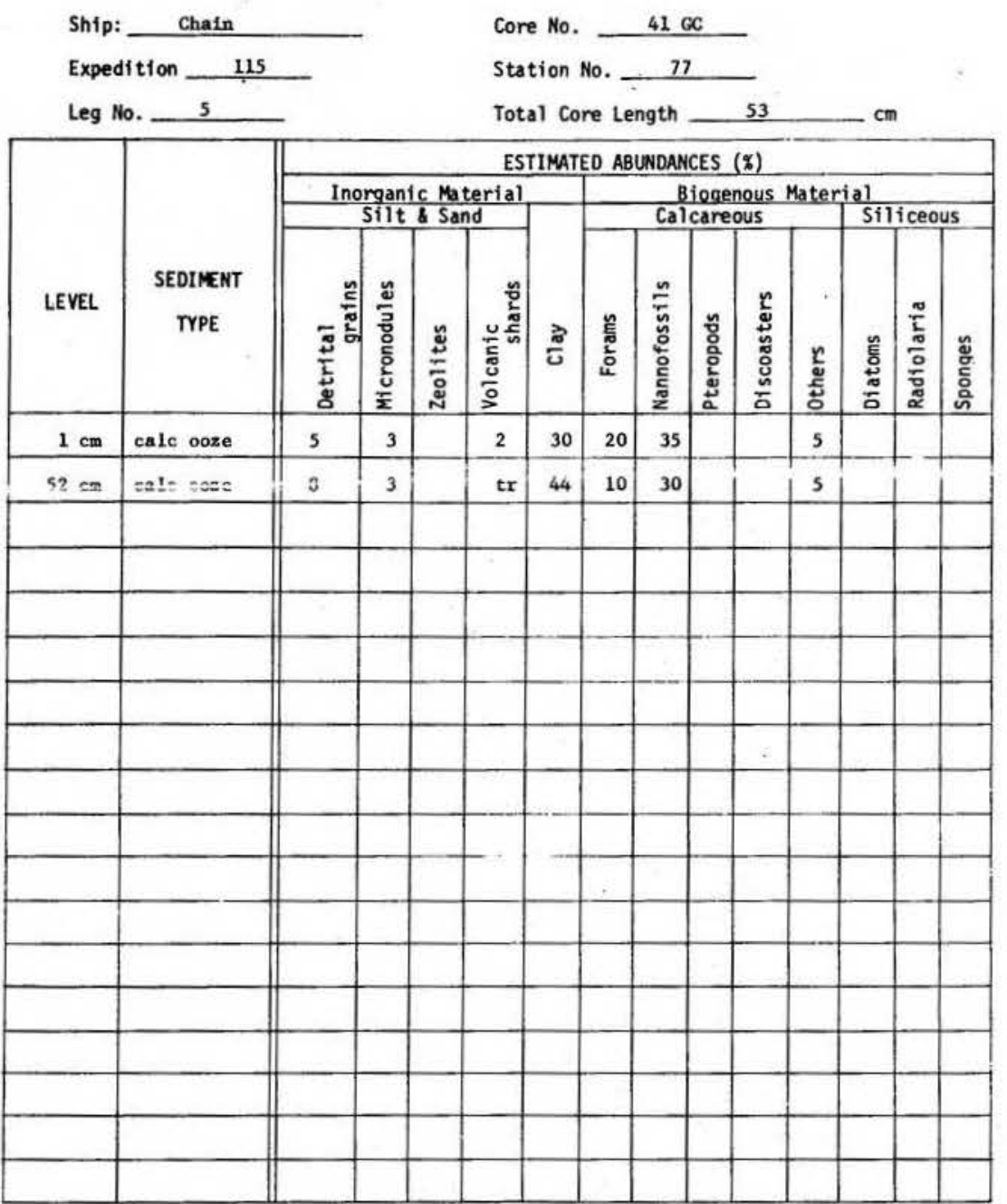

VISUAL CORE DESCRIPTION

Page 1 of 1

Ship CHAIN Cruise 115 Leg 5 sta. 80 Core No 42 PC Total Length $302 \mathrm{~cm}$. Lat. $310.41 .5 \mathrm{~g}^{2} \mathrm{~S}$ Long. $19^{\circ} 05.03 \mathrm{~W}$ Depth $3815 \mathrm{~m} \cdot \mathrm{um}$ Total Length $-502 \mathrm{~cm}$. Lat. $31041.58 \mathrm{~S}$ Long. $19^{\circ} 05.03 \mathrm{~N}$ Depth $\frac{3815 \mathrm{~m} \cdot 0 \mathrm{~s}}{\text { CXCELENT }}$ Physiographic location WEST FLNIK- MID ATLANTC RIDGE.

Lithologic

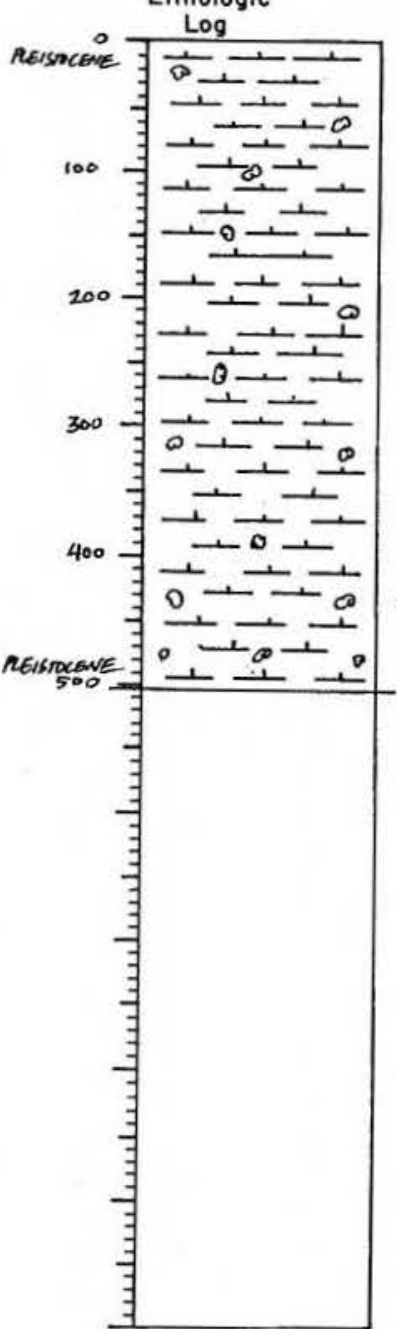

\section{Detailed Description}

CALC OOZE GEADES TO BARSO OOZE

Dredes to $8 / 3$ very pale brown

scattered but distinct, well formed white mottles throughout, a few elongated in the zone $60-200 \mathrm{~cm}$

ast sightly silty lutite with scattered forms grades to Btiffer plastic like lutite around $215 \mathrm{~cm}$; texture remain consistent frow there to end of core

Note; section $4,(0-57 \mathrm{~cm})$ somewhat disturbed in extrusion from core barrel, not ortented for paleonagnetics 


\section{2}

. SMEAR SLIDE DESCRIPTIONS - W.H.0.I. SEDIMENT CORES

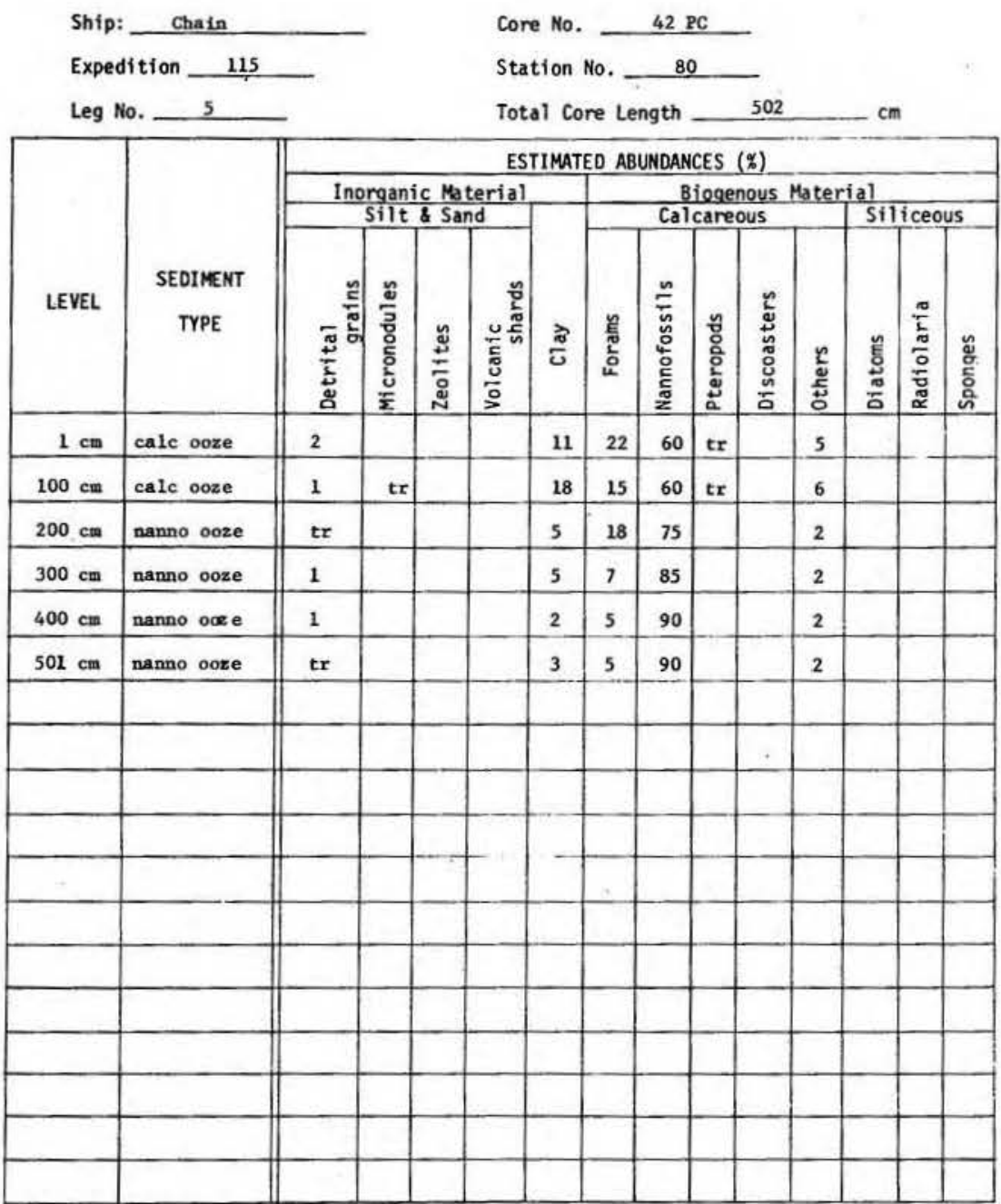

323

VISUAL CORE DESCRIPTION

Page 1 of 2

Ship CHAll Cruise 115 L 54 Core No 43 PC Total Length $699^{\circ} \mathrm{cm}$. Lat. $31^{\circ} 43.55^{\prime} \mathrm{S}$ Long. $20^{\circ} 52.42^{\prime} \omega_{\text {Depth }} 4141 \mathrm{COR} R \mathrm{~m}$ Core condition EXCEWENT Date Described 14 JULY75 by $R M^{0} G I R R$ Physiographic location West Flank, Mid-Atlantic Ridge

Lithologic

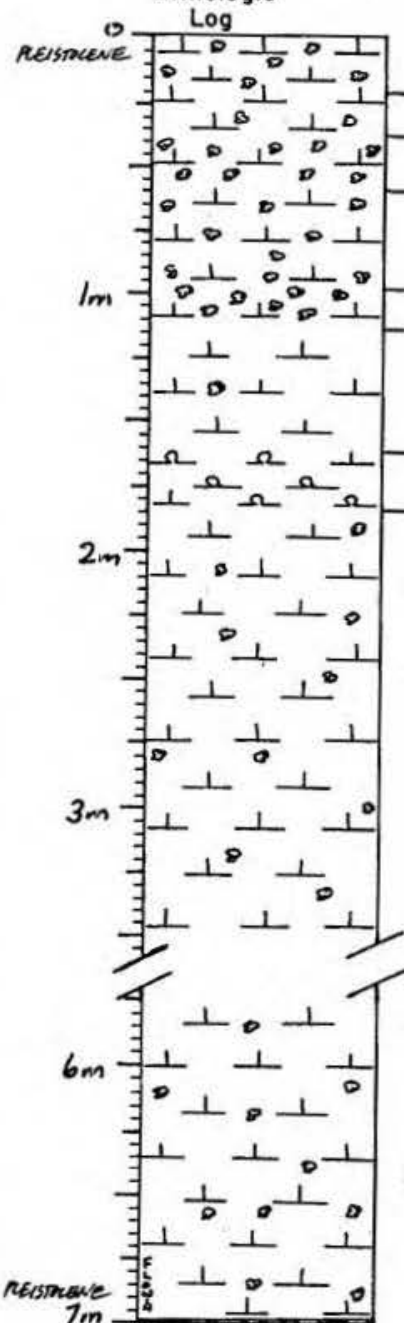

0-19 Detailed Description

CAIC OOZE

10YR $6 / 4$ 1ight yellowish brown

cormon mottling throughout, pale brow

(1rregular, nearly horizontal $1 \mathrm{~cm}$ thick laminations, brown 37 moted

$10 \mathrm{YR} 7 / 3$ and $8 / 3$ very pale browns

scattered mottling throughout, very pale brewn, mottled at upper contact, light yellowlsh brown moist silty lutite

37-59 mottled

CALC OOZE

1ORR $7 / 3$ very pale brown and $6 / 4$ 1ight yellowish brown

coumon motting thro ughout, yellowish brow

solst silty lutite

59-97 mottled

CAIC OOZE

CALC $0 O Z E$
$10 \mathrm{YR} 7 / 3$ and $8 / 3$ very pale brown

common intermottiling and marbling of the 2 major colors

1 patch of white at $86 \mathrm{~cm}$ 7-113

\section{CALC OOZE}

10YR $6 / 3$ pale brown

cokmon mottling throughout, very pale brown and brown silty lutite

113-161

CAIC OOZE

10 YR $7 / 3$ very pale brow

fitite mottles, pale brown one with a white halo, at $136 \mathrm{~cm}$

$3 \mathrm{~cm}$ wide lutite lamination at $117-120 \mathrm{~cm}$, thin wavy vhite Iamination, $148 \mathrm{~cm}$

161-184

\section{SILIC CALC OOZE}

10YR $8 / 2$ white

semi siliceous malch

184-699

ations throughout

CALC OOZE

LOXR $5 / 3$ brown alternating $7 / 4$ very pale brown

few to comonn mottles throughout of the two mafor colors

in 年 $\frac{1}{1}$ 
324

VISUAL CORE DESCRIPTION

Page 2 of 2

Ship CHAWN Cruise 115 Leg 5 Sto. 84 Core No. 43 PC

Lithologic

Log

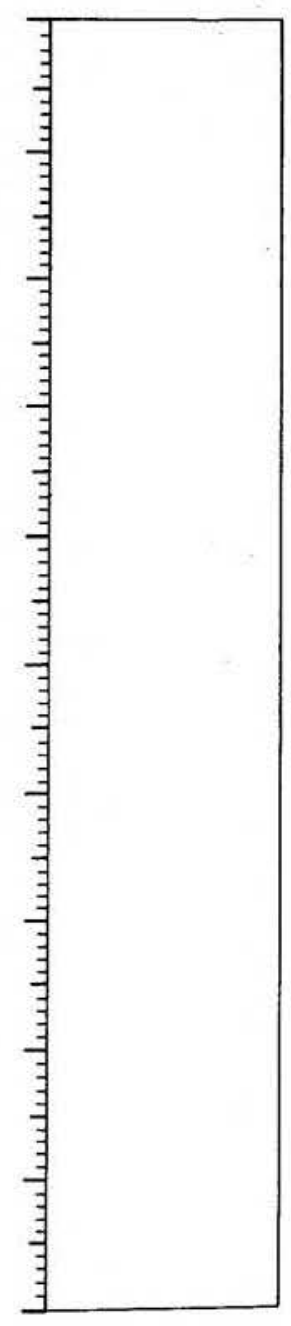

Detoiled Description

alternating 1ight and dark areas throughout; vertical streaks, 675-699 cm, probable flow in

end of core

\section{5}

SHEAR SLIDE DESCRIPTIONS - W.H.O.I. SEDIMENT CORES

Ship: Chain

Expedition 115

Leg No. 5
Core No. $43 \mathrm{PC}$

Station No. 84

Total Core Length 699

\begin{tabular}{|c|c|c|c|c|c|c|c|c|c|c|c|c|c|c|}
\hline \multirow[b]{3}{*}{ LEVEL } & \multirow[b]{3}{*}{$\begin{array}{c}\text { SEDIMENT } \\
\text { TYPE }\end{array}$} & \multicolumn{13}{|c|}{ ESTIMATED ABUNDANCES $(x)$} \\
\hline & & \multicolumn{4}{|c|}{$\begin{array}{l}\text { Inorganic Material } \\
\text { Silt Sand }\end{array}$} & \multirow[b]{2}{*}{$\frac{\pi}{6}$} & \multicolumn{5}{|c|}{$\begin{array}{l}\text { Biogenous Mater } \\
\text { Calcareous }\end{array}$} & \multicolumn{3}{|c|}{ Sfliceous } \\
\hline & & $\frac{5}{\frac{5}{5}}$ & 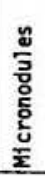 & $\frac{\$}{\frac{\pi}{2}}$ & 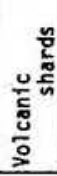 & & 宸 & 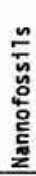 & 응 & 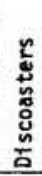 & $\begin{array}{l}r \\
\text { t } \\
\text { ț } \\
\end{array}$ & 嵌 & $\begin{array}{l}\frac{\pi}{2} \\
\frac{\pi}{0} \\
\frac{0}{0} \\
\frac{\pi}{0} \\
\frac{\pi}{2}\end{array}$ & 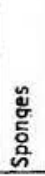 \\
\hline $1 \mathrm{~cm}$ & calc ooze & 5 & 3 & & 2 & 40 & 10 & 30 & & tr & 10 & tr & & \\
\hline $50 \mathrm{~cm}$ & calc oose & 3 & 3 & & 2 & 29 & 3 & 50 & & tr & 10 & & & \\
\hline $80 \mathrm{~cm}$ & calc ooze & 3 & 3 & & 1 & 30 & 3 & 55 & & $t x$ & 5 & & & \\
\hline $140 \mathrm{~cm}$ & calc ooze & 3 & 2 & & 3 & 22 & 10 & 50 & tr & tx & 10 & & & \\
\hline $175 \mathrm{~cm}$ & $\begin{array}{l}\text { silic } \\
\text { calc ooxe }\end{array}$ & 2 & tr & & & 20 & & 13 & & tx & 5 & 60 & & \\
\hline $200 \mathrm{~cm}$ & calc ooze & 3 & 4 & & tr & 50 & 5 & 28 & & tr & 10 & & & \\
\hline $300 \mathrm{~cm}$ & calc ooxe & 5 & 3 & & tr & 60 & 3 & 24 & & $t x$ & 5 & & & \\
\hline $400 \mathrm{~cm}$ & calc ooze & 3 & 3 & & 2 & 47 & 10 & 30 & & tx & 5 & & & \\
\hline $500 \mathrm{~cm}$ & calc ooze & 5 & 1 & & 1 & 60 & 2 & 21 & & $t x$ & 10 & & & \\
\hline $600 \mathrm{~cm}$ & calc ooze & 10 & tr & & -tr. & 43 & 2 & 40 & & tr & 5 & & & \\
\hline $692 \mathrm{~cm}$ & calc ooxe & 10 & 2 & & tr & 45 & 2 & 31 & & tr & 10 & & & \\
\hline & & & & & & & & & & & & & & \\
\hline & & & & & & & & & & & & & & \\
\hline & & & & & & & & & & & & & & \\
\hline & & & & & & & & & & & & & & \\
\hline & & & & & & & & & & & & & & \\
\hline & & & & & & & & & & & & & & \\
\hline & & & & & & & & & & & & & & \\
\hline
\end{tabular}

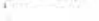


Ship CHAIN Cruise 115 Leg 5 Sto. 84 Core No. 43 PG Total Length $117 \mathrm{~cm}$. Lat. $31^{\circ} 43.55^{\prime} \mathrm{S}$ Long. $20^{\circ} 52.42^{\circ} \omega$ Depth $4141 \mathrm{CeRR}_{\mathrm{m}}$ Core condition EXCELENT Physiographic location West Flank, Mid-Atlantic Ridge

Lithologic

\section{Detailed Description}

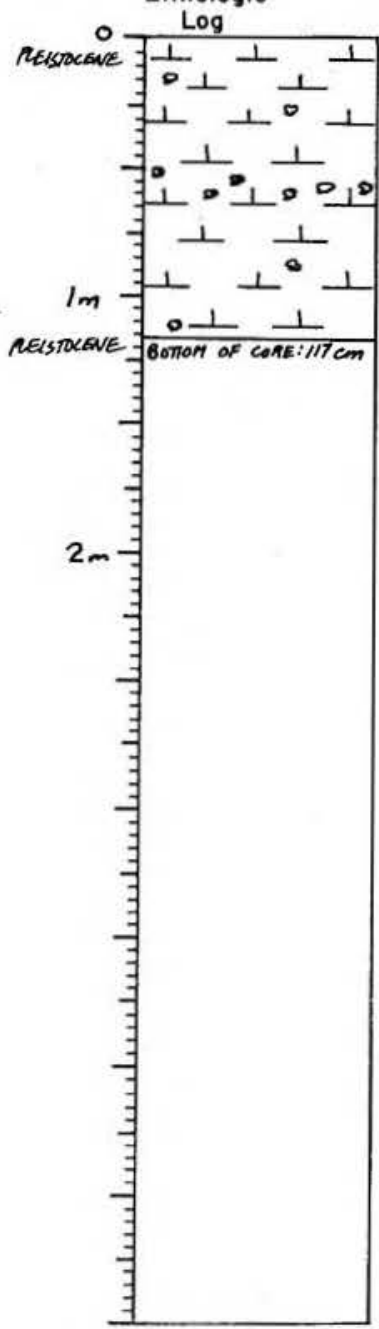

0-117 CALC OOZE

10YR $6 / 3$ pale brown extensive

core

molst silty lutite

(a) $25 \mathrm{~cm}, 33 \mathrm{~cm}, 46 \mathrm{~cm}, 67$

end of core

Ship: Chain

Expedition 115

Leg No. 5 Total Core Length 117

\begin{tabular}{|c|c|c|c|c|c|c|c|c|c|c|c|c|c|c|}
\hline \multirow[b]{3}{*}{ LEVEL } & \multirow[b]{3}{*}{$\begin{array}{c}\text { SEDIMENT } \\
\text { TYPE }\end{array}$} & \multicolumn{13}{|c|}{ ESTIMATED ABUNDANCES ( $(\varpi)$} \\
\hline & & \multicolumn{4}{|c|}{$\begin{array}{l}\text { Inorganic Material } \\
\text { Silt \& Sand }\end{array}$} & & \multicolumn{5}{|c|}{$\begin{array}{l}\text { Biogenous Mater } \\
\text { Calcareous }\end{array}$} & \multicolumn{2}{|c|}{$\frac{\partial}{\text { Siliceous }}$} & \\
\hline & & 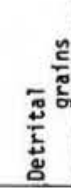 & 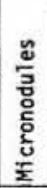 & 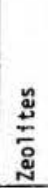 & $\frac{\sqrt{2}}{\frac{2}{2}}$ & $\frac{\bar{\sigma}}{\sigma}$ & 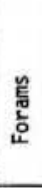 & 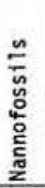 & 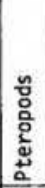 & 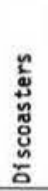 & 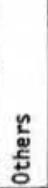 & 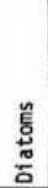 & $\frac{\frac{\pi}{2}}{\frac{\pi}{0}}$ & 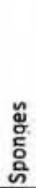 \\
\hline $1 \mathrm{~cm}$ & calc ooze & 5 & 3 & & tr & 22 & 15 & so & & tr & 5 & & & \\
\hline $111 \mathrm{~cm}$ & calc ooze & 5 & 3 & & 1 & 40 & 15 & 26 & & tr & 10 & & & \\
\hline & & & & & & & & & & & & & & \\
\hline & & & & & & & & & & & & & & \\
\hline & & & & & & & & & & & & & & \\
\hline & . & & & & & & & & & & & & & \\
\hline & & & & & & & & & & & & & & \\
\hline & & & & & & & & & & : & & & & \\
\hline & & & & & & & & & & & & & & \\
\hline & & & & & & & & & & & & & & \\
\hline & & & & & & & & & & & & & & \\
\hline & & & & & & & & & & & & & & \\
\hline & & & & & & & & & & & & & & \\
\hline & & & & & & & & & & & & & & \\
\hline & & & & & & & & & & & & & & \\
\hline & & & & & & & & & & & & & & \\
\hline & & & & & & & & & & & & & & \\
\hline & & & & & & & & & & & & & & \\
\hline
\end{tabular}


Ship CHAIN Cruise 115 Leg 5 Sta. 85 Core No. 44 PC Total Length $880 \mathrm{~cm}$. Lat. $31^{\circ} 36.49^{\prime}$ 'S Long. $22^{\circ} 26.73^{\prime} \omega_{\text {Depth }} 4411$ cerRm Core condition ExceLENT Date Described 10 Jub Vy by R MCGIRR Physiographic location Extreme Sevthern Brezilien Besin

Lithologic

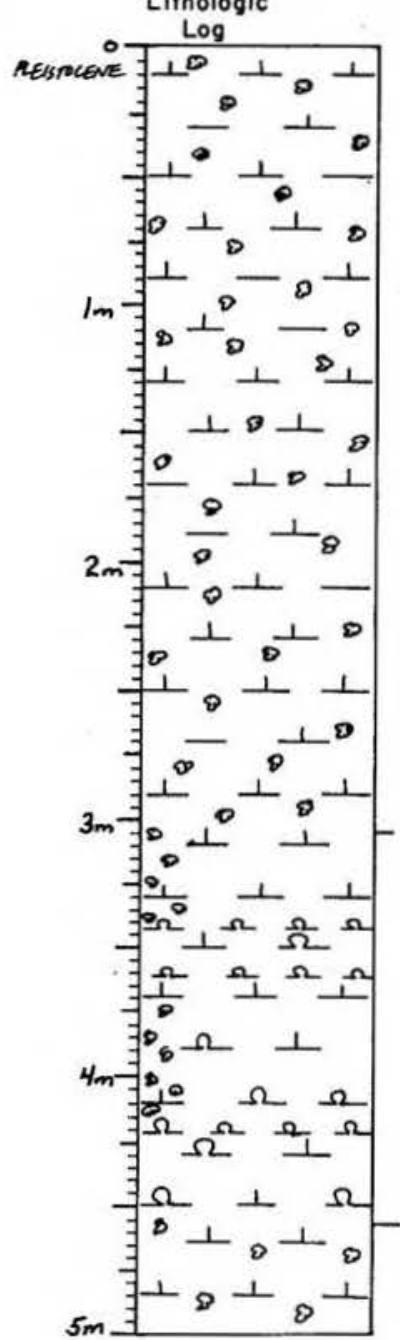

0-305 Detailed Description

COYR $6 / 4$ AND CALC CLAY brown and $4 / 4$ dark yellowish bro ane colors throughout fine silty lutite

ltemating light and dark ares vithin unit, with extensive mottling of the other color in each;

light

$0-5 \mathrm{~cm}$

$103-134 \mathrm{~cm} \quad 134-152 \mathrm{~cm}$

$152-159 \mathrm{~cm}$

$\begin{array}{ll}184-202 & \mathrm{~cm} \\ 232-273 \mathrm{~cm} & 202-232 \mathrm{~cm}\end{array}$

mottled bottom contact

SILIC CALC DOZE, wTTR LAMTNATTOE Of DIAZOM OOZE 10YR $7 / 3$ very pale brow

arge, connected mottles, white, along 1 side from

fim, hard molst silty lutite

thin (1 cm thick) convex up inations of silic mule $340-370 \mathrm{~cm}$, and 419-424 on, the bottom most several anina tions are very black, while most are light gray in color, several black, lutite lominations convex up $446-456 \mathrm{~cm}$ $\underset{456-880}{S}$ convex up

CALC 00ZE

large $(4 \times 4 \mathrm{~cm})$ white, 1rregular mottle at $578 \mathrm{~cm}$

alternating 11ght and dark areses throughout ent1re length of unit

11ght

$592-532$

dark

$555-591$

749-773

$787-831$

$850-877$

$864-880$ c

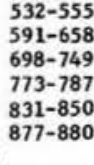

$698-74$

$773-787$

end of core

$877-880$

$$
\begin{aligned}
& \exists \Omega \Omega \\
& \exists \Omega \perp \Omega \\
& \exists \perp \perp 0 \\
& \exists \perp 0 \perp 0
\end{aligned}
$$

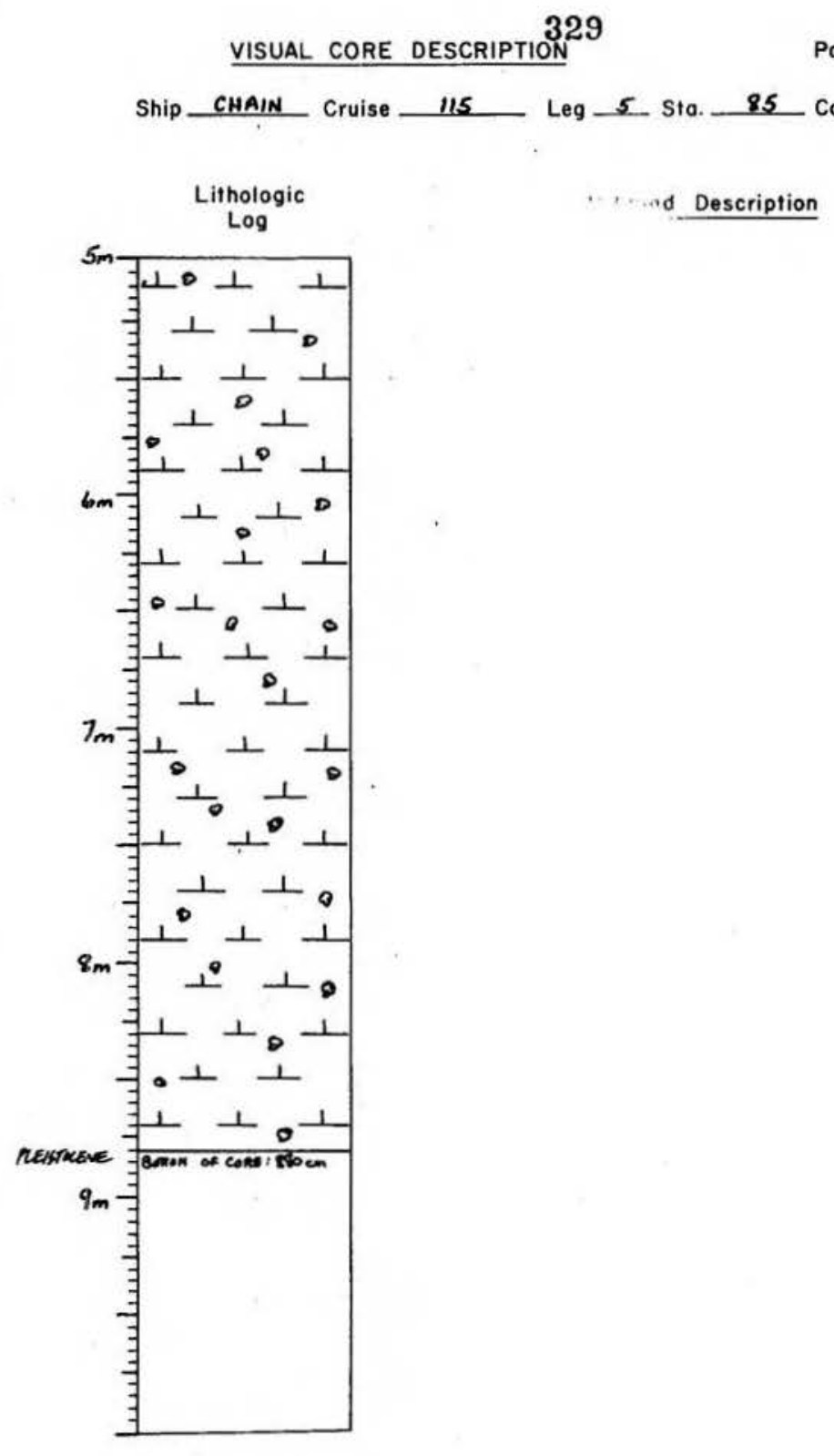


330

SMEAR SLIDE DESCRIPTIONS - W.H.O.I. SEDIMENT CORES

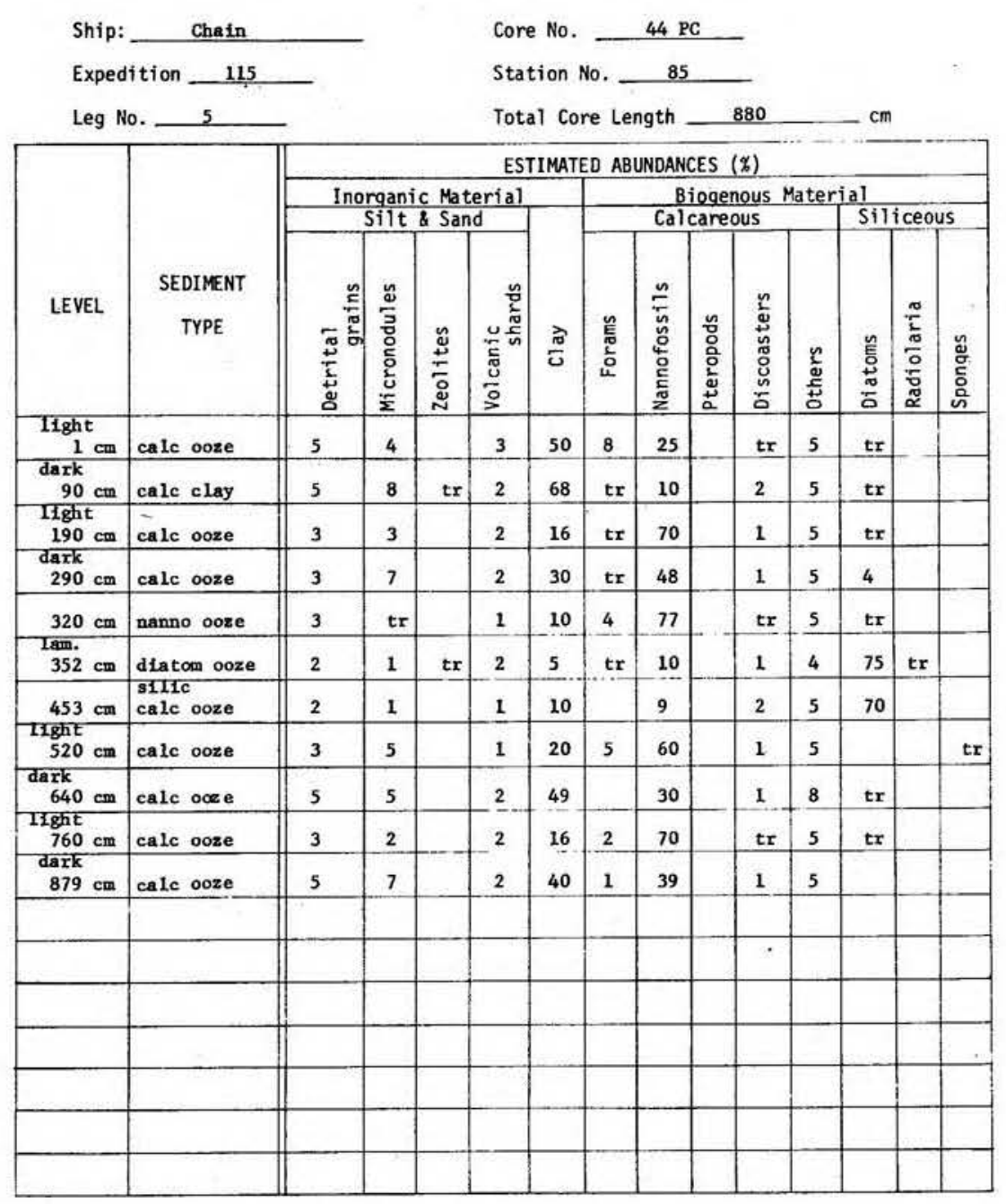

331

VISUAL CORE DESCRIPTION

Page 1 of 1

Ship CHAIN Cruise 115 Leg 5 Sta. 85 Core No. 44 PG Total Length $99 \mathrm{~cm}$. Lat. $31^{\circ} 31.49^{\prime} \mathrm{S}$ Long. $33^{\circ} 26.73^{\circ} \mathrm{W}$ Depth 4411 CoRR m Core condition EXCELLENT Date Described 11 JULY $7 S$ by $R$ M MIRR

Physiographic location Extreme Seothern Brazilien Basin

Lithologic

Detailed Description

Reispicere

ithologic

0-26 CALC OOZE

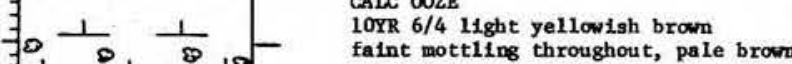

$10^{0} \frac{1}{0} 8^{2}-10$ moist silty lutite

$\exists \perp \therefore 10-26-58$

$\exists \perp \perp \perp$ CAIC OOZE

$\perp 11$ 10YR 5/4 yellowish brom

$\lim$

$100-10$

10XR $5 / 4$ yellowish brown $\mathrm{cm}$, and $50-55 \mathrm{~cm}$, common $42-50 \mathrm{~cm}$ extensive mott1

noist silty lutite

white, hortzontal streak $3 \mathrm{~cm}$ long, at $54 \mathrm{~cm}$

mottled botton contact

CALC OOZE

10YR 6/4 11ght yellowish brow

, yellowish brom, $1 \mathrm{~cm}$ wide mottle

pale brown halo molst silty lutite

$88-9$ irragular

HIGILY CALC CLAY

10YR $4 / 4$ dark yellowish brown

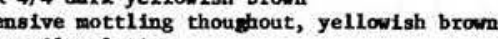

moist silty lutite

log records some washing at the top of end of core 
332

- SMEAR SLIDE DESCRIPTIONS - W.H.O.I. SEDIMENT CORES

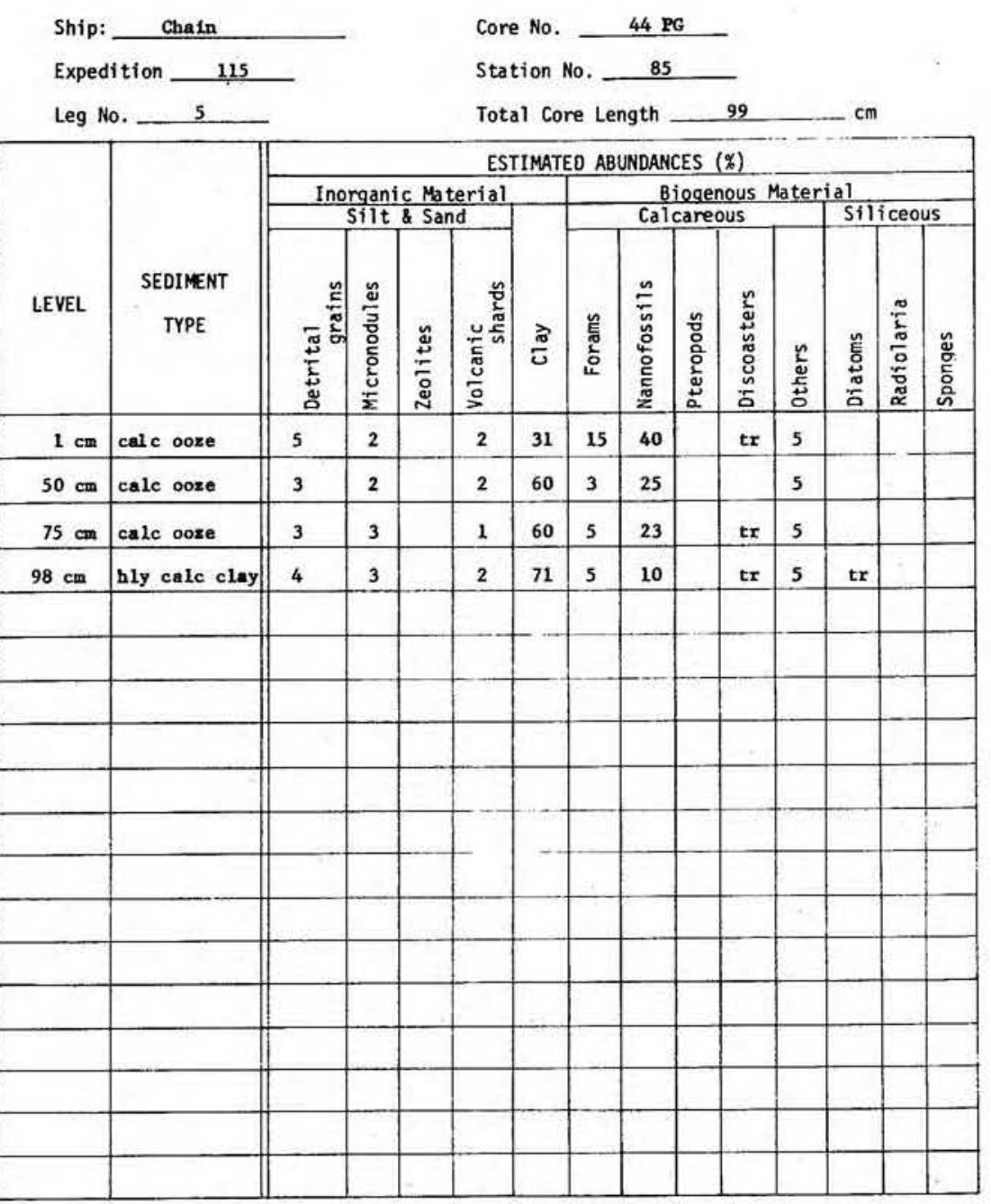

333

VISUAL CORE DESCRIPTION

Page_ 1 of 2

Ship CHAIN Cruise $115 \quad 5$ sta 86 Core No 45PC

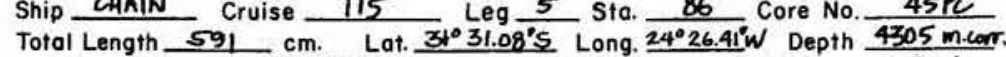

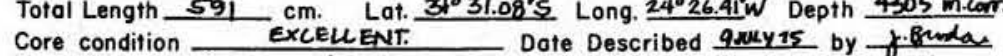
Physiographic location HUNTER CHANNEL.. EXTREME SWTHERN ORAZIL BSSW. Lithologic

Defailed Description
Ileistaine


VISUAL CORE DESCRIPTION 334

Ship CHAIN Cruise 115 Leg 5 Sto. 86 Core No. A5PC

Lithologic

Log

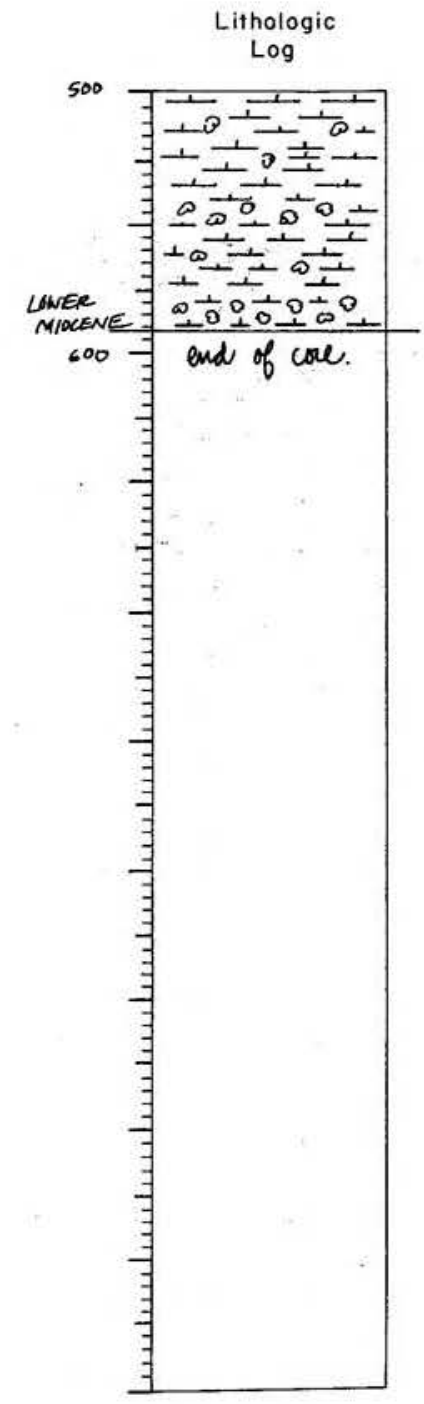

Detailed Description
335

SMEAR SLIDE DESCRIPTIONS - W.H.O.I. SEDIMENT CORES

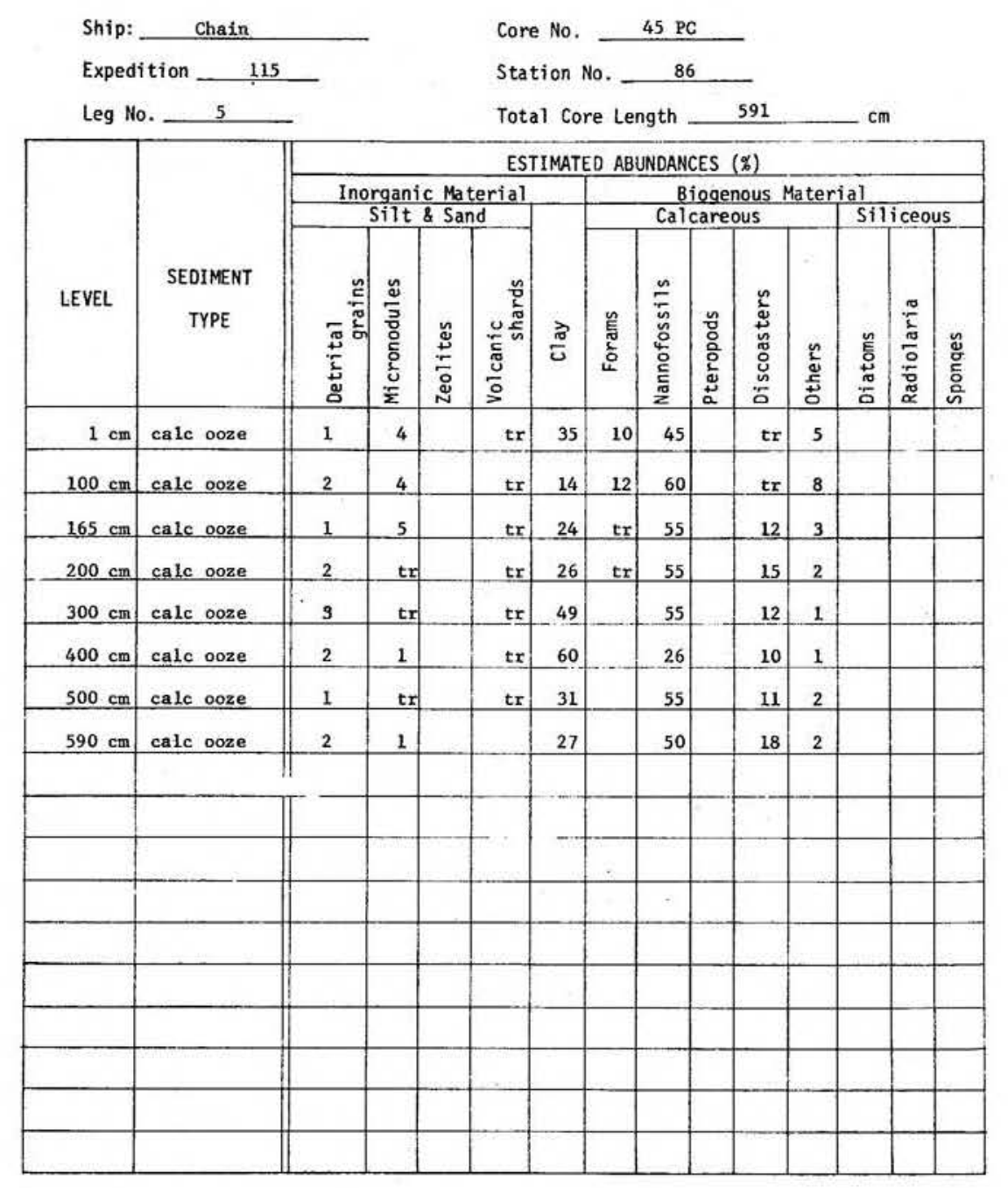


336

VISUAL CORE DESCRIPTION

Page 1 of 1

Ship CHAIN Cruise 115 Leg 5 Sta. 86 Core No. $45 \mathrm{PG}$ Total Length $152 \mathrm{~cm}$. Lat. $31^{\circ} 31.08^{\prime} \mathrm{S}$ Long. $\frac{24^{\prime} 26.4^{\prime} \mathrm{W}}{\mathrm{C}}$ Depth $4305 \mathrm{~m}$.cow

Core condition EXCELLENT Dote Described 9 JuLY 75 by $\gamma$ broda.

Physion HUNTER CHANNEL.... EXTREME SOUTHERN BRAZLL BASIN.

Lithologic

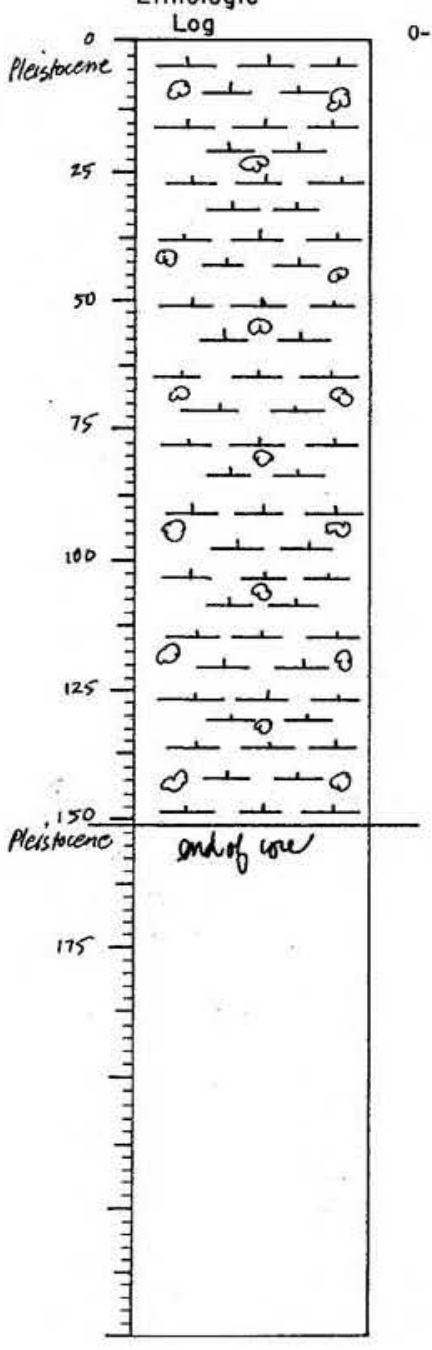

CALC 002B

10YR $6 / 3$ pale brown repeatedly grades to $5 / 3$ brown

common intermotting of the colors above with the occasional

$0-30 \mathrm{~cm}$ scattere

end of core
337

SMEAR SLIDE DESCRIPTIONS - W.H.O.I. SEDIMENT CORES

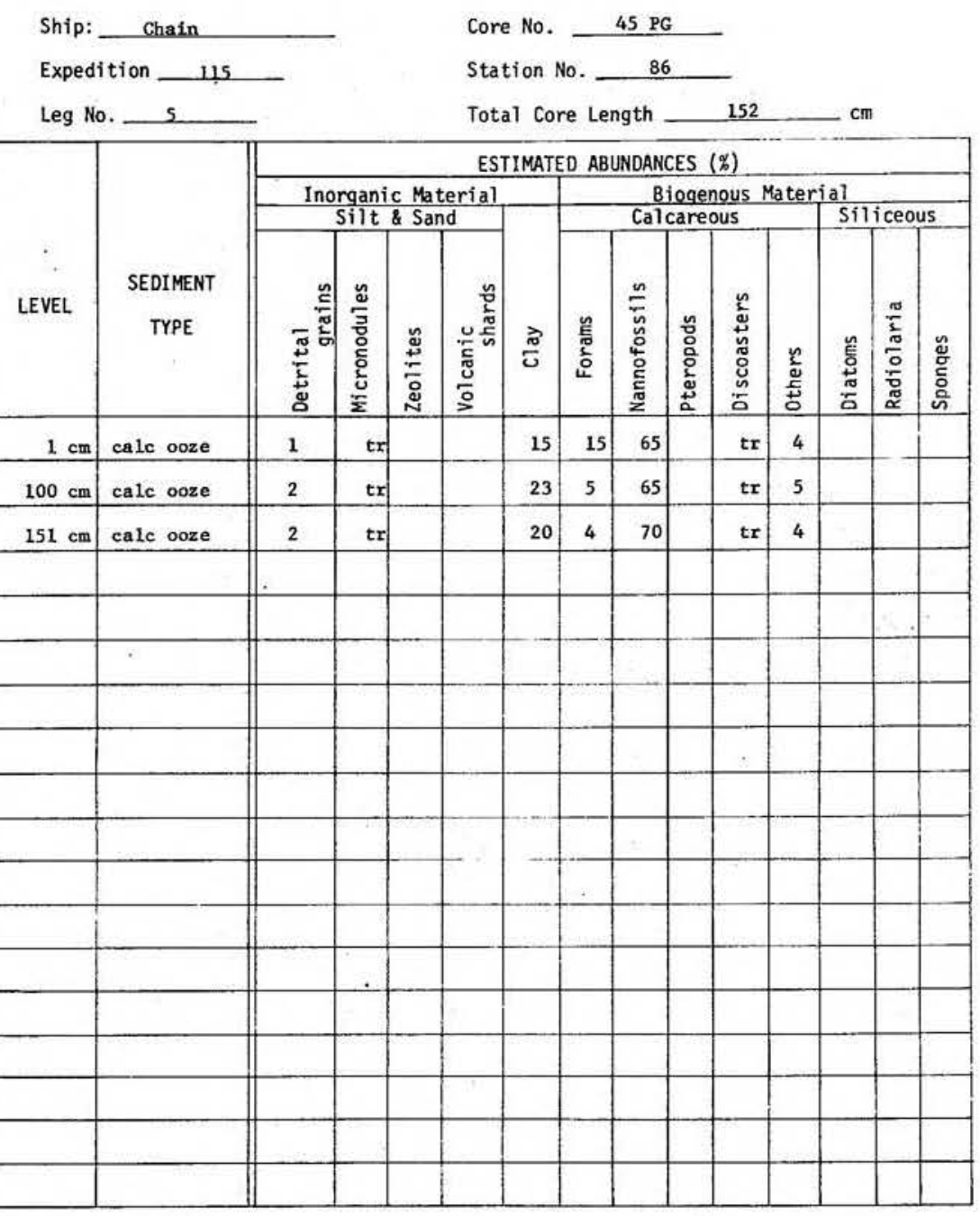


338

VISUAL CORE DESCRIPTION
Page_ 1 of 1

Ship CHAIN Cruise 115 Sore No 46 GC

Total Length $156^{\circ} \mathrm{cm}$. Lat. $29^{\circ} 53.41^{\circ} \mathrm{S}$ Long. $25^{\circ} 29.58^{\circ}$ W Depth $4833 \mathrm{CoBr} \mathrm{m}$ Core condition EXCELLENT Date Described ZJULY 7S by R MCGIBR Physiographic location Southern Brazilien Besin

Lithologic

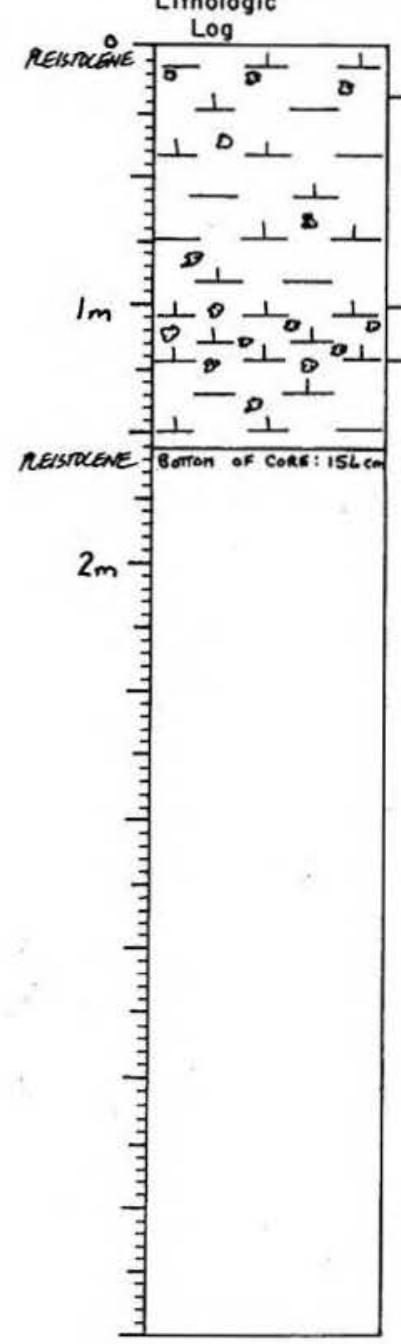

Detailed Description

$0-18$

HIGHYY CALC CLAY

OrR $4 / 4$ dark yellowlsh brown

firm slightly silty lutite

$G$ mottle
-102

HIGHLY CALC CLAY

IOYR $4 / 3$ dark brown and $4 / 4$ dark yellowish brown

oxe latermottilng or the two major colors-two mottles at

61 if $88 \mathrm{~cm}$, halo of light brown around a center of dark firown

alternations of the two major colors $S$ mottled

102-122

CAIC OOZE

10 R $5 / 3$ brown

extensive mottling throughout, dark brown and 11ght

firm slightly silty lutite

$S$ mottled

122-156

HIGaLY CALC CLAY $146 \mathrm{~cm}$

ottles at upper contact, 11ght yellowish brown; several

mottles $130-145 \mathrm{~cm}$, 11ght yellowith brown

singhtly silty lutite

end of core
1OYR $4 / 3$ dark brown becoming light yellowish brown $4 / 4$ at
339

SMEAR SLIDE DESCRIPTIONS - W.H.O.1. SEDIMENT CORES
Ship: Chain

Expedition 115

Leg No. 5

\begin{tabular}{|c|c|c|c|c|c|c|c|c|c|c|c|c|c|c|}
\hline \multirow[b]{4}{*}{ LEVEL } & \multirow[b]{4}{*}{$\begin{array}{c}\text { SEDIMENT } \\
\text { TYPE }\end{array}$} & \multicolumn{13}{|c|}{ Total Core Length $\quad 156$} \\
\hline & & \multicolumn{13}{|c|}{ ESTIMATED ABUWDANCES ( $(\boldsymbol{x})$} \\
\hline & & \multicolumn{4}{|c|}{$\begin{array}{l}\text { Inorganic Material } \\
\text { Silt \& Sand }\end{array}$} & & \multicolumn{5}{|c|}{$\begin{array}{l}\text { Biogenous Mater } \\
\text { Calcareous }\end{array}$} & \multicolumn{3}{|c|}{$\frac{\text { al }}{\text { Siliceous }}$} \\
\hline & & $\frac{5}{\frac{5}{5}}$ & 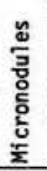 & 离 & 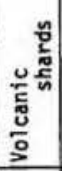 & $\frac{\pi}{\omega}$ & 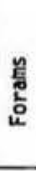 & 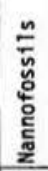 & 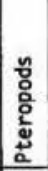 & 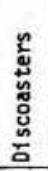 & 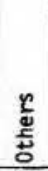 & 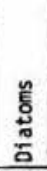 & $\frac{\frac{\pi}{i}}{\frac{\pi}{0}}$ & 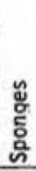 \\
\hline $1 \mathrm{~cm}$ & hly cale clay & 4 & 7 & & 2 & 58 & 5 & 20 & & tr & 3 & 1 & & \\
\hline $50 \mathrm{~cm}$ & hly cale clay & 10 & 6 & & 3 & 60 & $\mathrm{tr}$ & 16 & & tr & 5 & & & \\
\hline $110 \mathrm{~cm}$ & calc ooze & 10 & 6 & & 1 & so & 4 & 19 & & tr & 10 & & & \\
\hline \multirow[t]{2}{*}{$149 \mathrm{~cm}$} & hly cale olay & 10 & 6 & & 2 & 54 & 3 & 20 & & tr & 5 & & & \\
\hline & . & & & & & & & & & & & & & \\
\hline & & & & & & & & & & & & & & \\
\hline & & & & & & & & & & . & & & & \\
\hline & & & & & & & & & & & & & & \\
\hline & & & & & & & & & & & & & & \\
\hline & & & & & & & & & & & & & & \\
\hline & & & & & & & & & & & & & & \\
\hline & & & & & & & & & & & & & & \\
\hline & & & & & & & & & & & & & & \\
\hline & & & & & & & & & & & & & & \\
\hline & & & & & & & & & & & & & & \\
\hline & & & & & & & & & & & & & & \\
\hline & & & & & & & & & & & & & & \\
\hline
\end{tabular}


341

Ship CHAiN Cruise 115 Leg 5 Sta 88 Core No. 47 GC Total Length $105 \mathrm{~cm}$. Lat. $28^{\circ} 31.55^{\prime} \mathrm{S}$ Long. $26^{\circ} 22.27^{\prime} \omega_{\text {Depth }} 4826 \mathrm{CORR}$ Core condition EXCEUEHT Date Described 3 JuLY $7 S$ by $R$ MEGIRR Physiographic location Central Brazilian Basin

Lithologic

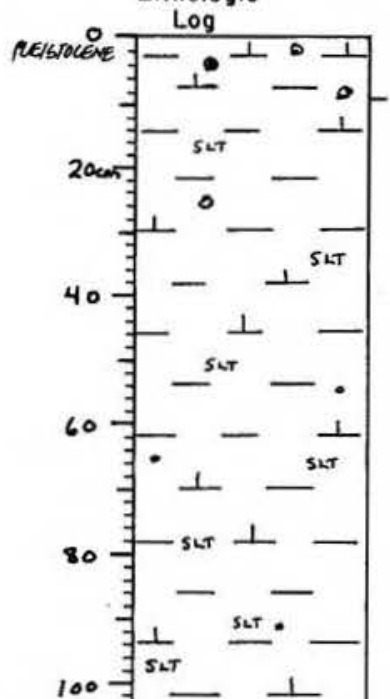

\section{Detailed Description}

\section{HIERLY CALC CIAY}

1OYR $4 / 3$ brown

cominon small mottles throughout, brown and dark brown fin lutite

$9-105$ mottled

SLICATLY CALC CLAY WITH DETRITUS

R $3 / 3$ dark brown

very small black specks scattered throughout

end of core

\section{PEISTOCOEET BOTON OF CORE:}

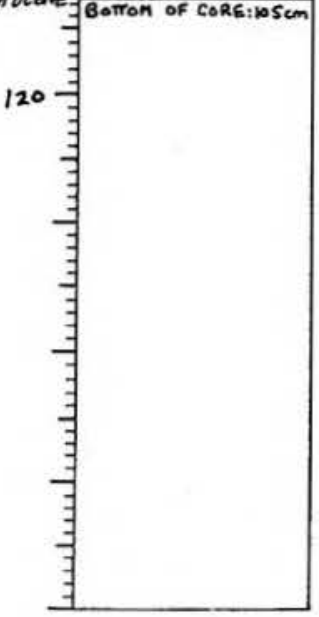

SIEAR SLIDE DESCRIPTIONS - W.H.O.I. SEDINENT CORES
Ship: Chain

Expedition 115

Leg No. 5
Core No. $47 \mathrm{GC}$

Station No. 88

Total Core Length 105

\begin{tabular}{|c|c|c|c|c|c|c|c|c|c|c|c|c|c|c|}
\hline \multirow[b]{3}{*}{ LEVEL } & \multirow[b]{3}{*}{$\begin{array}{c}\text { SEDIMENT } \\
\text { TYPE }\end{array}$} & \multicolumn{13}{|c|}{ ESTIMATED ABUNDANCES ( $(\Sigma)$} \\
\hline & & \multicolumn{4}{|c|}{$\begin{array}{c}\text { Inorganic Material } \\
\text { Silt \& Sand }\end{array}$} & \multirow[b]{2}{*}{$\frac{\pi}{6}$} & \multicolumn{5}{|c|}{$\begin{array}{l}\text { Biogenous } \\
\text { Calcareous } \\
\end{array}$} & \multicolumn{3}{|c|}{$\frac{\text { al }}{\text { Siliceous }}$} \\
\hline & & $\frac{5}{5}$ & 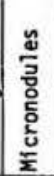 & 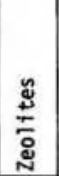 & 竎 & & 号 & 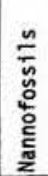 & 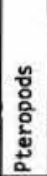 & 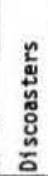 & $\begin{array}{l}\frac{r}{2} \\
\frac{5}{5}\end{array}$ & $\begin{array}{l}\tilde{\omega} \\
\frac{0}{0} \\
\bar{\sigma}\end{array}$ & $\frac{\pi}{\frac{\pi}{i}} \frac{\pi}{\frac{\pi}{0}}$ & ڤั \\
\hline $1 \mathrm{~cm}$ & hly calc clay & 5 & 10 & & 3 & 57 & tr & 20 & & tr & 5 & & & \\
\hline $30 \mathrm{~cm}$ & $\begin{array}{l}\text { S1 calc clay } \\
\text { with det }\end{array}$ & 20 & 10 & & 5 & 61 & tr & 2 & & & 2 & & & \\
\hline \multirow[t]{2}{*}{$104 \mathrm{~cm}$} & $\begin{array}{l}\text { S1 calc clay } \\
\text { with det }\end{array}$ & 20 & 12 & tr & 3 & 61 & & 2 & & & 2 & & & \\
\hline & * probably ze: & ites, & thou & exa & ct de & term & patic. & has & not & beer & made & & & \\
\hline & & & & & & & & & & & & & & \\
\hline & & & & & & & & & & & & & & \\
\hline & & & & & & & & & & . & & & & \\
\hline & & & & & & & & & & & & & & \\
\hline & & & & & & & & & & & & & & \\
\hline & & & & & & & . & & & & & & & \\
\hline & & & & & & & & & & & & & & \\
\hline & & & & & & & & & & & & & & \\
\hline & & & & & & & & & & & & & & \\
\hline & & & & & & & & & & & & & & \\
\hline & & & & & & & & & & & & & & \\
\hline & & & & & & & & & & & & & & \\
\hline & & & & & & & & & & & & & & \\
\hline
\end{tabular}


Ship CHaw Cruise 15 Leg 5 Sto. 89 Core No. $48 \mathrm{PC}$ Total Length $891 \quad \mathrm{~cm}$. Lat. $26^{\circ} 51.18^{\prime} \mathrm{S}$ Long. $27^{\circ} 23^{\circ} 44^{\prime} \omega$ Depth $\frac{5933 \operatorname{coks} \mathrm{m}}{2}$ Core condition EXEELENT Date Described S JULY 7S by R M.GiRe Physiographic location Central Brazilian Basin

Lithologic

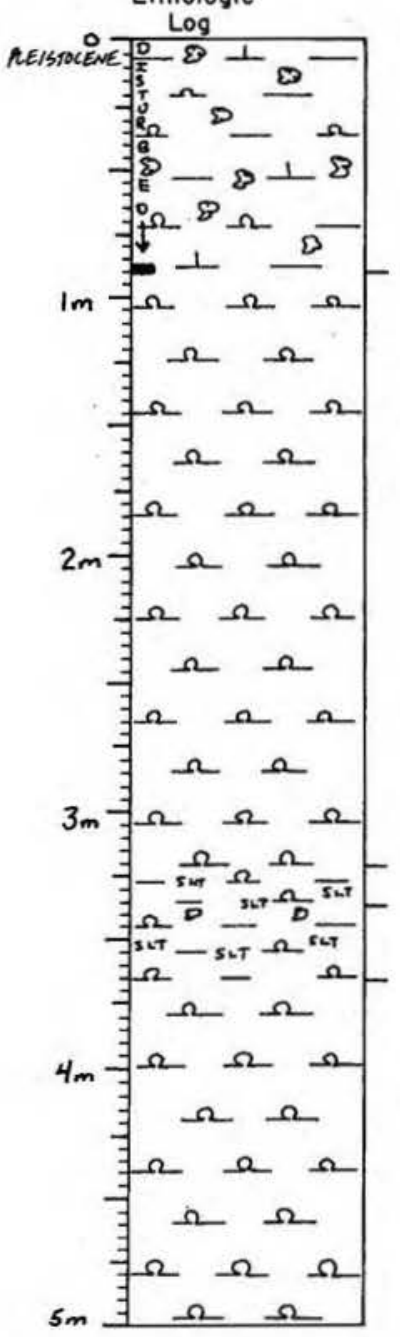

sorz, Detailed Description

The piston corer tripped while the corer was being lovered at speed 2, at fourXthe usual lowering speed. The effect of this may be manifested in the unusual appearance of the uppermost unit of this core. Because of th high speed of
entry, the sediment surface was most probably "blown of $\mathrm{f}^{\prime \prime}$ $-90$ and lost.

SLIGHTLY CAIC CIAY AND HIGHLY SILIC CLAY

10YR $6 / 4$ 11ght yellowish brown and $3 / 3$ dark brow extensive intermottling of the 2 major colors

slightly silty lutite
severely contorted intermixture of the 2 major colors; dsturbed looking unit, a very sharp, horizontal contact colors at $49 \mathrm{~cm}$

$90-32$ S Irregular

DIATOM OOZB

major colors are: $10 \mathrm{YR} 8 / 3$ and $7 / 3$ very pale brown, $8 / 1$ white, and $5 / 2$ grayish brown
sowewhat clumpy, spongy silic mulch

sowewhat clumpy, spongy silic mulch
unit is predominantly brown and grayish brown, 90-130 ca; over this interval the two colors are internottled $-130-320 \mathrm{~cm}$, all major colors plus similar shades are together, usually occuring in $1-2 \mathrm{~cm}$ thick layers, and convex upwards, several gray patches occur, $90-130,166-171,177,276,297-$
$303-1 \mathrm{~cm}$ wide drying crack at base of unit 5 irregular

320-336

SIIIC CLAX WITH DETRTTUS

$2.5 Y 6 / 2$ light brownish gray

very faint mottling at lower contact

G

SILIC CLAY WIMH DETRITUS

1OYR 6/4 1ight yellowish brown

scattered mottling at upper contact

the sequence of multi-colored, laminated unit to light gray

malt to light brown wat ao repeats in texture are noticeable

365-625 625

major colors are: 10 YR $6 / 3$ pale brown, $8 / 3$ very pale brown, $8 / 1$ white, and $6 / 1$ light gray somewhat clumpy spongy, silic mulc

VISUAL CORE DESCRIPTION 343

Page 2 of 2 .

Ship_CHAIN Cruise 115 Leg 5 Sta. 89 Core No. 48 PC

Lithologic

Log

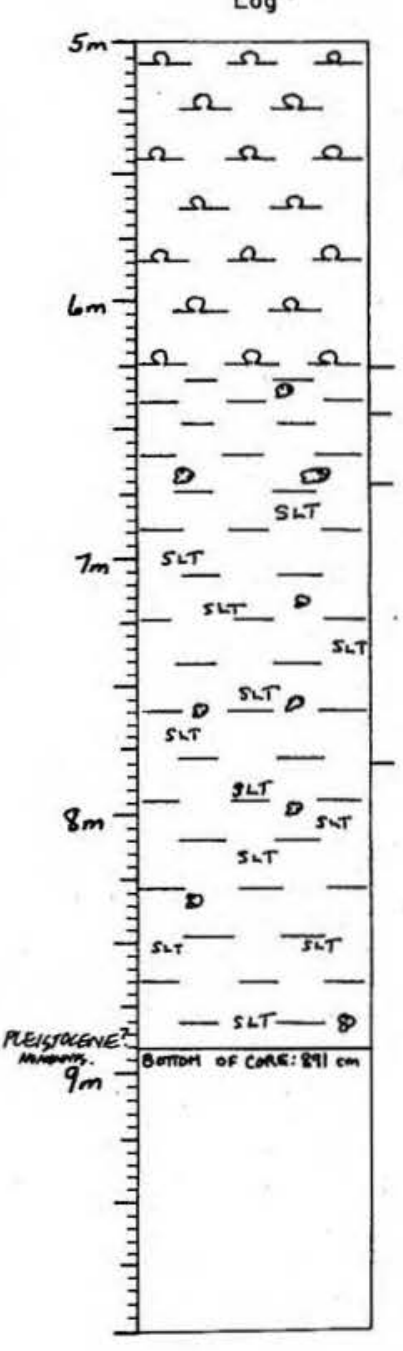

\section{Detailed Description}

charcteristics very similar to those of the multicolored unit observed further up core. This unit is composed primarily 年 gray imations in this unit.

625-644 irregular

UNPOSS CIAY

$2.5 \times 6 / 2$ 11ght brownish grey

faint mottling 625-634 cm, dark gray

firm lutite

$1 \mathrm{~cm}$ thick co

44-670

UNFOSS CLAY

10YR 6/4 11ght yellowish brow

several very large inclusions of lover unit, $664-670 \mathrm{~cm}$

same sequence of silic mulch, gray unit, then brown unit, s irregular

670-779

UNPOSS CTAY UITH DETRATUS

$10 \mathrm{YR} 4 / 3$ brown

scattered, somenhat hary mottles throughout, 1ight brown calmon mottling 760-764 cm dark brow

firm sllty lutite

779-891

UNFOSS CLAY WTII BETRTTUS

10YR $3 / 3$ dark brown

occasional mottles throughout 11ght brom, black specks

scattered throughout

B75-891 cy core cutte

end of core 
344

SIEAR SLIDE DESCRIPTIONS - W.H.O.1. SEDIMENT CORES

Ship: Chain

Expedition 115

Leg No. 5

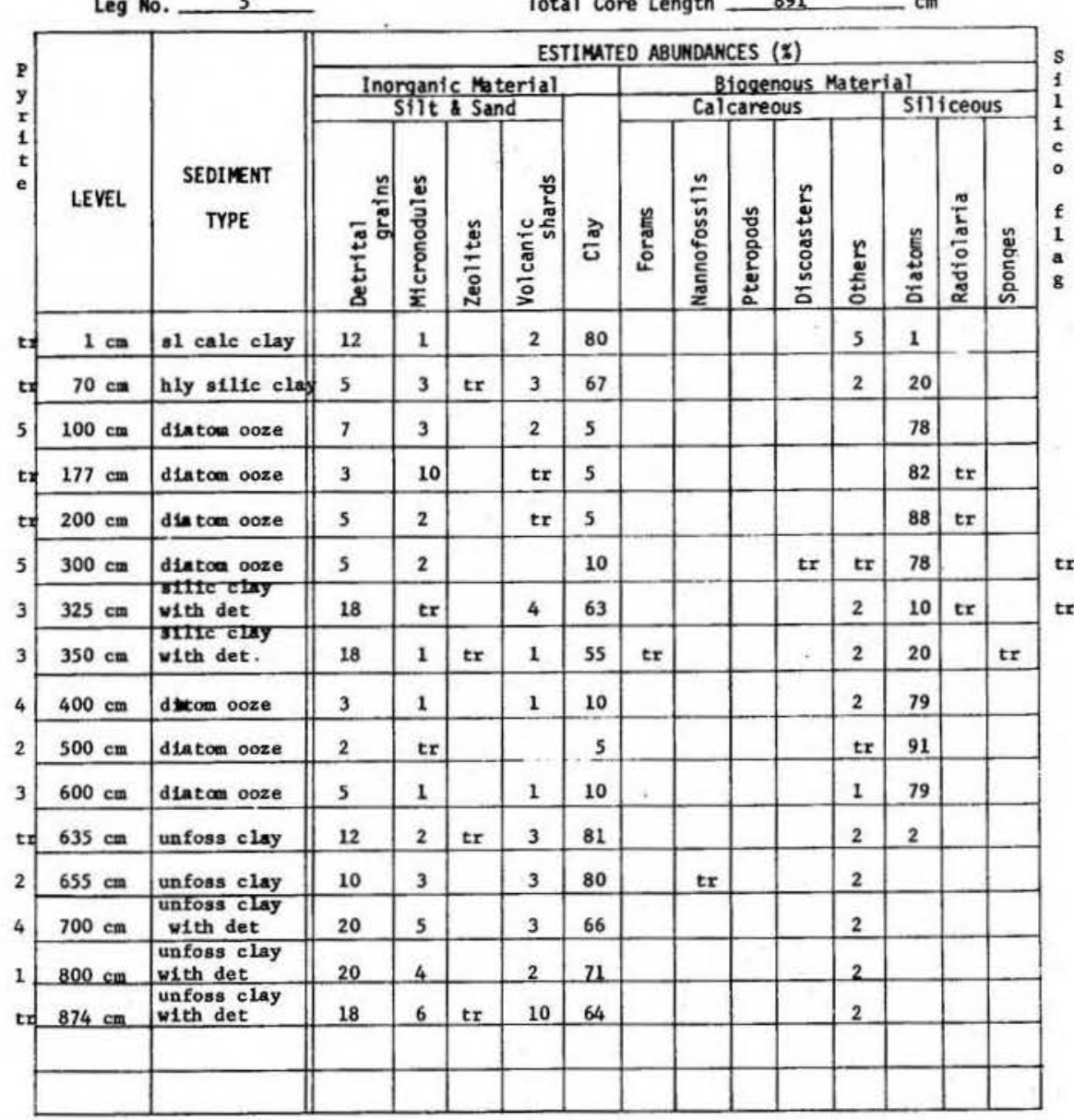

345

VISUAL CORE DESCRIPTION

Page 1 of 1

Ship CHAIN Cruise 115 Lea 5 sto 89 Core No. 48 PG Total Length $158 \mathrm{~cm}$. Lat. $22^{\circ} 51.18^{\prime} \mathrm{S}$ Long. $27^{\circ} 2344^{\prime}$ W Depth $5933 \mathrm{CaR} R \mathrm{~m}$

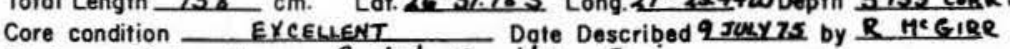
Physiographic location Central Brazilian Basin

Lithologic

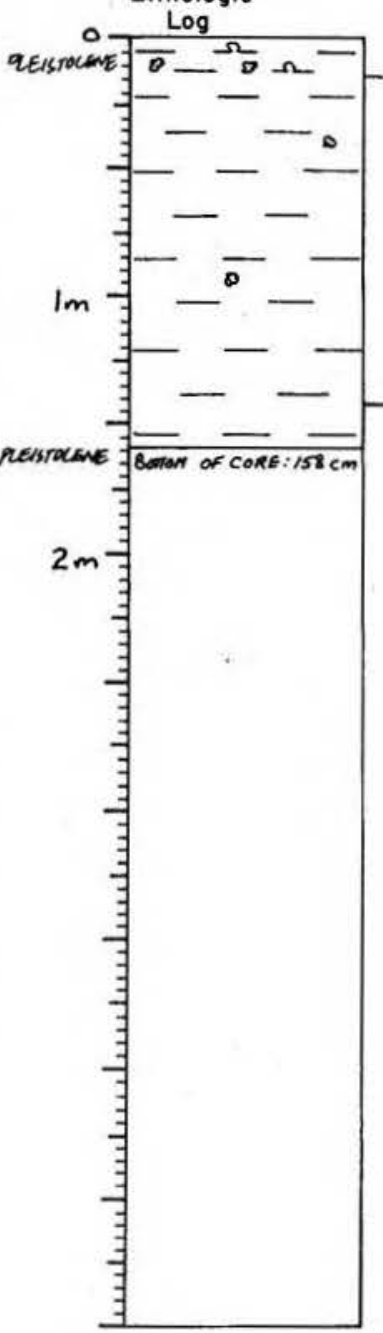

NoTE,

\section{Detailed Description}

2, rather than the usual 1 1. There is some possibility of disturbance in the upper

SLIGanX SILIC cLXX

1OYR $3 / 3$ dark brown
mottling at $8-11 \mathrm{~cm}, 11 \mathrm{ght}$ brow

moist, slightly silty lutite

thin, interrupted wavy lamination, 11ght brovu at $2 \mathrm{~cm}$ 3-142

\section{UNPOSS CIAY}

1 NOR $4 / 3$ brow

few mottles throughout, 1ight brown

moist, slightly silty lutite

$S$ inclined $5^{\circ}$

UNFOSS CLAY

(OYR $3 / 3$ derk brow

very faint mottlog, liaht bmin

poist, slightly silty lutite 0-13 part of the pilot gravity core. 
346

SIEAR SLIDE DESCRIPTIONS - W.H.O.I. SEDIMENT CORES

Ship: _Chain

Leg No. 5
Expedition 145

Core No. $48 \mathrm{PG}$

Station No. 89

Total Core Length $\quad 158 \quad \mathrm{~cm}$

\section{ESTIMATED ABUNDANCES (\%)}

\begin{tabular}{|c|c|c|c|c|c|c|c|c|c|c|c|c|c|c|}
\hline \multirow[b]{3}{*}{ LEVEL } & \multirow[b]{3}{*}{$\begin{array}{c}\text { SEDIMENT } \\
\text { TYPE }\end{array}$} & \multicolumn{13}{|c|}{ ESTIMATED ABUNDANCES (\%) } \\
\hline & & \multicolumn{4}{|c|}{$\begin{array}{c}\text { Inorganic Material } \\
\text { Silt \& Sand }\end{array}$} & & \multicolumn{5}{|c|}{$\begin{array}{l}\text { Biogenous Mater } \\
\text { Calcareous }\end{array}$} & \multicolumn{3}{|c|}{ Siliceous } \\
\hline & & $\frac{\tilde{5}}{5}$ & 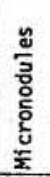 & $\frac{ \pm}{\frac{ \pm}{5}}$ & 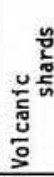 & $\frac{\vec{\pi}}{0}$ & $\begin{array}{l}\text { 崖 } \\
\text { 它 }\end{array}$ & 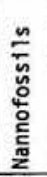 & 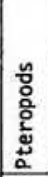 & 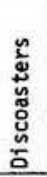 & $\begin{array}{l}n \\
a \\
\text { ș } \\
0 \\
0\end{array}$ & 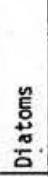 & $\begin{array}{l}\frac{\pi}{L} \\
\frac{\pi}{0} \\
\frac{0}{0} \\
\tilde{\pi} \\
\end{array}$ & 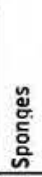 \\
\hline $1 \mathrm{~cm}$ & $\begin{array}{l}\text { 81 } \\
\text { s11ic clay }\end{array}$ & 8 & 6 & tr & 2 & 82 & & tx & & & $\operatorname{tr}$ & 2 & & \\
\hline $100 \mathrm{~cm}$ & unfoss clay & 13 & 8 & $\operatorname{tr}$ & 2 & 77 & tr & tr & tr & & $\mathrm{tr}$ & & & \\
\hline $152 \mathrm{~cm}$ & unfoss clay & 12 & 4 & tr & 2 & 82 & & tr & & & $\operatorname{tr}$ & tr & & \\
\hline & & & & & & & & & & & & & & \\
\hline & & & & & & & & & & & & & & \\
\hline & & & & & & & & & & & & & & \\
\hline & & & & & & & & & & & & & & \\
\hline & & & & & & & & & & . & & & & \\
\hline & & & & & & & & & & & & & & \\
\hline & & & & & & & & & & & & & & \\
\hline & & & & & & & & & & & & & & \\
\hline & & & & & & & & & & & & & & \\
\hline & & & & & & & & & & & & & & \\
\hline & & & & & & & & & & & & & & \\
\hline & & & & & & & & & & & & & & \\
\hline & & & & & & & & & & & & & & \\
\hline & & & & & & & & & & & & & & \\
\hline & & & & & & & & & & & & & & \\
\hline
\end{tabular}

347

VISUAL CORE DESCRIPTION

Page 1 of 1

Ship CHain Cruise $115 \quad$ Le 5 Sto 90 Core No $49 \mathrm{GC}$

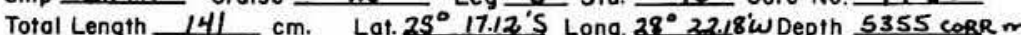
Core condition EXCE世ENT Date Described 3 JULY 75 by $R$ MCGIRR Physiographic location_Central Brazilian Besin

Lithologic

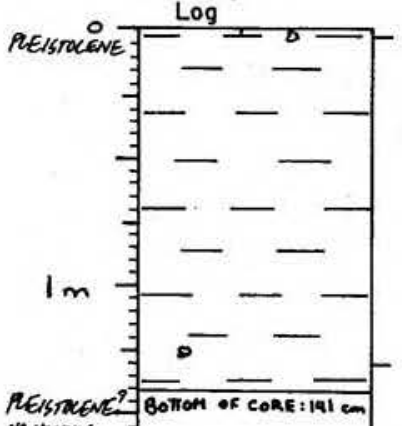

No manowos.

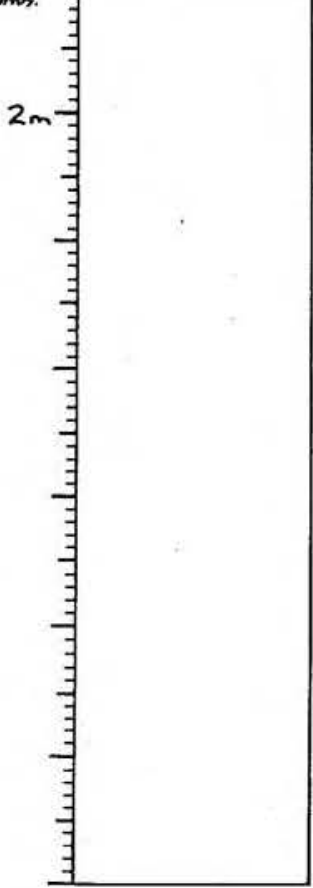

Detailed Description

CALC CLAY

IOYR $6 / 3$ pale brown

one mottle

moist lutite

$2-130$ hrzontal

UNFOSS CLAY

alo intermitcantly thin patches of pale brown lutite

along liner edge, scmewhat lnclined, $3 \mathrm{~cm}$ wide Layer of

palili $\mathrm{cm}$ late with black streaks ruming thru layers,

pale bm, large elongated gray motte at $9 \mathrm{~cm}$, small.

130-141

UNFOSS CLAX

10YR $4 / 3$ dark brown

large pale brown blotch 129-131 cm

manll pale brad

brown blotches $130-136 \mathrm{~cm}$

end of core 
Ship CHaiN Cruise 115 Leg 5 Sto. 91 Core No. 50 PC Total Length $173 \mathrm{~cm}$. Lat. $24^{\circ} 12.79^{\prime} \mathrm{S}$ Long. $29^{\circ} 06^{\circ} 37^{\circ}$ W Depth 5460 CeRr m Core condition EXCFLLENT, THe' QISTNRAEODate Described 2JeaY 7S by $R$ MCGIRR Physiogrophic location Ceatral Brazilian Basin

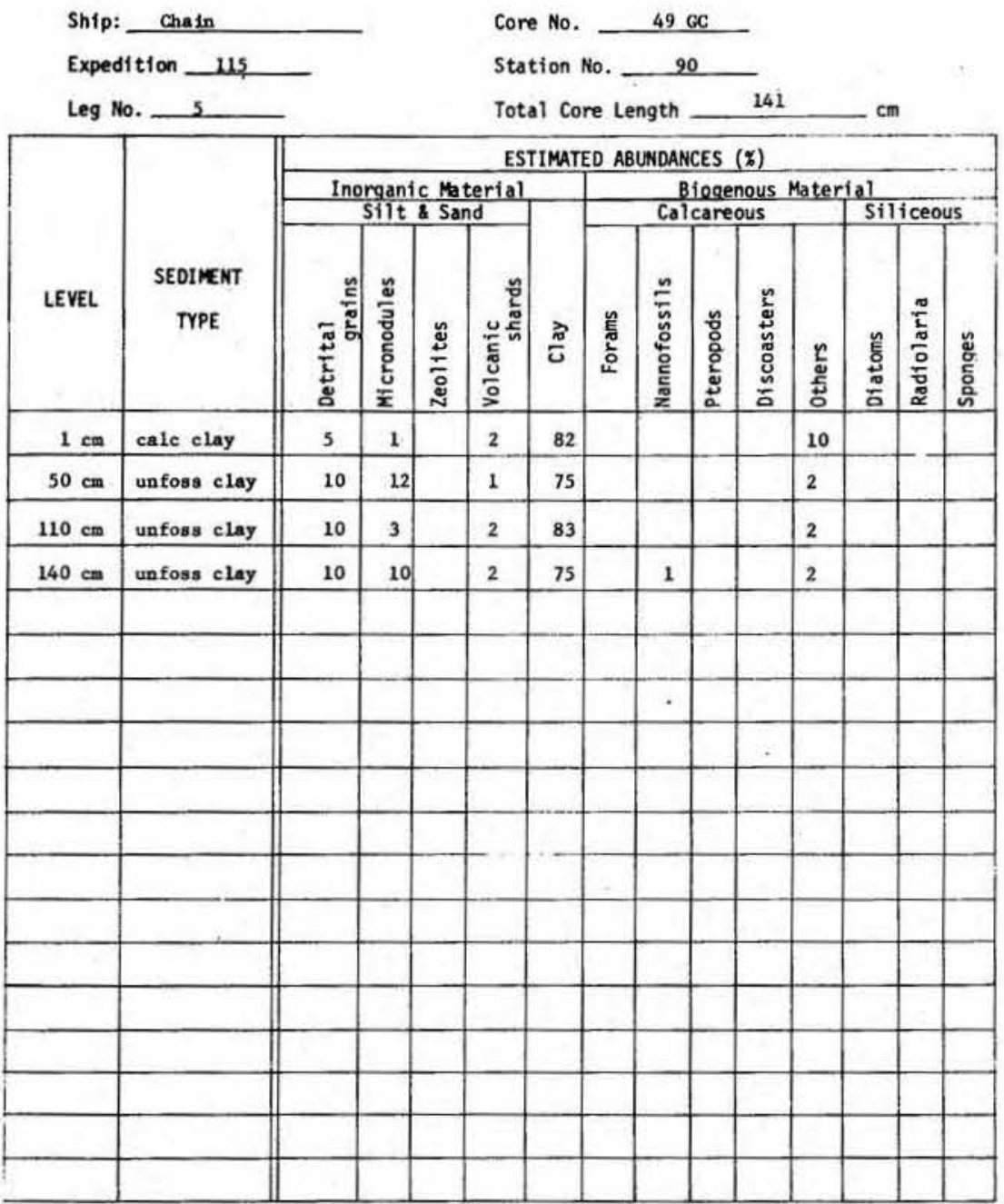

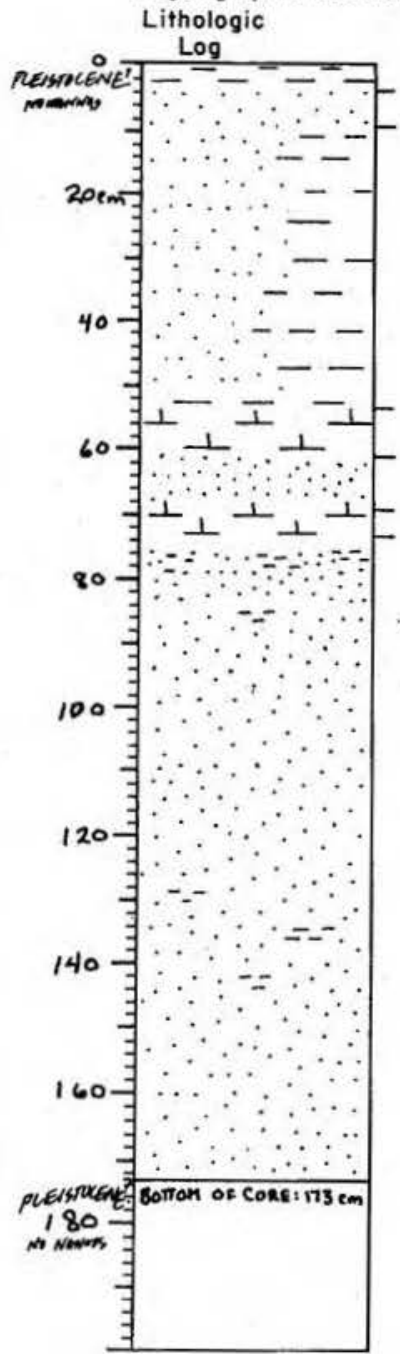

\section{Detailed Description}

Severe problems were encountered during the coring operation for core so PC. Notes from the shipboard log read i piston core held for 6 minutes, about 1 minute after that point was 5-10 feet. barrel.. sample on hand extruded froe widdle barrel...suspect flow-in in lover bent pipe, not extruded". The core appears to be disturbed. Two very different
1ithologic units, an abysal lutite and a detrital send ifthologic units, an abyssal lutite and a detrital sand, been highly altered, and that the sand units in particular are severely washed. There is some indication of graded bedding in the sand over the length of the entire core. The macroscopic description that follows was done with the above considerations in mind.

UNFOSS CLAY

common swirls from $2-4 \mathrm{~cm} 11 \mathrm{ght}$ brown molst lutite some vashed sand along very edge of unit 4-9 DETrerat sime

\section{DETRETAL SAND}

fine and pale brown $s$ irregular

$9-53$

DETRITAL SAND AND UNPOSS CIAY 1OrR $6 / 3$ pale brown (sand) and $3 / 3$ dark brown (Iutite) sand and lutite

litite 53-61 lutite 18 along one side and and atsd along the other

CAIC OOZE

2.5Y 7/2 11ght gray

extensive mottling throughout, dark brow moist lutite

61-69

DETRITAL SAND

10YR $6 / 3$ pale brown

fine and medutm sand

somewhat washed along liner at lower contact

69-73

CALC OOZE

$2.5 Y 6 / 2$ Light brownish gray

several small mottles, dark brown and 11ght brown 
350

VISUAL CORE DESCRIPTION

Page 2 of 2

Ship_CHN Cruise 115 Leg -5 Sta. 91 Core No. 50 PC

Lithologic

Log

Detailed Description

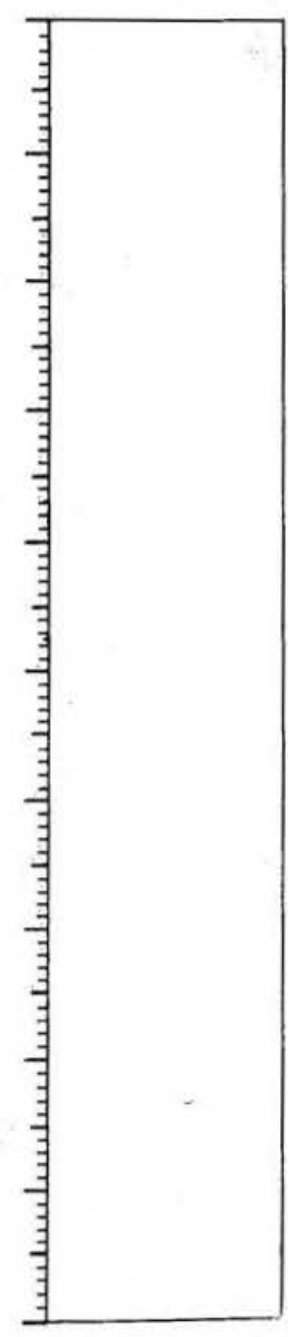

lutite

very hard, compact nodule of lutite $(2 \times 3 \times 3 \mathrm{~cm})$ at $73 \mathrm{~cm}$ $S$ irregular
-173

DETRITAL SAND AND CLUMPS OF UNPOSS CIAY $10 \mathrm{YR} 6 / 3$ pale brown

two patches of included lutite blobs, which do not extend to the liner and appear to be artificially emplaced here; $73-$ $85,126-146 \mathrm{~cm}$, appears to be a general size gradation over the length of the unit
351

SMEAR SLIDE DESCRIPTIONS - W.H.O.I. SEDIMENT CORES

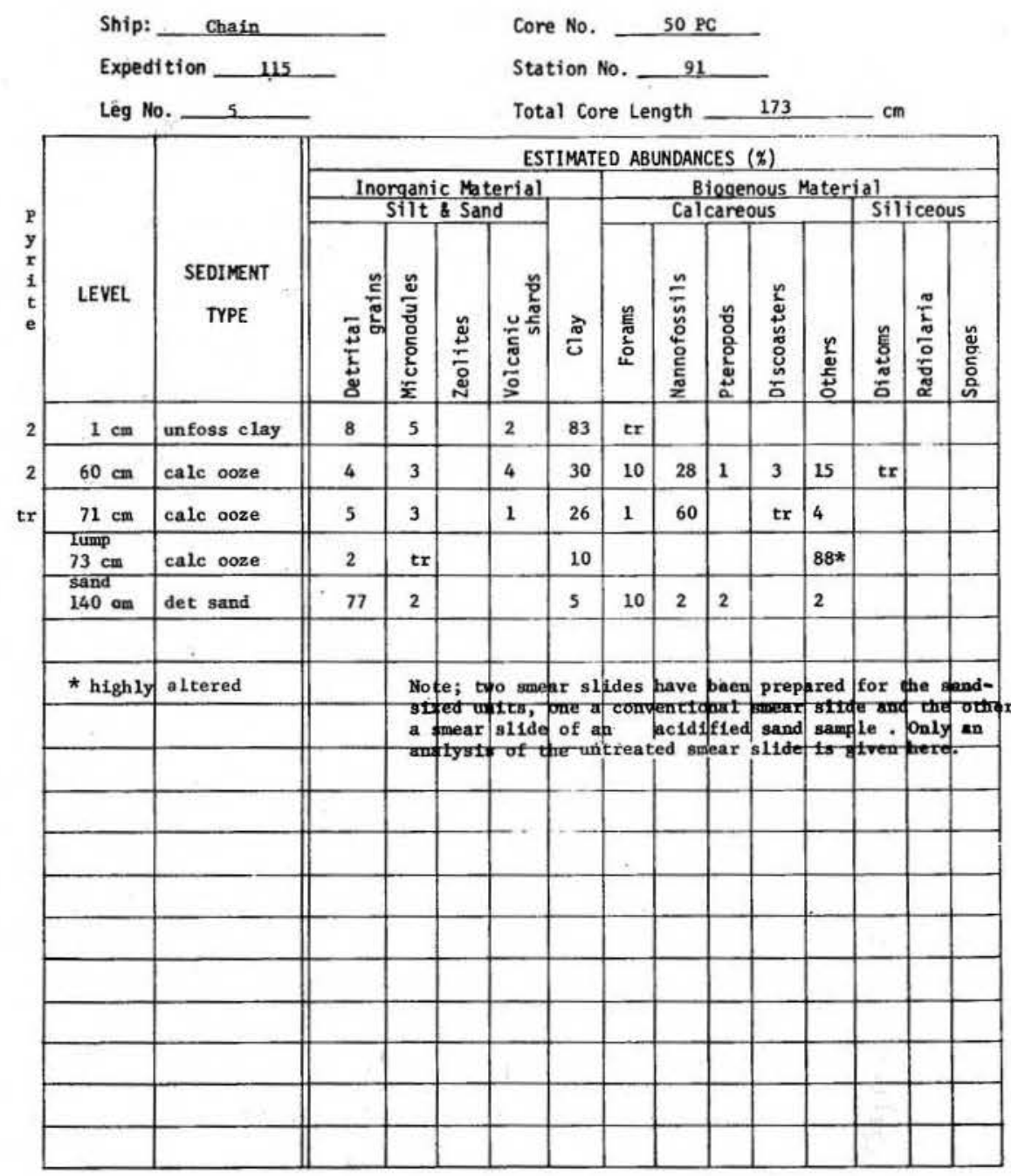


Ship CHAIN Cruise 115 Leg -5 Sto. 92 Core No. S1 GC

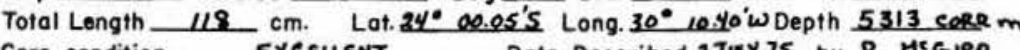
Core condition EXCSMENT Date Described 2JWy 75 by $R$ MEGIRR

Physiographic location Central Brazilian Basio

Lithologic

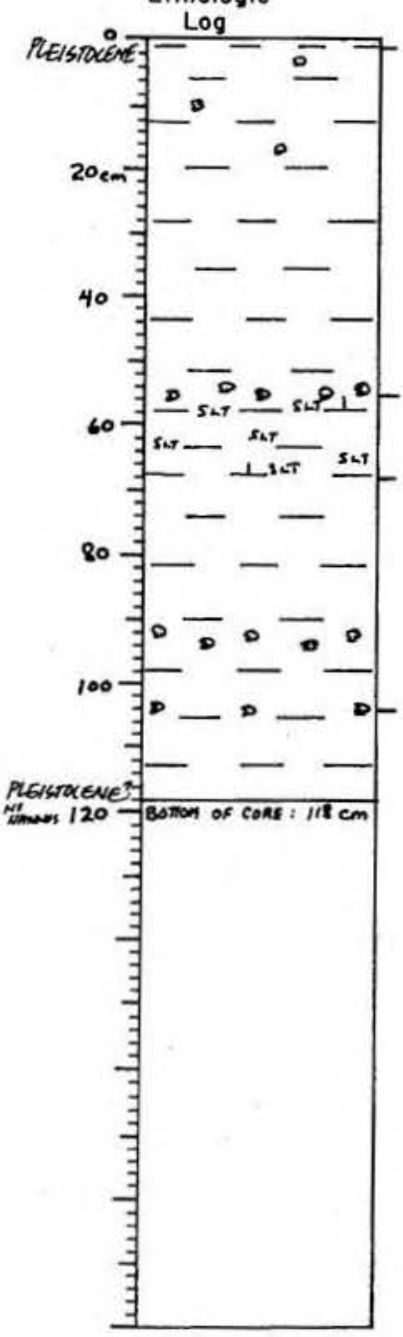

$0-$

Detailed Description

SLIGHTLY CALC-SILIC CLAY

faint black horleotal atreaking throughos irregular

$1-55$

UNPOSS CLAY

lack smeared mottles, $1-15 \mathrm{~cm}$

15-55 vertical, ameared lineations, 11ght brownish gray indication of disturbance

55-63 interval 50-60 cm

STICIIY CALC CLAY/DETRLTTS

mertact, dark brow

thin black microlaminations $60-64 \mathrm{~cm}$

8-104

UNPOSS CIAY

10YR $5 / 3$ brown

xtengive mottling 90-96 cm 11ght brownish gray, mottled at lower contace

mevhat vertical, smeared lineation, 11ght brounish gray $68-91 \mathrm{~cm}$, possible indication of disturbance

S irregular, mottled

4-118

INPOSS CLAY

end of core
SIEAR SLIDE DESCRIPTIONS - W.H.O.I. SEDIMENT CORES

Core No. $51 \propto$

Station No. 92

Total Core Length 118

\begin{tabular}{|c|c|c|c|c|c|c|c|c|c|c|c|c|c|c|}
\hline \multirow[b]{4}{*}{ LEVEL } & \multirow[b]{4}{*}{$\begin{array}{c}\text { SEDIMENT } \\
\text { TYPE }\end{array}$} & \multicolumn{13}{|c|}{ ESTIMATED ABUWDANCES $(x)$} \\
\hline & & \multirow{2}{*}{\multicolumn{4}{|c|}{$\begin{array}{c}\text { Inorganic Material } \\
\text { Silt \& Sand }\end{array}$}} & \multirow[b]{3}{*}{$\frac{\pi}{6}$} & \multirow{2}{*}{\multicolumn{5}{|c|}{$\begin{array}{l}\text { Biegenous Mater } \\
\text { Calcareous }\end{array}$}} & & & \\
\hline & & & & & & & & & & & & \multicolumn{3}{|c|}{ Siliceous } \\
\hline & & $\frac{n}{5}$ & 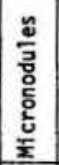 & ڤั & 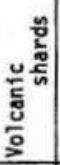 & & 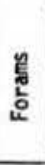 & 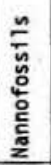 & 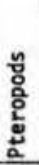 & 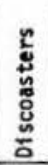 & 密 & 兽 & $\frac{\frac{\pi}{i}}{\frac{\pi}{0}}$ & $\begin{array}{l}\text { ू․ } \\
\text { 응 }\end{array}$ \\
\hline $1 \mathrm{~cm}$ & $\begin{array}{l}\text { slightly cal } \\
\text { silic_slay }\end{array}$ & 8 & 3 & & 4. & 83 & & 1 & & & tr & tx & & 1 \\
\hline $40 \mathrm{~cm}$ & unfoss clay & 10 & 10 & & 3 & 77 & & & & & & & & \\
\hline $58 \mathrm{~cm}$ & $\begin{array}{l}\text { S1 calc clay } \\
\text { det }\end{array}$ & $55 *$ & 4 & & 2 & 36 & & 3 & & & & & & $\mathrm{t}$ \\
\hline $80 \mathrm{~cm}$ & unfoss clay & 5 & 12 & & 2 & 81 & & & & & & tr & & $\mathrm{tr}$ \\
\hline \multirow[t]{3}{*}{$112 \mathrm{~cm}$} & unfoss clay & 8 & 10 & & 2 & 80 & & & & & & & & \\
\hline & . & & & & & & & & & & & & & \\
\hline & * most graing & have so & ne 5 & pe o & conet & ing & & & & & & & & \\
\hline & & & & & & & & & & . & & & & \\
\hline & & & & & & & & & & & & & & \\
\hline & & & & & & & & & & & & & & \\
\hline & & & & & & & & & & & & & & \\
\hline & & & & & & & & & & & & & & \\
\hline & & & & & & & & & & & & & & \\
\hline & & & & & & & & & & & & & & \\
\hline & & & & & & & & & & & & & & \\
\hline & & & & & & & & & & & & & & \\
\hline & & & & & & & & & & & & & & \\
\hline & & & & & & & & & & & & & & \\
\hline
\end{tabular}

Ship: Chain

Expedition 115

Expegtion 
354

VISUAL CORE DESCRIPTION
Page 1 of 1
355

SIEAR SLIDE DESCRIPTIONS - W.H.O.I. SEDIMENT CORES

Ship CHN Cruise 115 Leg 5 sta. 93 Core No. $52 \mathrm{GC}$

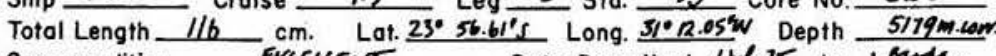

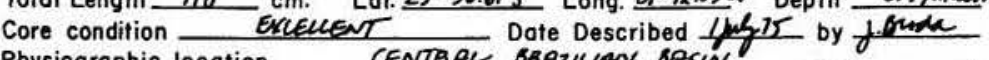
Physiogrophic location

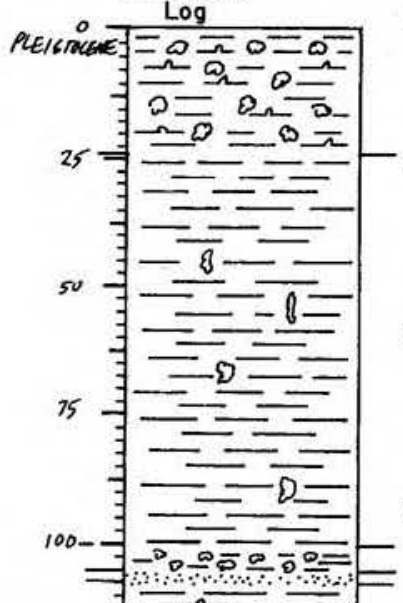

0-24 Detailed Description

SLIGHTIY SILIC CLAY

extengive pale brown and dark brountoh gray mottling

moist slick lutite

24-100

UNFOSS CLAY

odd vertically oriented pale brow streaking elongated

throughout unit moist slick lutit

100-105

UNFOSS CLAY

(OYR $6 / 3$ pale brown

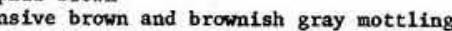

molst slick lutite

105-107

DETRITUS

OYR $5 / 2$ grayish brown

tiff compact silty lutite

REISTOCONE- $\frac{\text { endof core }}{107-116}$

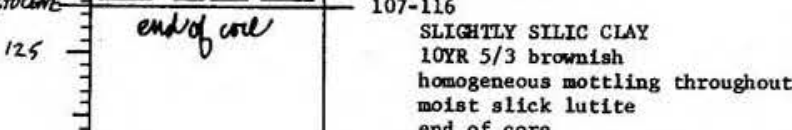

end of core
Ship: Chain

Expedition 115

Leg No. 5

\begin{tabular}{|c|c|c|c|c|c|c|c|c|c|c|c|c|c|c|c|}
\hline \multicolumn{2}{|l|}{ s. } & \multicolumn{14}{|c|}{ Fore Leng } \\
\hline \multirow[b]{3}{*}{ LEVEL } & \multirow[b]{3}{*}{$\begin{array}{c}\text { SEDIMENT } \\
\text { TYPE }\end{array}$} & \multicolumn{14}{|c|}{ ESTIMATED ABUNDANCES (q) } \\
\hline & & \multicolumn{4}{|c|}{$\begin{array}{c}\text { Inorganic Material } \\
\text { Silt \& Sand } \\
\end{array}$} & \multirow[b]{2}{*}{$\frac{\pi}{5}$} & & \multicolumn{5}{|c|}{$\begin{array}{l}\text { Biogenous Mater } \\
\text { Calcareous }\end{array}$} & \multicolumn{2}{|c|}{ Siliceous } & \\
\hline & & $\begin{array}{l}5 \\
\frac{5}{5} \\
\text { a } \\
\end{array}$ & 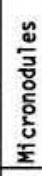 & ڤั & : & & & 峞 & 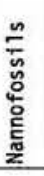 & 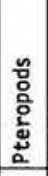 & 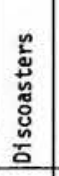 & 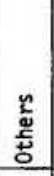 & 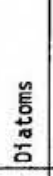 & $\begin{array}{l}\frac{\pi}{1} \\
\frac{\pi}{\pi} \\
\frac{0}{\tilde{J}} \\
\tilde{\alpha} \\
\end{array}$ & 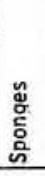 \\
\hline $1 \mathrm{~cm}$ & sl silic clas & 12 & 3 & & 2 & & & & tx & & & & $\operatorname{tr}$ & & 2 \\
\hline $60 \mathrm{~cm}$ & unfoss clay & 4 & 2 & & t & & & & & & & & $t x$ & & tr \\
\hline $106 \mathrm{~cm}$ & detritus & 83 & 2 & & & & & & & & & $\operatorname{tr}$ & $\operatorname{tr}$ & & tr \\
\hline \multirow[t]{3}{*}{$115 \mathrm{~cm}$} & sl silic clat & 3 & 3 & & 1 & & & & & & & & $\mathrm{tr}$ & & 1 \\
\hline & & & & & & & & & & & & & & & \\
\hline & . & & & & & & & & & & & & & & \\
\hline & & & & & & & & & & & & & & & \\
\hline & & & & & & & & & & & . & & & & \\
\hline & & & & & & & & & & & & & & & \\
\hline & & & & & & & & & & & & & & & \\
\hline & & & & & & & & . & & & & & $=$ & & \\
\hline & & & & & & & & & & & & & & & \\
\hline & & & & & & & & & & & & & & & \\
\hline & & & & & & & & & & & & & & & \\
\hline & & & & & & & & & & & & & & & \\
\hline & & & & & & & & & & & & & & & \\
\hline & & & & & & & & & & & & & & & \\
\hline & & & & & & & & & & & & & & & \\
\hline
\end{tabular}

Core No. $52 \mathrm{GC}$

Station No. 94

Total Core Length

(1)

STIMATED ABUNDANCES $(\boldsymbol{x})$ 
356

VISUAL CORE DESCRIPTION
Page 1 of 1

Ship $\mathrm{CHN}_{\mathrm{HW}}$ Cruise 115 Leg 5 Sta. 94 Core No. $53 \mathrm{GC}$

Totol Length $117 \mathrm{~cm}$. Lat. 23049.03'S Long. 33013.50 W Depth 4757 CoRam

Core condition ExcEULANT Dote Described 1 July 75 by $B$ MeGiRR

Physiographic location_Central Brazilian Basin

Lithologic

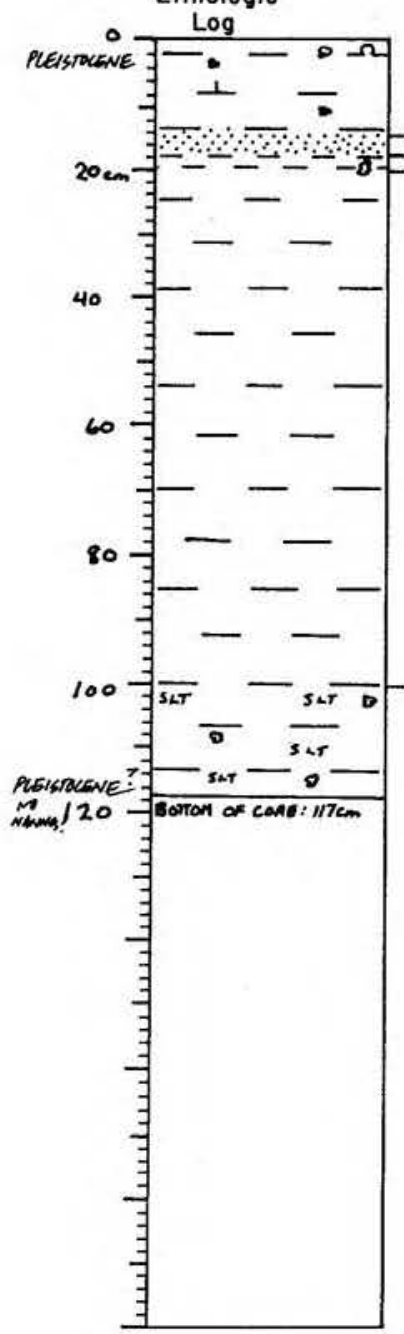

\section{0-14 Detailed Description}

SLIGHIXY SILIC CALC CLAY

compon motting brown

one small sand mottle at $12 \mathrm{~cm}$

14-17 1rxegular

DETRITUS

$2.5 Y 5 / 2$ grayish brown

medium and 1 rregular

17-20

UNFOSS CIAY

$2.5 Y 6 / 2$ 11ght brownish gray

grayish brown, one large elongated the extends thry entire unit and into unit below, dark graylsh brown

mottled, over $3 \mathrm{~cm}$ interva

20-100

\section{UNPOSS CLAY}

lishtly dark brown

numerous thin, black, fuzzy almost horizontal laminations throughout 100-117

\section{UNPOSS CLAY WITH DETRITUS}

1 OYR $5 / 3$ brown

coumon small mottles throughout, 11ght brow

tutite

$105-106 \mathrm{~cm}$, patches with numerous black specks slightly silty lutite
357

SHEAR SLIDE DESCRIPTIONS - W.H.O.I. SEDIMENT CORES

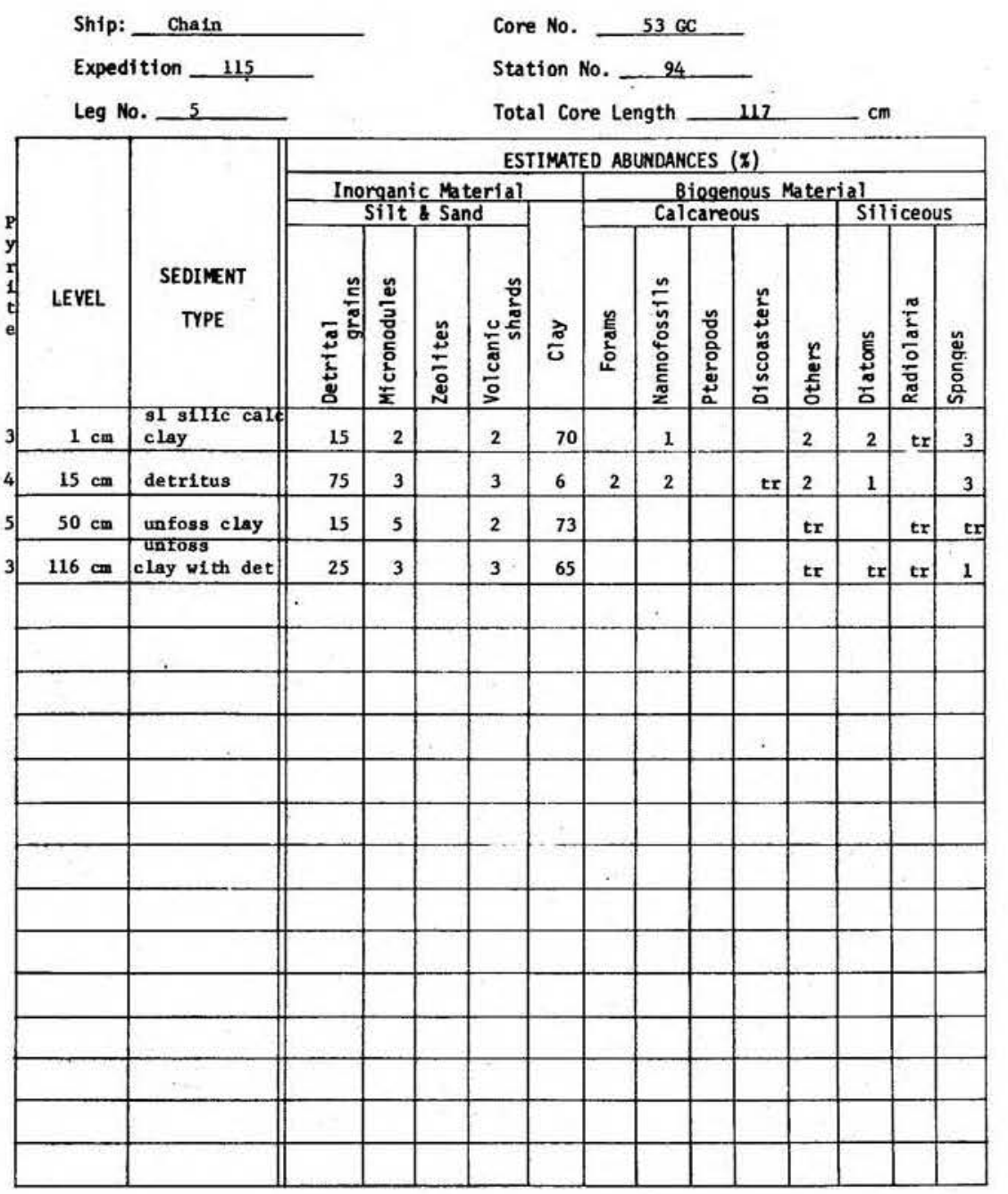


Ship CHN Cruise 115 Leg 5 Sta. 95 Core No. 54 aC Total Length $118 \mathrm{~cm}$. Lat. $23^{\circ} 39.17^{\prime} \mathrm{s}$ Long.35 $35^{\circ} 13.55^{\prime} \mathrm{w}$ Depth 4153 carr. Core condition Rxcellent Date Described 11 Nov 75 by HFarmer Physiographic location Continental Rise ofe Rio de JANEIRo

Lithologic

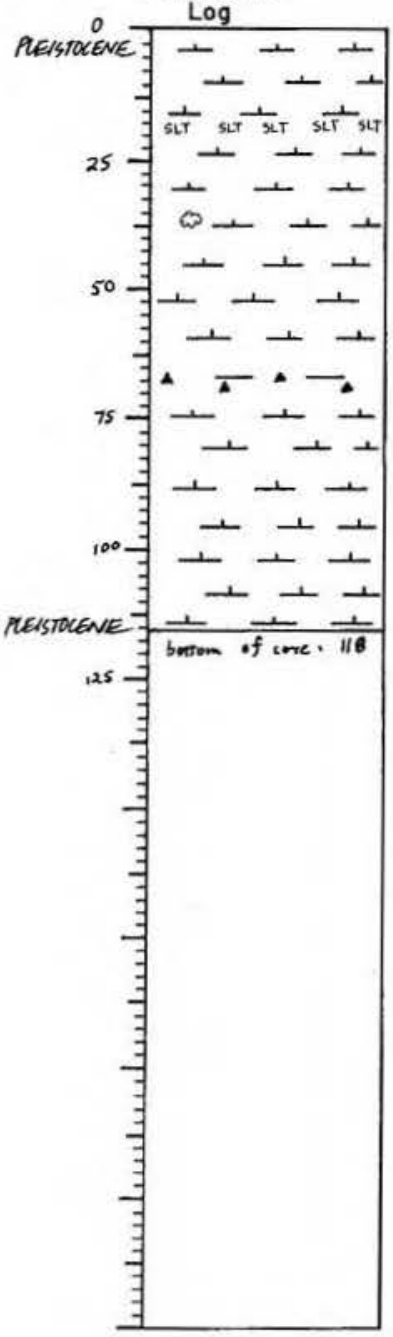

Detailed Description

$0-118$

CALC OOZE WITH LAMINATIONS OF PYRITE-RICH UNFOSSILI-

Pr $5 / 3$ brom $(0-17 \mathrm{~cm}), 10 \mathrm{YR} 6 / 3$ pale broun at $20 \mathrm{~cm}$ and back to pale brown by $\sim 90 \mathrm{~cm}$

$2-3$ min dark brown discontin.ous streaks cormon $(0-5 \mathrm{~cm})$; they are similar to laminse below and were probably disturbed as core entered sediment: $7 \mathrm{~cm}$ small dark brown clast of slick lutite: thin streaks, subtle from 20-45 em, below this, only the laminations are present, varying from $<1$ an to $5 \mathrm{~cm}(66-71 \mathrm{~cm})$ and irregularly spaced; laminations are 10 YR $4 / 2$, dark grayish brown slick lutite

whole core is a silck lutite with very few forams excep $17-20 \mathrm{~cm}$; dark brown ( $10 \mathrm{Tk} 4 / 3$ ) silt with sharp botnd of core
SMEAR SLIDE OESCRIPTIONS - W.H.O.I. SEDIMENT CORES
Ship:

Expedition

Leg No.
Core No. $\quad 54 \mathrm{GC}$

Station No. 95

Total Core Length $118 \quad \mathrm{~cm}$

\begin{tabular}{|c|c|c|c|c|c|c|c|c|c|c|c|c|c|c|c|c|}
\hline \multirow[b]{3}{*}{ LEVEL. } & \multirow[b]{3}{*}{$\begin{array}{c}\text { SEDIMENT } \\
\text { TYPE }\end{array}$} & \multicolumn{15}{|c|}{ ESTIMATED ABUNDANCES (\%) } \\
\hline & & \multicolumn{6}{|c|}{$\begin{array}{l}\text { Inorganic Material } \\
\text { Silt \& Sand }\end{array}$} & \multicolumn{6}{|c|}{$\begin{array}{l}\text { Biogenous Mater } \\
\text { Calcareous }\end{array}$} & \multicolumn{3}{|c|}{$\frac{\text { ial }}{\text { siliceous }}$} \\
\hline & & 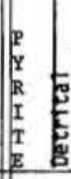 & ำำ & 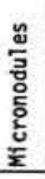 & ฏ & $\frac{5}{2}$ & the & & 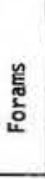 & 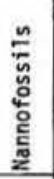 & 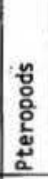 & 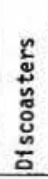 & 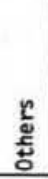 & 产 & $\begin{array}{l}\frac{\pi}{5} \\
\frac{\pi}{0} \\
\frac{0}{0} \\
0 \\
0 \\
\alpha\end{array}$ & 亗 \\
\hline 1 & calc ooze & 1 & 3 & tr & & 2 & 20 & & $\mathrm{tr}$ & 69 & & tr & 3 & tr & 1 & 2 \\
\hline 68 & unfoss. clay & 15 & & & & 1 & 84 & & & & & & & tr & tr & tr \\
\hline 116 & calc ooze & tx & 3 & & & .1 & 50 & & & 44 & & tr & 2 & tr & tr & \\
\hline & & . & & & & & & & & & & & & & & \\
\hline & & & & & & & & & & & & & & & & \\
\hline & & & & & & & & & & : & & & & & & \\
\hline & & & & & & & + & & & & & & & & & \\
\hline & & & & & s: & & & & & & & & & & & \\
\hline & & & & & & & & & & & & & & & & \\
\hline & & & & & & & & & & & & & & & & \\
\hline & & & & & & & & & & & & & & & & \\
\hline & & & & & & & & & & & & & & & & \\
\hline & & & & & & & & & & & & & & & & \\
\hline & & & & & & & & & & & & & & & & \\
\hline b & & & & & & & & & & & & & & & & \\
\hline & & & & & & & & & & & & & & & & \\
\hline & & & & & & & & & & & & & & & & \\
\hline & & & & & & & & & & & & & & & & \\
\hline
\end{tabular}


360

VISUAL CORE DESCRIPTION
Page__ of _ 1

Ship CHAIN Cruise 115 Leg 5 Sta. 96 Core No. S56C

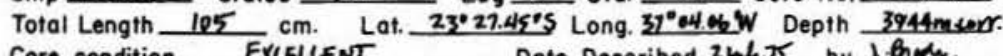

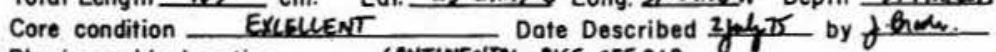
Physiographic location

Lithologic CONTINENTAL RISE OFF RIO

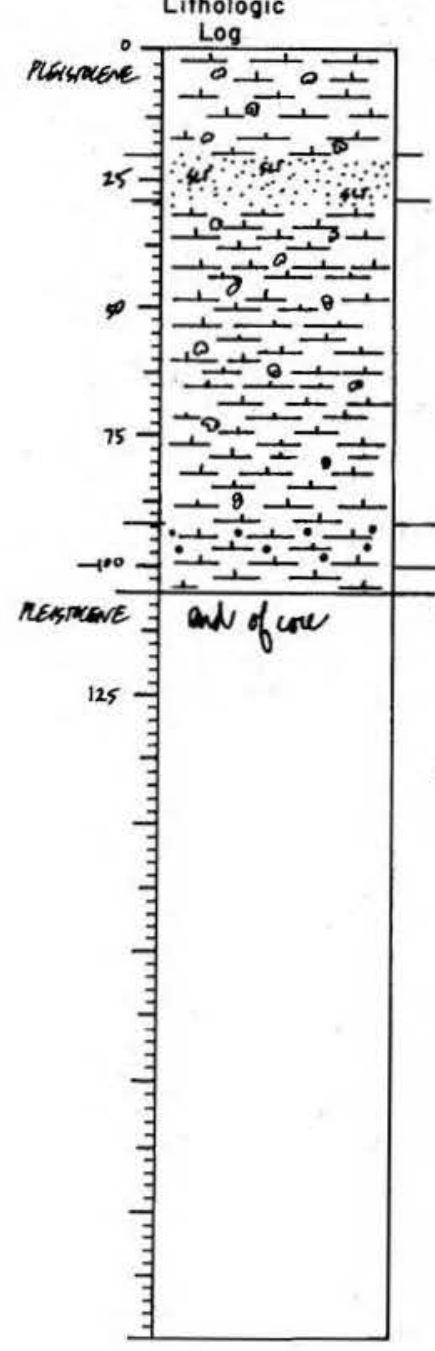

$0-20$ CALC DOZB

10YR $6 / 411$ ght yellowish brown grades to $6 / 3$ pale bown coxmon small light brown and bromaish gray mottling throughout molst slightly silty lutite with forams scattered irregularly throughout 5
S text

DETRITUS

10YR $5 / 2$ gmyish brown

roughout; one thick $1 \mathrm{~cm}$ uide dark

thates unit at $24 \mathrm{~cm}$ 29-92 inclined 10

CALC OOZE

10YR $6 / 2$ 11ght brownish gray grades to $6 / 3$ pale brown coomon faint 11ght yellowish brown and very pale brown

moist very slightly silty lutite with a feu forams scattered $s$ throughout

CALC OOZE WITH Th MICRO HODULBS

10YR $4 / 1$ dark 8 Tay

very abund. thin and thick laminations throughout, additional hues pale brown and light gray

moist silghlty silty lutite

100-105

CAIC OOZE

IOYR $6 / 2$ brownish gray

a fes faint gray hazy micro laminae throughout end of core
361

SMEAR SLIDE DESCRIPTIONS - W.H.O.I. SEDIIENT CORES

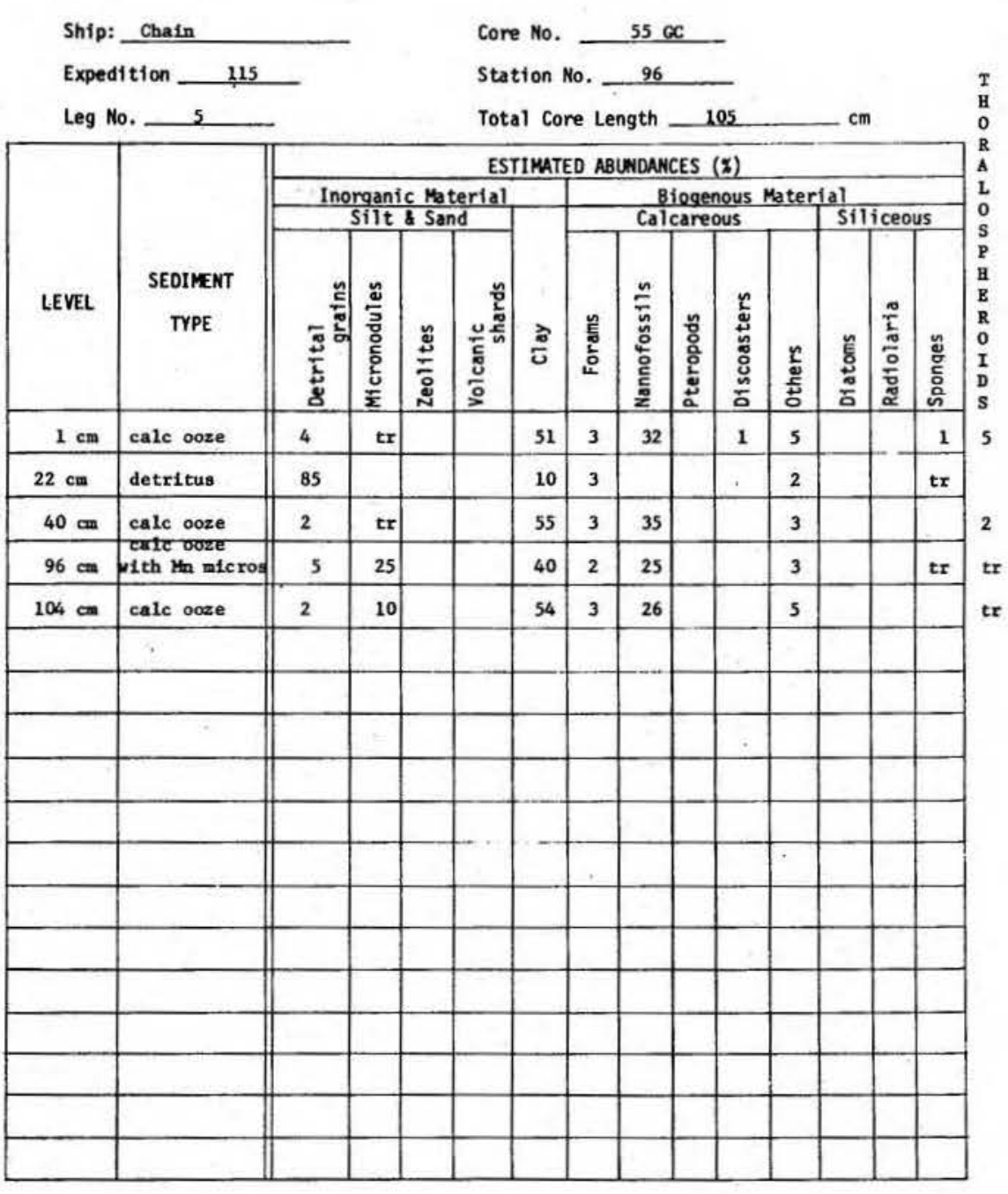

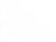

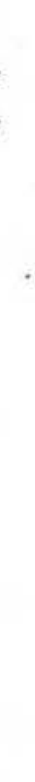


362

VISUAL CORE DESCRIPTION

Page 1 of 1

Ship CHAIN Cruise 115 Leg 5 Sta. 98 Core No. $57 \mathrm{GC}$ Total Length 105 cm. Lat. $23^{\circ}$ o5. 8 's Long. $40^{\circ}$ oq.11'W Depth $2595 \mathrm{CaRRm}$ Core condition EXCELLENT Date Described 243UNE ZS by R MEGIRB Physiographic location Comtinental Slope off Rio de Janeiro Lithologic

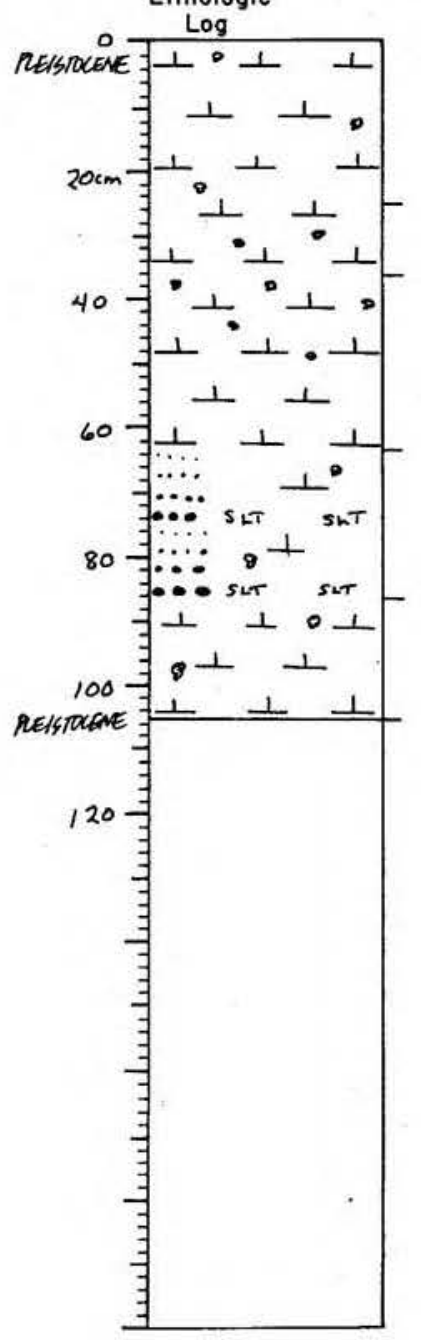

\section{Detailed Description}

CALC OOZE

$2.5 Y 6 / 2$ 11ght brownish gray

scattered small mottles throughout, gray

several small patches and layers of high-foram concentrations

$25-36$

CALC OOZE

$7.5 Y R 5 / 4$ brown

common small mottles throughout, 11ght brown

several small pockets of high-foram concentration $G$
$6-63$

6-63

CAIC OOZB

5 Y $6 / 3$ pale ollive

$36-50 \mathrm{~cm}$, extensive sma11, black mottling

several approximately horizontal fuzzy orange laminations, $S$ horizontal

63-86

CALC OOZE GRADES TO DETRITUS

$5 Y 5 / 2$ olive gray

occastona black specks throughout

63-74, 74-86 cm, sandy layer from $85-86$

86-105

CALC OOZE

$5 Y 6 / 1$ gray

specks throughout

two $1 \mathrm{~cm}$ wide, brown laminations, inclined $40^{\circ}$ at $90,94 \mathrm{~cm}$ end of core
363

SMEAR SLIDE DESCRIPTIONS - W.H.O.I. SEDIMENT CORES

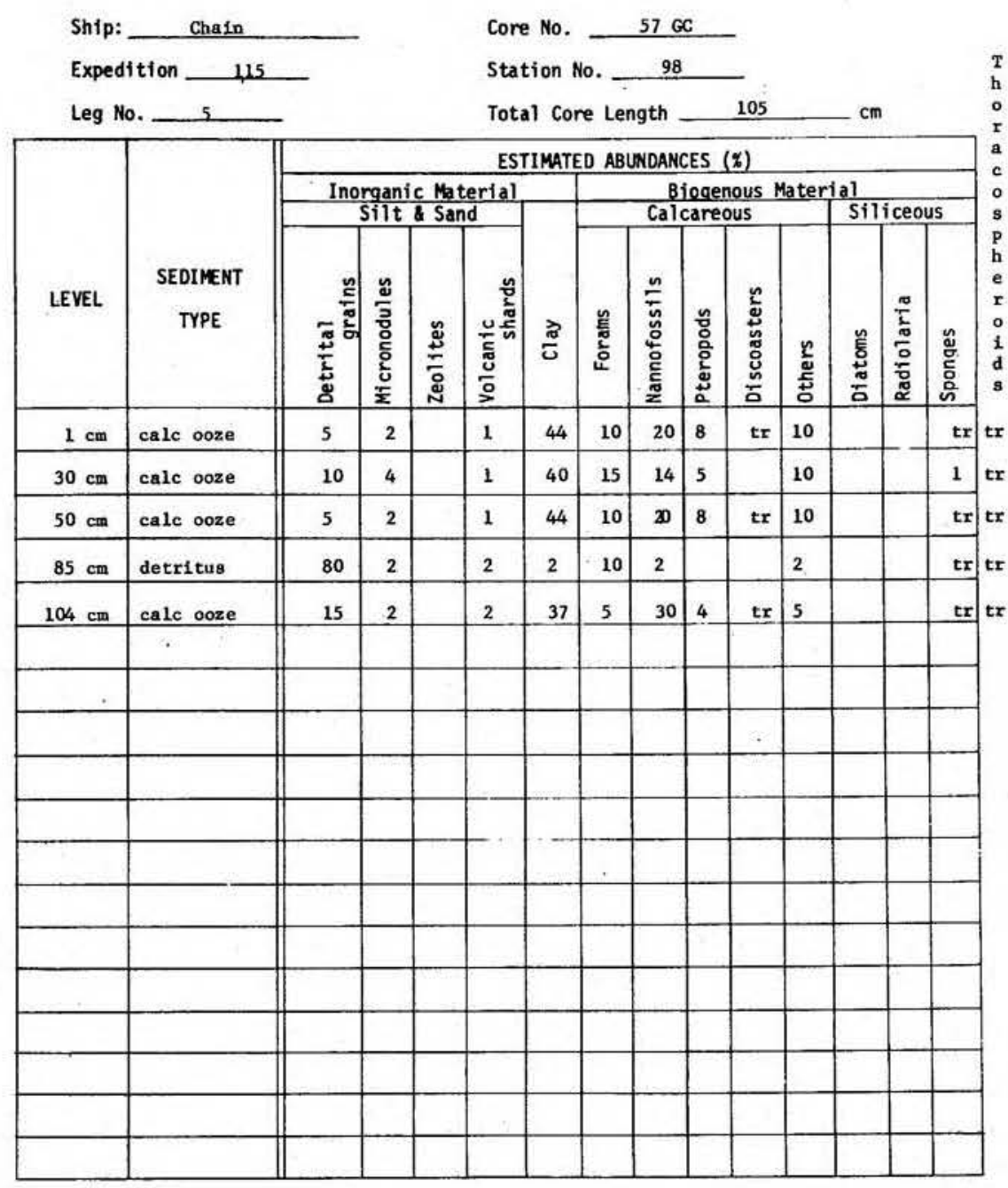


Ship CHAIN Cruise 115 Leg 5 Sta. 99 Core No. $586 \mathrm{C}$ Total Length $45 \mathrm{~cm}$. Lat. $24^{\circ} 16.79^{\prime} \mathrm{S}$ Long. $41^{\circ} 37.90 \mathrm{~W}$ Depth 1922 CaRB m Physiographic location Continental Slope off Rio de Janeiro

Lithologic

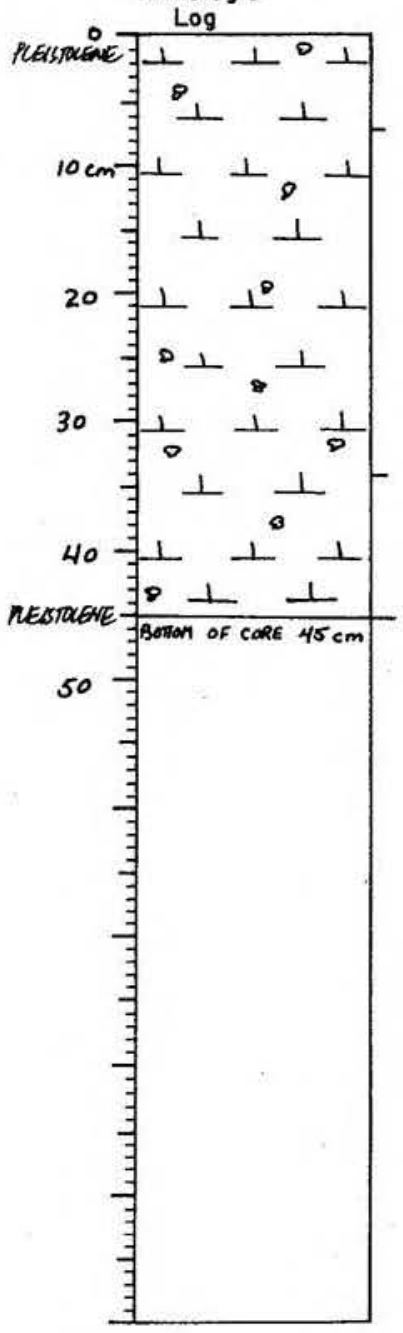

Detailed Description$$
\text { CALC OOZE }
$$

$5 Y 5 / 2$ ollve gray

scattered faint mottles throughout, light brownlsh gray

7-34

CALC OOZE

$2.5 \mathrm{Y} 6 / 2$ light brownish gray

scattered mottling throughout brownish gray, cormon $24-34 \mathrm{~cm}$ gray

1st silty lutite

2 approximately horizontal, fuzzy, dark laminations at 15 an $S$ irregular

34-45

CAIC OOZE

5Y $5 / 2$ olive gray

scattered mottling throughout, gray

core; 40-45 cm core cutter

end of core
SHEAR SLIDE DESCRIPTIONS - W.H.O.I. SEDINENT CORES

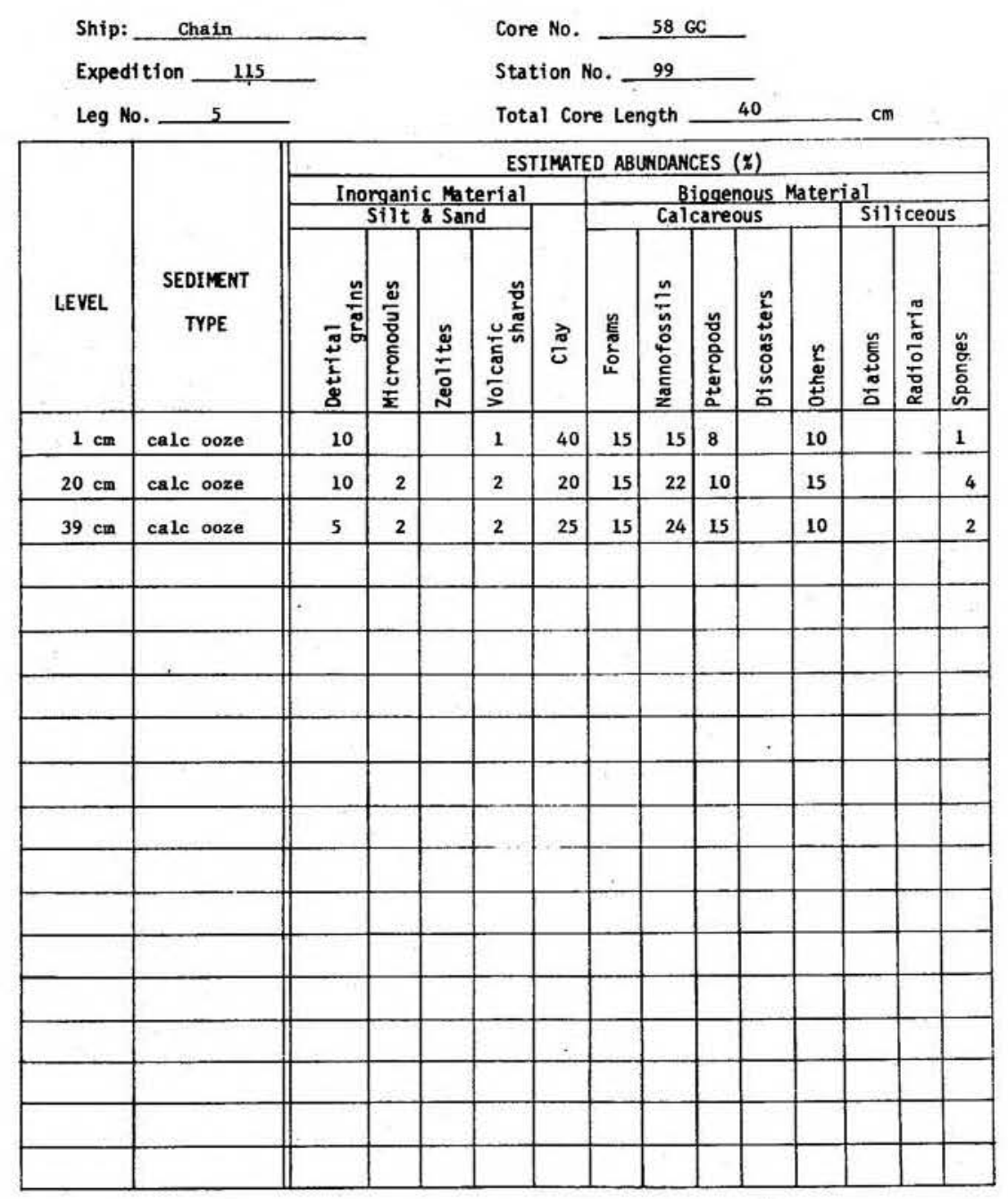


366

VISUAL CORE DESCRIPTION

Page 1 of 2

Ship CHN Cruise 1155 Leg 6 Sta. 102 Core No. SgPe

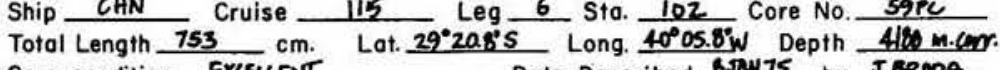
Core condition EXLEUENT Date Described BSW 75 by J.GROOA Physiographic location WESTERN VEMA CHANNEL

Lithologic

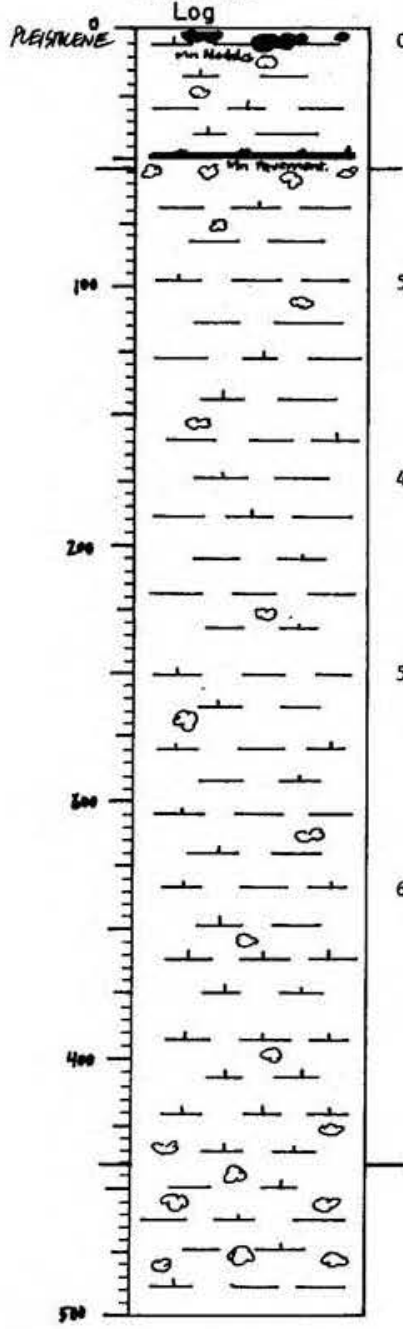

Detailed Description

$0-54$

LY CALC CLAY

1OYR $6 / 3$ pale brand gredes to $5 / 3$ brat

列 $6 / 4$ it yellowlsh brown, extensive

(ving

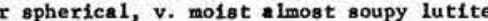

chetrated at $50 \mathrm{~cm}$

$G$ mottled

$-441$

SL CALC CIAY TO CALC OOZE

$.5 \times 5 / 2$ graylah brosn to $6 / 21$ t browainh gray

faint s1 mottling throughout anit, scattered black fleck (th

uicro?) and some brown found overall

molst firmer lutite with occasional small bits of lithified $\mathrm{s}, \mathrm{I} 15$

SL CALC CIAY

. gray mottl ing $441-470 \mathrm{~cm}$

irm smooth lutite

thin $(2 \mathrm{~mm})$ black lamination at bottom contact

$\mathrm{S}$,
$29-651$

SL CALC CIAY

$5 Y 5 / 3$ ollve, $2.5 Y 8 / 2$ dk graylgh brow

common marbling
ollive brown

inm smooth lutite, feu black flocks ocattered

peculiar interlocking arrangement of two different 1ithologien

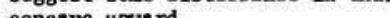

$51-720$

$2.5 Y 6 / 2$ it brownish gray

a number of various inclusions appear, $1.2 \times 3 \mathrm{~cm}$ smooth mottle 1OYR $6 / 2$ olive, 2. a few scattered white lithified nodules (0) can

firm moist lutite, abund. forams

disturbance (flow in is suspect) in this unit with no continuous features and an assortment of unusuál incfisions s, I $30^{\circ}$

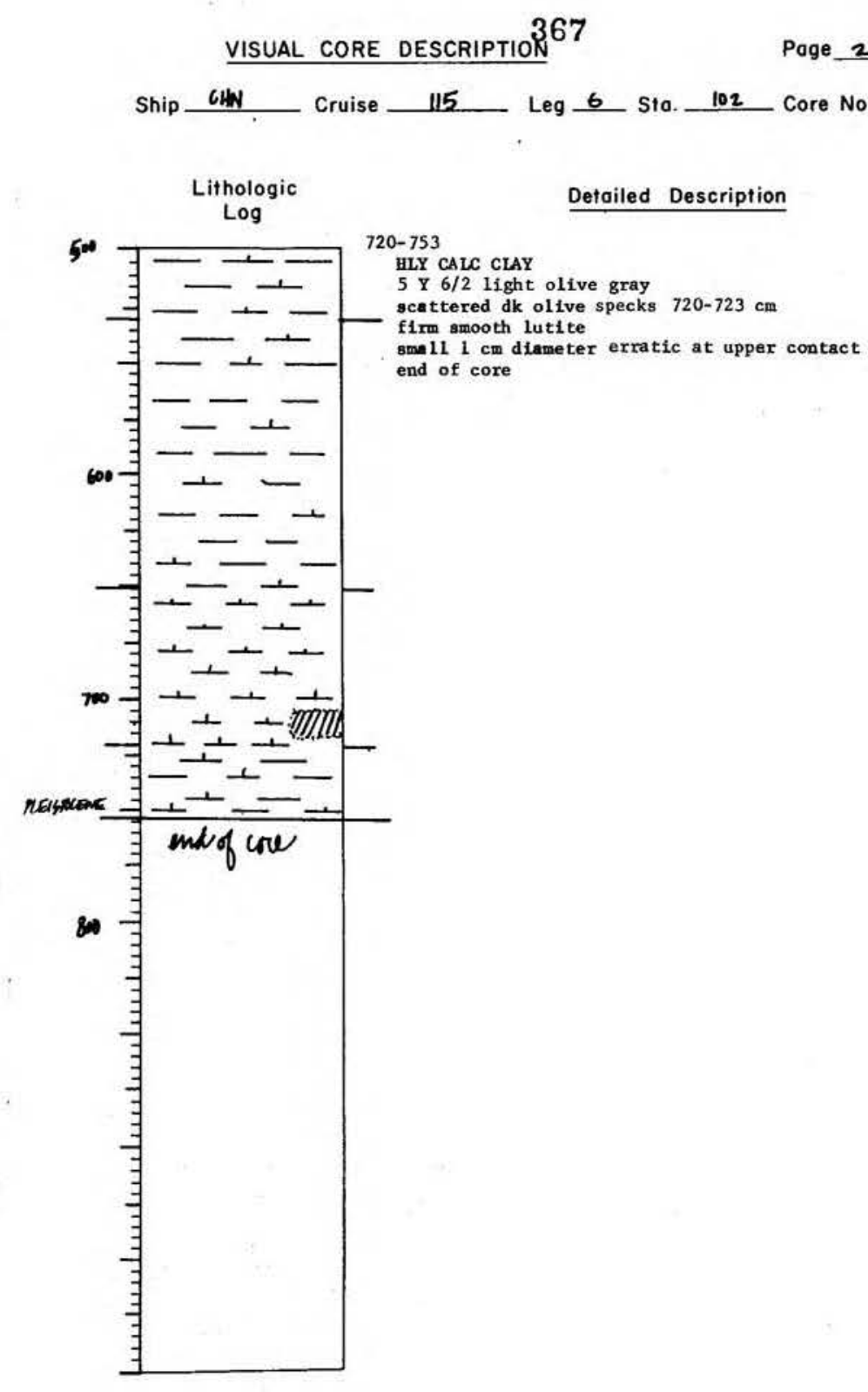


368

SIEAR SLIDE DESCRIPTIONS - W.H.0.I. SEDIMENT CORES

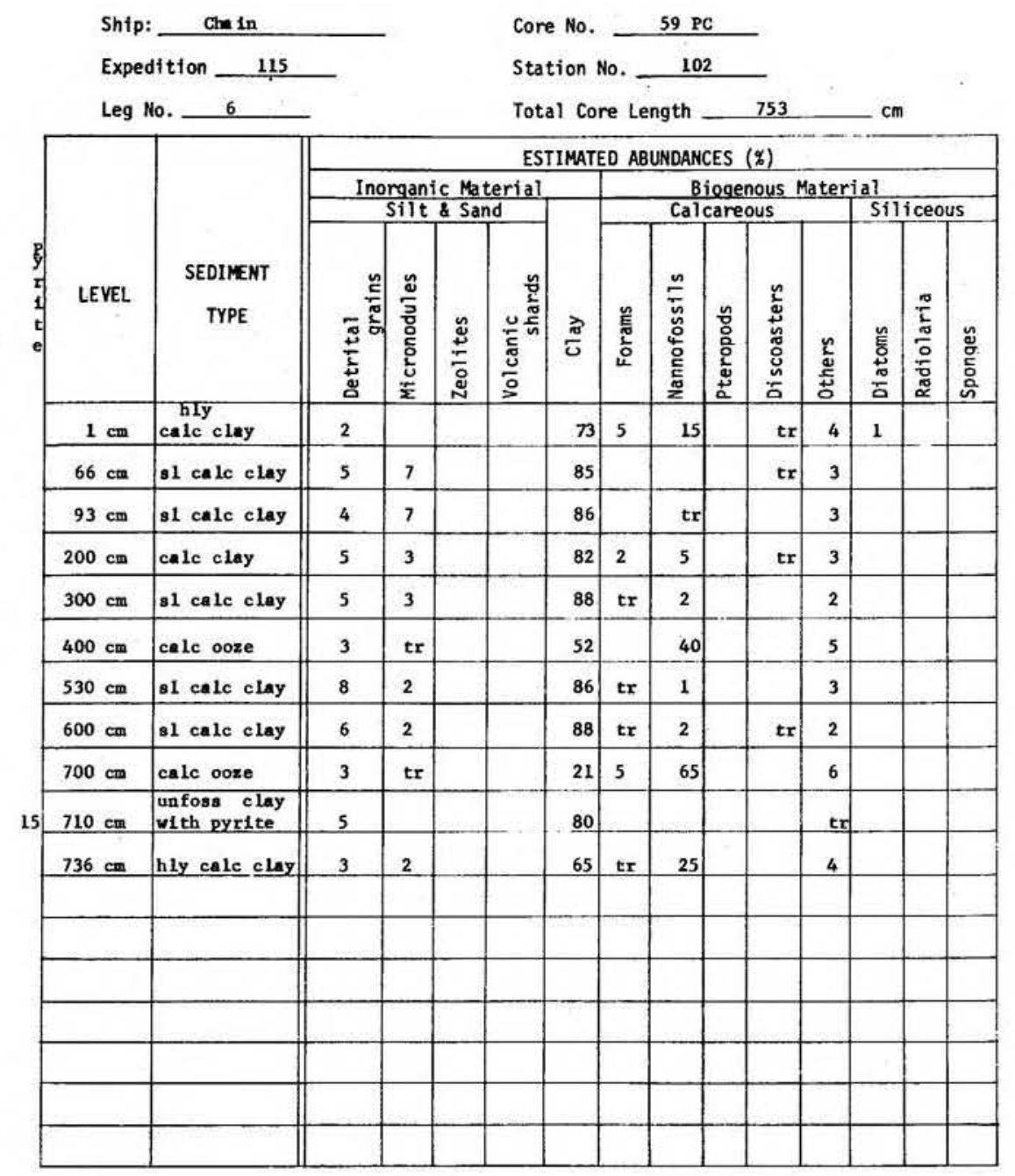

369

VISUAL CORE DESCRIPTION

Page 1 of 1

Ship GHN Cruise 115 Leg 6 Sta. 102 Core No. $59 \mathrm{PG}$ Total Length $52 \mathrm{~cm}$. Lat. $29^{\circ} 20.8^{\prime} \mathrm{S}$ Long. $40^{\circ} 05.8^{\circ} \mathrm{W}$ Depth $4198 \mathrm{~mm} \cdot \mathrm{cer}$. Core condition EXCELLENT Date Described 220N 75 by J.ERopA

Physiographic location WESTERN VEMA CHANNEL.

Lithologic

Detailed Description

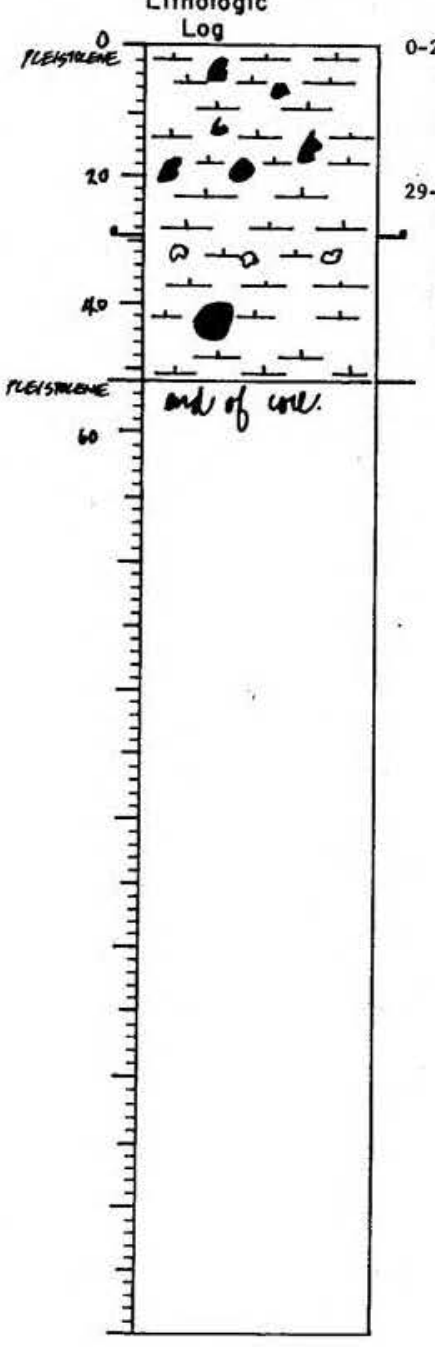

CALC OOZE

moist slick lutite, nine modules of assorted sizes

largest $3 \times 4 \mathrm{~cm}$, more fragments visible

$9-52$

oce

T/4 1t gellowish brown

comonon mottling $29-34 \mathrm{~cm}$ 10YR $5 / 4$ brow

moist firmer lutite vith scattered forens, one beautiful $4 \mathrm{~cm}$ dometer nodule $40-45 \mathrm{~cm}$ 


\section{0}

SMEAR SLIOE DESCRIPTIONS - W.H.O.I. SEDIMENT CORES

Ship: Chain

Expedition 115

Leg No. 6

\begin{tabular}{|c|c|c|c|c|c|c|c|c|c|c|c|c|c|c|}
\hline \multirow[b]{4}{*}{ LEVEL } & \multirow[b]{4}{*}{$\begin{array}{c}\text { SEDIMENT } \\
\text { TYPE }\end{array}$} & \multicolumn{13}{|c|}{ ESTIMATED ABUNDANCES (\%) } \\
\hline & & \multirow{2}{*}{\multicolumn{4}{|c|}{$\begin{array}{c}\text { Inorganic Material } \\
\text { Silt \& Sand }\end{array}$}} & \multirow[b]{3}{*}{$\frac{\pi}{\sigma}$} & & \multirow{2}{*}{\multicolumn{4}{|c|}{$\begin{array}{l}\text { Biegenous Mater } \\
\text { Calcareous }\end{array}$}} & \multirow{2}{*}{\multicolumn{3}{|c|}{ Siliceous }} \\
\hline & & & & & & & & & & & & & & \\
\hline & & 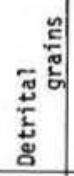 & $\begin{array}{l}\frac{\omega}{3} \\
\frac{3}{0} \\
\frac{0}{0} \\
\frac{0}{\delta} \\
\frac{\sigma}{\Sigma}\end{array}$ & 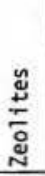 & 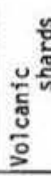 & & & 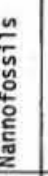 & 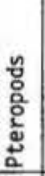 & 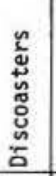 & 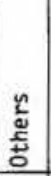 & 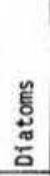 & $\frac{\frac{\pi}{2}}{\frac{\pi}{0}}$ & 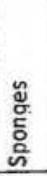 \\
\hline $1 \mathrm{~cm}$ & calc ooze & 3 & 2 & & & 5 & & 25 & & & 5 & & & \\
\hline $51 \mathrm{~cm}$ & calc ooze & 10 & 5 & & & 5 & & 10 & & & 5 & & & \\
\hline & & & & & & & & & & & & & & \\
\hline & & & & & & & & & & & & & & \\
\hline & & & & & & & & & & & & & & \\
\hline & & & & & & & & & & & & & & \\
\hline & & & & & & & & & & & & & & \\
\hline & & & & & & & & & & . & & & & \\
\hline & & & & & & & & & & & & & & \\
\hline & & & & & & & & & & & & & & \\
\hline & & & & & & & & & & & & & & \\
\hline & & & & & & & & & & & & & & \\
\hline & & & & & & & & & & & & & & \\
\hline & & & & & & & & & & & & & & \\
\hline & & & & & & & & & & & & & & \\
\hline & & & & & & & & & & & & & & \\
\hline & & & & & & & & & & & & & & \\
\hline & & & & & & & & & & & & & & \\
\hline
\end{tabular}

371

VISUAL CORE DESCRIPTION

Page 1 of 2

Ship CHAIN Cruise $115 \quad$ Leg 6 sto. 104 Core No. GoPC Total Length $547 \mathrm{~cm}$. Lat. $30^{\circ} 13.8^{\prime} \mathrm{S}$ Long. $39^{\circ} 14.6^{\circ} \mathrm{W}$ Depth $4310 \mathrm{~m} \cdot \mathrm{corr}$ Core condition EXEEULNT Date Described 9JAN.75 by J.GRODA. Physiographic location EASTERN VEMA CMANWEL

Lithologic

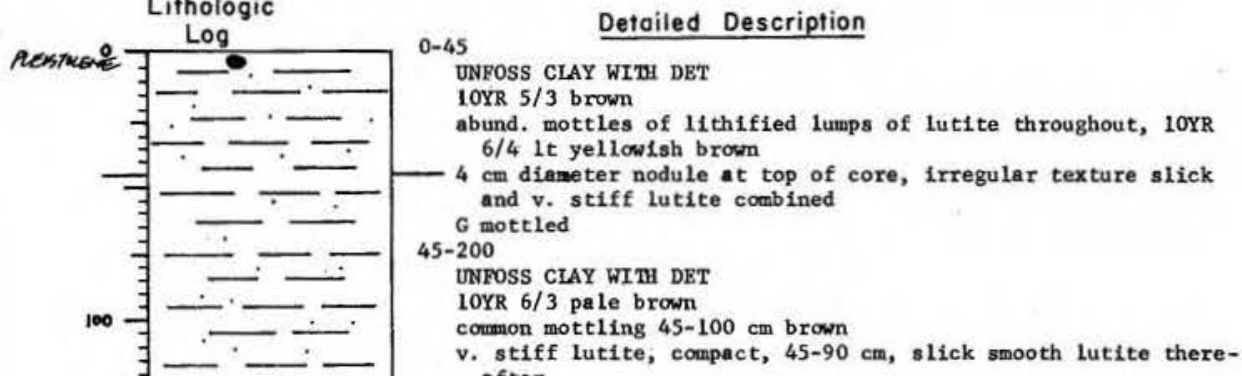

cormon mottling 45-100 $\mathrm{cm}$ brown

alick smooth lutite there-

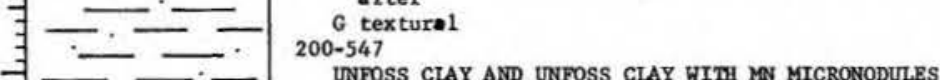

UNPOSS CLAY AND scattered rarely throughout

wo dk shadowy layers of sediment with fairly sharp upper and

- -1 - $\begin{aligned} & \text { two dk shadowy layers of sediment with } \\ & \text { lower conts 263-269 cm, 383-389 cm }\end{aligned}$

$-1-$

而 - -

-

-

근

$\exists-1-$

$\exists-\square$

- - -

$-\div . \overline{-}$

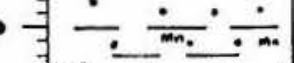

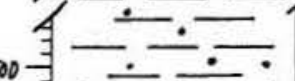

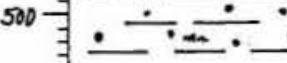

正 -

PLESTOCENE 
372

SMEAR SLIDE DESCRIPTIONS - W.H.O.I. SEDIMENT CORES

Ship: Chain

Expedition

\begin{tabular}{|c|c|c|c|c|c|c|c|c|c|c|c|c|c|c|}
\hline \multicolumn{2}{|c|}{ Leg No. 6} & \multicolumn{13}{|c|}{ Total Core Length 547} \\
\hline \multirow[b]{4}{*}{ LEVEL } & \multirow[b]{4}{*}{$\begin{array}{c}\text { SEDIMENT } \\
\text { TYPE }\end{array}$} & \multicolumn{13}{|c|}{ ESTIMATED ABUNDANCES (\%) } \\
\hline & & \multirow{2}{*}{\multicolumn{4}{|c|}{$\begin{array}{c}\text { Inorganic Material } \\
\text { Silit \& Sand }\end{array}$}} & \multirow[b]{3}{*}{$\frac{\pi}{0}$} & \multirow{2}{*}{\multicolumn{5}{|c|}{$\begin{array}{l}\text { Biogenous Mater } \\
\text { Calcareous }\end{array}$}} & \multirow{2}{*}{\multicolumn{3}{|c|}{ Siliceous }} \\
\hline & & & & & & & & & & & & & & \\
\hline & & 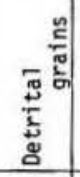 & 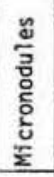 & 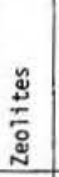 & 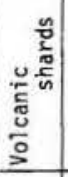 & & 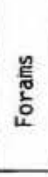 & 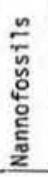 & 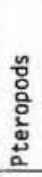 & 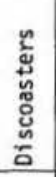 & 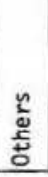 & 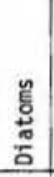 & $\frac{\frac{\pi}{2}}{\frac{\pi}{0}}$ & 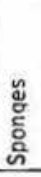 \\
\hline $5 \mathrm{~cm}$ & $\begin{array}{l}\text { unfoss clay } \\
\text { with det }\end{array}$ & 15 & 5 & $\operatorname{tr}$ & $\operatorname{tr}$ & 80 & & & & & & & & \\
\hline $60 \mathrm{~cm}$ & $\begin{array}{l}\text { unfoss clay } \\
\text { with det }\end{array}$ & 25 & 3 & $t r$ & tr & 72 & & & & & & & & \\
\hline $160 \mathrm{~cm}$ & $\begin{array}{l}\text { unfoss clay } \\
\text { with det }\end{array}$ & 15 & 5 & & $\mathrm{tr}$ & 80 & & & & & : & & & \\
\hline $260 \mathrm{~cm}$ & unfoss clay & 10 & 3 & & & 87 & $\mathrm{tr}$ & & & & & & & \\
\hline $360 \mathrm{~cm}$ & unfoss clay & 10 & 5 & & & 85 & tr & & & & & & & \\
\hline $386 \mathrm{~cm}$ & $\begin{array}{l}\text { unfoss clay } \\
\text { with Mn micro }\end{array}$ & 10 & 20 & & & 70 & tr & & & & & & & \\
\hline & $\begin{array}{l}\text { unfoss clay } \\
\text { with Mn micro }\end{array}$ & 5 & 15 & & & 80 & & & & & & & & \\
\hline $546 \mathrm{~cm}$ & unfoss clay & 10 & 10 & & & 80 & tr & & & - & & & & \\
\hline & & & & & & & & & & & & & & \\
\hline & & & & & & & & & & & & & & \\
\hline & & & & & & & & & & & & & & \\
\hline & & & & & & & & & & & & & & \\
\hline & & & & & & & & & & & & & & \\
\hline & & & & & & & & & & & & & & \\
\hline & & & & & & & & & & & & & & \\
\hline & & & & & & & & & & & & & & \\
\hline & & & & & & & & & & & & & & \\
\hline & & & & & & & & & & & & & & \\
\hline
\end{tabular}

373

VISUAL CORE DESCRIPTION

Page 1 of 1

Ship CHAin Cruise 115 Leg 6 Sta. 104 Core No. GopG Total Length $85 \mathrm{~cm}$. Lat. $30^{\circ} 13.8$ 's Long. $39 \% 14.6 \mathrm{~W}$ Depth $4310 \mathrm{micm}$ Core condition EXLEUENT Dote Described 2.JAN.75 by J.SRODA.

Physiographic location

Lithologic

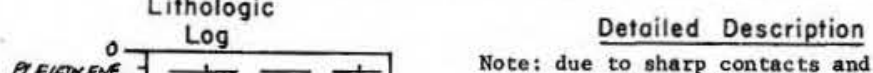

\section{Detailed Description}

Note: due to sharp contacts and non-continuous 1ithology it appears evident that this pilot core represents a multiple penetration $50-85 \mathrm{~cm}$ ). $0-36$ $\exists+1,0$

$207 \overline{6} \frac{\omega}{\infty}$

HLY CALC CIAY GRADES TO UNFOSS CIAY COYR $5 / 4$ yellowish brown

grayish brown inm molst lutite vith lithified lumps

$\exists \frac{0}{\sigma}-\begin{gathered}\text { firm molst } \\ \mathrm{G} \text { mottled }\end{gathered}$

$407 \frac{\infty}{3}-\frac{}{3}-36-50$

o 10 10YR $7 / 4 \mathrm{v}$. pale brown

作 $\begin{gathered}\text { brown } \\ \text { firm compact lutite, sl silty }\end{gathered}$ 60 政- $-\mathrm{firm}$ compact lutite, s1 silty

当 $-\frac{0}{0}{ }_{\text {UNFOSS CLAY }}^{50-77}$

$\exists 0-\infty-0$ 1OYR $5 / 4$ yellowish brown

o 7 - - numerous lithified mottles throughout, pale brown amerous lithified mottles throug $G$ mottled

\section{endof cove.} UNPOSS CLAY

1OYR $6 / 41 \mathrm{t}$ yellowish brown

$100-$ yelling at upper contact with yellowish brown firm conpact lutite end of core 
374

SHEAR SLIDE DESCRIPTIONS - W.H.O.I. SEDIMENT CORES

Ship: Chain

Expedition 115

Leg No. 6
Core No. $60 \mathrm{PG}$

Station No. 104

Total Core Length $\quad 85 \quad \mathrm{~cm}$

ESTIMATED ABUNDANCES (\%)

\begin{tabular}{|c|c|c|c|c|c|c|c|c|c|c|c|c|c|c|}
\hline \multirow[b]{3}{*}{ LEVEL } & \multirow{3}{*}{$\begin{array}{l}\text { SEDIMENT } \\
\text { TYPE }\end{array}$} & \multicolumn{13}{|c|}{ ESTIMATED ABUNDANCES ( $(\%)$} \\
\hline & & \multicolumn{4}{|c|}{$\begin{array}{c}\text { Inorganic Material } \\
\text { Silt \& Sand }\end{array}$} & \multirow[b]{2}{*}{$\frac{\pi}{6}$} & \multicolumn{5}{|c|}{$\begin{array}{l}\text { Biegenous Mater } \\
\text { Calcareous }\end{array}$} & \multicolumn{3}{|c|}{$\frac{a 1}{\text { siliceous }}$} \\
\hline & & 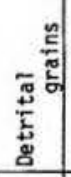 & 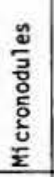 & 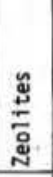 & 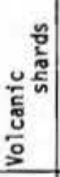 & & 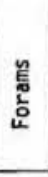 & 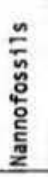 & 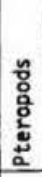 & 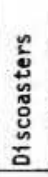 & $\begin{array}{l}r \\
\text { s. } \\
\text { ș } \\
0\end{array}$ & $\begin{array}{l}\text { 号 } \\
\mathbf{n} \\
\vdots \\
\end{array}$ & $\begin{array}{l}\frac{\pi}{2} \\
\frac{0}{0} \\
\frac{0}{2} \\
\frac{0}{0} \\
\end{array}$ & 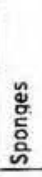 \\
\hline $1 \mathrm{~cm}$ & hly calc clay & 3 & 1 & & & 75 & 6 & 15 & & & & & & \\
\hline $49 \mathrm{~cm}$ & unfoss clay & 7 & $\mathrm{tr}$ & & & 93 & & & & & & & & \\
\hline $55 \mathrm{~cm}$ & unfoss clay & 6 & 12 & & & 82 & & & & & & & & \\
\hline $84 \mathrm{~cm}$ & unfoss clay & 12 & 3 & & & 85 & & & & & & & & \\
\hline & & & & & & & & & & & & & & \\
\hline & & & & & & & & & & & & & & \\
\hline & & & & & & & & & & & & & & \\
\hline & & & & & & & & & & . & & & & \\
\hline & & & & & & & & & & & & & & \\
\hline & & & & & & & & & & & & & & \\
\hline & & & & & & & . & & & & & & & \\
\hline & & & & & & & & & & & & & & \\
\hline & & & & & & & & & & & & & & \\
\hline & & & & & & & & & & & & & & \\
\hline & & & & & & & & & & & & & & \\
\hline & & & & & & & & & & & & & & \\
\hline & & & & & & & & & & & & & & \\
\hline & & & & & & & & & & & & & & \\
\hline
\end{tabular}

375

VISUAL CORE DESCRIPTION

Page 1 of 2

Ship CHAN Cruise 115 Leg 6 Sta. 105 Core No. GIPC Total Length $819 \mathrm{~cm}$. Lat. $30^{\circ} 15.5^{\prime} \mathrm{S}$ Long. $39^{\circ} 05.8^{\circ} \mathrm{W}$ Depth $4181 \mathrm{corm}$ Core condition ExCELAENT Date Described 10 JAN 15 by J.ERoph. Physiographic location EASTERN VEMA CHANNEL

Lithologic

retsoceave

\section{- +-1}

$\Delta+\infty+0$

$-\infty+\infty$

$+\div$

$+\div+$

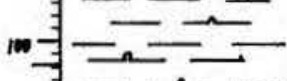

긍

$\rightarrow-\frac{1}{0}$

$\exists \rightarrow-$

再的高

2007 I. $1+1+$ $\omega$

-

G. $\div \cdot \div$

- $\rightarrow-$

A $-\frac{1}{2}$

300 - -

- $\sim$

-

그는

400 든

$1+$

든

두

고도

$\pm+$

든 te

brown burrow extending $24-30 \mathrm{~cm}$

two wide shadowy micro nodule(Mn) rich layers 86-90, $102-108 \mathrm{~cm}$

SL SILIC CLAY GRADES TO CALC OOZE TO SL SILIC CLAY WIT MN MICRO TO SL SIUIC CIAY

faint 2 lt brownsh gray

moist slick lutite, homogeneous texture throughout

wide $\mathrm{Mn}$ micro nodule rich band $339-353 \mathrm{~cm}$, scattered rusty flecks and splotches $260-290 \mathrm{~cm}$

350-510

CALC OOZE GRADES TO HIGHLY CALC CLAY

107R $6 / 3$ pele brown grades to $5 / 3$ brown

si faint mottling gcattered throughout, mostly it yellowish

and $y$, pale brow

gi darkening to brownish gray $435-450 \mathrm{~cm}$; another distinct zone 463-471 $\mathrm{cm}$ and two

$510-749$ (laminations)

SL. SILIC CLAY GRADES TO CALC OOZE

Cork $6 / 3$ pale brown grades to $6 / 4$ tt yellowish brown to $7 / 4$

ing 10YR 8/3 v. pale brown, $652-657$ cm sl shadowy (5) $610 \mathrm{~cm}$ and faint brown mott1ing $660-749 \mathrm{~cm}$

$\mathrm{s}$
$49-760$

749-760

UNPOSS CLAY/M MICRO
1OYR $3 / 1$ v. dk gray

sone marbling with pale brown throughout

1 wn rich lutite with scattered 1ithified flecks

S, H

UNFOSS CIAY

It ollve brown grades to $2.5 Y 5 / 2$ grayish brown si it gray motting $785-800 \mathrm{~cm}$

wo thin wel1-11 thified, almost $d r y$, concave-upverd lans 2 mi

780 and $795 \mathrm{~cm}$

end of core 
VISUAL CORE DESCRIPTION 376

Ship CHAIN Cruise $115 \quad$ Leg 6 Sta. 105 Core No. 6IPC

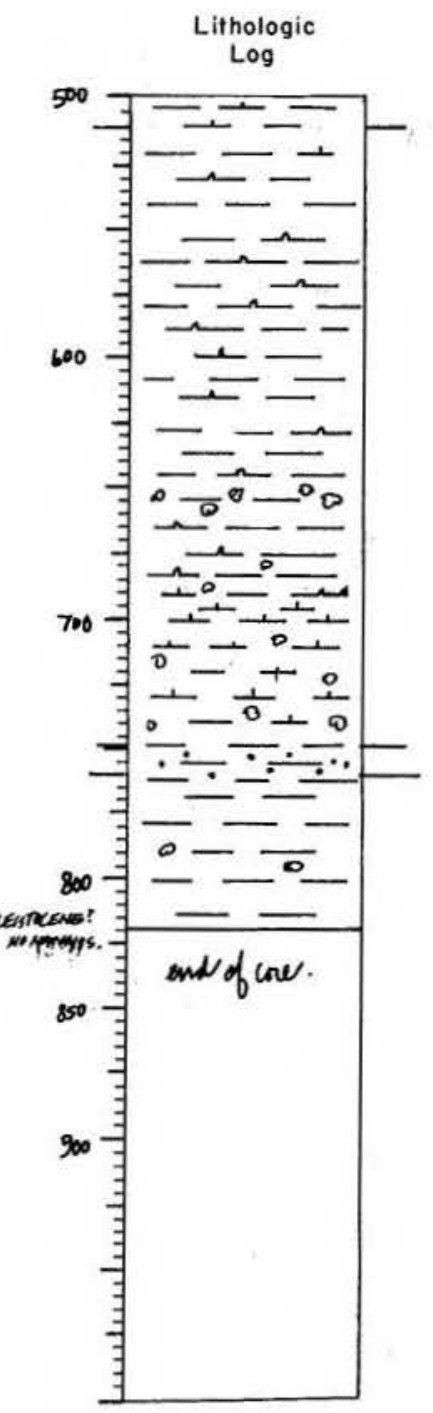

Detailed Description
377

SMEAR SLIDE DESCRIPTIONS - W.H.0.1. SEDIMENT CORES

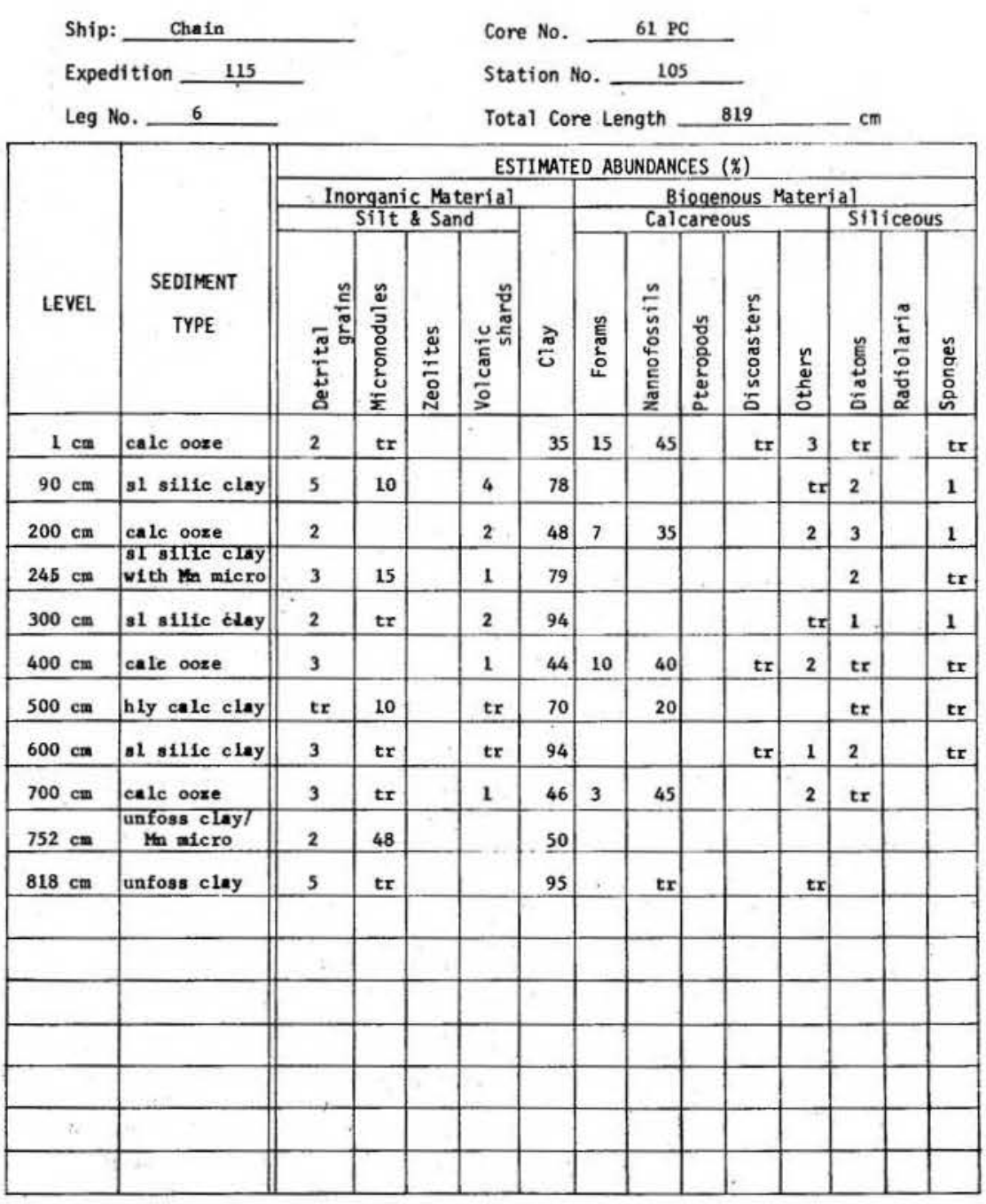


378

VISUAL CORE DESCRIPTION

Page 1 of 1

Ship CHAIN Cruise 115 Leg 6 Sta 105 Core No. 6IPG Total Length $151 \mathrm{~cm}$. Lat. $30^{\circ} 15.5^{\circ} \mathrm{S}$ Long. $39^{\circ} 05.8^{\circ} \mathrm{W}$ Depth $4181 \mathrm{meer}$ Core condition EXCELENT Date Described 13 JWW TS by S.RzOBA. Physiographic location EASTERN YEMA CHANNEL

Lithologic

Detailed Description

RESTOCENE J

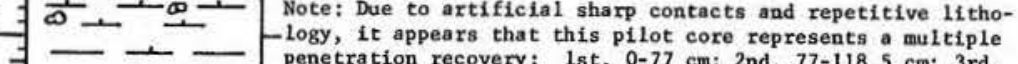

- - $\begin{aligned} & \text { penetration recovery: } 1 \mathrm{st}, 0-77 \mathrm{~cm} ; 2 \mathrm{nd}, 77-118.5 \mathrm{~cm} ; 3 \mathrm{rd} \text {, } \\ & 118.5-151 \mathrm{~cm} \text {. Also }\end{aligned}$

2. 1 - 1 (pushed) out of the top of the corer and neither of the two

2. - - - repenetrations have recovered this surface interval. This

- - - - resulted from resistance offered by the mud already in the

- - the initial sample to move and allow entry of new sedfments,

$40-1-1$ by which time the corer was already below the sediment

- -1 sur

- - CALC OOZE

- common v. pale brown mottlin

เ

-

$\mathrm{S}$ mottled

10YR $5 / 2$ graylsh brown grades to $6 / 3$ pale brown

7 -1 faint bands of brown mottling

글 hazy bands of the micro nodule rich sediment $6-18,41-58 \mathrm{~cm}$

으 $\quad$ s, I

$100=-1-$ sL sIITc ciny

- 10 10YR $6 / 4$ 1t yellowish brown

brown $s 1$ mottling and marbling

F $\div-\infty{ }_{85-118.5}^{G}$

$120=-\infty-118.5$

10YR $5 / 2$ grayish brown grades to $6 / 3$ pale brow

v. si faint bwown bands and mottling

我 - $-2 \mathrm{~cm}$ band of Mn nodule rich lutite 105-107 mooth luti

160

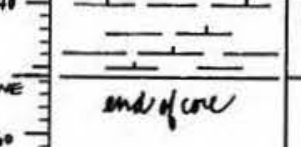
18.5-138

SL CALC CIAY

yellowish brwon

nt Inclined banding (brown) and mottling

138-151

HLY CALC CLAY

10YR $5 / 2$ grayish

faint graylsh brown banding found 81 throughout

end of eore lutite
379

SMEAR SLIDE DESCRIPTIONS - W.H.O.I. SEDIMENT CORES

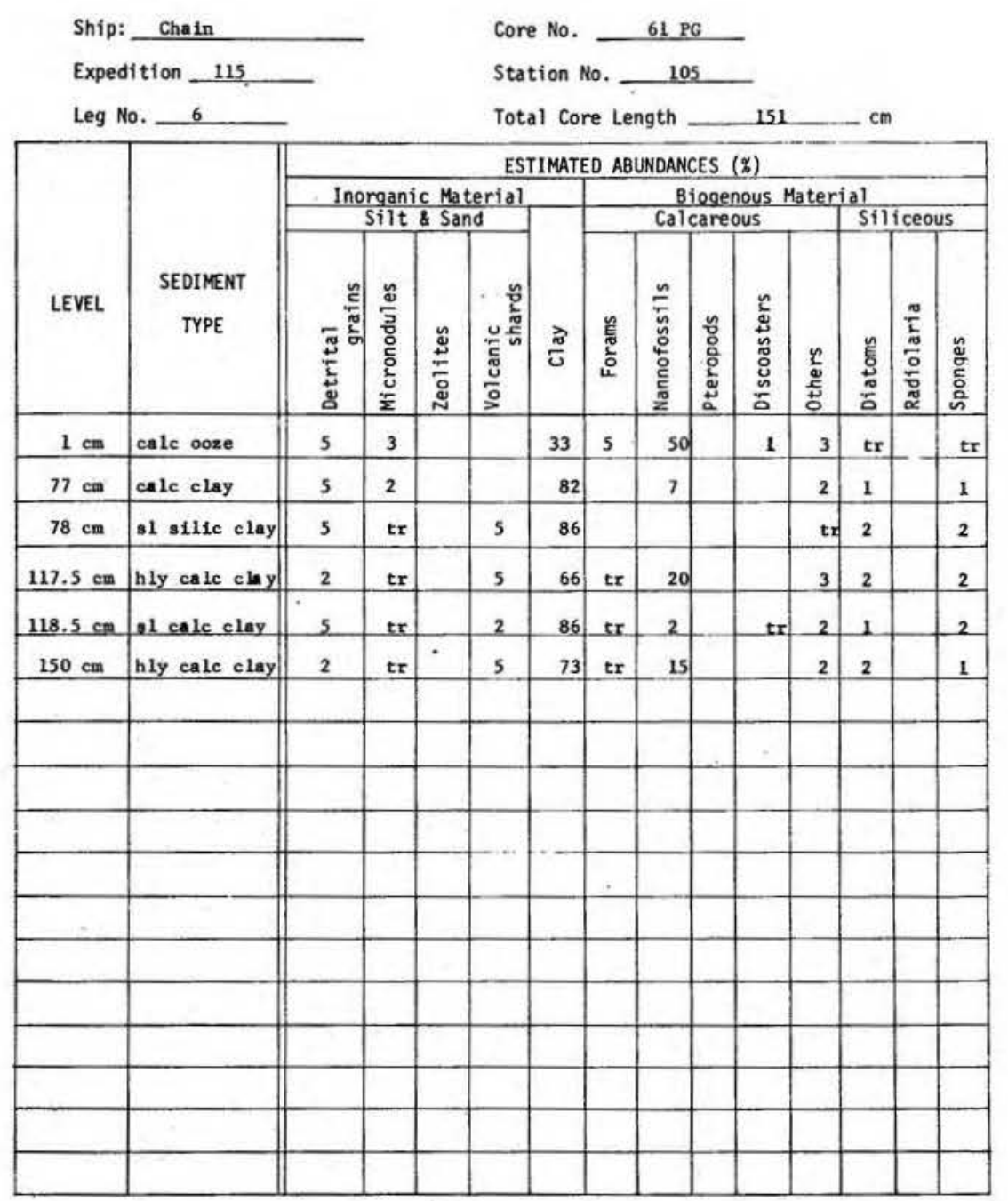



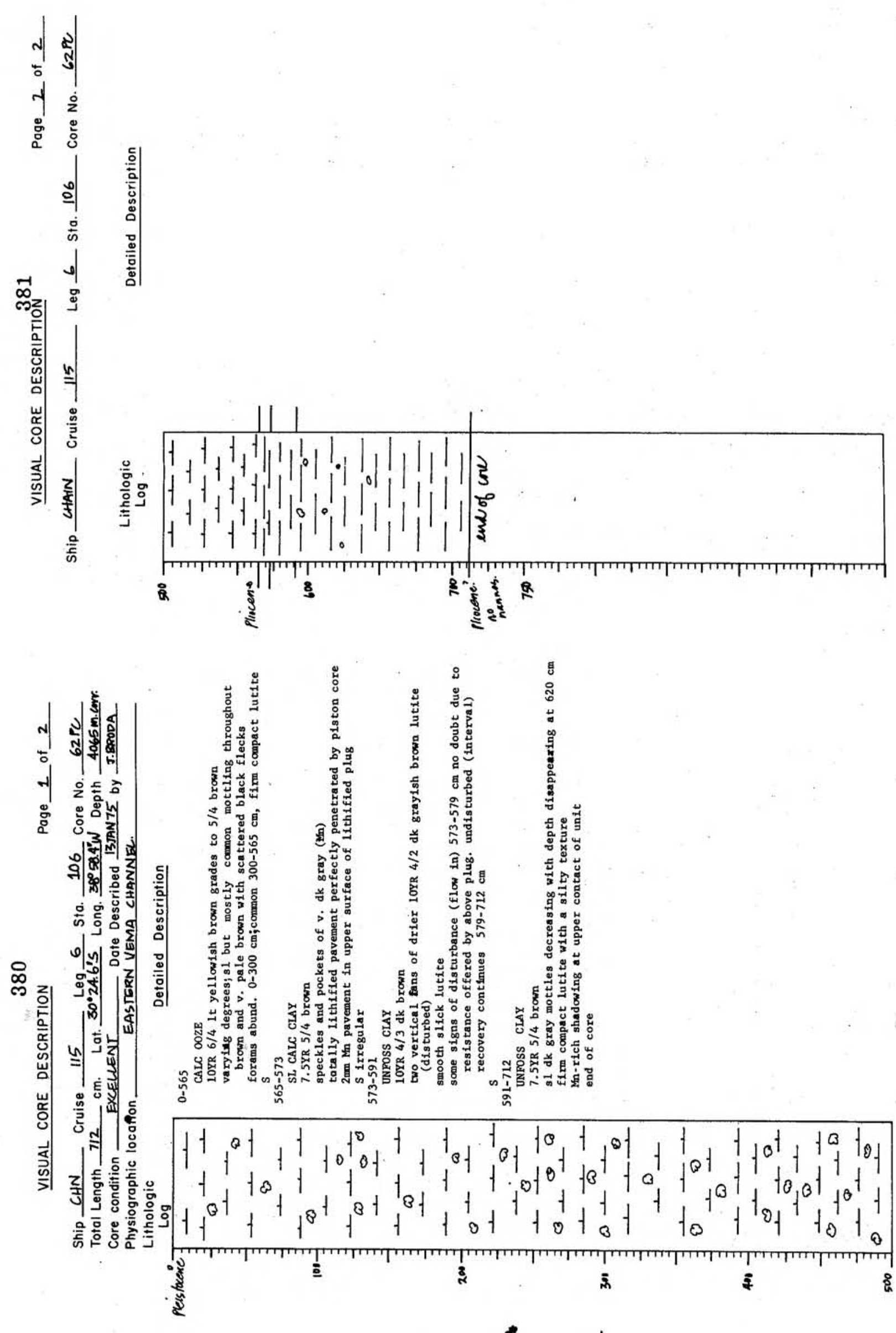
382

SMEAR SLIDE DESCRIPTIONS - W.H.O.I. SEDIMENT CORES

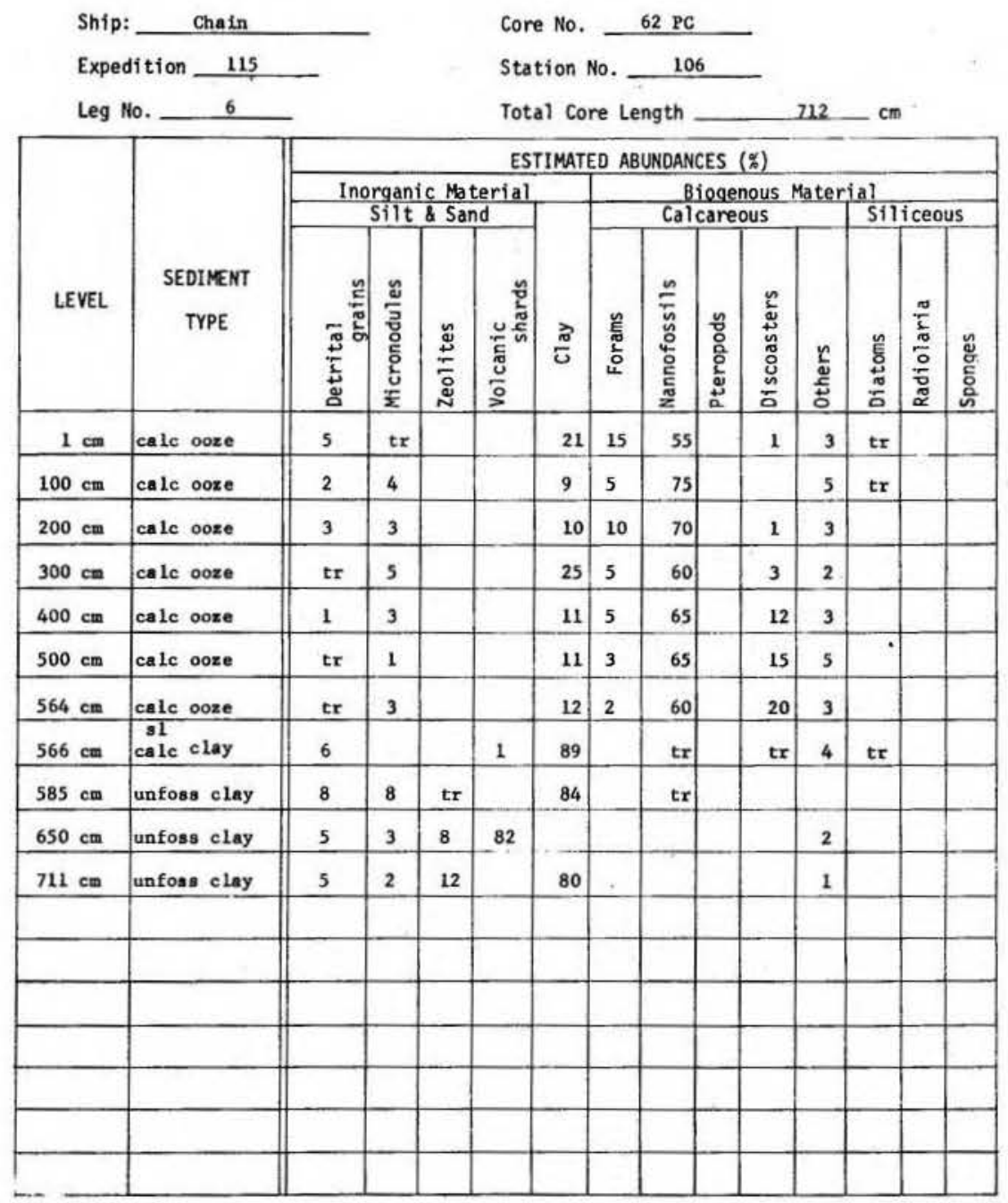

383

VISUAL CORE DESCRIPTION

Page 1 of 1

Ship CHAIN Cruise 115 Leg 6 Sta. 106 Core No 62PG Total Length $148 \mathrm{~cm}$. Lat. $30^{\circ} 24.6^{\prime \prime} \mathrm{S}$ Long. $38^{\circ} 58.4^{\prime} \mathrm{W}$ Depth $4065 \mathrm{~m} \cdot \mathrm{urr}$ Core condition EXCELLENT D. T.BRODA Physiographic location ENSTERN VEMA CHANNEL Lithologic

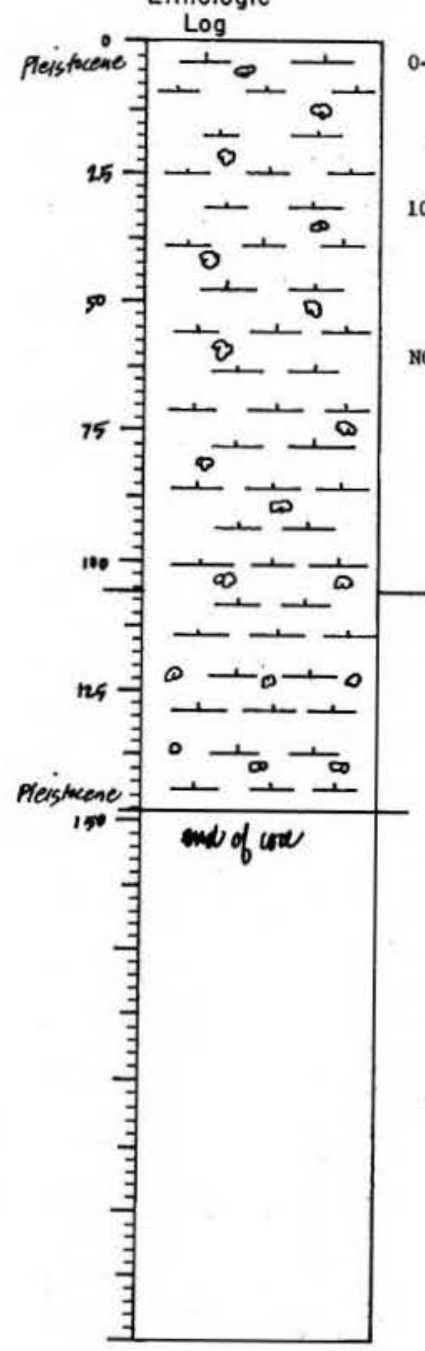

Detoiled Description CALC OOZE

1OYR $6 / 4$ 1t yellow1sh brown

abund formeting S, 1 H

CAIC OOZE

10YR $6 / 3$ pale brown grades to $5 / 4$ brown

and

abse of the nature of the contact mentioned logic features in this core, repenetrate

rebound) is suspected; 1st penetration, 0-106 cm -

in this case some of the near surface sediment has been shot out the top of the corer and lost; 2nd penetration, $106-10 \mathrm{~cm}$ - in this section the top couminan pale brown mottling $120-125 \mathrm{~cm}, 135-148 \mathrm{~cm}$ abund. forams in moist silty lutite 


\section{4}

SMEAR SLIDE DESCRIPTIONS - W.H.O.I. SEDIMENT CORES

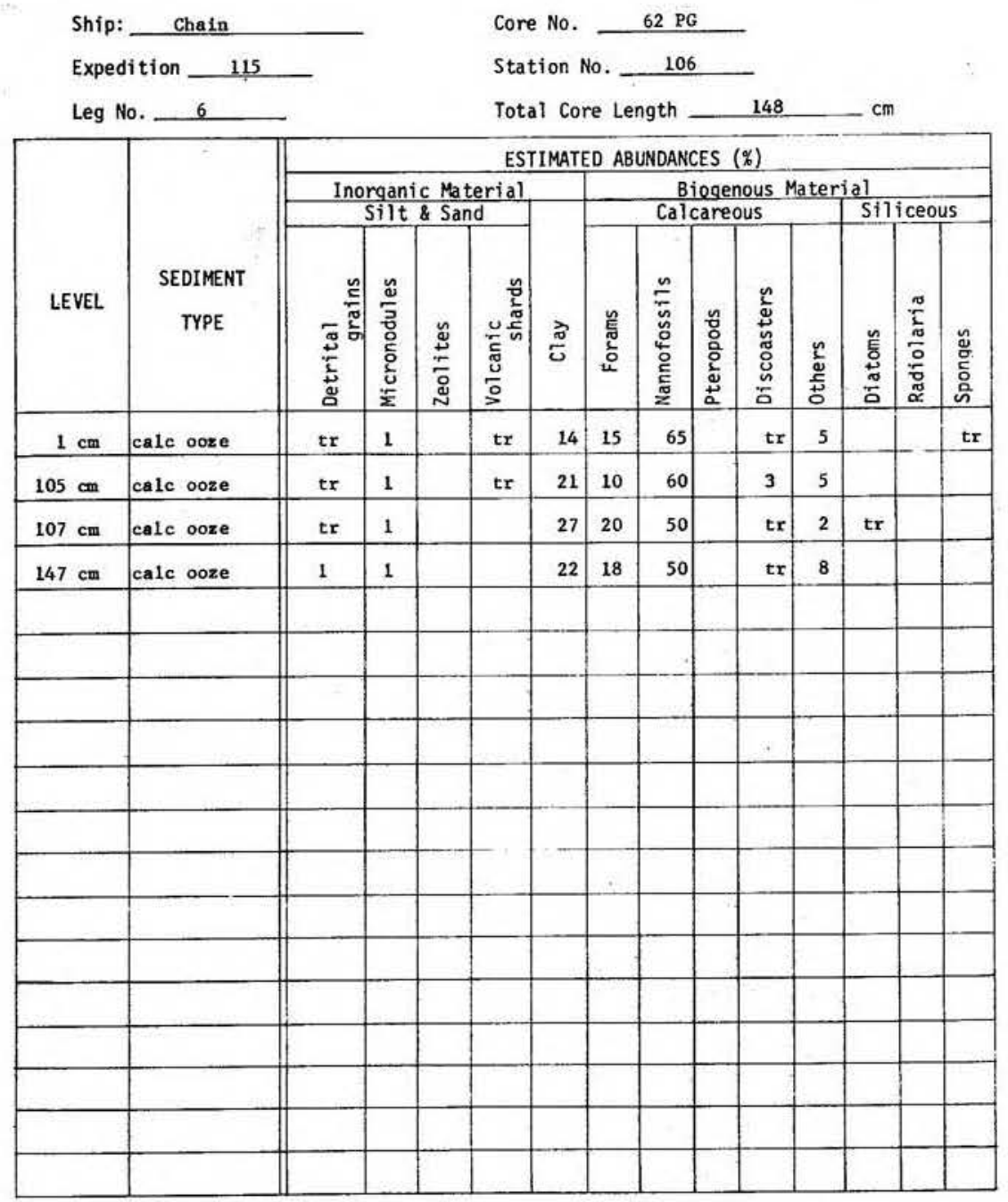

385

VISUAL CORE DESCRIPTION

Page 1 of 2

Ship CHAIN Cruise $115 \quad$ sta 108 Core No. $64 \mathrm{PC}$

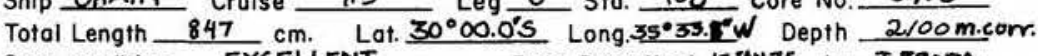
Core condition EXCELLENT Date Described 17JWN75 by J.ERODA Physiogrophic location NORTH FLANK OF DOGRANDE RISE

Lithologic

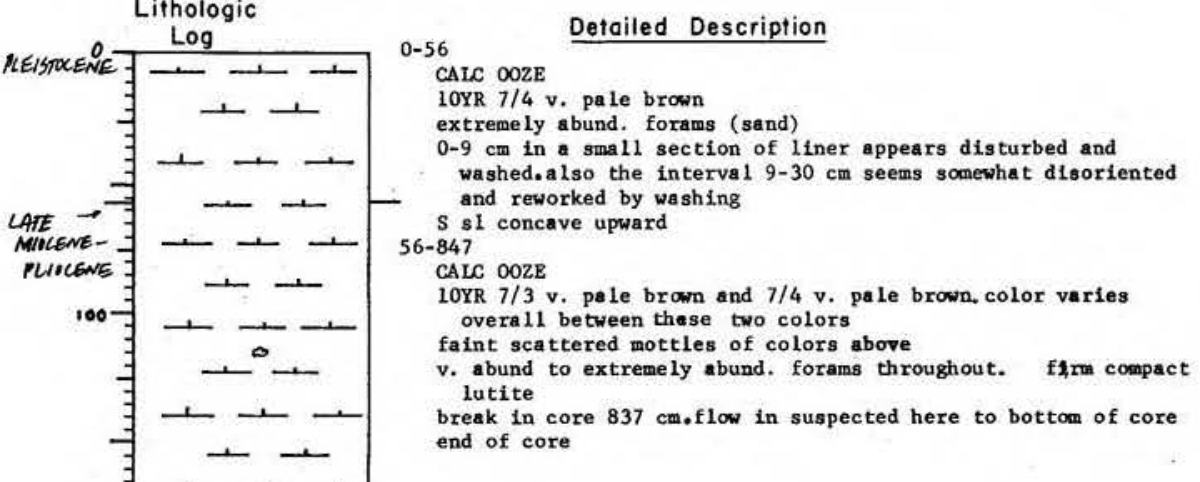




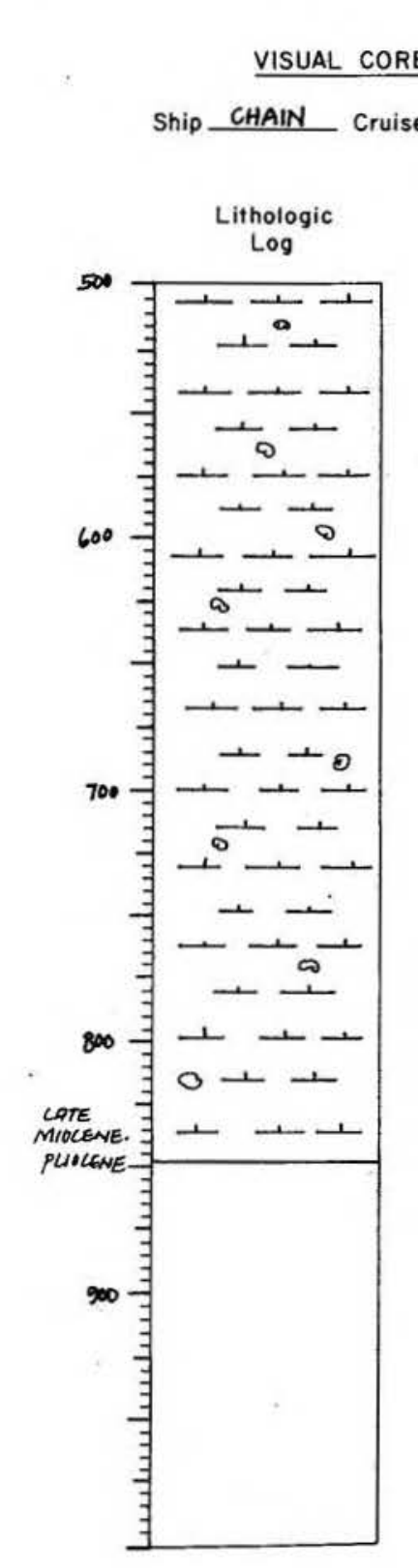

Page 2 of 2

108 Core No. $64 \mathrm{PL}$

Detailed Description 


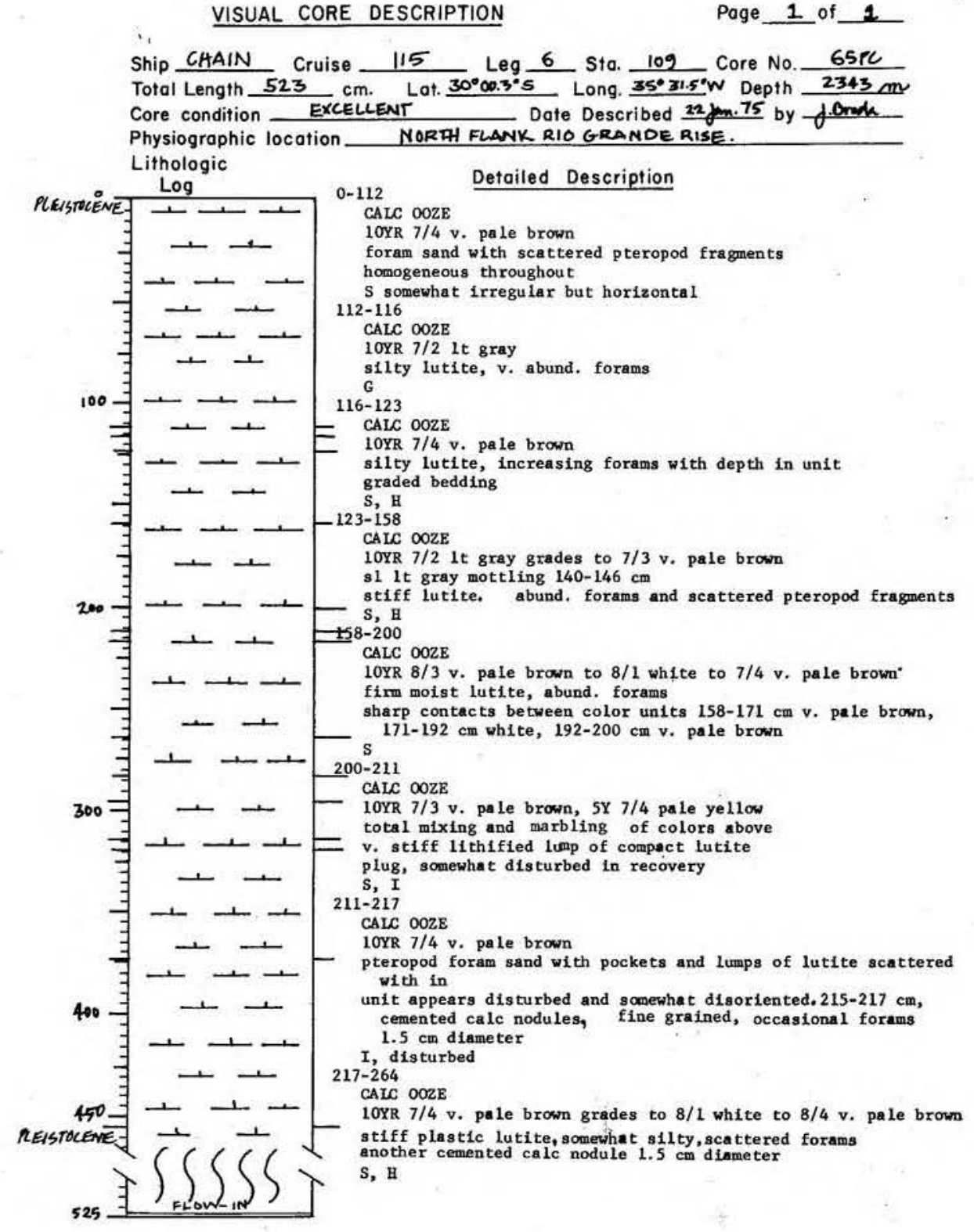

CoxCELLENT Core condition $\frac{\text { EXCELLENT }}{\text { Physiographic location NORTH FLANK RIO GRANDE RISE. }}$ Date Described $\frac{22}{210} .75$ by fork Lithologic trough but horizontal CALC 002 -12 gility lutite, increasing foram with depth in unit abs vale brown to $8 / 1$ white to $7 / 4$ v, pale brown s ing and marbling of colors above ALC OOZE it appears disturbed and gavewhat diacriented. $215-217 \mathrm{~cm}$, plastic lutite, somewhat silty, scattered fors. nother cemented calc nodule $1.5 \mathrm{~cm}$ diameter

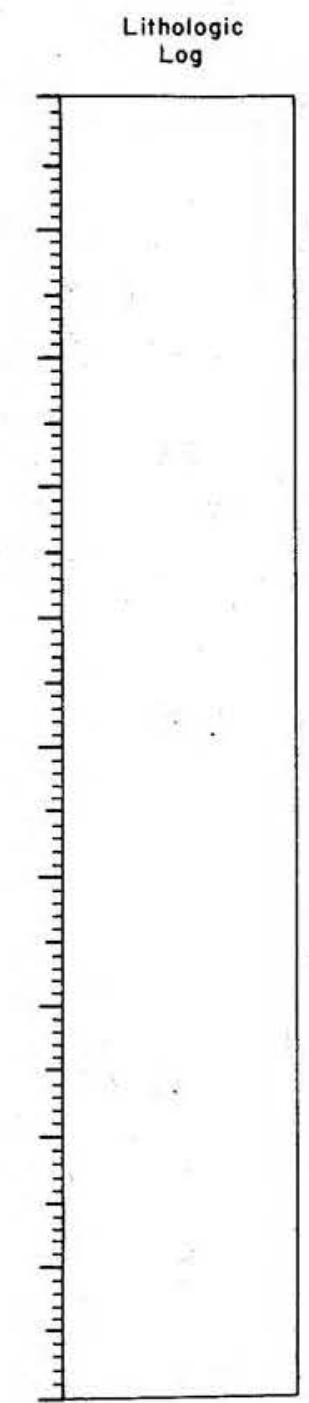

\section{Detailed Description}

264-283 CALC OOZE foram sand $s$ inclined $10^{\circ}$ and concave upward

283-295 CALC OOZB 10YR $6 / 4$ it yellowish brown ith scattered forams $\mathrm{S}$ concave upward CALC $002 \mathrm{E}$
IOYR $7 / 4$ v. pale brown foram sand with scattered pteropods and abund. smell lumpe of lutite lincluded $S$ convex upwar
a

CAIC OOZE

$7.5 \mathrm{Y} 8 / 2$ white

firm lutite less silty than above units firm
S,B
$319-373$
CAIC

319-373
CAIC OOZE

CAIC $00 Z$ E
10YR $7 / 4$ v. pale brown

foram sand with rare pteropods and fey scattered lutite limpo sand appears wasked and disturbed $319-330 \mathrm{~cm}$

373-455

CALC OOZE

10YR $8 / 4$ v. pale brown and $8 / 1$ white

colors above throughout unit lation

455-523

FLON IN

end of core 
390

SHEAR SLIDE DESCRIPTIONS - W.H.O.I. SEDIMENT CORES

$$
\text { Ship: Chain }
$$

Expedition 115

Leg No.

\begin{tabular}{|c|c|c|c|c|c|c|c|c|c|c|c|c|c|c|}
\hline & \multicolumn{13}{|c|}{ Total Core Length } \\
\hline \multirow[b]{3}{*}{ LEVEL } & \multirow[b]{3}{*}{$\begin{array}{c}\text { SEDIMENT } \\
\text { TYPE }\end{array}$} & \multicolumn{13}{|c|}{ ESTIMATED ABUNDANCES (\%) } \\
\hline & & \multicolumn{4}{|c|}{$\begin{array}{c}\text { Inorganic Material } \\
\text { Silt \& Sand }\end{array}$} & & \multicolumn{5}{|c|}{$\frac{\text { Biogenous Mater }}{\text { Calcareous }}$} & \multicolumn{3}{|c|}{$\frac{\text { al }}{\text { Siliceous }}$} \\
\hline & & 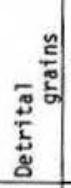 & 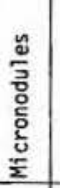 & 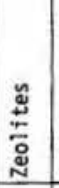 & 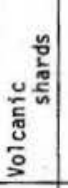 & $\frac{\vec{\pi}}{\sigma}$ & 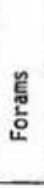 & 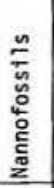 & 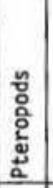 & 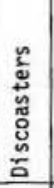 & 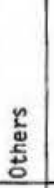 & 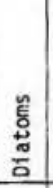 & $\begin{array}{l}\frac{\pi}{2} \\
\frac{\pi}{0} \\
\frac{0}{0} \\
\frac{\pi}{x} \\
\end{array}$ & 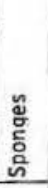 \\
\hline $1 \mathrm{~cm}$ & calc ooxe & $\mathrm{tr}$ & & & & 3 & 55 & 35 & 2 & & 5 & & & \\
\hline $111 \mathrm{~cm}$ & calc ooze & tr & & & & 19 & 8 & 70 & tr & $t r$ & 3 & & & \\
\hline $201 \mathrm{~cm}$ & cal c ooze & $\mathrm{tr}$ & & $\mathrm{tr}$ & & 30 & 3 & 65 & & & 2 & & & \\
\hline $214 \mathrm{~cm}$ & calc ooze & & & & & 15 & & & & & 85 & & & \\
\hline $245 \mathrm{~cm}$ & calc ooze & 1 & & & & 2 & 15 & 80 & $\mathrm{tr}$ & & 2 & & & \\
\hline $290 \mathrm{~cm}$ & calc ooze & 1 & & & & 2 & 10 & 84 & & & 3 & & & \\
\hline $317 \mathrm{~cm}$ & calc ooze & 1 & & & & 6 & 3 & so & & & 40 & & & \\
\hline $400 \mathrm{~cm}$ & calc ooze & tr & & & & 10 & 7 & 80 & $t r$ & 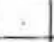 & 3 & & & \\
\hline $450 \mathrm{~cm}$ & calc ooze & & & & & 10 & 7 & 80 & $\mathrm{tr}$ & & 3 & & & \\
\hline $522 \mathrm{~cm}$ & calc ooze & & & & & 10 & 7 & 80 & $\operatorname{tr}$ & & 3 & & & \\
\hline & & & & & & & & & & & & & & \\
\hline & & & & & & & & & & & & & & \\
\hline & & & & & & & & & & & & & & \\
\hline & & & & & & & & & & & & & & \\
\hline & & & & & & & & & & & & & & \\
\hline & & & & & & & & & & & & & & \\
\hline & & & & & & & & & & & & & & \\
\hline & & & & & & & & & & & & & & \\
\hline
\end{tabular}

391

VISUAL CORE DESCRIPTION

Page 1 of 1

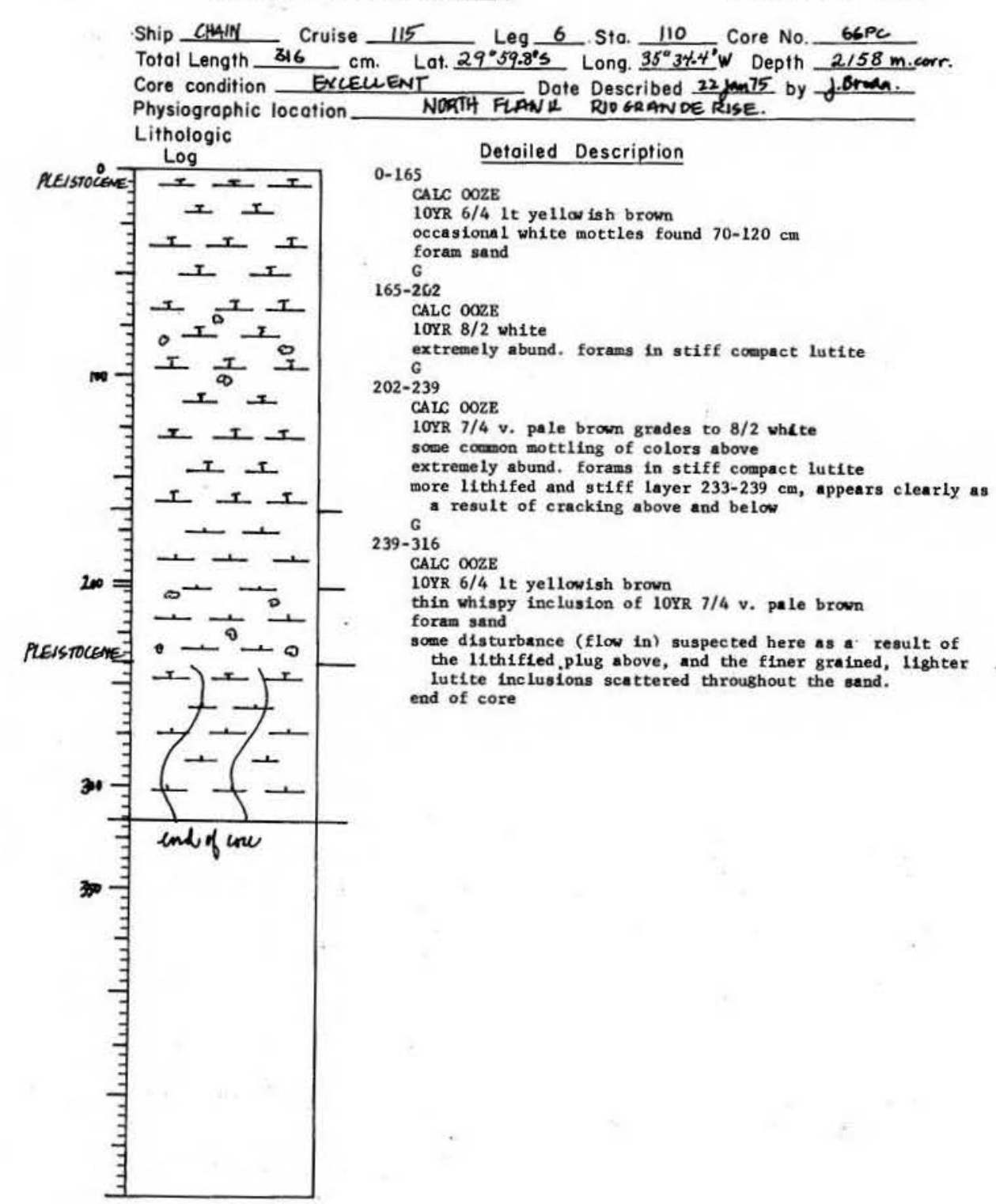




\section{2}

SMEAR SLIDE DESCRIPTIONS - W.H.O.I. SEDIMENT CORES

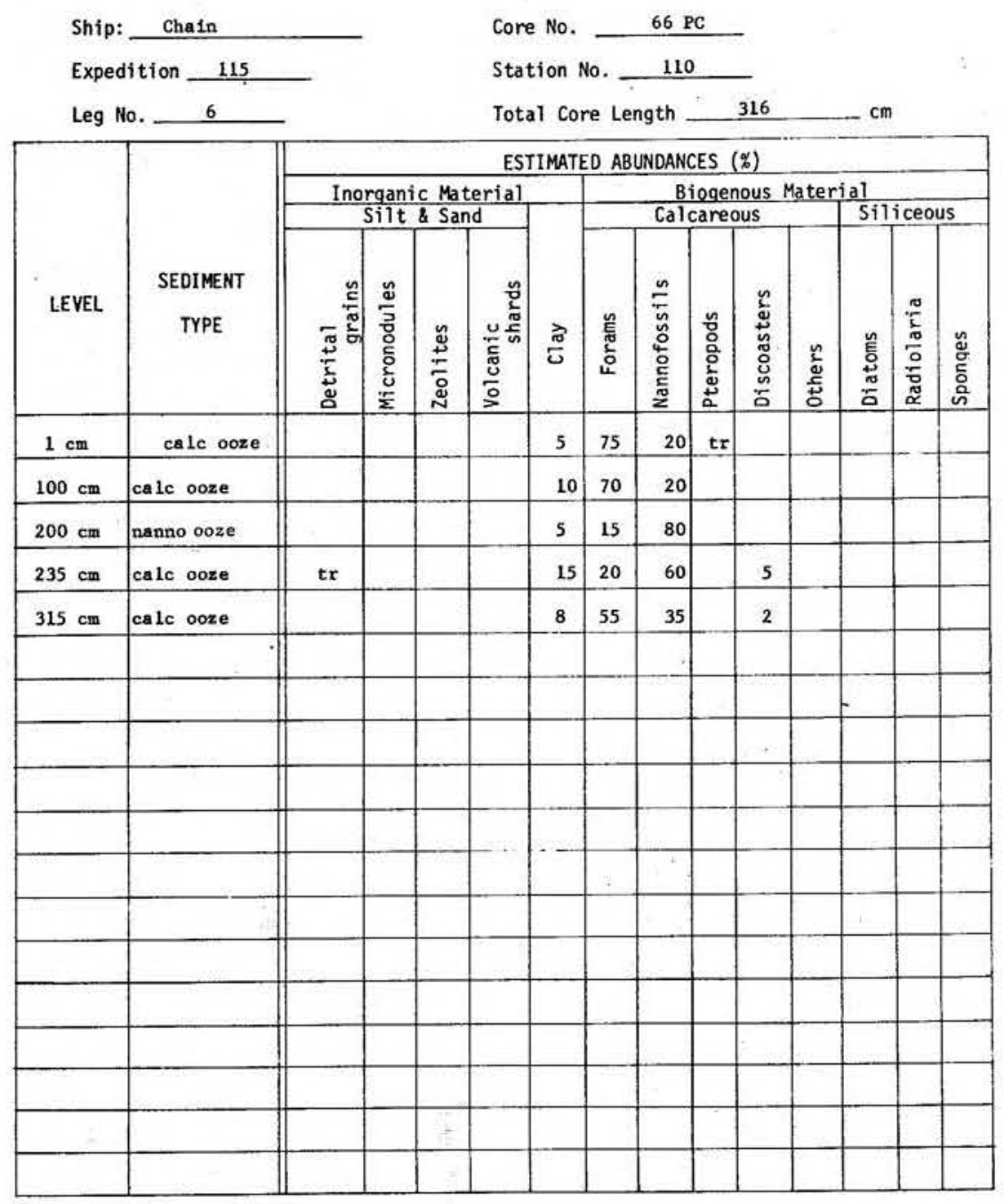

393

VISUAL CORE DESCRIPTION

Page 1 of 1

Ship CHAIN Cruise 115 Leg 6 Sta. III Core No. $67 \mathrm{PC}$ Total Length $716 \mathrm{~cm}$. Lat. $29^{\circ} 57^{\circ} 8^{\prime} \mathrm{S}$ Long. $35^{\circ} 338^{\circ} \mathrm{W}$ Depth $2195 \mathrm{micmu}$ Core condition EXCELLENT Date Described $23 j \mathrm{j} 75$ by $\gamma$. Brode Physiographic location NORTIt FLANK OF RIO GRANDE RISE Lithologic

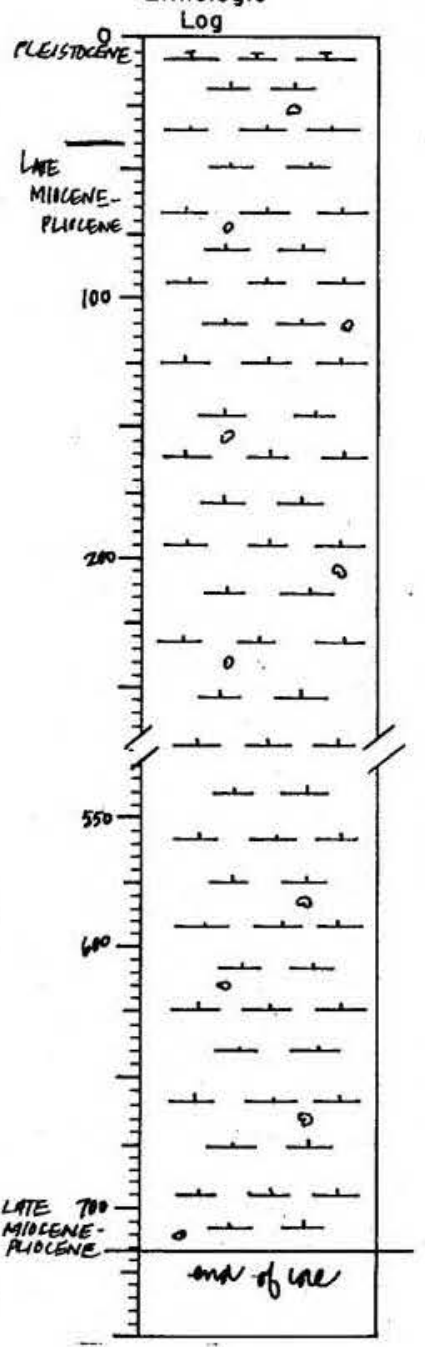

0-716 CALC OOZ

brown grades to $10 \mathrm{YR} 7 / 4 \mathrm{v}$, pale brow to $8 / 2$ whit

sl white and $v$. pale brown mottles

0-11 $\mathrm{cm} \cdot$ extremely abund, forams $11-125 \mathrm{~cm}, \mathrm{grad}$ ually decreasing to abund. then scattered at bottom of end

\section{Detailed Description}




\section{4}

SUEAR SLIDE DESCRIPTIONS - W.H.O.I. SEDIMENT CORES

$$
\text { Ship: Chain }
$$

Expedition 115

Core No. 111

Leg No.

\begin{tabular}{|c|c|c|c|c|c|c|c|c|c|c|c|c|c|c|}
\hline & \multirow{2}{*}{\multicolumn{13}{|c|}{ ESTIMATEO ABUNDANCES $(\xi)$}} \\
\hline \multirow[b]{3}{*}{ LEVEL } & \multirow[b]{3}{*}{$\begin{array}{c}\text { SEDIMENT } \\
\text { TYPE }\end{array}$} & & & & & & & & & & & & & \\
\hline & & \multicolumn{4}{|c|}{$\begin{array}{c}\text { Inorganic Material } \\
\text { Silt \& Sand }\end{array}$} & \multirow[b]{2}{*}{$\frac{\bar{\pi}}{6}$} & \multicolumn{5}{|c|}{$\begin{array}{l}\text { Biogenous Mater } \\
\text { Calcareous }\end{array}$} & \multicolumn{2}{|c|}{ Siliceous } & \\
\hline & & 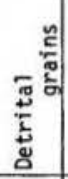 & 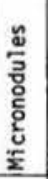 & $\frac{\stackrel{4}{u}}{\frac{ \pm}{2}}$ & 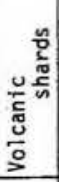 & & 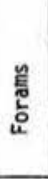 & 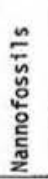 & 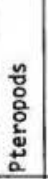 & 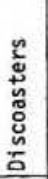 & 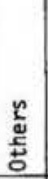 & 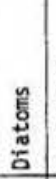 & $\begin{array}{l}\frac{\pi}{L} \\
\frac{\pi}{0} \\
\frac{0}{5} \\
\frac{a}{\alpha} \\
\end{array}$ & 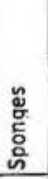 \\
\hline $1 \mathrm{~cm}$ & calc ooze & & & & & 8 & 35 & 50 & $\operatorname{tr}$ & 5 & 2 & & & \\
\hline $100 \mathrm{~cm}$ & calc ooze & $\mathrm{tr}$ & & & & 4 & 15 & 70 & & 8 & 3 & & & \\
\hline $200 \mathrm{~cm}$ & calc ooze & tr & & & & 8 & 10 & 65 & $\operatorname{tr}$ & 12 & 5 & & & \\
\hline $300 \mathrm{~cm}$ & calc ooze & tr & & & & 8 & 15 & 62 & $\mathrm{tr}$ & 12 & 3 & & & \\
\hline $400 \mathrm{~cm}$ & Calc ooze & $\mathrm{tr}$ & & & & 15 & 10 & 60 & tr & 10 & 5 & & & \\
\hline $500 \mathrm{~cm}$ & calc ooze & tr & & & & 5 & 10 & 67 & & 15 & 3 & & & \\
\hline $600 \mathrm{~cm}$ & calc ooze & tr & & & & 5 & 10 & 60 & & 15 & 10 & & & \\
\hline $715 \mathrm{~cm}$ & calc ocze & tr & & & & 10 & 8 & 67 & & 10 & 5 & & & \\
\hline 4 & & & & & & & & & & & & & & \\
\hline & & & & & & & & & & & & & & \\
\hline & & & & & & & & & & & & & & \\
\hline & & & & & & & & & & & & & & \\
\hline & & & & & & & & & & & & & & \\
\hline & & & & & & & & & & & & & & \\
\hline & & & & & & & & & & & & & & \\
\hline & & & & & & & & & & & & & & \\
\hline & & & & & & & & & & & & & & \\
\hline & & & & & & & & & & & & & & \\
\hline
\end{tabular}

395

VISUAL CORE DESCRIPTION

Poge 1 of 1

Ship CHAIN Cruise 115 Leg 6 Sta. 112 Core No. $68 \mathrm{PC}$ Total Length $193 \mathrm{~cm}$. Lat. $29^{\circ} 37.9^{\circ} \mathrm{s}$ Long. $\frac{33^{\circ} 33.6^{6} \mathrm{w}}{\mathrm{W}}$ Depth $2192 \mathrm{mumr}$. Core condition ExcEuENr Date Described 23 junts by flowa Physiographic location R10 GRANDE RISE

Lithologic

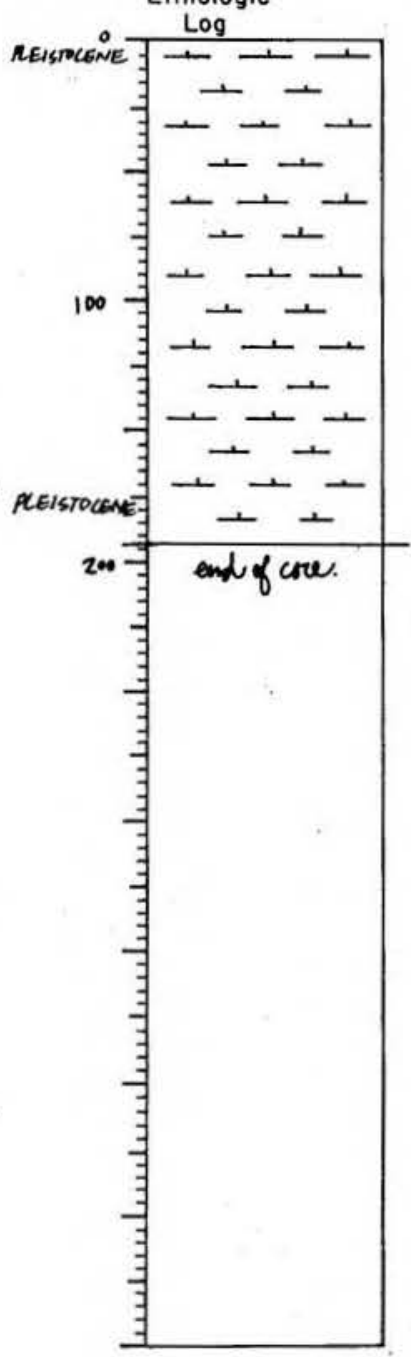

CALC OOZE

1. faint whiter mottles scattered throughout

$0-10 \mathrm{~cm}$ somewhat washed foram

end of core
$0-193$ Detailed Description 
408

SMEAR SLIDE DESCRIPTIONS - W.H.O.I. SEDIMENT CORES

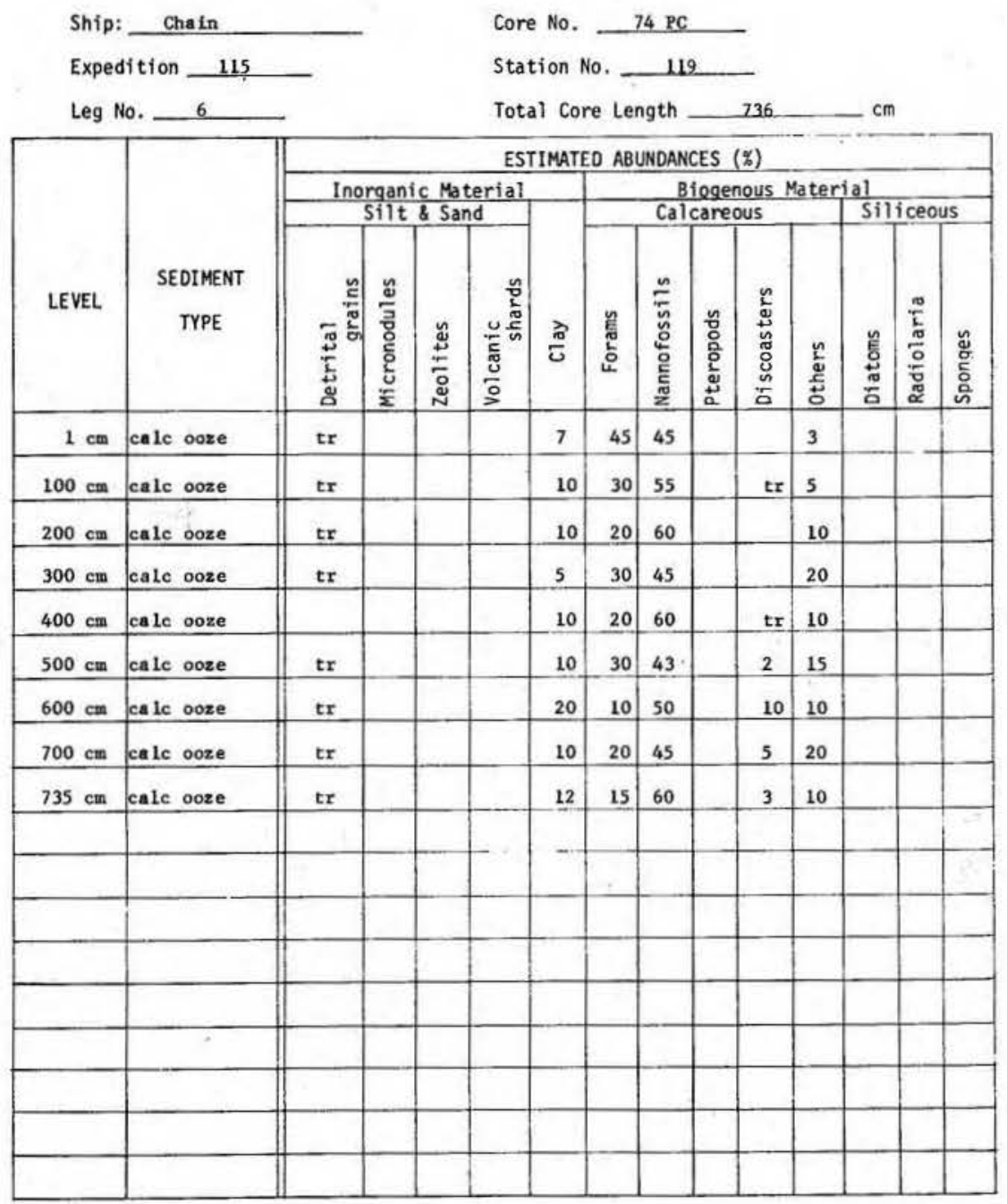

409

VISUAL CORE DESCRIPTION

Page 1 of 2

Ship GHAiN Cruise 115 Leg 6 Sta. 120 Core No. 75PC Total Length $686 \mathrm{~cm}$. Lat. $29^{\circ} 588^{\circ} \mathrm{s}$ Long. $35^{\circ} 32.9^{\circ} \mathrm{W}$ Depth $2260 \mathrm{Nccur}$ Core condition EXCELLEN Date Described 27 yas 75 by $\gamma$ Brode Core condition Lithologic Log
PLEISTCLENE $=-90$ Detailed Description
CALC OOZE $6 / 41 \mathrm{t}$ yellowish brown 10YR $6 / 4$ 1t yellowish brown occasional large white mottles found $40-60 \mathrm{~cm}$ extremely abund. forams in finm silty lutite

$\rightarrow+1$

$\exists+\bar{a}+\bar{a}$.

1 $\rightarrow$

$100=$ $+1$ $+\frac{1}{\infty}+$ $90-252$

OYR $6 / 4$ it yellowlsh brown grades slowly to $7 / 4 \mathrm{v}$. pale brow firm s1 silty lutite, scattered forams;a few black flecks 252-423

$\exists+1+$

두

두

10YR $8 / 2$ white grades to $8 / 4$ v. pale brown

1 white mottles and occesional black streaks and flecks v plastic sl silty lutite

3-429

CAIC $002 \mathrm{E}$

CALC OOZE

foram sand, somewhint graded

graded bedding

$+1+$

$+-1$

$+ \pm+$

$+1$

$+1+$

CALC OOZE

IOYR $8 / 2$ white

matrix finn si silty lutite with scattered forams

ote: a remarkable combination of elements exist in this unit pelecypod ohell frapments, large benthic forems, a fragent of $v$. brittle Mn pavement, a pyritised burrow, la rge $G$ reworked Oligocene forams and Bocene (?) nannos

444-45

CALC OOZE

LOYR $8 / 4$ v. pale brown

extensive white mottling at unit basal contact

inm sl silty lutite, scattered forams

456-582

CAlc 098

1OYR $8 / 1$ white grades to $8 / 2$ white

cormon faint white mottles throughout

moist amooth lutite

再 - -

$\exists=\frac{1}{x} \pm 1$

12 +

MOCEEE

工十 - 10YR $6 / 4$ 1t yellowlsh brown

v. s1 v. pale brown mottling

firm si silty lutite, more lithified $582-589 \mathrm{~cm}$

$500+\frac{1}{\infty}+\frac{1}{1}$ 


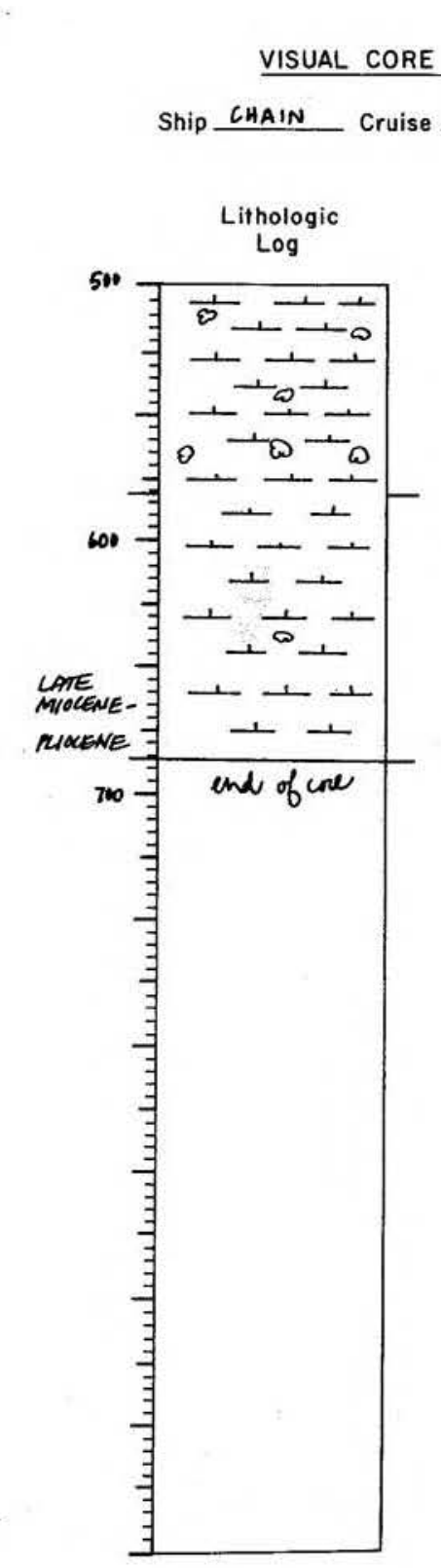

Page 2 of 2

$\exists \rightarrow$

$\exists+1$

$+-$

\begin{abstract}
115 Leg 6 Sta. 120 Core No. $75 \mathrm{PC}$
\end{abstract}
VISUAL CORE DESCRIPTION 410

\section{Detailed Description}

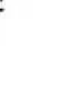




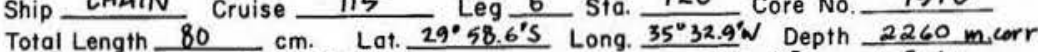
Core condition EXLEUENT. Date Described 21jen 15 by forded. Physiographic location_NURTH FLANK ... RIO GRANDE RISE.

Lithologic

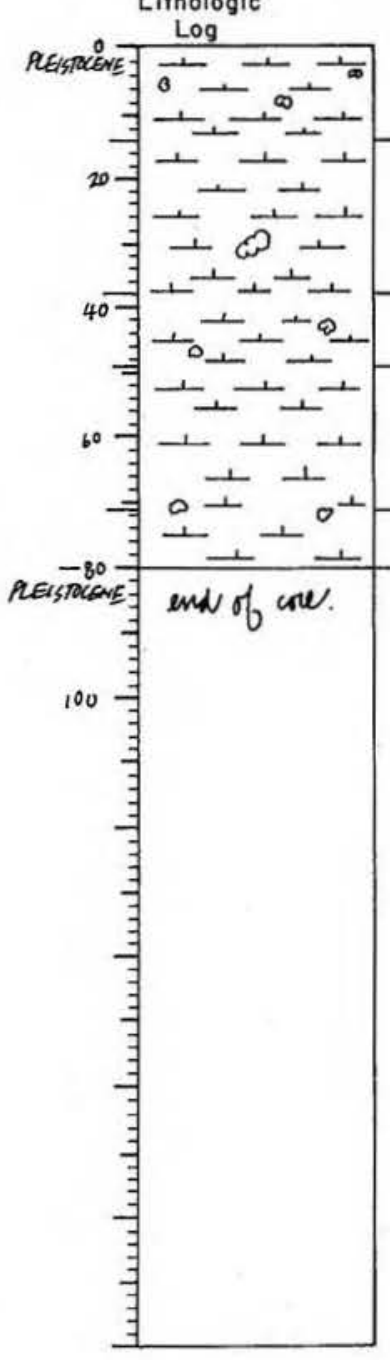

\section{Detoiled Description}

$$
\text { CALC OOZE }
$$

brown abund. forams throughout, silty lutite $G$ mottled

14-38

CALC OOZE

Coe large concentric $v$, pale brown mottle at $32 \mathrm{~cm}$

scattered forams in firm sl silty lutite $\mathrm{S}$ concave upward $38-49$ CALC OZE

10YR $7 / 4 \mathrm{v}$. pale brown

s1 faint le gray wotting $38-41 \mathrm{~cm}$

6 s1 mottled

CAIC OOZE

10YR $6 / 4$ 1t yellowish brown

si v. pale brown motting at

scattered foram in sl silty lutite

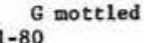

$1-80$ CALC OOZE

fark it vellowish brown mottling found sl throughout abund. forams in silty lutite

end of core

Note: Due to similiax combinations of Iithologies found in the sample it is suspected to be an example of a

tion 0-38 cm, repenetration $38-80 \mathrm{~cm}$

SMEAR SLIDE DESCRIPTIONS - W.H.0.1. SEDIMENT CORES

Ship: Chain

Expedition 115

Leg No. 6
Core No. 75 PG

Station No. 120

Total Core Length $\quad 80$ ESTIMATED ABUNDANCES (§)

\begin{tabular}{|c|c|c|c|c|c|c|c|c|c|c|c|c|c|c|}
\hline \multirow[b]{4}{*}{ LEVEL } & \multirow[b]{4}{*}{$\begin{array}{c}\text { SEDIMENT } \\
\text { TYPE }\end{array}$} & \multicolumn{13}{|c|}{ ESTIMATED ABUNDANCES (\%) } \\
\hline & & \multirow{2}{*}{\multicolumn{4}{|c|}{$\begin{array}{c}\text { Inorganic Material } \\
\text { Silt \& Sand }\end{array}$}} & \multirow[b]{3}{*}{$\frac{\pi}{0}$} & \multirow{2}{*}{\multicolumn{5}{|c|}{$\begin{array}{l}\text { biogenous mate } \\
\text { Calcareous }\end{array}$}} & & & \\
\hline & & & & & & & & & & & & \multicolumn{3}{|c|}{ Siliceous } \\
\hline & & 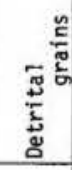 & 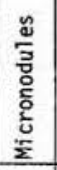 & 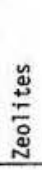 & 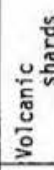 & & 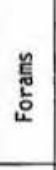 & 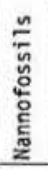 & 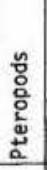 & 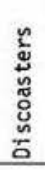 & 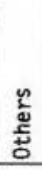 & 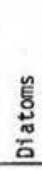 & 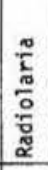 & 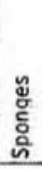 \\
\hline $1 \mathrm{~cm}$ & calc ooze & 1 & tr & & & 8 & 40 & 50 & & & 1 & & & \\
\hline $23 \mathrm{~cm}$ & calc ooze & 3 & 1 & & & 12 & 13 & 65 & & & 6 & & & \\
\hline $56 \mathrm{~cm}$ & calc ooze & 1 & & & & 15 & 19 & 60 & & & 5 & & & \\
\hline $40 \mathrm{~cm}$ & calc ooze & 2 & & & & 7 & 40 & 50 & & & 1 & & & \\
\hline $79 \mathrm{~cm}$ & calc ooze & 1 & & & & 15 & 20 & 60 & & & 4 & & & \\
\hline - & . & & & & & & & & & & & & & \\
\hline & & & & & & & & & & & & & & \\
\hline & & & & & & & & & & & & & & \\
\hline & & & & & & & & & & & & & & \\
\hline & & & & & & & & & & & & & & \\
\hline & & & & . & & & & & & & & & & \\
\hline & & & & & & & & & & & & & & \\
\hline & & & & & & & & & & & & & & \\
\hline & & & & & & & & & & & & & & \\
\hline & & & & & & & & & & & & & & \\
\hline & & & & & & & & & & & & & & \\
\hline & & & & & & & & & & & & & & \\
\hline & & & & & & & & & & & & & & \\
\hline
\end{tabular}


Ship CHAIN Cruise 115 Leg 6 Sta. 121 Core No. 76PC Total Length $671 \mathrm{~cm}$. Lat. $29^{\circ} 56.2^{\prime} \mathrm{S}$ Long. $35^{\circ} 33.0^{\circ} \mathrm{W}$ Depth $2315 \mathrm{~m} \cdot \mathrm{cor}$ Core condition EXCELENT Date Described $29 \mathrm{jan} 75$ by $\mathrm{f}$. ordex.

Physiographic location NRRH FLANK, R10 GRANDE RISE.

Lithologic

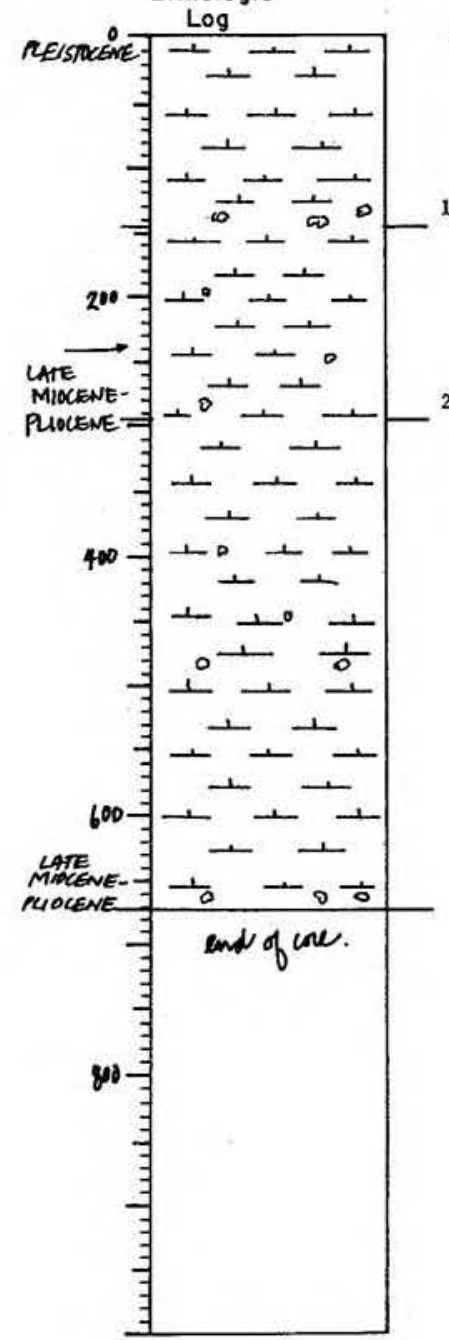

\section{Detailed Description}

$0-145$

CAIC OOZE

common v. pale brown mottling $0-40 \mathrm{~cm}, 132-145 \mathrm{~cm}$ common v. pale brown motting $0-4-145 \mathrm{~cm}, 132-145 \mathrm{~cm}$, scattered elsewhere in sl silty lutite 145-295 mottied

CAYR $7 / 4$ v. pale brown and $8 / 2$ white

ol white mottilng found occasionally throughout

a lutite

color grades in two cycles from v. pale brown to white then 95-671

CALC OOZE

1OYR $8 / 2$ white

10 600-67

firm plastic lutite $y i$ th forams abund. $383-500 \mathrm{~cm} ;$ scattered

throughout unit

end of core
SMEAR SLIDE DESCRIPTIONS - W.H.0.1. SEDIMENT CORES

Ship: Chain

Expedition 115

Leg No. 6

Core No. 76 PC

Station No. 121

Total Core Length $\quad 671$

\begin{tabular}{|c|c|c|c|c|c|c|c|c|c|c|c|c|c|c|}
\hline \multirow[b]{3}{*}{ LEVEL } & \multirow[b]{3}{*}{$\begin{array}{c}\text { SEDIMENT } \\
\text { TYPE }\end{array}$} & \multicolumn{13}{|c|}{ ESTIMATED ABUNDANCES (\%) } \\
\hline & & \multicolumn{4}{|c|}{$\begin{array}{c}\text { Inorganic Material } \\
\text { Silt \& Sand }\end{array}$} & & \multicolumn{5}{|c|}{$\begin{array}{l}\text { Biegenous Mate } \\
\text { Calcareous }\end{array}$} & \multicolumn{3}{|c|}{$\frac{\text { al }}{\text { Siliceous }}$} \\
\hline & & 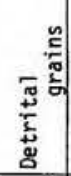 & 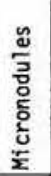 & 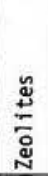 & 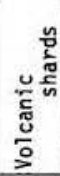 & $\frac{\pi}{0}$ & 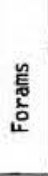 & 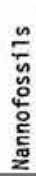 & $\begin{array}{l}n \\
0 \\
0 \\
0 \\
0 \\
0\end{array}$ & 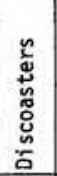 & 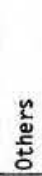 & 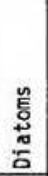 & 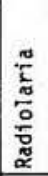 & 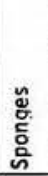 \\
\hline $1 \mathrm{~cm}$ & calc ooze & $\mathrm{tr}$ & & & & 10 & 30 & 55 & & & 5 & & & \\
\hline $100 \mathrm{~cm}$ & calc ooze & $t x$ & 2 & & $t r$ & 13 & 30 & so & & & 5 & & & \\
\hline $200 \mathrm{~cm}$ & calc ooze & 1 & & & $t r$ & 3 & 25 & 65 & & $t x$ & 6 & & & \\
\hline $300 \mathrm{~cm}$ & calc ooze & $t r$ & & & & 10 & 15 & 62 & & 5 & 8 & & & \\
\hline $400 \mathrm{~cm}$ & calc ooze & 2 & & & & 10 & 15 & 57 & & 10 & 6 & & & \\
\hline $500 \mathrm{~cm}$ & calc ooxe & $t r$ & & & & 12 & 15 & 60 & & 8 & 5 & & & \\
\hline $600 \mathrm{~cm}$ & calc ooxe & tr & & & & 15 & 20 & 56 & & 5 & 4 & & & \\
\hline $670 \mathrm{~cm}$ & calc ooze & tr & & & & 15 & 10 & 61 & & 10 & 4 & & & \\
\hline & & & & & & & & & & & & & & \\
\hline & & & & & & & & & & & & & & \\
\hline & & & & & & & & & & & & & & \\
\hline & & & & & & & & & & & & & & \\
\hline & & & & & & & & & & & & & & \\
\hline & & & & & & & & & & & & & & \\
\hline & & & & & & & & & & & & & & \\
\hline & & & & & & & & & & & & & & \\
\hline & & & & & & & & & & & & & & \\
\hline & & & & & & & & & & & & & & \\
\hline
\end{tabular}


416

VISUAL CORE DESCRIPTION

Page 1 of 1

Ship CHAIN Cruise 115 Leg 6 sto. 122 Core No. $77 \mathrm{PC}$

Total Length $423 \mathrm{~cm}$. Lat. $299^{\circ} 57.7^{\circ} \mathrm{S}$ Long. $35 \cdot 34.7^{\circ} \mathrm{W}$ Depth $2330 \mathrm{~m}$. Com

Core condition EXCEUENT. Date Described 29 jen 75 by j.brda.

Physiogrophic locotion NORTH FLANK RIO GRANDE RISE

Lithologic

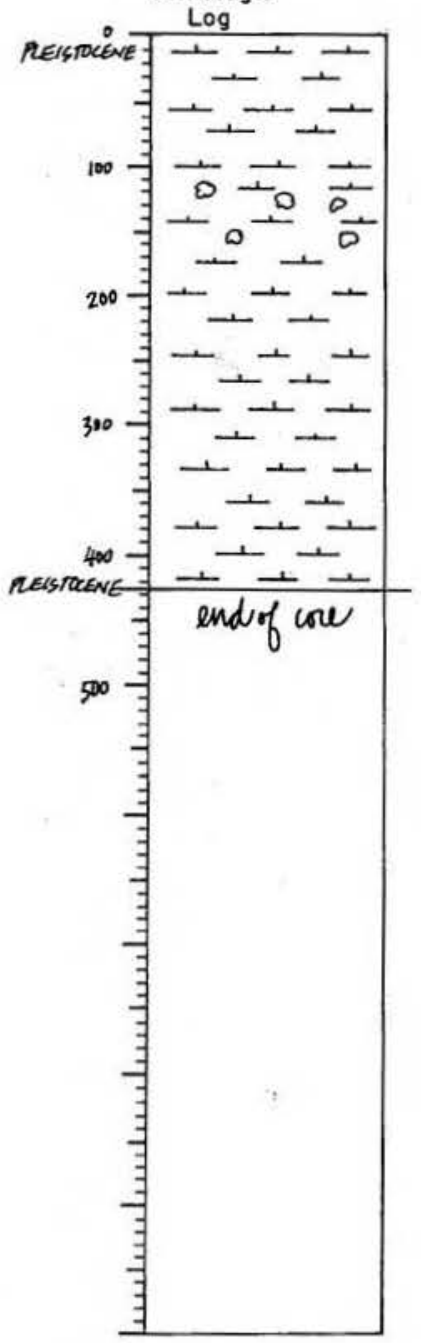

0-423 CALC OOZE

10YR $6 / 4$ 1t yellowish brown grades to $7 / 4 \mathrm{v}$, pale brown

foram sand grades to firm silty lutite with extremely abund.

forams
end of core

\section{Detailed Description}

417

SMEAR SLIDE DESCRIPTIONS - W.H.O.I. SEDIMENT CORES

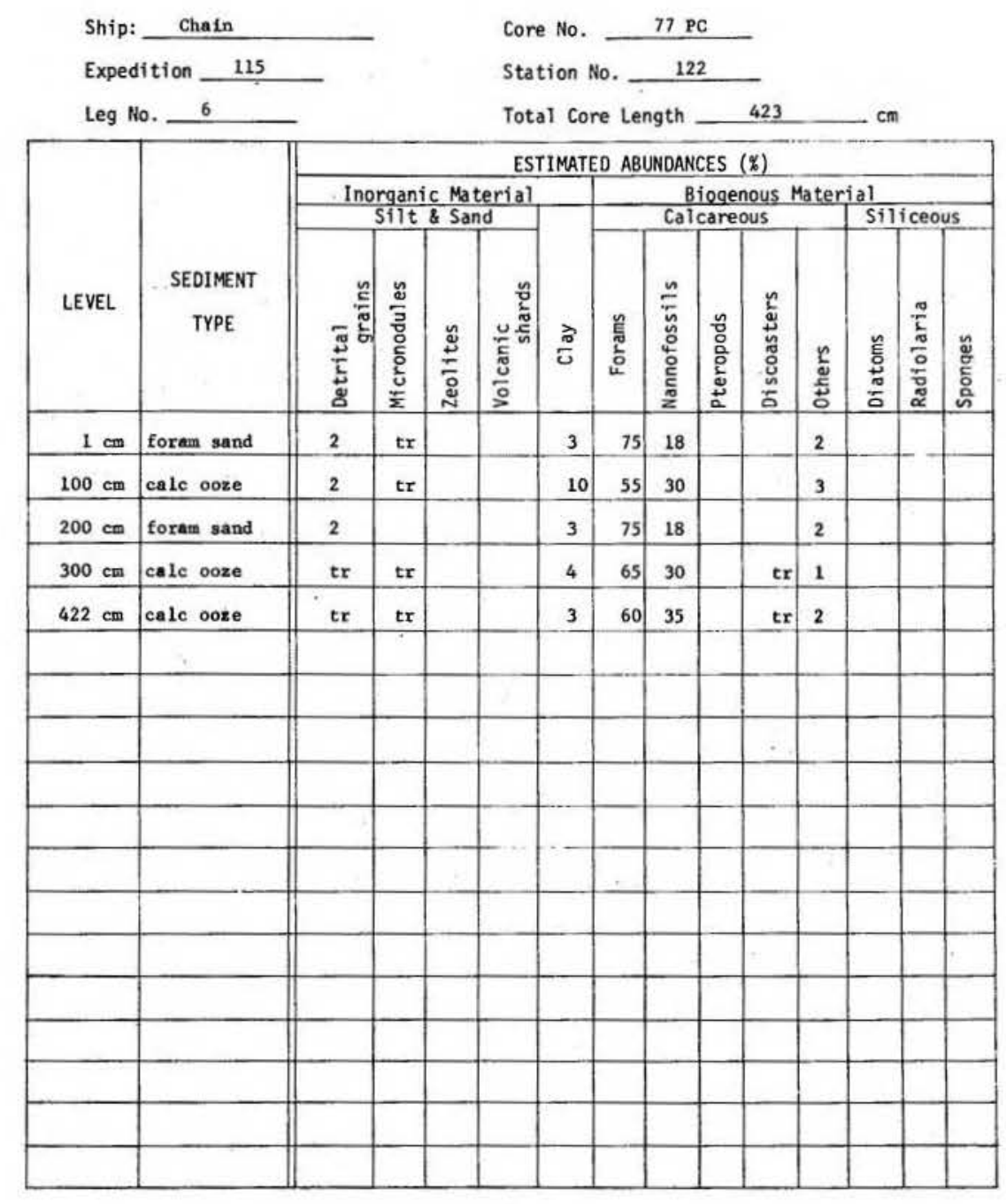


418

VISUAL CORE DESCRIPTION

Ship CHAIN Cruise 115 Leg 6 Sto. 123 Core No. 78P

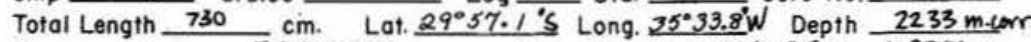
Core condition EXCELLENT Date Described 30 yw 75 by 7 .3MPA. Physiogrophic location NURTH PLANK: R1O GRANDE RISE

Lithologic

\section{Detailed Description}

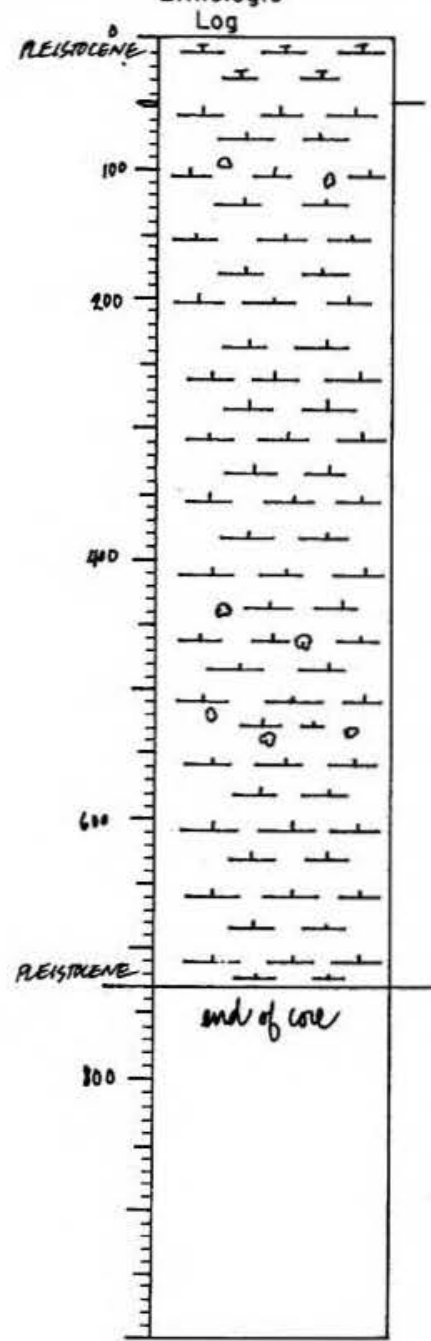

Page 1 of 1

419

SMEAR SLIDE DESCRIPTIONS - W.H.O.I. SEDIMENT CORES

CALC OOZE

OYR $7 / 4$ v. pale brow

top $15 \mathrm{~cm}$ disturbed and washed

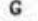

cA

COYR $7 / 4 \mathrm{v}$. pale brown grades internittently and irregularly chrougho wh $10 \mathrm{n}$ s/2 white

farnt white mothere $90-120 \mathrm{~cm}, 430-570 \mathrm{~cm}$

throughout nd of core

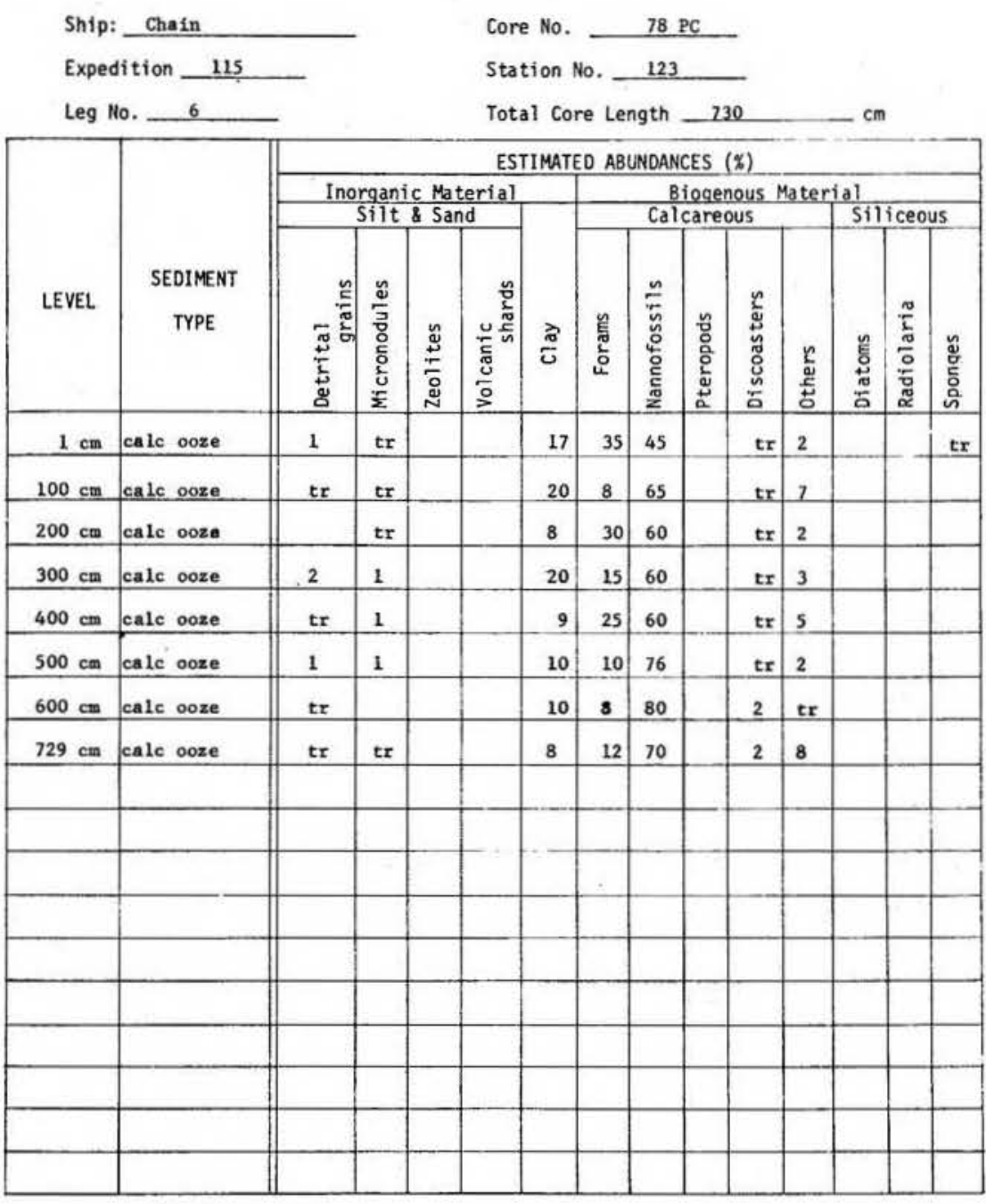


Ship CHAIN Cruise 115 Leg 6 Sto. 124 Core No. 79 PC Total Length $682 \mathrm{~cm}$. Lat. $29^{\circ} 57.6^{\circ} \mathrm{S}$ Long. $35^{\circ} 33.7^{\circ} \mathrm{w}^{\circ}$ Depth $2214 \mathrm{micom}$ Core condition ExLEUENT

Lithologic

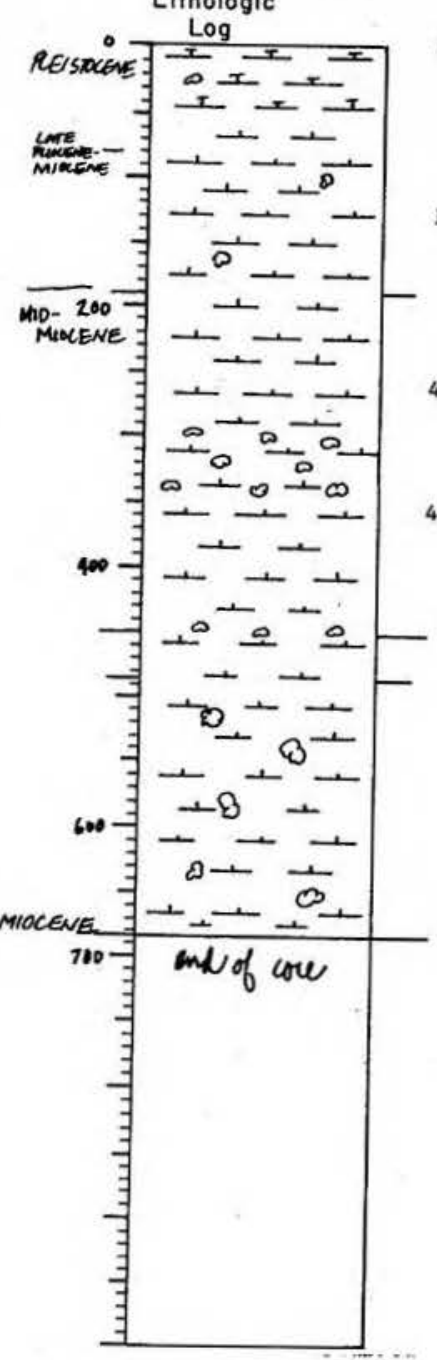

\section{Detailed Description}

CALC OOZE

$10 Y R 7 / 4 \mathrm{v}$. pale brown

. lutite with v. abund. foram

190-450

CALC OOZE

10XR $6 / 4$ it yellorish brown

abund. v. pale brown mottling $290-340 \mathrm{~cm}, 440-450 \mathrm{~cm}, \mathrm{v}$. gmall pale brown mottles scattered slightly otherwise
abund. forams in finm slightky silty lutite

$450-485$

CAIC OOZE

10YR $8 / 1$ white

abund. forases in

S concave upward

CAIC OOZE

10YR $7 / 4$ v. pale brown to $8 / 4 \mathrm{v}$, pale brown

large white mottles found comonly throughout, varying

hues of two colors above alternating

firm compact lutite with abund. forams

end of core
Leg No. 6

\begin{tabular}{|c|c|c|c|c|c|c|c|c|c|c|c|c|c|c|}
\hline \multirow[b]{3}{*}{ LEVEL } & \multirow[b]{3}{*}{$\begin{array}{c}\text { SEDIMENT } \\
\text { TYPE }\end{array}$} & \multicolumn{13}{|c|}{ ESTIMATEO ABUNDANCES ( $(\%)$} \\
\hline & & \multicolumn{4}{|c|}{$\frac{\text { Inorganic Material }}{\text { Silt \& Sand }}$} & & \multicolumn{5}{|c|}{$\begin{array}{l}\text { Biogenous Mater } \\
\text { Calcareous }\end{array}$} & \multicolumn{3}{|c|}{$\frac{\text { a) }}{\text { siliceous }}$} \\
\hline & & 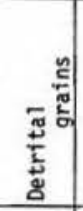 & 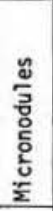 & 岕 & 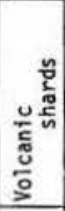 & $\frac{\pi}{6}$ & 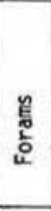 & 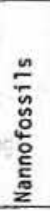 & 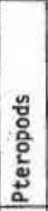 & 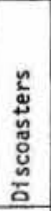 & 点 & 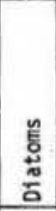 & 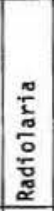 & 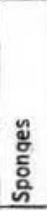 \\
\hline $1 \mathrm{~cm}$ & Calc ooze & tr & tr & & & 5 & 75 & 15 & & 3 & 2 & & & \\
\hline $100 \mathrm{~cm}$ & calc ooze & 1 & 1 & & & 12 & 3 & 70 & & 8 & 5 & & & \\
\hline $200 \mathrm{~cm}$ & calc ooze & tr & & & & 30 & 2 & 60 & & 3 & 5 & & & \\
\hline $300 \mathrm{~cm}$ & calc ooxe & 2 & & & & 20 & 3 & 62 & & 8 & 5 & & & \\
\hline $400 \mathrm{~cm}$ & calc ooxe & tr & & & & 30 & 2 & 60 & & 3 & 5 & & & \\
\hline $490 \mathrm{~cm}$ & calc ooze & 1 & 1 & & & 12 & 3. & 70 & & 8 & 5 & - & & \\
\hline $590 \mathrm{~cm}$ & calc ooze & $t x$ & & & & 20 & 2 & 70 & $\cdot$ & 3 & 5 & & & \\
\hline $682 \mathrm{im}$ & calc ooze & 2 & $\mathrm{tr}$ & & & 12 & 3 & 70 & & 8 & 5 & & & \\
\hline & & & & & & & & & & & & & & \\
\hline & & & & & & & & & & & & & & \\
\hline & & & & & & & . & & & & & & & \\
\hline & & & & & & & & & & & & & & \\
\hline & & & & & & & & & & & & & & \\
\hline & - & & & & & & & & & & & & & \\
\hline & & & & & & & & & & & & & & \\
\hline & & & & & & & se & & & & & & & \\
\hline & & & & & & & 2 & & & & & & & \\
\hline & & & & & & & & & & & & & & \\
\hline
\end{tabular}

Ship: _Chain

Expedition 115

SMEAR SLIDE DESCRIPTIONS - W.H.0.1. SEDIMENT CORES

Core No. $79 \mathrm{PC}$

Station No. 124

Total Core Length $682 \quad \mathrm{~cm}$ 
418

VISUAL CORE DESCRIPTION

Ship CHAIN Cruise 115 Leg 6 Sta. 123 Core No. 78P Total Length $730 \mathrm{~cm}$. Lat. $29^{\circ} 57.1^{\prime \prime S}$ Long. $35^{\circ} 33.8^{\circ} \mathrm{W}$ Depth $2233 \mathrm{~m}$.corr Core condition EXCELLENT Dote Described 30 yen 75 by $\gamma$. BRODA. Physiogrophic locotion NORTH FLANK: RIO GRANDE RISE.

Lithologic

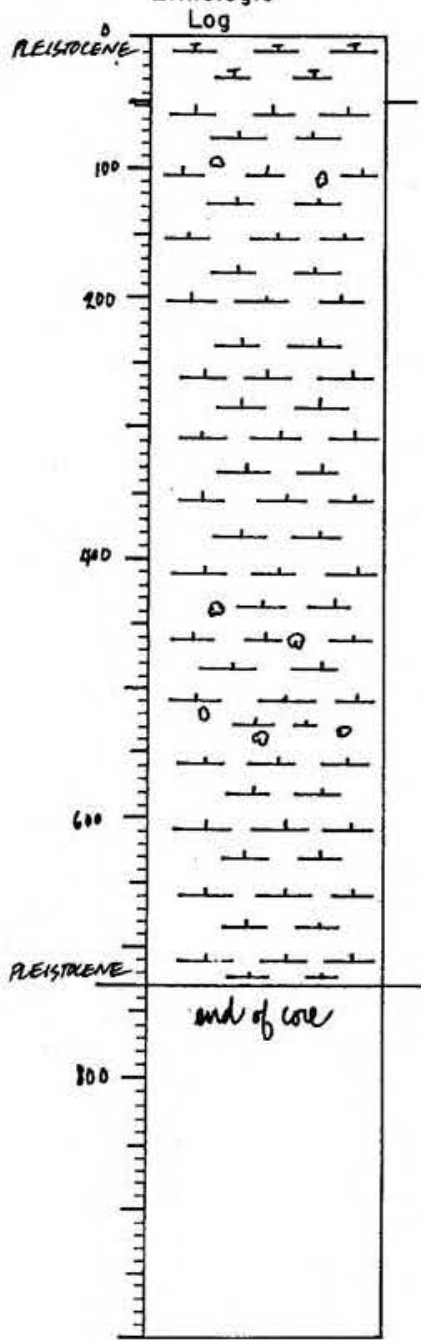

Page 1 of 1

Detailed Description

419

SMEAR SLIDE DESCRIPTIONS - W.H.O.I. SEDIMENT CORES

CALC OOZE

10YR $7 / 4$ v. pale brow

t5 $15 \mathrm{~cm}$ disturbed and washed

CALC OOZE

IOYR $7 / 4 \mathrm{v}$. pale brown grades intermittently and irregularly

faint white mottling scattered, $90-120 \mathrm{~cm}, 430-570 \mathrm{~cm}$

te with extremely abund. forams throughout nd of core

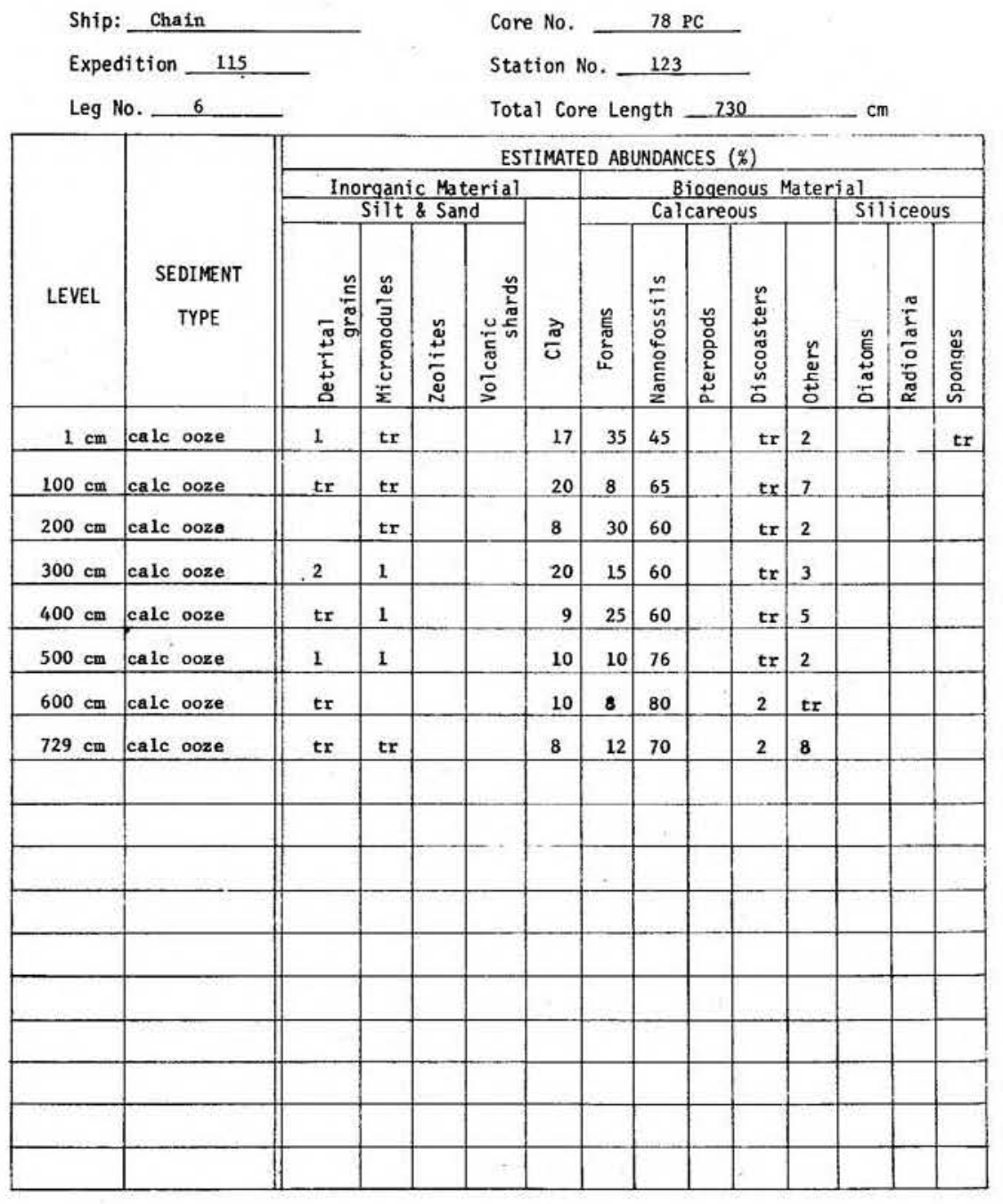




\section{1}

Ship CHAIN Cruise 115 Leg 6 Sto. 124 Core No. 79 PC Total Length $682 \mathrm{~cm}$. Lat. $29^{\circ} 57.6^{\circ} \mathrm{s}$ Long. $35^{\circ} 33.7^{\circ} \mathrm{W}$ Depth 2214 micorr Core condition ExCEUENT Date Described 24 Feb75 by Jorida. Physiographic location_NIRTH FLANK: RIO GRPNDE RISE

Lithologic

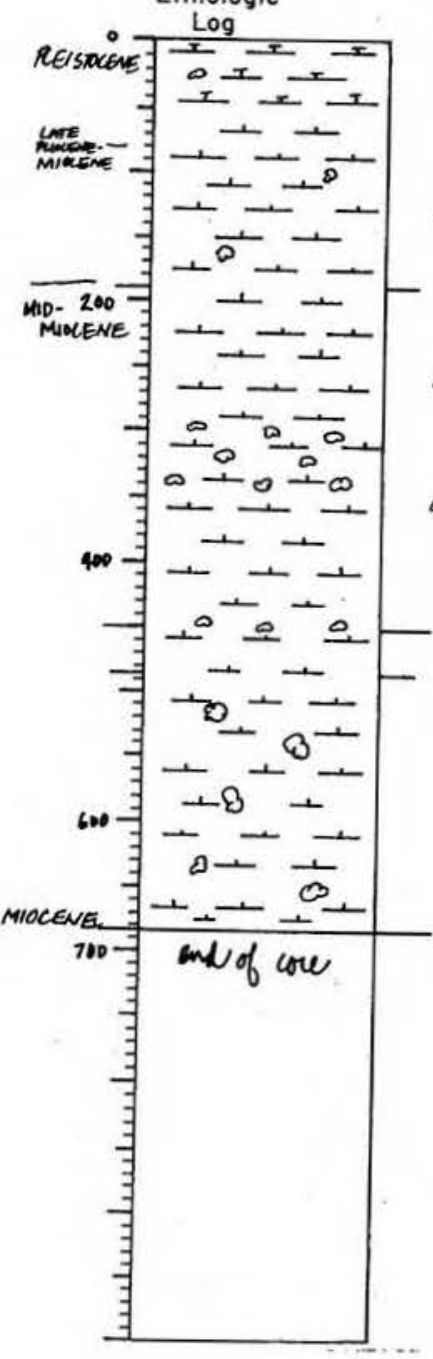

\section{Detailed Description}

CALC OOZE

10YR $7 / 4$ v. pale brown

. for sa $040 \mathrm{~cm}$ grades to silty lutite with v. abund. fora

$190-450$

$$
\text { CALC OOZE }
$$

OYR $6 / 4$ 1t yellowish brown

abund. v. pale brown mottling $290-340 \mathrm{~cm}, 440-450 \mathrm{~cm}$, v, small pale brown mottles scattered slightly otherwise abund. forams in firm slightky silty lutite 450-485 485 CALC OOZE
1OYR $8 / 1$ wh1te

irm silty lutite $S$ concave upward

485-682

CALC OOZE

OXR $7 / 4 \mathrm{v}$. pale brown to $8 / 4 \mathrm{v}$. pale brown

hues of two colors above alterly throughout, varyin ini compact lutite with abund, forams 
422

VISUAL CORE DESCRIPTION

Page 1 of 1

Ship CHAIN Cruise 115 Leg 6 sta. 124 Core No. $79 P G$ Total Length $24 \mathrm{~cm}$. Lat. $29^{\circ} 57.6^{\prime} \mathrm{S}$ Long. $35^{\circ} 33.7 \mathrm{~W}$ Depth 2214 mecorr.

Core condition EXCEUENT Date Described 24 Fob 15 by fbleda.

Physiographic location NORTH FLANK: R10 GRANDE RISE Lithologic

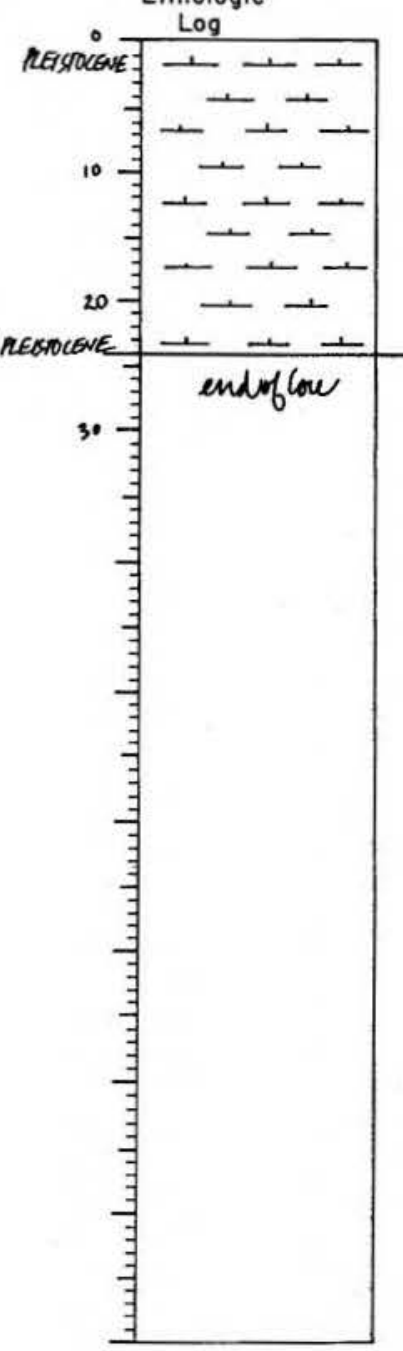

423

SMEAR SLIDE DESCRIPTIONS - W.H.0.1. SEDIMERT CORES

Detailed Description
Ship: Chain

Expedition 115

Leg No. 6
79 PG

Station No. 124

Tota1 Core Length 24 ESTIMATEO ABUNDANCES (\%) 10YR $7 / 4$ v. pale brown core appears aconets washed $0-10 \mathrm{~cm}$ end of core

\begin{tabular}{|c|c|c|c|c|c|c|c|c|c|c|c|c|c|c|}
\hline \multirow[b]{3}{*}{ LEVEL } & \multirow[b]{3}{*}{$\begin{array}{c}\text { SEDIMENT } \\
\text { TYPE }\end{array}$} & \multicolumn{13}{|c|}{ ESTIMATEO ABUNDANCES (\%) } \\
\hline & & \multicolumn{4}{|c|}{$\begin{array}{c}\text { Inorganic Material } \\
\text { Silt \& Sand }\end{array}$} & \multirow[b]{2}{*}{ 高 } & \multicolumn{5}{|c|}{$\begin{array}{l}\text { Biogenous Mater } \\
\text { Calcareous }\end{array}$} & \multicolumn{3}{|c|}{$\frac{\text { al }}{\text { Siliceous }}$} \\
\hline & & 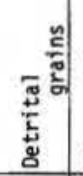 & 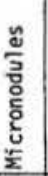 & 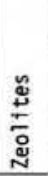 & 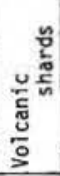 & & 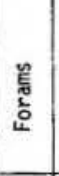 & 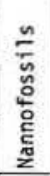 & 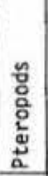 & 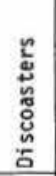 & 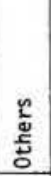 & 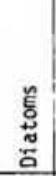 & $\frac{\pi}{\frac{\pi}{0}}$ & 气ूँ \\
\hline $1 \mathrm{~cm}$ & alc ooze & tr & & & & 10 & 65 & 20 & & 3 & 2 & & & \\
\hline $22 \mathrm{~cm}$ & alc ooze & tr & tr & & & 10 & 20 & 65 & & 2 & 3 & & & \\
\hline & & & & & & & & & & & & & & \\
\hline & & & & & & & & & & & & & & \\
\hline & & . & & & & & & & & & & & & \\
\hline & & & & & & & & & & & & & & \\
\hline & & & & & & & & & & & & & & \\
\hline & & & & & & & & & & & & & & \\
\hline & & & & & & & & & & & & & & \\
\hline & & & & & & & & & & & & & & \\
\hline & & & & & & & & & & & & & & \\
\hline & & & & & & & & & & & & & & \\
\hline & & & & & & & & & & & & & & \\
\hline & & & & & & & & & & & & & & \\
\hline & & & & & & & & & & & & & & \\
\hline & & & & & & & & & & & & & & \\
\hline & & & & & & & & & & & & & & \\
\hline & & & & & & & & & & & & & & \\
\hline
\end{tabular}


424

VISUAL CORE DESCRIPTION

Page 1 of 1

\section{5}

SMEAR SLIDE DESCRIPTIONS - W.H.O.I. SEDIMENT CORES

Ship CHAIN Cruise 115 Leg 6 Sta. 125 Core No. BOPC Total Length $462 \mathrm{~cm}$. Lot. $29^{\circ} 56^{\circ} 3^{\circ} \mathrm{S}$ Long. $35^{\circ} 33.6 \mathrm{~N}$ Depth 2293 corr.meters. Core condition EXCEUENT Date Described 25 Ees.75 by f. Buma Physiographic location NORTH FLANK: RIO GRANDE RISE

Lithologic

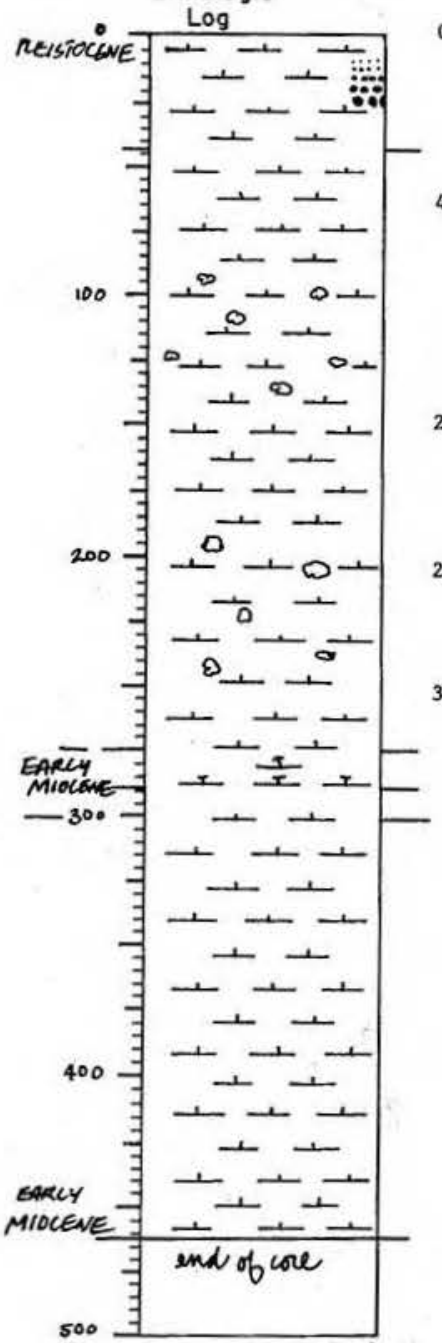

Detailed Description

CAIC OOZE

pale brom

graded pteropod ooze, silt size fragments to $1.5 \mathrm{~cm}$ long one v. well $\mathrm{S}$ textural

43-275

CAIC DOZE

OYR $6 / 4$ 1t yellowish brown grades slowly to $7 / 4 \mathrm{v}$. pale brown

cveral zones of faint, poorly define white and $y$, pale brown mottles, $90-109 \mathrm{~cm}, 120-130 \mathrm{~cm}$, overall 11ghtening

somewhat splotchy $190-240 \mathrm{~cm}$
moist silty lutite with forams abund. throughout S tex
75-289
CALC

CAIC OOZE

10YR $6 / 4$ it yellowish brown

sonewhat graded $s 1$ laminated foram sand

$1 \mathrm{~cm}$ break in core $275-276 \mathrm{~cm}$

horixontal, textural

289-300

CALC OOZE

6/4 1t. yellowish brown

cattered forams in $\mathrm{f} 1 \mathrm{rm}$, tacky, 31 silty lutite.

$300-462$

CAIC OOZE

1OYR $8 / 1$ white

inm,plastic, smooth lutite with a few v. scattered forains end of core

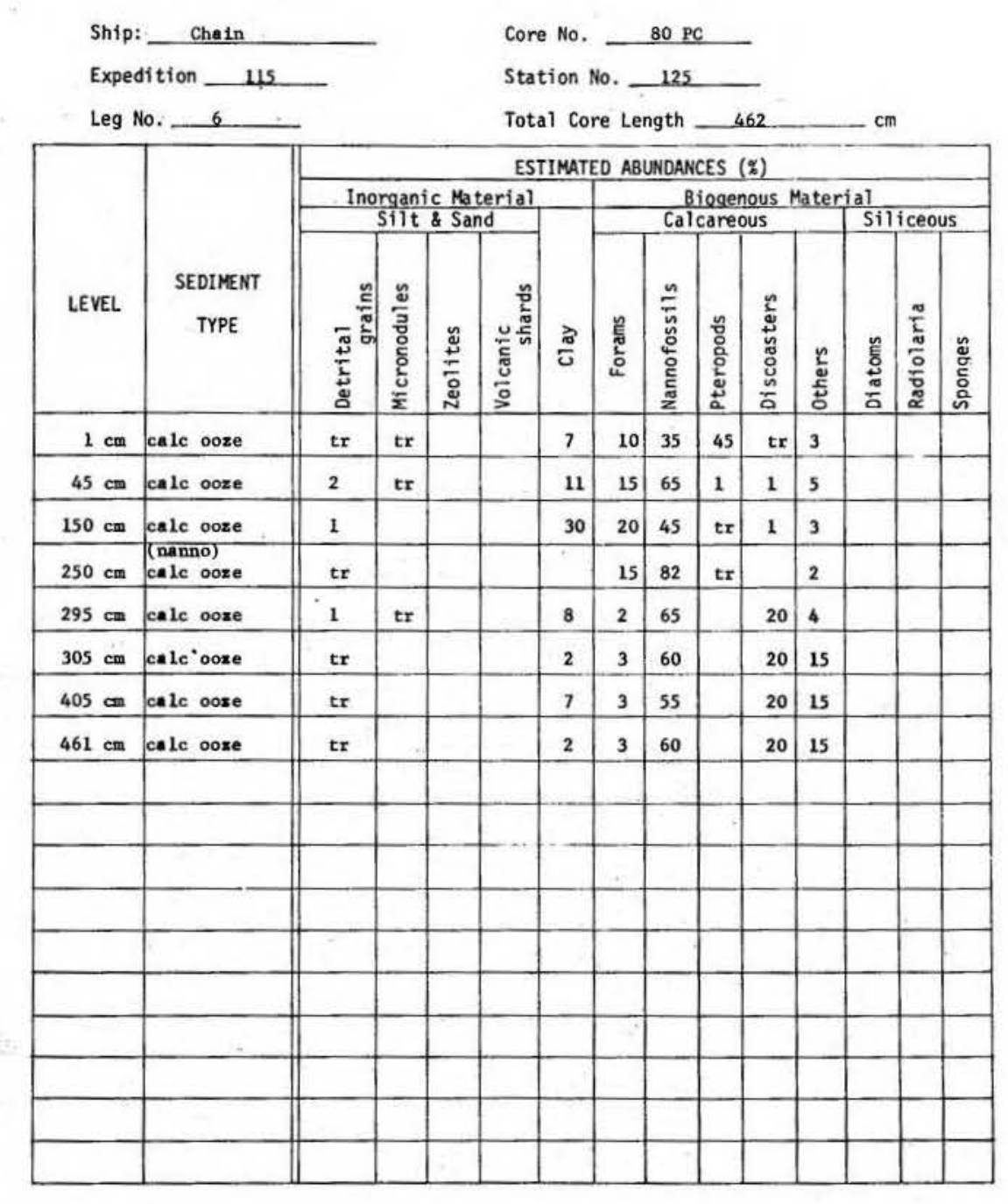


426

VISUAL CORE DESCRIPTION

Page 1 of 1

Ship CHAIN Cruise 115 Leg 6 sto. 125 Core No. BoPG

Total Length $80 \mathrm{~cm}$. Lat. $29956.3^{\circ} \mathrm{S}$ Long. $35^{\circ} 33.6^{\circ} \mathrm{N}$ Depth 2293 corr meturs

Core condition EXLELLENT Date Described 25 feb 75 by J.Bnda.

Physiographic location NORTH FLANK: RUO GRANDS RIsE

Lithologic

Detailed Description

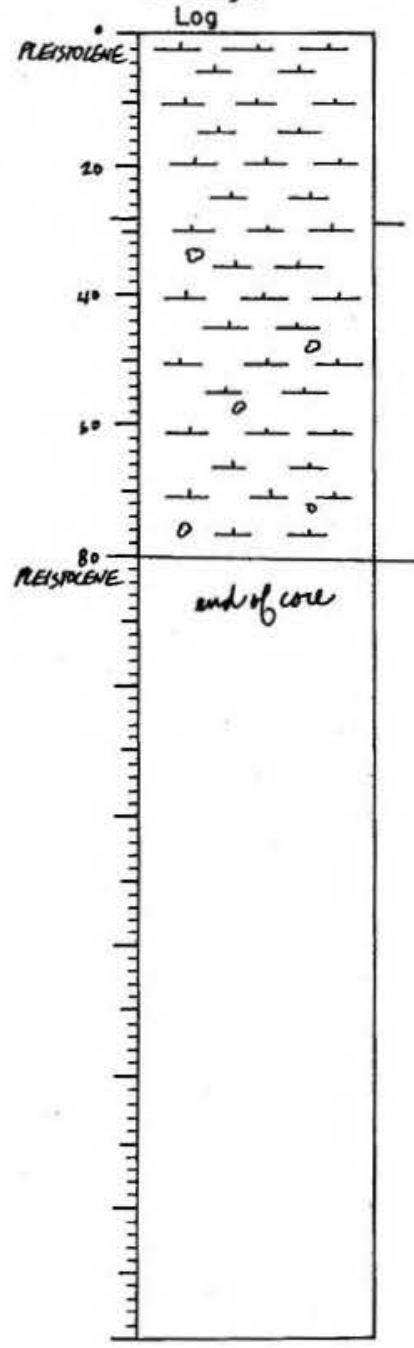

$0-28$ CALC OOZE

10YR $7 / 4$ v. pale brown

coarse pteropod ooze with one lamination of fine fragments one remarkably well preserved large test at $27-28 \mathrm{~cm}$ 28-80

CALC OOZE

it yellowish browm

v. pale brown mottling scattered throughout

lutite with abund. forams

nd of core

\section{7}

SMEAR SLIDE DESCRIPTIONS - W.H.O.I. SEDIMENT CORES
Ship: Chain
Expedition 115
Leg No. 6

Core No. 80 PG

Station No. 125

Total Core Length $\quad 80$

\begin{tabular}{|c|c|c|c|c|c|c|c|c|c|c|c|c|c|c|}
\hline \multirow[b]{3}{*}{ LEVEL } & \multirow[b]{3}{*}{$\begin{array}{c}\text { SEDIMENT } \\
\text { TYPE }\end{array}$} & \multicolumn{13}{|c|}{ ESTIMATED ABUNDANCES ( $(\%)$} \\
\hline & & \multicolumn{4}{|c|}{$\begin{array}{l}\text { Inorganic Material } \\
\text { Silt \& Sand }\end{array}$} & \multirow[b]{2}{*}{$\frac{\sqrt{0}}{6}$} & \multicolumn{5}{|c|}{$\begin{array}{l}\text { 8iogenous Mater } \\
\text { Calcareous }\end{array}$} & \multicolumn{3}{|c|}{$\frac{\text { al }}{\text { siliceous }}$} \\
\hline & & 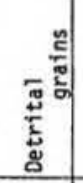 & 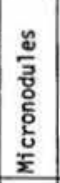 & 岕 & 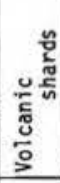 & & 崖 & 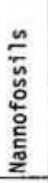 & 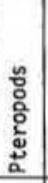 & 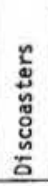 & 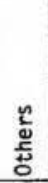 & 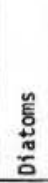 & $\frac{\pi}{\frac{\pi}{\pi}} \frac{\pi}{\frac{\pi}{\sigma}}$ & 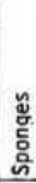 \\
\hline $1 \mathrm{~cm}$ & calc ooze & & & & & 5 & 20 & 15 & 60 & & $\mathrm{tr}$ & & & \\
\hline $30 \mathrm{~cm}$ & calc ooze & 2 & & & & 30 & 10 & 50 & 3 & & 5 & & & \\
\hline $79 \mathrm{~cm}$ & calc ooze & 2 & & & & 10 & 20 & so & 13 & & 5 & & & \\
\hline & & & & & & & & & & & & & & \\
\hline & & - & & & & & & & & & & & & \\
\hline & & & & & & & & & & & & & • & \\
\hline & & & & & & & & & & & & & & \\
\hline & & & & & & & & & & . & & & & \\
\hline & & & & & & & & & & & & & & \\
\hline & & & & & & & & & & & & & & \\
\hline & & & & & & & & & & & & & & \\
\hline & & & & & & & & & & & & & & \\
\hline & & & & & & & & & & & & & & \\
\hline & & & & & & & & & & & & & & \\
\hline & & & & & & & & & & & & & & \\
\hline & & & & & & & & & & & & & & \\
\hline & & & & & & & & & & & & & & \\
\hline & & & & & & & & & & & & & & \\
\hline
\end{tabular}


432

VISUAL CORE DESCRIPTION
Page 1 of 1

Ship CHAIN Cruise 115 Leg 6 Sta. 128 Core No. 83 PL Total Length 651 cm. Lat. $29^{\circ} 580^{\circ} \mathrm{s}$ Long. $35^{\circ} 33.7^{\circ} \mathrm{W}$ Depth $2189 \mathrm{~m}$.cer Core condition EXCEUENT Date Described 26Feb 75 by fruda.

Physiographic location NIRTH FLANIK: RIO GRANDE RKE.

Lithologic

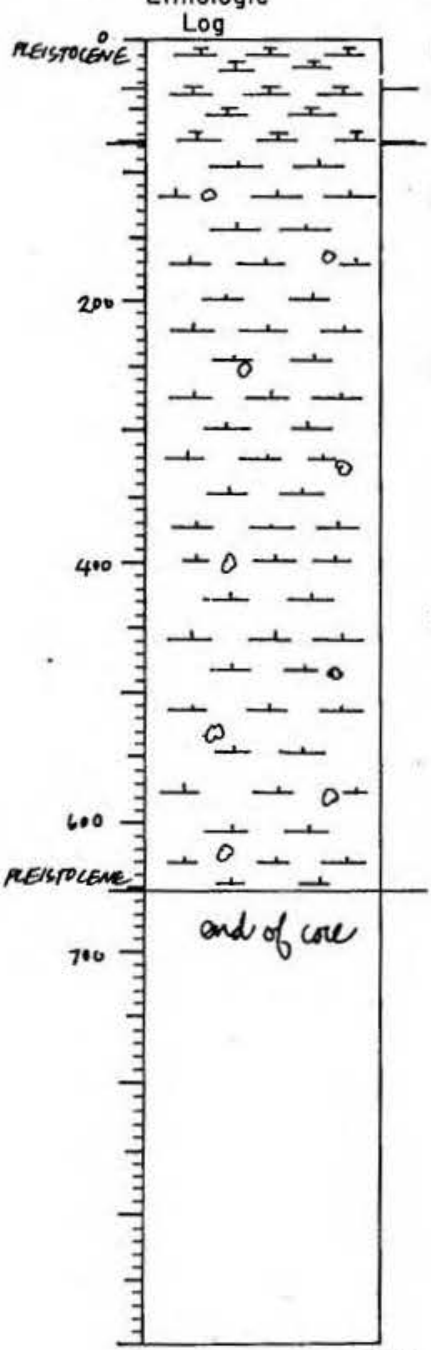

\section{Detailed Description}

$0-34$ CALC DOZE

1OYR $8 / 4$ v. pale brewn

ine to course foram sand

epparently washed due to recovery $0 \div 15 \mathrm{~cm}$ 4.73 1

CAIC OOZE

OYR $6 / 3$ pale brown grades to $8 / 4 \mathrm{v}$. pale brown

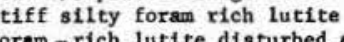

fitist distured and washed and mixed with forain sand $34-40 \mathrm{~cm}$

73-7

CALC OOZE

OYR $8 / 4$ v. pale brov

$S$ inclined $15^{\circ}$

Note: The sharpness of contact and similar inclinations above and below this foram sand layer appear to indicate that there was a break in core (probably during pullout) and the forams now included there washed down the side of the iner and filled the gap.

74-651 CALC OOZE

OYR $8 / 3$ v. pale brown to $7 / 4$.v. pale brown, color gradea tho hues

white mottling found occssionally throughout tiff compact silty lutite with v. abund. forams few scattered dk flecks and tiny mottles $373-380 \mathrm{~cm}$ end of core
433

SMEAR SLIDE DESCRIPTIONS - W.H.O.I. SEDIMENT CORES

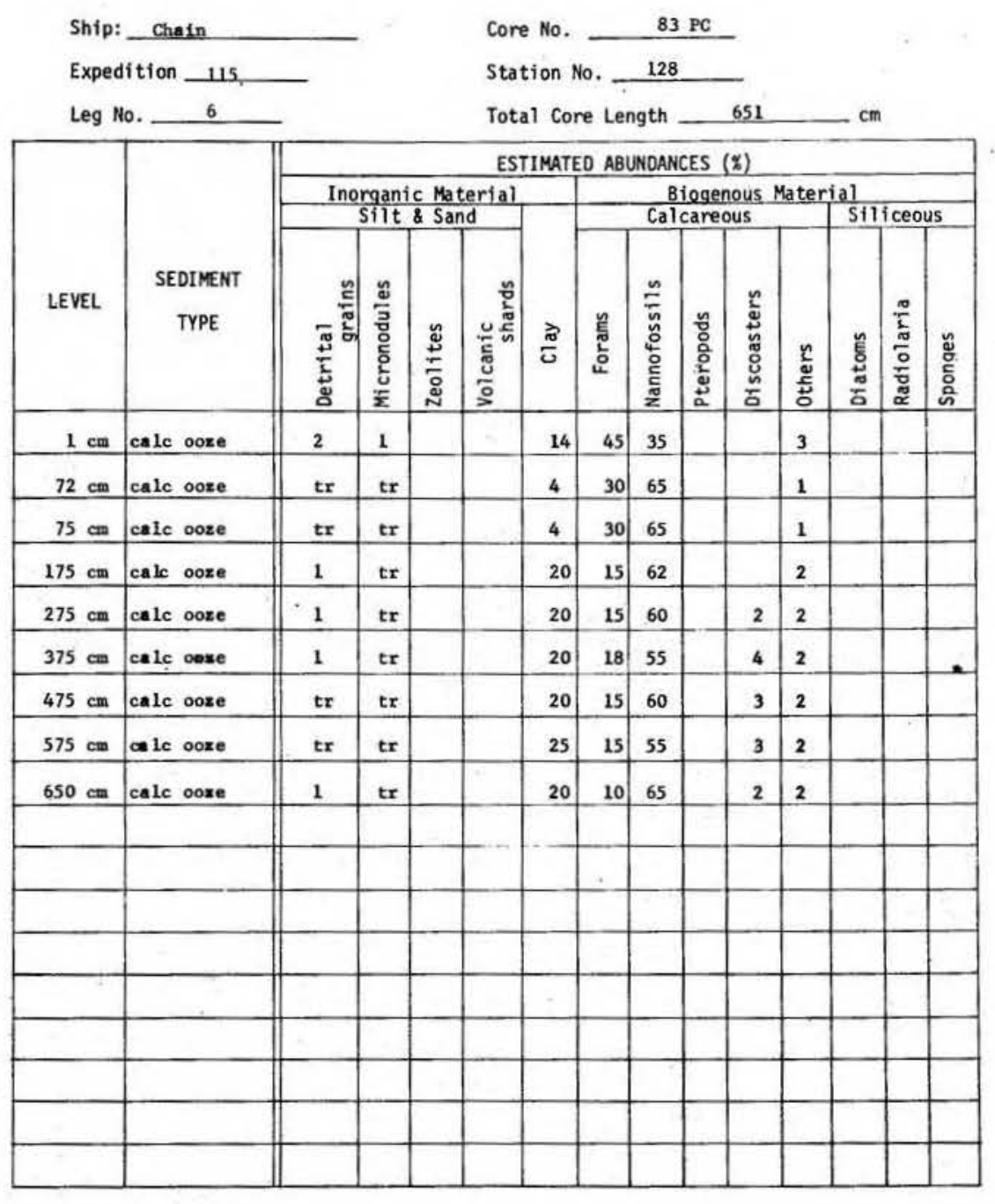


434

VISUAL CORE DESCRIPTION

Page 1 of 1

Ship CHAIN Cruise 115 Leg 6 sto. 129 Core No. 84 i Total Length $667 \mathrm{~cm}$. Lat. $29^{\circ} 599^{7} 7^{7}$ Long. $35^{\circ} 33.5 \mathrm{~W}$ Depth $2110 \mathrm{morr}$ Core condition EXCEUENT Date Described 3 Mar 75 by fouda Physiographic location NORTH FLANK: A10 GRANDE RISE

Lithologic

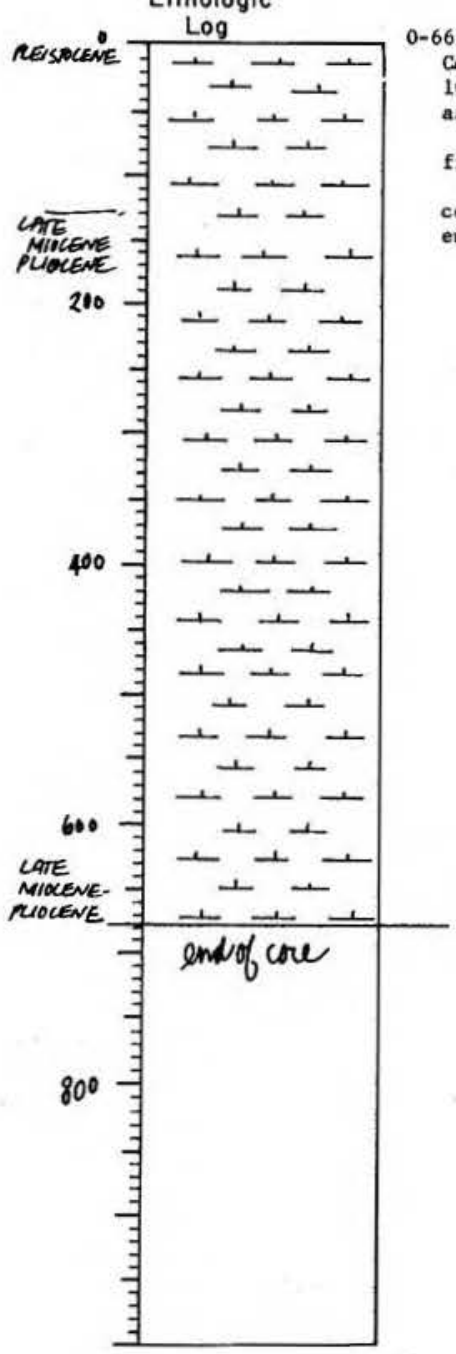

\section{Detailed Description}

CALC OOZE

OYR $7 / 3$ v. pale brown grades to $8 / 2$ white and back to $7 / 3$ aside from gradual color evolution, entire core ts of uniform 1ithology

firm compact lutite vith v. abund. forams throughout, generally a bit coarser $0-225 \mathrm{~cm}$

washed $0-12 \mathrm{~cm}$

\section{5}

SIEAR SLIDE DESCRIPTIONS - W.H.O.I. SEDIMENT CORES

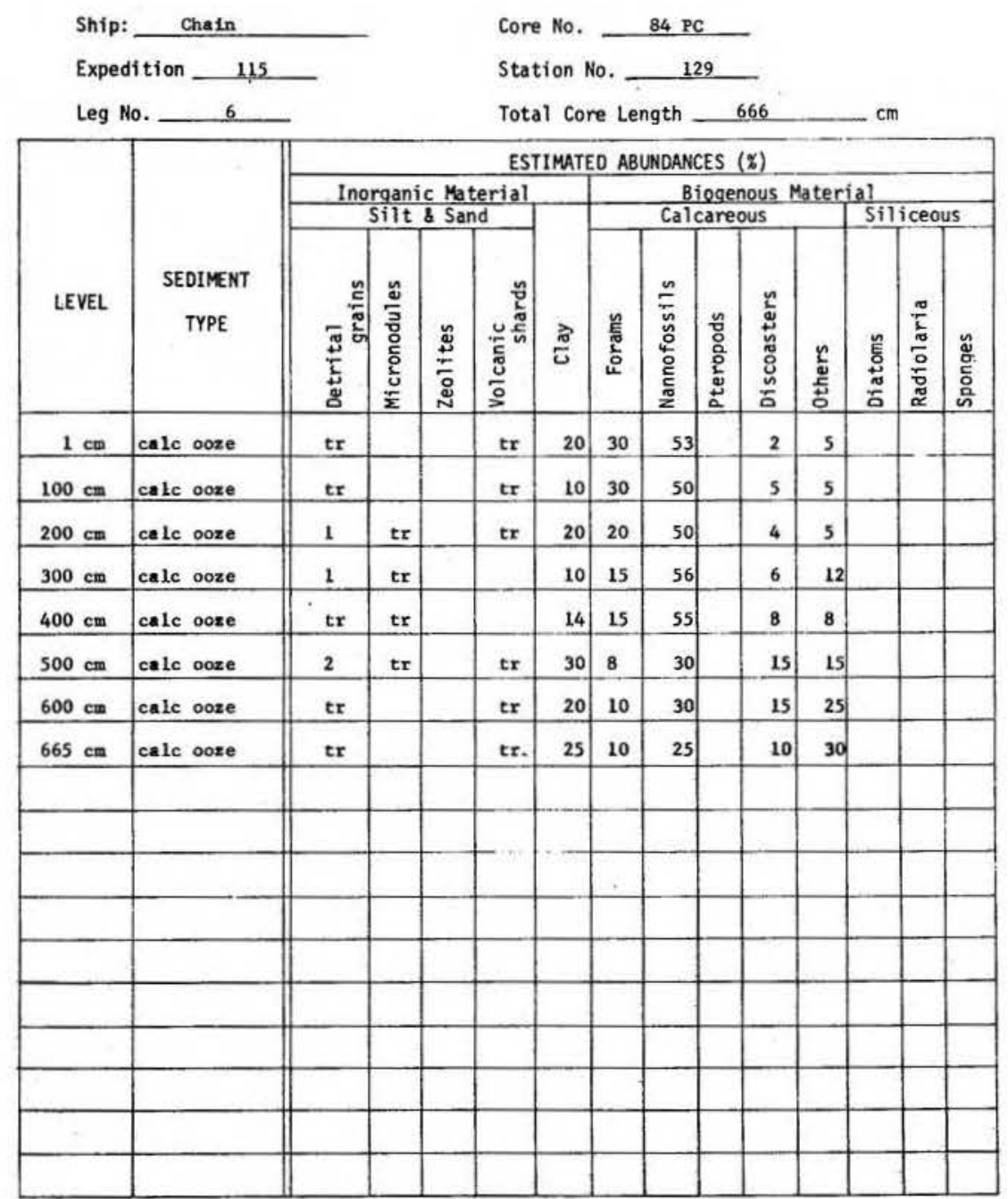


436

VISUAL CORE DESCRIPTION

Page 1 of 1

Ship CHAIN Cruise 115 Leg 6 Sta. 130 Core No. 85 PC Total Length $740 \mathrm{~cm}$. Lat. $29^{\circ} 59.3^{\prime} \mathrm{S}$ Long. $35^{\circ} 33.6^{\circ} \mathrm{W}$ Depth $2130 \mathrm{~m}$. Com Core condition EXLELENT Dote Described 3MAR 75 by f. Bhodw

Physiogrophic locotion NORTH FLANK: R10 GRANOE RISE

Lithologic

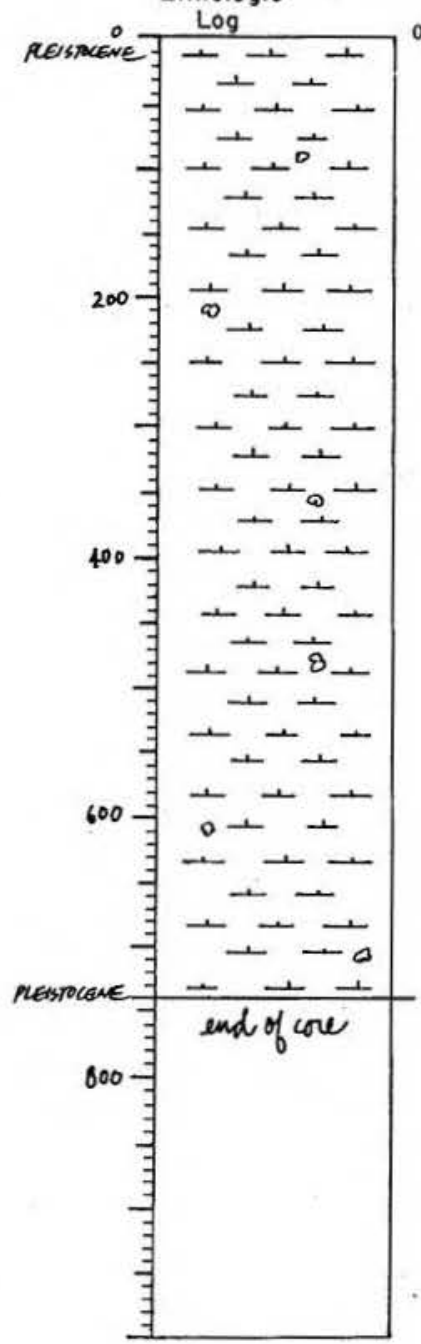

\section{Detailed Description}

CALC OOZE

OYR $8 / 2$ white to $7 / 4 \mathrm{v}$. pale brown color gently and repeatedly grades between these two color

the interval $0-20 \mathrm{~cm}$ appears to have been washed, disturbed during recovery

end of core
437

SMEAR SLIDE DESCRIPTIONS - W.H.O.I. SEDIMENT CORES

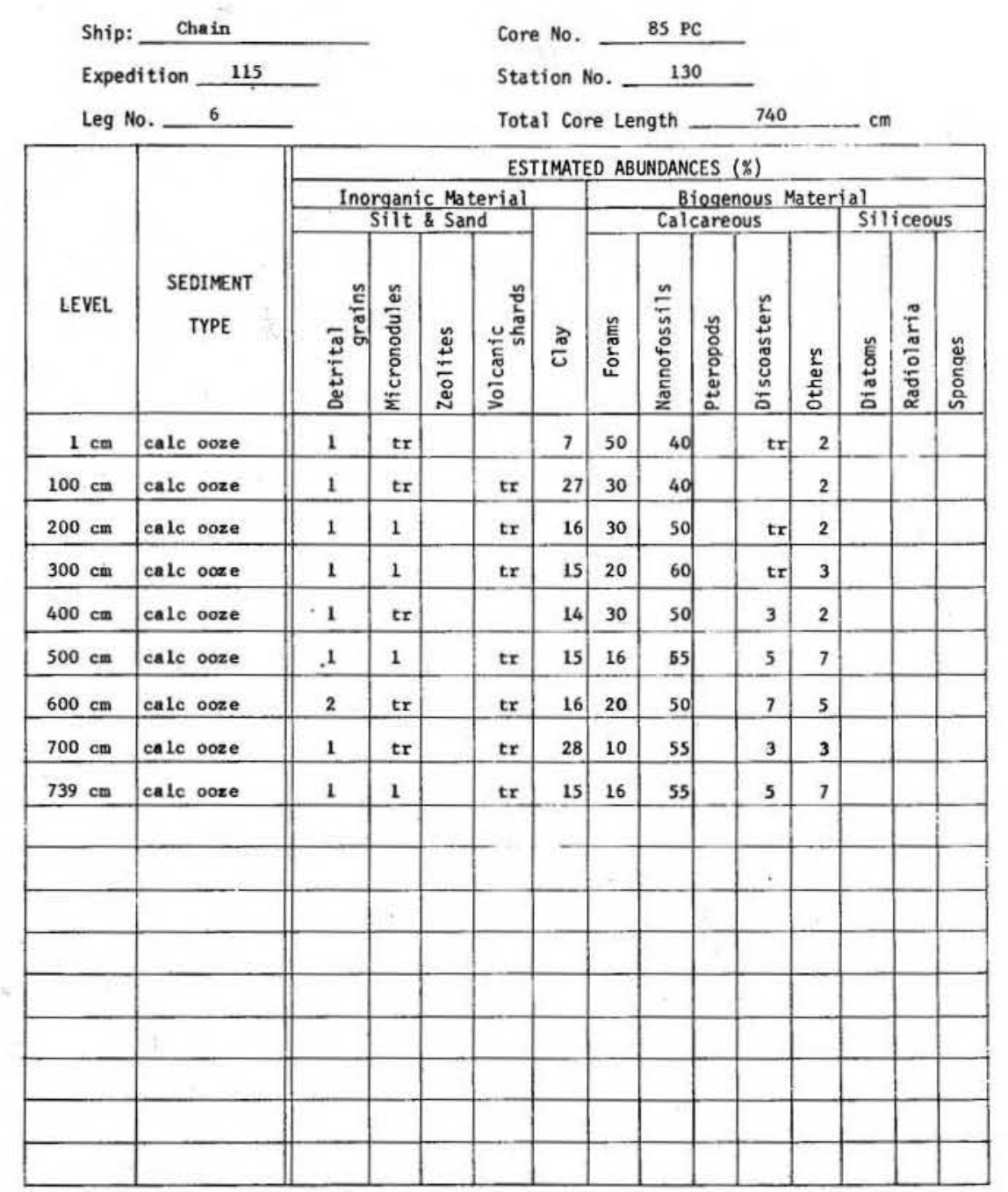




\section{8}

VISUAL CORE DESCRIPTION

Page 1 of 1

Ship CHAIN Cruise 115 Leg 6 Sta. 131 Core No. 86 PC

Total Length $740 \mathrm{~cm}$. Lat. $30^{\circ} 00.1^{\prime} \mathrm{S}$ Long. $35^{\circ} 33.7 \mathrm{~W}$ Depth $2090 \mathrm{~m}$. Corr

Core condition EXCELENT Dote Described 4MUR75 by JPhola

Physiogrophic location NORTH FLANK: RIO GRANDE RISE.

Lithologic
Log

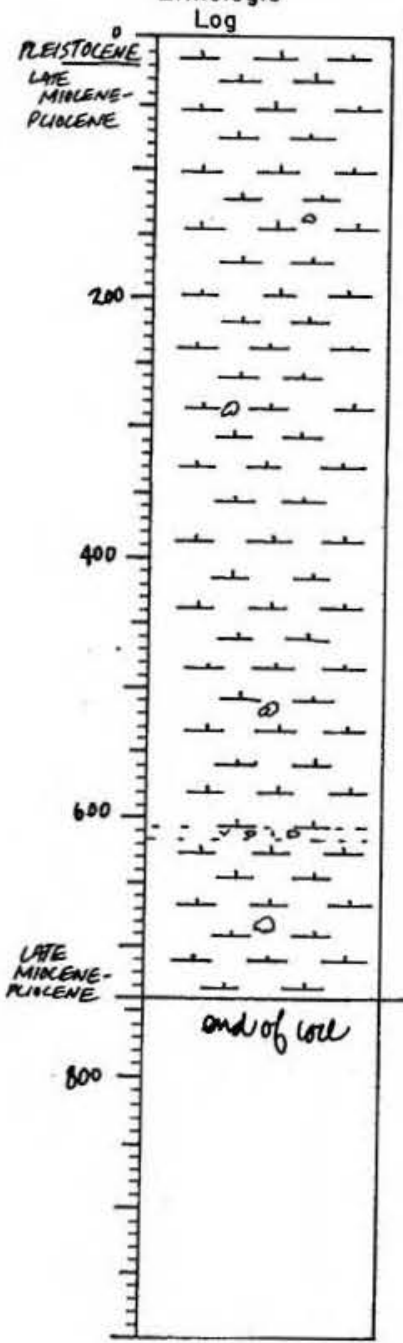

\section{CALC OOZE}

few whtte gently grades to $7 / 3$ v. pale brown

firm compect lutite

foram sand disturbed by washing in recovery

06-611 cmivold water pocket, removed before

end of core
439

SMEAR SLIDE DESCRIPTIONS - W.H.O.I. SEDIMENT CORES

Ship: Chain

Expedition 115

Leg No. 6

Core No. $86 \mathrm{PC}$

Station No. 131

Total Core Length $\quad 740 \quad \mathrm{~cm}$

\begin{tabular}{|c|c|c|c|c|c|c|c|c|c|c|c|c|c|c|}
\hline \multirow[b]{4}{*}{ LEVEL. } & \multirow[b]{4}{*}{$\begin{array}{c}\text { SEDIMENT } \\
\text { TYPE }\end{array}$} & \multicolumn{13}{|c|}{ ESTIMATEO ABUNDANCES ( $(\xi)$} \\
\hline & & \multirow{2}{*}{\multicolumn{4}{|c|}{$\begin{array}{c}\text { Inorganic Material } \\
\text { silt \& Sand }\end{array}$}} & \multirow[b]{3}{*}{$\frac{\pi}{0}$} & \multirow{2}{*}{\multicolumn{5}{|c|}{$\begin{array}{l}\text { Biogenous Mater } \\
\text { Calcareous }\end{array}$}} & \multirow{2}{*}{\multicolumn{3}{|c|}{ Siliceous }} \\
\hline & & & & & & & & & & & & & & \\
\hline & & $\begin{array}{l}\frac{n}{\tilde{c}} \\
\frac{5}{5} \\
\frac{5}{5} \\
8\end{array}$ & 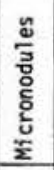 & 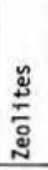 & 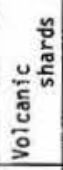 & & 鵕 & 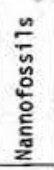 & 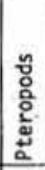 & 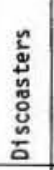 & 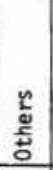 & \begin{tabular}{l} 
产 \\
\multirow{+}{0}{} \\
$\vdots$ \\
\end{tabular} & $\frac{\pi}{\frac{\pi}{0}}$ & 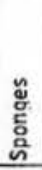 \\
\hline $1 \mathrm{~cm}$ & calc ooze & $\mathrm{tr}$ & $\mathrm{tr}$ & & & 12 & 35 & 45 & & 3 & 5 & & & \\
\hline $100 \mathrm{~cm}$ & calc oose & tr & tr & & $\mathrm{tr}$ & 20 & 15 & 40 & & 10 & 15 & & & \\
\hline $200 \mathrm{~cm}$ & calc ooze & 1 & tr & & & 22 & 15 & so & & 7 & 5 & & & \\
\hline $300 \mathrm{~cm}$ & cale ooze & 2 & $\operatorname{tr}$ & & tr & 21 & 12 & s5 & & 5 & 5 & & & \\
\hline $400 \mathrm{~cm}$ & calc ooze & 1 & 1 & & & 20 & 10 & 58 & & $s$ & 5 & & & \\
\hline $500 \mathrm{~cm}$ & calc ooze & 1 & $\mathbf{t r}$ & & tr & 15 & 10 & 52 & & 10 & 12 & & & \\
\hline $600 \mathrm{~cm}$ & calc ooze & 1 & 1 & & tr & 43 & 5 & 15 & & 15 & 25 & & & \\
\hline $700 \mathrm{~cm}$ & calc ooze & $\operatorname{tr}$ & $\mathrm{tr}$ & & & 35 & 5 & 25 & & 10 & 25 & & & \\
\hline $739 \mathrm{~cm}$ & calc ooze & tr & tr & & & 30 & 5 & 25 & & 15 & 25 & & & \\
\hline & & & & & & & & & & & & & & \\
\hline & & & & & & & & & &. & & & & \\
\hline & & & & & & & & & & & & & & \\
\hline & & & & & & & & & & & & & & \\
\hline & & & & & & & & & & & & & & \\
\hline & & & & & & & & & & & & & & \\
\hline & & & & & & & & & & & & & & \\
\hline & & & & & & & & & & & & & & \\
\hline & & & & & & & & & & & & & & \\
\hline
\end{tabular}


440

\section{VISUAL CORE DESCRIPTION}

Poge 1 of 1

Ship CHAIN Cruise 115 Leg 6 Sto. 131 Core No. 86PG

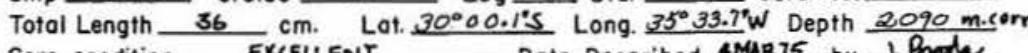

Core condition EXCELENT Dote Described 4 MRR75 by Jooda

Physiographic location NORTH FLANK: RIO GRANDE RISE

Lithologic

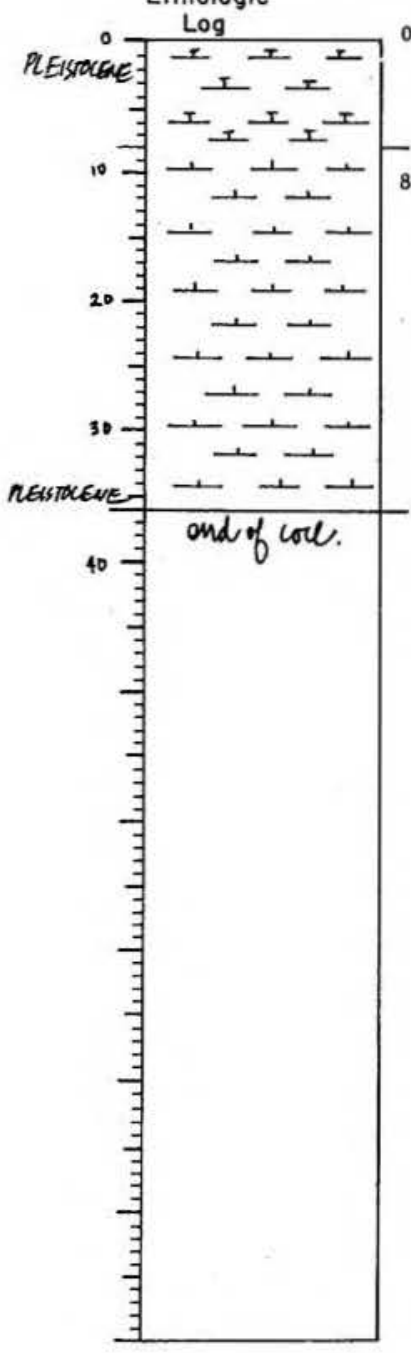

0-8

Detailed Description

CALC OOZE

$7 / 3$ v. pale brown grades to $6 / 2$ it brownish gray chin gradational laminations throughout

fairly coarse foram send

S, textural

CALC OOZE

10YR $8 / 2$ white

ind

nd of core 
Ship CHN Cruise 115 Leg 6 Sto. 134 Core No. 87 PC Total Length $\sim 2 \quad \mathrm{~cm}$. Lat. $29^{\circ} 46.3^{\prime} \mathrm{S}$ Long. $35^{\circ} 36.0^{\prime} \mathrm{W}$ Depth 3302 censm Core condition _- Dote Described 21 May 75 by $R$ MCGiRe Physiographic location Cenyen trending NE, Nerth Flank of Rio Grende Rise Lithologic

Detailed Description

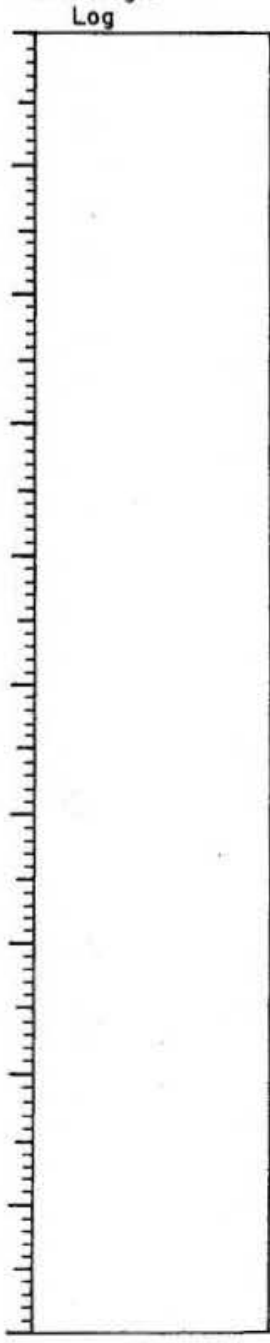

Ship CHAIN Cruise 115 Leg $\frac{6}{15}$ Sta. $\frac{137}{38^{\circ}}$ Core No. $88 \mathrm{PC}$ Total Length $704 \mathrm{~cm}$. Lat. $30^{\circ} 55.0^{7} \mathrm{~S}$ Long. $38^{\circ} 048^{\prime} \mathrm{W}$ Depth $2941 \mathrm{~m}$ corr Core condition EXLELLENT Date Described $\frac{5 M A R .75}{\text { by }}$ byoda. Physiogrophic location EAST SIDE OF VEMA CHANNEL

Lithologic Detailed Description

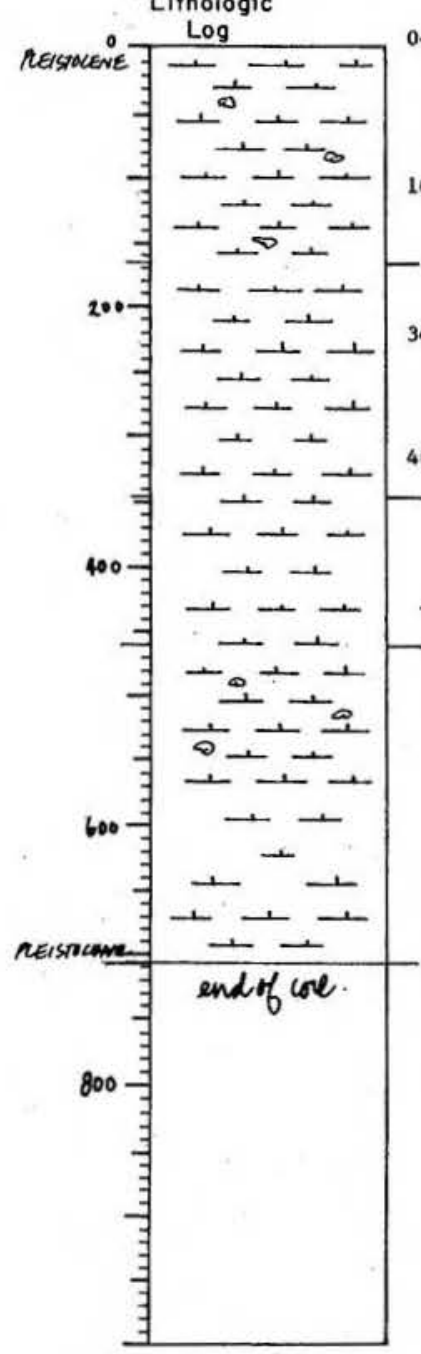

165 v. Poorly defined white mottling $0-90 \mathrm{~cm}$ mo1st silty lutite with scattered forams $165-344$ NANNO OOZE

$8 / 2$ white stiffer more plastic-1ike lutite with abund. forams $s$ textural $344-460$

CALC OOZE GRADES TO NANAO DOZE

(O) $7 / 4$ v. pale brown grades to $8 / 2$ white 60-704

CALC DOZE GRADES TO NANNOO OOZE LOYR $7 / 4$ v. pale brou grades to $6 / 4$ 1t yellowish brown then back to $7 / 4$ v. pale brown moist lutite with scattered forams becomes more plastic 645-691 ch near end or of core
LYR $6 / 4$ it yellowish brown grades to $7 / 4 \mathrm{v}$, pale brown moist less compact lutite with scattered forams 
444

SMEAR SLIDE DESCRIPTIONS - W.H.O.I. SEDIMENT CORES

Ship: Chain

Expedition 115

Leg No. 6

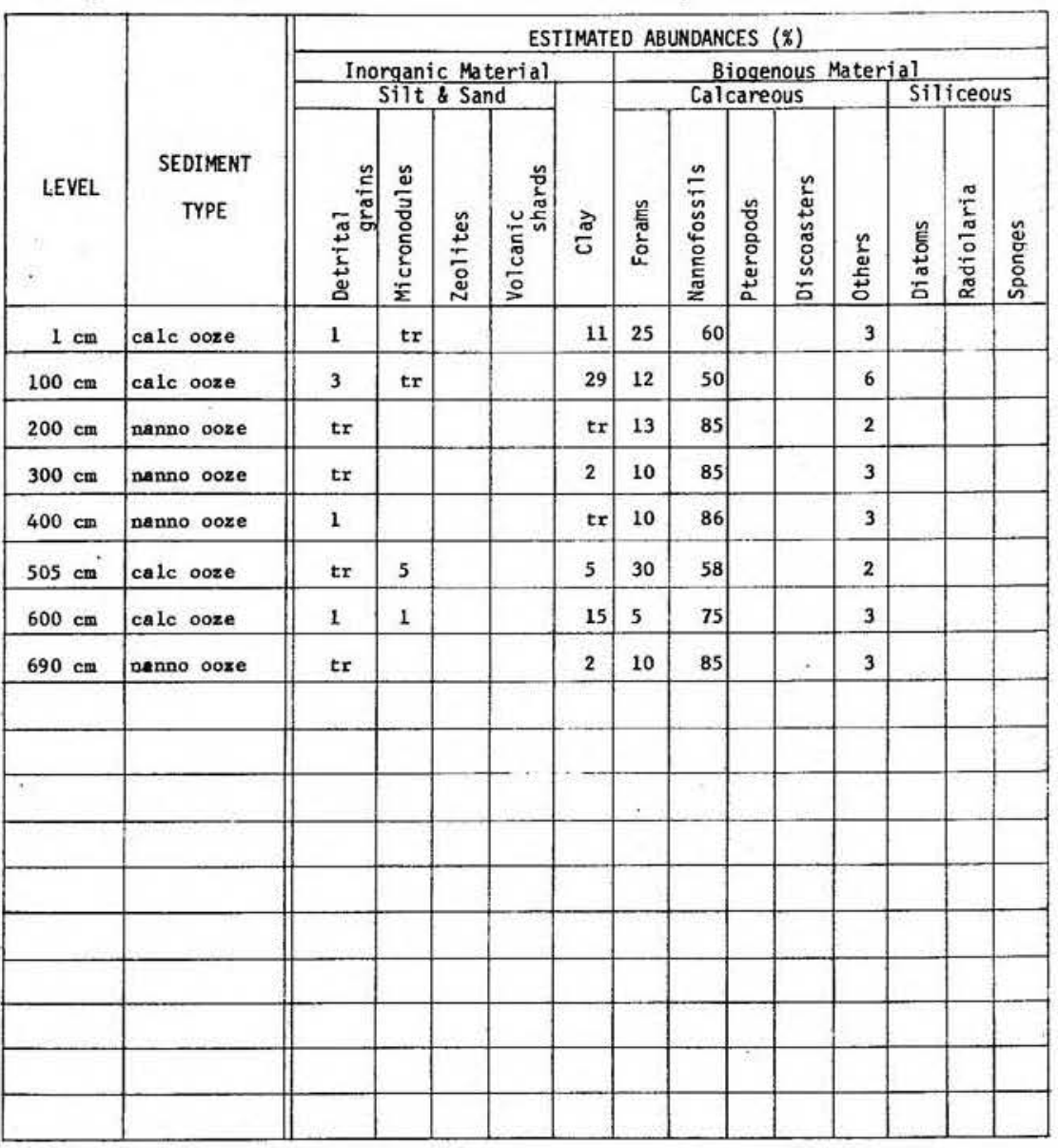

445

VISUAL CORE DESCRIPTION

Page 1 of 1

Ship CHAIN Cruise 115 Leg 6 Sta. 137 Core No. $88 \mathrm{PG}$ Total Length $128 \mathrm{~cm}$. Lat. $30^{\circ} \mathrm{E5} .0^{\prime} \mathrm{S}$ Long. $38^{\circ} 04.8^{\prime} \mathrm{W}$ Depth 2941 Core condition EXCEUENT Dote Described 5MAR75 by J Binda, Physiographic location EAST SIDE OF YEMACHANNEL. Lithologic Detailed Description

reisricone $\begin{gathered}\text { Log } \\ \text { A }\end{gathered}$
CALC OOZE 10XR $7 / 4$ v. pale brown

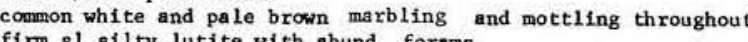
66-128

CALC OOZE 10R $6 / 1$ it gray grades to $6 / 3$ pale brown to $6 / 4$ It yellowish common $v$. pale brown mottling found in each of the 1t yellowish brown areas

molat atite with abund. forams throughout the gray top portion of the end of core

$\mathrm{cm}, 102-126 \mathrm{~cm}$ 
446

SMEAR SLIDE DESCRIPTIONS - W.H.O.I. SEDIMENT CORES

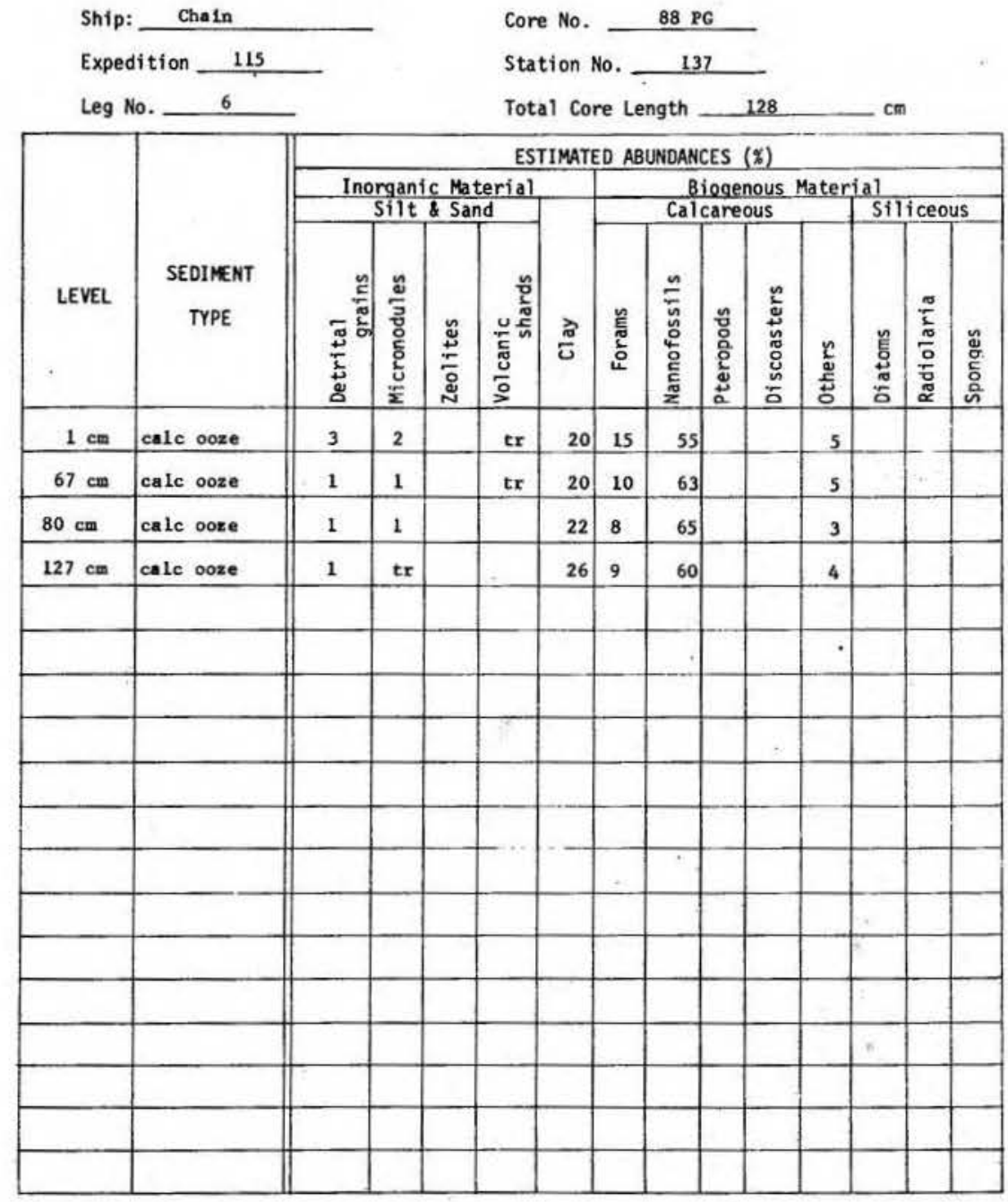

447

VISUAL CORE DESCRIPTION

Page 1 of 1

Ship CHAIN Cruise 115 Leg 6 sto. 139 Core No Bap Total Length $672 \mathrm{~cm}$. Lat. $30 \circ 528^{\prime \prime S}$. $38^{\circ} 11.8^{\prime} \mathrm{W}$ Depth $3152 \mathrm{~m} \cdot \mathrm{corr}$. Core condition EXLEUENT. Date Described EMay75 by frudul. Physiographic location

Lithologic VEMA CHANNEL

$$
\begin{gathered}
\text { Lithologic } \\
\text { Log }
\end{gathered}
$$

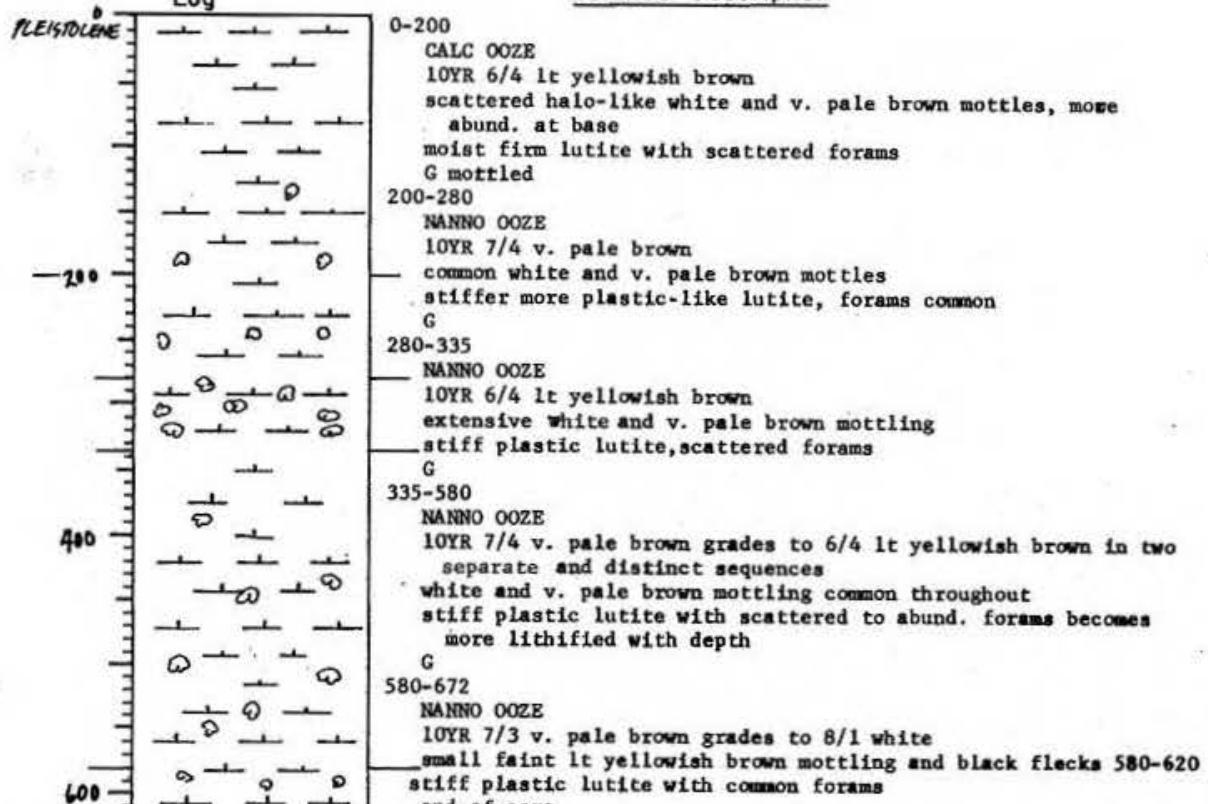

800

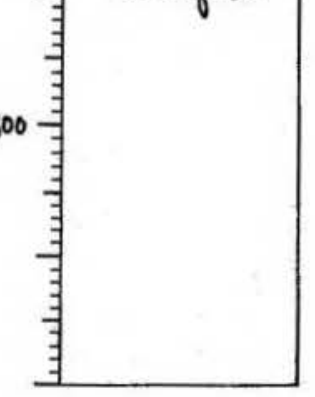


448

SMEAR SLIDE DESCRIPTIONS - W.H.O.I. SEDIMENT CORES

Ship: Chain

Expedition 115

Leg No. 6

\begin{tabular}{|c|c|c|c|c|c|c|c|c|c|c|c|c|c|c|}
\hline & & & & & & & & & & & & & & \\
\hline \multirow[b]{3}{*}{ LEVEL } & \multirow[b]{3}{*}{$\begin{array}{c}\text { SEDIMENT } \\
\text { TYPE }\end{array}$} & \multicolumn{13}{|c|}{ ESTIMATED ABUNDANCES ( $(x)$} \\
\hline & & \multicolumn{4}{|c|}{$\begin{array}{l}\text { Inorganic Material } \\
\text { Silt \& Sand }\end{array}$} & \multirow[b]{2}{*}{ 商 } & \multicolumn{5}{|c|}{$\begin{array}{l}\text { Biogenous Mater } \\
\text { Calcareous }\end{array}$} & \multicolumn{3}{|c|}{$\frac{\text { al }}{\text { Siliceous }}$} \\
\hline & & 总 & 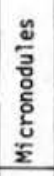 & 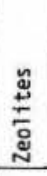 & 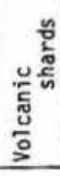 & & 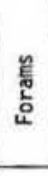 & 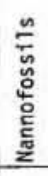 & 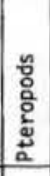 & 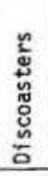 & 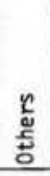 & 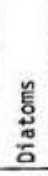 & $\begin{array}{l}\frac{\pi}{2} \\
\frac{\pi}{0} \\
\frac{0}{0} \\
\frac{\pi}{\pi} \\
\end{array}$ & 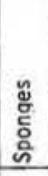 \\
\hline $1 \mathrm{~cm}$ & calc ooze & 1 & 1 & & & 20 & 35 & 4. & & & 3 & & & \\
\hline $100 \mathrm{~cm}$ & calc ooxe & 1 & 1 & & tr & 26 & 20 & 4 & & & 12 & & & \\
\hline $200 \mathrm{~cm}$ & nanno ooze & tr & 1 & & & 8 & 7 & 8 & & & 4 & & & \\
\hline $300 \mathrm{~cm}$ & nanno ooze & 1 & & & & 6 & 8 & $8:$ & & $\operatorname{tr}$ & 3 & & & \\
\hline $400 \mathrm{~cm}$ & nanno ooze & $\operatorname{tr}$ & 1 & & & 8 & 7 & 8 & & & 4 & & & \\
\hline $500 \mathrm{~cm}$ & nanno ooze & tr & tr & & & 2 & 5 & 96 & & & 3 & & & \\
\hline $600 \mathrm{~cm}$ & nanno ooze & 1 & & & & 8 & 5 & $8:$ & & $\operatorname{tr}$ & 4 & & & \\
\hline $670 \mathrm{~cm}$ & nanno ooze & tr & & & & 2 & 2 & 9: & & . & 1 & & & \\
\hline & & & & & & & & & & & & & & \\
\hline & & & & & & & & & & & & & & \\
\hline & & & & & & & & s. & & & & & & \\
\hline & & & & & & & & & & & & & & \\
\hline & & & & & & & & & & & & & & \\
\hline & & & & & & & & & & & & & & \\
\hline & & & & & & & & & & & & & & \\
\hline & & & & & & & & & & & & & & \\
\hline & & & & & & & & & & & & & & \\
\hline & & & & & & & & & & & & & & \\
\hline
\end{tabular}

449

VISUAL CORE DESCRIPTION

Page 1 of 1

Ship CHAIN Cruise $115 \quad$ Leg 6 sto 140 Core No. 9OPL Total Length $664 \mathrm{~cm}$. Lat. $30^{\circ} 51.0^{\circ} \mathrm{S}$ Long. $38^{\circ} 22.3^{2} \mathrm{~W}$ Depth 3384 marr. Core condition EXCEUENT Date Described 6MAB.75 by fouda. Physiographic location EAGTERN FLANK: VEMA CHANNEL.

Lithologic

Detailed Description

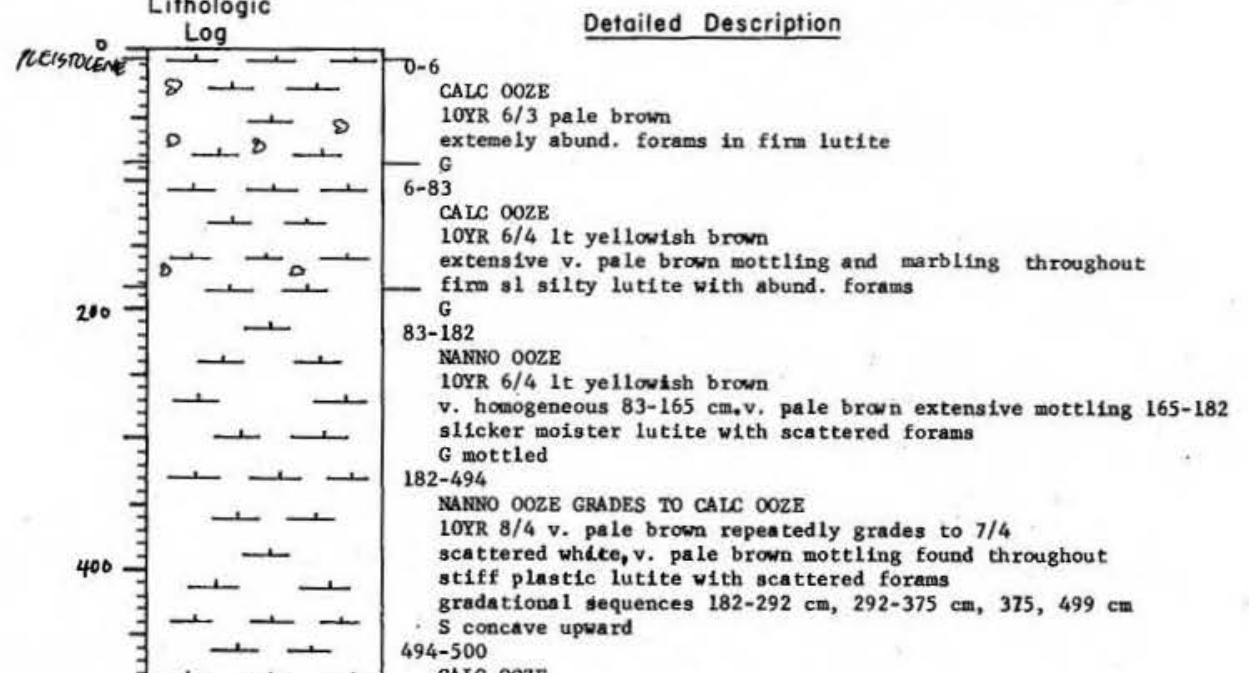

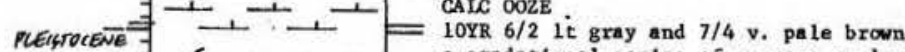

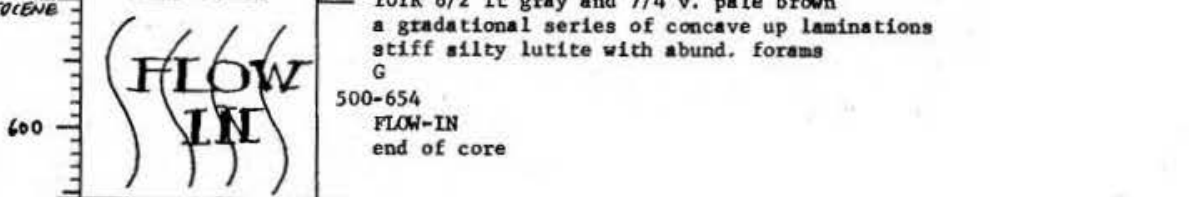

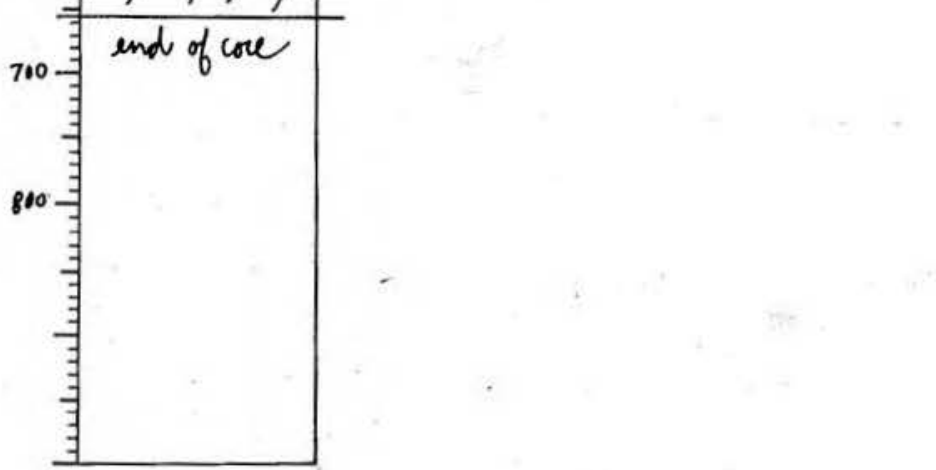


450

SMEAR SLIDE DESCRIPTIONS - W.H.O.I. SEDIMENT CORES

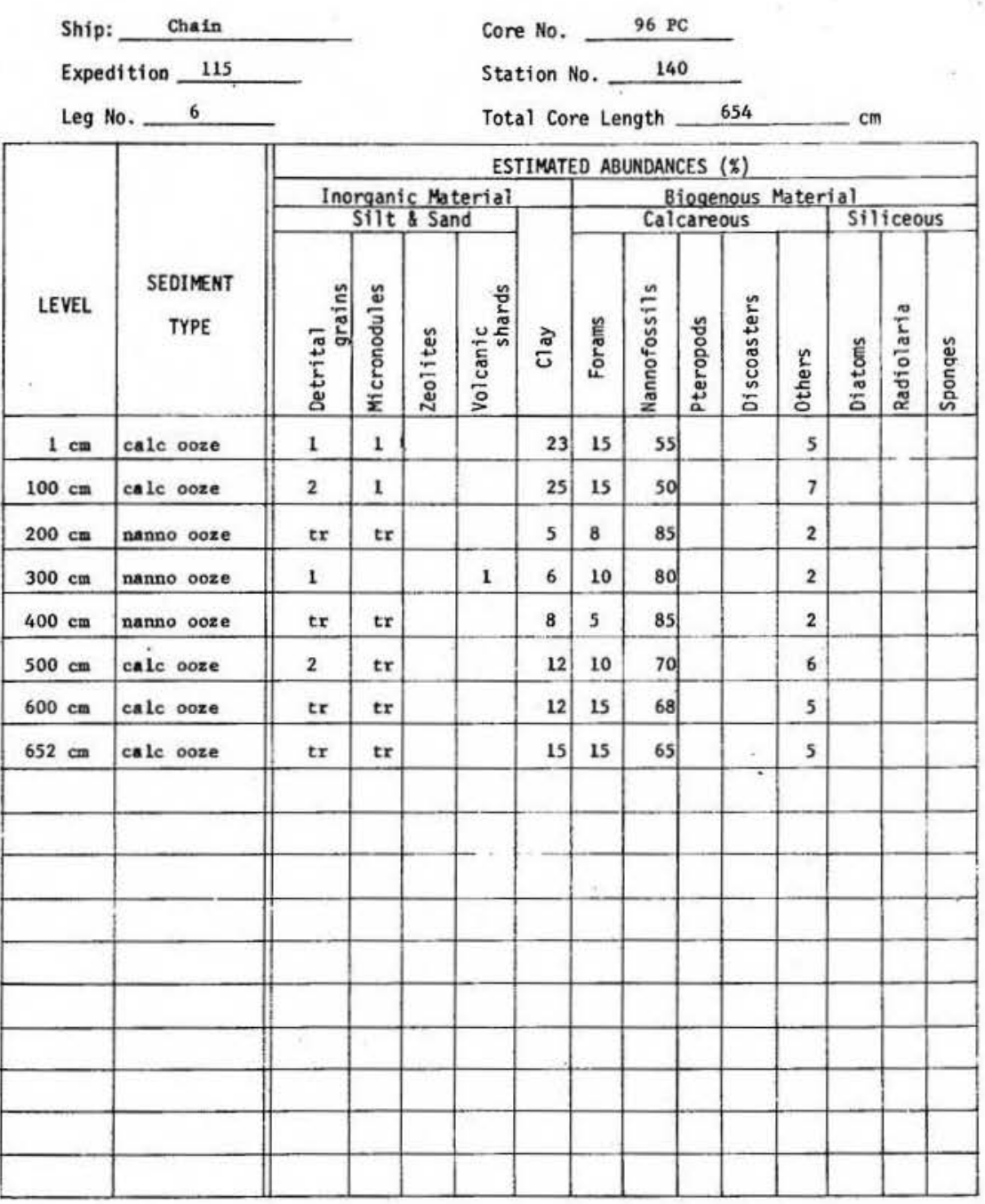

451

VISUAL CORE DESCRIPTION

Page 2 of 1

Ship CHAIN Cruise 115 Leg 6 sta. 140 Core No. 90PG Total Length 51 cm. Lat. $30^{\circ} 51.0^{\circ}$ 'S Long. $38^{\circ} 223^{\circ} \mathrm{W}$ Depth $3384 \mathrm{~m}$. Lorr Core condition EXLELLENT Date Described GMARTS by j.bode Physiographic location EASIERN FLANK: VEMA CHANNEL.

Lithologic

Detoiled Description

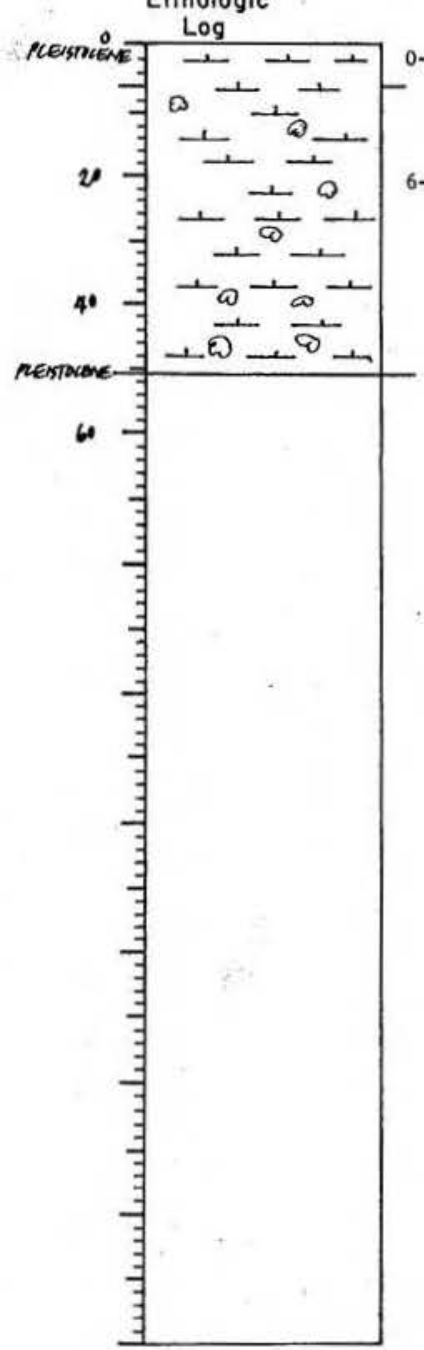

$0-6$

10YR $6 / 3$ pale brown

extremely abund. forams in firm kutite CAIC OOZE

10YR $6 / 4$ 1t yellowish brown

extensive $y$. pelo brown mottling, marbling throughout

firm si silty lutite with abund, forams end of core 

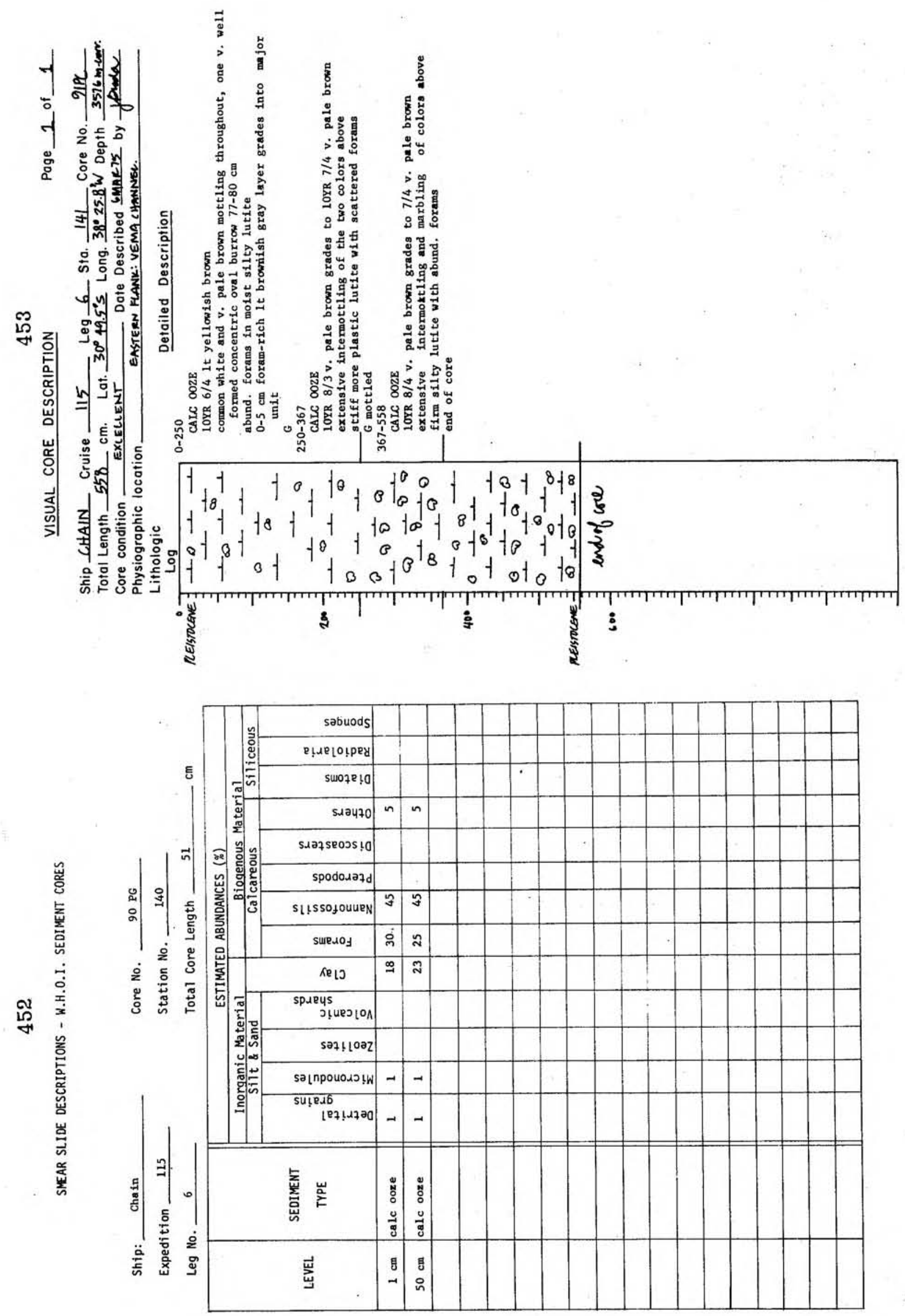


\section{4}

SIEAR SLIDE DESCRIPTIONS - W.H.0.1. SEDIMENT CORES

Ship: Chain

Expedition 115

Leg No. 6

\begin{tabular}{|c|c|c|c|c|c|c|c|c|c|c|c|c|c|c|}
\hline \multirow[b]{3}{*}{ LEVEL } & \multirow[b]{3}{*}{$\begin{array}{c}\text { SEDIMENT } \\
\text { TYPE }\end{array}$} & \multicolumn{13}{|c|}{ ESTIMATED ABUNDANCES ( $($ ) } \\
\hline & & \multicolumn{4}{|c|}{$\frac{\text { Inorganic Material }}{\text { Silt \& Sand }}$} & \multirow[b]{2}{*}{$\frac{\pi}{6}$} & \multicolumn{5}{|c|}{$\begin{array}{l}\text { Biogenous Mater } \\
\text { Calcareous }\end{array}$} & \multicolumn{3}{|c|}{ siliceous } \\
\hline & & $\frac{5}{5}$ & 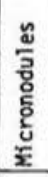 & 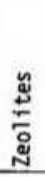 & 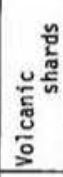 & & 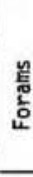 & 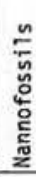 & $\begin{array}{l}5 \\
0 \\
0 \\
\vdots \\
0\end{array}$ & 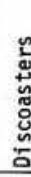 & 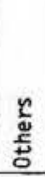 & 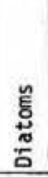 & $\frac{\frac{\pi}{2}}{\frac{\pi}{0}}$ & 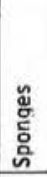 \\
\hline $1 \mathrm{~cm}$ & calc ooze & 1 & 1 & & & 25 & 20 & & & & 3 & & & \\
\hline $100 \mathrm{~cm}$ & calc ooxe & 1 & 3 & & & 35 & 15 & & & & 6 & & & \\
\hline $200 \mathrm{~cm}$ & nanno ooze & tr & tr & & & 10 & 5 & & & & 3 & & & \\
\hline $300 \mathrm{~cm}$ & nanno ooxe & & tr & & & 5 & 3 & & & & 2 & & & \\
\hline $400 \mathrm{~cm}$ & nanno ooze & tr & tr & & & 8 & 5 & & & & 2 & & & \\
\hline $500 \mathrm{~cm}$ & calc ooze & 1 & 1 & & & 25 & 20 & & & & 3 & & & \\
\hline $542 \mathrm{~cm}$ & nanno ooze & tr & tr & & & 10 & 5 & & & & 3 & & & \\
\hline & & & & & & & & & & & & & & \\
\hline & & & & & & & & & & & & & & \\
\hline & & & & & & & & & & & & & & \\
\hline & & & & & & & & . & & & & & & \\
\hline & & & & & & & & & & & & & & \\
\hline & & & & & & & & & & & & & & \\
\hline & & & & & & & & & & & & & & \\
\hline & & & & & & & & & & & & & & \\
\hline & & & & & & & & & & & & & & \\
\hline & & & & & & & & & & & & & & \\
\hline & & & & & & & & & & & & & & \\
\hline
\end{tabular}

455

VISUAL CORE DESCRIPTION

Page 1 of 1

Ship CHAIN Cruise 115 Leg 6 Sta. 141 Core No. 91PG Total Length $112 \mathrm{~cm}$. Lat. $30^{\circ} 49.5^{\circ} \mathrm{S}$ Long. $38^{\circ} 25.8^{\circ} \mathrm{W}$ Depth $3576 \mathrm{~m} . \mathrm{com}$. Core condition EXUEVENT Date Described GMAR.75 by fBuda

Physiographic location EAGERN FLANK: VEMA CHANNST

Lithologic

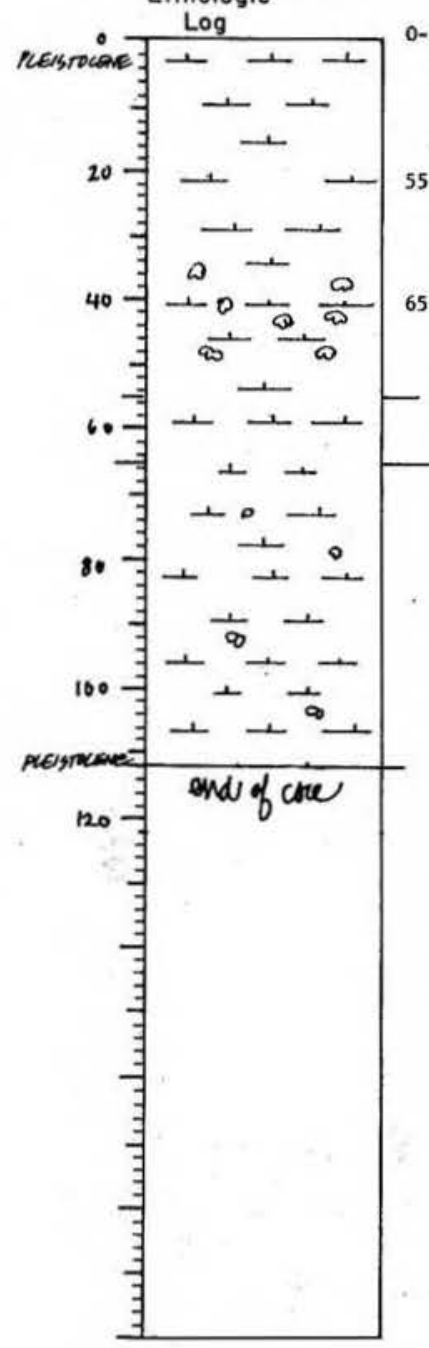

0-55 Detailed Description

CALC OOZE

G/4 1t yellowish brown

oist sl silty luttte with scattered foram

S, irregular

CAIC OOZE

CAIC OOZB

moist silty lutite with v. abund. forams

5-112

CALC OOZE

OYR $6 / 4$ 1t yellowish brown

combon faint $v$. pale brown mottling throughout

cist si silty lutite with scat

作

extected

lat penetration: 0-55 cm, 2nd 55-112 $\mathrm{cm}$

end of core 
SMEAR SLIDE DESCRIPTIONS - W.H.O.I. SEDIMENT CORES

Ship: Chain

Expedition 115

Leg No.

\begin{tabular}{|c|c|c|c|c|c|c|c|c|c|c|c|c|c|c|c|}
\hline \multirow[b]{3}{*}{ LEVEL } & \multirow[b]{3}{*}{$\begin{array}{c}\text { SEDIMENT } \\
\text { TYPE }\end{array}$} & \multicolumn{13}{|c|}{ ESTIMATED ABUNDANCES (\%) } & \\
\hline & & \multicolumn{4}{|c|}{$\begin{array}{l}\text { Inorganic Material } \\
\text { Silt \& Sand }\end{array}$} & \multirow[b]{2}{*}{ ढ़ } & \multicolumn{5}{|c|}{$\begin{array}{l}\text { Biogenous Mater } \\
\text { Calcareous }\end{array}$} & & \multicolumn{3}{|c|}{ Stliceous } \\
\hline & & $\frac{5}{5}$ & 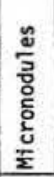 & 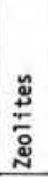 & 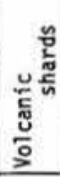 & & 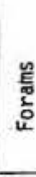 & & & & 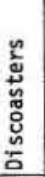 & 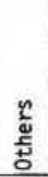 & 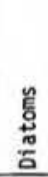 & 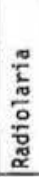 & 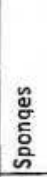 \\
\hline $1 \mathrm{~cm}$ & calc ooze & 1 & I & & & 25 & 20 & & & & & 3 & & & \\
\hline $56 \mathrm{~cm}$ & calc ooze & 1 & 1 & & & 25 & 15 & & & & & 8 & & & \\
\hline $111 \mathrm{~cm}$ & calc ooze & 1 & tr & & & 20 & 25 & & & & & 4 & & & \\
\hline & & & & & & & & & & & & & & & \\
\hline & & & & & & & & & & & & & & & \\
\hline & $\cdot$ & & & & & & & & & & & & & & \\
\hline & & & & & & & & & & & & & & & \\
\hline & & & & & & & & & & & + & & & & \\
\hline & & & & & & & & & & & & & & & \\
\hline & 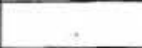 & & & & & & & & & & & & & & \\
\hline & & & & & & & . & & & & & & & & \\
\hline is & & 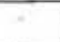 & & & & & & & & & & & & & \\
\hline & & & & & & & & & & & & & & & \\
\hline & & & & & & & & & & & & & & & \\
\hline & & & & & & & & & & & & & & & \\
\hline & & & & & & & & & & & & & & & \\
\hline & & & & & & & & & & & & & & & \\
\hline & & & & & & & & & & & & & & & \\
\hline
\end{tabular}

Station No. 141
VISUAL CORE DESCRIPTION

Page 1 of 1

Ship CHAIN Cruise 115 Leg 6 Sta. 144 Core No. $92 \mathrm{PC}$ Total Length $734 \mathrm{~cm}$. Lat. $30^{\circ} 25.7^{\circ} \mathrm{S}$ Long. $38^{\circ} 5^{\circ} .3^{\circ} \mathrm{W}$ Depth 3934 mearr. Core condition EXLELLENT Date Described GMAR75 by Jenedar

Physiographic location VEMA CHANNEL.

Lithologic

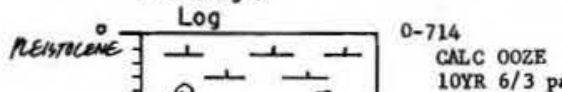

Detailed Description

Core No. $91 \mathrm{PG}$

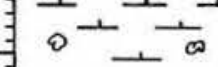

$\exists \rightarrow \frac{1}{\infty}$

$\infty+1$

-

궁

$200 \exists \div \overrightarrow{0}$

$\exists 0^{-1}-\frac{1}{\omega}$

$\exists+\infty+\infty$

$+\frac{1}{\omega}+$

$\theta+\frac{1}{c}$

$400-\perp^{\circ} \overline{0}^{\infty}$.

$\infty+1$

$\div+\frac{1}{\circ}$

$\exists+\infty$

$\vec{\infty}+\frac{1}{\infty}$

$+1$

$600 \exists+\infty$

$\exists \omega+\infty$

土 $^{\infty}+1$

$\exists$ जे

naspreate $f$ end of cree

800

手

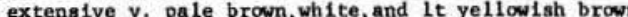
mottling throughou a fey black flecks can be seen 100-120 cm mo1st s1 silty lutite with foram abund.varying s1 throughout from colmon to abund.

ovarall hue occasionally and gradually lightens to $7 / 4 \mathrm{v}$, pale brown in the zones $230-255 \mathrm{~cm}, 290-330 \mathrm{~cm}, 520-580 \mathrm{~cm}, 600-$ at 0 
458

SMEAR SLIDE DESCRIPTIONS - W.H.O.I. SEDIMENT CORES

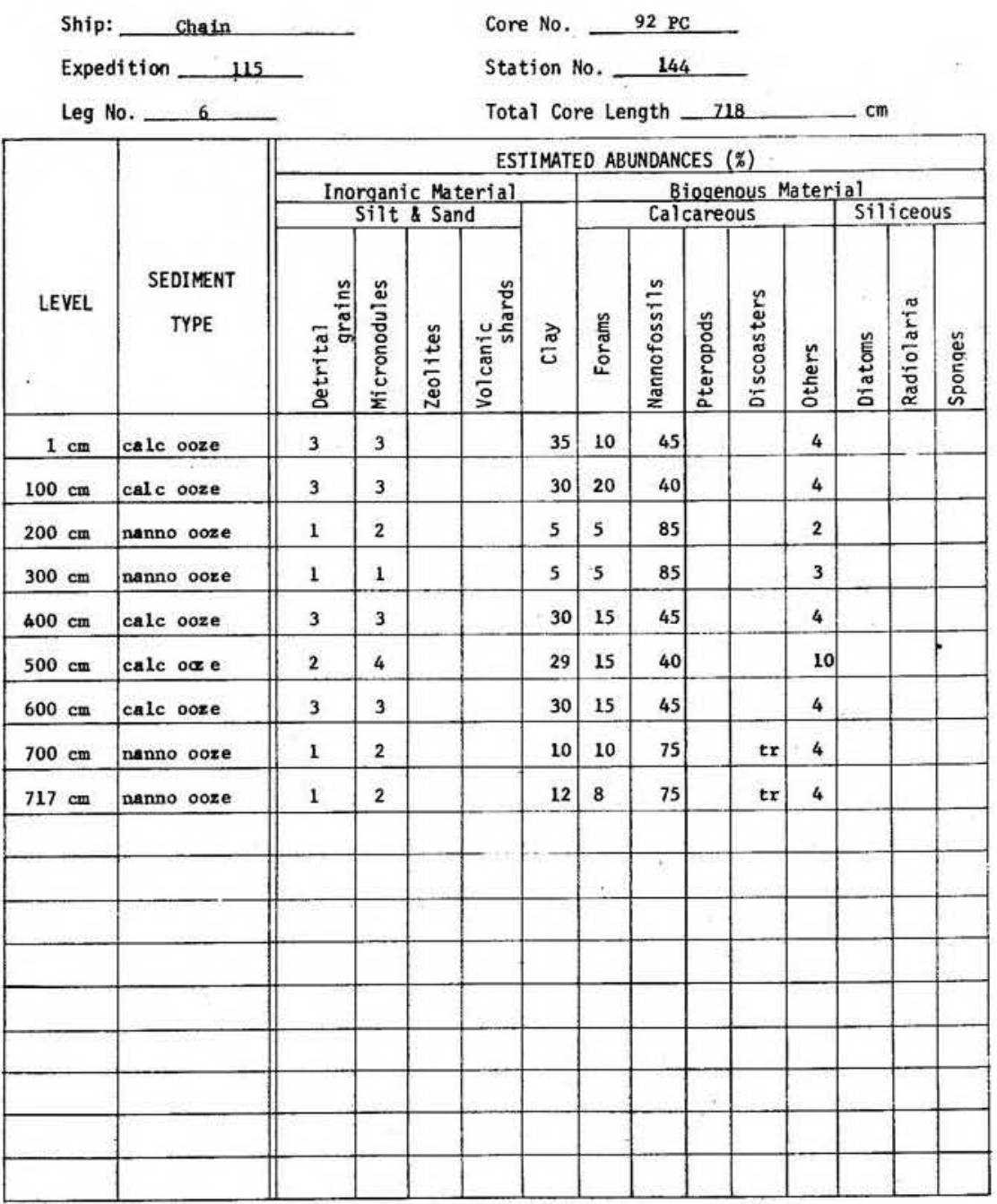

459

VISUAL CORE DESCRIPTION

Page 1 of 1

Ship CHAIN Cruise 115 Leg 6 Sta. 144 Core No. 92PG Lat. $30^{\circ} 25.7^{\prime} \mathrm{s}$ Long. $38^{\circ} 50.3^{\circ} \mathrm{W}$ Depth $3934 \mathrm{~m}$. Corr Core condition EXCEUENT Date Described 6 MAR. 75 by jower Physiographic location VEMA CHANNEL.

Lithologic REISTCOVI

Detoiled Description

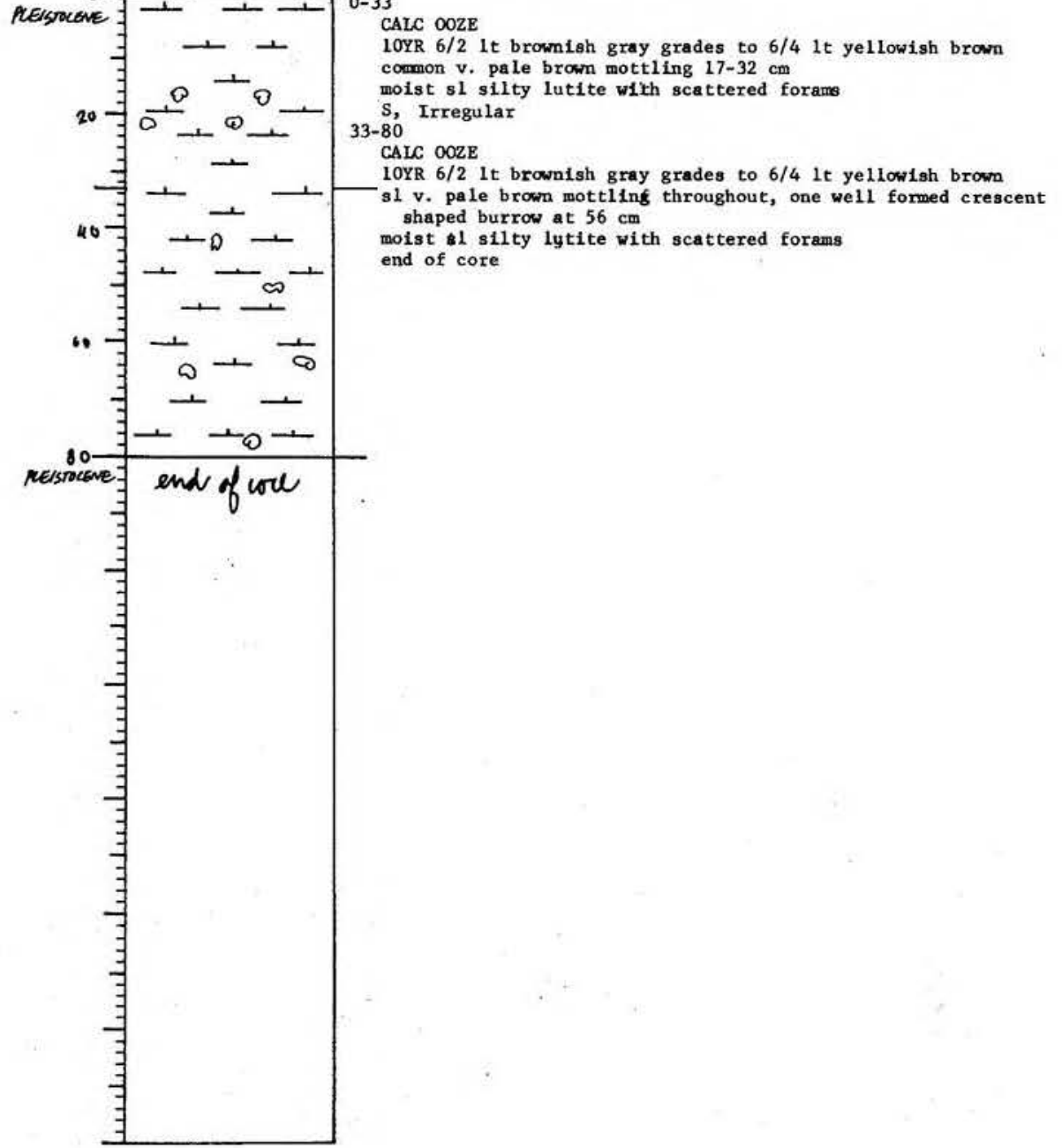


460

SMEAR SLIDE DESCRIPTIONS - W.H.O.I. SEDIMENT CORES

Ship: Chain

Expedition 115

Leg No. 6
Core No. 92 PG

Station No. 144

Total Core Length $\quad 80 \quad \mathrm{~cm}$ ESTIMATED ABUNDANCES (\%)

\begin{tabular}{|c|c|c|c|c|c|c|c|c|c|c|c|c|c|c|}
\hline \multirow[b]{3}{*}{ LEVEL. } & \multirow[b]{3}{*}{$\begin{array}{c}\text { SEDIMENT } \\
\text { TYPE }\end{array}$} & \multicolumn{13}{|c|}{ ESTIMATED ABUNDANCES (\%) } \\
\hline & & \multicolumn{4}{|c|}{$\begin{array}{c}\text { Inorganic Material } \\
\text { Silt \& Sand }\end{array}$} & \multirow[b]{2}{*}{$\frac{\pi}{6}$} & \multicolumn{5}{|c|}{$\begin{array}{l}\text { Biegenous Mater } \\
\text { Calcareous }\end{array}$} & \multicolumn{3}{|c|}{$\frac{11}{\text { Siliceous }}$} \\
\hline & & 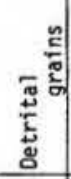 & 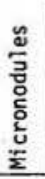 & $\frac{\stackrel{4}{*}}{\frac{ \pm}{2}}$ & $\frac{n}{\frac{n}{2}}$ & & 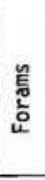 & 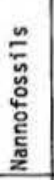 & 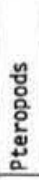 & 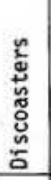 & 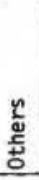 & 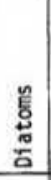 & $\begin{array}{l}\frac{\pi}{2} \\
\frac{\pi}{0} \\
\frac{0}{0} \\
\tilde{\pi} \\
\end{array}$ & 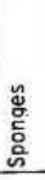 \\
\hline $1 \mathrm{~cm}$ & calc ooze & 3 & 3 & & & 25 & 25 & 40 & & & 4 & & & \\
\hline $79 \mathrm{~cm}$ & calc ooze & 2 & 5 & & & 22 & 20 & 45 & & & 6 & & & \\
\hline & & & & & & & & & & & & & & \\
\hline & & & & & & & & & & & & & & \\
\hline & & & & & & & & & & & & & & \\
\hline & & & & & & & & & & & & & & \\
\hline & & & & & & & & & & & & & & \\
\hline & & & & & & & & & & & & & & \\
\hline & & & & & & & & & & & & & & \\
\hline & 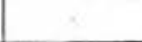 & & & & & & & & & & & & & \\
\hline & & & & & & & & & & & & & & \\
\hline & & & & & & & & & & & & & & \\
\hline & & & & & & & & & & & & & & \\
\hline & & & & & & & & & & & & & & \\
\hline & & & & & & & & & & & & & & \\
\hline & & & & & & & & & & & & & & \\
\hline & & & & & & & & & & & & & & \\
\hline & & & & & & & & & & & & & & \\
\hline
\end{tabular}

461

VISUAL CORE DESCRIPTION

Page 1 of 1

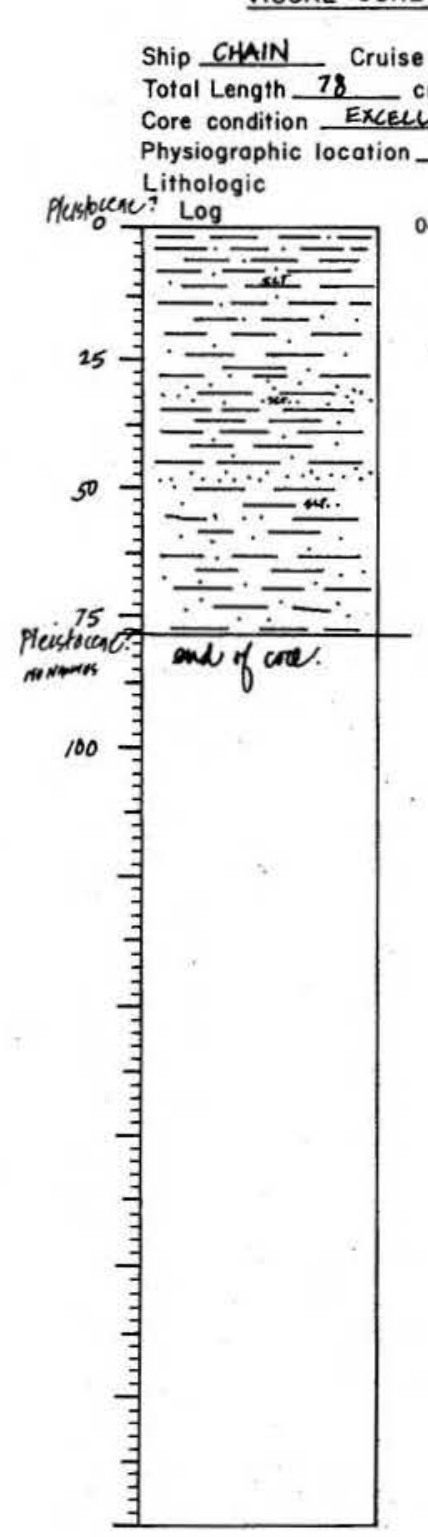

Leg \& Sta. 159 Core No. $936 \mathrm{C}$ Long. $48^{\circ} 50.9^{\circ} \mathrm{W}$ Depth Sm.corr Date Described $1730 L 475$ by frude CONTINENTAL SHELF OFF BRAZIL.

\section{Detailed Description}

UNFOSS CLAY/BETRITUS WITH LAMLNATIONS OF DETRITUS a few very faint grayish bown inclined laminationa in the zone $42-52 \mathrm{~cm}$ very moist, varying from slightly silty to silty throughout end of core 
462

SMEAR SLIDE. DESCRIPTIONS - W.H.O.I. SEDIMENT CORES

Ship: Chain

Expedition 115

Leg No. 8

Core No. $93 \mathrm{GC}$

Station No. 159

Total Core Length 78

\begin{tabular}{|c|c|c|c|c|c|c|c|c|c|c|c|c|c|c|}
\hline & \\
\hline \multirow[b]{3}{*}{ LEVEL. } & \multirow[b]{3}{*}{$\begin{array}{c}\text { SEDIMENT } \\
\text { TYPE }\end{array}$} & \multicolumn{13}{|c|}{ ESTIMATED ABUNDANCES (\%) } \\
\hline & & \multicolumn{5}{|c|}{$\begin{array}{c}\text { Inorganic Material } \\
\text { Silt \& Sand }\end{array}$} & \multicolumn{5}{|c|}{$\begin{array}{l}\text { Biogenous Mater } \\
\text { Calcareous }\end{array}$} & \multicolumn{3}{|c|}{ ial } \\
\hline & & & 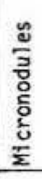 & 岕 & 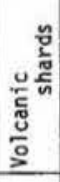 & $\frac{\pi}{0}$ & 望 & 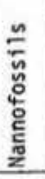 & 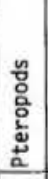 & 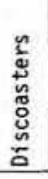 & 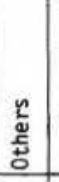 & 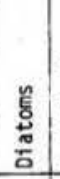 & $\begin{array}{l}\frac{\pi}{i} \\
\frac{\pi}{0} \\
\frac{0}{0} \\
\frac{0}{0} \\
\tilde{\alpha} \\
\end{array}$ & 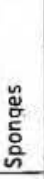 \\
\hline $1 \mathrm{~cm}$ & unfoss clay/de & 35 & 2 & & $\mathrm{tr}$ & 63 & & & & & tr & tr & & \\
\hline $43 \mathrm{~cm}$ & detritus & 85 & tr & & & 15 & & & & & & & & $\mathrm{tr}$ \\
\hline $77 \mathrm{~cm}$ & unfoss clay/de & 45 & 2 & & tr & 53 & & & & & $t x$ & tr & & tr \\
\hline & & & & & & & & & & & & & & \\
\hline & & & & & & & & & & & & & & \\
\hline & & & & & & & & & & & & & & \\
\hline & & & & & & & & & & & & & & \\
\hline & & & & & & & & & & & & & & \\
\hline & & & & & & & & & & & & & & \\
\hline & & & & & & & & & & & & & & \\
\hline & & & & & & & & & & & & & & \\
\hline & & & & & & & & & & & & & & \\
\hline & & & & & & & & & & & & & & \\
\hline & & & & & & & & & & & & & & \\
\hline & & & & & & & & & & & & & & \\
\hline & & & & & & & & & & & & & & \\
\hline & & & & & & & & & & & & & & \\
\hline & & & & & & & & & & & & & & \\
\hline
\end{tabular}

463

VISUAL CORE DESCRIPTION

Page 1 of 1

Ship CHAIN Cruise 115 Leg 8 Sta. 160 Core No. $94 \mathrm{GC}$ Total Length $128 \mathrm{~cm}$. Lat.010 $37.5^{\prime} \mathrm{N}$ Long. $49^{\circ} 010^{\prime} \mathrm{w}$ Depth $10 \mathrm{~m}$ Core condition EXCELLENT Date Described 17 JULY 75 by R MIGIRR Physiographic location_Brazilian Shelf

Physiographic

Lithologic

Pentricio Log Detailed Description

Nowhane

:

- very small individual black specks throughout

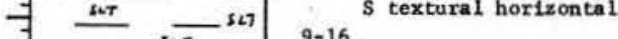

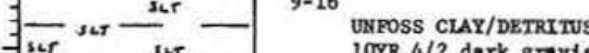

10YR $4 / 2$ dark grayish brown

$\operatorname{lm}$ 年

several thin late

tions and small patches of silt

SLT SLT SLT $16-38$

Plistuceret. textural inclined $10^{\circ}$

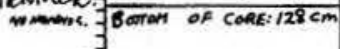
DETRITUS

dark grayish brown

very silty lutite grades to sil

38-89

textural, inclined $30^{\circ}$

\section{UNFOSS CLAY/DETRITUS}

10YR $4 / 2$ dark grayish brown

several $1 \mathrm{~cm}$ thick silt laminations, inclined $30^{\circ}$ at 71,81 -

$\mathrm{S}$, textural, inclined $10^{\circ}$

$39-128$

THS CLAY/DETRITUS

Y $4 / 2$ dark grayish brown

moist silty lutite

several very thin wavy ailt laminations $110-118 \mathrm{~cm}$ 
SMEAR SLIDE DESCRIPTIONS - W.H.O.I. SEDIMENT CORES

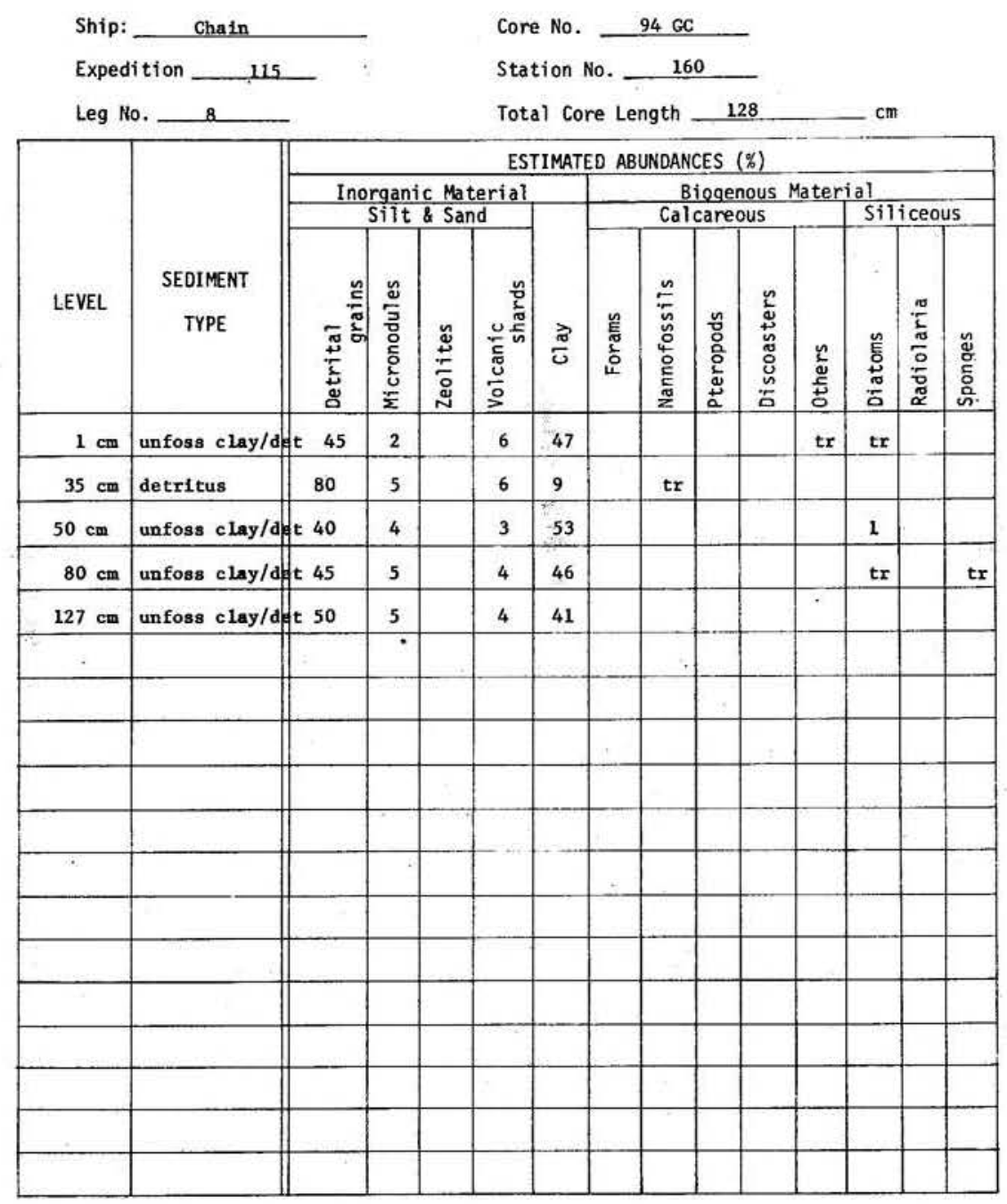




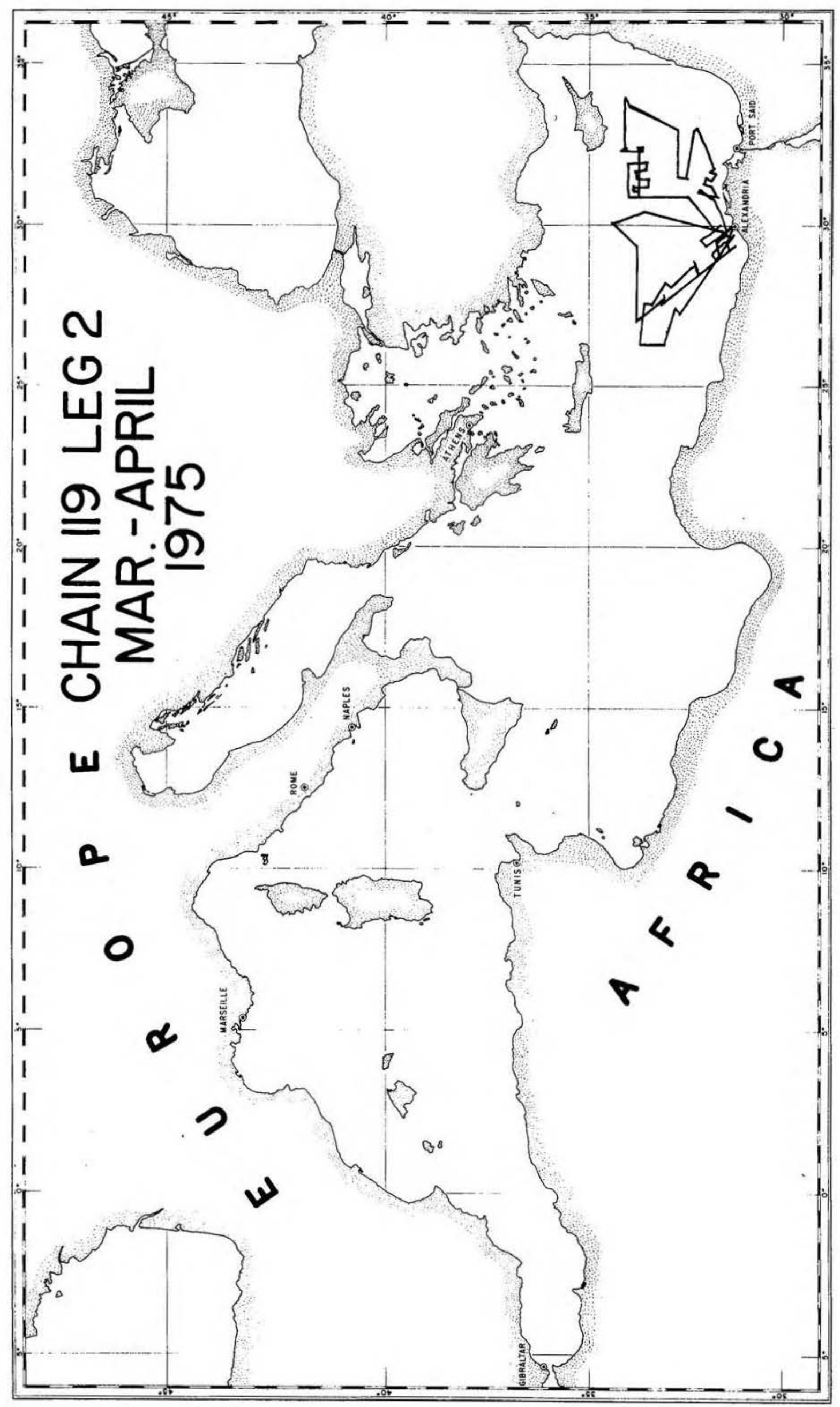


SAMPLE DE- DATE

LENGTH DREDGE ROCK

MARS. CORE $Q R$ OR $Q R$ PHYSIO. $\theta R$

LATITUDE LONGITUDE FIX DEN DREDGE DPH END SAMPLE GRAPHIC SEO. VITA

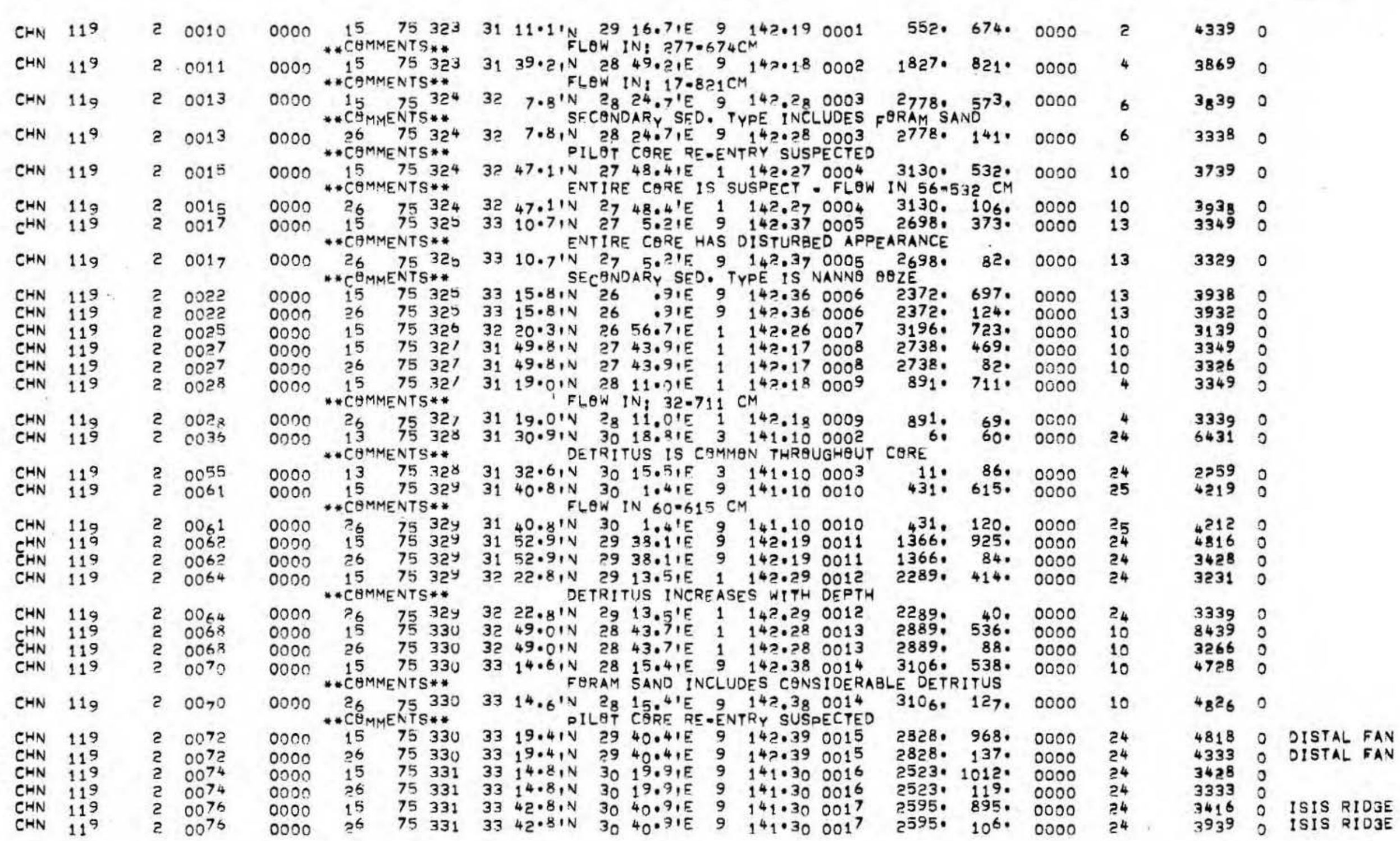




$\begin{array}{llll}\text { CHN } & 119 & 2 & 0078 \\ \text { CHN } & 119 & 2 & 0078 \\ \text { CHN } & 119 & 2 & 0080 \\ \text { CHN } & 119 & 2 & 0080 \\ \text { CHN } & 119 & 2 & 0082 \\ \text { CHN } & 119 & 2 & 0082 \\ \text { CHN } & 119 & 2 & 0083 \\ \text { CHN } & 119 & 2 & 0083 \\ \text { CHN } & 119 & 2 & 0087 \\ \text { CHN } & 119 & 2 & 0109 \\ \text { CHN } & 119 & 2 & 0115 \\ \text { CHN } & 119 & 2 & 0115 \\ \text { CHN } & 119 & 2 & 0116 \\ \text { CHN } & 119 & 2 & 0115 \\ \text { CHN } & 119 & 2 & 0119 \\ \text { CHN } & 119 & 2 & 0118 \\ \text { CHN } & 119 & 2 & 0120 \\ \text { CHN } & 119 & 2 & 0120 \\ \text { CHN } & 119 & 2 & 0121 \\ \text { CHN } & 119 & 2 & 0121 \\ \text { CHN } & 119 & 2 & 0122 \\ \text { CHN } & 119 & 2 & 0127 \\ \text { CHN } & 119 & 2 & 0129 \\ \text { CHN } & 119 & 2 & 0129 \\ \text { CHN } & 119 & 2 & 0131 \\ \text { CHN } & 119 & 2 & 0131 \\ \text { CHN } & 119 & 2 & 0133 \\ \text { CHN } & 119 & 2 & 0133 \\ \text { CHN } & 119 & 2 & 0135 \\ \text { CHN } & 119 & 2 & 0135 \\ \text { CHN } & 119 & 2 & 0137 \\ \text { CHN } & 119 & 2 & 0137 \\ \text { CHN } & 119 & 2 & 0139 \\ \text { CHN } & 119 & 2 & 0139 \\ \text { CHN } & 119 & 2 & 0141 \\ & & & \end{array}$

\section{0}

\section{(2000}

0000

0000

0000

0000

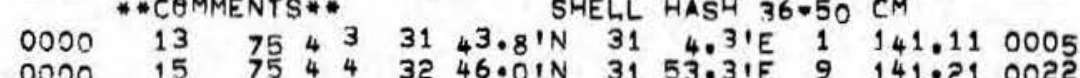

$\begin{array}{llllll}15 & 75 & 4 & 1 & 34 & 20.81 \mathrm{~N} \\ 26 & 75 & 4 & 1 & 34 & 20.81 \mathrm{~N}\end{array}$

$15 \quad 754$

26 754

$15754 \quad 4$

$\begin{array}{lllllll}6 & 75 & 4 & 1 & 33 & 13.91 & 13\end{array}$

$\begin{array}{lll}5 & 75 & 4 \\ 15 & 75 & 4\end{array}$

$\begin{array}{lllllll}7 & 34.91 N & 31 & 16.31 E & 9 & 141.21 & 0021 \\ 3 & 34.91 & 31 & 16.31 E & 9 & 141.21 & 0021\end{array}$

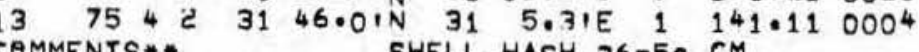
SHELL HASH $36.50 \mathrm{CM}$

$0000 \quad 26 \quad 7544 \quad 3246.01 N$ 31 $53.31 E$ 9 141.210022

$\begin{array}{lllllllllllllll}0000 & 15 & 75 & 4 & 4 & 33 & 30.51 N & 31 & 52.61 E & 9 & 141.31 & 0023\end{array}$

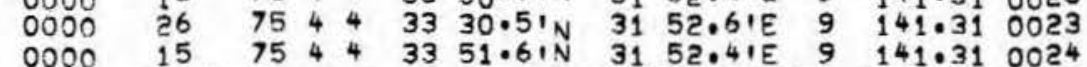

* COMMENTS** SECONDARY SED. TYPE INCLUDES
INCLES

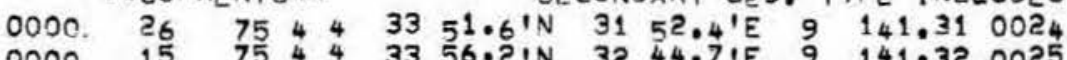

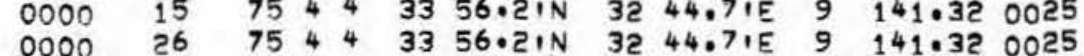

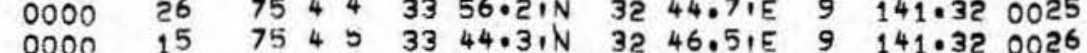

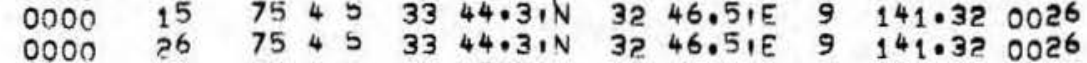

$0000 \quad 13754$ b $3345.71 \mathrm{~N}$ 32 $48.01 E 99141.320006$ * COMMENTS** SECONDARY SED. TYPE IS NANNO $9 \theta Z$

$000013 \quad 754$ Y $3340.71 N 3240.91 E 99141.320007$ *COMMENTS**

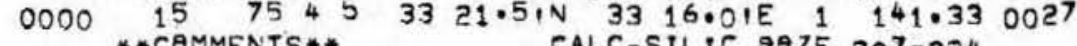
$\theta \theta Z E^{88}$

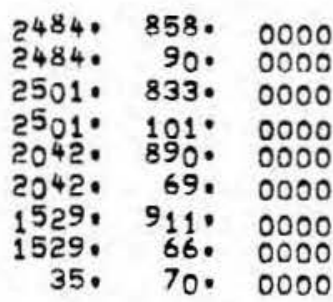

21. 97. 0000

1581. 931. 0000

1581.113 .0000

$\begin{array}{lll}2142, & 322^{\circ} & 0000 \\ 2142, & 70 . & 0000\end{array}$

2636. 711. 0000 QRAM SAND

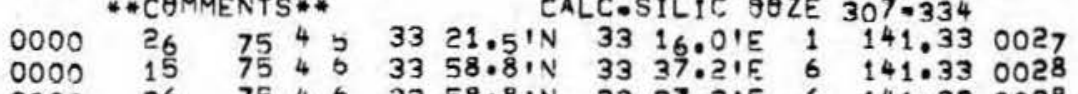

$\begin{array}{llllllllllll}0000 & 26 & 75 & 4 & 6 & 33 & 58.81 N & 33 & 37.21 E & 6 & 141.33 & 0028 \\ 0 & 15 & 75 & 4 & 6 & 34 & 9.11 \mathrm{~N} & 34 & 23.01 E & 9 & 141.44 & 0029\end{array}$

2636.76 .0000

1827. 456. 0000

1827. 94. 0000

898. 506. 0000

898. 77. 0000

89. 46. 0000 882.

43. 0000

629. 698. 0000

1829. 76. 0000

2द20. 700. 0000 2220. 142. 0000 2073. 1294 . 0000

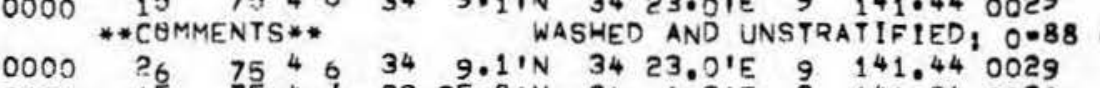

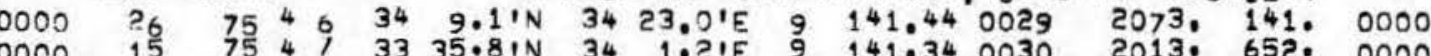

S 34 1.2UE 140.340030 2013, 652. 0000 RAM SAND

$0000 \quad 26754,3335.81 N^{34} 1.21 E, 9141.340030$ 2013.111. 0000 *COMMENTS** 7535.8 SECONDARY SED. TYPE INCLUDES FORAM SAND

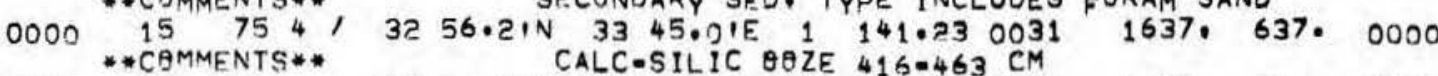

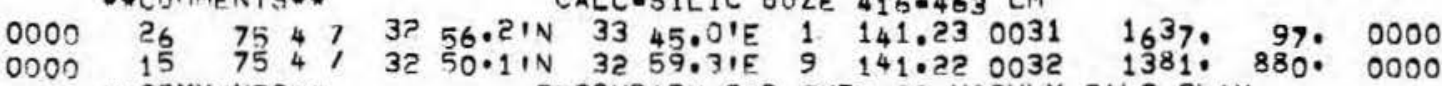
* *CEMMENTS** SECONDARY SED TYPE IS HIGHLY CALC CLAY

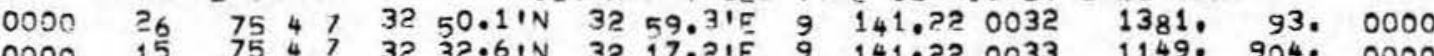

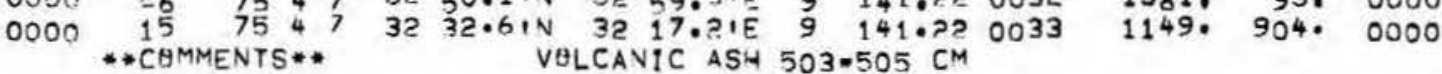

$\begin{array}{lll}13 & 3438 & 0 \\ 13 & 3938 & 0 \\ 24 & 3438 & 0 \\ 24 & 3938 & 0 \\ 24 & 3238 & 0 \\ 24 & 3338 & 0 \\ 24 & 3818 & 0 \\ 24 & 3338 & 0 \\ 24 & 2465 & 0\end{array}$

ISIS RIDGE

SIS RIDGE

ISIS RIOGE

ISIS RIOGE

2.4690

ISIS RIDGE

3432 ISIS RIOGE

3348 O ISIS RIOGE

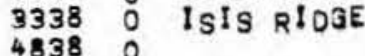

48330

393

39330

33430

$\begin{array}{ll}3322 & 0 \\ 3332 & 0\end{array}$

33320

33380

33280

43380

4339
3738

37310

38190

38180

33320

34320

$\begin{array}{lll}3338 & 0 & \\ 3338 & 0 & \text { ISIS RIDGE }\end{array}$ 


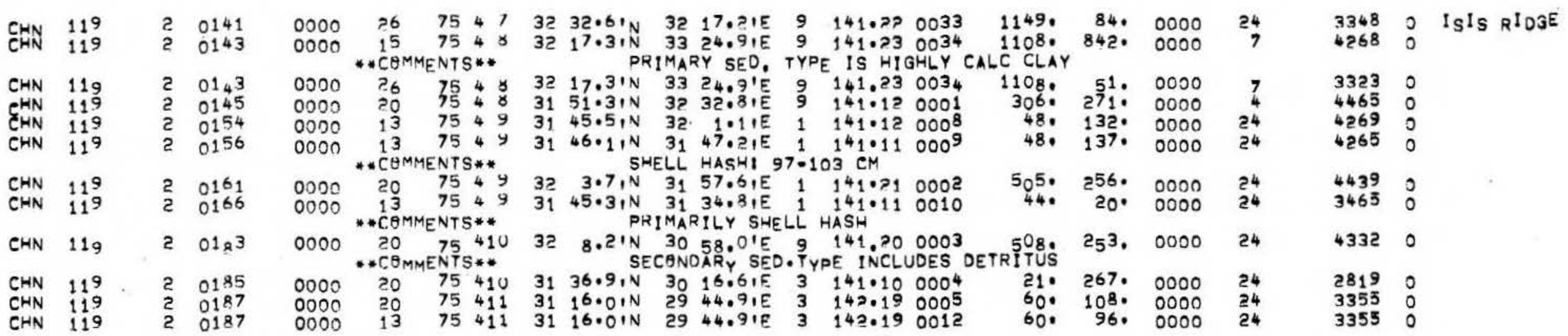




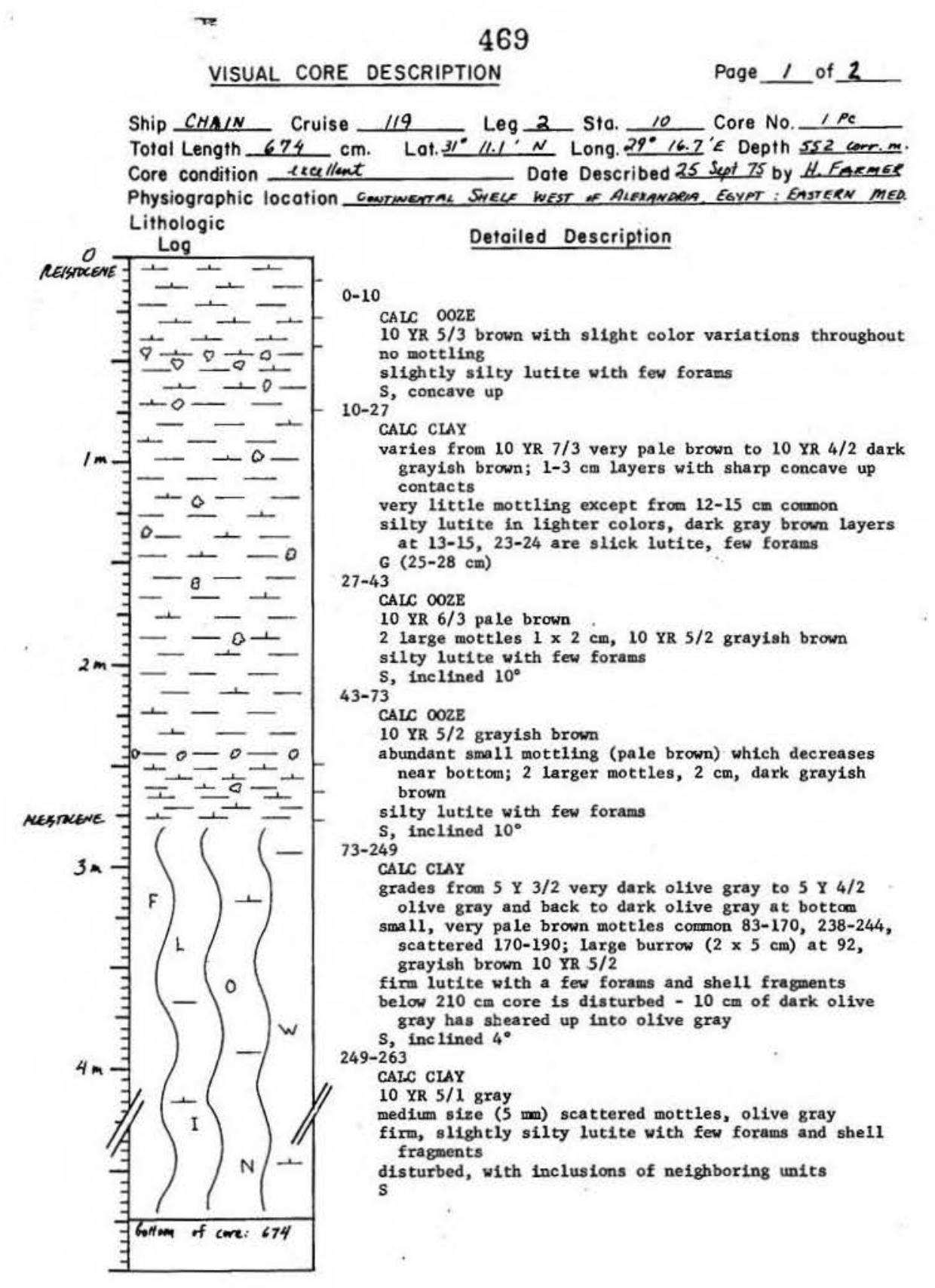
Core condition excellent Dote Described 25 Sept 75 by H. Faremes

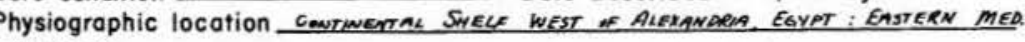
ithologic

Detailed Description

10 YR $5 / 3$ brown with slight color variations throughout grayish brown very little mottling except from $12-15 \mathrm{~cm}$ coumon ty lutite in lighter colors, dark gray brown layers $(25-28 \mathrm{~cm})$

CALC 0028

(pale brown) which decrease

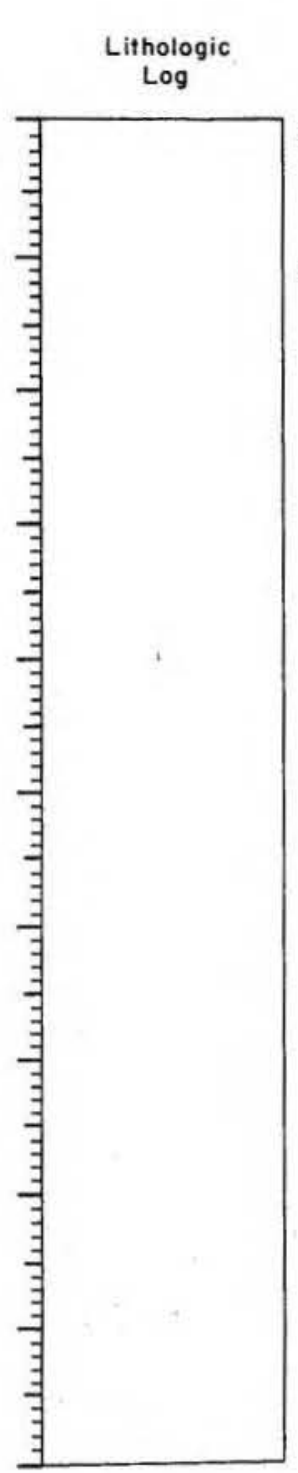

\section{Detailed Description}

263-277

CALC CLAY

10 YR 4/1 dark gray

no mottling

277-674

CALC CLAY GRADES TO HIGHLY CALC CIAY

10 YR $4 / 1$ dark gray, turns to $6 / 2$ light brownish gray $(\sim 386 \mathrm{~cm})$

flow in 
SIEAR SLIDE DESCRIPTIONS - W.H.O.I. SEDIMENT CORES

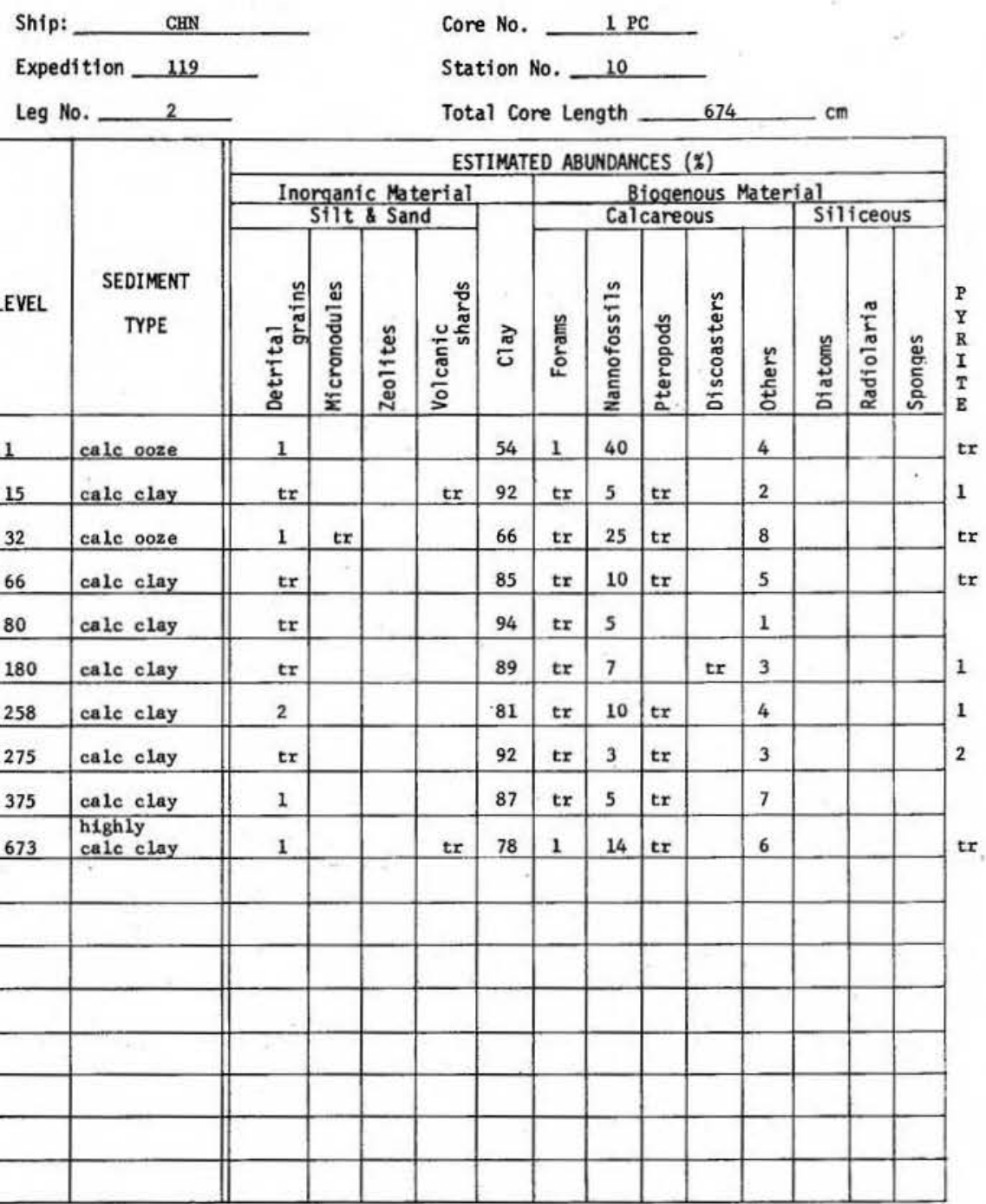

Ship CHAIN Cruise 119 Leg 2 Sta. II Core No. 2 PC Total Length $821 \quad \mathrm{~cm}$. Lat.31. 39.2' $\mathrm{N}$ Long. $28^{\circ} 49.2^{\prime} \mathrm{E}$ Depth $1827 \mathrm{carc}$ Core condition exallent Date Described 26 Sept 15 by H. Farmer Physiographic location CONTINENTAL SNELF NW of ALENANPRIA, EOVIT Lithologic

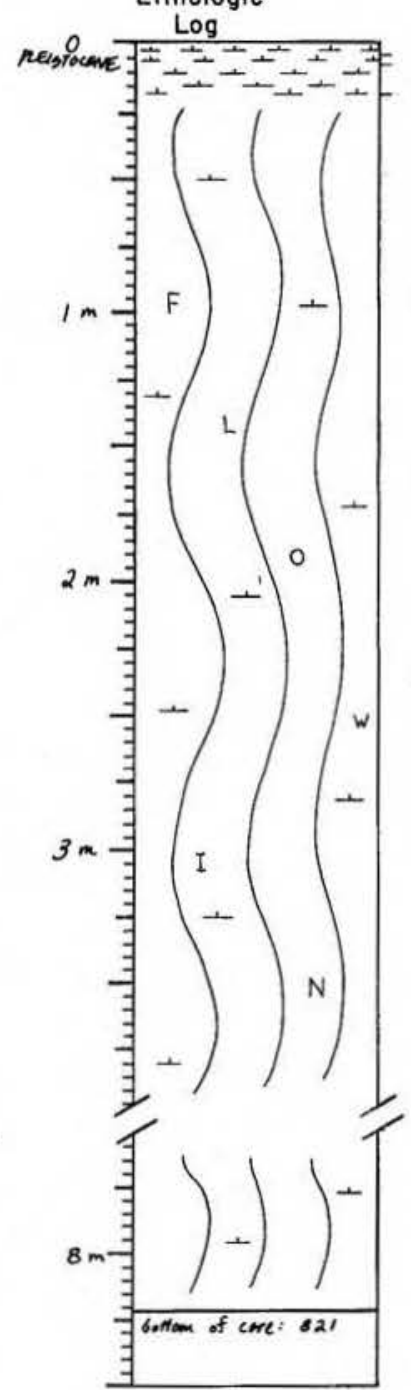

Detailed Description

10 YR $6 / 4$ 1ight yellow brow

no mottling

wedges in top $2 \mathrm{~cm}-$ somewhat disturbed up to $6 \mathrm{~cm}$

3-7 CALC OOZE

10 YR $6 / 2$ light olive gray

no mottling very silty lutite s, concave

$7-17$ CALC OOZE CALC OOZE
5 Y $5 / 2$ olive gray no mottiling

tite with few foram

laminated from $2-5 \mathrm{~mm}$ thick;pyrite nodule at 10 $(2 \times 2 \mathrm{~mm})$

irregular,

CALC OOZE

5 Y $6 / 2$ light ollve gray

no mottling

very silty lutite

end of core 
SHEAR SLIDE DESCRIPTIONS - W.H.O.I. SEDIMENT CORES

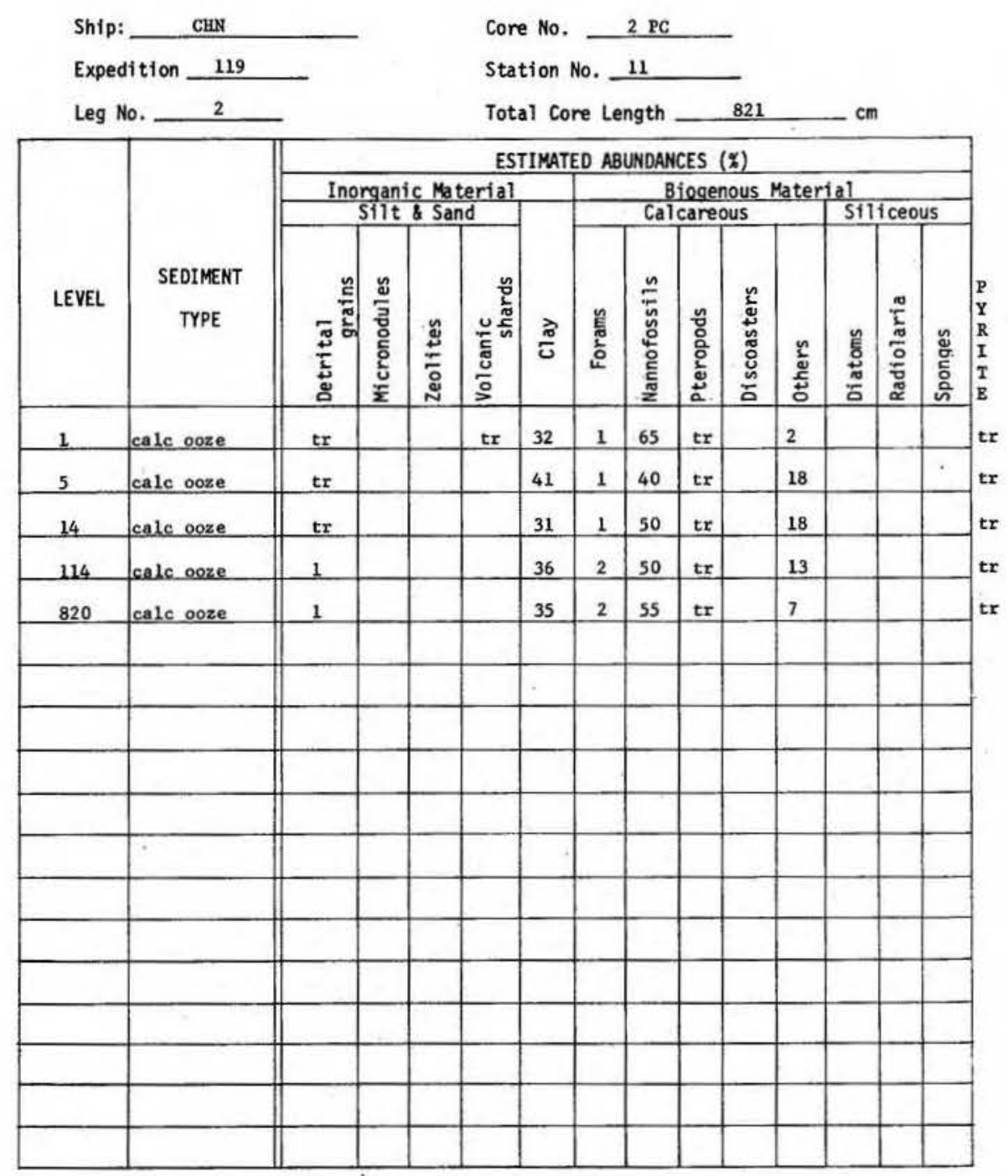

Ship CHAIN Cruise 119 Leg 2 Sta. 13 Core No. 3 PC Total Length $573 \mathrm{~cm}$. Lat. $32^{\circ} 07.8^{\prime} N$ Long. $28^{\circ} 24.7^{\prime}$ \& Depth $2778^{\circ} \mathrm{corm}$ Core condition excullent Dote Described 3 Dec 75 by $T$ Farmer Physiographic location LowER ContINENTAL Slope NW OF ALERANDRIA, ESVVPT - MEO SEA Lithologic

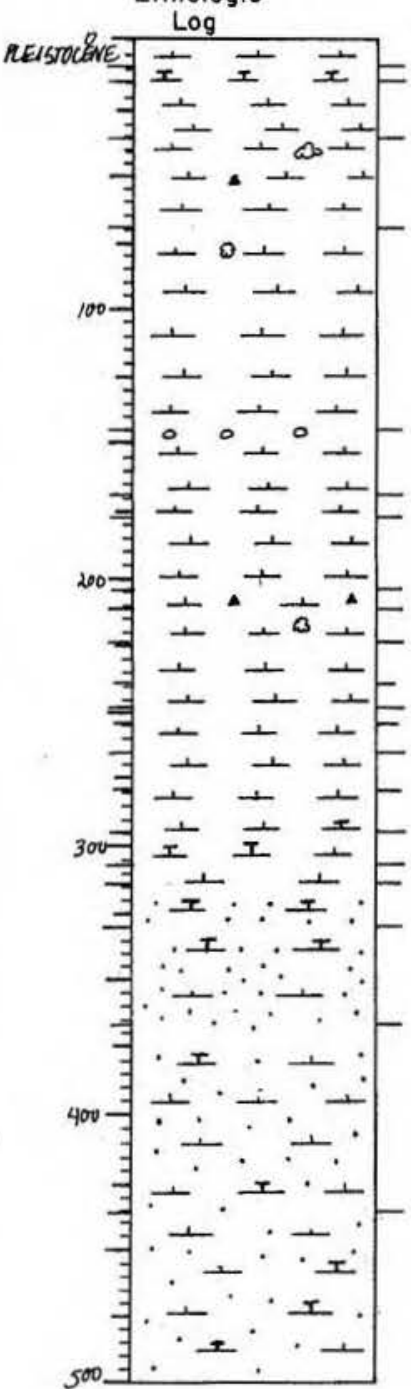


475

VISUAL CORE DESCRIPTION

Ship CHAIN Cruise

119

Leg 2 Sta. 13 Core No. 3 PC

Lithologic

Log

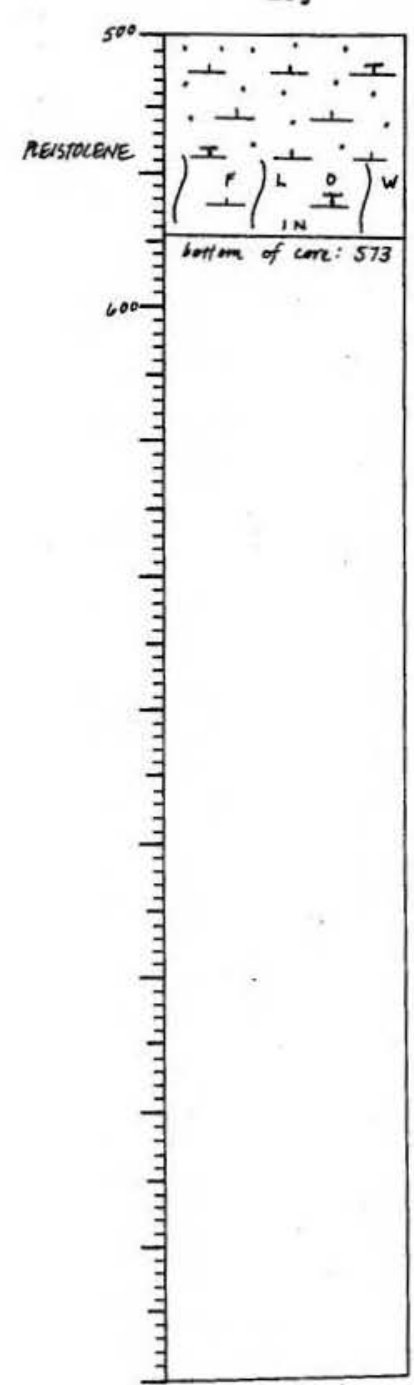

\section{Detailed Description}

145-168

silty lutite with scattered for genic sand (161-168) with pebbles ( $1 \times 2 \mathrm{~cm})$ of lamis

168-177

distorted bands of 5 Y 5/2,4/2,3/2 olive gray to dark olive gray (sapropel)

no mottling apparent

lutite with scattered foram

$\mathrm{S}$,
$7-204$

CAIC 007

CALC OOZE

$5 \times 4 / 4$ olive

homogeneous lutite with occasional very fine, dark distorted laminations

177-181 interbedded with above

204-211

CALC OOZE

5 Y $3 / 2$ dark olive gray

no mottling

slightly silty lutite with abundant forams and

pteropods
sapropel-like material with faint, very fine laminations

11-22

CALC OOZE

5 Y $4 / 2$ olive gray grades to $6 / 2$ light olive gray

scattered dark mottles from above

sequence from $204-224$ repeated $224-248,248-265,265-$ 294

294-30

307

10 YR $7 / 3$ very pale brown

mottiing

gand, grades from very fine to fine stle color laminations
476

VISUAL CORE DESCRIPTION

Page 3 of 3

Ship CHAIN Cruise 119 Leg 2 Sto. 13 Core No. $3 \mathrm{PC}$

Lithologic

Log

Detailed Description

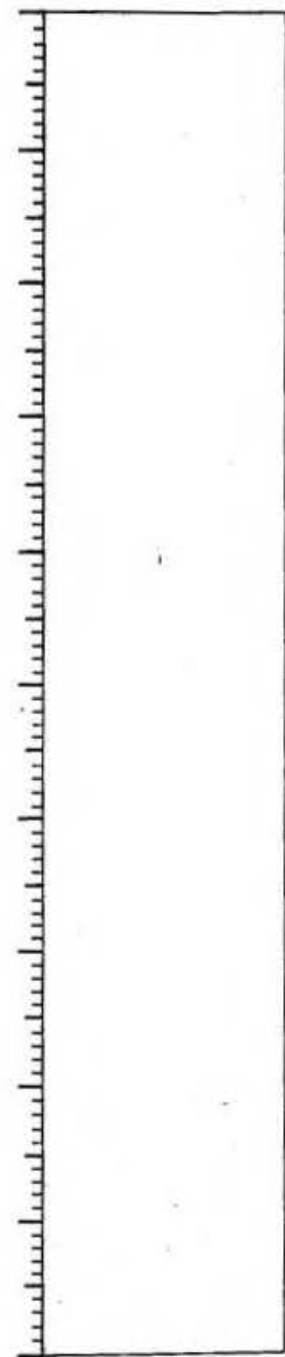

307-314

CAIC OOZE

5 I $6 / 2$ 11ght olive gray

silty lutite with scattered forams

S14-330

CALC OOZE/DETRITUS

2.5 Y $4 / 4$ olive brown

no mottling

326-330 pale brown lutite clast mixed with coarse $s$ $\stackrel{-2}{S}$

DETRTTUS/CALC OOZE

2.5 Y $5 / 4$ 1ight olive brown

no mottling

medium sand

340-355 intermixed gradational bands of dark gray $s$

367-463

CALC OOZE/DETRITUS

dark gray brown 2.5 Y $4 / 2$

fine to medium homogeneous detrital sand

436-543 laminations ( $2-10 \mathrm{~mm})$ of dark gray brown $(2.5$ Y $4 / 2)$
silt-fine sand (CAIC OOZE/DETRITUS) and olive ( 5 X 4/4) silty lutite (HIGHLY CALC CLAY)

flow in of above material, sandy lutite (CALC OOZE) of core 
SIEAR SLIDE DESCRIPTIONS - W.H.O.I. SEDIMENT CORES
Ship: CHN

Expedition 119
Leg No. 2
Core No. 3 PC
Station No. 13

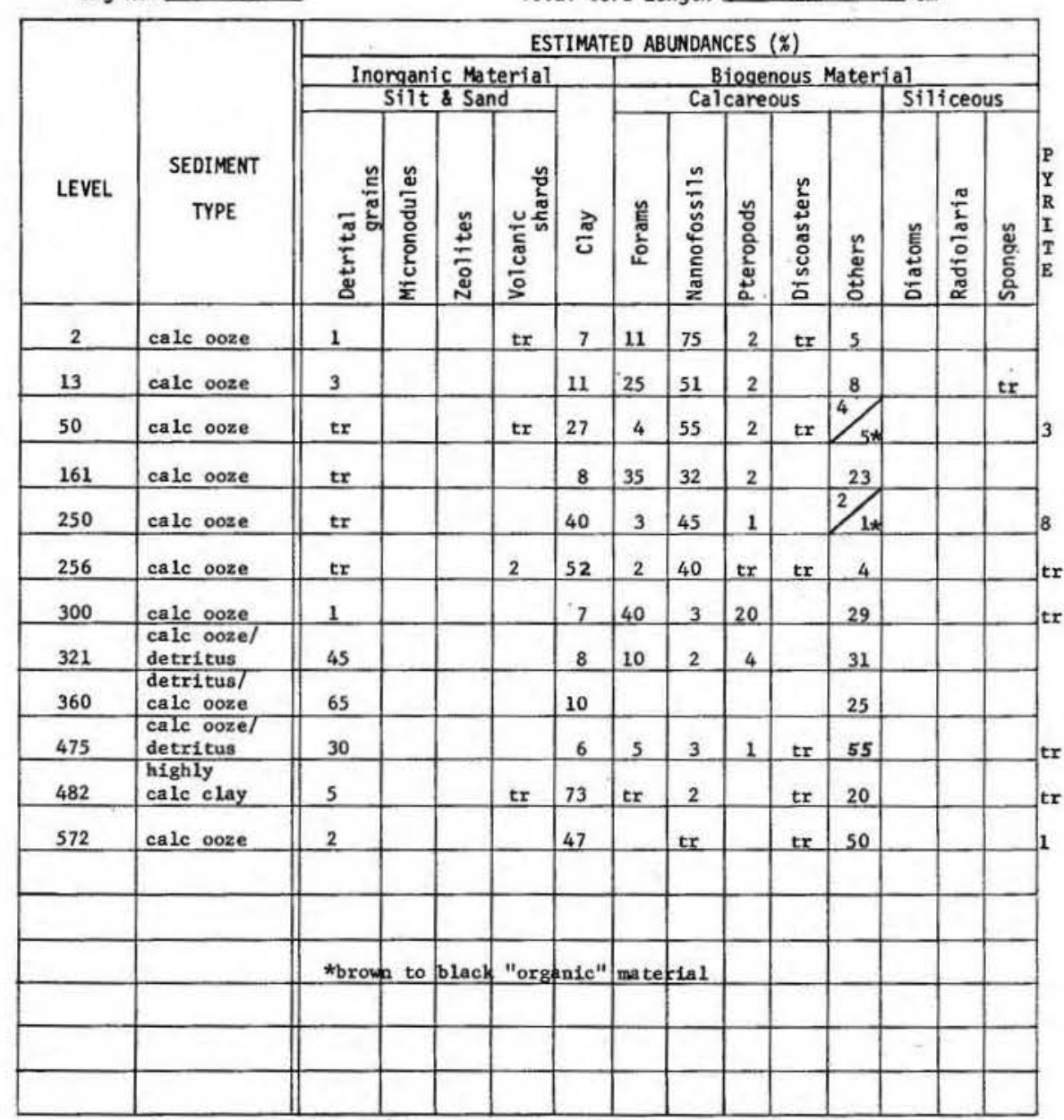

VISUAL CORE DESCRIPTION

Page 1 of 2

Ship CHAIN Cruise 119 Leg 2 Sto. 13 Core No. 3 PG

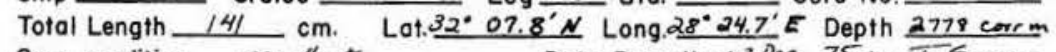
Core condition eteellent Date Described 3 Dec 75 by 1 Farmer Physiographic location kower Continental Slope NW of Alexandria, Egypt. Hed. Sa Lithologic

REISTRENE L Log Detailed Description

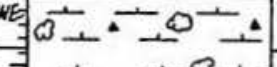

正 $+\infty+$

$\exists I x$

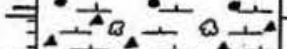

正土+

$\exists+1$

$1007+\div$

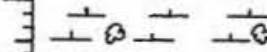

REIGTOLNE bottion of core: 141 penetration (plilot core rebound) is suspected. Bxcepting the top $J S$ cal $(\mathrm{DG})$ which were not recovered by the piston core, there lo excellent correlation between the lithologies of the 2 cores, with the pilot compacted to $1 / 2$ the However, the lowest $16 \mathrm{~cm}$ of the pilot repeat the sequence previously sampled from 68-108 PG, whereas the piston core sampled $7 \mathrm{~cm}$ of foram sand at this depth. Thus, after the PG "rebounded" and began 1ts second penetration, it apparent1y "blew by" the top sediment and did not begin sampling until $68 \mathrm{~cm} P G$ (due to the inertia of the mud already reduring the previous penetration).

$0-1$

CALC OOZE

5 Y $3 / 2$ dark olive gray

scattered small very pale brown mottles

with pteropods

void at $18 \mathrm{~cm}$

18-53

CALC OOZE

gre 5 Y $5 / 2$ olive gray to 10 YR $7 / 4$ very

pate 10 YR $6 / 4$ light yellow brow

silty lutite with scattered pteropods and foram

laminations of foram silt/sand 40,42-43, dark brown S
3-69

CAIC OOZE WITH PYRITE

5 Y $3 / 2$ dark olive gray

with scattered forams and pteropods

sapropel-1ike material

69-11

CALC OOZE

grades from 5 Y $5 / 2$ olive gray to 10 YR $7 / 4$ very pale

brown

no mottling

政 olive brown 102-104 
Ship CHAIN Cruise 119 Leg 2 Sta. 13 Core No. 3 PG

Lithologic

Log

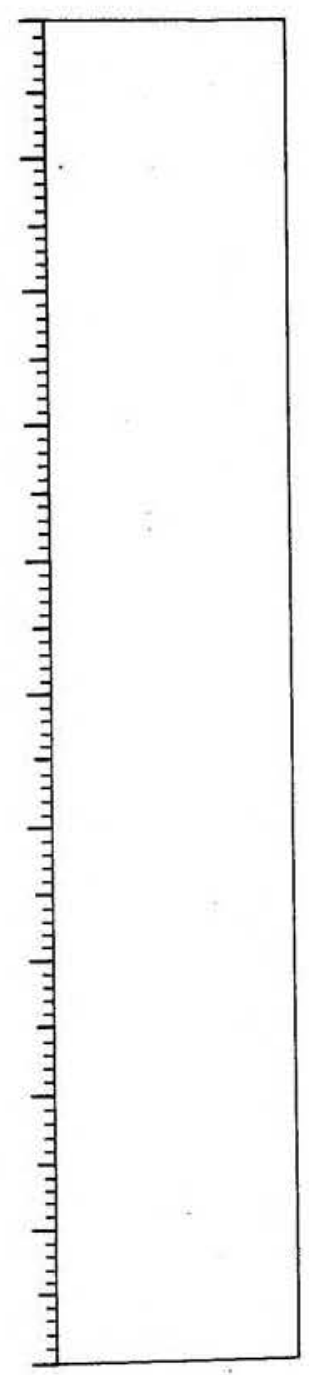

Detailed Description

$110-126$

CALC OOZE

10 YR $6 / 3$ pale brown grades to $7 / 4$ very pale brown

126-141

repeat of sequence from $68-110$

end of core
SMEAR SLIDE DESCRIPTIONS - W.H.O.I. SEDIMENT CORES
Core No. $3 \mathrm{PG}$

Station No. 13

Total Core Length $141 \_\mathrm{cm}$

ESTIMATED ABUNDANCES (\%)

\begin{tabular}{|c|c|c|c|c|c|c|c|c|c|c|c|c|c|c|}
\hline \multirow[b]{4}{*}{ LEVEL } & \multirow[b]{4}{*}{$\begin{array}{c}\text { SEDIMENT } \\
\text { TYPE }\end{array}$} & \multicolumn{13}{|c|}{ ESTIMATED ABUNDANCES (\%) } \\
\hline & & \multirow{2}{*}{\multicolumn{4}{|c|}{$\begin{array}{c}\text { Inorganic Material } \\
\text { Silt \& Sand }\end{array}$}} & & \multirow{2}{*}{\multicolumn{5}{|c|}{$\begin{array}{l}\text { Biogenous Mate } \\
\text { Calcareous }\end{array}$}} & & & \\
\hline & & & & & & & & & & & & \multicolumn{3}{|c|}{ Siliceous } \\
\hline & & 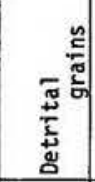 & 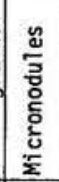 & 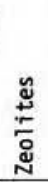 & $\frac{5}{5}$ & తె & 崖 & 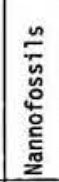 & 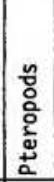 & 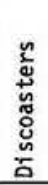 & 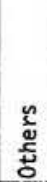 & 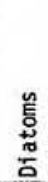 & 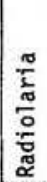 & $\begin{array}{l}\text { ڤ. } \\
\text { Бे } \\
\text { हn }\end{array}$ \\
\hline 1 & calc ooze & tr & & & tx & 40 & 2 & 51 & tr & & $3 / 2$ & & & \\
\hline 50 & $\begin{array}{l}\text { calc ooze w/ } \\
\text { in nodules }\end{array}$ & tr & 15 & & & 7 & 5 & 65 & 1 & & 7 & & & \\
\hline 65 & $\begin{array}{l}\text { calc ooze w/ } \\
\text { pyrite }\end{array}$ & tr & & & & 15 & 4 & 45 & 1 & & $5 / 5$ & & & \\
\hline 80 & nanno ooze & tx & & & & 5 & 1 & 90 & tr & tr & 4 & & & \\
\hline 115 & calc ooze & $\operatorname{tr}$ & & & & 30 & 2 & 58 & $\operatorname{tr}$ & tr & 10 & & & \\
\hline 124 & calc ooze & tx & & & & 30 & 5 & 48 & 2 & tr & 15 & & & \\
\hline \multirow[t]{4}{*}{140} & calc ooze & 2 & & & & 12 & 1 & 64 & 1 & & 20 & & & \\
\hline & & & & & & & & & & & & & & \\
\hline & . & & & & & & & & & & & & & \\
\hline & & *brown & sapr & pel: & ("o & $\operatorname{gan}$ & m & teri & 1 & & & & & \\
\hline & & & & & & & & & & & & & & \\
\hline & & & & & & & & & & & & & & \\
\hline & & & & & & & & $=$ & & & & & & \\
\hline & & & & & & & & & & & & & & \\
\hline & & & & & & & & & & & & & & \\
\hline & & & & & & & & & & & & & & \\
\hline & & & & & & & & & & & & & & \\
\hline & & & & & & & & & & & & & & \\
\hline
\end{tabular}


Ship GMALN Cruise 119 Leg $\frac{2}{19}$ Sta. $\frac{15}{27^{\circ}}$ Core No. $\frac{4 P C}{3130}$ Total Length $532 \mathrm{~cm}$. Lot. $32^{\circ} 47.1^{\prime} \mathrm{N}$ Long. $27^{\circ} 48.4^{\prime} \mathrm{z}$ Depth $3130 \mathrm{cmr}$. Core condition excelleat, stratigraphy suspect Date Described 16 Nev 75 by T. Farmer Physiographic location Herodotus Basin Eastern Med. Sea Lithologic

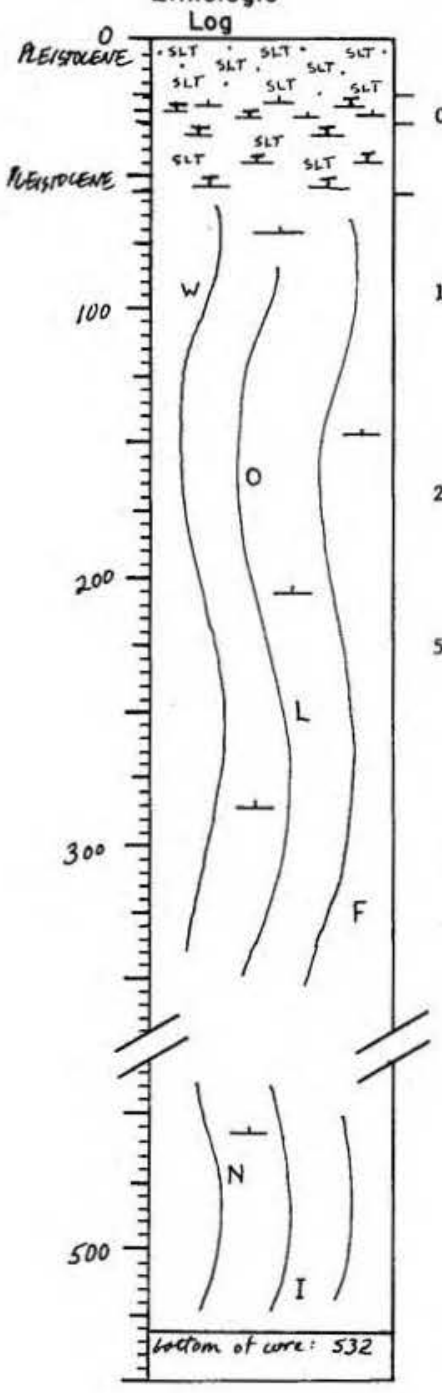

Detailed Description

$0-19$

CALC OOZE/DETRITUS

10 YR $3 / 3$ dark brown

grades from very fine to fine sand

ppears to have been washed in core line

9-29 CALC OOZE

10 YR $7 / 3$ very pale brown and $6 / 2$ light brownish gray lutite

9-26 original laminations are folded and distorted, dge of 10 YR $3 / 2$ very dark gray-brown 29-56 slick lutite, 26-29 fairly true

CAIC OOZE/DETRIIUS

YR $4 / 2$ dark grayish brown - somewhat 1ighter towards bottom

grades from slick lutite to medium sand

56-532

CALC OOZE

soupy lutite

This in

The ence of fine and at the top (absent in pilot) and the absence of a sapropelic layer between the thind snd fourth unit (present from 37-so in pilot) are end of core
10 YR $4 / 2$ dark grayish brown
SMEAR SLIDE DESCRIPTIONS - W.H.O.I. SEDIMENT CORES
Ship: _ CBAIN

Expedition 119

Leg No. 2
Core No. $4 \mathrm{PC}$

Station No. 15

Total Core Length $\quad 532$

\begin{tabular}{|c|c|c|c|c|c|c|c|c|c|c|c|c|c|c|}
\hline \multirow[b]{4}{*}{ LEVEL } & \multirow[b]{4}{*}{$\begin{array}{c}\text { SEDIMENT } \\
\text { TYPE }\end{array}$} & \multicolumn{13}{|c|}{ ESTIMATED ABUNDANCES ( $(x)$} \\
\hline & & \multirow{2}{*}{\multicolumn{4}{|c|}{$\frac{\text { Inorganic Material }}{\text { Silt \& Sand }}$}} & \multirow[b]{3}{*}{$\frac{\pi}{\sigma}$} & \multirow{2}{*}{\multicolumn{5}{|c|}{$\begin{array}{l}\text { Biogenous Mater } \\
\text { Calcareous }\end{array}$}} & & & \\
\hline & & & & & & & & & & & & \multicolumn{3}{|c|}{ Siliceous } \\
\hline & & 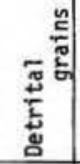 & 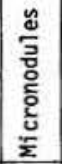 & $\frac{\$}{\frac{\$}{2}}$ & & & 高 & 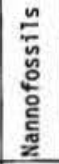 & 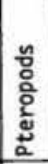 & 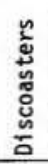 & $\begin{array}{l}r \\
5 \\
\text { sँ } \\
0\end{array}$ & 蓆 & $\frac{\frac{\pi}{\frac{\pi}{\sigma}}}{\frac{\sigma}{0}}$ & $\begin{array}{l}\text { แूँ } \\
\text { हैं } \\
\text { ڤั }\end{array}$ \\
\hline 1 & $\begin{array}{l}\text { calc ooze/ } \\
\text { detritus }\end{array}$ & 50 & & & & & 17 & 2 & 1 & & 30 & & & \\
\hline 18 & calc ooze & 1 & & & & 5 & 29 & 30 & 5 & & 30 & & & \\
\hline 32 & $\begin{array}{l}\text { calc oozel } \\
\text { detritus }\end{array}$ & 30 & & & & 1 & 23 & 4 & 5 & & 37 & & & \\
\hline 70 & $\begin{array}{l}\text { calc ooze } \\
\text { vith detritus }\end{array}$ & 15 & & & & 30 & 2 & 43 & 2 & & 8 & & & \\
\hline 531 & calc ooze & 7 & & & & 35 & $t r$ & 54 & & & 4 & & & \\
\hline & & & & & & & & & & & & & & \\
\hline & & & & & & . & & & & & & & & \\
\hline & & & & & & & & & & & & & & \\
\hline & & & & & & & & & & & & & & \\
\hline & & & & & & & & & & & & & & \\
\hline & & & & & & & & & & & & & & \\
\hline & & & & & & & & & & & & & & \\
\hline & & & & & & & & & & & & & & \\
\hline & & & & & & & & & & & & & & \\
\hline & & & & & & & & & & & & & & \\
\hline & & & & & & & & & & & & & & \\
\hline & & & & & & & & & & & & . & & \\
\hline & & & & & & & & & & & & & & \\
\hline
\end{tabular}


Ship CHAIN Cruise 119 Leg 2 Sto. 15 Core No. 4 PG Total Length $106 \mathrm{~cm}$. Lot. $32^{\circ} 47.1^{\prime} \mathrm{N}$ Long. $27^{\circ} 48.4^{\prime}=$ Depth $3 / 30$ comm Core condition excellent Date Described 16 Nov 75 by I Farmer

Physiographic location Herodotus Basin, Eastern med. Sea

\section{Lithologic \\ Detailed Description}

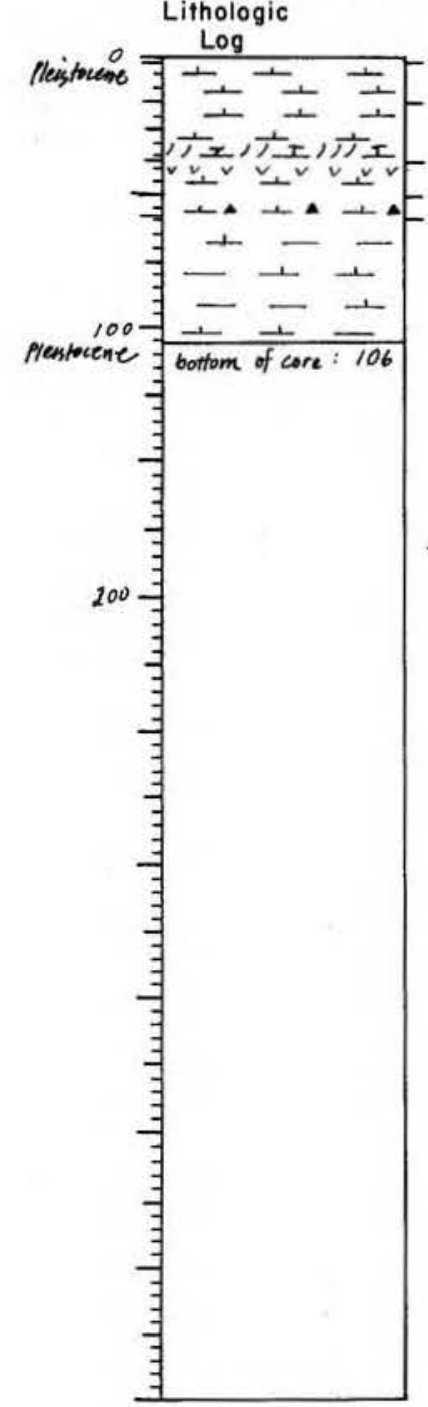

$0-2$

void produced as top sediment vas compacted because no end cep on core liner

CALC OOZE

10 YR $7 / 3$ very pale brown

light gray mottles down to $8 \mathrm{~cm}$, fine, light gray and red-brown laminae, 8-15 smooth, slick lutite

compaction features evident 2-6

$15-37$ horizontal

CALC OOZE

10 YR $6 / 2$ light brownish gray

lutite with cross-bedded, laminated foram

S, slightly inclined

CALC OOZE

10 YR $6 / 4$ 1ight yellowish brown, slightly paler nea

botton

subdued grayish mottles comonon 46-50

$41 \mathrm{~cm}-3 \mathrm{~mm}$ yellow-brown bed of volcanic ash

s, slightly inclined

$50-58$

CALC 0OZE (saprope

2.5 y $3 / 2$ vexy dark grayish brown

silck lutite with common forams and pteropods S, horizonta 1

-106

CALC CIAY

5 Y $3 / 2$ dark ollve gray

slick, smooth lutite

$90-1 \mathrm{~cm}$ bed of above unit

end of core

SMEAR SLIDE DESCRIPTIONS - W.H.O.I. SEDIMENT CORES

hip:

Expedition 119

Leg No. 2
Core No. $\quad 4$ PG

Station No. 15

Total Core Length 106

ESTIMATED ABUNDANCES (q)

\begin{tabular}{|c|c|c|c|c|c|c|c|c|c|c|c|c|c|c|}
\hline \multirow[b]{3}{*}{ LEVEL } & \multirow[b]{3}{*}{$\begin{array}{c}\text { SEDIMENT } \\
\text { TYPE }\end{array}$} & \multicolumn{13}{|c|}{ ESTIMATED ABUNDANCES ( $($ ) } \\
\hline & & \multicolumn{4}{|c|}{$\begin{array}{l}\text { Inorganic Materia } \\
\text { Silt \& Sand }\end{array}$} & \multirow[b]{2}{*}{$\frac{\pi}{\omega}$} & \multicolumn{5}{|c|}{$\begin{array}{l}\text { Biegenous Mate } \\
\text { Calcareous }\end{array}$} & \multicolumn{3}{|c|}{$\frac{\text { al }}{\text { siliceous }}$} \\
\hline & & 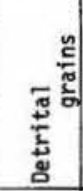 & 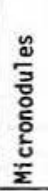 & 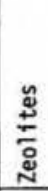 & $\frac{{ }^{\frac{n}{2}}}{\frac{0}{2}}$ & & $\begin{array}{l}\text { 岕 } \\
\text { : }\end{array}$ & 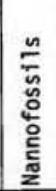 & 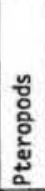 & 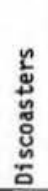 & $\begin{array}{l}\frac{n}{5} \\
\frac{\omega}{5} \\
\text { के }\end{array}$ & 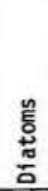 & $\frac{\frac{\pi}{i}}{\frac{\pi}{0}} \frac{0}{0}$ & 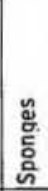 \\
\hline 4.5 & calc_ooze & 1 & & & & 53 & 5 & 30 & 1 & tr & 10 & & & \\
\hline 32 & $\begin{array}{l}\text { calc ooze } \\
\text { (foram sand) }\end{array}$ & 2 & & & & 5 & 50 & 28 & 3 & $\mathrm{tr}$ & 12 & & & tr \\
\hline 41. & volcanic ash & 2 & & & 92 & & 1 & 2 & & & 3 & & & \\
\hline 46 & calc ooze & 1 & & & & 21 & 2 & 70 & 1 & & 5 & & & \\
\hline 55 & calc ooze & 4 & & & & 10 & 4 & 62 & 1 & & 8 & & & \\
\hline 105 & cale clay & 10 & tr & & 2 & 71 & $\mathrm{tr}$ & 6 & & & 8 & & & \\
\hline & & & & & & . & & & & & & & & \\
\hline & & & & & & & & & & & & & & \\
\hline & & & & & & & & & & & & & & \\
\hline & & & & & & & & & & & & & & \\
\hline & & & & & & & & & & & & & & \\
\hline & & & & & & & & & & & & & & \\
\hline & & & & & & & & & & & & & & \\
\hline & & & & & & & & & & & & & & \\
\hline & t & & & & & & & & & & & & & \\
\hline & & & & & & & & & & & & & & \\
\hline & & & & & & & & & & & & & & \\
\hline & & & & & & & & & & & & & & \\
\hline
\end{tabular}


Ship CHAIN Cruise 119 Leg 2 Sta. 17 Core No. 5PC

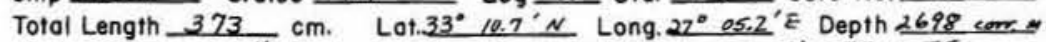
Core condition exeellent Date Described/7 Nov 75by 7 Farmer

Physiographic location LOWER SoWN FLANK, MEDITERRANEAN RIDGE, E. MEO. SEM

Lithologic

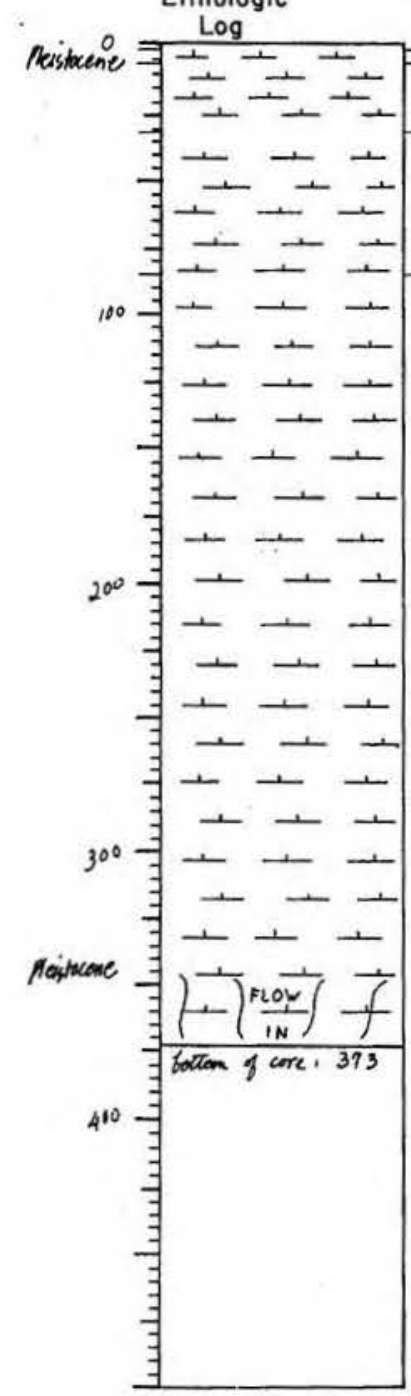

\section{Detailed Description}

$0-1$

CALC OOZE

10 YR $6 / 6$ brownish yellow

silty lutite

$1-6$

CALC OOZE

5 Y $7 / 1$ light gray

silty lutite with scattered foram

$6-31$

NANNO OOZE

5 Y $8 / 1$ white

very homogeneous throughout
stiff silty lutite with scattered forams

void at 31, was initially bigger but has been filled by slumping of white unit

31-85

CALC OOZE

5 Y $7 / 1$ light gray grades into $6 / 1$ gray $\sim 53 \mathrm{~cm}$ fim motty lutite

an, silty latite with scattered forams $31-53$ an $1 \mathrm{~cm}$ void at 51

S

CALC OOZE

5 Y $3 / 2$ dark olive gray, 5 Y $8 / 1$ white, $7 / 1$ light

gray, and $6 / 1$ gray

tremely apparent below 85 where intense marbling

occurrs between white, 11ght gray and gray 168-169,

milty lutite with scattered and conmon for

sapunt from 95-110

348-373

(sheared and distorted beds?)

flow in
SMEAR SLIDE DESCRIPTIONS - W.H.O.I. SEDIMENT CORES
Ship:

Expedition 1

Leg No. 2
Core No. $5 . \mathrm{PC}$

Station No. 17

Total Core Length 373

\begin{tabular}{|c|c|c|c|c|c|c|c|c|c|c|c|c|c|c|}
\hline \multirow[b]{3}{*}{ LEVEL } & \multirow[b]{3}{*}{$\begin{array}{c}\text { SEDIMENT } \\
\text { TYPE }\end{array}$} & \multicolumn{13}{|c|}{ ESTIMATED ABUNDANCES ( $(\%)$} \\
\hline & & \multicolumn{4}{|c|}{$\begin{array}{c}\text { Inorganic Material } \\
\text { Silt \& Sand }\end{array}$} & \multirow[b]{2}{*}{$\frac{\pi}{6}$} & \multicolumn{5}{|c|}{$\begin{array}{l}\text { Biogenous Mater } \\
\text { Calcareous }\end{array}$} & \multicolumn{3}{|c|}{ Silliceous } \\
\hline & & 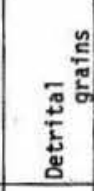 & 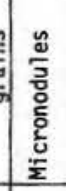 & 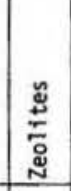 & 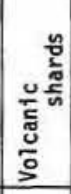 & & 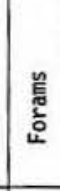 & 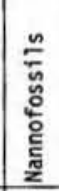 & 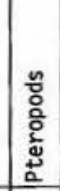 & 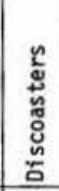 & 乩 & 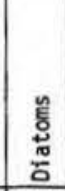 & 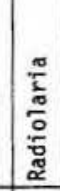 & 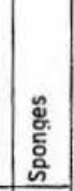 \\
\hline 1 & calc ooze & 2 & tr & & tr & 32 & 3 & 60 & tr & & 3 & & & \\
\hline 18 & nanno ooze & tr & & & & 4 & tr & 95 & tr & $t x$ & 1 & & & \\
\hline 80 & calc ooze & 1 & & & & 24 & 15 & 53 & 1 & & 4 & & & \\
\hline 160 & nanno ooze & 1 & & & & 9 & 1 & 88 & tr & tr & 1 & & & \\
\hline 272 & calc ooze & tr & & & & $41^{*}$ & 7 & $45^{\prime}$ & * $t r$ & tr & 2 & & & \\
\hline 372 & calc ooze & tr & $\mathrm{tr}$ & & & 24 & 3 & 70 & tr & tr & 3 & & & \\
\hline & & & & & & + & & & & & & & & \\
\hline & & & & & & & & & & & & & & \\
\hline & & & & & & & & & & & & & & \\
\hline & & ${ }^{*}$ about & $1 / 2$ & of the & clay & and & pann & $\operatorname{arc}$ & e sta & Ined & py a & 11.ght & to-to- & \\
\hline & & dark & trown & disce & 1orat & Lon & & & & & & & & \\
\hline & & & & & & & & & & & & & & \\
\hline & & & & & & & & & & & & & & \\
\hline & & & & & & & & & & & & & & \\
\hline & & & & & & & & & & & & & & \\
\hline & & & & & & & & & & & & & & \\
\hline & & & & & & & & & & & & & & \\
\hline & & & & & & & & & & & & & & \\
\hline
\end{tabular}


VISUAL CORE DESCRIPTION

Page__ of 1

Ship CHAIN Cruise 119 Leg 2 sta. 17 Core No. 5 PG Total Length $97 \mathrm{~cm}$. Lat. $33^{\circ} 10.7^{\prime} \mathrm{N}$ Long. $27^{\circ} 05.2^{\prime} ' E$ Depth $2698 \mathrm{com}$ Core condition excellent Date Described 12 Nov 75 by 11 . Farmer Physiogrophic location LOWER SOUTH. FLANK, MED. RIDGE, E. MED. SEA Lithologic

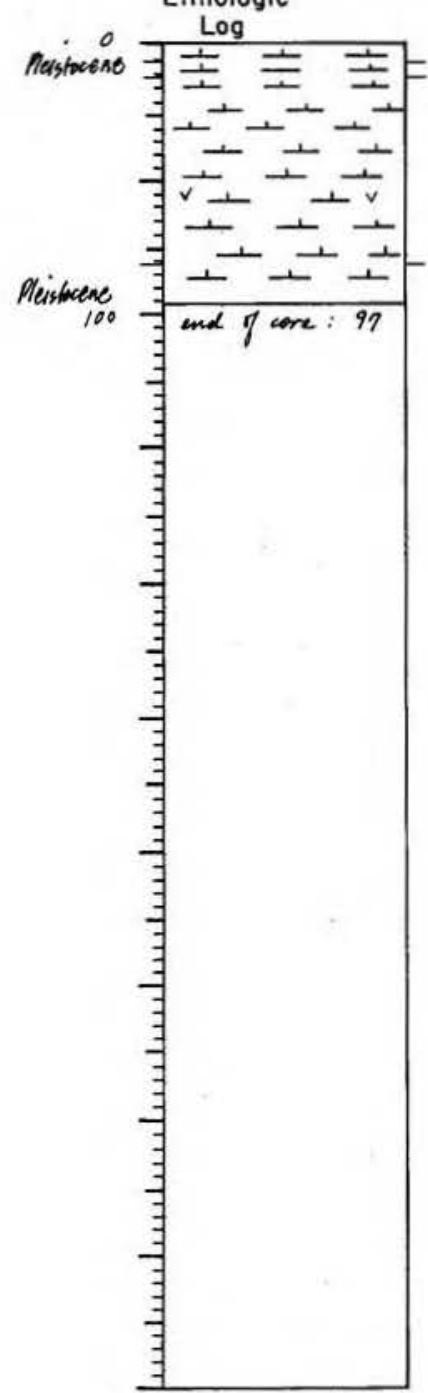

Detailed Description

$0-5.5$

RANNO OOZE

YR $6 / 4$ light yellow brow

silty lutite with scattered forams

S, horizontal

CALC OOZE

10 YR $7 / 3$ very pale brown

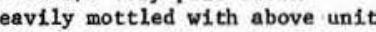

silty lutite with scattered forams, gradational contact (1)

CALC OOZE

$10 \mathrm{YR} 6 / 3$ pale brown, grades generally into $10 \mathrm{YR} 5 / 6$

yellow-brown, but an array of intermediate shades are

eral cm; trends back to $6 / 4$ light yellow brown and pale

brown from $61-81 \mathrm{~cm}$

very faint mottles are scattered throughout the subdu-

ed laminations

silty lutite with scattered forams canic ash - very gradational contace

82-97

CALC OOZE

10 YR $6 / 2$ light brownish-gray, grades smoothly into

$7 / 2$ light gray

few very dark gray fine laminae and a couple medium

end of core size mottles of same color
SMEAR SLIDE DESCRIPTIONS - W.H.0.1. SEDIMENT CORES

Ship:

CHN

Expedition 119

Leg No. 2

Station No. 17

Total Core Length __ 97

\begin{tabular}{|c|c|c|c|c|c|c|c|c|c|c|c|c|c|c|}
\hline \multirow[b]{3}{*}{ LEVEL } & \multirow[b]{3}{*}{$\begin{array}{c}\text { SEDIMENT } \\
\text { TYPE }\end{array}$} & \multicolumn{13}{|c|}{ ESTIMATED ABUNDANCES (\%) } \\
\hline & & \multicolumn{4}{|c|}{$\frac{\text { Inorganic Material }}{\text { Silt \& Sand }}$} & \multirow[b]{2}{*}{ ढे } & \multicolumn{5}{|c|}{$\begin{array}{l}\text { Biogenous Mater } \\
\text { Calcareous } \\
\end{array}$} & \multicolumn{3}{|c|}{$\frac{\text { al }}{\text { Silliceous }}$} \\
\hline & & $\frac{\frac{n}{\pi}}{5}$ & 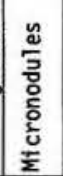 & 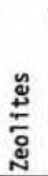 & 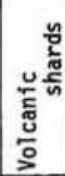 & & 号 & 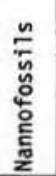 & 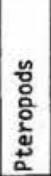 & 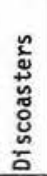 & 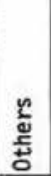 & 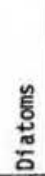 & 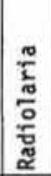 & 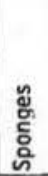 \\
\hline 1 & nanno ooze & $\operatorname{tr}$ & $\mathrm{tr}$ & & tr & 19 & 2 & 75 & 2 & & 2 & & & \\
\hline 13 & calc ooze & 2 & $t r$ & & & 18 & 2 & 75 & $t x$ & tr & 3 & & & \\
\hline 55.5 & $\begin{array}{l}\text { highly } \\
\text { calc clay }\end{array}$ & tr & tr & & $5^{*}$ & 71 & $t x$ & 20 & tr & & 4 & & & \\
\hline 95 & calc ooze & 3 & & & & 42 & 4 & 40 & 1 & tr & 10 & & & \\
\hline & & & & & & & & & & & & & & \\
\hline & & & & & & & & & & & & & & \\
\hline & & ${ }^{*}$ very & fine & sil & cious & nee & Les & & & & & & & \\
\hline & & & & & & & & & & & & & & \\
\hline & & & & & & & & & & & & & & \\
\hline & & & & & & & & & & & & & & \\
\hline & & & & & & & & & & & & & & \\
\hline & & & & & & & & & & & & & & \\
\hline & & & & & & & & & & & & & & \\
\hline & & & & & & & & & & & & & & \\
\hline & & & & & & & & & & & & & & \\
\hline & & & & & & & & & & & & & & \\
\hline & & & & 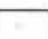 & & & & & & & & & & \\
\hline & & & & & & & & & & & & & & \\
\hline
\end{tabular}


Ship CHN Cruise 119 Leg 2 Sta. 22 Core No. $6 P_{C}$ Totol Length $697 \mathrm{~cm}$. Lat. $33^{\circ} 15.8^{\prime} \mathrm{N}$ Long. $26^{\circ} 00.9^{\prime} E$ Depth 2372 comm Core condition excellent Date Described 3 Nov 75 by H. FARemer Physiographic location MEDITERRANEAN RIOGE, EASTERN MED. SEA Lithologic

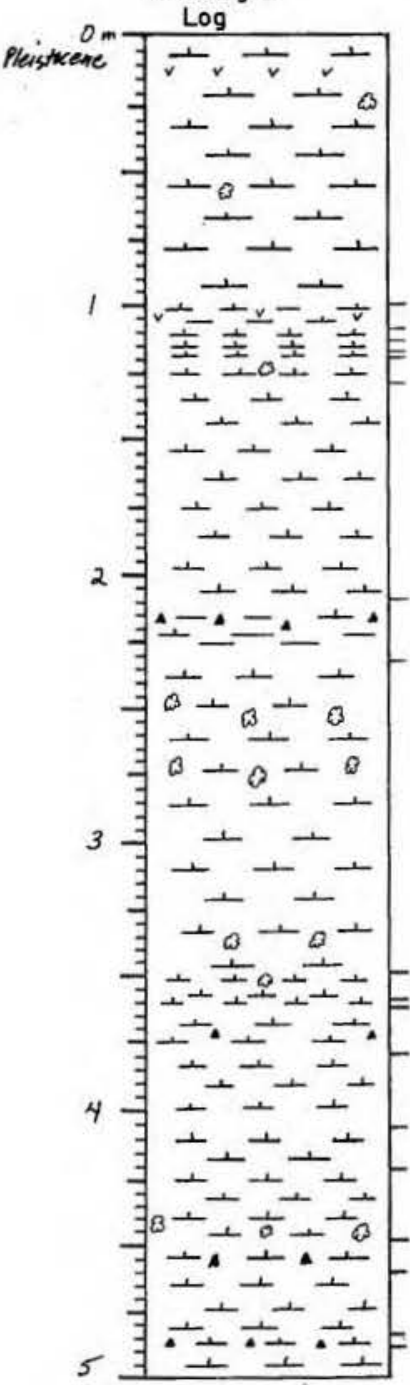

Detoiled Description

$0-100$ CALC OOZE (10 YR $6 / 4$ light yellow brown, fewer bands of 10 YR $7 / 4$ very pale broun in top 25 can

few mottles throughout 14-15 crumbly layer, lutite, dark brown; 39-40 subdued $\mathrm{s}$

-108

HIGHLY CALC CLAY WITH VOLCANIC ASB

10 YR $3 / 2$ very dark gray brown no mottiing

Iithified lutite

108-113

CALC OOZE

10 YR $6 / 3$ pale brom and 10 YR $3 / 2$ very dark gray brown (in fine laminations?) gmeared together with slight influence of lower unit

Thing of 10 YR $5 / 6$ and $5 / 8$ ye1lowish

with a few very fine gray to white laminations at bottom

S
$117-119$

7-119

CALC OOZE
5 Y $3 / 2$ dark ollive gray

few pale brown mottles

s

119-128

\section{CALC OOZE}

5 Y $6 / 1$ gray to 1ight gray

cattered, very dark

silty lutite with scattered forams only at top, and

few pteropods

sharp, mottled with lower un1

CALC OOZE

gradational banded unit primarily of 10 XR $7 / 3$ very

pale browns with 10 YR $6 / 2$ 1ight brownish gray 164-174 medium light gray mottles $174-177$

silty lutite with scattered forans except abundant 202-

207 and common pteropods 169-174

151-153. light olive brown band

Ship CHN Cruise 119 Leg 2 Sta. 22 Core No. 6 PC

7

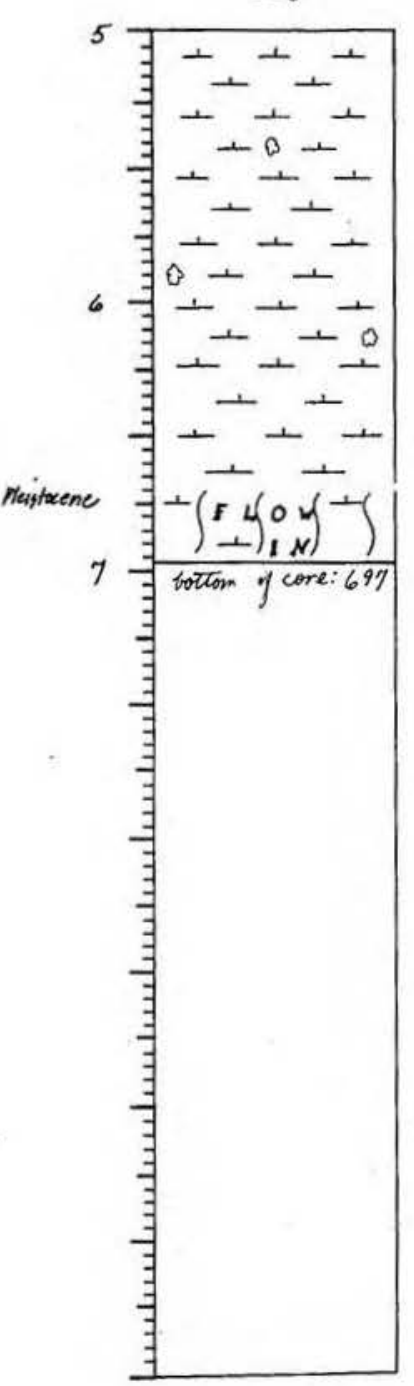

Lithologic

Log
Detailed Description

209-232

CALC CLAY

10 YR $2.5 / 1$ black becomes slightly browner in lower $5 \mathrm{~cm}$
abundan

bundant small pale brown mottles 209-218 (or incomplete microlams?)

very fine humus texture (organic lutite)

sapropelic material

32-349 - CALC OOZE

5 Y $7 / 1$ light gray grades into $(283 \mathrm{~cm}) 10$ YR $6 / 3$ pale bundant medium gray mottles 232-283 and scattered mot-

few pteropods $\stackrel{\text { S }}{\mathrm{S}}$

CALC OOZE

$2.5 \times 4 / 2$ dark grayish brown in somewhat disturbed

pattern with 10 YR $5 / 2$ grayish brown

a few mottles of above color

S

CALC OOZE

5 Y $6 / 2$ light olive gray

slightly

S -378

CAIC OOZE

5 Y $3 / 2$ dark olive gray

no mottles

, very silty lutite with scattered forams

with extremely fine microlaminations (?) 368 -

S

CALC OOZE

5 Y $7 / 1$ light gray grading to 10 YR $7 / 3$ very pale brown a very few small black mottles

firm lutite with few forams

S, bottom

5 Y $3 / 2$ dark ollive gray

5 Y $3 / 2$ dark olive gray
abundant mottles grading to few very pale brown mottles

(incomplete microlaminations?) 407-414

(

418-419 abundant

sapropelic material 
Lithologic

Log

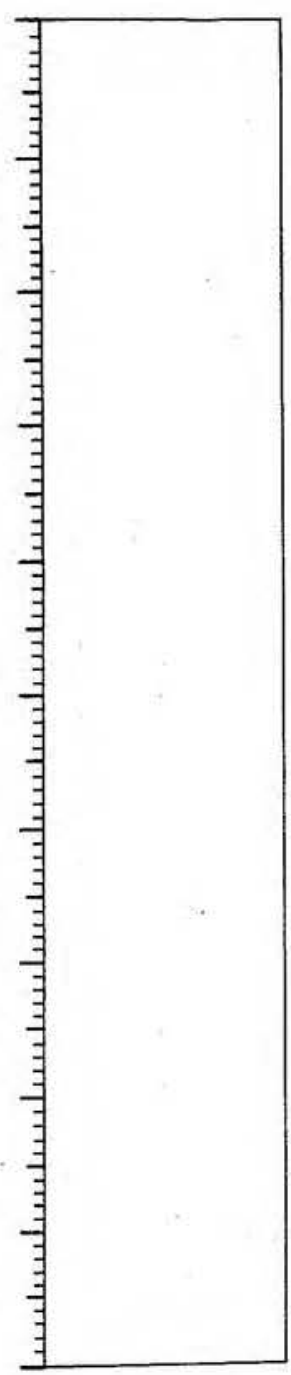

\section{Detailed Description}

421-448

CALC OOZE

grades from 5 Y $6 / 1$ gray to $7 / 2$ light gray to $6 / 2$ light olive gray with subdued banding

sma11, very light grey mottles common 425-427; abundant

few to abundant (438448-460

CALC OOZE

5 Y $3 / 2$ dark olive gray

456-460 abundant, very pale small brown mottles

very silty organic lutite

dued fine laminations $(452-460)$; irregular wedges of 10 YR $4 / 2$ dark grayish

460-483

CALC OOZE

$6 / 3$ pale brown, $7 / 4$ light gray

463-465 5 × $6 / 1$ small mottles cormon

firm lutite with few forams, except 477-483 common

483-487

CALC OOZE

5 Y $3 / 2$ dark olive gray

scattered small very pale brown mottles

sapropelic material

S

CALC OOZE

10 YR $5 / 1$ gray grading to $6 / 2$ litht brownish gray to bands of various pale browns $(6 / 3)$, bands of $6 / 4$ and 5/4 yellow brom also present

mottles 487-489; sma11 mottles of above colors scattered to common in many areas

stiff, slightly silty lutite with few forams, except

common at 536, 577-591, 650-660

581-586 gray layer similar to previous ones that under-

670-697

ANNO OOZE

10 YR $6 / 6$ brownish yellow

no mottling

slightly silty lutite with scattered forams

end of core
Ship:

Expedition 119

Leg No. 2

\begin{tabular}{|c|c|c|c|c|c|c|c|c|c|c|c|c|c|c|}
\hline \multirow[b]{3}{*}{ LEVEL } & \multirow[b]{3}{*}{$\begin{array}{c}\text { SEDIMENT } \\
\text { TYPE }\end{array}$} & \multicolumn{13}{|c|}{ ESTIMATED ABUNDANCES $(\%)$} \\
\hline & & \multicolumn{4}{|c|}{$\begin{array}{c}\text { Inorganic Material } \\
\text { Silt \& Sand }\end{array}$} & & \multicolumn{5}{|c|}{$\begin{array}{l}\text { Biogenous Mater } \\
\text { Calcareous }\end{array}$} & ial & Siliceous & \\
\hline & & 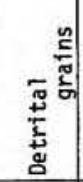 & 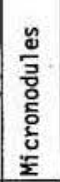 & 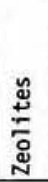 & 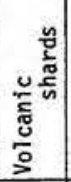 & $\frac{\pi}{\sigma}$ & 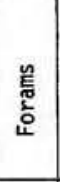 & 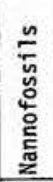 & 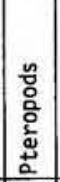 & 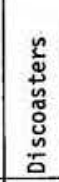 & 咅 & 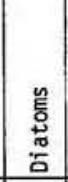 & 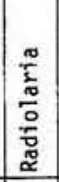 & 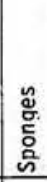 \\
\hline 1 & calc ooze & 4 & tr & & & 32 & 3 & 40 & 1 & & 16 & & & \\
\hline 14.5 & $\begin{array}{l}\text { highly calc } \\
\text { clay w/vol.as }\end{array}$ & tr & & & 20 & 60 & $\operatorname{tr}$ & tr & & & 20 & & & \\
\hline 106 & $\begin{array}{l}\text { highly calc } \\
\text { clay w/vol.as }\end{array}$ & 1 & tr & & 15 & 55 & tr & 8 & $\mathrm{tr}$ & & 21 & & & \\
\hline 111 & calc ooze & tr & 1 & & & 40 & 3 & 50 & 1 & tx & 6 & & & \\
\hline 115 & calc ooze & 1 & & & & 40 & 2 & 50 & 1 & & 8 & & & \\
\hline 118 & calc ooze & tr & & & & 43 & 4 & 40 & $\mathrm{tr}$ & $\operatorname{tr}$ & 13 & & & \\
\hline 124 & calc ooze & 2 & & & & 54 & 3 & 30 & $t x$ & tr & 11 & & & \\
\hline 195 & calc ooze & tr & & & & 53 & 2 & 30 & $\mathrm{tr}$ & & 15 & & & \\
\hline 223 & calc clay & 2 & & & & $10 / 64$. & 4 & 2 & & tr & 3 & & & \\
\hline 244 & calc ooze & tr & & & & 36 & 1 & 50 & $t x$ & & 12 & & & \\
\hline 325 & calc ooze & 1 & tr & & & 58 & 1 & 30 & $\operatorname{tr}$ & & 10 & & & \\
\hline 352 & calc ooze & 1 & & & 1 & 15 & 10 & 65 & 1 & & 5 & & & \\
\hline 370 & calc ooze & tr & $t x$ & & & $8 / 15^{\circ}$ & 5 & 63 & 1 & tr & 4 & & & \\
\hline $409 *$ & calc ooze & 1 & $\mathrm{tx}$ & & & $1 / 10^{\circ}$ & 4 & 15 & 1 & & 10 & & & \\
\hline-442 & calc ooze & tr & tr & & & 45 & 10 & 37 & 1 & & 7 & & & \\
\hline (cont & b & & & & & & & & & & & & & \\
\hline $\begin{array}{l}\text { ligh } \\
\text { or } \mathrm{n} t\end{array}$ & $\begin{array}{l}\text { o dark brown } \\
\text { pos, or even }\end{array}$ & $\begin{array}{l}\text { cained } \\
\text { it size }\end{array}$ & (sap & ope1 & $\begin{array}{l}\mathrm{mat}_{\text {ticle }} \\
\text { ticl }\end{array}$ & erial & that & appe & ars & somet & mes & $\mathrm{Asc1}$ & Layey & \\
\hline$\frac{\text { sami }}{\text { sam }}$ & 8 & & & & & 1. & & & & & & & & \\
\hline
\end{tabular}

Core No. 6 PC

(n)


Page 2

SMEAR SLIDE DESCRIPTIONS - W.H.O.I. SEDIMENT CORES

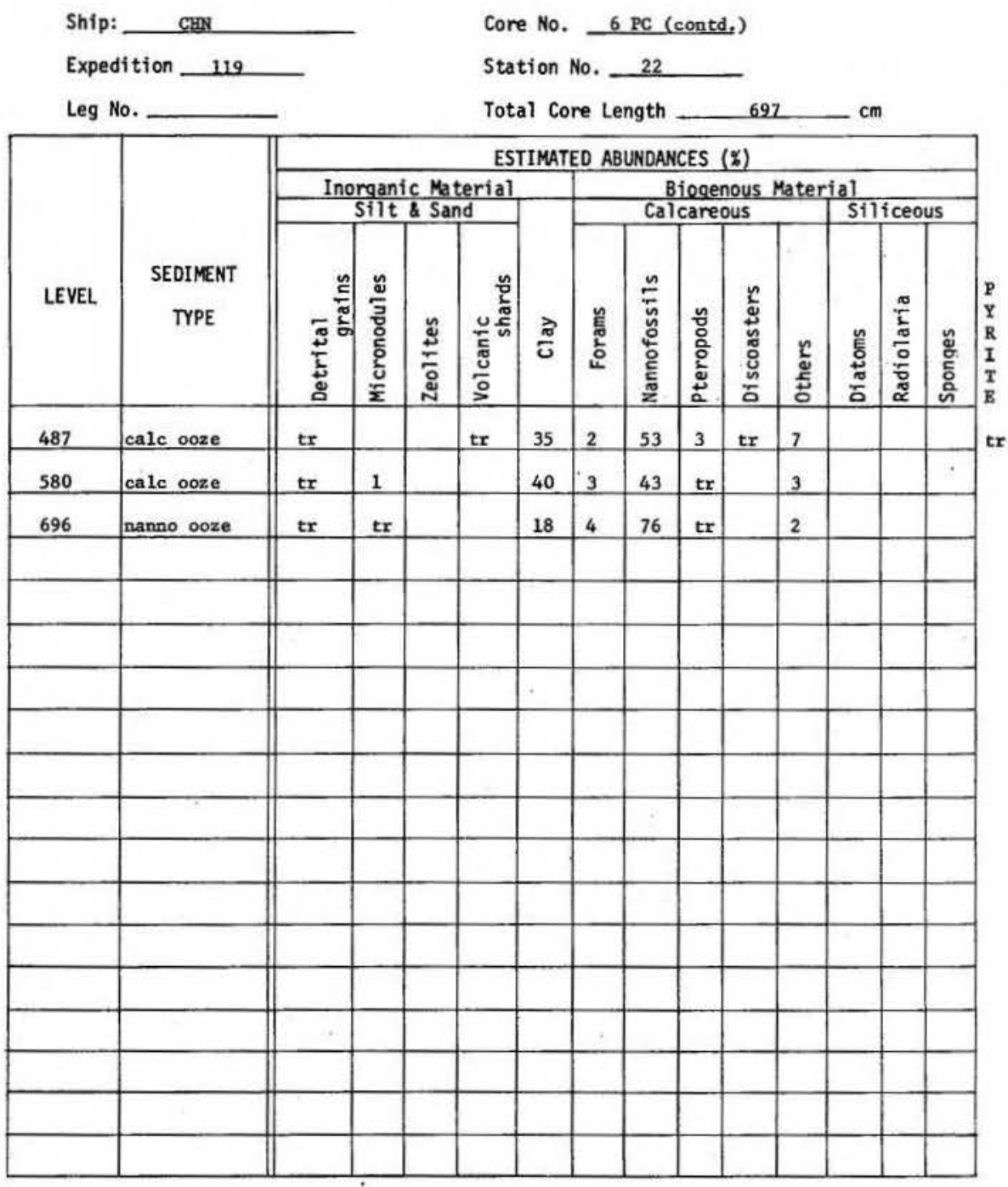

494

VISUAL CORE DESCRIPTION

Page 1 of 1

Ship CHAIN Cruise 119 Leg 2 Sta. 22 Core No. 6 PG Total Length $124 \mathrm{~cm}$. Lat. $33^{\circ} 15.8^{\prime} \mathrm{N}$ Long. $26^{\circ}$ oa.' $E$ Depth $2.372 \mathrm{Cor} m$ Core condition excellent Physiogrophic location MERITERRANEAN RIOGE, EASTERN MED.

Physiogrophic location MEPITERRANEAN RIOGE, ENSTERN
Lithologic Detailed Description

Mesiscene

Log

$+\perp+\perp+\mid=0-14$

$1+++$

$1+10$ YR $6 / 4$ light yellowish brown

-1

++-+ slightly silty lutite with scattered forams and

steropods

$14-19$

CALC 0O2E

5 Y $3 / 2$ dark olive gray

abundant, sma11, pale brown mottling

slightly silty lutite with forams and pteropods common
sapropel zone

19-30

CALC OOZE

5 Y 6/1 light gray (grades from dark to 1ight)

bundant, sma11, dark gray mottles

silty lutite with common forams

30-124

CALC $002 E$

10 YR $6 / 4$ light yellow-brown appears as subdued

sma11 mottles are scattered from 40-60 and $72-75 \mathrm{~cm}$

ilty lutite with a few pteropods

rumbly lutite

$104-109$ 11ght gray (5 Y $7 / 2$ ), thin, convoluted layer end of core 
SMEAR SLIDE DESCRIPTIONS - W.H.O.I. SEDIMENT CORES

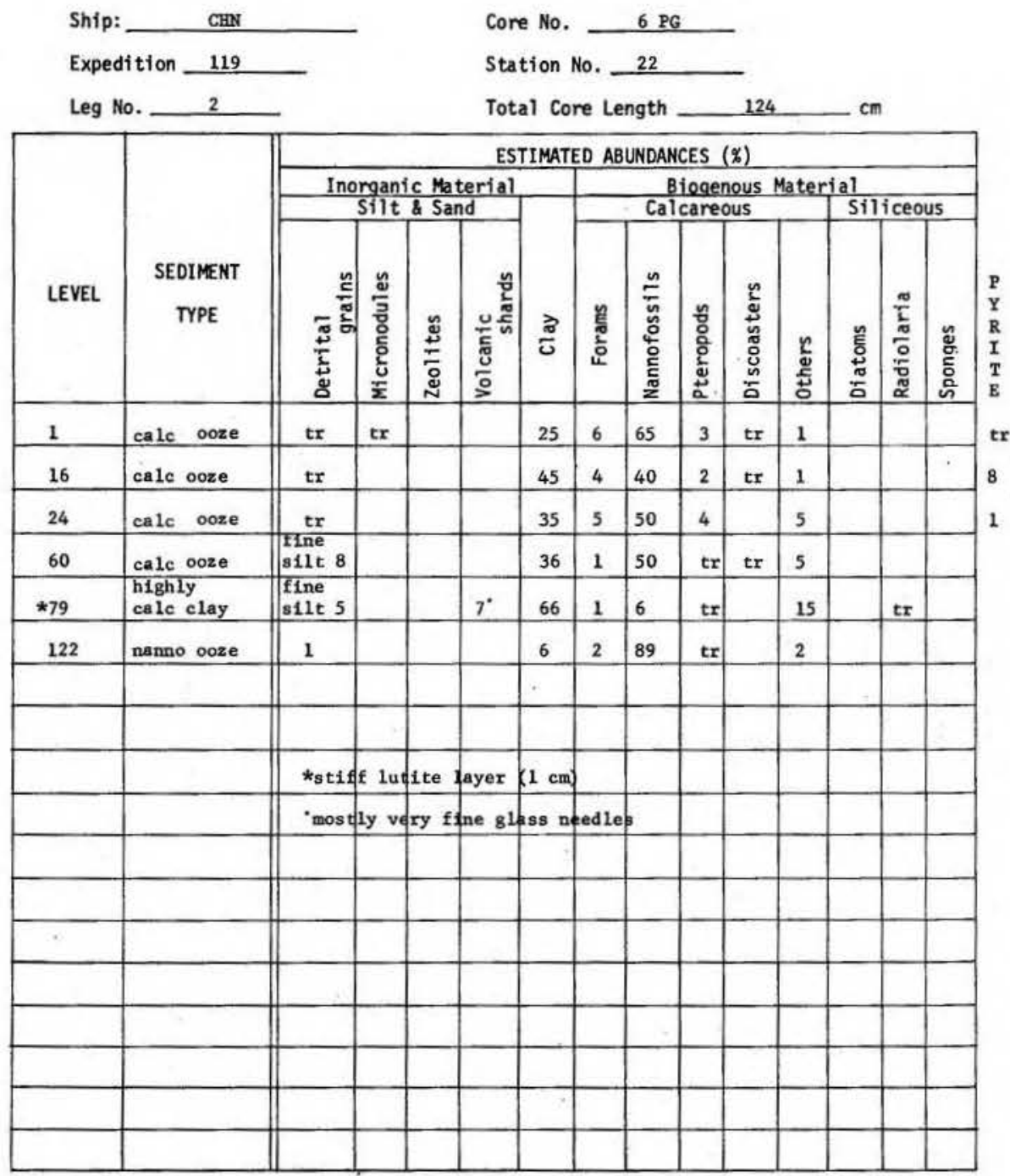

Ship CMAIN Cruise 119 Leg 2 sto. 25 Core No. $7 \mathrm{PC}$ Total Length $723 \mathrm{~cm}$. Lat. $32^{\circ} 20.3^{\circ} \mathrm{N}$ Long. $26^{\circ} 56.7^{\prime} \mathrm{F}$ Depth $3 / 96 \mathrm{corr} . \mathrm{m}$ Core condition elecllent Date Described 28 Spt 75 by $H$. Farmer Physiographic location Heropotus BASIN, EASTERN MED. SEA

Lithologic

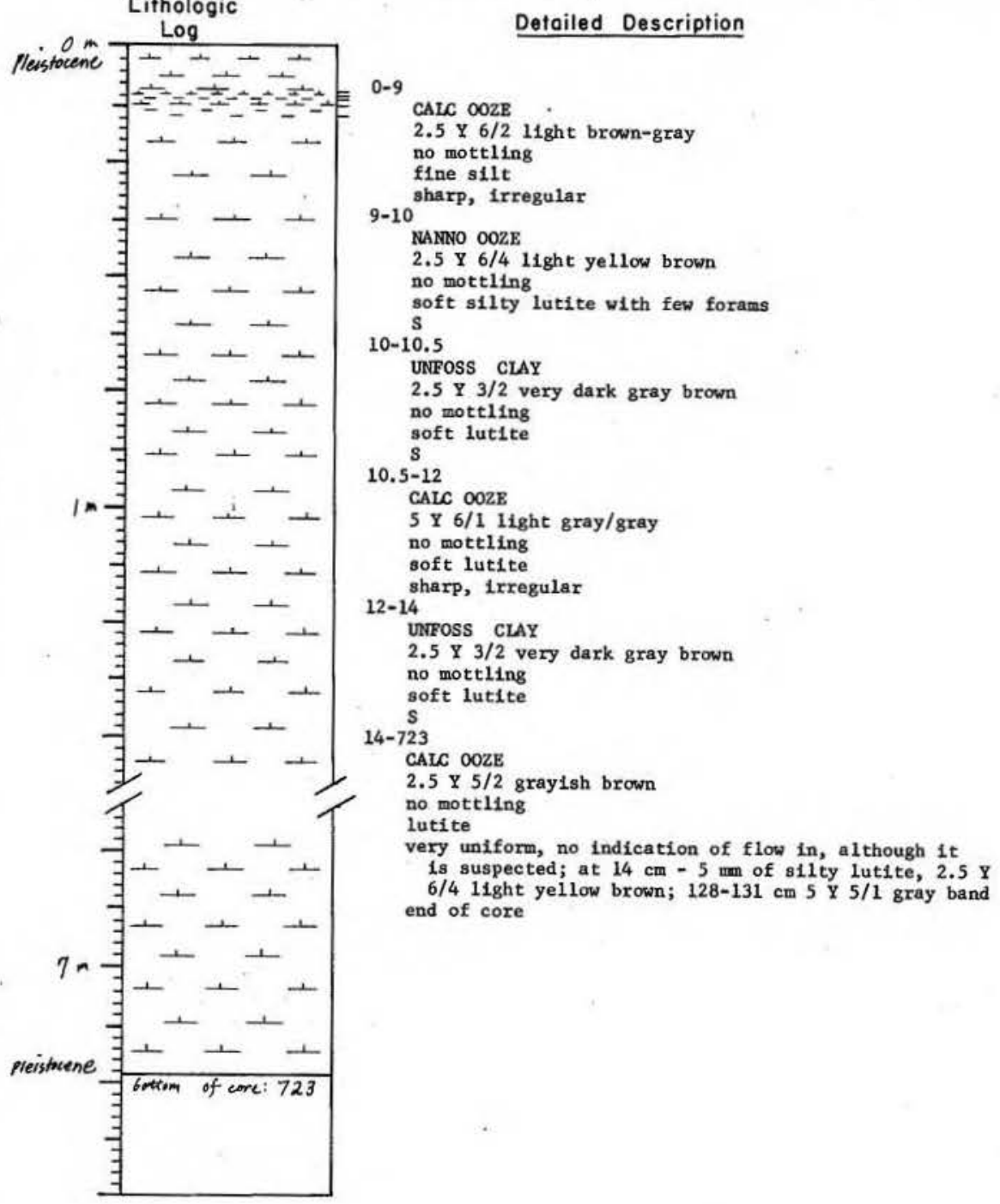


SMEAR SLIDE DESCRIPTIONS - W.H.O.I. SEDIMENT CORES

Ship:

Expedition 119

Leg No.

\begin{tabular}{|c|c|c|c|c|c|c|c|c|c|c|c|c|c|c|}
\hline \multirow[b]{4}{*}{ LEVEL } & \multirow[b]{4}{*}{$\begin{array}{c}\text { SEDIMENT } \\
\text { TYPE }\end{array}$} & \multicolumn{13}{|c|}{ ESTIMATED ABUNDANCES ( $(z)$} \\
\hline & & \multirow{2}{*}{\multicolumn{4}{|c|}{$\begin{array}{c}\text { Inorganic Material } \\
\text { Silit \& Sand }\end{array}$}} & & \multirow{2}{*}{\multicolumn{5}{|c|}{$\begin{array}{l}\text { Biogenous Mater } \\
\text { Calcareous }\end{array}$}} & \multirow{2}{*}{\multicolumn{3}{|c|}{ Siliceous }} \\
\hline & & & & & & & & & & & & & & \\
\hline & & $\begin{array}{c}\frac{n}{\pi} \\
\frac{5}{5} \\
\frac{5}{5} \\
\frac{5}{2}\end{array}$ & 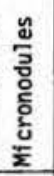 & 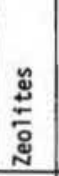 & 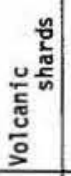 & $\frac{\pi}{6}$ & 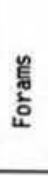 & 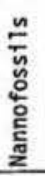 & 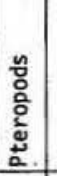 & 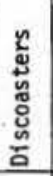 & $\begin{array}{l}\frac{r}{2} \\
\frac{5}{ \pm} \\
0\end{array}$ & 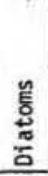 & $\begin{array}{l}\frac{\pi}{2} \\
\frac{\pi}{0} \\
\frac{0}{0} \\
\approx \\
\end{array}$ & 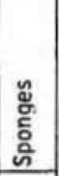 \\
\hline 1 & calc ooze & tr & tr & & $\operatorname{tr}$ & 26 & 2 & 40 & 2 & $\operatorname{tr}$ & 30 & & & $\mathrm{tr}$ \\
\hline 9.5 & nanno ooze & 1 & tr & & & 6 & 2 & 86 & 1 & tr & 4 & & & - \\
\hline 11 & calc ooze & 1 & & & & 50 & tr & 8 & $\mathrm{tr}$ & tr & 40 & & & \\
\hline 13 & unfoss. clay & 10 & & & $\mathrm{tr}$ & 90 & & $\mathrm{tr}$ & & tr & $\mathrm{tr}$ & & & \\
\hline 113 & calc ooze & 3 & & & tr & 45 & tr & 45 & $\operatorname{tr}$ & tr & 7 & & & \\
\hline 213 & calc ooze & 2 & & & & 60 & tr & 35 & $\mathrm{tr}$ & $\operatorname{tr}$ & 3 & & & \\
\hline 313 & calc ooze & 3 & & & & 47 & tr & 45 & $\mathrm{tr}$ & tr & 5 & & & \\
\hline 413 & calc ooze & 1 & & & & 60 & & 36 & $\operatorname{tr}$ & tr & 3 & & & \\
\hline 513 & calc ooze & 3 & & & & 57 & $t r$ & 35 & $\mathrm{tr}$ & $\mathrm{tr}$ & 5 & & & \\
\hline 613 & calc ooze & 1 & & & & 37 & tr & 59 & 1 & tr & 2 & & & \\
\hline 722 & calc ooze & 1 & & & & 66 & tr & 30 & 1 & tr & 2 & & & \\
\hline & & & & & & & & & & & & & & \\
\hline & & & & & . & & & & & & & & & \\
\hline & & & & & & & & & & & & & & \\
\hline & & & & & & & & & & & & & & \\
\hline & & & & & & & & & & & & & & \\
\hline & & & & & & & & & & & & & & \\
\hline & & & & & & & & & & & & & & \\
\hline
\end{tabular}

Core No.
Ship CHAIN Cruise 119

Leg 2 Sta. 27 Core No. 8 PL Total Length $469 \mathrm{~cm}$. Lat. $31^{\circ} 49.8^{\circ} \mathrm{N}$ Long. $27^{\circ} 43.9^{\circ} \mathrm{E}$ Depth $2738 \mathrm{~m}$.corr Core condition EXCELLENT Date Described IINor.75 by f.barda Physiographic location HERODOTUS BASIN, E. MEDITERR AMEAN SEA. Lithologic

Detailed Description

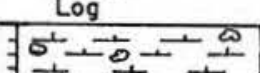

寻立吉 $-^{0-18}$

CALC OOZE

F 10 YR $6 / 4$ 1ight yellowish brown and $7 / 4$ very pale brown

intermottling and marbling of the colors above plus
very dark gray and dark grayish brown inclusions

(disturbed laminae) in the zone $8-14 \mathrm{~cm}$

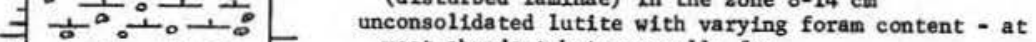

$\exists \rightarrow+\frac{1}{1}=$

most, abundant, but generally few

(in short 1iner) is somewhat disturbed

and disoriented. Stratigraphic features are preserve

but horiz ons are inclined sharply or broken. $1 \mathrm{~cm}$ wide dark laminations are found in the zone $8-14 \mathrm{~cm}$.

WOIE: After couparison with pilot core it appears

that the first $12 \mathrm{~cm}$ of the piston core represent the

in a compacted seditint recovered by the pllot core was imnobile during intital moments of penetratist and recovered (and compacted) only as much sediment would fit in the catcher cutter assembly (approximately

$12 \mathrm{~cm}+$ water). The rest of the core represents what

was recovered after the piston began to move - and by

the p1lot's record 1t would seem that at least $50 \mathrm{~cm}$ have been missed or "blown by".

Meistocene

$=$

$+0, \div$ S, 1rregula:

CALC OOZE

$2.5 \times 7 / 2$ 11ght gray

stiff, somewhat silty lutite with sma11 lithified 1 mos

included; also a wedge of foram sand appears $22-24 \mathrm{~cm}$ stratification questionable 26-40,

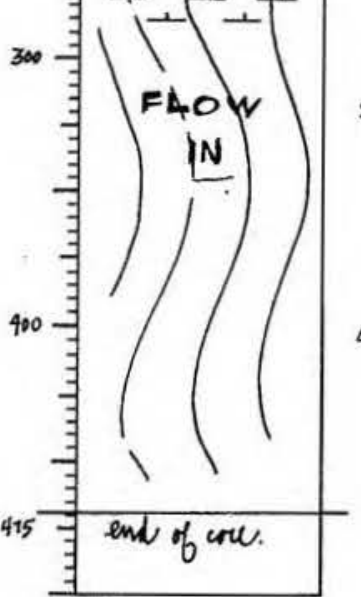
horizontal

CAIC OOZE

S $7 / 2$ 1ight gray and $6 / 1$ gray ray inclusions in the zone

tutite intermittentiy contacts $S$
45

CAIC OOZE

YR $6 / 4$ light yellowish brown grades to $7 / 4$ very pale no mottling

firm, smooth lutite with scattered forams 
Lithologic Log

Detailed Description

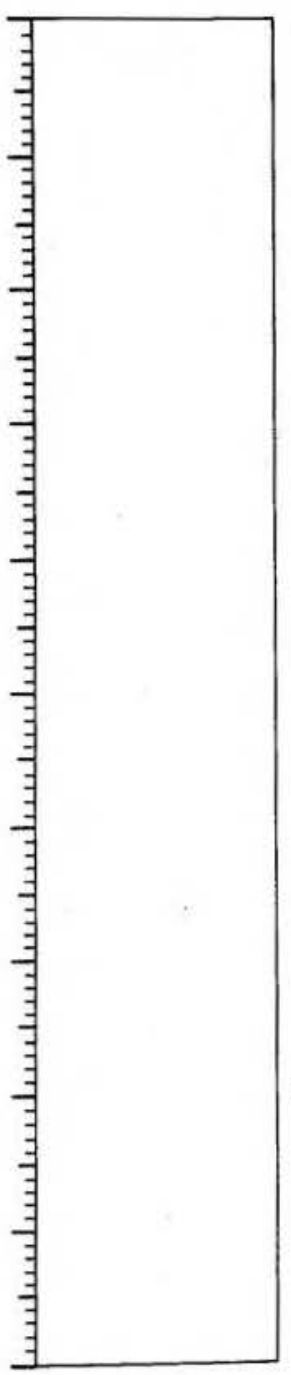

$10 \mathrm{YR} 6 / 3$ pale brown

very silghtly silty lutite
55-80

CALC OOZE

$2.5 \times 7 / 4$ pale yellow

gray mottles $65-80 \mathrm{~cm}$

$80-91$,

with a few forams $70-80 \mathrm{~cm}$

OOzE

homogeneous throughout

91-94

CALC OOZE

10 YR $8 / 1$ white

homogeneous throughout

fine compact form sand

94-124,

CALC OOZE

varying hues of $10 \mathrm{YR} 7 / 4$ very pale brown, $6 / 4$ 1ight

a few very small foram-rich light mottles scattered

throughout

allty lutite with scattered foram

$S$, horizontal

124-155

CALC OOZE

$2.5 \times 7 / 4$ pale yellow grades to 10 YR $6 / 4$ 1ight yellow1sh brown

numerous very small yellowish brown speck-1ike mottles 124-128 cm, also brosd banded laminations of the 11 hitly oflty luts

scattered forams

$155-204$

CALC OOZE

10 YR $6 / 4$ 11ght yellow1sh brown

firm, slightly silty lutite with scattered foram

195-204 marbled and disturbed - inner barrel suck up 204-232

CALC OOZE

2.5 × 7/4 pale yellow

faint irregular intermottling and maxbling with unit above

firm, silty lutite with abundant forams

again, this unit appears flowed, disturbed, and un-

s, horizontal
Ship CAAIN Cruise 119 Leg 2 Sta. 27 Core No. BPC

Lithologic

Log

Detailed Description

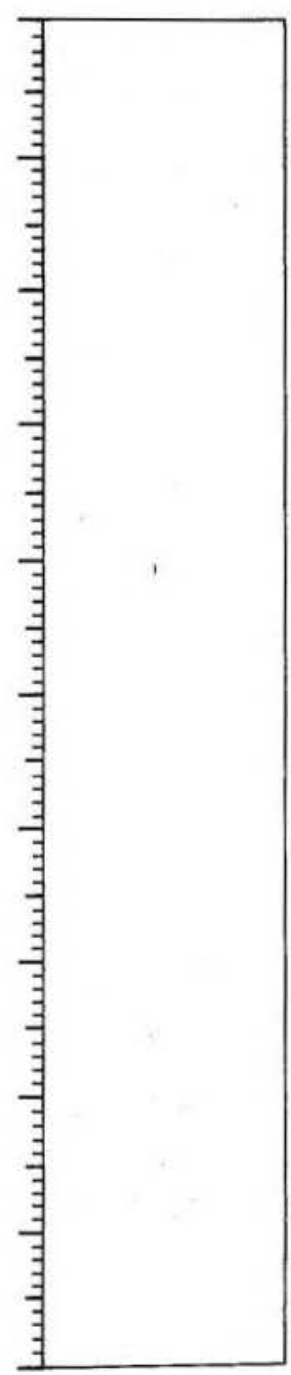

232-245

CALC OOZE

2.5 Y $6 / 2$ light brownish gray grades to $7 / 2$ 11ght gray finm, silghtly silty lutite with scattered forams $245-469$

FLOW-TN

end of core 
". SIEAR SLIDE DESCRIPTIONS - W.H.O.I. SEDINENT CORES

Ship: CHN

Expedition 119

Leg No. 2

\begin{tabular}{|c|c|c|c|c|c|c|c|c|c|c|c|c|c|c|}
\hline \multirow[b]{4}{*}{ LEVEL } & \multirow[b]{4}{*}{$\begin{array}{c}\text { SEDIMENT } \\
\text { TYPE }\end{array}$} & \multicolumn{13}{|c|}{ ESTIMATED ABUNDANCES $(x)$} \\
\hline & & \multirow{2}{*}{\multicolumn{4}{|c|}{$\begin{array}{c}\text { Inorganic Material } \\
\text { Silt \& Sand }\end{array}$}} & \multirow[b]{3}{*}{$\stackrel{\frac{\pi}{U}}{0}$} & \multirow{2}{*}{\multicolumn{5}{|c|}{$\begin{array}{l}\text { Biogenous Mater } \\
\text { Calcareous }\end{array}$}} & \multirow{2}{*}{\multicolumn{3}{|c|}{ Siliceous }} \\
\hline & & & & & & & & & & & & & & \\
\hline & & 要 & 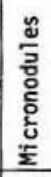 & 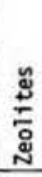 & 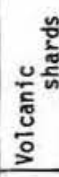 & & 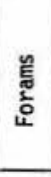 & 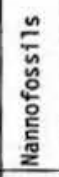 & 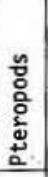 & 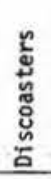 & 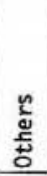 & 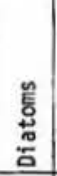 & $\begin{array}{l}\frac{\pi}{\vdots} \\
\frac{\pi}{0} \\
\frac{\pi}{0} \\
\frac{\pi}{\alpha}\end{array}$ & 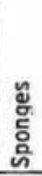 \\
\hline 1 & calc ooze & 2 & $\mathrm{tr}$ & & & 33 & 8 & 50 & 2 & & 5 & & & \\
\hline 10 & calc ooze & 2 & 5 & & & 15 & 10 & 60 & $t x$ & & 8 & & &. \\
\hline 28 & calc ooze & 3 & $t r$ & & & 50 & 5 & 30 & $t r$ & & 12 & & & \\
\hline 39 & calc ooze & 4 & & & & 44 & 10 & 14 & 2 & & 26 & & & \\
\hline 85 & nanno ooze & $\mathrm{tr}$ & tr & & & 16 & 4 & 75 & & & 5 & & & \\
\hline 93 & calc ooze & 3 & & & & 33 & 30 & 22 & & & 12 & & & \\
\hline 150 & calc ooze & $\mathrm{tr}$ & & & & 18 & 3 & 70 & & & 9 & & & \\
\hline 230 & calc ooze & tr & & & & 18 & 4 & 60 & & & 18 & & & \\
\hline 270 & calc ooze & 3 & tr & & & 39 & 8 & 40 & & & 10 & & & \\
\hline 465 & calc ooze & 2 & 1 & & & 35 & 12 & 36 & & & 14 & & & \\
\hline & & & & & & & & & & & & & & \\
\hline & & & & & . & & & & & & & & & \\
\hline & & & & & & & & & & & & & & \\
\hline & & & & & & & & & & & & & & \\
\hline & & & & & & & & & & & & & & \\
\hline & & & & & & & & & & & & & & \\
\hline & & & & & & & & & & & & & & \\
\hline & & & & & & & & & & & & & & \\
\hline
\end{tabular}

Core No. 8 PC

Station No. 27

Total core Length
AIN Cruise 119

Leg 2 Sto. 27 Core No. 8 PG

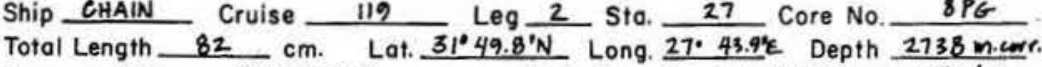
Core condition EXCEULENT Date Described 11 Nov 75 by - . broda. Physiographic location HERodeTUS BASIN; EASTERN MEDITERRANEAN SEA. Lithologic

Detailed Description

Preistacene $=\stackrel{\log }{+}$

正十 $+0-20$

ss

के 1

CALC OOZE

varying hues of 10 YR $6 / 4$ light yellowish brown and 7/4 very pale brown

the hues above unconsolidated slightly silty lutite with a few silty

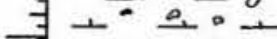

$\rightarrow+-1$

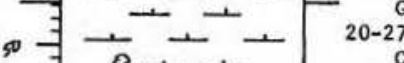

a十+ CALC OOZB

$+\quad+10$ YR $4 / 3$ brown grades to $5 / 6$ yellowish brown

-

15 고느․

Presifocenc 7 - 5 - 5 , , colors above sick lutite with a few scattered forams S, horizontal ende of core CAIC OOZE WITH PYRITE

10 YR $4 / 1$ dark gray silty lutite(sapropel) with scattered forams and ptero pods one remarkably well-preserved pteropod cese 1 sharply $G$ also 42-82 CALC OOZE

10 YR $6 / 1$ gray grades to $6 / 3$ pale brown a few faint 11ght brownish gray mottles scattered

firmer slightly silty lutite with scattered foram end of core 
SMEAR SLIDE DESCRIPTIONS - W.H.O.I. SEDIMENT CORES

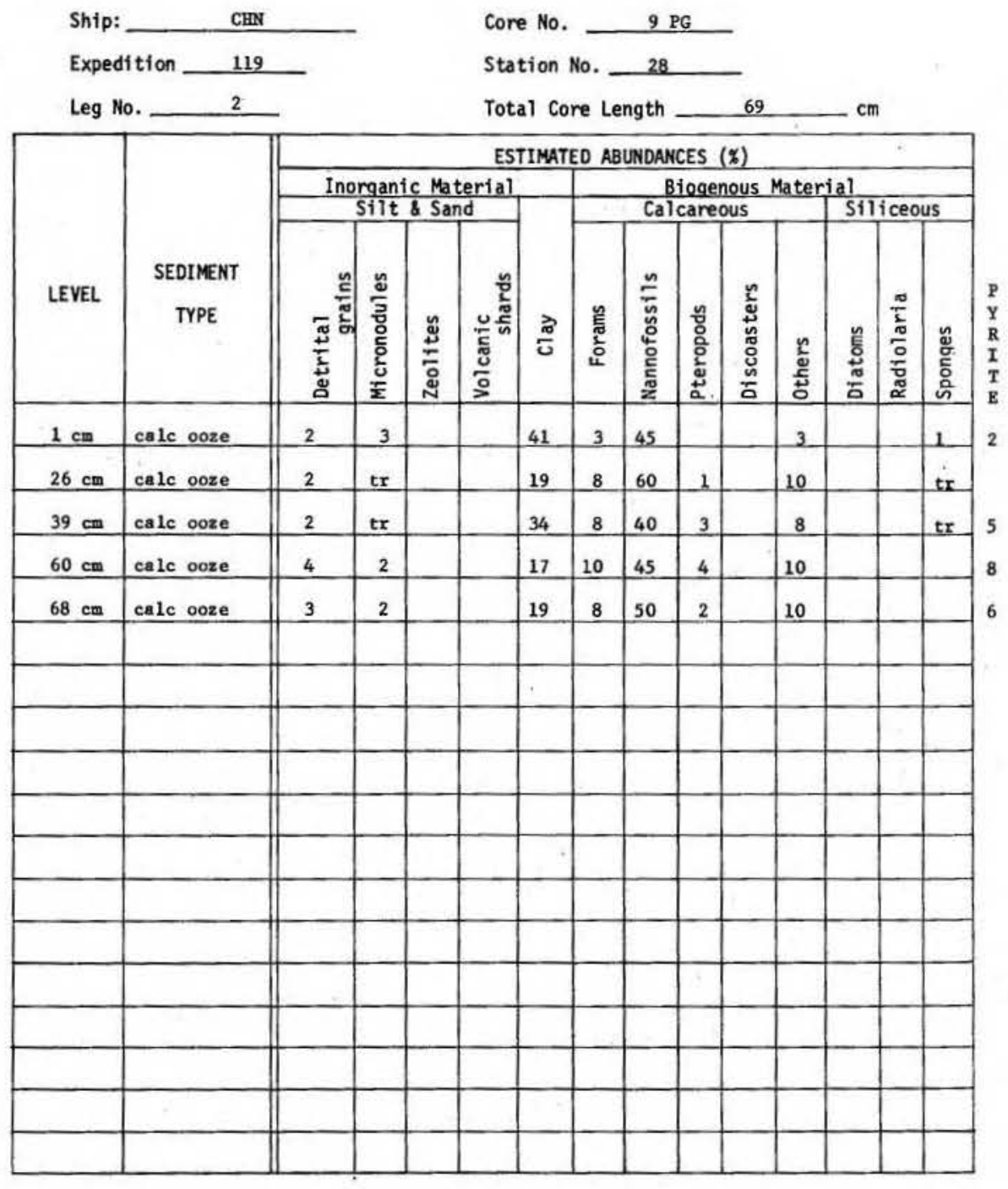

\section{VISUAL CORE DESCRIPTION}

Page_L of 1

Ship CHAW Cruise 119 Leg 2 Sto. 36 Core No. 2 GC Total Length $86^{\circ} \mathrm{cm}$. Lat. $31^{\circ} 309^{\prime} \mathrm{N}$ Long. $30^{\circ} 18.8 \mathrm{E}$ Depth 6 CeRR m Core condition EXCELENT Date Described 15 Aug 75 by $R$ MeGirr Physiographic location Abv Quer Bay, Eqyptian Contiaental Shelf, Eastern Med Lithologic

$$
\begin{aligned}
& 0 \text { Log Detailed Description } \\
& \text { Neristoceme }
\end{aligned}
$$

SLICHTLY SILIC CLAY WITH DETRITUS 2.5 Y $3 / 2$ very dark grayish brown

no mott1ing

moist, firm silty lutite

1t becomes allghtly darker with depth - se

nearly horizontal 1 -cm thick laminations through-

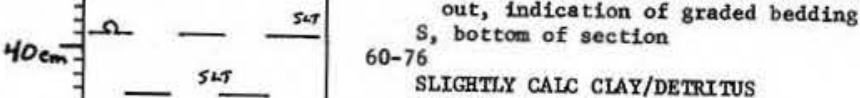

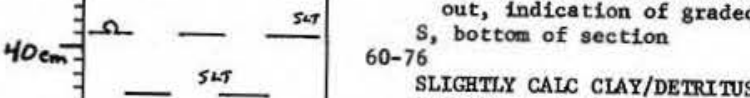

5 Y $3 / 2$ dark olive gray

no mottling

firm, silty lutite, becoming siltier with depth

S, textural, horizontal

$60 \mathrm{~cm}^{-1}$ SLT - ${ }_{S L T}$ 工 SLT 76 -

SSLT SLT SLIGHTIY CALC CLAY/DETRITUS

F the sur 5 × $3 / 2$ dark olive gray

son- Sw- Sw

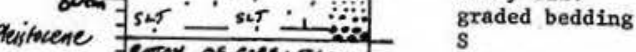

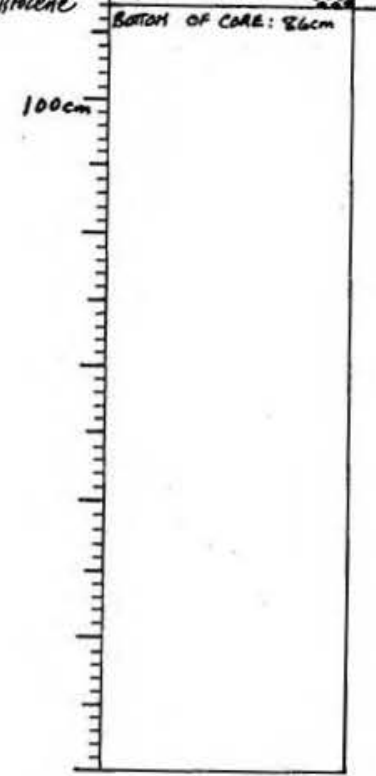

bottom of core 
509

SMEAR SLIDE DESCRIPTIONS - W.H.O.I. SEDIMENT CORES

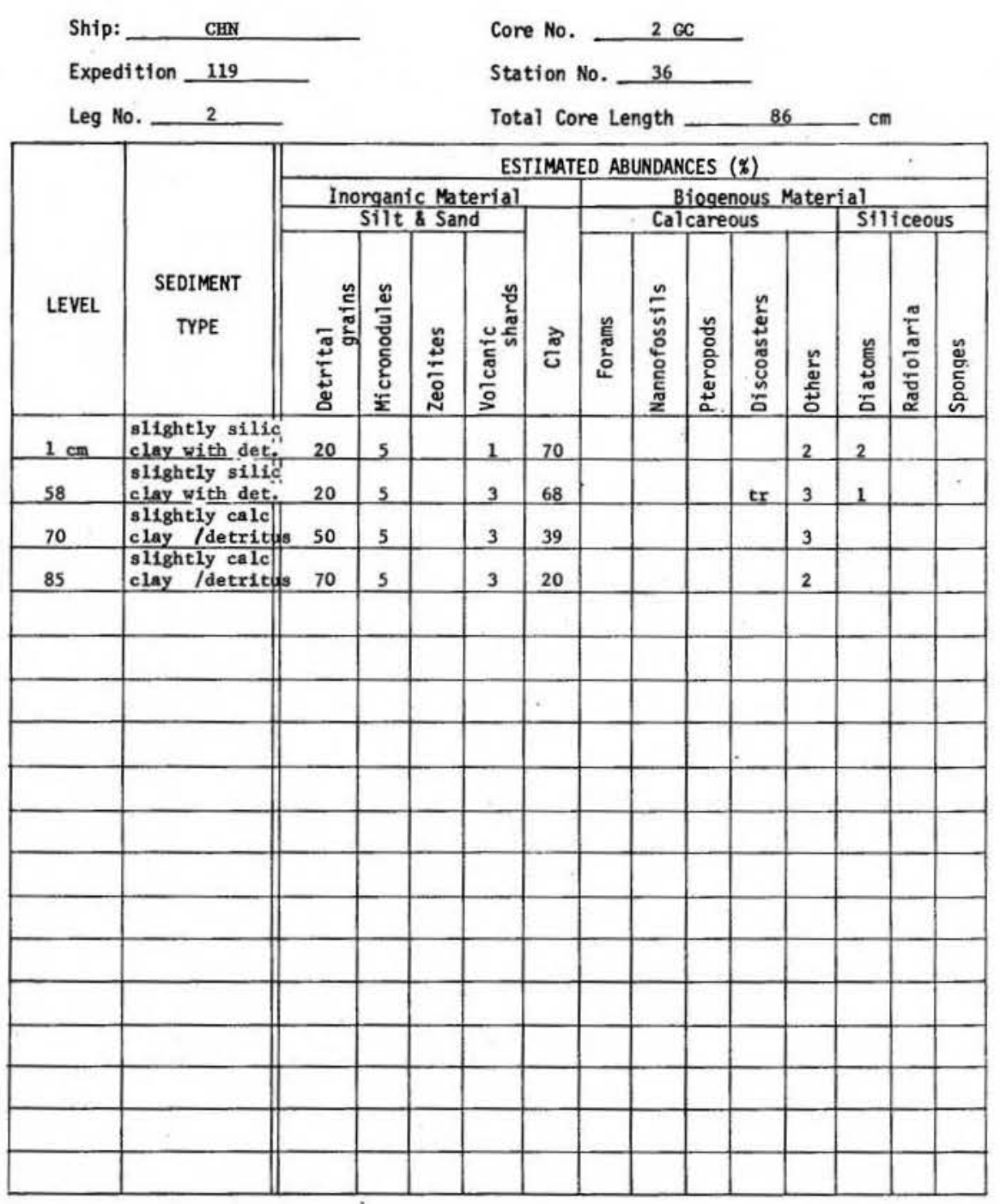

510

VISUAL CORE DESCRIPTION

Poge 1 of 1

Ship C NAIN Cruise 119 Leg 2 Sta. 55 Core No. 3 ac Total Length $-8.3 \mathrm{~cm}$. Lat. $31^{\circ} 32.4^{\prime} \mathrm{N}$ Long. $30^{\circ} 15.5^{\prime} E$ Depth 12 carm $\mathrm{m}$. Core condition exeelleat Date Described 17 Syf '75 by H. Faremes

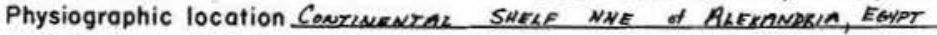
Lithologic

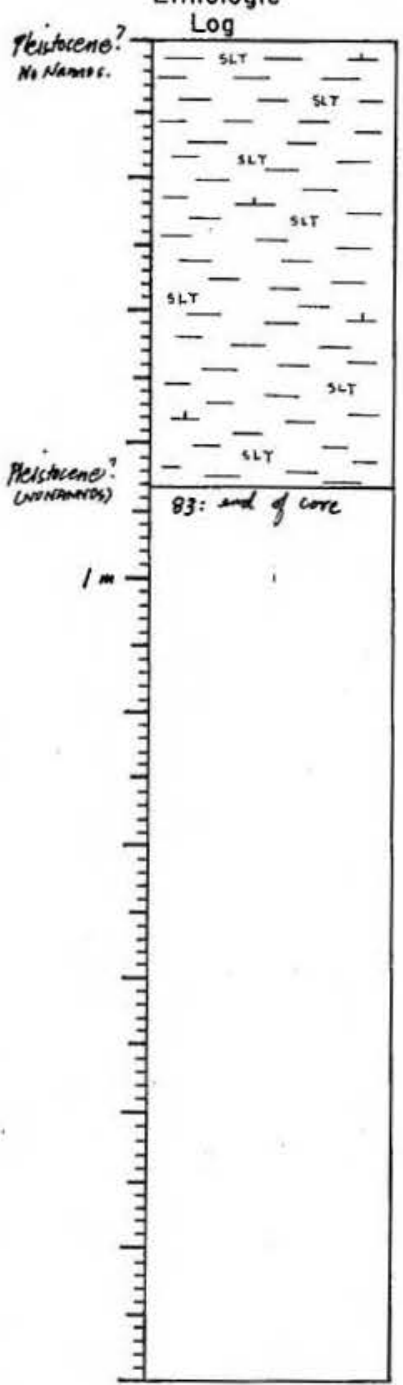

Detailed Description

$0-83$

CALC CIAY WITH DETRITUS

.5 $3 / 2$ very dark gray brown laminated with 2.5 Y dark gray brown

teting

laminae vary from $.5-3.0 \mathrm{~cm}$ with sharp contacts 


\section{1}

SMEAR SLIDE DESCRIPTIONS - W.H.O.I. SEDIMENT CORES

Ship:

\begin{tabular}{|c|c|c|c|c|c|c|c|c|c|c|c|c|c|c|c|}
\hline \multirow[b]{4}{*}{ LEVEL } & \multirow[b]{4}{*}{$\begin{array}{c}\text { SEDIMENT } \\
\text { TYPE }\end{array}$} & \multicolumn{14}{|c|}{ ESTIMATED ABUNDANCES (\%) } \\
\hline & & \multirow{2}{*}{\multicolumn{4}{|c|}{$\begin{array}{c}\text { Inorganic Material } \\
\text { Silt \& Sand }\end{array}$}} & \multirow[b]{3}{*}{$\frac{\pi}{6}$} & \multirow{2}{*}{\multicolumn{6}{|c|}{$\begin{array}{l}\text { Biogenous Mater } \\
\text { Calcareous }\end{array}$}} & ial & & \\
\hline & & & & & & & & & & & & & \multicolumn{3}{|c|}{ Siliceous } \\
\hline & & 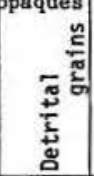 & 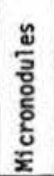 & 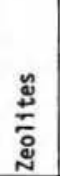 & 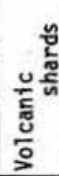 & & & है & 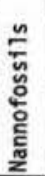 & 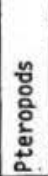 & 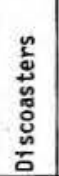 & $\begin{array}{l}\frac{r}{d} \\
\frac{5}{\Delta}\end{array}$ & 产 & 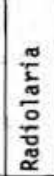 & 产 \\
\hline 1 & $\begin{array}{l}\text { slightly calc } \\
\text { clay w/detritu }\end{array}$ & $\frac{20}{10}$ & & & 1 & 67 & & & tr & & $t r$ & 2 & tr & tr & \\
\hline $18 *$ & $\begin{array}{l}\text { silghtly calc } \\
\text { clay w/detritu }\end{array}$ & $\frac{17}{8}$ & & & 1 & 70 & & $x$ & tr & & & 2 & & tr & . \\
\hline \multirow[t]{4}{*}{81} & $\begin{array}{l}\text { slightiy calc } \\
\text { clay w/detritu }\end{array}$ & $\frac{20}{10}$ & & & 1 & 67 & & & tr & & & 2 & & & \\
\hline & $=$ & & & & & & & & & & & & & & \\
\hline & & & & & & & & & & & & & & & \\
\hline & & $\star_{\text {from }}$ & ight & $r \mathrm{co}$ & lored & $1 \mathrm{am}$ & pe & & & & & & & & \\
\hline & & & & & & . & & & & & & & & & \\
\hline & & & & & & & & & & & & & & & \\
\hline & & & & & & & & & & & & & & & \\
\hline & & & & & & & & & & & & & & & \\
\hline & & & & & & & & & & & & & & & \\
\hline & & & & & & & & & & & & & & & \\
\hline & & & & & & & & & & & & & & & \\
\hline & & & & & & & & & & & & & & & \\
\hline & & & & & & & & & & & & & & & \\
\hline & & & & & & & & & & & & & & & \\
\hline & & & & & & & & & & & & & & & \\
\hline & & & & & & & & & & & & & & & \\
\hline
\end{tabular}

Core No. $3 \mathrm{GC}$

Station No. 55

Total Core Length

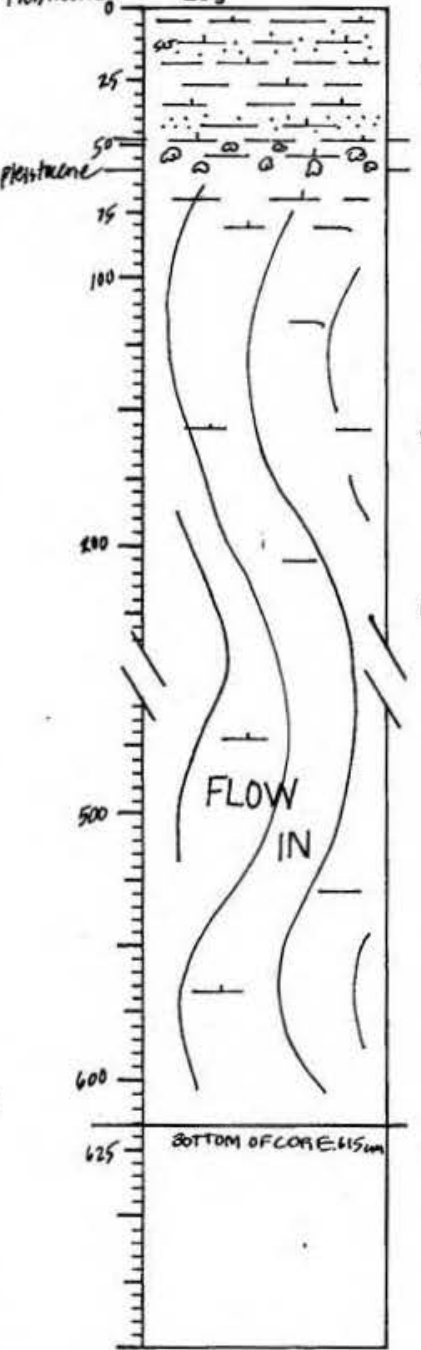

$-512$

VISUAL CORE DESCRIPTION

Page 1 of 1

Ship CHAIM Cruise 119 L II Sta 6I Core No $109 \mathrm{C}$

Total Length $615 \mathrm{~cm}$. Lat. $311^{\circ} 40.8^{\prime} \mathrm{N}$ Long. $30^{\circ} \mathrm{Ol} .4^{\prime} \mathrm{E}$ Depth $431 \mathrm{~m} \cdot$ carr . Core condition ExLEU. ENT. Date Described 24 Murit5s by forda. Physiographic locotion ALEXANDRLA CAIYOW IN ROGEVTA FAN, EAKTERN MEAT ERRAWIEAN. Lithologic

Detailed Description

$0-49$

HIGHLY CALC CLAY WITH IAMINATIONS OF HIGHYY CALC CLAY/DETRITUS

10 YR $4 / 2$ dark grayish brown

repeated grayish brown and light brownish gray lamina-

tions and beds; many are inclined and irregular, some are incomplete (do not span entire liner)
slick lutite interbeds with slightly to very silty lutite

NOTE: All the features in this unit appear quite compacted this whole $49 \mathrm{~cm}$ surfact After comparison with pilot core, and shortened sample of the first meter of the sediment. $s$, Irregular

HIGHLY CALC CLAY

10 YR $4 / 2$ dark grayish brown

extensive light brownish gray mottling

all features concave upward $60-615$

\section{CALC OOZE}

FLON-IN

end of core 
SMEAR SLIDE DESCRIPTIONS - W.H.O.I. SEDIMENT CORES

Ship:

Expedition 11

- Leg No. 2
Core No. $10 \mathrm{PC}$

Station No. 61

Total Core Length $\quad 615$

ESTIMATED ABUNDANCES (\%)

\begin{tabular}{|c|c|c|c|c|c|c|c|c|c|c|c|c|c|c|}
\hline \multirow[b]{4}{*}{ LEVEL. } & \multirow[b]{4}{*}{$\begin{array}{c}\text { SEDIMENT } \\
\text { TYPE }\end{array}$} & \multicolumn{13}{|c|}{ ESTIMATED ABUNDANCES (\%) } \\
\hline & & \multirow{2}{*}{\multicolumn{4}{|c|}{$\begin{array}{c}\text { Inorganic Material } \\
\text { Silt \& Sand }\end{array}$}} & & \multirow{2}{*}{\multicolumn{5}{|c|}{$\begin{array}{l}\text { Biogenous Mater } \\
\text { Calcareous }\end{array}$}} & \multirow{2}{*}{\multicolumn{3}{|c|}{ Siliceous }} \\
\hline & & & & & & & & & & & & & & \\
\hline & & 总 & $\begin{array}{l}\text { y } \\
\frac{3}{3} \\
\frac{0}{0} \\
\frac{2}{0} \\
\frac{1}{\Sigma}\end{array}$ & 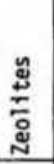 & 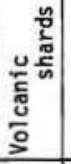 & $\frac{\pi}{5}$ & 衰 & 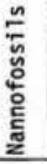 & 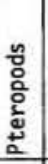 & 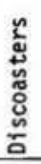 & 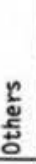 & 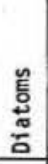 & $\frac{\frac{\pi}{2}}{\frac{\pi}{0}}$ & $\begin{array}{l}\text { ळूँ } \\
\text { हूँ } \\
\text { के }\end{array}$ \\
\hline 1 & $\begin{array}{l}\text { highly } \\
\text { calc clay }\end{array}$ & 7 & tr & & 2 & 66 & 3 & 12 & & & 7 & $\mathrm{tr}$ & & tr \\
\hline 19 & $\begin{array}{l}\text { highly calc } \\
\text { clay/detritus }\end{array}$ & 32 & 1 & & 3 & 40 & 4 & 8 & & & 8 & tr & & tr \\
\hline 35 & calc clay & 6 & tr & & 2 & 79 & $\mathrm{tr}$ & 4 & & & 4 & & & tr \\
\hline 60 & $\begin{array}{l}\text { highly } \\
\text { calc clay }\end{array}$ & 5 & 1 & & $\mathrm{tr}$ & 66 & 2 & 18 & & & 4 & & & tr \\
\hline 614 & calc ooze & 3 & tr & & tr & 47 & 6 & 35 & & & 5 & & & tr \\
\hline & & & & & & & & & & & & & & \\
\hline & & & & & & . & & & & & & & & \\
\hline & & & & & & & & & & & & & & \\
\hline & & & & & & & & & & & & & & \\
\hline & & & & & & & & & & & & & & \\
\hline & & & & & & & & & & & & & & \\
\hline & & & & & & & & & & & & & & \\
\hline & & & & & & & & & & & & & & \\
\hline & & & & & & & & & & & & & & \\
\hline & & & & & & & & & & & & & & \\
\hline & & & & & & & & & & & & & & \\
\hline & & & & & & & & & & & & & & \\
\hline & & & & & & & & & & & & & & \\
\hline
\end{tabular}

Ship CHAiN Cruise 119 Leg II sto. 61 Core No. 10PG Total Length $120 \mathrm{~cm}$. Lat. $31040.8 \mathrm{~N}$ Long. $30^{\circ} 01.4^{\circ} \mathrm{E}$ Depth $431 \mathrm{m.corr}$ Core condition EKLELENT Tat Described 25NV75 by fordw.

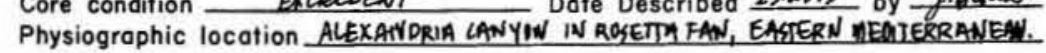
Physiographic location ALEXANDRIA LANYOW IN ROEETMATAN,
Lithologic Detailed Description

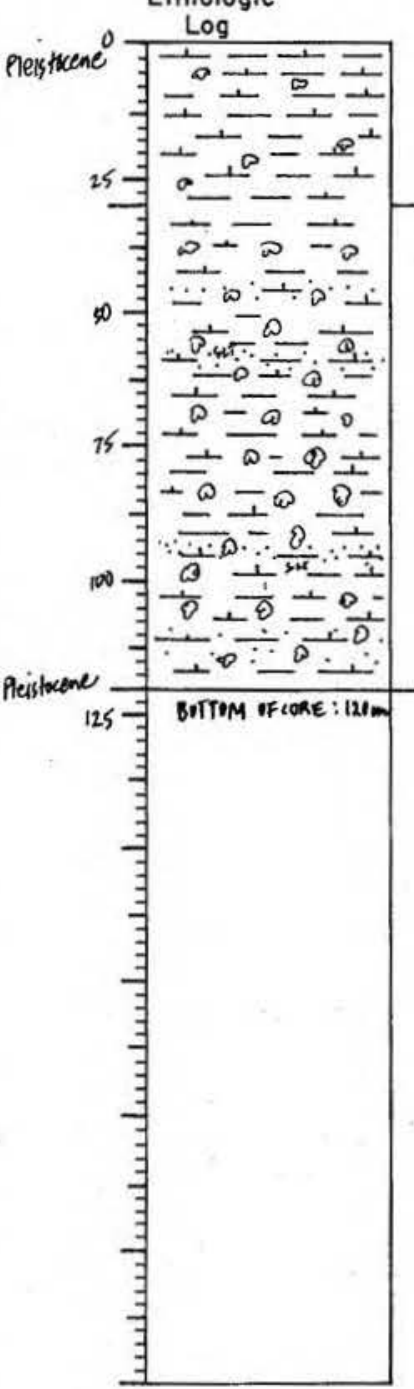

$0-30$

HIGHLY CALC CLAY GRADES TO CALC CLAY

10 YR $4 / 2$ dark grayish brown

faint, Irregular and poorly defined light brownish-

molst, unconsolidated slick lutite s, mottled

(120

HIGHLY CALC CLAY WITH LAMINATIONS OF HIGHLY CALC CLAY/DETRITUS

YR $4 / 2$ dark grayish brown and $5 / 2$ grayish brown and rownish gray to extensive inter-mottling throughout

moist, slightly silty lutite with occasional zones of very silty material; also scattered Mn-rich flecks

zones of highly silty material: $59-65 \mathrm{~cm}, 92-94 \mathrm{~cm}$ and scattered, thin, irregular silty lenses, 102end of core repeated sequences of beds of the colors above with 
SIEAR SLIDE DESCRIPTIONS - W.H.O.I. SEDIMENT CORES

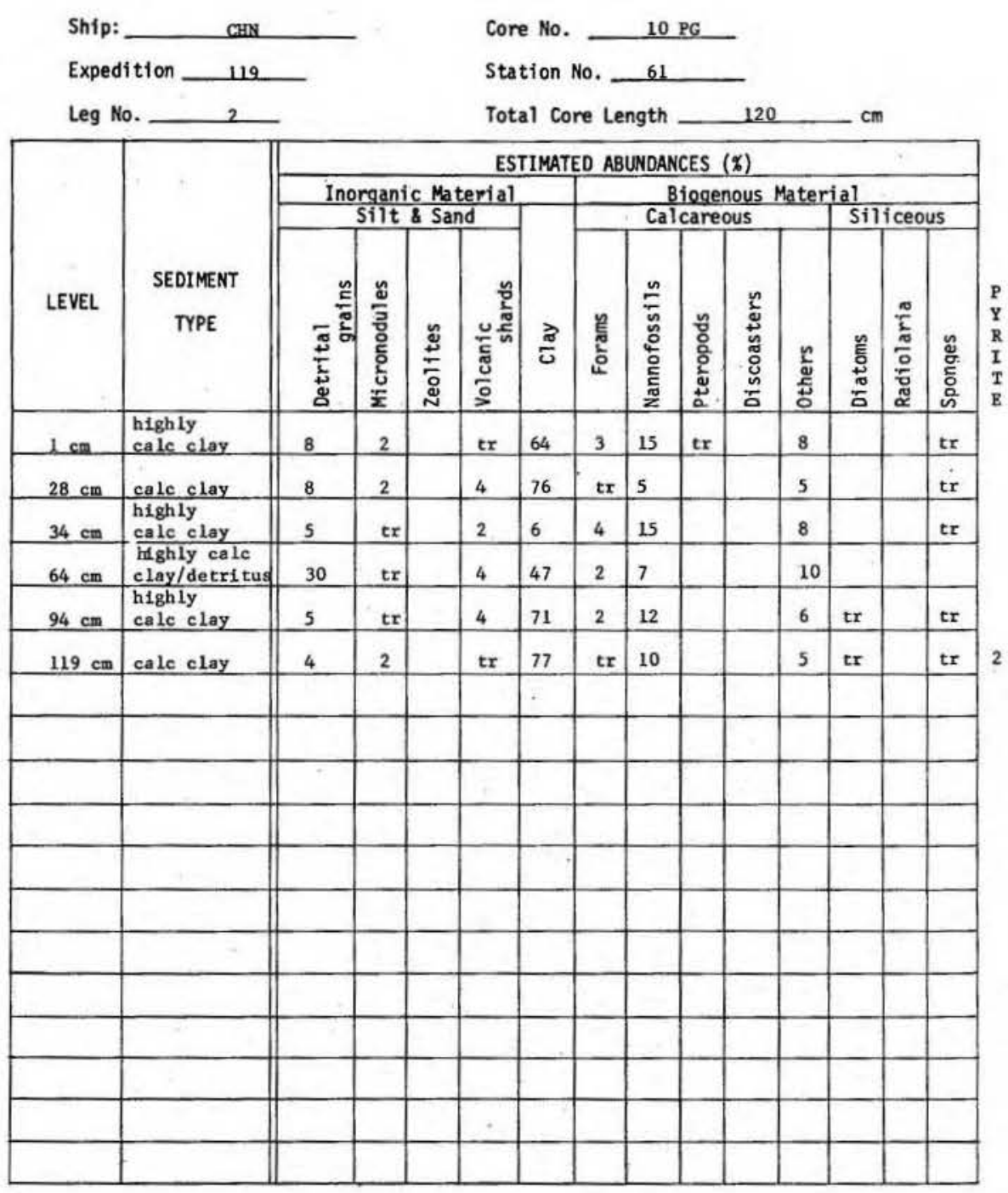

Ship CHAIN Cruise 119 Leg II Sto. 62 Core No. II TC Total Length $925^{\circ} \mathrm{cm}$. Lat. $31^{\circ} 52.92^{\circ} \mathrm{N}$ Long. $29^{\circ} 38.10^{\circ} \mathrm{E}$ Depth $1366 \mathrm{~m}$. Corr Core condition EXLELLENT. Date Described 8 fun 75 by 7 . Brona. Physiographic location MOGEMTA FAN (AILE LONE) N.W. A ALEXAN DRIA EGYPT, E. MEOTT ERRANEAN. Lithologic

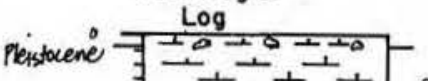

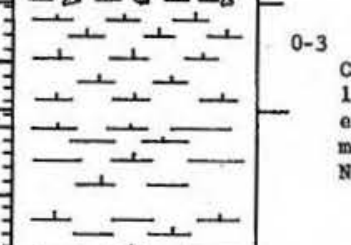

Detailed Description

$0-3$ ALC OOZE

10 YR $6 / 4$ 1ight yellowish brown extensive, dark olive gray intermarbling oist, unconsolidated lutite

this unit appears disturbed and unstratified; also, on comparison with pilot core, it seems likely that the piston core missed ("blew by") the first 35 or $40 \mathrm{~cm}$ of sediment and then compacted the next 30 or $40 \mathrm{~cm}$ in

100 -1- the top $15 \mathrm{~cm}$ of 1 ts recovery

- $3-43$ , irregula

CALC OOZE

5 Y $3 / 2$ dark olive gray

numerous, faint and sonewhat 1rregular olive gray moist, slick lutite with a few fine wedges and lense of silt

$+\div$

N 0

SLIGETLY CALC CLAY WITH LAMIRATIONS OF CALC OOZE AND DETRTTUS/PYRTTE

Innumerable and re gray white, light gray, and olive gray microlaminae, generally carbonate in composition, but silt-sized detrital laminations are also common: some remarkably fine feafirm, smooth lutite throughout, with occasional silty few of the

carbonate laminae found throughout are somewhat lithified and brittle

300 $-\div-$ 630

350-630

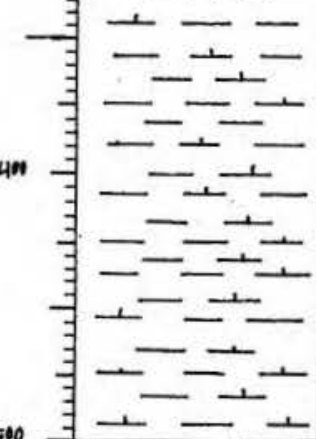

SLIGETLY CALC CLAY, GRADES TO CALC CLAY WITH LAMTNATIONS AND BANDS OP CAIC OOZE

5 Y $3 / 2$ dark olive gray

laminations continue, but they are considerably fainter and in general not so well-defined: there are a number of broad bands of olive brown lutite (373-375.5 cm) (396-399 cm) (422-425 cm) (488-490 cm) $(633-634 \mathrm{~cm})$ and beds occurs at $512 \mathrm{~cm}$ change in firm, smooth lutite with occasional thin silty laminations 
517

VISUAL CORE DESCRIPTION

Page 2 of 2

Ship CHAIN Cruise 119 Leg II Sta. 62 Core No. IIPC

Lithologic

Log

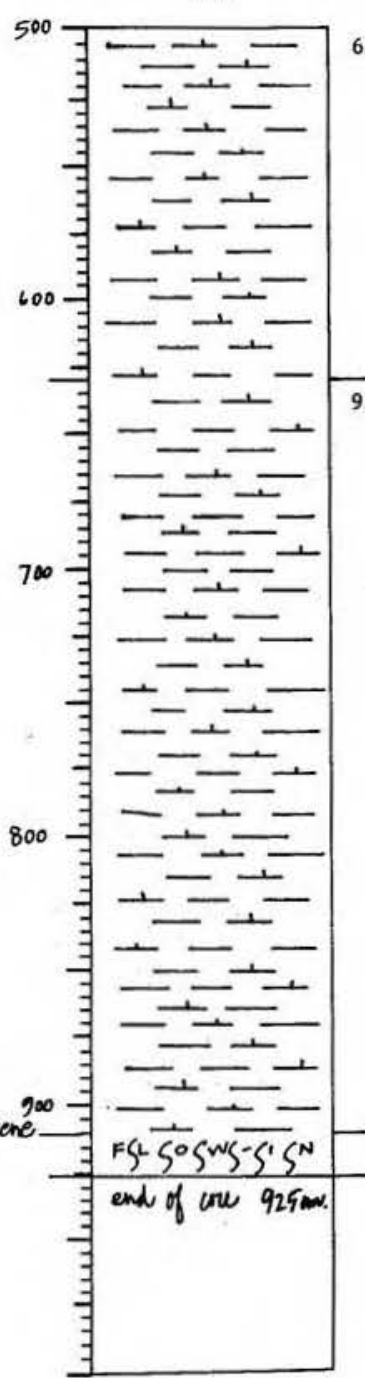

Detailed Description

630-910 CALC CLAY WITH LAMINATIONS OF CALC OOZE AND DETRITUS/ 5 Y $3 / 2$.

light gray and olive gray laminations continue, with the addition of two dark grayish brown, $1-1.5 \mathrm{~cm}$, bed t very stiff, lithified lutite, also, the fine, silty laminations take on a rusty hue from here to bottom of

irmer, more compact, smooth lutite, with occasional

Dits in the zone $823-910 \mathrm{~cm}$

910-925

FLOW-IN

end of core
SHEAR SLIDE DESCRIPTIONS - K.H.O.I. SEDIMENT CORES

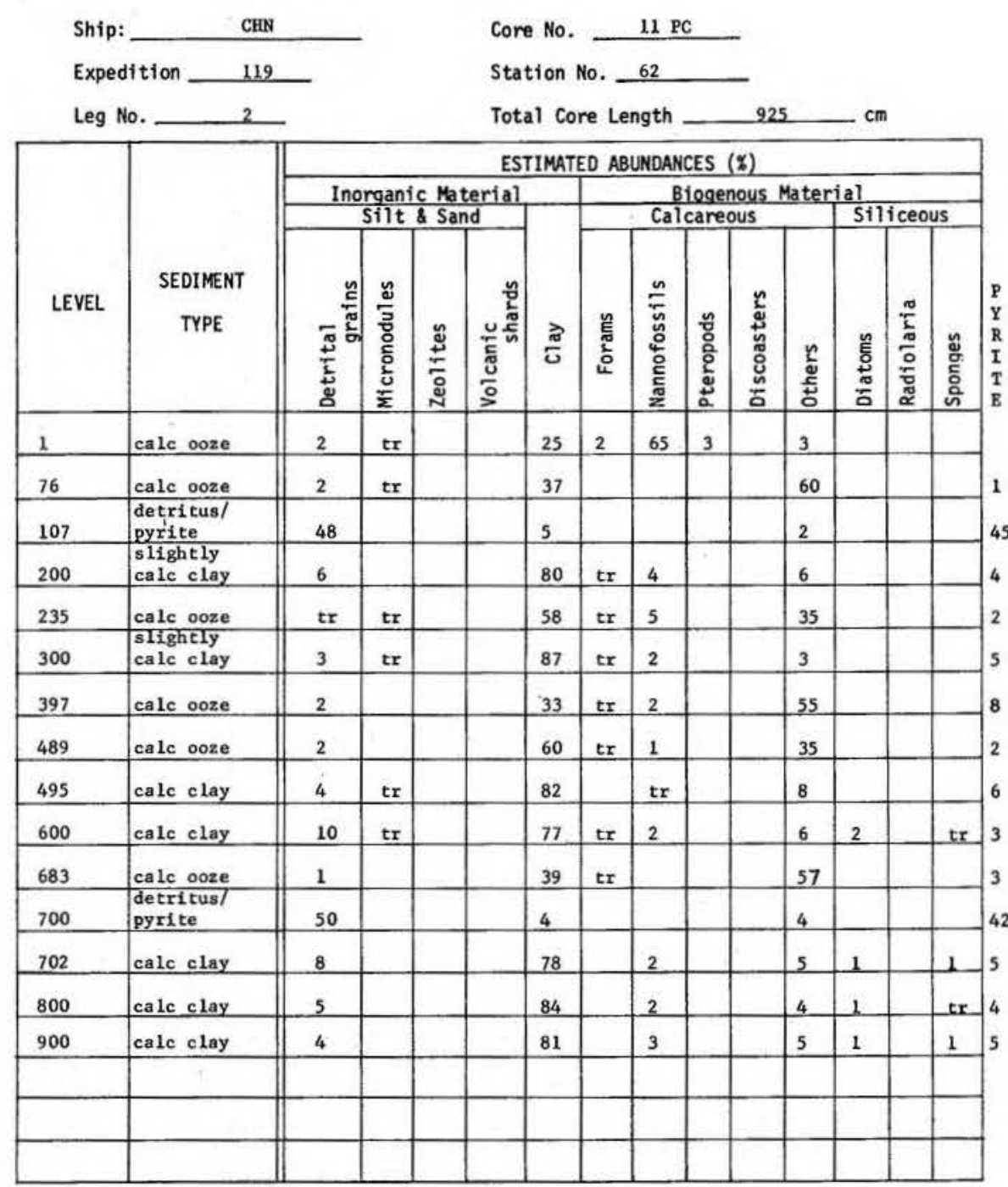




\section{VISUAL CORE DESCRIPTION}

Ship CHAIN Cruise 119 Leg II Sta. 62 Core No. IIPG Total Length $84 \mathrm{~cm}$. Lat. $31^{\circ} 52.92^{\prime} \mathrm{N}$ Long. $29^{\circ} 380^{\circ} \mathrm{E}$ Depth $1366 \mathrm{micar}$ Core condition ExGEWISNT Date Described 6 Ju 75 by friodar.

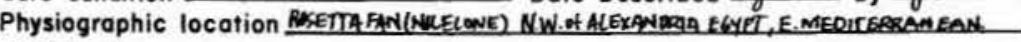
Lithologic

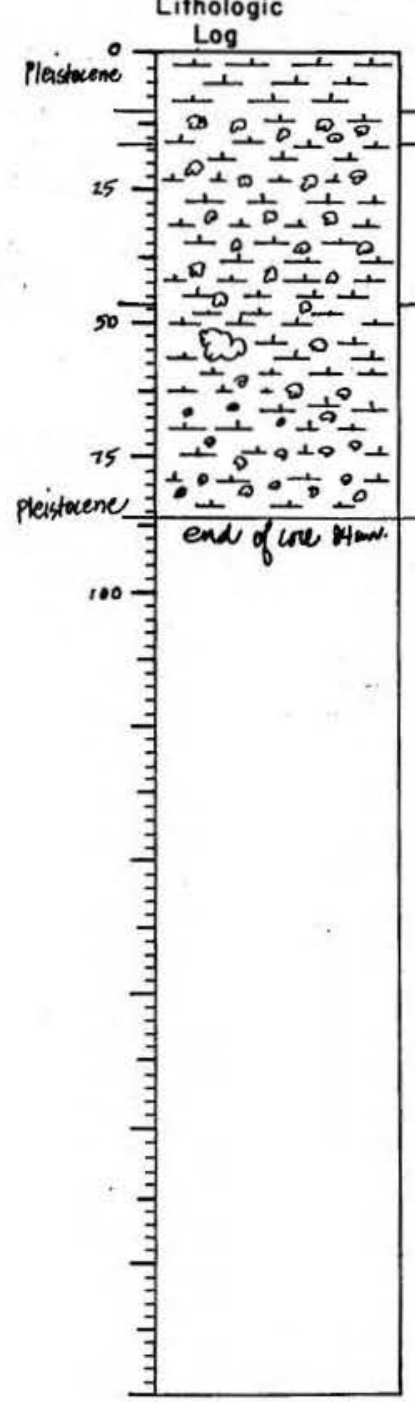

\section{Detailed Description}

0-10

\section{$10 \mathrm{YR} 4 / 3$ dark brown}

homogeneous throughout upper $5 \mathrm{~cm}$ a bit washed and upset: large pteropod inirregular

0-16, irreguiar

$10 \mathrm{YR} 6 / 3$ pale brown

extensive, fine, dark brown mottling and burrowing

a bit fimer, slightly silty lutite with a few forans s, mottled

\section{ALC OOZE}

10 YR $6 / 4$ light yellowish brown, grades to $5 / 2$ grayish brown

extensive, very pale brown and 1 ght grayish brown mottling and burrowing throughout

moist, very slightly silty lutite with scattered forams

and pteropods

fine bed of small pteropod fragments is found $16-17 \mathrm{~cm}$ $s$, inclined $10^{\circ}$

CALC OOZE GRADES TO CAIC CLAY

5 Y $3 / 2$ dark olive gray

fle pale brown mottles, burrows,

very large, irregular, 11ght

a few fine laminae appear $79-84 \mathrm{~cm}$

end of core ist, slightly silty lutite
SMEAR SLIDE DESCRIPTIONS - W.H.O.I. SEDIMENT CORES
Ship: Expedition

Leg No. 2
Core No. $11 \mathrm{PG}$

Station No. 62

Total Core Length $\quad 84-\mathrm{cm}$

\begin{tabular}{|c|c|c|c|c|c|c|c|c|c|c|c|c|c|c|}
\hline \multirow[b]{3}{*}{ LEVEL } & \multirow[b]{3}{*}{$\begin{array}{c}\text { SEDIMENT } \\
\text { TYPE }\end{array}$} & \multicolumn{13}{|c|}{ ESTIMATED ABUNDANCES ( $(\%)$} \\
\hline & & \multicolumn{4}{|c|}{$\frac{\text { Inorganic Material }}{\text { Silt \& Sand }}$} & & \multicolumn{5}{|c|}{$\begin{array}{l}\text { Biogenous Mater } \\
\text { Calcareous }\end{array}$} & \multicolumn{3}{|c|}{$\frac{\text { al }}{\text { Siliceous }}$} \\
\hline & & 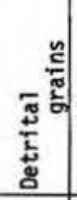 & 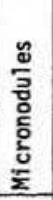 & 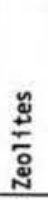 & 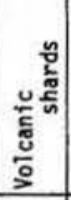 & $\frac{\pi}{5}$ & 望 & 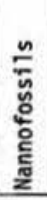 & 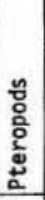 & 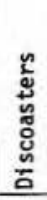 & $\begin{array}{l}\frac{c}{2} \\
\text { sँ๊ } \\
\end{array}$ & $\begin{array}{l}\tilde{E} \\
\stackrel{ \pm}{0} \\
\bar{\sigma}\end{array}$ & $\frac{\frac{\pi}{2}}{\frac{\pi}{0}}$ & 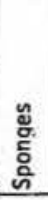 \\
\hline 1 & calc ooze & 6 & tr & & 1 & 44 & 2 & 35 & 4 & & 7 & $\mathrm{tr}$ & & 1 \\
\hline 20 & calc ooze & 2 & & & $t x$ & 24 & 5 & 60 & 3 & & 6 & & & \\
\hline 50 & calc ooze & 1 & & & & 42 & 2 & 50 & 3 & & 2 & & & \\
\hline 83 & calc clay & 6 & $\mathrm{tr}$ & & & 70 & $\mathrm{tr}$ & 10 & $\mathrm{tr}$ & & 4 & & & \\
\hline & & & & & & & & & & & & & & \\
\hline & & & & & & & & & & & & & & \\
\hline & & & & & & . & & & & & & & & \\
\hline & & & & & & & & & & & & & & \\
\hline & & & & & & & & & & & & & & \\
\hline & & & & & & & & & & & & & & \\
\hline & & & & & & & & & & & & & & \\
\hline & & & & & & & & & & & & & & \\
\hline & & & & & & & & & & & & & & \\
\hline & & & & & & & & & & & & & & \\
\hline & & & & & & & & & & & & & & \\
\hline & & & & & & & & & & & & & & \\
\hline & & & & & & & & & & & & & & \\
\hline & & & & & & & & & & & & & & \\
\hline
\end{tabular}


Ship CHAlN Cruise 119 Leg 2 Sto. $\frac{64}{29}$ Core No. 12 PC Total Length $414 \mathrm{~cm}$. Lot. $32^{\circ} 22.8^{\prime} \mathrm{N}$ Long. $29^{\circ} 13.5^{\circ} \mathrm{E}$ Depth $2289 \mathrm{corcm}$. Core condition excellent Date Described $70 x \quad 75$ by H. Former Physiographic location Rosetts Fan (Nre Cowe), Nw of Alexandria, Egypt

Lithologic

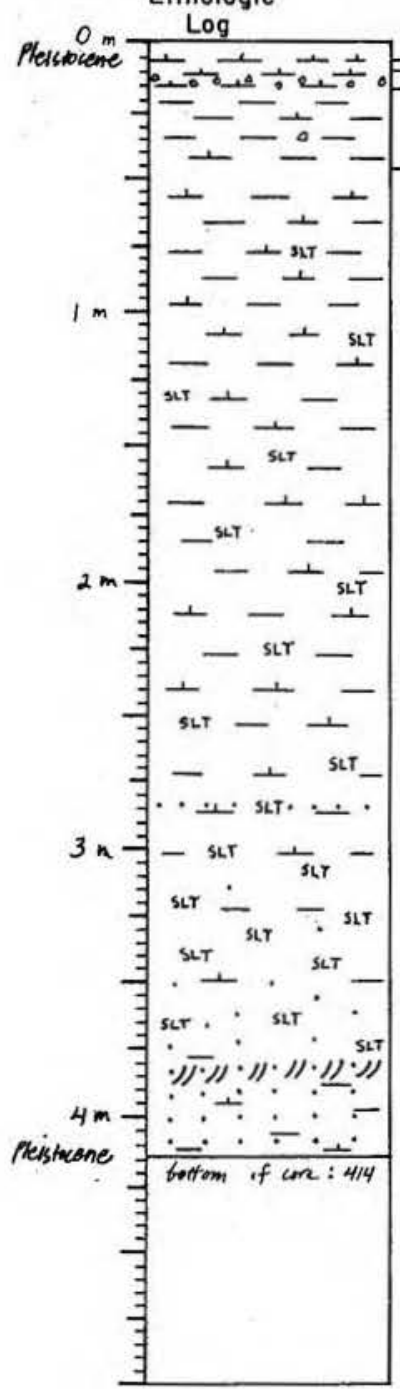

Detailed Description

$0-6$

10 YR $6 / 4$, light yellow brow

slightly silty lutite with few forams

CALC OOZE

10 YR $4 / 4$, dark yellow brown

alightly silty lutite with few forams

${ }^{6}{ }^{6}$

\section{CALC OOZE}

2,5 × $3 / 2$, very derk gray brow

very abundent small mottles throughout

slightly silty lutite

thin $(3 \mathrm{~mm})$ sand layer.at bottom

16-46

SLIGHTLY CALC CLAY

few sma il moteline gray 2 larger irregular sand

clasts $40-49 \mathrm{~cm}$

lutite, 24-27 can bioclastic fra silt/fine send laminations from 30-38 cm $(\sim 2-4$ 46-414

CALC CLAY WITH DETRITUS GRADES TO SLIGHTLY CALC CLAY/ DETRITUS

generally few forams but scattered small patches with generally few forang but scatte common; becomes very gradually more silty/sandy a fter $250 \mathrm{~cm}$, until last $50 \mathrm{~cm} \mathrm{is}$ a fine sand: cross-bedded laminations from $382-388$; large lutite/ silty lutite intraclasts $352-358,372-384,403-408$; thin dark yellow-brown sand bed $(2 \mathrm{~mm})$ at 289 . end of core
SMEAR SLIDE DESCRIPTIONS - W.H.O.I. SEDIMENT CORES

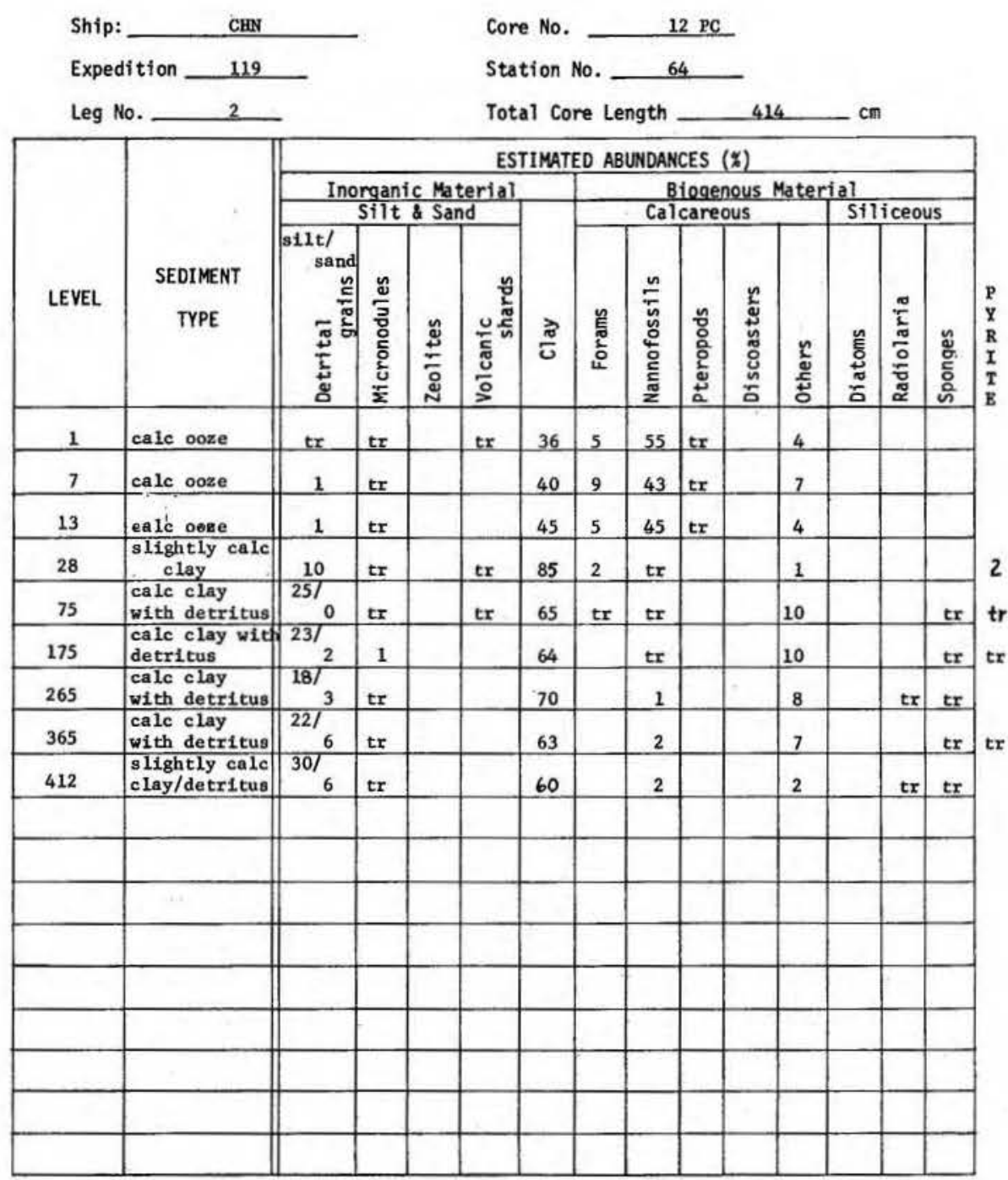


Ship CHAIN Cruise 119 Leg 2 Sta. 64 Core No. 12 PG Total Length $40 \mathrm{~cm}$. Lat. $32^{\circ} 22.8^{\prime} \mathrm{V}$ Long. $29^{\circ} 13.5^{\prime} \mathrm{E}$ Depth $2289 \mathrm{corrm}$ Core condition excellent Date Described 80075 by $T$ Farmer Physiographic location Rosetta Fan (NILE Cove) NW of Alexandria, Egypt

Lithologic

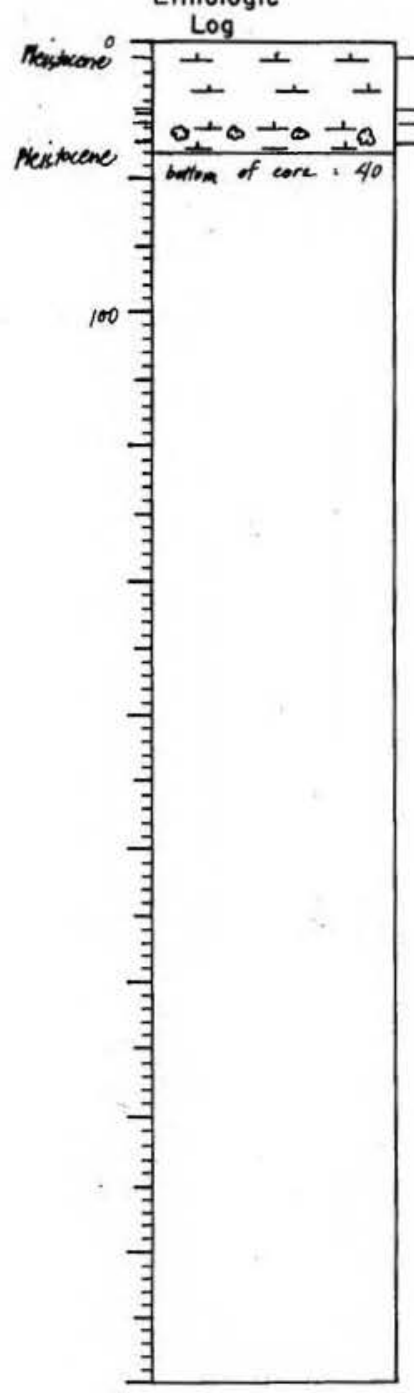

\section{Detailed Description}

\section{CAIC OOZE}

XR 6/3, pale brown

smooth, stightly oilty lutite, very fey forams $\mathrm{s}$, horizontal $5-5.5$

CALC OOZE

$2.5 \times 5 / 4$, light olive brown

pteropod hash in lutite matrix S. $5-23$

CALC OOZE

$2.5 \times 5 / 4$, light olive brown

mottling apparent

23-27

CALC OOZE

10 YR $5 / 3$, brow

25-27 laminated with dark brown and scattered very

pale brown mottes with scattered foram $-36$

5 Y $3 / 2$, dark olive gray

slightly silty lutite with scattered forams, thin sand lense near top s

IC OOZE/DETRITUS

SY $3 / 2$, dark olive gray

no mond

, horizontal

MIGHYY CALC CLAY

5 Y $3 / 2$, dark olive gray

no motties

lutite, no forams

end of core
SIEAR SLIDE DESCRIPTIONS - W.H.O.I. SEDIIENT CORES

$\begin{array}{lll}\text { Ship: } & \text { CHAIN } & \text { Core No. } \frac{12 ~ P G}{6} \\ \text { Expedition } 119 & \text { Station No. } & 64\end{array}$

Leg No. 2 Total Core Length $\quad 40$

\begin{tabular}{|c|c|c|c|c|c|c|c|c|c|c|c|c|c|c|}
\hline \multirow{4}{*}{ LEVEL. } & \multirow[b]{4}{*}{$\begin{array}{c}\text { SEDIMENT } \\
\text { TYPE }\end{array}$} & \multicolumn{13}{|c|}{ ESTIMATED ABUNDANCES $(x)$} \\
\hline & & \multirow{2}{*}{\multicolumn{4}{|c|}{$\frac{\text { Inorganic Material }}{\text { Silt \& Sand }}$}} & \multirow[b]{3}{*}{$\frac{\pi}{\sigma}$} & \multirow{2}{*}{\multicolumn{5}{|c|}{$\begin{array}{l}\text { Biogenous Mater } \\
\text { Calcareous }\end{array}$}} & \multirow{2}{*}{\multicolumn{3}{|c|}{ Siliceous }} \\
\hline & & & & & & & & & & & & & & \\
\hline & & 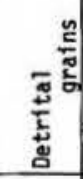 & $\begin{array}{l}\frac{\omega}{3} \\
\frac{0}{3} \\
\frac{0}{0} \\
\frac{0}{2} \\
\frac{0}{z}\end{array}$ & 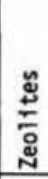 & 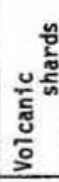 & & 产 & 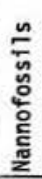 & 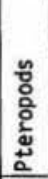 & 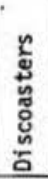 & $\begin{array}{l}\frac{r}{2} \\
\frac{\omega}{ \pm} \\
0 \\
0\end{array}$ & 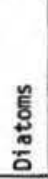 & $\frac{\frac{\pi}{i}}{\frac{\pi}{0}}$ & $\begin{array}{l}\text { まे } \\
\text { हें } \\
\text { जั }\end{array}$ \\
\hline 2 & calc ooze & tr & & & & 30 & 1 & 60 & 2 & tr & 7 & & & tr \\
\hline 18 & calc ooze & $t x$ & & & & 38 & 5 & so & 2 & & 5 & & & \\
\hline 24 & callc ooze & $t x$ & 5 & & & 40 & 7 & 40 & 2 & & 6 & & & \\
\hline 30 & calc ooze & 1 & & & tr & 55 & 2 & 30 & 2 & & 5 & & & \\
\hline 39 & $\begin{array}{l}\text { highly } \\
\text { calc clay }\end{array}$ & 10 & & & $\mathrm{tr}$ & 59 & & 1 & & & 25 & & & \\
\hline & & & & & & & & & & & & & & \\
\hline & & & & & & & & & & & & & & \\
\hline & & & & & & & & & & & & & & \\
\hline & & & & & & & & & & & & & & $\cdot$ \\
\hline & & & & & & & & & & & & & & \\
\hline & & & & & & & & & & & & & & \\
\hline & & & & & & & & & & & & & & \\
\hline & & & & & & & & & & & & & & \\
\hline & & & & & & & & & & & & & & \\
\hline & - & & & & & & & & & & & & & \\
\hline & & & & & & & & & & & & & & \\
\hline & & & & & & & & & & & & & & \\
\hline & & & & & & & & & & & & & & \\
\hline
\end{tabular}




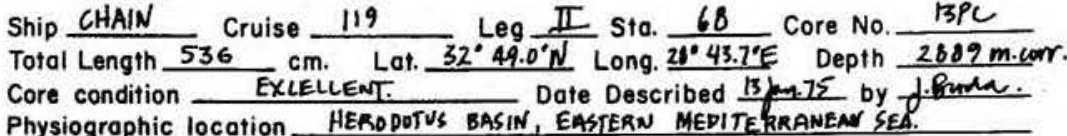

Physiographic location HERODOTUS BASIN, EASTERN MEDITERRANEAN SEC.

Lithologic

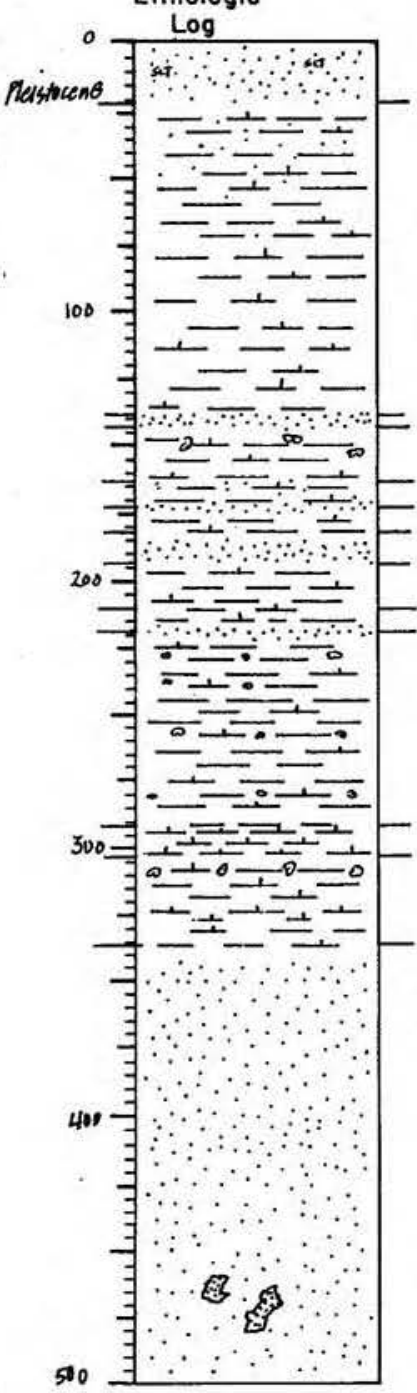

\section{Detailed Description}

NOTE: due to the lithology of the pilot gravity core on this station and the generally dubious appearance of unusthe upper 1.4 meters of this piston core is question-

$0-21$ able

\section{DETRITUS/CALC CLAY}

2.5 Y $4 / 2$ dark grayish brown

homogeneous throughout

large, $3 \mathrm{~cm}$, frregular pale brown and gray inclusion S ( (textural), irtegutic

SLIGHTLY CALC CIAX WITH DETRITUS GRADES TO CALC CLAY 5 Y $3 / 2$ dark olive gray

several zones of disturbed marbling of pale brown an olive gray lutite

rregular texture throughout: some zones laminated with highly silty material, $35-48 \mathrm{~cm}, 125-135 \mathrm{~cm}$; degree of consolidation varies, but generally firm, evere disturbance evident $65-78$ con

$\mathrm{cm}, 107-123 \mathrm{~cm}$ G

DETRITUS

2.5 Y $4 / 2$ dark gxayish brown

somewhat graded bed with a few faint color laminations $\mathrm{S}$

CALC CLAY

$5 \times 5 / 3$ olive, grades to $3 / 2$ dark olive gray

145-47. also fine of the zone firm, slick lutite throughout

63-173

73 textural

CALC CIAY WITH DETRITUS, GRADES TO DETRITUS

5 Y $3 / 1$ very dark gray, grades to $3 / 2$ dark olive gray

one fine, dark lamination at upper unit contact; other-

silty lutite, grades to stiff, compact, pure silt
, textural

173-182

$$
\text { CALC CIAY }
$$

Ship CHAIN Cruise 119 Leg II Sta. 68 Core No. I3PC

Lithologic

Log

\section{Detailed Description}

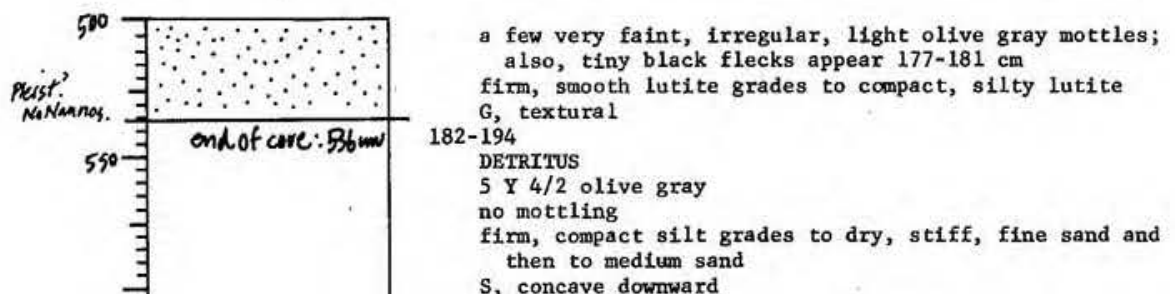
194-210 CALC CIAY

5 Y $7 / 3$ pale yellow and $4 / 2$ ollve gray

light-colored, firm lutite is mixed and whirled with

entire unit disturbed and unstratified $210-219$

CALC CIAY WITH DETRITUS, GRADES TO DETRITUS

5 Y $4 / 2$ olive gray

a few falnt dark laninations and bands $211-218 \mathrm{~cm}$ extremely silty lutite, grades to stiff, very compact s, textural

219-292

SLIGHTLY CALC CLAY

5 X $4 / 2$ olive gray and $3 / 2$ dark olive gray

several zones of very fine sapropel-like light olive gray flecks and specks (mottles) $220-227 \mathrm{~cm}, 235-$

$236 \mathrm{~cm}, 244-254 \mathrm{~cm}, 260-277 \mathrm{~cm}$

firm, fairly compact lutite with varying amounts of silt; also a few pteropod fragments found in the $G$

292-303

CALC OOZE

5 Y $6 / 3$ pale olive

smooth, slick lutite

303-336

CALC CIAY

5 Y $5 / 2$ olive gray

fine, pale olive mottling and flecks 303-315; large, 
527

VISUAL CORE DESCRIPTION

Page 3 of 3 Ship CHAIN Cruise 119 Leg 2 Sta. 68 Core No. 13PC
Lithologic

Log

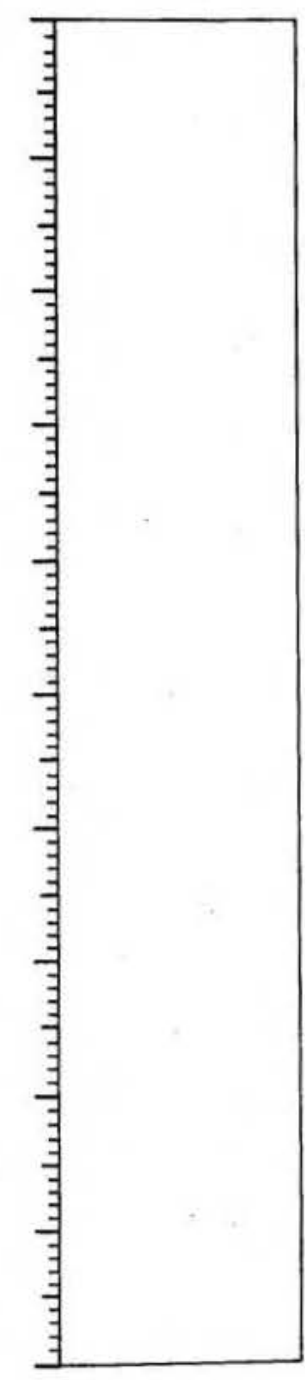

336-536

DETRITUS

5 Y $4 / 2$ ollive gray

no mottiing

compact, fine silt, grades slowly and evenly to

$460-480-\operatorname{sev} x$

large, cemented sand stones are end of core

\section{Detailed Description}

SHEAR SLIDE DESCRIPTIONS - W.H.O.I, SEDINENT CORES

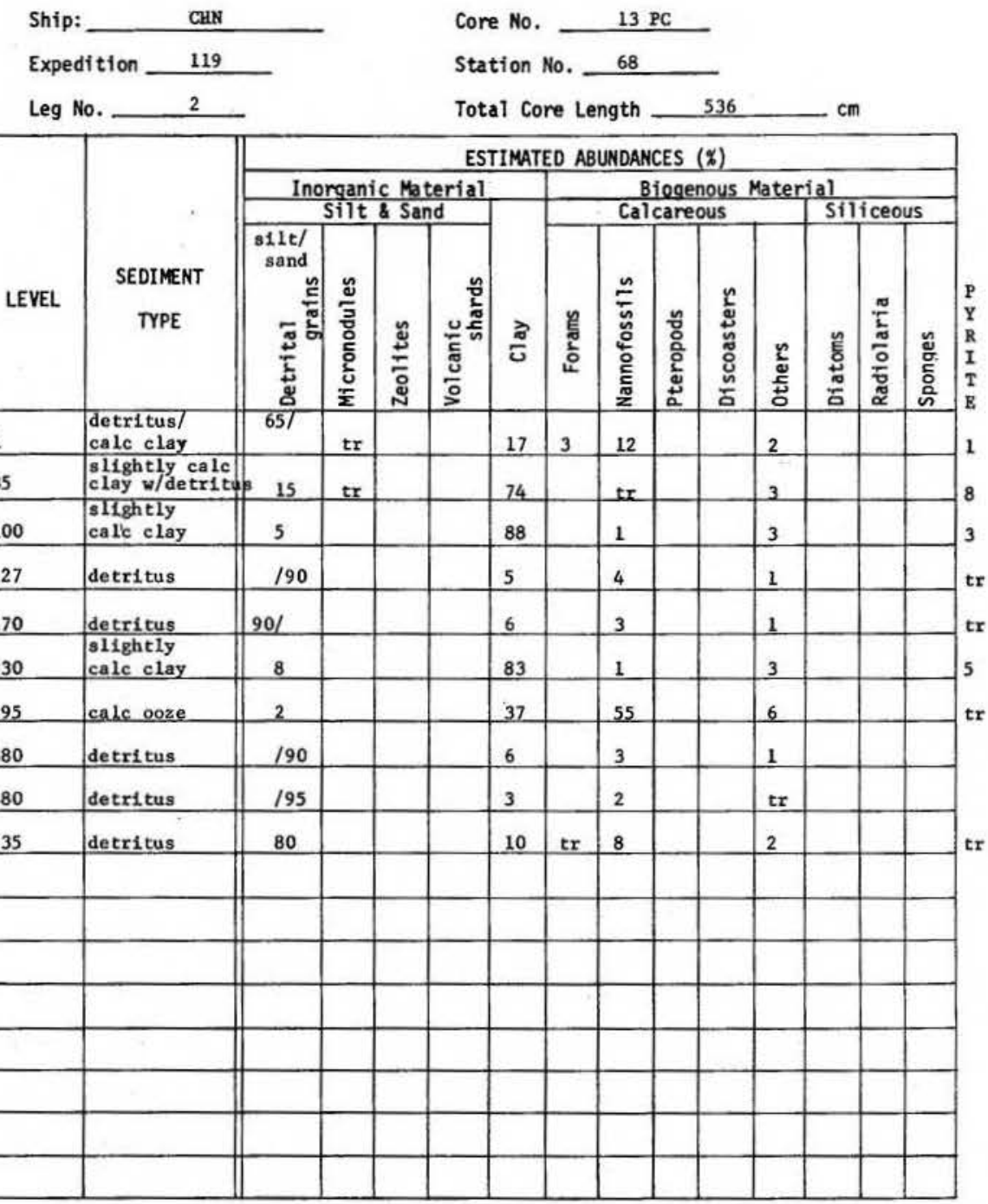


529

VISUAL CORE DESCRIPTION

Page 1 of 1

Ship CHAIN Cruise 119 Leg II Sta. 68 Core No. 13PG Total Length $88 \mathrm{~cm}$. Lat. $32^{\circ} 49.0^{\circ} \mathrm{N}$ Long. $28^{\circ} 43.7^{\circ} \mathrm{E}$ Depth $2889 \mathrm{mcarr}$ Core condition EXLEUENT Date Described $13 \mathrm{fm} 75$ by 7 . Gradar. Physiogrophic location HERODOTUS BASIN, EASTERN MEOATERRANEAN SEA. Lithologic

Presinticare

\section{Detailed Description}

$0-37$

CALC OOZE

$7 / 4$ very pale brown

innumerable, fine brown and light yellowish-brown

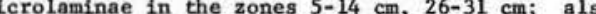
two broader $(1.5 \mathrm{~cm})$ bands, light brownish-gray and light gray at lower unit contact S, horizonta1

CALC OOZE, GRADES TO CALC OOZE WITH PYRITR, TO CALC few broad, textural bands found throughout

several zones of mulchy, sapropel-1ike pteropod-rich material $36-41 \mathrm{~cm}, 58-55 \mathrm{~cm}, 61-63 \mathrm{~cm}, 71-75 \mathrm{~cm}$;

otherwise moist, smooth lutite becoming more silty at base

one very large, well-preserved pteropod test at $55 \mathrm{~cm}$ CALC CIAY

CALC CIAY
5 Y $4 / 2$ olive gray

compact, extremely silty lutite

end of core moist, silck, smooth lutite with a few forams $10-23 \mathrm{~cm}$

\section{0}

SMEAR SLIDE DESCRIPTIONS - W.H.O.I. SEDIMENT CORES

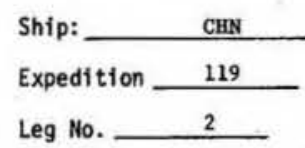

Core No. 13 PG

Station No. 68

Total Core Length $\quad 88$

\begin{tabular}{|c|c|c|c|c|c|c|c|c|c|c|c|c|c|c|}
\hline \multirow[b]{3}{*}{ LEVEL } & \multirow[b]{3}{*}{$\begin{array}{c}\text { SEDIMENT } \\
\text { TYPE }\end{array}$} & \multicolumn{13}{|c|}{ ESTIMATED ABUNDANCES (\%) } \\
\hline & & \multicolumn{4}{|c|}{$\frac{\text { Inorganic Material }}{\text { Silt \& Sand }}$} & \multirow[b]{2}{*}{ 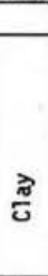 } & \multicolumn{5}{|c|}{$\frac{\text { Biogenous Mater }}{\text { Calcareous }}$} & \multicolumn{3}{|c|}{ Siliceous } \\
\hline & & $\frac{5}{\frac{5}{5}}$ & $\begin{array}{l}\frac{\omega}{\frac{\omega}{z}} \\
\frac{5}{0} \\
\frac{0}{0} \\
\frac{0}{\Sigma}\end{array}$ & 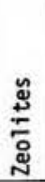 & 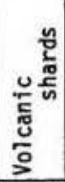 & & 鵕 & 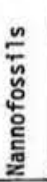 & 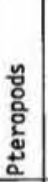 & 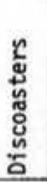 & 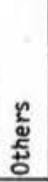 & 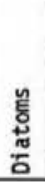 & $\begin{array}{l}\frac{\pi}{\pi} \\
\frac{\pi}{0} \\
\frac{\pi}{0} \\
\alpha\end{array}$ & 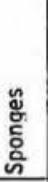 \\
\hline 1 & calc ooze & 2 & $\operatorname{tr}$ & & & 35 & tr & 60 & & & 3 & & & $\mathrm{tr}$ \\
\hline 35 & calc ooze & $\operatorname{tr}$ & $\operatorname{tr}$ & & & 26 & 4 & 65 & 2 & & 3 & & & $\mathrm{tr}$ \\
\hline 52 & $\begin{array}{l}\text { calc ooze } \\
\text { w/pyrite }\end{array}$ & 3 & & & & 45 & 2 & 30 & 3 & & 2 & & & \\
\hline 80 & $\begin{array}{l}\text { calc ooze w/ } \\
\text { pyrite and }\end{array}$ & 15 & & & & 33 & 3 & 30 & 2 & & 2 & & & \\
\hline & detritus & & & & & & & & & & & & & \\
\hline 87 & calc clay & 4 & & & & 76 & tr & 7 & 1 & & tr & & & \\
\hline & & & & & & & & & & & & & & \\
\hline & & & & & & & & & & & & & & \\
\hline & & & & & & & & & & & & & & \\
\hline & & & & & & & & & & & & & & \\
\hline & & & & & & & & & & & & & & \\
\hline & & & & & & & & & & & & & & \\
\hline & & & & & & & & & & & & & & \\
\hline & & & & & & & & & & & & & & \\
\hline & ' & & & & & & & & & & & & & \\
\hline & & & & & & & & & & & & & & \\
\hline & & & & & & & & & & & & & & \\
\hline & & & & & & & & & & & & & & \\
\hline
\end{tabular}


Ship CHN Cruise 119 Leg 2 Sta. 70 Core No. $14 \mathrm{PC}$ Total Length $538 \mathrm{~cm}$. Lat. $33^{\circ} \cdot 14.6^{\prime} \mathrm{N}$ Long. $28^{\circ} 15.4 \mathrm{E}$ Depth 3106 corr. Core condition sxcellent Date Described 6 Jan $7 k$ by H. Fienex Physiographic location CENTEAL HEReotTus BASIN EAST. ME. SEA

Lithologic

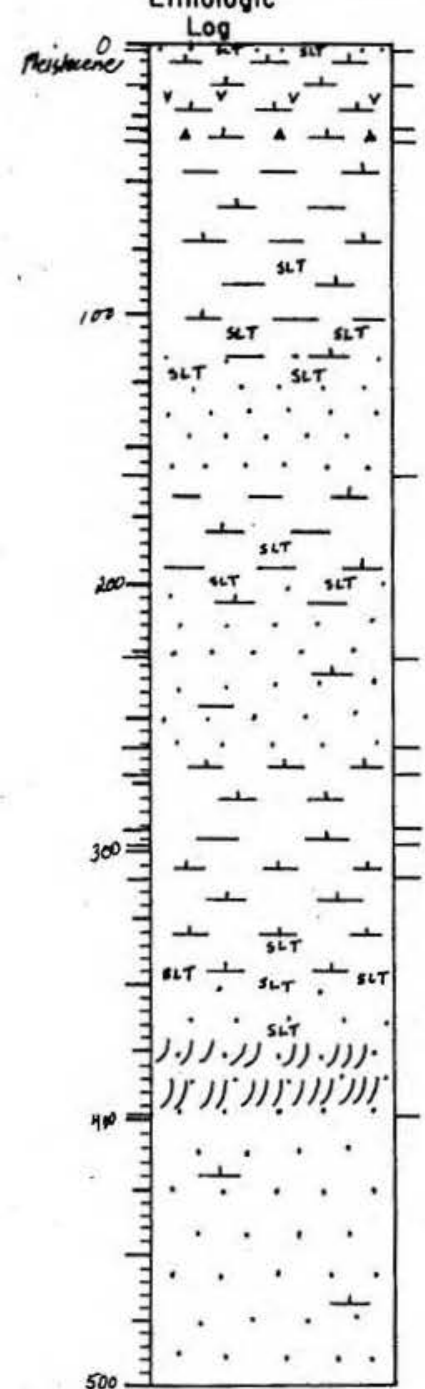

Detailed Description

0-2

\section{CAIC OOZE/DETRITUS}

2.5 Y $4 / 2$ dark gray1sh brow

washed and unstratified, soupy sand with lutite $-14^{\mathrm{S}}$

CAIC OOZE

10 YR $7 / 3$ very pale brown

of dark brown from 5-13 cm

ite, except thin, fine silty laye S$$
\text { S }
$$

10 YR $6 / 4$ 11ght yellowish brown grades to $6 / 6$ brownish

no mottling
silty, grades to slightly silty lutite, forams grade

from scattered to con

brown), 2 m

$29.5-34$

CAIC OOZE WITH PYRITE

5 Y $3 / 2$ dark olive gray

no mottling

silty lutite, abundant pteropod fragments

sapropel-1ike materia

$\mathrm{S}$, but disturbed

CALC CLAY GRADES TO EIGHLY CALC CLAY/DETRITUS

5 Y $3 / 2$ dark olive gray

no mottling

smooth, slick lutite grades smoothly to silty lutite $(\sim 100 \mathrm{~cm})$ and sand $(\sim 125 \mathrm{~cm})$

$1 \mathrm{~cm}$ sapropel with abundant pteropods at $68 \mathrm{~cm}$ 160-227

CAIC CLAY GRADES TO HIGHLY CALC CIAY/DETRITUS 5 Y $3 / 2$ dark olive gray

smooth, slick lutite grades smoothly to very silty lutite

S27-262

CALC CLAY/DETRTTUS

2.5 × $4 / 2$ dark grayish brown

sand with very subdued laminae
VISUAL CORE DESCRIPTION

Page 2 of 2

Ship_CHN Cruise 119 Leg 2 Sto. 70 Core No. $14 \mathrm{PC}$

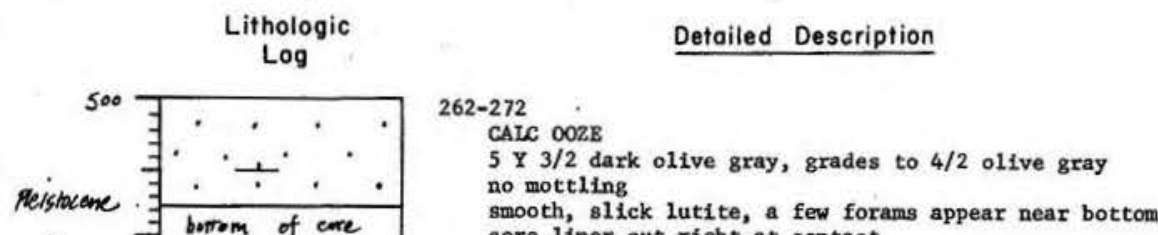

272-291

CAIC OOZE

5 Y $3 / 2$ dark olive gray

no mottling

gades to slightly silty lutite

291-297

CALC CLAY

5 Y $4 / 2$ ollive gray grades to $5 / 3$ olive

marbled throughout

silty lutite with forams common

S, mottled contact

\section{CAIC 007}

5 Y $4 / 2$ olive gray, becomes somewhat darker toward

sma11, scattered mottles from above

slick lutite, few forams present at top but disappear shortly

310-398

CAIC OOZE GRADES TO CALC OOZE/DETRITUS

5 Y $3 / 2$ dark olive gray, except near bottom, sands grade into brown of lower unit

noteling

lutite grades smoothly to fine and medium sand; sands show cross-bedded laminations 作

398- $\frac{\text { very }}{538}$ (5)

10 YR $4 / 3$ brown

10 YR $4 / 3$ brow

homogeneous,

endium, very hard sand 
SMEAR SLIDE DESCRIPTIONS - W.H.O.I. SEDIMENT CORES

Ship: Expedition 119

Leg No.

\begin{tabular}{|c|c|c|c|c|c|c|c|c|c|c|c|c|c|c|}
\hline \multirow[b]{3}{*}{ LEVEL } & \multirow[b]{3}{*}{$\begin{array}{c}\text { SEDIMENT } \\
\text { TYPE }\end{array}$} & \multicolumn{13}{|c|}{ ESTIMATED ABUNDANCES ( $(x)$} \\
\hline & & \multicolumn{4}{|c|}{$\begin{array}{c}\text { Inorganic Material } \\
\text { Silt \& Sand }\end{array}$} & \multirow[b]{2}{*}{$\frac{\pi}{0}$} & \multicolumn{5}{|c|}{$\frac{\text { Biogenous Mater }}{\text { Calcareous }}$} & \multicolumn{3}{|c|}{ Siliceous } \\
\hline & & $\frac{5}{\frac{5}{5}}$ & 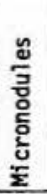 & 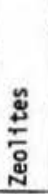 & 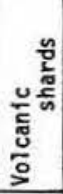 & & 崖 & 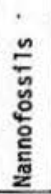 & 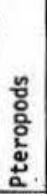 & 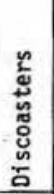 & $\begin{array}{l}\frac{r}{2} \\
\frac{5}{a}\end{array}$ & 产 & $\frac{\frac{\pi}{\pi}}{\frac{\pi}{0}}$ & 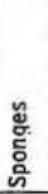 \\
\hline 2.5 & calc ooze & & & & & 50 & tr & 35 & $\mathrm{tr}$ & & 15 & & & \\
\hline 19.5 & volcanic ash & & & & 85 & 5 & tr & 10 & & & & & & . \\
\hline 25 & calc ooze & tr & $\mathrm{tr}$ & & & 42 & 3 & 48 & 2 & & 5 & & & \\
\hline 32 & $\begin{array}{l}\text { calc ooze } \\
\text { with pyrite }\end{array}$ & & . & & & 50 & 7 & 21 & 2 & & 5 & & & \\
\hline 40 & calc clay & 10 & & & & 74 & tr & 1 & tr & & 1.3 & & & \\
\hline 120 & $\begin{array}{l}\text { highly calc } \\
\text { clay w/detrit }\end{array}$ & 25 & & & & 47 & tr & 5 & $\mathrm{tr}$ & & 19 & & & \\
\hline 272 & calc ooze & 1 & & & & 66 & 2 & 25 & 1 & & 5 & & & \\
\hline 348 & $\begin{array}{l}\text { calc ooze w/ } \\
\text { detritus }\end{array}$ & 18 & & & & 15 & 2 & 14 & 1 & & 50 & & & \\
\hline 537 & $\begin{array}{l}\text { calc ooze/ } \\
\text { detritus }\end{array}$ & 40 & & & & 8 & & 2 & & & 50 & & & \\
\hline & & & & & & & & & & & & & & \\
\hline & & & & & & & & & & & & & & \\
\hline & & & & & & & & & & & & & & \\
\hline & & & & & & & & & & & & & & \\
\hline & & & & & & & & & & & & & & \\
\hline & & & & & & & & & & & & & & \\
\hline & & & & & & & & & & & & & & \\
\hline & & & & & & & & & & & & & & \\
\hline & & & & & & & & & & & & & & \\
\hline
\end{tabular}

VISUAL CORE DESCRIPTION

Page 1 of 1

Ship CHN Cruise 119 Sta. 70 Core No. 14 PG Total Length $127 \mathrm{~cm}$. Lat. $33^{\circ} 14.6^{\prime} \mathrm{N}$ Long. $28^{\circ} 15.4^{\prime}$ E Depth 3106 carr. Core condition Acellent Date Described E Tan ht by I FARemef

Physiographic locotion CENTRTL HERODOTUS RASIN EAST. ME). SEA

Lithologic

Log Detailed Description

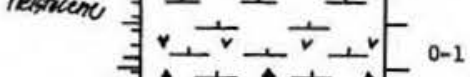

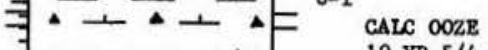

- - -10 YR $5 / 4$ yellowish brown

+- no mottling

土 $\overline{\text { SLT }}$

SLT. - . - SLT $1-13$

$\mathrm{s}$, but disturbed

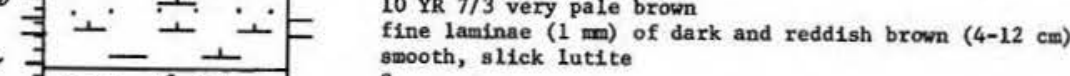
botrom of core 3 -28

\section{CALC OOZE}

$10 \mathrm{YR} 6 / 4$ 1ight yellowish brown grades to $6 / 6$ brownno mottling

$3 \mathrm{~mm}$ of shell hash overlies a silty lutite which becomes somewhat less silty with depth; forams grade from scattered to common 2 man bed brown volcanic ash at $19 \mathrm{~cm}$ 28-34

\section{CALC OOZE WITH PYRTTE}

5 Y $3 / 2$ dark olive gray

no mottling

od fragments abundant

CALC CLAY GRADES TO HIGHYY CALS CLAY/DETRLTUS 5 Y $3 / 2$ dark olive gray

no mottling

( 90 silty lutite by fine sand

it at $60 \mathrm{~cm}$

108-114

sequence frow 3 to $20 \mathrm{~cm}$ is repeated, although compressed to $1 / 3$ of 1 ts original thicknes $\stackrel{\text { S, }}{114-127}$

sequence from $\sim 90$ to $108 \mathrm{~cm}$ is repeated

due to repetition of distinctive stratigraphic features which are not repeated in the p1ston core, rebound of the pillot core is assumed

end of core 
SMEAR SLIDE DESCRIPTIONS - W.H.O.I. SEDIMENT CORES

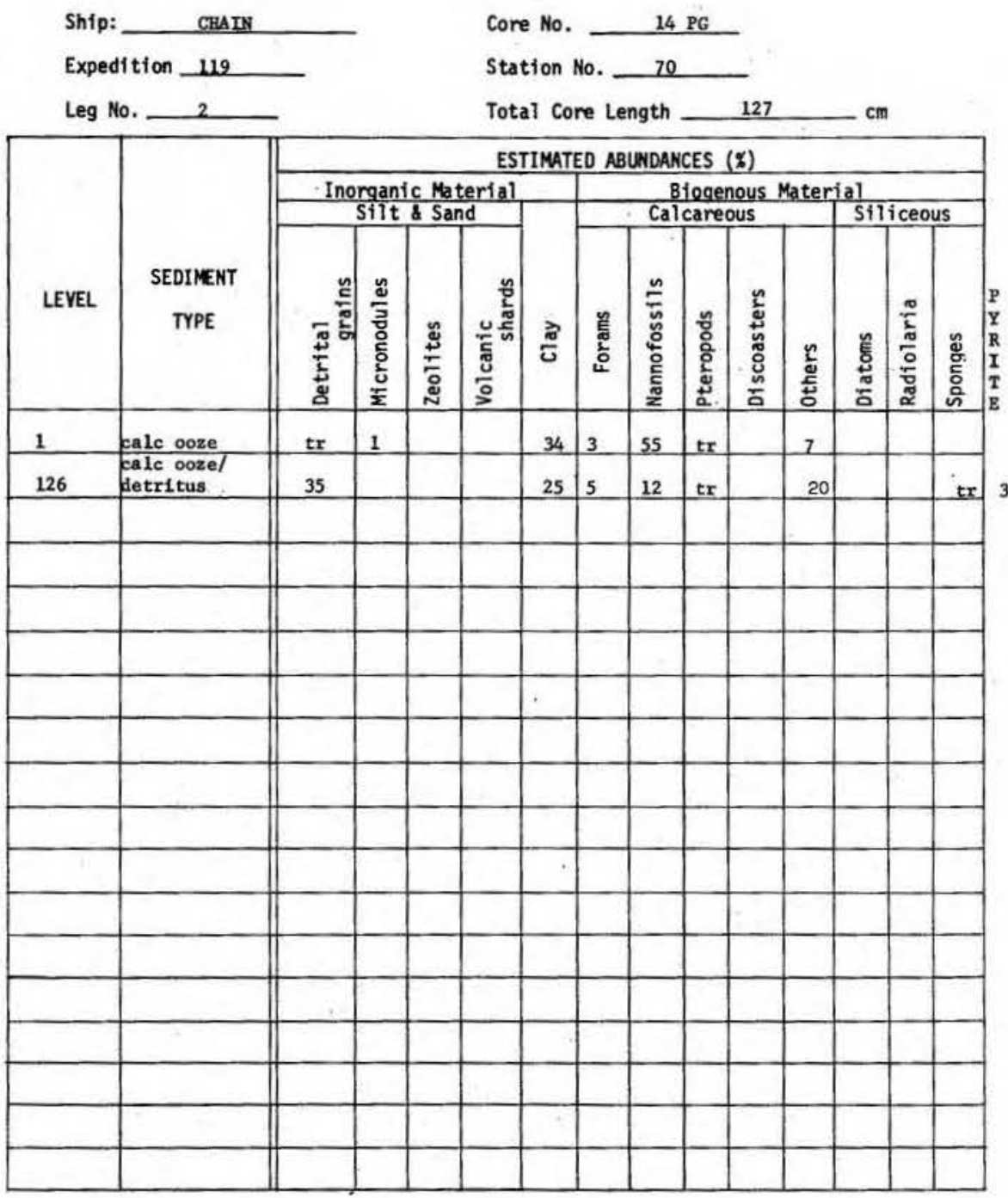

VISUAL CORE DESCRIPTION

Page 1 of 3

Ship CHAIN Cruise 119 II sto 72 Core No. $15 \mathrm{PC}$

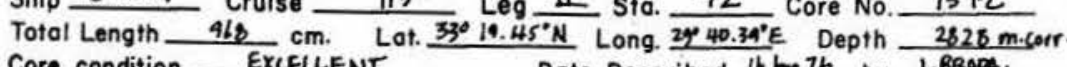
Core condition EXCEULNT Date Described Hf Jen 76 by J.BRopa. Physiographic location DLSTAL NILE CONE, NEIH OF ALEXAMDRH, EAGERN MED. Lithologic

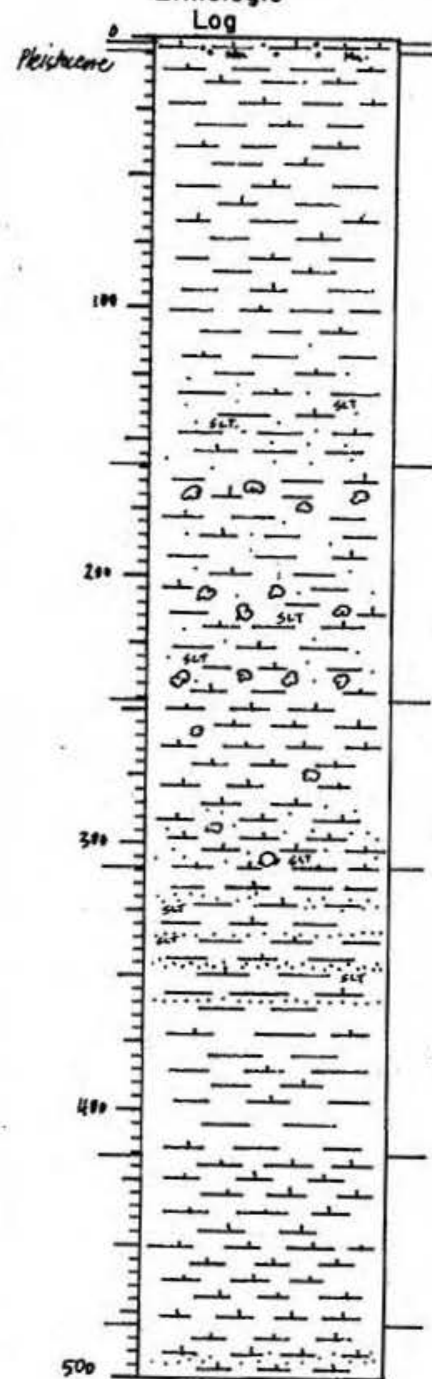

Detailed Description

$0-2$

CALC OOZE

10 $6 / 4$ light yellowish brown

Iutite with a few foram

2-4 orizontal

CALC OOZE WITH M MICRONODULES

10 YR $4 / 4$ dark yellowish brown

innumerable, fine microlaminae; dark and light yellow-

brown alternate

$S$
$4-159$

ooth, moist lutite

HIGHLY CALC CLAY, GRADES TO CALC CIAY WITH DETRITUS

5 Y $3 / 1$ very dark gray and $3 / 2$ dark olive gray

appear 4-5 cm, $12-18 \mathrm{~cm}, 36-39 \mathrm{~cm} 57-67 \mathrm{~cm}, 110-114 \mathrm{~cm}$ very mo1st, slick lutite throughout with the above mentioned exceptions

$\underset{159-246}{S, \text { textura }}$

CALC CIAY WITH DETRITUS

5 Y $3 / 1$ very dark gray and $5 / 2$ olive gray

extensive intermarbling of the colors above in zones moist, smooth lutite with pteropo s, very horizontal

46-308

CALC OOZE GRADFS TO CAIC OOZE/DETRITUS

2.5 Y $6 / 4$ light yellowish brown

slight, faint, light brownish gray mottling scattered

nore compact,

lightly silty lutite with increasing

"silt" (calc fragments); content grades to fine (calc S, very horizonta

- 416

CALC CIAY WITH LAMINATIONS OF DETRITUS

5 Y $3 / 1$ very dark gray

few zones of olive gray banding and fine flecks,

smooth, firm lutite with

and calc fragments (310 few fine beds of pteropod

coarse pteropod ooze $415-416$

$\mathrm{s}$, very 
537

VISUAL CORE DESCRIPTION

Page 2 of 3

Ship CHAIN Cruise 119 Leg II Sta. 72 Core No. 15PC

Lithologic

Log

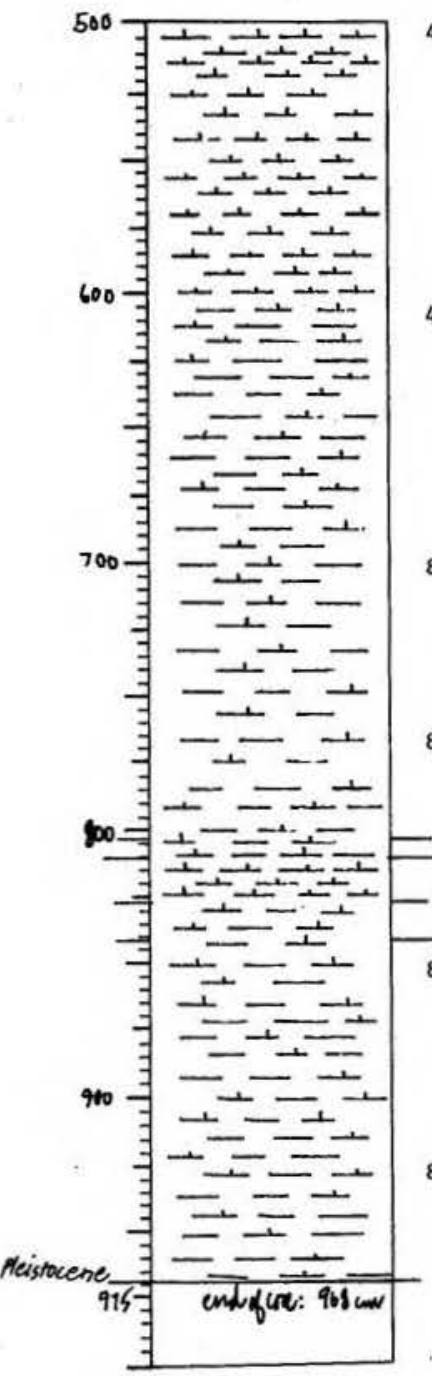

\section{Detailed Description}

$416-480$

CALC OOZE

varying hues of 2.5 Y 5/2 grayish brown, $6 / 2$ light brownish gray, and $6 / 4$ light yellowish brown alternating beds of the colors above with two 11ght yellowlsh-brown zones dominating the unit at 439$452 \mathrm{~cm} ; 455-460 \mathrm{~cm}$, also very fine, light brown mottling scattered throughout
generally smooth, compact lutite throughout with a S

CALC OOZE WITH LAMINATIONS OF DETRITUS, GRADES TO SLIGHTLY CALC CIAY, TO CALC CIAY

5 Y $3 / 1$ very dark gray

umerous and repeated dark olive gray, pteropod-rich beds $480-660 \mathrm{~cm}$

compact, smooth lutite with a number of fine, silty laminations 480-660 cist emented gastropod shell found at $798 \mathrm{~cm}$

3, concave

CALC CLAY

5 Y $5 / 2$ olive gray and $5 / 1$ gray

several broad laminations of the colors above, plus some fine intermottling in the zone $804-807 \mathrm{~cm}$ smooth, compact lutite

810-827 concave upward

\section{ALC OOZE}

5 Y $6 / 2$ light olive gray, grades to $7 / 2$ light gray

few poorly defined, olive gray mottles and one lami-

nation occur at upper unit contact

in, stiff, foram-rich lutite becomes

then totally lithified and brittle

remarkably stiff zone has sheared horizontally at $819 \mathrm{~cm}$

327-84

CALC CLAY

5 I $4 / 2$ olive gray and $6 / 2$ light olive gray

of the colors

forams

$$
\mathrm{s}, \text { frregular }
$$

$81-968$

5 Y $3 / 2$ dark olive gray

scattered and irregular olive-gray mottling, marbling,
538

VISUAL CORE DESCRIPTION

Page 3 of 3

Ship CAAIN Cruise 119 Leg II Sta. 72 Core No. 15N

Lithologic

Log

Detailed Description

841-968 (cont.)

$$
\text { and lamination }
$$

compact, slightly silty lutite with scattered, silty

laminations and zones of pteropod-rich saprope1-

like material 
SMEAR SLIDE DESCRIPTIONS - W.H.O.I. SEDIMENT CORES

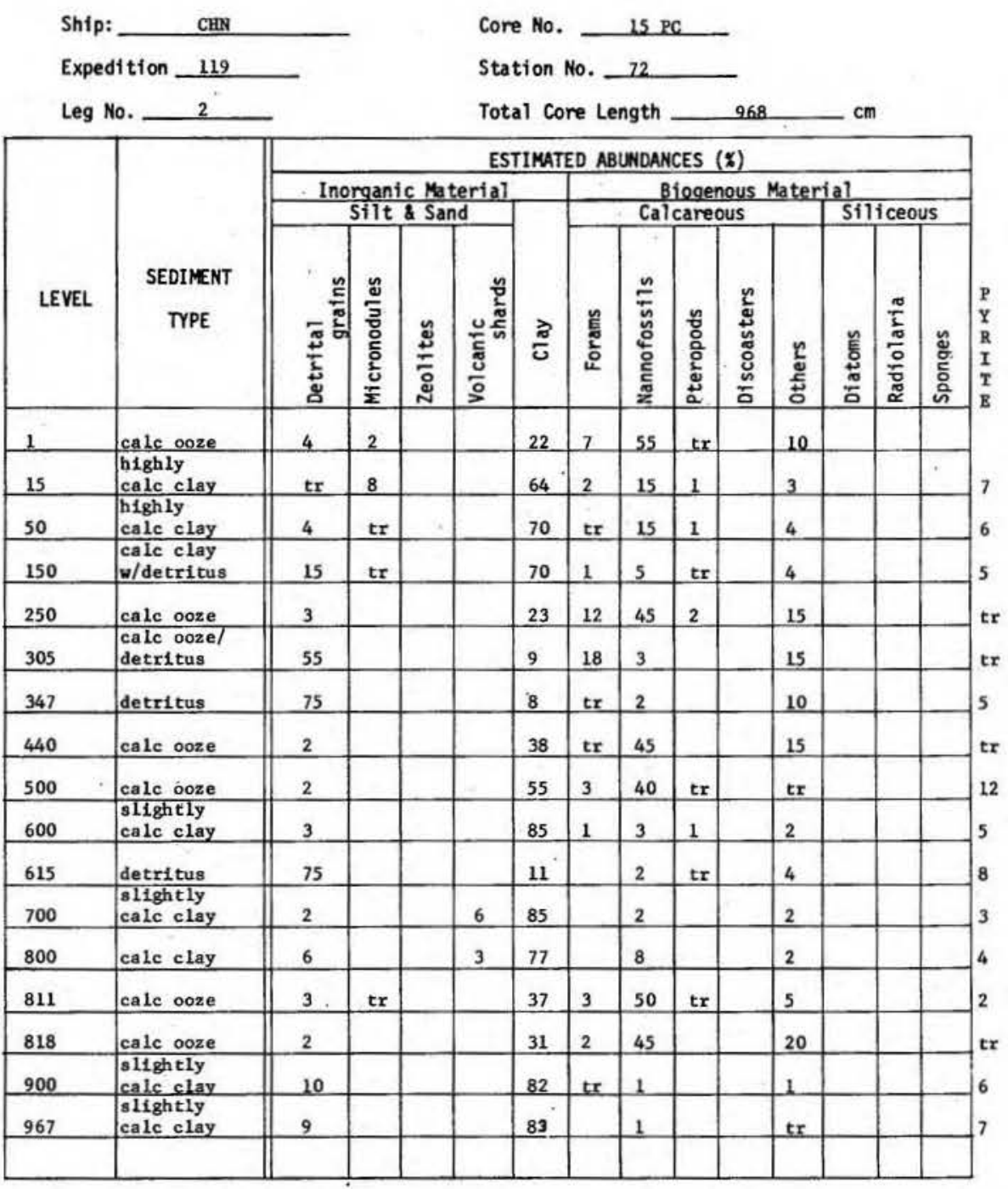

VISUAL CORE DESCRIPTION

Page 1 of 1

Ship CHAIN Cruise 119 Leg II Sta. 72 Core No. ISPG Total Length $138 \mathrm{~cm}$. Lat. $33^{\circ} 19.45^{\circ} \mathrm{N}$ Long. $29^{\circ} 40^{\circ} .39^{\circ} \mathrm{E}$ Depth $2828 \mathrm{~m}$-Corr. Core condition EXCELENT Date Described $1 / 4 / m .76$ by fouda. Physiographic location DSTRL NLLE CANE NIRTH OF ALEXINPRIA , EASTERY, MED. Lithologic Log Detailed Description

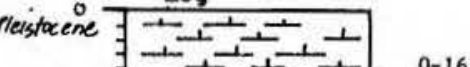

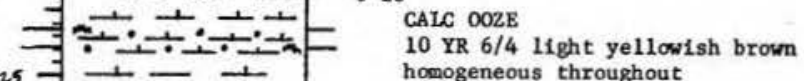

$25 \div-1-1$ homogeneous throughout

moist, unconsolidated lutite with scattered foram S, horizontal

16-1

50
$10 \mathrm{YR} 4 / 4$ dark yellowish brown

nnumerable, fine microlaminae; dark and 11ght yellow1sh brown alternate

斗- - $\begin{gathered}\text { smo } \\ \text { s }\end{gathered}$

25 -1 - - HIGHLY CAIC CLAY

5 Y $3 / 1$ very dark gray and $3 / 2$ dark olive gra dark olive gray sapropel-1ike, pteropod-rich band appear $19-21 \mathrm{~cm}, 30-38 \mathrm{~cm}, 50-52 \mathrm{~cm}, 67-75 \mathrm{~cm}$, very moist, slick lutite throughout, with the above

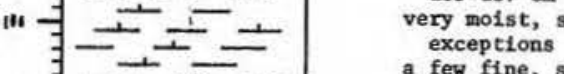
a few fine, silty laminations appear $90-135 \mathrm{~cm}$

reisticene $\exists-1$

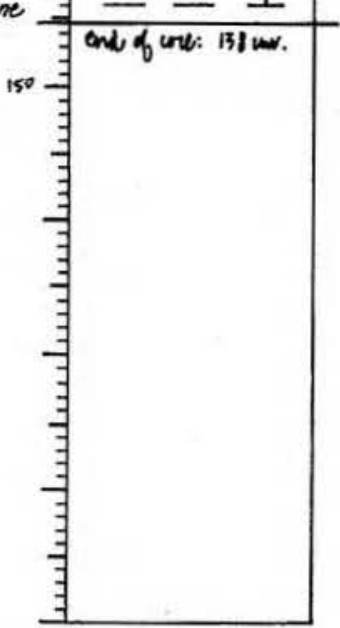


SMEAR SLIDE DESCRIPTIONS - W.H.O.I. SEDIIENT CORES

Ship:

can

Expedition 119

Leg No. _ 2

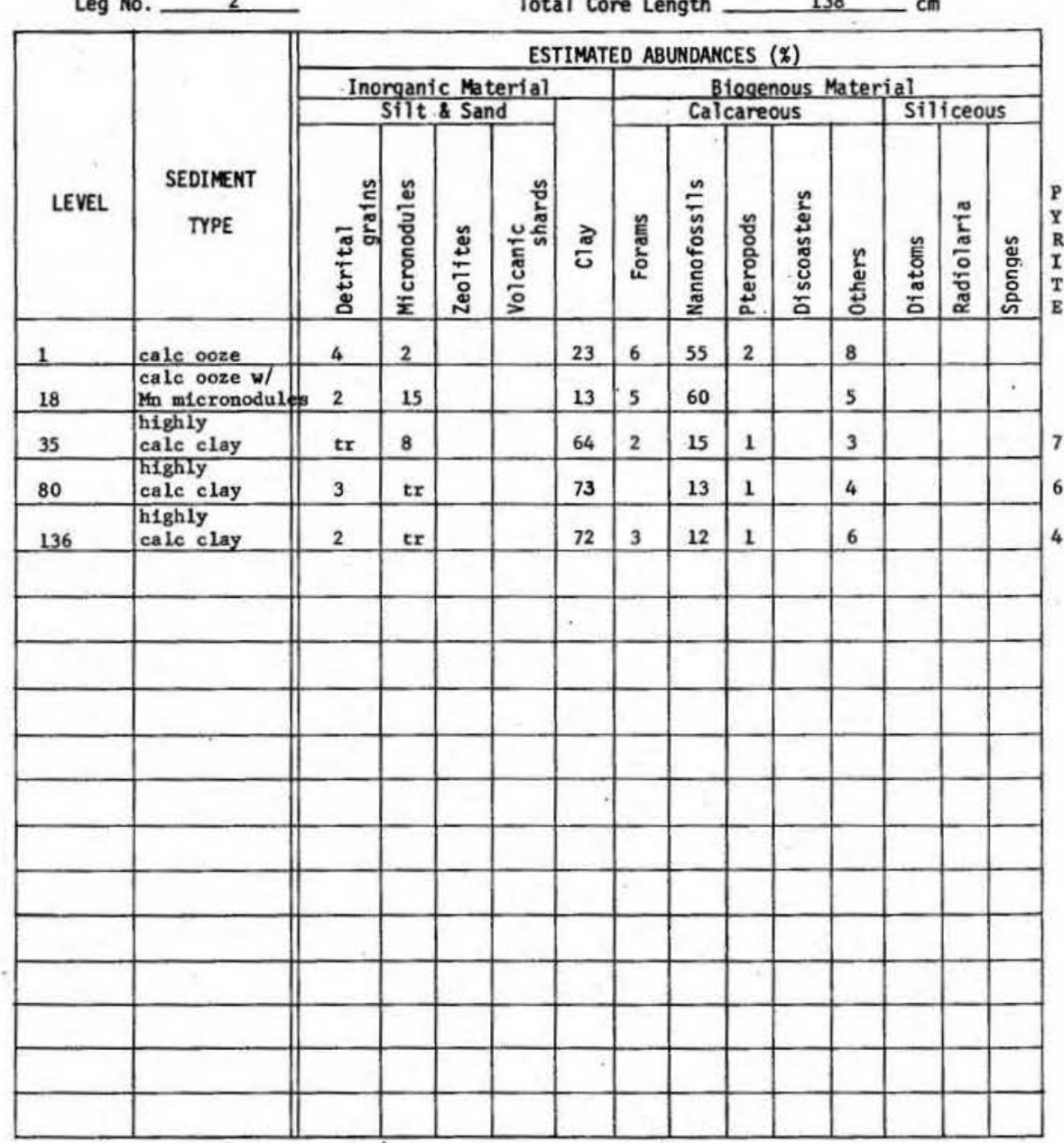

Core No. 15 PG

Station No. 72

Station No. 72

Total Core Length $138-\mathrm{cm}$ 
543

VISUAL CORE DESCRIPTION

Page 2 of 4 Ship CHAIN Cruise 119 Leg II Sta. 74 Core No. 16PC

Lithologic

Log

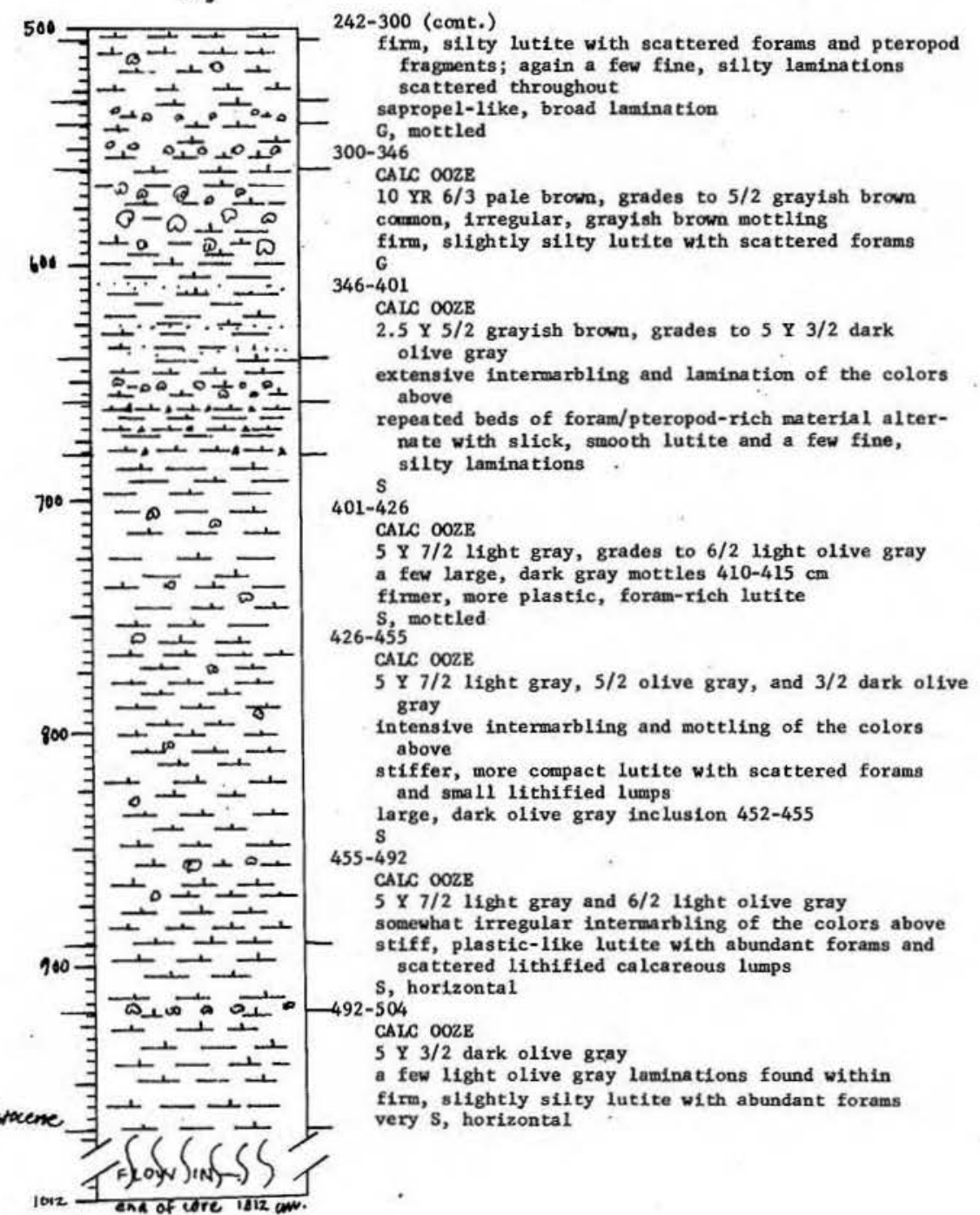

544

VISUAL CORE DESCRIPTION

Page 3 of 4

Ship CHAIN Cruise 119 Leg II Sta. 74 Core No. I6PC

Lithologic

Log

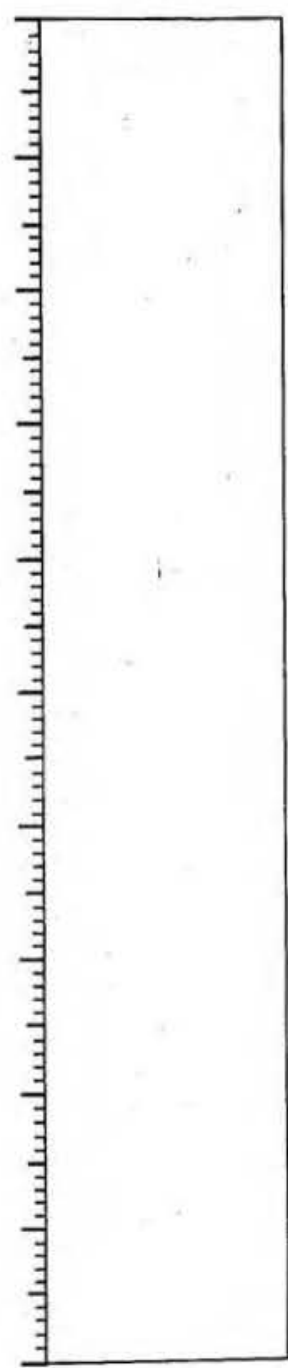

\section{Detailed Description}

$504-528$

CAIC OOZE

5 Y $7 / 2$ light gray, grades to $6 / 2$ light olive gray colors above

stiff lutite with abundant foram

$528-539$

CALC OOZE

5 Y $3 / 2$ dark olive gray

fine, very small light olive gray mottling and fleck lutite with abundant forams and pteropod fragments horizontal
hon

539-559

CALC OOZE

5 Y $7 / 2$ light gray, grades to $6 / 3$ pale olive extensive, very fine, light gray mottling $545-548 \mathrm{~cm}$ and a few laxge, dark gray burrows $S$, horizontal

559-640

CALC CIAY WITH LAMINATIONS OF DETRITUS

5 Y $3 / 1$ very dark gray, grades to $3 / 2$ dark olive gray extensive (sma11) fine, and (large) 1rregular mottling and burrowing 560-594; fine laminations $594-640 \mathrm{~cm}$ firm, compact, smooth lutite with many fine, wellpreserved silt $(.5 \mathrm{~cm})$ lawinas forams abundant in some laminae 595-620 cm very S, horizonta 1

CALC OOZE

5 Y $6 / 2$ light olive gray

intensive, fine mottling and burrowing 653-658 cm stiff, conpact, slightly silty lutite with foram common $640-653 \mathrm{~cm}$

very

CALC OOZE WITH PYRITE, ALTERRATES WITH SLIGATLY CALC CLAY

5 Y $3 / 1$ very dark gray and $3 / 2$ dark olive gray repeated, even, sharp (1 cm wide) laminations of the

$663 \mathrm{~cm}$
tiff, smooth lutite inter-laminated with foram/pteropod-rich, slightly silty lutite

$\frac{v e r y}{S}$ nice preservation of cyclical sedimentary process 
545

VISUAL CORE DESCRIPTION

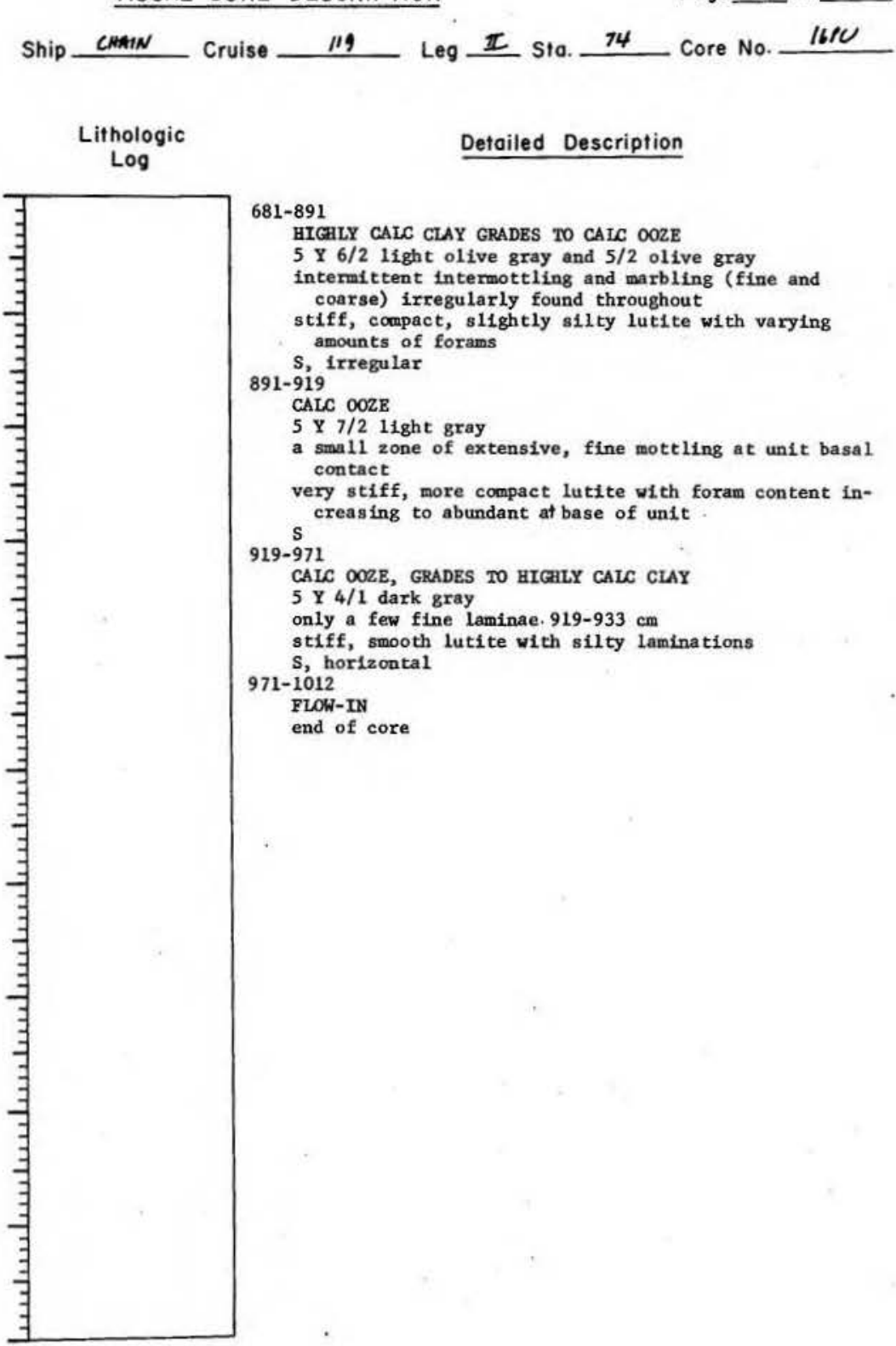

546

SMEAR SLIDE DESCRIPTIONS - W.H.O.I. SEDIMENT CORES

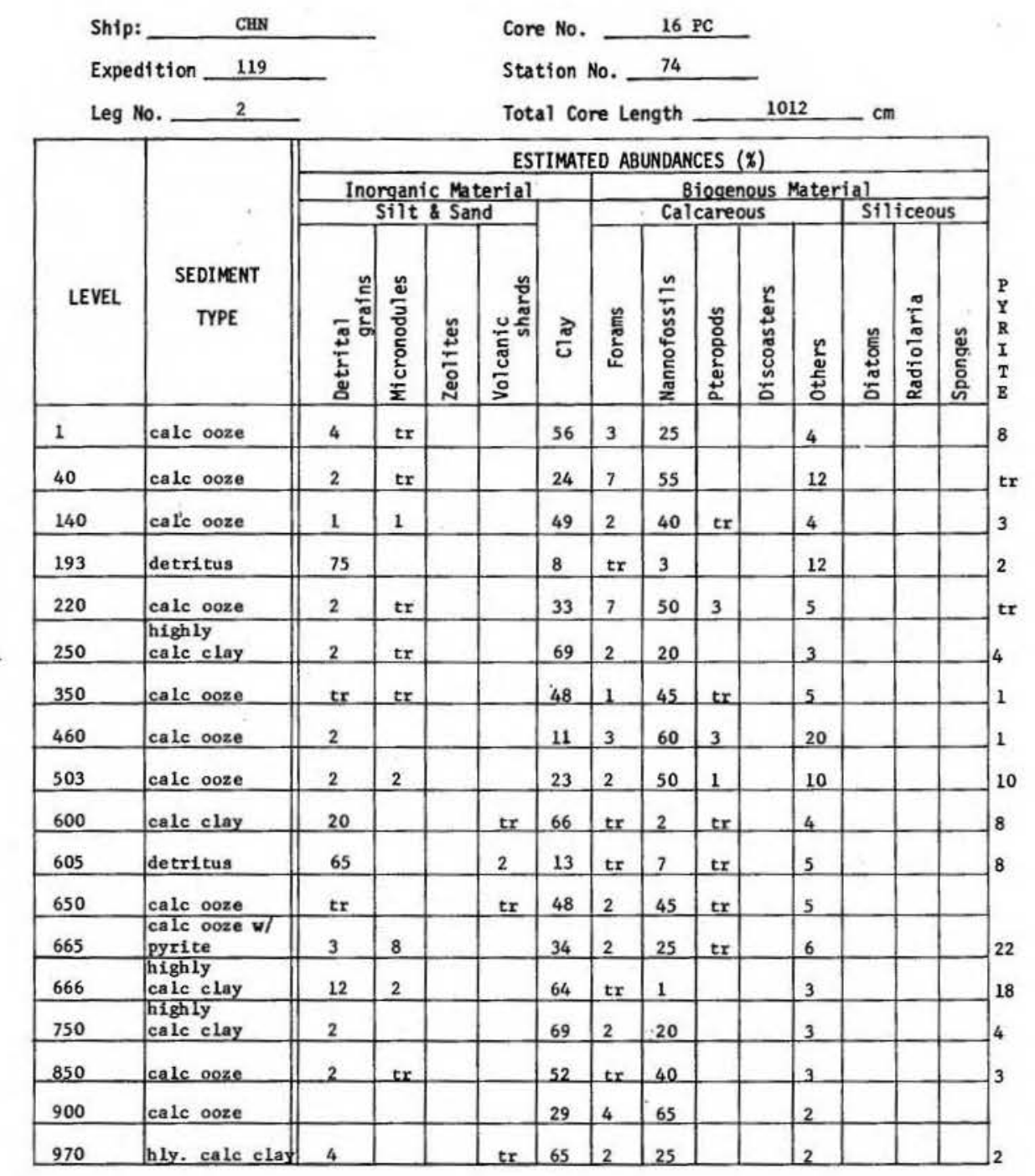




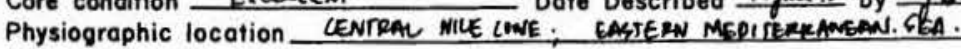

Lithologic

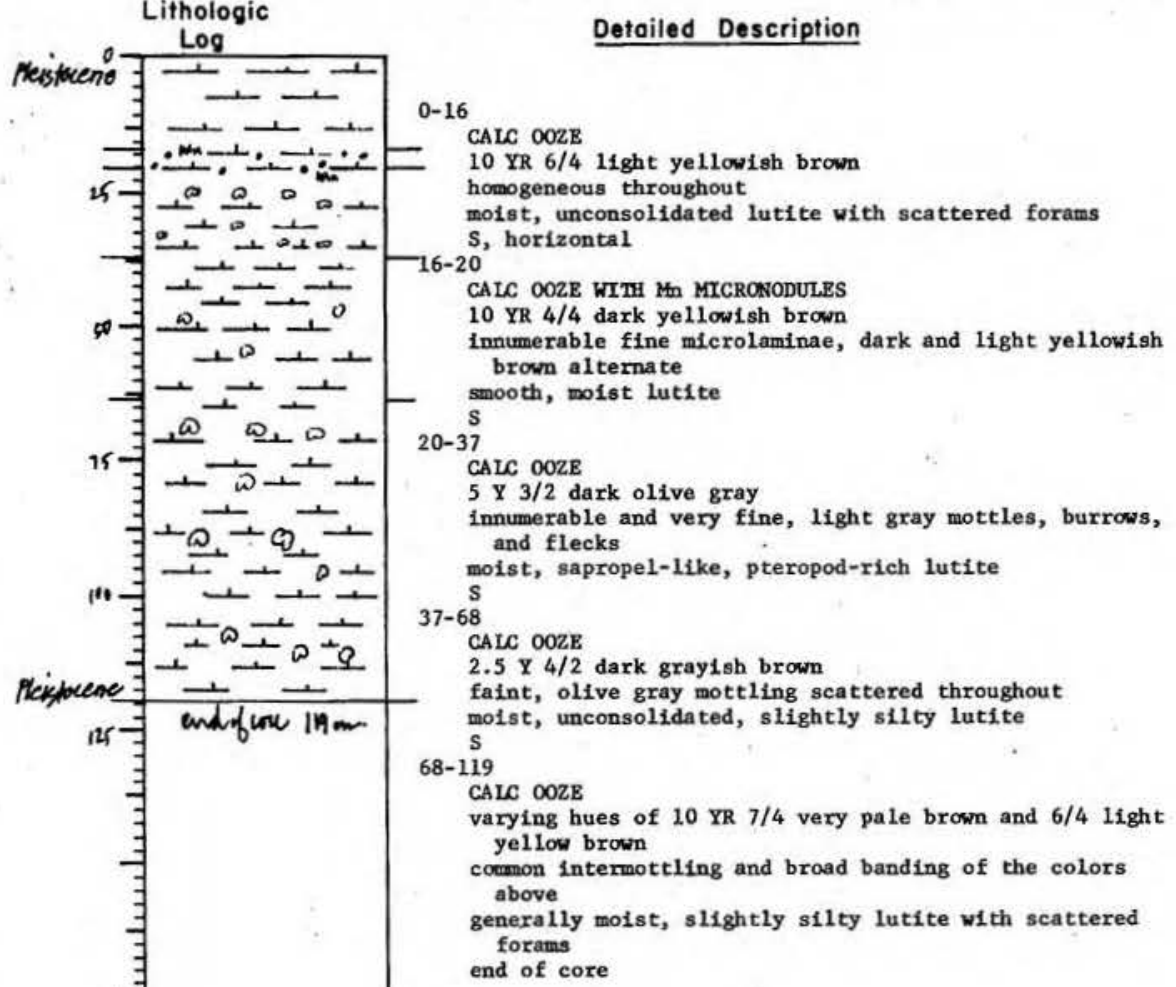


Ship CHN Cruise 119 Leg 2 Sta. 76 Core No. $171 \mathrm{C}$ Total Length $895 \mathrm{~cm}$. Lat. $39^{\circ} 42.752^{\prime} \mathrm{N}$ Long. $30^{\circ} 40.921$ ' $E$ Depth 2595 cars Core condition EKCELENT Dote Described is Jw 26 by I FARMER

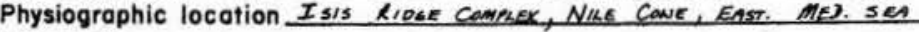
Lithologic

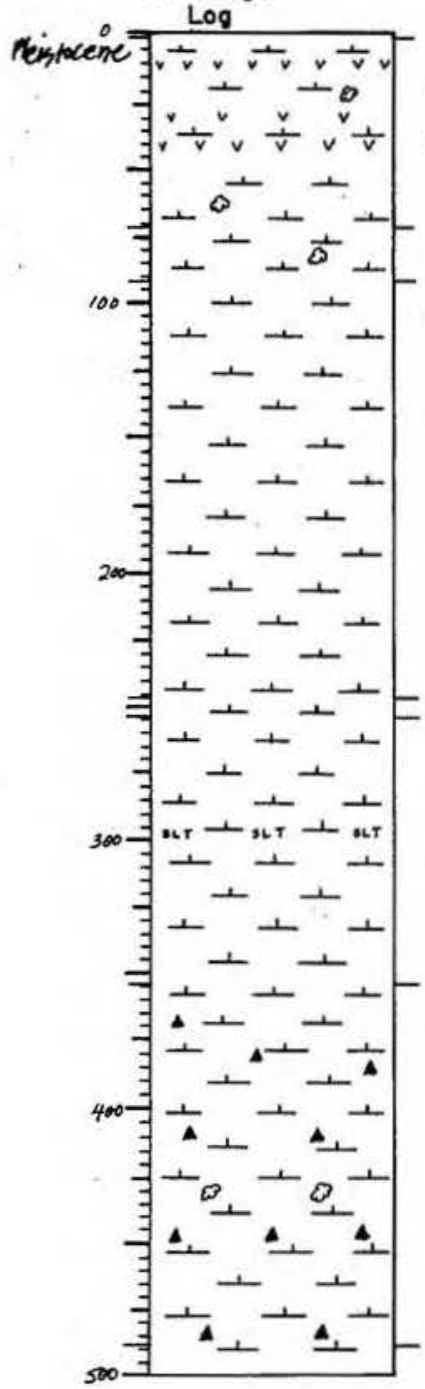

vold

CAIC OOZE

10 YR $6 / 4$ light yellow brown, $6 / 3$ pale brown, and $7 / 3$

very pale brown; gradational bands $(2-5 \mathrm{~cm})$ of above very top

scattered intermottling of above colors

very silty lutite with scattered forams

$10.5-11 \mathrm{~cm}, 42-42.5 \mathrm{~cm}, 28-29 \mathrm{~cm}$ volcanic ash; severa 1 large calcareous fragments at $31 \mathrm{~cm}$

$\mathrm{s}, \mathrm{d}$

thole core below this point has a very disturbed appearance, some of uhich is clearly due to coring, uhile the rema inder is possibly caused by severe bioturbation CALC OOZE

10 YR $5 / 3$ brow

scattered mottles of pale brow

silty lutite with scattered to common forams

G 246

2-246 0928

$10 \mathrm{YR} 7 / 1$ to $5 / 1$, 11ght gray to gray, and $6 / 2$ light

brownish gray

intermarbling and distorted laminations of above color are common; 2 beds of 5 Y 3/2 dark olive gray appear at $145-150 \mathrm{~cm}$ and $204-208 \mathrm{~cm}$

generally silty to very silty lutite with forams common S $46-254$

CALC OOZB

1 light gray and $5 / 1$ gray

no mottling

S

CAIC OOZE 2.5 Y $5 / 2$ grayish brown grades to $10 \mathrm{YR} 7 / 1$ and then to $6 / 1$ (light grays) with periodic sharply contacted beds of $6 / 2$ light brownish gray both brownish grays and several larger burrow-1ike

mottles are also present

silby to very silty lutite with common to abundant bio-

genic grains maxbe colors is common

Ship CHN Cruise 119 Leg 2 Sta. 76 Core No. $17 P_{C}$

Lithologic

Log

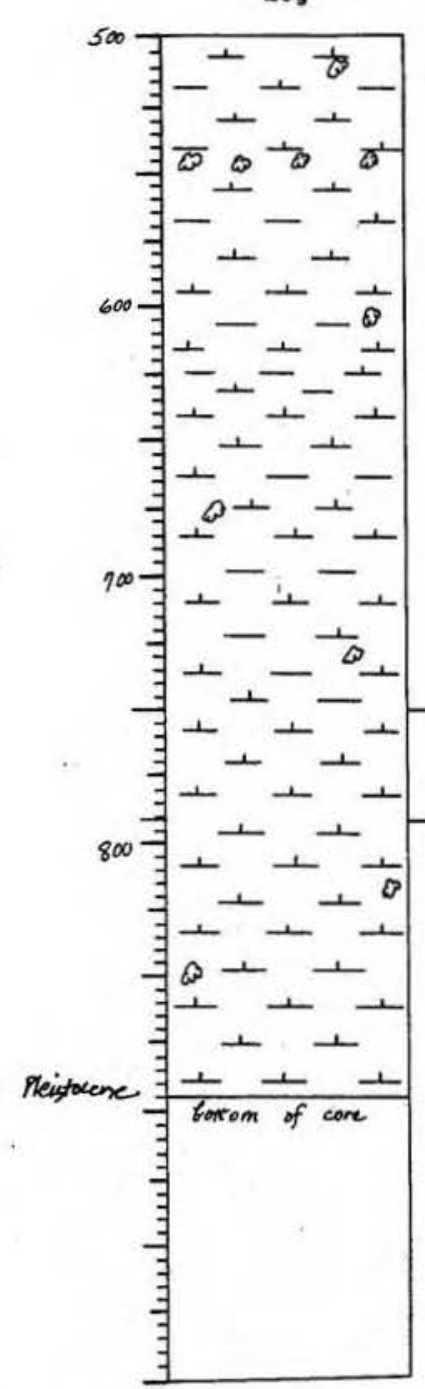

\section{Detailed Description}

$254-351$ (cont.)

silt beds, dark gray (5 Y 4/1) and dark olive gray, appear at $294-295$ and $297-299 \mathrm{~cm}$

S54-488

5 Y $7 / 1$ 1ight gray, $6 / 2$ 1ight olive gray, $3 / 2$ dark cycles (354-38bove colors appear in 4 consecutive degree of mottling varies considerably between sapropels; 444-464 cm sapropel is interbedded with slick lutite, 5 Y $4 / 1$ dark gray

marbling and disturbance are present, as are severa large burrow-1ike structures; motting is present over only small sections

he sapropels have a humus-1ike organic lutite texture, lutites; forams vary from ocattered to abundant in

488-750

CALC OOZE INTERBEDDED WITH CALC CLAY

5 Y 7/1, 6/1 light grays grade gently to various hues of light ollve gray (6/2) below $580 \mathrm{~cm}$; numerous bands ( $\sim 1-4 \mathrm{~cm}$ ) of $5 / 1$ gray (calc clay) Interrupt the above collors, lin betulng with depth

throught, intengely mottled from 540-552 common laminae are clearly sheared and not burrowed predominately a silty lutite with scattered to common forams, the darker beds being somewhat less silty; 621-634 cm slightly silty lutite ( 5 Y $3 / 1$, very dark gray - slightly calc clay - interbedded with dark $G$

olive gray (3/2) sapropelic lutite

CAIC OOZE GRADES TO NAMNO OOZE

5 Y $7 / 2$ light gray grades to $8 / 1$ white and back to $7 / 2$ minor mott ling

stiff, very silty lutite with scattered forams

very gradationa 1

CALC OOZE

$5 \times 7 / 2$ light gray and $6 / 2$ light olive gray appear in microlaminations

silty to very silty lutite, forams vary from abundant to scattered

end of core 
SHEAR SLIDE DESCRIPTIONS - W.H.0.1. SEDINENT CORES

\section{Ship: _ CHAIN}

Expedition 119

Leg No.

\begin{tabular}{|c|c|c|c|c|c|c|c|c|c|c|c|c|c|c|}
\hline \multirow[b]{3}{*}{ LEVEL } & \multirow[b]{3}{*}{$\begin{array}{c}\text { SEDIMENT } \\
\text { TYPE }\end{array}$} & \multicolumn{13}{|c|}{ ESTIMATED ABUNDANCES ( $(\boldsymbol{)})$} \\
\hline & & \multicolumn{4}{|c|}{$\begin{array}{l}\text { Inorganic Material } \\
\text { Silt \& Sand }\end{array}$} & & \multicolumn{5}{|c|}{$\begin{array}{l}\text { Biogenous } \\
\text { Calcareous } \\
\end{array}$} & \multicolumn{3}{|c|}{ Sifliceous } \\
\hline & & $\frac{\text { हn }}{\frac{5}{6}}$ & 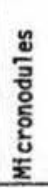 & 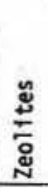 & 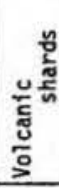 & ढె & 战 & 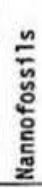 & 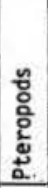 & 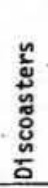 & 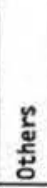 & 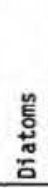 & $\frac{\pi}{\frac{\pi}{2}}$ & 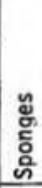 \\
\hline 2 & calc ooze & 1 & 1 & & & 26 & 2 & 60 & $t x$ & & 8 & & & \\
\hline 63 & calc ooze & 1 & tr & & & 26 & 2 & 53 & 1 & & 17 & & & . \\
\hline 152 & calc ooze & $\mathrm{tr}$ & & & & 58 & 1 & 30 & $\mathrm{tr}$ & & 6 & & & \\
\hline 254 & calc ooze & & & & & 15 & tr & 15 & & & 63 & & & \\
\hline 270 & calc ooze & tr & tr & & & 39 & 7 & 40 & 1 & & 13 & & & \\
\hline 312 & calc ooze & 1 & & & & 25 & 1 & 50 & 1 & & 21 & & & \\
\hline 410 & calc ooze & & & & & 55 & 6 & 28 & $\mathrm{tr}$ & & 2 & & & \\
\hline 425 & calc ooze & 1 & 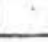 & & & 55 & 6 & 34 & $t r$ & & 4 & & & \\
\hline 484 & calc ooze & tr & & & & 59 & 4 & 25 & $t x$ & & 2 & & & \\
\hline 591 & calc ooze & 1 & tr & & & 50 & 3 & 43 & tr & & 3 & & & \\
\hline 626 & $\begin{array}{l}\text { slightly } \\
\text { calc clay }\end{array}$ & 14 & & & & 77 & $t_{x}$ & 1 & & & 4 & & & \\
\hline 700 & calc clay & 12 & & & & 77 & & 10 & & & 1 & & & \\
\hline 780 & nanno ooze & tr & & & & 5 & 3 & 90 & tr & & 2 & & & \\
\hline 835 & calc ooze & 3 & & & & 32 & 1 & 38 & tr & & 25 & & & \\
\hline 894 & calc ooze & 3 & tr & & & 31 & 1 & 50 & & & 15 & & & \\
\hline & & & & & & & & & & & & & & \\
\hline & . & & & & & & & & & & & & & \\
\hline & & & & & & & & & & & & & & \\
\hline
\end{tabular}

Ship CWAN Cruise 119 Leg 2 Sta. 76 Core No. $17 \mathrm{P}_{6}$ Total Length $106 \mathrm{~cm}$. Lat. $33^{\circ} 42.752^{\prime} \mathrm{N}$ Long. $30^{\circ} 40.22{ }^{\prime} \mathrm{E}$ Depth $255^{2} \mathrm{comm}$ Core condition $E_{\text {xeeuent }}$ Date Described 15 Jan 76 by T. Farmer Physiogrophic location ISIS RIDGE COMPLES, NILE CONE, EnST. MED. SEA Lithologic

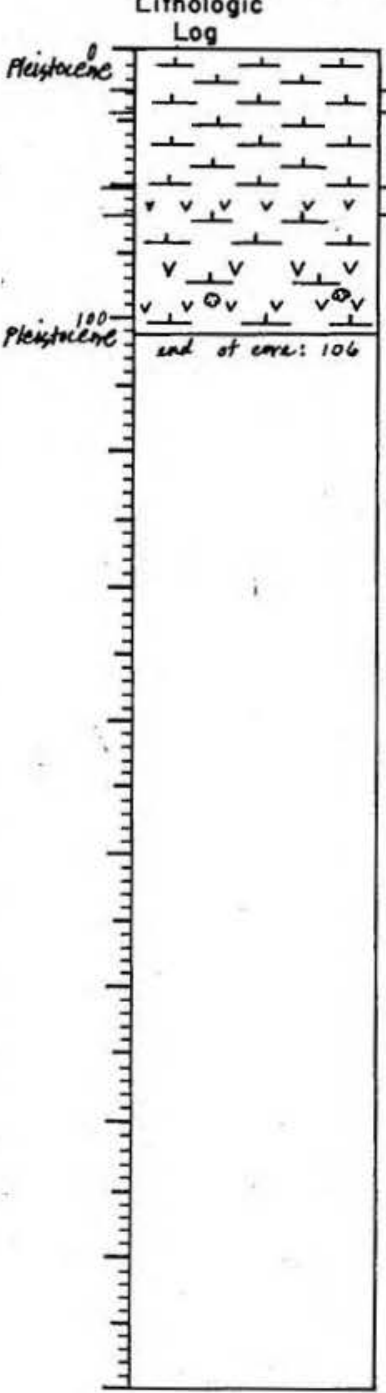

\section{Detailed Description}

$0-14$

CALC OOZE

10 YR $6 / 4$ light yellowish brown

no mottling
very silty lutite with scattered forams $14-22$

CALC OOZE

moist, silty lutite with scattered forams saprope1-1ike material

22-52

CALC OOZE

10 YR $5 / 2$ grayish brown, pales somewhat with depth no mottling

silty lutite, with scattered forams

52-62, large burrow has disturbed contact

CALC OOZE

no mottling extremely silty, stiff lutite, forams fairly comon

$58 \mathrm{~cm}-5$ min dark brown volcanic ash

CAIC OOZE

10 YR $5 / 2$ grayish brown

pale, sma 11 mottling (90-94) scattered below light gray lamination

silty lutite with scattered forams and pteropod

$82.5-84 \mathrm{~cm}, 10$ YR $6 / 2$ light brownish gray volcanic ash; $96 \mathrm{~cm}, 5 \mathrm{~mm}$ volcanic ash $10 \mathrm{YR} 4 / 2$ dark grayend of core
5 Y. $3 / 2$ dark olive gray

10 YR $7 / 2$ 1ight gray 
SHEAR SLIDE DESCRIPTIONS - W.H.O.I. SEDIMENT CORES

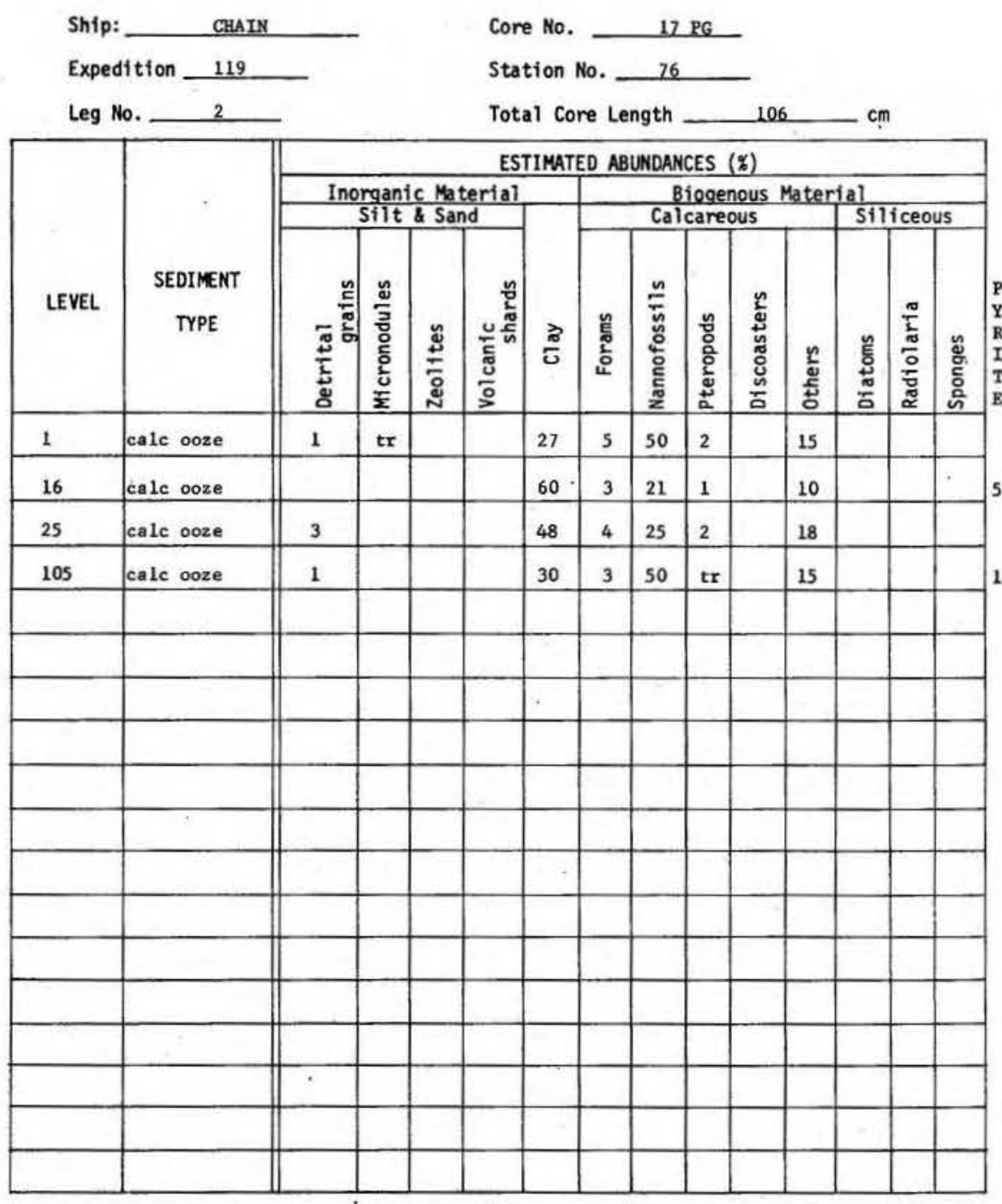

Ship CHAIN Cruise 119 Leg 2 Sto. 78 Core No. 18 PC Total Length $858 \mathrm{~cm}$. Lat. $34^{\circ} 20.84^{\prime} \mathrm{N}$ Long. $30^{\circ} 55.84^{\prime} \mathrm{E}$ Depth $2484 \mathrm{crrm}$ Core condition EXCELLENT Date Described 9 Dec 75 by I Foremex

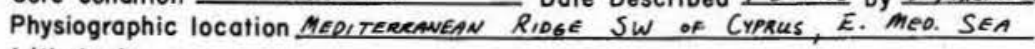
Lithologic

CALC 00zE
10 YR $6 / 4$ light yellow brown grades to $4 / 4$ dark yellow
brown and back to $7 / 4$ very pale brown
no mottling
very silty lutite with scattered forams
s


Ship CHAIN Cruise 119 Leg 2 Sta. 78 Core No. 18 PC

Lithologic

Log

Detailed Description

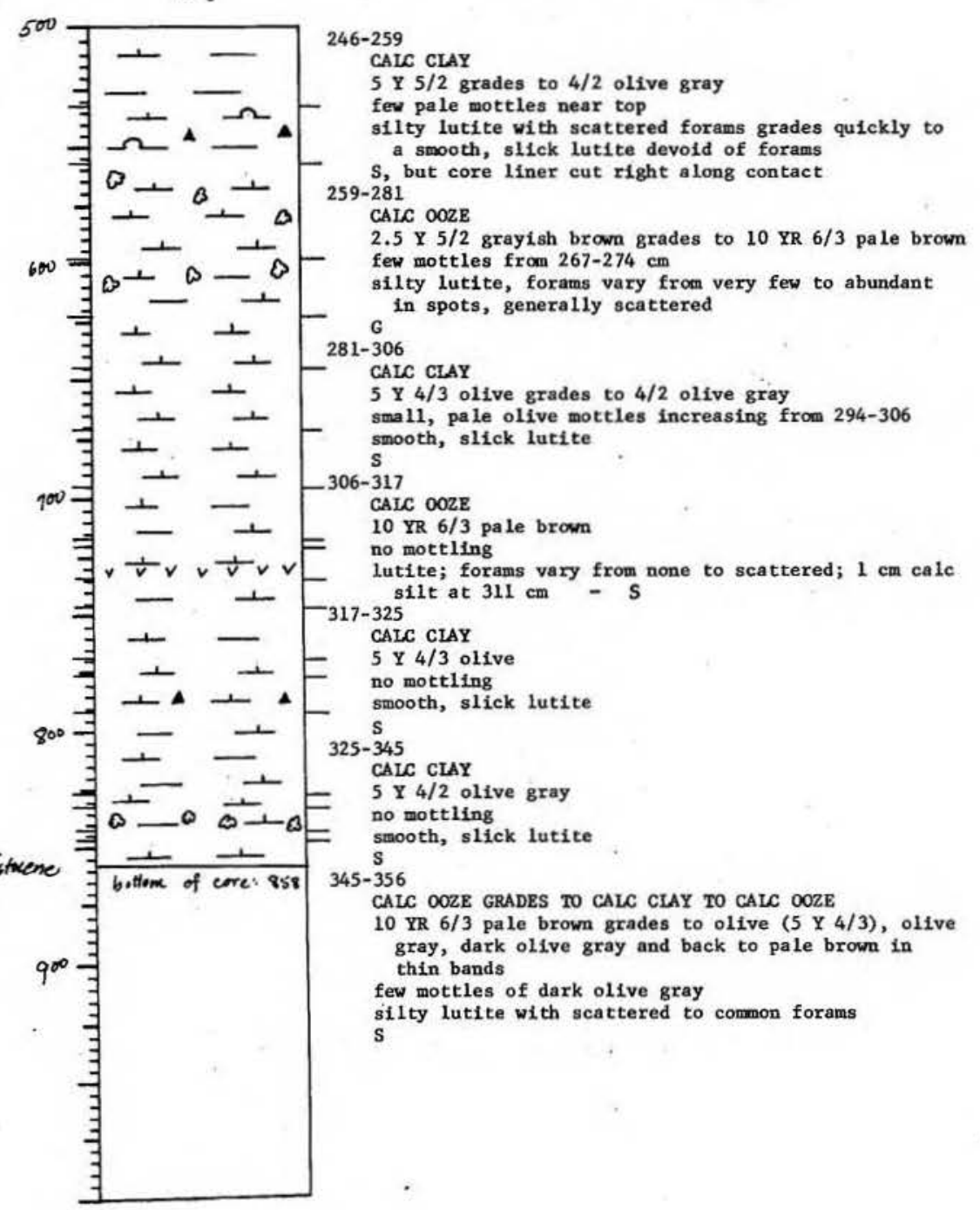

Lithologic Log

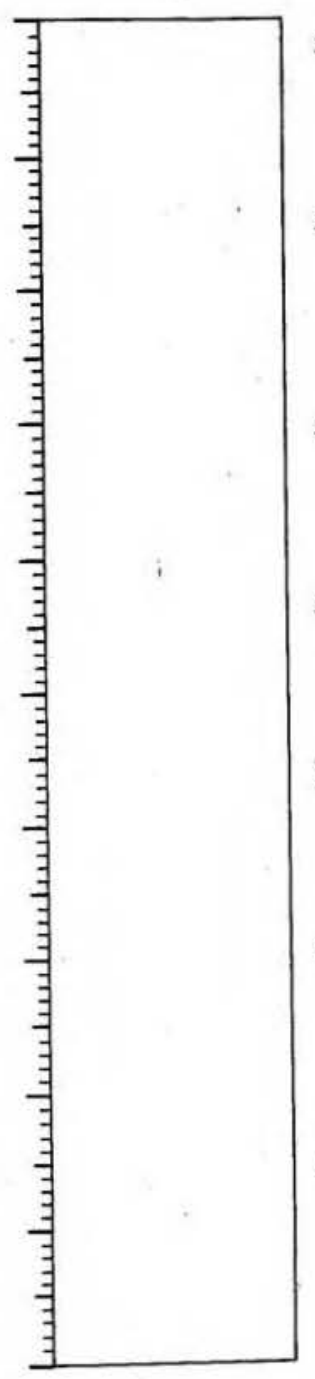

Page 3 of 5 Leg 2 Sta. 78 Core No. $10 \mathrm{PC}$

\section{Detailed Description}

356-361

$$
\text { CAIC CIAY }
$$

5 y $3 / 2$ dark olive gray

no mottling

lutite with fine organic humus texture sapropelic material

$\underset{361-533}{\mathrm{~S}}$

CALC CIAY AND CALC OOZE (pale browns)

repeated cycles of 10 YR $6 / 3$ pale brown, 5 Y $5 / 2$ and $4 / 2$ olive grays, with sharp contacts occasional mottling of pale brown into lower bands smooth, slick lutite except forams generally scattered

s

\section{HIGHLY SILIC-CALC CLAY}

Y $3 / 2$ dark olive gray

fine, organic humus texture

S

7-598

CALC OOZE
5 Y $6 / 1$ gray grades into 10 YR $7 / 3$ very pale brown

5 Y $6 / 1$ gray grades into 10 YR $7 / 3$ very pale brown 591 medightly silty lutite with scattered forams and few pteropods $\mathrm{S}^{\mathrm{p}}$

ILY CALC CLAY GRADES TO CALC CLAY

10 YR $4 / 2$ grayish brown grades to $5 / 2$ dark grayish

heavily mottled from above

smooth, slick lutite

623-645

CALC OOZE

$10 \mathrm{YR} 7 / 3$ very pale brown

no mottling

lutite with scattered forams

$632-633 \mathrm{~cm}$ sandy unit of volcanic ash, forams and $G$ detritus; 640-643 sandy unit of volcanic ash

645-671

CAIC OOZE $10 \mathrm{YR} 6 / 2$ light brownish gray grades to $7 / 3$ very pale 

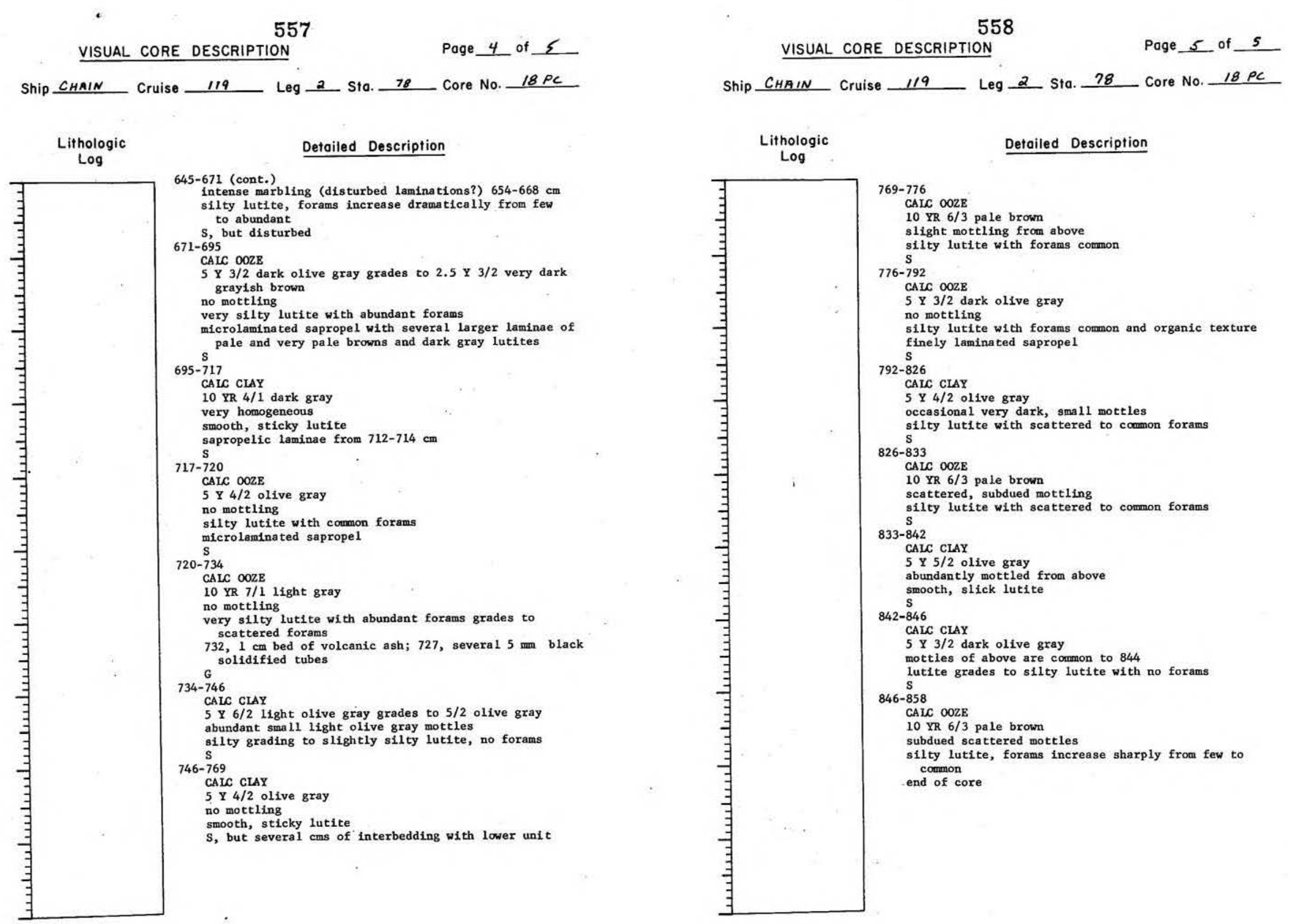
SMEAR SLIDE DESCRIPTIONS - W.H.O.I. SEDIMENT CORES

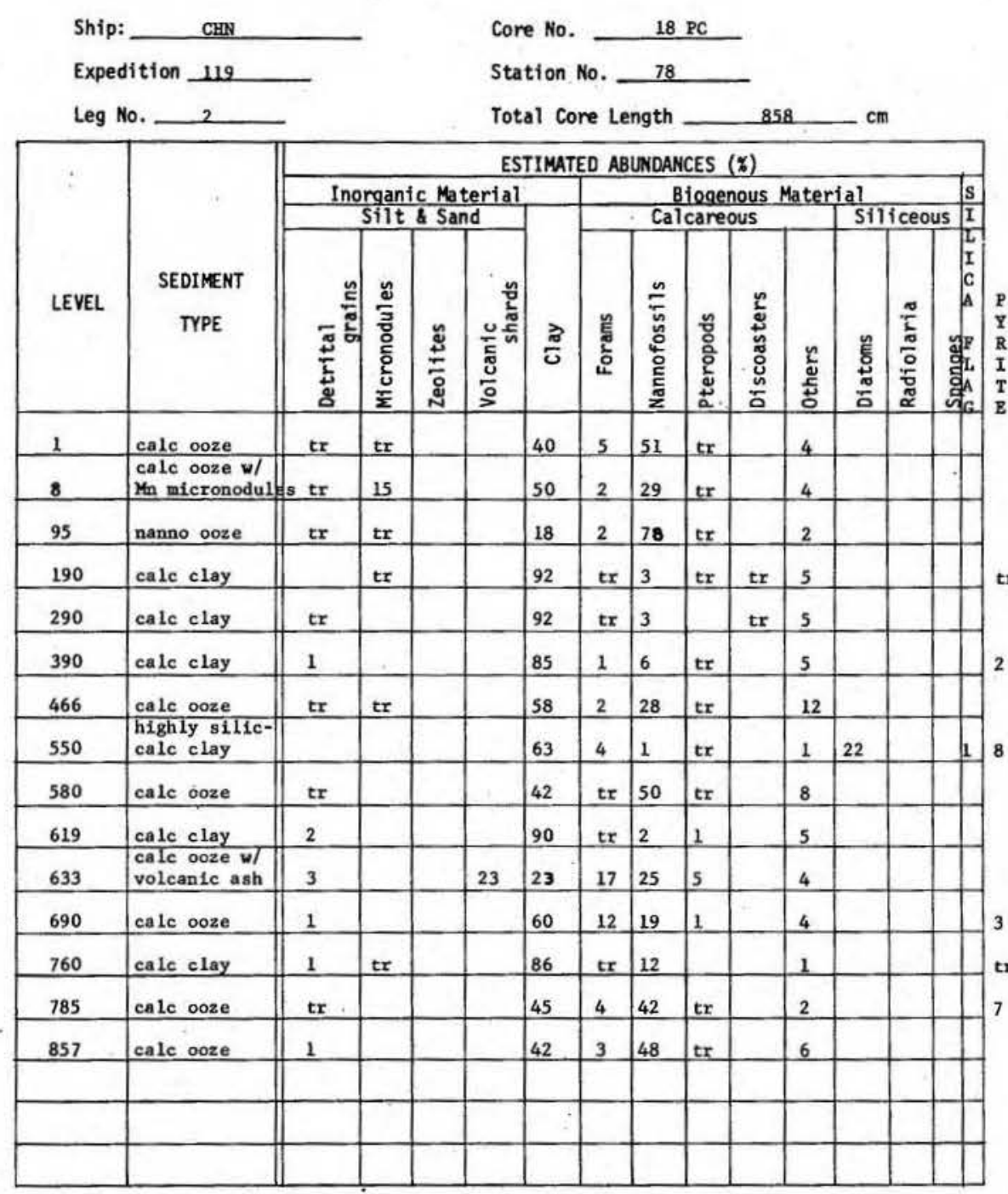

Ship CHAIN Cruise 119 Leg 2 Sta. 78 Core No. 18 PG Total Length $90 \mathrm{~cm}$. Lat. $34^{\circ} 20.84^{\circ} \mathrm{N}$ Long. $30^{\circ} \mathrm{55.2} 4^{\prime} \mathrm{E}$ Depth $2484 \mathrm{com}$. Core condition Excesuent Date Described 9 are '75 by $T$ Farmer Physiographic location MERITERRANEAN RIPSE SW of CYrous, E. MED. SEA Lithologic

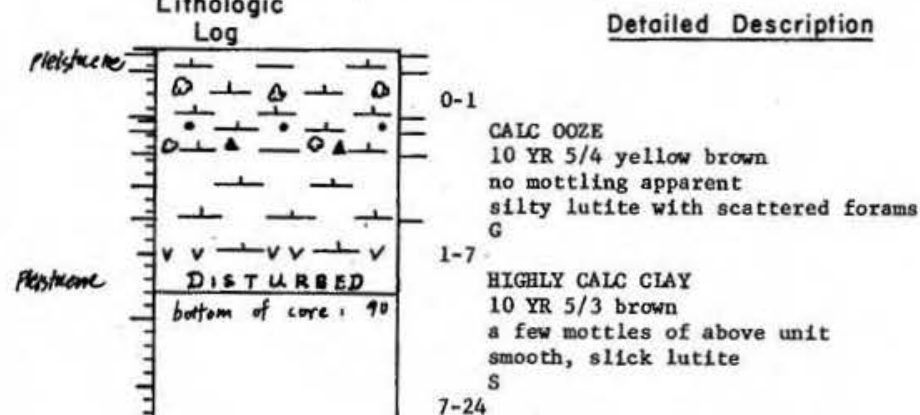

Pestrome

HIGHLY CALC CIAY

0 YR $5 / 3$ brown

few mottles of above un1t smooth, slick lutite

$7-24$

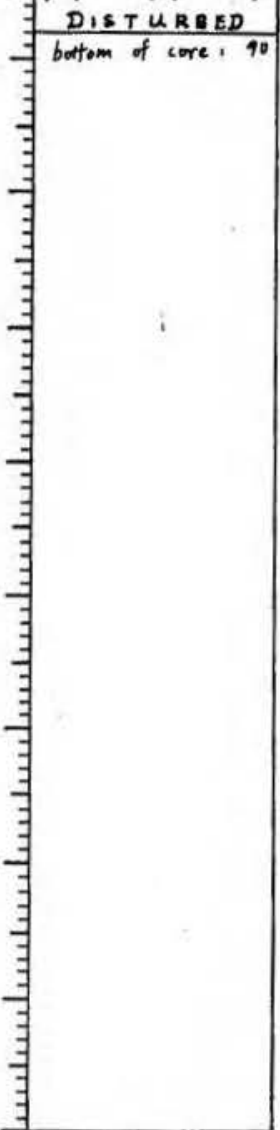

10 YR $6 / 4$ light yellow brown, grades to $5 / 4$ and back to $6 / 4$

8-10 cm intense mottling from above unit

silty lutite with scattered forams very gradationa

24-28.5.

CALC OOZE WITH MN MECRONODULES

grades from 10 YR $6 / 4$ light yellow brown to 5 YR $3 / 3$ dark reddish brown

silty lutite with scattered foram

$\mathrm{S}$
$28.5-38.5$

HICHLY CALC CLAY WITH PYRITE

5 × $3 / 2$ dark olive gray

abundant very pale brown mottles

lightly silty lutite with scattered foram

38.5-63

CALC OOZE

2.5 Y $5 / 2$ graylsh brown grades to 10 YR $6 / 3$ pale brown few small mottles throughout

silty lutite, forams grade from scattered to few; $1 \mathrm{~cm}$ $G$

CALC OOZE

10 YR $6 / 4$ lifoht yellow brom

a few mottles throughout

ate forams grade from coumon to scattered 4-77.5 cm dark grayish brown (10 YR 4/2) volcanic ash bed; 83-87 1rregular void with an inclusion of volcanic ash (10 YR $6 / 2$ light brownish gray) straddling end of core thin bed of pteropod shell hash $(10 \mathrm{~cm})$ 
SMEAR SLIDE DESCRIPTIONS - W.H.O.I. SEDINENT CORES

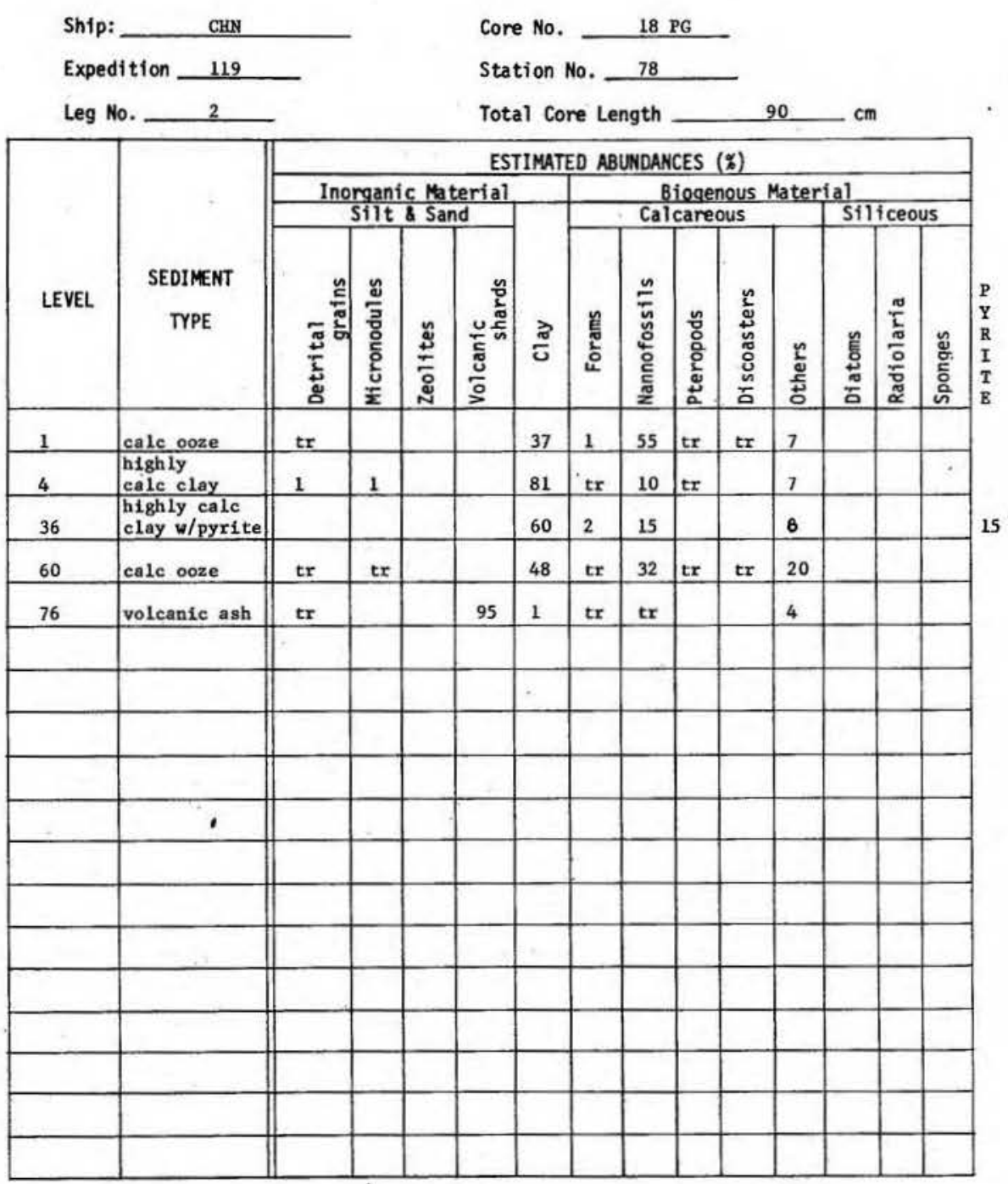

Ship CHN Cruise 119 Leg 2 Sta. 80 Core Nc. 19 PC Total Length $833 \mathrm{~cm}$. Lat. $33^{\circ} 48.8^{\prime} \mathrm{N}$ Long. $31^{\circ} 14.5^{\prime} \mathrm{E}$ Depth 2501 carr.m Core condition excellent Date Described 12 Jax 76 by I FAKmER. Physiographic location ISIS RIDGE COMPLEX, NILE CONE, EASTERN MEP. SEA Lithologic

$$
\text { Log Detailed Description }
$$

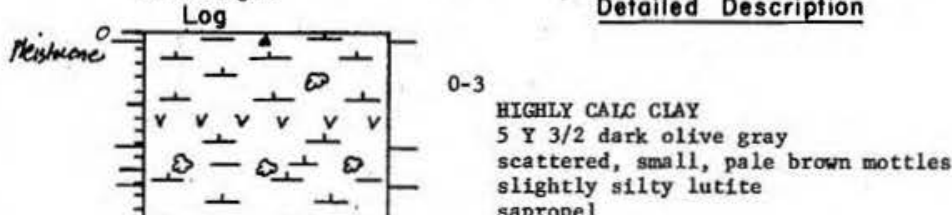

stiff lutite with scattered forams "natural?" curvilinear laminations $(2 \mathrm{~mm})$ s
natura
cis

CAIC CLAY

5 Y $3 / 2$ dark olive gray

slightly silty lutite with a few forams

very fine microlaminations

S

CALC OOZE

S Y $4 / 2$ olive gray, grades to 10 YR $6 / 3$ pale brown

intermotting between above colors

silty to very silty lutite with forams comon
s, mottled contact 
Ship CHN Cruise Wh Core No. 19 Pe

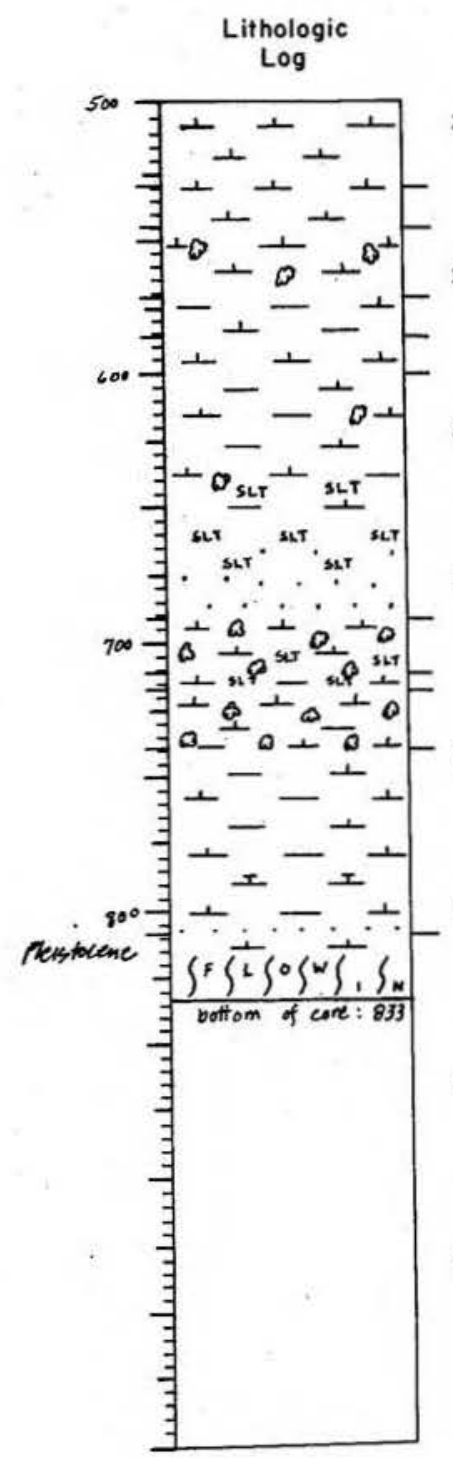

Detailed Description

211-218

CALC OOZE

10 YR $5 / 3$ brown

no mottling

mooth, slick lutite

218-232

CALC OOZB

5 × $5 / 3$ olive

silty lutite with scattered forams

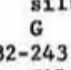

CALC OOZE

$5 \times 5 / 3$ olive

no mottling

$\mathrm{S}$
3. 328

CALC CLAY

5 Y $3 / 2$ dark olive gray

作 smocth, slick lutite, except $320-328$
microlaminated, very silty lutite 28-334

CALC OOZE

5 Y $5 / 2$ olive gray

silty lutite with scattered forams

S

\section{CALC CLAY WITH PYRITE}

5 Y $3 / 2$ dark olive gray

no mottling

silghtly silty lutite

340-379

CALC OOZE

5 Y $4 / 2$ olive gray

no mottling

smooth, sticky lutite, grades smoothly into laminas

CALC OOZE

5 Y $5 / 2$ olive gray grades to $6 / 2$ light olive gray

silty lutite with scattered forams, grades to smooth

s
Ship CHAIN Cruise 1199 Leg 2 Sta. 80 Core No. 19 PC

Lithologic

Log

Detailed Description

407-416

CALC OOZE

5 Y $4 / 2$ olive gray

no mottling

$S$, but distorted

416-427

CALC OOZE

silty lutite, forams increase with depth

S

427-461

5 × $2.5 / 2$ black

lutite with organic (humus) texture; interbedded with

dark gray silty lutite $457-461 \mathrm{~cm}$

microlaminated saprope1

$461-529$

CALC OOZE

5 Y $6 / 1$ gray, grades to varying hues of $10 \mathrm{XR} 7 / 3$ pale

minor intermottling between above colors

ilty to very silty lutite with forams, grading from few to abundant over two cycles

529-545

CALC OOZE

2.5 Y $3 / 2$ very dark grayish brown

very silty lutite

finely laminated sapropel interbedded with gray

545-572

CALC OOZE

10 YR $7 / 1$ light gray, grades to 5 Y $5 / 2$ and $4 / 2$ dark olive gray

intermotting of above colors coumon

very silty lutite; lutite becomes less silty as forams grade from abundant to very few

572-586

CALC CLAY

5 Y $3 / 2$ dark olive gray
no mottling 
565

VISUAL CORE DESCRIPTION

Page 4 of 5

Ship_CHaIN Cruise 119 Leg 2 Sta. 80 Core No. 19 PC

Lithologic Log

Detailed Description

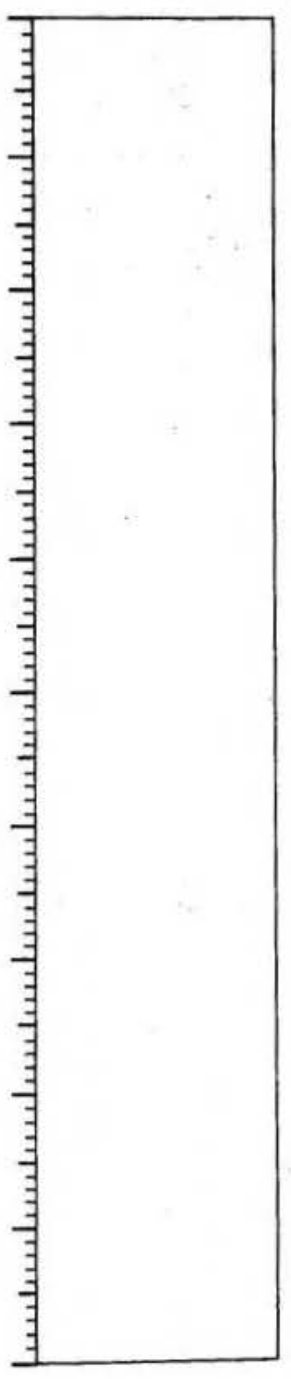

572-586 (cont.)

ooth, slick lutite, except 576-579 foram-rich saproS

586-600

$$
\begin{aligned}
& \text { CALC } 0 \text { YZE } \\
& \text { n } 3 / 2 \text { dark olive gray } \\
& \text { organic-ring } \\
& \text { microlaminated sapropel }
\end{aligned}
$$

600-691 Y $4 / 2$ olive gray

cattered medium-sized, dark olive gray mottles (600-

smooth, sticky lutite, grades smoothily to very silty s

691-711

CALC OOZE GRADES TO CALC OOZE/DETRTTUS

5 Y $4 / 2$ olive gray, grades to $6 / 2$ light ollve gray, and

very intense, pale mottling becomes siltier at base of unit

silty lutite
top $\mathrm{cm}$ straddle end of core sections and are disturbed

711-717

IIGHLY CALC CLAY/DETRITUS

5 Y $3 / 2$ dark olive gray

scattered, pale mottles
silty lutite, grades to fine sand

s, erosional

717-739

CALC OOZE GRADES TO HIGHIY CALC CLAY

macrolaminated unit of $10 \mathrm{YR} 7 / 3$ very pale brown, 5 Y

$6 / 2,5 / 2,4 / 2$ olive grays

intense, pale mottiing

silty lutite, forams abundant in two discreet bands

739-808

TETRTIUS

5 Y $3 / 1$ very dark gray, grades to $4 / 1$ dark gray

smooth, slightly silty lutite interbedded with $.5-2 \mathrm{~cm}$

siltier with several fine sand laminae in lowest 15

$\mathrm{cm}$; occasional foram sand lenses and laminations oc-

cur in conjunction with sapropelic layers

$s$, erosional
HIGHLY CALC CLAY, GRADES TO HIGHLY CALC CIAY/DETRITUS

5 Y $3 / 2$ dark olive gray, sapropelic layers; becomes

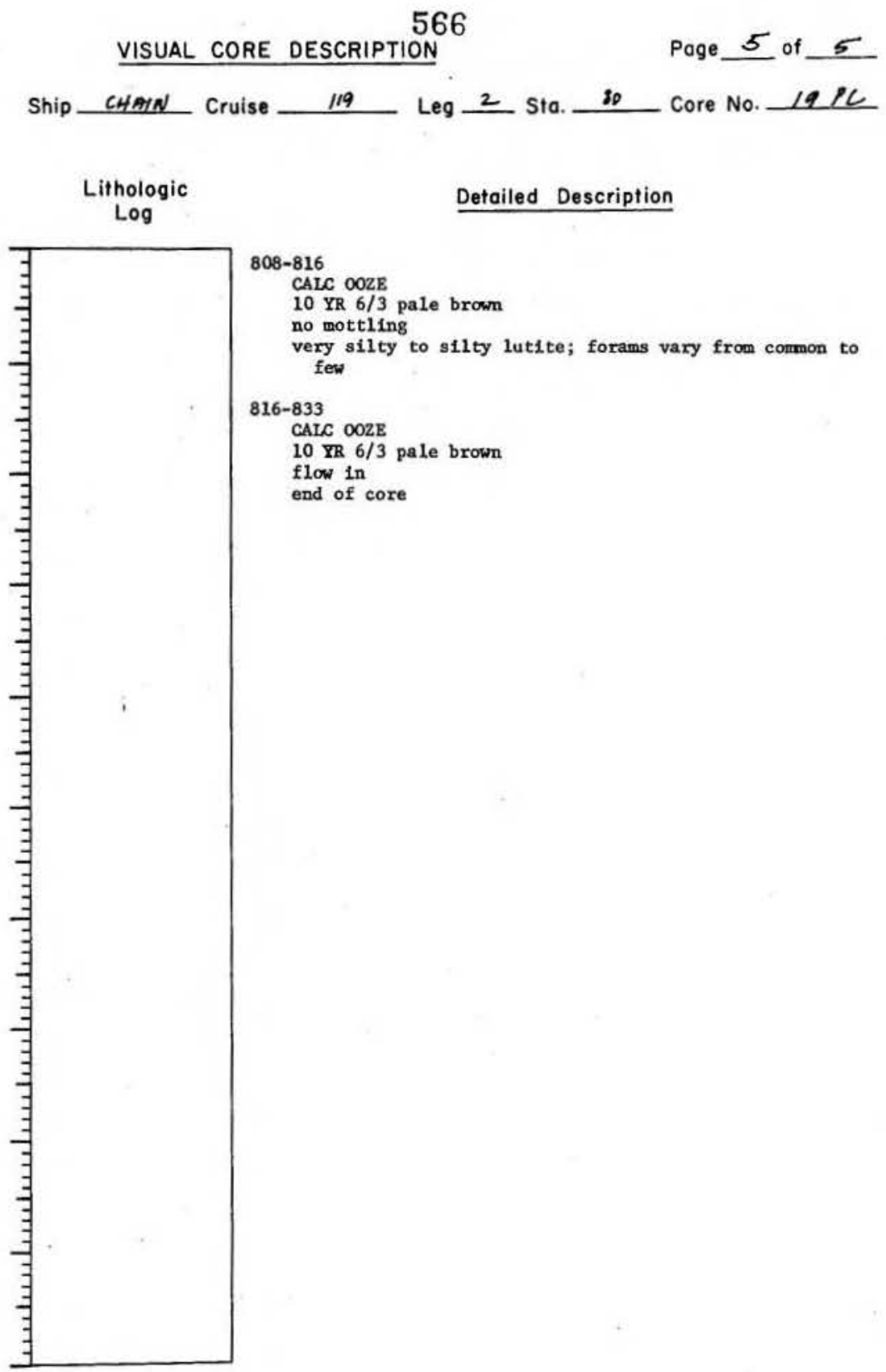


SMEAR SLIDE DESCRIPTIONS - W.H.O.I. SEDIMENT CORES

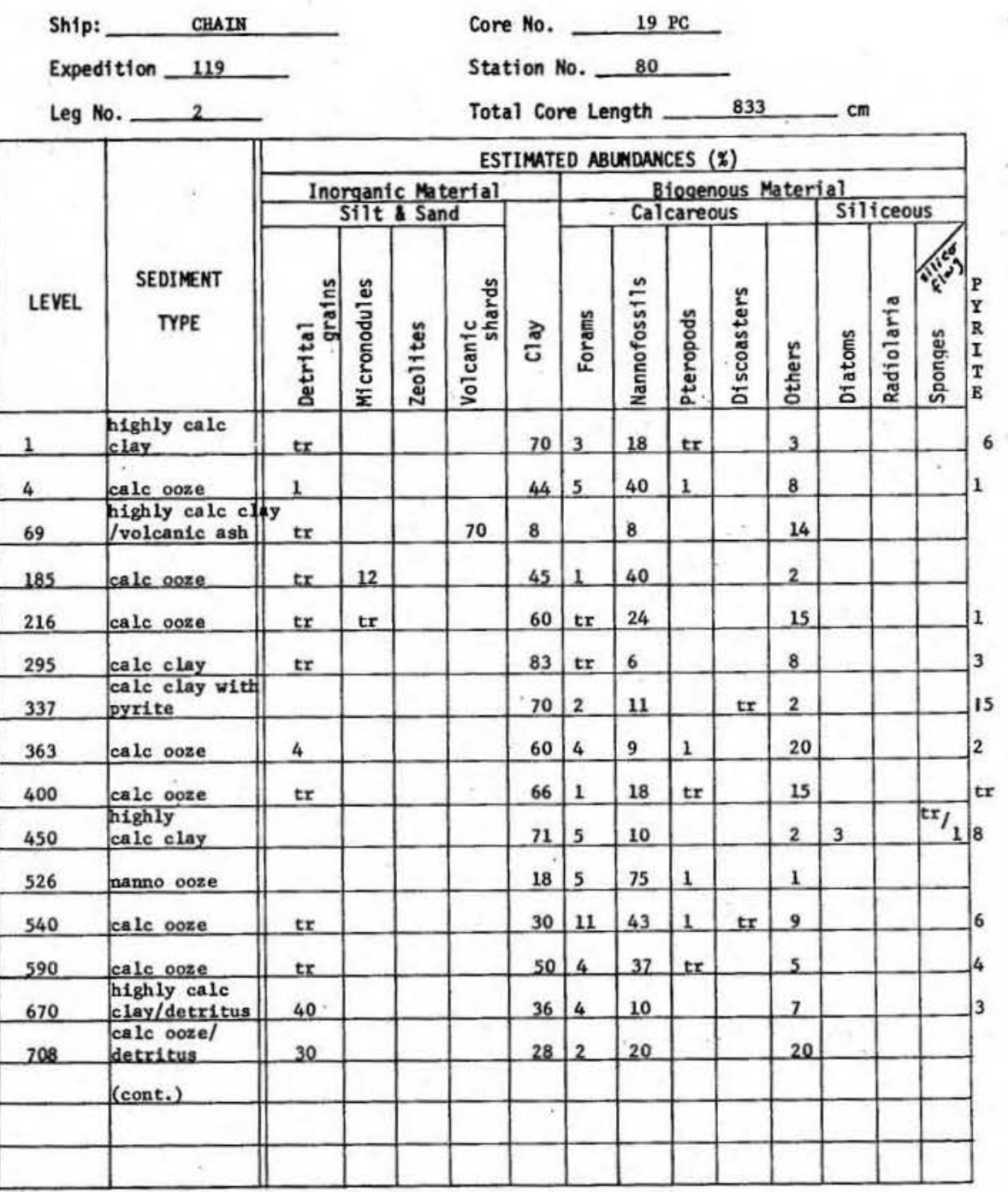

SMEAR SLIDE DESCRIPTIONS - W.H.O.I. SEDIMENT CORES

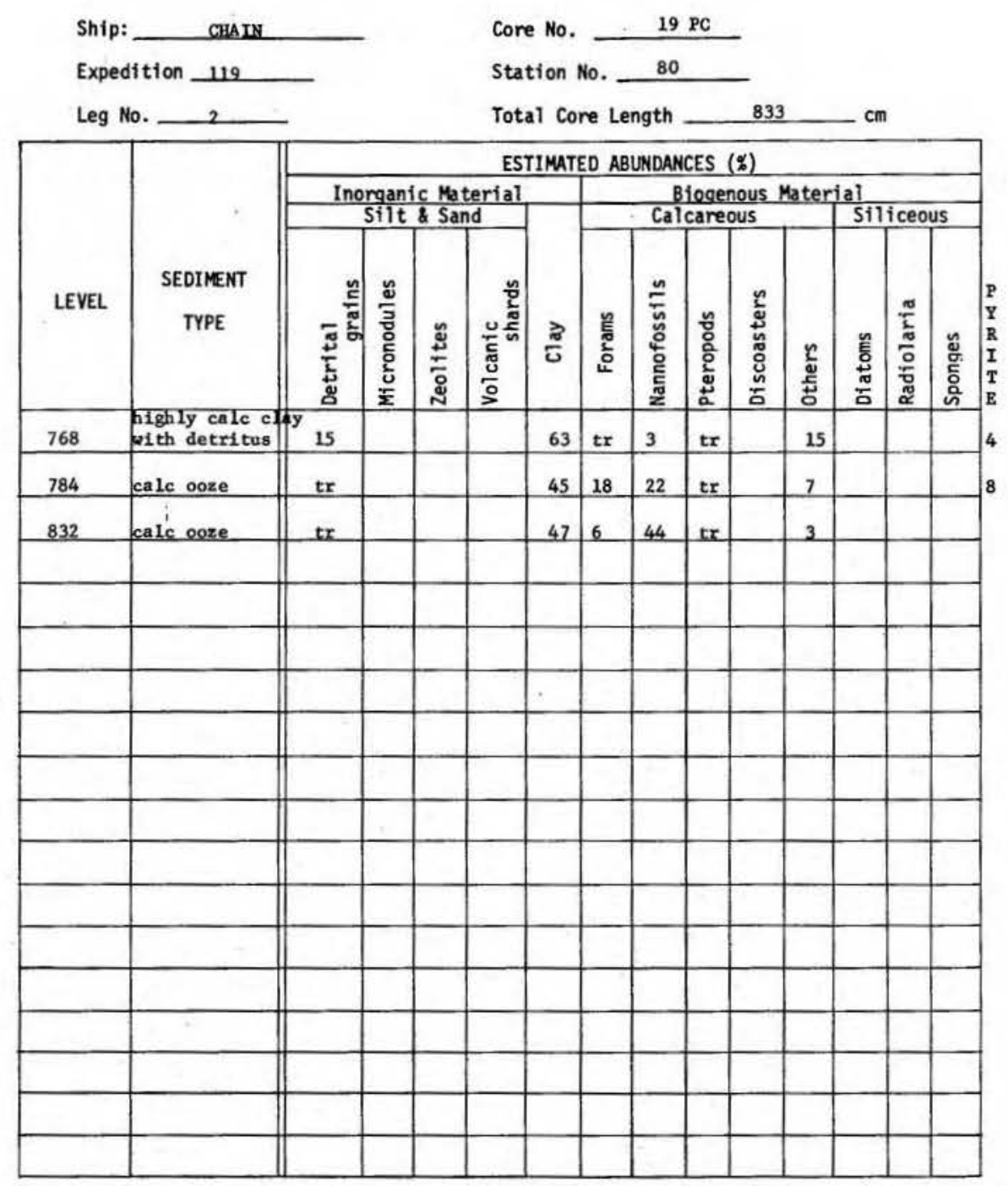


Ship CHN Cruise 119 Leg 2 Sta. $\frac{80}{30}$ Core No. 19 PG Total Length $101, \mathrm{~cm}$. Lat. $33^{\circ} 48.8^{\prime}, \mathrm{N}$ Long. $31^{\circ} 14.5^{\prime}, E$ Depth 2501 corr. $m$ Core condition EXCELENT Dat Physiographic location ISIS RUSE COMPLEX, NILE CONE, EAST. MED. SEA Lithologic

Merstocene

\section{Detailed Description}

$-14$

10 YR $6 / 4$ light yellowish brown

no mottling

th scattered forams and pteropod fragments

$14-20$

HIGHLY CALC CLAY WITH MN MICRONODULES

5 YR $4 / 4$ reddish brown to $3 / 4$ dark reddish brown no mottling

very fine laminae of pale yellow brown

20-27

HIGHLY CALC CIAY

5 Y $3 / 2$ dark olive gray

vilty lutite with scaitered

$27-70$

$2.5 \times 5 / 2$ grayish brown, grades to various hues of pale brown 10 YR $6 / 3$

smali, pale brown mottles scattered through top $10 \mathrm{~cm}$; small grayish mottles scattered through pale browns silty lutite with scattered to common forams

HIGHLY CAIC CIAY

10 YR $4 / 3$ dark brown from above, decreas

small, pale brow

smooth, slick lutite, becomes very silty lutite $77-80 \mathrm{~cm}$ S

CALC OOZE

10 YR $6 / 3$ various hues of pale brovm

no mottling

silty lutite, with scattered to conmon forams

$91-92 \mathrm{~cm}$ volcanic ash

end of core
SMEAR SLIDE DESCRIPTIONS - W.H.O.I. SEDIMENT CORES

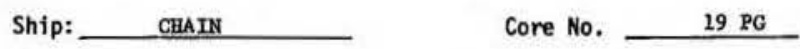

Expedition 119

Station No. 80

Leg No. 2 Total Core Length $101-\mathrm{cm}$

\begin{tabular}{|c|c|c|c|c|c|c|c|c|c|c|c|c|c|c|}
\hline \multirow[b]{4}{*}{ LEVEL } & \multirow[b]{4}{*}{$\begin{array}{c}\text { SEDIMENT } \\
\text { TYPE }\end{array}$} & \multicolumn{13}{|c|}{ ESTIMATED ABUNDANCES (\%) } \\
\hline & & \multirow{2}{*}{\multicolumn{4}{|c|}{$\begin{array}{c}\text { Inorganic Material } \\
\text { Silt \& Sand }\end{array}$}} & \multirow[b]{3}{*}{$\frac{\pi}{\sigma}$} & \multirow{2}{*}{\multicolumn{5}{|c|}{$\begin{array}{l}\text { Biegenous Mater } \\
\text { Calcareous }\end{array}$}} & & & \\
\hline & & & & & & & & & & & & \multicolumn{3}{|c|}{ Siliceous } \\
\hline & & 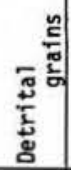 & 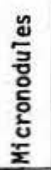 & 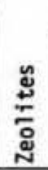 & 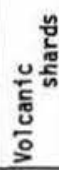 & & 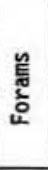 & 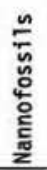 & 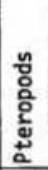 & 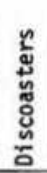 & 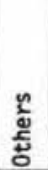 & 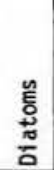 & 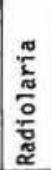 & 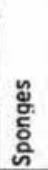 \\
\hline 1 & calc ooze & tr & 1 & & & 22 & 2 & 66 & & & 9 & & & \\
\hline 18 & $\begin{array}{l}\text { highly calc } \\
\text { clay with } \text { Hn }_{\text {n }}\end{array}$ & tr & 15 & & & 60 & 1 & 21 & & & 3 & & & \\
\hline 22 & $\begin{array}{l}\text { highly } \\
\text { calc clay }\end{array}$ & tr & & & & 70 & 1 & 16 & & & 3 & & & \\
\hline 76 & $\begin{array}{l}\text { highly } \\
\text { calc clay }\end{array}$ & 3 & & & & 72 & tr & 15 & & & 10 & & s & \\
\hline 99 & calc ooze & 1 & & & & 45 & I & 48 & & & 5 & & & \\
\hline & & & & & & & & & & & & & & \\
\hline & & & & & & . & & & & & & & & \\
\hline & & & & & & & & & & & & & & \\
\hline & & & & & & & & & & & & & & \\
\hline & & & & & & & & & & & & & & \\
\hline & & & & & & & & & & & & & & \\
\hline & & & & & & & & & & & & & & \\
\hline & & & & & & & & & & & & & & \\
\hline & & & & & & & & & & & & - & & \\
\hline & & & & & & & & & & & & & & \\
\hline & & & & & & & & & & & & & & \\
\hline & & & & & & & & & & & & & & \\
\hline & & & & & & & & & & & & & & \\
\hline
\end{tabular}


Ship CHANN Cruise 119 Leg 2 Sto. 82 Core No. 20 PC

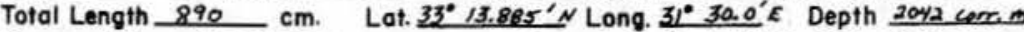
Core condition ExcelLeyt Date Described 19 Jen 76 by TFarmer Physiographic location ISIS RNAE COMREX, NIME CONE, EAST. MED. SEA Lithologic

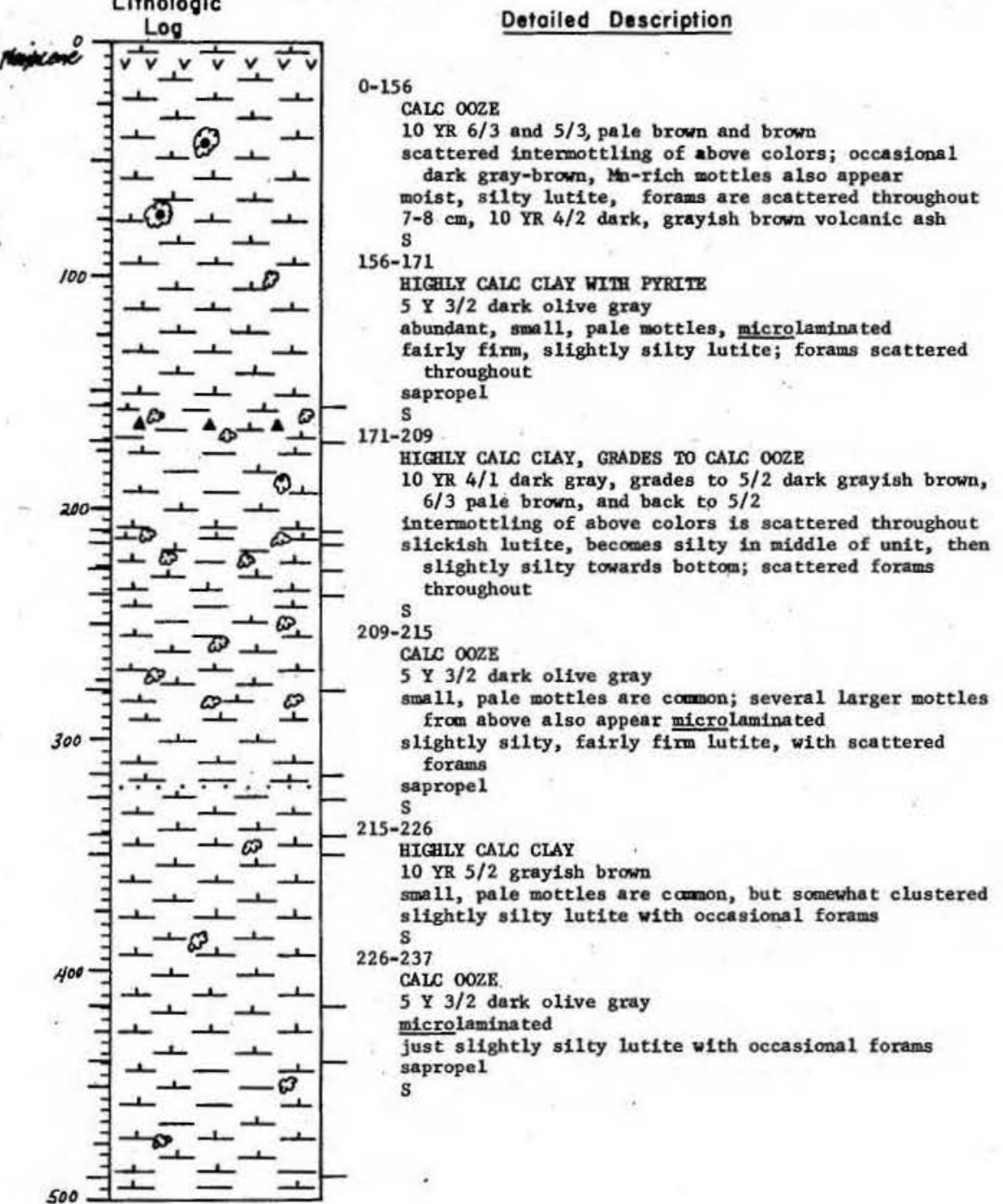

VISUAL CORE DESCRIPTION

Page 2 of 4 Ship CHAN Cruise 119 Leg 2 Sto. 82 Core No. 20 PC

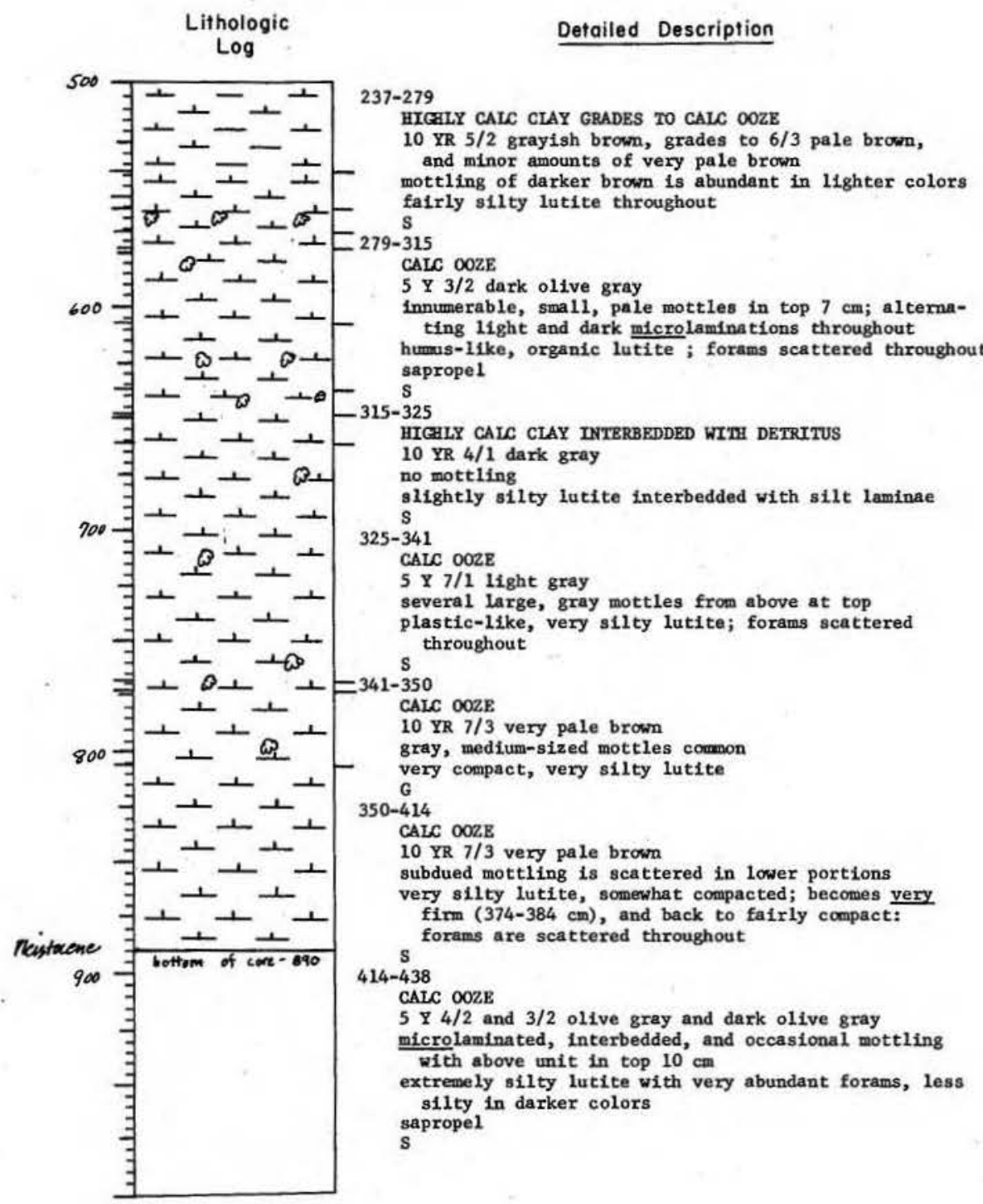


Ship CHAIN Cruise 19 Leg 2 Sta. 82 Core No. 20 PC

\section{Lithologic}

\section{Detailed Description}

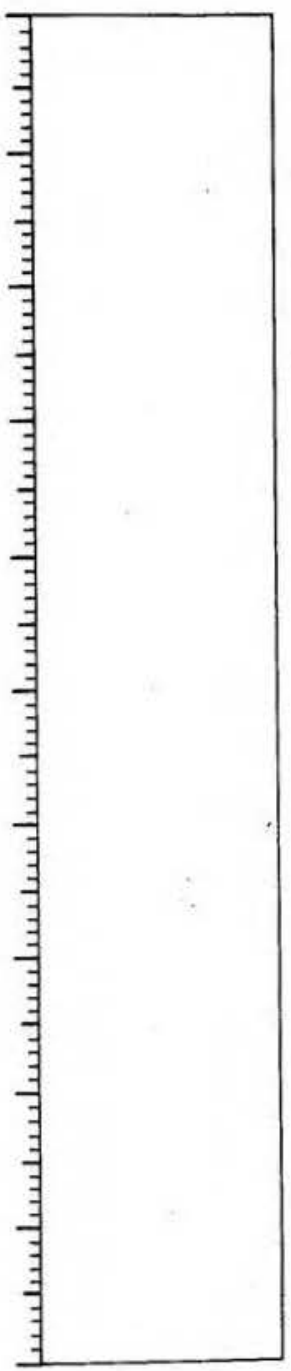

$38-490$

CALC CLAY GRADES TO CAIC OOZE

10 YR $6 / 2$ light brownish gray and 2.5 Y $5 / 2$ grayish

browns appear as gradational bands

small, pale mottles are common in the darker bands, while larger mottling between above colors is fixm, silty lutite, becomes somewhat less silty with depth $\underset{490-538}{-2}$

HIGHLY CALC CIAY

5 Y $3 / 2$ dark olive gray

microlaminated sapropelic material

broken occasionally by firm, smooth, lutite lamination

538-556

CALC OOZE

10 YR $6 / 2$ light brownish gray, grades to 2.5 Y $6 / 2$ light brownish gxay

minor mottling

silty lutite, becomes very silty as forams increase with depth

$\underset{556-566}{S}$

CALC OOZE

5 Y $3 / 2$ dark olive gray

slightly silty, sapropel-1ike lutite interrupted by smooth lutite smooth
saprope1

566-573 CALC OOZE

5 × $4 / 2$ olive gray

homogeneous throughout

$G$, mottled contac

573-608

5 Y $3 / 2$ dark olive gray

alternately light and dark microlaminae; several large silty, humus-like lutite broken in middle by smooth lutite $(2 \mathrm{~cm})$

574

VISUAL CORE DESCRIPTION

Ship CHAN Cruise 119 Leg $q$ Sta. 82 Core No. $20 \mathrm{PC}$

Lithologic

Log

Detailed Description

608-637

CALC OOZE

5 Y $5 / 2$ ollve gray, grades to 2.5 × $6 / 2$ varying hues of light brownish gray small mottles are combon in the paler colors in the middle of the unit

silty lutite; forams are coumo

$637-648$

CALC OOZE

5 Y $3 / 2$ dark olive gray

intense, pale mottling in upper $7 \mathrm{~cm}$ of sapropel

silty, humus-1ike lutite, forams are common

$\underset{648-661}{\mathrm{~s}}$

CALC OOZE

5 Y $5 / 2$ olive gray grades to 10 YR $5 / 2$ grayish brown no mottling

sily lutite with few forams

661-769

CALC OOZE

2.5 Y $5 / 2$ varying hues of grayish brown appear in gradational bands with 10 YR $6 / 3$ various hues of pale brown
intermottling of above colors is generally abundant throughout

lightly silty litite, forams are scattered $\mathrm{S}$
$\mathrm{C}$
$69-773$

CALC OOZE

5 × $3 / 2$ dark olive gray

smal1, pale mottles are counon

slightly silty lutite with scattered forams

G

CAIC OOZE

5 Y $5 / 2$ olive gray grades to 2.5 Y $5 / 2$ grayish brown

slightly silty lutite, becomes fairly smooth as forams $G$

806-890

CAIC OOZE

2.5 Y $5 / 2$ various hues of grayish brown appear in gradational bands with 10 YR $6 / 3$ pale browns

mottling is generally scarce except near top; several

olive yellowish discolorations also appear $749-754 \mathrm{~cm}$

silty to silghtly silty lutite, foram a 
Ship CHAW Cruise 119 Leg II Sto. 83 Core No. 21P

SMEAR SLIDE DESCRIPTIONS - W.H.O.I. SEDINENT CORES

Lithologic

Log

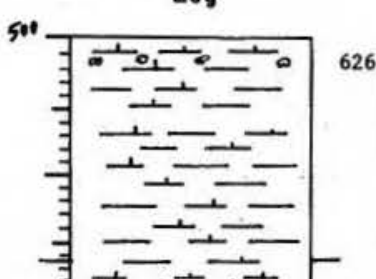

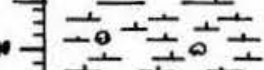

立立高立

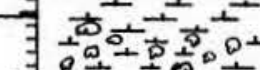

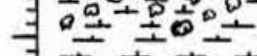

ज十+工十

$\exists--1$

100 丰过-

$\exists+\overline{0}-\overline{0} 0$

正二

$\exists$ 느

드- -

$\exists$ 士

in $\exists$ -

$\exists-1+\div$

$\exists \overline{1} \div \overline{\mathrm{C}}$

$\exists \div-\div$

$\rightarrow \div-$

... $\overline{-502} \div \ldots$

plastuene

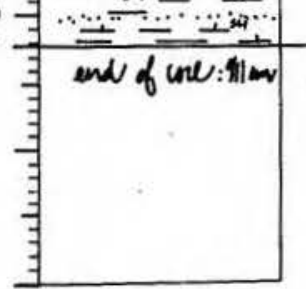

\section{Detailed Description}

626-662 CAIC OOZE

5 Y $4 / 2$ olive gray

motilng oll olive of innumerable hues with some very derk gray and black

firm, compact lutite with abundant forams and lithified calcareous fragments S, horizontal

HICHYY CALC CLAY

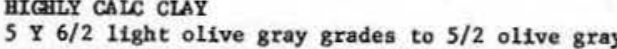
very fine, light gray mottling abundant at unit basal

firm, compact, plastic-1ike lutite with abundant forams 716-911

HICHLY CALC CLAY WITH LAMINATIONS OF DETRITUS GRADES TO SLICHTLY CALC CLAY WITH LAMINATIONS OP DETRITUS 5 Y $3 / 2$ dark olive gray

thick and thin laminations abundant $850-911 \mathrm{~cm} . ;$ als some foram-rich grayish-brown mottling $716-730 \mathrm{~cm}$. irm, slightly silty lutite with a number of varied

tiffly lithified ind sone very silty, others

bit of coring disturbance is evident in shearing of horizontal laminae $850-880 \mathrm{~cm}$ end of core

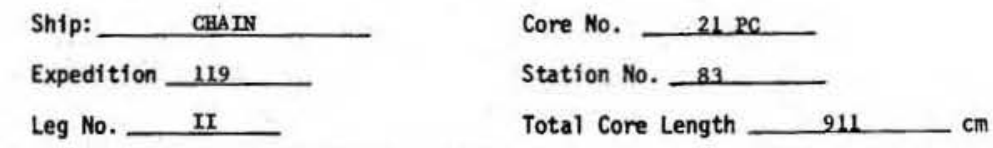

\begin{tabular}{|c|c|c|c|c|c|c|c|c|c|c|c|c|c|c|}
\hline \multirow[b]{3}{*}{ LEVEL } & \multirow[b]{3}{*}{$\begin{array}{c}\text { SEDIMENT } \\
\text { TYPE }\end{array}$} & \multicolumn{13}{|c|}{ ESTIMATED ABUNDANCES (\$) } \\
\hline & & \multicolumn{4}{|c|}{$\begin{array}{c}\text { Inorganic Material } \\
\text { Silt \& Sand }\end{array}$} & $\frac{\pi}{6}$ & \multicolumn{5}{|c|}{$\begin{array}{l}\text { Biegenous Mater } \\
\text { Calcareous }\end{array}$} & \multicolumn{2}{|c|}{ Stliceous } & \\
\hline & & 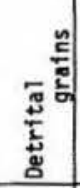 & 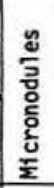 & 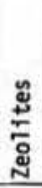 & 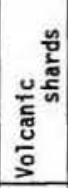 & . & 产 & 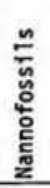 & 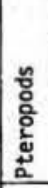 & 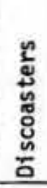 & 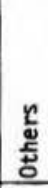 & 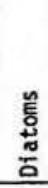 & 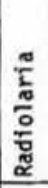 & 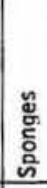 \\
\hline $1 \mathrm{~cm}$ & calc oozé & 2 & 1 & & & 43 & 5 & 40 & 1 & & 5 & & & \\
\hline 53 & $\begin{array}{l}\text { highly } \\
\text { calc ooze }\end{array}$ & 4 & 1 & & & 71 & 4 & 15 & tr & & 4 & & & \\
\hline 150 & calc ooze & 2 & tr & & & 52 & 4 & 35 & 1 & & 6 & & & \\
\hline 250 & $\begin{array}{l}\text { highly } \\
\text { calc clay }\end{array}$ & 4 & tr & & & 67 & 1 & 20 & 1 & & 7 & & & \\
\hline 300 & calc ooze & 3 & tr & & & 40 & 6 & 45 & 1 & & 5 & & & \\
\hline 400 & \begin{tabular}{|l|} 
highly \\
calc clay
\end{tabular} & 3 & tr & & tr & 68 & 1 & 22 & 1 & & 4. & & & \\
\hline 500 & calc ooze & 2 & $t z$ & & & 57 & 2 & 25 & 1 & & 3 & & & \\
\hline 581 & calc clay & 2 & tr & & tr & 84 & $t x$ & 10 & tr & & 2 & & ? & \\
\hline 583 & calc ooze & 2 & $t x$ & & & 21 & 8 & 60 & 3 & & 6 & & & \\
\hline 650 & calc ooze & 2 & 1 & & & 13. & 9 & 58 & 2 & & 5 & & & \\
\hline 750 & $\begin{array}{l}\text { highly } \\
\text { calc clay }\end{array}$ & 4 & & & & 67 & 1 & 20 & 1 & & 6 & & & \\
\hline 850 & $\begin{array}{l}\text { highly } \\
\text { calc clay }\end{array}$ & 2 & & & & 77 & tr & 15 & tr & & 4. & & & \\
\hline 898 & detritus & 78 & tr & & & 10 & & 3 & & & 5 & $=$ & & \\
\hline 910 & $\begin{array}{l}\text { s1ightly } \\
\text { calc clay }\end{array}$ & 6 & & & & 84 & $\mathrm{~m}$ & 2 & tr & & 3 & & & \\
\hline & & & & & & & & & & & & & & \\
\hline & & & & & & & & & & & & & & \\
\hline & & & & & & & & & & & & & & \\
\hline & & & & & & & & & & & & & & \\
\hline
\end{tabular}


581

VISUAL CORE DESCRIPTION

Page 1 of 1

Ship CHNN Cruise 14 Le II Sta. \& Core No. $21 \mathrm{PG}$ Total Length $66 \quad \mathrm{~cm}$. Lat. $30^{\circ} 54.877^{\mathrm{N}}$ Long. $31^{\circ} 16.30^{\circ} \mathrm{E}$ Depth 1529 m.10ers Core condition EXIELEENT Date Described 20jan 76 by f-Bader Physiographic location CENRRL MLE WNE. EASEKN MEATERRANEAN.

Lithologic

Detailed Description

Log

$\stackrel{\log }{\longrightarrow} \stackrel{1}{\perp}$

斗立实 $0-2$

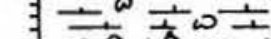

$0-24$ CALC OOZE

C $6 / 4$ 1 1 ght yellowish brova

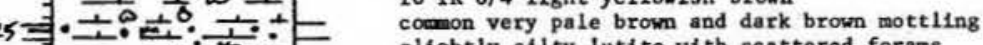

14-26

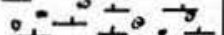

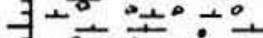

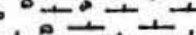

mesituenc

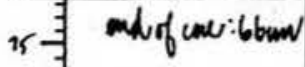

CALC OOZE

YR $4 / 4$ dark yellowish brow

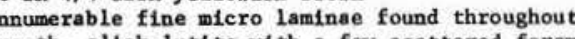
, $10^{\circ}$ the with

CAIC OOZE WITH Ha MICRONODULPS

5 Y $3 / 2$ dark ollve gray

intense, very fine light gray mottling and flecks $26-50 \mathrm{~cm}$

moist, smooth lutite with scattered forams in the

motting zone
end of core

青
SMEAR SLIDE DESCRIPTIONS - W.H.O.I. SEDIIENT CORES

Ship: _ CBA

Expedition 115

Leg No. II II

83

Total Core Length _ 66

$\mathrm{cm}$

\begin{tabular}{|c|c|c|c|c|c|c|c|c|c|c|c|c|c|c|}
\hline \multirow[b]{4}{*}{ LEVEL } & \multirow[b]{4}{*}{$\begin{array}{c}\text { SEDIMENT } \\
\text { TYPE }\end{array}$} & \multicolumn{13}{|c|}{ ESTIMATED ABUNDANCES ( $($ ) } \\
\hline & & \multirow{2}{*}{\multicolumn{4}{|c|}{$\begin{array}{c}\text { Inorganic Material } \\
\text { Silt \& Sand }\end{array}$}} & & \multirow{2}{*}{\multicolumn{5}{|c|}{$\begin{array}{l}\text { Biogenous Mater } \\
\text { Calcareous }\end{array}$}} & \multirow{2}{*}{\multicolumn{3}{|c|}{ siliceous }} \\
\hline & & & & & & & & & & & & & & \\
\hline & & 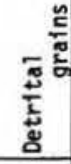 & 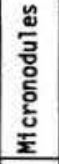 & 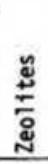 & 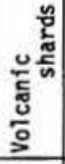 & के & 嵩 & 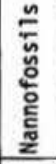 & 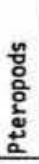 & 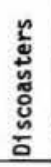 & $\begin{array}{l}\frac{c}{v} \\
\text { sँ } \\
0\end{array}$ & 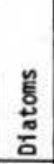 & $\frac{\frac{\pi}{2}}{\frac{\pi}{0}}$ & 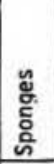 \\
\hline 1 & calc ooze & 3 & tr & & & 26 & 6 & 55 & 2 & & 6 & & & \\
\hline 25 & \begin{tabular}{|l|} 
calc ooze $\mathrm{w} / \mathrm{M}$ \\
micronodules
\end{tabular} & $t r$ & 15 & & & 13 & 8 & 55 & 4 & & 5 & & & \\
\hline 40 & calk ooze & 2 & 1 & & & 46 & 2 & 45 & 1 & & & & & \\
\hline 65 & calc ooze & 2 & tr & & & 64 & $\mathrm{tr}$ & 30 & tr & & 2 & & & \\
\hline & & & & & & & & & & & & & & \\
\hline & & & & & & & & & & & & & & \\
\hline & & & & & & . & & & & & & & & \\
\hline & & & & \pm & & & & & & & & & & \\
\hline & & & & & & & & & & & & & & \\
\hline & & & & & & & & & & & & & & \\
\hline & & & & & & & & & & & & & & \\
\hline & & & & & & & & & & & & & & \\
\hline & & & & & & & & & & & & & & \\
\hline & & & & & & & & & & & & & & \\
\hline & & & & & & & & & & & & & & \\
\hline & & & & & & & & & & & & & & \\
\hline & & & & & & & & i & & & & & & \\
\hline & & & & & & & & & & & & & & \\
\hline
\end{tabular}


583

VISUAL CORE DESCRIPTION

Page 1 of 1 Ship CMAIN Cruise 119 Leg 2 Sta. 87 Core No. 4 GC

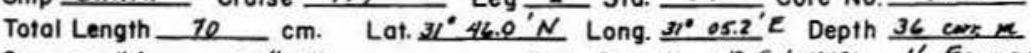
Core condition reallent Date Described 19 Sot 1976 by H. Fiemese

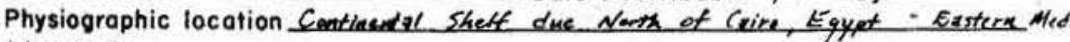
Lithologic

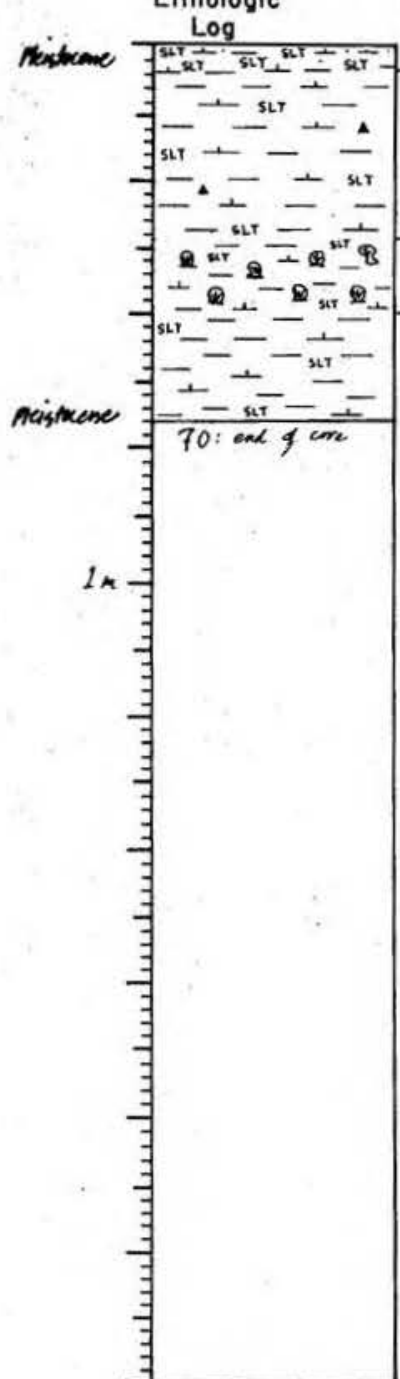

Detailed Description

\section{CAIC CLAY/DETRITUS}

Aark gray-brown

very coarse silt at top, grades into next unit; a few $G$

4-36

\section{HIGHIY CALC CLAY/DETRITUS}

5 Y $3 / 2$ dark ollve gray

(n)

above unit, except lower $10 \mathrm{~cm}$, shells are more cow into lower unit

arregularly-shaped patch of above color extends from 4-20

36-50

SHELL HASH IN MATRIX OF HIGHLY CALC CLAY/DETRITUS 5 Y $3 / 2$ dark olive gray (lutite fraction) primarily shells (pteropods and others) with sall amount of lutite

$50-70$

IGHLY CALC CLAY WITH DETRITUS

5 Y $3 / 2$ dark olive gray

fim lutite

$51-52$ slightly lighter colored with coumon bloclastic and a few bioclastic grains; $53-57 \mathrm{~cm}$ very silty end of core grains; $5 \mathrm{~mm}$ layer at $60 \mathrm{~cm}$ with small black spots

\section{4}

SIEAR SLIDE DESCRIPTIONS - W.H.O.I. SEDINENT CORES

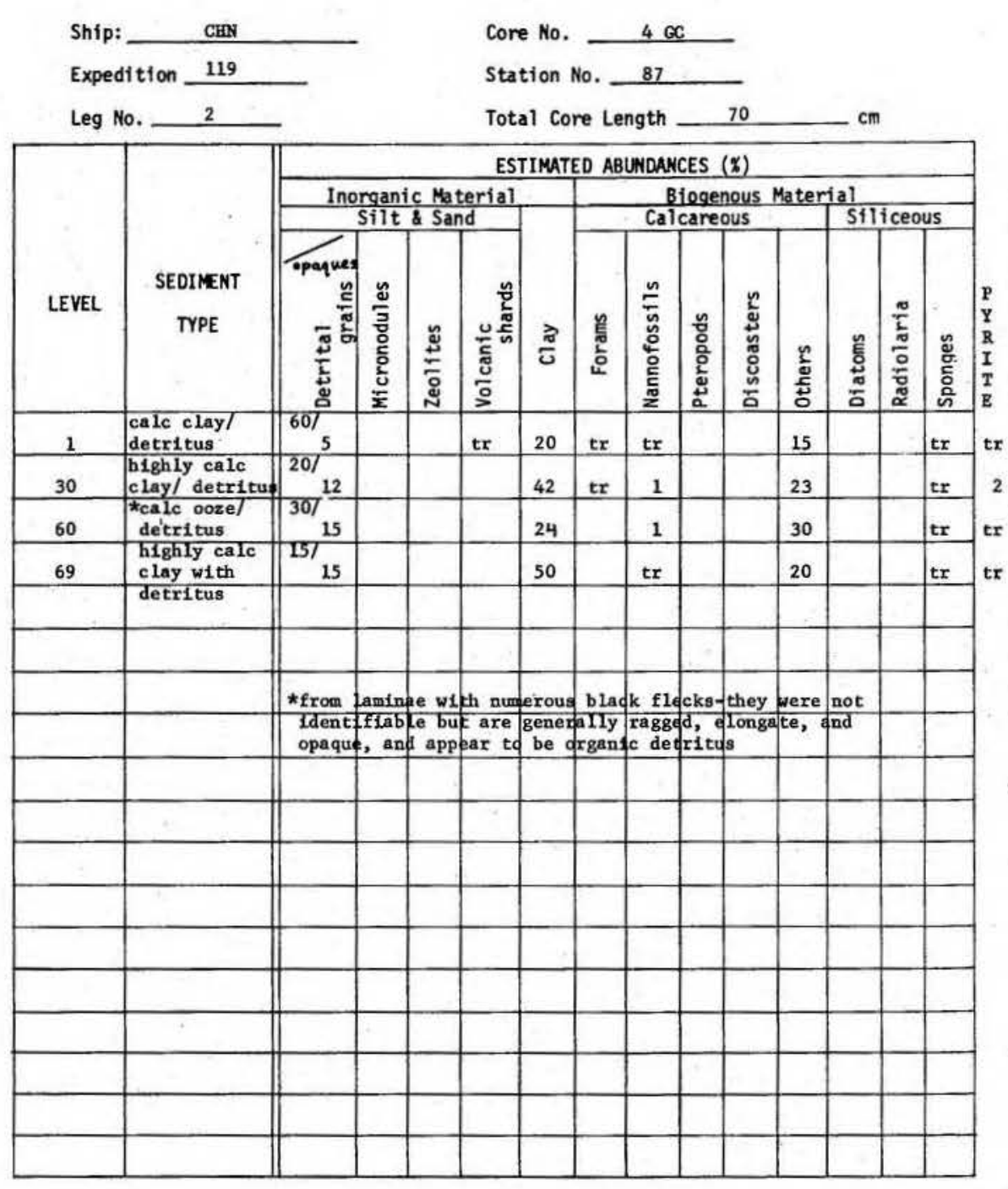


585

VISUAL CORE DESCRIPTION

Poge 1 of 1

Ship C AniN Cruise 119 Leg 2 Sta. 109 Core No. 5 ac Total Length $97 \quad \mathrm{~cm}$. Lat.31. $43.8^{\circ} \mathrm{N}$ Long. $31^{\circ} .04 .25^{\prime} E$ Depth $20 \mathrm{cmr}$ Core condition excellent Date Described $/ 8$ Sad 75 by H. Faem5R Physiogrophic location CowTInENTAR SWRF NOATH of CAMRe, EGYPT

Lithologic

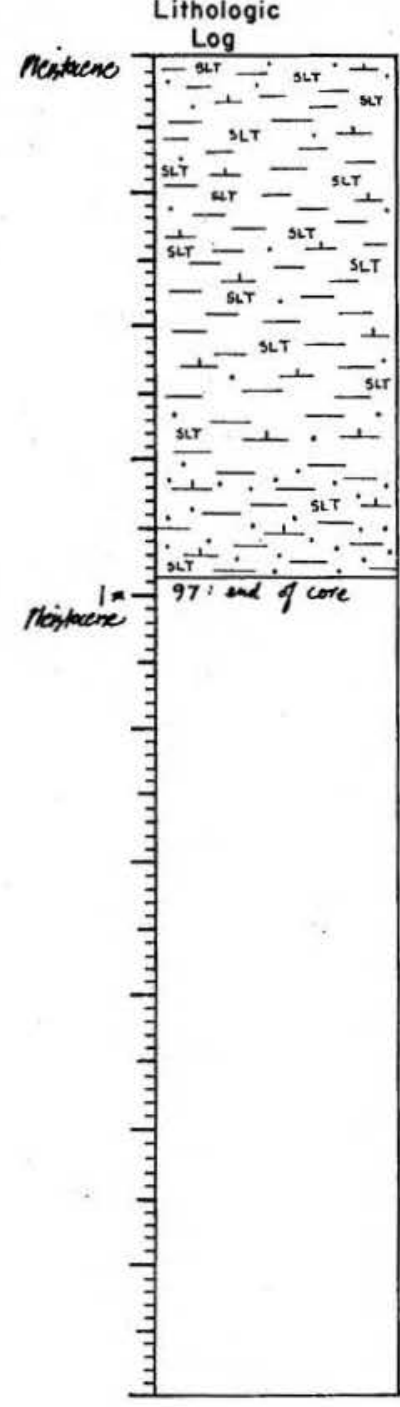

Detailed Description

0-97

CALC CIAY/DETRITUS

10 YR $3 / 2$ very dark gray brown

silt with sone intermixed lutite, and a few shell

small gastropod at $2 \mathrm{~cm}$

end of core

586

SMEAR SLIDE DESCRIPTIONS - W.H.O.I. SEDIMENT CORES

Ship:

Leg No. 2
Expedition

Core No. $5 \mathrm{GC}$

Station No. 109

Total Core Length 97 ESTIMATED ABUNDANCES $(\boldsymbol{q})$

\begin{tabular}{|c|c|c|c|c|c|c|c|c|c|c|c|c|c|c|}
\hline \multirow[b]{3}{*}{ LEVEL } & \multirow[b]{3}{*}{$\begin{array}{c}\text { SEDIMENT } \\
\text { TYPE }\end{array}$} & \multicolumn{13}{|c|}{ ESTIMATED ABUNDANCES ( $(\%)$} \\
\hline & & \multicolumn{4}{|c|}{$\begin{array}{c}\text { Inorganic Material } \\
\text { Silt \& Sand }\end{array}$} & \multirow[b]{2}{*}{$\frac{\vec{\partial}}{\sigma}$} & \multicolumn{5}{|c|}{$\begin{array}{l}\text { Biogenous Mater } \\
\text { Calcareous }\end{array}$} & \multicolumn{3}{|c|}{ Sfliceous } \\
\hline & & 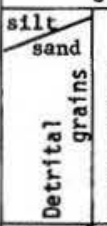 & $\begin{array}{l}\frac{u}{3} \\
\frac{5}{0} \\
\frac{0}{0} \\
\frac{0}{\Sigma} \\
\frac{5}{\Sigma}\end{array}$ & 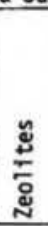 & 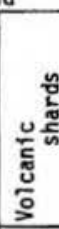 & & 崖 & 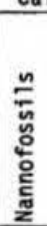 & 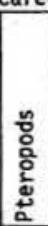 & 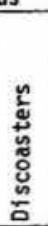 & 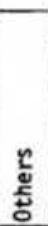 & 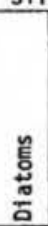 & $\frac{\frac{\pi}{\pi}}{\frac{\pi}{0}} \frac{0}{0}$ & 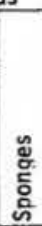 \\
\hline 1 & $\begin{array}{l}\text { calc clay/ } \\
\text { detritus }\end{array}$ & & & & & 45 & & $t x$ & & & 10 & & & \\
\hline 64 & $\begin{array}{l}\text { calc clay w/ } \\
\text { detritus }\end{array}$ & & tr & & tr & 65 & & tr & & & 10 & & & \\
\hline 96 & $\begin{array}{l}\text { calc clay/ } \\
\text { detritus }\end{array}$ & & & & & 40 & tr & tr & & & 15 & & & \\
\hline & & & & & & & & & & & & & & \\
\hline & & & & & & & & & & & & & & \\
\hline & & & & & & & & & & & & & & \\
\hline & & & & & & . & & & & & & & & \\
\hline & & & & & & & & & & & & & & \\
\hline & & & & & & & & & & & & & & \\
\hline & & & & & & & & & & & & & & \\
\hline & & & & & & & & & & & & & & \\
\hline & & & & & & & & & & & & & & \\
\hline & & & & & & & & & & & & & & \\
\hline & & & & & & & & & & & & & & \\
\hline & & & & & & & & & & & & & & \\
\hline & & & & & & & & & & & & & & \\
\hline & & & & & & & & & & & & & & \\
\hline & & & & & & & & & & & & & & \\
\hline
\end{tabular}


Ship CHAIN Cruise 119 Leg $\frac{2}{2}$ Sta. $\frac{115}{31^{\circ}}$ Core No. $\frac{22 \text { PC }}{1581 \text { com }}$ Totol Length $953 \mathrm{~cm}$. Lat. $32^{\circ} 46.0^{\prime} \mathrm{N}$ Long. $31^{\circ} 53.3^{\prime} \mathrm{E}$ Depth 1581 carr. $\mathrm{m}$. Core condition EXCELLENT Dote Described 22 Jan z\& by I. Former Physiographic location GRABEN IN ISIS RDGE COMPLEK, NILE CONE, ENST: ME J. SEA Lithologic

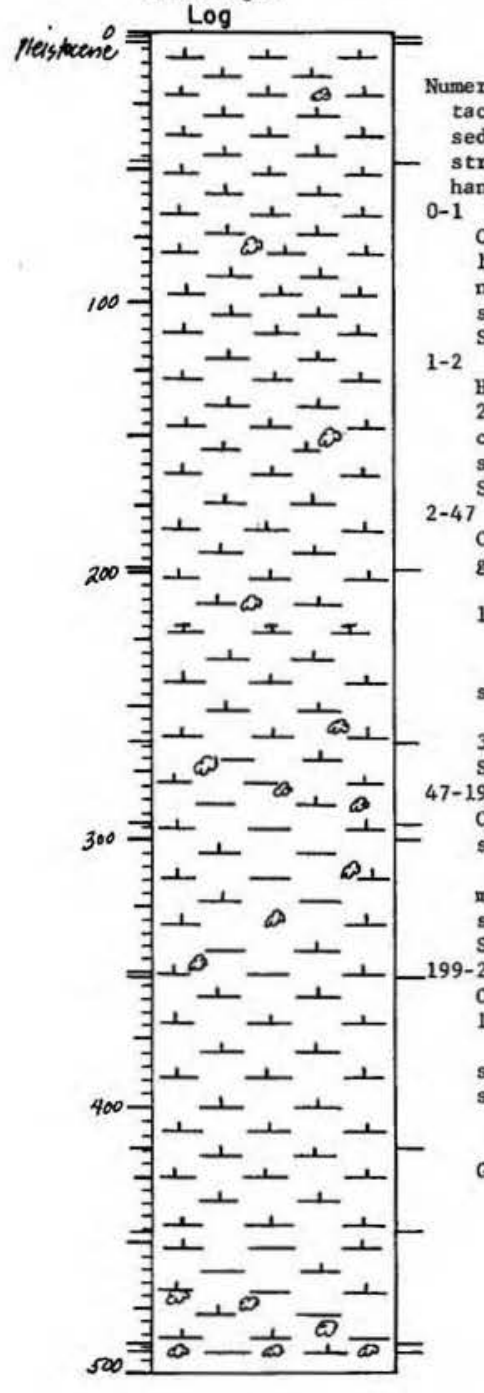

\section{Detailed Description}

Numerous sheared laminations, highly distorted, inclined concacts, and irregular conbinations of units suggest the tratigraphic position either in the coring, oo posstery

CALC OOZE

10 YR $4 / 4$ dark yellowish brown

no mottling

silty lutite, scattered forans

$1-2$

HIGHLY CALC CLAY

.5 Y $3 / 2$ very dark gray brown

lightly silty lutite with scattered foram

$2-47$ , inclined

CALC OOZE

grades from 2.5 Y $4 / 2$ dark gray ish brown, to $10 \mathrm{YR}$

$5 / 3$ brown

- $13 \mathrm{~cm}$ abundant, small, very dark brown mottles; 19 -

$1 \mathrm{~cm}$ pale brown lamination with mottles extending to

ilty lutite, sonewhat siltier towards bottom, with scattered forans and pteropods very silty, somewhat compacted laye S, CALC OOZE

subtle hues of 10 YR $4 / 2$ dark grayish brown and $4 / 3$ brown

inor mottling of above colors is scattered throughout fightly silty lutite with few to scattered forams S

CALC OOZE

$10 \mathrm{YR} 5 / 3$ brown, tends towards pale brown $6 / 3$, then

$5 / 2$ and $4 / 2$ grayish browns

scattered, subtle, medium mottles $203-222 \mathrm{~cm}, 257-261 \mathrm{~cm}$ ightly silty lutite, becomes smoother with depth; gen-

$209 \mathrm{~cm}$, and abundant forams and pteropods from $217-225 \mathrm{~cm}$ handling stages
Ship CHAIN

Cruise 119

Leg 22 Sta. 115

Detailed Description

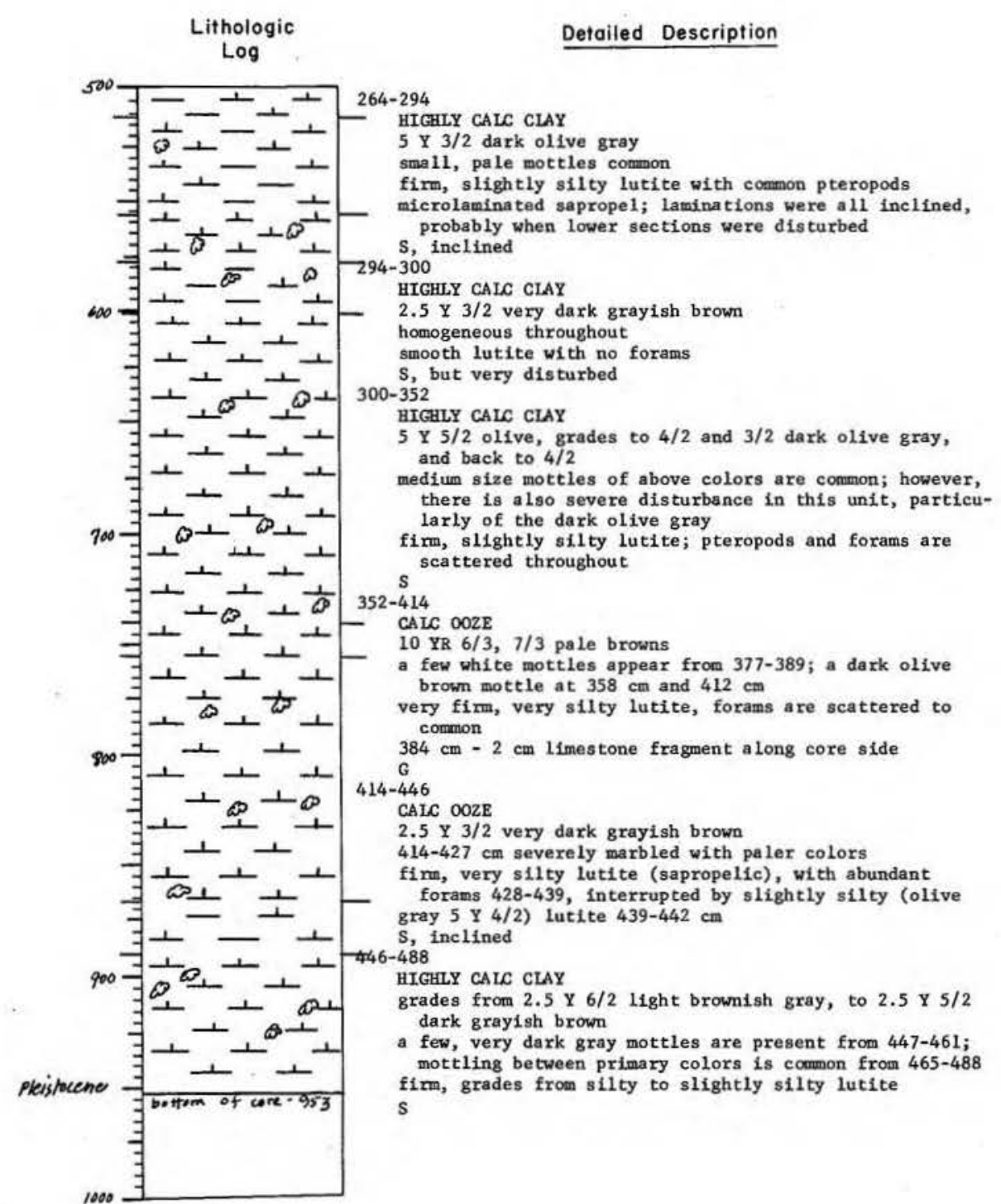


Lithologic Log

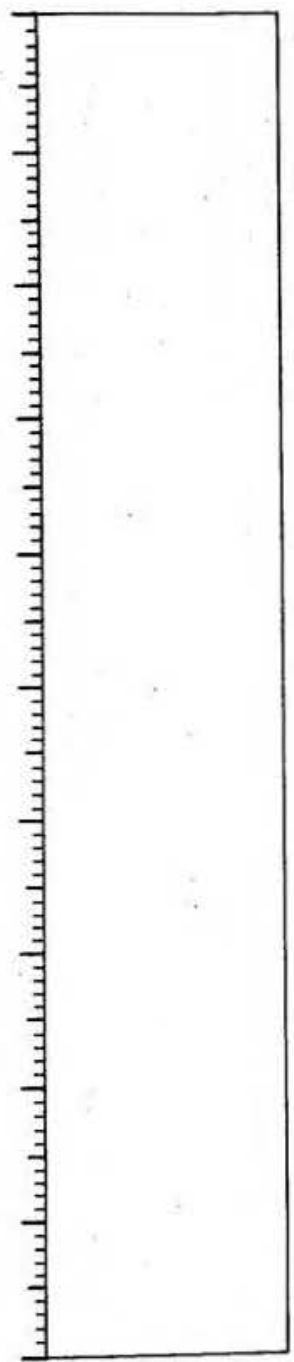

\section{Detailed Description}

$88-491$

HIGaIY CALC CLAY

Y

fairly smooth lutite, with few forams

491-512

HIGHY CALC CIAY

5 Y $4 / 2$ varying hues of olfve

scattered, pale mottling from above; also an olive mottle is present at $510 \mathrm{~cm}$

cattered forams

$\stackrel{-}{\mathrm{C}}$

HIGLYY CALC CLAY

5 Y $3 / 2$ dark olive gray and 10 XR $3 / 2$ very dark grayish brown

$520-545 \mathrm{~cm}$; however, a severely distorted lamination (?)

interbedded, firm sapropel (humus-11ke lutite) and very

mooth lutite; forams are fey to scattered in the tremate 11 h

ingt and dark microlaminations in sapropels 557-577

CAIC OOZE

S $6 / 2$ light olive gray

three large, dark olive gray mottles appear in middle; shecle, very small mottles are cormon throughout $S$, inclined

HICHLY CAIC CLAX

merous, gma11, pale mottles in top $12 \mathrm{~cm}$; also, several large burrows are present silty, humus-1ike lutite, with forams and pteropods $s$, but shows en echelon faulting

CALC OOZE

10 XR $4 / 2$ dark grayish brown, grades to $5 / 2$ grayish

ors; motting of daxker brown occurs in lighter colslightly silty lutite with few forams

VISUAL CORE DESCRIPTION

Page 4 of 4 Ship CAAIN Cruise 119 Leg 2 Sto. 115 Core No. 22 N

Lithologic

Log

\section{Detailed Description}

740-755

CAIC OOZE

5 Y $5 / 3$ olive this unit into top of lower unit fairly smooth lutite $S$, distorted

755-867

CALC OOZE

varying hues of 5 y $5 / 2$ olive gray and $5 / 3$ ollve

small and large moteles of above colors are coumon; $\mathrm{S}$,
$867-890$ inclined $70^{\circ}$

HICHLY CAIC CLAY

5 Y $3 / 2$ dark olive gray

several large, pale olive mottles and laminations are present

silty lutite, forams are comnon

S, distorted CALC OOZE

5 Y $4 / 2$ olive gray grades to $5 / 2$ (olive gray) and back to $4 / 2$

dark olive gray mottles cluster at various points

smooth, slightly silty lutite, forams occasionaliy end of 
SMEAR SLIDE DESCRIPTIONS - W.H.O.I. SEDIMENT CORES

Ship:

Leg No. 2
Expedition 119

Core No. 22 PC

Station No. 115

Total Core Length 953

\section{ESTIMATED ABUNDANCES (\%)}

\begin{tabular}{|c|c|c|c|c|c|c|c|c|c|c|c|c|c|c|}
\hline \multirow[b]{3}{*}{ LEVEL. } & \multirow[b]{3}{*}{$\begin{array}{c}\text { SEDIMENT } \\
\text { TYPE }\end{array}$} & \multicolumn{13}{|c|}{ ESTIMATED ABUNDANCES ( $(\%)$} \\
\hline & & \multicolumn{4}{|c|}{$\begin{array}{c}\text { Inorganic Material } \\
\text { Silt \& Sand }\end{array}$} & \multirow[b]{2}{*}{$\frac{\pi}{\sigma}$} & \multicolumn{5}{|c|}{$\begin{array}{l}\text { Biogenous Mater } \\
\text { Calcareous }\end{array}$} & \multicolumn{3}{|c|}{$\frac{a l}{\text { Siliceous }}$} \\
\hline & & 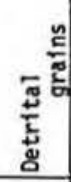 & 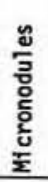 & 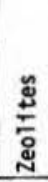 & 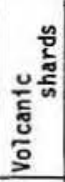 & & 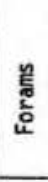 & 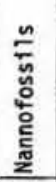 & 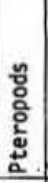 & 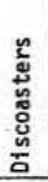 & $\begin{array}{l}\frac{n}{w} \\
\frac{N}{5} \\
\end{array}$ & 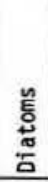 & $\frac{\frac{\pi}{2}}{\frac{\pi}{0}}$ & 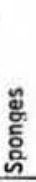 \\
\hline 1 & calc ooze & $t r$ & 3 & & & 47 & 2 & 44 & $\mathrm{tr}$ & & 4 & & & \\
\hline 90 & calc ooze & 1 & tr & & tx & 56 & 1 & 38 & tr & & 4 & & & . \\
\hline 152 & $\begin{array}{l}\text { highly } \\
\text { calc clay }\end{array}$ & 6 & & & & 65 & tr & 22 & & & 7 & & & \\
\hline 245 & calc ooze & 1 & & & & 62 & tr & 35 & $\mathrm{tr}$ & & 2 & & & \\
\hline 306 & $\begin{array}{l}\text { highly } \\
\text { calc clay }\end{array}$ & 3 & 1 & & $\mathrm{tr}$ & 69 & 2 & 21 & $\mathrm{tr}$ & & 4 & & & \\
\hline 338 & $\begin{array}{l}\text { highly } \\
\text { calc clay }\end{array}$ & tr & & & tr & 83 & tr & 15 & $\mathrm{tr}$ & & 2 & & & \\
\hline 367 & calc ooze & 3 & & & & 34 & 8 & 38 & 7 & & 10 & & & \\
\hline 433 & calc ooze & tr & & & & 61 & 10 & 25 & $\mathrm{tx}$ & & 2 & & & \\
\hline 465 & $\begin{array}{l}\text { highly } \\
\text { calc clay }\end{array}$ & 1 & tr & & & 72 & tr & 25 & $\mathrm{tr}$ & & 2 & & & \\
\hline 546 & $\begin{array}{l}\text { highly } \\
\text { cale clay }\end{array}$ & & & & tr & 70 & 1 & 26 & $\mathrm{tr}$ & & & & & \\
\hline 615 & $\begin{array}{l}\text { highly } \\
\text { calc clay }\end{array}$ & 2 & tx & & & 70 & tr & 26 & $\mathrm{tr}$ & & & & 2 & \\
\hline 690 & calc ooze & 1 & tr. & & & 66 & $t r$ & 30 & & & 3 & & & \\
\hline 752 & calc ooze & $t x$ & $t x$ & & & 39 & tr. & 60 & & & 1 & & & \\
\hline 842 & calc ooze & 2 & 1 & & & 43 & tx & 50 & & & 4 & & & \\
\hline 880 & $\begin{array}{l}\text { highly } \\
\text { calc clay }\end{array}$ & & & & & 72 & 1 & 23 & $+x$ & & 1 & & & \\
\hline 952 & calc ooze & 1 & $t_{r}$ & & & 68 & tr & 28 & & & 3 & & & \\
\hline & & & & & & & & & & & & & & \\
\hline & & & & & & & & & & & & & & \\
\hline
\end{tabular}

VISUAL CORE DESCRIPTION

Page 1 of 1

Ship CHAIN Cruise 119 Leg 2 sto 115 Core No. 22 PG Total Length $113 \mathrm{~cm}$. Lat. $32^{\circ} 460^{\circ} N$ Long. $31^{\circ} 53.3^{\prime} \mathrm{E}$ Depth $158^{\prime}$ Carr m. Core condition EKCELLENT Date Described $22 \mathrm{Jen}$ Zh by $T$ Fermer Physiographic location GRABEN in ISIS RIXGE COMPLEX, NILE CONE, EAST. MEX SEA Lithologic

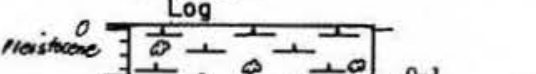

\section{Detailed Description}

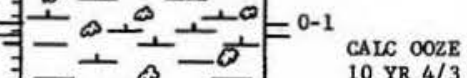

$\exists=10$ YR $4 / 3$ brown

smooth, slick lutite

$\exists+1+{ }_{1-17^{5}}$

는 CALC OOZE

(100

我, 10 YR $5 / 4$ yellowish brown

reases with depth of yellowish brown 1s common;

$=$ bottem of corc - $1 / 3$

pteropods common at con$G$, and heavily mottled

$12-22$

CALC OOZE

10 YR $4 / 4$ dark yellowish brown

silty lutite, increasingly silty with depth, forams S

HITHIY CALC CLAY

5 Y $3 / 2$ dark olive gray

(anumerable, small, pale mottles; several large, grayis̈hbrown burrows in top $15 \mathrm{~cm}$

slick, slightly silty lutite with forams and pteropods S

50-113

CALC OOZE

10 YR $4 / 2$ dark grayish brown, tends toward $5 / 2$ with depth scattered intermottling of above color

slick, slightly silty lutite with scattered forams and pteropods; becomes considerably siltier in lowest $10 \mathrm{~cm}$ end of core 
SIEAR SLIDE DESCRIPTIONS - W.H.0.I. SEDINENT CORES

\begin{abstract}
Ship: Expedition 119
\end{abstract}

Leg No. 2

\begin{tabular}{|c|c|c|c|c|c|c|c|c|c|c|c|c|c|c|}
\hline \multirow[b]{3}{*}{ LEVEL. } & \multirow[b]{3}{*}{$\begin{array}{c}\text { SEDIMENT } \\
\text { TYPE }\end{array}$} & \multicolumn{13}{|c|}{ ESTIMATED ABUNDANCES ( $(z)$} \\
\hline & & \multicolumn{4}{|c|}{$\begin{array}{c}\text { Inorganic Material } \\
\text { Silt \& Sand }\end{array}$} & \multirow[b]{2}{*}{$\frac{\pi}{5}$} & \multicolumn{5}{|c|}{$\begin{array}{l}\text { Biogenous Mater } \\
\text { Calcareous }\end{array}$} & \multicolumn{3}{|c|}{$\frac{\text { al }}{\text { siliceous }}$} \\
\hline & & 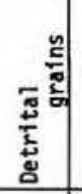 & 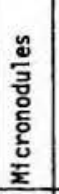 & 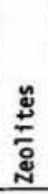 & 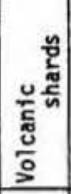 & & 点 & 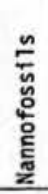 & 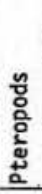 & 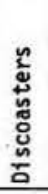 & $\begin{array}{l}\frac{n}{2} \\
\frac{5}{ \pm} \\
0\end{array}$ & 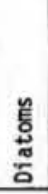 & $\begin{array}{l}\frac{\pi}{2} \\
\frac{\pi}{0} \\
\frac{0}{0} \\
\alpha\end{array}$ & 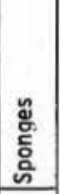 \\
\hline 1 & calc ooze & 1 & $t r$ & & & 66 & 1 & 27 & & & 5 & & & \\
\hline 7 & calc ooze & 1 & 1 & & & 40 & 3 & 47 & 2 & & 6 & & & : \\
\hline 19 & calc ooze & tr & 4 & & & 53 & 2 & 40 & tr & & 1 & & & \\
\hline $\begin{array}{c}\text { (pale } \\
35 \text { mottle) }\end{array}$ & calc ooze & tr & $\mathrm{tr}$ & & & 60 & 1 & 34 & 1 & & 3 & & & \\
\hline $\begin{array}{c}\text { (dark } \\
46 \text { area) }\end{array}$ & $\begin{array}{l}\text { highly } \\
\text { calc clay }\end{array}$ & & & & & 82 & 1 & 15 & tr & & 1 & & & \\
\hline 109 & calc ooze & 2 & $\mathrm{tr}$ & & & 60 & 2 & 30 & tr & & 5 & & & \\
\hline & & & & & & + & & & & & & & & \\
\hline & & & & & & & & & & & & & & \\
\hline & . & & & & & & & & & & & & & \\
\hline & 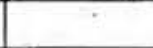 & & & & & & & & & & & & & \\
\hline & & & & & & & & & & & & & & \\
\hline & & & & & & & & & & & & & & \\
\hline & & & & & & & & & & & & & & \\
\hline & & & & & & & & & & & & & & \\
\hline & - & & & & & & & & & & & & & \\
\hline & & & & & & & & & & & & & & \\
\hline & ' & & & & & & & & & & & & & \\
\hline & & & & & & & & & & & & & & \\
\hline
\end{tabular}

Core No. 22PG

Station No. 11

Total core Length

-

Ship CHALN Cruise 119 Leg II Sta. 116 Core No. 23 PC Total Length $322 \mathrm{~cm}$. Lat. $33^{\circ} 30.5^{\circ} \mathrm{N}$ Long. $31^{\circ} 52.6^{\prime} \mathrm{E}$ Depth $2142 \mathrm{~m}$. comr Core condition EXLEUENT Date Described 26 fa. 76 by f.Buda Physiographic location ISIS RIDGE COMREX, NILE CONE, EAETERN MEE.

Lithologic

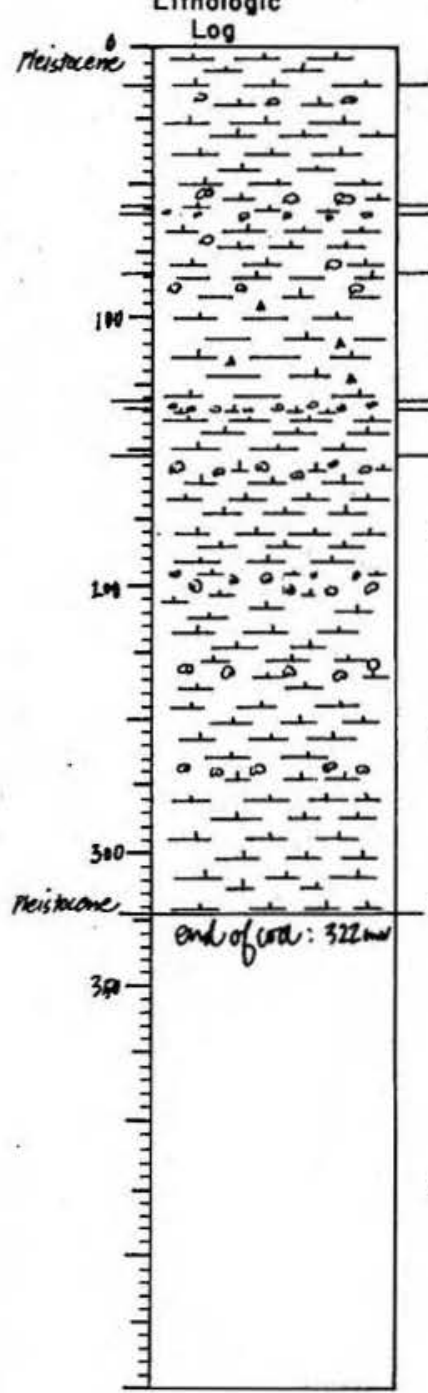

\section{Detailed Description}

$0-13$

\section{CALC OOZE}

$10 \mathrm{YR} 6 / 4$ 1ight yellowish brown and 5 Y $3 / 2$ very

irregular mixing of the two colors above

moist, smooth lutite with an irregular ashy inclusion

at unit basal contact

entire unit disturbed, compacted, and disoriented

3-58 $\mathrm{S}$, irregular

CALC OOZE

10 YR $6 / 3$ pale brow

irregular, 1ight olive brown mottling $13-18 \mathrm{~cm}, 51-56 \mathrm{~cm}$

ry $\mathrm{s}$, horizontal

CALC OOZE

5 Y $3 / 2$ dark olive gray

innumerable, very fine, light gray mottles and flecks slightly silty foram/pteropod-rich, sapropel-1ike lutit distinct, light gray band, 1 can wide, at unit basa itregular

62-83 CALC OOZE

5 Y $6 / 2$ light olive gray small lithified, white, calcareous lumps 5 , inclined $5^{\circ}$

\section{MIGILY CALC CLAY WITH PYRITE}

5 Y $3 / 2$ very dark ollve gray nae throughout: also a bit of pale olive mottling common, $83-88 \mathrm{~cm}$

stiffer, more compact, nearly mulch-like, foram-rich lutite with a few scattered, white, lithified calcareous fragments sapropel zone: coring disturbance evident $124-131 \mathrm{~cm}$ 131-134

\section{CAIC OOZE}

2.5 y N $7 /$ light gray

no mottling slight, faint, white marbiling and mottling throughout firmer, more compact lutite with scattered forams and 
Ship CHAIN Cruise 119 Leg II Sto. 116 Core No. $23 P C$

SIEAR SLIDE DESCRIPTIONS - H.H.O.I. SEDINENT CORES

Lithologic Log

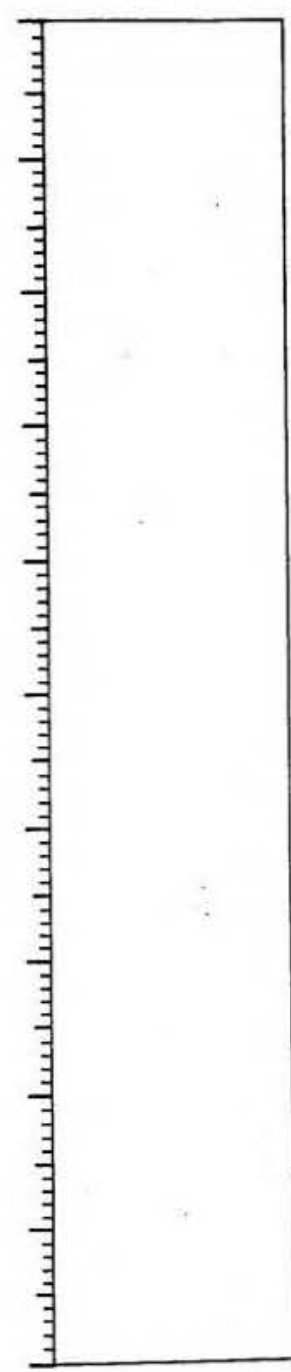

\section{Detailed Description}

131-134 (cont.)

completely lithified, brittle and fragmented zone of 1 imestone and cemented lutite

\section{CALC 002}

5 y $6 / 2$ light olfve gray

homogeneous throughout

finm, plastic-1Lke lutite with scattered foram very $\mathrm{s}$, horizontal

cinc

CAIC OOZE

5 Y $4 / 2$ olive gray, grades to $3 / 2$ dark ollve gray of extensive, 1ight olive gray mottlin also, two very distin, $226-236 \mathrm{~cm}, 264-270 \mathrm{~cm}$ : break up sapropel $218-225 \mathrm{~cm}, 294-307 \mathrm{~cm}$ : some fine suggestions of Laminations are found 183-194 cm

stiff, more compact, foram-rich lutite with extremely

abundant tests in the zones $205-213 \mathrm{~cm}, 280-283 \mathrm{~cm}$; end of core
Ship: CHAIN

Expedition 119

Leg No. 2
Core No. 23 PC

Station No. 11

Total Core Length 322

\begin{tabular}{|c|c|c|c|c|c|c|c|c|c|c|c|c|c|c|}
\hline \multirow[b]{3}{*}{ LEVEL. } & \multirow{3}{*}{$\begin{array}{l}\text { SEDIMENT } \\
\text { TYPE }\end{array}$} & \multicolumn{13}{|c|}{ ESTIMATED ABUNDANCES ( $(\boldsymbol{x})$} \\
\hline & & \multicolumn{4}{|c|}{$\begin{array}{c}\text { Inorganic Materfal } \\
\text { Silt \& Sand } \\
\end{array}$} & & \multicolumn{5}{|c|}{$\begin{array}{l}\text { Biogenous Mater } \\
\text { Calcareous }\end{array}$} & \multicolumn{3}{|c|}{ Siliceous } \\
\hline & & 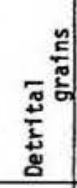 & 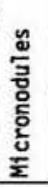 & 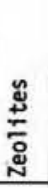 & 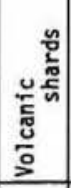 & $\frac{\pi}{6}$ & 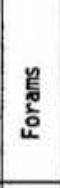 & 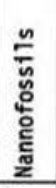 & 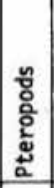 & 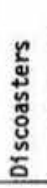 & $\begin{array}{l}\frac{c}{2} \\
\frac{\Sigma}{5} \\
\end{array}$ & 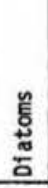 & $\begin{array}{l}\frac{\pi}{2} \\
\frac{\pi}{0} \\
\frac{0}{0} \\
\frac{\alpha}{\alpha} \\
\end{array}$ & 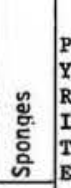 \\
\hline 1 & ealc ooze & 3 & 1 & & & 25 & 11 & 45 & $\mathrm{tr}$ & tr & 15 & & & \\
\hline 30 & alc ooze & 2 & $\operatorname{tr}$ & & & 31 & 10 & 40 & 7 & & 10 & & & \\
\hline 70 & salć ooze & 2 & $\mathrm{tr}$ & & & 50 & 8 & 30 & $\mathrm{tr}$ & & 10 & & & \\
\hline 100 & $\begin{array}{l}\text { Ighly calc } \\
\text { Elay w/pyrite }\end{array}$ & 2 & & & & 57 & 12 & 10 & $\mathrm{tr}$ & & 3 & & & 1 \\
\hline 133 & Eale ooze & tr & & & & 10 & 1 & 8 & & & 81 & & & t \\
\hline 145 & falc ooze & 3 & tr & & & 27 & 6 & 55 & 2 & & 7 & & & 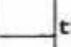 \\
\hline 220 & alc ooze & 5 & tr & & & 56 & 5 & 30 & $t x$ & & 4 & & & \\
\hline 250 & alc ooze & 2 & & & & 49 & 4 & 28 & 4 & & 3 & & & 1 \\
\hline 321 & alc ooze & 2 & 1 & & & 33 & 8 & 40 & 3 & & 5 & & & 8 \\
\hline & & & & & & & & & & & & & & \\
\hline & & & & & & & & & & & & & & \\
\hline & & & & & & & & & & & & & & \\
\hline & & & & & & & & & & & & & & \\
\hline & & & & & & & & & & & & & & \\
\hline & & & & & & & & & & & & & & \\
\hline & & & & & & & & & & & & & & \\
\hline & . & & & & & & & & & & & & & \\
\hline & & & & & & & & & & & & & & \\
\hline
\end{tabular}


597

VISUAL CORE DESCRIPTION

Page 1 of 1

Ship CHAIN Cruise $119 \quad 2$ Sta. 116 Core No. 23P6 Total Length $70 \mathrm{~cm}$. Lat. $33^{\circ} 30.5^{\circ} \mathrm{N}$ Long. $31^{\circ} 52.6^{\prime} \mathrm{E}$ Depth $2142 \mathrm{~m}$.corr.

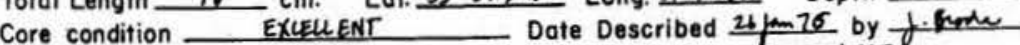
Physiographic location. ISIS RIDGE COMRLEX, NILE CONE. EASTERN MEO.

Lithologic

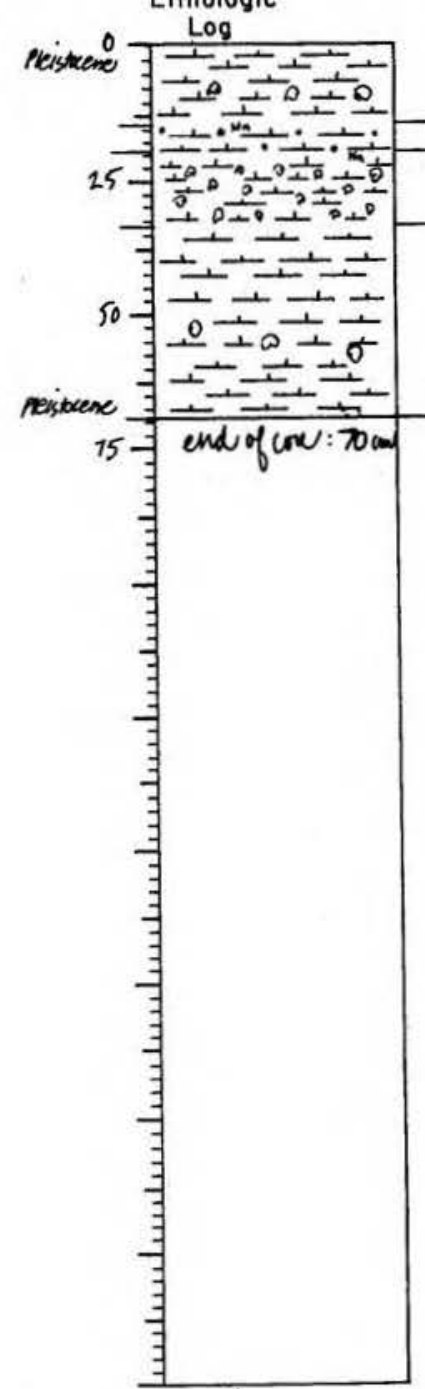

Detailed Description

$0-14$

CALC OOZE

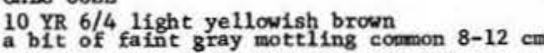
slightly silty lutite with scattered forams $\mathrm{s}$, horizontal

14-19

CALC OOZE WITH Yn MICRONODULES

10 YR $4 / 4$ dark yellowish brown smooth lutite with just a few forams s, horizontal

19-33 CALC OOZB

5 Y $3 / 2$ dark olive gray

intense, tiny, white and 1ight gray mottles and flecks throughout sapropel zone moist, slick lutite with scattered forams and white she11/pteropod fragment 33-70 CALC OOZE

10 YR $6 / 3$ pale brown, grades to $6 / 4$ light yellowish broun

comon, very pale brown mottling 50-64 $\mathrm{c}$

silghtly silty lutite with scattered forams and ashy pockets
end of core
598

SHEAR SLIDE DESCRIPTIONS - W.H.O.I. SEDIMENT CORES

Ship: _ CHAIN

Expedition 119

Core No.

$23 \mathrm{PG}$

Leg №. 2

Station No. 116

Total Core Length $\quad 70$

\begin{tabular}{|c|c|c|c|c|c|c|c|c|c|c|c|c|c|c|}
\hline \multirow[b]{4}{*}{ LEVEL } & \multirow[b]{4}{*}{$\begin{array}{c}\text { SEDIMENT } \\
\text { TYPE }\end{array}$} & \multicolumn{13}{|c|}{ ESTIMATED ABUNDANCES ( $(\%)$} \\
\hline & & \multirow{2}{*}{\multicolumn{4}{|c|}{$\begin{array}{c}\text { Inorganic Material } \\
\text { Silt \& Sand }\end{array}$}} & \multirow[b]{3}{*}{$\frac{\pi}{0}$} & \multirow{2}{*}{\multicolumn{5}{|c|}{$\begin{array}{l}\text { Biogenous Mater } \\
\text { Calcareous }\end{array}$}} & & & \\
\hline & & & & & & & & & & & & \multicolumn{3}{|c|}{ Siliceous } \\
\hline & & $\frac{5}{5}$ & 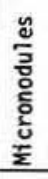 & 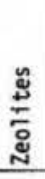 & 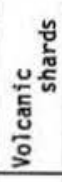 & & 薦 & 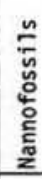 & 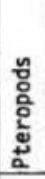 & 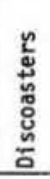 & $\frac{n}{2}$ & 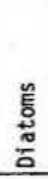 & \begin{tabular}{|l}
$\frac{\pi}{2}$ \\
$\frac{\pi}{0}$ \\
$\frac{\pi}{\sigma}$ \\
$\frac{\pi}{\alpha}$
\end{tabular} & 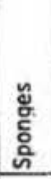 \\
\hline 1 & calc ooze & 2 & 3 & & & 23 & 8 & 55 & 4 & & 5 & & & \\
\hline 16 & $\begin{array}{l}\text { calc ooze w/Man } \\
\text { micronodules }\end{array}$ & 2 & 15 & & & 41 & 3 & 35 & tr & & 4 & & & \\
\hline 25 & cal'c ooze & 1 & & & & 18 & 7 & 55 & 3 & & 4 & & & \\
\hline 69 & calc ooze & 2 & tr & & 10 & 26 & 4 & 50 & 2 & & 6 & & & \\
\hline & & & & & & & & & & & & & & \\
\hline & & & & & & & & & & & & & & \\
\hline & & & & & & . & & & & & & & & \\
\hline & & & & & & & & & & & & & & \\
\hline & & & & & & & & & & & & & & \\
\hline & & & & & & & & & & & & & & \\
\hline & & & & & & & & & & & & & & \\
\hline & & & & & & & & & & & & & & \\
\hline & & & & & & & & & & & & & & \\
\hline & & & & & & & & & & & & & & \\
\hline & & & & & & & & & & & & & & \\
\hline & & & & & & & & & & & & & & \\
\hline & & & & & & & & & & & & & & \\
\hline & & & & & & & & & & & & & & \\
\hline
\end{tabular}


Ship CHAIN Cruise 119 Leg 2 Sta. 118 Core No. 24 PC Total Length 7111 cm. Lat. $33^{\circ} 51.6^{\prime} N$ Long. $31^{\circ} 52.4^{\prime} E$ Depth $2636 \mathrm{corrm}$ Core condition EXceweNT Date Described $31 \operatorname{Tan} 76$ by TFARER Physiogrophic location ABYSSAL HILLS JUE W. of ERATOSTHENES SEAMEUNT, EMS. Lithologic

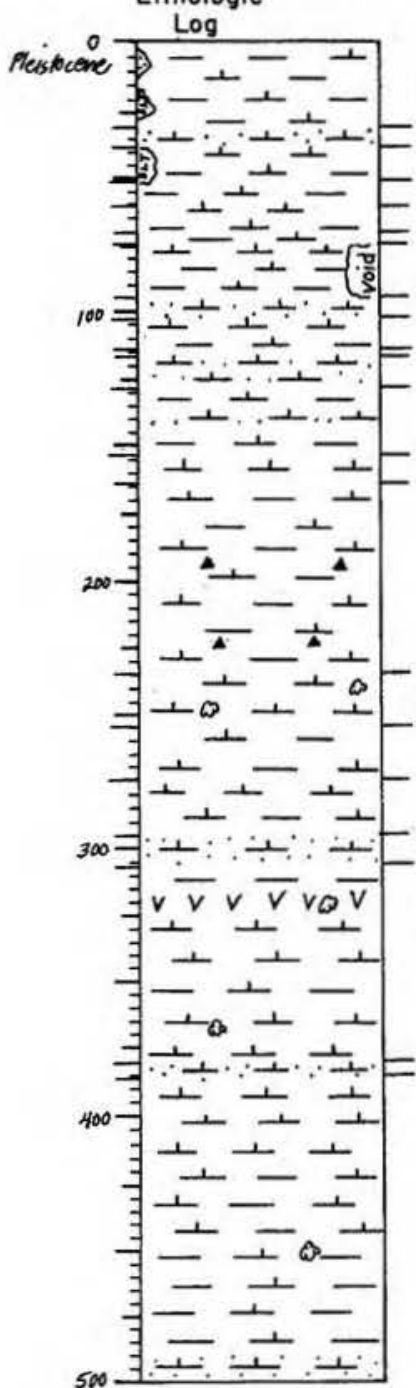

Detailed Description

$-30$

CALC CIAY

10 YR $4 / 2$ dark grayish brown

homogeneous, except for two silt clasts along side of core liner, and a few, pale brown marbles indicating smooth, slick, unconsolidated lutite $\mathrm{s}$
-38

CAIC OOZE/DETRTTUS

10 YR $4 / 2$ dark' grayish brown

ogeneous throughout

silt

38-51

CAIC OOZE OVERLIES CALC CLAY

10 YR $6 / 3$ pale brown $(1 \mathrm{~cm})$, followed by 2.5 × $4 / 4$

olive brown

minor mottling of pale brown into olive brown; dark grayish brown, silty lutite clast extends along

silty lutite with pteropods common (pale brown) over-

lies a smooth, slick, unconsolidated lutite

51-61

AIC CIAY

10 YR $4 / 2$ dark grayish brow

homogeneous throughout

consolidated lutite $\mathrm{s}$
-70

CALC OOZE OVERLTES CATC CTAY

10 YR $6 / 3$ pale brown $(1 \mathrm{~cm})$ overlies 2.5 Y $4 / 4$ olive brown

minor mottling of pale brown into olive brown

silty lutite with common biogenous grains (pale brown) $\mathrm{s}$

CALC CLAY

10 YR $4 / 2$ dark grayish brown

smooth, slightly silty, unconsolidated lutite

4-95

CALC OOZE OVERLIES CALC CLAX

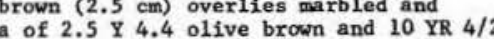

dark grayish brown

$5007 \div \div \div$
Ship CHAIN Cruise 119

Leg 2 Sta. 118 Core No. $24 \mathrm{PC}$

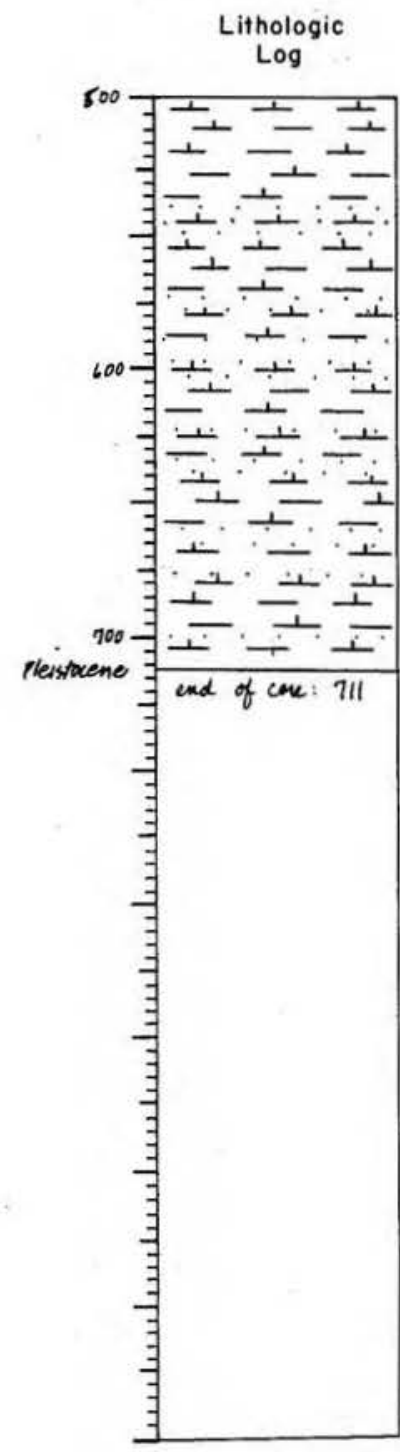

\section{Detailed Description}

74-95 (cont.)

cattered mottling of pale browns in rest of unit to $f$ ll a s all vold in core a resite of aly lity lutite with pteropods and

and forams common (pale brown)

95- 103

CALC OOZE/DETRITUS

10 YR $4 / 2$ dark grayish brown

no mottling

grades to silty lutite in last $2 \mathrm{~cm}$

103-114

CALC OOZE OVERLIRS CALC CLAY

10 YR $6 / 3$ pale brown $(2 \mathrm{~cm}$ ) overlies 2.5 Y $4 / 4$ olive brown

minor mottling of pale brown into olive brow

silty lutite with comon blogenic grains (pale brown) $s$

114-116

10 YR $4 / 2$ dark graylsh brown

homogeneous throughout

smooth, slick, unconsolidated lutite

116-128

CALC OOZE/DETRITUS

10 YR $4 / 2$ dark grayish brown

no mottling

thery silty lutite

128-153

HIGHLY CAIC CLAY GRADES TO CALC OOZE/DETRITUS AND BACK TO HIGHLY CALC CIAY

no mottling

smooth, slick lutite, grades to silt at $143 \mathrm{~cm}$ and back 53-16 to very silty lutite

10 YR $6 / 3$ pale brown with a hint of $6 / 2$ light brownish gray

ainor subdued lnternotting

slightly silty lutite; forams and pteropods are common 
601

Page 3 of 4

VISUAL CORE DESCRIPTION

Ship CHAIN Cruise

119

Leg II Sta. 118 Core No. $24 P C$

Lithologic

Log

\section{Detailed Description}

164-235

HIGHLY CALC CLAY INTERBEDDED WITH HIGHLY CALC CIAY 10 YR $4 / 2$ dark grayish brown and 5 Y $3 / 2$ dark olive

gray
the slick lutites are generally homogeneous, excepting a few, tiny, black mottles 184-194; some of the sapropels show subdued lamination

mooth, slick latite lnterbedded with silty, humuscommon in the sapropels; $198-202 \mathrm{~cm}$, silt bed with gradational contacts $\mathrm{S}$

$\stackrel{\text { s }}{235-255}$

$10 \mathrm{YR} 5 / 2$ grayish brown, grades to $6 / 3$ pale brown

intermotting is comon

$255-275$

HIGHLY CALC CLAY

2.5 Y $4 / 2$ dark grayish brown

a scattering of pale brown mottles appeax in two

clusters s

275-295

10 YR $5 / 3$ brown, grades to 2.5 Y $4 / 2$ dark grayish

brown

few pale mottles

silty lutite, with coumon bioclastic material $(2 \mathrm{~cm})$ $\mathrm{s}^{\mathrm{g}}$

295-307

CALC OOZE/DETRTTUS

2.5 Y $4 / 2$ dark grayish brown

no mottling

S
$07-380$

CAIC OOZE WITH MINOR AYSOUNT OF HIGHLY CALC CLAY

10 YR $6 / 3$ pale brown, $5 / 3$ brown, and minor amount or 4/2 dark grayish brown; above colors appeax ln macrolaminated unit with the darkest color internteling ws cattered through

for collor cor very $319-323 \mathrm{~cm}$ volcanic ash

$G$ (texturally)

602

VISUAL CORE DESCRIPTION

Page 4 of 4

Ship CHAIN Cruise 119 Leg I Sta. 118 Core No. 24 PC

Lithologic

Log

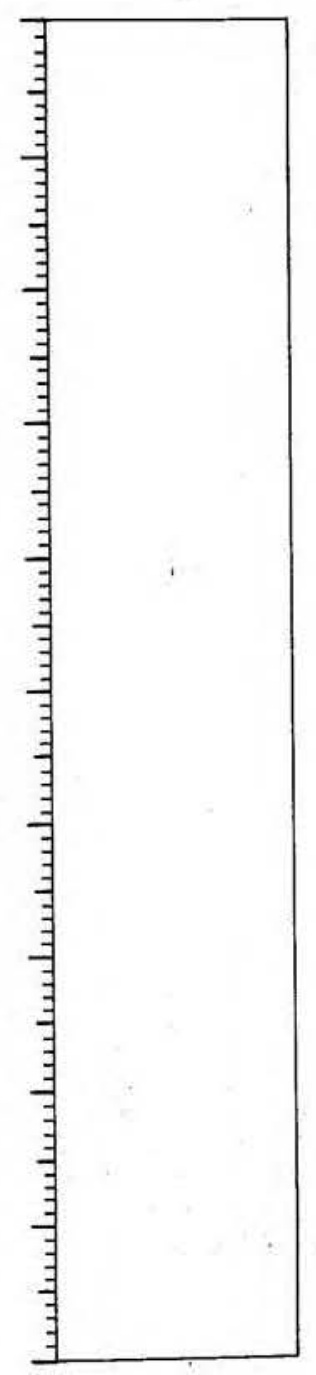

\section{Detailed Description}

380-386

CAIC OOZE/DETRITUS

10 YR $4 / 2$ dark grayish brown

very, very silty lutite with several distorted, pale, s

CAIC OOZE (PALE LAMTNATIONS), GRADES TO HICHLY CALC CIAY, CALC CIAY, AND PINALLY CALC OOZE/DETRITUS 10. YR $5 / 3$ and $4 / 3$ browns, and lesser amounts of $6 / 3$ pale brown, and $4 / 2$ dark grayish brown

these colors appear in repeated cycles of macrolaminations, generally grading from light to dark colors: mottling is scattered here and there throughout, but

the pale browns abundant at top are generally silty lutites with forams and pteropods tairly common: the the repeated lutite cycles commonly end with a laminated, grayish brown silt; these occur at 490-500, 539$550,570-576,580-583,593-603,621-624,633-641,658-$ $666,673-677,699-702$

contacts between laminations are generally sharp, although occasionally disturbed: $695-700 \mathrm{~cm}, 5 \mathrm{Y} 3 / 2$ $679 \mathrm{~cm}$, thin bed of shell hash

of NOTE: After comparison with the pilot core, it seems
likely that the piston core missed the top 70$75 \mathrm{~cm}$ of sediment. 


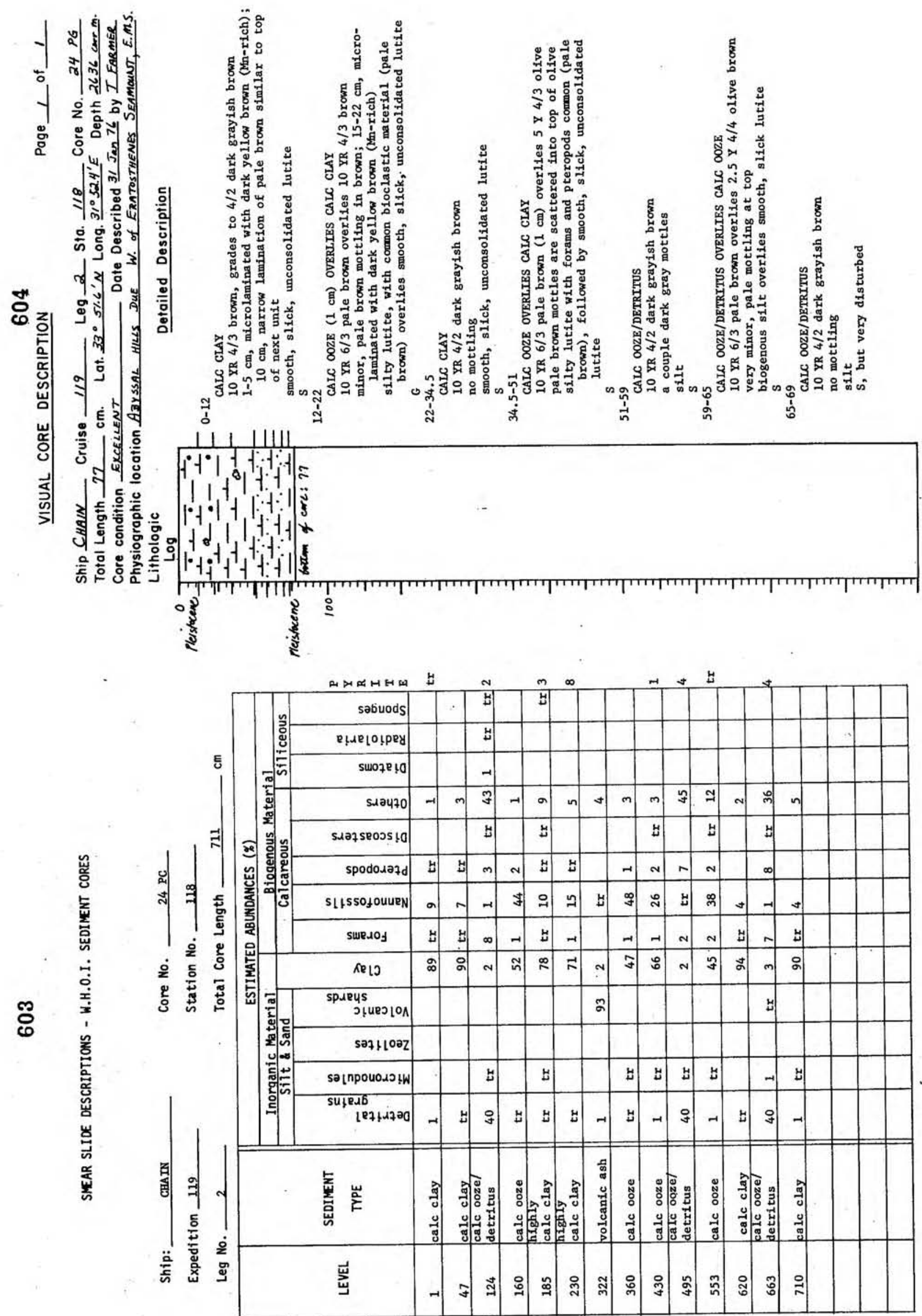


605

VISUAL CORE DESCRIPTION

Ship CHAIN Cruise 119 Leg II Sta. 118 Core No. 24 PG

Lithologic

Log

Detailed Description

69-73

HICHIY CALC CLAY

5 Y $4 / 3$ olive

smooth, slick lutite

73- 77

CAIC CLAY

10 YR $4 / 2$ dark grayish brown

homogeneous throughout

smooth, silick lutit

Poge 2 of 2

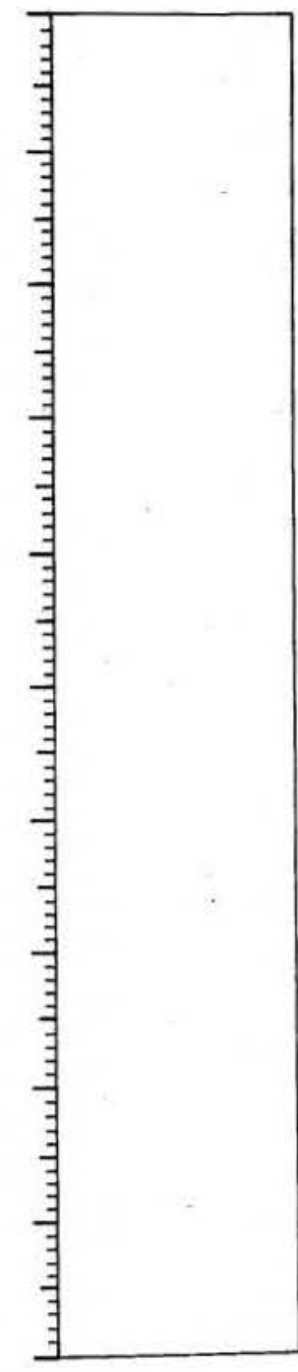

606

SMEAR SLIDE DESCRIPTIONS - W.H.O.I. SEDIMENT CORES

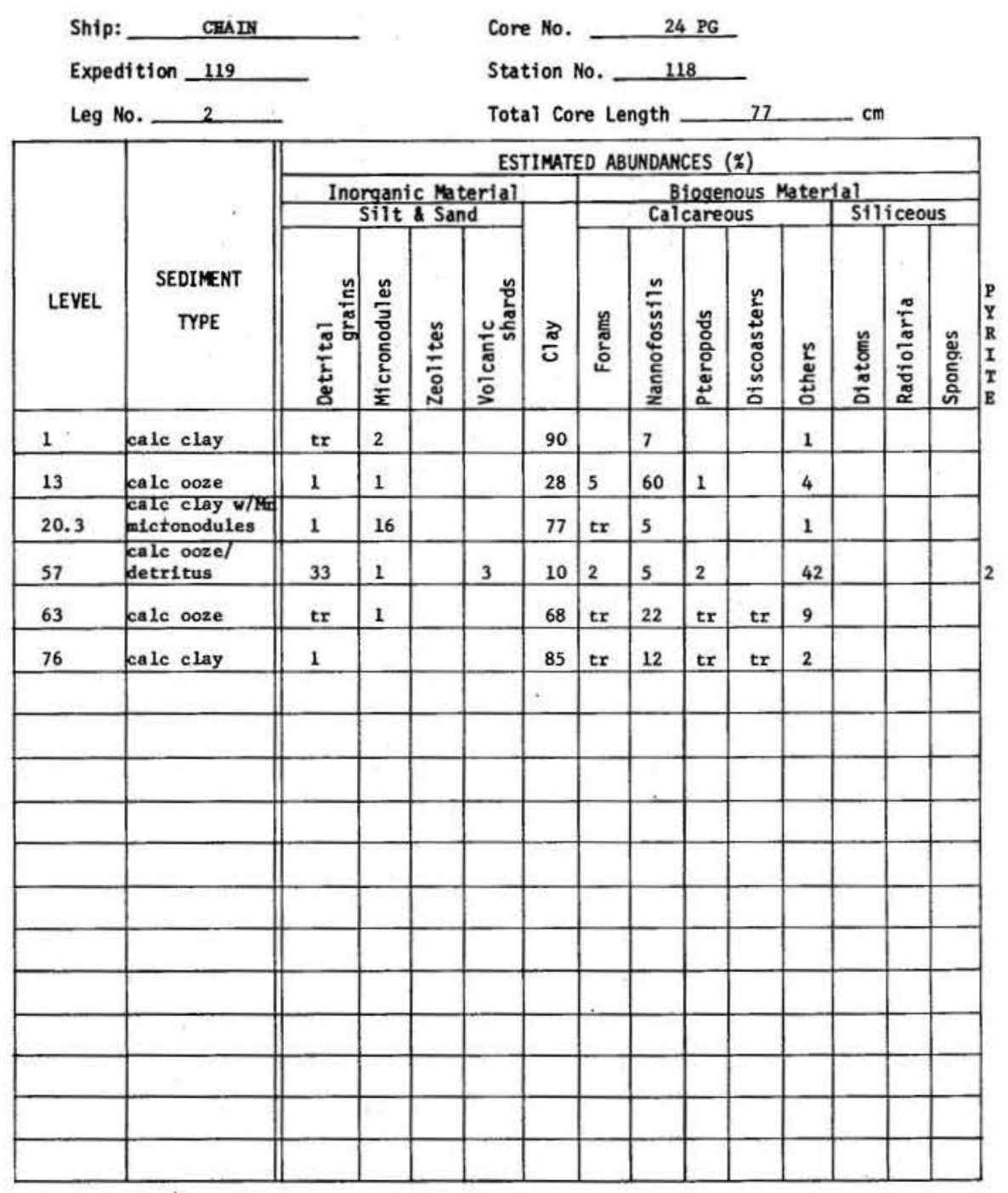




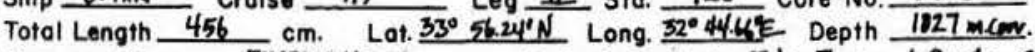
Core condition EXCEUENI Date Described $27 \mathrm{pm} \pi$ by f.Phola Physiographic location. NGRTH FIANR OF ERATES THENES GEAMOUNT, EXSERN MED Lithologic

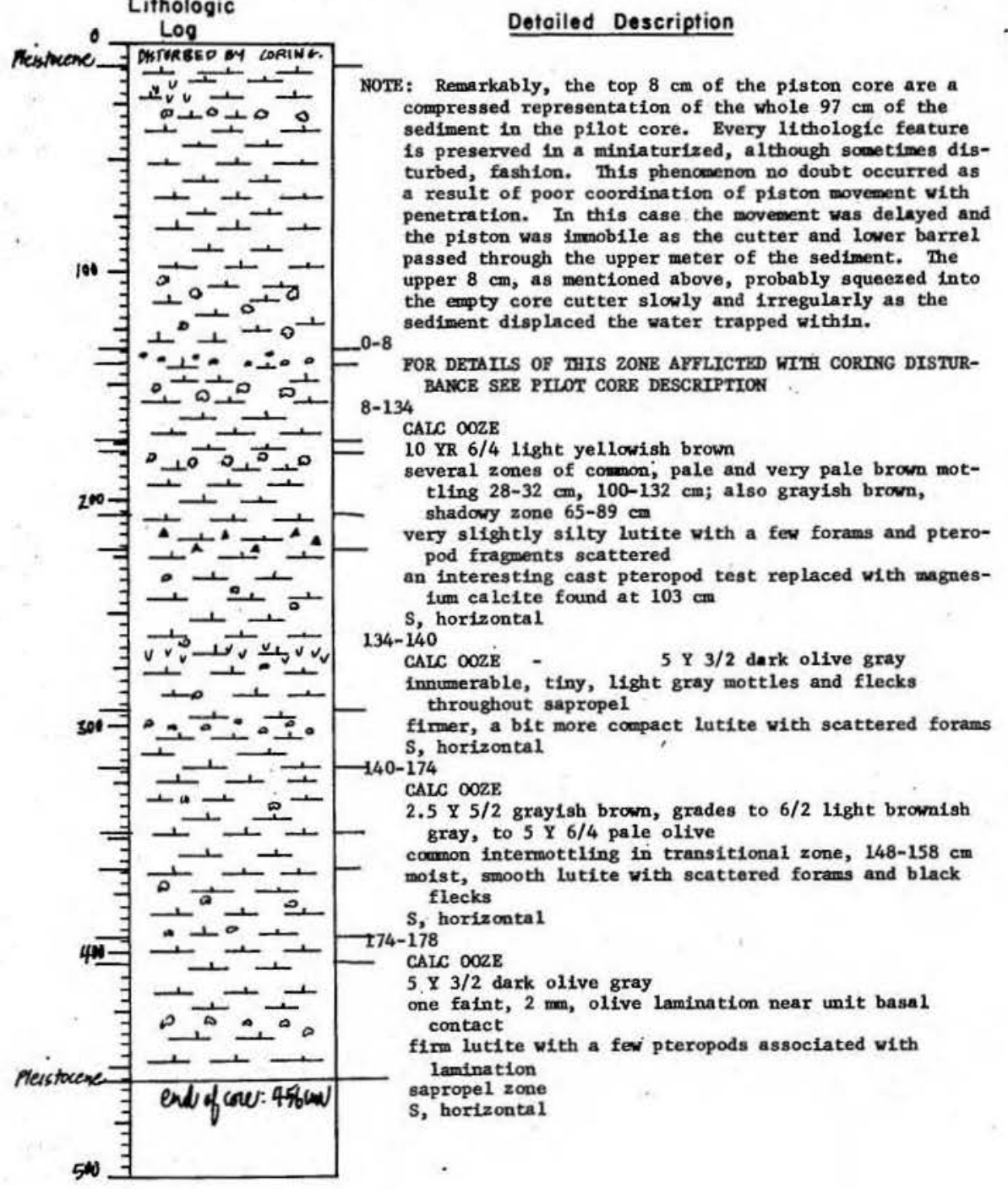

Lithologic$$
\text { Log }
$$

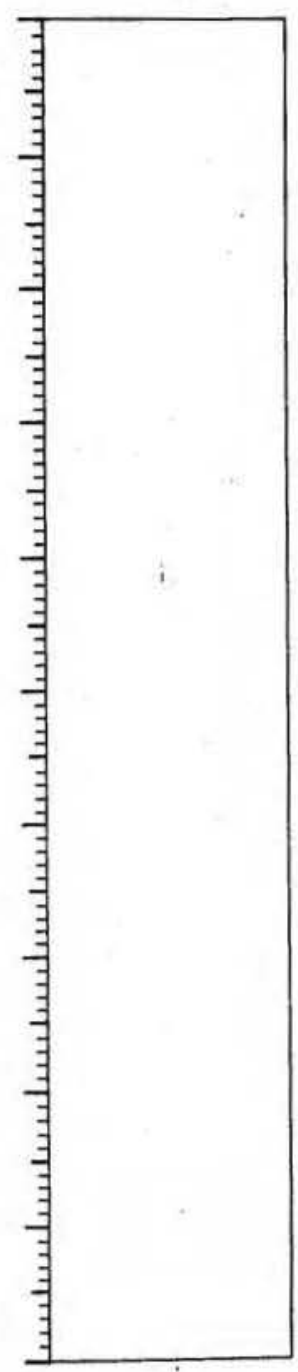

\section{Detailed Description}

178-206

2.5 × $5 / 2$ grayish brown, grades to $6 / 2$ light browntsh coumon intermottling in transitional zone, 184-188 cm slightly silty lutite with scattered forams, pteropod $S$, horizontal

CALC OOZB WITH PYRTTE

fine, light gray laminations and flecks $206-212 \mathrm{~cm}$; two $1 \mathrm{~mm}$ laminations at $221.5 \mathrm{~cm}$ somewhat coarse, mulchy lutite with abundant forams and scattered pteropods $S$, horizontal

222-293

CALC OOZE WITH A ZONE OP CALC OOZE/ASH

5 Y $7 / 2$ light gray, grades to $7 / 3$ pale yellow

a few faint, light gray mottles and burrows scattered throughout

inm, becoming more compact, slightly silty lutite with scattered, white, lithified calc lumps and an ash stiff zone, $267-274 \mathrm{~cm}$

293-318 s, mottled

CATC OOZE

2.5 Y $4 / 2$ dark grayish brown, grades to 5 Y $3 / 2$ dark olive gray

extensive, grayish brown and ollve brown mottling 293$307 \mathrm{~cm}$; finely laminated thereafter

scmewhat coarse, mulchy lutite with abundant forams and pteropod fragments sapropel zone

$318-348$

CALC OOZE

5 Y $6 / 2$ light olive gray, grades to 2.5 Y $6 / 2$ light

brownish gray

slightly silty lutite in transition zone

S, irregular

CALC OOZE

5 Y $3 / 2$ dark olive gray

fine, thy, 1ight gray flecks and mottles

chy lutite with abundant forams and

$$
\text { sap }
$$


609

VISUAL CORE DESCRIPTION

Poge 3 of 3 Ship CHAIN Cruise 119 Leg II Sta. 120 Core No. 25 PC

Lithologic

Log

Detailed Description

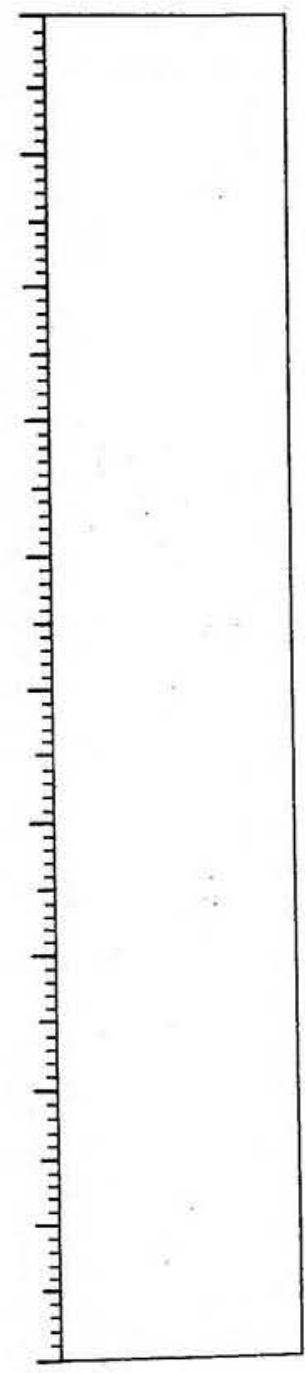

364-394

CALC OOZE

5 y $5 / 2$ ollve gray, grades to 2.5 × $6 / 2$ light grayish

smal1,

intermottling of above colors throughout lutite with scattered forams throughout, 394-405 CALC OOZE

5 Y $3 / 2$ dark ollive gray

tes $397-400 \mathrm{~cm}$

with abundant forams $\mathrm{s}$, horizontal

CALC OOZ

2.5 Y $5 / 2$ grayish brown, grades to $6 / 4$ 1ight yellowish brown

firm, slightly silty lutite with scattered forams NOT:

fyc above combinations of lithologies should not be overlooked
610

SMEAR SLIDE DESCRIPTIONS - W.H.O.I. SEDIMENT CORES

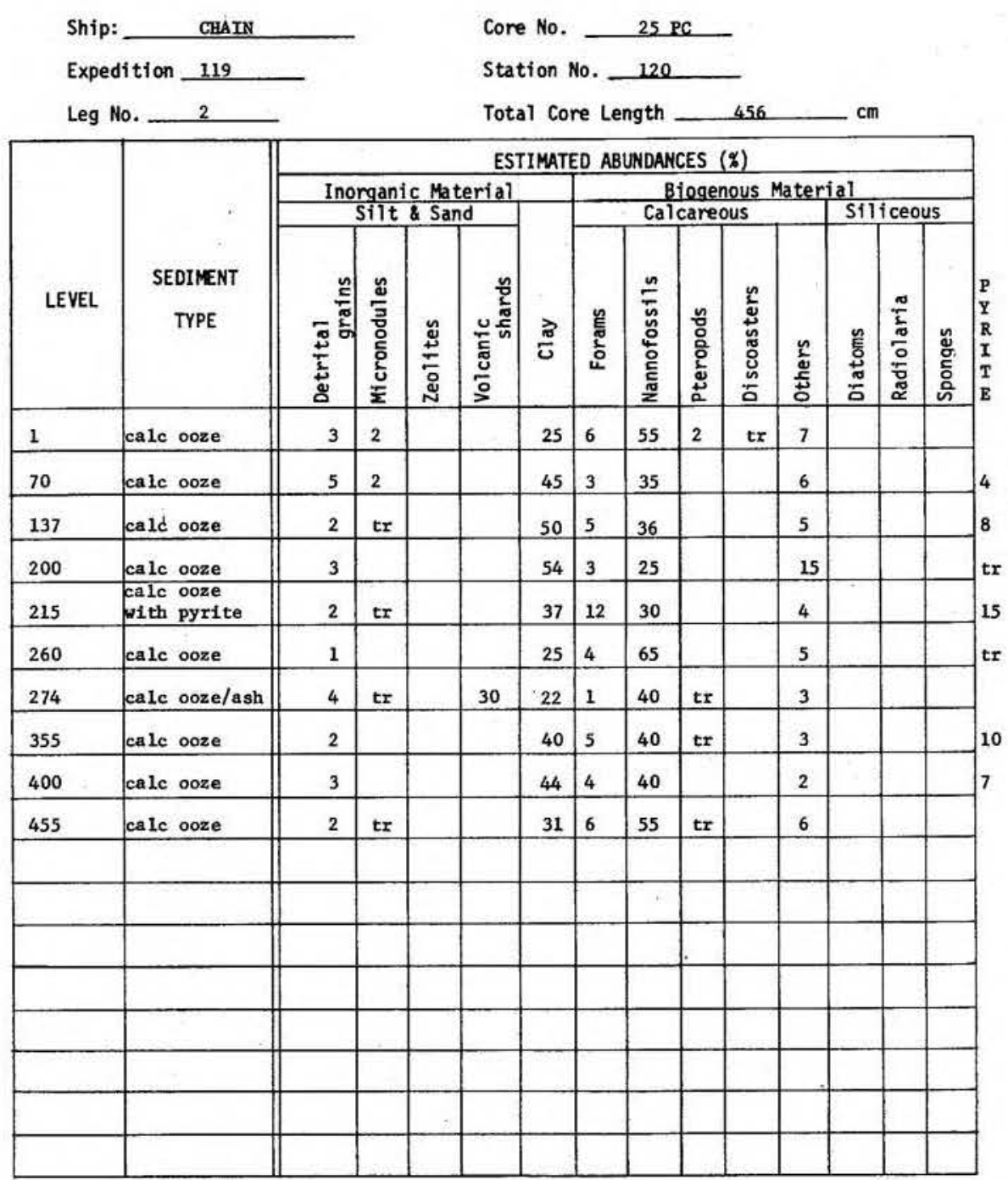


Ship CHAIN Cruise $119 \quad 120$ Core No $25 \mathrm{PF}$

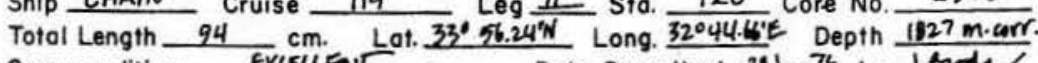
Core condition EXUEUEN Date Described 28/an 76 by forde Physiographic location NORTH FLANK OF ERATOSTHENES SEAmOVWT, EATTERN MEP. Lithologic

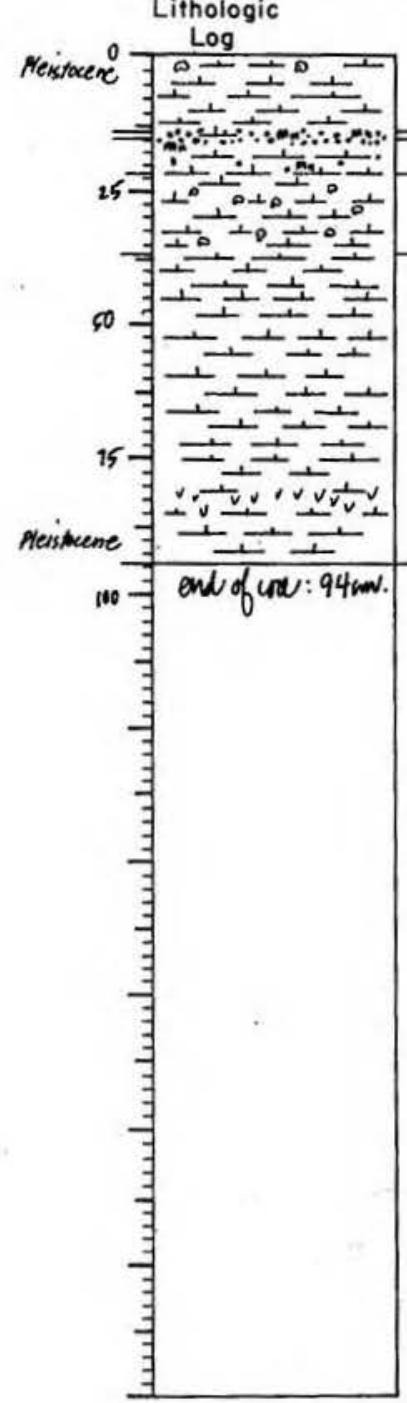

\section{Detailed Description}

$0-13$

CALC OOZE

10 YR $6 / 4$ light yellowish brow forams

13-14.5

10 YR $2.5 / 1$ black

homogeneous throughout (slight mixing at upper unit

contact)

slick and silty lutite combined

$14.5-21$

CAIC OOZE WITH Mn MTCRONODULES
10 YR $4 / 4$ dark yellowish brown

innumerable, fine, dark brown microlaminae throughout

smooth lutite with just a few forams

21-36

S, horizon tal

5 Y $3 / 2$ dark olive gray

ight gray mottles and flecks

moist, slick lutite with scattered forame

36-94

CALC OOZE WITH BRD OF CALC OOZE/ASH

YR $6 / 2$ light brownish gray, grades to $6 / 4$ light

yellowish brown

homogeneous throughout

silghtly silty latite with gcattered forams

somewhat graded ash bed $80-83 \mathrm{~cm}$ s
SMEAR SLIDE DESCRIPTIONS - W.H.O.I. SEDIMENT CORES

\section{Expedition 1 (1)}

Leg No.

119

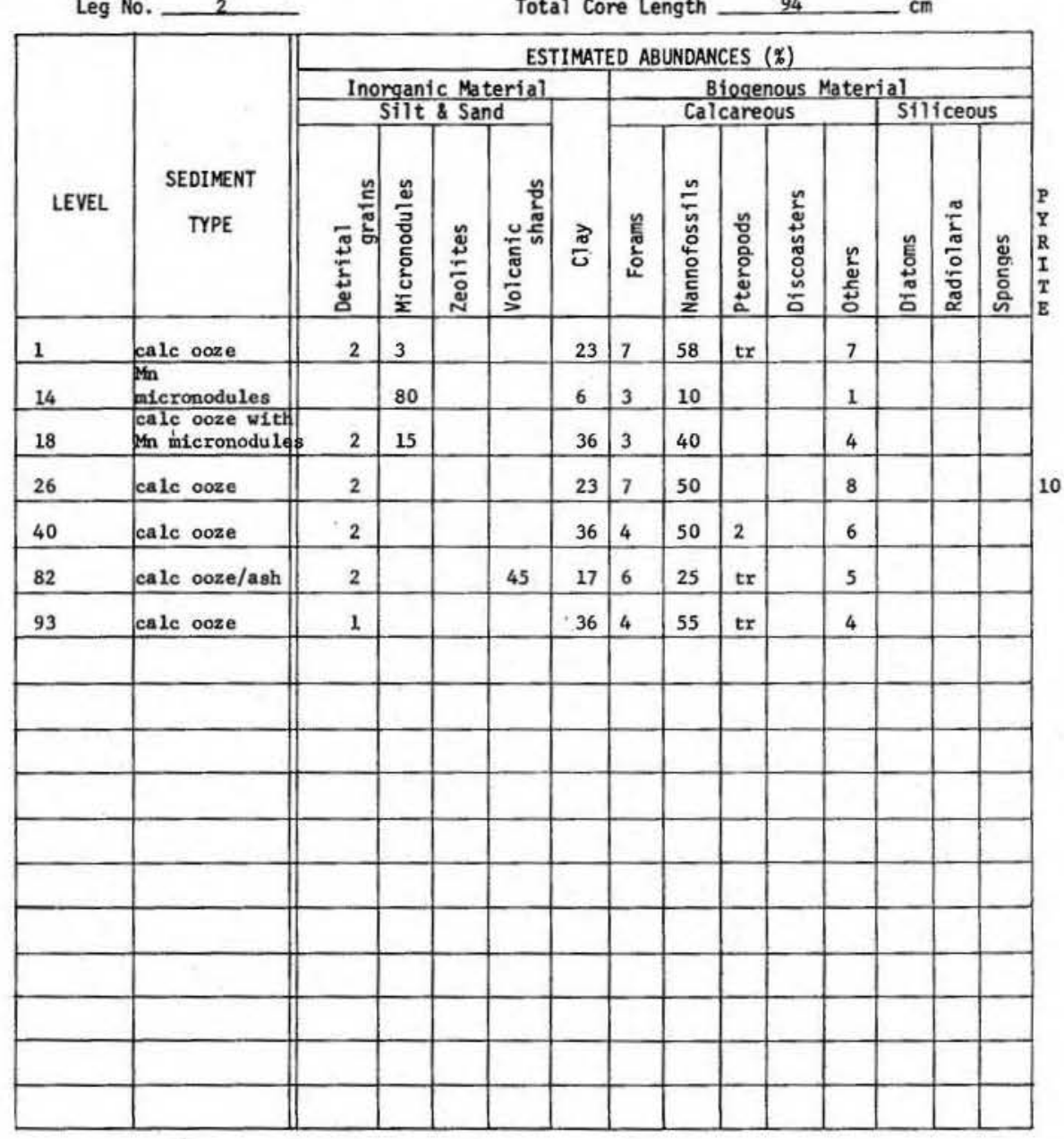

Core No. $25 \mathrm{PG}$

Station No. 1 
Ship CHAIN Cruise 119 Leg 2 Sto. 121 Core No. 26 pc Total Length $506 \mathrm{~cm}$. Lat. $33^{\circ} 44.3^{\circ} \mathrm{N}$ Long. $22^{\circ} 46.5^{\prime} E$ Depth $898 \mathrm{corm}$.

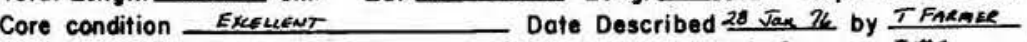

Physiographic location ERnTestuenes SEnmount south + Cypeus, EMS. Lithologic

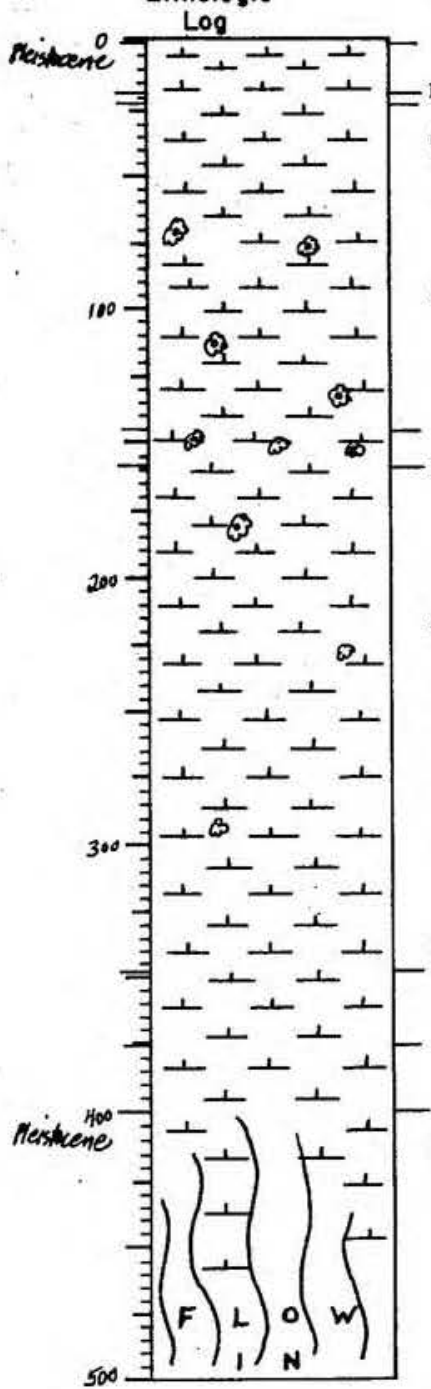

Detailed Description

Note: whole core has disturbed appearance indicated by

CALC OOZB

homogeneous throughout

very silty lutite, forams are common

$1-18$

CALC OOZE

YR $6 / 4$ light yellowish brow

very silty Iutite, forams are $s$, but disturbed

18-22

CALC OOZE

10 YR $6 / 3$ pale brown

no mottling

very, very silty lutite; becomes foram sand from $20-21 \mathrm{~cm}$ $22-145$

CALC OOZE

10 YR $7 / 3$ very pale brown, $6 / 4$ light yellow brown

contorted laminations $(\sim 0.5-4 \mathrm{~cm})$ in varying hues of

above colors appear throughout; occasional lamina-

tions of $6 / 6$ brownish yellow appear in lower portions:

small, scattered, black mottles (m-rich) are present from $68-79 \mathrm{~cm}$ and $105-145 \mathrm{~cm}$

generally a very sulty lut $c$, with

patan

al piece of coral (?) recovered at $92 \mathrm{~cm}$

$\mathrm{S}$, but heavily mottled

CALC OOZE

10 YR $7 / 4$ very pale broun

heavily mottled with above unit; sma11, black mottles

(Mn-rich) are also scattered throughout

silty, compacted, plastic-like lutite; forams are $\mathrm{S}$, but mottled

158-348

\section{CALC OOZE}

10 YR $6 / 4$ 11ght yellowlsh brown, and $6 / 3$ pale brown;

$7 / 4$ and $7 / 3$ very pale browns become more prominent

with depth

VISUAL CORE DESCRIPTION

Page 2 of 2

Ship CHAIN Cruise 119 Leg 2 Sta. 121 Core No. 26 PC

Lithologic

Log

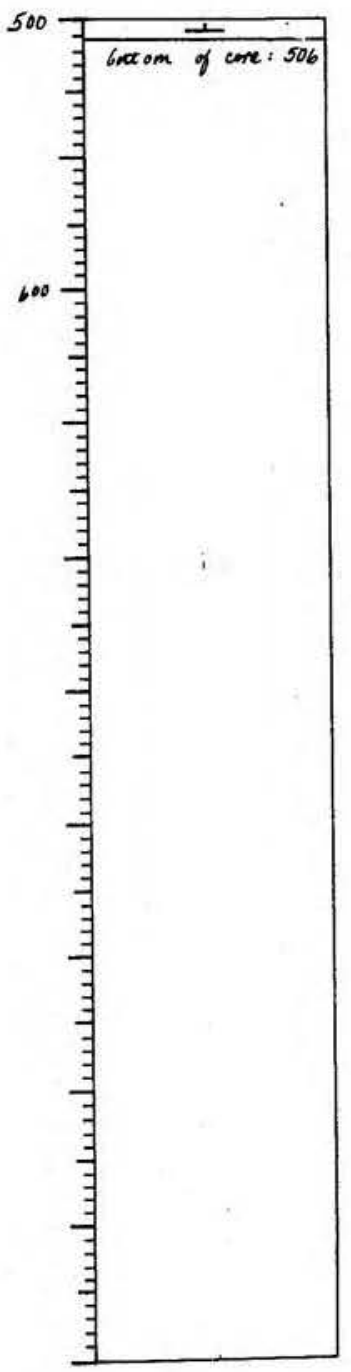

\section{Detailed Description}

$158-348$ (cont.)

ormal and contorted laminstions, and scattered intermoting of above colors appear throughout; small, (to tiff lutite, varies from silty to very silty as for abundant

$S$, but severely disturbed

8-376

CALC OOZE

2.5 Y $7 / 4$ pale yellow, grades to $6 / 6$ olive yellow no mottling

very silty lutite; forams are common S

CALC OOZE

5 Y $5 / 3$ olive

several subdued, yellowish laminae from above inter-

bedded with upper portions of this unit

very firm, very silty lutite; forams are scattered to 400-506

flow in of above unit

end of core 
SNEAR SLIDE DESCRIPTIONS - W.H.0.1. SEDIIENT CORES

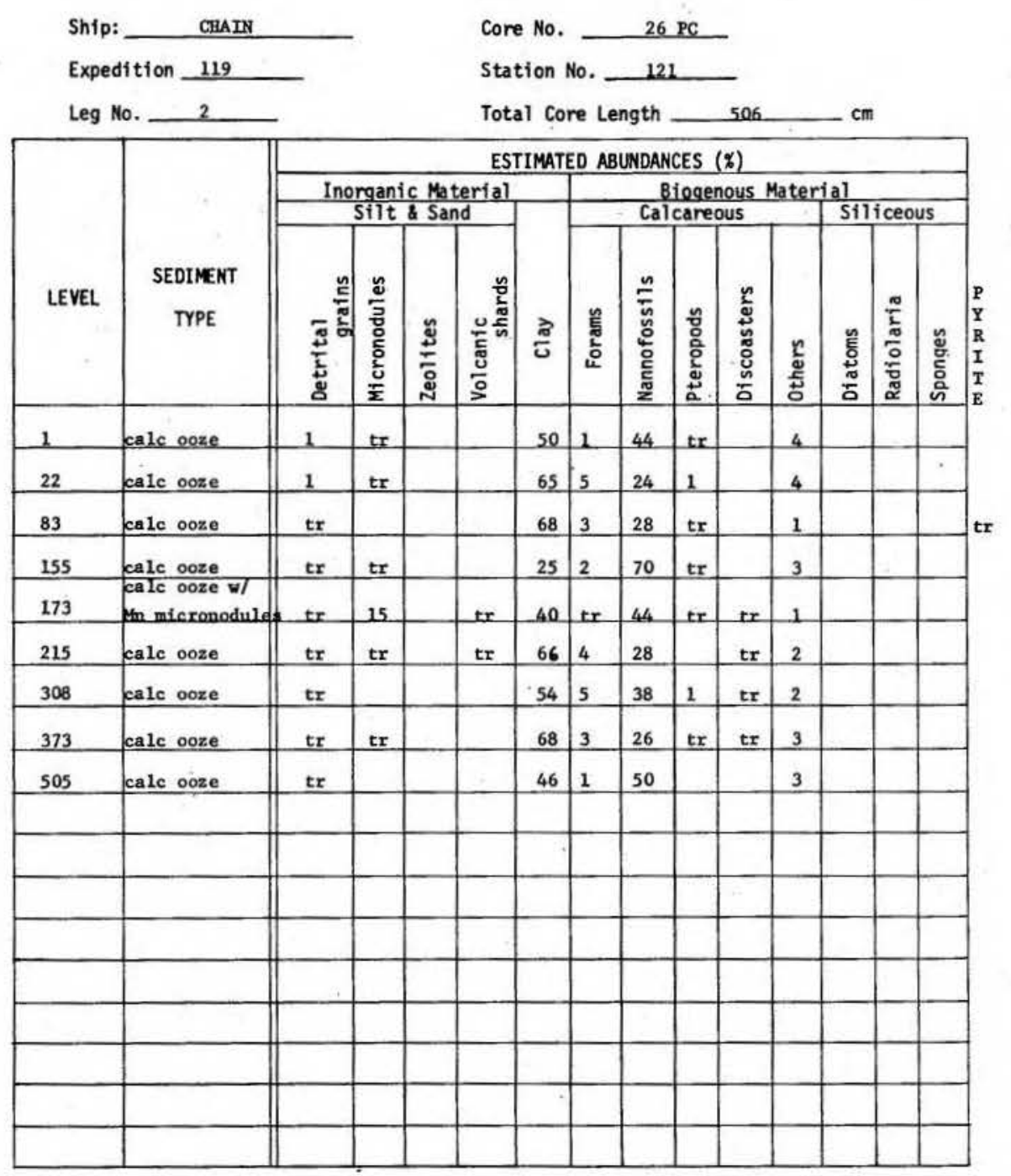

Ship CMAN Cruise 119 Leg 2 Sta. 121 Core No. 26 P6 Total Length $77 \mathrm{~cm}$. Lat. $33^{\circ} 443^{\prime} \mathrm{N}$ Long. $32^{\circ} 46.5^{\prime} \mathrm{E}$ Depth $898 \mathrm{carr} \mathrm{m}$. Core condition Exceuent Physiographic location Eeatestuenes SEAmawT Sourh of Cypeks, E.M.S. Lithologic

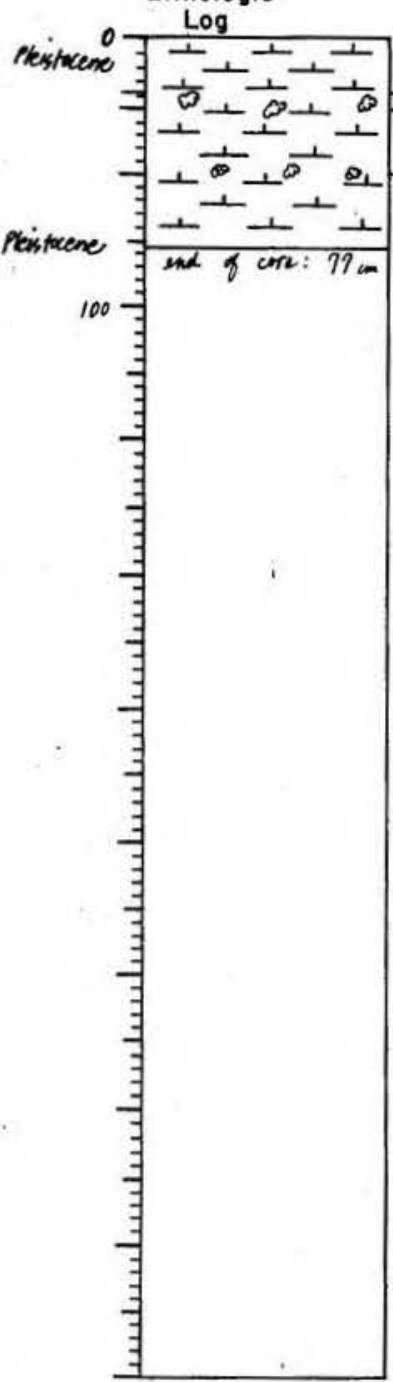

Detailed Description

$0-19$

\section{CALC OOZE}

portion, and darker

slight, pale mottling in uppe

silty lutite with scattered foram

19-25

\section{CALC OOZE}

YR $4 / 4$ dark yellowish brown silty lutite, sma11, pale mottles

25-50 CALC OOZE

10 YR $6 / 3$ pale brow

47-50 cm extremely intense, gray1sh brown (10 YR 5/2) mottling; some subtle marbling of paler hues indicates silty lutite, forame are cor

50-77

CALC OOZE

10 YR $7 / 3$ very pale brown, grades to $6 / 3$ pale brown scattered intermottling of pale browns very silty, plastic-like lutite, becomes somewhat les dite with depth 
SIEAR SLIDE DESCRIPTIONS - W.H.O.I. SEDINENT CORES

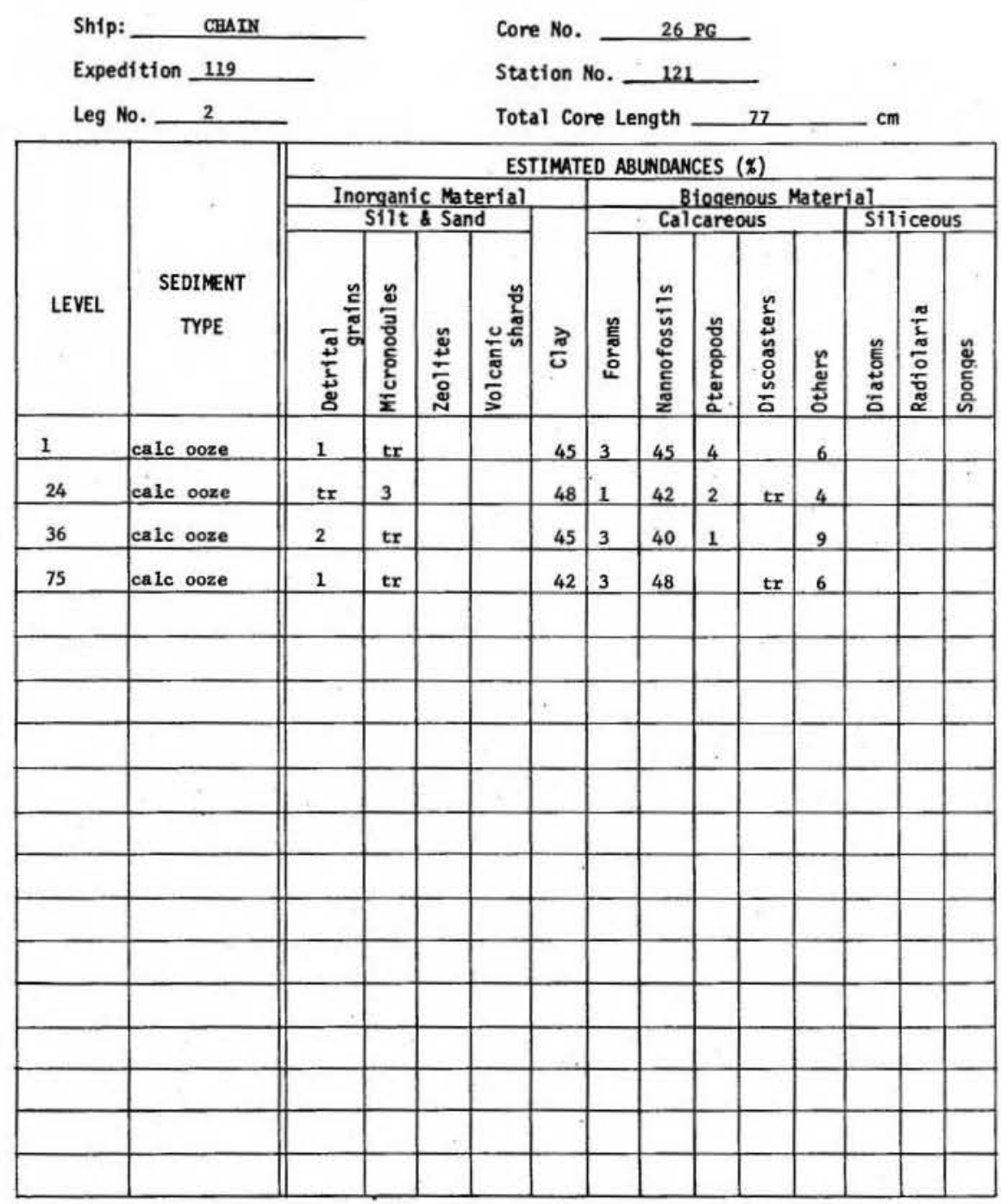

Ship CHAIN Cruise 119 Leg 2 Sta. 122 Core No. 6 GC Total Length $46 \quad \mathrm{~cm}$. Lat. $33^{\circ} 45.7^{\prime} \mathrm{N}$ Long. $32^{\circ} 48.0^{\prime} E$ Depth $894 \mathrm{~cm} . \mathrm{m}$

Core condition exallent Lat Lescribed $19 \mathrm{sed} 75$ by H. Faneres

Physiographic location ERATOSTHENES SEAMOUNT - EASTERN MED. SEA Lithologic

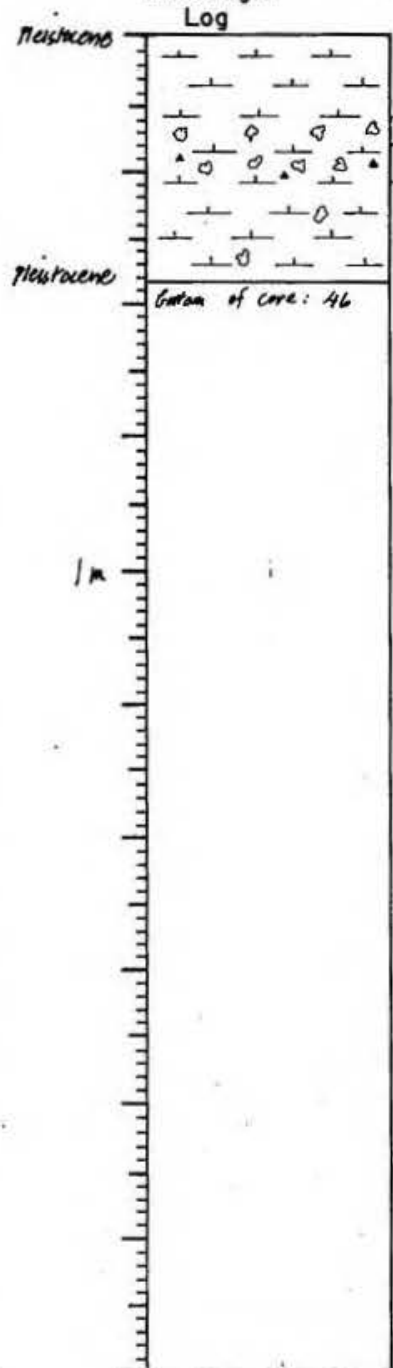

\section{Detailed Description}

$0-14$

NANNO OOZE

10 YR $5 / 6$ yellow brown

no mottling

slightly silty lutite with scattered forams

mottled contact from 12-14

CALC OOZE

10 YR $4 / 4$ dark yellow brown; $18-20$ primarily yellow

brown

sma11, abundant mottling with upper unit slightly silty lutite with scattered foram

20-27

CALC OOZE

IR $3 / 3$ dark brown

brown mottling

with few forams

27-46

CAIC OOZE

grades from 10 YR $5 / 3$ brown to 10 YR $6 / 3$ pale brown few, small pale brown and dark brown mottles silghty silty lutite with few forams pedimin subdued patches 
SMEAR SLIDE DESCRIPTIONS - W.H.0.I. SEDIMENT CORES

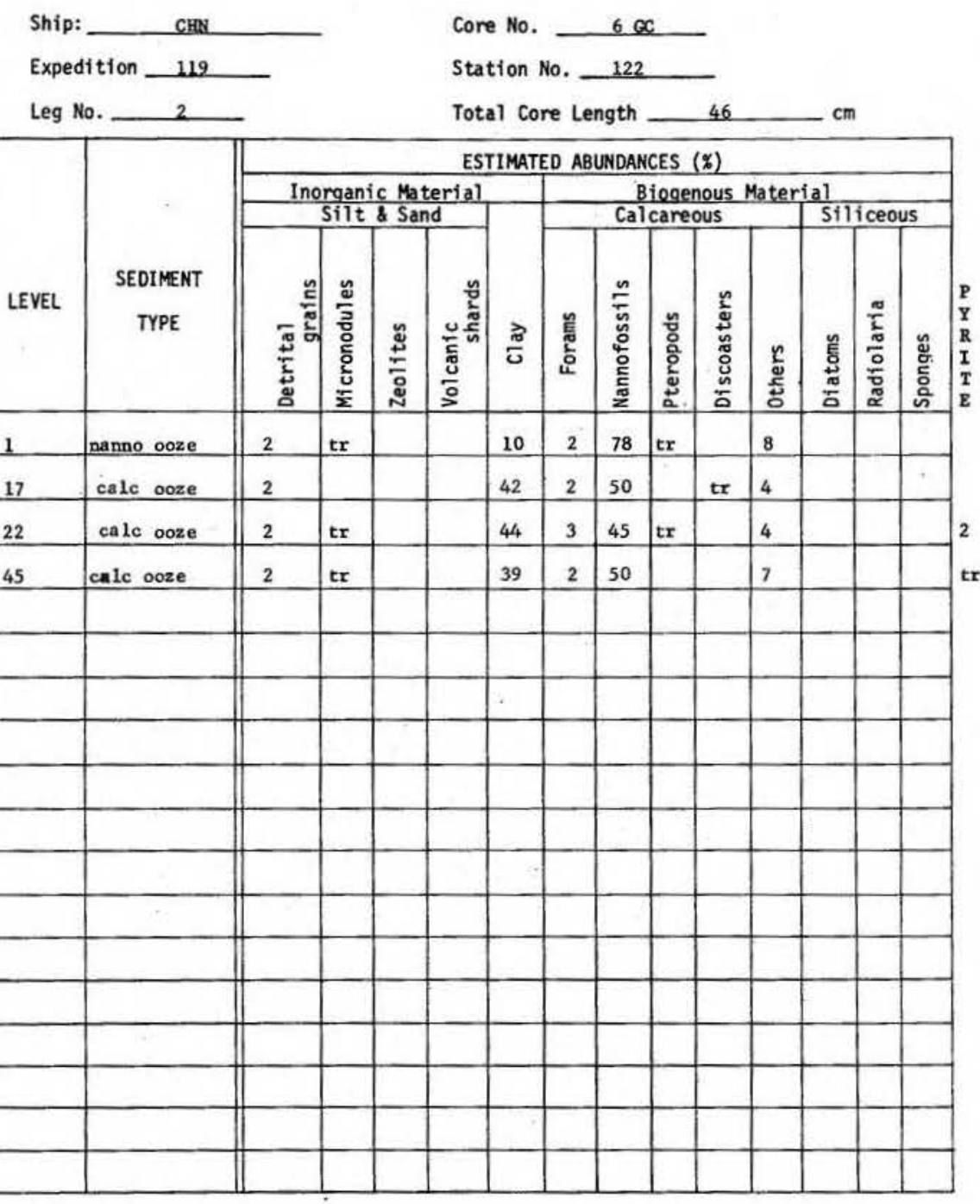

Ship CNAIN Cruise 119 Leg 2 Sto. 127 Core No. $7 \mathrm{AC}$ Total Length 43 cm. Lat. $33^{\circ} 40.7^{\prime} \mathrm{N}$ Long. $33^{\circ} 40.9^{\prime}$ ' E Depth 882 corcm Core condition ereullent Date Described is sept 75 by H. Fasmer Physiogrophic location ERATOSTHENES SERMOUNT - EASTERN MEDITERRANEAN SEA Lithologic

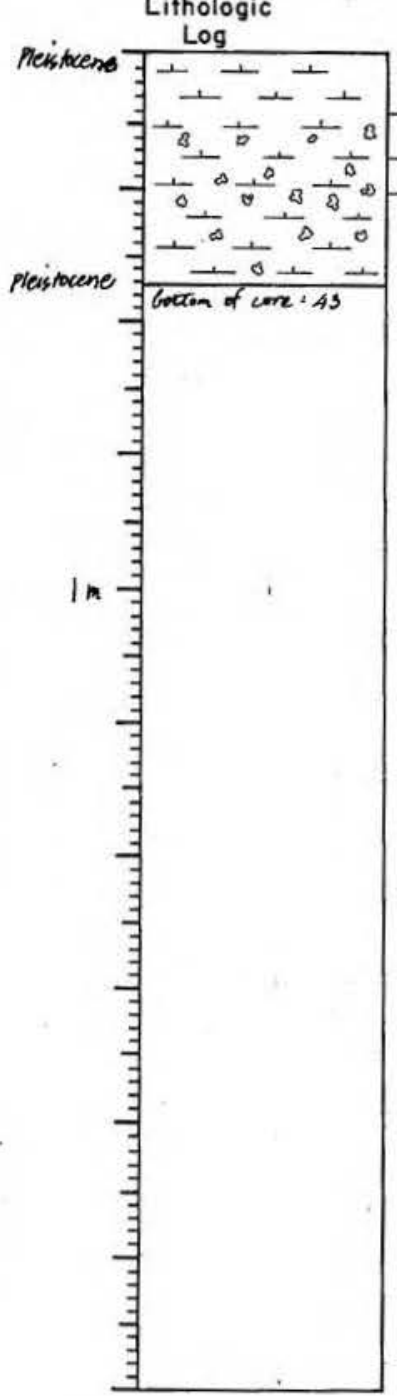

\section{Detailed Description}

$0-11$

MANEO OOZE

fR $5 / 6$ yellowish brown

lutite with scattered forams, common from $8-12 \mathrm{~cm}$

severely burrowed contact from $8-12 \mathrm{~cm}$

11-19

CALC OOZE

10 YR 4/4 dark yellow brown; 17-19 primarily yellow brown

small, coumon mottles of above colo utite, forams very abundant 12-13, scattered 18-19

$19-26$

CALC OOZE

10 YR $3 / 3$ dark brown

small, abundant (brown) mottles

gmooth lutite with few forams G, irregular

26-43 CALC OOZE

10 XR $5 / 3$ brown, getting somewhat paler at bottom small, abundant mottling at top, grading to few at lightly

作, forams 
SIEAR SLIDE DESCRIPTIONS - K.H.O.I. SEDINENT CORES

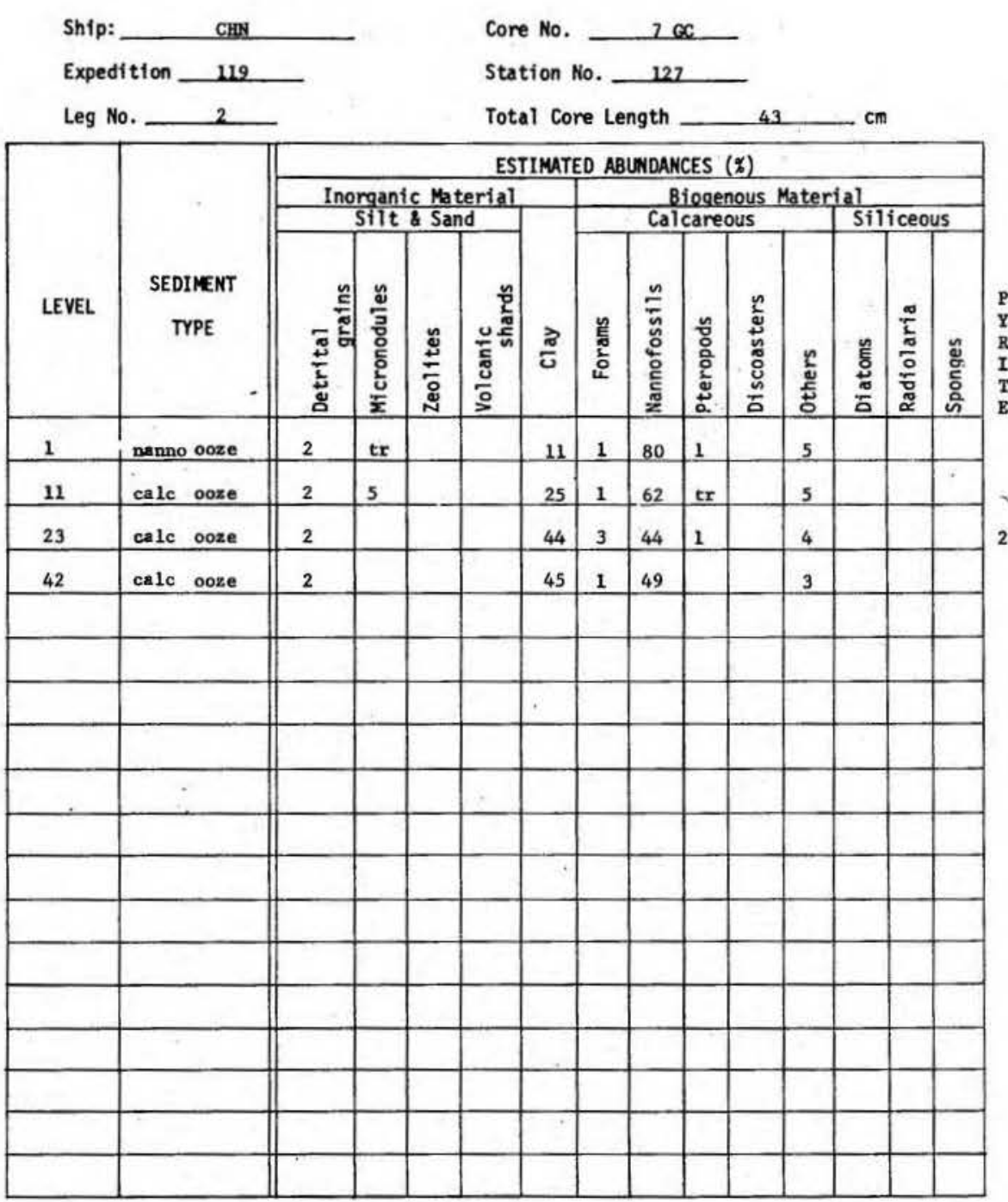

Ship CHAIN Cruise 119 Leg II Sta. 129 Core No. 27 PC

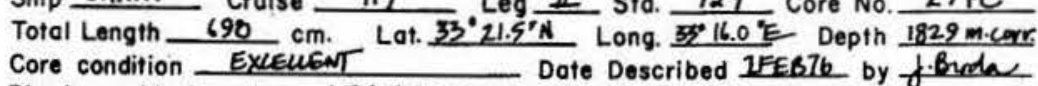
Physiographic location LEVAWT PLATFERM S SE OF ERATSTHENES SEAMOUNT. EATEFN hologic
Neistoceñ Detailed Description

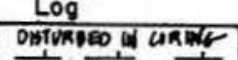

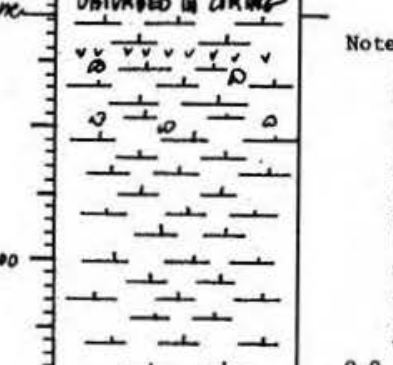

: As in core $25 \mathrm{PC}$ the top $7-8 \mathrm{~cm}$ of this piston a the the pllot core. Every

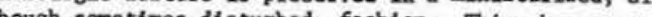
o doubt occurred as a result of poor la phenomenon piston movement with penetration. In this case the movement was delayed and the piston was immobile as the cutter and lower barrel passed through the upper meter of the sediment. The upper $8 \mathrm{~cm}$, as mentioned above, probable squeezed lanto the empty core cutter slowly and 1rregularly as the sediment displaced the

$0-8$ wer trapped within.

$\exists+1$,

$\exists \circ \pm$ 工我

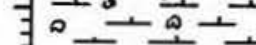

200 청ㅎㅎ

DISTURBED IN CORING - SEE PILOT CORE DESCRIPTION 8-204 FOR DETAILS

CAIC OOZE

10 YR $6 / 4$ 11ght yellow brown, grades to $6 / 3$ pale brown, to 2.5 Y $7 / 4$ pale yellow brown motting 20-30 cm, 44-50 cm, 160-180 cm, 194$204 \mathrm{~cm}$; a lao grayish brown -rich shadowing $104-$

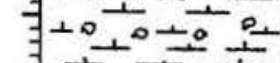

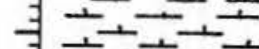

$\Rightarrow+ \pm+1+1=$

块 三 三 $204-2$

(largish) pteropods scattered throughout 204-210

CALC OOZE

等 5 Y $3 / 2$ dark olive gray

我声车

innumerable, thy, ifs gray mottles and fleck

throughout
firm, a bit more conpact lutite with scattered forams s, horizonta.

$\exists+$ CALC

2.5 × $5 / 2$ grayish brown grades to $6 / 2$ 11ght brownish

common intermottling in transitional zone $226-237 \mathrm{~cm}$

400 돋 moist, smooth lutite with scattered forams and a few flecks and pteropods

$\exists \div \frac{1}{0} \div-\frac{1}{0}$

F CALC OOZE

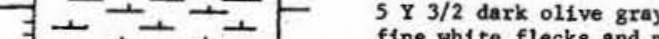

fine white flecks and microlaminations (poorly defined) f1rm, compact lutite with scattered pteropod fragments
sapropel zone 
Ship CHAIN Cruise 119 Leg II Sta. 129 Core No. $27 \% \mathrm{C}$

Lithologic

Log

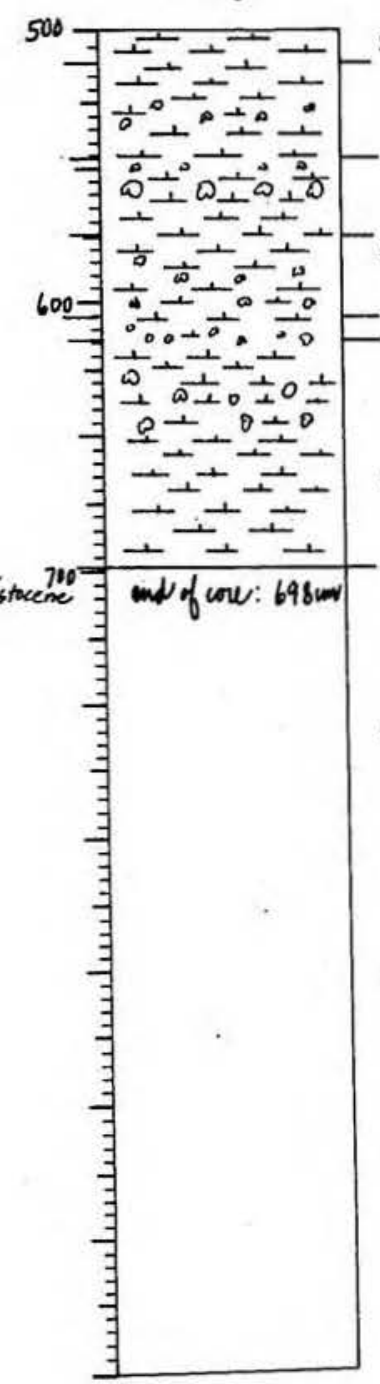

Detailed Description

267-307

2.5 y $5 / 2$ grayish brown, grades to $6 / 2$ light brownish

gray

slightly silty lutite with scattered forams, pteropod fragments and white lithified calc lumps

307-334

CALC SIIIC OOZE WITH PYRIT

5 Y $3 / 2$ dark olive gray

ine, light olive gray laminations and flecks 307-313, darker ones throughout uni

abundant forams and scatred pteropods

$G$, horizontal

$334-420$

CALC OOZE

5 Y $7 / 2$ 1ight gray, grades to $7 / 3$ pale yellow, to $6 / 2$

few faint, 1 ight gray mottles and burrows scattered throughout

lutite, stiffens with depth, and silty (ash) conwhite calcareous lump and forams are found throughout $\mathrm{s}$, mottled

2.5 Y $4 / 2$ dark grayish brosm, grades to 5 Y $3 / 2$ dark

olive gray
extensive grayish brown and olive brown mottling $420-$ $435 \mathrm{~cm}$; finely laminated thereafter

hat stif, wulchy lutite ith abundant forams apropel zone

very sharp horizontal

480-490

CALC OOZE

5 × $6 / 2$ light olive gray, grades to 2.5 × $6 / 2$ light brownish gray

very slight intermottling in transition zone

tiny, brittle, $1 \mathrm{~cm}$ long, pyritized worm burrows scat-

tered throughout

$s$, irregular
Ship CHAIN Cruise 119 Leg II Sta. 129 Core No. 27 PC
Lithologic

Log

\section{Detailed Description}

490-510

CALC OOZE

5 Y $3 / 2$ dark olive gra

few fine, gray flecks and mottles

wh abundant foram sapropel zone

510-546

CALC OOZE

5 Y $5 / 2$ olive gray, grades to 2.5 × $6 / 2$ 1ight grayish

coumon, fine intermottling of the colors above in

transitional zone

irm, silty lutite with scattered forams; grades to

546-574

CALC OOZE

5 Y $3 / 2$ dark olive gray

extensive, tiny, grayish-brown mottles $546-548 \mathrm{~cm}$;

large, gray mottles 548-560; finely laminated (olive gray) $560-574 \mathrm{~cm}$ sapropel zone

574-605

CALC OOZE

5 Y $5 / 2$ olive gray, grades to 2.5 Y $6 / 2$ light grayish

coumon intermottling (well-defined) of the colors above throughout

finm, silty lutite with scattered forams; becomes mor 605-614 CALC OOZE

5 Y $3 / 2$ dark olive gray

5 Y $3 / 2$ dark olive gray
innumerable, fine, light ollve gray burrows and mottles mulchy, firm, foram-rich lutite

$s$, horizontal

614-698

CALC OOZE
5 Y $5 / 2$ olive gray, grades to 2.5 Y $6 / 2$ light gray 1 sh

common intermottling of the colors above $620-650 \mathrm{~cm}$ irm, slightly silty lutite with scattered forams and a few pteropods

nature of the above combinations of end of core 
SIEAR SLIDE DESCRIPTIONS - W.H.O.I. SEDIMENT CORES

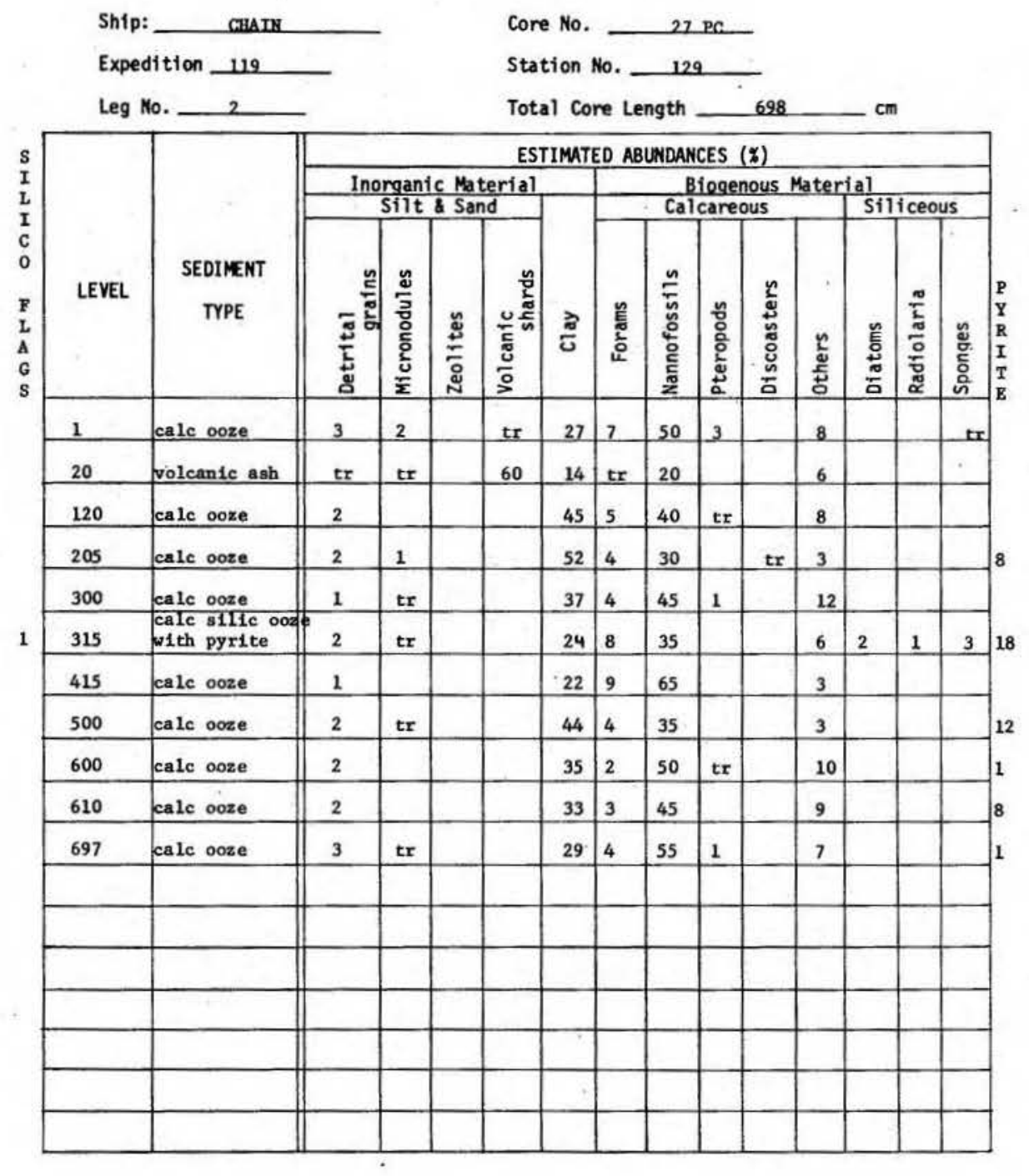

Ship CHAIN Cruise 119 Leg II Sta. 129 Core No. 27P6

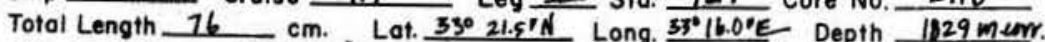

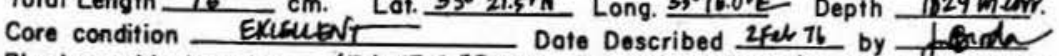

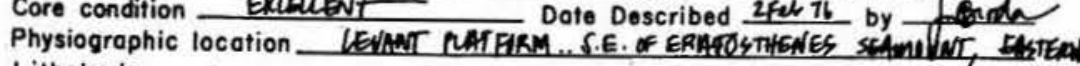
Lithologic

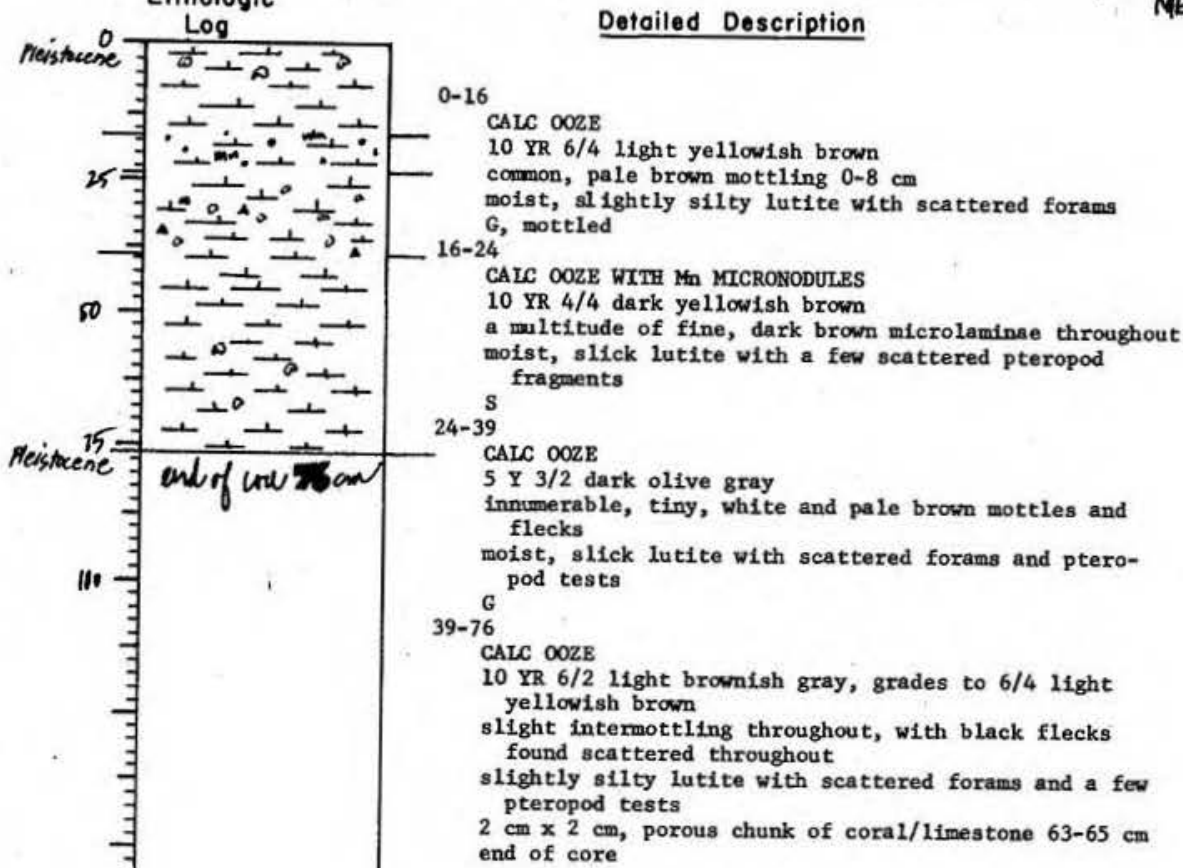


SMEAR SLIDE DESCRIPTIONS - W.H.O.I. SEDIMENT CORES

Ship: CHAIN

Leg No. 2
Expedition we

Core No. 27 PG

Station No. 129

Total Core Length $76 \quad \mathrm{~cm}$

\section{ESTIMATED ABUNDANCES (\%)}

\begin{tabular}{|c|c|c|c|c|c|c|c|c|c|c|c|c|c|c|}
\hline \multirow[b]{4}{*}{ LEVEL } & \multirow[b]{4}{*}{$\begin{array}{c}\text { SEDIMENT } \\
\text { TYPE }\end{array}$} & \multicolumn{13}{|c|}{ ESTIMATED ABUNDANCES (\%) } \\
\hline & & \multirow{2}{*}{\multicolumn{4}{|c|}{$\begin{array}{c}\text { Inorganic Material } \\
\text { Silt \& Sand }\end{array}$}} & \multirow[b]{3}{*}{$\frac{\pi}{5}$} & \multirow{2}{*}{\multicolumn{5}{|c|}{$\begin{array}{l}\text { Biogenous Mater } \\
\text { Calcareous } \\
\end{array}$}} & al & & \\
\hline & & & & & & & & & & & & \multicolumn{3}{|c|}{ Sfliceous } \\
\hline & & 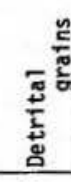 & $\begin{array}{l}\frac{\omega}{3} \\
\frac{3}{0} \\
\frac{0}{0} \\
\frac{0}{\Sigma}\end{array}$ & 岇 & 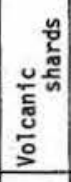 & & 言 & 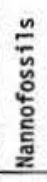 & 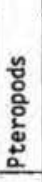 & 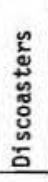 & $\begin{array}{l}\frac{r}{5} \\
\text { ș } \\
0 \\
\end{array}$ & $\begin{array}{l}\text { 产 } \\
\text { to } \\
\frac{0}{6} \\
\end{array}$ & 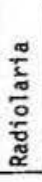 & 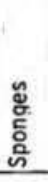 \\
\hline 1 & calc ooze & 2 & 3 & & & 22 & 8 & 60 & tr & & 5 & & & \\
\hline 20 & $\begin{array}{l}\text { calc ooze } w / M / 4 \\
\text { micronodules }\end{array}$ & 2 & 18 & & & 18 & 4 & 55 & & & 3 & & & $\cdot$ \\
\hline 30 & calc ooze & 2 & & & & 23 & 7 & 50 & tr & & 8 & & & \\
\hline 65 & $\begin{array}{l}\text { calc ooze/othe } \\
\text { (1imestone) }\end{array}$ & & & & & & & & & & 100 & & & \\
\hline 75 & calc ooze & 1 & $t r$ & & & 31 & 5 & 60 & & & 3 & & & \\
\hline & & & & & & & & & & & & & & \\
\hline & & & & & & . & & & & & & & & \\
\hline & 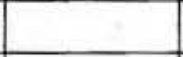 & & & & & & & & & & & & & \\
\hline & & & & & & & & & & & & & & \\
\hline & & & & & & & & & & & & & & \\
\hline & & & & & & & & & & & & & & \\
\hline & & & & & & & & & & & & & & \\
\hline & & & & & & & & & & & & & & \\
\hline & & & & & & & & & & & & & & \\
\hline & . & & & & & & & & & & & & & \\
\hline & & & & & & & & & & & & & & \\
\hline & & & & & & & & & & & & & & \\
\hline & & & & & & & & & & & & & & \\
\hline
\end{tabular}

VISUAL CORE DESCRIPTION

Page 1 of 2

Ship ChaIN Cruise $119 \quad$ Leg 2 Sto 131 Core No 28 PC Total Length $700 \mathrm{~cm}$. Lat. $33^{\circ} 58.8^{\prime} \mathrm{N}$ Long. $33^{\circ} 37.2^{\prime} E$ Depth $22.20 \mathrm{cmrim}$. Core condition EXCEUENT Date Described 4 FCb 76 by T. FARMES Physiogrophic location CENTRAL CYPRUS BASIN, E. OA ERATOSTHENES SEAMOWNT, EMS. Lithologic

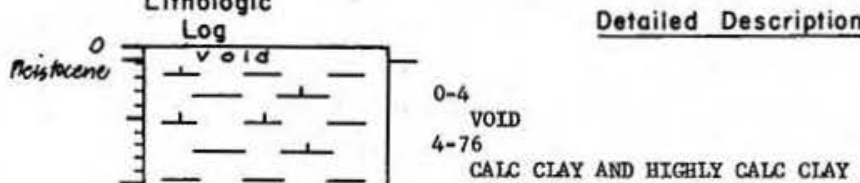

CALC CIAY AND HIGHLY CALC CIAY hounts of $Y 4 / 2$ olive gray and 10 YR $6 / 3$ pale brown in the lower half of the unit; above colors
appear in varying hues in sharply contacted macrolaminations
very little mottling; very fine, dark brown laminations are present from $10-12 \mathrm{~cm}$

are present slick, unconsolidated lutite, except the thin, pale brown laminations which are slightly silty lutites with scattered forams and pteropods $\stackrel{\text { S6-163 }}{\text { S6- }}$

CALC CLA

5 Y $4 / 2$ varying hues of olive gray, somewhat darke from $122-141 \mathrm{~cm}$

homogeneous, except $5 \mathrm{~mm}$ bed of sapropelic, slightly silty lutite (5 Y $3 / 2$ dark olive gray) at $86.5 \mathrm{~cm}$ smooth, very slick, unconsolidated lutite

200

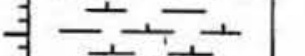

$\exists+163-236$

$\exists+-12+$ HIGHLY CALC CLAY, CALC OOZE, AND MINOR AMOUNTS OP HIGLY CALC

10 YR $6 / 3$ pale brown, $2.5 \times 4 / 4$ olive brown, 10 YR 5/3 brown, and $2.5 Y 3 / 2$ colors appeax as macrolaulnations in a somewhat cyciic sequence as listed above

throughout

the brows and pale browns tend to be slightly silty ile the other lani-

slick lutites $36-258$

HIGHLY CALC CLAY

5 Y $5 / 2$ olive gray, grades to 2.5 Y $4 / 4$ olive brow scattered, dark ollive gray to totles from above common; grades to sticky lutite

$400=$

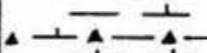
58- 355

CAIC OOZE

10 YR $5 / 3$ brown and $6 / 3$ pale brown; varying hues of above colors appear as gradational macrolamination.

scattered intermottling
silightly silty lutite with scattered forams 
629

VISUAL CORE DESCRIPTION

Ship $C_{\text {HAIN }}$ Cruise 119 Leg 2 Sta. 131 Core No. 28 PC

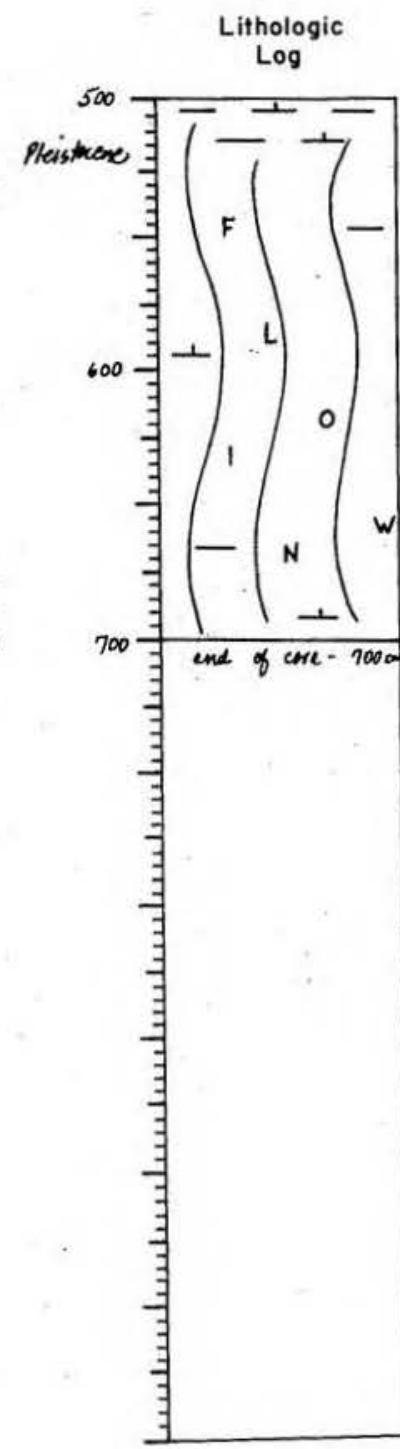

Detailed Description

\section{5-366}

CALC OOZE

Y olive gray

-11ke, sapropelic lutite; foram

are comon and pteropods abundant

366-407

MIGRLY CALC CLAY

5 Y $4 / 2$ olive gray

homogeneous, except few small mottles near top

firm, slightiy silty lutite

tion of calc ooze/pyrite

Y $3 / 2$ dark olive gray

407-45

CALC OOZE

5 Y $4 / 2$ and $5 / 2$ olive grays, grades to $5 / 3$ olive; colors

appear as macrolsminations

intermottling is coumon in top $20 \mathrm{~cm}$.

tions are less silty

$436-440 \mathrm{~cm}$, shell hash (calc ooze with detritus)

$\underset{455-498}{\mathrm{~S}}$

CAIC CLAY

5 Y $3 / 2$ varying hues of dark olive gray, and $4 / 2$ olive gray, 490-493 cm

very small, pale mottles are coumon in top $13 \mathrm{~cm}$

wooth, compact, sticky lutite, except two thin (5 m)

coumon forams

498-505

CALC CIAY

no mottling

smooth, compact, sticky lutite

(n)

flow in of above

end of core
5 Y $4 / 2$ olive gray
630

SMEAR SLIDE DESCRIPTIONS - W.H.O.I. SEDIMENT CORES

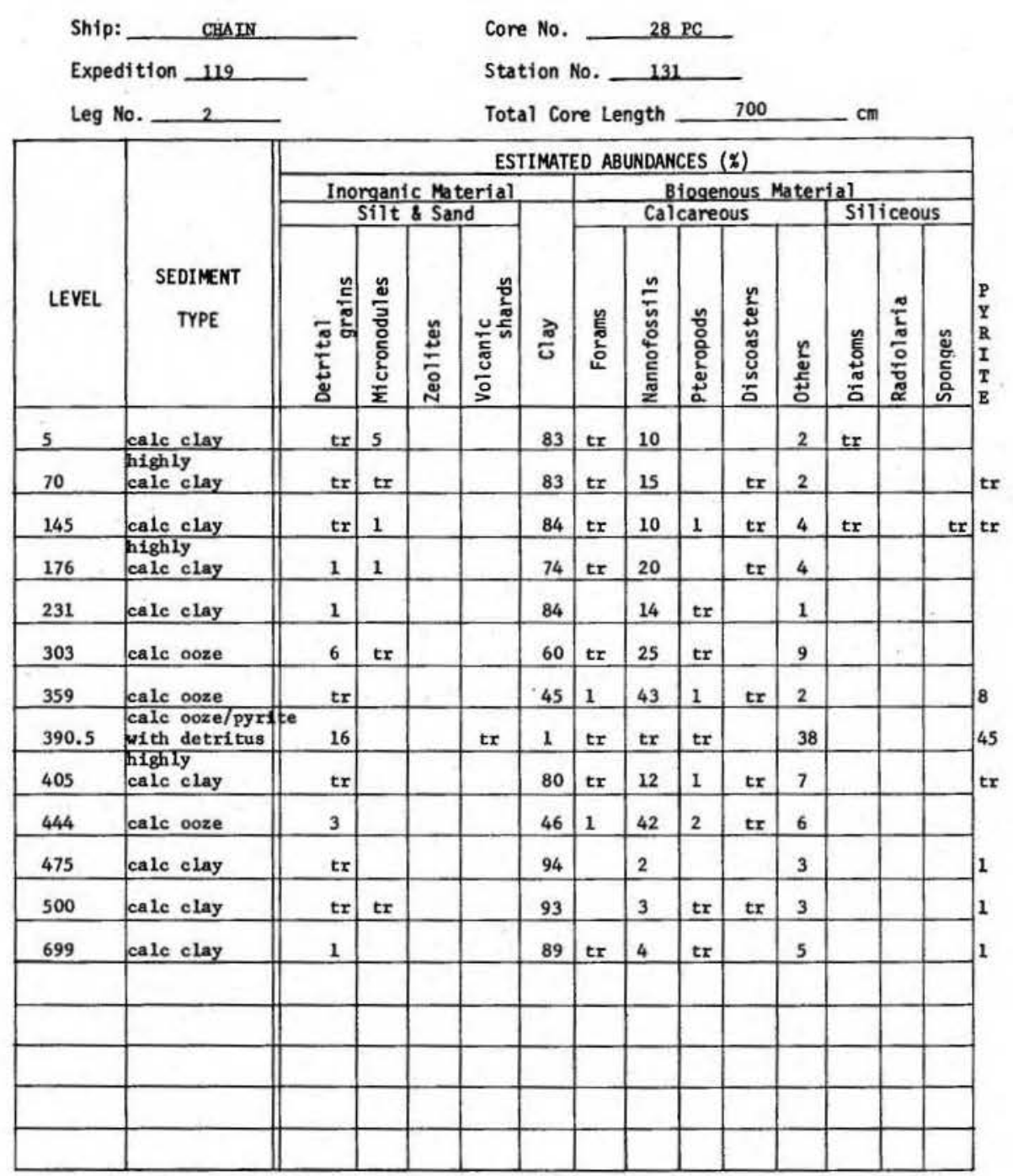


631

VISUAL CORE DESCRIPTION

Page 1 of 1

Ship CHAIN Cruise 119 Leg 2 Sto. 131 Core No. 28 PG Total Length $140 \mathrm{~cm}$. Lat. $33^{\circ} 58.8^{\prime} \mathrm{N}$ Long. $33^{\circ} 37.2^{\prime} \mathrm{E}$ Depth $2230 \mathrm{cem} . \mathrm{m}$ Core condition ExCELEENT Date Described 4 Fet. 76 by I FARMER Physiogrophic location CENTEAL CYPRUS BASIN, E. of ERATOSTMENES SEAmounT, EMS Lithologic

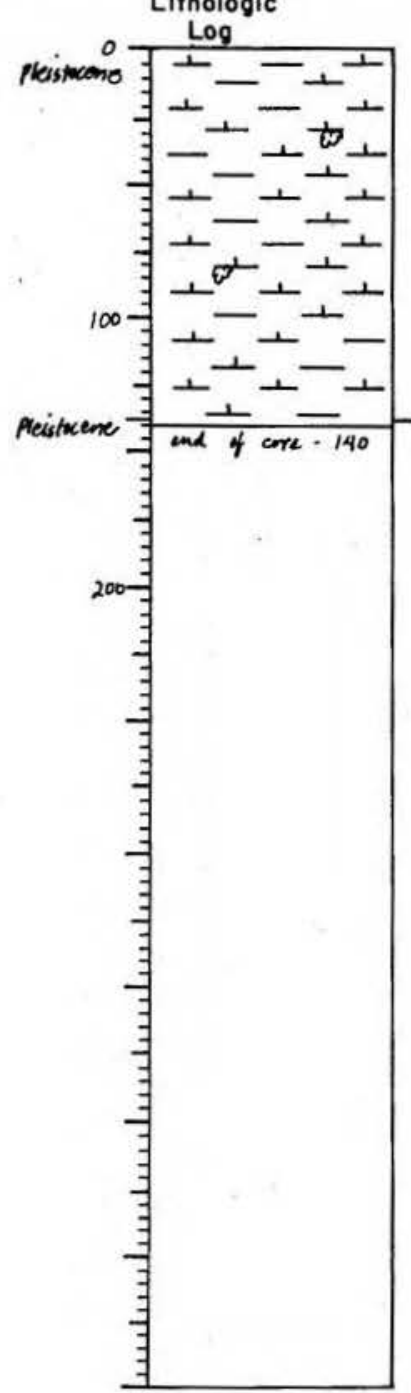

\section{Detailed Description}

$0-138$ 10 YR $4 / 3$ brown, $4 / 2$ dark grayish brown, with lesser amounts of 5 Y $4 / 2$ ollve gray and $10 \mathrm{YR} 6 / 3$ pale brown in the lower half of the unit; variations of light mottling is scattered throughor

oghout; 10-13 cm dark mooth, very slick,

except the pale brown laminations which are slightiy silty lu$\underset{38-140}{S}$

CALC CLAY

5 Y $4 / 2$ olive gray

homogeneous throughout

sucth, very sllck, unconsolidated lutite
HIGHLY CALC CLAY AND MDNOR AMOUNTS OF CALC OOZE
SIEAR SLIDE DESCRIPTIONS - W.H.O.I. SEDIMENT CORES

Ship: CHAIN

Expedition 119

Leg No. 2

\begin{tabular}{|c|c|c|c|c|c|c|c|c|c|c|c|c|c|c|}
\hline \multirow[b]{3}{*}{ LEVEL } & \multirow[b]{3}{*}{$\begin{array}{c}\text { SEDIMENT } \\
\text { TYPE }\end{array}$} & \multicolumn{13}{|c|}{ ESTIMATED ABUNDANCES (\%) } \\
\hline & & \multicolumn{4}{|c|}{$\begin{array}{c}\text { Inorganic Material } \\
\text { Silt \& Sand }\end{array}$} & & \multicolumn{5}{|c|}{$\begin{array}{l}\text { Biogenous Mater } \\
\text { Calcareous }\end{array}$} & \multicolumn{3}{|c|}{$\frac{\text { al }}{\text { Siliceous }}$} \\
\hline & & & 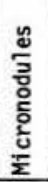 & 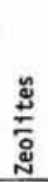 & 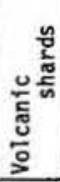 & $\frac{\pi}{6}$ & 望 & 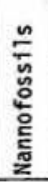 & 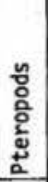 & 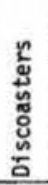 & $\frac{n}{2}$ & 崖 & $\frac{\stackrel{0}{E}}{\frac{\pi}{0}}$ & 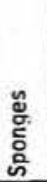 \\
\hline 1 & $\begin{array}{l}\text { highly } \\
\text { calc clay }\end{array}$ & 1 & 5 & & & 77 & tr & 15 & & & 2 & & + & \\
\hline 62 & calc ooze & $\operatorname{tr}$ & tr & & & 34 & 2 & 60 & 2 & tr & 2 & & & \\
\hline 130 & $\begin{array}{l}\text { hrighry } \\
\text { cale clay }\end{array}$ & $\mathrm{tr}$ & tr & & & 80 & tr & 18 & & & 2 & & & \\
\hline 139 & calc clay & $\mathrm{tr}$ & & & & 86 & & 10 & tr & tr & 3 & & & $t x$ \\
\hline & & & & & & & & & & & & & & \\
\hline & & & & & & & & & & & & & & \\
\hline & & & & & & . & & & & & & & & \\
\hline & & & & & & & & & & & & & & \\
\hline & & & & & & & & & & & & & & \\
\hline & & & & & & & & & & & & & & \\
\hline & & & & & & & & & & & & & & \\
\hline & & & & & & & & & & & & & & \\
\hline & & & & & & & & & & & & & & \\
\hline & & & & & & & & & & & & & & \\
\hline & & & & & & & & & & & & & & \\
\hline & & & & & & & & & & & & & & \\
\hline & & & & & & & & & & & & & & \\
\hline & & & & & & & & & & & & & & \\
\hline
\end{tabular}

Core No. 28 PG

Station No. 131

are Length

$-140$ 
Ship CHAN Cruise 119 Leg 2 Sto. $\frac{133}{34^{\circ} 70^{\circ}}$ Core No. $248 \mathrm{C}$

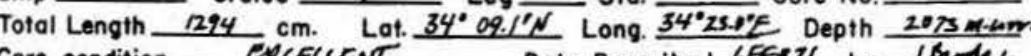
Core condition DPCEUENT Date Described GFE 76 by frodv.

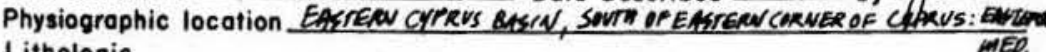
Lithologic

Detailed Description

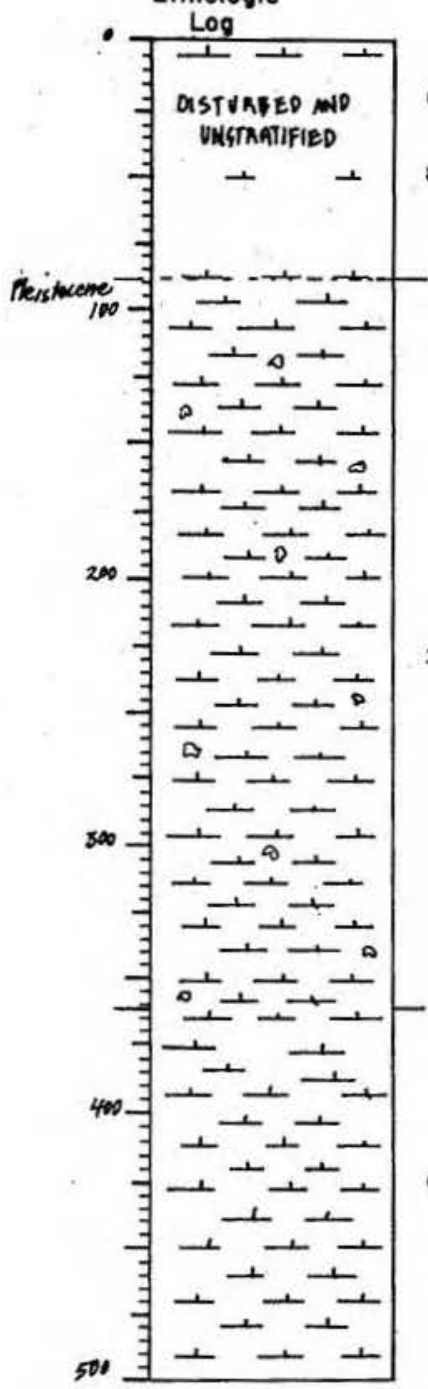

EATIRE TOP SECTION (9) WASHED, DISTURBED, AND UNSTRATIFIED 88-362 CALC OOZE

ALC OOZE brown, and $6 / 2$ light brownish gray

brown, $6 / 3$ pale slight intermottling of the colors above with the addition of grayish brown burrows found scattered throughout: numerous, sharply defined, textural beds are described below

smooth, very slightly silty lutite predominates with a number of very pale brown and grayish brown, somewhat calcareous fragments: the following intervals define the ma jor beds: 156-163 cm (ends with an olive yellow contact $\approx 25^{\circ}$ ); 258-264; $\mathrm{cm} ; 270-277 \mathrm{~cm}$ (ends with ol1ve yellow lemination); 279-282 com (washed, frregular basal contact); 297-301 cm; $310-313 \mathrm{~cm} ; 315-322 \mathrm{~cm} ; 331-$ $335 \mathrm{~cm}$ S, ho h-693

\section{CALC OOZE WITH A NUMBER OF BEDS OF CALC OOZE WITH} DETRTTUS

arying hues of 2.5 × $4 / 2$ dark grayish brown and $5 / 2$ graylsh brown

extenthe addition of $5 \mathrm{y}$ islamination and bedding, with the intervals below

firm, sightly silty lutite with a few forams dominates. but olive gray and graylsh brown silt and fragmented pteropods and foram beds are coumon: $367-370 \mathrm{~cm}$ (sapropel (267-472 cm; 483-498 and (nicely ed shell hash and detritus); 560-566 (irregular basal contact); thin laminations again $570-630 \mathrm{~cm}$; 638-648 cm (well-graded); 665-672 cm (slightly graded); 688s $693 \mathrm{~cm}$ ( some medium sand)

\section{3-1294}

end of core graded beds of a little detrital silt and predominantly tions in the zone $505-532 \mathrm{~cm} ; 542-550$ (bed of weather-

VISUAL CORE DESCRIPTION

Page 2 of 2

Ship CHAIN Cruise 119 Leg II Sta. 133 Core No. 29 PC

Lithologic

Log

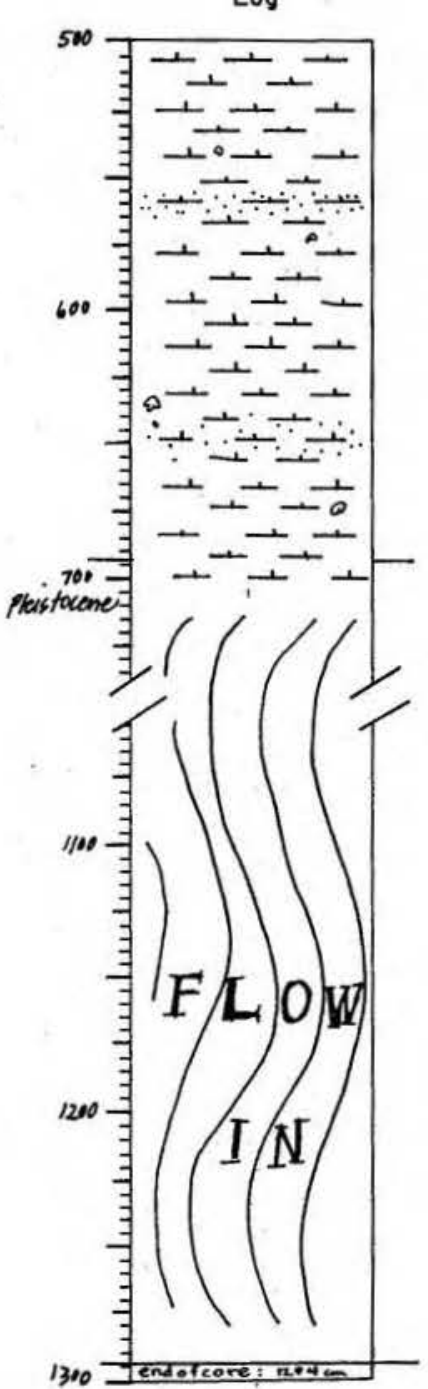

Detailed Description 
SMEAR SLIDE DESCRIPTIONS - W.H.O.I. SEDIMENT CORES

Ship:

2

\begin{tabular}{|c|c|c|c|c|c|c|c|c|c|c|c|c|c|c|}
\hline \multirow[b]{3}{*}{ LEVEL } & \multirow{3}{*}{$\begin{array}{c}\text { SEDIMENT } \\
\text { TYPE }\end{array}$} & \multicolumn{13}{|c|}{ ESTIMATED ABUNDANCES (ซ) } \\
\hline & & \multicolumn{4}{|c|}{$\begin{array}{c}\text { Inorganic Material } \\
\text { Silt \& Sand }\end{array}$} & \multirow[b]{2}{*}{$\frac{\pi}{6}$} & \multicolumn{5}{|c|}{$\begin{array}{l}\text { Biogenous Mater } \\
\text { Calcareous }\end{array}$} & \multicolumn{2}{|c|}{$\frac{\text { al }}{\text { Siliceous }}$} & \\
\hline & & 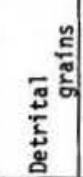 & 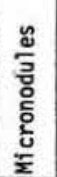 & 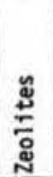 & 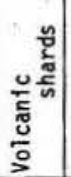 & & 崖 & 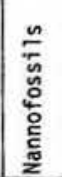 & 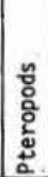 & 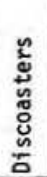 & $\begin{array}{l}\frac{r}{\omega} \\
\frac{\tilde{J}}{0}\end{array}$ & 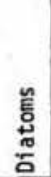 & $\begin{array}{l}\frac{\pi}{2} \\
\frac{\pi}{0} \\
\frac{0}{0} \\
\ddot{0} \\
\ddot{0}\end{array}$ & 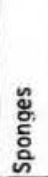 \\
\hline 1 & calc ooze & 3 & $\mathrm{tr}$ & & & 29 & 10 & 45 & 2 & & 7 & & & \\
\hline 90 & calc ooze & 5 & tr & & & 49 & 4 & 30 & & & 12 & & & $\cdot$ \\
\hline 160 & calc ooze & 7 & tr & & & 16 & 45 & 6 & 1 & & 25 & & & \\
\hline 250 & calc ooze & 3 & 1 & & & 48 & 1 & 35 & & $\mathrm{tr}$ & 12 & & & \\
\hline 260 & calc ooze & 8 & 1 & & & 14 & 40 & 8 & 4 & & 25 & & & \\
\hline 360 & calc ooze & 3 & tr & & & 54 & 4 & 30 & 1 & & 8 & & & \\
\hline $375^{*}$ & alc ooze & 2 & tr & & & 60 & tr & 30 & tx & & 4 & & & \\
\hline 475 & calc ooze & 2 & tr & & & 56 & 2 & 35 & $t x$ & & 5 & & & \\
\hline 545 & calc ooze & 3 & $\mathrm{tr}$ & & & 10 & 12 & 5 & 15 & & +55 & & & \\
\hline 635 & calc ooze & 6 & & & & 54 & 2 & 30 & $t x$ & & 8 & & & \\
\hline 645 & $\begin{array}{l}\text { ale ooze with } \\
\text { detritus }\end{array}$ & 15 & tr & & & 36 & 12 & 25 & & & 10 & & & \\
\hline 692 & falc ooze & 6 & tr & & & 49 & 7 & 20 & 2 & & 12 & & & \\
\hline 1293 & ale clay & 8 & tr & & & 73 & tr & 12 & tr & & 3 & & & \\
\hline & & & & & & & & & & & & & & \\
\hline & & * & ins & & nifi & ant & ex & atage & & & eis & sene & & hos \\
\hline & & sh & 1 & omm & ts & & & & & & & & & \\
\hline & & & & & & & & & & & & & & \\
\hline & & & & & & & & & & & & & & \\
\hline
\end{tabular}

Core No. $29 \mathrm{PC}$

Station No. 133

lone leng

Total Core Length
VISUAL CORE DESCRIPTION

Page 1 of 1

Ship CHAIN Cruise 119 Leg II Sta. 133 Core No. 29PG Total Length $141^{1} \mathrm{~cm}$. Lat. $34^{\circ} 09.1^{\circ} \mathrm{N}$ Long. $34^{\circ} 23.0^{\circ} \mathrm{E}$ Depth $2073 \mathrm{~m}$.carr. Core condition EXCEUENT Date Described GFEB 76 by firde

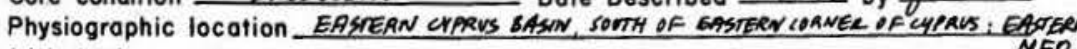
Lithologic

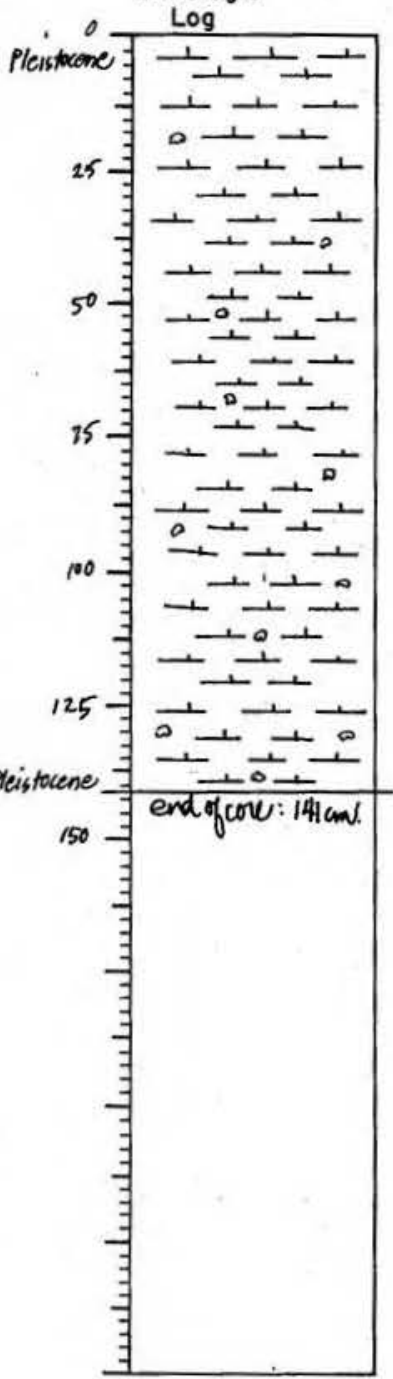

Detailed Description

0-141

varying hues of 10 YR $4 / 3$ dark brown, $4 / 2$ dark grayish

light, faint intermottling of the colors above through-

oist, unconsolidated, very slightly silty lutite domiwhat graded)

$118 \mathrm{~cm}$ (interlaminated and nicely graded)

end of core 
SMEAR SLIDE DESCRIPTIONS - W.H.O.I. SEDIMENT CORES

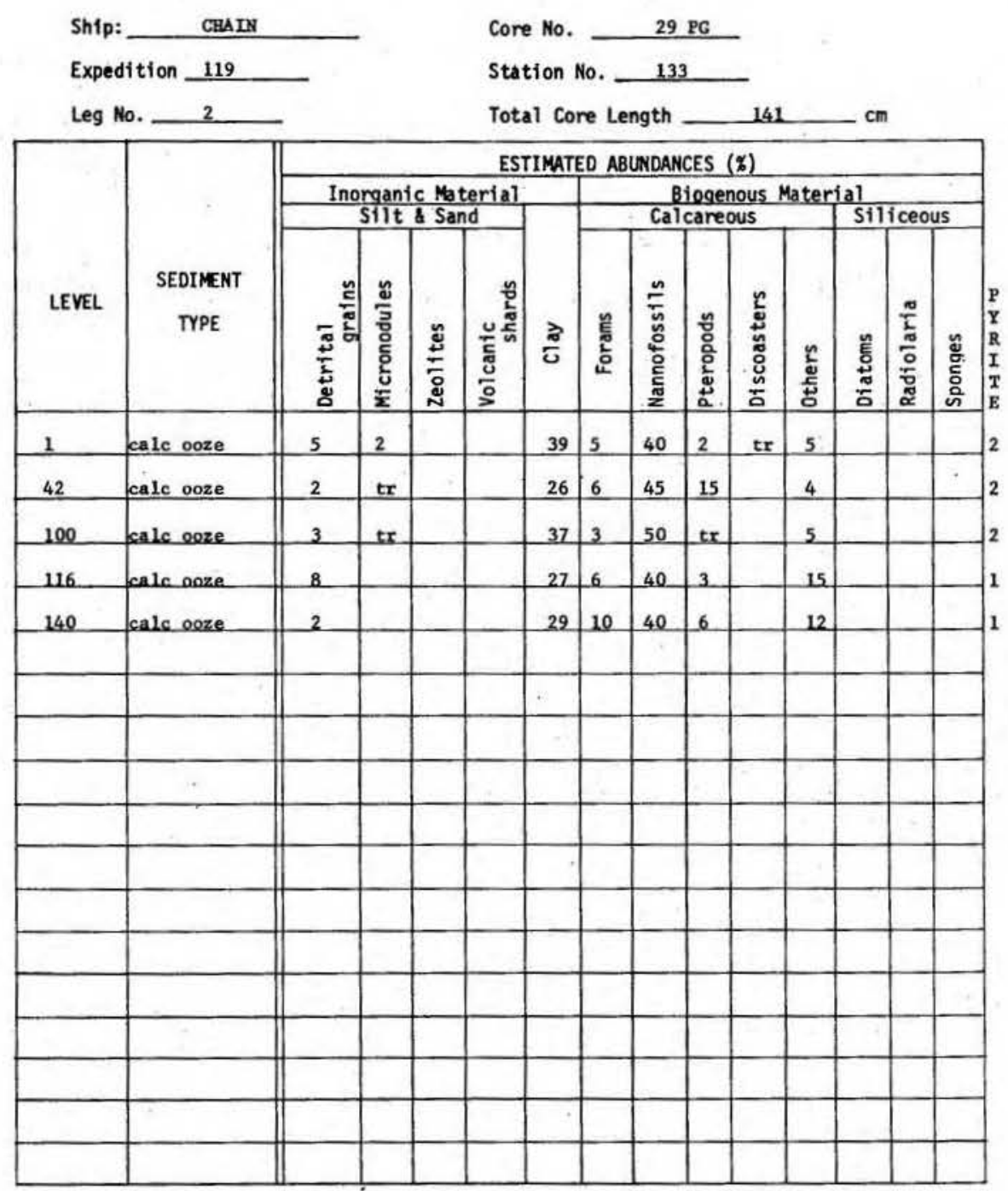

VISUALL CORE DESCRIPTION

Page 1 of 3

Ship CHAIN Cruise 119 Core condion.

Core condition EXCELLENT

Leg 2 Sta. 135 Core No. 30 AC Date Described $3 \sqrt{26} 6$ 16 by H. Famer Lithologic

Log 0

Log

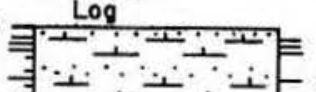

Detailed Description

NOTE: On comparison with the pilot core, the top $17 \mathrm{~cm}$ do

$\exists+-+{ }^{+} \quad$ not appear stratigraphically continuous. Rather, it

$\exists+\perp \perp$

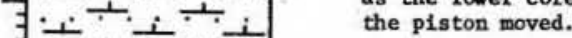

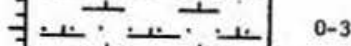

我本上

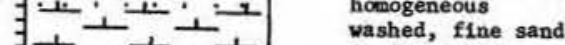

는도

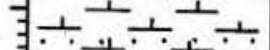

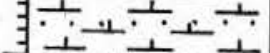

$\exists+1+\frac{1}{1+\infty}$

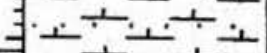

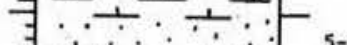

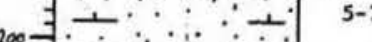

$\exists \div \div \quad 2.5$ Y $3 / 2$

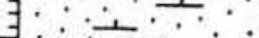

고 노 느

노도

$1+1$

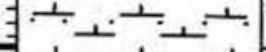

$300 \exists \div+\div \perp \div$

前走前

3-5

10 YR $5 / 3$ pale brown

subtle, scattered mottlin

1ightly silty, slick lutite with scattered foran

2.5 Y $3 / 2$ very dark grayish brown

ociewhat disturbed, subele Laninations

(5)

10 YR $6 / 3$ pale brown, $5 / 2$ and $4 / 2$ grayish browns; colors appear as sowevhat disturbed and mottled macrolaminations no mottling

alightly silty lutite with few forams, except 14-17 cm a fine sand bed washed and distorted along core liner s.

․․ - -1 CALC OOZE INTERBEDDED HITH CAIC OOZE/DETRITUS

$\perp-10$ YR $6 / 3$ pale brown and $5 / 3$ brown with minor lamina$\exists+-\quad \begin{aligned} & \text { tions of } 7 / 3 \text { very pale brown; colors appear as grada- } \\ & \text { tional macrolaminations, generally becoming darker }\end{aligned}$ with depth

scattered mottling of pale brown and brown occurs silty to slightly ofity lutites uith are scattered

interbedded uith very pale brom scatered forams 56-59, 74-76, 103-107, 136-139, 168-171 med sands inclusions of these fine sands occur elsewhere in

그ㄱㅗㅗ 판

$\exists \div \div \div$.

$\exists+1-1$

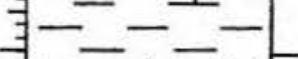


Ship CHAIN Cruise 119 Leg 2 Sto. 135 Core No. 30 PC

Lithologic

Log

Detailed Description

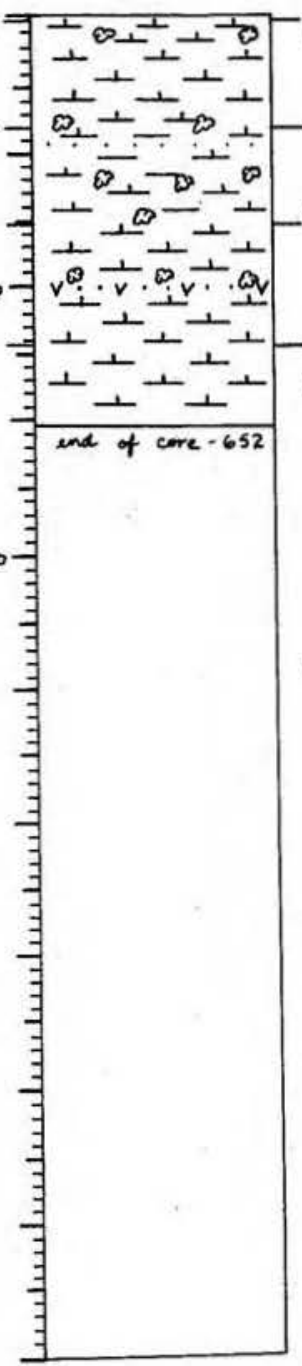

180-224

YR $5 / 3$ brown

laminated in hues of brown, becoming grayer with depth fine, but sonewhat disturbed sand, lutite clast from

lover unit present at $209 \mathrm{~cm}$ $\mathrm{s}$, but very disturbed

CAIC O0e

5 Y $5 / 3$ olive

no mottling

silty lutite with sandy clasts indicating

mith above unit 232-298

CALC OOZE INTBRBEDDED WITH DETRITUS

Y $4 / 2$ (predominant), $5 / 2$ olive grays and $3 / 2$ dark olive gray $(246-248 \mathrm{~cm})$

macrolaminations with no mottling, but some coring

silty to very silty lutite interbedded with fine sand; forams and pteropods are abundant $23 \mathrm{~g}-242 \mathrm{~cm}$, bat s

CALC OOZE TRTERBEDDED WITH CALC OOZB/DETRITUS Y $5 / 2$ olive gray (predorinant) with lesser amounts of $4 / 2$ and $3 / 2$ olive gray and dark ollve gray

macrolaminations with minor, localized mottling 314-316, $341-346 \mathrm{~cm}$

Lutites vary from slightly to very silty, interbedded crease rapidly in remainder of lutite S

CALC CLAY

sattered, small, pale olive mottles throughout smooth, firm, sticky lutite

379-424

CAIC OOZE

.5 × $6 / 2$ light brownish gray

moting is common in the top $7 \mathrm{~cm}$; below this to $410 \mathrm{~cm}$ are numerous, very pale mottles and sandy clasts that appear to have come from several

1ty, torted into isolated clasts

\section{CALC OOZE/DETRITUS}

5 Y $3 / 2$ dark olive gray
Ship CHAIN Cruise 119 Leg II Sta. 135 Core No. 30 PC

Lithologic
Log

424-490

\section{Detailed Description}

\section{CAIC OOZE AND HIGHLY CALC CLAY}

2.5 Y $6 / 2$ light brownish gray, grades to 10 YR $5 / 3$ brown extensive marbling and mixing with lower unit of $10 \mathrm{YR}$ $4 / 2$ dark grayish brown

ticky, slightly silty lutite becomes less silty with depth; dark grayish brown unit is a smooth lutite; $\mathrm{s}$, disturbed

5 Y $5 / 2$ olive gray

homogeneous

fine sand

$\mathrm{S}, \mathrm{di}$

HIGHLY CALC CLAY

10 YR $3 / 2$ very dark grayish brown

slight mottling from below

smooth, sticky lutite

S, very distorted

CALC OOZE

5 Y $5 / 2$ olive gray

dark grayish brown mottles are coumon in top $10 \mathrm{~cm}$ and bottom $14 \mathrm{~cm}$

ty, sticky lutite

S, but severely burrowed

HTGLY CALC CIAY AND CALC OOZP

Y $3 / 2$ dark olive gray (primarily $540-547 \mathrm{~cm}$ ), and $4 / 2$ olive gray due to burrowing; however, coring disturbance is clear above and below and may be partially responsible detrital sands at 546 and $548 \mathrm{~cm}$
dightly silty, stick lutite with fow $\mathrm{s}$, very distorted

576-622

CALC OOZE

silty lutite with few forams, except fine sand (calc ooze/volcanic ash/detritus) $597-602 \mathrm{~cm}$ S

CALC OOZE

5 Y $6 / 2$ light olive gray and $5 / 2$ olive gray

severely disturbed, probably flow in

silty lutite with few forams

end of core severe marbling of above colors appears to be mostly

5 y 5/2 olive gray, becomes somewhat paler with depth dark olive gray mottling is common from $588-597 \mathrm{~cm}$, 
SHEAR SLIDE DESCRIPTIONS - W.H.O.I. SEDINENT CORES

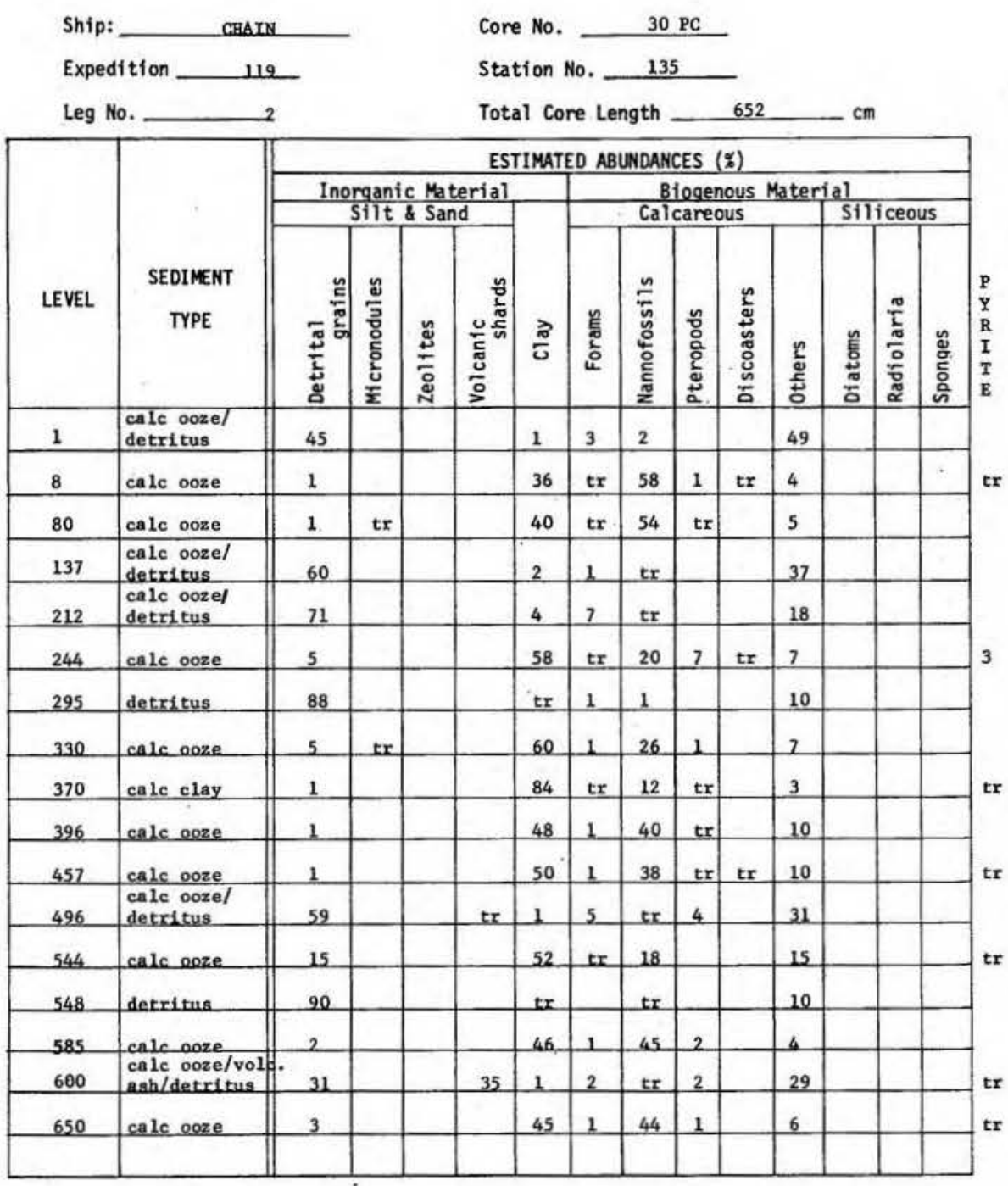

Ship CHAIN Cruise 119 Leg 2 Sta. 135 Core No. 30 PG Total Length III Core condition EXCELLENT Dote Described 3 Feb 76 by 1 . FARMER Physiographic location EASTERN CYPRUS BASIN, $E$. Of ERATOSTMENES SEMMOUNT, Lithologic

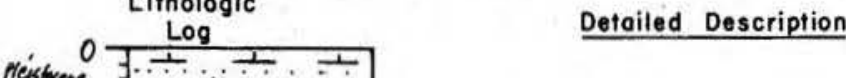

Detailed Description EMS

CAIC OOZE AND CALC OOZE/DETRITUS

10 YR $5 / 3$ brown, grades to pale brown $6 / 3$ below $19 \mathrm{~cm}$

Thered motting in lower $10 \mathrm{~cm}$

slick, unconsolidated lutite with scattered forams and

pteropods; interbedded with brown, pteropod-rich

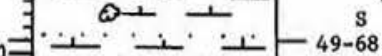

Pleisincene
end of cole: 111 sand at $7 \mathrm{~cm}, 17.5-19 \mathrm{~cm}, 23-25 \mathrm{~cm}$

bedded with laminations of grayish brom very smal1, pale mottles are coumon in top $8 \mathrm{~cm}$ and very

futites interbedded with fine sand and very, very $G$

68-95

CALC OOZE

10 YR $5 / 2$ gray1sh brown, grades to $6 / 3$ varying hues of pale brown, with lesser amounts of $4 / 2$ dark grayish

scattered mottling between macrolaminations of above

ilty lutite with scattered forams; dark grayish browns at bottom

$\underset{95-111}{\mathrm{~s}}$

CALC OOZE

10 YR $5 / 3$ brown, tends towards $6 / 3$ pale brown

ered forams

end of core 
SMEAR SLIDE DESCRIPTIONS - W.H.O.I. SEDINENT CORES

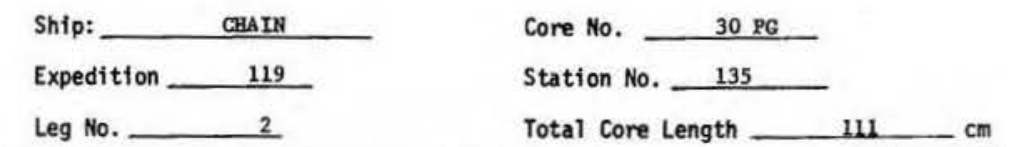

\begin{tabular}{|c|c|c|c|c|c|c|c|c|c|c|c|c|c|c|}
\hline \multirow[b]{4}{*}{ LEVEL } & \multirow{4}{*}{$\begin{array}{l}\text { SEDIMENT } \\
\text { TYPE }\end{array}$} & \multicolumn{13}{|c|}{ ESTIMATED ABUNDANCES ( $(\%)$} \\
\hline & & \multirow{2}{*}{\multicolumn{4}{|c|}{$\begin{array}{c}\text { Inorganic Material } \\
\text { Silt \& Sand }\end{array}$}} & & \multirow{2}{*}{\multicolumn{5}{|c|}{$\begin{array}{l}\text { Biogenous Mate } \\
\text { Calcareous }\end{array}$}} & \multirow{2}{*}{\multicolumn{3}{|c|}{ Siliceous }} \\
\hline & & & & & & & & & & & & & & \\
\hline & & 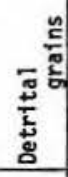 & $\begin{array}{l}\frac{\tilde{\omega}}{3} \\
\frac{3}{0} \\
\frac{0}{0} \\
\frac{0}{2}\end{array}$ & 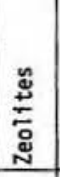 & 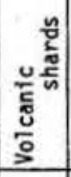 & $\frac{\pi}{0}$ & 萨 & 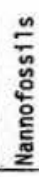 & 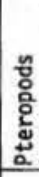 & 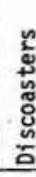 & : & 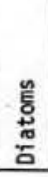 & $\begin{array}{l}\frac{\pi}{2} \\
\frac{\pi}{0} \\
\frac{\pi}{\pi} \\
\alpha\end{array}$ & 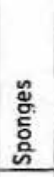 \\
\hline 1 & \multirow{2}{*}{$\begin{array}{l}\text { calc ooze } \\
\text { calc oozel } \\
\text { detritus } \\
\end{array}$} & 2 & $t x$ & & & 55 & $t x$ & 40 & $t$ & & 3 & & & \\
\hline 19 & & 70 & $\mathrm{tr}$ & & & tr & 7 & $t_{1}$ & 8 & & 15 & & & . \\
\hline 51 & calc ooze & tr & & & & 47 & 1 & 47 & $t$ & & 2 & & & \\
\hline 63 & \begin{tabular}{|l} 
calc ooze/ \\
detritus \\
\end{tabular} & 60 & & & & $t x$ & 5 & ti & 2 & & 32 & & & \\
\hline 109 & calc ooze & tr & & & & 50 & & 46 & & & 4 & & & \\
\hline & & & & & & & & & & & & & & \\
\hline & & & & & & . & & & & & & & & \\
\hline & & & & & & & & & & & & & & \\
\hline & & & & & & & & & & & & & & \\
\hline & & & & & & & & & & & & & & \\
\hline & & & & & & & & & & & & & & \\
\hline & & & & & & & & & & & & & & \\
\hline & & & & & & & & & & & & & & \\
\hline & & & & & & & & & & & & & & \\
\hline & & & & & & & & & & & & & & \\
\hline & & & & & & & & & & & & & & \\
\hline & & & & & & & & & & & & & & \\
\hline & & & & & & & & & & & & & & \\
\hline
\end{tabular}

VISUAL CORE DESCRIPTION

Page 1 of 2

Ship CHAiN Cruise 119 Leg II Sta. 137 Core No. 31PC Total Length $637 \mathrm{~cm}$. Lat. $32^{\circ} 56.2^{\circ} \mathrm{N}$ Long. $33^{\circ} 45.0^{\circ} \mathrm{E}$ Depth $1637 \mathrm{m.cove}$ Core condition EXLEUENT Date Described 10FES/6 by frude.

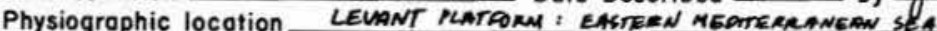
Lithologic

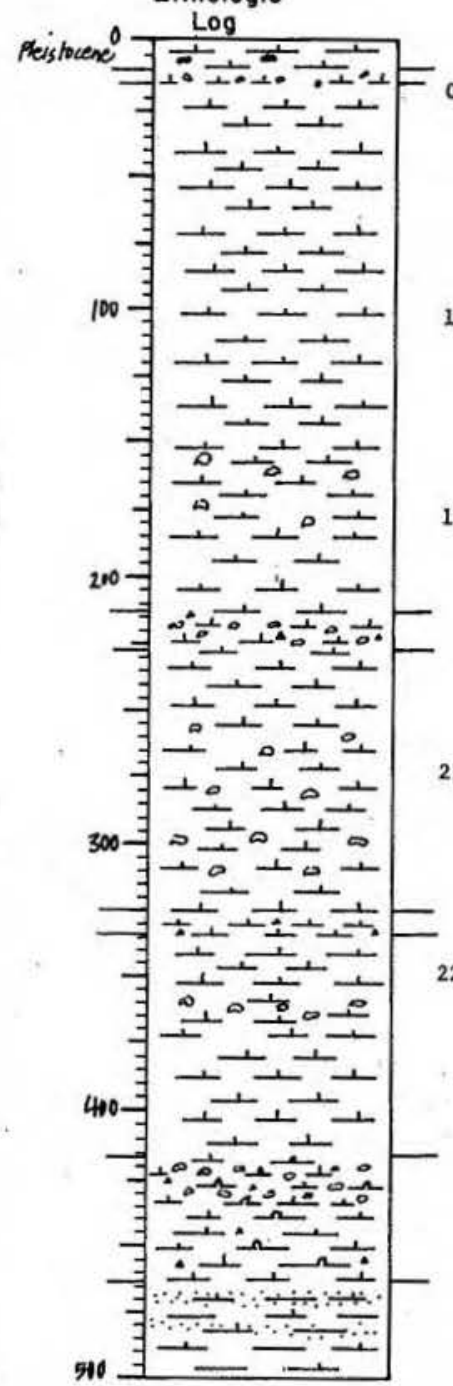

Detailed Description

$0-10$

10 YR $6 / 3$ pale brown, grades to $6 / 1$ light gray

a bit or line lntermoteling at $6 \mathrm{~cm}$

slick lutite with a few forams

the $97 \mathrm{~cm}$ of the sur0-15

CALC 002 B

5 Y $3 / 2$ dark olive gray

extensive, fine, light gray mottling and microlaminamoist, slick lutite with scattered forams

S

CALC OOZE

10 YR $6 / 4$ 1ight yellowish brown, grades to $6 / 3$ pale brown

scattered, slight grayish brown motting $150-180 \mathrm{~cm}$ smooth Lutite, becomep more compact with increasing depth; a few pteropod and shell fragments (pelecypod) scattered, then a bed and a few microlaminations of foram sand

213-226

CAIC OOZE

5 Y $3 / 2$ dark olive gray

innumerable, fine, light gray mottles, burrows, and then microlaminae found throughout

firm, compact lutite with scattered pteropods and foram sapropel zone 26-325

\section{CAIC OOZE}

10 YR $5 / 2$ grayish brown, grades to $6 / 3$ pale brown, back to $5 / 2$ grayish brown

coumon, fine intermottling $250-310 \mathrm{~cm}$

firm, compact, smooth lutite with scattered forams and pteropod fragments

pteropods very abundant $315-325$, and a hash of fragments is found in dark olive gray, very slick lutite s, irregular contact 
645

VISUAL CORE DESCRIPTION

Ship CHAIN Cruise 119 Leg III Sto. 137 Core No. 31PC

Lithologic

Log

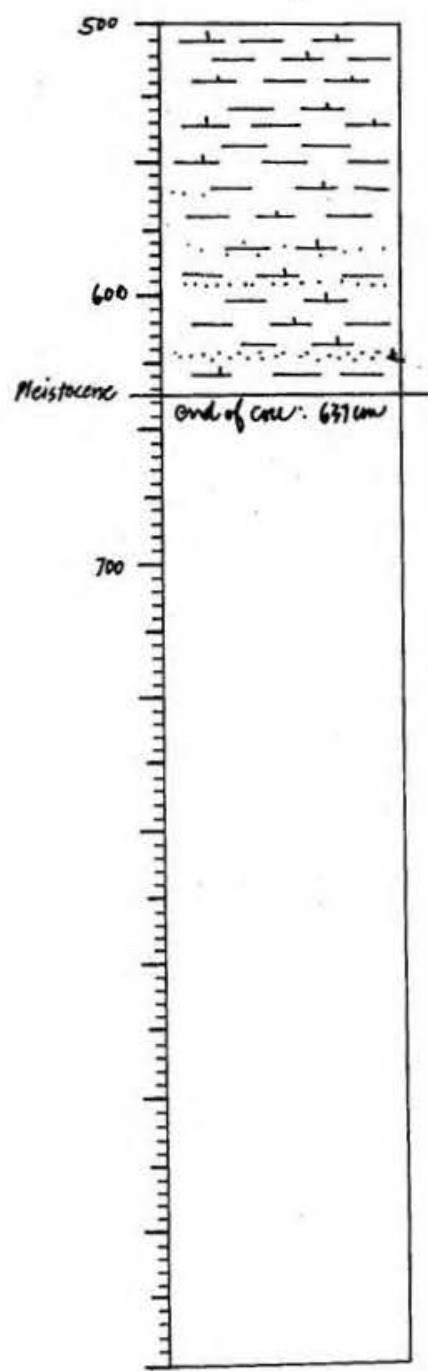

Detailed Description

325-334

CALC OOZE

5 X $3 / 2$ dark olive gray

(1) 330-334 cm

ilick, compact lutite

slick, compact lutst
laminated zone sapropel zone

$334-416$

CALC OOZE

10 XR $5 / 2$ grayish brown, grades to $6 / 3$ pale brown

cormon intermottling in the transitional zone $356-365 \mathrm{~cm}$ finm, slightly silty lutite with a few forams, becoming more abundant with depth

416-463

CALC SIIIC OOZE

T Y $3 / 2$ dark ollve gray basically homogeneous $431-463$, with a few fine microlaminae
slick lutite with abundant forams and scattered ptero-

pods
sapropel zone saprope

463-637

HIGHYY CALC CLAY WITH BEDS OF CALC CLAY/DETRITUS; GRADES TO CALC CIAY WITH BEDS AND IAMTINATIONS OF CALC CLAY/DETRTTUS

10 YR $4 / 1$ dark gray textural anomalies

compact, slick lutite with several silty beds and laminations: broad beds - $468-472 \mathrm{~cm}, 478-482 \mathrm{~cm}, 620-$ $624 \mathrm{~cm}$; laminations - throughout the zone $515-615 \mathrm{~cm}$
end of core several shadowy, very dark gray zones associated with
646

SHEAR SLIDE DESCRIPTIONS - W.H.O.I. SEDIMENT CORES

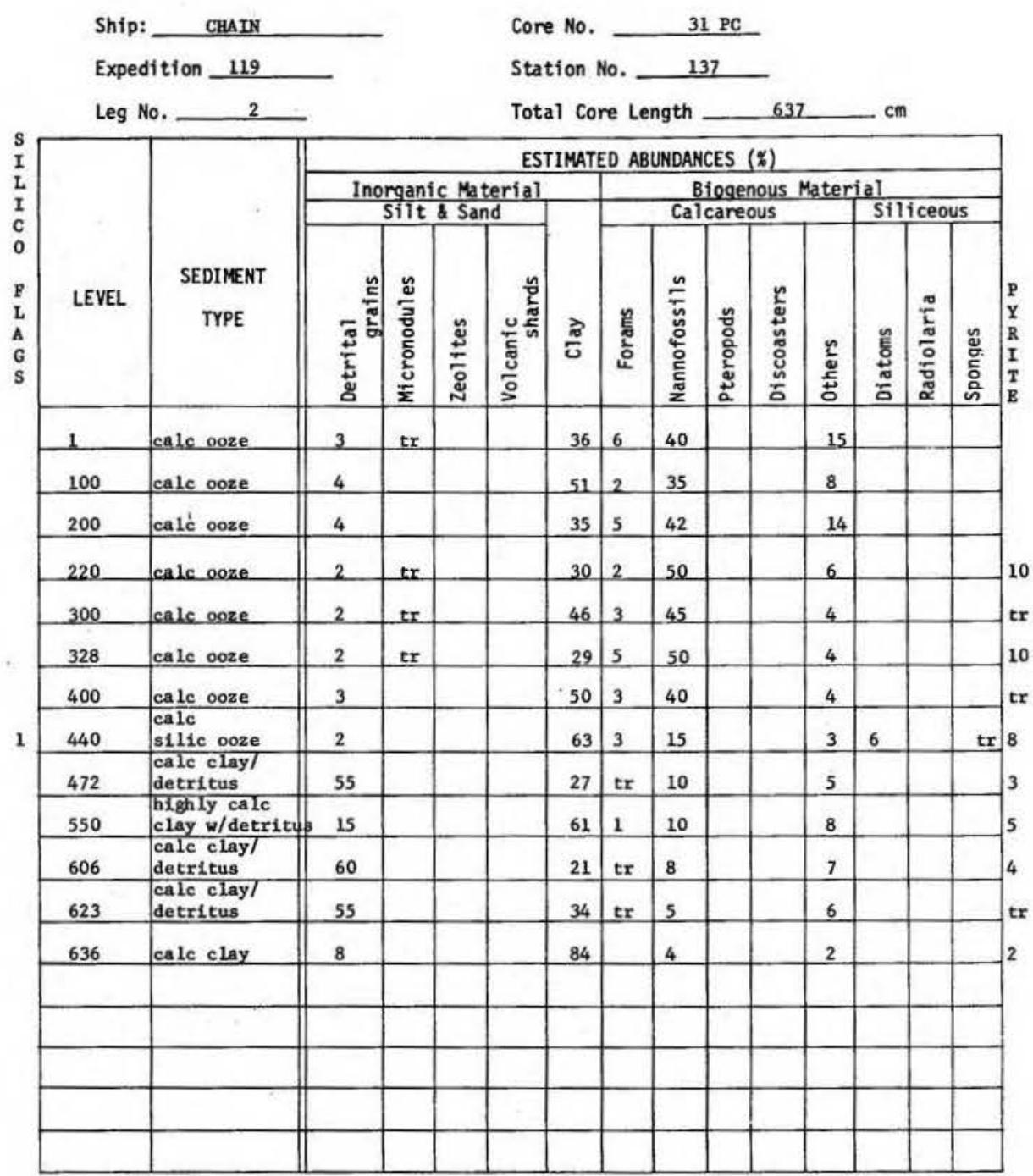




\section{VISUAL CORE DESCRIPTION}

Page 1 of 1

Ship CHAIN Cruise 119 Leg II Sto. 137 Core No. 3IPG Total Length $97 \mathrm{~cm}$. Lat. $3 x^{\circ} 56.2^{\prime \prime N}$ Long. $33^{\circ} 45.0^{\prime} E$ Depth 1637 meorr. Core condition ExLEUENT Lithologic

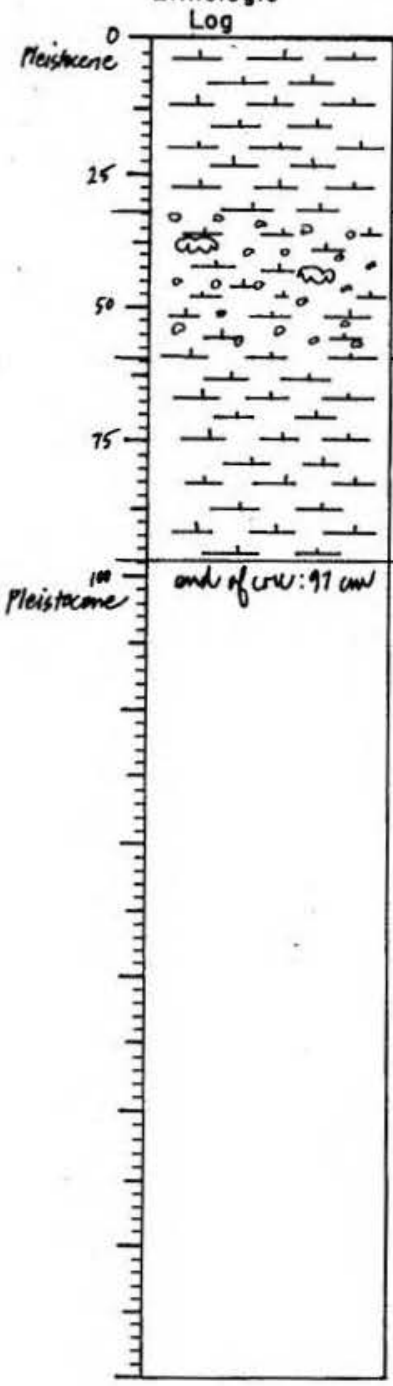

\section{Detailed Description}

0-32

\section{CALC OOZE}

10 YR 6/4 Iight yellowish brown

howogeneous throughout, except for some slight inter-

mottling at basal contact s, mottled

S2-59, mottle

CALC $002 E$

Y $3 / 2$ dark olive gray

tense, very fine, 1ight olive mottling and flecking throughout, with two very large, grayish-brown bur-

rows spanning liner $37-38 \mathrm{~cm}, 41-42 \mathrm{~cm}$

moist, slick lutite with abundant forams and scattered pteropods 59-97 noist, slick, unconsolidated lutite with a few scat-

\section{CALC OOZE}

10 YR $5 / 2$ grayish brown, grades to $6 / 3$ pale brown, to $6 / 4$ 1ight yellowish brom

slight, fine intermottiing throughout

moist, slick lutite with a few scattered foram

end of core
SHEAR SLIDE DESCRIPTIONS - W.H.O.I. SEDIMENT CORES

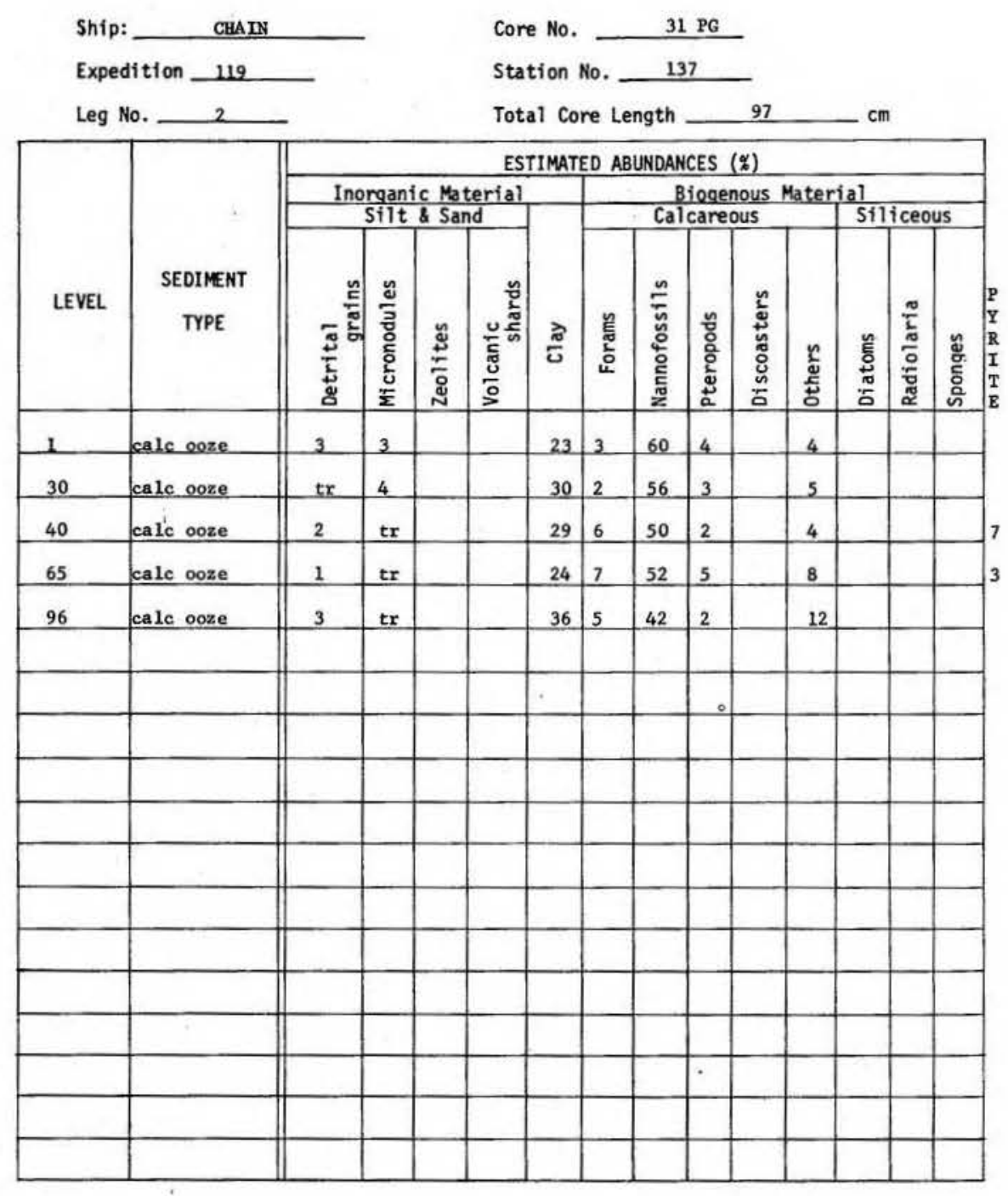


Ship $C_{\text {HAIN }}$ Cruise 119 Leg 2 Sto. 139 Core No. 32 PC Core condition ExceuENT Date Described 2 Feb 76 by T FARMER Physiographic location Edge of LEVANT PLATFaRM, N. of Part Said, Egypt; EMS. Lithologic

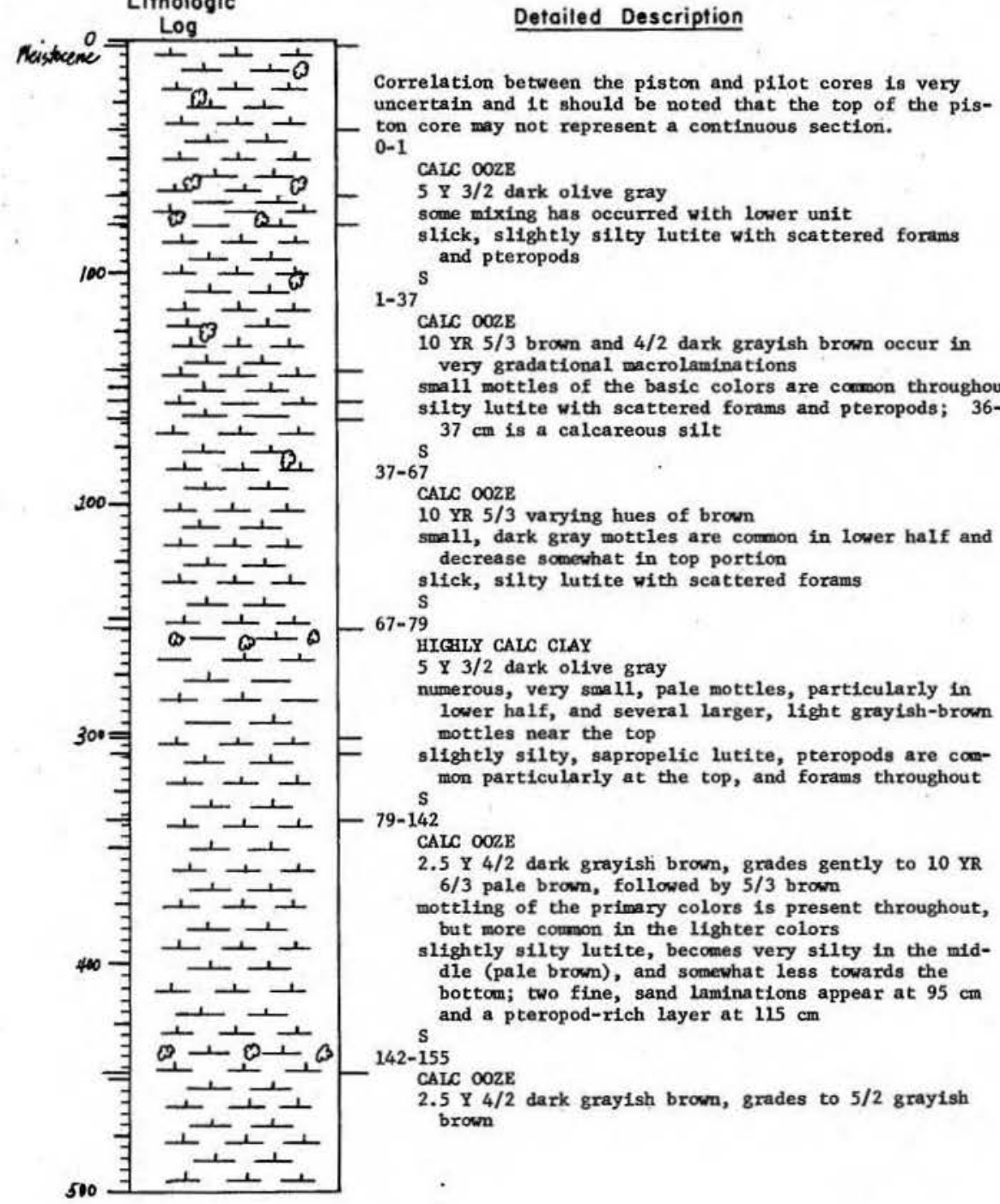

Ship CHAIN Cruise 119 Leg 2 Sta. 139 Core No. 32 PC

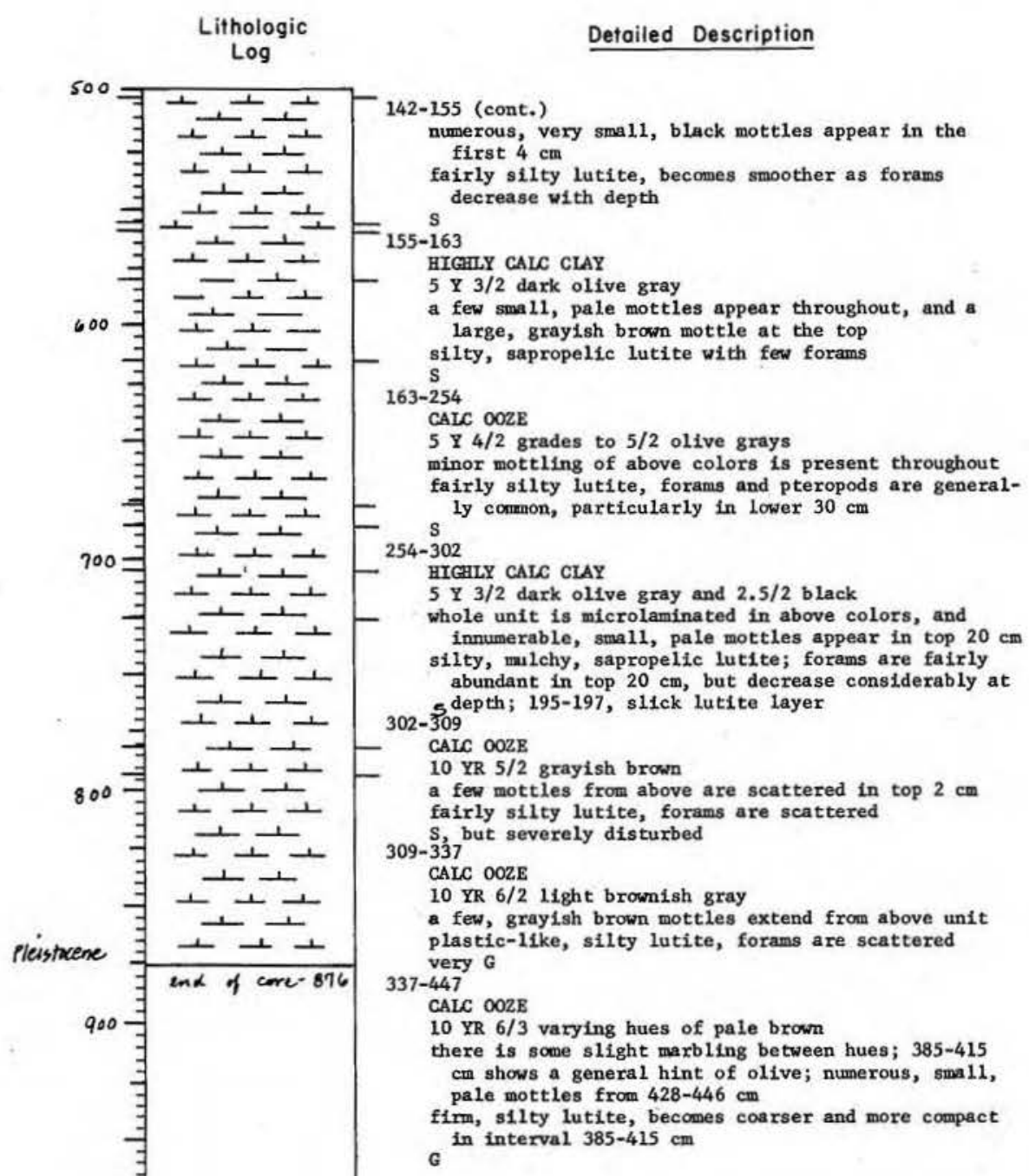


651

VISUAL CORE DESCRIPTION

Ship CHAIN Cruise 119 Leg II Sta. 139 Core No. $32 \mathrm{PC}$

Lithologic

Detailed Description

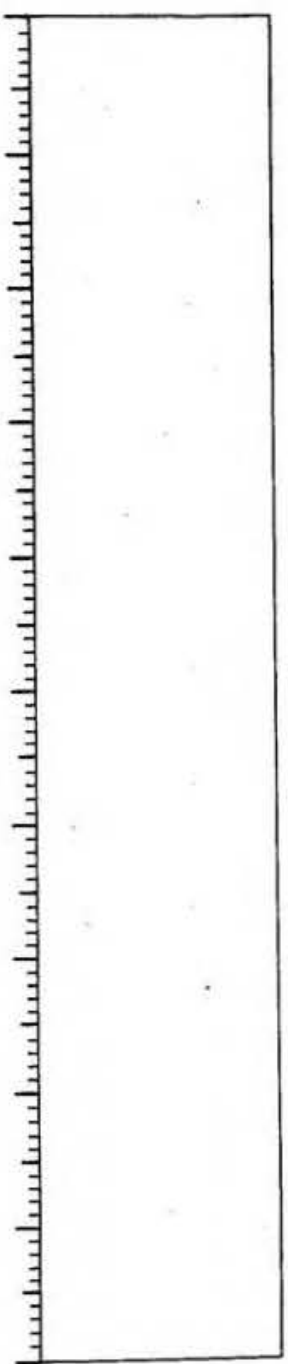

Page 3 of 4

$47-503$

CALC OOZE

5 Y $4 / 2$ olive gray, $3 / 2$ dark ollve gray laminations in lower half

innumerable, fine microlaminations are particularly

inm, silty, mulchy, sapropelic lutite; forams vary 503-556

CALC OOZE

5 Y $6 / 2$ light olive gray, grades to $5 / 2$ olive gray innumerable, small, very subtle mottles appear from

very silty lutite, grades to fairly slick lutite with S

HTGLYY CAIC CIAY

5 Y $3 / 2$ dark olive gray

innumerable, sma11, pale mottles throughout

finn, fairly slick lutite, forams are scattered S, but severely disoriented

$560-576$

CAIC OOZE

5 Y $5 / 2$ grades to $4 / 2$ olive grays

slightly silty lutite with common forams

S, b

HTCHLY CALC CIAY

5 Y $3 / 2$ dark olive gray

innumerable, well-deflned microlaminations

silty, mulch-1ike, foram-rich sapropel, grades to a $\mathrm{S}$
-677

CAL 677 . 008

5 Y $4 / 2$ grades to $5 / 2$ olive grays, and $6 / 2$ 11ght

olive gray

scattered, intermottling is present, particularly

with a darker lamination 642-648 cm

irm, fairly silty lutite, becomes very silty in

s

CALC OOZE
5 Y $3 / 2$ dark olive gray, grades to $4 / 2$ olive gray

numerous, $\operatorname{sma11}$, pale mottles throughout foram-rich, sapropelic lutite, becomes less silty and

s, but severely distorted

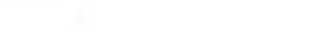

652

VISUAL CORE DESCRIPTION

Page 4 of 4

Ship CHAIN Cruise 119 Leg II Sta. 139 Core No. 32 PC

Lithologic
Detailed Description

686-705 CALC OOZE

5 Y $4 / 2$ olive gray

cormon, small, subtle mottles throughout

firm, very silty lutite with comon forams

705-725

CALC OOZE

5 Y $3 / 2$ dark ollve gray

microlaminations

mulchy, sapropelic lutite; forams are numerous to abundant

$25-78$

CALC OOZE

5 Y $5 / 2$ olive gray, grades to $5 / 3$ olive

extensive marbling $1 \mathrm{~s}$ present from $740-775 \mathrm{~cm}$; a 2 th forams more

781-793

CALC OOZE

5 Y $3 / 2$ dark olive gray $\mathrm{s}$

793-876

CALC OOZE

YR $4 / 2$ dark grayish brown, grades to $5 / 3$ hues of brown $5 / 2$ grayish brown

middle (813-855) romewhat slick lutite; forams are abumdant near the top, but scattered in the remainder of the unit end of core

NOTE: the cyclic nature of the above combination of lifthologies should not be overlooked 
SHEAR SLIDE DESCRIPTIONS - W.H.O.I. SEDIEENT CORES

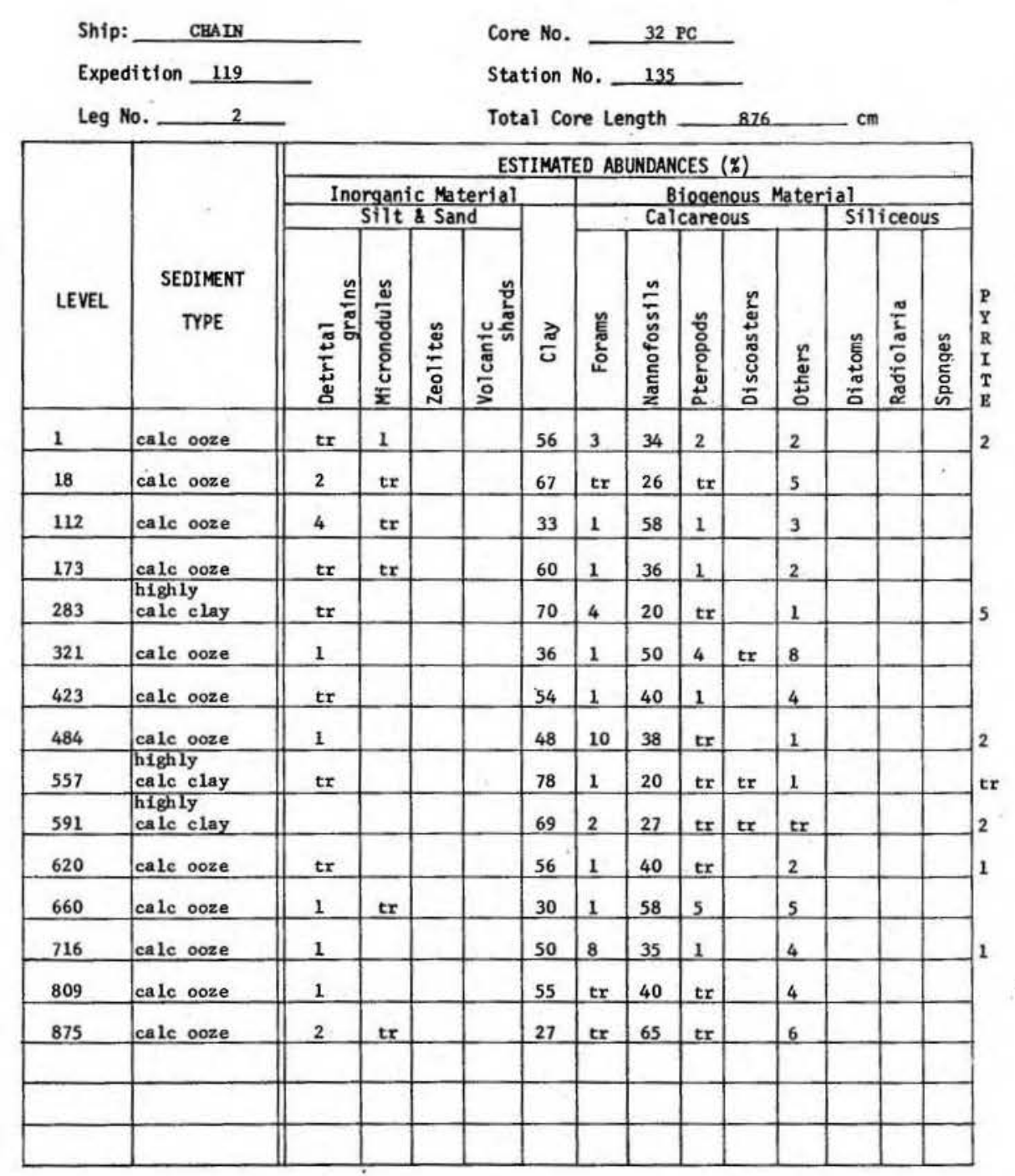

Ship CHAIN Cruise 119 Leg 2 Sto. 139 Core No. 32 PG Total Length $93 . \mathrm{cm}$. Lat.32. $50.07^{\prime} \mathrm{N}$ Long. $32^{\circ} 59.27^{\prime} \mathrm{E}$ Depth $1381 \mathrm{umm}$. Core condition ExceweNT Date Described $9 \mathrm{Feb} 76$ by T. Facmes Physiographic location Edge of LEVANT PLATrFoem, N. of Rort Said, Egypt; E. M.S. Lithologic

$$
\begin{aligned}
& \text { Pleisticene Defailed Description } \\
& \text { Pleiticene }
\end{aligned}
$$


SIEAR SLIDE DESCRIPTIONS - W.H.O.I. SEDIMENT CORES

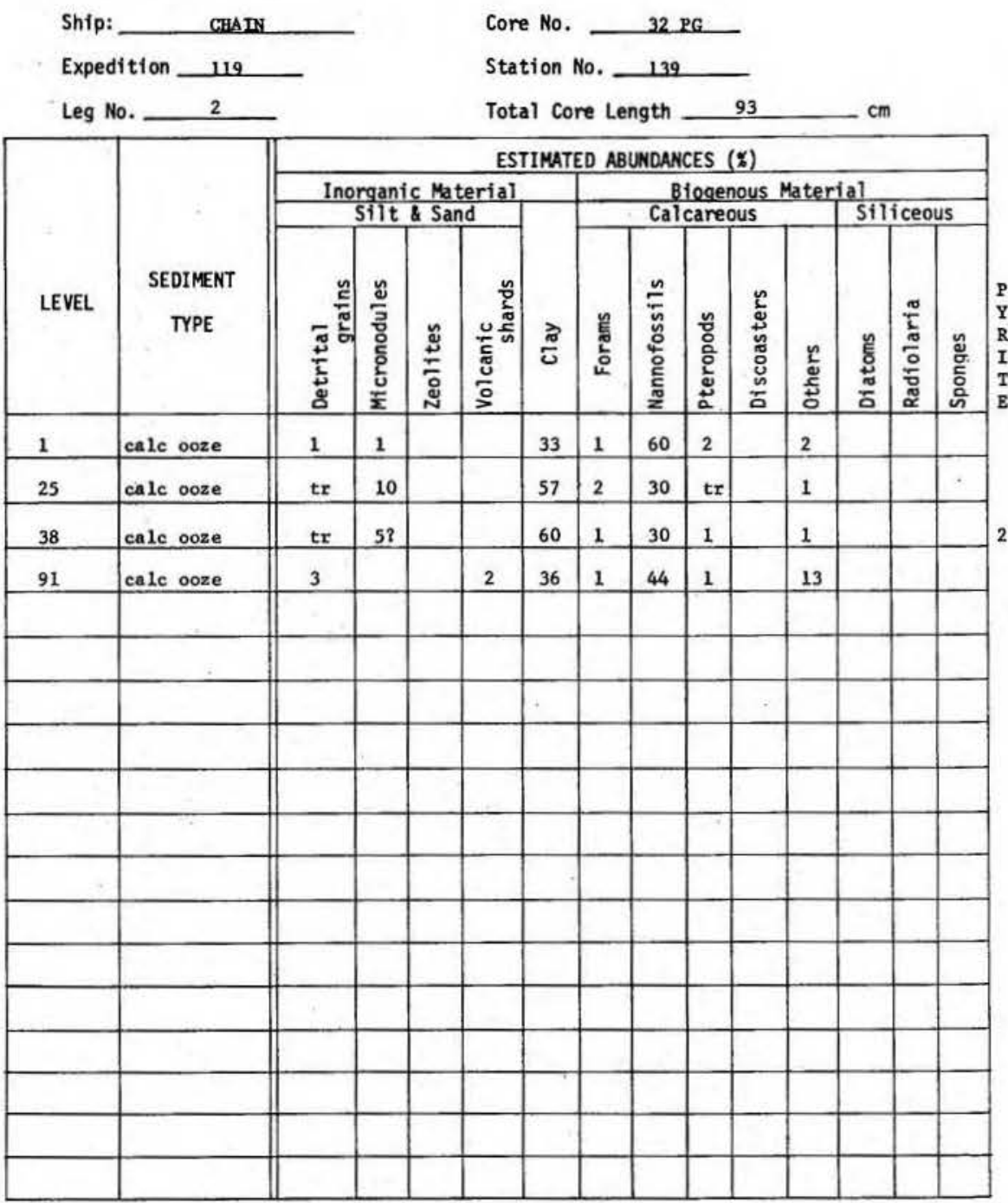
Ship $\frac{\text { CHAIN }}{\text { Total Length }}$ Cruise $\frac{119}{904} \mathrm{~cm}$. Lat. $32^{\circ}{ }_{32.6^{\prime} \mathrm{N}}$ Long. $\frac{141}{32^{\circ} 17.2}$ Core No. $\frac{33 \mathrm{PC}}{1149 \text { Core. }} \mathrm{m}$. Core condition Exievent - Date Described 12 Fes 76 by frota Physiographic location ISIS RIDGE GIMREXX, N. of PORT SAID, EAST. MEDITERRANOW Lithologic

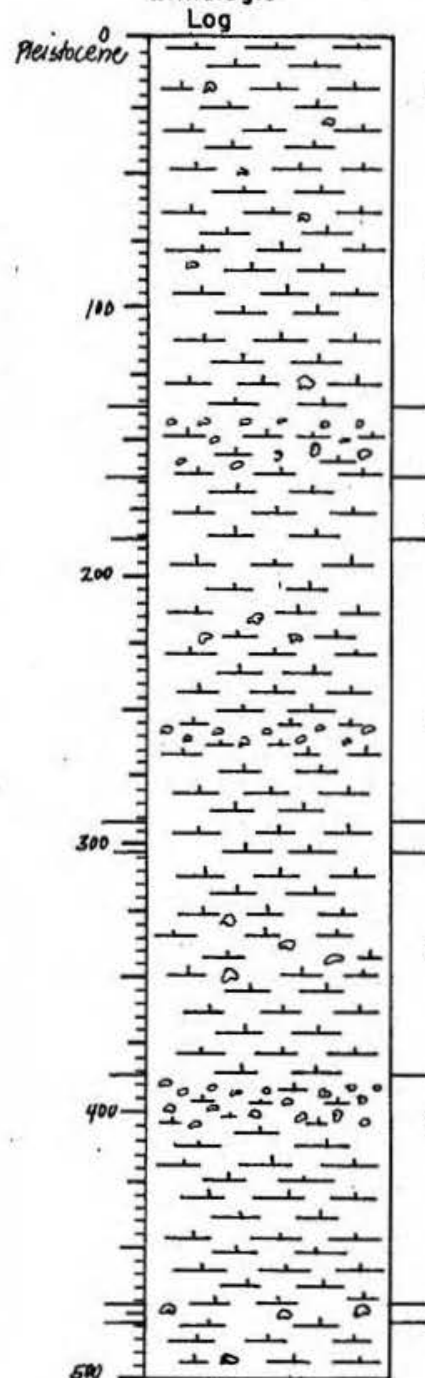

Detailed Description

$0-137$

CAIC OOZE

varying hues of 10 YR $5 / 2$ grayish brown and $6 / 3$ pale

slight, irregular intermottling found scattered throughout moist, slick lutite with scatered forams, a few pteropods $G$

137-164

CALC OOZE

5 Y $3 / 2$ dark olive gray

ive gray mottling and flecks

fine microlaminae are found near

asal contact

bit more compact, slick lutite with scattered forams and pteropods

164-186

CALC OOZE

10 YR $5 / 2$ grayish brown

homogeneous throughout, except for one thin, pale brown lethet $176 \mathrm{cos}$

lutite with scattered forams and a silty lamination at basal contact 186-29

CAIC OOZE

10 YR $6 / 3$ pale brown, grades to $6 / 4$ light yellowish

common intermotting of the colors above in transitional

zones

extensively mottled sapropel-1ike zone (5 X 5/3) $255-265 \mathrm{~cm}$
exteristy extent

291-302

CALC OOZE

2.5 Y $3 / 2$ very dark grayish brown

homogeneous throughout

compact, silty lutite with abundant forams

sapropel-like zone

302-386

CALC OOZE

10 XR $5 / 2$ grayish brown, grades to $6 / 3$ pale brown

common intermottling of colors above in transitional zone

1ick lutite with scattered forams, irregular lenses and

inclusions of silty carbonate fragments in disturbed

core appears disturbed and unstratified $321-341 \mathrm{~cm}$

$G$, mottled 
657

VISUAL CORE DESCRIPTION

Page 2 of 3

Ship CAAIN Cruise 119 Leg II Sta. 141 Core No. 33PC

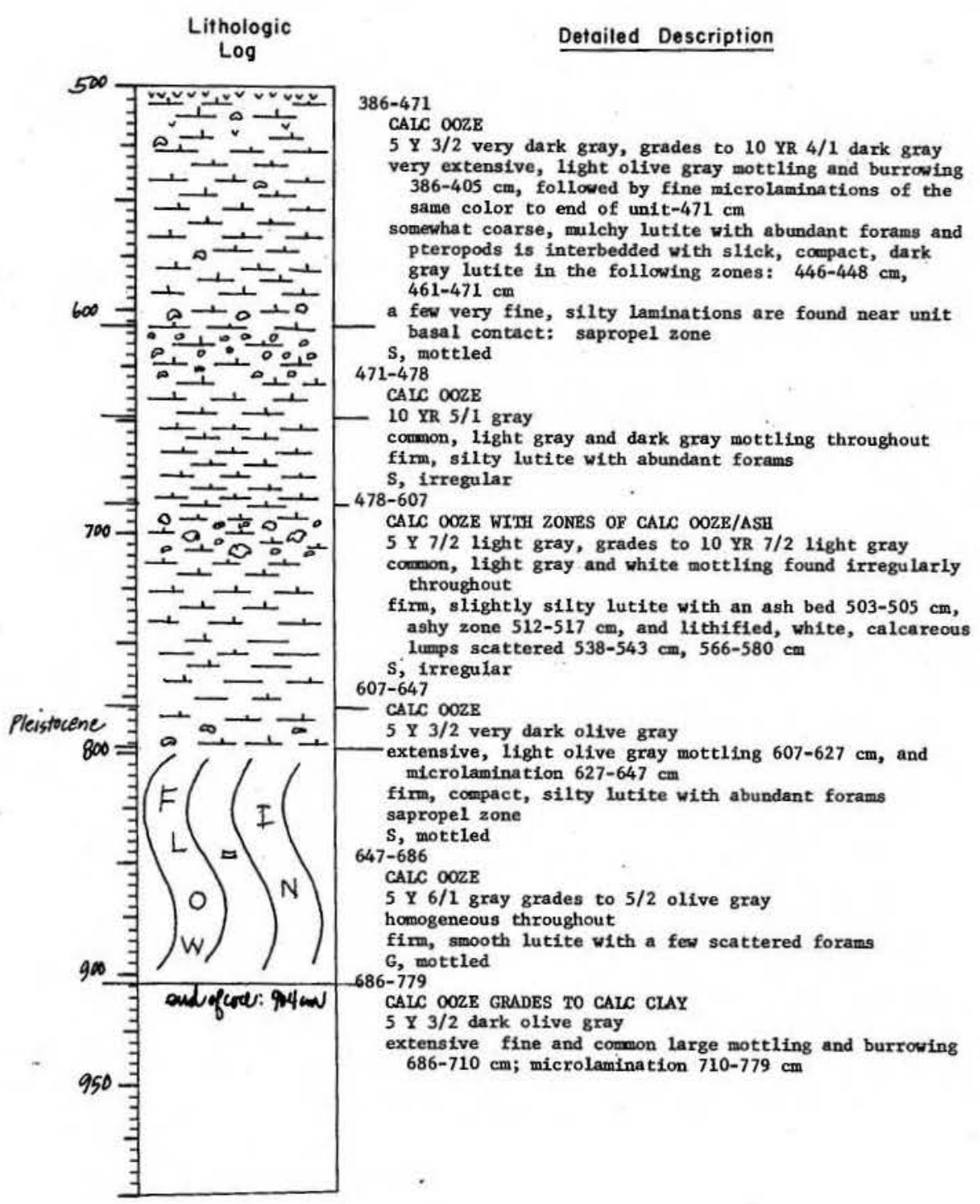

658

VISUAL CORE DESCRIPTION

Page 3 of 3 Ship CHAIN Cruise 119 Leg II Sta. 141 Core No. 33 PC

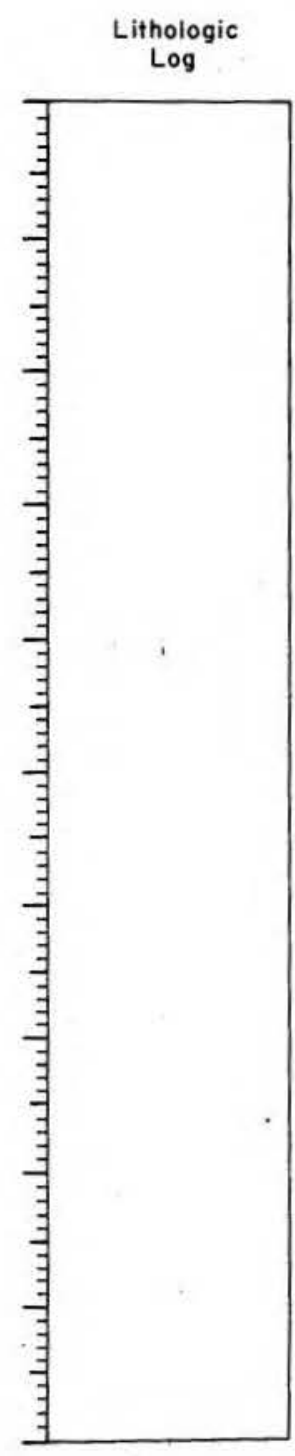

\section{Detailed Description}

686-779 (cont.)

firm, compact, silty lutite with abundant forams sapropel zone

S, horiz onta
$779-796$

CALC OOZE

10 YR $6 / 3$ pale brown

faint, light gray mottling found throughout

firm, compact, slightly silty lutite with scattered s, irregula

796-904 ${ }^{2}$

FLOW IN

end of core 
SIEAR SLIDE DESCRIPTIONS - W.H.O.I. SEDINENT CORES

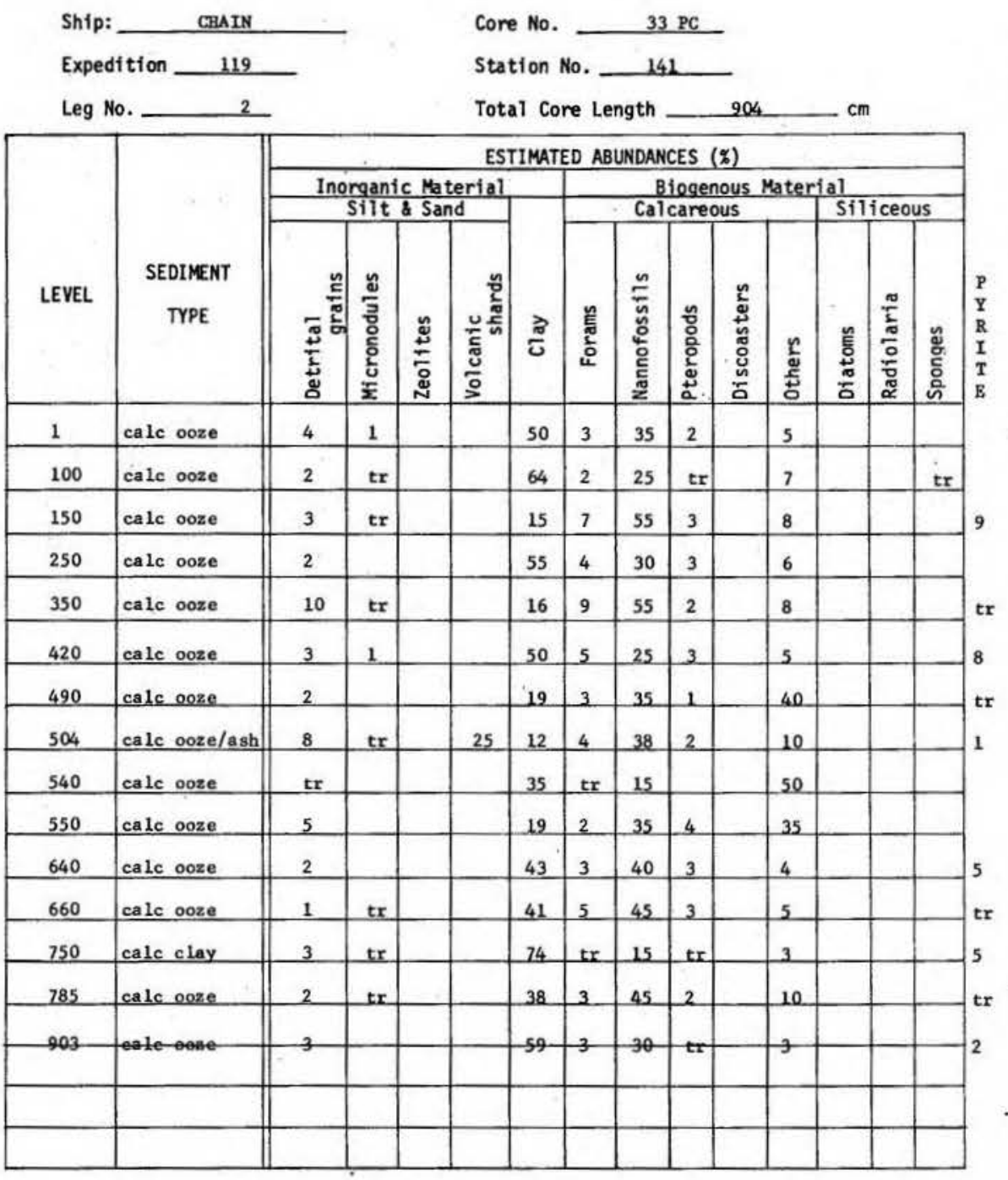

VISUAL CORE DESCRIPTION

Page 1 of 1

Ship CHAIN Cruise $119 \quad$ Leg $I I$ Sta. 141 Core No. $339 \mathrm{G}$ Total Length $84 \mathrm{~cm}$. Lat. $32^{\circ}{ }_{32.6^{\prime} \mathrm{N} \text { Long. } 32^{\circ} 17.2}^{\prime} \mathrm{E}$ Depth 1149 CoRR $\mathrm{m}$ Core condition EXCEUENT Date Described ILFES 76 by f. Burder

Physiographic location Is/S RIDGE CIMPCEX, N. Of PORT SAID, EAST. MED. SEA Lithologic

$$
\begin{gathered}
\text { Lithologic Lotailed Description } \\
\text { Log }
\end{gathered}
$$

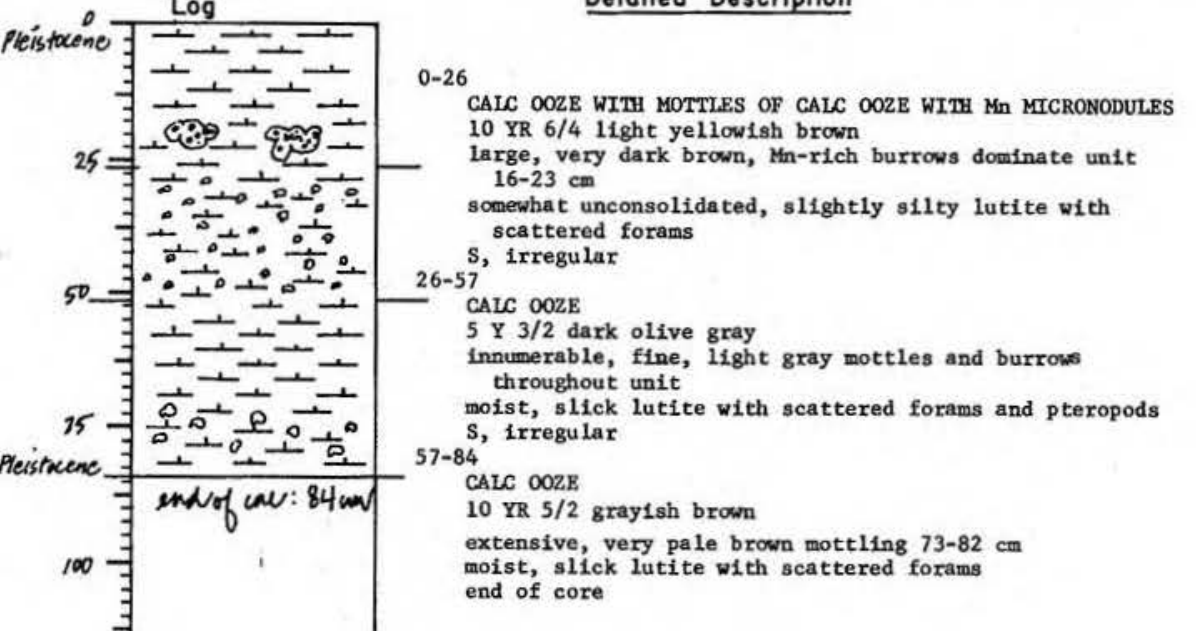
MICRONODUL somewhat unconsolidated, slightly silty lutite with extensive, very pale brown mottilng $73-82 \mathrm{~cm}$ end of core

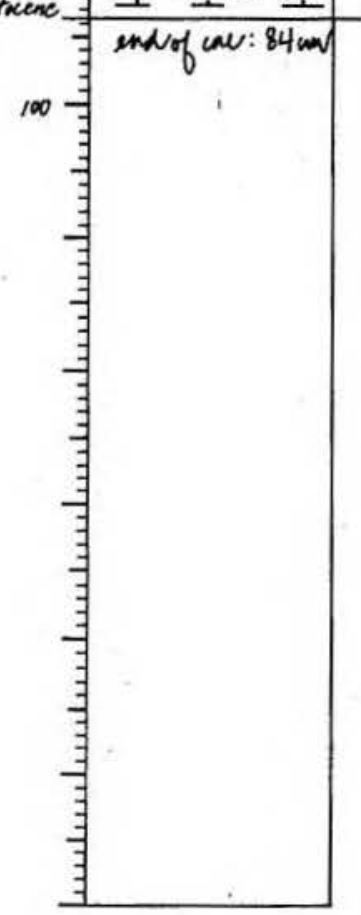


SMEAR SLIDE DESCRIPTIONS - W.H.O.I. SEDINENT CORES

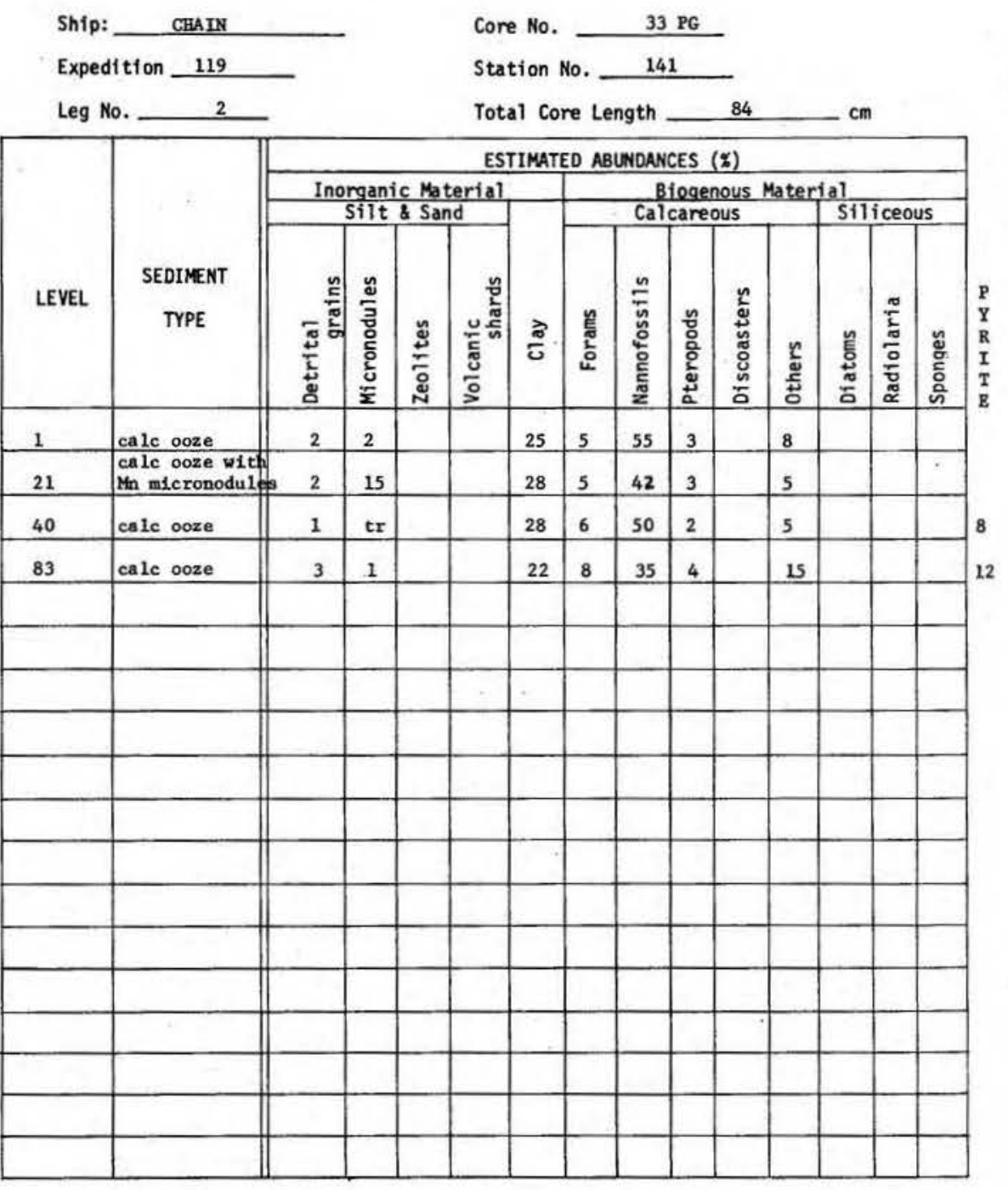

Ship CHAIN Cruise 119 Leg 2 Sto. 143 Core No. 34 PC Total Length $842 \mathrm{~cm}$. Lat. $32^{\circ} 17.3^{\circ}, \frac{2}{\mathrm{~N}}$ Long. $33^{\circ} 24.9^{\prime} \mathrm{E}$ Depth $1108 \mathrm{com} \mathrm{m}$ Core condition EXCELLENT Date Described 10 EES 76 by T FAXMER

Physiographic location SMaLLow PART of LEVANT PLATFOAM, E.M.S.

Lithologic

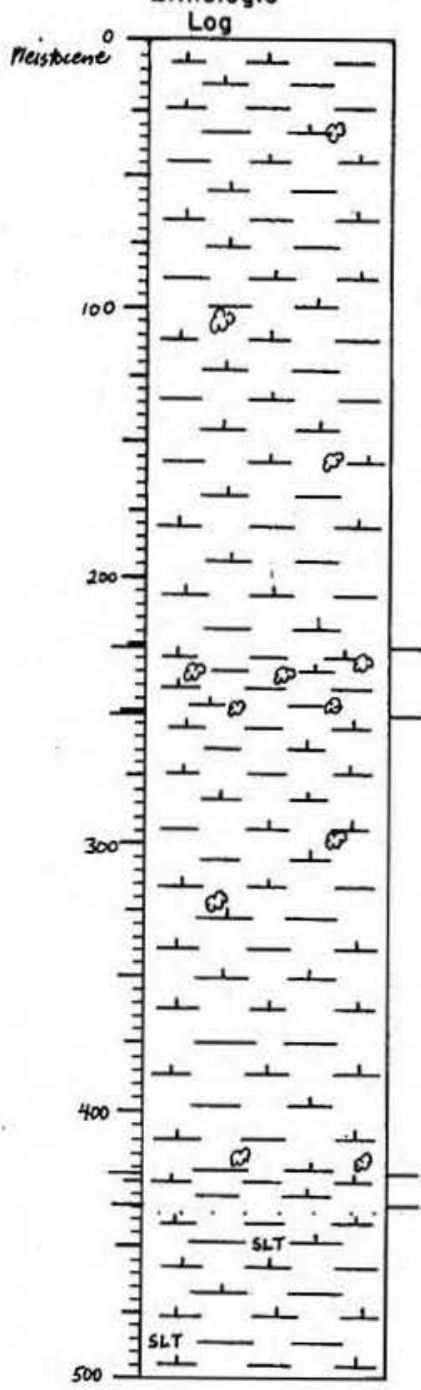

Detailed Description

$0-226$

HIGHLY CALC CLAY

5 Y $4 / 2$ and $5 / 2$ olive grays; various hues of above colors appear as gradational bands with $5 / 2$ more promInent below $125 \mathrm{~cm}$

mottling, generally of the darker colors in the paler bands, is scattered throughout

somewhat sifck, silty lutite, becomes more compact with deastonally, faint olive yellored appear in olive grays of this core

226-251

HIGHLY CALC CLAY

Y $3 / 2$ dark olive gray

innumerable, small, light olive gray mottles throughout, and a few black (pyrite-rich) mottles strewn about

$\mathrm{S}_{251-423}$

BIGHLY CALC CLAY

Y $4 / 2$, grades to $5 / 2$ olive grays, and $5 / 3$ olive (310$350 \mathrm{~cm}$ ), and back to $4 / 2$

dn paler areas; abundant small mottles from lower unit in bottom $10 \mathrm{~cm}$

aber forams are scattered throughout; $386 \mathrm{~cm}-5 \mathrm{~mm}$ dis$6^{\text {tu }}{ }^{423-434}$

HIGHLYY CALC CLAY

5 Y $3 / 2$ dark olive gray

fim, somewhat sticky, slightly silty lutite with few fora:

434-580

HIGHLY CALC CLAY WITH DETRITUS

5 Y $5 / 2$ ollve gray grades to $5 / 3$ olive in lowest $25 \mathrm{~cm}$ very few, subtle mottles, except in bottom $25 \mathrm{~cm}$ wher they are abundant, small, dark olive gray

firm, slightly silty to silty lutite, forams and ptero$G$ pods are few; thin send lense at $437 \mathrm{~cm}$ 
663

VISUAL CORE DESCRIPTION

Ship CHAIN Cruise 119 Leg 2 Sta. 143 Core No. 34 PC

Lithologic

Log

Detailed Description

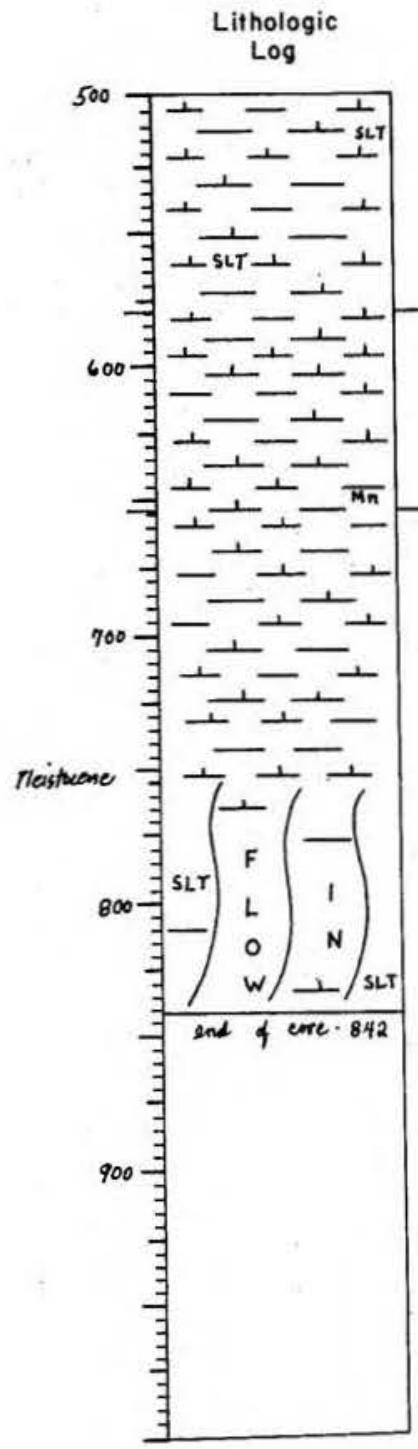

atensely burrowed and mottled in top $10 \mathrm{~cm}$; below,

664

SIEAR SLIDE DESCRIPTIONS - K.H.O.I. SEDINENT CORES

Ship:

Expedition 119

Y $3 / 2$ dark olive gray, grades very gently to $4 / 2$ $11 y$ few mottles, but innumerable, $8 \mathrm{~cm}$ - thin, black, Manextends across half of core firm, compct, slightly silty sapropelic lutite with forams common; becomes smoother as color pales very gradationa 1

654-754

IGHLY CALC CLAY

5 Y $4 / 2$ olive gray

no mottilng

lutite uith scattered silt lenses and

clasts, and several detrital silts of $2-5 \mathrm{~cm}$

HICur

CALC CIAY WITH DETRTTUS

low in of above

end of core

Leg No. 2

\begin{tabular}{|c|c|c|c|c|c|c|c|c|c|c|c|c|c|c|c|}
\hline \multirow[b]{3}{*}{ LEVEL. } & \multirow[b]{3}{*}{$\begin{array}{c}\text { SEDIMENT } \\
\text { TYPE }\end{array}$} & \multicolumn{14}{|c|}{ ESTIMATED ABUNDANCES ( $(x)$} \\
\hline & & \multicolumn{4}{|c|}{\begin{tabular}{|c|} 
Inorganic Material \\
Silt \& Sand \\
\end{tabular}} & \multirow[b]{2}{*}{$\frac{\pi}{4}$} & \multicolumn{6}{|c|}{$\begin{array}{l}\text { Biogenous Mater } \\
\text { Calcareous }\end{array}$} & \multicolumn{3}{|c|}{ al } \\
\hline & & 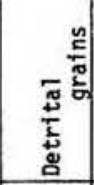 & 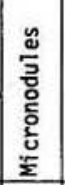 & 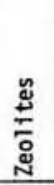 & 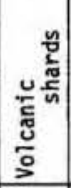 & & 宸 & & & 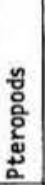 & 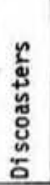 & c & 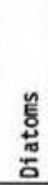 & $\frac{\pi}{2}$ & 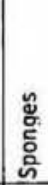 \\
\hline 1 & $\begin{array}{l}\text { highly } \\
\text { calc clay }\end{array}$ & 10 & & & & 62 & $t x$ & & & & & 10 & & & \\
\hline 100 & $\begin{array}{l}\text { highiy } \\
\text { calc clay }\end{array}$ & 10 & & & & 61 & tr & & & 1 & & 18 & & & \\
\hline 188 & $\begin{array}{l}\text { highly } \\
\text { calc_clny }\end{array}$ & 10 & tr & & & 64 & $t x$ & & & 1 & & 10 & & & \\
\hline 240 & $\begin{array}{l}\text { calc ooze/ } \\
\text { pyrite }\end{array}$ & & & & & 15 & & & & & & & & & \\
\hline 243 & $\begin{array}{l}\text { highly } \\
\text { calc clay }\end{array}$ & $\mathrm{tr}$ & & & & 75 & 1 & & & $\mathrm{tr}$ & & 2 & & & \\
\hline 340 & $\begin{array}{l}\text { highly } \\
\text { calc clay }\end{array}$ & 10 & & & & 61 & 2 & & & 2 & & 8 & & & \\
\hline 430 & $\begin{array}{l}\text { high ly } \\
\text { calc clay }\end{array}$ & $\mathrm{tr}$ & & & & 67 & 1 & & & tr & & 1 & & & \\
\hline 530 & $\begin{array}{l}\text { highly calc } \\
\text { clay w/detritu }\end{array}$ & 16 & & & & 64 & tr & & & & tx & 10 & & & \\
\hline 593 & $\begin{array}{l}\text { highiy } \\
\text { calc clay }\end{array}$ & & & & & 70 & 1 & & & tr & & tr & & & \\
\hline 648 & micronodules & $\mathrm{tr}$ & $100 ?$ & & & tr & & & & & & tr & & & \\
\hline 698 & $\begin{array}{l}\text { highly } \\
\text { calc clay }\end{array}$ & 10 & tr & & & 72 & tr & & & & & 13 & & & \\
\hline 740 & detritus & 83 & & & & 2 & & & & & & 15 & & & \\
\hline 841 & $\begin{array}{l}\text { highly calc } \\
\text { clay with det }\end{array}$ & Ltus 16 & $t x$ & & & 61 & tr & & & & & 22 & & & \\
\hline & & & & & & & & & & & & & & & \\
\hline & & & & & & & & & & & & & & & \\
\hline & & & & & & & & & & & & & & & \\
\hline & & & & & & & & & & & & & & & \\
\hline & . & & & & & & & & & & & & & & \\
\hline
\end{tabular}

Total Core Length $\quad 842$

STIMATED ABUMDANCES

Core No. 34 . PC

Station No. 143

Station No. 
Ship CHAIN Cruise 119 Leg 2 Sto. 143 Core No. 34 PG Total Length $51 \quad \mathrm{~cm}$. Lat. $32^{\circ} 17.3^{\prime} \mathrm{N}$ Long. $33^{\circ} 24.9^{\prime} \mathrm{E}$ Depth $1108 \mathrm{~cm}$. Core condition ExCELENT Date Described 10 Feb 76 by TFARMER Physiographic location SHALLOW PANT OF LEVANT PLATFORM. EMS

Lithologic

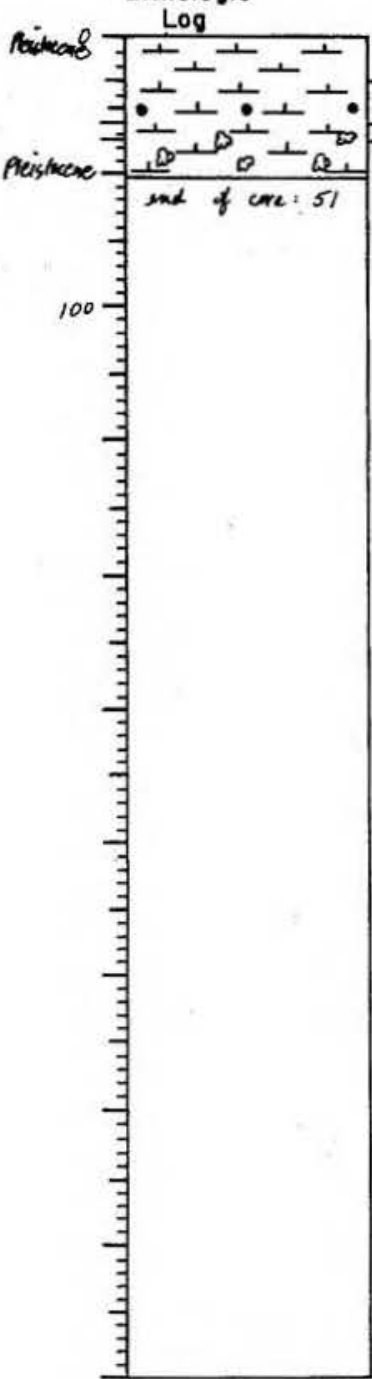

Detailed Description

CALC $00 Z$

$10 \mathrm{YR} 5 / 3$ brown, becomes somewhat paler with depth somewhat unconsolidated, slightly silty lutite, with scattered pteropods and forams

5-30

CALC OOZE AND CALC OOZE WTTH Mn MICRONODULES

10 YR $6 / 3$ pale brown, and $3 / 3$ several hues of dark

brom; the two colors are mixed and marbled; pale lightly ailty lutite with ocettered foran G

CALC OOZE

10 YR $5 / 2$ grayish brown, grades to $4 / 2$ dark grayish brown sma11, 11ght brownish gray mottles are coumon in bottom ear lower unit

$37-5$

CALC OOZE

$5 \times 3 / 2$ dark olive gray

innumerable, small, 1ight brownish gray mottles, and several larger ones scattered about; a large burro from above extends several cms into this unit; a well-preserved lamination in aane color as moteling 1s present at $48.5 \mathrm{~cm}$. few to abundant

end of core

SMEAR SLIDE DESCRIPTIONS - W.H.O.I. SEDIMENT CORES

Ship: CHA

Leg No. 2
Expedition 11

Core No. 34 PG

Station No. 134

Total Core Length _ 50

ESTIMATED ABUNDANCES ( $($ )

\begin{tabular}{|c|c|c|c|c|c|c|c|c|c|c|c|c|c|c|}
\hline \multirow[b]{4}{*}{ LEVEL } & \multirow[b]{4}{*}{$\begin{array}{c}\text { SEDIMENT } \\
\text { TYPE }\end{array}$} & \multicolumn{13}{|c|}{ ESTIMATED ABUNDANCES ( $(\%)$} \\
\hline & & \multirow{2}{*}{\multicolumn{4}{|c|}{$\begin{array}{c}\text { Inorganic Material } \\
\text { Silt \& Sand }\end{array}$}} & \multirow[b]{3}{*}{$\frac{\overrightarrow{0}}{\sigma}$} & \multirow{2}{*}{\multicolumn{5}{|c|}{$\begin{array}{l}\text { Biogenous Mate } \\
\text { Calcareous }\end{array}$}} & & & \\
\hline & & & & & & & & & & & & \multicolumn{2}{|c|}{ Siliceous } & \\
\hline & & $\frac{5}{\frac{5}{5}}$ & 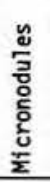 & 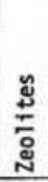 & 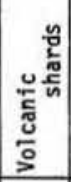 & & 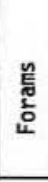 & 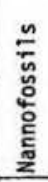 & 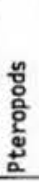 & 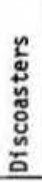 & L & 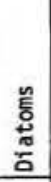 & $\begin{array}{l}\frac{\pi}{5} \\
\frac{\pi}{0} \\
\frac{0}{0} \\
\frac{\pi}{\alpha}\end{array}$ & 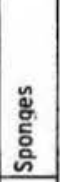 \\
\hline 1 & calc ooze & tr & tr & & & 30 & tr & 66 & 1 & & 3 & & & \\
\hline 25 & $\begin{array}{l}\text { calc ooze w/Ar } \\
\text { micronodules }\end{array}$ & tr & 15 & & & 36 & tr & 45 & tr & & 4 & & & \\
\hline 49 & calc ooze & $\operatorname{tr}$ & & & & 65 & tr & 30 & 1 & & 2 & & & \\
\hline & & & & & & & & & & & & & & \\
\hline & & & & & & & & & & & & & & \\
\hline & & & & & & & & & & & & & & \\
\hline & & & & & & . & & & & & & & & \\
\hline & & & & & & & & & & & & & & \\
\hline & & & & & & & & & & & & & & \\
\hline & & & & & & & & & & & & & & \\
\hline & & & & & & & & & & & & & & \\
\hline & & & & & & & & & & & & & & \\
\hline & & & & & & & & & & & & & & \\
\hline & & & & & & & & & & & & & & \\
\hline & & & & & & & & & & & & & & \\
\hline & & & & & & & & & & & & & & \\
\hline & & & & & & & & & & & & & & \\
\hline & 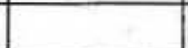 & & & & & & & & & & & & & \\
\hline
\end{tabular}


VISUAL CORE DESCRIPTION

Page 1 of 1

Ship CHanN Cruise 119 Leg 2 Sta. 145 Core No. I KC Total Length $271 \quad \mathrm{~cm}$. Lat. $31^{\circ} 51.87^{\prime} \mathrm{N}$ Long. $322^{\circ} 33.66^{\prime} \mathrm{E}$ Depth $348 \mathrm{com} . \mathrm{m}$

Core condition ExcEMENT Date Described 13 Feb 76 by $I$ EARMER Physiographic location BAReNIL ESCARPAENT eff POAT SAOC, EMS.

Lithologic

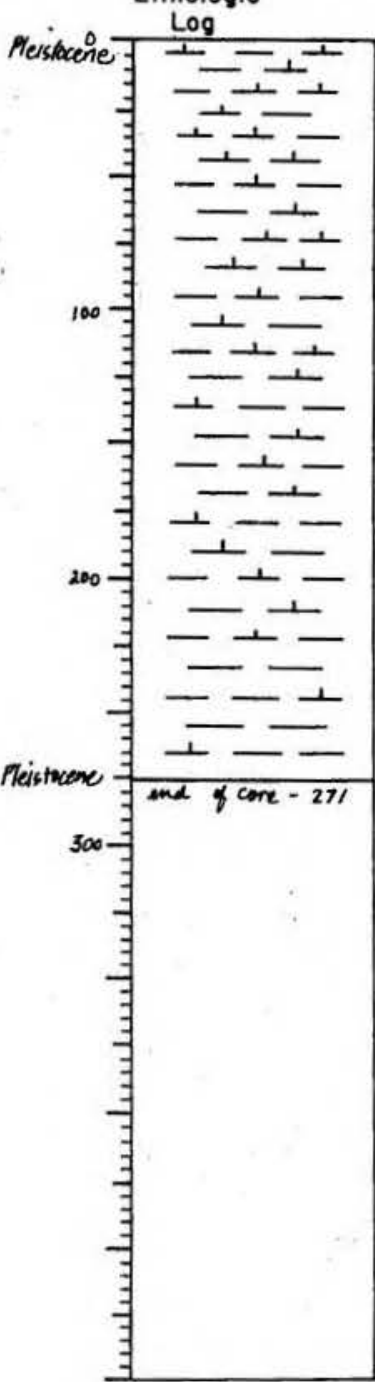

Detailed Description

$0-271$

HICHLY CALC CIAY GRADES TO SLIGHTLY CALC OLAY 2.5 Y $4 / 2$ dark grayish brown grades very smoothly to $3 / 2$ very dark grayish brown $(\sim 200 \mathrm{~cm})$ no mottling

silty, unconsoldated lutite grades gently to a slick, smooth lutite below $\sim 100 \mathrm{~cm}$; several sea urchin fragments are present at $23 \mathrm{~cm}$
SHEAR SLIDE DESCRIPTIONS - W.H.O.I. SEDINENT CORES

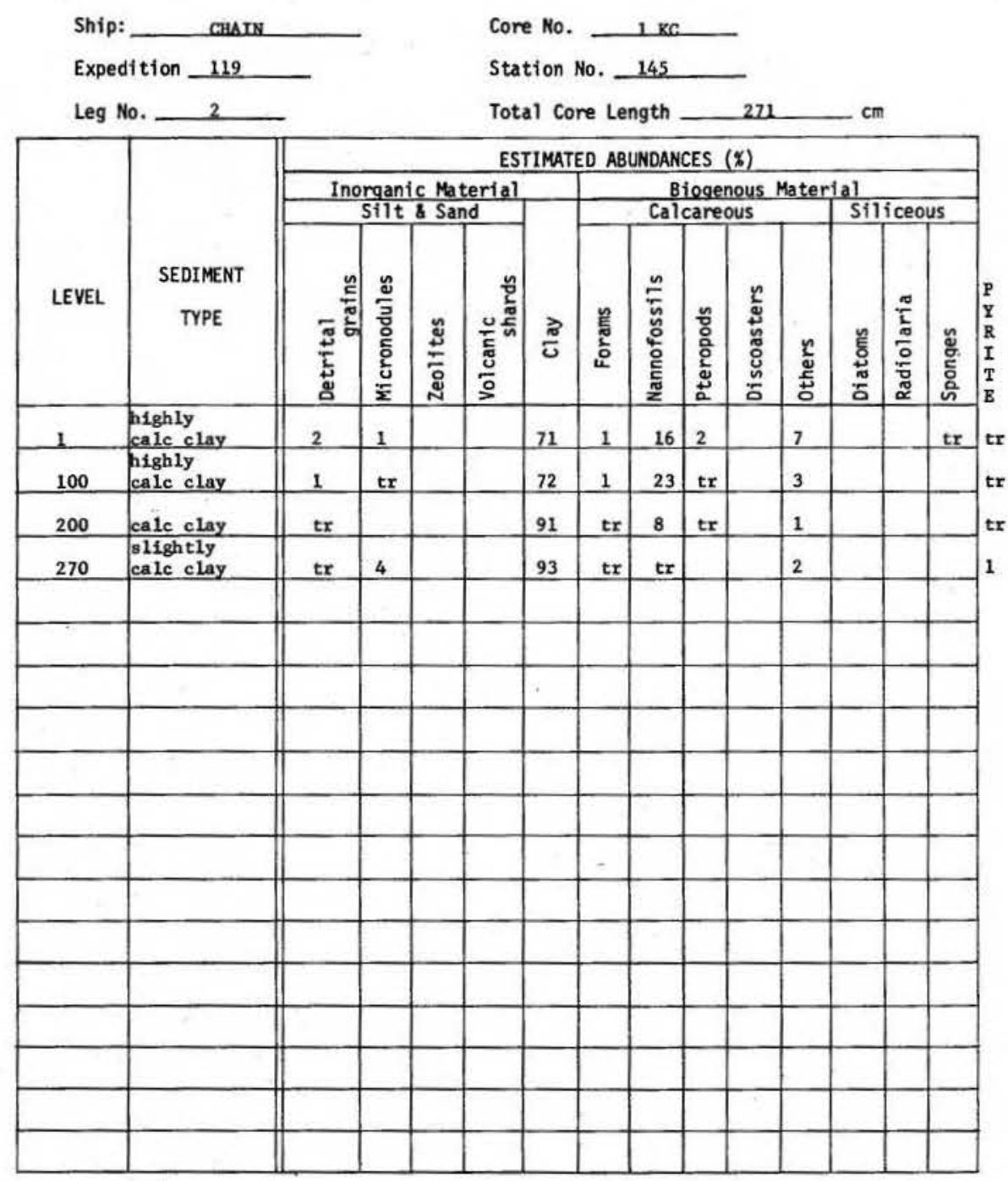


669

VISUAL CORE DESCRIPTION

Page_1 of _ 1

Ship CHAIN Cruise 119 Sta. 154 Core No. \& GC

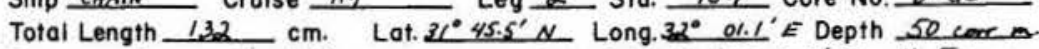
Core condition exallent Date Described 23 Sept'75 by H. Farmer

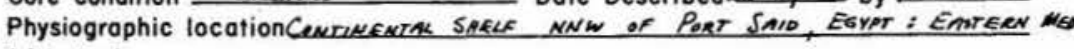
Lithologic

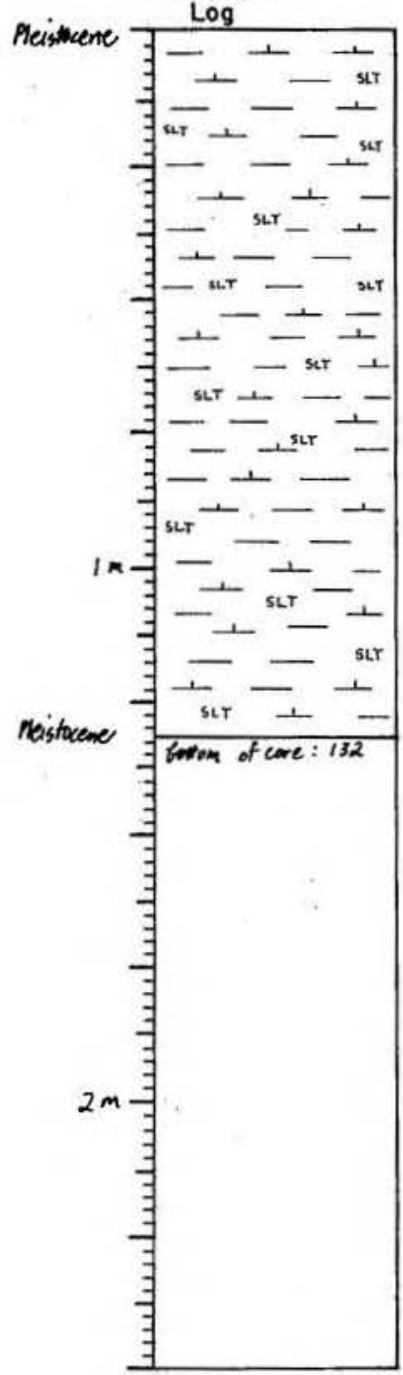

Detailed Description

0-132

HICHIY CALC CLAY HITH DETRITUS

10 YR $3 / 3$ dark brown

very uniform slick lutite, few bioclastic grains

end of core
670

SMEAR SLIDE DESCRIPTIONS - W.H.O.I. SEDIMENT CORES

Ship:

Expedition 119

Leg No. 2

Core No. $8 \propto C$

Station No. 15
Total Core Length $132 \mathrm{~cm}$

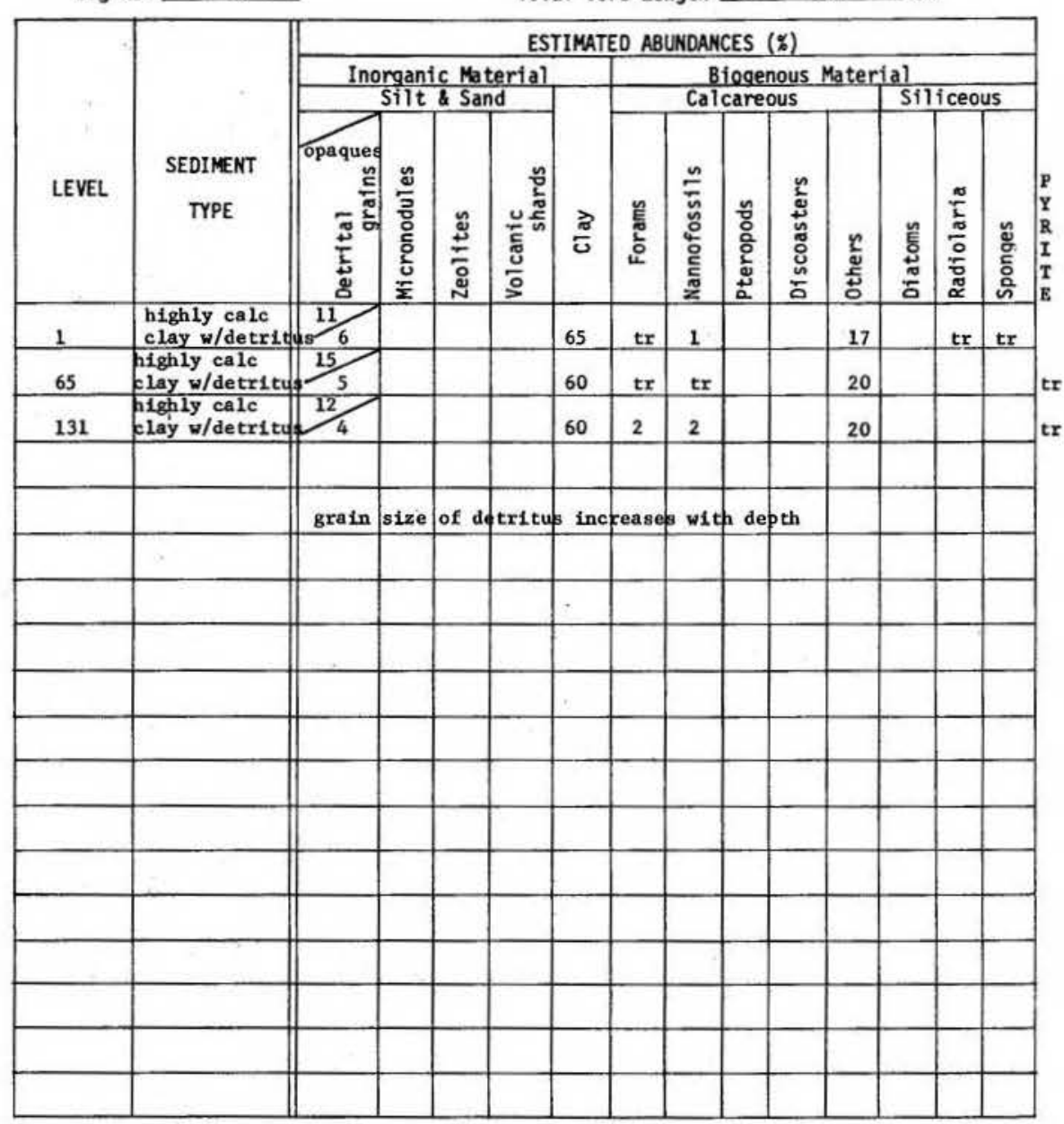


VISUAL CORE DESCRIPTION

Ship CHain Cruise 119 Leg 2 Sta. 156 Core No. 9 GC Total Length $137 \mathrm{~cm}$. Lat. $37^{\circ} 4 \mathrm{k}^{\circ} 1^{\prime} \mathrm{N}$ Long. $31^{\circ} 472^{\prime} \mathrm{K}$ Depth $50 \mathrm{~cm} \mathrm{~m}$ Core condition reallent Date Described 24 Iopt ' 75 by H. Fnemes Physiographic location Continendal Shelf NW of Port Sald, Eqypt : Eastera med. Lithologic

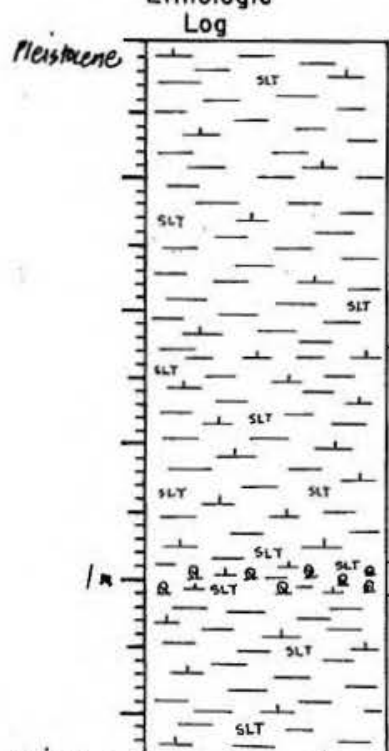

Plextwene $=\frac{1}{137 \text { i send of core }}$

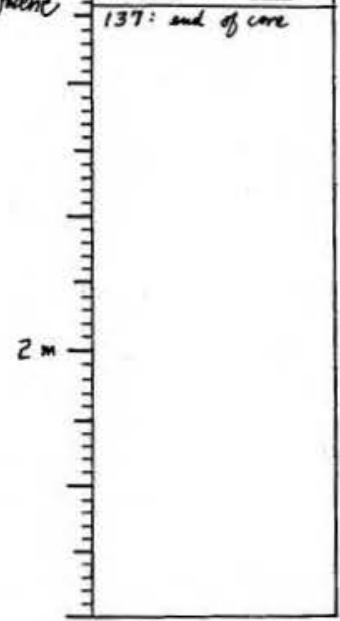

\section{Detailed Description}

$0-57$

\section{CIAY}

10 YR $3 / 3$ dark brown

no mottling 6 occasionally concentrated

HIGHLY CALC CLAY WITH DETRITUS

2.5 Y $3 / 2$ very dark gray brown

no mottling

sightly silty lutite, scattered forams

$97-103$

SHELL HASH IN HICHLY CAIC CIAY WITH DETRTTUS

2.5 Y $3 / 2$ very dark gray brown (lutite fraction)

no motting

large rounded imestone fragment, $2 \times 3 \mathrm{~cm}$ (in working

103-137

CAIC CLAY

5 Y $3 / 2$ dark olive gra

no mottling

slightly silty lutite

section with $1 / 2$ of core missing; 33-134 shell hash

end of core
65-68 sharp band of above unit
SMEAR SLIDE DESCRIPTIONS - W.H.O.I. SEDIMENT CORES

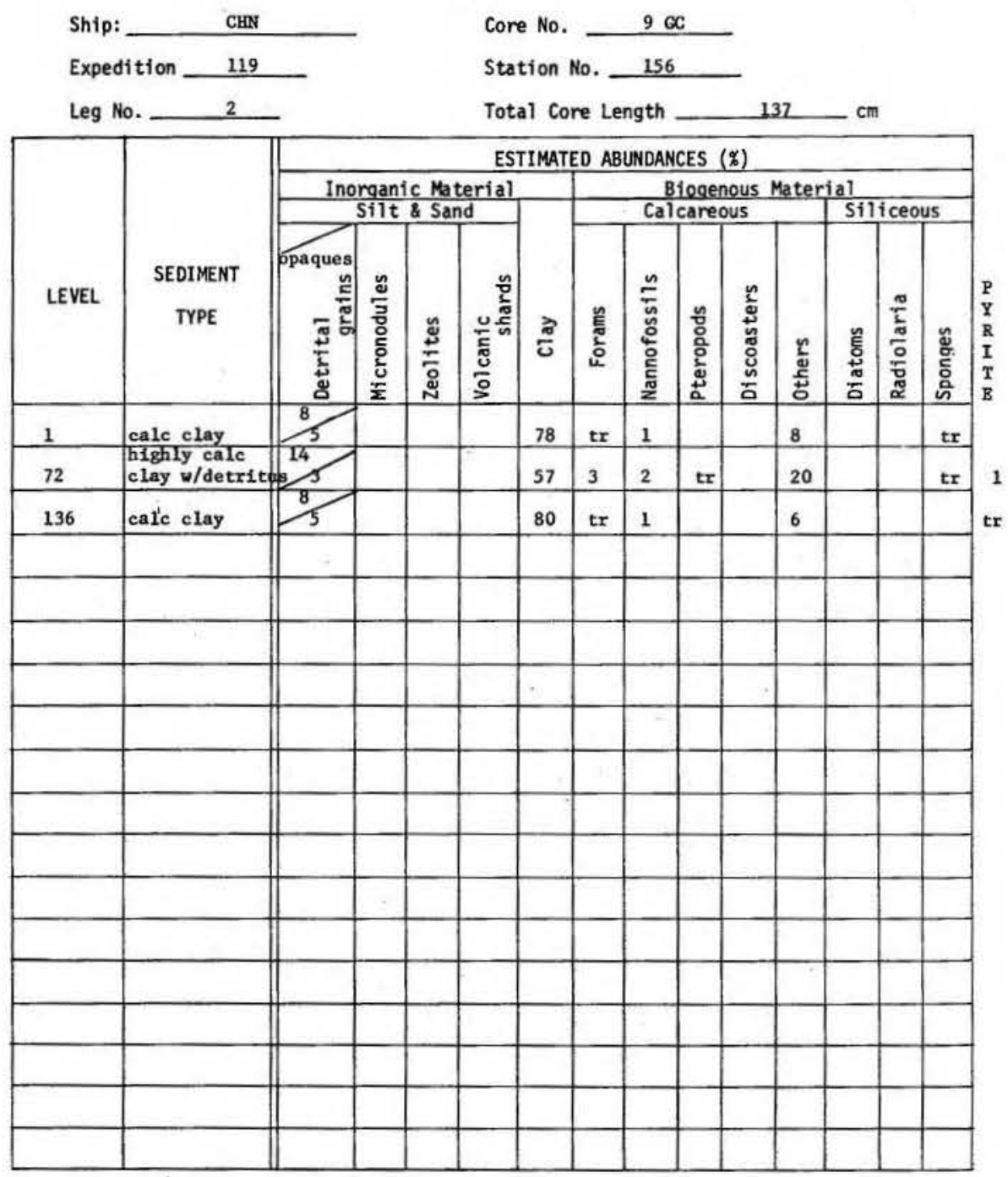


673

VISUAL CORE DESCRIPTION

Page 1 of 1
674

SIEAR SLIDE DESCRIPTIONS - W.H.O.I. SEDIMENT CORES

Ship Cuan Cruise 119 Leg 2 Sta. $16 /$ Core No. $2 \mathrm{KC}$ Total Length $256-\mathrm{cm}$. Lat. $32^{\circ} 03.7^{\prime} \mathrm{N}$ Long. $31^{\circ} 57^{\prime} 6^{\prime} \mathrm{E}$ Depth $505 \mathrm{carr} \mathrm{m}$. Core condition EXCELLENT Date Described $13 \mathrm{Fik} 76$ by $T$ FARMER

Physiographic location EAST. MED. SEA, NILE CONE, EGYPTINW CONTNENTAL SHELF Lithologic

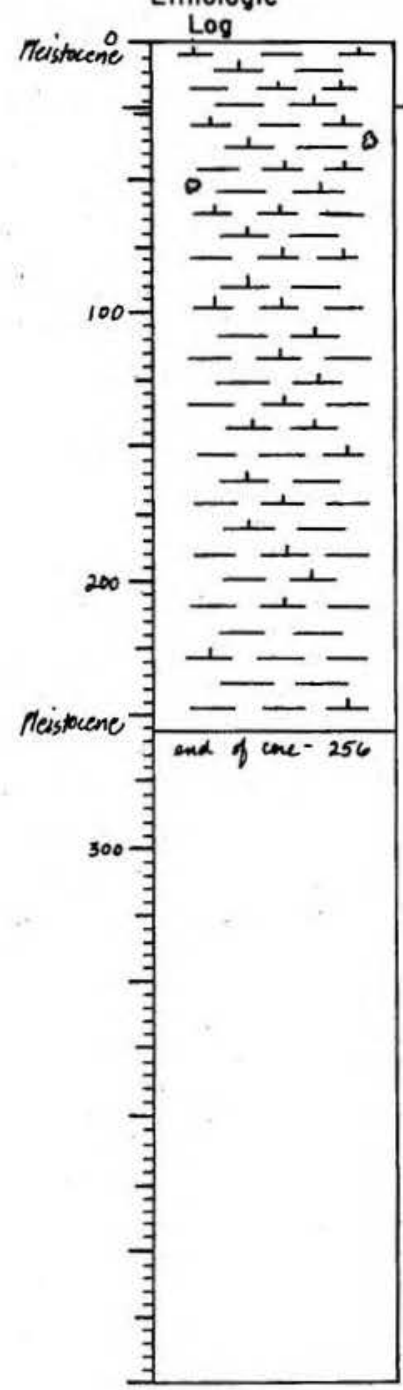

$0-23$

5 Y $4 / 3$ olive

slick, slightly silty lutite with very few forams slick, slightly silty

23-256 HTGHY CAIC CIAY GRADES TO SLTGHTTY CALC CTAY 5 Y $5 / 3$ olive grades gently to 2.5 × $4 / 2$ dark grayish brown

small, olive mottles are scattered from upper contact down to $00 \mathrm{~cm}$

lick, slightly silty lutite, gently becomes slicker, with very litele silt: there are few forams above end of core

\section{BIGHLY CALC CLAY}

Ship: $\frac{\text { CBAIN }}{2}$

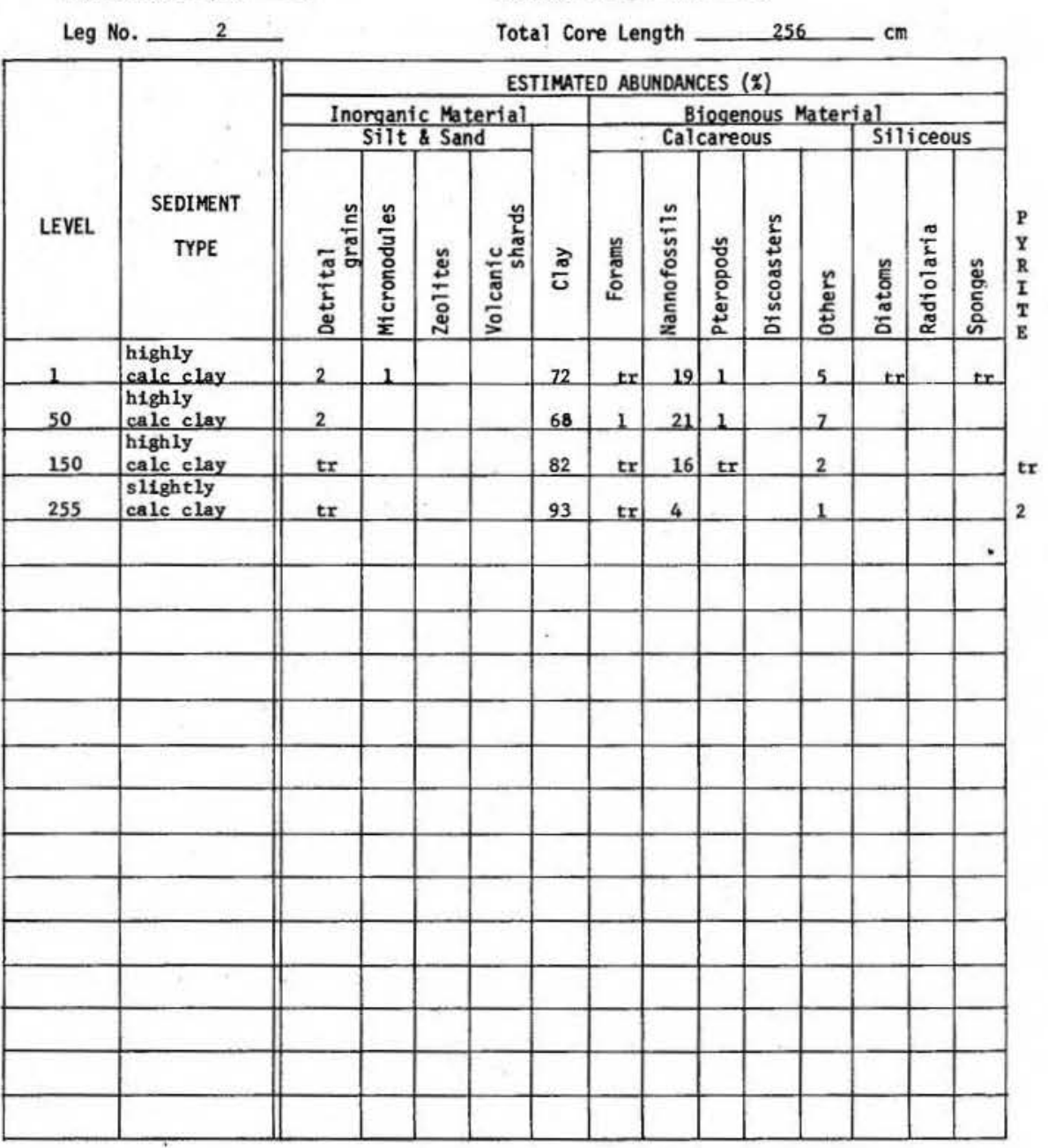


Page 1 of 1

Ship Chain Cruise 119 Leg 2 Sto. 166 Core No. $10 \mathrm{GC}$ Total Length $20 \mathrm{~cm}$. Lat. $31^{\circ} 45.3^{\prime} \mathrm{N}$ Long. $31^{\circ} 34.8^{\prime} \mathrm{E}$ Depth $44 \mathrm{corr} . \mathrm{m}$. Core condition ExcEUENT Date Described 10 Feb 76 by T. Fmence. Physiographic location DemiETTh BRANCA of NuE COME, EAST. MES. SEA Lithologic

Detailed Description

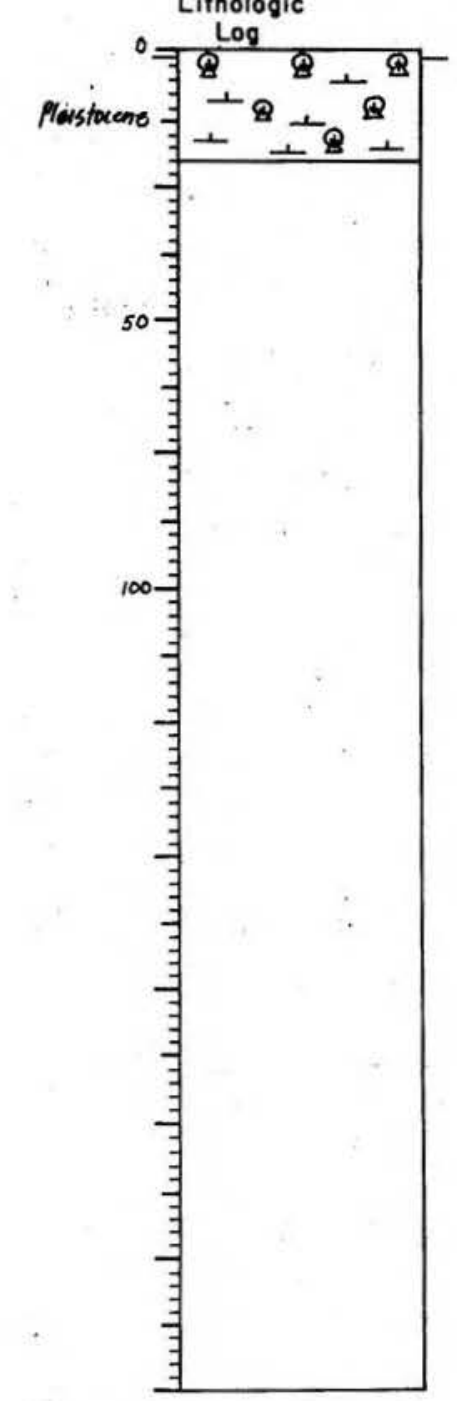

CALC OOZE

shell hash of wel1-preserved she11s up to $1 \mathrm{~cm}$ $\mathrm{S}$
$\mathbf{1} 20$

CAIC OORE

10 YR $3 / 2$ very dark grayish brown

(utite matrix, lutite

7 increases in bottom $3 \mathrm{~cm}$
SIEAR SLIDE DESCRIPTIONS - W.H.O.I. SEDIMENT CORES

Expedition 11

Leg No.

\begin{tabular}{|c|c|c|c|c|c|c|c|c|c|c|c|c|c|c|}
\hline \multirow[b]{3}{*}{ LEVEL } & \multirow{3}{*}{$\begin{array}{l}\text { SEDIMENT } \\
\text { TYPE }\end{array}$} & \multicolumn{13}{|c|}{ ESTIMATED ABUNDANCES (\%) } \\
\hline & & \multicolumn{4}{|c|}{$\begin{array}{c}\text { Inorganic Material } \\
\text { Silt \& Sand }\end{array}$} & & \multicolumn{5}{|c|}{$\begin{array}{l}\text { Biogenous Mater } \\
\text { Calcareous }\end{array}$} & \multicolumn{3}{|c|}{$\frac{\text { al }}{\text { Stliceous }}$} \\
\hline & & 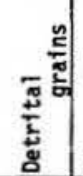 & 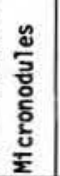 & 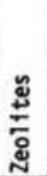 & 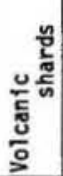 & $\frac{\pi}{6}$ & $\begin{array}{l}\text { 嗕 } \\
\text { 훈 }\end{array}$ & 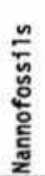 & 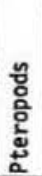 & 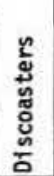 & 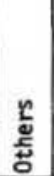 & 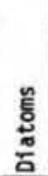 & 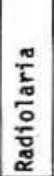 & 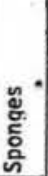 \\
\hline 19 & calc ooze & 6 & & & & 60 & 2 & 18 & 8 & & 10 & & & $t x$ \\
\hline & i & & & & & & & & & & & & & \\
\hline & & & & & & & & & & & & & & \\
\hline & & & & & & & & & & & & & & \\
\hline & & & & & & & & & & & & & & \\
\hline & & & & & & . & & & & & & & & \\
\hline & & & & & & & & & & & & & & \\
\hline & & & & & & & & & & & & & & \\
\hline & & & & & & & & & & & & & & \\
\hline & & & & & & & & & & & & & & \\
\hline . & & & & & & & & & & & & & & \\
\hline & & & & & & & & & & & & & & \\
\hline & & & & & & & & & & & & & & \\
\hline & & & & & & & & & & & & & & \\
\hline & & & & & & & & & & & & & & \\
\hline & & & & & & & & & & & & & & \\
\hline & & & & & & & & & & & & & & \\
\hline
\end{tabular}

Core No. $10 \mathrm{GC}$

Station No. 166

Total core Length 
Ship CHAIN Cruise 119 Leg 2 Sta. 183 Core No. $3 K \mathrm{C}$

Total Length $253 \mathrm{~cm}$. Lat. $32^{\circ}$ 08. $2^{\prime} \mathrm{N}$ Long. $30^{\circ} 58.0^{\prime} \mathrm{E}$ Depth $508 \mathrm{corr} . \mathrm{m}$. Core condition EXCELENT Date Described 13 Feb 76 by 2 FARMER Physiographic location EAST. MED. SEA, NILE CAVE, EGYMTIAN CONTINENTRK SIJHF Lithologic

Detailed Description

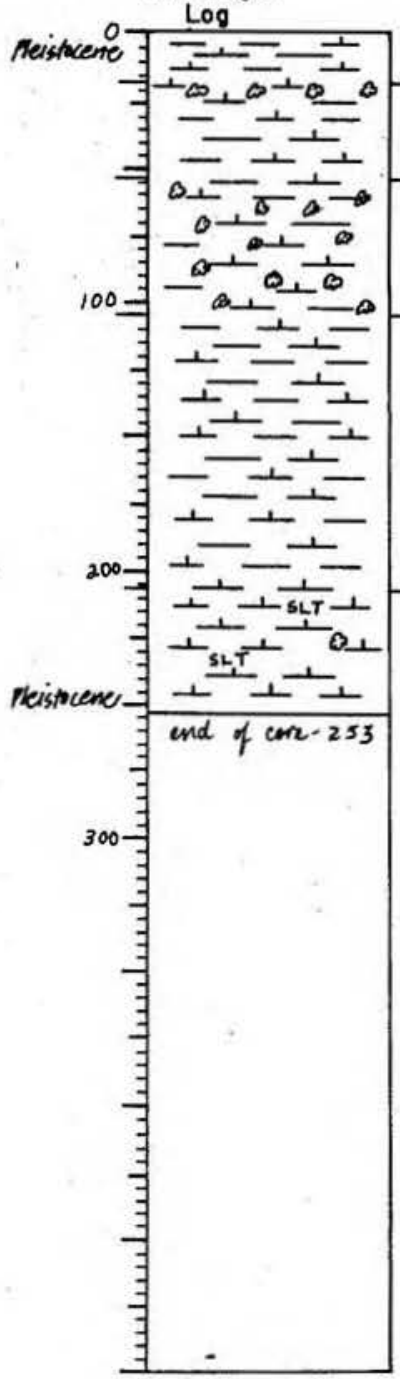

CALC CIAY 2.5 Y $4 / 2$ dark grayish brown

ing with depth firm, slightly silty lutite with very few forams $\mathrm{S}$, mottled $18-53$ HIGHIY CALC CLAY

10 YR $5 / 2$ grayish brown and 2.5 Y $4 / 2$ dark grayish brown

extensive, dark grayish brown intermottling at upper compact, slightly silty lutite with a few forams 53-105 $G$, mottied

CALC CIAY

dark gray brow

innumexable, fine, light grayish brown burrows and mottles throughout the saprope 1

compact, somewhat mulchy, slightiy silty lutite with very few foram

enteropod test found at $83 \mathrm{~cm}$ (removed from working half)

105-207 CALC CIAY

5 Y $3 / 2$ dark olive gray grades to 2.5 Y $3 / 2$ very dark gray brown
virtually hom

geneous throughout; only a few, faint, scattered, 1rregular mottles are found very sharp inclined lutite

207-253

CALC OOZE WITI DETRITUS

5 Y $5 / 2$ olive grades to $4 / 2$ olive gray

scattered, faint, fine, light olive gray (sapropel-

firm, compact, silty lutite grades to stiffer, even more compact, extremely silty lutite end of core
SUEAR SLIDE DESCRIPTIONS - K.H.O.I. SEDINENT CORES
Ship:

Expedition

Leg No.
Core No. $\quad 3 \mathrm{KC}$

Station No. 183
Total Core Length 253

\begin{tabular}{|c|c|c|c|c|c|c|c|c|c|c|c|c|c|c|}
\hline \multirow[b]{3}{*}{ LEVEL } & \multirow[b]{3}{*}{$\begin{array}{c}\text { SEDIMENT } \\
\text { TYPE }\end{array}$} & \multicolumn{13}{|c|}{ ESTIMATED ABUNDANCES ( $(x)$} \\
\hline & & \multicolumn{4}{|c|}{$\begin{array}{c}\text { Inorganic Material } \\
\text { Silt \& Sand }\end{array}$} & & \multicolumn{5}{|c|}{$\begin{array}{l}\text { Biogenous Mater } \\
\text { Calcareous }\end{array}$} & \multicolumn{3}{|c|}{$\frac{\text { al }}{\text { Silliceous }}$} \\
\hline & & 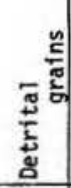 & 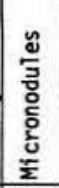 & 迹 & 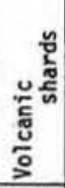 & $\frac{\pi}{6}$ & 厸 & 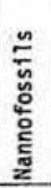 & 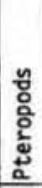 & 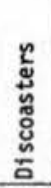 & 品 & 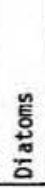 & $\begin{array}{l}\frac{\pi}{\frac{\pi}{0}} \\
\frac{0}{0} \\
\frac{\pi}{\alpha}\end{array}$ & 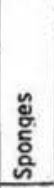 \\
\hline 1 & calc clay & tr & 3 & & & 91 & tr & 5 & & & 1 & & & \\
\hline 30 & $\begin{array}{l}\text { highly } \\
\text { calc clay }\end{array}$ & $\mathrm{tr}$ & & & & 74 & tr & 24 & $t r$ & & 2 & & & \\
\hline 66 & cal'c clay & tr & & & & 88 & $\mathrm{tr}$ & 8 & tr & & 3 & & & \\
\hline 145 & calc clay & 3 & & & & 91 & $\mathrm{tr}$ & 2 & & & 4 & & & \\
\hline 208 & \begin{tabular}{|l|} 
calc ooze with \\
detritus
\end{tabular} & 17 & & & & 12 & 1 & 2 & 4 & & 64 & & & \\
\hline 252 & calc ooze & 5 & & & & 36 & 1 & 4 & 2 & & 52 & & & tr \\
\hline & & & & & & . & & & & & & & & \\
\hline & & & & & & & & & & & & & & \\
\hline & & & & & & & & & & & & & & \\
\hline & & & & & & & & & & & & & & \\
\hline & & & & & & & & & & & & & & \\
\hline & & & & & & & & & & & & & & \\
\hline & & & & & & & & & & & & & & \\
\hline & & & & & & & & & & & & & & \\
\hline & & & & & & & & & & & & & & \\
\hline & & & & & & & & & & & & & & \\
\hline & & & & & & & & & & & & & & \\
\hline & & & & & & & & & & & & & & \\
\hline
\end{tabular}




\section{VISUAL CORE DESCRIPTION}

Poge 1 of 1 Ship CHAIN Cruise 19 Leg $\pi$ Sta. 185 Core No. $4 \mathrm{KC}$

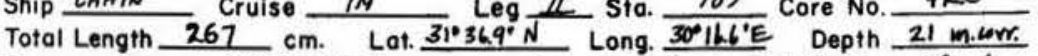

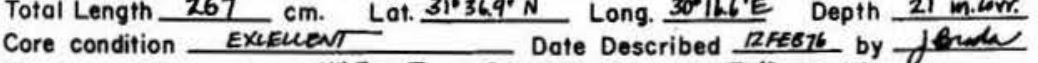
Physiographic location NILELONE.. OFF MLEKANDRIA - CONTINENTAC SAECTE.

Lithologic

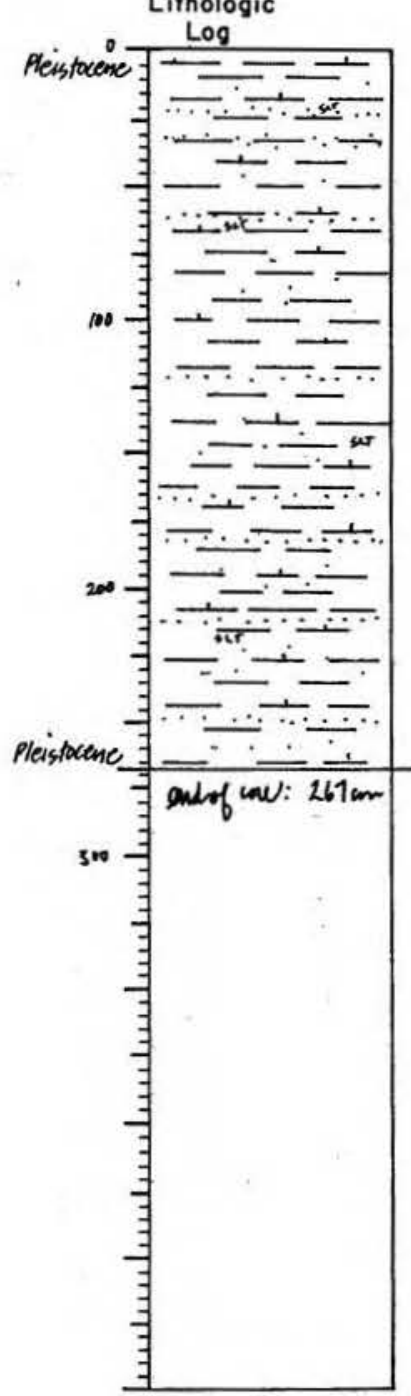

$0-2.67$

SLIGHTLY CALC CLAX WITH DETRITUS AND BEDS OF DETRITUS 10 YR $4 / 2$ dark grayish brown

slightly darker, silty laminations at the intervals smooth; faint, irregular, dark gray mottling 0-110 cm beds and lenses; the dominant ons, inclined, silty $29 \mathrm{~cm}, 38-39 \mathrm{~cm}, 46-47 \mathrm{~cm}, 62-65 \mathrm{~cm}, 67-68 \mathrm{~cm}, 121-$ $122 \mathrm{~cm}, 166-167 \mathrm{~cm}, 181-182 \mathrm{~cm}, 210-211 \mathrm{~cm}, 249-250 \mathrm{~cm}$ end of core
SIEAR SLIDE DESCRIPTIONS - W.H.O.I. SEDIMENT CORES

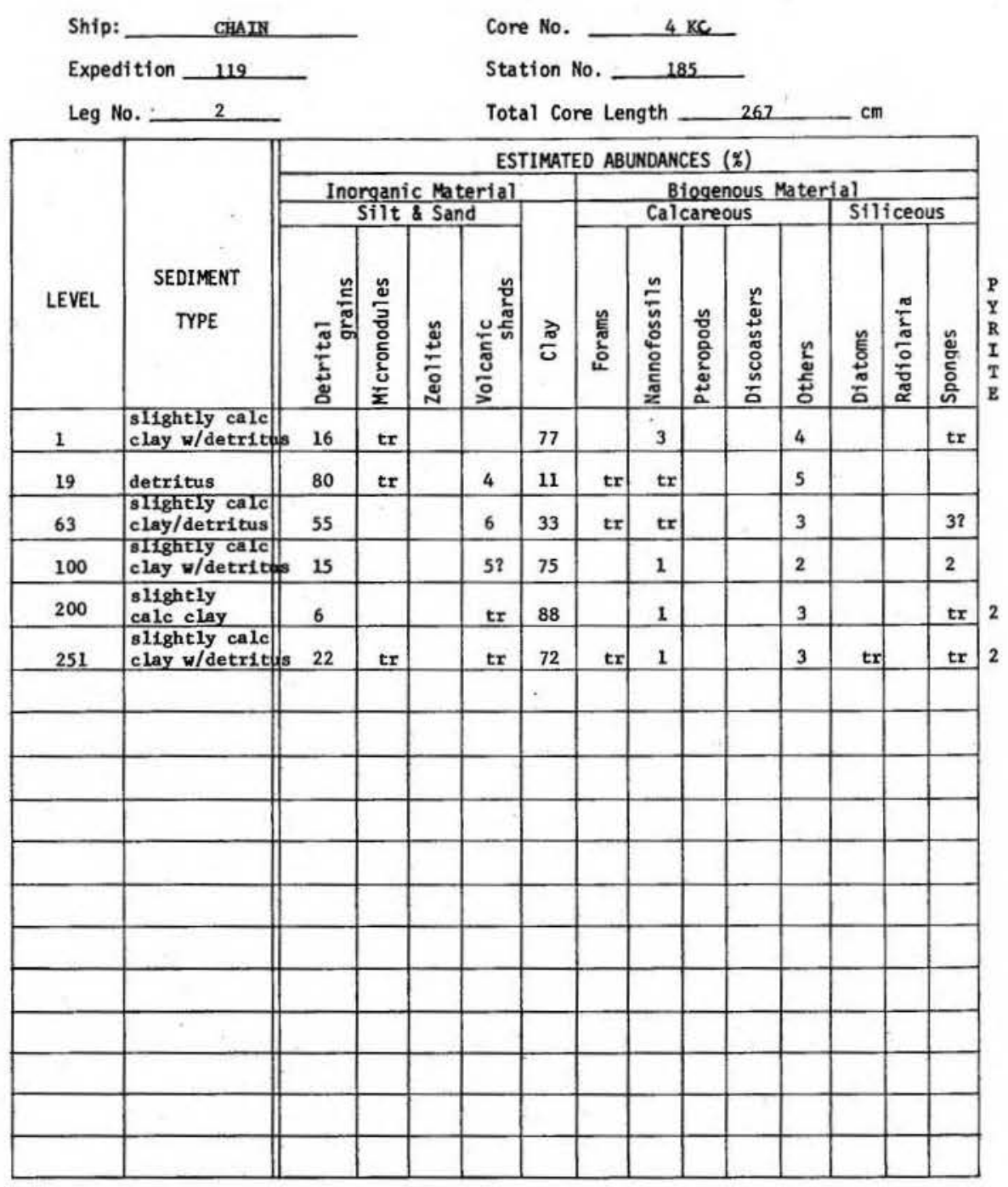


681

VISUAL CORE DESCRIPTION

Page 1 of 1

Ship CHAN Cruise 119 Leg II Sto. 187 Core No. $5 \mathrm{KC}$

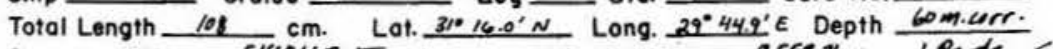
Core condition ExLEUEN Date Described BFES 76 by ferde

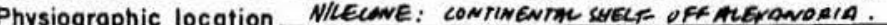
Lithologic

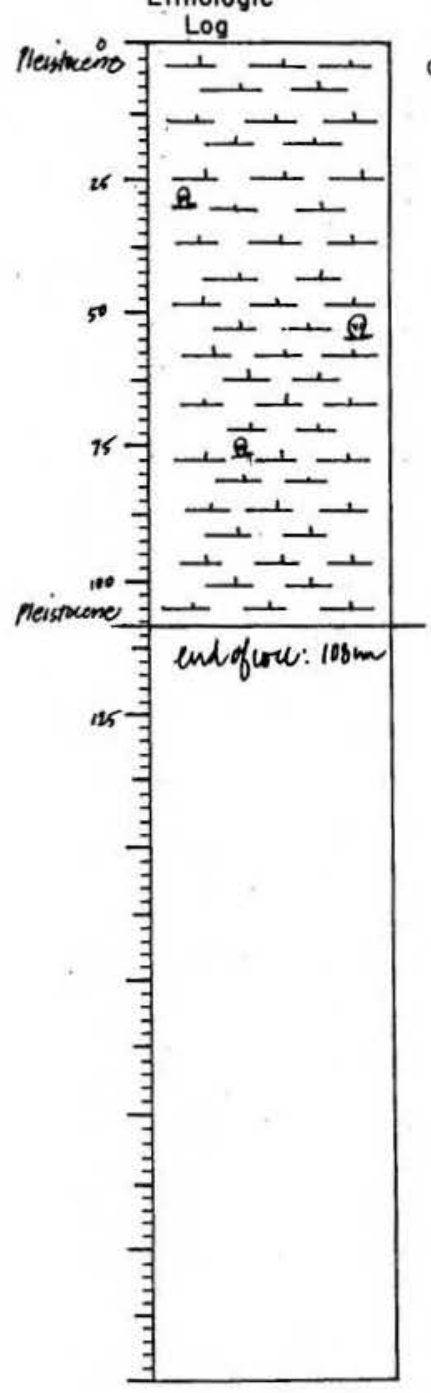

\section{Detailed Description}

$0-108$

CALC OOZE

homogeneous throughout

thich compact, silty latite with scattered forams,

calcareous lumps; a few well preserved coral structures are also present end of core
5 Y $7 / 1$ light gray, grades to $2 / 2$ light gray
SIEAR SLIDE DESCRIPTIONS - W.H.O.I. SEDIMENT CORES

Ship: CHAIN

Expedition 119

Core No. $5 \mathrm{KC}$

Leg No. Station No. 187

Total Core Length $\quad 108-\mathrm{cm}$

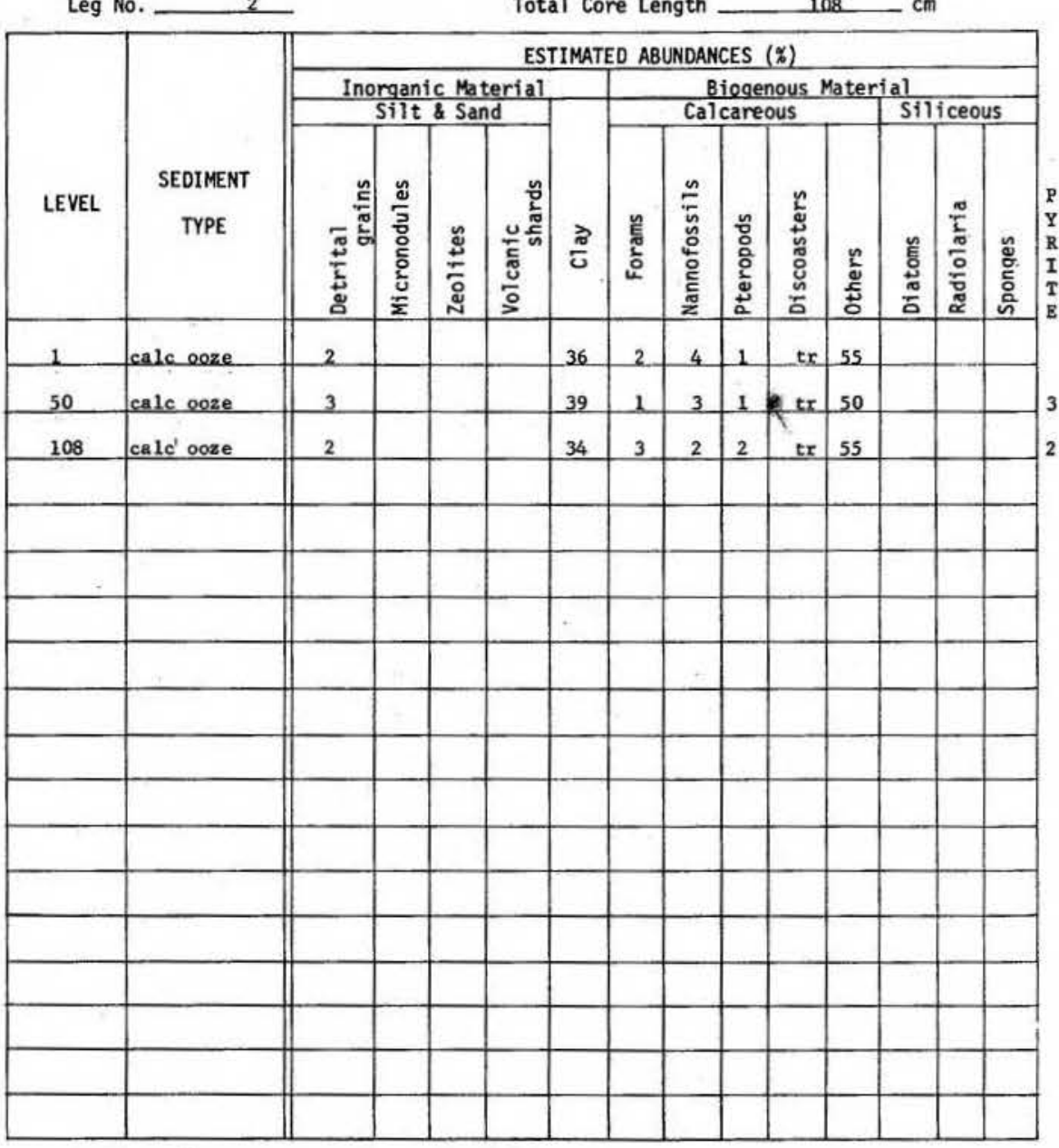


683

VISUAL CORE DESCRIPTION

Page 1 of 1

Ship CHAIN Cruise 119 Leg II Sta. 187 Core No. $12 \mathrm{GC}$

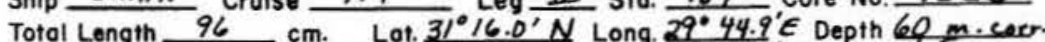
Core condition ExcELENT Date Described 1 Sept 16 by H. FinemER

Physiographic location EGYPTIAN CONTINENTAL SIELLF - NILE CONE Lithologic

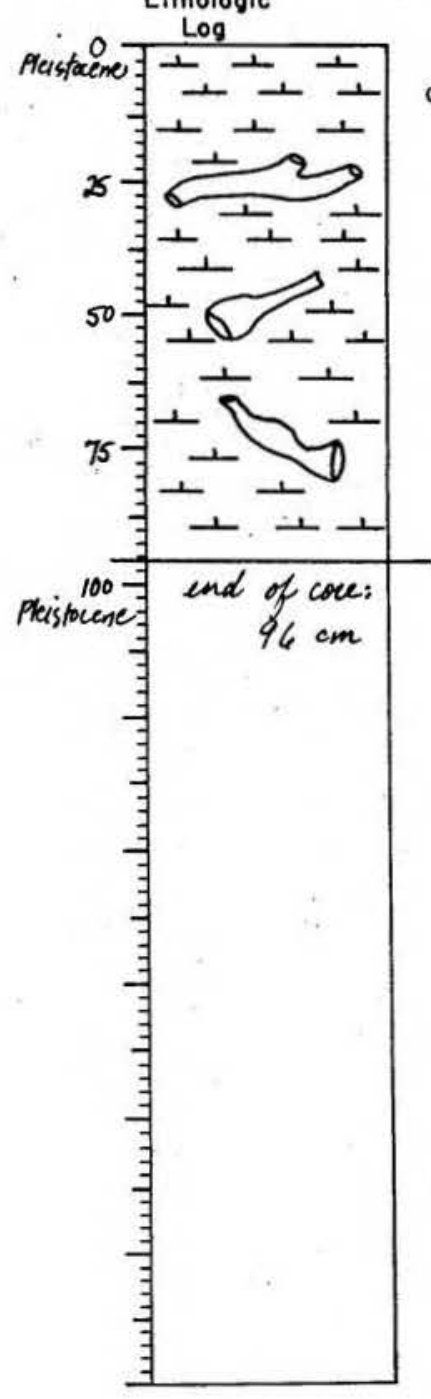

684

SIEAR SLIDE DESCRIPTIONS - W.H.O.I. SEDIMENT CORES
Ship: CHN

Expedition 219

CAIC $002 E$ $48-61 \mathrm{~cm}$

frm, plastic, silty lutite with foram-size, biogenic material coumon; several small shells (pelecypods)

large pieces of coral span the core liner at $23-27 \mathrm{~cm}$,

end of core
Leg No. several medium size, pale maxbles are apparent from
Core No. $12 \mathrm{GC}$

Station No. 187

Total Core Length $\quad 96 \quad \mathrm{~cm}$

\begin{tabular}{|c|c|c|c|c|c|c|c|c|c|c|c|c|c|c|}
\hline \multirow[b]{4}{*}{ LEVEL. } & \multirow{4}{*}{$\begin{array}{l}\text { SEDIMENT } \\
\text { TYPE }\end{array}$} & \multicolumn{13}{|c|}{ ESTIMATED ABUNDANCES ( $(\alpha)$} \\
\hline & & \multirow{2}{*}{\multicolumn{4}{|c|}{$\begin{array}{c}\text { Inorganic Material } \\
\text { Silt \& Sand }\end{array}$}} & & \multirow{2}{*}{\multicolumn{5}{|c|}{$\begin{array}{l}\text { Blogenous Mate } \\
\text { Calcareous }\end{array}$}} & \multirow{2}{*}{\multicolumn{3}{|c|}{ Siliceous }} \\
\hline & & & & & & & & & & & & & & \\
\hline & & 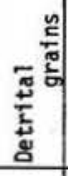 & 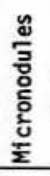 & 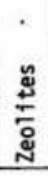 & 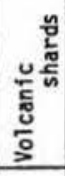 & $\frac{\pi}{6}$ & 宸 & 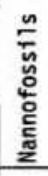 & 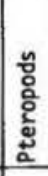 & 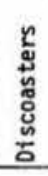 & $\begin{array}{l}\text { है } \\
\text { है } \\
\text { ثै }\end{array}$ & $\begin{array}{l}\text { 产 } \\
\stackrel{\pi}{0} \\
\end{array}$ & $\begin{array}{l}\frac{\pi}{2} \\
\frac{\pi}{0} \\
\frac{0}{0} \\
\alpha \\
\end{array}$ & 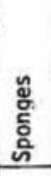 \\
\hline 1 & calc, ooze & 1 & & & & 60 & 2 & 2 & & & 35 & $t r$ & & tr \\
\hline 50 & calc ooze & 1 & & & & 54 & 2 & 2 & 1 & & 40 & & & $\mathrm{tr}$ \\
\hline 95 & calc ooze & 1 & & & & 60 & 2 & 2 & tr & & 35 & & & \\
\hline & & & & & & & & & & & & & & \\
\hline & & & & & & & & & & & & & & \\
\hline & & & & & & & & & & & & & & \\
\hline & & & & & & 8 & & & & & & & & \\
\hline & & & & & & & & & & & & & & \\
\hline & & & & & & & & & & .. & & & & \\
\hline & & & & & & & & & & & & & & \\
\hline & & & & & & & & & & & & & & \\
\hline & & & & & & & & & & & & & & \\
\hline & & & & & & & & & & & & & & \\
\hline & & & & & & & & & & & & & & \\
\hline & & & & & & & & & & & & & & \\
\hline & & & & & & & & & & & & & & \\
\hline & & & & & & & & & & & & & & \\
\hline & & & & & & & & & & & & & & \\
\hline
\end{tabular}




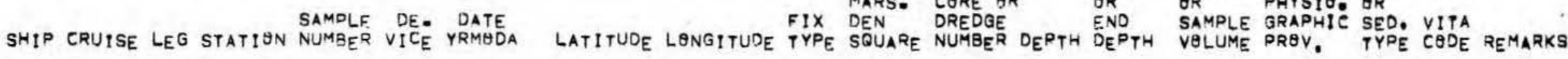

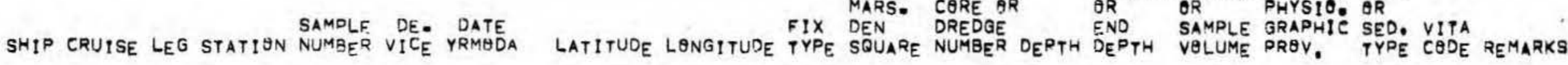

\begin{tabular}{lllll} 
CHN & 119 & 2 & 0001 & 0000 \\
CHN & 119 & 2 & 0002 & 0000 \\
CHN & 119 & 2 & 0003 & 0000 \\
CHN & 119 & 2 & 0004 & 0000 \\
CHN & 119 & 2 & 0005 & 0000 \\
CHN & 119 & 2 & 0006 & 0000 \\
CHN & 119 & 2 & 0007 & 0000 \\
CHN & 119 & 2 & 0008 & 0000 \\
CHN & 119 & 2 & 0009 & 0000 \\
CHN & 119 & 2 & 0030 & 0000 \\
CHN & 119 & 2 & 0031 & 0000 \\
CHN & 119 & 2 & 0032 & 0000 \\
CHN & 119 & 2 & 0033 & 0000 \\
CHN & 119 & 2 & 0034 & 0000 \\
CHN & 119 & 2 & 0035 & 0000 \\
CHN & 119 & 2 & 0036 & 0000 \\
CHN & 119 & 2 & 0038 & 0000 \\
CHN & 119 & 2 & 0039 & 0000 \\
CHN & 119 & 2 & 0041 & 0000 \\
CHN & 119 & 2 & 0042 & 0000 \\
CHN & 119 & 2 & 0045 & 0000 \\
CHN & 119 & 2 & 0047 & 0000 \\
CHN & 119 & 2 & 0048 & 0000 \\
CHN & 119 & 2 & 0049 & 0000 \\
CHN & 119 & 2 & 0050 & 0000 \\
CHN & 119 & 2 & 0051 & 0000 \\
CHN & 119 & 2 & 0052 & 0000 \\
CHN & 119 & 2 & 0053 & 0000 \\
CHN & 119 & 2 & 0054 & 0000 \\
CHN & 119 & 2 & 0055 & 0000 \\
CHN & 119 & 2 & 0058 & 0000 \\
CHN & 119 & 2 & 0059 & 0000 \\
CHN & 119 & 2 & 0086 & 0000 \\
CHN & 119 & 2 & 0087 & 0000 \\
CHN & 119 & 2 & 0089 & 0000 \\
CHN & 119 & 2 & 0090 & 0000 \\
CHN & 119 & 2 & 0091 & 0000 \\
CHN & 119 & 2 & 0093 & 0000 \\
CHN & 119 & 2 & 0094 & 0000 \\
CHN & 119 & 2 & 0096 & 0000 \\
CHN & 119 & 2 & 0097 & 0000 \\
CHN & 119 & 2 & 0098 & 0000 \\
CHN & 119 & 2 & 0099 & 0000 \\
CHN & 119 & 2 & 0100 & 0000 \\
CHN & 119 & 2 & 0101 & 0000 \\
CHN & 119 & 2 & 0102 & 0000 \\
& & & & \\
\hline
\end{tabular}

\begin{tabular}{|c|c|c|c|c|c|c|c|c|}
\hline & 22 & 31 & 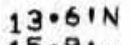 & 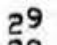 & $46.71 \mathrm{E}$ & 1 & 19 & \\
\hline & $\begin{array}{l}322 \\
322\end{array}$ & 31 & $\begin{array}{l}15.91 \mathrm{~N} \\
19.7, \mathrm{~N}\end{array}$ & $\begin{array}{l}29 \\
29\end{array}$ & $\begin{array}{l}45.11 E \\
43.3, E\end{array}$ & 1 & 142.19 & \\
\hline & 322 & & $55 \cdot 5_{1 N}$ & 29 & $50.61 E$ & & $142 \cdot 19$ & \\
\hline & 300 & & & 20 & OIE & & & \\
\hline & 322 & 1 & In & 29 & $71 E$ & & $142 \cdot 19$ & 000 \\
\hline & 322 & & $16.71 \mathrm{~N}$ & $2^{9}$ & $56.81 E$ & & & \\
\hline & उटूट & & $20.01 \mathrm{~N}$ & 29 & $57.21 E$ & & & \\
\hline & 322 & 1 & & 30 & $1.81 E$ & & $142 \cdot 10$ & 0 \\
\hline & $32^{7}$ & 1 & $31.81 \mathrm{~N}$ & 99 & $58.41 E$ & & 14 & \\
\hline & 327 & & $29.51 \mathrm{~N}$ & 30 & $1.01 E$ & & & \\
\hline & 328 & & $26.81 \mathrm{~N}$ & 30 & . & & 10 & 001 \\
\hline & 328 & 1 & $24.31 \mathrm{~N}$ & & If & & 14 & 01 \\
\hline 5 & $32^{\gamma}$ & 31 & $21.71 \mathrm{~N}$ & 0 & $11 \cdot 3^{\prime} E$ & & & \\
\hline & 328 & 31 & $18.11 \mathrm{~N}$ & 10 & & & & \\
\hline & 328 & & $13.61 \mathrm{~N}$ & 7 & TE & & 19 & 000 \\
\hline & $32^{\gamma}$ & $\sqrt{1}$ & $2^{9 \cdot 8, N}$ & 0 & IE & & 14 & 000 \\
\hline 5 & $32^{x}$ & & $33 \cdot 3.1 \mathrm{~N}$ & & & & & \\
\hline & 328 & 31 & $37.11 \mathrm{~N}$ & & IE & & 10 & 000 \\
\hline & 328 & & $41.01 \mathrm{~N}$ & & IE & & 14 & \\
\hline & 328 & 31 & $45.01 \mathrm{~N}$ & & & & $141 \cdot 10$ & 0006 \\
\hline & $32^{8}$ & 31 & $52 \cdot 41 \mathrm{~N}$ & & & & $141 \cdot 10$ & \\
\hline & 328 & & $56.41 \mathrm{~N}$ & & IE & & 14 & 000 \\
\hline & 328 & & $53.01 \mathrm{~N}$ & 30 & $11.11 E$ & 3 & 14 & 001 \\
\hline & $32^{8}$ & 31 & $48 \cdot 3, N$ & 30 & $1 E$ & 1 & $141 \cdot 10$ & 001 \\
\hline & $32^{6}$ & 30 & $45 \cdot 0^{1 N}$ & & IE & & & \\
\hline & 328 & 31 & 40. & & & & & \\
\hline & згу & 1 & $36.81 \mathrm{~N}$ & & IE & & & \\
\hline & 328 & 31 & $33.4, \mathrm{~N}$ & & IE & & $141 \cdot 10$ & \\
\hline & $32^{8}$ & 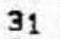 & $32.61 \mathrm{~N}$ & & & & & \\
\hline & $32^{y}$ & & 34. & & & & & \\
\hline & $32 y$ & 31 & $36.9, N$ & & $E$ & & 10 & \\
\hline & 2 & 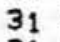 & $50^{\circ} \mathrm{O}^{1 \mathrm{~N}}$ & & E & & & \\
\hline & 2 & & $46.01 \mathrm{~N}$ & & & & & \\
\hline & 2 & & $36.81 \mathrm{~N}$ & & & & 14 & \\
\hline 3 & 2 & 3 & $33.71 \mathrm{~N}$ & & IE & & & \\
\hline 75 & & & $36.51 \mathrm{~N}$ & & & & & \\
\hline & ? & 31 & $41 \cdot$ & & & 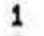 & $141 \cdot 10$ & 002 \\
\hline & & 31 & $44^{\circ}$ & 30 & & 3 & $141 \cdot 10$ & $002 ?$ \\
\hline 80 & 2 & & $55.31 \mathrm{~N}$ & & & & & \\
\hline 5 & 2 & 3 & $59.1, \mathrm{v}$ & & $36.31 E$ & & 14 & \\
\hline$\ldots$ & & & $59.7, \mathrm{~N}$ & & & & & \\
\hline $7=$ & 2 & 31 & $55 \cdot 31 \mathrm{~N}$ & & 'E & & & \\
\hline & 2 & & $52 \cdot$ & & & & & $=$ \\
\hline & & & & & & & & \\
\hline
\end{tabular}

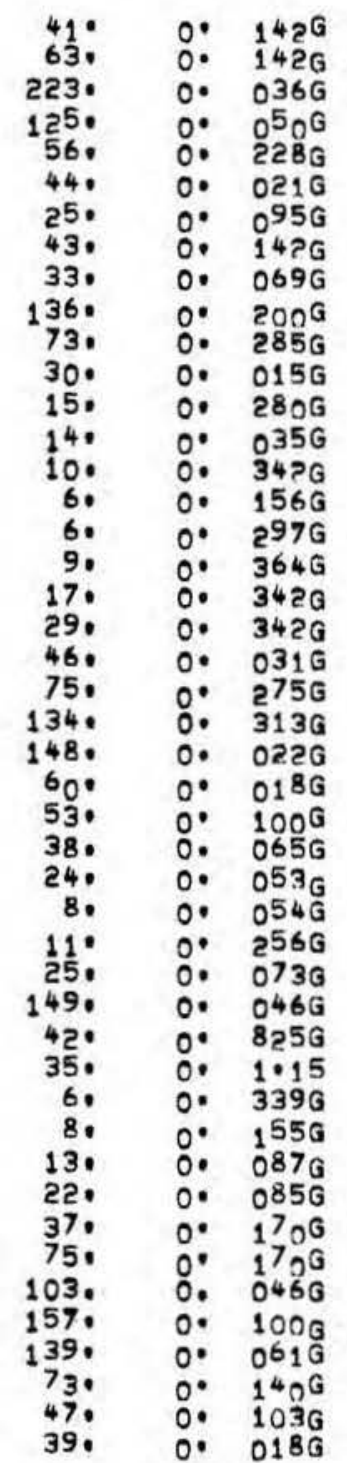

$\begin{array}{lll}24 & 3369 & 0 \\ 24 & 3365 & 0 \\ 24 & 3365 & 0 \\ 24 & 3365 & 0 \\ 24 & 3369 & 0 \\ 24 & 3365 & 0 \\ 24 & 3365 & 0 \\ 24 & 3365 & 0 \\ 24 & 3365 & 0 \\ 24 & 3369 & 0 \\ 24 & 4469 & 0 \\ 24 & 8869 & 0 \\ 24 & 2469 & 0 \\ 24 & 8469 & 0 \\ 24 & 8869 & 0 \\ 2 & 8469 & 0 \\ 24 & 8869 & 0 \\ 24 & 8469 & 0 \\ 24 & 8469 & 0 \\ 24 & 8469 & 0 \\ 24 & 3365 & 0 \\ 24 & 3869 & 0 \\ 2 & 3365 & 0 \\ 24 & 3369 & 0 \\ 24 & 3365 & 0 \\ 24 & 2469 & 0 \\ 24 & 2469 & 0 \\ 24 & 2469 & 0 \\ 24 & 2269 & 0 \\ 24 & 2469 & 0 \\ 24 & 8869 & 0 \\ 24 & 2469 & 0 \\ 24 & 8869 & 0 \\ 24 & 2469 & 0 \\ 24 & 8869 & 0 \\ 24 & 8869 & 0 \\ 24 & 2269 & 0 \\ 24 & 8869 & 0 \\ 24 & 8365 & 0 \\ 24 & 3265 & 0 \\ 24 & 3265 & 0 \\ 24 & 3265 & 0 \\ 24 & 3265 & 0 \\ 24 & 3265 & 0 \\ 24 & 3469 & 0 \\ 24 & 2469 & 0\end{array}$




\section{6}

*************************
STATION UATA RETRIEVAL DATE: $17: 32$ JUN 03,177

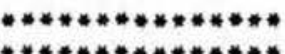

PAGE ?

* WHOI**

CORE

MARS. CORE $Q R$ LENGTH QREDGE PHYSIO. RQCK

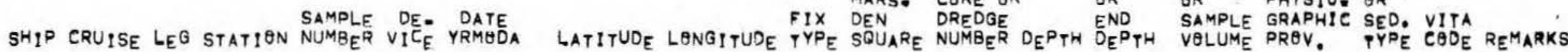

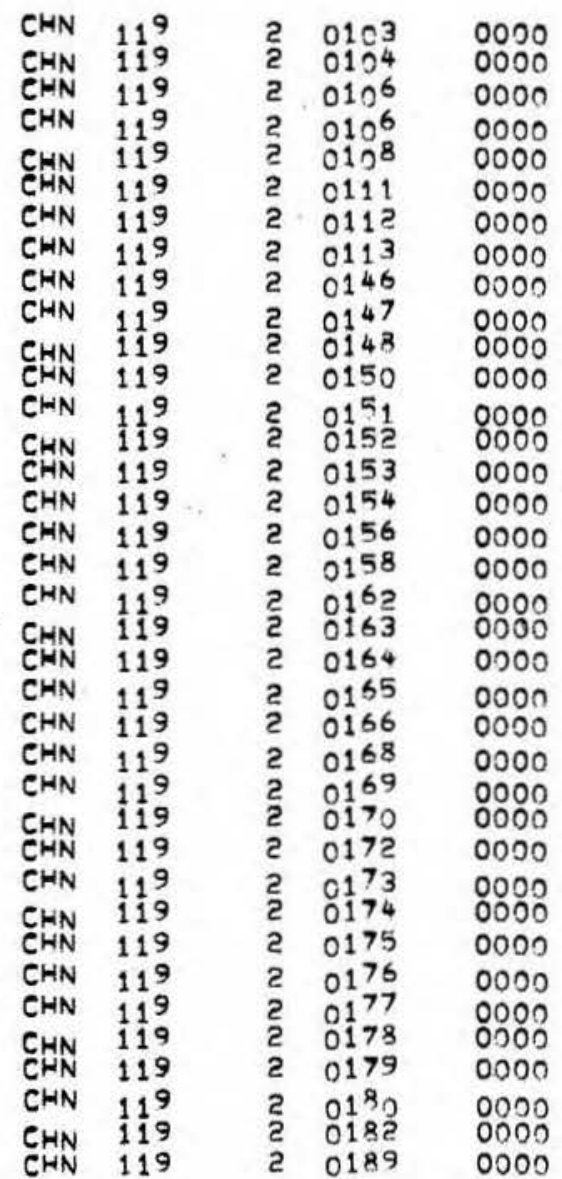

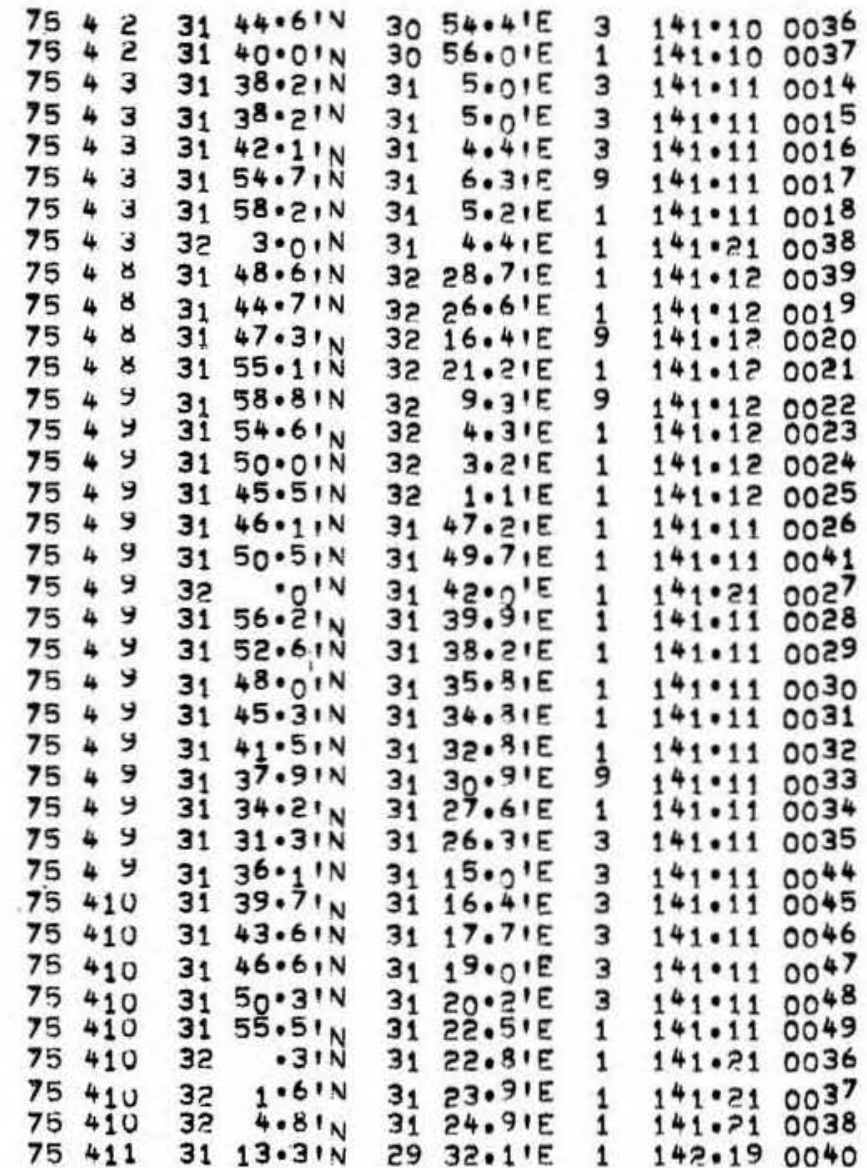

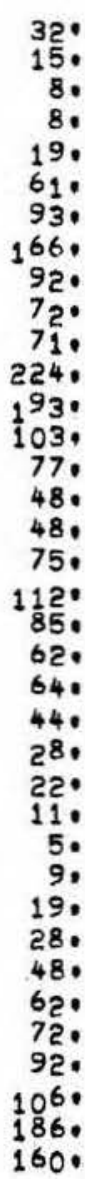

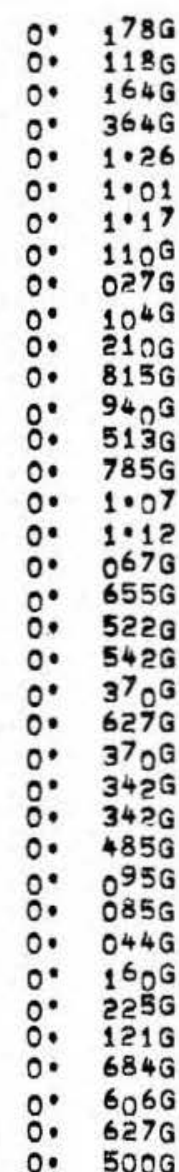

24
24
24
24
24
24
24
24
24
24
24
4
4
24
24
24
24
24
24
24
24
24
24
24
24
24
24
24
24
24
24
24
24
24
24
24
24

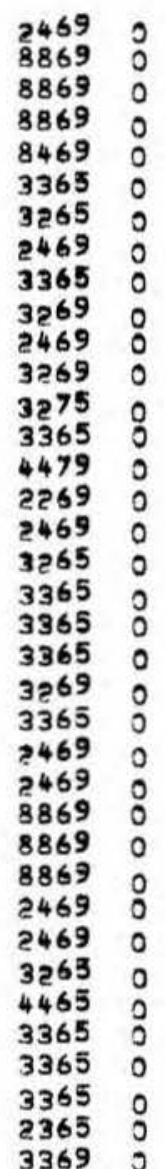




\section{7}

NILE DELTA UNDERWAY and GRAB SAMPLES

CHAIN 119 , LEG 2

\begin{tabular}{|c|c|c|c|c|c|c|}
\hline $\begin{array}{l}\text { Station } \\
\text { Number }\end{array}$ & $\begin{array}{l}\text { Sample } \\
\text { Number }\end{array}$ & Color & $\begin{array}{l}\text { Sand-Silt- } \\
\text { Clay }\end{array}$ & $\% \mathrm{CaCO}_{3}$ & $\% c_{\text {org }}$ & $\begin{array}{l}\text { Depth } \\
\text { corr. } \mathrm{m} .\end{array}$ \\
\hline 1 & U/W 1 & $\begin{array}{l}10 \text { YR } 7 / 2 \\
\text { light gray }\end{array}$ & $56-28-16$ & 86 & .0 & 41 \\
\hline 2 & U/W 2 & $\begin{array}{l}10 \text { YR } 7 / 2 \\
\text { light gray }\end{array}$ & $42-36-22$ & 83 & .3 & 63 \\
\hline 3 & $0 / W 3$ & $\begin{array}{l}10 \mathrm{YR} 5 / 3 \\
\text { brown }\end{array}$ & $34-32-34$ & 71 & .3 & 223 \\
\hline 4 & U/W 4 & $\begin{array}{l}10 \text { YR } 6 / 2 \\
\text { light brownish } \\
\text { gray }\end{array}$ & $25-44-31$ & 71 & .5 & 125 \\
\hline 5 & U/W 5 & $\begin{array}{l}10 \text { YR } 7 / 1 \\
\text { light gray }\end{array}$ & $2 l_{i}-50-26$ & 79 & .5 & 56 \\
\hline 6 & U/W 6 & $\begin{array}{l}10 \text { YR } 8 / 1 \\
\text { white }\end{array}$ & $94-3-3$ & 90 & 1.0 & 44 \\
\hline 7 & $\mathrm{U} / \mathrm{W} 7$ & $\begin{array}{l}10 \text { YR } 5 / 8 \\
\text { yellow1sh brown }\end{array}$ & $99-0-1$ & $i^{92}$ & .1 & 25 \\
\hline 8 & $\mathrm{U} / \mathrm{W} 8$ & $\begin{array}{l}10 \text { YR } 6 / 2 \\
\text { light brownish } \\
\text { gray }\end{array}$ & $59-23-19$ & 76 & .5 & 43 \\
\hline 9 & U/W 9 & $\begin{array}{l}10 \text { YR } 5 / 3 \\
\text { brown }\end{array}$ & $96-2-2$ & 70 & .1 & 33 \\
\hline 30 & U/W 10 & $\begin{array}{l}10 \text { YR } 4 / 3 \\
\text { brown }\end{array}$ & $27-40-33$ & 52 & .7 & 136 \\
\hline 31 & - U/W 11 & $\begin{array}{l}10 \text { YR } 3 / 3 \\
\text { dark brovn }\end{array}$ & $5 / 58 / 37$ & 22 & 1.1 & 73 \\
\hline 32 & $\mathrm{U} / \mathrm{W} 12$ & $\begin{array}{l}10 \text { YR } 5 / 3 \\
\text { brown }\end{array}$ & $71-21-8$ & 6 & .2 & 30 \\
\hline 34 & U/W 14 & $\begin{array}{l}10 \text { YR } 4 / 4 \\
\text { dark yellowish } \\
\text { brown }\end{array}$ & $89-7-4$ & 12 & .1 & 14 \\
\hline 35 & $\mathrm{U} / \mathrm{W} 15$ & $\begin{array}{l}10 \text { YR } 5 / 4 \\
\text { yellowish brown }\end{array}$ & $99-1-0$ & 4 & .0 & 10 \\
\hline 36 & GR 1 & $\begin{array}{l}10 \text { YR } 3 / 2 \\
\text { very dark grayish } \\
\text { brown }\end{array}$ & $1-60-39$ & 4 & .7 & 6 \\
\hline
\end{tabular}


688

\begin{tabular}{|c|c|c|c|c|c|c|}
\hline $\begin{array}{l}\text { Station } \\
\text { Number }\end{array}$ & $\begin{array}{l}\text { Sample } \\
\text { Number }\end{array}$ & Color & $\begin{array}{l}\text { Sand-Silt- } \\
\text { Clay }\end{array}$ & $\% \mathrm{CaCO}_{3}$ & $\% \mathrm{C}_{\text {org }}$ & $\begin{array}{l}\text { Depth } \\
\text { corr. } m \text {. }\end{array}$ \\
\hline 38 & GR 2 & $\begin{array}{l}10 \mathrm{YR} 4 / 3 \\
\text { brown }\end{array}$ & $92-6-2$ & 1 & .0 & 6 \\
\hline 39 & GR 3 & $\begin{array}{l}10 \text { YR } 3 / 2 \\
\text { very dark grayish } \\
\text { brown }\end{array}$ & $1-59-40$ & 5 & .9 & 9 \\
\hline 41 & GR 4 & $\begin{array}{l}10 \text { YR } 3 / 2 \\
\text { very dark grayish } \\
\text { brown }\end{array}$ & $16-54-30$ & 4 & .6 & 17 \\
\hline 42 & GR 5 & $\begin{array}{l}5 \text { Y } 3 / 2 \\
\text { dark olive gray }\end{array}$ & $9-56-35$ & 6 & 1.0 & 29 \\
\hline 45 & GR 6 & $\begin{array}{l}10 \text { YR } 3 / 2 \\
\text { very dark grayish } \\
\text { brown }\end{array}$ & $70-16-14$ & 85 & .3 & 46 \\
\hline 47 & GR 8 & $\begin{array}{l}10 \text { YR } 4 / 2 \\
\text { very dark grayish } \\
\text { brown }\end{array}$ & $45-29-26$ & 31 & .6 & 75 \\
\hline 48 & GR 9 & $\begin{array}{l}10 \mathrm{YR} 4 / 2 \\
\text { very dark grayish } \\
\text { brown }\end{array}$ & $58-16-25$ & 50 & .6 & 134 \\
\hline 49 & U/W 16 & $\begin{array}{l}2.5 \text { Y } 5 / 2 \\
\text { grayish brown }\end{array}$ & $46-27-27$ & 48 & .6 & 148 \\
\hline 50 & U/W 17 & $\begin{array}{l}10 \text { YR } 4 / 4 \\
\text { dark yellowish } \\
\text { brown }\end{array}$ & $94-3-2$ & 88 & .3 & 60 \\
\hline 51 & U/W 18 & $\begin{array}{l}10 \text { YR } 3 / 2 \\
\text { very dark grayish } \\
\text { brown }\end{array}$ & $5-54-41$ & 12 & 1.0 & 53 \\
\hline 52 & U/W 19 & $\begin{array}{l}10 \text { YR } 3 / 2 \\
\text { very dark grayish } \\
\text { brown }\end{array}$ & $8-67-25$ & 9 & .9 & 38 \\
\hline 53 & $\mathrm{U} / \mathrm{H} 20$ & $\begin{array}{l}10 \text { YR } 3 / 2 \\
\text { very dark grayish } \\
\text { brown }\end{array}$ & $0-31-69$ & 5 & 1.4 & 24 \\
\hline 54 & U/W 21 & $\begin{array}{l}10 \text { YR } 3 / 2 \\
\text { very dark grayish } \\
\text { brown }\end{array}$ & $1-43-56$. & 2 & .9 & 8 \\
\hline 55 & GR 10 & $\begin{array}{l}10 \text { YR } 3 / 2 \\
\text { very dark grayish } \\
\text { brown }\end{array}$ & $1-55-44$ & 5 & 1.1 & 11 \\
\hline
\end{tabular}


689

\begin{tabular}{|c|c|c|c|c|c|c|}
\hline $\begin{array}{l}\text { Station } \\
\text { Number }\end{array}$ & $\begin{array}{l}\text { Sample } \\
\text { Number }\end{array}$ & Color & $\begin{array}{l}\text { Sand-Silt- } \\
\text { Clay }\end{array}$ & $\% \mathrm{CaCO}_{3}$ & $\% c_{\text {org }}$ & $\begin{array}{l}\text { Depth } \\
\text { corr. } \mathrm{m} \text {. }\end{array}$ \\
\hline 58 & v/W 22 & $\begin{array}{l}10 \text { YR } 4 / 3 \\
\text { brown }\end{array}$ & $59-34-7$ & 2 & .1 & 25 \\
\hline 59 & U/W 23 & $\begin{array}{l}10 \text { YR } 4 / 2 \\
\text { dark gray brown }\end{array}$ & $3-70-27$ & 16 & 1.1 & 149 \\
\hline 86 & GR II & $\begin{array}{l}10 \text { YR } 5 / 6 \\
\text { yellowish brown }\end{array}$ & $98-0-2$ & 15 & .1 & 42 \\
\hline 87 & GR 12 & $\begin{array}{l}10 \text { YR } 4 / 2 \\
\text { dark grayish } \\
\text { brown }\end{array}$ & $27-42-31$ & 11 & .9 & 35 \\
\hline 89 & GR 13 & $\begin{array}{l}10 \text { YR } 3 / 3 \\
\text { dark brown }\end{array}$ & $89-7-4$ & 2 & .0 & 6 \\
\hline 90 & U/W 24 & $\begin{array}{l}5 \text { Y } 7 / 2 \\
\text { light gray }\end{array}$ & $98-1-1$ & 0 & .0 & 8 \\
\hline 91 & U/W 25 & $\begin{array}{l}10 \text { YR } 3 / 2 \\
\text { very dark gray } \\
\text { brown }\end{array}$ & $36-48-16$ & 4 & .5 & 13 \\
\hline 93 & U/W 26 & $\begin{array}{l}10 \text { YR } 6 / 4 \\
\text { light yellowish } \\
\text { brown }\end{array}$ & $97-1-2$ & 2 & .0 & 22 \\
\hline 94 & U/W 27 & $\begin{array}{l}10 \text { YR } 4 / 4 \\
\text { dark yellow brown }\end{array}$ & $84-9-7$ & 35 & .2 & 37 \\
\hline 96 & U/W 29 & $\begin{array}{l}10 \text { YR } 4 / 2 \\
\text { dark gray brown }\end{array}$ & $67-20-13$ & 63 & .5 & 75 \\
\hline 97 & U/W 30 & $\begin{array}{l}10 \text { YR } 4 / 2 \\
\text { dark gray brown }\end{array}$ & $47-29-24$ & 47 & .6 & 103 \\
\hline 98 & U/W 31 & $\begin{array}{l}10 \text { YR } 4 / 2 \\
\text { derk gray brown }\end{array}$ & $31-32-37$ & 43 & .6 & 157 \\
\hline 99 & U/W 32 & $\begin{array}{l}10 \text { YR } 4 / 2 \\
\text { dark gray brown }\end{array}$ & $43-28-29$ & 51 & .5 & 139 \\
\hline $100^{\circ}$ & U/W 33 & $\begin{array}{l}10 \text { XR } 4 / 2 \\
\text { gray brown }\end{array}$ & $60-21-19$ & 66 & .5 & 73 \\
\hline 101 & $\mathrm{U} / \mathrm{w} 34$ & $\begin{array}{l}10 \text { YR } 6 / 4 \\
\text { light yellow } \\
\text { brown }\end{array}$ & $99-0-1$ & 11 & .0 & 47 \\
\hline 102 & U/W 35 & $\begin{array}{l}10 \text { YR } 2.5 / 1 \\
\text { black }\end{array}$ & $30-25-45$ & 8 & .7 & 39 \\
\hline 103 & U/W 36 & $\begin{array}{l}5 \times 3 / 2 \\
\text { dark olive gray }\end{array}$ & $26-43-31$ & 9 & 1.0 & 32 \\
\hline
\end{tabular}




\section{0}

\begin{tabular}{|c|c|c|c|c|c|c|}
\hline $\begin{array}{l}\text { Station } \\
\text { Number }\end{array}$ & $\begin{array}{l}\text { Sample } \\
\text { Number }\end{array}$ & Color & $\begin{array}{l}\text { Sand-Silt- } \\
\text { Clay }\end{array}$ & $\% \mathrm{CaCO}_{3}$ & $\% \mathrm{C}_{\text {org }}$ & $\begin{array}{l}\text { Depth } \\
\text { corr. } m .\end{array}$ \\
\hline 104 & U/W 37 & $\begin{array}{l}10 \text { YR } 5 / 6 \\
\text { yellowish brown }\end{array}$ & $99-0-1$ & 3 & .0 & 15 \\
\hline 106 & GR 14 & $\begin{array}{l}10 \text { YR } 4 / 2 \\
\text { dark grayish } \\
\text { brown }\end{array}$ & $88-9-3$ & 1 & .1 & 8 \\
\hline 106 & GR 15 & $\begin{array}{l}10 \mathrm{YR} 4 / 2 \\
\text { dark grayish } \\
\text { brown }\end{array}$ & $87-9-4$ & 3 & .1 & 8 \\
\hline 108 & GR 26 & $\begin{array}{l}10 \text { YR } 4 / 2 \\
\text { dark grayish } \\
\text { brown }\end{array}$ & $61-20-19$ & 13. & .3 & 19 \\
\hline 111 & GR 17 & $\begin{array}{l}10 \text { YR } 4 / 2 \\
\text { dark grayish } \\
\text { brown }\end{array}$ & $75-15-10$ & 72 & .6 & 61 \\
\hline 112 & GR 18 & $\begin{array}{l}10 \text { YR } 5 / 3 \\
\text { brown }\end{array}$ & $91-3-5$ & 76 & .3 & 93 \\
\hline 113 & U/W 38 & $\begin{array}{l}10 \text { YR } 4 / 2 \\
\text { dark gray brown }\end{array}$ & $11-28-61$ & 19 & $\cdot 3$ & 166 \\
\hline 146 & U/W 39 & $\begin{array}{l}10 \text { YR } 4 / 3 \\
\text { brown }\end{array}$ & $82-9-9$ & 183 & .0 & 92 \\
\hline 147 & GR 19 & $\begin{array}{l}10 \text { YR } 4 / 2 \\
\text { dark gray brown }\end{array}$ & $54-25-21$ & 39 & .6 & 72 \\
\hline 148 & GR 20 & $\begin{array}{l}10 \text { YR } 4 / 2 \\
\text { dark gray brown }\end{array}$ & $3-40-57$ & 7 & 1.0 & 71 \\
\hline 150 & GR 21 & & & & & 224 \\
\hline & top & $\begin{array}{l}10 \mathrm{YR} 4 / 3 \\
\text { brown }\end{array}$ & $25-42-33$ & 34 & .4 & \\
\hline & bottom & $\begin{array}{l}10 \text { YR } 4 / 3 \\
\text { brown }\end{array}$ & $36-32-32$ & 42 & .4 & \\
\hline 151 & $\begin{array}{l}\text { GR } 22 \\
\text { top }\end{array}$ & $\begin{array}{l}10 \text { YR } 4 / 3 \\
\text { brown }\end{array}$ & $56-23-21$ & 53 & .4 & 193 \\
\hline & bottom & $\begin{array}{l}10 \mathrm{YR} 4 / 2 \\
\text { dark grayish } \\
\text { brown }\end{array}$ & $6-43-51$ & 18 & .8 & \\
\hline 152 & GR 23 & $\begin{array}{l}10 \text { YR } 5 / 3 \\
\text { brown }\end{array}$ & $81-7-12$ & 79 & .3 & 103 \\
\hline
\end{tabular}


691

\begin{tabular}{|c|c|c|c|c|c|c|}
\hline $\begin{array}{l}\text { Station } \\
\text { Number }\end{array}$ & $\begin{array}{l}\text { Sample } \\
\text { Number }\end{array}$ & Color & $\begin{array}{l}\text { Sand-Silt- } \\
\text { Clay }\end{array}$ & $\% \mathrm{CaCO}_{3}$ & $\% \mathrm{C}_{\text {org }}$ & $\begin{array}{l}\text { Depth } \\
\text { corr. } \mathrm{m} .\end{array}$ \\
\hline \multirow[t]{3}{*}{153} & GR 24 & & & & & 77 \\
\hline & top & $\begin{array}{l}10 \text { YR } 4 / 3 \\
\text { brown }\end{array}$ & & . & & \\
\hline & bottom & $\begin{array}{l}10 \text { YR } 4 / 2 \\
\text { dark gray browm }\end{array}$ & $23-31-46$ & 27 & .8 & \\
\hline 154 & GR 25 & $\begin{array}{l}10 \text { YR } 4 / 3 \\
\text { brown }\end{array}$ & $1-47-52$ & 4 & 1.1 & 48 \\
\hline 156 & GR 26 & $\begin{array}{l}10 \text { YR } 4 / 2 \\
\text { dark gray brown }\end{array}$ & $1-44-55$ & 7. & .9 & 48 \\
\hline 158 & U/W 41 & $\begin{array}{l}10 \text { YR } 4 / 2 \\
\text { dark gray brown }\end{array}$ & $52-22-26$ & 58 & .0 & 75 \\
\hline 162 & GR 27 & $\begin{array}{l}10 \mathrm{YR} 5 / 3 \\
\text { brown }\end{array}$ & $35-3-62$ & 85 & $\cdot 3$ & 112 \\
\hline \multirow[t]{2}{*}{163} & $\begin{array}{l}\text { GR } 28 \\
\text { top }\end{array}$ & $\begin{array}{l}10 \text { YR } 4 / 4 \\
\text { dark yellow brown }\end{array}$ & & & & 85 \\
\hline & bottom & $\begin{array}{l}10 \text { YR } 4.3 \\
\text { brown }\end{array}$ & $75-12-14$ & 77 & .4 & \\
\hline 164 & GR 29 & $\begin{array}{l}10 \text { YR } 4 / 4 \\
\text { dark yellowish } \\
\text { brown }\end{array}$ & $91-4-5$ & 76 & .2 & 62 \\
\hline 165 & GR 30 & $\begin{array}{l}10 \text { YR } 4 / 2 \\
\text { dark gray brown }\end{array}$ & $42-23-35$ & 39 & .8 & 64 \\
\hline 166 & GR 31 & $\begin{array}{l}10 \text { YR } 4 / 2 \\
\text { dark gray brown }\end{array}$ & $77-10-13$ & 85 & .2 & 44 \\
\hline 168 & GR 32 & $\begin{array}{l}10 \text { YR } 3 / 2 \\
\text { very dark gray } \\
\text { brown }\end{array}$ & $4-53-43$ & 5 & 1.1 & 28 \\
\hline 169 & GR 33 & $\begin{array}{l}10 \text { YR } 3 / 2 \\
\text { very dark gray } \\
\text { brown }\end{array}$ & $10-60-30$ & 5 & .8 & 22 \\
\hline 170 & GR 34 & $\begin{array}{l}10 \text { YR } 3 / 3 \\
\text { dark brown }\end{array}$ & $75-15-6$ & 2 & .1 & 11 \\
\hline 172 & GR 35 & $\begin{array}{l}10 \text { YR } 3 / 3 \\
\text { dark brown }\end{array}$ & $88-9-3$ & 2 & .0 & 5 \\
\hline 173 & U/W 44 & $\begin{array}{l}10 \text { YR } 4 / 2 \\
\text { dark gray brown }\end{array}$ & $92-5-2$ & 2 & .0 & 9 \\
\hline
\end{tabular}


692

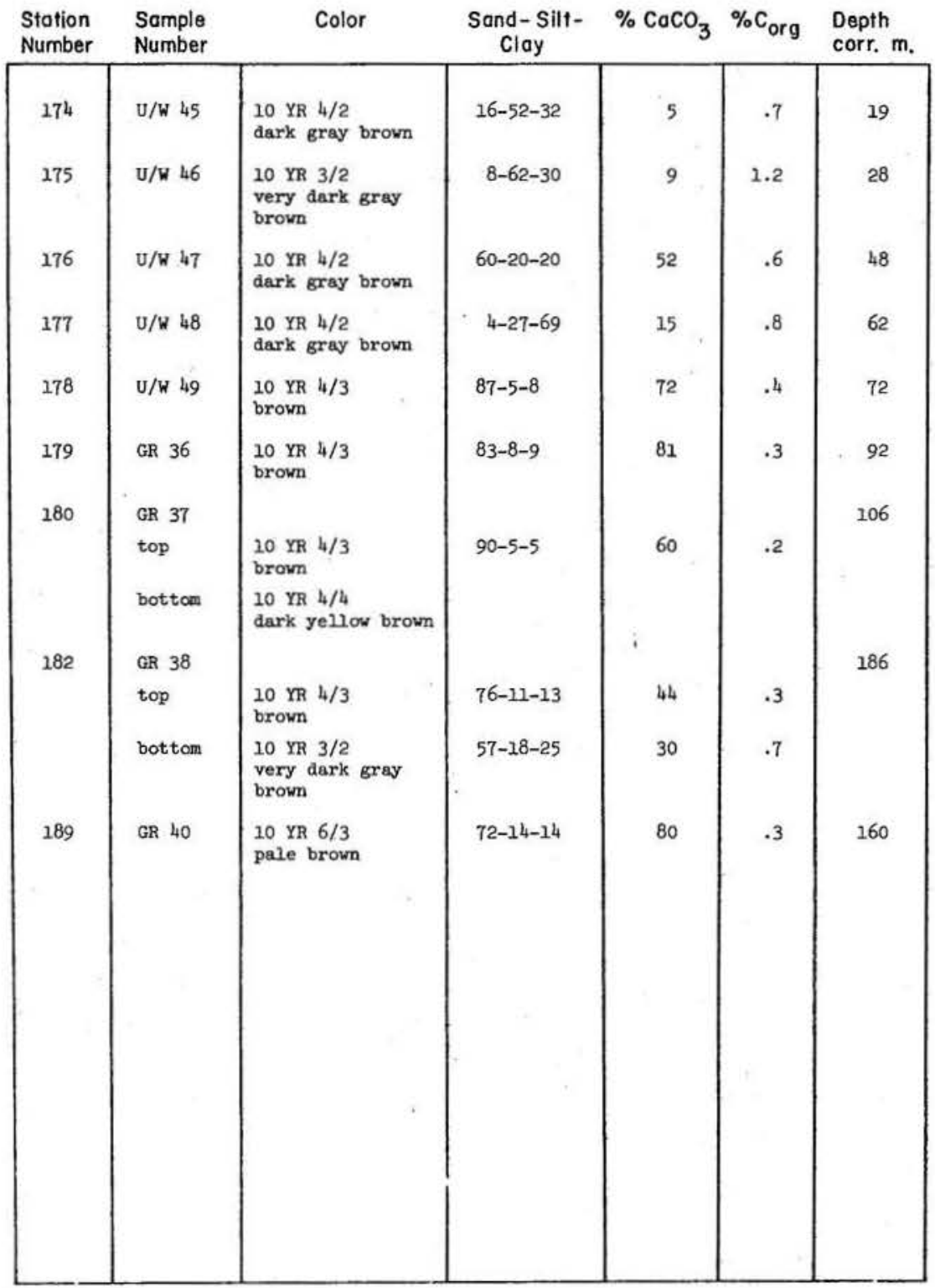


I:

I

I

।

1

I

1

I

1

I

I

1

1

1

I

I

I

1 
693

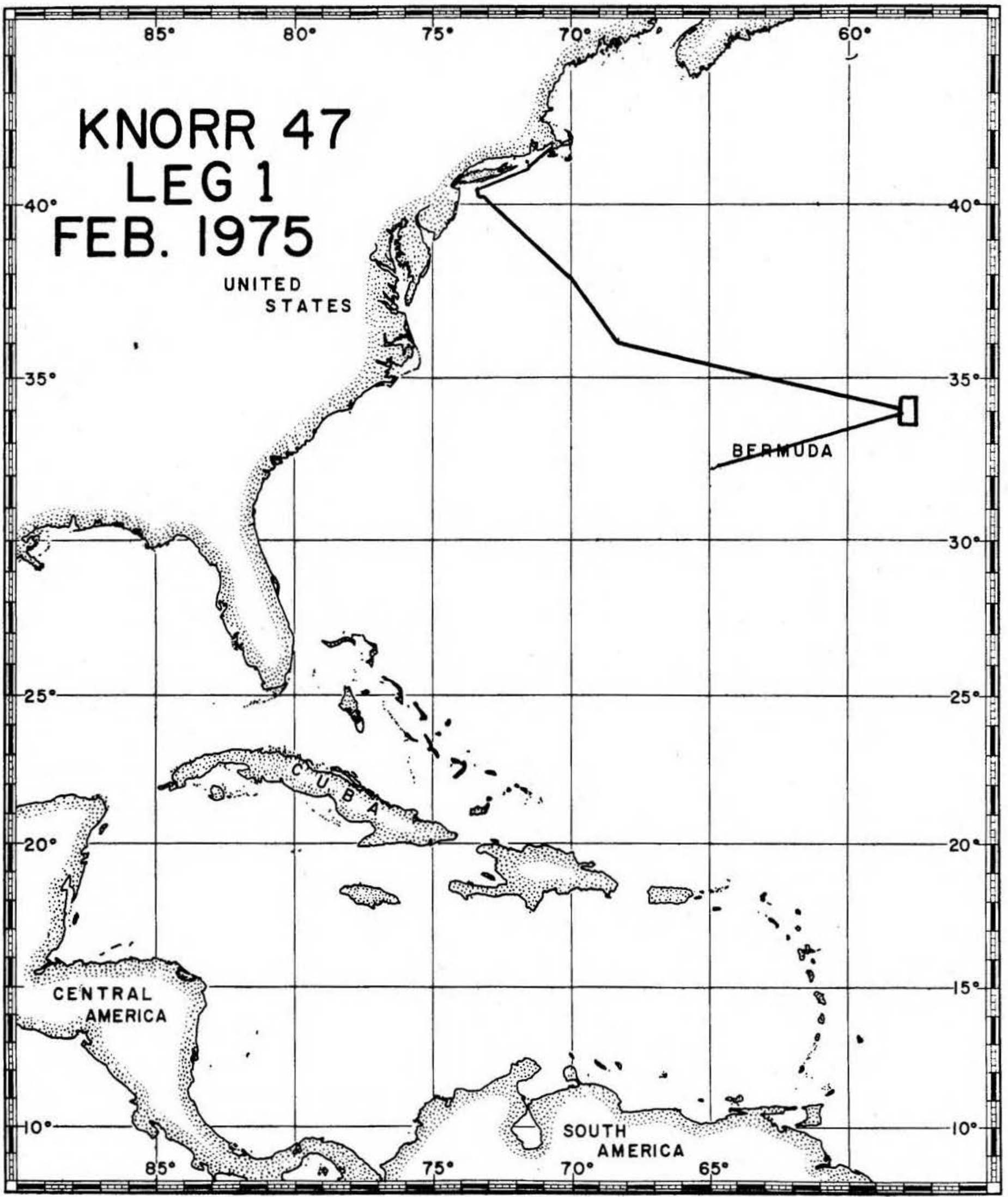


$\Gamma$

$\Gamma$

$\Gamma$

1

$\Gamma$

1

1

1

1

1

I

1

1

I

1

$I$

I

1 


\section{4}

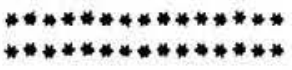

STATION UATA RETRIEVAL

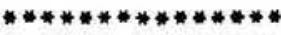

PAGE DATE: 17:20 JUN 03,177

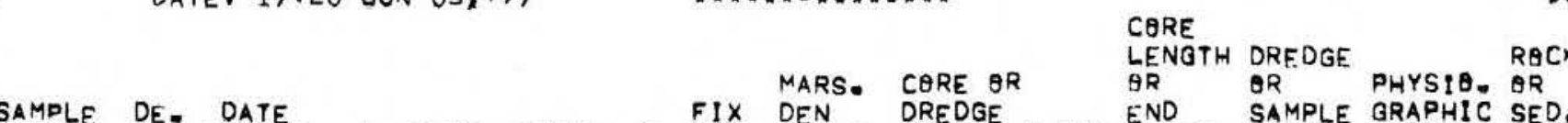

END SAMPLE GRAPHIC SED, VITA

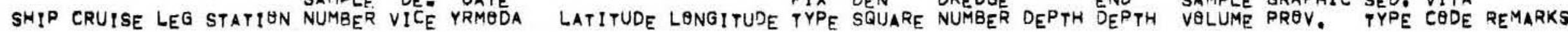

\begin{tabular}{|c|c|c|c|c|c|c|c|c|c|c|c|c|c|c|c|c|c|c|}
\hline KNR & 47 & 1 & 0001 & 0000 & $\begin{array}{l}15 \\
C \theta M\end{array}$ & $\begin{array}{l}75 \\
\text { ENTS }\end{array}$ & 210 & 33 & $50.61 \mathrm{~N}$ & $\begin{array}{l}57 \\
53 \cdot 1 \\
\text { IMEROUS HYDRE }\end{array}$ & $\stackrel{9}{\theta P R E}$ & 8ILITE L & OOMOINAT & $\begin{array}{l}42^{84} 4^{\circ} \\
10 N S\end{array}$ & 1155. & 0000 & 13 & 4660 \\
\hline $\begin{array}{l}\text { KNR } \\
\text { KNR }\end{array}$ & $\begin{array}{l}47 \\
47\end{array}$ & $\begin{array}{l}1 \\
1\end{array}$ & $\begin{array}{l}0001 \\
0002\end{array}$ & $\begin{array}{l}0000 \\
0000\end{array}$ & $\begin{array}{l}26 \\
15 \\
\text { COMM }\end{array}$ & $\begin{array}{c}75 \\
75 \\
\text { ENTS }\end{array}$ & $\begin{array}{l}210 \\
211 \\
3 * *\end{array}$ & $\begin{array}{l}33 \\
33\end{array}$ & $\begin{array}{r}50.6^{1 N} \\
40.81 \mathrm{~N} \\
\mathrm{NU}\end{array}$ & $\begin{array}{r}5733.11 W \\
57 \\
40.31 W \\
\text { JMERQUS HYDRE }\end{array}$ & $\begin{array}{l}9 \\
6 \\
\text { QTRE }\end{array}$ & $\begin{array}{l}114.37 \\
114.37 \\
\text { OILITE L }\end{array}$ & $\begin{array}{l}0001 \\
\text { OOOZ } \\
\text { LAMINAT }\end{array}$ & $\begin{array}{l}4284^{\circ} \\
4619^{\circ} \\
\text { IONS }\end{array}$ & $\begin{array}{r}122 . \\
11950\end{array}$ & $\begin{array}{l}0000 \\
0000\end{array}$ & $\begin{array}{l}13 \\
13\end{array}$ & $\begin{array}{l}3369 \\
4660\end{array}$ \\
\hline $\begin{array}{l}\text { KNR } \\
\text { KNR }\end{array}$ & $\begin{array}{l}47 \\
47\end{array}$ & 1 & $\begin{array}{l}0002 \\
000^{4}\end{array}$ & $\begin{array}{l}0000 \\
0000\end{array}$ & $\begin{array}{l}26 \\
15 \\
\text { COMME }\end{array}$ & $\begin{array}{c}75 \\
75 \\
\text { ENTS }\end{array}$ & $\begin{array}{l}211 \\
215 \\
5 * *\end{array}$ & $\begin{array}{l}33 \\
33\end{array}$ & $\begin{array}{r}40.81 \mathrm{~N} \\
54.81 \mathrm{~N}\end{array}$ & $\begin{array}{l}57,40.31 W \\
57.230 .31 W \\
\text { JMERQUS HYDRE }\end{array}$ & $\begin{array}{l}6 \\
6 \\
\text { OTRE }\end{array}$ & $\begin{array}{l}114.37 \\
114.37 . \\
\text { OILITE L }\end{array}$ & $\begin{array}{l}0002 \\
\text { OOOO4 } \\
\text { LAMINAT }\end{array}$ & $\begin{array}{l}4619 . \\
4792 . \\
10 N S\end{array}$ & $\begin{array}{l}370 \\
7760\end{array}$ & $\begin{array}{l}0000 \\
0000\end{array}$ & $\begin{array}{l}13 \\
13\end{array}$ & $\begin{array}{l}3339 \\
1342\end{array}$ \\
\hline $\begin{array}{l}\text { KNR } \\
\text { KNR }\end{array}$ & $\begin{array}{l}47 \\
47\end{array}$ & $\begin{array}{l}1 \\
1\end{array}$ & $\begin{array}{l}0004 \\
0005\end{array}$ & $\begin{array}{l}0000 \\
0600\end{array}$ & 26 & $\begin{array}{r}75 \\
75 \\
\text { ENTS }\end{array}$ & $\begin{array}{l}215 \\
216 \\
3 * *\end{array}$ & $\begin{array}{l}33 \\
33\end{array}$ & $\begin{array}{r}54.81 \mathrm{~N} \\
30 \cdot 6, \mathrm{~N} \\
\mathrm{NU}\end{array}$ & $\begin{array}{c}5723.31 W \\
5749.41 W \\
\text { JMEROUS HYDRE }\end{array}$ & $\begin{array}{l}6 \\
6 \\
\text { QTRE }\end{array}$ & $\begin{array}{l}114.37 \\
114.37 \\
\text { OILITE L }\end{array}$ & $\begin{array}{l}0004 \\
\text { OOO5 } \\
\text { LAMINAT }\end{array}$ & $\begin{array}{l}4792 . \\
4618^{\circ} \\
10 N S\end{array}$ & 1189. & $\begin{array}{l}0000 \\
0000\end{array}$ & $\begin{array}{l}13 \\
13\end{array}$ & $\begin{array}{l}335^{3} \\
4340\end{array}$ \\
\hline $\begin{array}{l}\text { KNR } \\
\text { KNR }\end{array}$ & $\begin{array}{l}47 \\
47\end{array}$ & $\begin{array}{l}1 \\
1\end{array}$ & $\begin{array}{l}0005 \\
0033\end{array}$ & $\begin{array}{l}0000 \\
0000\end{array}$ & 26 & 75 & $\begin{array}{l}216 \\
216\end{array}$ & $\begin{array}{l}33 \\
34\end{array}$ & $\begin{array}{l}30.61 \mathrm{~N} \\
10.31 \mathrm{~N}\end{array}$ & $\begin{array}{ll}57 & 49.41 \mathrm{~W} \\
57 & 11.51 \mathrm{w}\end{array}$ & $\begin{array}{l}6 \\
6\end{array}$ & $\begin{array}{l}114.37 \\
114.47\end{array}$ & $\begin{array}{l}0005 \\
0006\end{array}$ & $\begin{array}{l}46180 \\
5500 .\end{array}$ & $\begin{array}{r}680^{\circ} \\
160 .\end{array}$ & $\begin{array}{l}0000 \\
0000\end{array}$ & $\begin{array}{l}13 \\
13\end{array}$ & $\begin{array}{l}3359 \\
1328\end{array}$ \\
\hline
\end{tabular}


11

\lceil

$\Gamma$

I

1

1

1

1

I

1

1

1

1

1

I

$\frac{1}{1}$ 
Ship KNORR Cruise 47 Leg I Sta. 1 Core No. 1 PC Total Length $1155 \mathrm{~cm}$. Lat. $33^{\circ} 50.6^{\prime} \mathrm{N}$ Long. $57^{\circ} 33.1$ 'W Depth 4204 corr. $\mathrm{m}$ Core condition EXCELLENT Date Described 23 Apail 76 by H. Farmer Physiographic location Lithologic

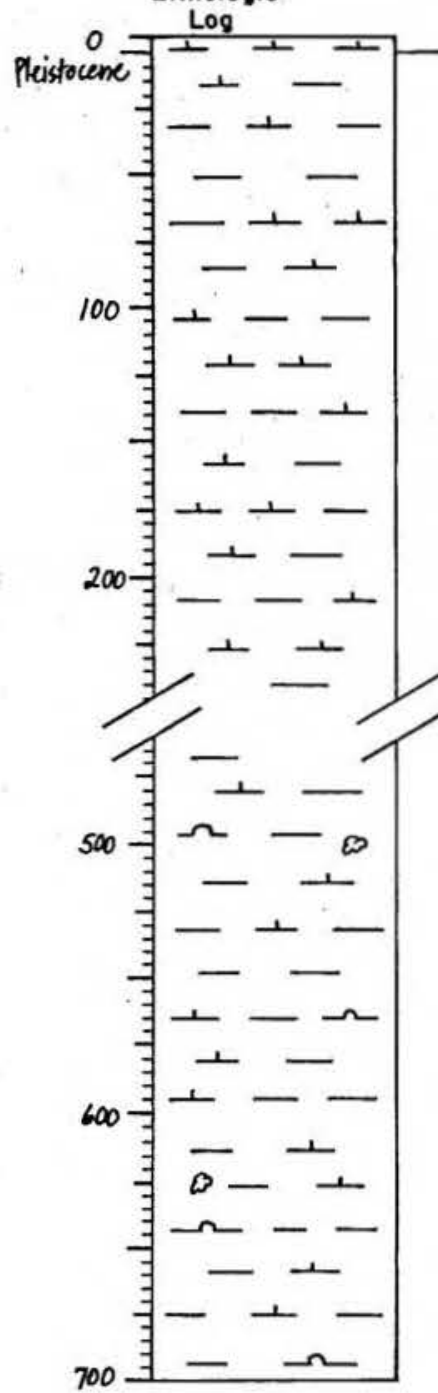

Detailed Description

cate

10 YR $6 / 3$ pale brown

soft, slightly silty lutite, few forams

5-1155

CAIC CLAY GRADES TO CAIC OOZE

YR $5 / 3$ brown ; grades very gently to $5 / 2$ grayish brown $(\approx 700 \mathrm{~cm})$ which pales to $6 / 2$ 1ight bromith gray $(\approx 1060 \mathrm{~cm})$

very sma1l, dark brown mottles are scattered fairly sparsely through the top $80 \mathrm{~cm}$ and very rarely through the next $400 \mathrm{~cm}$; from there, slightly bigger, dark brown mottles are scattered to the end of the core
fairly slick, slightly silty lutite with only occasiona fairly sick, slightly silty lutite with only occasion firmer and less slick; a number of thin $(\boldsymbol{F} 1 \mathrm{~cm})$, firmer laminations are found below $600 \mathrm{~cm}$ such as $608,630,732,758,782,798,822,866,895,910,933$ and $970 \mathrm{~cm}$. These are coumonly a bit pale and sometimes are followed by somewhat darker layers such as $867-887 \mathrm{~cm}, 910-915 \mathrm{~cm}, 934-944 \mathrm{~cm}$ and $970-976 \mathrm{~cm}$ ceaslonal lsolated, canpact, yellowlsh brom $1 \mathrm{mps}$ appear $125-260 \mathrm{~cm}$, scoe with black, llthifled naclel, while occasional black fragnents appear without the yellowlish crust

points out that aumerous hydrotroilite layers were present

end of core
Ship KNeRR Cruise 47 Leg 1 Sta. 1 Core No. I PC

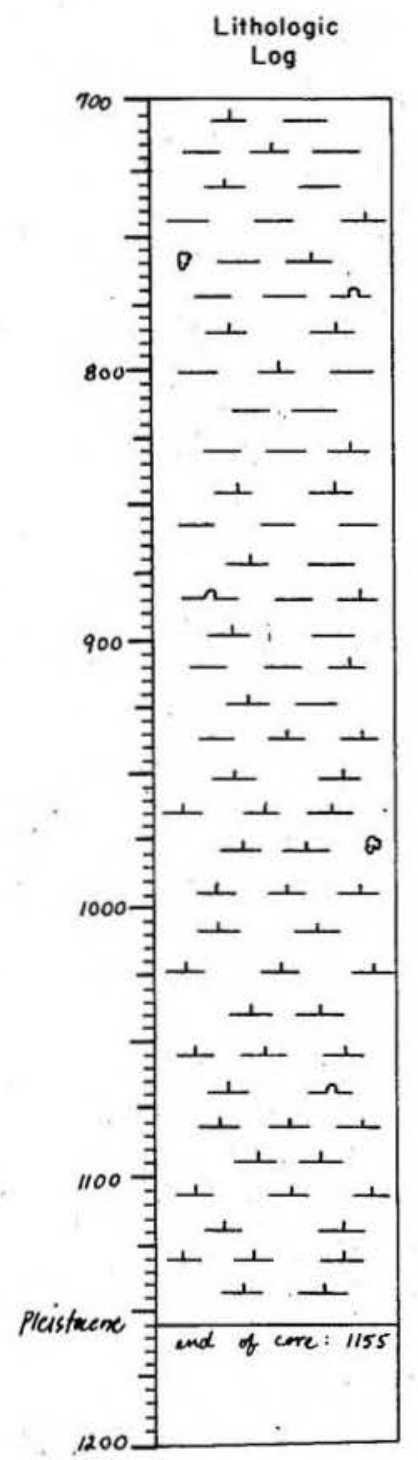

Detailed Description 
SHEAR SLIDE DESCRIPTIONS - W.H.O.I. SEDIMENT CORES

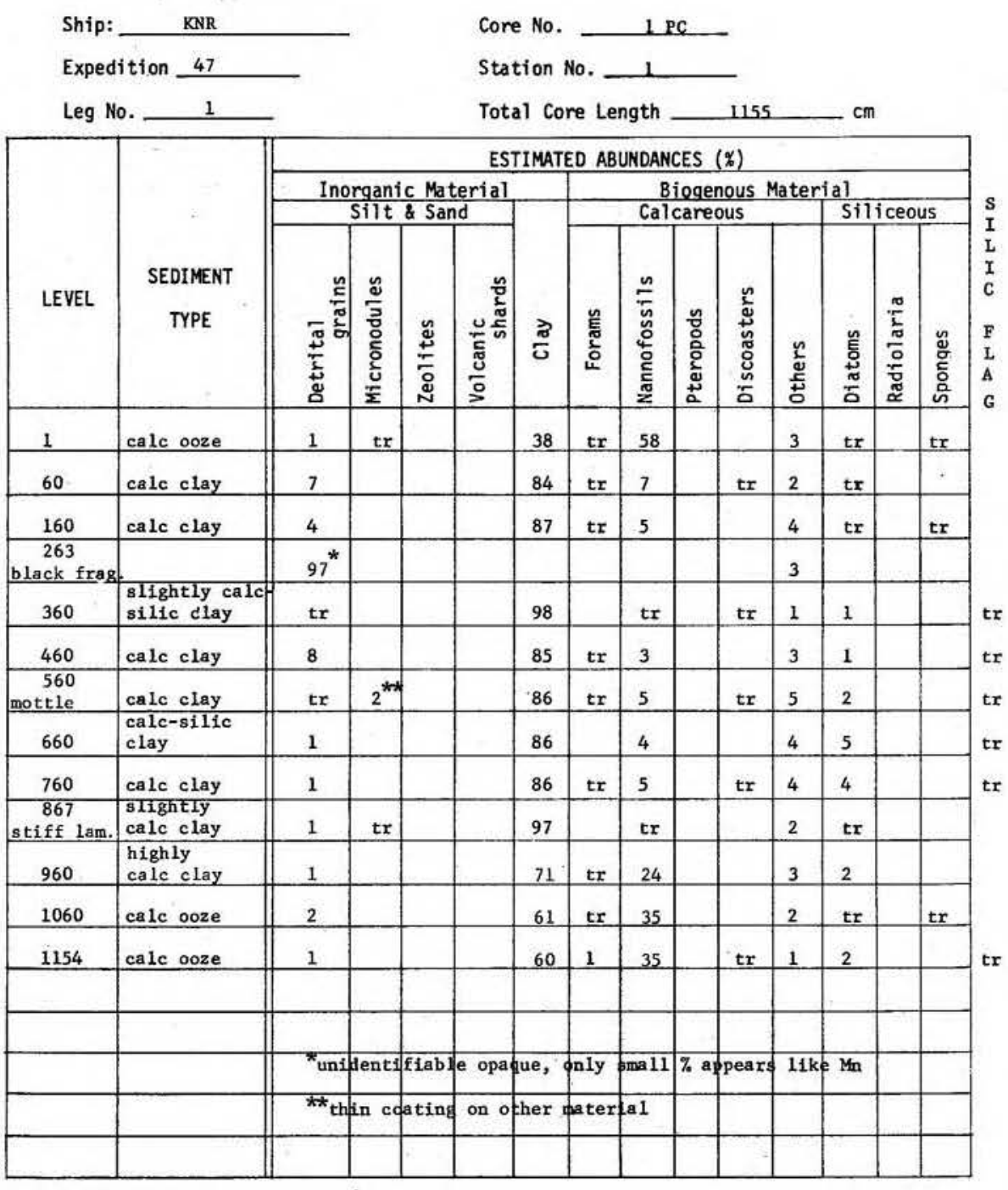

Ship KNORR Cruise 47 Leg 1 Sto. 1 Core No. 1 PG Total Length $122 \mathrm{~cm}$. Lat. $33^{\circ} 50.6^{\prime} \mathrm{N}$ Long. $57^{\circ} 33.1^{\prime}$ 'W Depth 4284 cora. $\mathrm{m}$ Core condition EXCELLENT Date Described 23 April76 T T FARMER Physiogrophic location PGATEAU ON BERMUDA RISE, AREn OF LAMINATED SEDIMENTI Lithologic

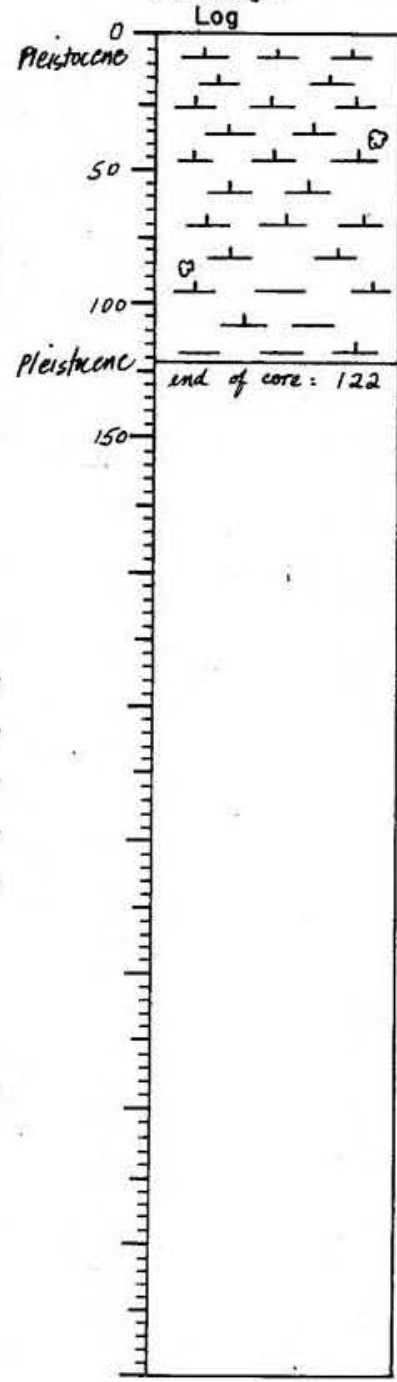

$0-122$

CALC DOZE

10 YR $5 / 3$ brown, grades to $6 / 3$ pale brown below $65 \mathrm{~cm}$ a few, small, grayish-brown mottles appear clustered at the following depths: $20,49,72-82,113$ somewhat $\mathrm{fim}$, slightly silty lutite, forams are scattered

$119 \mathrm{~cm}-3 \mathrm{~mm}$ bed of $10 \mathrm{YR} 3 / 3$ dark brown, slightly silty than end of core
Detailed Description 
SIEAR SLIDE DESCRIPTIONS - W.H.O.I. SEDIIENT CORES

Ship: KRP

Expedition 47

Leg No. 1

\begin{tabular}{|c|c|c|c|c|c|c|c|c|c|c|c|c|c|c|}
\hline \multirow[b]{3}{*}{ LEVEL } & \multirow[b]{3}{*}{$\begin{array}{c}\text { SEDIMENT } \\
\text { TYPE }\end{array}$} & \multicolumn{13}{|c|}{ ESTIMATED ABUNDANCES ( $(x)$} \\
\hline & & \multicolumn{4}{|c|}{$\frac{\text { Inorganic Material }}{\text { Silt \& Sand }}$} & \multirow[b]{2}{*}{$\frac{\pi}{6}$} & \multicolumn{5}{|c|}{$\begin{array}{l}\text { Biogenous Mater } \\
\text { Calcareous }\end{array}$} & \multicolumn{2}{|c|}{$\frac{19}{\text { Siliceous }}$} & \\
\hline & & $\frac{\underbrace{\circ}}{\frac{5}{5}}$ & 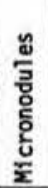 & 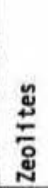 & 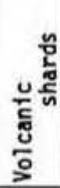 & & 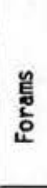 & 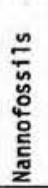 & 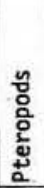 & 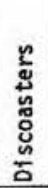 & $\begin{array}{l}\frac{r}{g} \\
\frac{5}{\Delta} \\
\end{array}$ & 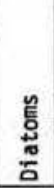 & $\frac{\pi}{\frac{\pi}{2}} \frac{\pi}{\frac{\pi}{0}}$ & 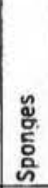 \\
\hline 1 & calc ooze & tr & $t x$ & & & 68 & tr & 30 & & & 2 & tr & & $t x$ \\
\hline 78 & calc ooze & tr & 3 & & & 60 & 2 & 34 & & & 1 & tr & & tr \\
\hline 119 & $\begin{array}{l}\text { slightly } \\
\text { calc clay }\end{array}$ & 1 & 4 & & & 94 & tr & $\operatorname{tr}$ & & & 1 & tr & & \\
\hline & & & & & & & & & & & & & & \\
\hline & & & & & & & & & & . & & & & \\
\hline & $=$ & & & & & & & & & & & & & \\
\hline & & & & & & & & & & & & & & \\
\hline & & & & & & & & & & & & & & \\
\hline & & & & & & & & & & & & & & \\
\hline & & & & & & & & & & & & & & \\
\hline & & & & & & , & & & & & & & & \\
\hline & & & & & & & & & & & & & & \\
\hline 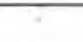 & & & & & & & & & & & & & & \\
\hline & & & & & & & & & & & & & & \\
\hline & & & & & & & & & & & & & & \\
\hline & & . & & & & & & & & & & & & \\
\hline & & & & & & & & & & & & & & \\
\hline & & & & & & & & & & & & & & \\
\hline
\end{tabular}

Core No. 1 PG

Station No. 1
Ship KNORR - Cruise 47 Total Length $1179 \mathrm{~cm}$. Lat. $33^{\circ} 40.8^{\prime} \mathrm{N}$ Long. $57^{*} 40.3^{\prime} \mathrm{W}$ Depth $46 / 9$ cree. Core condition ExcrLEENT Date Described 27 Aani 16 by $T$ FMemes. Physiographic location BERMUDA RISE, AREA OF ACOUSTIC LAMINATIONS Lithologic

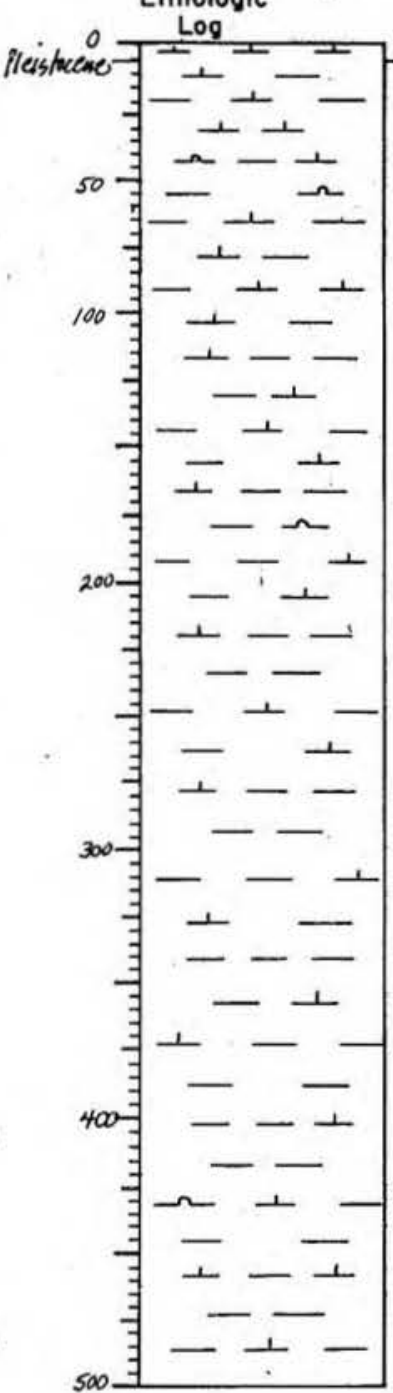

Detailed Description

CALC OOZE

a few, very subdued laminations appear in somewhat dis-

(1ightly silty lutite; few forams are present s

CAIC CIAY GRADES TO CALC-SILIC CIAY

gradational hues of 10 YR $4 / 3$ and $5 / 3$ browns, below 850 $\mathrm{cm}$ the color turns slowly to $5 / 2$ grayish brown

small, dark graylsh brown mottles are scattored sparcely down to $110 \mathrm{~cm}$, and even less commonly from $160-285 \mathrm{~cm}$ again in the lowest $2.5 \mathrm{~m}$ a few small clusters of mot-

slick, slightly silty lutite, with some variations in tions: blow $300 \mathrm{~cm}$, the sediment is more unf form and

230-870 cm - 1solated, rusty brown, somewhat compacted lumps, lens, and laminations are scattered sparingly $940-1015 \mathrm{~cm}$ several interesting, black, $1 \mathrm{~cm}$ long, horizontal lying, needle-like objects vere noted (pyritized ?)

NOTE: Initial shipboard descriptions indicate that (a) Many of the previously noted, rusty brom lumps and lenses correspond to these hydrotroilite layers. end of core
YR $6 / 3$, pale brow forams are apparent 
701

VISUAL CORE DESCRIPTION

Page 2 of 2

Ship KNORR Cruise 47 Leg 1 Sta. 2 Core No. 2 PC

Lithologic

Log

Detailed Description

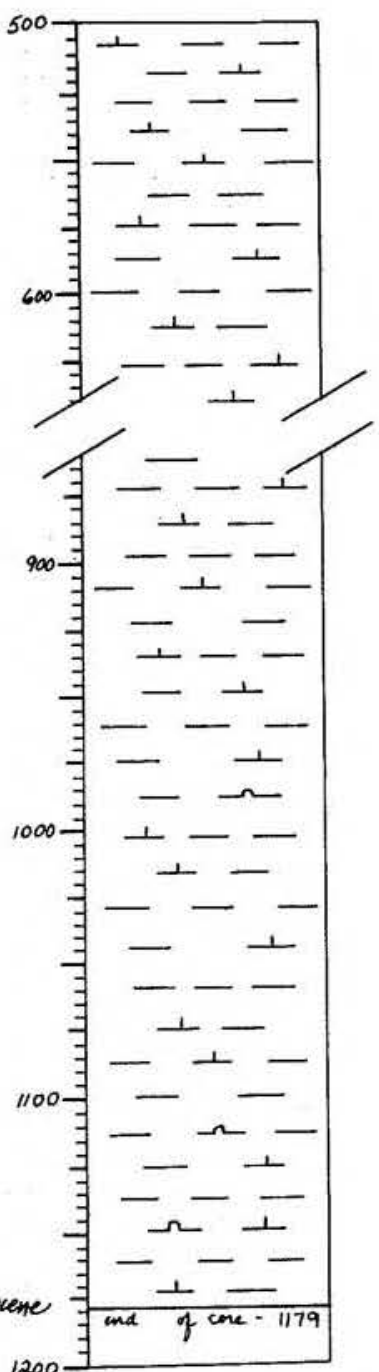

702

SMEAR SLIDE DESCRIPTIONS - W.H.O.I. SEDIMENT CORES

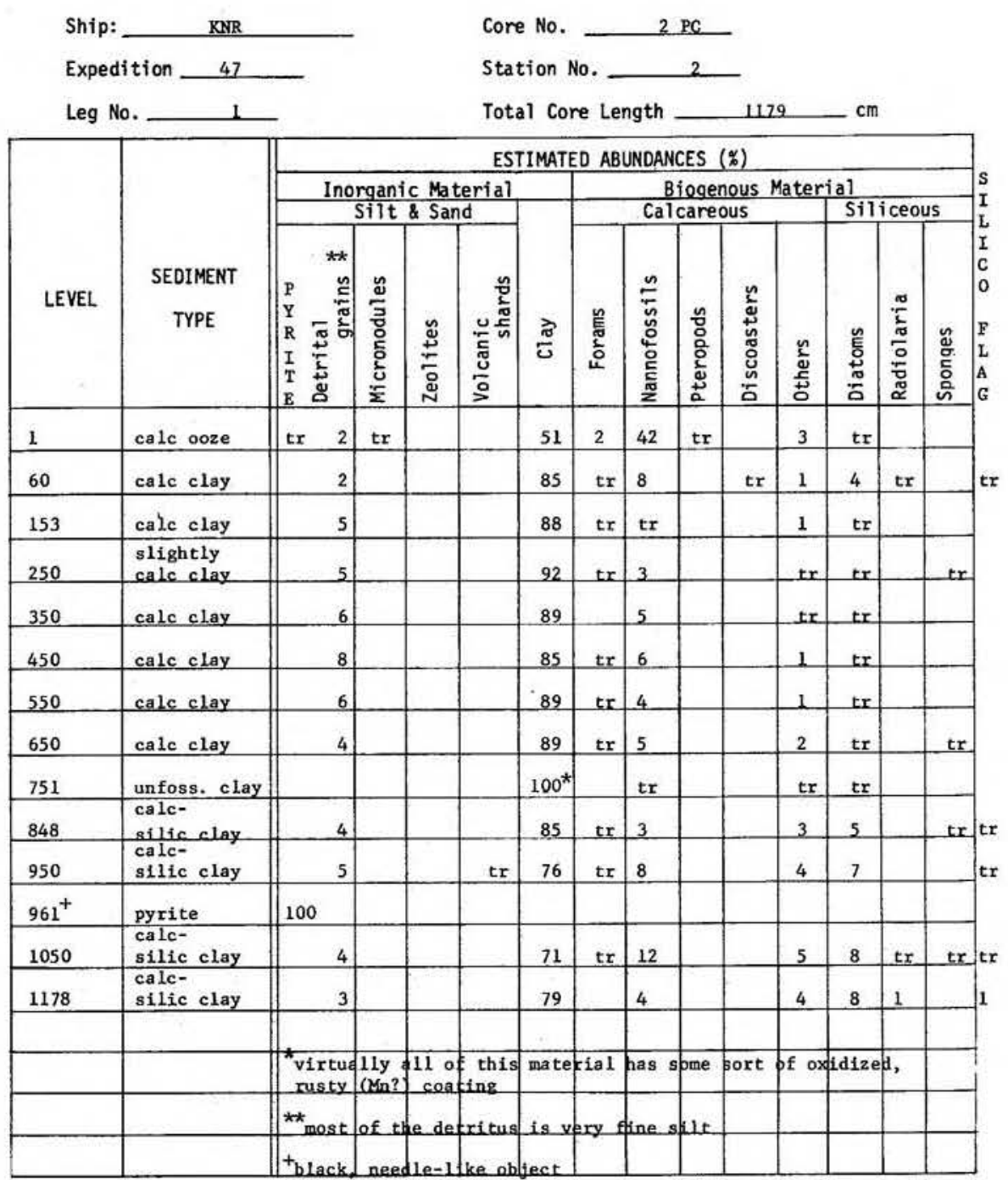


703

VISUAL CORE DESCRIPTION

Page 1 of 1

Ship KMORR Cruise 47 Leg 1 Sto. 2 Core No. $2 \mathrm{PG}$ Total Length $37 \mathrm{~cm}$. Lat. $33^{\circ} 40.8^{\prime} \mathrm{N}$ Long. $57^{\circ} 40.3^{\prime}$ w Depth 4619 caee.m Core condition ExcensenT Date Described 27 Apeil 76 by I FARmer

Physiographic location BERMWDA RISE, AREA of ACOUTTC LAMINATTONS Lithologic Meispene $\left.\frac{\text { Log }}{\frac{1}{11}+\frac{1}{1}}\right|^{0-3}$

50 ind of core - 37

$\exists$

$75 \div$

$100-$

王

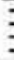

$\exists$

1

$\exists$

$\exists$

$\exists$

$\exists$
$0-30$

$10 \mathrm{YR} 5 / 3$

homogeneous, except for thin, dark grayish brown mottling from $19-21 \mathrm{~cm}$
slick lutite; a few forams are scattered throughout 30-37

casc 0038

CALC OOZE
$10 \mathrm{YR} 6 / 3$ pale brown

two large, irregular patches of brown sediment are mixed in the upper $5 \mathrm{~cm}$ of this unit

less slick, slightly silty lutite; some forams are present end of core

704

SMEAR SLIDE DESCRIPTIONS - W.H.O.I. SEDINENT CORES

Ship:

\section{Expeditt}

Leg No.
KXORR

47

-
Core No. 2

Station No. 2

Total Core Length $\quad 37 \quad \mathrm{~cm}$

ESTIMATED ABUNDANCES ( $(x)$

\begin{tabular}{|c|c|c|c|c|c|c|c|c|c|c|c|c|c|c|}
\hline \multirow[b]{4}{*}{ LEVEL } & \multirow[b]{4}{*}{$\begin{array}{c}\text { SEDIMENT } \\
\text { TYPE }\end{array}$} & \multicolumn{13}{|c|}{ ESTIMATED ABUNDANCES ( $(x)$} \\
\hline & & \multirow{2}{*}{\multicolumn{4}{|c|}{$\begin{array}{c}\text { Inorganic Material } \\
\text { Silt \& Sand }\end{array}$}} & & \multirow{2}{*}{\multicolumn{5}{|c|}{$\begin{array}{l}\text { Biogenous Mater } \\
\text { Calcareous }\end{array}$}} & \multirow{2}{*}{\multicolumn{3}{|c|}{$\frac{\text { al }}{\text { Siliceous }}$}} \\
\hline & & & & & & & & & & & & & & \\
\hline & & 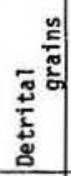 & 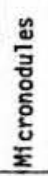 & 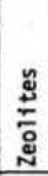 & 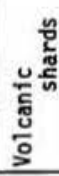 & ढ़ & 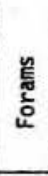 & 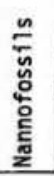 & 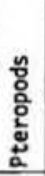 & 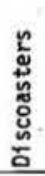 & 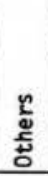 & 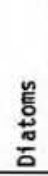 & $\frac{\pi}{\frac{\pi}{2}}$ & 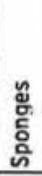 \\
\hline 1 & calc ooze & $t x$ & tr & & & 33 & 1 & 65 & & & 1 & tr & & $t x$ \\
\hline 36 & calc ooze & tr & $\mathrm{tr}$ & & & 49 & tr & 50 & & & 1 & tr & & tr \\
\hline & i & & & & & & & & & & & & & \\
\hline . & & & & & & & & & & & & & & \\
\hline & & & & & & & & & & & & & & \\
\hline & & & & & & & & & & & & & & \\
\hline & & & & & & & & & & & & & & \\
\hline & & & & & & & & & & & & & & \\
\hline & & & & & & & & & & & & & & \\
\hline & & & & & & & & & & & & & & \\
\hline & & & & & & & & & & & & & & \\
\hline & & & & & & & & & & & & & & \\
\hline & & & & & & & & & & & & & & \\
\hline & & & & & & & & & & & & & & \\
\hline & & & & & & & & & & & & & & \\
\hline & & & & & & & & & & & & & & \\
\hline & & & & & & & & & & & & & & \\
\hline & & & & & & & & & & & & & & \\
\hline
\end{tabular}


Ship KNoRR Cruise 47 Leg I Sta. 4 Core No. 4-PC Total Length $776 \mathrm{~cm}$. Lat. $33^{\circ} 54^{\circ} 8^{\prime} \mathrm{N}$ Long. $57^{\circ} 23.3^{\prime}$ WDepth 4792 core. $\mathrm{m}$ Core condition EXCELLENT Dote Described 4 Juve 76 by H. Former Physiographic location Bermuda Rise

Lithologic

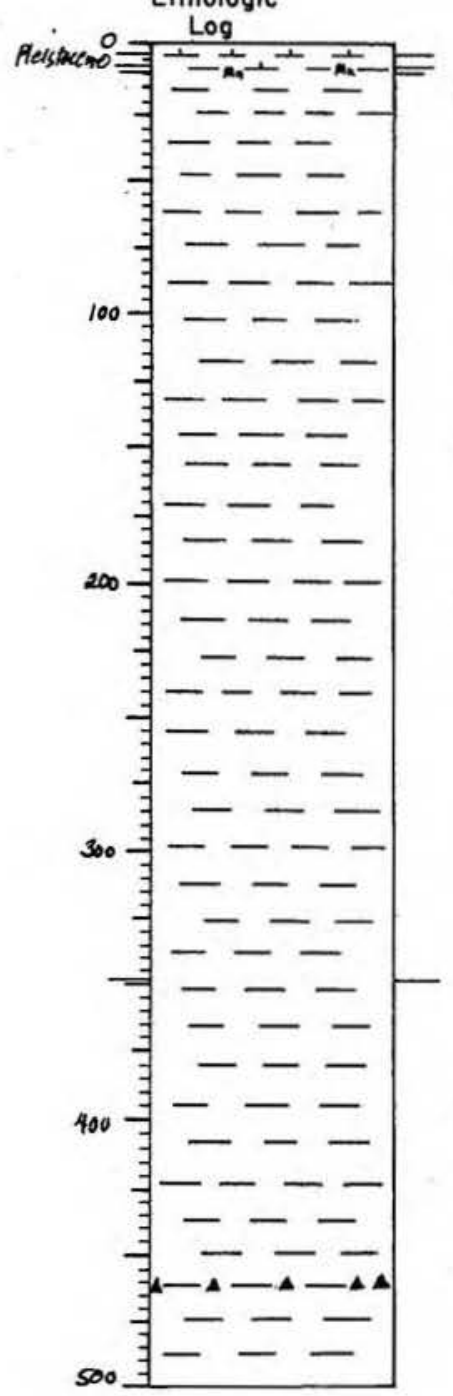

\section{Detailed Description}

$0-3$

\section{CALC OOZE}

$10 \mathrm{YR} 5 / 3$ brown

no mottling

consolidated, slightly silty lutite, but distorted

3-6

CAIC OOZE

0 YR $6 / 4$ light yellowish brown

relatively

arively unconsolidated, silty lutite, forams ery Sharp, slightly distorted

6-6.5

10 YR $5 / 4$ yellowish brown

no mottling

silck lutite, very few forams

very Sharp, but disturbed

NFOSSILIFEROUS CLAY

$\mathrm{Y} 6 / 2$ and $6 / 3$ light olive gray and olive gray

umerous compacted, elongate mottles (and possibly

ish gray and dartions?) of $5 \mathrm{GY} 5 / 1$ and $4 / 1$ green-

zones of yellow greenish gray; also, a few $\operatorname{sma}$

$13 \mathrm{~cm}$ ); and a few very small, black mottles (Mn rich) are present in this zone

generally a very slick lutite except the yellowish zones which are semi-1ithified, lumpy, and not so

OTE: The above $14 \mathrm{~cm}$ appear to be a compressed representation of as much as the top 2 meters of sedidination of the probably is a result or prey have penetrated up to 2 meters before the piston moved and nomal sampling began. However, the core cutter $(\approx 16 \mathrm{~cm})$ seems to have sampled a portion(?) of 14-348

UNFOSSILIFEROUS CIAY

10 YR $6 / 2$ light olive gray

several large, irregularly shaped mottles of $5 \mathrm{GY} 4 / 1$ dark greenish gray are present to $50 \mathrm{~cm}$; these generally show both sharp and smeared out gradational contacts with the primary color; below this large
VISUAL CORE DESCRIPTION

Page 2 of 2 Ship KNORR Cruise 47 Leg I Sto. 4 Core No. 4-PC

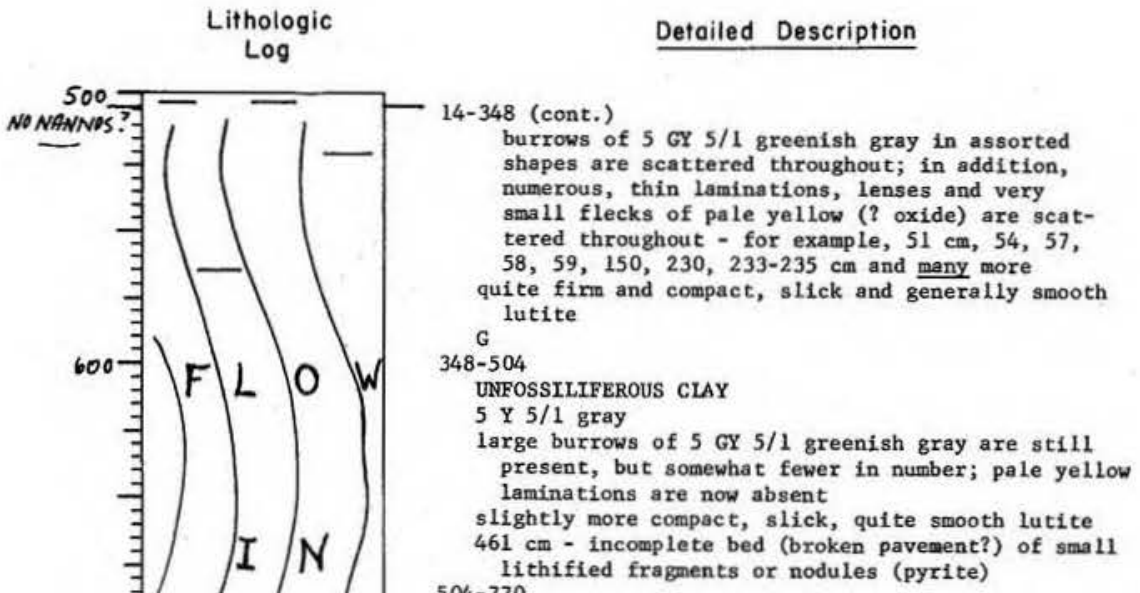
$504-770$

NFOSSILIFERous CIAY

700

5 Y $5 / 1$ gray

low in of above unit

end of core 
SMEAR SLIDE DESCRIPTIONS - W.H.O.I. SEDINENT CORES

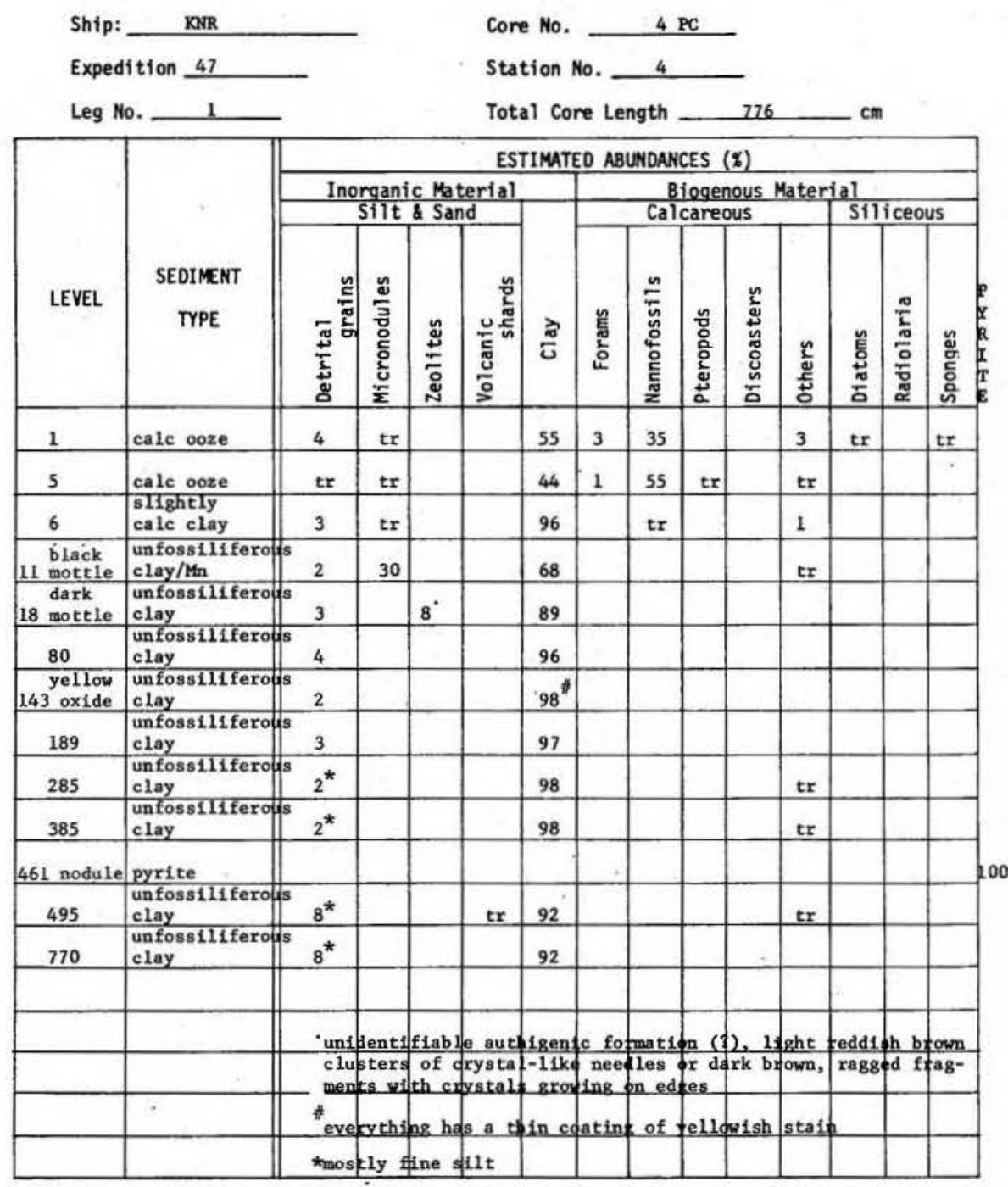

Ship KNORR Cruise 47 Leg I sta. 4 Core No. 4 - PG Total Length $62 \mathrm{~cm}$. Lat. $33^{\circ} 54.8^{\circ} \mathrm{N}$ Long. $57^{\circ} 23.3^{\prime}$ w Depth 4792 cesem Core condition ExCELLENT Date Described 4 Iues 76 by $H$. Farmer Physiographic location Bermud 2 Rise Lithologic

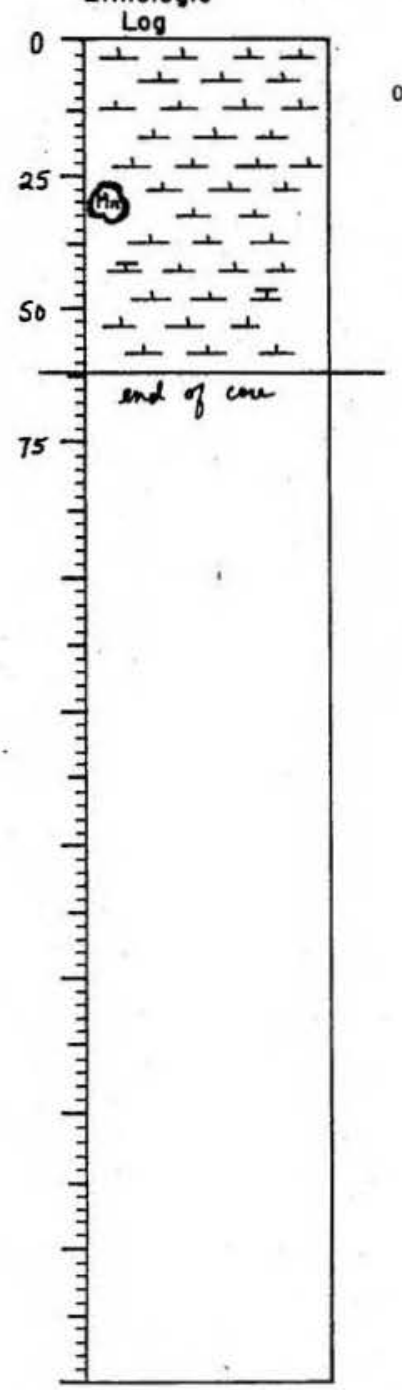

Detailed Description

$0-62$ CALC OOZE $40-50 \mathrm{~cm}$ is slightly darker brown with faint mottles or brown

ty to silty lutite; forams are generally comon, except abundant in numerous pockets and from $33-53 \mathrm{~cm}$

列 $27 \mathrm{~cm}$ end of core 
SIEAR SLIDE DESCRIPTIONS - W.H.O.I. SEDIMENT CORES

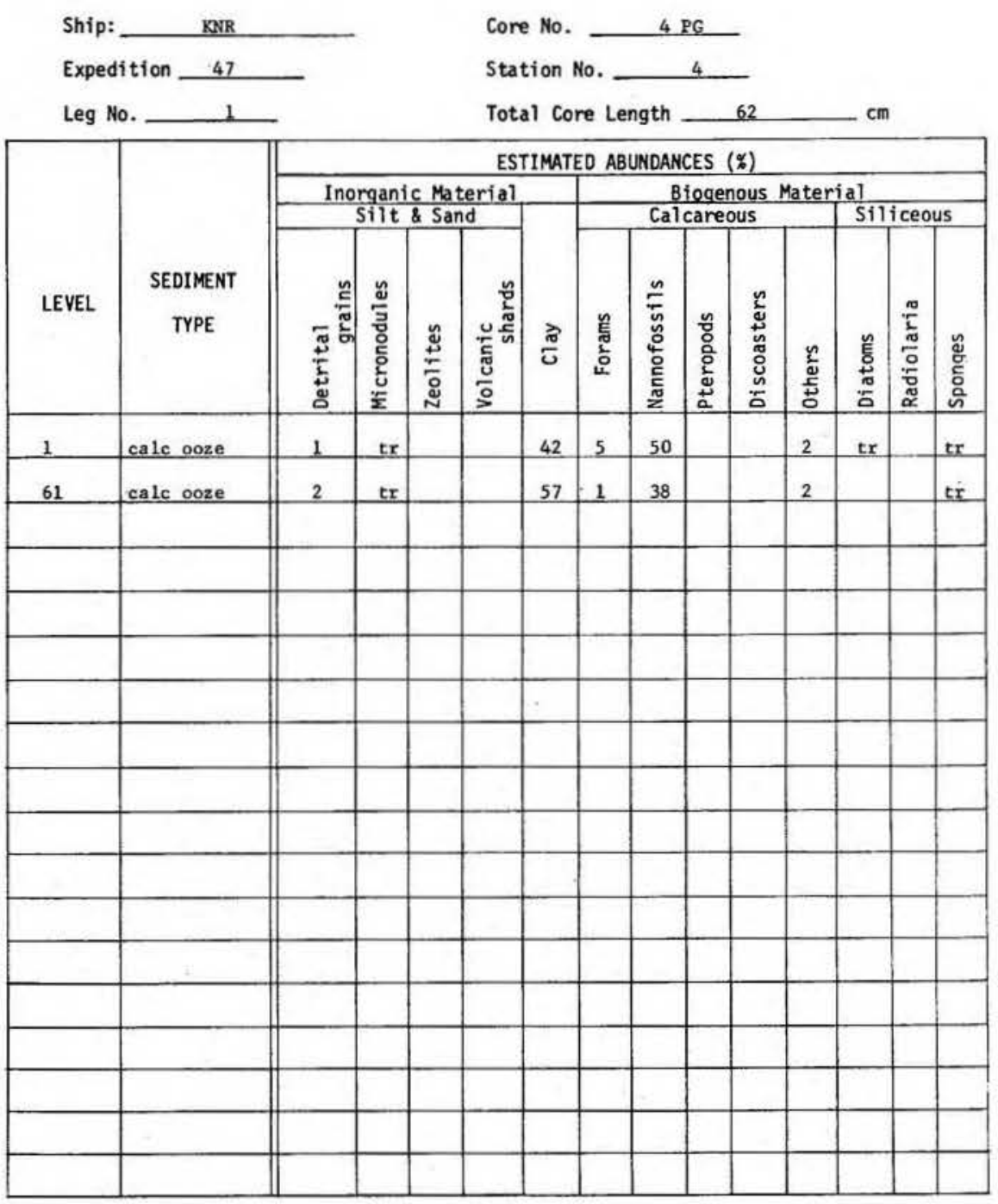

Ship KNORR Cruise 47 Leg I Sta. 5 Core No. 5-PC Total Length $1173 \mathrm{~cm}$. Lat. $33^{\circ} 30.6^{\prime} \mathrm{N}$ Long. $57^{\circ} 49.4^{\prime}$ 'W Depth $46 / 8 \mathrm{corr} . \mathrm{m}$ Core condition EXCELLENT Date Described 30 Apil 76 by H. Farmer Core condition EXCELENT Date

Physiographic location BERMUDA RISE
Lithologic

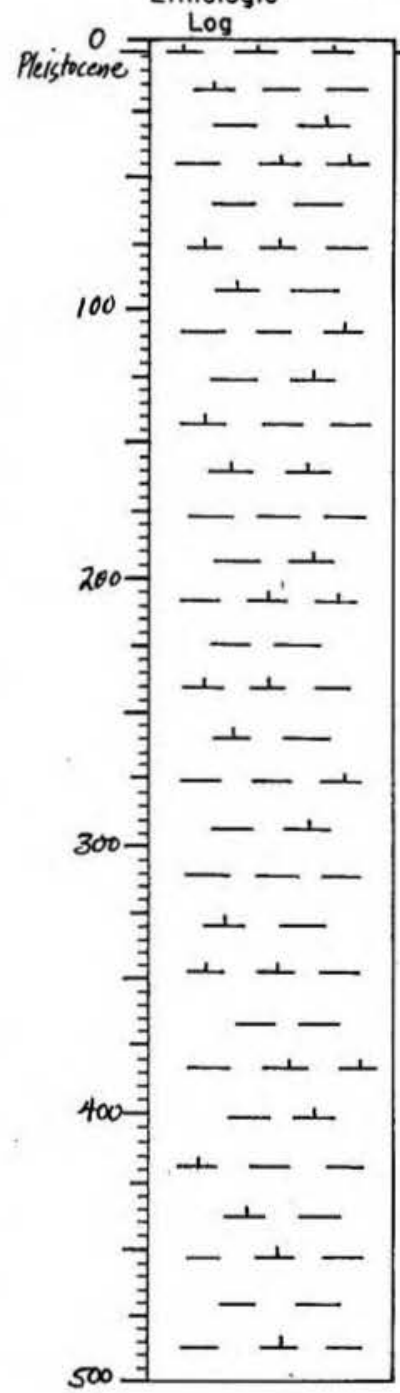

CALC OOZE

no mottling, but some color variations appear as disturbed laminations

lick, smooth, slightly silty lutite

3-1173

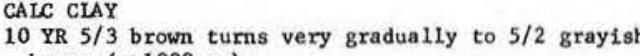
mall, very dark $\mathrm{g}$

Fish brown mottles are clustered 列 $13-25,31-39,59-64,84-93$, and 650-780; are: Mottle-free areas are 200-610 zones, such as: $19-26,114-130$ denotes subtle 18-327, 335-343, 393-404, 454-462, 483-496, 875892 , and $897-899$ on

silck lutite; generally the sedisomevhat firmer towards the bottom of the core

conted, also appear as incomplete bedding features that extend horizontally across part of the core; two black, horizontal, tube-like objects were observed at $859 \mathrm{~cm}$ and $1044 \mathrm{~cm}$ (pyritized worm burrows)

NOIE: Initial shipboard descriptions indicate that numerous black, often semi-lithified, hydrotroilite laminations were present from $200-810 \mathrm{~cm}$. Many of and Iaminations noted above

end of core $S$, mildly disturbed ment below $200 \mathrm{~cm}$ appears more uniform and becomes 
711

VISUAL CORE DESCRIPTION

Ship KNERR Cruise 47 Leg 1 Sta. 5 Core No. 5 PC

$$
\begin{gathered}
\text { Lithologic } \\
\text { Log }
\end{gathered}
$$

Detailed Description
Page_2 of 2

712

Page 1 of 2

SMEAR SLIDE DESCRIPTIONS - H.H.O.I. SEDIVENT CORES

\begin{tabular}{|c|c|c|c|c|c|c|c|c|c|c|c|c|c|c|c|}
\hline Ship: & $\mathrm{K}$ & & & & Core & No. & & 5 & & & & & & & \\
\hline Expe & ition & & & & Stat & ion 1 & 10. & - & 5 & & & & & & \\
\hline Leg & o. & & & & Tota & $17 \mathrm{Cor}$ & e Ler & ngth & 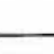 & 1173 & 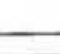 & $-\mathrm{cm}$ & & & \\
\hline & & & & & EST & IMATE & $D$ AB: & UNDAN & CES & & & & & & \\
\hline & & Inor & rgani & ic Mat & erial & & & & ieger & lous 1 & lateri & al & & & I \\
\hline & & & Silt & 2 San & & & & $\mathrm{Cal}$ & care & us & & Sil & iceo & & $\mathrm{L}$ \\
\hline LEVEL & $\begin{array}{c}\text { SEDIMENT } \\
\text { TYPE }\end{array}$ & 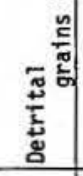 & 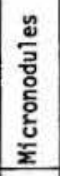 & 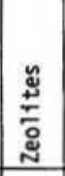 & 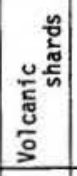 & $\frac{\pi}{0}$ & 呂 & 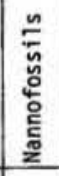 & 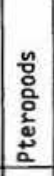 & 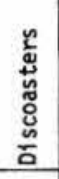 & 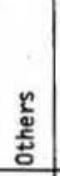 & 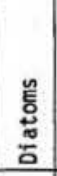 & $\frac{\pi}{\frac{\pi}{2}}$ & 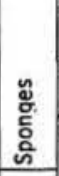 & $\begin{array}{l}\mathrm{F} \\
\mathrm{L} \\
\mathrm{A} \\
\mathrm{G}\end{array}$ \\
\hline 1 & calc ooze & tr & & & & 56 & 3 & 40 & tr & & 1 & & & & \\
\hline $22^{\circ}$ & calc clay & 7 & & & & 82 & tr & 8 & & & 3 & tr & & & \\
\hline 37 & $\begin{array}{l}\text { highly } \\
\text { calc clay }\end{array}$ & 4 & & & & 71 & 1 & 20 & & & 3 & 1 & & & \\
\hline $125^{\circ}$ & calc clay & 3 & & & & 89 & $\mathrm{tr}$ & 5 & & & 3 & $t_{r}$ & & & \\
\hline $\begin{array}{l}154 \\
\text { mottle }\end{array}$ & calc clay & 7 & $2^{*}$ & & & 81 & $t r$ & 4 & & & 6 & $t x$ & & & \\
\hline $\begin{array}{l}227 \\
\text { lutite 1 }\end{array}$ & $\begin{array}{l}\text { unfoss. } \\
\text { ap clay }\end{array}$ & 5 & & & & $95^{*}$ & & & & & $t r$ & tr & & & \\
\hline 250 & calc clay & 9 & tr & & & 86 & $\mathrm{tr}$ & 3 & & & 2 & $t x$ & & & \\
\hline 350 & calc clay & 2 & & & & 92 & $-5 x$ & 3 & & & 3 & $t x$ & & tr & \\
\hline 450 & calc clay & 3 & & & & 89 & $t r$ & 5 & & & 3 & $t x$ & & $t x$ & \\
\hline $458^{\circ}$ & calc clay & 7 & & & & 85 & tr & 4 & & & 4 & tx & & tr & \\
\hline 550 & calc clay & 1 & & & & 90 & tr & 5 & & & 4 & tr & & & \\
\hline 650 & $\begin{array}{l}\text { slightly } \\
\text { calc clay }\end{array}$ & 6 & & & & 91 & $t x$ & 2 & & & 1 & $\mathrm{tr}$ & & & \\
\hline (cont.) & & & & & & & & & & & & & & & \\
\hline 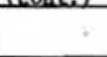 & & ${ }^{8}$ this & mat & rial & is pro & imari & & pe & & lze & & & & & \\
\hline & & redg & ish, & ones & & & & & & & & & & & \\
\hline 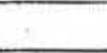 & & * rust $^{\circ}$ & $y($ OH & 2) $\mathrm{co}$ & ating & on $v$ & riet & of & bbje & ts & & & & & \\
\hline & & ${ }^{* * *}$ the & $n$ & (?) & oating & 3 cla & & & & & & & & & \\
\hline & & & & & & & & & & & & & & & \\
\hline
\end{tabular}


SMEAR SLIDE DESCRIPTIONS - W.H.O.I. SEDIMENT CORES

Ship:

ition 47

Leg №. 1
Core No. SPC

Station No. 5

Total Core Length 1173

ESTIMATED ABUNDANCES (\%)

\begin{tabular}{|c|c|c|c|c|c|c|c|c|c|c|c|c|c|c|}
\hline \multirow[b]{3}{*}{ LEVEL } & \multirow[b]{3}{*}{$\begin{array}{c}\text { SEDIMENT } \\
\text { TYPE }\end{array}$} & \multicolumn{13}{|c|}{ ESTIMATED ABUNDANCES ( $(\%)$} \\
\hline & & \multicolumn{4}{|c|}{$\begin{array}{c}\text { Inorganic Material } \\
\text { Silt \& Sand }\end{array}$} & & \multicolumn{5}{|c|}{$\begin{array}{l}\text { Biogenous Mate } \\
\text { Calcareous }\end{array}$} & \multicolumn{3}{|c|}{$\frac{\text { al }}{\text { Silíceous }}$} \\
\hline & & 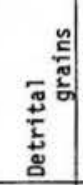 & 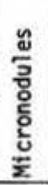 & $\frac{ఖ}{\frac{\pi}{0}}$ & $\frac{0}{\circ}$ & $\stackrel{\pi}{4}$ & 薦 & 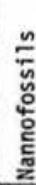 & 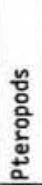 & 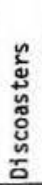 & 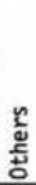 & $\begin{array}{l}\text { E } \\
\frac{0}{0} \\
\frac{0}{a}\end{array}$ & $\begin{array}{l}\frac{\pi}{2} \\
\frac{\pi}{0} \\
\frac{0}{0} \\
\frac{\pi}{\alpha}\end{array}$ & 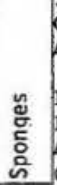 \\
\hline 750 & $\begin{array}{l}\text { slightly } \\
\text { calc clay }\end{array}$ & 6 & & & & 9 & tr & 2 & & & 1 & tr & & $\mathrm{tr}$ \\
\hline 850 & calc clay & 3 & & & & 8 & tr & 5 & & & 6 & tr & & $\therefore$ \\
\hline 950 & $\begin{array}{l}\text { calc- } \\
\text { silic clay }\end{array}$ & 1 & & & & 8 & tr & 4 & & & 4 & 7 & & \\
\hline 1050 & calc clay & $\mathrm{tr}$ & & & & 9 & tr & 4 & & & 4 & 2 & tr & \\
\hline 1172 & calc clay & $\mathrm{tr}$ & & & & 8 & tr & 8 & & & 2 & 1 & & \\
\hline & & & & & & & & & & & & & & \\
\hline & & & & & & & & & & & & & & \\
\hline & & & & & & & & & & & & & & \\
\hline & & & & & & & & & & & & & & \\
\hline & & & & & & & & & & & & & & \\
\hline & & & & & & & & & & & & & & \\
\hline & & & & & & & & & & & & & & \\
\hline & & & & & & & & & & & & & & \\
\hline & & & & & & & & & & & & & & \\
\hline & & & & & & & & & & & & & & \\
\hline & & & & & & & & & & & $=$ & & & \\
\hline & & & & & & & & & & & & & & \\
\hline & & & & & & & & & & & & & & \\
\hline
\end{tabular}

Ship KNORR Cruise 47 Leg 1 sta 5 Core No 5 PG Total Length $68 \mathrm{~cm}$. Lat. $33^{\circ} 30.6^{\circ} \mathrm{N}$ Long. $57^{\circ} 49.4^{\prime} \mathrm{W}$ Depth $46 / 8$ are m Core condition Exedleat Date Described 30 Agril'16 by H. FARmER Physiographic location BERMUSA RISE

Lithologic

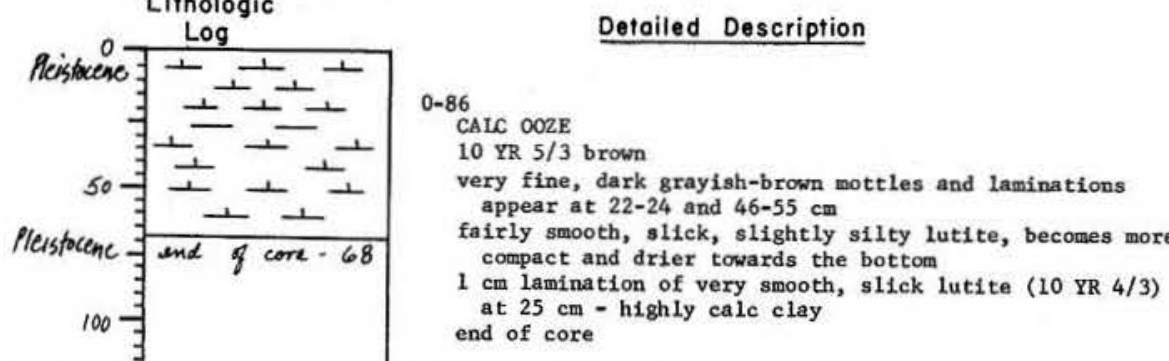


SMEAR SLIDE DESCRIPTIONS - W.H.O.I. SEDINENT CORES

Ship:

Expedition

Leg No.

\begin{tabular}{|c|c|c|c|c|c|c|c|c|c|c|c|c|c|c|}
\hline \multirow[b]{4}{*}{ LEVEL, } & \multirow[b]{4}{*}{$\begin{array}{c}\text { SEDINENT } \\
\text { TYPE }\end{array}$} & \multicolumn{13}{|c|}{ ESTIMATED ABUNDANCES ( $($ ) } \\
\hline & & \multirow{2}{*}{\multicolumn{4}{|c|}{$\begin{array}{c}\text { Inorganic Material } \\
\text { Silt \& Sand }\end{array}$}} & & \multirow{2}{*}{\multicolumn{5}{|c|}{$\begin{array}{l}\text { Biegenous Mater } \\
\text { Calcareous }\end{array}$}} & \multirow{2}{*}{\multicolumn{3}{|c|}{ Silliceous }} \\
\hline & & & & & & & & & & & & & & \\
\hline & & - & 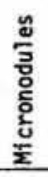 & 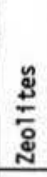 & 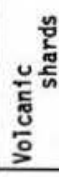 & ढे & 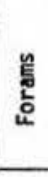 & 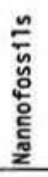 & 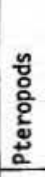 & 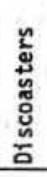 & 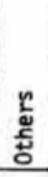 & $\begin{array}{l}\text { E. } \\
\stackrel{+}{0} \\
\bar{\sigma} \\
\end{array}$ & 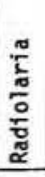 & 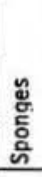 \\
\hline 1 & calc ooze & 1 & tr & & & 33 & tr & 65 & & & 1 & tr & & tr \\
\hline $25^{*}$ & $\begin{array}{l}\text { highly } \\
\text { calc clay }\end{array}$ & 1 & $\mathrm{tx}$ & & & 82 & tr & 15 & & & 2 & tr & & tix \\
\hline \multirow[t]{3}{*}{66} & calc ooze & $t x$ & tr & & & 25 & & 75 & & & $t x$ & $t x$ & & tr \\
\hline & & & & & & & & & & & & & & \\
\hline & & $*_{\text {fro }}$ & $s 1$ & ck 1 & ninat & on & & & & & & & & \\
\hline & & & & & & & & & & & & & & \\
\hline & & & & & & . & & & & & & & & \\
\hline & . & & & & & & & & & & & & & \\
\hline & & & & & & & & & & & & & & \\
\hline & & & & & & & & & & & & & & \\
\hline & & & & & & & & & & & & & & \\
\hline & & & & & & & & & & & & & & \\
\hline & & & & & & & & & & & & & & \\
\hline & & & & & & & & & & & & & & \\
\hline & & & & & & & & & & & & & & \\
\hline & & & & & & & & & & & & & & \\
\hline & & & & & & & & & & & & & & \\
\hline & & & & & & & & & & & & & & \\
\hline
\end{tabular}

Core No. 5 PG

Station No. 5

Total Core Length $\quad 68$
Ship KNORR Cruise 47

Leg 1 sta. 33 Core No. $6 G \mathrm{C}$ Total Length $160 \mathrm{~cm}$. Lat. $34 \% 10.28^{\circ} \mathrm{N}$ Long. $57^{\circ 11.51 \%} \mathrm{~W}$ Depth $5500 \mathrm{~m} . \mathrm{com}$

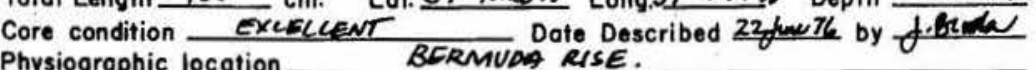

Physiographic location

Lithologic

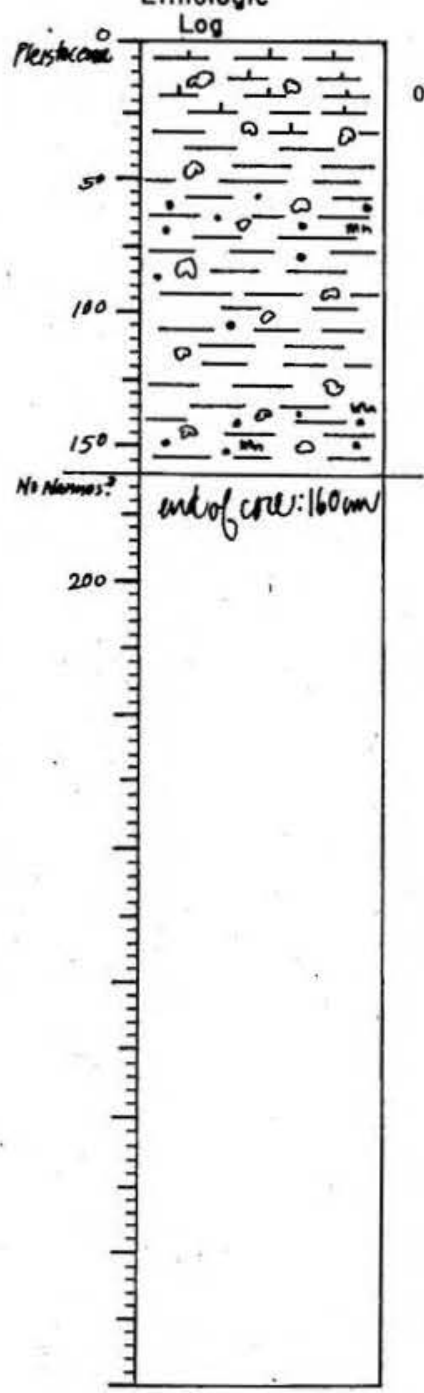

Detailed Description

CALC OOZE GRADES TO UNFOSSILIFEROUS CLAY WITH Mn MICRONODULES

7.5 YR $5 / 4$ brown grades to 10 YR $6 / 4$ light yellowish brown conmon pale brown mottling, burrowing and marbiling

tered forams from there to base of core

end of core 
SMEAR SLIDE DESCRIPTIONS - W.H.O.I. SEDIMENT CORES

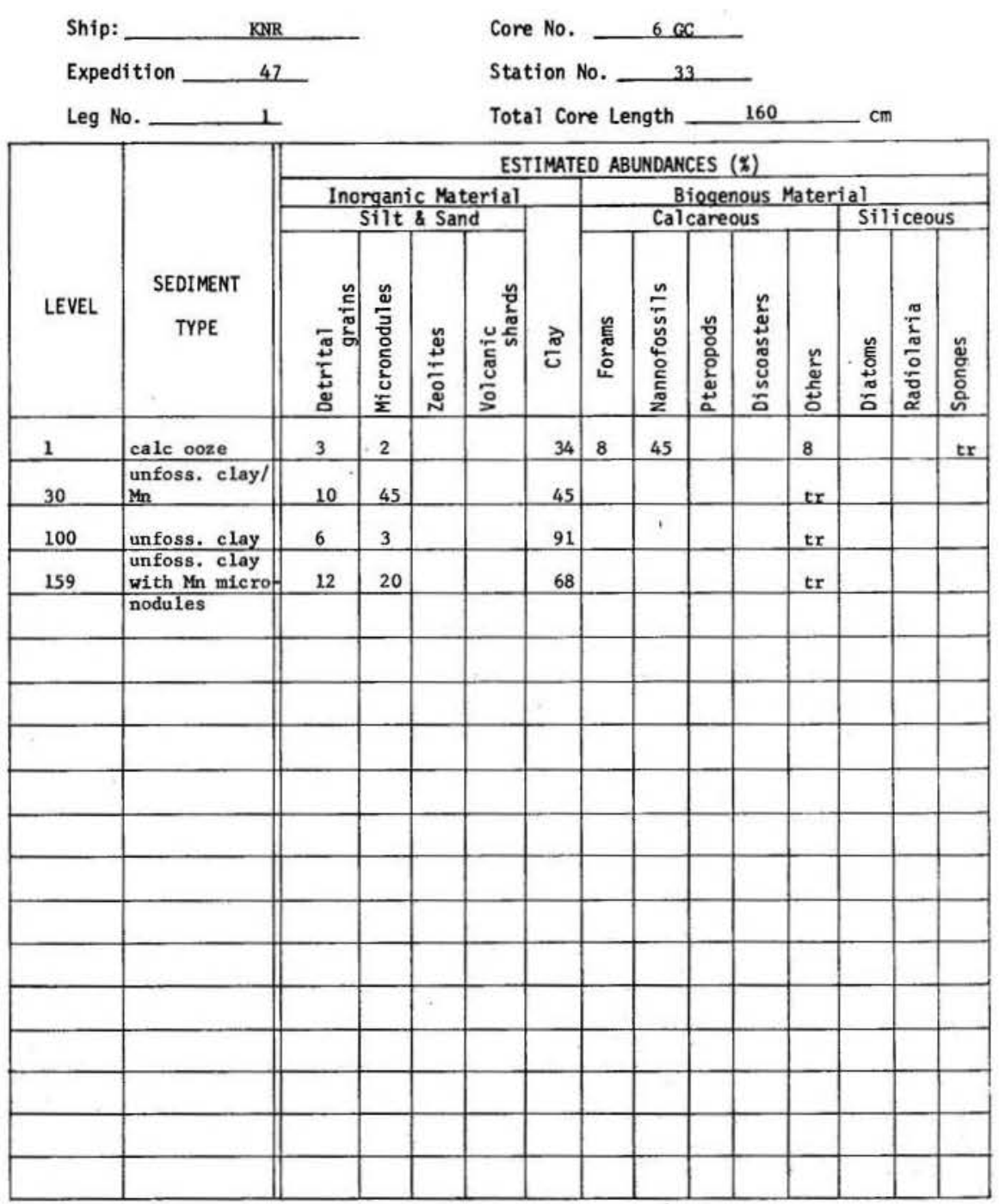




\section{8}

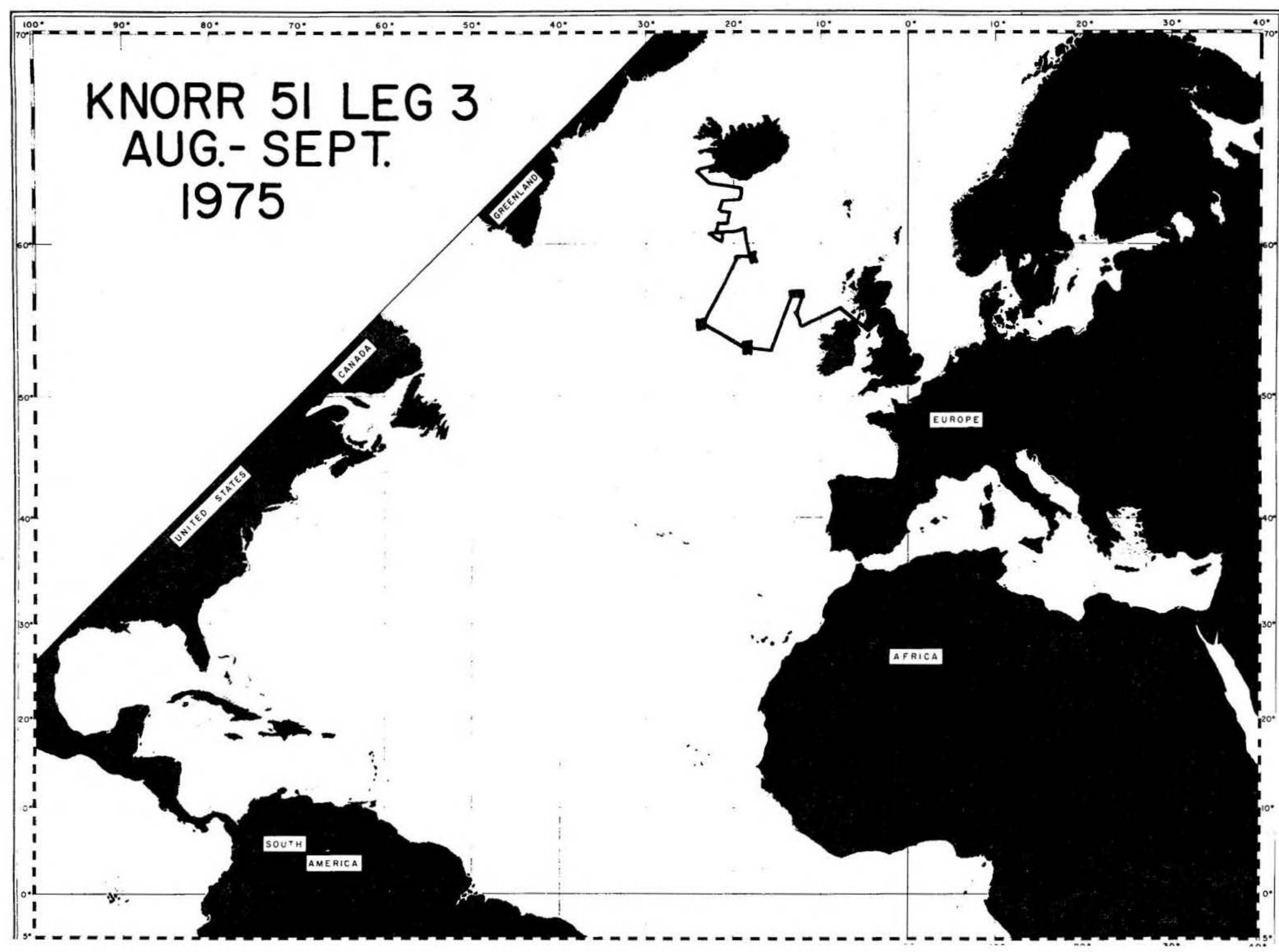


1

1

1

I

I

1

1

1

1

1

1

1

1

1

1

1

1

1

I 
SAMPLF. DE- DATE

MARS. CQRE QR

CORE

LENGTH DREDGE PHYSTO ROCK

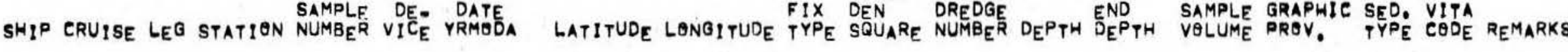

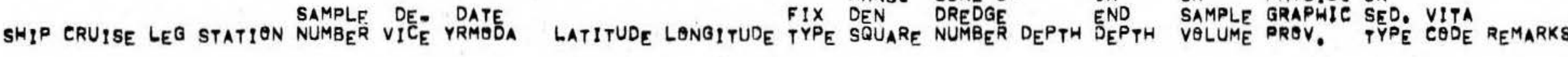
$\begin{array}{ll}\text { OR } & \text { OR } \\ \text { END } & \text { SAMPL }\end{array}$

SAMPLE GRAPHIE SEO. VITA

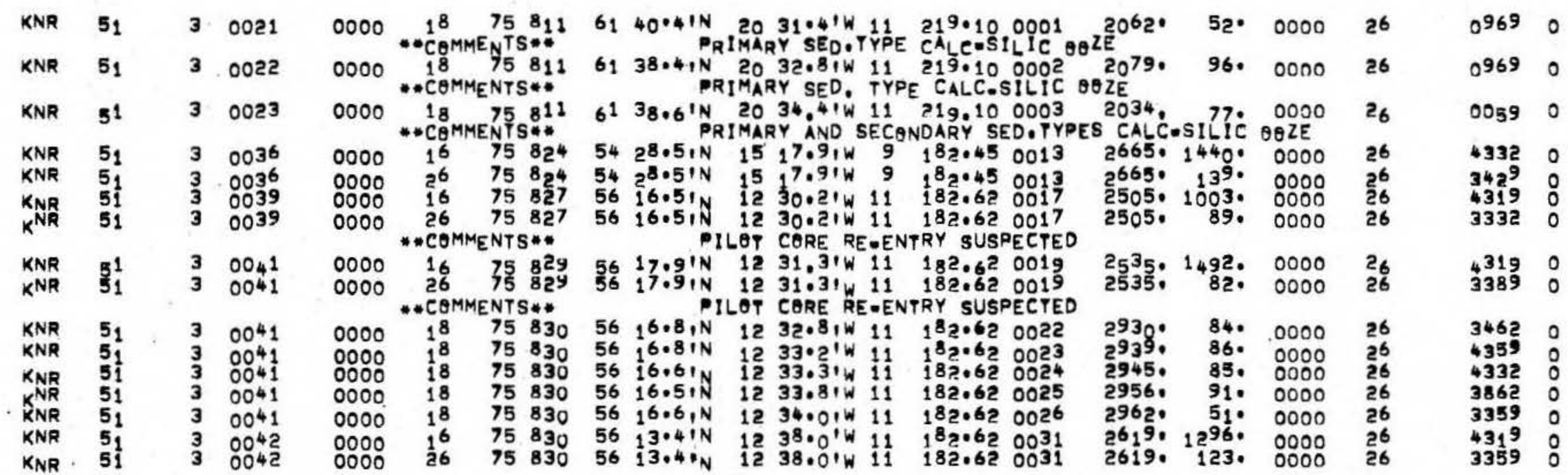


1

1

I.

f.

1

I

1

I

1

1

1

1

1

1

1

1

I

1 
720

VISUAL CORE DESCRIPTION

Page 1 of 1

Ship KNR Cruise 51 Leg III Sto. 21 Core No. IFF Total Length $\mathrm{SZ}_{2} \mathrm{~cm}$. Lat. $11^{\circ} 40^{\circ} 4^{\circ} \mathrm{N}$ Long. $20^{\circ} 31.4 \mathrm{~W}$ Depth $2062 \mathrm{m.corr}$ Core condition EXCECLENT Dote Described T.BRoDef by 4 JWE 76

Physiographic location MNRY CHANNEL MEAWOER.

Lithologic

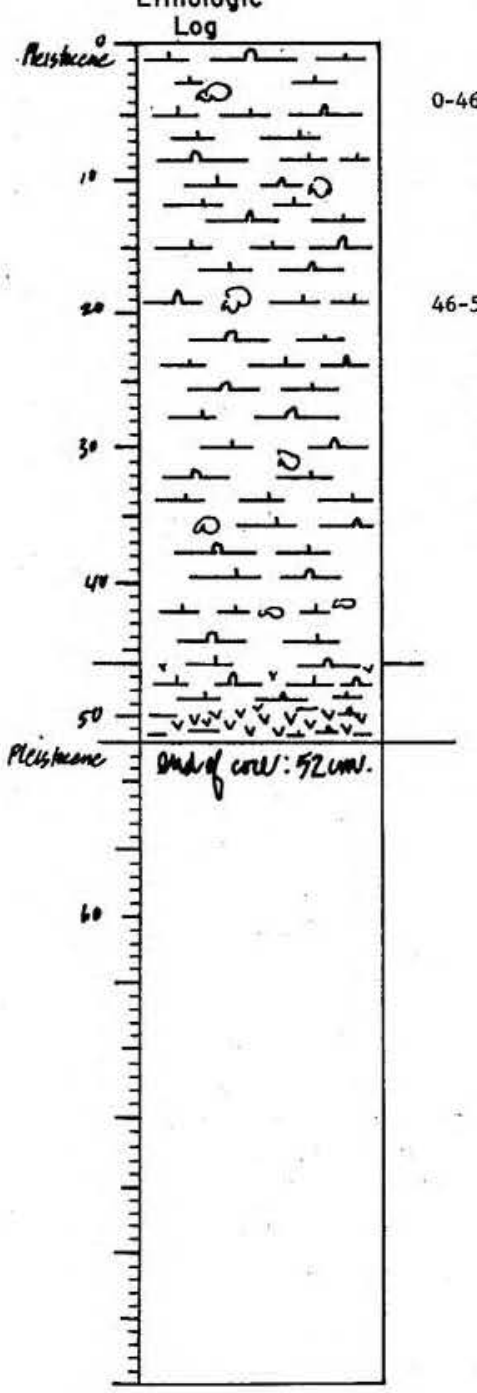

\section{Detailed Description}

CALC SILIC OOZE

dark grayish brown

few, faint, grayish brom mottles and burrows

and white calc fragments

$S$, irregular

CALC SILIC OOZB WITH ASH AND A ZONE OF HIGHLY CALC SILIC CIAY/ASH

inclusion (see below)

aped, found at

$50-51.5 \mathrm{~cm}$

end of core mist, very silty lutite with a few scattered foram
721

SMEAR SLIDE DESCRIPTIONS - W.H.O.I. SEDIMENT CORES

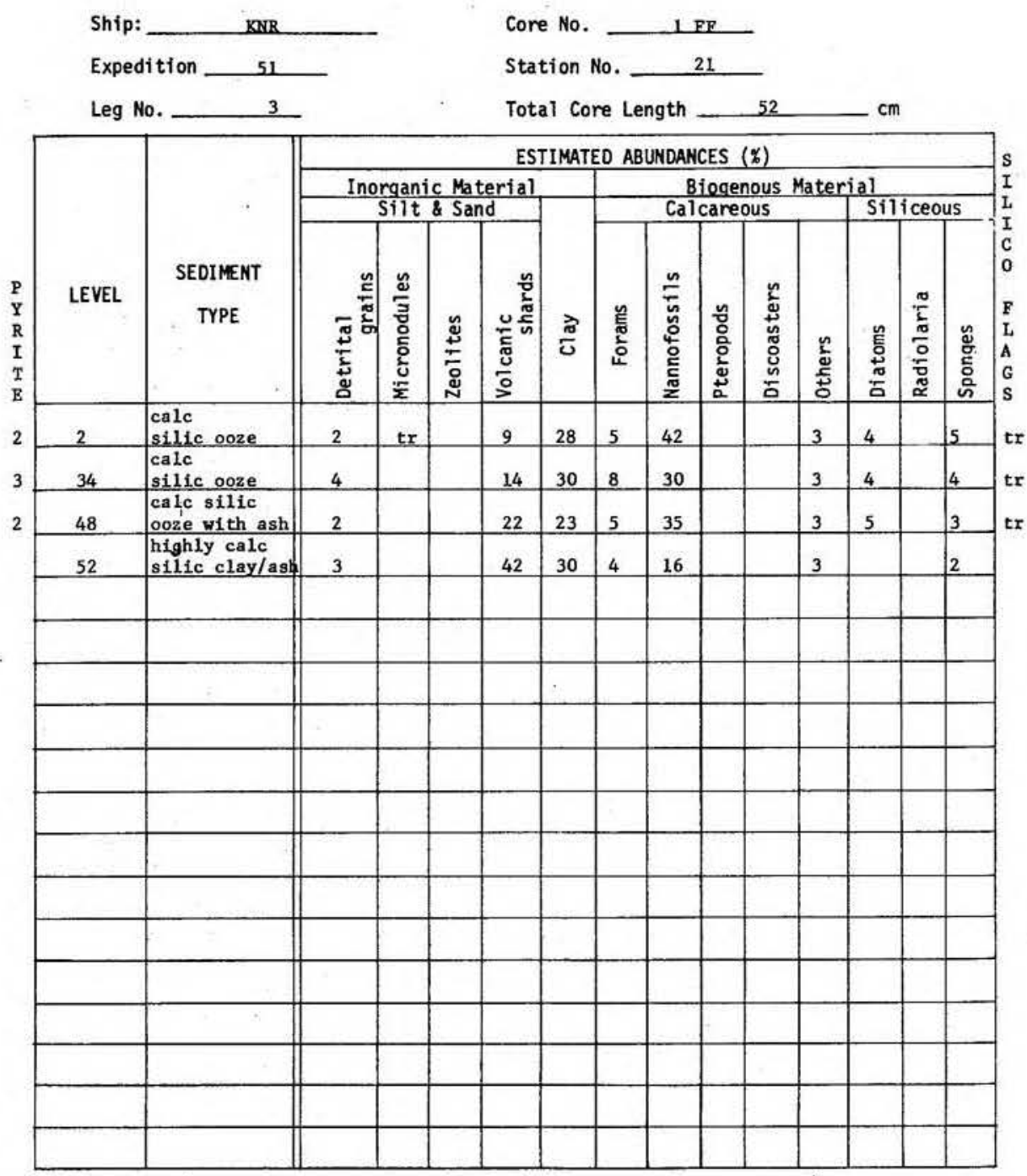




\section{VISUAL CORE DESCRIPTION}

Page 1 of 1

Ship KMIRR Cruise 51 Leg III Sta. 22 Core No. ZFF Total Length $96 \mathrm{~cm}$. Lat. $61^{\circ} 38.4 \mathrm{~N}$ Long. $20^{\circ} 32.8 \mathrm{~W}$ Depth 2079 atem

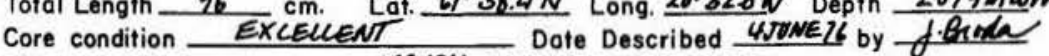
Physiographic location MAURY CHANNEL MEANDER Lithologic

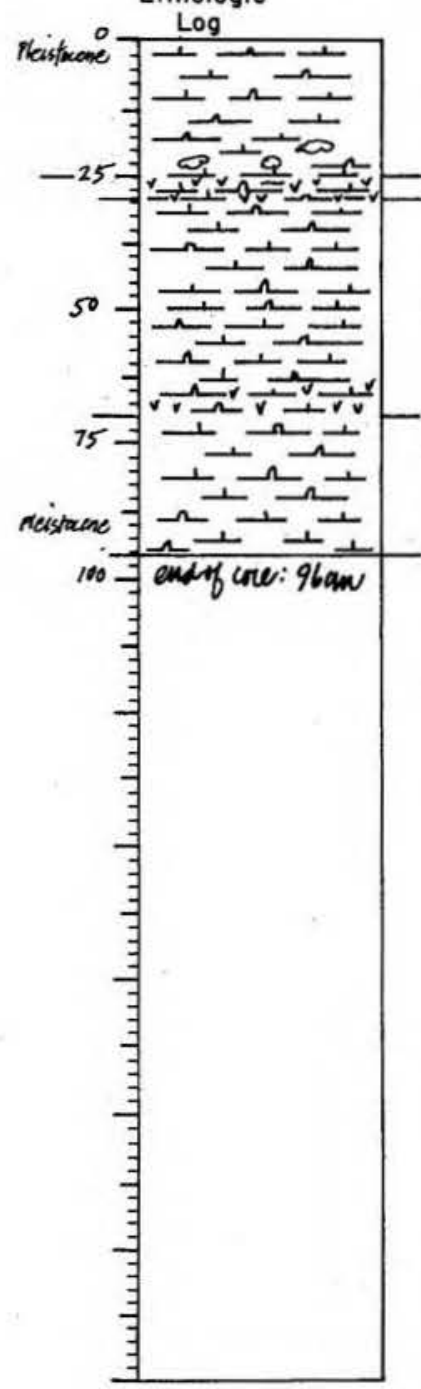

\section{Detailed Description}

$0-25$

CALC SILIC OOZE

few darker burrows and mottles found at unit basal contact

very moist, watery, silty lutite with very few foram slightly disturbed and washed $0-13 \mathrm{~cm}$ S, irregular

HIGHLY CALC SILIC CLAY/ASH

. 5 N2.5/ black

molst bed of nearly pure

very S, slightly disturbed

29-70

CALC SILIC OOZE GRADES TO CALC SILIC OOZE/ASH

2.5 Y $4 / 2$ dark grayish brown
a few, yellowish, siliceous laminations found at $32 \mathrm{~cm}$, (talnt),

color grades to $2.5 \mathrm{Y} 3 / 2$ very dark grayish brown

$0-96$

CALC SILIC OOZE

2.5 × $4 / 2$ dark graydent

oist, silty, slightly mulchy lutite

end of core several lightex brownish-gray laminations and zones

SMEAR SLIDE DESCRIPTIONS - W.H.O.I. SEDIMENT CORES

Ship:

Expedition

Leg No.

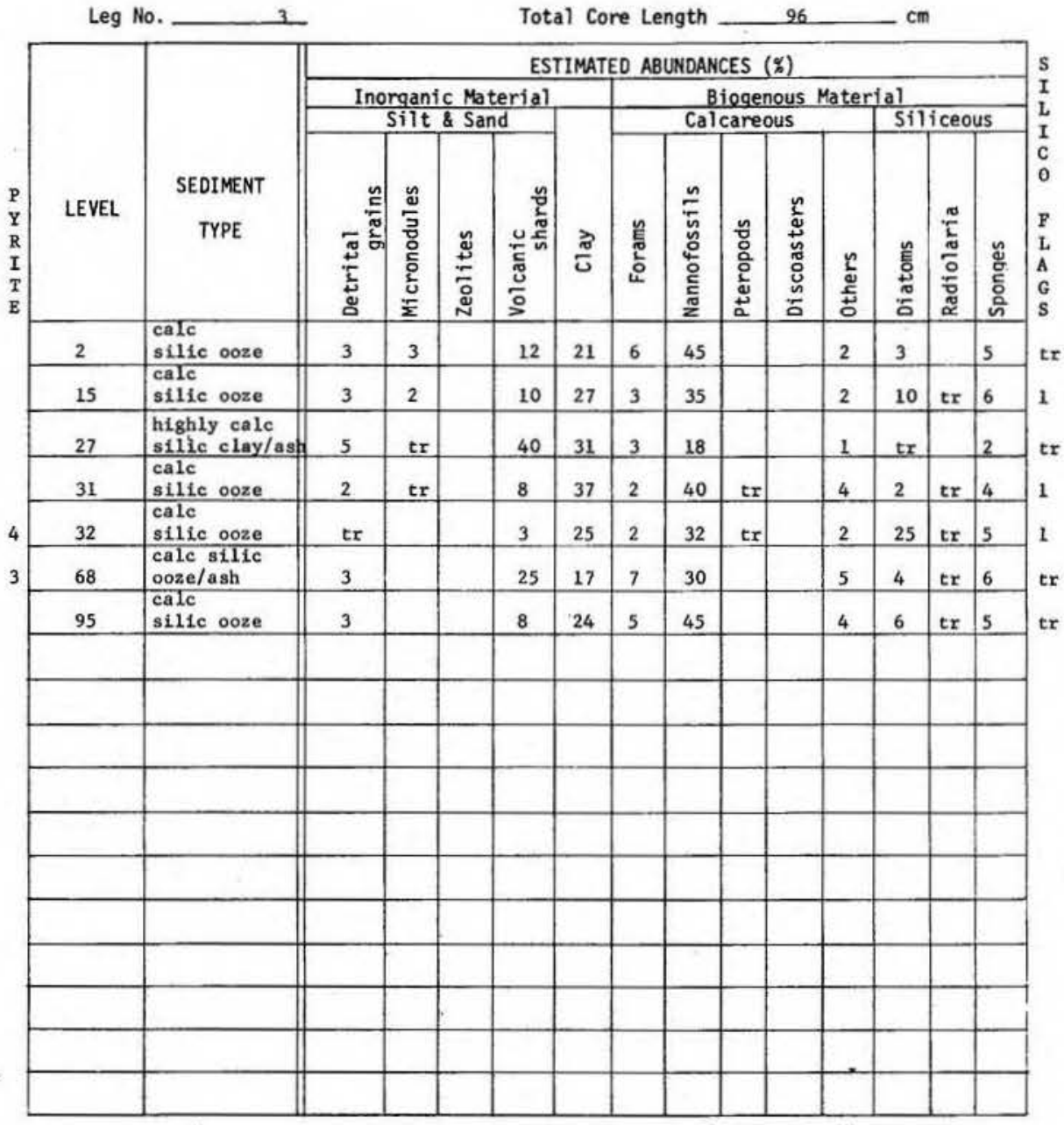

Core No. $2 \mathrm{FE}$

Station No. 21

Core Length 96

ESTIMATED ABUNDANCES (\%) 
724

VISUAL CORE DESCRIPTION

Page 1 of 1

Ship KNAR 51 TIL sto 23 3FF Total Length $77 \mathrm{~cm}$. Lat. $610^{\circ} 38.6 \% \mathrm{~N}$ Long. $20^{\circ} 34.4^{\circ} \mathrm{W}$ Depth $2034 \mathrm{mancm}$.

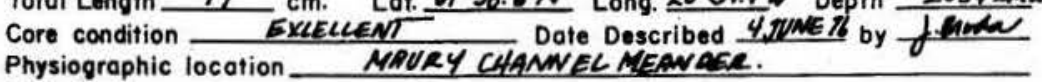

Lithologic

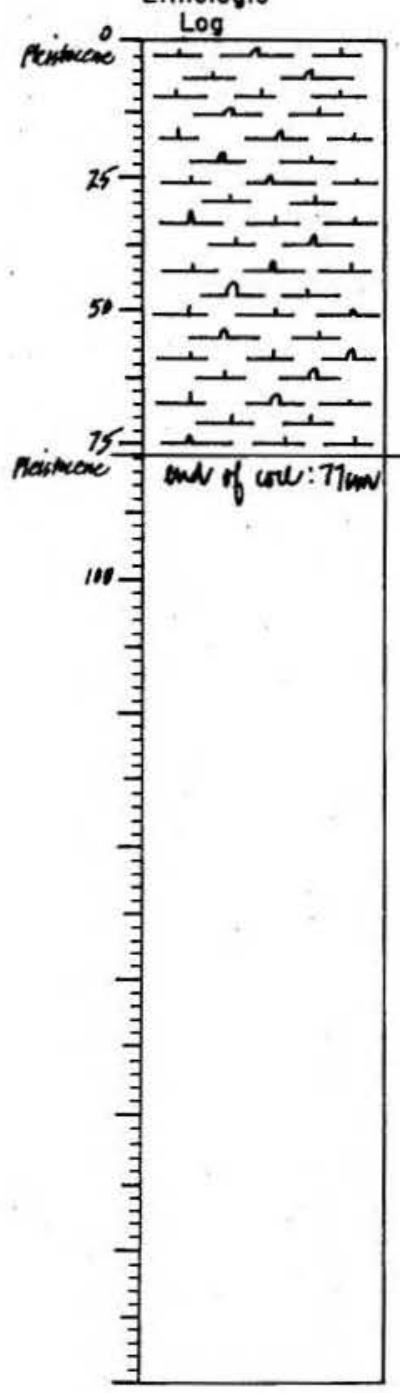

Detoiled Description

0-77

CALC SILIC OOZB

rayish broun and $5 / 2$ grayish brown

the colors above found throughout grada10-15 cm, $35-42 \mathrm{~cm}$

ilightly mulchy lutite throughout

end of core vo1d, top of liner, filled with foam - no sediment
SMEAR SLIDE DESCRIPTIONS - W.H.O.I. SEDIIENT CORES

\begin{tabular}{|c|c|}
\hline Ship: & Core No. $3 \mathrm{FI}$ \\
\hline Expedition & Station No. \\
\hline
\end{tabular}

\begin{tabular}{|c|c|c|c|c|c|c|c|c|c|c|c|c|c|c|}
\hline \multirow[b]{4}{*}{ LEVEL } & \multirow[b]{4}{*}{$\begin{array}{c}\text { SEDIMENT } \\
\text { TYPE }\end{array}$} & \multicolumn{13}{|c|}{ ESTIMATED ABUNDANCES (\%) } \\
\hline & & \multirow{2}{*}{\multicolumn{4}{|c|}{$\frac{\text { Inorganic Material }}{\text { Silt \& Sand }}$}} & & \multirow{2}{*}{\multicolumn{5}{|c|}{$\begin{array}{l}\text { Biogenous Mater } \\
\text { Calcareous }\end{array}$}} & \multirow{2}{*}{\multicolumn{3}{|c|}{ Stliceous }} \\
\hline & & & & & & & & & & & & & & \\
\hline & & 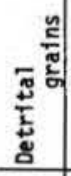 & $\begin{array}{l}\frac{n}{3} \\
\frac{0}{0} \\
\frac{5}{0} \\
\frac{\partial}{2}\end{array}$ & 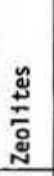 & 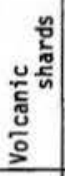 & $\stackrel{\frac{\pi}{6}}{\sigma}$ & 鵕 & 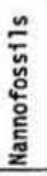 & 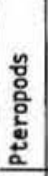 & 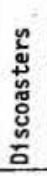 & 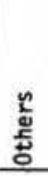 & 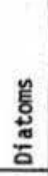 & 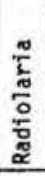 & 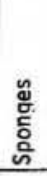 \\
\hline 1 & $\begin{array}{l}\text { calc } \\
\text { silic ooze }\end{array}$ & 3 & $\operatorname{tr}$ & & 6 & 47 & 3 & 32 & $\operatorname{tr}$ & & 4 & 3 & $\operatorname{tr}$ & 2 \\
\hline 17 & $\begin{array}{l}\text { calc } \\
\text { silic ooze }\end{array}$ & 2 & $\mathrm{tr}$ & & 5 & 42 & 6 & 35 & $t x$ & & tr & 3 & 1 & 6 \\
\hline 38 & $\begin{array}{l}\text { calc } \\
\text { silic ooze }\end{array}$ & 5 & & & 9 & 40 & 2 & 30 & & & 3 & 4 & tr & 7 \\
\hline 70 & $\begin{array}{l}\text { calc } \\
\text { silic ooze }\end{array}$ & 3 & $\operatorname{tr}$ & & 5 & 32 & 7 & 42 & & & tr & 3 & tr & 8 \\
\hline 77 & $\begin{array}{l}\text { calc } \\
\text { silic ooze }\end{array}$ & 2 & $\mathrm{tr}$ & & 5 & 39 & 4 & 40 & & & 2 & 2 & tr & 6 \\
\hline & & & & & & & & & & & & & & \\
\hline & & & & & & . & & & & & & & & \\
\hline 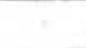 & & & & & & & & & & & & & & \\
\hline & & & & & & & & & & & & & & \\
\hline & & & & & & & & & & & & & & \\
\hline & & & & & & & & & & & & & & \\
\hline & & & & & & & & & & & & & & \\
\hline & & & & & & & & & & & & & & \\
\hline & & & & & & & & & & & & & & \\
\hline & & & & & & & & & & & & & & \\
\hline & 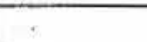 & & & & & & & & & & & & & \\
\hline & & & & & & & & 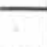 & & & & & & \\
\hline & & & & & & & & & & & & & & \\
\hline
\end{tabular}


Ship KNORR Cruise 51 Leg III Sta. 36 Core No. 13-GPC Total Length $1440 \mathrm{~cm}$. Lat. $54^{\circ} 28.5^{\prime} \mathrm{N}$ Long. $15^{\circ} / 7.9^{\prime} \mathrm{W}$ Depth 2665 care. $\mathrm{m}$ Core condition EXCEULENT Dote Described $7 J_{\text {une }} 76$ by H. Farmer Physiographic locotion FENI DRIFT - EAsTERV NoRTH ATLPWTIC Lithologic

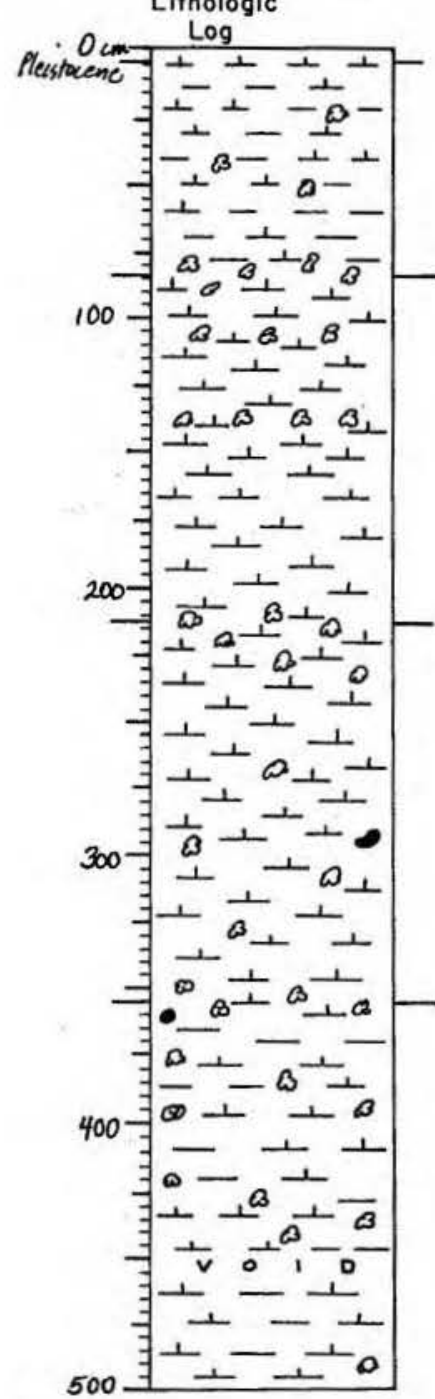

Detailed Description

$0-4$

CALC OOZE

2.5 Y $6 / 2$ 1ight brownish gray

peous throughout

falrly firm, very silty lutite; forams are comon

4-83

IGHLY CALC CLAY

$2.5 \times 5 / 2$ grayish brown

of various brownish grays and suble light and dark laminations appear

compact, very silty lutite, forams vary from scattered to quite common but are particularly abundant from very distorted lamination - appears along edge of core liner from $8-11 \mathrm{~cm}$

$\mathrm{S}$, mottled from $76-88 \mathrm{~cm}$

IIE: Upon inspection of the pilot core, the top and disturbed representation of approxinately the

83-213

CALC OOZE

grades from 10 YR $7 / 1$ or $7 / 2$ light gray to 2.5 Y $6 / 2$ and $5 / 2$ brownish grays; three cycles of the above sequence appear from $84-104 \mathrm{~cm}, 104-140 \mathrm{~cm}$ and 140 $213 \mathrm{~cm}$ with the paler colors being much more cormon mottling and marbling of the above colors are comon, piff, very silty lutite, with the darker areats

soreas being are particularly abundant in the light gray zones but are quite conmon throughout S13-35s , well mottled

CALC OOZE

YR $7 / 1$ light gray grades towards $8 / 1$ white (255$295 \mathrm{~cm}$ ) and then darkens to $7 / 1$ again

into the scattered terval compact, quite silty lutite except somewhat less
silty in the white zone; forams are abundant in nummerous patches throughout the unit

ery $\mathrm{G}$, mottled from $350-362 \mathrm{~cm}$ $17-18 \mathrm{~cm}, 24-29 \mathrm{~cm}$, and $75-78 \mathrm{~cm}$; a clast of silt f

VISUAL CORE DESCRIPTION

Page 2 of 3

Ship KNORR Cruise 51 Leg III Sta. 36 Core No. 13-GPC

Lithologic

Log

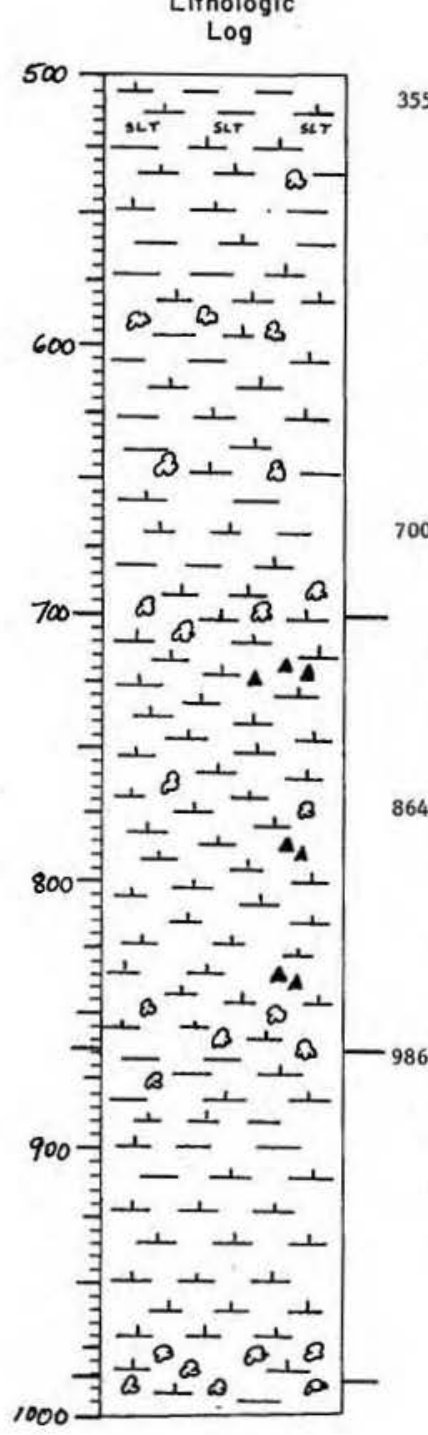

Detailed Description

$355-700$

HIGHLY CALC CLAY

predominant color is somewhat paler than $2.5 \times 5 / 2$ grayish brown except transitional zone with upper unit $(355-370 \mathrm{~cm}) 2.5$ Y $6 / 2$ light brownish gray

wide laminations of 2.5 Y $5 / 2$ grayish brown appear at $373-386 \mathrm{~cm}, 420-440 \mathrm{~cm}$, and $692-699 \mathrm{~cm}$; pale band of $6 / 2$ light brownish gray at $586-697 \mathrm{~cm}$; mottling
and marbling are present throughout but more common near above lrregularities

compact, silty lutite; forams increase from common to (detritus/calc ooze) oding up into silty le sand and laminations at $518 \mathrm{~cm}$

sma11, well rounded pebble at $362 \mathrm{~cm}$; small pumice (?) fragment at $399 \mathrm{~cm}$; void $-452-454 \mathrm{~cm}$

$\mathrm{S}$, but very mottled

CALC OOZE

10 YR $7 / 1$ light gray grades to 2.5 Y $6 / 2$ light brownish gray $(\approx 750 \mathrm{~cm})$ and back to $7 / 1(\approx 780 \mathrm{~cm})$

intermottling of above colors is scattered throughout quite $\mathrm{firm}$, compact, very silty

very dark gray-brown lens at $720 \mathrm{~cm}$ and a couple
mottle-1ike features at $790 \mathrm{~cm}$ and $842 \mathrm{~cm}$ (calc ooze with pyrite) very

CALC CIAY GRADES TO NANNO OOZE

2.5 Y $5 / 2$ grayish brown pales to 10 YR $7 / 1$ 1ight subtle mottling is

apparent as it grasent throughout but particularly des to light gray; 1ight brownish sility lutite becomes quite firm and siltier as color

$G$ and extensively mottled $(975-990 \mathrm{~cm})$

HIGHLY CALC CIAY

2.5 Y $5 / 2$ grayish brown

clasts of dark of light gray and a few very small

comon forams harp, mottled 
VISUAL CORE DESCRIPTION

Page 3 of 3

Ship KNORR Cruise 51 Leg III Sta. 36 Core No. 13- GPC

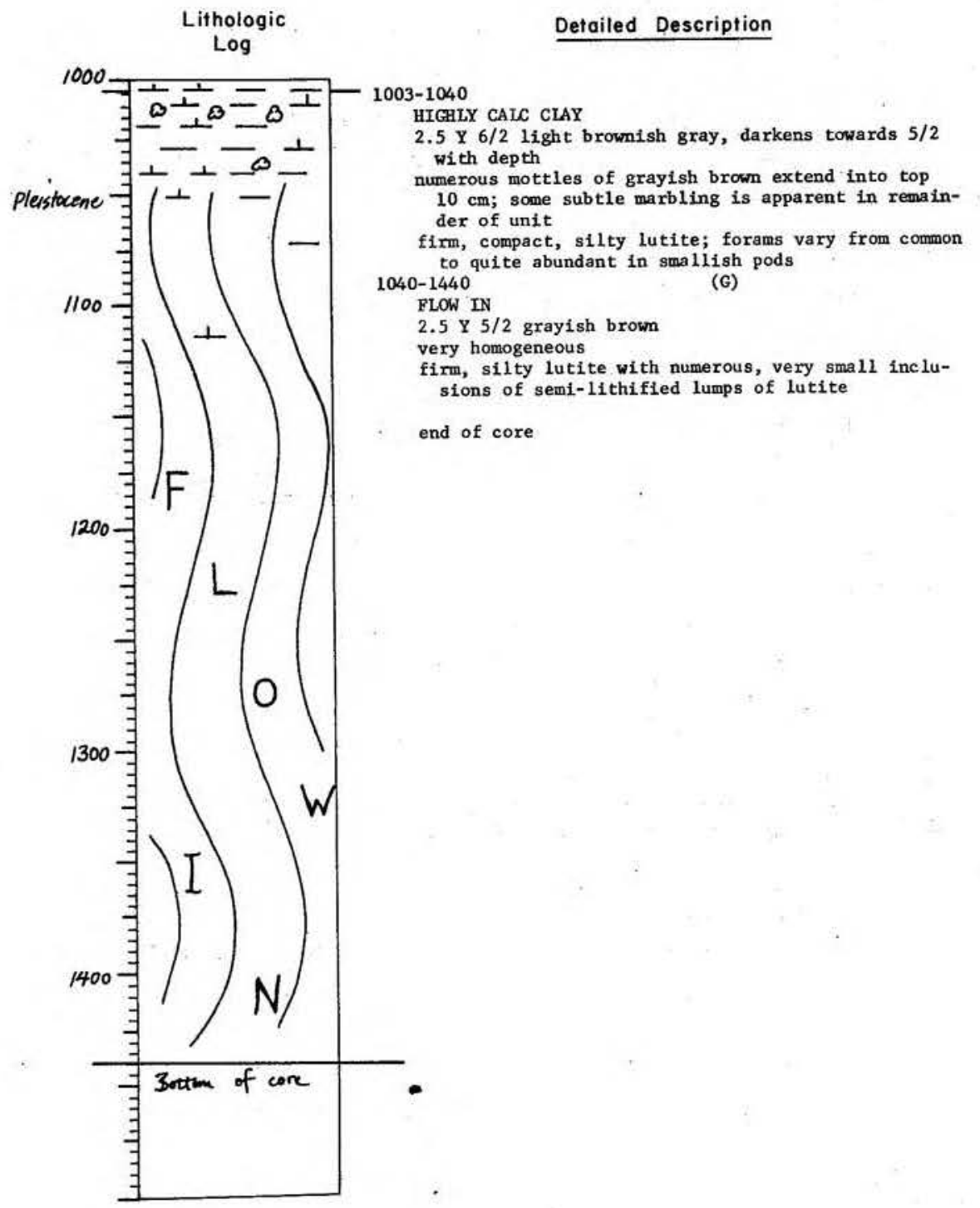

SIEAR SLIDE DESCRIPTIONS - W.H.O.I. SEDIMENT CORES

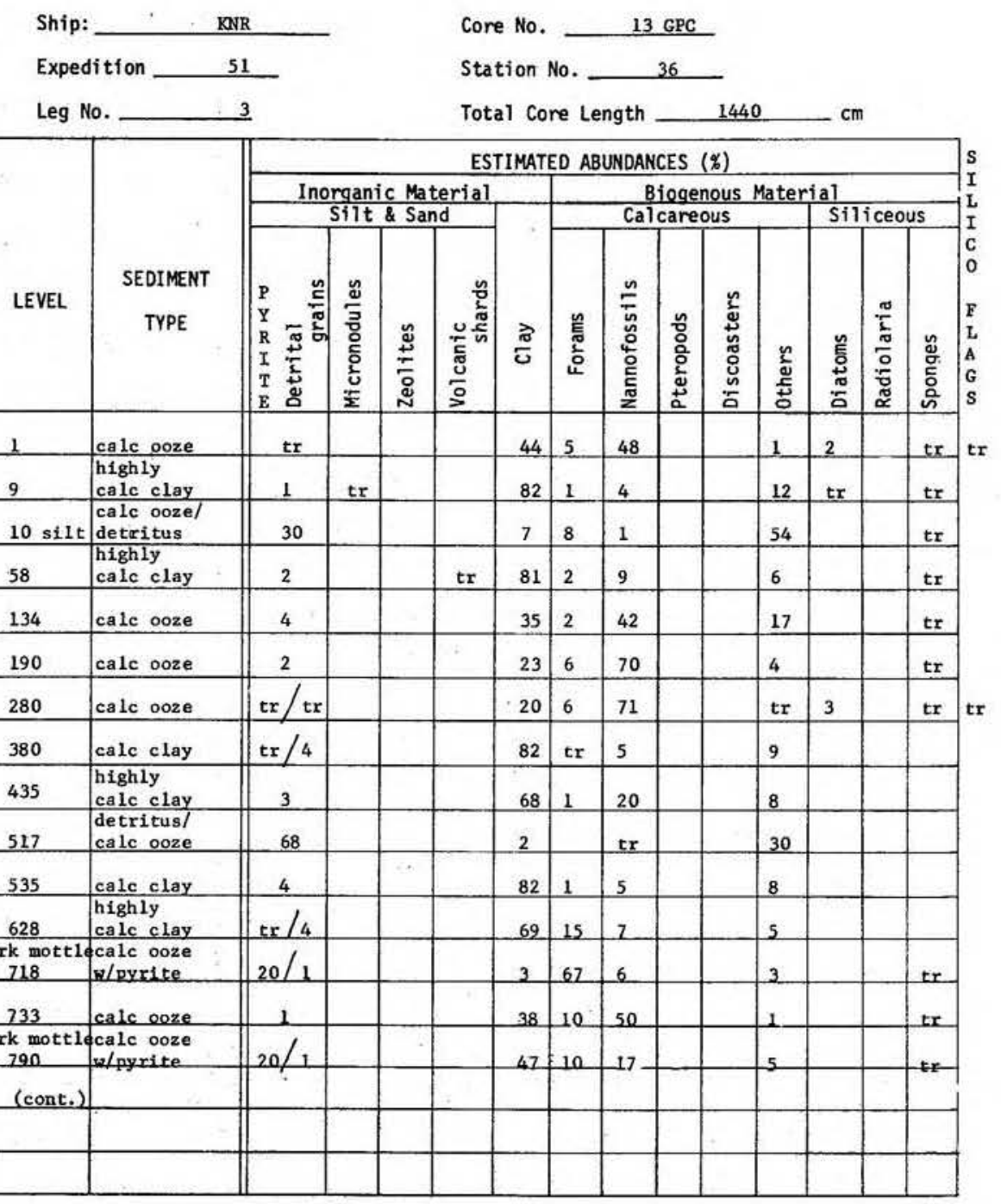


SEENR SLIE OESCRIPTIONS - M.H.O.I. SEDINEMT CORES

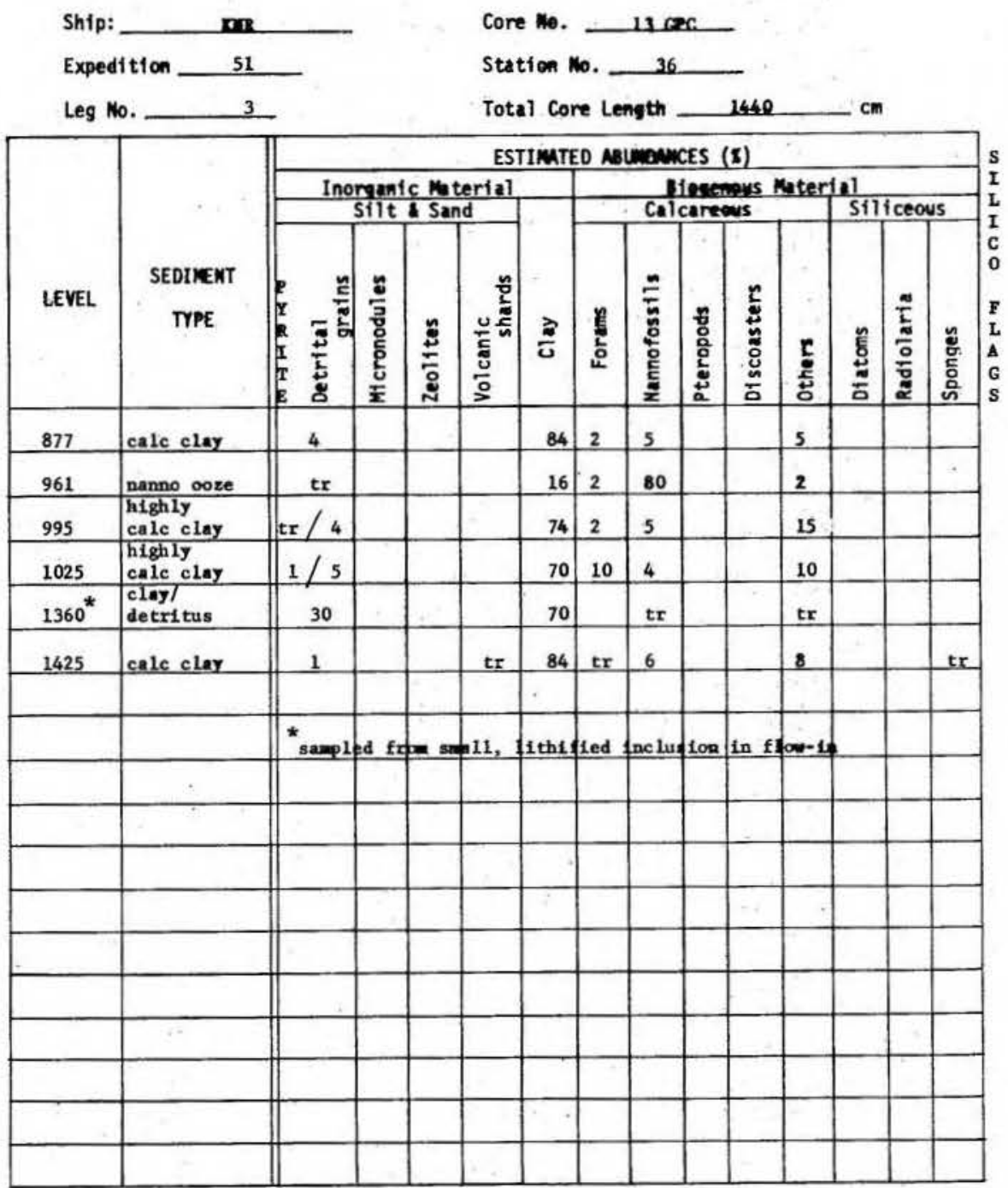

Ship KNORR Cruise 51 Leg II Sta. 36 Core No. 13-AG

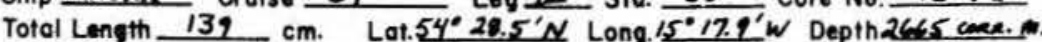
Core condition Exceused Date Described 2Jime 74 by H. Farmer Physiographic location FENI DRIET- EAsTERe NiRTH ARminc

Lithologic

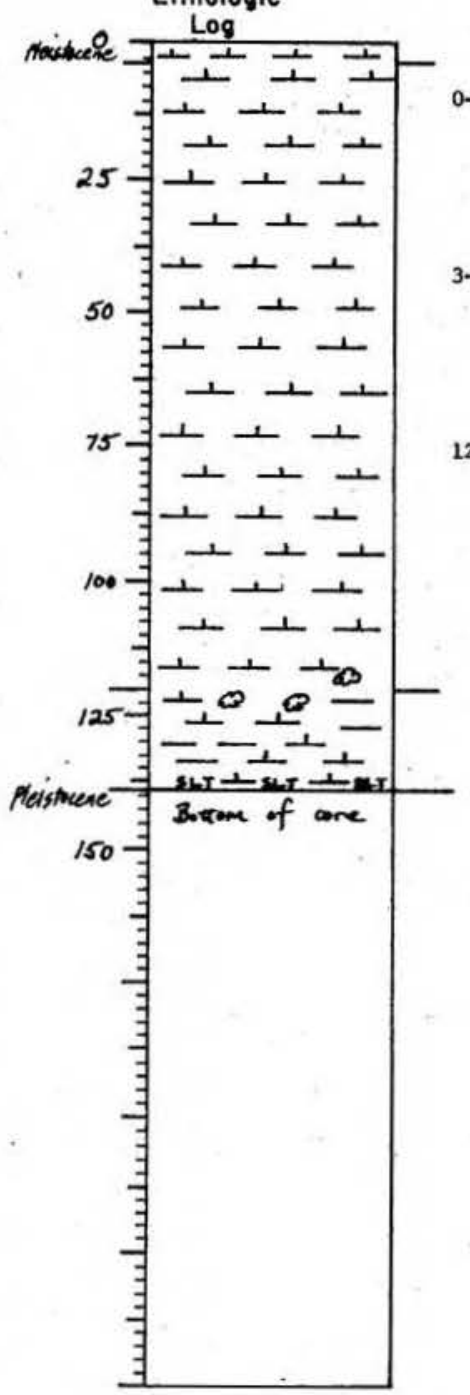

Detailed Description

CALC OOZE

10 YR $6 / 3$ pele brown

no mottling

void: $0-2$ cal

$S$, distorted

$-120$ CAIC OOZE

10 YR $7 / 1$ 11ght gray, darkens very smoothly with depth tovards 2.5 Y 6 / 2 light brownish gray co, very, silty lutite with numerous foran $G$, very mottled

120-139

HIGHIY CAIC CIAY

2.5 Y $6 / 2$ light bromish gray grades to $5 / 2$

grayish brown

arge mottling with the upper unit appears from $115-135$ cas

thin silt lamination (calc ooze/detritus) at base of unit

end of core 
SMEAR SLIDE DESCRIPTIONS - W.H.O.I. SEDIMENT CORES

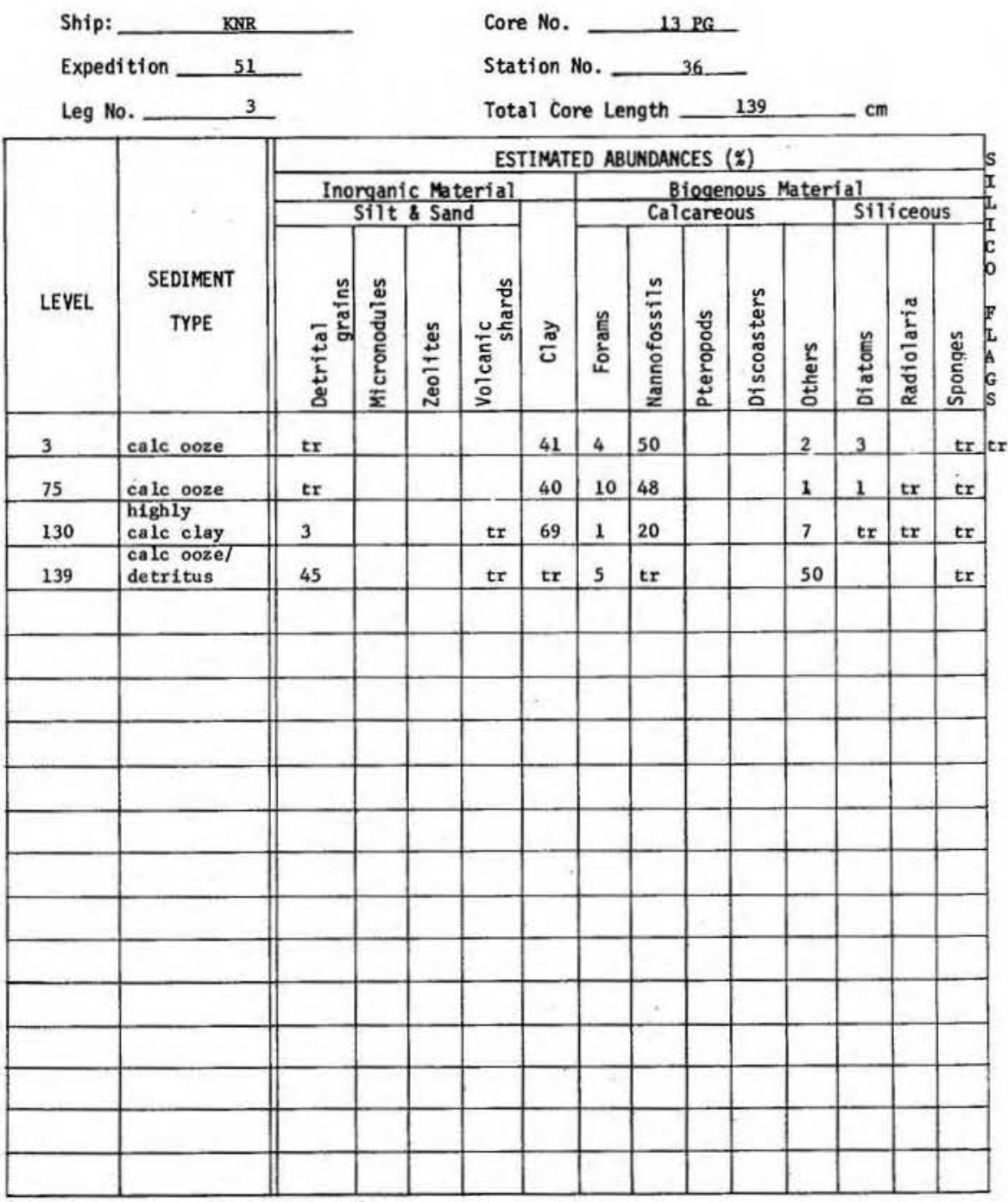

Ship KNORR Cruise 51 Leg 3 Sto. 39 Core No. I7ER

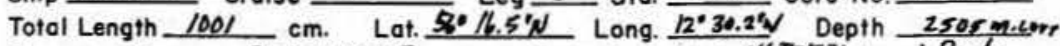
Core condition EXCELLENT Date Described 14 JNE 76 by f.brede. Physiographic location ROCKACL TROV $C H$

Lithologic

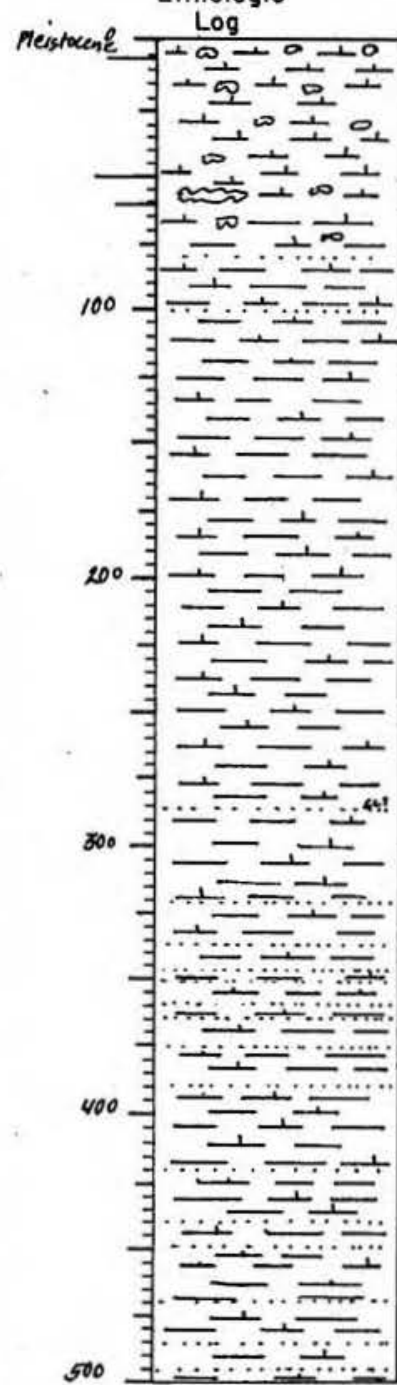

Detailed Description

\section{CALC OOZE}

10 YR $6 / 3$ pale brown

complete (somewhat disturbed) intermottling and marbling with the unit

fairly coarse, silty lutite with abundant forams entire unit slightly disturbed and washe

$6-50$ CALC OOZE

10 YR $7 / 2$ light gray

coumon, pale brown and very pale brown mottling and

burrowing

firm, slightly silty lutite with abundant foram $G$, mottled

CAIC OOZE

10 YR $5 / 2$ grayish brow

few, faint light gray mottles scattered throughout and one outstanding, very pale brown, foram-xich burrow

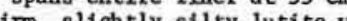

with forans coumon in burscattered in matrix

S, extural CIAY GRADES TO CAIC CLAY WITH IAMLMATIONS OF DETRTTUS TO CAIC OOZB 10 YR $5 / 2$ grayish brown

few light gray mottles $61-75 \mathrm{~cm}$; also an excellent, well-defined foram-rich, very pale brown burrow $(1 \mathrm{~cm}$
wide) $77-78 \mathrm{~cm}$ : from there on a series of silt laminawile) 77 -78 can: froml there on a serles of silt laming intervals throughout the core. The ma jor laninations are found: $80 \mathrm{~cm}$, fine and somewhat 1rregular; $100 \mathrm{~cm}$; $286 \mathrm{~cm}$, again irregular in thickness; $320-321 \mathrm{~cm}$, rusty, yellowish-brown and concave upward; $336-337 \mathrm{~cm}$, $1 \mathrm{~cm}$ thick; $345-365 \mathrm{~cm}, 2$ to $3 \mathrm{~mm}$ thick a $1 \mathrm{~cm}$ interval $374 \mathrm{~cm}, 1 \mathrm{~cm}$ wide, distorted by coring (concave downward); $377-390 \mathrm{~cm}$ - many thin, distorted laminations; thick), very similar ones found $417,420,426,440,442$, $444,465,475,478,484,487,491,518,520 \mathrm{~cm}$ and so on throughout the unit: color of the matrix sediment in a heavily ... burrowed zone (gray and pale brown) $830-1000 \mathrm{~cm}$. Near the base of the core the small mottles have a rusty, frecklelike appearance. 
Ship KNoRe

Cruise

Leg

Leg Sta. 39 Core No. 176ne

Detailed Description

ithologic

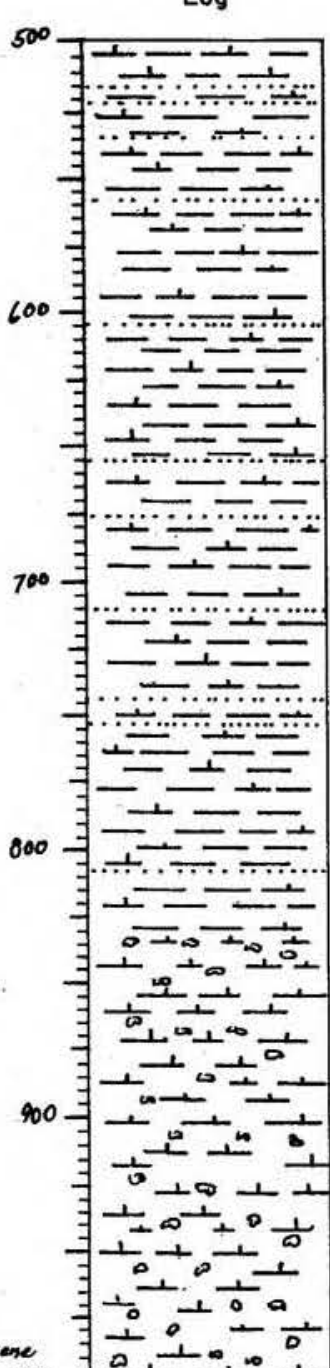

Meismene $=\frac{0}{0}+\infty-\frac{1}{0}$
61-1001 (cont.)

NOTE: Due to the density of laminations throughout the unit, only a portion of the features are depicted

in the lick, cold cream-11ke lutite with silt laminations mentioned above, grades slowly to more compact, slightly

end of core

end ofcore $1001 \mathrm{~cm}$.
SIEEAR SLIDE DESCRIPTIONS - W.H.O.I. SEDIEENT CORES

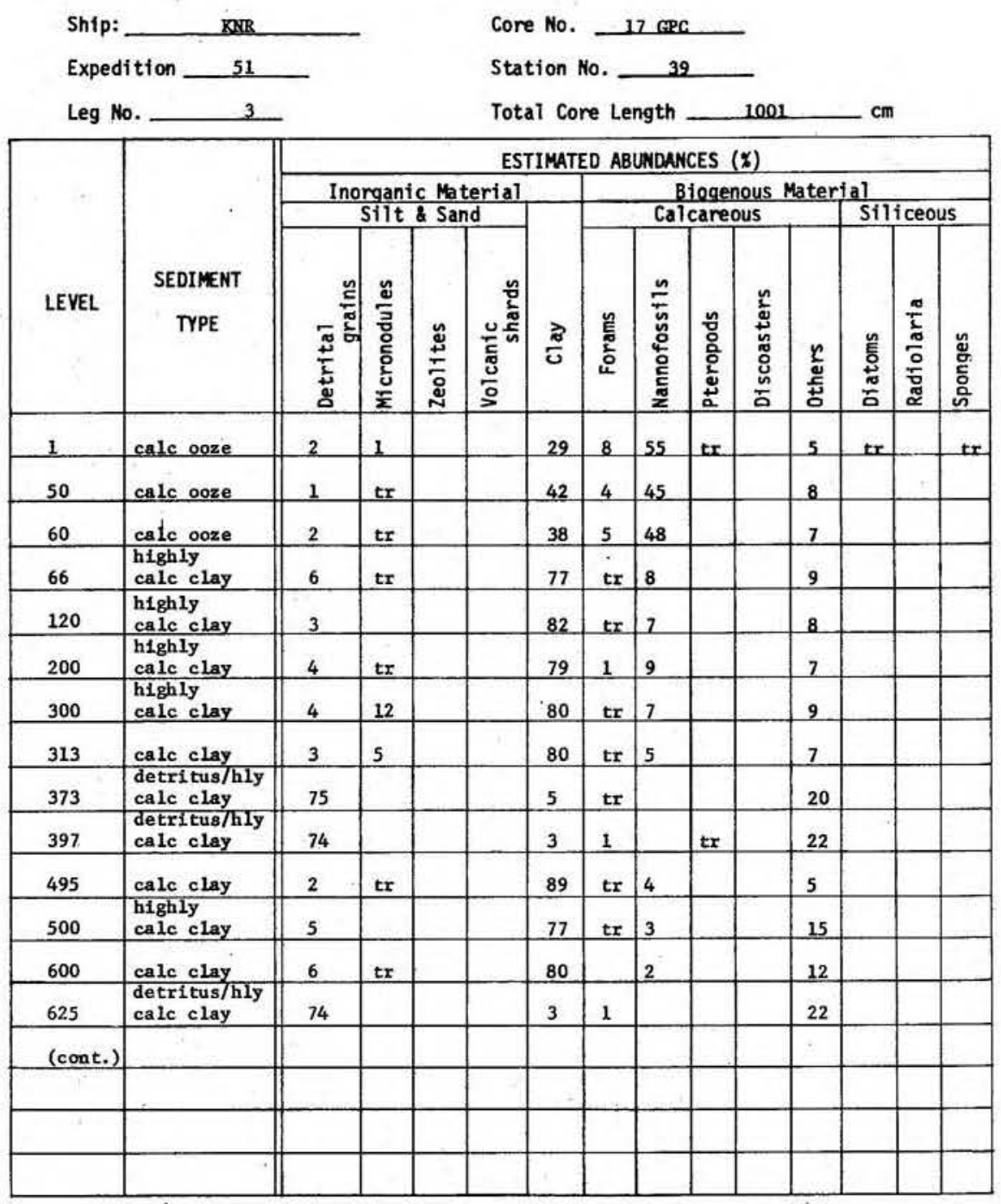


SMEAR SLIDE DESCRIPTIONS - W.H.O.1. SEDIMENT CORES

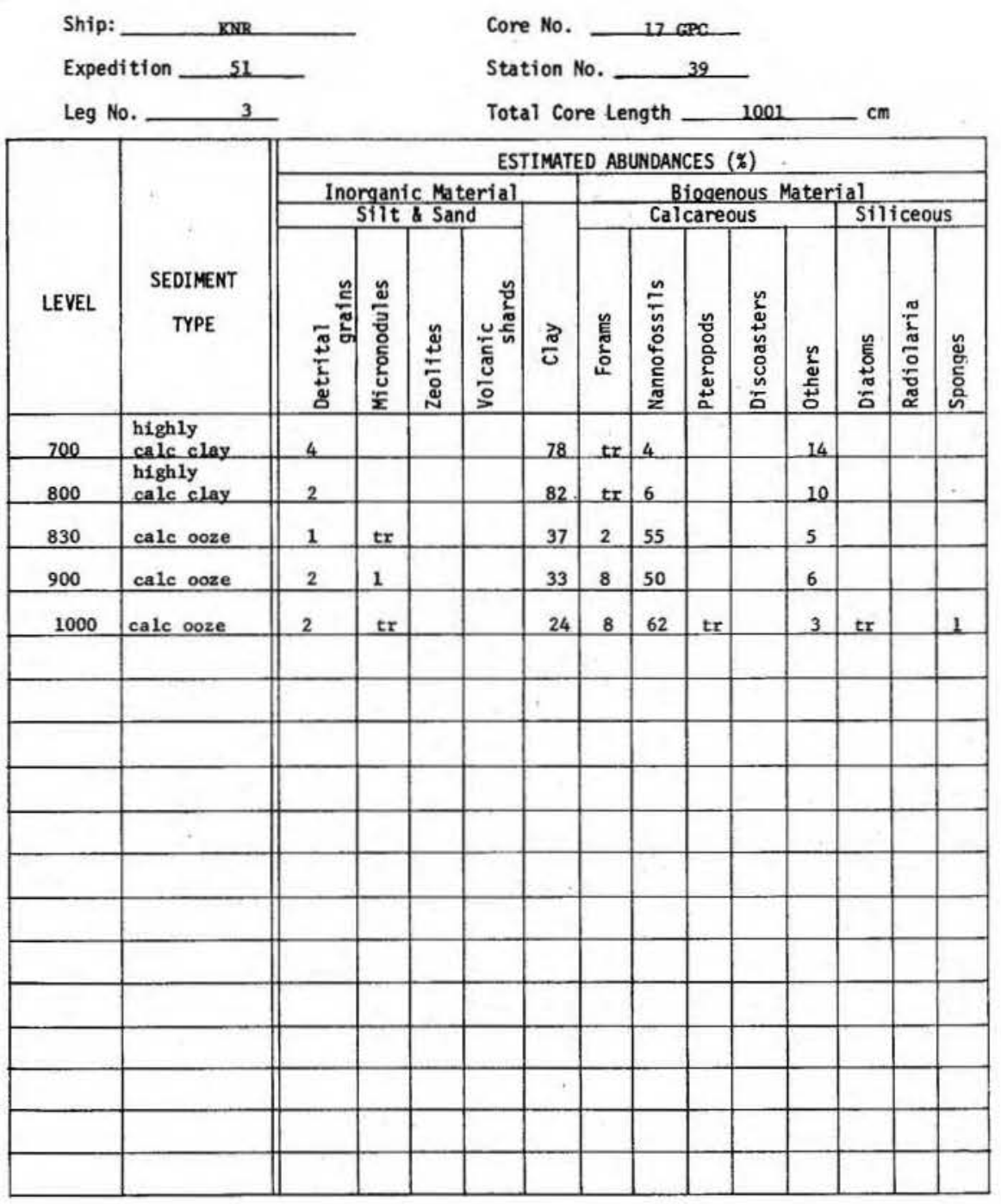

Ship KNORR Cruise 51 Leg 3 sta. 39 Core No. $17 P G$ Total Length $89^{-} \mathrm{cm}$. Lat. $66^{\circ} 16^{\circ} 5^{\circ} \mathrm{N}$ Long. $12^{\circ} 30.2^{\prime} \mathrm{W}$ Depth $2505 \mathrm{~m}$. cam Core condition EXCELENT Date Described 14 pme 76 by Jute. Physiographic location Lithologic

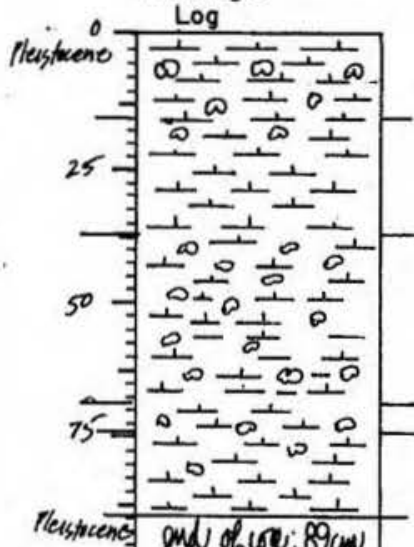

Mersincente

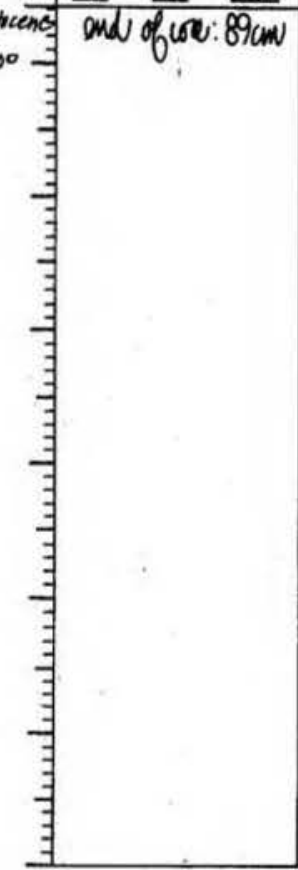

RoLrel TROU CA.

\section{Detailed Description}

NOTE: Due to a repetative sequence of similar lithologies this core is suspected to be an example of multiple penetration (pilot rebound) recovery CALC OOZE

10 YR $6 / 3$ pale brown grades to $5 / 3$ brown

common intermottling of the colors above through the zone $6-15 \mathrm{~cm}$ (a few light gray mottles are also included) firm, slightly silty lutite with scattered forams s, horizontal

a few faint, pale brown mottles near top of unit, otherwise homogeneous throughout

firm, slightly silty lutite with scattered forams

CALC OOZE GRADES TO CALC CLAY

10 YR $5 / 2$ grayish brown grades to $4 / 2$ dark grayish brown extensive, pale brown and grayish brown mottling and slightly silty lutite pith forams exclusively in to smooth, slick lutite Very Sharp, slightly irregular

68-74 ALC OOZE

10 YR $5 / 3$ brown

cormon, pale brown mottling and marbling throughout firm, slightly silty lutite with scattered forams

74-89, CALC OOZE

10 YR $6 / 2$ light brownish gray

a few, pale brown and gray mottles at top of unit, then homogeneous to base

firm, slightly silty lutite with scattered to abundant forams

end of core 
SIEAR SLIDE DESCRIPTIONS - W.H.O.I. SEDIMENT CORES

Ship: Expedition 51

Leg No.

\begin{tabular}{|c|c|c|c|c|c|c|c|c|c|c|c|c|c|c|}
\hline \multirow[b]{3}{*}{ LEVEL } & \multirow[b]{3}{*}{$\begin{array}{c}\text { SEDIMENT } \\
\text { TYPE }\end{array}$} & \multicolumn{13}{|c|}{ ESTIMATED ABUWDANCES ( $(\chi)$} \\
\hline & & \multicolumn{4}{|c|}{$\begin{array}{c}\text { Inorganic Material } \\
\text { Silt \& Sand }\end{array}$} & \multirow[b]{2}{*}{$\frac{\pi}{0}$} & \multicolumn{5}{|c|}{$\begin{array}{l}\text { Biogenous Mater } \\
\text { Calcareous }\end{array}$} & \multicolumn{3}{|c|}{$\frac{\text { al }}{\text { Silliceous }}$} \\
\hline & & $\frac{5}{5}$ & $\begin{array}{l}\frac{\omega}{3} \\
\frac{\vec{z}}{0} \\
\frac{0}{0} \\
\frac{0}{\Sigma}\end{array}$ & 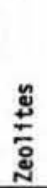 & 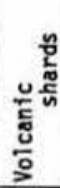 & & 童 & 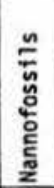 & 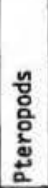 & 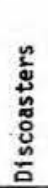 & $\begin{array}{l}\frac{r}{2} \\
\text { ș } \\
\text { s. }\end{array}$ & 感 & 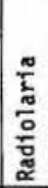 & 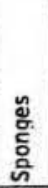 \\
\hline 1 & calc ooze & 2 & 1 & & & 20 & 12 & 55 & 2 & & 5 & 2 & & 1 \\
\hline 25 & calc ooze & tr & 1 & & & 25 & 14 & 50 & 1 & & 8 & tr & & 1 \\
\hline 67 & calc clay & 2 & $\mathrm{tr}$ & & & 91 & tr & 3 & & & 4 & & & \\
\hline 88 & calc ooze & 3 & tr & & & 36 & 8 & 50 & tr & & 5 & tr & & tr \\
\hline & . & & & & & & & & & & & & & \\
\hline & & & & & & & & & & & & & & \\
\hline & & & & & & . & & & & & & & & \\
\hline & & & & & & & & & & & & & & \\
\hline & 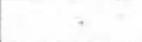 & & & & & & & & & & & & & \\
\hline & & & & & & & & & & & & & & \\
\hline & & & & & & & & & & & & & & \\
\hline & & & & & & & & & & & & & & \\
\hline & & & & & & & & & & & & & & \\
\hline & & & & & & & & & & & & & & \\
\hline & & & & & & & & & & & & & & \\
\hline & & & & & & & & & & & & & & \\
\hline & . & & & & & & & & & & & & & \\
\hline & & & & & & & & & & & & & & \\
\hline
\end{tabular}

Core No. $17 \mathrm{PG}$

Station No: 31
Ship Kmese Cruise S7 Leg II Sto. 41 Core No. 19-GPC Total Length $1492 \mathrm{~cm}$. Lat. $56^{\circ} 17.9^{\prime} \mathrm{N}$ Long. $2^{\circ} 31.3 \mathrm{~s}^{\prime} \mathrm{w}$ Depth $2535^{\circ}$ carr. Core condition ExceuLENT Date Described 18 Jume 76 by H. Farmer

Physiographic location RoCKALL TROUGA - EASTERN NORTH ATLANTTC.

Lithologic

Log Detailed Description

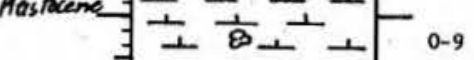

$\perp \perp$ CALC 0OZz

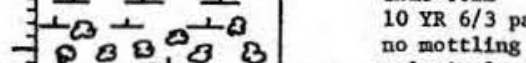

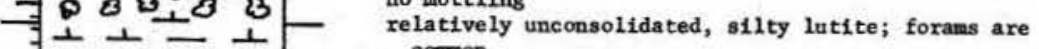

$\rightarrow-1-1$

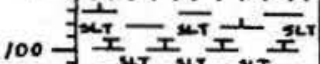

s, but very disturbed

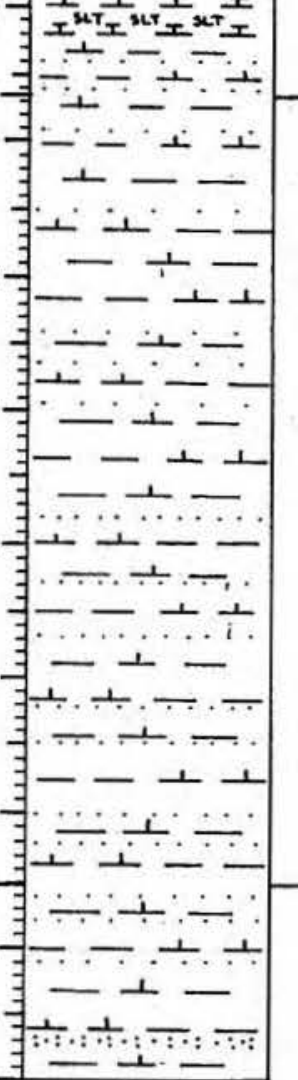

\section{CALC OOZE}

slightly darker marbling and mottling are scattered

through the top $20 \mathrm{~cm}$ while extensive internottling

with the lower unit is predowinant in the basal $15 \mathrm{~cm}$ somevhat firmer, silty lutite; forams are generally $\mathrm{s}$, motte com

57-76, 5 , mottle

HICHIY CALC CLAY

2.5 Y $5 / 2$ grayish brown

few light brownish gray mottles appear near the top, and a $5 \mathrm{~mm}$, slightly distorted lamination of light brownish gray is situated at $70 \mathrm{~cm}$

firm, slightily silty lutite; forams are scattered lightly throughout; poorly defined zone with a num $\begin{array}{ll}\text { ber of very small shell fragments mixed with lu- } \\ \text { tite } 73-75 \mathrm{~cm} & \text { (S) }\end{array}$ $76-1.33$ tite $73-75 \mathrm{~cm}$

CAIC CIAY WITH LAMDNATIONS OP DETRITUS/CAIC OOZE 2.5 Y $4 / 2$ dark grayish brown $2 \times 2 \mathrm{~cm}$ mottle of the same color appear at $76 \mathrm{~cm}$ and $96 \mathrm{~cm}$; a partially eroded lamination at $99 \mathrm{~cm}$ and a complete but slightly disturbed lamination from $102-103$ are $5 / 2$ grayish brown

quite smooth, slick, cold cream-like lutite with the following exceptions: thin $(\approx 3 \mathrm{~mm})$, somewhat disturded tus to foram sand) vith aharp, erostomal basal contacts and relatively sharp upper contacts at 95-99 $\mathrm{cm}$ and 103-108 $\mathrm{cm}$ (s, disturbed)

$133-425$

HIGHLY CALC CLAY WITH LAMINATIONS OF DETRITUS/CALC OOZE 2.5 Y 5/2 grayish brown

a somewhat paler hue of the above color is also present and the two are intemottled and subtly laminslow spoto generally uith a compact, partiy lithified center (calc clay) are found scattered from 220-320 $\mathrm{cm}$ and more coumonly below that to $425 \mathrm{~cm}$
2.5 Y $6 / 2$ light brownish gray

another $5 \mathrm{~mm}$, light brownish gray lamination and a 


\section{Lithologic}

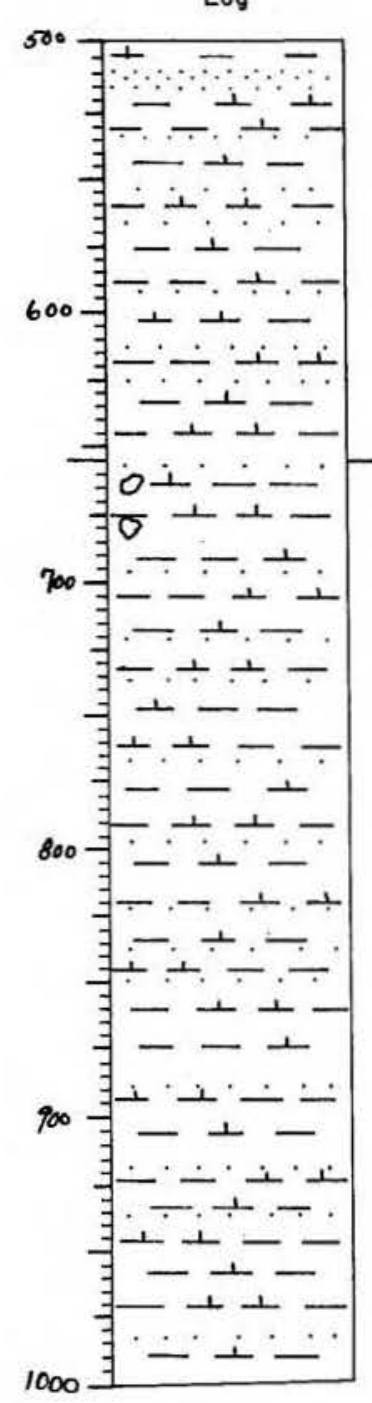

Detailed Description

133-425 (cont.)

compact and fairly smooth, slick lutite; a few forams are scattered throughout with the following exceptions: fine silt laminations similar $288,316,321,331,334,337,359,375,387,400$ $406,407,411$ and more

$425-654$

HIGLY CALC CLAY WITH LAMIXATIONS OF DETRITUS/ CAIC OOZB

$2.5 \times 5 / 2$ grayish brown

no mottling

very similar to above unit but smoother and more uniform - no forams or rusty, dark yellow spots:

fine laminations of silt are still numerous and two large, somewhat disturbed beds of well-sorted silt $\stackrel{-9}{\mathrm{C}}$

HIGHLY CALC CLAY WITH IAMINATIONS OF DETRITUS/ CAIC OOZE

$2.5 \times 5 / 2$ grayish brown (however, this unit is distinctly lighter than the above unit and quite similar to the one above $425 \mathrm{~cm}$ )

as before, there are two shades of grayish brown that appear as very large $(20-40 \mathrm{~cm})$ and often sharply contacted laminations: several of the dark yellow, rusty spots of very compact lut the

do again, fine laminations of silt are spaced anywhe

2 good-sized $(2 \times 2)$, fairly angular pebbles: 665 and $1140-1492 \mathrm{~cm}$

FLOW IN of above material

end of core

NOTB: Initial shipboard description indicates that numerous (at $5 \mathrm{~cm}$ ) hydrotroilite layers were present throughout most of this core immediately after extrusion. Notable zones devoid of these layers wer $0-160 \mathrm{~cm}$ and $460-660 \mathrm{~cm}$.
VISUAL CORE DESCRIPTION

Poge 3 of 3 Ship KNORR Cruise 51 Leg III Sto. 41 Core No. 19-GPC

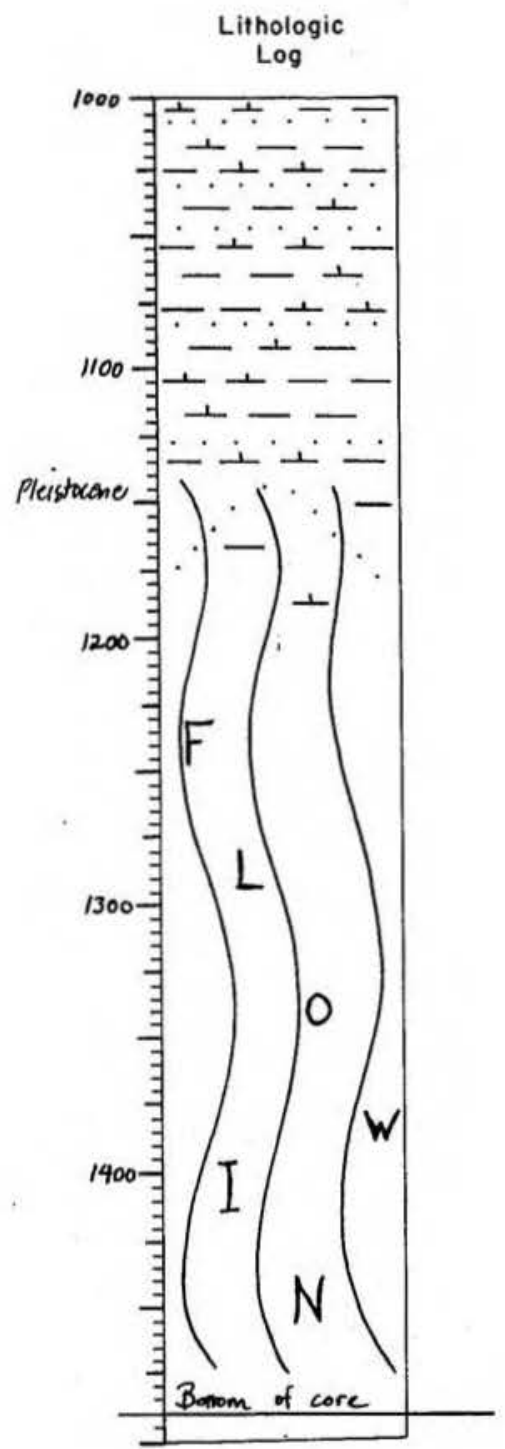

Detailed Description 
SIEAR SLIDE DESCRIPTIONS - W.H.O.I. SEDIMENT CORES

Ship:

Expedition 51

Leg No. 3

\begin{tabular}{|c|c|c|c|c|c|c|c|c|c|c|c|c|c|c|}
\hline \multirow[b]{3}{*}{ LEVEL } & \multirow[b]{3}{*}{$\begin{array}{c}\text { SEDIMENT } \\
\text { TYPE }\end{array}$} & \multicolumn{13}{|c|}{ ESTIMATED ABUNOANCES ( $(\boldsymbol{)})$} \\
\hline & & \multicolumn{4}{|c|}{$\begin{array}{c}\text { Inorganic Material } \\
\text { Silt \& Sand } \\
\end{array}$} & \multirow[b]{2}{*}{ ढ़े } & \multicolumn{5}{|c|}{$\begin{array}{l}\text { Biogenous Mater } \\
\text { Calcareous }\end{array}$} & \multicolumn{2}{|c|}{ Siliceous } & \\
\hline & & 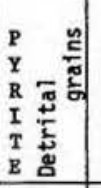 & 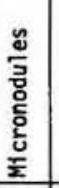 & 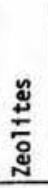 & 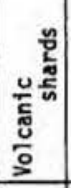 & & 唇 & $\begin{array}{l}\frac{n}{\bar{n}} \\
\frac{\tilde{u}}{2} \\
\frac{0}{E} \\
\frac{0}{z}\end{array}$ & 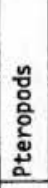 & 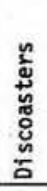 & 䒾 & 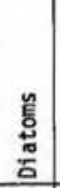 & 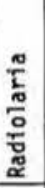 & 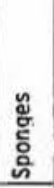 \\
\hline 4 & calc ooze & 1 & 2 & & & 34 & 9 & 47 & & & 2 & 5 & & tr \\
\hline 26 & calc ooze & 1 & & & & 39 & 7 & 50 & & & 4 & 2 & & tr \\
\hline 67 & $\begin{array}{l}\text { highly } \\
\text { calc clay }\end{array}$ & 2 & $\mathrm{tr}$ & & & 69 & 1 & 22 & & & 6 & & & $t \pi$ \\
\hline 80 & $\begin{array}{l}\text { detritus/ } \\
\text { cale ooze }\end{array}$ & 70 & $\operatorname{tr}$ & & tx & tx & & & & & 30 & & & \\
\hline 95 & $\begin{array}{l}\text { calc ooze/ } \\
\text { detritus }\end{array}$ & 30. & & & & 4 & 10 & 1 & & & 55 & & & \\
\hline 99 & forsm ooze & 10 & & & & 5 & 70 & 5 & & & 10 & & & \\
\hline 117 & calc clay & $t r$ & $\mathrm{tr}$ & & & 89 & & 1 & & & 10 & & & \\
\hline 170 & $\begin{array}{l}\text { highly } \\
\text { calc clay }\end{array}$ & 3 & & & & 78 & tr & 3 & & & 16 & & & \\
\hline 176 & \begin{tabular}{|l|} 
detritus/ \\
calc ooze
\end{tabular} & 63 & 1 & & tr & tr & 1 & tr & & & 35 & & & tr \\
\hline 246 & $\begin{array}{l}\text { detrituis/ } \\
\text { calc ooze }\end{array}$ & 65 & $t x$ & & $t x$ & tr & & tr & & & 35 & & & \\
\hline 272 & $\begin{array}{l}\text { highly } \\
\text { calc clay }\end{array}$ & 2 & & & & 80 & tr & 3 & & & 15 & & & \\
\hline 380 & $\begin{array}{l}\text { highly } \\
\text { calc clay }\end{array}$ & 3 & & & & & tr. & 2 & & & 15 & & & \\
\hline $392^{*}$ & calc clay ${ }^{*}$ & 1 & & & & 89 & & 3 & & & 1 & & & \\
\hline & (cont.) & & & & & & & & & & & & & \\
\hline & & & & & & & & & & & & & & \\
\hline & & & & & & & & & & & & & & \\
\hline & & & & & & & & & & & & & & \\
\hline - & & & & & & & & & & & & & & \\
\hline
\end{tabular}

Core No. $19 \mathrm{GPC}$

Station No. 41
SMEAR SLIDE DESCRIPTIONS - W.H.O.I. SEDIMENT CORES

Ship:

Expedition si

Leg No. 3

Station No. 41

Total Core Length 1492

\begin{tabular}{|c|c|c|c|c|c|c|c|c|c|c|c|c|c|c|}
\hline \multirow[b]{3}{*}{ LEVEL } & \multirow[b]{3}{*}{$\begin{array}{c}\text { SEDIMENT } \\
\text { TYPE }\end{array}$} & \multicolumn{13}{|c|}{ ESTIMATED ABUNDANCES ( $(\boldsymbol{\alpha})$} \\
\hline & & \multicolumn{4}{|c|}{$\frac{\text { Inorganic Material }}{\text { Silt \& Sand }}$} & \multirow[b]{2}{*}{$\frac{\pi}{6}$} & \multicolumn{5}{|c|}{$\frac{\text { Biogenous Mater }}{\text { Calcareous }}$} & & Siliceous & \\
\hline & & 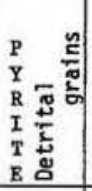 & 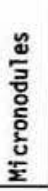 & 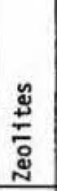 & 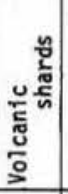 & & 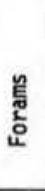 & 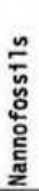 & 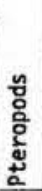 & 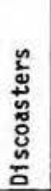 & 先 & 产 & $\frac{\pi}{\frac{\pi}{2}}$ & 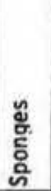 \\
\hline 495 & $\begin{array}{l}\text { highly } \\
\text { calc clay. }\end{array}$ & 6 & & & & 73 & & 1 & & & 20 & & & \\
\hline 601 & $\begin{array}{l}\text { highly } \\
\text { calc clay }\end{array}$ & 3 & & & & 82 & $t r$ & tr & & & 15 & & & \\
\hline 656 & $\begin{array}{l}\text { highly } \\
\text { calc clay }\end{array}$ & 6 & & & & 70 & tr & 1 & & & 23 & & & \\
\hline 754 & $\begin{array}{l}\text { highly } \\
\text { calc clay }\end{array}$ & 7 & & & & 76 & tr & 2 & & & 15 & & & \\
\hline 846 & calc clay & 1 & & & & 86 & & 1 & & & 12 & & & \\
\hline 943 & $\begin{array}{l}\text { highly } \\
\text { calc clay }\end{array}$ & 6 & & & & 68 & tr & 7 & & & 19 & & & \\
\hline 1033 & $\begin{array}{l}\text { detritus/ } \\
\text { calc ooze }\end{array}$ & 69 & & & 1 & tr & tr & tx & & & 30 & & & \\
\hline 1050 & $\begin{array}{l}\text { highly } \\
\text { calc clay }\end{array}$ & 5 & & & & 79 & tr & 1 & & & 15 & & & \\
\hline 1138 & $\begin{array}{l}\text { highly } \\
\text { calc clay }\end{array}$ & 8 & & & & 68 & 1 & 2 & & & 21 & & & \\
\hline & & & & & & & & & & & & & & \\
\hline & & & & & & & & & & & & & & \\
\hline & & & & & & & & & & & & & & \\
\hline & & & & & & & & & & & & & & \\
\hline & & & & & & & & & & & & & & \\
\hline & & & & & & & & & & & & & & \\
\hline & & & & & & & & & & & & & & \\
\hline & & & & & & & & & & & & & & \\
\hline & & & & & & & & & & & & & & \\
\hline
\end{tabular}


Ship KNORR Gruise 51 Leg III Sta. 41 Core No. 19-PG Total Length $82 \mathrm{~cm}$. Lat $56^{\circ} 17.9^{\prime \prime N}$ Long. $12^{\circ} 31.3$ 'w Depth $2535 \mathrm{cerr} . \mathrm{m}$.

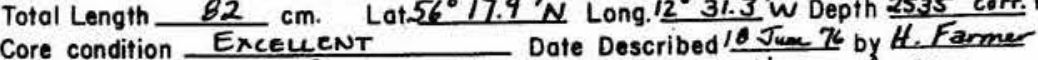
Physiographic location RockALL TROUGH, EASTERN NORTN ATLANTIC Lithologic

Detailed Description

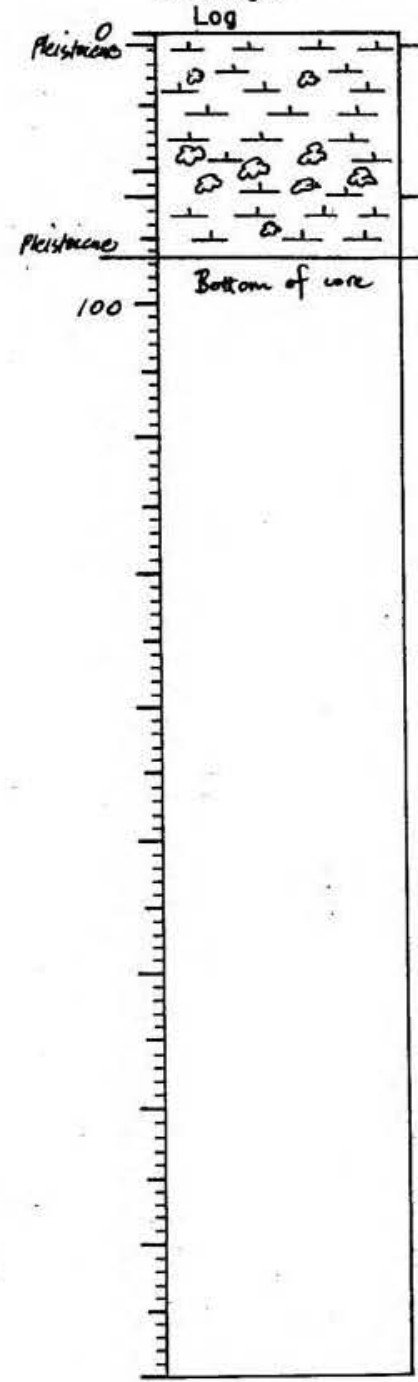

$0-3$

CALC OOZE

10 YR $6 / 3$ pale brown

$5 / 3$ brown appears at base of unit ilty lutite; forams are coumon; the core liner from $0-3 \mathrm{~cm}$

$3-60$

CALC OOZE

2.5 Y $6 / 2$ light brownish gray

in the zone fow $40-60 \mathrm{~cm}$ is extensively mottled in a similar color

irm, compact, fairly silty lutite; forams or other

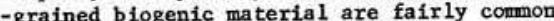
S, but distorted

CALC OOZE

2.5 Y $6 / 2$ light brownish gray

distorted sliver of 10 YR $6 / 3$ pale brown lies at the upper contact; a lew graylsh brown nottles appear in the zone $62-64$ cm and a simlar marblin In the basal $4 \mathrm{cr}$

lutite; forams or other biogenic meterial are fairly common

end of core

NOIE: After comparison with the giant piston core, the contact between the two units described at $60 \mathrm{~cm}$ repenetration of the pilot core.
SHEAR SLIDE DESCRIPTIONS - W.H.0.I. SEDIMENT CORES

\begin{tabular}{|c|c|c|c|c|c|c|c|c|c|c|c|c|c|c|c|}
\hline \multicolumn{16}{|c|}{$19 \mathrm{PG}$} \\
\hline Exped & ition & & & & \multirow{2}{*}{\multicolumn{5}{|c|}{$\begin{array}{l}\text { Station No. } \\
\text { Total Core Length }\end{array}$}} & \multirow{2}{*}{\multicolumn{5}{|c|}{$82 \quad \mathrm{~cm}$}} & \\
\hline \multicolumn{5}{|c|}{ Leg No. 3} & & & & & & & & & & & \\
\hline \multirow[b]{3}{*}{ LEVEL } & \multirow[b]{3}{*}{$\begin{array}{c}\text { SEDIMENT } \\
\text { TYPE }\end{array}$} & \multicolumn{13}{|c|}{ ESTIMATED ABUNDANCES (\%) } & $s$ \\
\hline & & \multicolumn{4}{|c|}{$\frac{\text { Inorganic Material }}{\text { Silt \& Sand }}$} & & \multicolumn{5}{|c|}{$\begin{array}{l}\text { Biogenous Mater } \\
\text { Calcareous }\end{array}$} & \multicolumn{3}{|c|}{ Siliceous } & $\frac{I}{2}$ \\
\hline & & $\frac{-\frac{c}{\pi}}{\frac{\pi}{5}}$ & 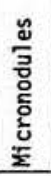 & 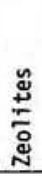 & 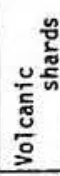 & $\frac{\pi}{6}$ & 望 & 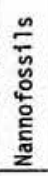 & 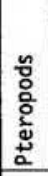 & 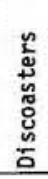 & 营 & 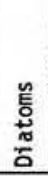 & $\frac{\frac{\pi}{\pi}}{\frac{\pi}{0}}$ & 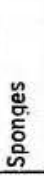 & \begin{tabular}{|l} 
F \\
L \\
A \\
G \\
S
\end{tabular} \\
\hline 1 & calc ooze & 1 & tx & & & 35 & 7 & 50 & & & 2 & 5 & tr & $t x$ & \\
\hline & calc ooze & tr & tr & & & 41 & 8 & 50 & & & 1 & tr & & tr & $t_{1}$ \\
\hline $\begin{array}{l}\text { mottle } \\
59\end{array}$ & $\begin{array}{l}\text { highly } \\
\text { calc clay }\end{array}$ & 2 & tr & & & 69 & 2 & 22 & & & 5 & & & tr & \\
\hline 81 & calc ooze & 1 & & & & 40 & 8 & 50 & & & 1 & tr & & tx & $t x$ \\
\hline & & & & & & & & & & & & & & & \\
\hline & & & & & & & & & & & & & & & \\
\hline & & & & & & . & & & & & & & & & \\
\hline & & & & & & & & & & & & & & & \\
\hline & & & & & & & & & & & & & & & \\
\hline & & & & & & & & & & & & & & & \\
\hline & & & & & & & & & & & & & & & \\
\hline & & & & & & & & & & & & & & & \\
\hline & & & & & & & & & & & & & & & \\
\hline & & & & & & & & & & & & & & & \\
\hline & & & & & & & & & & & & & & & \\
\hline & & & & & & & & & & & & & & & \\
\hline & & & & & & & & & & & & & & & \\
\hline & & & & & & & & & & & & & & & \\
\hline
\end{tabular}


Ship RNORE Cruise 57 Leg III Sta $\frac{41}{4}$ Core No. $22 F F$

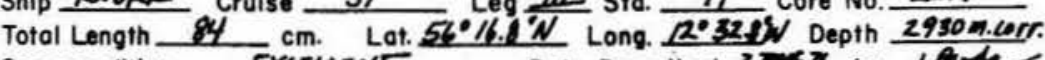

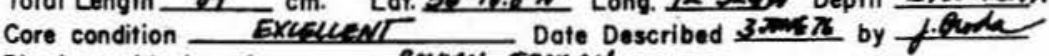
Physiographic location

Lithologic

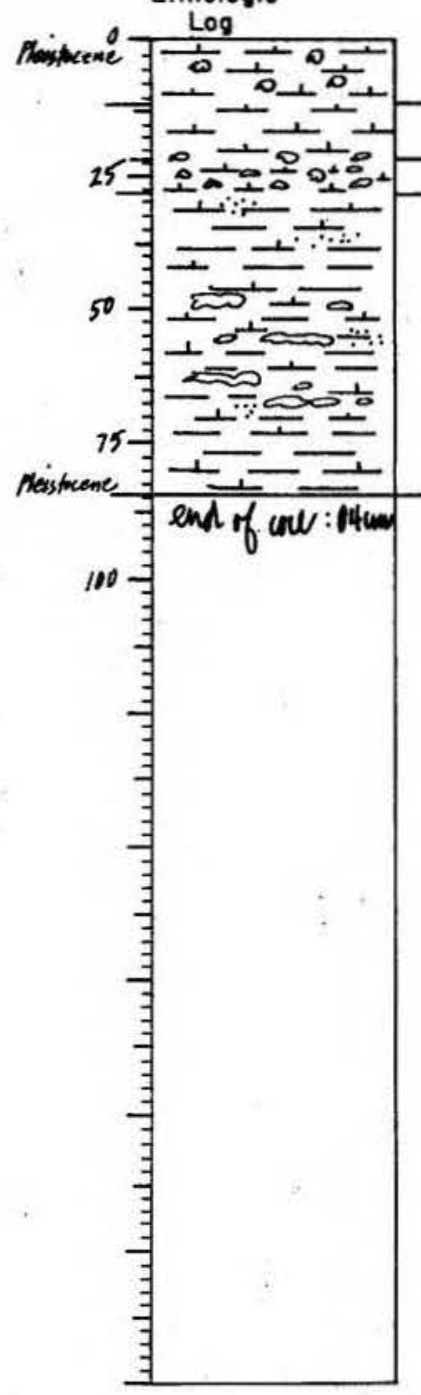

\section{Detailed Description} $0-12$ CALC OOZE

10 XR $7 / 3$ very pale broum, 10 YR $6 / 3$ pale brown, 10 YR $5 / 4$ brown

intermottling and marbling of the three colors above in an irregular fashion

firm, silty lutite with abundant forams $12-22$ G, Irregular

10 YR $6 / 2$ light brownish gray

slight grayish brown (5/2) burrowing near unit basal

contact
firm, compact, silty lutite with scattered to abundant forams

5 ,

CALC OOZE

complete ond and mott11ng

moist, slick lutite as a watrix with silty, foram-ric $G$

28-84

CALC CLAY WITH INCLUSIONS OF CAIC OOZE/DETRITUS

10 YR 5/2 gray1sh brown, grades to $6 / 3$ pale brown $54-55 \mathrm{~cm}, 62-63 \mathrm{~cm}, 66-67 \mathrm{~cm}$

mostly s11ck, mo1st lutite with few forams throughout; lutite within laminations and burrows is more compact

few, fine, silty inclusions and lenses found $28 \mathrm{~cm}$, 35 con, $61 \mathrm{~cm}$

end of core
SIEAR SLIDE DESCRIPTIONS - W.H.O.I. SEDINENT CORES
Ship:

Expedition

Leg No.
Core No. $22 \mathrm{FF}$

Station No. 41

Total Core Length $\quad 84 \quad \mathrm{~cm}$

\begin{tabular}{|c|c|c|c|c|c|c|c|c|c|c|c|c|c|c|}
\hline \multirow[b]{3}{*}{ LEVEL. } & \multirow[b]{3}{*}{$\begin{array}{c}\text { SEDIMENT } \\
\text { TYPE }\end{array}$} & \multicolumn{13}{|c|}{ ESTIMATED ABUNDANCES ( $(\%)$} \\
\hline & & \multicolumn{4}{|c|}{$\frac{\text { Inorganic Material }}{\text { Silt \& Sand }}$} & \multirow[b]{2}{*}{$\frac{\pi}{6}$} & \multicolumn{5}{|c|}{$\begin{array}{l}\text { Biogenous Mater } \\
\text { Calcareous }\end{array}$} & \multicolumn{3}{|c|}{$\frac{\text { Ial }}{\text { Siliceous }}$} \\
\hline & & $\frac{5}{5}$ & 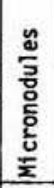 & $\frac{\check{\Perp}}{\frac{ \pm}{\sigma}}$ & 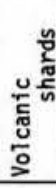 & & 鵕 & 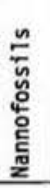 & 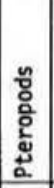 & 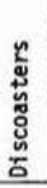 & 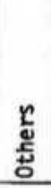 & 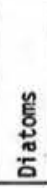 & $\begin{array}{l}\frac{\pi}{i} \\
\frac{\pi}{0} \\
\frac{0}{0} \\
\frac{0}{0} \\
\alpha\end{array}$ & 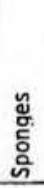 \\
\hline 1 & calc ooze & $\mathrm{tr}$ & & & & 24 & 12 & 60 & & & 2 & 2 & & 1 \\
\hline 15 & calc ooze & 1 & & & & 23 & 15 & 55 & $\mathrm{tr}$ & & 4 & 1 & & 1 \\
\hline 32 & calc clay & 5 & tr & & & 86 & 1 & 6 & & & 2 & tr & & \\
\hline 35 & $\begin{array}{l}\text { calc ooze/ } \\
\text { detritus }\end{array}$ & 45 & & & & 21 & tr & 4 & & & 30 & & & tr \\
\hline 66 & calc ooze & tr & & & & 33 & 12 & 50 & tr & & $s$ & $t r$ & & \\
\hline 83 & calc clay & 5 & & & & 83 & 3 & 3 & & & 6 & & & \\
\hline & & & & & & . & & & & & & & & \\
\hline & & & & & & & & & & & & & & \\
\hline & & & & & & & & & & & & & & \\
\hline & & & & & & & & & & & & & & \\
\hline & & & & & & & & & & & & & & \\
\hline & & & & & & & & & & & & & & \\
\hline & & & & & & & & & & & & & & \\
\hline & & & & & & & & & & & & & & \\
\hline & & & & & & & & & & & & & & \\
\hline & & & & & & & & & & & & & & \\
\hline & & & & & & & & & & & & & & \\
\hline & & & & & & & & & & & & & & \\
\hline
\end{tabular}


748

VISUAL CORE DESCRIPTION

Page 1 of 1

Ship KNeRR Cruise 51 Leg II Sto. 41 Core No. 23 fF

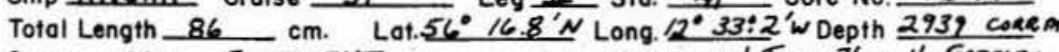
Core condition EXCELLENT Date Described $1 J_{\text {WE }} \mathrm{V}_{\text {by }}$ H. FARMER

Physiographic location ROCKALL TKOLGH

Lithologic

Detailed Description

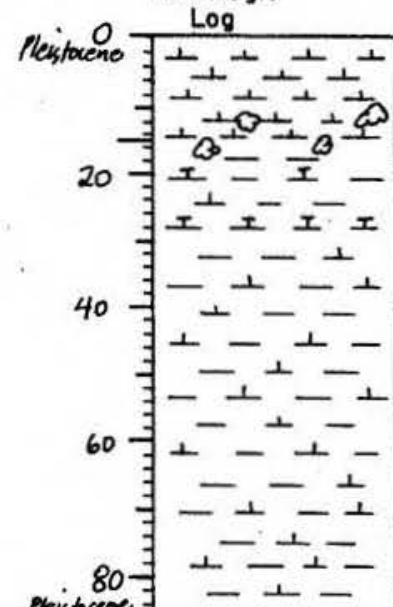

Plesstocen

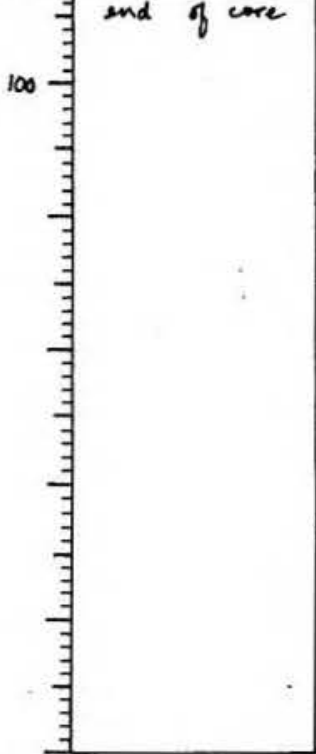

0-1 voID

-1-15 CALC OOZE

.5 Y $6 / 2$ light brownish gray pales with depth

mottled with lower unit from $13-18 \mathrm{~cm}$

no mottling, but two very distorted laminations of 2.5 $\mathrm{Y} 4 / 2$ dark grayish brown appear from $20-27 \mathrm{~cm}$ and $32-37$ $\mathrm{cm}$; sediment is very uniform below $37 \mathrm{~cm}$

lick lutite with few forass interrupted by foram sand inclusions $(23 \mathrm{~cm}, 26 \mathrm{~cm})$ and distorted beds $(19-21 \mathrm{~cm}$ and

pumice fragment in lens of fine calcend of core

\section{9}

SMEAR SLIDE DESCRIPTIONS - W.H.O.I. SEDIMENT CORES
Ship:

Expedition 51

Leg No. 3
Core No. $23 \mathrm{FR}$

Station No. 41

Total Core Length $86 \quad \mathrm{~cm}$

\begin{tabular}{|c|c|c|c|c|c|c|c|c|c|c|c|c|c|c|c|}
\hline \multirow[b]{4}{*}{ LEVEL } & \multirow[b]{4}{*}{$\begin{array}{c}\text { SEDIMENT } \\
\text { TYPE }\end{array}$} & \multicolumn{14}{|c|}{ ESTIMATED ABUNDANCES ( $(\alpha)$} \\
\hline & & \multirow{2}{*}{\multicolumn{4}{|c|}{$\frac{\text { Inorganic Material }}{\text { Silt \& Sand }}$}} & & & \multirow{2}{*}{\multicolumn{5}{|c|}{$\begin{array}{l}\text { Biogenous Mater } \\
\text { Calcareous }\end{array}$}} & & & \\
\hline & & & & & & \multirow{2}{*}{\multicolumn{2}{|c|}{$\frac{\pi}{U}$}} & & & & & & \multicolumn{3}{|c|}{ Siliceous } \\
\hline & & 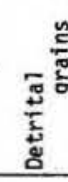 & $\begin{array}{l}\frac{y}{3} \\
\frac{0}{3} \\
\frac{0}{2}\end{array}$ & 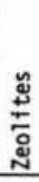 & $\frac{0}{\frac{0}{c}}$ & & & 参 & 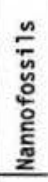 & 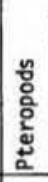 & 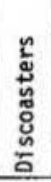 & $\begin{array}{l}\frac{c}{2} \\
\frac{5}{4} \\
0\end{array}$ & 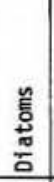 & $\frac{\frac{\pi}{d}}{\frac{\pi}{0}}$ & 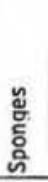 \\
\hline 2 & calc ooze & tr & tr & & t & & 42 & 8 & so & & & tr & $\mathrm{tr}$ & & tr \\
\hline 22 & calc clay & 1 & & & & & 39 & & $\operatorname{tr}$ & & & 10 & & & \\
\hline 50 & $\begin{array}{l}\text { pumice } \\
\text { fragement }\end{array}$ & & & & & & tr & & $\mathrm{tr}$ & & & tr & & & \\
\hline 85 & calc clay & 1 & & & & & 39 & & tr & & & 10 & & & \\
\hline & & & & & & & & & & & & & & & \\
\hline & & & & & & & & & & & & & & & \\
\hline & & & & & & . & & & & & & & & & \\
\hline & & & & & & & & & & & & & & & \\
\hline & & & & & & & & & & & & & & & \\
\hline & & & & & & & & & & & & & & & \\
\hline & & & & & & & & & & & & & & & \\
\hline & & & & & & & & & & & & & & & \\
\hline & & & & & & & & & & & & & & & \\
\hline & & & & & & & & & & & & & & & \\
\hline & & & & & & & & & & & & & & & \\
\hline & & & & & & & & & & & & & & & \\
\hline & & & & & & & & & & & & & & & \\
\hline & & & & & & & & & & & & & & & \\
\hline
\end{tabular}


Ship KNORR Cruise 51 Leg III Sto. 41 Core No. 24 Ff

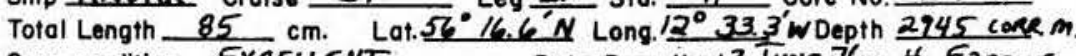
Core condition EXCELENT Date Described 2 JuNe $76_{\text {by }}$ H. Ferener

Physiographic location RockALE TROMGH

Lithologic

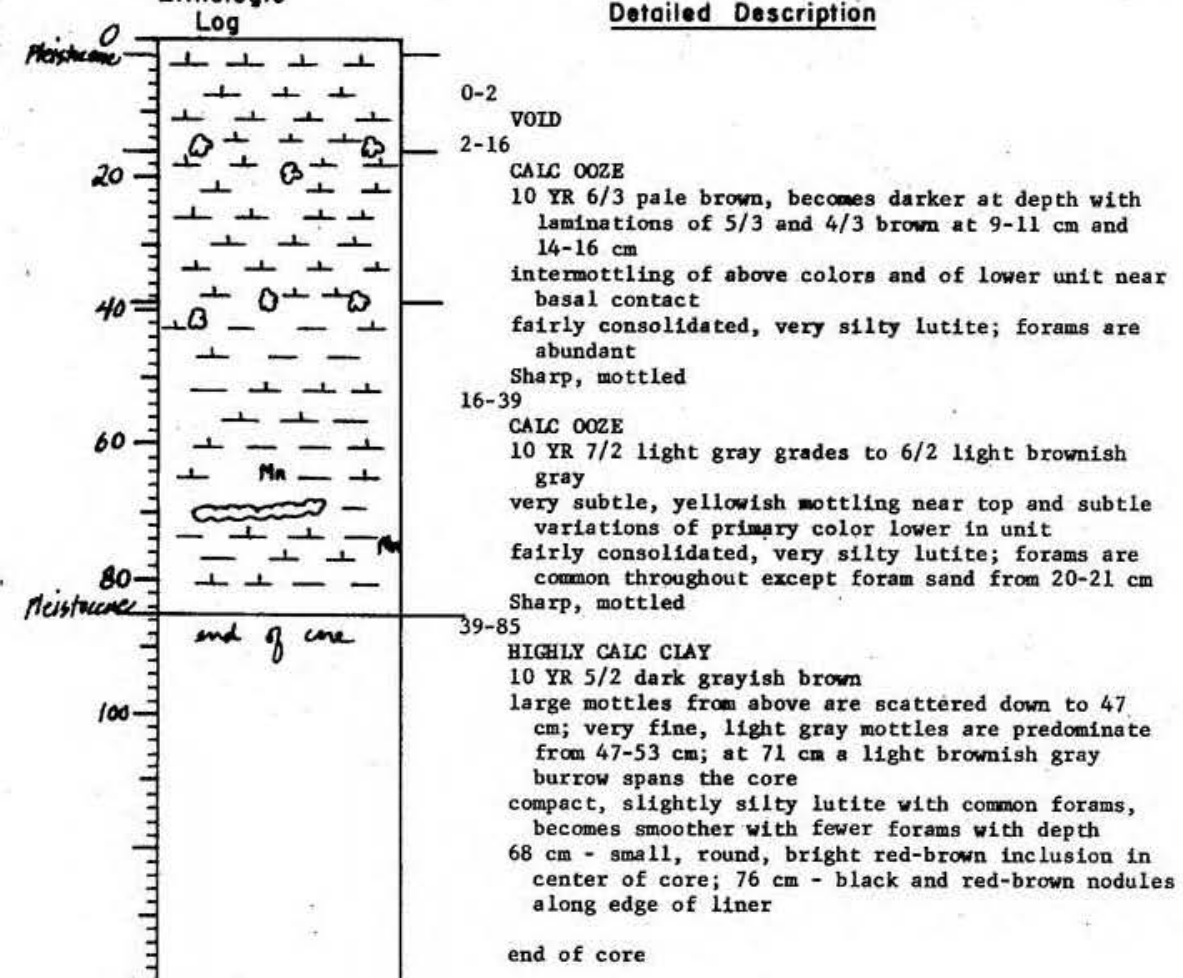

end of core
751

SMEAR SLIDE DESCRIPTIONS - W.H.0.I. SEDIMENT CORES

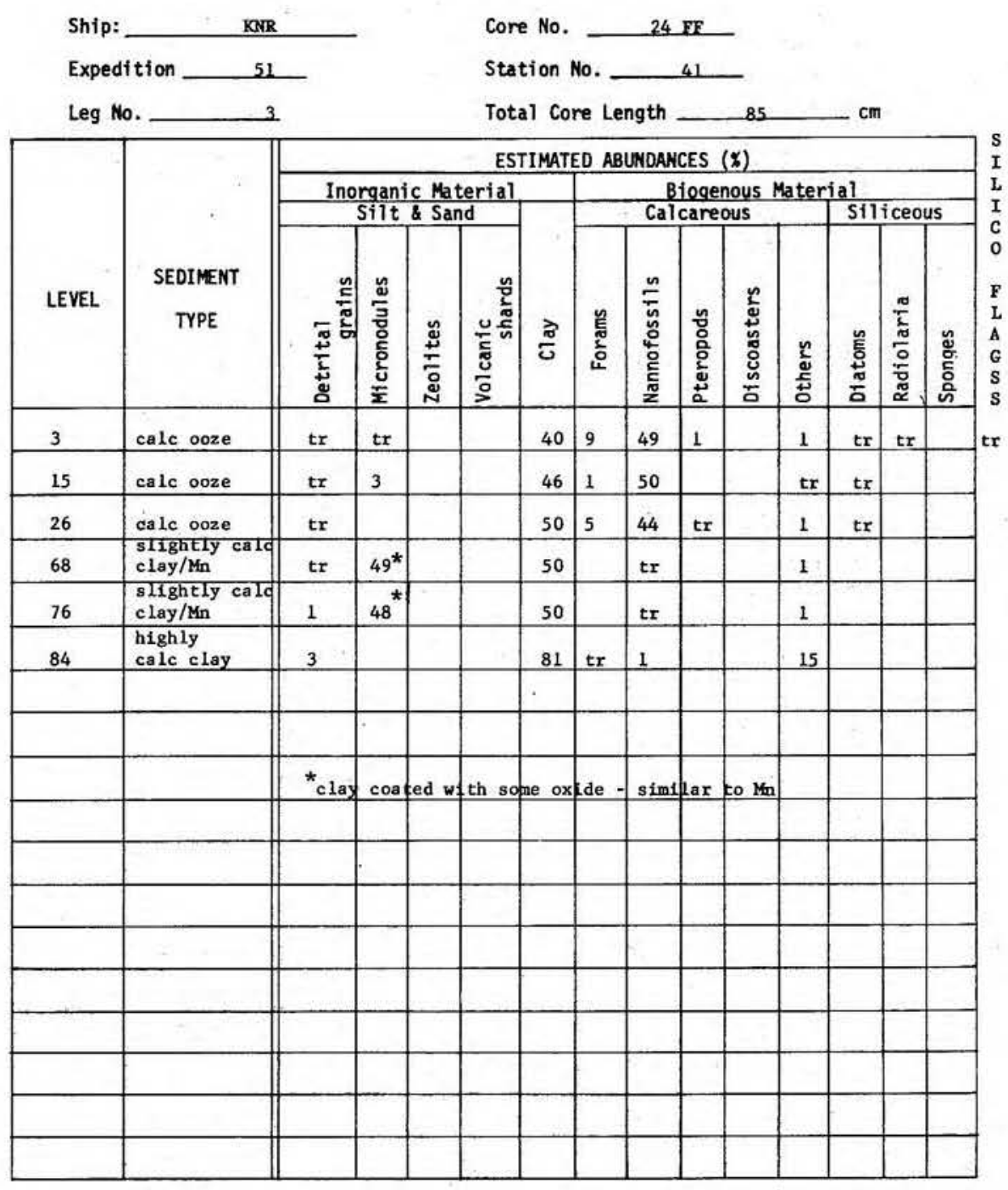


752

VISUAL CORE DESCRIPTION

Page 1 of 1
753

SMEAR SLIDE DESCRIPTIONS - W.H.O.I. SEDIMENT CORES

Ship KAORR Cruise 51 Leg III Sta. 41 Core No. $25 \mathrm{FE}$ Total Length 91 cm. Lat. $56^{\circ} 16.5 \mathrm{~W}$ Long. $12^{\circ} 331$ iw Depth $2956 \mathrm{mem}$

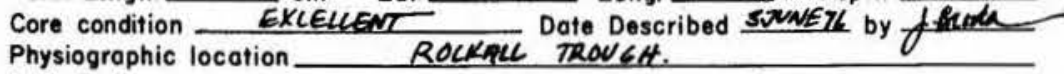

Lithologic

Detailed Description

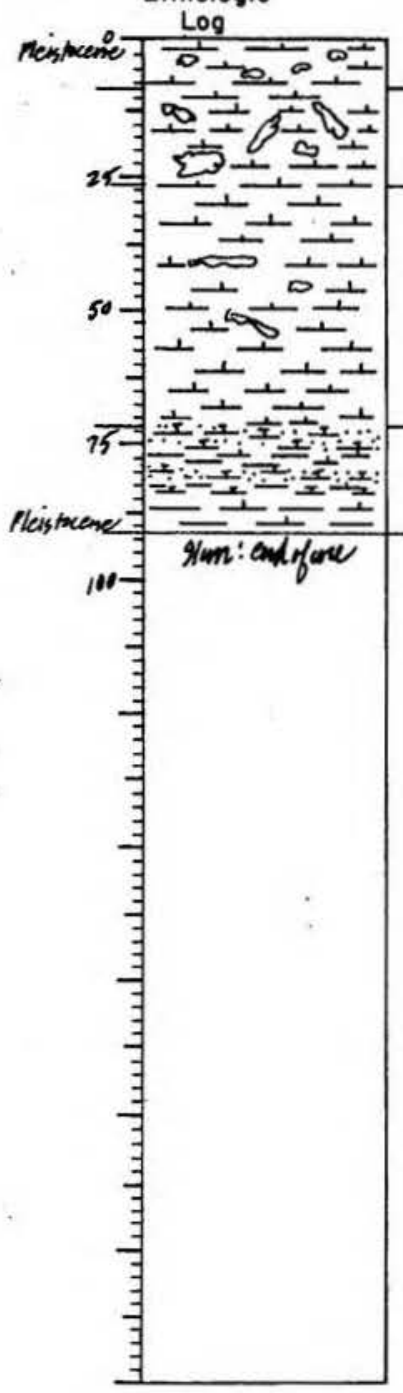

\section{CALC OOZE}

10 YR $6 / 3$ pale brown, grades to $5 / 2$ grayish brown

common intermottling of the two colors above

firm, silty lutite with abundant forams, especially in mottle pocket:

8-26

CALC OOZE

10 YR $7 / 2$ light gray, grades to $6 / 2$ light brownish gray extensive burrow-mottling $15-26 \mathrm{~cm}$. trated in burrows

CAIC OOZE WITH DETRITUS

10 YR $5 / 1$ gray ach spanning liner throughout

very slightly silty lutite with only a very Sharp, slightly inclined

$72-91$

CALC CLAY WITH ZONES OF CALC OOZE/DETRITUS 10 YR $5 / 1$ gray

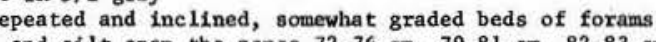
a the coarse at base to fine foram (fractured) sand at top

end of core few, faint, light brownish gray, elongated burrows
Ship: KNR

Expedition

Leg No. 51 3

Station No. 41

Total Core Length _ $\quad \mathrm{cm}$

\begin{tabular}{|c|c|c|c|c|c|c|c|c|c|c|c|c|c|c|}
\hline \multirow[b]{3}{*}{ LEVEL } & \multirow[b]{3}{*}{$\begin{array}{c}\text { SEDIMENT } \\
\text { TYPE }\end{array}$} & \multicolumn{13}{|c|}{ ESTIMATED ABUNDANCES $(\%)$} \\
\hline & & \multicolumn{4}{|c|}{$\begin{array}{c}\text { Inorganic Material } \\
\text { Silt \& Sand }\end{array}$} & \multirow[b]{2}{*}{$\frac{\pi}{\tilde{c}}$} & \multicolumn{5}{|c|}{$\begin{array}{l}\text { Biogenous Mater } \\
\text { Calcareous }\end{array}$} & \multicolumn{3}{|c|}{$\frac{\text { al }}{\text { Siliceous }}$} \\
\hline & & $\frac{\stackrel{\frac{n}{5}}{\frac{5}{5}}}{\frac{5}{5}}$ & 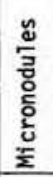 & 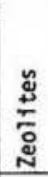 & $\begin{array}{l}\frac{n}{2} \\
\frac{\pi}{5} \\
\frac{\pi}{n} \\
\frac{5}{5} \\
\frac{5}{5}\end{array}$ & & 高 & 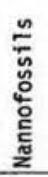 & 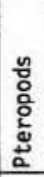 & 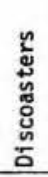 & 产 & 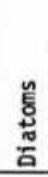 & $\begin{array}{l}\frac{\pi}{L} \\
\frac{\pi}{0} \\
\frac{\pi}{0} \\
\frac{\pi}{\alpha}\end{array}$ & 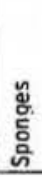 \\
\hline 1 & calc ooze & 2 & & & & 27 & 12 & 55 & & & 2 & 1 & tr & 1 \\
\hline 40 & $\begin{array}{l}\text { calc ooze } \\
\text { with detritus }\end{array}$ & 20 & & & & 25 & 3 & 15 & & & 35 & tr & & 2 \\
\hline 74 & $\begin{array}{l}\text { calc oozel } \\
\text { detritus }\end{array}$ & 30 & & & & 15 & 45 & 12 & & & 3 & & & \\
\hline 90 & calc clay & 4 & tr & & & 88 & tr & 2 & & & 6 & & & \\
\hline & & & & & & & & & & & & & & \\
\hline & & & & & & & & & & & & & & \\
\hline & & & & & & : & & & & & & & & \\
\hline & & & & & & & & & & & & & & \\
\hline & & & & & & & & & & & & & & \\
\hline & & & & & & & & & & & & & & \\
\hline & & & & & & & & & & & & & & \\
\hline & & & & & & & & & & & & & & \\
\hline & & & & & & & & & & & & & & \\
\hline & & & & & & & & & & & & & & \\
\hline & & & & & & & & & & & & & & \\
\hline & & & & & & & & & & & & & & \\
\hline & , & & & & & & & & & & & & & \\
\hline & & & & & & & & & & & & & & \\
\hline
\end{tabular}


VISUAL CORE DESCRIPTION

Page 1 of 1

Ship KNORR Cruise 51 Leg III Sta. 41 Core No. 26 FF Total Length $51 \mathrm{~cm}$. Lat. $56^{\circ} 16.6^{\prime N}$ Long. $12^{\circ} 34.0^{\prime}$ WDepth 2962 corer. $M$. Core condition EXCELLENT Date Described 2JumE 76 by H. Farmer Physiographic location RockAle TROCGH

Lithologic

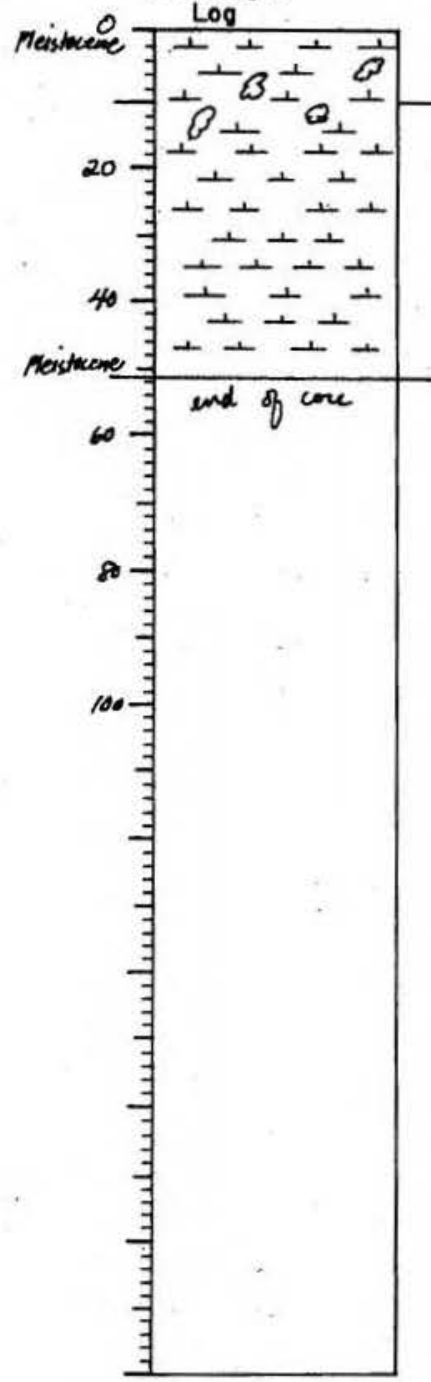

SMEAR SLIDE DESCRIPTIONS - W.H.O.I, SEDIMENT CORES

Ship:

Expedition

Leg No. CAIC OOZE

10 YR $6 / 3$ pale brown with distorted laminations of $5 / 3$ and $4 / 3$ brown $(3 \mathrm{~cm}, 5-6 \mathrm{~cm}, 9-10 \mathrm{~cm}$ )

from 5-10 and large, tube-11ke burrous irom $5-10$ cms

ite with numerous foram , somenhat mottled

CAIC OOZE

10 YR $6 / 2$ light brownigh gray

$8 \mathrm{~cm}$ and a few, very $\operatorname{sinall}$ mottles are scattered throughout ccmpact, silty lutite vith scattered forams; forans

end of core

\begin{tabular}{|c|c|c|c|c|c|c|c|c|c|c|c|c|c|c|}
\hline \multirow[b]{3}{*}{ LEVEL } & \multirow[b]{3}{*}{$\begin{array}{c}\text { SEDIMENT } \\
\text { TYPE }\end{array}$} & \multicolumn{13}{|c|}{ ESTIMATED ABUNDANCES ( $(\boldsymbol{\alpha})$} \\
\hline & & \multicolumn{4}{|c|}{$\begin{array}{c}\text { Inorganic Material } \\
\text { Silt \& Sand }\end{array}$} & & \multicolumn{5}{|c|}{$\begin{array}{l}\text { Biogenous Mater } \\
\text { Calcareous }\end{array}$} & \multicolumn{2}{|c|}{ S1liceous } & \\
\hline & & 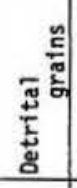 & $\begin{array}{l}\frac{0}{3} \\
\frac{\pi}{0} \\
\vdots \\
\frac{0}{0} \\
\frac{0}{\Sigma}\end{array}$ & 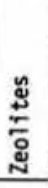 & 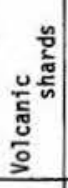 & $\frac{\pi}{6}$ & 产 & 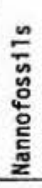 & 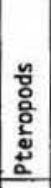 & 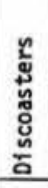 & $\begin{array}{l}\frac{n}{2} \\
\frac{5}{5} \\
\text { ț } \\
\end{array}$ & 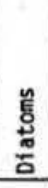 & $\frac{\frac{\pi}{2}}{\frac{\pi}{0}}$ & 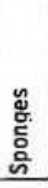 \\
\hline 1 & calc ooze & tr & 1 & & & 44 & 5 & 50 & & & tr & tr. & tr & \\
\hline 9 & calc ooze & tr & 1 & & & 53 & 3 & 43 & tr & & tr & $t r$ & tr & \\
\hline 17 & calc ooze & $\mathrm{tr}$ & $\operatorname{tr}$ & & & 40 & 6 & 53 & & & 1 & tr & $\mathrm{tr}$ & tr \\
\hline 50 & calc ooze & 1 & & & & 36 & 2 & 60 & & & 1 & & & tr \\
\hline & & & & & & & & & & & & & & \\
\hline & & & & & & & & & & & & & & \\
\hline & & & & & & . & & & & & & & & \\
\hline & & & & & & & & & & & & & & \\
\hline & & & & & & & & & & & & & & \\
\hline & & & & & & & & & & & & & & \\
\hline & & & & & & & & & & & & & & \\
\hline & & & & & & & & & & & & & & \\
\hline & & & & & & & & & & & & & & \\
\hline & & & & & & & & & & & & & & \\
\hline & & & & & & & & & & & & & & \\
\hline & & & & & & & & & & & & & & \\
\hline & & & & & & & & & & & & & & \\
\hline & & & & & & & & & & & & & & \\
\hline
\end{tabular}

Core No. 26 ng

Station No. 41

Total Core Length ESTIMATED ABUNDANCES ( 
Ship KNORR Cruise 51 Leg III Sta. 42 Core No. $31 \mathrm{GPC}$ Total Length $1296 \mathrm{~cm}$. Lat. $56^{\circ} 13.4^{\prime} \mathrm{N}$ Long. $12^{\circ} 38.0^{\prime} \mathrm{W}$ Depth $26 / 9$ Conem. Core condition EXCELCENT Dote Described \& MAY 76 by H. FORMER Physiogrophic location RockAll TKOUGH, NORTH ATLANTIC

Lithologic

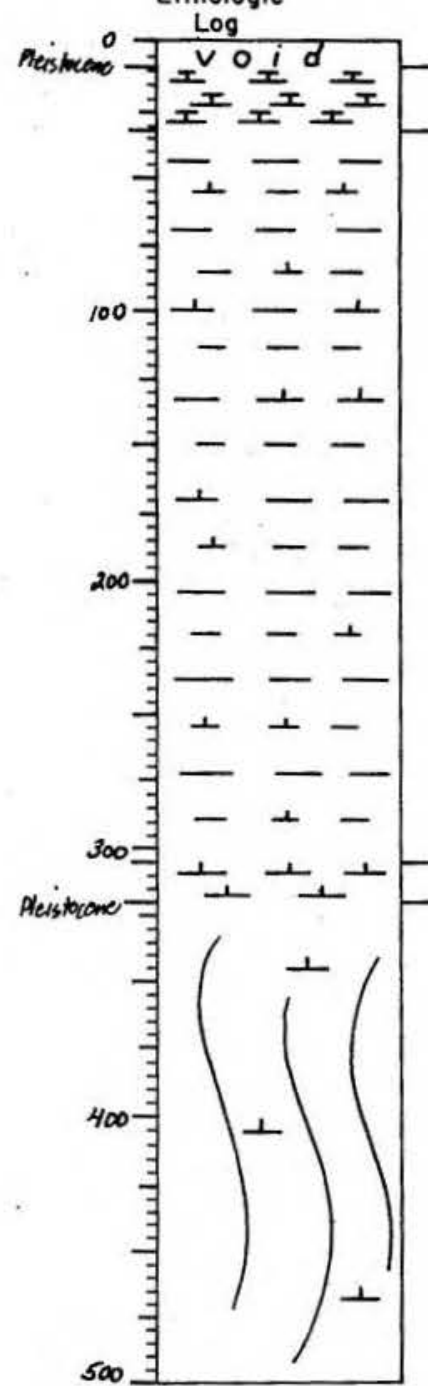

\section{Detailed Description}

\section{vorn}

CALC OOZE

10 YR $6 / 3$ pale brown

no mottling, but a few gray and dark gray laminations

Sharp, but non-planar (either disturbed or erosional)

$32-305$

MINA TIONS OP SLIGHTLY CALC CLAY (5 Y 4/2) AND

CALC CLAY $(5 / 2)$

varying hues of the above (olors appear as large band ( $5-20 \mathrm{~cm}$ vide); s1x 5 laminations (somewhat distorted) of 5 Y $6 / 2$ light olive gray are present between $37-48 \mathrm{~cm}$; light ollve gray also appears in a layer band from $278-281 \mathrm{~cm}$ (calc ooze)

mottling is scarce, but very fine olive gray (4/2) flecks show up in some of the 1ighter colored bands at $37-48 \mathrm{~cm}, 71-90 \mathrm{~cm}, 160-170 \mathrm{~cm}, 200-212 \mathrm{~cm}, 274-$ (1) $\mathrm{cm}$

ker bands are a smooth lutite with few, if any, forams, whereas the lighter ones tend to be silty tions) with common foram can be found; two beds of laminated, detrital silt and fine sand appear at $217-224 \mathrm{~cm}$ and $252-257 \mathrm{~cm}$ (1-2 cm crack has developed in this bed)

many or the contocts betopen banda are concave or S, distorted

NANNO O0Z

$5 \times 7 / 2$ 1ight gray and $6 / 2$ light olive gray, probably originated as distinct laminations but are severely mixed now

no mottling

very silty lutite

$320-1296$

flow-in of above unit

end of core

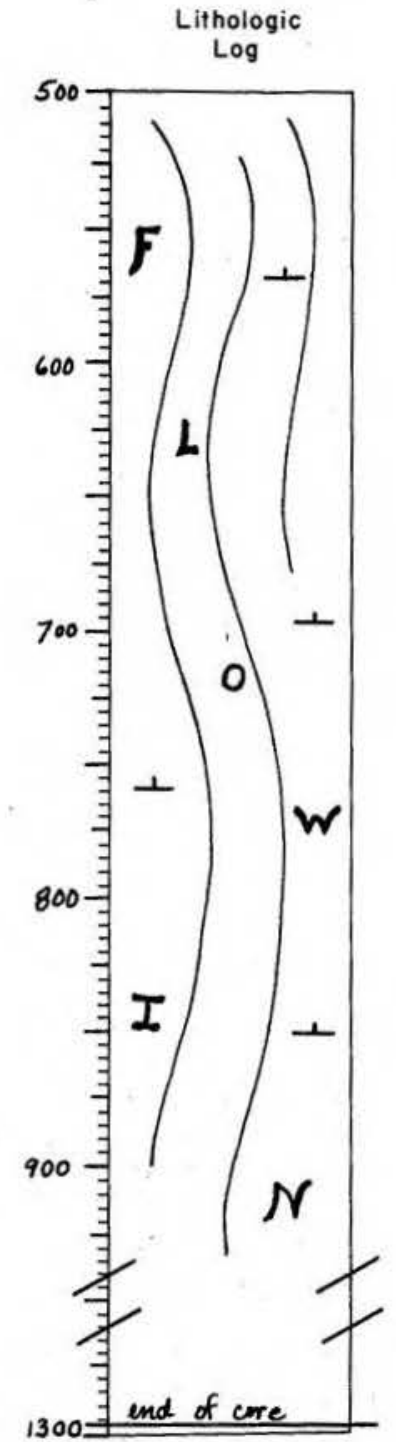

Page 2 of 2

Ship KNORR Cruise 51 Leg III Sto. 42 Core No. 31 GPC

Detailed Description 
SIEAR SLIDE DESCRIPTIONS - W.H.O.I. SEDIMENT CORES

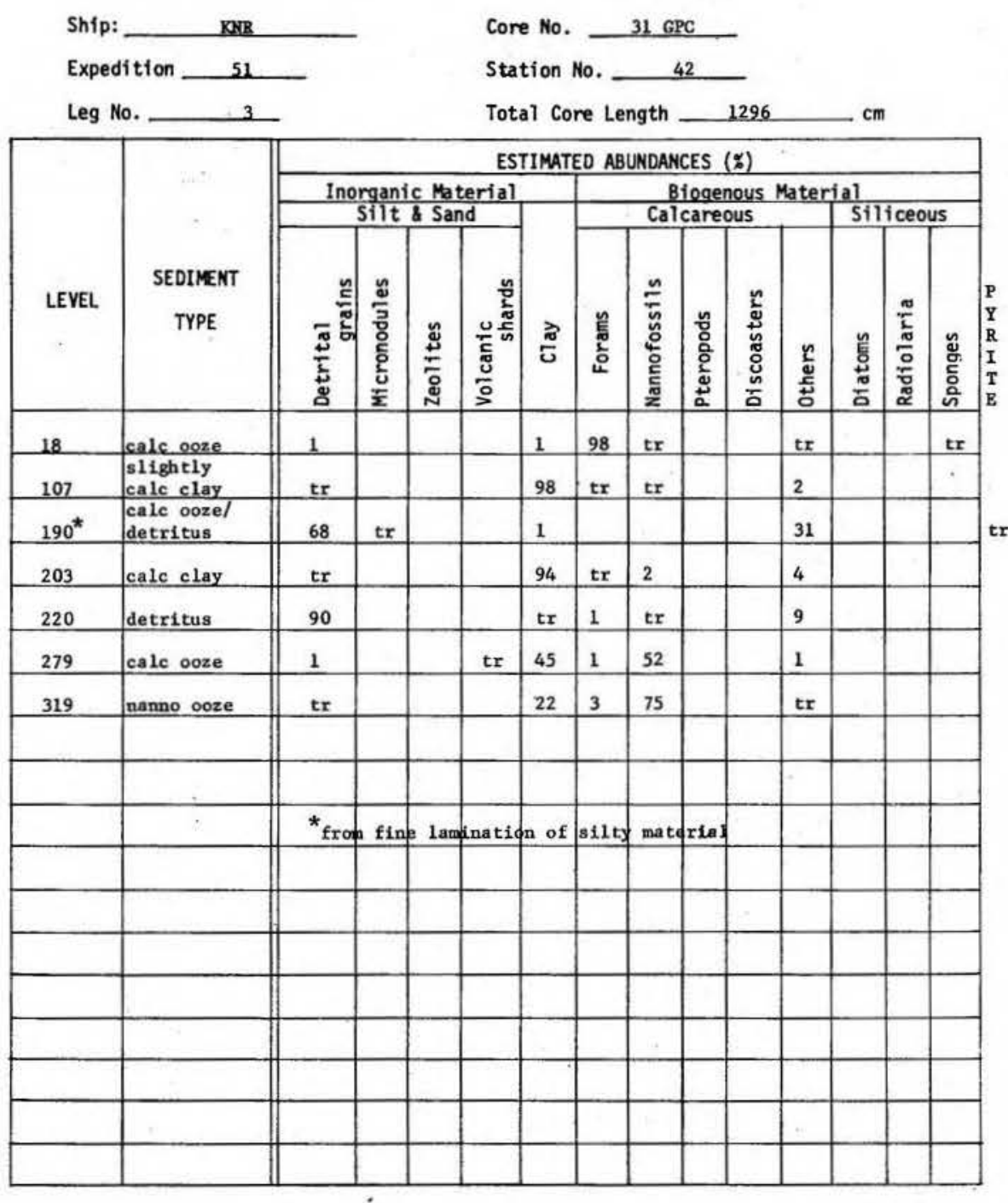

VISUAL CORE DESCRIPTION

Page 1 of 1

Ship KNORR Cruise 51 Leg II Sto. 42 Core No. 31 PG Total Length $124^{\circ} \mathrm{cm}$. Lat. $56^{\circ} 13.4^{\prime} \mathrm{N}$ Long. $12^{\circ} 38.0^{\prime}$ W Depth 2619 core.m. Core condition EXCELLENT Date Described 4 MAY 76 by H. FARMER

Physiographic location ROCKALL TROUGH, NORTH ATLANTIC

Lithologic

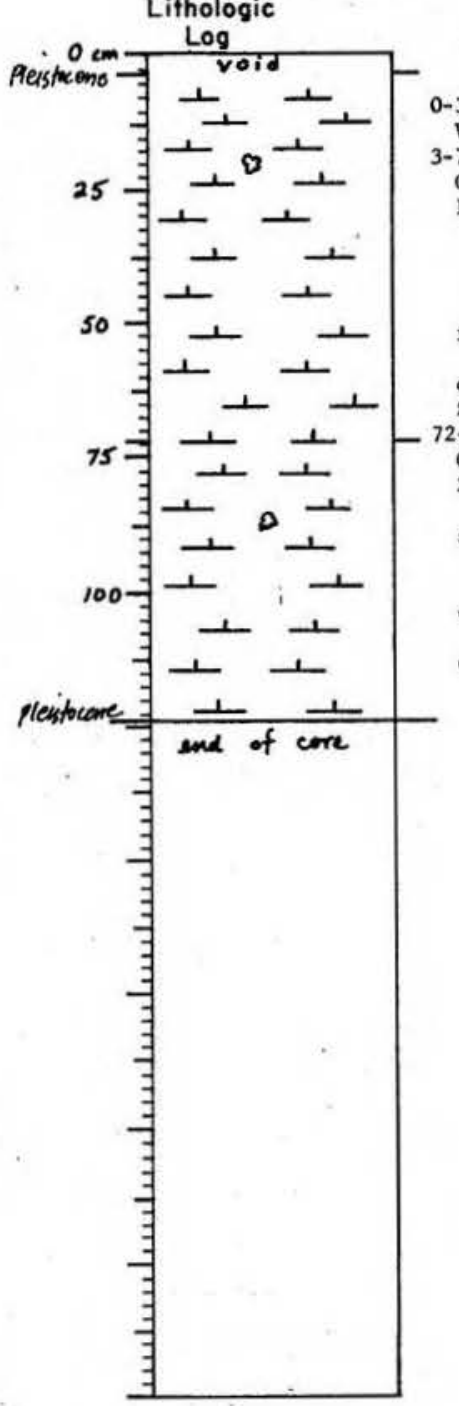

Detailed Description 0-3 $-72$

TR $6 / 3$ pale brown grades gently to $6 / 2$ 1ight brownIsh gray: in the basal $5 \mathrm{~cm}$, this grades sharply to 12 grayish brown and is marbled somewhat with the ediment above: two 5 mm laminations (slightly disturbed) at 6 and $8 \mathrm{~cm}$ are brown (5/3) intermottling from $19-25 \mathrm{~cm}$ compact, silty lutite S 124.5 (ATC 0078 2.5 Y $6 / 2$ light brownish gray grades to 5 Y $6 / 2$ light olive gray $(91 \mathrm{~cm})$ zone $(84-94 \mathrm{~cm})$ between the above colors; also two narrow brown laminations appear at 82 and $83 \mathrm{~cm}$ compact, silty lutite end of core in general, the colors are smooth, except for some 
SMEAR SLIDE DESCRIPTIONS - W.H.O.I. SEDIMENT CORES

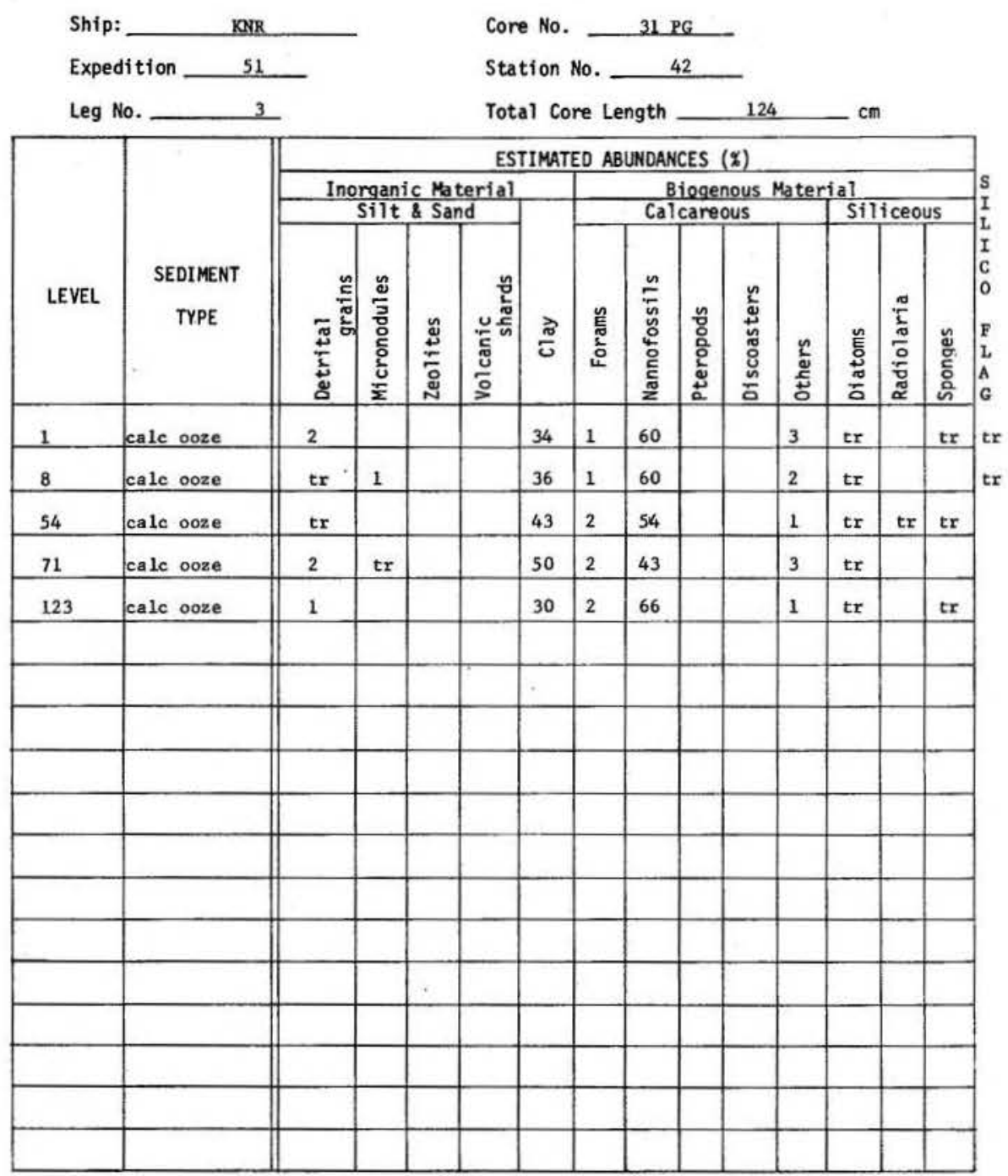




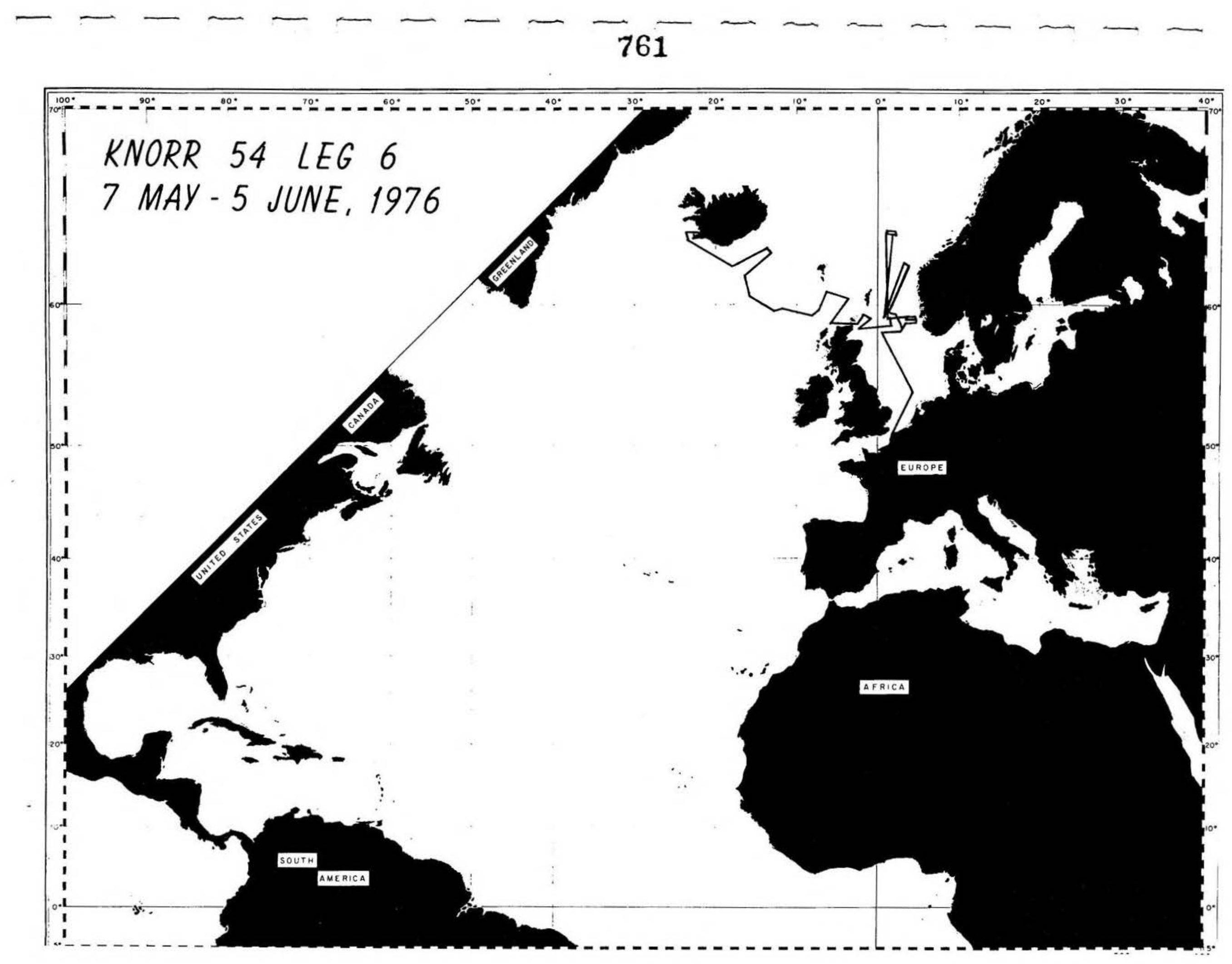


1.

I

I

1

I

1

1

1

I

I

1

I

I

1

1

I

1 


\section{2}

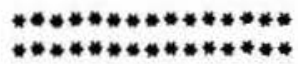

STATION DATA RETRIEVAL

***************

PAGE 1 DATE: 17120 JUN 03,177

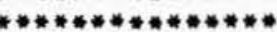

* WHOI **

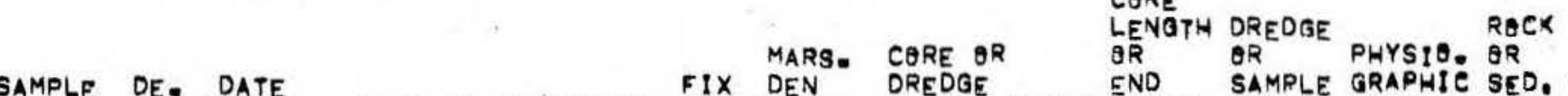

SHIP CRUISE LEG STATION NUMBER VICE YRMODA LATITUDE LONGITUDE TYPE SQUARE NUMBER DEPTH DEPTH VOLUME PROV. TYPE COOE REMARKS

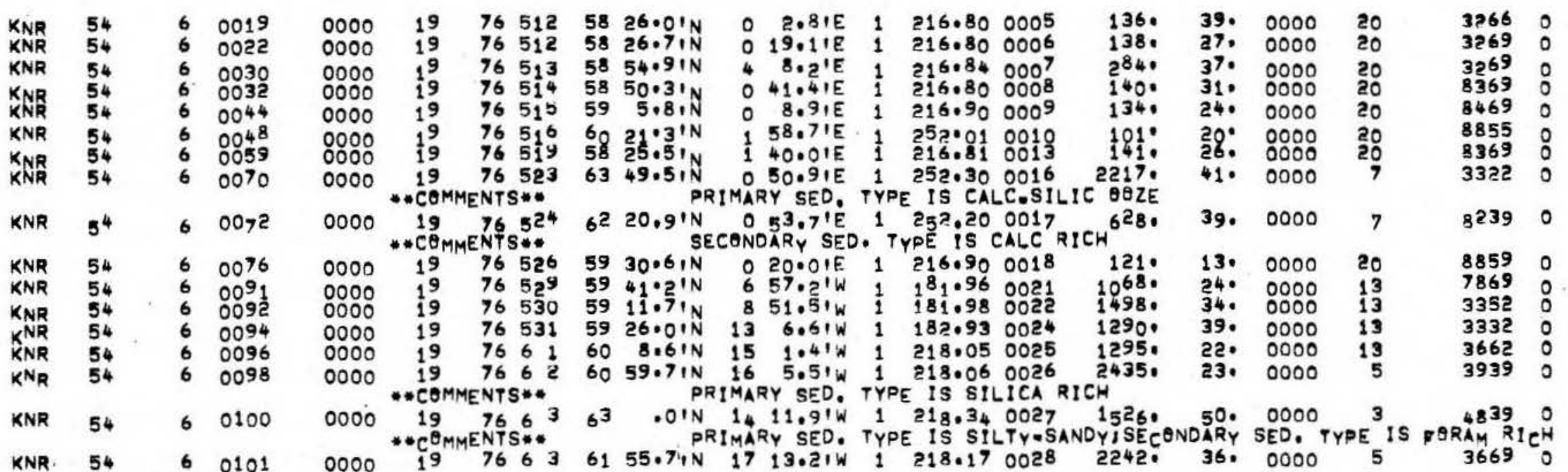


I

1

I

1

1

1

1

1

1

1

I

1

I

1

1

I

1

1

1 


\section{3}

VISUAL CORE DESCRIPTION

Page 1 of 1

Ship KNORR Cruise 54 Leg 6 Sto. $\frac{19}{6}$ Core No. $5 \cdot 8 \mathrm{C}$ Total Length $39 \mathrm{~cm}$. Lat. $58^{\circ} 26^{\prime} \mathrm{N}$ Long. $R^{\circ} 2.8^{\prime} \mathrm{K}$ Depth $136 \mathrm{carr}$ metions Core condition Excewent Date Described 30 Nav 76 by H. Ferears

Physiographic location N__ Nexty SEA Lithologic

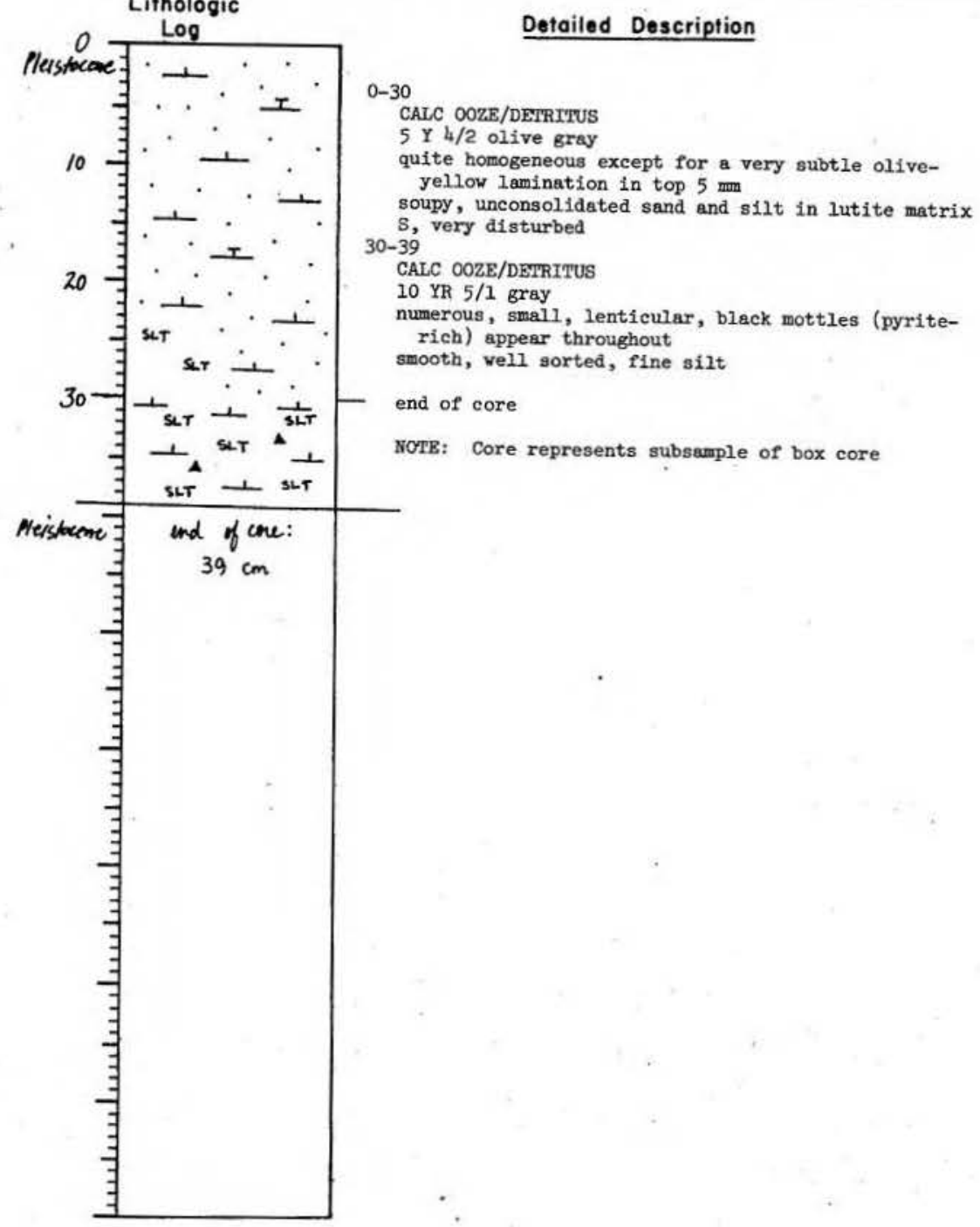

764

SMEAR SLIDE DESCRIPTIONS - W.H.O.I. SEDIMENT CORES

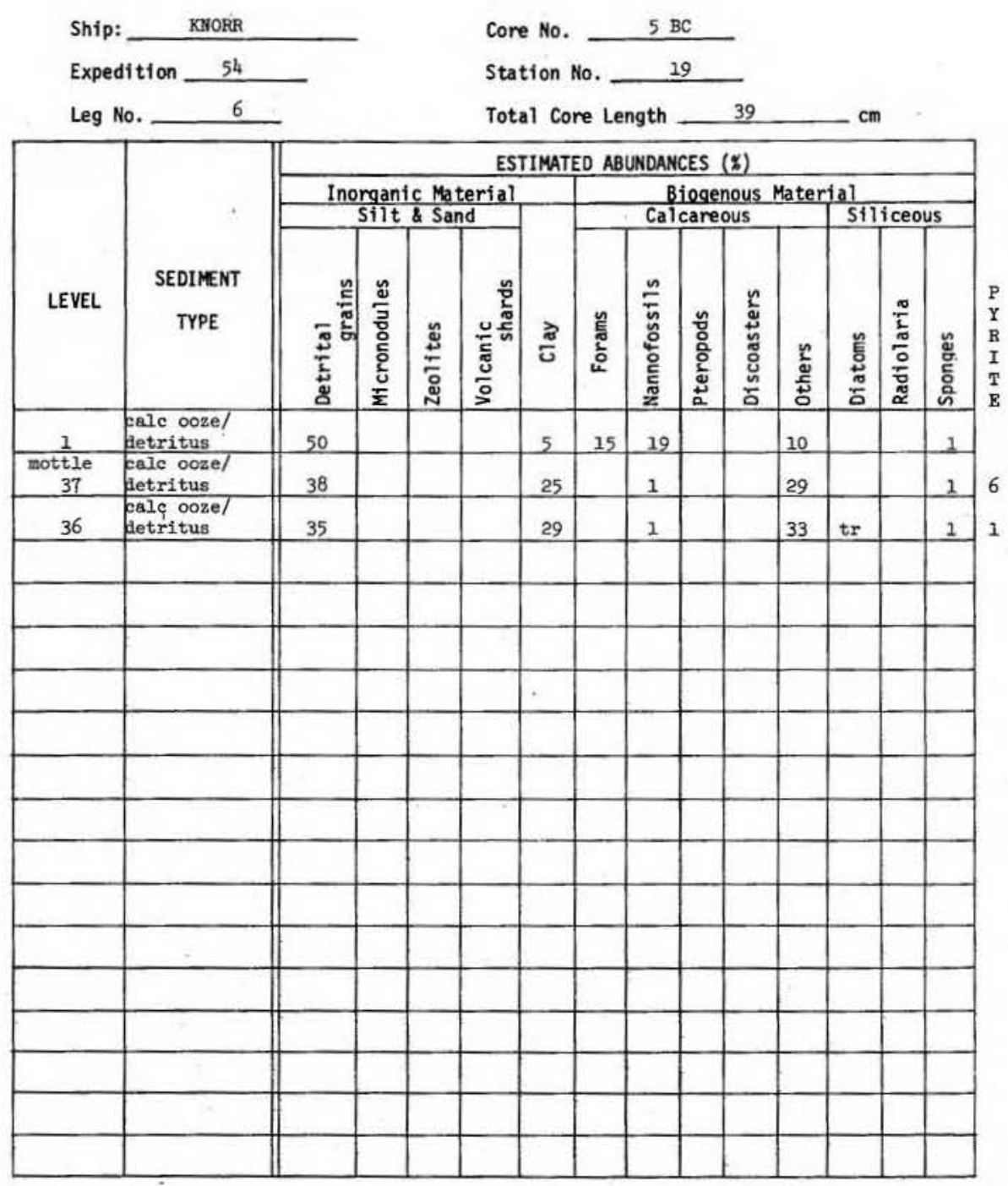


765

VISUAL CORE DESCRIPTION

Page 1 of 1

Ship KNORR Cruise 54 Leg $\frac{6}{7}$ Sta. $\frac{22}{0^{\circ}}$ Core No. $\frac{6-B C}{138}$ Total Length $27 \mathrm{~cm}$. Lat. $58^{\circ} 26.7^{\circ} \mathrm{N}$ Long. $0^{\circ} 19.1^{\prime} \mathrm{E}$ Depth 138 corr.meters Core condition EXCELLENT Date Described $30 \mathrm{NW} 76$ by $\mathrm{H}$. Freancs

Physiographic location NoRTH SEA

Lithologic

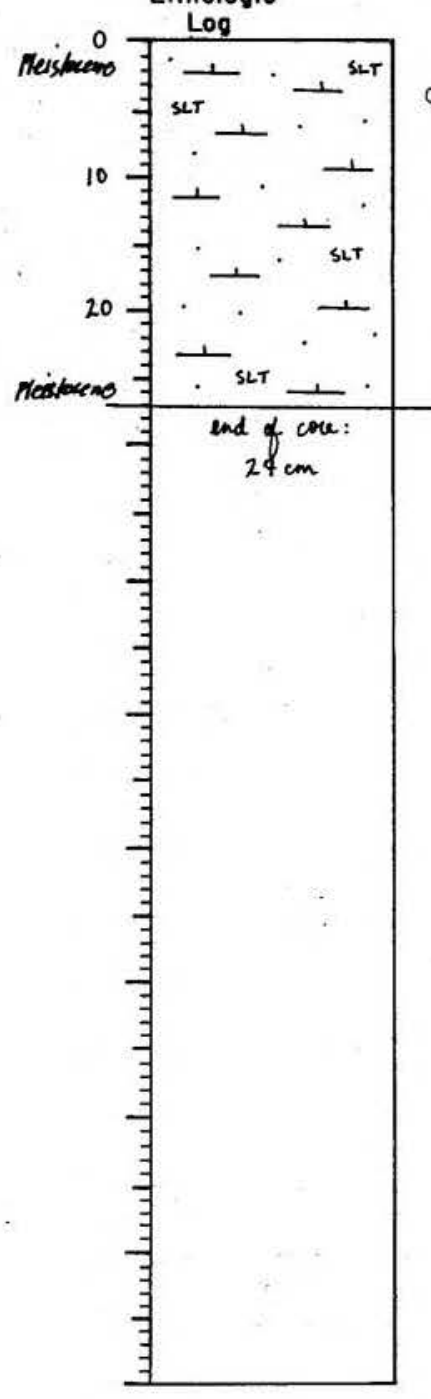

\section{Detailed Description}

\section{CALC OOZE/DETRITUS}

ray

quite homogeneous excepting a very subtle, olive-yellow lamination in the top 5 ma

silt in lutite matrix

end of core

NOTE: Core represents subsample of box core
SMEAR SLIDE DESCRIPTIONS - W.H.0.I. SEDIMENT CORES

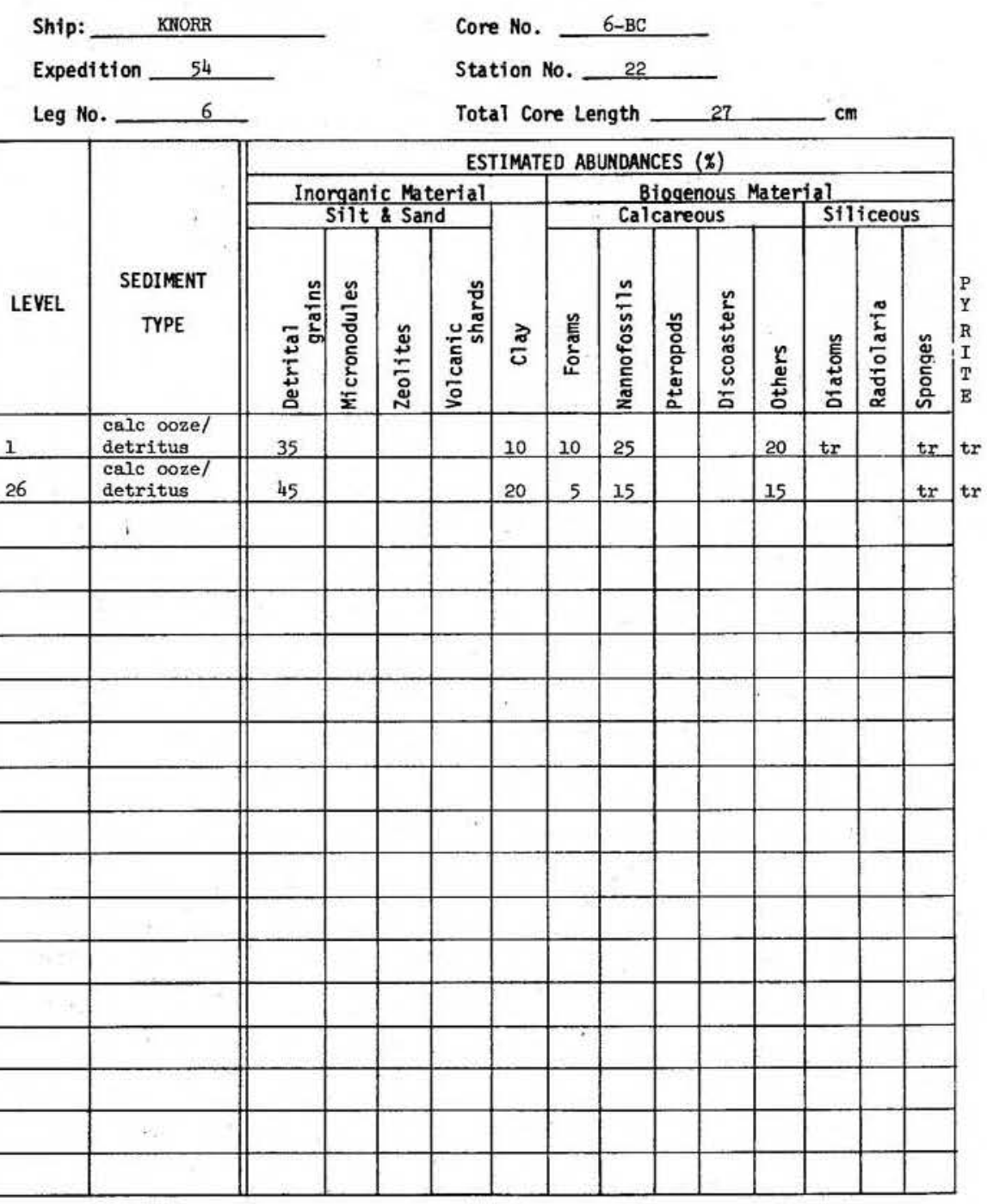


VISUAL CORE DESCRIPTION

Ship KNoRR Cruise 54 L $30 \mathrm{~A}$ Core No $7 . \mathrm{BC}$ Total Length $37 \mathrm{~cm}$. Lat. $58^{\circ} 54^{\circ} 9^{\prime} \mathrm{N}$ Long. $4^{\circ} 08.2^{\prime} E$ Depth 284 corr. mekers Core condition ExcELENT Date Described 30 Ner 76 by 14 . Fremere. Physiogrophic location NoRTA SEA

Lithologic

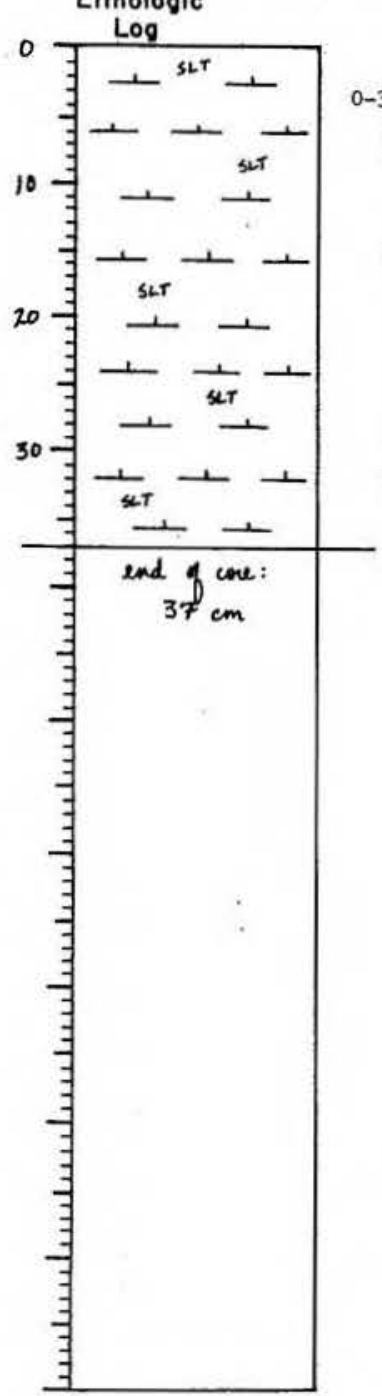

Detailed Description

$0-37$

CALC OOZE WITH DETRITUS

grayish brown grades to 5 Y $3 / 2$ dark $(\sim 3 \mathrm{~cm})$, then $4 / 2$ olive grey $(\sim 10 \mathrm{~cm})$
ation of very dark grayish brown $(10 \mathrm{YR}$ 3/2) st $0.5 \mathrm{~cm}$

soft, unconsolidated, very silty/sandy lutite; very small, ellipsoidal pellets or a composition apparentiy ldentical (?) to the primary sediment are abundant throughout; also, pumerous, very pine, transparent spicules are scattered throughout the

end of core

NOTE: Core represents subsemple of box core
SMEAR SLIDE DESCRIPTIONS - W.H.O.I. SEDIMENT CORES

Ship:

KMORR

Expedition 54

Leg No. 6

Station No. 30 A

Total Core Length

\begin{tabular}{|c|c|c|c|c|c|c|c|c|c|c|c|c|c|c|}
\hline \multirow[b]{3}{*}{ LEVEL } & \multirow[b]{3}{*}{$\begin{array}{c}\text { SEDIMENT } \\
\text { TYPE }\end{array}$} & \multicolumn{13}{|c|}{ ESTIMATED ABUKDANCES ( $(x)$} \\
\hline & & \multicolumn{4}{|c|}{$\begin{array}{c}\text { Inorganic Material } \\
\text { Silt \& Sand }\end{array}$} & \multirow[b]{2}{*}{ సे } & \multicolumn{5}{|c|}{$\frac{\text { Biogenous Mate }}{\text { Calcareous }}$} & \multicolumn{3}{|c|}{$\frac{\text { al }}{\text { Sfliceous }}$} \\
\hline & & $\frac{5}{\frac{5}{\pi}}$ & 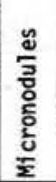 & 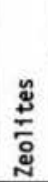 & $\frac{\sqrt{2}}{\frac{2}{2}}$ & & 哀 & 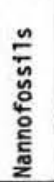 & 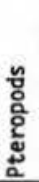 & 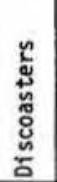 & $\frac{c}{\frac{c}{2}}$ & 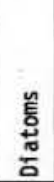 & 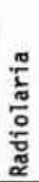 & $\begin{array}{l}\text { ㅎ․ } \\
\text { 음 }\end{array}$ \\
\hline .5 & $\begin{array}{l}\text { highly cale of } \\
\text { with detritus }\end{array}$ & 20 & $2^{*}$ & & & 57 & & 1 & & & 20 & tr & & tr \\
\hline 2.5 & calc ooze & 10 & & & & 45 & 2 & 3 & & & 40 & & & tr \\
\hline 5 & $\begin{array}{l}\text { calc ooze with } \\
\text { det'ritus }\end{array}$ & 20 & & & & 25 & 2 & 12 & & & 35 & & & 5 \\
\hline 36 & $\begin{array}{l}\text { calc ooze with } \\
\text { detritus }\end{array}$ & 20 & & & & 43 & & 15 & & & 20 & tr & & 2 \\
\hline & & & & & & & & & & & & & & \\
\hline & & & & & & & & & & & & & & \\
\hline & & & & & & . & & & & & & & & \\
\hline & & & & & & & & & & & & & & \\
\hline & & & $*$ & $\mathrm{yth}$ & e & & $y$ & & & or & F & & & \\
\hline & & & & & & & & & & & & & & \\
\hline & & & & & & & & & & & & & & \\
\hline & & & & & & & & & & & & & & \\
\hline & & & & & & & & & & & & & & \\
\hline & & & & & & & & & & & & & & \\
\hline & & & & & & & & & & & & & & \\
\hline & & & & & & & & & & & & & & \\
\hline & & & & & & & & & & & & & & \\
\hline & & & & & & & & & & & & & & \\
\hline
\end{tabular}


Ship KNops Cruise 54 Leg 6 Sta. $\frac{32}{2}$ Core No. $\frac{8-8 C}{140}$ Total Length 31 cm. Lat. $58^{\circ} 50.3^{\prime} \mathrm{N}$ Long. $0^{\circ} 41^{\prime} 4^{\prime} \mathrm{E}$ Depth 140 com meters Core condition ExcELLENT Date Described I Dec 76 by Al. FARTER

Physiographic location NORTH SEA

Lithologic

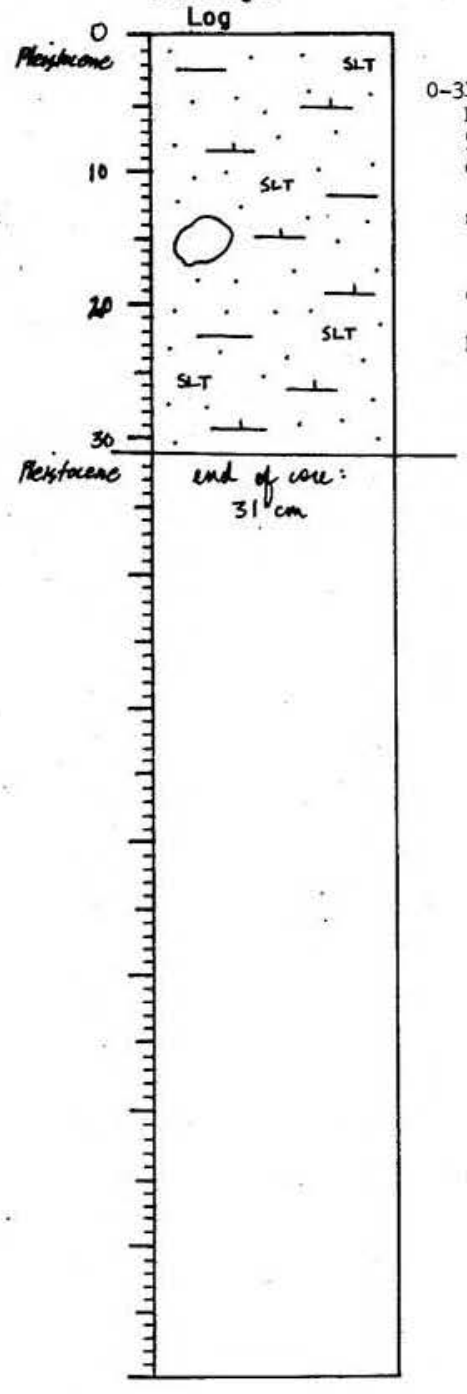

Detailed Description

$0-31$

HIGHLY CALC CLAY/DETRITUS

5 Y $4 / 2$ olive gray

quite hamogeneous excepting a very subtle, $3 \mathrm{~mm}$, olive-

yellow lamination at $0.5 \mathrm{~cm}$

and silt in lutite

;arge, $2 \mathrm{~cm}$ diameter, rounded stone at $16 \mathrm{~cm}$

end of core

NOTE: Core represents subsample of box core

SUEAR SLIDE DESCRIPTIONS - W.H.O. SEDINENT CORES

Ship:

Expeditio

Leg No. 54 6

\begin{tabular}{|c|c|c|c|c|c|c|c|c|c|c|c|c|c|c|}
\hline \multirow[b]{4}{*}{ LEVEL } & \multirow[b]{4}{*}{$\begin{array}{c}\text { SEDIMENT } \\
\text { TYPE }\end{array}$} & \multicolumn{13}{|c|}{ ESTIMATED ABUNDANCES (\%) } \\
\hline & & \multirow{2}{*}{\multicolumn{4}{|c|}{$\begin{array}{c}\text { Inorganic Material } \\
\text { Siit \& Sand }\end{array}$}} & \multirow[b]{3}{*}{$\frac{\pi}{\omega}$} & \multirow{2}{*}{\multicolumn{5}{|c|}{$\begin{array}{l}\text { Biogenous Mater } \\
\text { Calcareous }\end{array}$}} & \multirow{2}{*}{\multicolumn{3}{|c|}{ stliceous }} \\
\hline & & & & & & & & & & & & & & \\
\hline & & 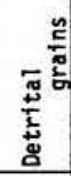 & 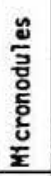 & 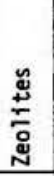 & 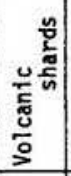 & & 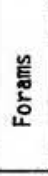 & 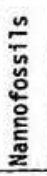 & 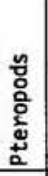 & 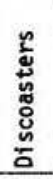 & 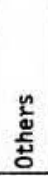 & 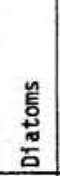 & $\frac{\pi}{\frac{\pi}{\pi}}$ & 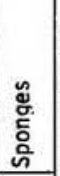 \\
\hline 1 & $\begin{array}{l}\text { highly calc } \\
\text { cley/detritus }\end{array}$ & 65 & & & & 10 & 5 & 10 & & & 10 & tr & & tr \\
\hline \multirow[t]{2}{*}{30} & $\begin{array}{l}\text { highly calc } \\
\text { cley/detritus }\end{array}$ & 65 & & & & 12 & 5 & 8 & & & 10 & & & tr \\
\hline & i & & & & & & & & & & & & & \\
\hline & . & & & & & & & & & & & & & \\
\hline & & & & & & & & & & & & & & \\
\hline & & & & & & & & & & & & & & \\
\hline & & & & & & . & & & & & & & & \\
\hline & & & & & & & & & & & & & & \\
\hline & & & & & & & & & & & & & & \\
\hline & & & & & & & & & & & & & & \\
\hline & & & & & & & & & & & & & & \\
\hline & & & & & & & & & & & & & & \\
\hline & & & & & & & & & & & & & & \\
\hline & & & & & & & & & & & & & & \\
\hline & & & & & & & & & & & & & & \\
\hline & - & & & & & & & & & & & & & \\
\hline & & & & & & & & & & & & & & \\
\hline & & & & & & & & & & & & & & \\
\hline
\end{tabular}

Core No. 8-BC

Station No. 32

Station No.

Total Core Length

ESTIMATED ABUNDANCES (\%)

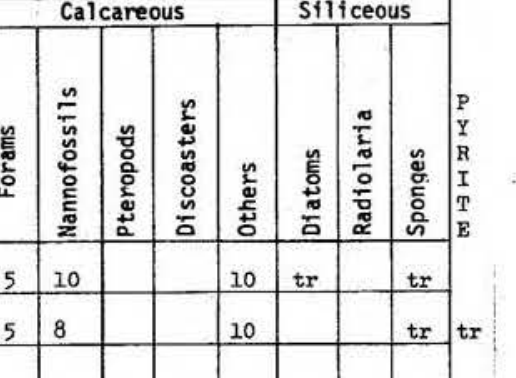
.

. 
771

VISUAL CORE DESCRIPTION

Page 1 of 1

Ship KNORR Cruise 54 Leg 6 Sta. 44 Core No. 9- BC Total Length $24 \mathrm{~cm}$. Lat. $59^{\circ} 5.8^{\prime} \mathrm{N}$ Long. $0^{\circ} 8.9^{\prime} \mathrm{E}$ Depth $\frac{134 \text { cerr. meter }}{14}$ Core condition Exceusent Date Described IDec 76 by H. Fiemge Physiographic location NorTh SEA

Lithologic

Detailed Description

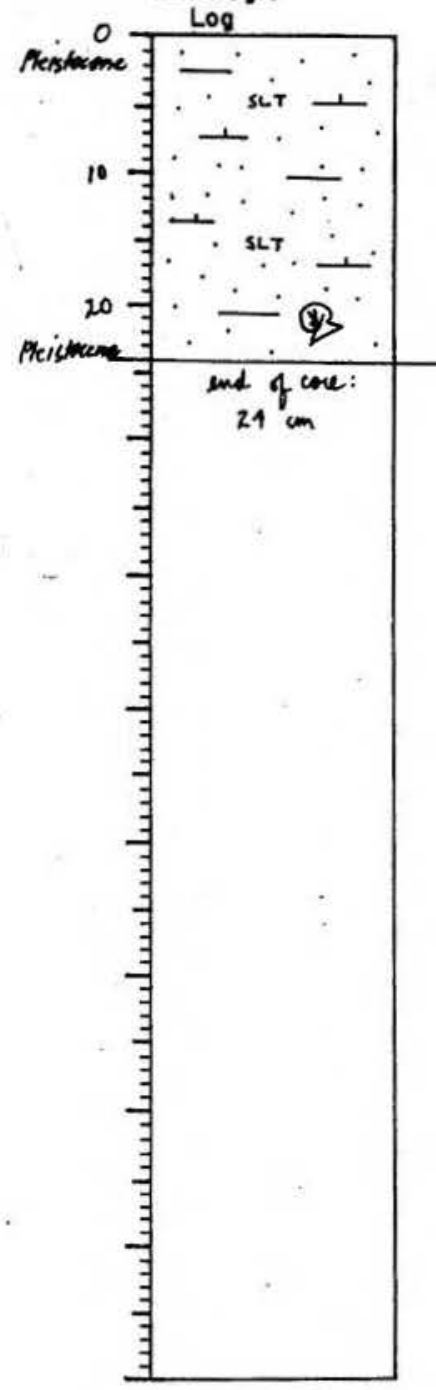

$0-24$

HIGHLY CALC CLAY/DETRTTUS

5 Y $4 / 2$ olive gray

soft, unconsolidated, fine sand and silt in lutite

matrix; large, vhite frapments of bivalve at $21 \mathrm{~cm}$

end of core

NOTE: Core represents subsample of box core

772

SMEAR SLIDE DESCRIPTIONS - W.H.O.I. SEDINENT CORES

Ship:

Expedition

Leg No.
Core No. 9-BC

Station No. L

Total Core Length

ESTIMATED ABUWDWICES ( $($ )

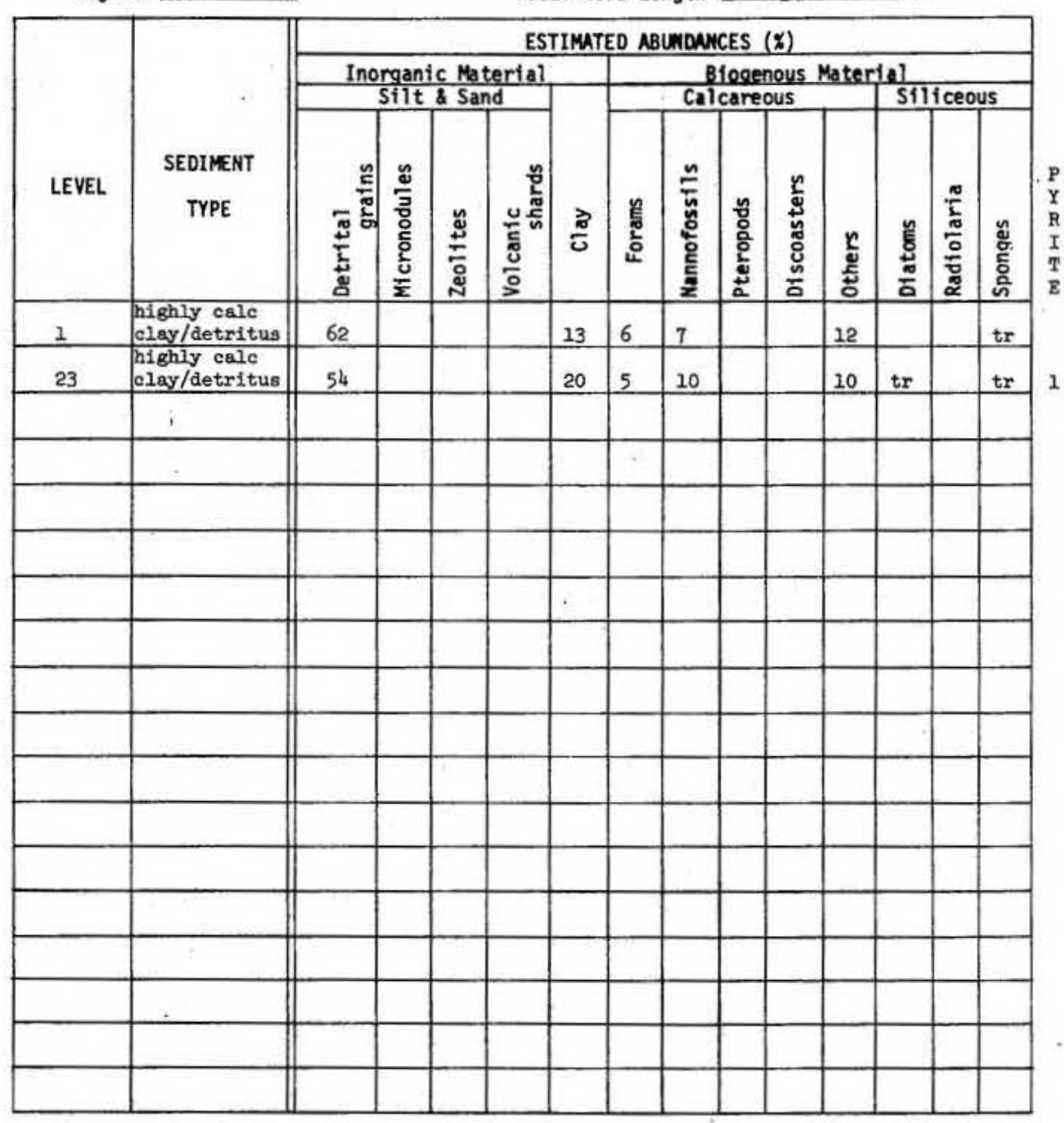


773

VISUAL CORE DESCRIPTION

Page 1 of 1

Ship KNORR Cruise 54 Leg 6 Sta. 48 Core No. $10-8 \mathrm{C}$

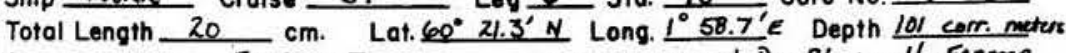

Core condition EXCELLENT Date Described Dec 76 by H, FAerexR

Physiographic location NoRTh SEA

Lithologic

Detailed Description

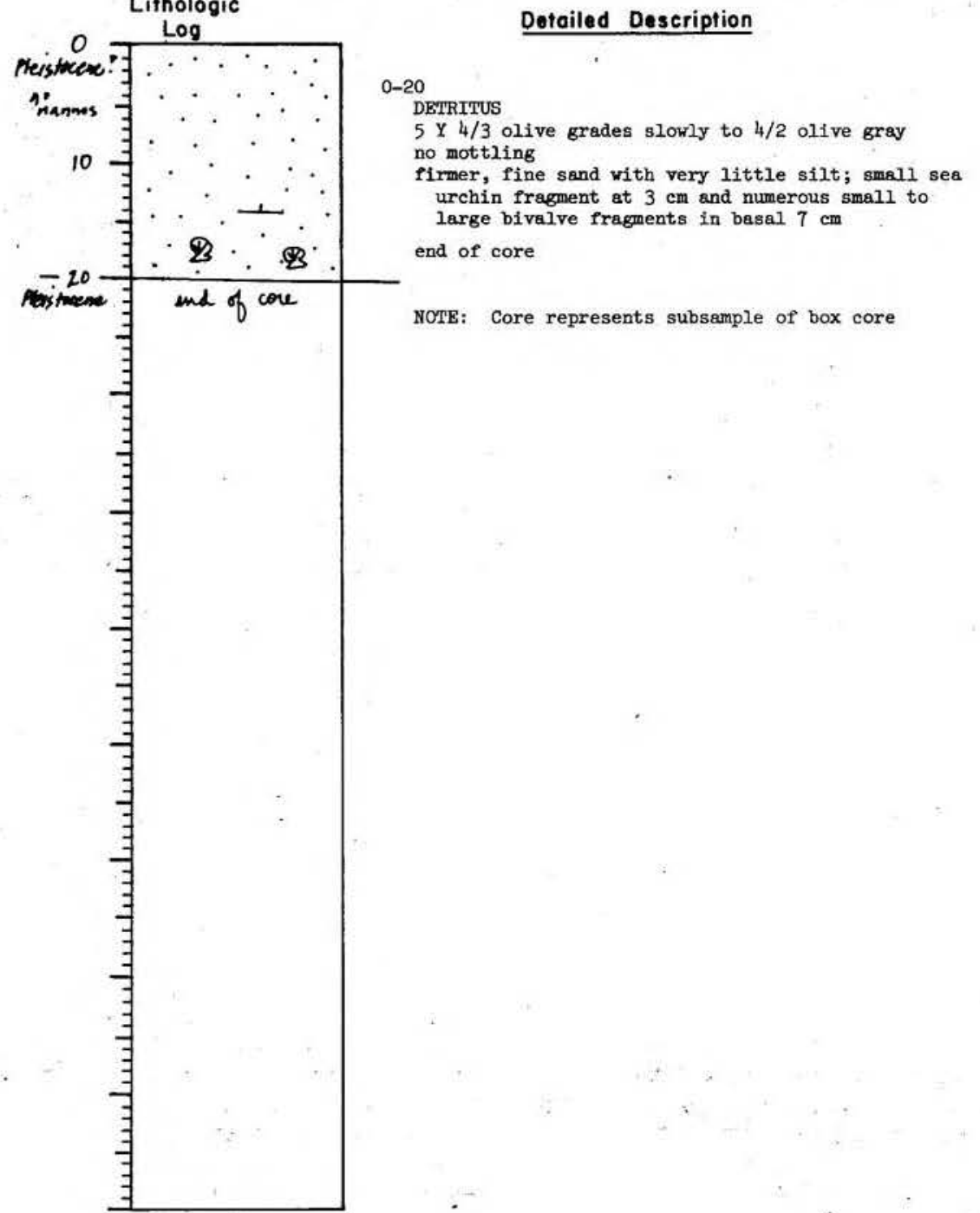

774

SMEAR SLIDE DESCRIPTIONS - W.H.O.I. SEDINENT CORES

Ship: _ _ KNORR

Expedition 54

Leg No. 6

ESTIMATED ABUNDANCES (q)

Core No. 10 BC

Station No. 48
Total Core Length _ 20

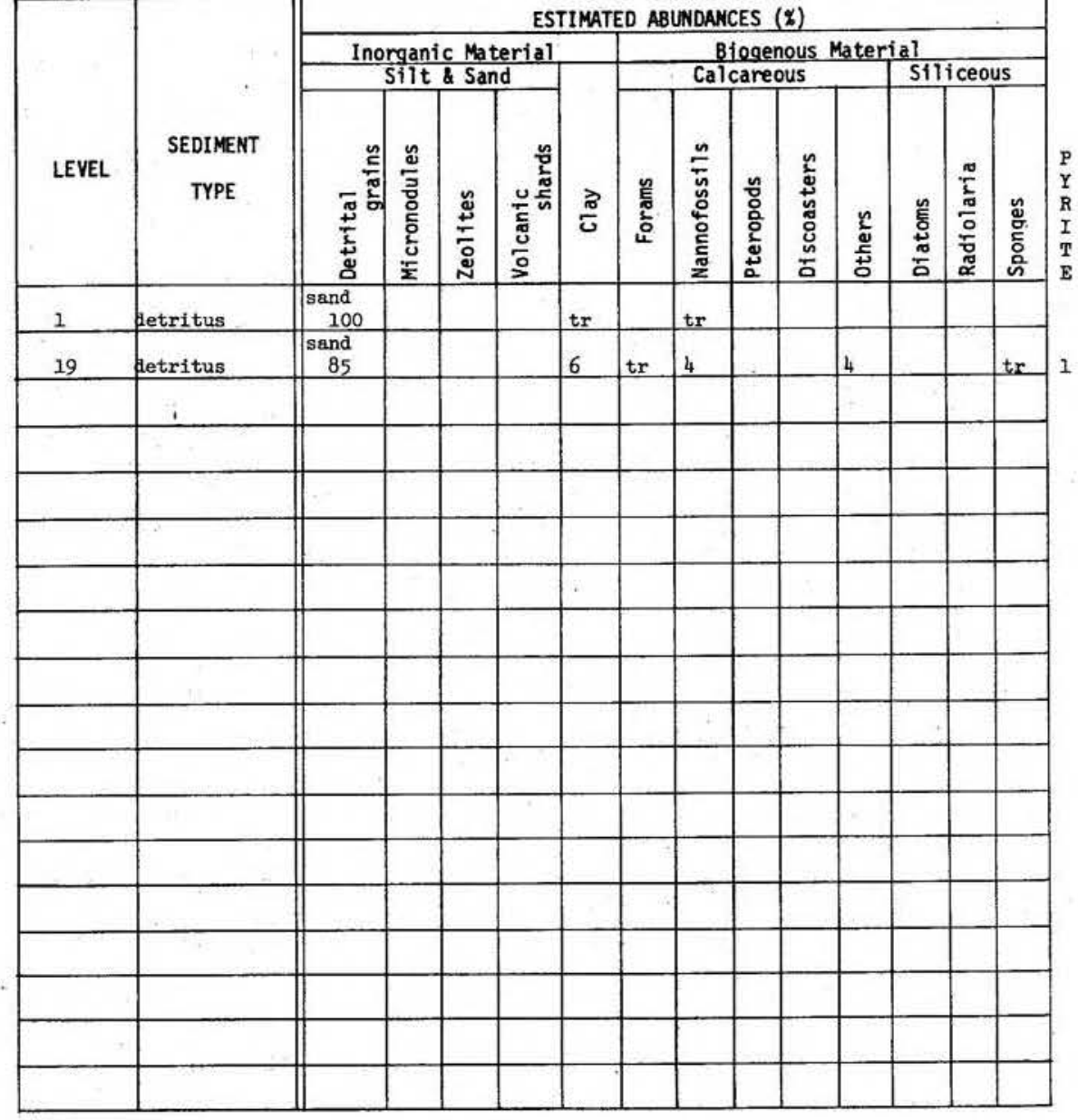


775

VISUAL CORE DESCRIPTION

Page 1 of 1

Ship KNesR Cruise 54 Leg 6 Sta. 59 Core No. 13 - BC Total Length $26 \quad \mathrm{~cm}$. Lat. $58 \cdot 25.5^{\prime} \mathrm{N}$ Long. $20.0^{\prime} \mathrm{E}$ Depth 141 corr, meters

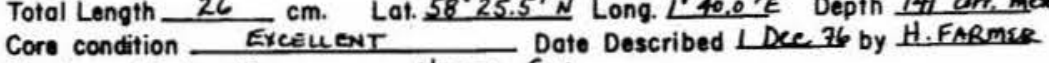
Physiographic location NORTH SEA

Detailed Description

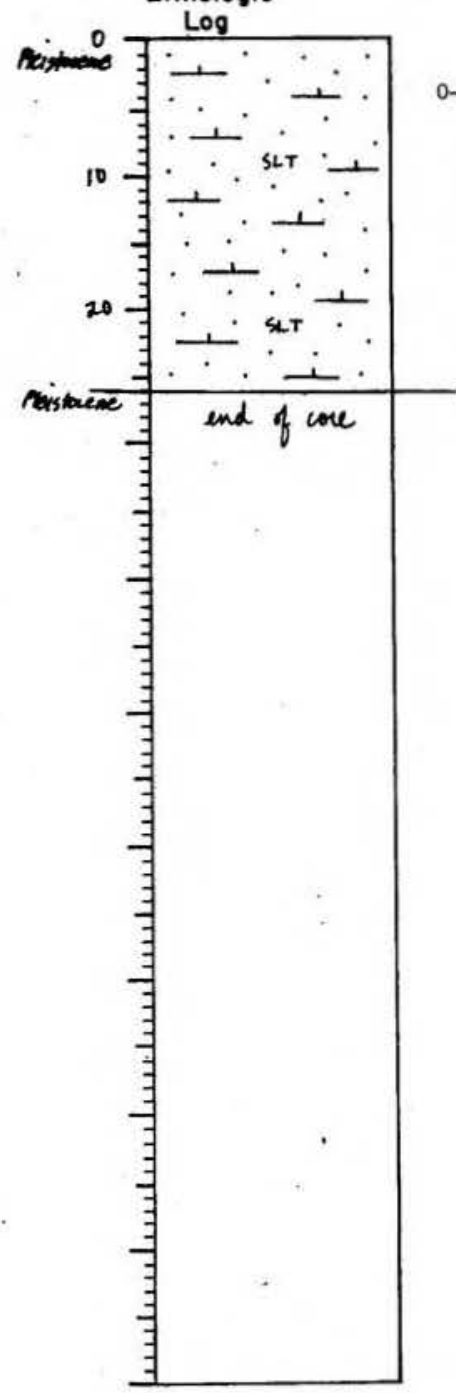

$0-\frac{10}{20}$

CALC OOZE/DETRITUS

(1)

lutite matrix

end of core

NoTE: Core represents subsample of box core very soft, unconsolidsted, fine sand and silt in

SHEAR SLIDE DESCRIPTIONS - W.H.O.I. SEDINENT CORES

\begin{tabular}{|c|c|}
\hline KMORR & Core No. $\quad 13-B C$ \\
\hline Expedition & Station No. \\
\hline
\end{tabular}

\begin{tabular}{|c|c|c|c|c|c|c|c|c|c|c|c|c|c|c|}
\hline \multirow[b]{4}{*}{ LEVEL } & \multirow[b]{4}{*}{$\begin{array}{c}\text { SEDIMENT } \\
\text { TYPE }\end{array}$} & \multicolumn{13}{|c|}{ ESTIMATED ABUNDANCES ( $(x)$} \\
\hline & & \multirow{2}{*}{\multicolumn{4}{|c|}{$\begin{array}{c}\text { Inorganic Material } \\
\text { Silt \& Sand }\end{array}$}} & & \multirow{2}{*}{\multicolumn{5}{|c|}{$\begin{array}{l}\text { Biogenous Mater } \\
\text { Calcareous }\end{array}$}} & \multirow{2}{*}{\multicolumn{3}{|c|}{$\begin{array}{l}\text { Ial } \\
\text { Silliceous }\end{array}$}} \\
\hline & & & & & & & & & & & & & & \\
\hline & & $\frac{5}{\frac{5}{5}}$ & $\begin{array}{l}\frac{\omega}{5} \\
\frac{3}{0} \\
\frac{0}{0} \\
\frac{0}{2}\end{array}$ & 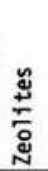 & 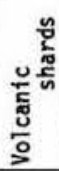 & $\frac{\pi}{6}$ & 崖 & 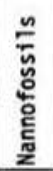 & 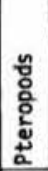 & 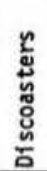 & 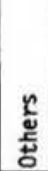 & 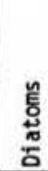 & $\frac{\frac{\pi}{L}}{\frac{\pi}{0}} \frac{0}{\frac{\pi}{\sigma}}$ & 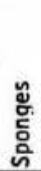 \\
\hline 1 & $\begin{array}{l}\text { calc ooze/ } \\
\text { detritus }\end{array}$ & 45 & & & & 14 & 5 & 15 & & & 20 & & & 1 \\
\hline \multirow[t]{2}{*}{25} & $\begin{array}{l}\text { calc ooze/ } \\
\text { detritus }\end{array}$ & 45 & & & & 13 & 15 & 7 & & & 20 & & & tr \\
\hline & ; & & & & & & & & & & & & & \\
\hline & & & & & & & & & & & & & & \\
\hline & & & & & & & & & & & & & & \\
\hline & & & & & & & & & & & & & & \\
\hline & & & & & & . & & & & & & & 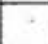 & \\
\hline & & & & & & & & & & & & & & \\
\hline & & & & & & & & & & & & & & \\
\hline & & & & & & & & & & & & & & \\
\hline & & & & & & & & & & & & & & \\
\hline & & & & & & & & & & & & & & \\
\hline & & & & & & & & & & & & & & \\
\hline & & & & & & & & & & & & & & \\
\hline & & & & & & & & & & & & & & \\
\hline & & & & & & & & & & & & & & \\
\hline & & & & & & & & & & & & & & \\
\hline & $\cdot$ & & & & & & & & & & & & & \\
\hline
\end{tabular}


777

VISUAL CORE DESCRIPTION

Page 1 of 1

Ship KNoRR Cruise 54 Leg 6 Sta. 70 Core No. $16-3 \mathrm{C}$

Total Length 41 cm. Lat. $63^{\circ} 49.5^{\prime} \mathrm{N}$ Long. $0^{\circ} 50.9^{\prime} \mathrm{E}$ Depth $2217 \mathrm{corr}$ meters Core condition EXCELLENT Date Described 2 DeC 76 by H. Fanemce Physiographic location SOuTHERN TIE VORING PLATEAK, NORMEGMN SEA

Lithologic Detailed Description

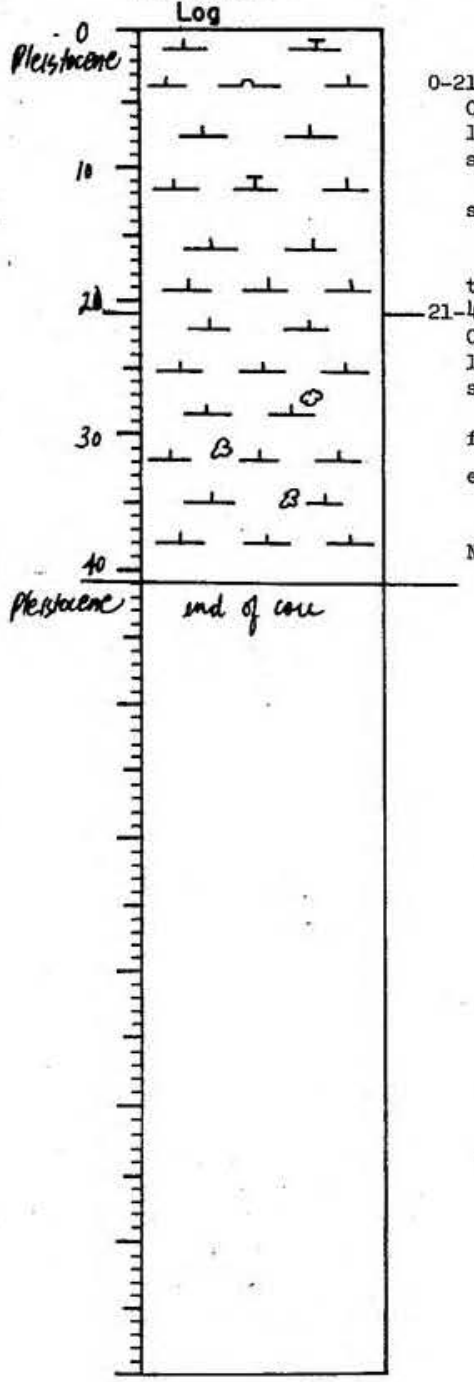

778

SMEAR SLIDE DESCRIPTIONS - W.H.O.I. SEDINENT CORES
Ship:

Expedition

Leg No.
KYORR

CALC-SILIC OOZE

10 YR $5 / 3$ brown

of slightly darker grayish

from $11-20 \mathrm{~cm}$ trix; numerous, coorse, sand-size, white shell throughout texturally Gradational

CALC $00 \mathrm{ZZ}$

20 TR $5 / 3$ brown

of dark grayish brown are

pirly silty lutite end of core

NOTE: Core represents subsample of box core

\begin{tabular}{|c|c|c|c|c|c|c|c|c|c|c|c|c|c|c|}
\hline \multirow[b]{3}{*}{ LEVEL. } & \multirow[b]{3}{*}{$\begin{array}{c}\text { SEDIMENT } \\
\text { TYPE }\end{array}$} & \multicolumn{13}{|c|}{ ESTIMATED ABUWDANCES ( $(x)$} \\
\hline & & \multicolumn{4}{|c|}{$\begin{array}{c}\text { Inorganic Material } \\
\text { Silt \& Sand }\end{array}$} & & \multicolumn{5}{|c|}{$\begin{array}{l}\text { Biogenous Mater } \\
\text { Calcareous }\end{array}$} & \multicolumn{3}{|c|}{$\frac{\text { ial }}{\text { Stliceous }}$} \\
\hline & & 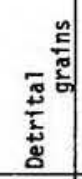 & 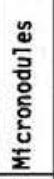 & 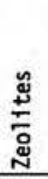 & 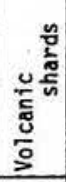 & $\frac{\pi}{0}$ & 鵕 & 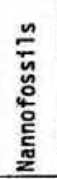 & 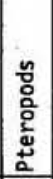 & 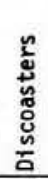 & 咅 & $\begin{array}{l}\frac{n}{5} \\
\frac{0}{0} \\
\frac{0}{6}\end{array}$ & $\frac{\frac{\pi}{2}}{\frac{\pi}{0}}$ & 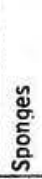 \\
\hline 1 & $\begin{array}{l}\text { colc-silic } \\
\text { ooze }\end{array}$ & 2 & & & & 25 & 25 & 38 & & & 2 & & 8 & tr \\
\hline 39 & calc ooze & 5 & $\operatorname{tr}$ & & & 60 & tr & 1 & & & 34 & & & \\
\hline & i & & & & & & & & & & & & & \\
\hline & & & & & & & & & & & & & & \\
\hline & $f$ & & & & & & & & & & & & & \\
\hline & & & & & & & & & & & & & & \\
\hline & & & & & & . & & & & & & . & & \\
\hline & & & & & & & & & & & & & & \\
\hline & & & & & & & & & & & & & & \\
\hline & & & & & & & & & & & & & & \\
\hline & & & & & & & & & & & & & & \\
\hline & & & & & & & & & & & & & & \\
\hline & & & & & & & & & & & & & & \\
\hline & & & & & & & & & & & & & & \\
\hline & . & & & & & & & & & & & & & \\
\hline & & & & & & & & & & & & & & \\
\hline & & & & & & & & & & & & & & \\
\hline & & & & & & & & & & & & & & \\
\hline
\end{tabular}

Station No. 70

Total Core Length $\quad 41$ 
779

VISUAL CORE DESCRIPTION

Page 1 of 1

Ship KNoRR Cruise 54 Leg 6 sto. 72 Core No. $17 \cdot B \mathrm{C}$ Total Length $39 \mathrm{~cm}$. Lat. $62^{\circ} 20.9^{\circ} \mathrm{N}$ Long. $e^{\circ} 53.7^{\prime} \mathrm{E}$ Depth $628 \mathrm{cam}$. meter Core condition EXCELENT Date Described 2 Dec 76 by H. Finemse

Physiographic location Sounteen To VQRING PLATEM, NORWESIAN SEA Lithologic

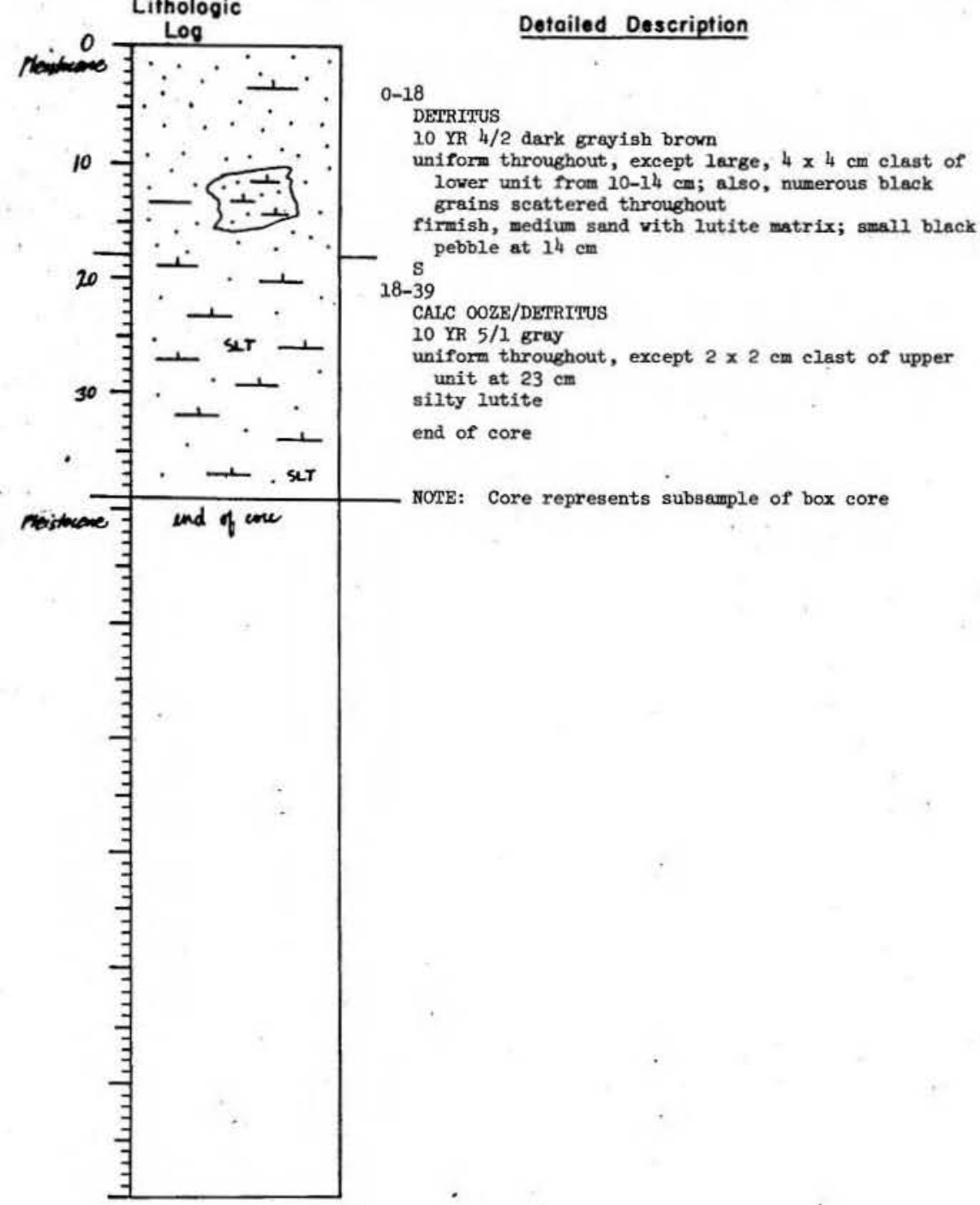

780

SMEAR SLIDE DESCRIPTIONS - W.H.O.I. SEDIIENT CORES

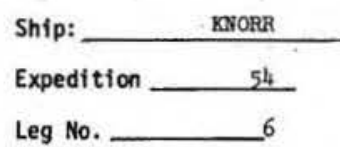

Core No. $17-B C$

Station No. 72

Total Core length $39 \quad \mathrm{~cm}$

\begin{tabular}{|c|c|c|c|c|c|c|c|c|c|c|c|c|c|c|c|}
\hline & & & & & & & & & & & & & & & \\
\hline \multirow[b]{3}{*}{ LEVEL. } & \multirow[b]{3}{*}{$\begin{array}{c}\text { SEDINENT } \\
\text { TYPE }\end{array}$} & \multicolumn{14}{|c|}{ ESTIMATED ABUNDANCES ( $($ ) } \\
\hline & & \multicolumn{4}{|c|}{$\begin{array}{l}\text { Inorganic Material } \\
\text { Silt \& Sand }\end{array}$} & \multirow[b]{2}{*}{$\frac{\pi}{\omega}$} & & \multicolumn{5}{|c|}{$\begin{array}{l}\text { Biogenous Mater } \\
\text { Calcareous }\end{array}$} & \multicolumn{3}{|c|}{$\frac{\text { al }}{\text { Silliceous }}$} \\
\hline & & & $\begin{array}{l}\frac{\omega}{3} \\
\frac{\vec{z}}{0} \\
\frac{0}{0} \\
\frac{0}{2} \\
\frac{1}{2}\end{array}$ & $\frac{\$}{\frac{\$}{2}}$ & 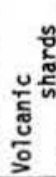 & & & & 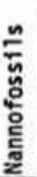 & 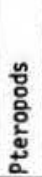 & 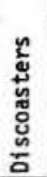 & 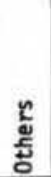 & $\begin{array}{l}\tilde{E}_{0} \\
\frac{\pi}{\sigma} \\
\end{array}$ & $\begin{array}{l}\frac{\pi}{\pi} \\
\frac{\pi}{0} \\
\frac{\pi}{\sigma} \\
\frac{\pi}{\alpha}\end{array}$ & 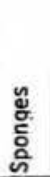 \\
\hline 1 & detritus. & 73 & & & & 8 & & 4 & 12 & & & 2 & 1 & & $\mathrm{tr}$ \\
\hline \multirow[t]{2}{*}{38} & $\begin{array}{l}\text { calc ooze/ } \\
\text { detritus }\end{array}$ & 35 & & & & 3 & & & $t x$ & & & 30 & & & tr \\
\hline & i & & & & & & & & & & & & & & \\
\hline & & & & & & & & & & & & & & & \\
\hline & & & & & & & & & & & & & & & \\
\hline & & & & & & & & & & & & & & & \\
\hline & & & & & & . & & & & & & & & & \\
\hline & & & & & & & & & & & & & & & \\
\hline & & & & & & & & & & & & & & & \\
\hline & & & & & & & & & & & & & & & \\
\hline & & & & & & & & & & & & & & & \\
\hline & & & & & & & & & & & & & & & \\
\hline & & & & & & & & & & & & & & & \\
\hline & & & & & & & & & & & & & & & \\
\hline & & & & & & & & & & & & & & & \\
\hline & & & & & & & & & & & & & & & \\
\hline & & & & & & & & & & & & & & & \\
\hline & & & & & & & & & & & & & & & \\
\hline
\end{tabular}


781

VISUAL CORE DESCRIPTION

Page 1 of 1

Ship KNosR Cruise 54 Leg 6 sto. 76 Core No. 18 - BC Total Length $13 \quad \mathrm{~cm}$. Lat. $59^{\circ} 30.6^{\prime} \mathrm{N}$ Long. $\frac{0^{\circ} 20.0^{\prime} \mathrm{F}}{\mathrm{c}}$ Depth 121 corr. meters Core condition EXceiLENT Date Described 2Dec 36 by H.FARmce Physiographic location Norent SEA

Lithologic

Detailed Description

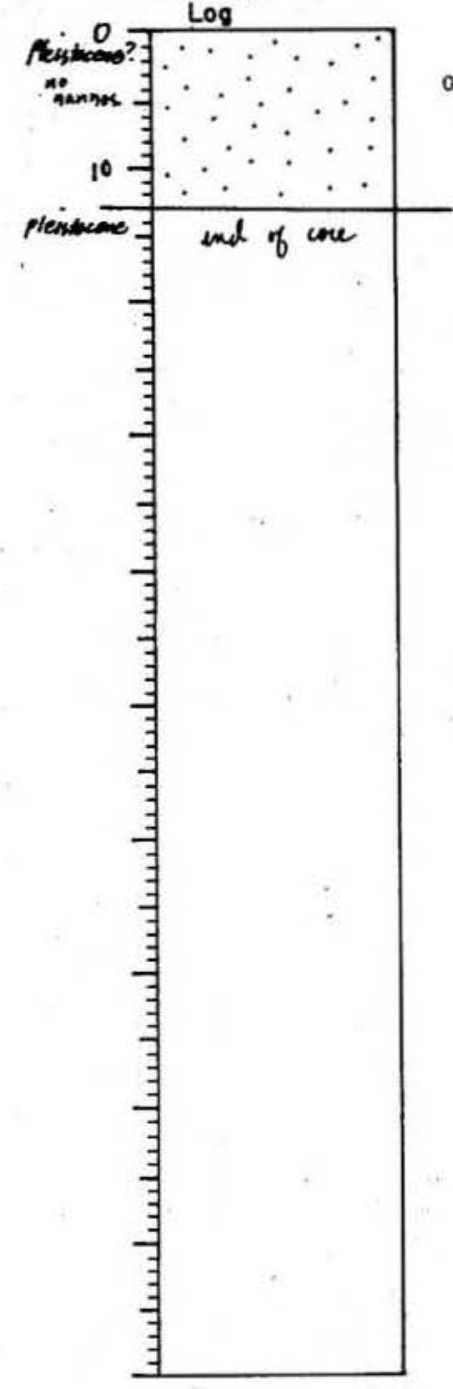

0-13

DEIRITUS

Y

medium sand vith scone lutite

end of core

NOTE: Core represents subsample of box core

.
SMEAR SLIDE DESCRIPTIONS - W.H.O.I. SEDIMENT CORES

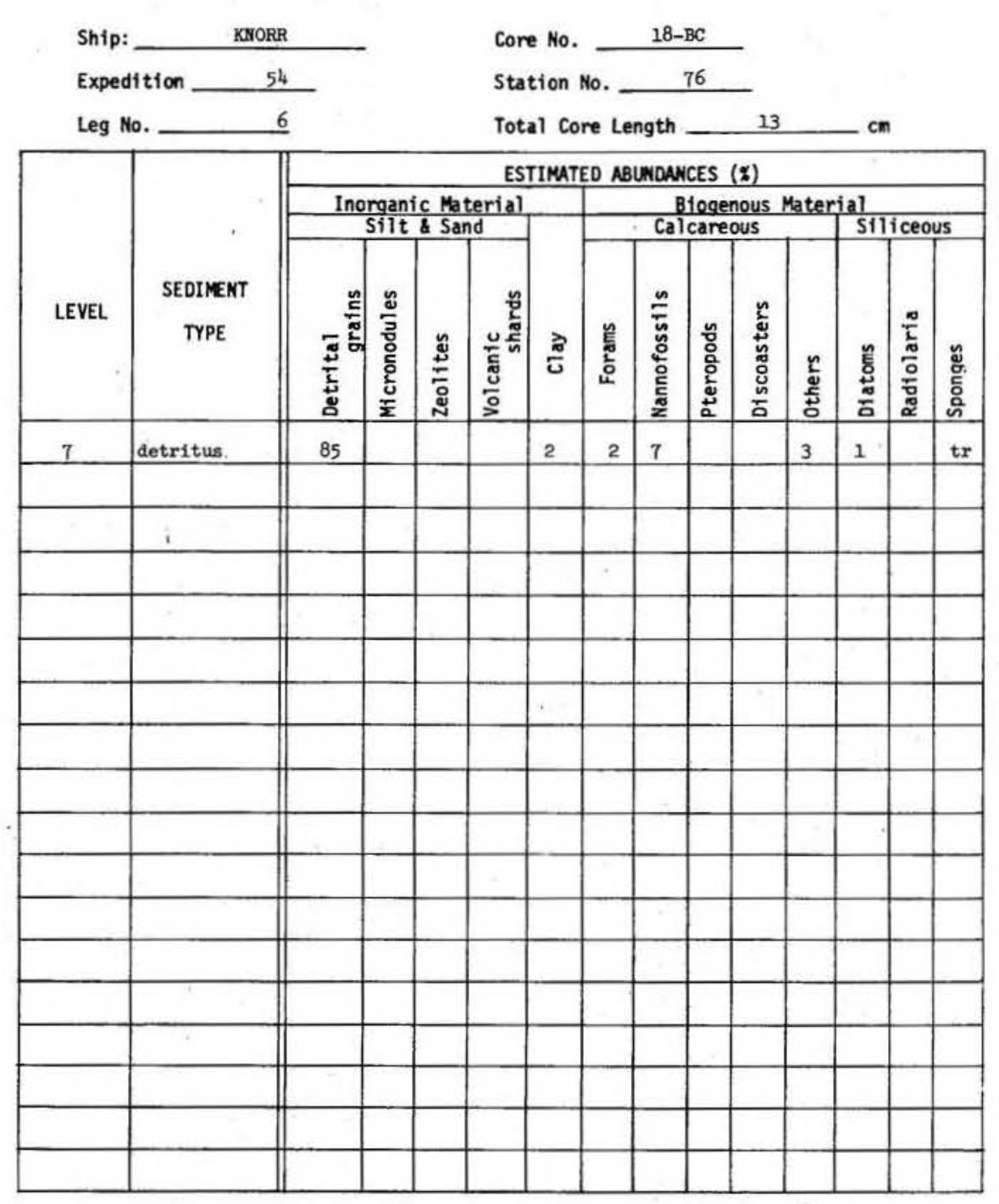


783

VISUAL CORE DESCRIPTION

Page_ 1 of 1

Ship KNORR Cruise 54 Leq 6 Sto. 91 Core No. $21-8 \mathrm{C}$

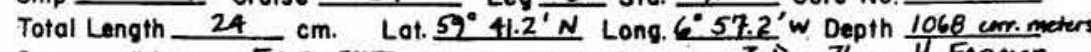

Core condition EXCELENT Date Described 3 Dee 76 by H. FARMER

Physiographic location Wrvilue THemeson Ribce, NoRtr ATLawnic

Lithologic

Detailed Description

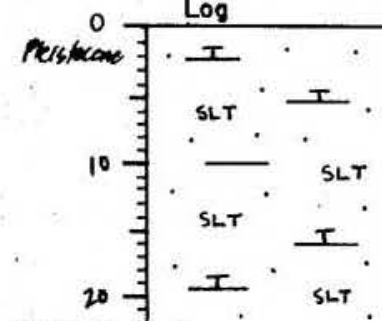

$0-24$

CALC OOZE/DETRITUS

5 Y $5 / 2$ olive gray

firm, fine-medium sand

end of core

Aessticare

\begin{tabular}{l|l|l}
\hline SLT & NOTE: Core represents subsample of box core \\
\hline if & end cone &
\end{tabular}
784

SMEAR SLIDE DESCRIPTIONS - K.H.O.I. SEDIMENT CORES

$\begin{array}{lll}\text { Ship: } & \text { KNORR } & \text { Core No. } \frac{21-B C}{54} \\ \text { Expedition } & \text { Station No. } \frac{21}{24} \\ \text { Leg No. } & 6 & \text { Total Core Length } \quad \text { cm }\end{array}$

\begin{tabular}{|c|c|c|c|c|c|c|c|c|c|c|c|c|c|c|}
\hline \multirow[b]{3}{*}{ LEVEL } & \multirow[b]{3}{*}{$\begin{array}{l}\text { SEDIMENT } \\
\text { TYPE }\end{array}$} & \multicolumn{13}{|c|}{ ESTIMATED ABUNDANCES ( $\boldsymbol{\xi}$ ) } \\
\hline & & \multicolumn{4}{|c|}{$\begin{array}{c}\text { Inorganic Material } \\
\text { Silt \& Sand }\end{array}$} & & \multicolumn{5}{|c|}{$\begin{array}{l}\text { Biogenous Mater } \\
\text { Calcareous }\end{array}$} & \multicolumn{3}{|c|}{$\frac{\text { aI }}{\text { Siliceous }}$} \\
\hline & & 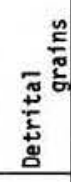 & 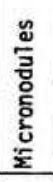 & 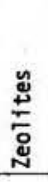 & 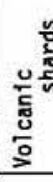 & ฮె & 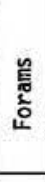 & 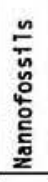 & 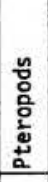 & 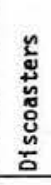 & 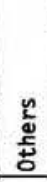 & 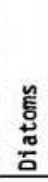 & 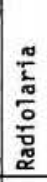 & 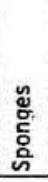 \\
\hline 1 & $\begin{array}{l}\text { calc ooze/ } \\
\text { detritus. }\end{array}$ & 44 & & & & 5 & 36 & 10 & & 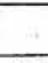 & 4 & $t x$ & & 1 \\
\hline 23 & $\begin{array}{l}\text { calc oozel } \\
\text { detritus }\end{array}$ & 30 & & & & 15 & 38 & 15 & & & 2 & & & tr \\
\hline & , & & & & & & & & & & & & & \\
\hline & & & & & & & & & & & & & & \\
\hline & & & & & & & & & & & & & & \\
\hline & & & & & & & & & & & & & & \\
\hline & & & & & & . & & & & & & & & \\
\hline & & & & & & & & & & & & & & \\
\hline & & & & & & & & & & & & & & \\
\hline & & & & & & & & & & & & & & \\
\hline & & & & & & & & & & & & & & \\
\hline & & & & & & & & & & & & & & \\
\hline & & & & & & & & & & & & & & \\
\hline & & & & & & & & & & & & & & \\
\hline & & & & & & & & & & & & & & \\
\hline & & & & & & & & & & & & & & \\
\hline & & & & & & & & & & & & & & \\
\hline & & & & & & & & & & & & & & \\
\hline
\end{tabular}


785

VISUAL CORE DESCRIPTION

Page 1 of 1

Ship KNORR Cruise 54 Leg 6 Sta. 92 Core No. $22-B C$

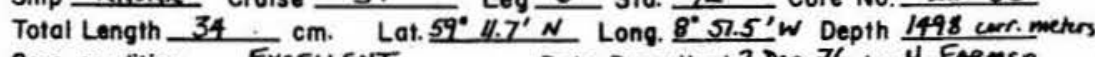

Core condition EXCELLENT Date Described 2Dee 76 by H. FAemse

Physiographic location NeRTHERN RocknLL PLATENL

Lithologic

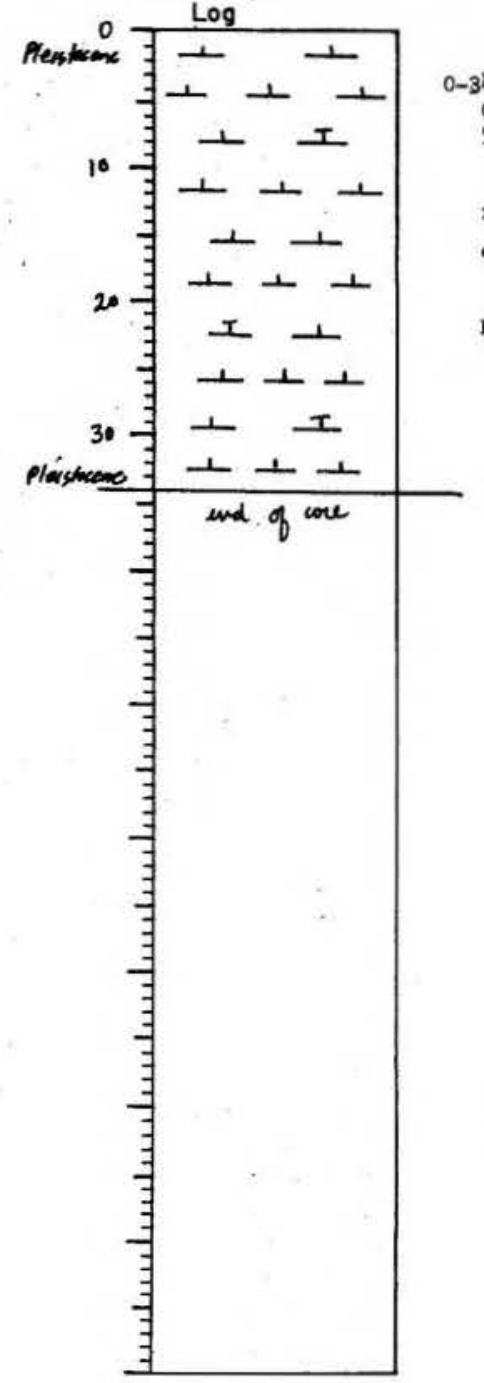

\section{Detailed Description}

CALC OOZE

5 Y $6 / 2$ light olive gray and 5/2 olive gray; inter-

marbing or the sbove colors throughout with

end of core

NOTE: Core represents subsample of box core
786

SIEAR SLIDE DESCRIPTIONS - W.H.O.I. SEDIMENT CORES

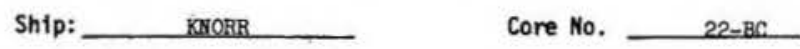

Expedition 54

Station No.

Leg No. 6

Total Core Length $34 \quad \mathrm{~cm}$

\begin{tabular}{|c|c|c|c|c|c|c|c|c|c|c|c|c|c|c|}
\hline \multirow[b]{3}{*}{ LEVEL. } & \multirow[b]{3}{*}{$\begin{array}{c}\text { SEDIMENT } \\
\text { TYPE }\end{array}$} & \multicolumn{13}{|c|}{ ESTIMATED ABUNDANCES ( $($ ) } \\
\hline & & \multicolumn{4}{|c|}{$\begin{array}{c}\text { Inorganic Material } \\
\text { Silt \& Sand }\end{array}$} & & \multicolumn{5}{|c|}{$\begin{array}{l}\text { Biogenous Mater } \\
\text { Calcareous }\end{array}$} & \multicolumn{3}{|c|}{$\frac{\text { al }}{\text { Stliceous }}$} \\
\hline & & 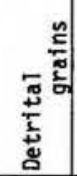 & 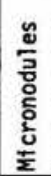 & 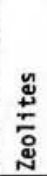 & $\frac{\mathrm{u}}{\mathrm{c}}$ & & 望 & 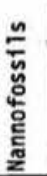 & 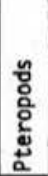 & 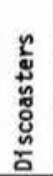 & 点 & $\begin{array}{l}\text { 产 } \\
\frac{0}{0} \\
\frac{0}{5}\end{array}$ & $\begin{array}{l}\frac{\pi}{L} \\
\frac{\pi}{0} \\
\frac{0}{0} \\
\frac{\pi}{0}\end{array}$ & 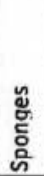 \\
\hline 1 & calc ooze & 3 & & & & 3 & 20 & 43 & & & 3 & & & 1 \\
\hline 33 & calc ooze & 5 & & & & 3 & 15 & 40 & & & 10 & & & tr \\
\hline & ; & & & & & & & & & & & & & \\
\hline & & & & & & & & & & & & & & \\
\hline & & & & & & & & & & & & & & \\
\hline & & & & & & & & & & & & & & \\
\hline & & & & & & . & & & & & & & & \\
\hline & & & & & & & & & & & & & & \\
\hline & & & & & & & & & & & & & & \\
\hline & & & & & & & & & & & . & & & \\
\hline & & & & & & & & & & & & & & \\
\hline & & & & & & & & & & & & & & \\
\hline & & & & & & & & & & & & & & \\
\hline & & & & & & & & & & & & & & \\
\hline & & & & & & & & & & & & & & \\
\hline & & & & & & & & & & & & & & \\
\hline - & & & & & & & & & & & & & & \\
\hline & & & . & & & & & & & & & & & \\
\hline
\end{tabular}




\section{7}

VISUAL CORE DESCRIPTION

Ship KNoRR Cruise 54 Leg 6 Sta. 94 Core No. $24-8 C$ Total Length $39^{\prime} \mathrm{cm}$. Lat. $59^{\circ} 26.0^{\prime} \mathrm{N}$ Long. $13^{\circ} 6.6^{\prime} \mathrm{W}$ Depth 1290 cerce meters EXeEENT Date Described 4 Dec 76 by H. FARme Physiographic location RockALL PLATEAL, N. ATLANTK

Lithologic

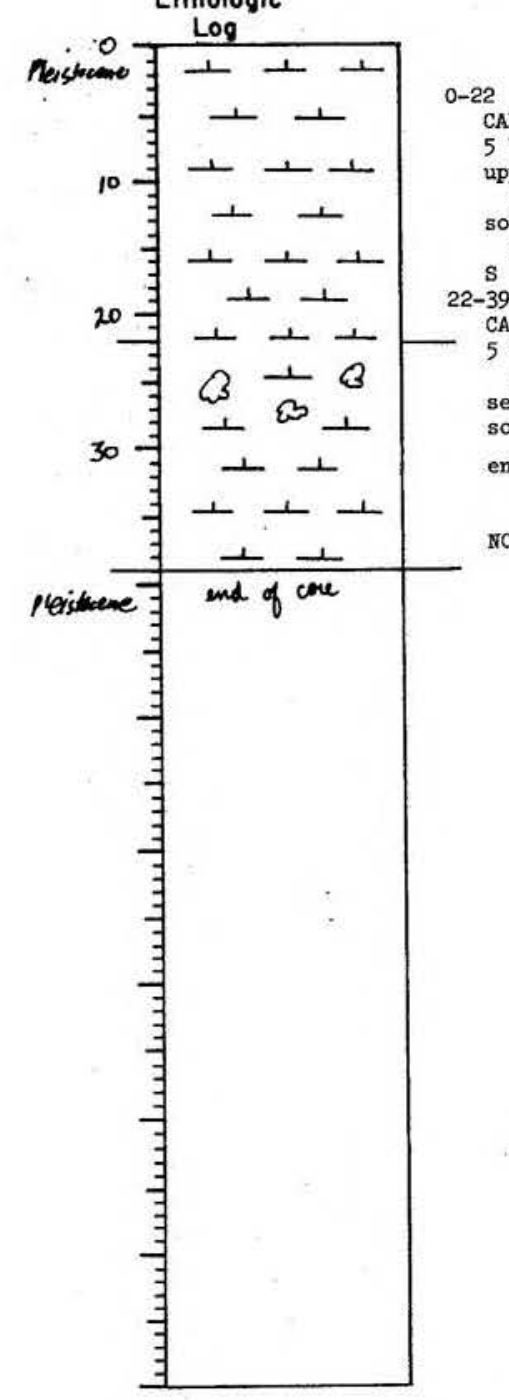

788

SMEAR SLIDE DESCRIPTIONS - W.H.O.I. SEDIMENT CORES

Detailed Description
Ship: $\quad$ KNORR

Expedition

Leg No. 6
Core No. 24-BC

Station No. 84

Total Core Length 39

ESTIMATED ABUNDANCES (\%)

5 Y $6 / 1$ gra

are slightly browner with a brown mottle

at $5 \mathrm{~cm}$

, silty lutite; small, black, angular pebble fragment at $16 \mathrm{~cm}$

CALC 0078

5 Y $5 / 2$ olive gray, grades slovly to 2.5 Y $5 / 2$

greyish brown

ereral down to $32 \mathrm{~cm}$ soft, silty lutite becomes less silty in basal $7 \mathrm{~cm}$ end of core

\$OTE: Core represents subsemple of box core

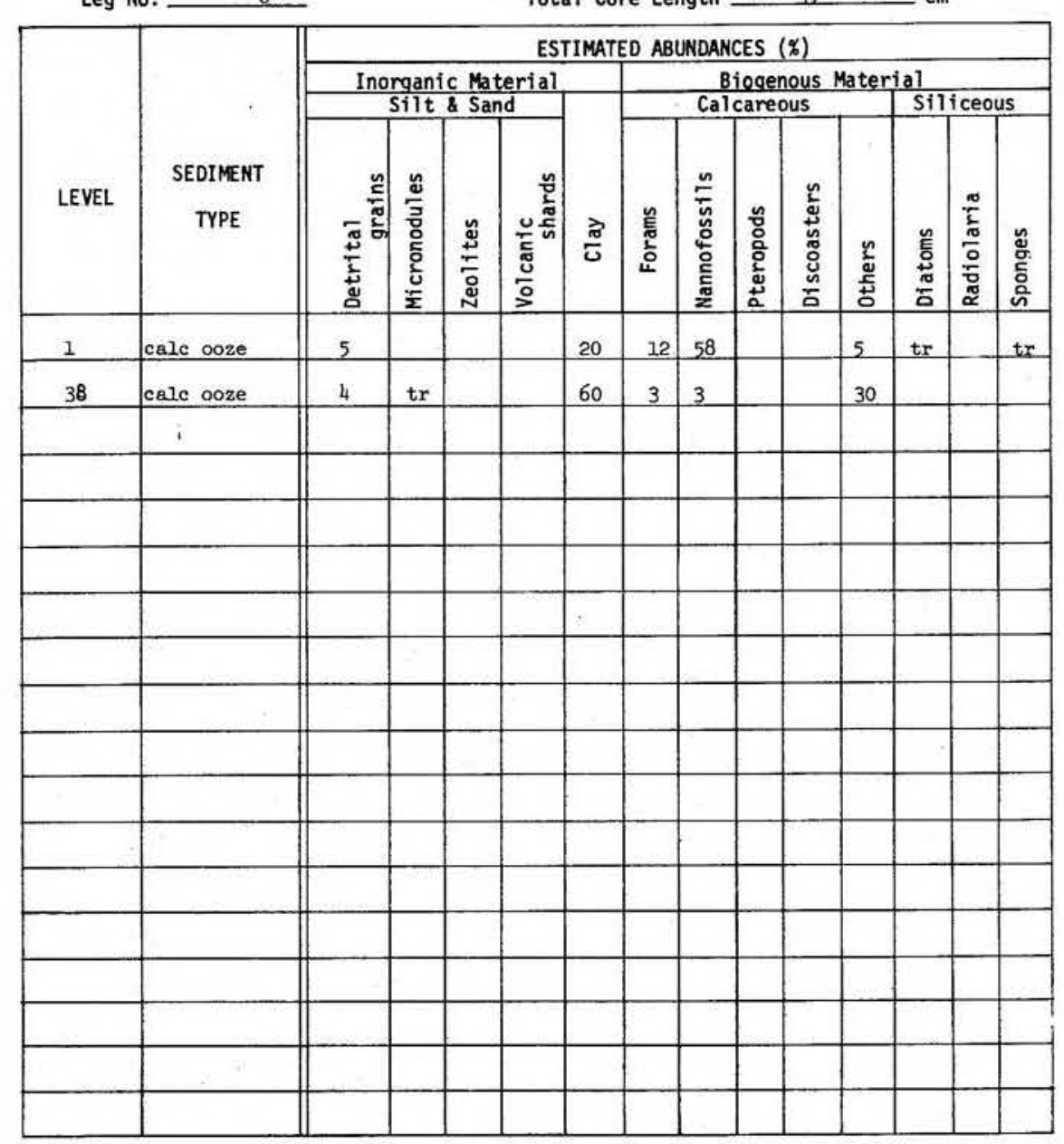




\section{VISUAL CORE DESCRIPTION}

Ship Kmoks Cruise 54 Leg 6 Sto. 96 Core No. $25-8 \mathrm{C}$ Total Length $22 \mathrm{~cm}$. Lat. $60^{\circ} 86^{\prime} \mathrm{N}$ Long. $15^{\circ} 1.4^{\prime} \mathrm{W}$ Depth 1295 carr. meters Core condition EXCELENT Date Described 4DeC 76 by H. FARERER Physiographic locotion ROCKALL PLATEAK, N. ATRANTRC Lithologic

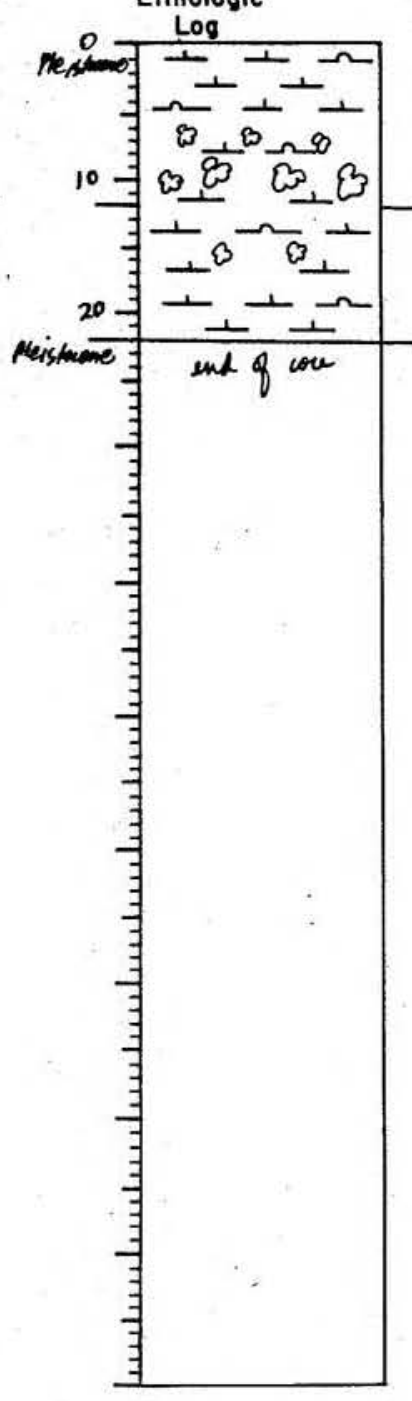

\section{Detailed Description}

0-12

CALC-SILIC OOZE

maing is present from $5-12 \mathrm{~cm}$ soft, unconsolidated, very silty lutite $2-22$

CAIC-SILIC $00 Z$ E

$10 \mathrm{YR} 7 / 1$ light gray

small, yellowish-brown mottles appear from 14-16 cm soft, very silty lutite

end of core

NOTE: Core represents subsample of box core
SUEAR SLIDE DESCRIPTIONS - W.H.O.I. SEDTMENT CORES

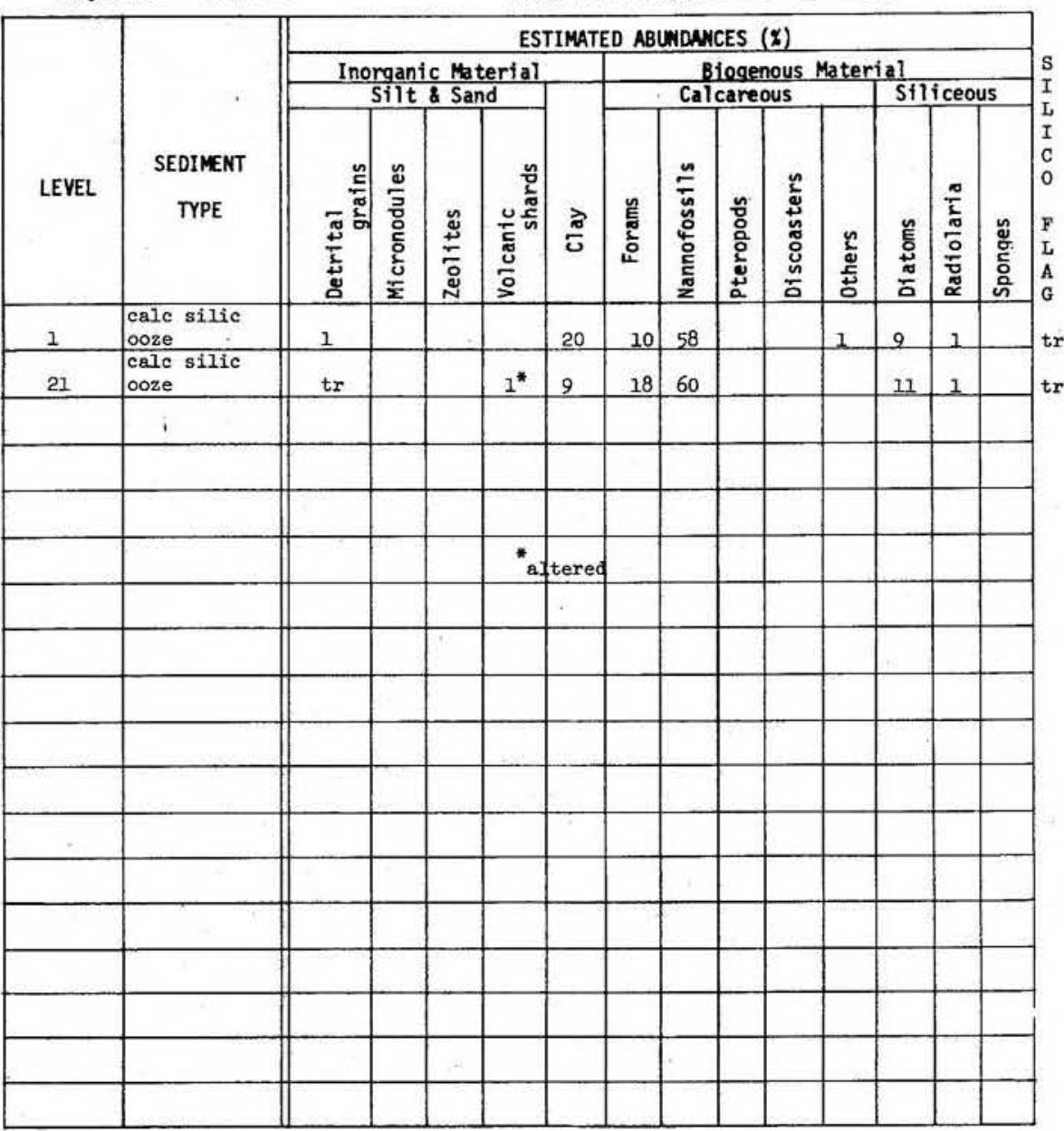

Ship: $\frac{\text { KNORR }}{\text { Expedition } \quad 54^{-}}$

Leg No. 6

Core No. $25-\mathrm{BC}$

Station No. 96

Total Core Length ___ 22

STIMATED ABUWDANCES $(\boldsymbol{x})$ 
791

VISUAL CORE DESCRIPTION

Page 1 of 1

Ship KMORR Cruise 54 Leg 6 Sto. 98 Core No. $26-8 \mathrm{C}$ Total Length $23 \mathrm{~cm}$. Lat. $60^{\circ} 59.7 \frac{\mathrm{N}}{2}$ Long. $16^{\circ} 5.5^{\prime} \mathrm{w}$ Depth 2435 comemeters Core condition EXCEUENT Date Described 5 Dec $\mathcal{H}$ by H. FarereR

Physiogrophic location ICELANDIC INSULAR RUSE, NORTH ATLANTK

Lithologic

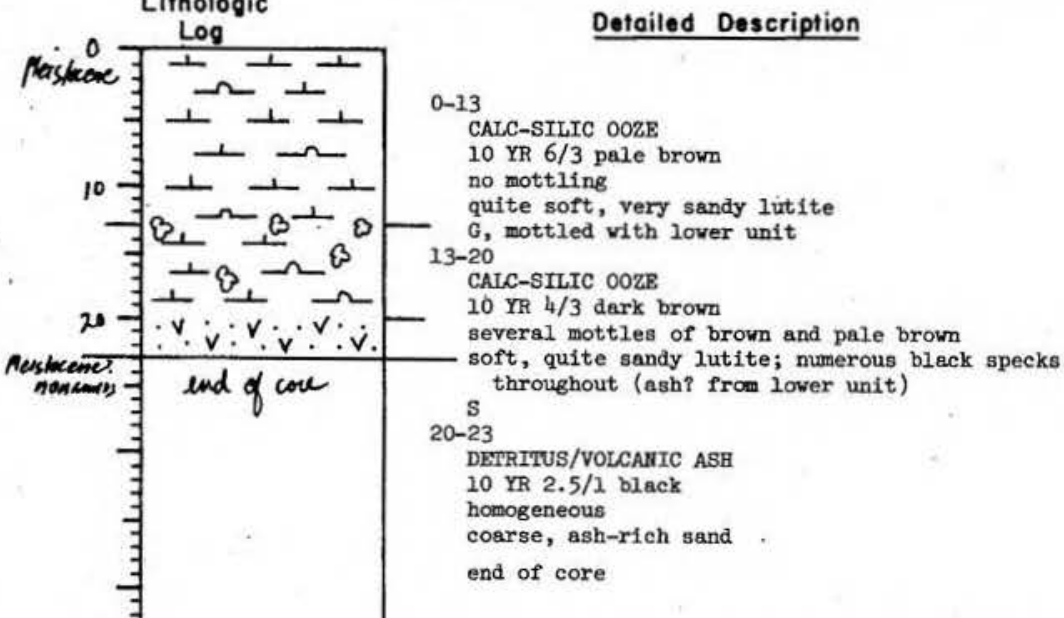

HOTE: Core represents subsample of box core
792

SMEAR SLIDE DESCRIPTIONS - W.H.O.I. SEDINENT CORES
Ship:

Expedition

Leg No.

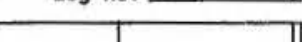

Core No. $26-\mathrm{BC}$

Station No. 98

Total Core Length 23

\begin{tabular}{|c|c|c|c|c|c|c|c|c|c|c|c|c|c|c|}
\hline \multirow[b]{3}{*}{ LEVEL } & \multirow[b]{3}{*}{$\begin{array}{c}\text { SEDIMENT } \\
\text { TYPE }\end{array}$} & \multicolumn{13}{|c|}{ ESTIMATED ABUNDANCES ( $(\boldsymbol{\alpha})$} \\
\hline & & \multicolumn{4}{|c|}{$\begin{array}{l}\text { Inorganic Material } \\
\text { Silt \& Sand }\end{array}$} & & \multicolumn{5}{|c|}{$\begin{array}{l}\text { Biogenous Mate } \\
\text { Calcarreous }\end{array}$} & \multicolumn{3}{|c|}{ Silliceous } \\
\hline & & 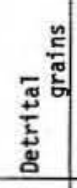 & 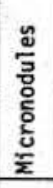 & 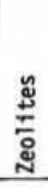 & 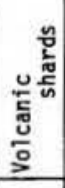 & $\frac{\pi}{6}$ & 产 & 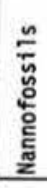 & 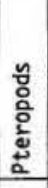 & 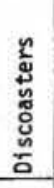 & 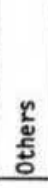 & 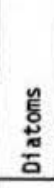 & $\begin{array}{l}\frac{\pi}{\pi} \\
\frac{\pi}{0} \\
0 \\
0 \\
0 \\
\pi \\
\end{array}$ & 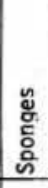 \\
\hline 1 & $\begin{array}{l}\text { calc-silic } \\
\text { ooze }\end{array}$ & 3 & & & $2^{*}$ & 14 & 10 & 60 & & & 2 & 10. & tx & tr \\
\hline 18 & $\begin{array}{l}\text { calc-silic } \\
\text { ooze }\end{array}$ & 5 & 2 & & $3^{*}$ & 13 & 15 & 50 & & & 2 & 10 & tr & tr \\
\hline 22 & $\begin{array}{l}\text { detritus/ } \\
\text { volcanic ash }\end{array}$ & 44 & & & $44^{*}$ & 1 & 10 & 1 & & & & 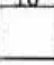 & I & tr \\
\hline & & & & & & & & & & & & & & \\
\hline & & & & & *alt & ered & & & & & & & & \\
\hline & & & & & & & & & & & & & & \\
\hline & & & & & & . & 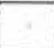 & & & & & & & \\
\hline & & & & & & & & & & & & & & \\
\hline & & & & & & & & & & & & & & \\
\hline & & & . & & & & & & & & & & & \\
\hline & & & & & & & & & & & & & & \\
\hline & & & & & & & & & & & & & & \\
\hline & & & & & & & & & & & & & & \\
\hline - & & & & & & & & & & & & & & \\
\hline & & & & & & & & & & & & & & \\
\hline & & & & & & & & & & & & & & \\
\hline & & & & & & & & & & & & & & \\
\hline & & & & & & & & & & & & & & \\
\hline
\end{tabular}


793

VISUAL CORE DESCRIPTION

Page 1 of 1

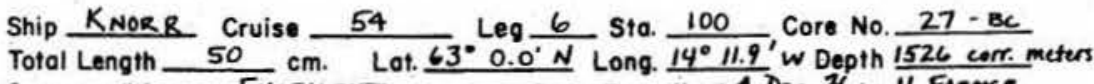
Core condition ExCELENT Date Described 4 Dec 76 by H. FARMES

Physiographic location ICELANDIC SLOPE

Lithologic

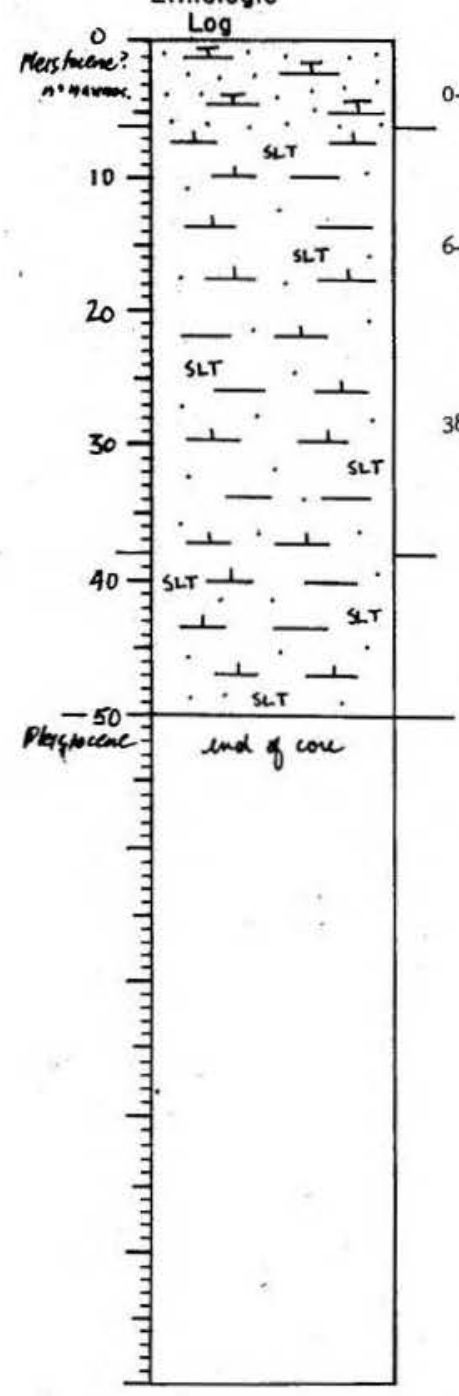

Detailed Description

DEIRITUS

10 YR $4 / 3$ brow

no mottling

$6-38$

HIGHLY CALC CLAY/DETRTTUS

$10 \mathrm{YR} 5 / 3$ brown

no mottling

somevhat compacted, silty lutite; several Iithified

lumps of lutite are found between 14-27 cm

38-50

HIGHLY CALC CLAY/DETRITUS

5 Y $4 / 2$ olive gray

ility lutite

end of core

WOTE: Core represents subsample of box core 
VISUAL CORE DESCRIPTION

Page 1 of 1

Ship KNORR Cruise 54 Leg 6 Sta. 101 Core No. $28-B C$ Total Length $36 \mathrm{~cm}$. Lat. $61^{\circ} 55.7^{\prime} \mathrm{N}$ Long. $17^{\circ} 13.2$ 'W Depth 2242 corr. meter Core condition EXCELLENT Date Described 4 DeC 76 by H. FARMER Physiographic location ICELANDIC INSULAR RISE, NORTH ARANTIC

Lithologic

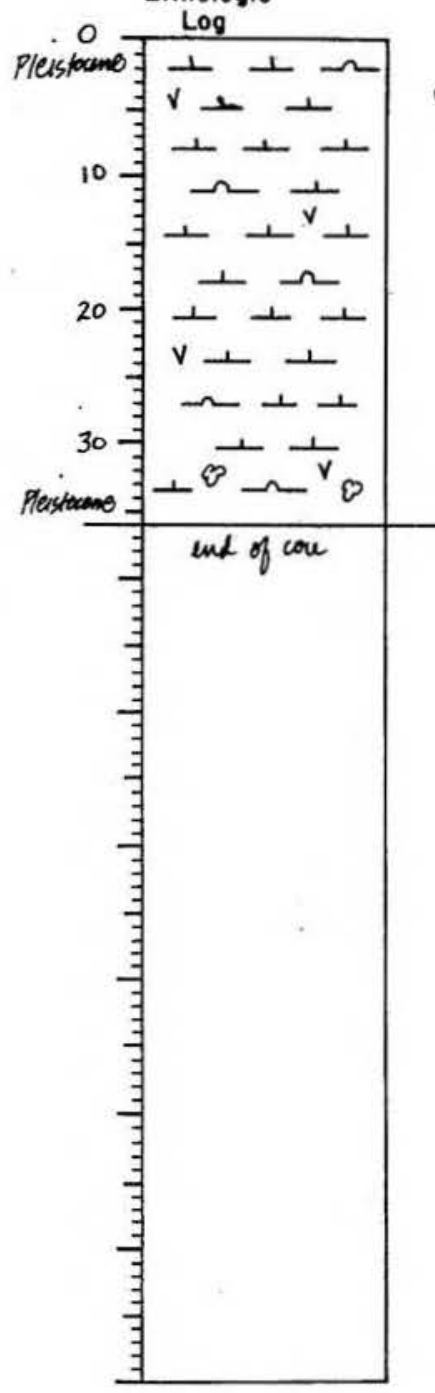

\section{Detailed Description}

CALC-SILIC OOZE

gray, grades to $4 / 1$ dark gray $(\sim 6 \mathrm{~cm})$

, very sandy lutite

end of core

NOTE: Core represents subsample of box core
SMEAR SLIDE DESCRIPTIONS - W.H.O.I. SEDIMENT CORES

\begin{tabular}{|c|c|}
\hline Ship: $\quad$ KNORR & Core No. $28-B C$ \\
\hline Expedition & Station No. 201 \\
\hline Leg No. & Total Core Length \\
\hline
\end{tabular}

\begin{tabular}{|c|c|c|c|c|c|c|c|c|c|c|c|c|c|c|}
\hline \multirow[b]{3}{*}{ LEVEL } & \multirow[b]{3}{*}{$\begin{array}{c}\text { SEDIMENT } \\
\text { TYPE }\end{array}$} & \multicolumn{13}{|c|}{ ESTIMATED ABUNDANCES ( $(x)$} \\
\hline & & \multicolumn{4}{|c|}{$\begin{array}{c}\text { Inorganic Material } \\
\text { Silt \& Sand }\end{array}$} & \multirow[b]{2}{*}{ 厄ٓ } & \multicolumn{5}{|c|}{$\begin{array}{l}\text { Biogenous Mater } \\
\text { Calcareous }\end{array}$} & \multicolumn{3}{|c|}{ Siliceous } \\
\hline & & 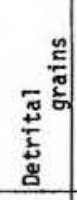 & 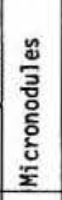 & 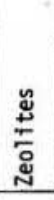 & 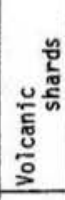 & & 高 & 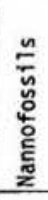 & 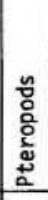 & 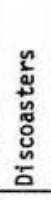 & 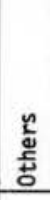 & 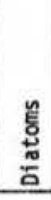 & 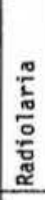 & 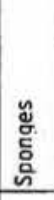 \\
\hline 1 & \begin{tabular}{|l|} 
calc- \\
ailic ooze
\end{tabular} & 5 & & & 7 & 25 & 20 & 28 & & & 5 & 9 & $t_{r}$ & 1 \\
\hline 35 & $\begin{array}{l}\text { calc- } \\
\text { silic ooze }\end{array}$ & 10 & & & 7 & 20 & 10 & 33 & & & 10 & 10 & $t x$ & tr \\
\hline & . & & & & & & & & & & & & & \\
\hline & & & & & & & & & & & & & & \\
\hline & & & & & & & & & & & & & & \\
\hline & & & & & & & & & & & & & & \\
\hline & & & & & & 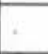 & & & & & & & & \\
\hline & & & & & & & & & & & & & & \\
\hline & & & & & & & & & & & & & & \\
\hline & & & & & & & & & & & & & & \\
\hline & & & & & & & & & & & & & & \\
\hline & & & & & & & & & & & & & & \\
\hline & & & & & & & & & & & & & & \\
\hline & & & & & & & & & & & & & & \\
\hline & & & & & & & & & & & & & & \\
\hline & & & & & & & & & & & & & & \\
\hline & & & & & & & & & & & & & & \\
\hline & & & & & & & & & & & & & & \\
\hline
\end{tabular}


SECURITY CLASSIFICATION OF THIS PAGE (When Date Entered)

\begin{tabular}{|c|c|}
\hline REPORT DOCUMENTATION PAGE & $\begin{array}{l}\text { READ INSTRUCTIONS } \\
\text { BEFORE COMPLETING FORM } \\
\end{array}$ \\
\hline \begin{tabular}{|l|l|} 
REPORT NUMAER & 2. GOVT ACCESSION NO. \\
WHOI $-77-26$ & \\
\end{tabular} & 3. MTCIPIENT'S CATALOG NUMEEA \\
\hline $\begin{array}{l}\text { 4. TITLE (and Subtitle) } \\
\text { DESCRIPTIONS OF WHOI SEDIMENT CORES, VOLUME } 5\end{array}$ & $\begin{array}{l}\text { 5. TYPE OF REPORT A PERIOD COVEREO } \\
\text { TEChnical }\end{array}$ \\
\hline . & 6. PERFORMING ORG. REPORT NUMBER \\
\hline $\begin{array}{l}\text { 7. AUTHOR(s) } \\
\text { Prepared by Staff of Sea Floor Samples Laboratory; } \\
\text { edited by D. A. Johnson and A. H. Driscoll }\end{array}$ & $\begin{array}{l}\text { D. CONTAACT OAGRANT NUMEER(D) } \\
\text { NOOO14-74-C-0262; } \\
\text { OCE 76-81488 }\end{array}$ \\
\hline $\begin{array}{l}\text { 9. PERFORMING ORGANIZATION NAME AND ADDRESS } \\
\text { WOOdS Hole OCEanOgraphic Institution } \\
\text { Woods Hole, MA } 02543\end{array}$ & $\begin{array}{l}\text { 10. PROGRAMELEMENT. PROJECT, TASK } \\
\text { AREA A WORK UNTT NUMEERS } \\
\text { NR } 083-004\end{array}$ \\
\hline $\begin{array}{l}\text { II CONTROLLING OFFICE NAME AND ADORESS } \\
\text { NORDA }\end{array}$ & $\begin{array}{l}\text { 12. REPORT OATE } \\
\text { April } 1977\end{array}$ \\
\hline $\begin{array}{l}\text { National Space Technology Laboratory } \\
\text { Bay St. Louis, MS } 39529\end{array}$ & $\begin{array}{l}\text { 13. NUMBER OF PAGES } \\
796 \text { pages } \\
\end{array}$ \\
\hline 14 MONITORING AGENCY NAME ADDRESS(It dilforant from Controlline Otlice) & $\begin{array}{l}\text { 18. SECURITY CLASS. (ol thio roport) } \\
\text { UnClaSSIEIEd } \\
\text { 15. DECLASSIFICATION/DOWNGAADING } \\
\text { SCHEDULE }\end{array}$ \\
\hline $\begin{array}{l}\text { 16. DISTRIBUTION STATEMENT (ol thlo Roport) } \\
\text { Prepared for public release; distribution unlim }\end{array}$ & ted. \\
\hline 17. DISTRIBUTION STATEMENT (of the abotrect entored In Block 20, II difforent fro & \\
\hline 18 SUPPLEMENTARY NOTES & - \\
\hline 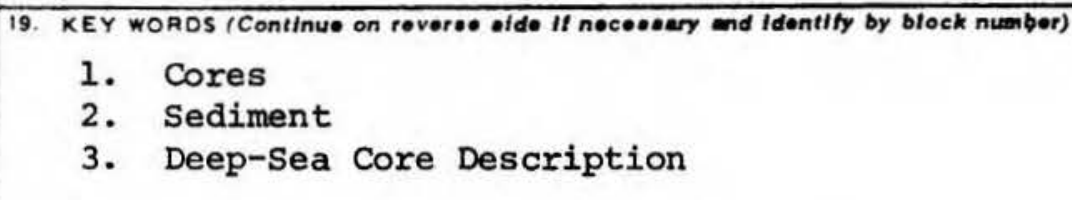 & . \\
\hline 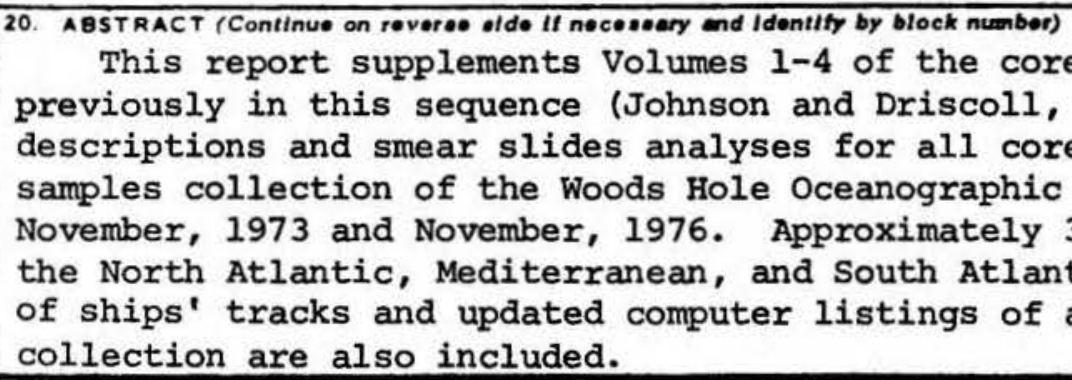 & $\begin{array}{l}\text { descriptions published } \\
1975) \text {. It contains visual } \\
\text { es received in the geological } \\
\text { Institution between } \\
368 \text { sample localities from } \\
\text { tic are represented. Charts } \\
\text { all cores in the W.H.O.I. }\end{array}$ \\
\hline
\end{tabular}




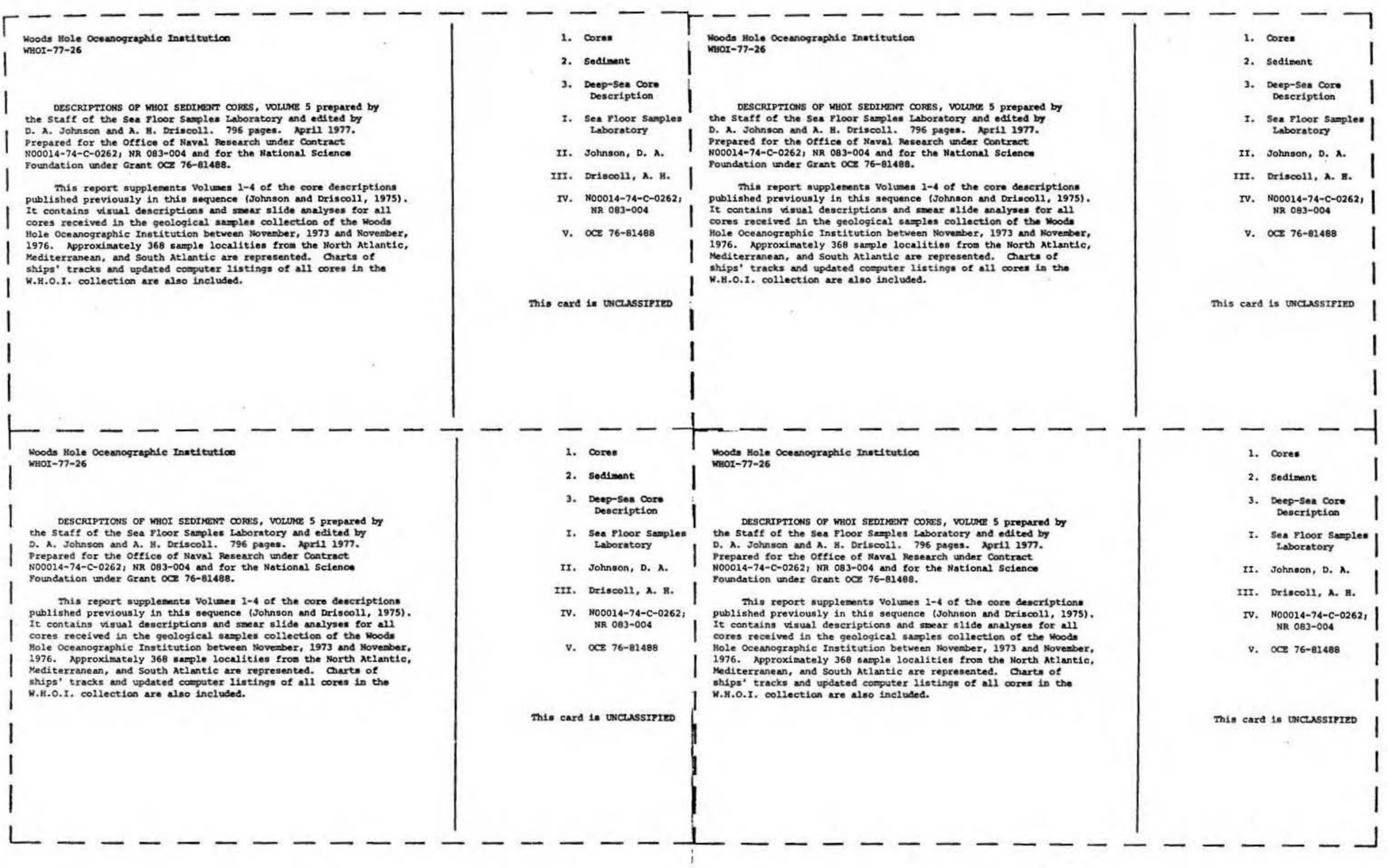


